\title{
EMERGING
}

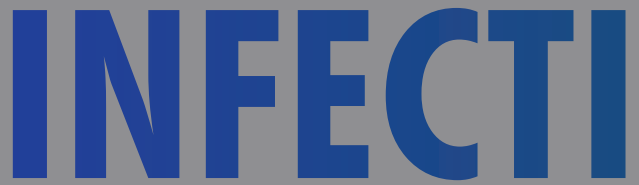

Zoonotic Infections

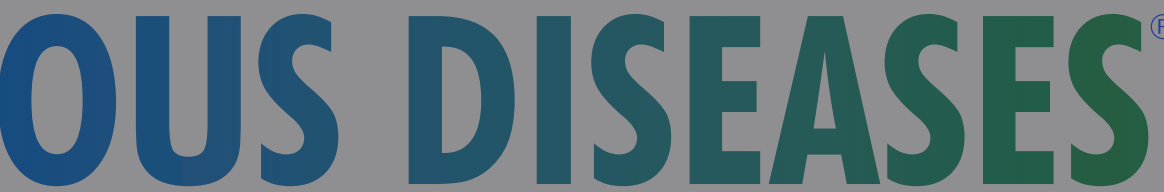

December 2018

2.

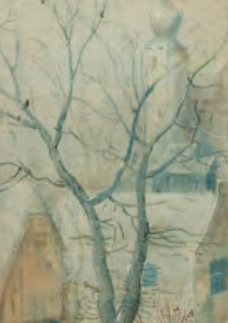

in of 5

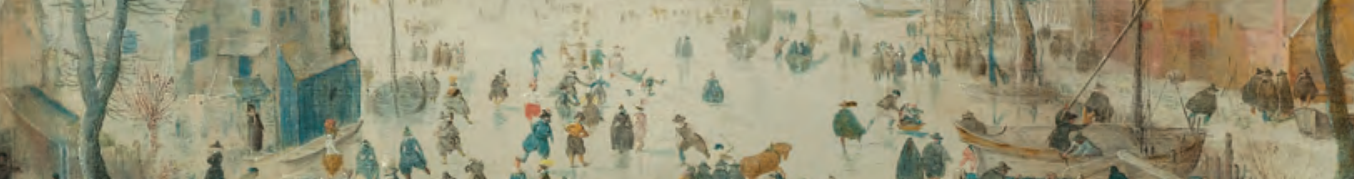

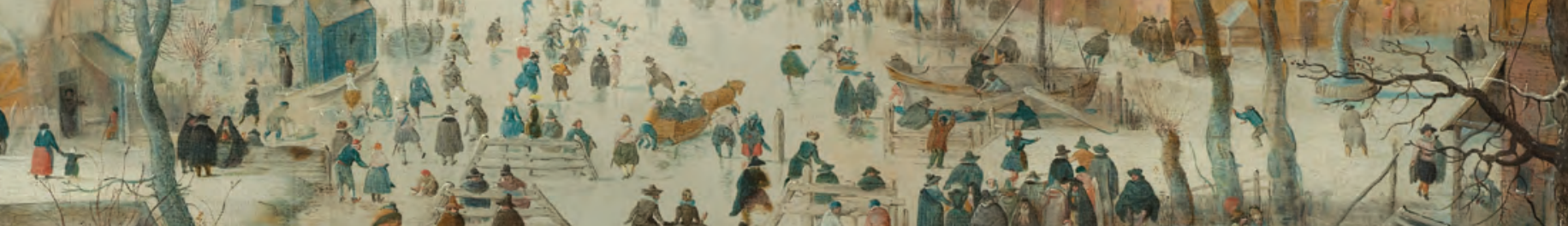

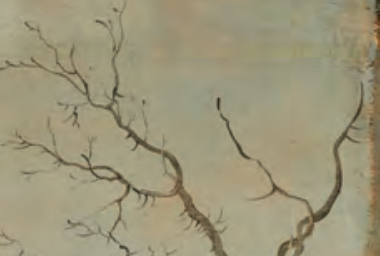

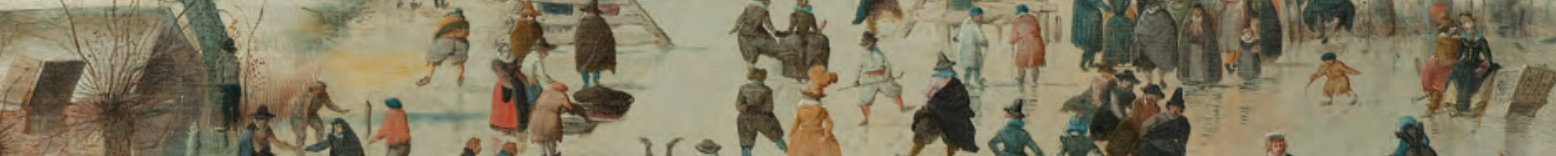

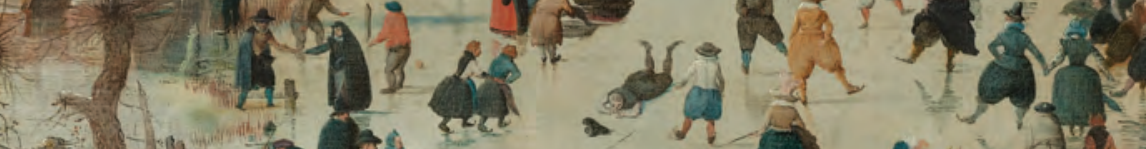

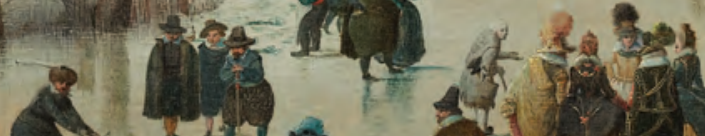

A buthen

$-x^{2} x^{2}$

किसे

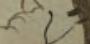

$1)$

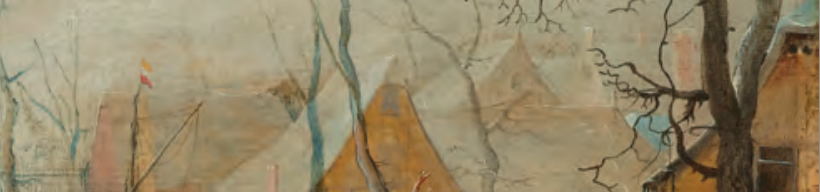

$\rightarrow 10+\frac{1}{2}+2$

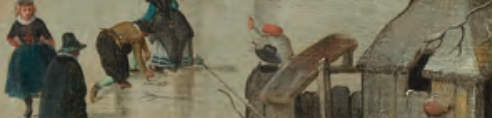




\section{EMERGING INFECTIOUS DISEASES}

\section{EDITOR-IN-CHIEF D. Peter Drotman}

\author{
Associate Editors \\ Paul Arguin, Atlanta, Georgia, USA \\ Charles Ben Beard, Fort Collins, Colorado, USA \\ Ermias Belay, Atlanta, Georgia, USA \\ David Bell, Atlanta, Georgia, USA \\ Sharon Bloom, Atlanta, Georgia, USA \\ Mary Brandt, Atlanta, Georgia, USA \\ Corrie Brown, Athens, Georgia, USA \\ Charles Calisher, Fort Collins, Colorado, USA \\ Michel Drancourt, Marseille, France \\ Paul V. Effler, Perth, Australia \\ Anthony Fiore, Atlanta, Georgia, USA \\ David Freedman, Birmingham, Alabama, USA \\ Peter Gerner-Smidt, Atlanta, Georgia, USA \\ Stephen Hadler, Atlanta, Georgia, USA \\ Matthew Kuehnert, Edison, New Jersey, USA \\ Nina Marano, Atlanta, Georgia, USA \\ Martin I. Meltzer, Atlanta, Georgia, USA \\ David Morens, Bethesda, Maryland, USA \\ J. Glenn Morris, Gainesville, Florida, USA \\ Patrice Nordmann, Fribourg, Switzerland \\ Ann Powers, Fort Collins, Colorado, USA \\ Didier Raoult, Marseille, France \\ Pierre Rollin, Atlanta, Georgia, USA \\ Frank Sorvillo, Los Angeles, California, USA \\ David Walker, Galveston, Texas, USA \\ J. Todd Weber, Atlanta, Georgia, USA

\section{Managing Editor} \\ Byron Breedlove, Atlanta, Georgia, USA
}

Copy Editors Kristina Clark, Dana Dolan, Karen Foster, Thomas Gryczan, Michelle Moran, Shannon O’Connor, Jude Rutledge, P. Lynne Stockton, Deborah Wenger

Production Thomas Eheman, William Hale, Barbara Segal, Reginald Tucker

Editorial Assistants Kristine Phillips, Susan Richardson

Communications/Social Media Sarah Logan Gregory,

Tony Pearson-Clarke, Deanna Altomara (intern)

Founding Editor

Joseph E. McDade, Rome, Georgia, USA

Emerging Infectious Diseases is published monthly by the Centers for Disease Control and Prevention, 1600 Clifton Rd NE, Mailstop H16-2, Atlanta, GA 30329-4027, USA. Telephone 404-639-1960, fax 404-639-1954, email eideditor@cdc.gov.

The conclusions, findings, and opinions expressed by authors contributing to this journal do not necessarily reflect the official position of the U.S. Department of Health and Human Services, the Public Health Service, the Centers for Disease Control and Prevention, or the authors' affiliated institutions. Use of trade names is for identification only and does not imply endorsement by any of the groups named above.

All material published in Emerging Infectious Diseases is in the public domain and may be used and reprinted without special permission; proper citation, however, is required.

\section{EDITORIAL BOARD}

Timothy Barrett, Atlanta, Georgia, USA

Barry J. Beaty, Fort Collins, Colorado, USA

Martin J. Blaser, New York, New York, USA

Richard Bradbury, Atlanta, Georgia, USA

Christopher Braden, Atlanta, Georgia, USA

Arturo Casadevall, New York, New York, USA

Kenneth C. Castro, Atlanta, Georgia, USA

Benjamin J. Cowling, Hong Kong, China

Vincent Deubel, Shanghai, China

Christian Drosten, Charité Berlin, Germany

Isaac Chun-Hai Fung, Statesboro, Georgia, USA

Kathleen Gensheimer, College Park, Maryland, USA

Rachel Gorwitz, Atlanta, Georgia, USA

Duane J. Gubler, Singapore

Richard L. Guerrant, Charlottesville, Virginia, USA

Scott Halstead, Arlington, Virginia, USA

Katrina Hedberg, Portland, Oregon, USA

David L. Heymann, London, UK

Keith Klugman, Seattle, Washington, USA

Takeshi Kurata, Tokyo, Japan

S.K. Lam, Kuala Lumpur, Malaysia

Stuart Levy, Boston, Massachusetts, USA

John S. MacKenzie, Perth, Australia

John E. McGowan, Jr., Atlanta, Georgia, USA

Jennifer H. McQuiston, Atlanta, Georgia, USA

Tom Marrie, Halifax, Nova Scotia, Canada

Nkuchia M. M'ikanatha, Harrisburg, Pennsylvania, USA

Frederick A. Murphy, Bethesda, Maryland, USA

Barbara E. Murray, Houston, Texas, USA

Stephen M. Ostroff, Silver Spring, Maryland, USA

Marguerite Pappaioanou, Seattle, Washington, USA

Johann D. Pitout, Calgary, Alberta, Canada

Mario Raviglione, Geneva, Switzerland

David Relman, Palo Alto, California, USA

Guénaël Rodier, Saône-et-Loire, France

Connie Schmaljohn, Frederick, Maryland, USA

Tom Schwan, Hamilton, Montana, USA

Rosemary Soave, New York, New York, USA

P. Frederick Sparling, Chapel Hill, North Carolina, USA

Robert Swanepoel, Pretoria, South Africa

Phillip Tarr, St. Louis, Missouri, USA

Duc Vugia, Richmond, California

John Ward, Atlanta, Georgia, USA

Jeffrey Scott Weese, Guelph, Ontario, Canada

Mary E. Wilson, Cambridge, Massachusetts, USA

Use of trade names is for identification only and does not imply endorsement by the Public Health Service or by the U.S. Department of Health and Human Services.

EMERGING INFECTIOUS DISEASES is a registered service mark of the U.S. Department of Health \& Human Services (HHS).

$\infty$ Emerging Infectious Diseases is printed on acid-free paper that meets the requirements of ANSI/NISO 239.48-1992 (Permanence of Paper) 


\section{EMERGING}

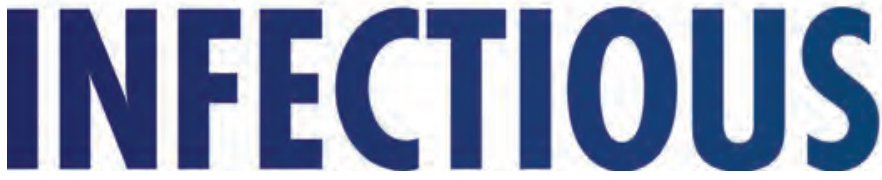

Zoonotic I nfections

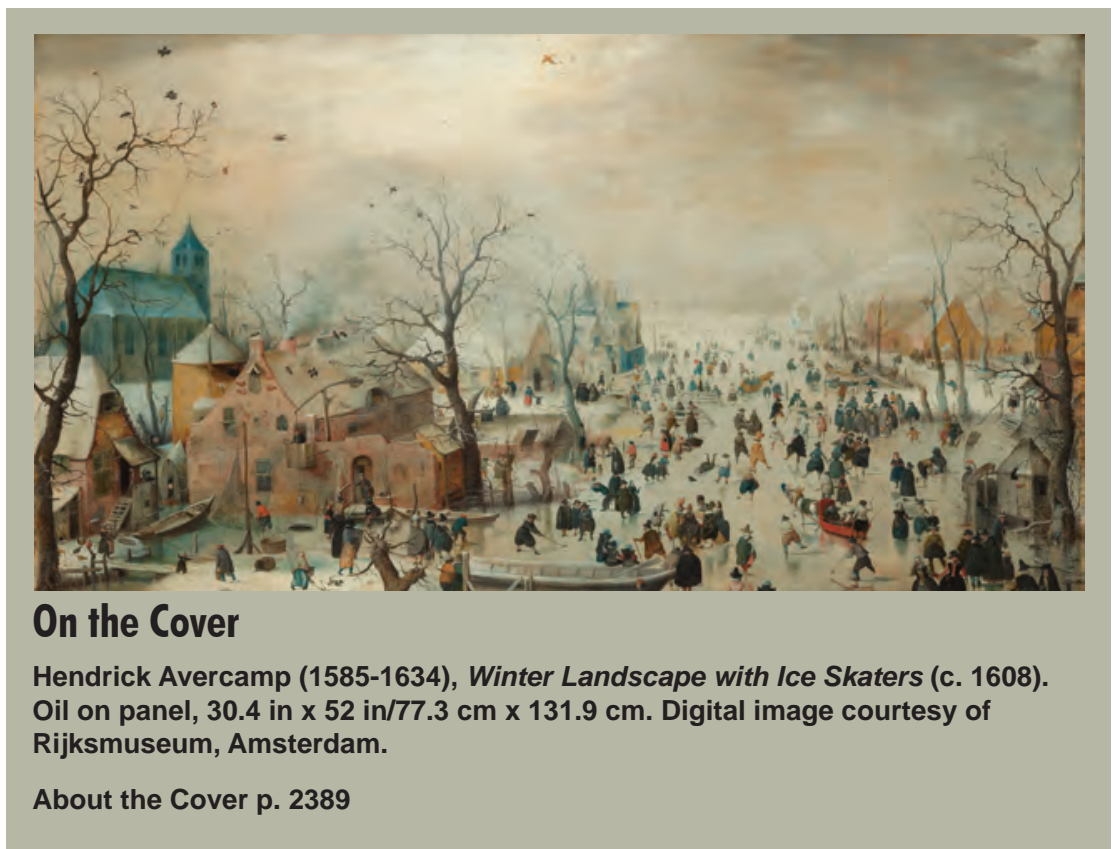

\section{Synopses}

Outbreak of HIV I nfection Linked to Nosocomial Transmission, China, 2016- 2017

$X$. Pan et al.

\section{Medscape}

ADUCATION

Reemergence of St. Louis Encephalitis Virus in the Americas

Reemerging virus in the western United States may have been mediated via migrating infected birds from southern South America, where it reemerged most recently in 2002

$$
\text { A. Diaz et al. }
$$

Autochthonous Human Case of Seoul Virus I nfection, the Netherlands

C. Swanink et al.

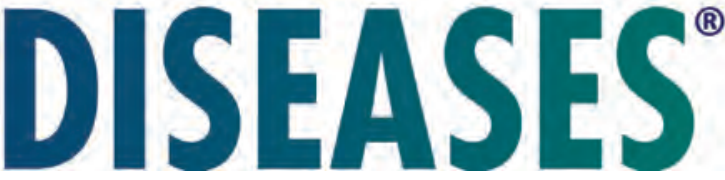

December 2018
Terrestrial Bird Migration and West Nile Virus Circulation, United States

D. Swetnam et al.

Capnocytophaga canimorsus Capsular Serovar and Disease Severity, Helsinki Hospital District, Finland, 2000-2017

E. Hess et al.

Related material available online: http://wwwnc.cdc.gov/eid/ article/24/12/17-2060_article

Crimean-Congo Hemorrhagic Fever Virus, Mongolia, 2013- 2014

M.A. Voorhees et al. 2202

Related material available online: http://wwwnc.cdc.gov/eid/ article/24/12/18-0175_article

Novel Type of Chronic Wasting Disease Detected in Moose (Alces alces), Norway L. Pirisinu et al. Grades and Salmonella I nfections, New York, New York, USA

M.J. Firestone, C.W. Hedberg

2164

\section{Research}

Spatial Analysis of Wildlife Tuberculosis Based on a Serologic Survey Using Dried Blood Spots, Portugal

N. Santos et al.

Rat Lungworm I nfection in Rodents across Post-Katrina New Orleans, Louisiana, USA

R.C. Rael et al.

2176

Related material available online: http://wwwnc.cdc.gov/eid/ article/24/12/18-0056 article
Related material available online: http://wwwnc.cdc.gov/eid/ article/24/12/18-0702 article

\section{Genomic Characterization} of $\beta$-Glucuronidase-Positive Escherichia coli 0157:H7 Strain Producing Stx $2 a$

Y. Ogura et al.

Related material available online: http://wwwnc.cdc.gov/eid/ article/24/12/18-0404 article

Survey of Ebola Viruses in Frugivorous and I nsectivorous Bats in Guinea, Cameroon, and the Democratic Republic of the Congo, 2015-2017

H.M. De Nys et al.

Related material available online:

http://wwwnc.cdc.gov/eid/ article/24/12/18-0740 article 
Rat Hepatitis E Virus as Cause of Persistent Hepatitis after Liver Transplant

S. Sridhar et al.

Related material available online: http://wwwnc.cdc.gov/eid/ article/24/12/18-0937_article

Influences of Community I nterventions on Zika Prevention Behaviors of Pregnant Women, Puerto Rico, J uly 2016-J une 2017 G. Earle-Richardson et al.

Related material available online: http://wwwnc.cdc.gov/eid/ article/24/12/18-1056_article

Emerging Multidrug-Resistant Hybrid Pathotype Shiga ToxinProducing Escherichia coli 080 and Related Strains of Clonal Complex 165, Europe

A. Cointe et al.

Related material available online: http://wwwnc.cdc.gov/eid/ article/24/12/18-0272 article

Comparison of 2016- 17 and Previous Epizootics of Highly Pathogenic Avian Influenza H5 Guangdong Lineage in Europe

P. Alarcon et al.

Related material available online: http://wwwnc.cdc.gov/eid/ article/24/12/17-1860_article

CTX-M-65 Extended-Spectrum $\beta$-Lactamase-Producing Salmonella enterica Serotype I nfantis, United States

A.C. Brown et al.

Related material available online: http://wwwnc.cdc.gov/eid/ article/24/12/18-0500_article

\section{Medscape ACTIVITY}

Substance Use and Adherence to HIV Preexposure Prophylaxis for Men Who Have Sex with Men

Substance and alcohol use were not associated with decreased adherence.

M. Hoenigl et al.

Related material available online: http://wwwnc.cdc.gov/eid/ article/24/12/18-0400_article

Highly Pathogenic Clone of Shiga Toxin-Producing Escherichia coli 0157:H7, England and Wales

L. Byrne et al.

Related material available online:

http://wwwnc.cdc.gov/eid/ article/24/12/18-0409 article

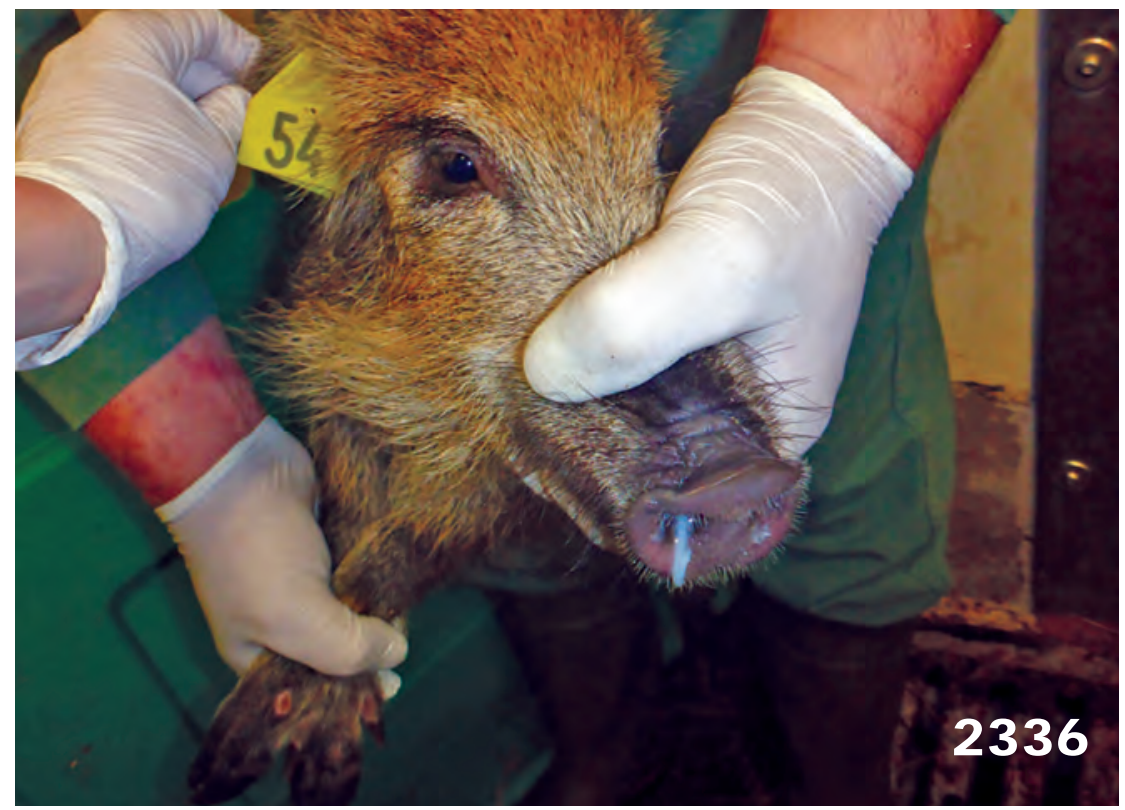

Prevalence of Avian Influenza

$A(H 5)$ and $A(H 9)$ Viruses in Live Bird Markets, Bangladesh

Y. Kim et al.

2309

Related material available online:

http://wwwnc.cdc.gov/eid/ article/24/12/18-0879_article

Human Exposure to Novel Bartonella Species from Contact with Fruit Bats

Y. Bai et al.

\section{Historical Review}

Emergent Sand Fly- Borne Phleboviruses in the Balkan Region

N. Ayhan, R.N. Charrel

2324

\section{Dispatches}

\section{I solation of Burkholderia pseudomallei from a Pet Green I guana, Belgium \\ T. Hellebuyck et al.}

2331

Neglected Hosts of Small Ruminant Morbillivirus

C. Schulz et al.

2334 Related material available online: http://wwwnc.cdc.gov/eid/ article/24/12/18-0507_article
Vaccinia Virus among

Domestic Dogs and Wild Coatis, Brazil, 2013- 2015

G. Barbosa Costa et al.

2338

Related material available online: http://wwwnc.cdc.gov/eid/ article/24/12/17-1584_article

Highly Pathogenic Avian Influenza Virus A(H5N6) in Domestic Cats, South Korea K. Lee et al.

Related material available online: http://wwwnc.cdc.gov/eid/ article/24/12/18-0290_article

Candidatus Cryptoplasma Associated with Green Lizards and I xodes ricinus Ticks, Slovakia, 2004- 2011

B. Kočíková et al.

Excess Mortality and Causes Associated with Chikungunya, Puerto Rico, 2014- 2015

A.R.R. Freitas et al.

Borrelia miyamotoi I nfections in Small Mammals, California, USA

D.J. Salkeld et al. 2356 
I n-Host Adaptation of Salmonella enterica Serotype Dublin during Prosthetic Hip J oint I nfection

F. El Sayed et al. 2360 Related material available online: http://wwwnc.cdc.gov/eid/ article/24/12/18-0214_article

Risk Factors for Acquiring Scrub Typhus among Children in Deoria and Gorakhpur Districts, Uttar Pradesh, I ndia, 2017

J.W.V. Thangaraj et al.

Related material available online:

http://wwwnc.cdc.gov/eid/

article/24/12/18-0695_article

Using PCR-Based Sequencing to Diagnose Haycocknema perplexum I nfection in Human Myositis Case, Australia

A.V. Koehler et al.

\section{Research Letters}

Chuzan Virus in Yaks, Qinghai-Tibetan Plateau, China

M. Wang et al.

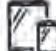

Related material available online: http://wwwnc.cdc.gov/eid/ article/24/12/17-1414_article

\section{Conservation of White}

Rhinoceroses Threatened by

Bovine Tuberculosis,

South Africa, 2016- 2017

M.A. Miller et al.

2373

Related material available online:

http://wwwnc.cdc.gov/eid/

article/24/12/18-0293 article

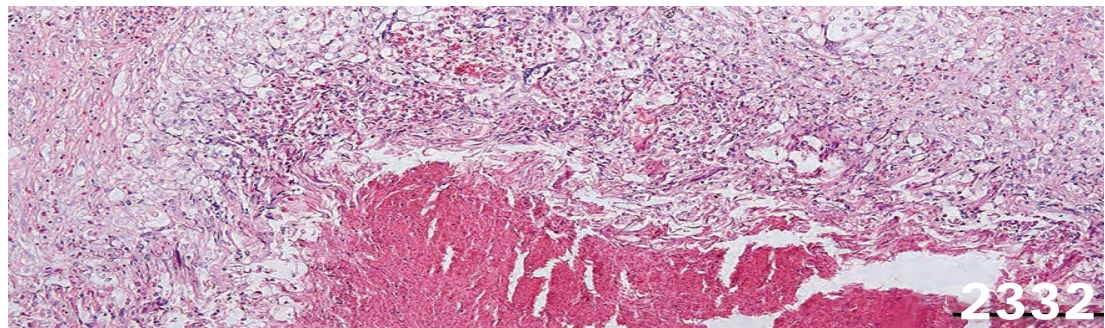

Lung I nvolvement in Chronic Schistosomiasis with Bladder Squamous Cell Carcinoma

A. Saade et al.

2375

Strongyloidiasis and Culture-

Negative Suppurative Meningitis, Japan, 1993- 2015

M. Mukaigawara et al.

2378

Related material available online:

http://wwwnc.cdc.gov/eid/ article/24/12/18-0375 article

Use of Next-Generation Sequencing for Diagnosis of West Nile Virus I nfection in Patient Returning to Belgium from Hungary by

E. Wollants et al.

Disseminated Spiroplasma apis I nfection in Patient with Agammaglobulinemia, France N. Etienne et al. article/24/12/18-0567 article
Mycoplasma ovipneumoniae in Wildlife Species beyond Subfamily Caprinae

M.A. Highland et al. 2384 Related material available online: http://wwwnc.cdc.gov/eid/ article/24/12/18-0632 article

Locally Acquired Leptospirosis in Expedition Racer, Manitoba, Canada

S.S. Kassim et al. 2386

\section{About the Cover}

An I cy Vista from a Golden Age

B. Breedlove

2389

\section{Etymologia}

\section{Capnocytophaga canimorsus}

R. Henry

2201

Reviewer Appreciation

2391

\section{Thank You EI D Reviewers \\ We couldn't do it without you.}

We only maintain high standards because of your support.

EID's 2018 Impact Factor of 7.42 ranked it 1st among open-access infectious disease journals and 4 th out of 88 infectious disease journals.

The Google Scholar h-Index is 86 ; 2 nd of top 20 publications in Epidemiology and

2nd among open-access journals; ranked 3rd among top 20 publications in

Communicable Diseases and 1st among open-access journals.

The electronic table of contents goes to 1,262,951 subscribers each month.

All articles published in the Emerging Infectious Diseases journal are peer-reviewed by volunteers from around the globe, enabling us to bring you high-quality content

about new and emerging infectious diseases and trends world-wide.

A list of reviewers is posted at http: //wwwnc.cdc.gov/eid/page/reviewers 


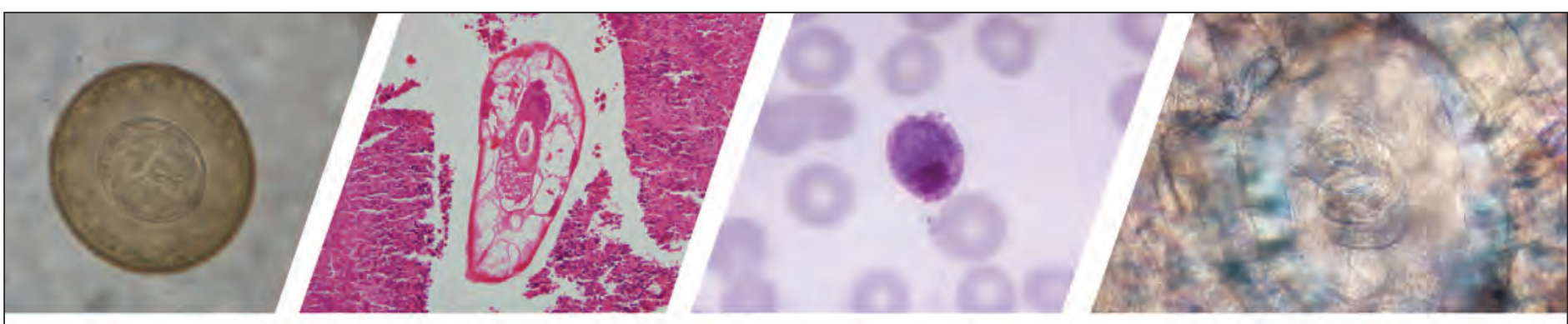

\section{(3DPDx}

\section{Diagnostic Assistance and Training in Laboratory Identification of Parasites}

A free service of CDC available to laboratorians, pathologists, and other health professionals in the United States and abroad

(2) Diagnosis from photographs of worms, histological sections, fecal, blood, and other specimen types

(10.) Expert diagnostic review 量旸 Formal diagnostic laboratory report

n Submission of samples via secure file share

Visit the DPDx website for information on laboratory diagnosis, geographic distribution, clinical features, parasite life cycles, and training via Monthly Case Studies of parasitic diseases.

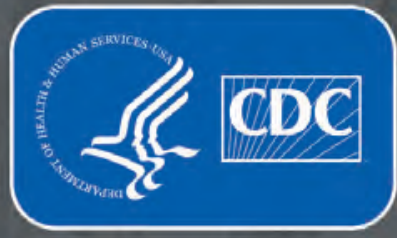




\title{
Outbreak of HIV I nfection Linked to Nosocomial Transmission, China, 2016-2017
}

\author{
Xiaohong Pan, ${ }^{1}$ Jianmin Jiang, ${ }^{1}$ Qiaoqin Ma, Jiafeng Zhang, Jiezhe Yang, Wanjun Chen, \\ Xiaobei Ding, Qin Fan, Zhihong Guo, Yan Xia, Shichang Xia, ${ }^{2}$ Zunyou Wu ${ }^{2}$
}

On January 25, 2017, a physician from ZC Hospital in Hangzhou, China, reported to the Zhejiang Provincial Center for Disease Control and Prevention that a potential HIV outbreak might have occurred during lymphocyte immunotherapy (LIT) performed at the hospital on December 30, 2016. We immediately began investigating and identified the index case-patient as an LIT patient's husband who donated lymphocytes for his wife's LIT and later screened HIV-reactive. Subsequent contamination by a technician resulted in the potential exposure of 34 LIT patients. Acute HIV infection was diagnosed in 5 persons. Phylogenetic analysis confirmed that the HIV-1 gag, pol, and env gene sequences from the index and outbreak-related cases had $\geq 99.5 \%$ similarity. Rapid investigation and implementation of effective control measures successfully controlled the outbreak. This incident provides evidence of a lapse in infection control causing HIV transmission, highlighting the need for stronger measures to protect patients from infectious disease exposure.

L ymphocyte immunotherapy (LIT) to treat recurrent $\checkmark$ miscarriage involves receipt of lymphocytes to a patient from a donor, usually the patient's male partner. Although the European Society of Human Reproduction and Embryology (1), the Royal College of Obstetricians (2), and the American College of Obstetricians and Gynecologists (3) have issued clear guidance against LIT, supported by a 2014 Cochrane review (4), more recent metaanalyses support its use $(5,6)$, as do 4 newer intervention control studies conducted in China (7-10). Although the number of LIT recipients in China is estimated to be large, no statistics are available. Within China's healthcare

\footnotetext{
Author affiliations: Zhejiang Provincial Center for Disease Control and Prevention, Hangzhou, China (X. Pan, J. Jiang, Q. Ma, J. Zhang, J. Yang, W. Chen, X. Ding, Q. Fan, Z. Guo, Y. Xia, S. Xia); National Center for AIDS/STD Control and Prevention, Beijing, China (Z. Wu); University of California, Los Angeles, California, USA (Z. Wu)
}

DOI: https://doi.org/10.3201/eid2412.180117 system, LIT is a category III medical service, meaning that each hospital regulates itself (11).

On January 24, 2017, a woman receiving LIT at ZC Hospital in Hangzhou, China, called a hospital staff member, Dr. X, asking if she had risk for HIV infection. She explained that her husband had just received a confirmed diagnosis of HIV infection and that on December 30, 2016, she had received LIT using lymphocytes her husband donated. Dr. X immediately reported this information to the hospital's deputy director, who informed the clinical medical laboratory director, Dr. Y. At $\approx 4: 00$ PM the same day, Dr. Y informed the responsible laboratory technician, Dr. Z, and requested that she stop LIT. One hour later, Dr. Z voluntarily reported to Dr. Y that she had deviated from protocol on December 30 and that other patients who received LIT on the same day might have been exposed. At 5:30 PM, the director of ZC Hospital called an emergency meeting with department directors, who decided to request help from the Zhejiang Provincial Center for Disease Control and Prevention (Zhejiang CDC). On January 25, 2017, Zhejiang CDC epidemiologists began investigating a possible HIV outbreak among LIT recipients at ZC Hospital. We report on the investigation conducted, control measures implemented, and outcomes observed.

\section{Methods}

The potential HIV outbreak at ZC Hospital was declared a public health emergency, and a formal investigation began on January 25, 2017, supported by provincial (Zhejiang Health Commission and Zhejiang CDC) and national (National Health Commission and National Center for AIDS/STD Control and Prevention, Chinese Center for Disease Control and Prevention [China CDC]) authorities and resources. Neither institutional review board approval nor individual informed consent was required for the investigation. Routine informed consent for HIV,

\footnotetext{
${ }^{1}$ These first authors contributed equally to this article.
}

${ }^{2}$ These senior authors contributed equally to this article. 
hepatitis B virus (HBV), hepatitis C virus (HCV), and syphilis testing (oral or written) and for contact tracing (oral) was obtained.

\section{Case Definition, Case Finding, and Contact Tracing}

We defined an outbreak-related case as a newly diagnosed laboratory-confirmed HIV infection, with evidence of acute infection suggesting occurrence of transmission on December 30, 2016, among women who had received LIT at ZC Hospital that day or their secondary contacts, with HIV gene sequence highly related to that of the index casepatient. Initial case finding began among all women who received LIT at ZC Hospital on December 30. A trained public health specialist conducted interviews on HIV risk behavior during December 30, 2016-January 25, 2017, to assess the possibility that HIV infection had been acquired by means other than LIT and that HIV already had been transmitted to others.

\section{HIV, HBV, HCV, and Syphilis Testing}

All potential outbreak-related case-patients and their contacts were provided free testing and counseling at ZC Hospital. Persons in whom HIV infection was diagnosed were referred to treatment. For HIV, serologic screening was conducted at ZC Hospital's laboratory using the Anti-HIV (1+2) 4th-generation antigen/antibody enzyme immunoassay (EIA) kit (Shanghai Kehua Bio-Engineering, Shanghai, China) and the HIV 1/2/O Tri-Line HIV Rapid Test Device (ABON Biopharm, Hangzhou). If reactive, new venous blood specimens were collected and sent to the Hangzhou Center for Disease Control and Prevention laboratory for confirmatory serologic testing by Western blot (WB; MP Biomedicals, Singapore). In parallel, plasma specimens were sent to the Zhejiang CDC, where HIV nucleic acid testing was conducted using COBAS AmpliPrep/COBAS TaqMan HIV-1 Test v2.0 kits (Roche, Branchburg, NJ, USA).

$\mathrm{HBV}, \mathrm{HCV}$, and syphilis testing were performed at ZC Hospital's laboratory. For HBV, samples were screened for 5 indicators (i.e., hepatitis B surface antigen, hepatitis B surface antibody, hepatitis B e-antigen, hepatitis B e-antibody, hepatitis B c-antibody) using EIA kits (InTec Products, Xiamen, China). For HCV, samples were screened for antibodies using an EIA kit (Zhuhai Livzon Diagnostics, Zhuhai, China). For syphilis, samples were initially screened by Toluidine Red Untreated Serum Test (TRUST, Shanghai Rongsheng Biotech, Shanghai, China). Reactive samples were confirmed by Treponema pallidum particle agglutination assay (Fujirebio Inc., Nagasaki, Japan).

\section{Laboratory Audit}

An audit of the hospital laboratory began immediately on January 25 and lasted 6 days. It was conducted by 3 trained compliance specialists and 3 public health officials from independent institutions. They thoroughly reviewed all relevant records: staffing, training, qualification, certification, security, inventory, equipment, LIT protocol, compliance, supervision, and infection control procedures, as well as records generated during the execution of LIT-related procedures. The audit also included private interviews with all laboratory staff and direct observation of staff rehearsing LIT procedures; investigation of other potential violations of protocol that might have caused nosocomial transmission; and a check of baseline laboratory tests for HIV, HBV, HCV, and syphilis for all 34 women and their husbands.

\section{Molecular Phylogeny Analysis}

All HIV sequencing was performed at the Zhejiang CDC laboratory using plasma specimens. Two technicians in separate laboratory areas extracted HIV RNA from specimens, each using a different method: one used the QIAamp Viral RNA Mini Kit (QIAGEN, Hilden, Germany), the other a viral RNA/DNA extraction kit on an automatic extraction platform (Suzhou Tianlong, Suzhou, China). Partial sequences for the HIV genes gag, pol, and env were amplified by reverse transcription PCR and nested PCR using GUX/GDX primers for gag, 5 different pairs of primers for pol, and M13F/M13R primers for env. PCR products were confirmed by $1 \%$ agarose gel electrophoresis and then purified and sequenced.

We analyzed sequences with Sequencher v5.0 (Gene Codes Corporation, Ann Arbor, MI, USA), examined them for similarity, and aligned them to reference sequences using BioEdit v7.2.0 (Ibis Therapeutics, Carlsbad, CA, USA). Two sets of reference sequences for each gene were selected for comparison to outbreak-related consensus sequences. The first set was international reference sequences obtained from the Los Alamos National Laboratory (https://www. hiv.lanl.gov). The second was representative of strains circulating in the area at the time of the outbreak. We used the neighbor-joining tree method (Kimura 2-parameter model) to determine HIV subtype and phylogenetic relationships and genetic distance between sequences. Two technicians blindly and independently analyzed 2 specimens from each patient. Neighbor-joining phylogenetic trees were constructed using MEGA 6.0 (https://www.megasoftware.net) with 1,000 replicate bootstrap alignments. We defined a transmission cluster as having a bootstrap value $\geq 90 \%$ and a mean genetic distance of $\leq 0.015$.

\section{Results}

\section{Epidemiologic Investigation}

Mrs. P0, age 36, is the wife of the index-case patient, P0, and had been enrolled in LIT starting June 21, 2016. At 
enrollment, P0 and Mrs. P0 both underwent behavioral health screening, physical examination, and HIV testing (both tested HIV negative). Mrs. P0 received LIT on July 19, August 16, September 13, October 14, November 11, December 2, and December 30, 2016, at ZC Hospital, each time with lymphocytes donated by her husband 3 days before her LIT dates. Later in the day after her December 30 treatment, Mrs. P0 learned that her husband had screened HIV reactive. On December 31, Mrs. P0 went to XX Hospital in Hangzhou, which treats persons living with HIV, where she informed the physician she had just discovered she was pregnant and had great concern about possible exposure to HIV by LIT because her husband had just screened HIV-reactive, which she worried she might transmit to her unborn baby. She was strongly encouraged to immediately begin postexposure prophylaxis (PEP), using a regimen of 3 antiretroviral medications (tenofovir, lopinavir/ritonavir, and lamivudine) for 4 weeks. She started PEP the same day.

Upon attending antenatal care shortly thereafter, Mrs. P0 tested negative for HIV, HBV, HCV, and syphilis. On January 24, 2017, after her husband received a confirmed diagnosis of HIV infection, Mrs. P0 alerted staff at ZC Hospital that she was concerned she was exposed to HIV via LIT on December 30, 2016; this report was the initiating event that sparked the outbreak investigation. As part of the investigation, Mrs. P0 was followed and regularly tested for HIV, HBV, HCV, and syphilis. All results were repeatedly negative. Her PEP regimen was converted to a prevention of mother-to-child transmission (PMTCT) program (same medicines extended to 8 weeks after delivery).
Mrs. P0’s baby was born on July 9, 2017, and Mrs. P0 stopped all use of antiretroviral therapy (ART) on the same day. Her most recent HIV screening test was performed on March 9, 2018, at 9 months since she stopped ART and $>14$ months since her exposure. Her results remained negative.

\section{Index Case-Patient}

P0 was the 40-year-old husband of Mrs. P0. Public health workers constructed a 2-month timeline related to his HIV exposure and testing (Figure 1). His most recent negative HIV screening result was on November 27,2016 . The residual specimen was retested using a 4th-generation antigen/antibody test during the investigation and confirmed negative. The exposure event that probably led to P0's HIV infection was traced to December 1, 2016, when he had condomless, receptive anal sex with a man he did not know at a gay bathhouse. Approximately 2 weeks later, fever developed, and he began to suspect HIV infection.

P0 went to the Yiwu CDC for HIV testing on Friday, December 23, but his specimen was not tested until December 29 (HIV testing by both antibody-only and antibody/antigen EIA is only performed on Thursdays at Yiwu CDC). He was not informed of his reactive result until on December 30, at which time he was encouraged to return for confirmatory testing. However, P0 instead sought rescreening at another facility. He returned for confirmatory testing on January 3, 2017. His first WB result was indeterminate (gp160/p24). His second result (using a new specimen obtained on January 23) was positive (gp160/gp120/ p66/p31/p24), and his viral load (VL) was 121,000 copies/

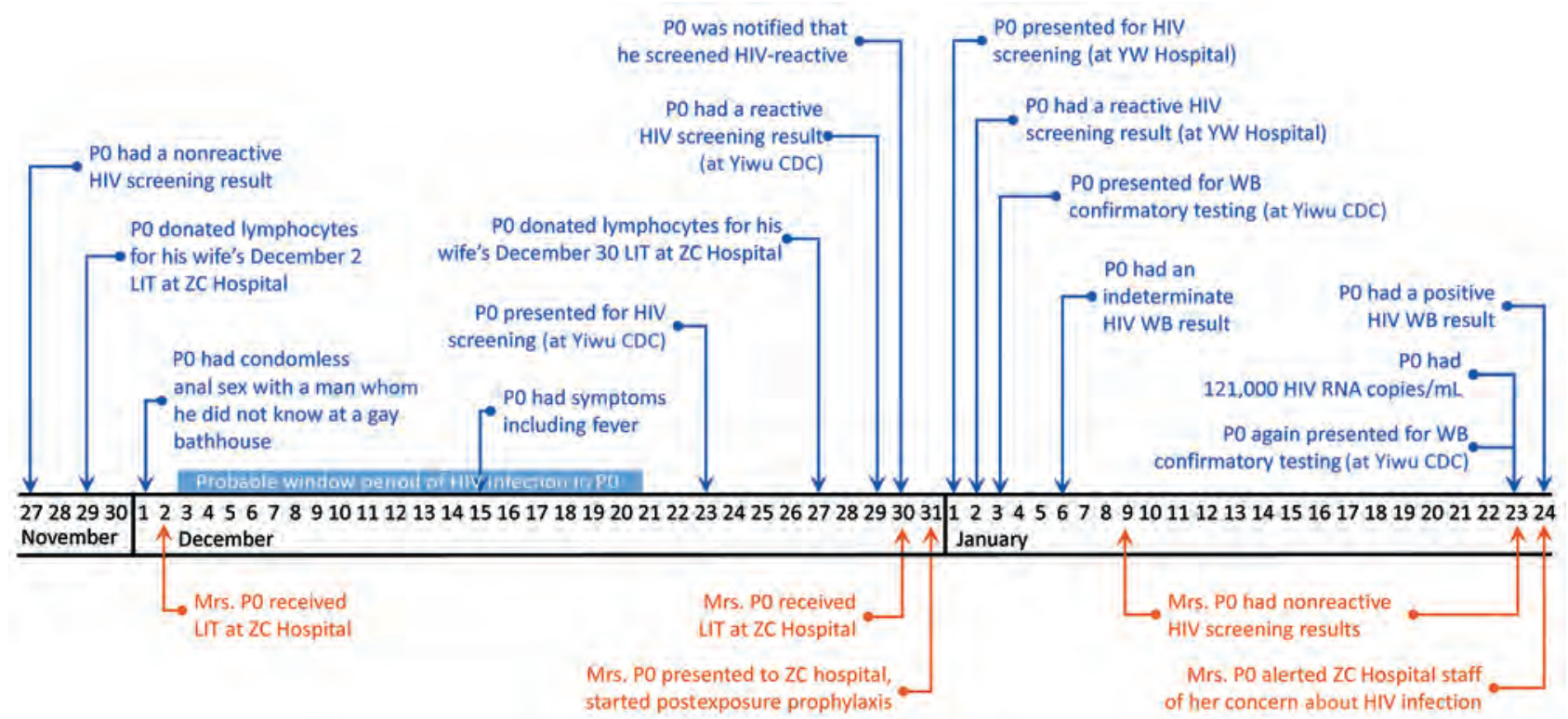

Figure 1. Timeline of HIV exposure and HIV diagnosis of the index case-patient, P0 (blue), and the HIV exposure of his wife, Mrs. P0 (orange), Hangzhou, China, November 27, 2016-January 24, 2017. CDC, Center for Disease Control and Prevention; LIT, lymphocyte immunotherapy; P, patient; WB, Western blot. 
mL. P0 was informed of his HIV diagnosis on January 24 and started treatment the next day. He tested negative for $\mathrm{HBV}, \mathrm{HCV}$, and syphilis. Investigators determined that P0 made no other donations of fluids, cells, or tissues after his exposure on December 1, 2016.

\section{Laboratory Investigation}

The audit of the LIT laboratory resulted in 5 main findings. First, although the appropriate protocol was used and requires that each disposable sterile tube for lymphocyte processing is used only once, the laboratory experienced a shortage of these tubes for 1 day on December 30 . To provide LIT to 34 women, $\geq 136$ tubes were needed (34 for moving lymphocytes from culture containers to washing plates and 102 for washing the 34 cultures 3 times each). Approximately 100 tubes were available on December 30. Second, instead of stopping and calling this issue to the attention of a supervisor, the laboratory technician processing donated lymphocytes for LIT on December 30 deviated from protocol and reused tubes. Deviations occurred in 2 procedures: tubes were used repeatedly for moving lymphocytes from culture containers to washing plates and for washing the lymphocyte cultures. Third, the technician failed to properly document the work performed and upon interview, admitted to reusing disposable tubes "a few times," but could not remember how many times or for which couples. Fourth, no deviation occurred on December 27 that could have caused contamination during blood specimen collection, lymphocyte separation, or lymphocyte culturing. Finally, LIT was performed at ZC Hospital only $\approx 1$ time each month. No LIT had yet been conducted during December 30, 2016-January 25, 2017. No evidence of these failures, or other failures that could have similarly resulted in nosocomial transmission of HIV, was found before December 30 or during December 31-January 25.

The auditors concluded that lymphocyte processing deviated from the protocol on December 30 and that the technician responsible contaminated an unknown number of patients' prepared lymphocytes on December 30 with lymphocytes from the index case-patient. Thus, all patients who received LIT on December 30 should be tested as if they had potentially been exposed. Auditors recommended that all the women and their husbands be tested for HIV, HBV, HCV, and syphilis.

\section{Contact Tracing}

Along with Mrs. P0, 33 other women received LIT at ZC Hospital on December 30. A medical records review found that all 33 had tested negative for HIV, HBV, HCV, and syphilis before beginning LIT in 2016. None reported any HIV risk behavior other than sexual contact, and none reported sexual contact with anyone other than their husbands. All 33 women (possible primary contacts) and their husbands (possible secondary contacts) were tested for HIV, HBV, HCV, and syphilis. Five cases of HIV were found (Figure 2).

\section{Case-Patient 1}

P1, age 35 (not pregnant) at the time of the investigation, received LIT at ZC Hospital on November 4, December 2, and December 30, 2016. She reported having fever, sore throat, and other symptoms, for which she had been given penicillin at a local clinic for a suspected bacterial infection. HIV serologic results were nonreactive, but virologic results were positive with VL of 756,000 copies/

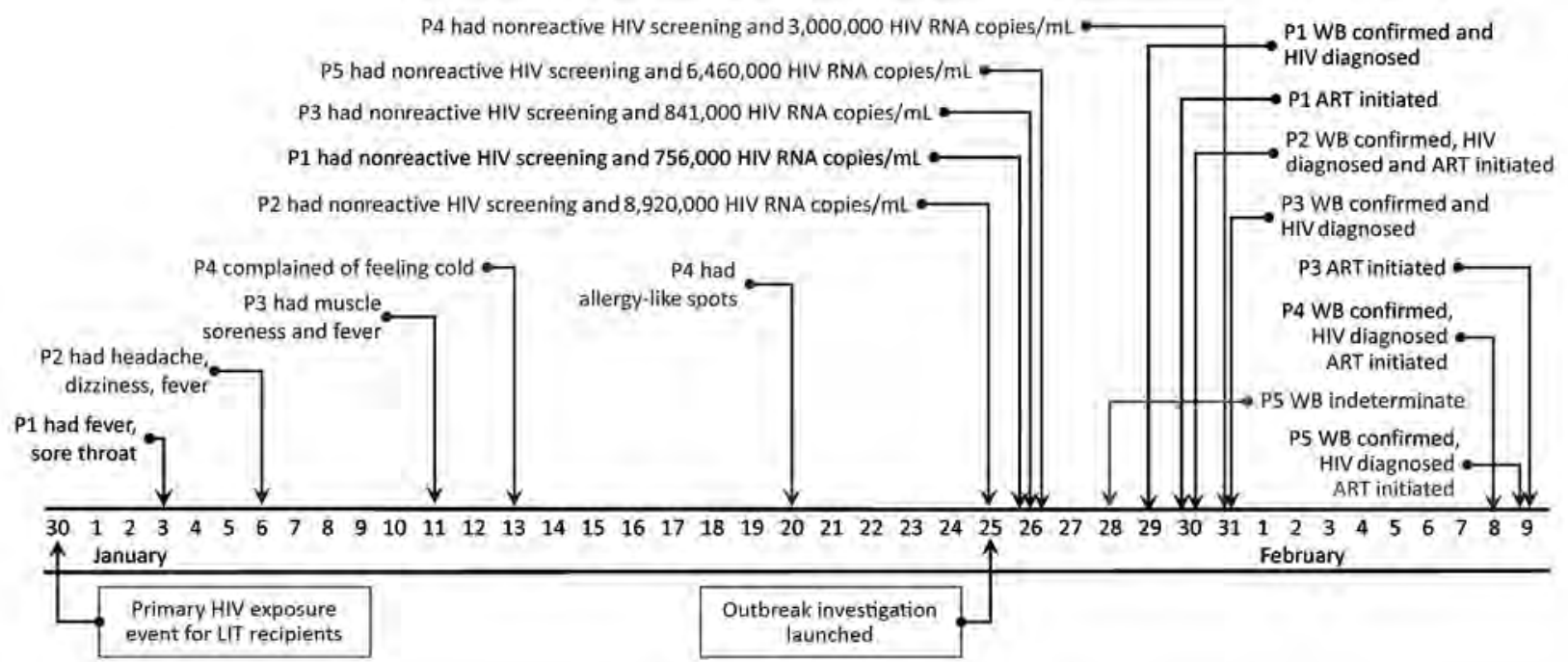

Figure 2. Timeline of HIV exposure, symptoms, diagnosis, and treatment initiation for the 5 HIV-infected women during nosocomial HIV outbreak, Hangzhou, China, December 30, 2016-February 9, 2017. ART, antiretroviral therapy; LIT, lymphocyte immunotherapy; $\mathrm{P}$, patient; WB, Western blot. 
$\mathrm{mL}$ on January 26, 2017, suggesting acute infection. Later confirmatory WB result was positive (gp160/gp120/p41/ p24). P1 was informed of her diagnosis on January 29, and she initiated ART the next day.

\section{Case-Patient 2}

P2, age 28 and pregnant (10 weeks' gestation) at the time of the investigation, received LIT at ZC Hospital on July 22, August 19, September 16, October 14, November 11, December 9, and December 30, 2016. She reported symptoms including headache, dizziness, and fever but sought no care. HIV screening results were nonreactive, but virologic results were positive with VL of 8,920,000 copies/mL on January 25, 2017, suggesting acute infection. Confirmatory WB result was positive (gp160/gp120/p24). P2 was informed of her HIV diagnosis, and she enrolled in PMTCT and initiated ART on January 30.

\section{Case-Patient 3}

P3, age 34 (not pregnant) at the time of the investigation, received LIT at ZC Hospital on October 10, November 4, December 2, and December 30, 2016. She had muscle soreness and fever but did not seek care. HIV screening results were nonreactive; however, virologic results were positive with VL of 841,000 copies/mL on January 26, 2017, suggesting acute infection. Confirmatory WB result was positive (gp160/gp120/p24) on January 31, and she was informed of her diagnosis on the same day. She began ART on February 9, 2017.

\section{Case-Patient 4}

P4, age 28 and pregnant (17 weeks' gestation) at the time of the investigation, received LIT at ZC Hospital on March 4, April 1, April 29, May 27, June 24, July 29, August 19, November 18, December 9, and December 30, 2016. She reported symptoms including feeling cold and red allergy-like spots on her chest but did not see a doctor. HIV screening results were nonreactive, but virologic results were positive with VL of 3,000,000 copies/mL on January 31, 2017, suggesting acute infection. First confirmatory WB result was indeterminate (gp160/p24); second was positive (gp160/gp120/p24). P4 was informed of her diagnosis, enrolled in PMTCT, and initiated on ART on February 2.

\section{Case-Patient 5}

$\mathrm{P} 5$, age 34 (not pregnant) at the time of the investigation, received LIT at ZC Hospital only on December 30, 2016. She reported no symptoms. HIV screening results were nonreactive, but virologic results were positive with VL of 6,460,000 copies/mL on January 26, 2017, suggesting acute infection. First confirmatory WB result was indeterminate (gp160), but the second was positive (gp160/gp120/p24).
P5 was informed of her diagnosis and initiated ART on February 9.

\section{Other Contacts}

The remaining 29 women, including Mrs. P0, the wife of the index case-patient, all had multiple negative serologic and virologic results during follow-up (Table 1, https:// wwwnc.cdc.gov/EID/article/24/12/18-0117-T1.htm). None had HBV, HCV, or syphilis.

Although all 33 husbands were tested initially, 3 husbands of the 5 women with newly diagnosed HIV infection, all of whom who reported sexual contact with their wives after December 30, 2016, were followed and provided HIV serologic and virologic testing, as well as HBV, HCV, and syphilis testing. All results were negative. Additionally, the infants of Mrs. P0 (born July 9, 2017), P2 (born August 14, 2017), and P4 (born July 3, 2017) were tested for HIV by early infant diagnosis (EID; by PCR) and were HIV-negative.

\section{Phylogenetic Investigation}

The HIV sequences derived from the index case-patient and the 5 women with newly diagnosed HIV infection shared a very high degree of similarity: mean of $99.95 \%$ for gag, $99.48 \%$ for pol, and $99.92 \%$ for env (Table 2). The gag and pol sequences were consistent with HIV1 subtype CRF01_AE, and env sequences were subtype as $C$, indicating that all 6 persons were infected with a recombinant CRF01_AE/C strain. Phylogenetic trees of gag, pol, and env sequences (Figure 3 ) indicate a very close genetic relationship between the virus present in the 5 women with newly diagnosed infection and the index casepatient. The sequences of all 3 genes map to monophyletic clusters in $100 \%$ of bootstrap replicates with genetic distances of $<0.015$.

\section{Summary of Outbreak Response}

In response to this outbreak, the National Health Commission immediately suspended all LIT services nationwide, and all 34 couples involved were provided counseling and support. The epidemiologic investigation found that a lymphocyte donor (P0) had become infected with HIV before donation on December 27 and that laboratory contamination occurred on December 30, which together caused 5 women to become infected with HIV. HIV phylogenetic investigation confirmed the causal relationship. All 5 women had initiated ART as of February 9, 2017, only 15 days after the investigation began. All remaining 29 women who initially screened nonreactive and the husbands of the 5 infected women were followed up for 6 months; no additional HIV infections were found. The 3 pregnant women were provided PMTCT; their newborn infants were followed 
Table 1. Interval between HIV exposure and follow-up HIV tests for the 37 persons followed up after initially screening HIV-nonreactive in investigation of nosocomial HIV outbreak, Hangzhou,China, 2016-2017*

\begin{tabular}{|c|c|c|c|c|c|c|c|c|}
\hline \multirow[b]{2}{*}{ Potential contact } & \multicolumn{8}{|c|}{ Days between HIV exposure and follow-up HIV tests } \\
\hline & 1st test & 2nd test & 3rd test & 4th test & 5th test & 6th test & 7 th test & 8th test \\
\hline \multicolumn{9}{|l|}{ Primary† } \\
\hline Mrs. P0‡ & 24 & 29 & 45 & 68 & 103 & 130 & 191 & 464 \\
\hline Q1 & 28 & 48 & 82 & & & & & \\
\hline Q2 & 28 & 58 & 86 & 186 & & & & \\
\hline Q3 & 27 & 29 & 59 & 84 & 182 & & & \\
\hline Q4 & 28 & 58 & 86 & 192 & & & & \\
\hline Q5 & 28 & 45 & 86 & 192 & & & & \\
\hline Q6 & 28 & 56 & 94 & 188 & & & & \\
\hline Q7 & 27 & 28 & 57 & 99 & 211 & & & \\
\hline Q8 & 29 & 58 & 100 & 189 & & & & \\
\hline Q9 & 27 & 57 & 45 & 85 & & & & \\
\hline Q10 & 27 & 28 & 60 & 93 & 187 & & & \\
\hline Q11 & 28 & 64 & 106 & 202 & & & & \\
\hline Q12 & 29 & 55 & 83 & 185 & & & & \\
\hline Q13 & 27 & 29 & 58 & 89 & & & & \\
\hline Q14 & 27 & 35 & 62 & 92 & 199 & & & \\
\hline Q15 & 27 & 33 & 64 & 93 & 212 & & & \\
\hline Q16 & 27 & 45 & 93 & & & & & \\
\hline Q17 & 27 & 33 & & & & & & \\
\hline Q18 & 27 & 34 & 59 & 90 & 189 & & & \\
\hline Q19 & 27 & 33 & 63 & 91 & 187 & & & \\
\hline Q20 & 28 & 60 & 90 & 194 & & & & \\
\hline Q21 & 30 & 55 & 80 & 192 & & & & \\
\hline Q22 & 31 & 45 & 93 & 186 & & & & \\
\hline Q23 & 31 & 53 & 81 & 188 & & & & \\
\hline Q24 & 33 & 55 & 89 & 187 & & & & \\
\hline Q25 & 32 & 63 & 92 & 196 & & & & \\
\hline Q26 & 33 & 63 & 95 & 189 & & & & \\
\hline Q27 & 33 & 47 & 97 & 193 & & & & \\
\hline Q28 & 33 & 60 & & & & & & \\
\hline \multicolumn{9}{|l|}{ Secondary§ণ } \\
\hline P3's husband & 7 & 30 & 57 & 97 & 182 & & & \\
\hline P4's husband & 11 & 28 & 52 & 98 & 180 & & & \\
\hline P5's husband & 2 & 18 & 25 & 32 & 60 & 196 & & \\
\hline Infant PO & 3 & 42 & 90 & 242 & & & & \\
\hline Infant P2 & 3 & 43 & & & & & & \\
\hline Infant P4 & 1 & 44 & & & & & & \\
\hline \multicolumn{9}{|c|}{$\begin{array}{l}\text { *First HIV test was conducted using both nucleic acid testing and EIA. All subsequent HIV tests were conducted using EIA only. Blank cells indicate no } \\
\text { further HIV test. EIA, enzyme immunoassay; LIT, lymphocyte immunotherapy; P, patient infected with HIV; Q, women who might have been exposed to } \\
\text { HIV but were not infected. } \\
\text { tBeginning of the time interval was counted from contaminated LIT at ZC Hospital on December } 30,2016 \text {. } \\
\text { fMrs. PO is the wife of the index case-patient, PO. She is continuing to be followed once a year for at least } 3 \text { years since she discontinued antiretroviral } \\
\text { therapy. Her most recent HIV test, on March } 9,2018 \text {, was again negative. } \\
\text { SFor husbands, beginning of the time interval was counted from the most recent sexual contact with the wife from the wife's exposure on December } 30 \text {, } \\
2016, \text { through the start of the investigation on January } 25,2017 \text {. Husbands received both serologic (4th-generation Ag/Ab EIA, Shanghai Kehua Bio- } \\
\text { Engineering, Shanghai, China) and virologic testing (HIV-1 RNA, COBAS AmpliPrep/COBAS TaqMan HIV-1 Test V2.0, Roche, Branchburg, NJ, USA). } \\
\text { ๆFor infants, beginning of the time interval was counted as the date of birth. The first } 3 \mathrm{HIV} \text { tests for infants were early infant diagnosis tests by a standard } \\
\text { nucleic acid testing protocol. The fourth test for infant PO was a 4th-generation Ag/Ab EIA. }\end{array}$} \\
\hline
\end{tabular}

up, and no HIV infection was found. The laboratory technician was sentenced to 2.5 years in prison. The hospital director, deputy director, and division chief accountable for the laboratory were dismissed. LIT services were suspended until a new guideline was issued on December 22, 2017 (12).

\section{Discussion}

These epidemiologic and phylogenetic investigations used techniques similar to those used during HIV outbreak investigations including a famous case of a Florida, USA, dentist (13-16); several criminal cases (17-20); a prison outbreak in Scotland, UK (21); 2 nosocomial outbreaks

Table 2. Similarity of HIV genetic sequence of viral nucleic acid from the index case-patient and the 5 women infected by during nosocomial HIV outbreak, Hangzhou, China, 2016-2017

\begin{tabular}{lccccc}
\hline & \multicolumn{5}{c}{ Sequence similarity, \% } \\
\cline { 2 - 6 } Region & Case-patient 1 & Case-patient 2 & Case-patient 3 & Case-patient 4 & Case-patient 5 \\
\hline gag & 99.70 & 100.00 & 100.00 & 100.00 & 100.00 \\
pol & 99.50 & 99.40 & 99.50 & 99.50 & 99.95 \\
env & 100.00 & 100.00 & 100.00 & 100.00 & 99.50 \\
\hline
\end{tabular}



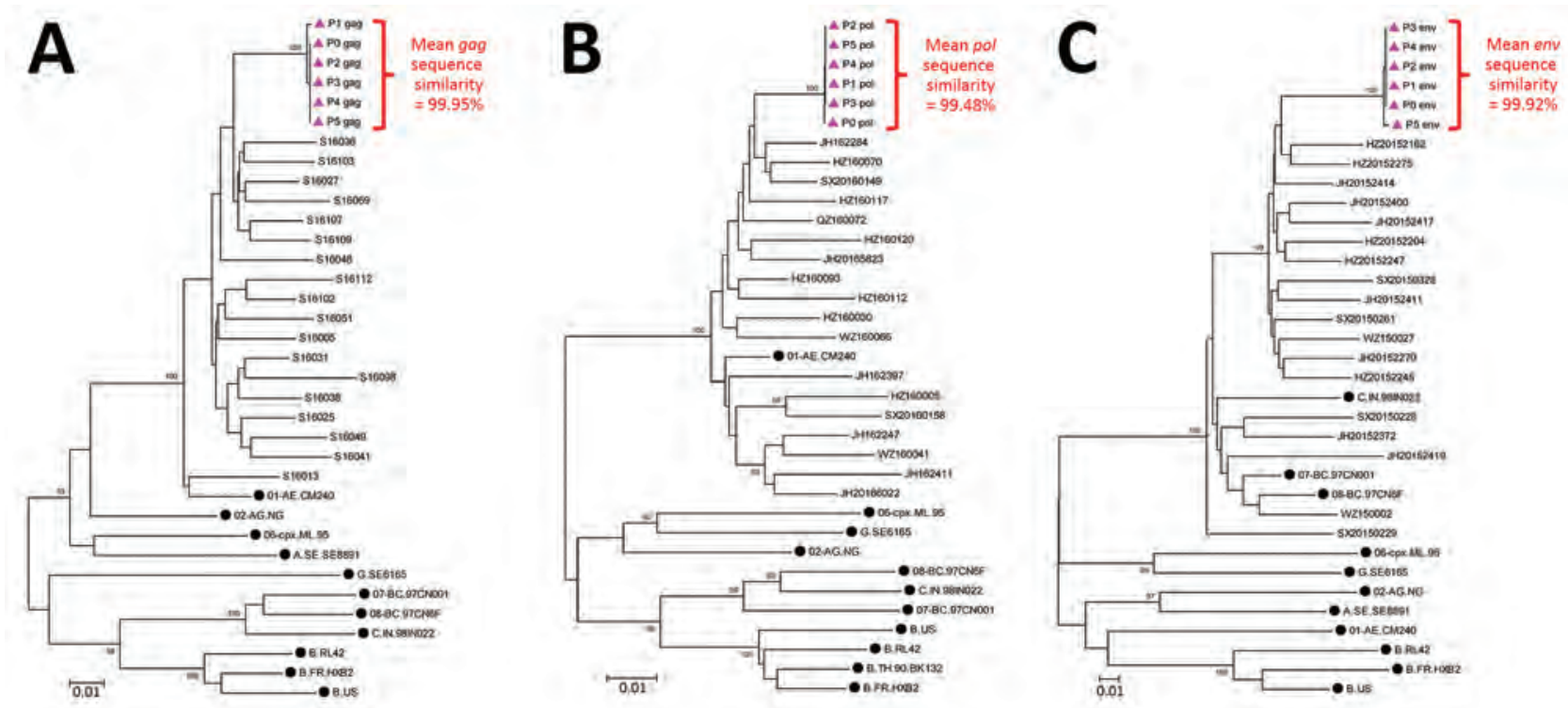

Figure 3. Phylogenetic trees showing relationships between HIV-1 gene sequences from index case-patient and 5 women infected during nosocomial HIV outbreak, Zhejiang Province, China, 2016-2017, and reference sequences. Bootstrap values $\geq 90 \%$ only are shown for gag sequences (A), pol sequences (B), and env sequences (C). Triangles indicate index case-patient (P0) and 5 women found to have HIV infection (P1-5); dots indicate international reference sequences. Scale bars indicate nucleotide substitutions per site. P, patient.

$(22,23)$; and a recent outbreak in Indiana, USA, associated with injection drug use (24). These investigations identified $\mathrm{P} 0$ as the index case-patient for this nosocomial HIV outbreak and demonstrate that deviation from protocol and lapse in infection control during LIT were the cause. We have yet to detect HIV infection in Mrs. P0, the wife of the index case-patient, suggesting that her immediate initiation of PEP might have averted infection. However, we are unable to definitively determine Mrs. P0's HIV status because she has been followed for only 9 months since she discontinued ART, and evidence of viremic rebound nearly 30 months after ART cessation was observed in the case of a child in Mississippi, USA $(25,26)$. These results underscore the critical importance of quickly investigating a suspected outbreak. Among the 34 women potentially exposed, only 5 acquired infections, and potential onward transmission of HIV to their husbands and infants was averted.

This study was subject to at least 2 limitations. First, follow-up HIV testing for the 29 potentially exposed women was voluntary, and some declined to have third and fourth HIV tests. For example, Q17 was followed up at 27 days (with nucleic acid testing and antigen/antibody EIA) and 33 days (antigen/antibody EIA only) and, although unlikely, it is possible that she had undetected HIV infection. Second, as noted, we were unable to definitively ascertain Mrs. P0's HIV status within the scope of this study. Hence, HIV infection linked to this outbreak might not yet have been diagnosed.

The results of this outbreak investigation offer important lessons that China must not ignore. First, the unacceptably long process of HIV diagnosis in China directly contributed to this outbreak. Ample evidence of the substantial benefit of streamlining and accelerating China's HIV care continuum already exists (27-29). However, although a rapid 1-visit testing, diagnosis, clinical staging, and ART initiation protocol has been adopted, China must accelerate the pace at which these changes are implemented if it is to avoid another, similar outbreak.

Second, China must implement more frequent and thorough training for medical professionals on the risks of nosocomial HIV transmission. The finding that Mrs. P0's attending physician at XX Hospital was concerned enough to start her on PEP but not to alert public health officials and the finding that the laboratory technician did not consider reusing sterile tubes to be unsafe both indicate that education about the risks for nosocomial HIV transmission is still lacking. China must act quickly to fill this gap.

Finally, laboratories in medical settings must be placed under stricter controls. Immediate supervision and monitoring and thorough and frequent laboratory audits should be implemented immediately in China's medical laboratories. A high level of vigilance in the medical laboratory setting is critical if China is to prevent similar future nosocomial outbreaks.

\section{Acknowledgments}

We are very grateful to Jennifer McGoogan for her invaluable comments, suggestions, and English editing of many revisions of the paper; Roger Detels and Julio Montaner for their helpful 
comments; the national and Zhejiang provincial panels for this emergency response; and the staff of Yiwu Municipality CDC, Hangzhou Municipality CDC, Shangcheng District CDC, ZC Hospital, and Xixi Hospital for their participation and support in the epidemiologic investigation, as well as HIV testing, counseling, and treatment.

The study was funded by the Emergency Response Funds from the National Health and Family Planning Commission, People's Republic of China; the Emergency Response Funds from the Zhejiang Health and Family Planning Commission; China National Technical Support and Operational Research for HIV/AIDS Prevention, Treatment and Care (grant no. 13113-000 105-01); National Science and Technology Major Project on Prevention and Treatment of Major Infectious Diseases Including AIDS and Viral Hepatitis (grant no. 2012ZX10001-007); and Zhejiang Provincial Key Programs for Science and Technology (grant no. 2013C03047-1). The funding agencies were actively involved in the field and laboratory investigations, data collection, analysis, and interpretation. They did not contribute to manuscript development.

\section{About the Author}

Dr. Pan is the director of the Division of HIV/STD at the Zhejiang Provincial CDC. Her research interests are monitoring and responding to HIV, sexually transmitted diseases, and HCV epidemics. Her current research priority is the HIV epidemic among men who have sex with men in China.

\section{References}

1. The ESHRE Guideline Group on RPL, Atik RB, Christiansen OB, Elson J, Kolte AM, Lewis S, et al. ESHRE guideline: recurrent pregnancy loss. Hum Reprod Open. 2018; 2018:1-12. https://doi.org/10.1093/hropen/hoy004

2. Royal College of Obstetricians and Gynaecologists. The investigation and treatment of couples with recurrent first-trimester and second trimester miscarriage. Green-top Guideline No. 17. 2011 [cited 2018 Oct 17]. https://www.rcog.org.uk/globalassets/ documents/guidelines/gtg_17.pdf

3. American College of Obstetricians and Gynecologists. Practice bulletin: early pregnancy loss. 2015; Number 150 [cited 2018 Oct 17]. https://www.acog.org/-/media/Practice-Bulletins/ Committee-on-Practice-Bulletins----Gynecology/Public/pb150.pdf

4. Wong LF, Porter TF, Scott JR. Immunotherapy for recurrent miscarriage. Cochrane Database Syst Rev. 2014;(10):CD000112.

5. Cavalcante MB, Sarno M, Araujo Júnior E, Da Silva Costa F, Barini R. Lymphocyte immunotherapy in the treatment of recurrent miscarriage: systematic review and meta-analysis. Arch Gynecol Obstet. 2017;295:511-8. http://dx.doi.org/10.1007/ s00404-016-4270-Z

6. Liu Z, Xu H, Kang X, Wang T, He L, Zhao A. Allogenic lymphocyte immunotherapy for unexplained recurrent spontaneous abortion: a meta-analysis. Am J Reprod Immunol. 2016;76:443-53. http://dx.doi.org/10.1111/aji.12511

7. Liu X, Tian L. Level of the blocking antibodies in women with recurrent spontaneous abortion and the effect of immunotherapy with lymphocytes. Chinese Journal of Healthy Birth \& Child Care. 2012;18:276-8.
8. Yang Z, Zhou C, Li X, Wang M, Tan Y. Clinical observation of lymphocyte active immunization combined with dydrogesterone in women with recurrent spontaneous abortion caused by blocking antibody-negative. J Reprod Med. 2014;23:128-30.

9. Liang X, Qiu T, Qiu L, Wang X, Zhao A, Lin Q. Female third party lymphocytes are effective for immunotherapy of patients with unexplained primary recurrent spontaneous abortion: a retrospective analysis of outcomes. Eur J Contracept Reprod Health Care. 2015;20:428-37. http://dx.doi.org/10.3109/ 13625187.2015.1046593

10. Chen JL, Yang JM, Huang YZ, Li Y. Clinical observation of lymphocyte active immunotherapy in 380 patients with unexplained recurrent spontaneous abortion. Int Immunopharmacol. 2016;40:347-50. http://dx.doi.org/10.1016/j.intimp.2016.09.018

11. The National Health and Family Planning Commission of the People's Republic of China. The announcement of calling for cancellation of approval requirement for clinical use of third category of medical services in China. Announcement no. 71 in 2015. The National Health and Family Planning Commission of People's Republic of China. June 29, 2015 [cited 2018 May 17]. http://www.nhfpc.gov.cn/yzygj/s3585/201507/c529dd6bb8084e09883ae417256b3c49.shtml

12. The Center for Drug Evaluation, State Food and Drug Administration. The guiding principles for cell therapeutic products used in scientific research and technical evaluation. The Center for Drug Evaluation, State Food and Drug Administration. December 22, 2017 [2018 Jun 25]. http://www.cde.org.cn/zdyz.do?method=largePage\&id=251

13. Ou CY, Ciesielski CA, Myers G, Bandea CI, Luo CC, Korber BT, et al. Molecular epidemiology of HIV transmission in a dental practice. Science. 1992;256:1165-71. http://dx.doi.org/10.1126/ science.256.5060.1165

14. Smith TF, Waterman MS. The continuing case of the Florida dentist. Science. 1992;256:1155-6. http://dx.doi.org/10.1126/ science.256.5060.1155

15. DeBry RW, Abele LG, Weiss SH, Hill MD, Bouzas M, Lorenzo E, et al. Dental HIV transmission? Nature. 1993;361:691. http://dx.doi.org/10.1038/361691a0

16. Hillis DM, Huelsenbeck JP. Support for dental HIV transmission. Nature. 1994;369:24-5. http://dx.doi.org/10.1038/369024a0

17. Machuca R, Jørgensen LB, Theilade P, Nielsen C. Molecular investigation of transmission of human immunodeficiency virus type 1 in a criminal case. Clin Diagn Lab Immunol. 2001; 8:884-90.

18. Metzker ML, Mindell DP, Liu XM, Ptak RG, Gibbs RA, Hillis DM. Molecular evidence of HIV-1 transmission in a criminal case. Proc Natl Acad Sci U S A. 2002;99:14292-7. http://dx.doi.org/10.1073/pnas.222522599

19. Lemey P, Van Dooren S, Van Laethem K, Schrooten Y, Derdelinckx I, Goubau P, et al. Molecular testing of multiple HIV-1 transmissions in a criminal case. AIDS. 2005;19:1649-58. http://dx.doi.org/10.1097/01.aids.0000187904.02261.1a

20. Birch CJ, McCaw RF, Bulach DM, Revill PA, Carter JT, Tomnay J, et al. Molecular analysis of human immunodeficiency virus strains associated with a case of criminal transmission of the virus. J Infect Dis. 2000;182:941-4. http://dx.doi.org/10.1086/315751

21. Yirrell DL, Hutchinson SJ, Griffin M, Gore SM, Leigh-Brown AJ, Goldberg DJ. Completing the molecular investigation into the HIV outbreak at Glenochil prison. Epidemiol Infect. 1999;123:277-82. http://dx.doi.org/10.1017/S0950268899002848

22. Blanchard A, Ferris S, Chamaret S, Guétard D, Montagnier L. Molecular evidence for nosocomial transmission of human immunodeficiency virus from a surgeon to one of his patients. J Virol. 1998;72:4537-40.

23. de Oliveira T, Pybus OG, Rambaut A, Salemi M, Cassol S, Ciccozzi M, et al.; Benghazi Study Group. Molecular epidemiology: 
HIV-1 and HCV sequences from Libyan outbreak. Nature. 2006;444: 836-7. http://dx.doi.org/10.1038/444836a

24. Peters PJ, Pontones P, Hoover KW, Patel MR, Galang RR, Shields J, et al.; Indiana HIV Outbreak Investigation Team. HIV infection linked to injection use of oxymorphone in Indiana, 2014-2015. N Engl J Med. 2016;375:229-39. http://dx.doi.org/ 10.1056/NEJMoa1515195

25. Persaud D, Gay H, Ziemniak C, Chen YH, Piatak M Jr, Chun TW, et al. Absence of detectable HIV-1 viremia after treatment cessation in an infant. N Engl J Med. 2013;369:1828-35. http://dx.doi.org/ 10.1056/NEJMoa1302976

26. Luzuriaga K, Gay H, Ziemniak C, Sanborn KB, Somasundaran M, Rainwater-Lovett K, et al. Viremic relapse after HIV-1 remission in a perinatally infected child. N Engl J Med. 2015;372:786-8. http://dx.doi.org/10.1056/NEJMc1413931

27. Wu Z, Zhao Y, Ge X, Mao Y, Tang Z, Shi CX, et al. Simplified HIV testing and treatment in China: analysis of mortality rates before and after a structural intervention. PLoS Med. 2015;12:e1001874. http://dx.doi.org/10.1371/journal.pmed.1001874

28. Wu Z, Tang Z, Mao Y, Van Veldhuisen P, Ling W, Liu D, et al. Testing and linkage to HIV care in China: a cluster-randomised trial. Lancet HIV. 2017;4:e555-65. http://dx.doi.org/10.1016/ S2352-3018(17)30131-5

29. Ma Y, Dou Z, Guo W, Mao Y, Zhang F, McGoogan JM, et al. The human immunodeficiency virus care continuum in China: 1985-2015. Clin Infect Dis. 2018;66:833-9. http://dx.doi.org/ 10.1093/cid/cix911

Address for correspondence: Shichang Xia, Zhejiang Provincial Center for Disease Control and Prevention, 3399 Binsheng Rd, Hangzhou, 310051, Zhejiang, China; email: scxia@cdc.zj.cn; Zunyou Wu, National Center for AIDS/STD Control and Prevention, 155 Changbai Rd, Beijing, 102206, China; email: wuzy@263.net

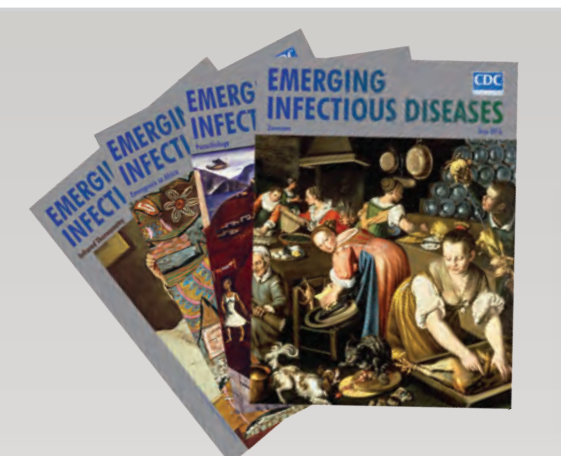

EMIERGING INFECTIOUS DISEASES

\section{Zoonoses}

- Ferrets as Models for Influenza Virus Transmission Studies and Pandemic Risk Assessments

- Occupation-Associated Fatal Limbic Encephalitis Caused by Variegated Squirrel Bornavirus 1, Germany, 2013

- Use of Bead-Based Serologic Assay to Evaluate Chikungunya Virus Epidemic, Haiti

- Widespread Treponema pallidum Infection in Nonhuman Primates, Tanzania

- Genomic Epidemiology of Global Carbapenemase-

Producing Enterobacter spp., 2008-2014

- Influenza D Virus Infection in Feral Swine Populations, United States

- Prion Disease in Dromedary Camels, Algeria

- Frequent Implication of Multistress-Tolerant Campylobacter jejuni in Human Infections

- Bioclinical Test to Predict Nephropathia Epidemica Severity at Hospital Admission

- Hepatitis E in Long-Term Travelers from the Netherlands to Subtropical and Tropical Countries, 2008-2011

\section{J une 2018}

- Novel Parvovirus Related to Primate Bufaviruses in Dogs

- Novel Poxvirus in Proliferative Lesions of Wild Rodents in East Central Texas, USA

- Rickettsia parkeri in Dermacentor parumapertus Ticks, Mexico

- Foot-and-Mouth Disease in the Middle East Caused by an A/ASIA/G-VII Virus Lineage, 2015-2016

- Novel Salmonella enterica Serovar Typhimurium Genotype Levels as Herald of Seasonal Salmonellosis Epidemics

- Urban Wild Boars and Risk for Zoonotic Streptococcus suis, Spain

- Human Endophthalmitis Caused by Pseudorabies Virus Infection, China, 2017

- Pulmonary Infections with Nontuberculous Mycobacteria, Catalonia, Spain, 1994-2014

- Westward Spread of Highly Pathogenic Avian Influenza A(H7N9) Virus among Humans, China

- Importation of Human Seoul Virus Infection to Germany from Indonesia 


\title{
Reemergence of St. Louis Encephalitis Virus in the Americas
}

\author{
Adrián Diaz, Lark L. Coffey, Nathan Burkett-Cadena, Jonathan F. Day
}

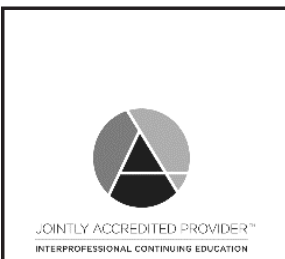

\section{$\underset{\text { MDUCATION }}{\text { Medscape ACTIVITY }}$}

In support of improving patient care, this activity has been planned and implemented by Medscape, LLC and Emerging Infectious Diseases. Medscape, LLC is jointly accredited by the Accreditation Council for Continuing Medical Education (ACCME), the Accreditation Council for Pharmacy Education (ACPE), and the American Nurses Credentialing Center (ANCC), to provide continuing education for the healthcare team.

Medscape, LLC designates this Journal-based CME activity for a maximum of 1.00 AMA PRA Category 1 Credit(s) ${ }^{\mathrm{TM}}$. Physicians should claim only the credit commensurate with the extent of their participation in the activity.

All other clinicians completing this activity will be issued a certificate of participation. To participate in this journal CME activity: (1) review the learning objectives and author disclosures; (2) study the education content; (3) take the post-test with a 75\% minimum passing score and complete the evaluation at http://www.medscape.org/journal/eid; and (4) view/print certificate. For CME questions, see page 2400 .

Release date: November 14, 2018; Expiration date: November 14, 2019

Learning Objectives

Upon completion of this activity, participants will be able to:

- Examine the history of St. Louis encephalitis virus epidemiology in the United States

- Evaluate the emergence and reemergence of St. Louis encephalitis virus in South America

- $\quad$ Assess the reemergence of St. Louis encephalitis virus in the western United States

\section{CME Editor}

Deborah Wenger, MBA, Copyeditor, Emerging Infectious Diseases. Disclosure: Deborah Wenger, MBA, has disclosed no relevant financial relationships.

CME Author

Laurie Barclay, MD, freelance writer and reviewer, Medscape, LLC. Disclosure: Laurie Barclay, MD, has disclosed the following relevant financial relationships: owns stock, stock options, or bonds from Pfizer.

\section{Authors}

Disclosures: Adrián Diaz, PhD; Lark L. Coffey, PhD; Nathan Burkett-Cadena, PhD; and Jonathan F. Day, PhD, have disclosed no relevant financial relationships.
\end{abstract}

We summarize and analyze historical and current data regarding the reemergence of St. Louis encephalitis virus (SLEV; genus Flavivirus) in the Americas. Historically, SLEV caused encephalitis outbreaks in the United States; however, it was not considered a public health concern in the rest of the

Author affiliations: Universidad Nacional de Córdoba, Córdoba, Argentina (A. Diaz); University of California, Davis, California, USA (L.L. Coffey); University of Florida, Vero Beach, Florida, USA (N. Burkett-Cadena, J.F. Day)

DOI: https://doi.org/10.3201/eid2412.180372
Americas. After the introduction of West Nile virus in 1999, activity of SLEV decreased considerably in the United States. During 2014-2015, SLEV caused a human outbreak in Arizona and caused isolated human cases in California in 2016 and 2017. Phylogenetic analyses indicate that the emerging SLEV in the western United States is related to the epidemic strains isolated during a human encephalitis outbreak in Córdoba, Argentina, in 2005. Ecoepidemiologic studies suggest that the emergence of SLEV in Argentina was caused by the introduction of a more pathogenic strain and increasing populations of the eared dove (amplifying host). 
$\mathrm{T}$ he disease known as St. Louis encephalitis (SLE) is caused by St. Louis encephalitis virus (SLEV), identified as the causative agent of a mosquitoborne viral epidemic in St. Louis, Missouri, USA, during the summer of 1933 (1). SLEV is transmitted by numerous mosquito species in the genus Culex and is amplified by passerine and columbiform avian species (1). Phylogenetic analysis based on the full-length E gene sequences grouped SLEV strains into 8 genotypes (2). Genotypes I and II are prevalent in the United States and genotype $\mathrm{V}$ is widely distributed in South America. Other genotypes have limited distribution: genotype III is in southern South America, IV is limited to Colombia and Panama, VI is in Panama, VII is in Argentina, and VIII has been detected only in the Amazon region of Brazil (2).

A retrospective analysis revealed that 38 human cases and 14 deaths were caused by SLEV in Paris, Illinois, USA, during the summer of 1932 (3). A 1933 SLE epidemic resulted in 1,095 clinical human cases and 201 deaths (3). Because subclinical cases are not identified or reported, retrospective serosurveys were conducted to determine the ratio of subclinical to clinical infections, which was determined to be $300: 1$ (3). Using this ratio, the actual number of SLE cases during the 1933 SLE epidemic was $\approx 328,500$, affecting nearly $40 \%$ of the city's 821,960 inhabitants, based on US census data for 1930 (4).

Since SLEV was first identified, 4 human SLE transmission scenarios have been reported (5). First, during most years, no human SLE infections are reported. However, SLEV transmission to sentinel animals and virus isolation from mosquito pools is documented in the absence of human cases. Second, small numbers of spatially and temporally isolated human SLE cases occur. For example, in 1993, 8 human SLE cases were reported in Lee and Collier counties, Florida, USA; 5 of the cases reported onset during October (6). Third, sporadic transmission occurs as widely dispersed (temporally and spatially) individual human cases. For example, in 1997, 9 human SLE cases were reported from 6 Florida counties, ranging from Brevard County on the central Atlantic Coast south to Lee County on the southern Gulf Coast. Onset for these cases ranged from July through late October (6). Finally, epidemic transmission occurs as focused (in space and time) clusters of human clinical cases: for example, an extensive 1975 epidemic that occurred along the Mississippi and Ohio River basins from Ontario, Canada; Cleveland, Ohio; and Chicago, Illinois, in the north to Birmingham, Alabama, and Mississippi in the south. Well-documented SLE outbreaks include the 1933 St. Louis epidemic and the 1959, 1961, 1962, 1977, and 1990 epidemics in southern Florida. Other epidemics of note occurred in St. Louis (1937); Hidalgo County, Texas (1954); High Plains, Texas; Louisville, Kentucky; and Cameron County, Texas (1956); and Houston,
Texas (1964). There was also a 1990 SLE epidemic in Florida (5), and, most recently, a 2015 epidemic in the Phoenix area of Arizona (Figure 1).

Epidemics of SLE are promoted by environmental factors including summer temperature, rainfall, snowmelt, and surface water conditions $(7,8)$. One of the most notable environmental drivers for SLEV activity is the cycling of rainfall and drought. The wet-dry cycle can affect the epidemiology of SLEV by forcing gravid floodwater Culex vectors to delay oviposition long enough to complete viral development (extrinsic incubation) in a single gonotrophic cycle, thus making them capable of viral transmission during their second blood meal (8). Drought has also been linked to urban SLE and West Nile virus (WNV) epidemics involving vectors in the $C x$. pipiens complex (9).

\section{Transmission Cycles}

The 4 primary vectors of SLEV in the United States are $C x$. pipiens pipiens Linnaeus, $C x$. pipiens quinquefasciatus Say, $C x$. tarsalis Coquillett, and $C x$. nigripalpus Theobald mosquitoes (1). Vector species distribution determines the geographic distribution of SLEV, and affects whether epidemics are urban or rural. Urban SLE epidemics usually involve $C x$. pipiens pipiens and $C x$. pipiens quinquefasciatus ( $C x$. pipiens complex) mosquitoes, species that oviposit in the permanent aquatic habitats provided by storm drains, sewage treatment facilities, and wastewater retention ponds. Rural SLE epidemics usually involve floodwater species, such as $C x$. tarsalis and $C x$. nigripalpus mosquitoes (1). Urban and rural epidemic transmission patterns are best demonstrated by the history of SLE in Florida, where urban human SLEV cases were detected in Miami in 1952 and 1954, followed by St. Petersburg in 1959, Tampa in 1961, and Sarasota in 1962. In 1977, SLEV epidemic transmission in Florida shifted from urban to rural areas (7). The 1977 and 1990 Florida SLE epidemics both started in Indian River County and then spread throughout the Florida peninsula (7). The temporal shift from urban to rural epidemic transmission in Florida was facilitated by changes in $C x$. nigripalpus oviposition preference and behavior. The $C x$. nigripalpus mosquito is a widespread subtropical species and a highly opportunistic blood feeder that oviposits in freshly flooded temporary aquatic habitats. In urban habitats, $C x$. nigripalpus mosquitoes oviposit in wastewater retention ponds and open wastewater outflow ditches (10). The shift to rural transmission in 1977 and 1990 was facilitated by an increase in citrus farming, as citrus groves were designed to be maintained by flood irrigation (10). In the 1970s through the 1990s, rural citrus grove drainage furrows became the preferred oviposition site for $C x$. nigripalpus mosquitoes (11). The proclivity of $C x$. nigripalpus mosquitoes to blood-feed on birds as well as mammals and the tendency of females to delay oviposition 


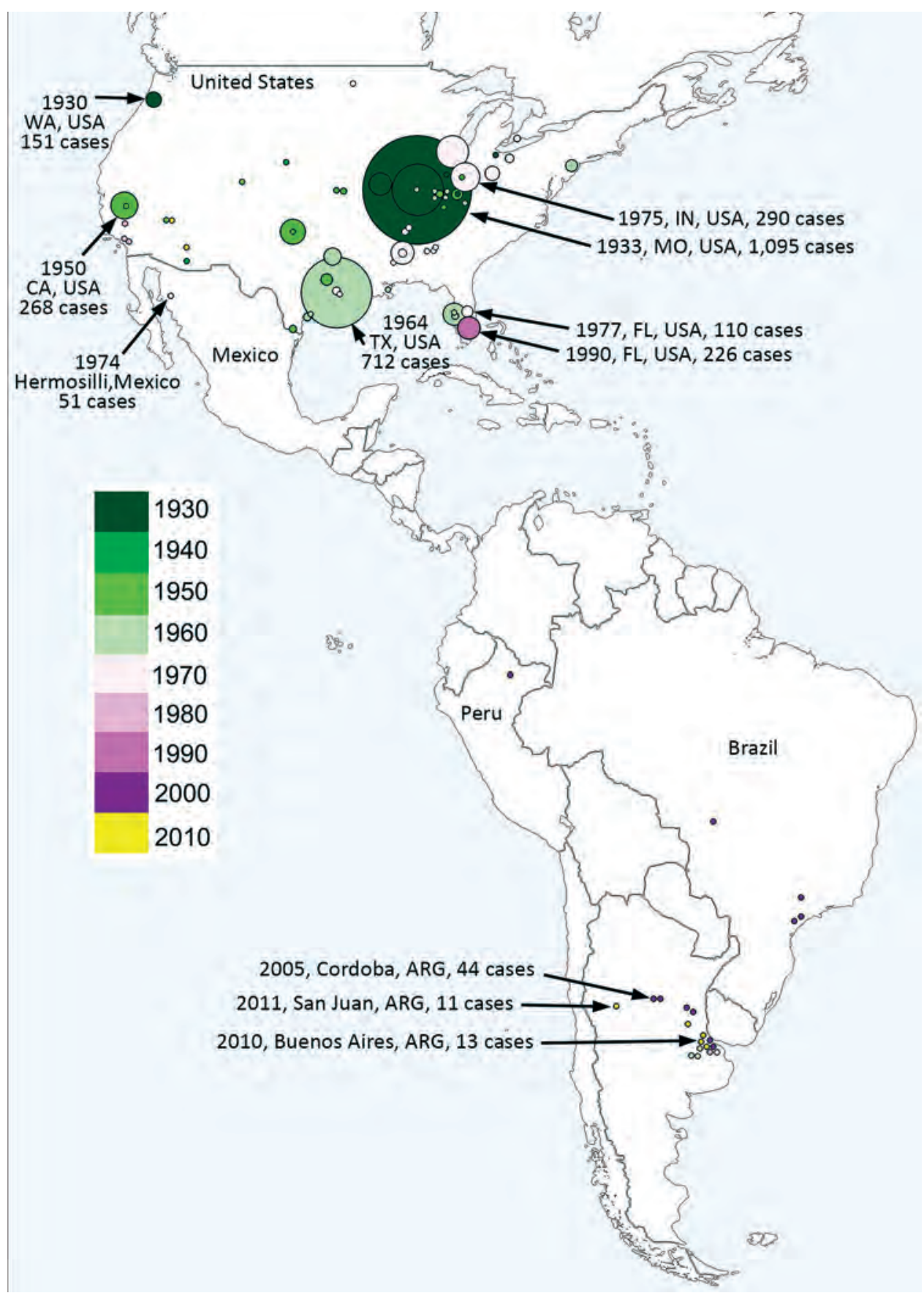

Figure 1. Geographic distribution of historical St. Louis encephalitis human cases reported in the Americas through November 2017. Dot size represents the number of human cases reported in each episode. Colors represent year of detection.

until the proper aquatic oviposition habitats are created by heavy summer rainfalls make it an excellent vector of SLEV (11).

The introduction of WNV into the United States in 1999 promoted debate about how the presence of WNV would affect the continued transmission of SLEV, given the serologic cross-reactivity of the 2 viruses in avian hosts. Laboratory studies demonstrated that house finches (Haemorhous mexicanus) experimentally infected with WNV developed neutralizing immunity that prevented infection after rechallenge with WNV or SLEV (12). In contrast, house finches first exposed to SLEV showed lower subsequent viremias after rechallenge with WNV. This suggests that WNV could competitively exclude SLEV from amplification in shared avian hosts like house finches (13). Indeed, transmission of SLEV in Florida decreased notably following the introduction and establishment of WNV (13). For competitive exclusion to suppress SLEV transmission, considerable host overlap between SLEV and WNV must occur. In Florida, avian species in the families 
Cardinalidae (northern cardinal, Cardinalis cardinalis), Columbidae (mourning dove, Zenaida macroura), Corvidae (blue jay, Cyanocitta cristata), and Icteridae (common grackle, Quiscalus quiscula) are frequently exposed to SLEV (14). For WNV, house sparrows (Passer domesticus) and blue jays are highly infectious to mosquitoes; in contrast, mourning doves were found to be 1 of the least competent WNV hosts of 25 bird species examined experimentally (15). Even if mourning doves are poor WNV hosts, being exposed to WNV may provide cross neutralization to SLEV and preempt amplification of SLEV, provided that doves are exposed to WNV early in the transmission season. In California, sparrows, finches, and corvids (house finch, house sparrow, purple finch [Haemorhous purpureus]); song sparrow (Melospiza melodia); western scrub jay (Aphelocoma californica); and white-crowned sparrow (Zonotrichia leucophrys) are considered major amplifying hosts of WNV and SLEV $(1,16)$. As in Florida, host overlap of WNV and SLEV likely contributed to the initial disappearance of SLEV from the western United States (17). Of these avian hosts, only house sparrows, house finches, American crows (Corvus brachyrhynchos), and western scrub jays were found to be amplifying hosts capable of developing viremias above the threshold for infection of $C x$. tarsalis, the principal vector of WNV and SLEV throughout much of California (16). Further work is needed to characterize the specific avian hosts fed on by $C x$. pipiens quinquefasciatus and $C x$. tarsalis mosquitoes in epidemic and nonepidemic settings to determine whether additional host species may be involved in amplification of SLEV and to what degree host overlap contributes to the competitive exclusion or reemergence of SLEV in areas of the United States outside California.

Since the introduction of WNV into the United States, human SLE cases continue to occur throughout the country. During 2004-2013, 92 clinical SLE cases were reported (18). Most cases were located in the Gulf Coast states of Louisiana (10 cases), Mississippi (13 cases), and Texas (16 cases). During 2014-2016, a total of 32 human SLE cases were reported (19). Most of these cases were reported in the Phoenix area, where a SLE epidemic resulted in 23 confirmed human cases, including 1 fatality (19). SLEV has remained endemic throughout much of the United States despite the introduction and establishment of WNV.

\section{Emergence and Reemergence in South America}

Historically, SLEV has not been considered a major public health threat in the Americas, other than in the United States. Human SLE cases have been reported sporadically throughout Latin America, but no human epidemics were reported until 2005. In Argentina, Charosky et al. (20) reported a neurologic human SLEV case in 1968. Two years later, Mettler et al. (21) isolated SLEV from a febrile human suspected to have Argentinean hemorrhagic fever. Human encephalitis cases were reported sporadically until 2005, when an unprecedented outbreak of SLE was reported in Córdoba City, Argentina (22). During this outbreak, signs and symptoms associated with a neurologic infection, including headache, sensory depression, temporal-spatial disorientation, tremors, and changes in consciousness, were reported. A correlation between age and sign or symptom severity was detected (Spearman coefficient $=0.74)(22)$. The frequency of encephalomyelitis varied from $80 \%$ of the cases in patients $<20$ years of age to $95 \%$ in those $>60$ years of age (22). A total of 47 probable and confirmed human cases were reported. Of these, 45 patients were hospitalized; 9 died, 125 years of age and $8>50$ years of age (22).

The SLEV strains CbaAr-4006 and CbaAr-4006 were isolated from $C x$. pipiens quinquefasciatus mosquitoes collected during the outbreak in the backyard of the index case-patient in Córdoba City (23). Molecular classification and phylogenetic analyses indicated that the strains isolated during the outbreak were closely related to a genotype III SLEV strain (79V-2533) that had been isolated in Santa Fe Province, Argentina (23). A 3-year retrospective phylogenetic analysis of SLEV genomes from mosquitoes collected in Córdoba City, designed to find an enzootic progenitor of the outbreak strains, indicated no circulation of genotype III before the 2005 outbreak (24). The extensive sampling effort detected low levels of SLEV transmission of genotypes other than genotype III. The CbaAr-4005 strain was more virulent and produced higher viremias in avian and murine models. In house sparrows, CbaAr-4005 produced viremias that were 2.2 logs higher and lasted for 2 days longer than its closest relative, the $197879 \mathrm{~V}-2533$ strain. Because mosquitoes show a dose response to infection, house sparrows inoculated with SLEV strain CbaAr-4005 will theoretically produce viremia sufficient to infect 10 times more mosquitoes than those inoculated with a nonepidemic strain (25).

In adult Swiss mice, the CbaAr-4005 strain of SLEV resulted in $100 \%(10 / 10)$ illness and death compared with sympatric nonepidemic SLEV strains isolated previously in Córdoba and Santa Fe Province, Argentina (26). Inoculation of only 1 plaque-forming unit in 10-day-old mice or 2 plaque-forming units in 21-day-old mice caused a $50 \%$ death rate with the strain CbaAr-4005 (lethal dose 50 $\left[\mathrm{LD}_{50}\right]$ in 10-day-old mice $=0.02$ ), proving it to be the most lethal strain compared in the study $\left(78 \mathrm{~V}-6507_{\mathrm{LD} 50}=1.75\right.$; CorAn-9275 ${ }_{\mathrm{LD} 50}=3.90$ ). That evidence supports the hypothesis that a more virulent SLEV strain was introduced into Córdoba City in early 2005 from Santa Fe (Argentina) or São Paulo (Brazil) (Figure 2). Serologic studies of wild birds sampled before and during the 2005 outbreak indicated that $99 \%(434 / 437)$ of the avian population lacked a SLEV-neutralizing antibody and were therefore 


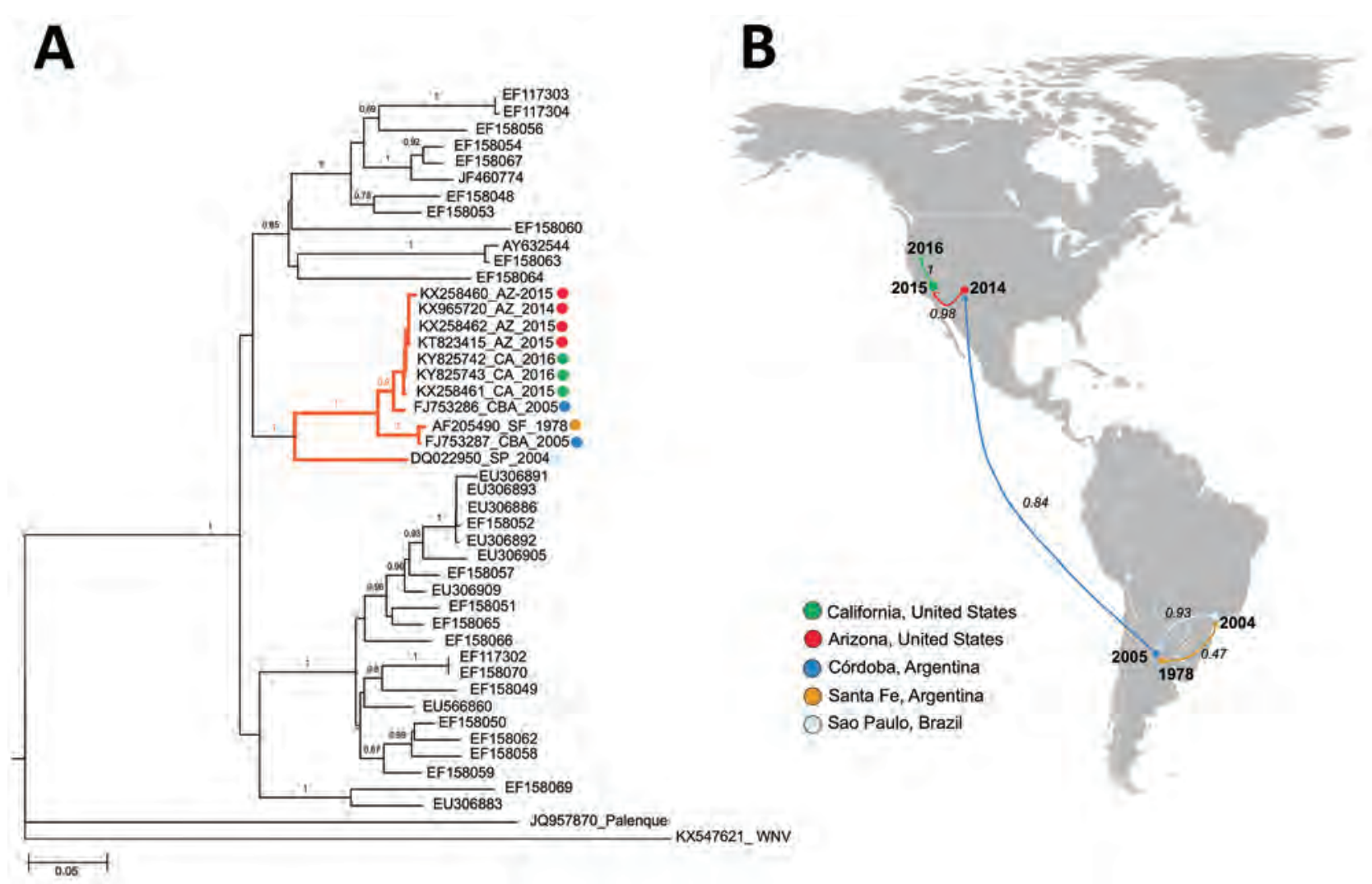

Figure 2. Phylogeny and spread of St. Louis encephalitis virus (SLEV) in the Americas. A) Multiple sequence alignment of 44 complete envelope protein SLEV sequences obtained from GenBank. The orange highlighted cluster contained the emerging SLEV strains isolated in Argentina, Brazil, and western United States. Alignment was performed by ClustalX, followed by tree generation using a neighbor-joining algorithm using MEGA 6 software (https://www.megasoftware.net). Sequences are labeled by their GenBank accession number. Sequences belonging to lineage III also contain place and year of isolation data. Bootstrap support values are given for each node. Scale bar represents nucleotide substitutions per site. B) Geographic spread of SLEV. A discrete Bayesian phylogeographic reconstruction for SLEV lineage III was made using 11 envelope protein sequences (highlighted cluster in Figure 2, panel A). We applied a constant-size coalescent tree before the phylogeny and a TNF93 nt substitution model. The Monte Carlo Markov chain model was obtained after 30 million iterations and subsampling every 20.000 iterations. Analyses were made using Beast version 1.8 .3 software (http://tree.bio.ed.ac.uk/software/beast/). Numbers over the arrows indicate the probability for the postulated viral dispersion in that pathway. Color of arrows indicates the location origin for the strain introduced.

susceptible to infection with SLEV, potentially serving as amplification hosts (27). The absence of natural immunity in the wild avian population may have promoted the 2005 SLE outbreak in Córdoba City. A host competence study in avian species showed that the eared dove (Zenaida auriculata) and picui ground-dove (Columbina picui) produce high levels of viremia compared with other avian species tested (28). Moreover, that study confirmed the role as amplifying host of these 2 dove species during the 2005 SLEV outbreak in Córdoba. Additional studies indicate that the population of eared doves has been increasing in the central region of Argentina during the past 10 years because of agricultural geographic expansion (29).

After the 2005 outbreak, additional SLE outbreaks in Argentina occurred in Parana (2006), Buenos Aires (2010), and San Juan (2011) (Pan American Health Organization, http://www.paho.org/hq/dmdocuments/2010/ alert_ epi_2010_31_marz_encefalitis_san_luis.pdf), but no ecologic studies focused on these outbreaks. Phylogenetic analysis of SLEV genomes associated with human cases in Buenos Aires confirmed the presence of SLEV genotype III (30).

SLE was diagnosed in a febrile human in São Paulo State, Brazil, in 2004, by viral isolation and molecular detection (31). Molecular characterization classified the isolate as SLEV genotype III. During a dengue virus outbreak in São José do Rio Preto (São Paulo State, Brazil) in 2006, Mondini et al. (32) reported the first outbreak of SLE in Brazil. Human cases were diagnosed by molecular detection of SLEV RNA in serum or cerebrospinal fluid. All SLEV-infected patients (6) had an initial diagnosis of dengue fever or viral encephalitis; 3 cases were diagnosed as viral meningoencephalitis, and the other 3 patients had signs of hemorrhagic disease (32). This finding was the 
first reported link of SLE infection and human hemorrhagic disease (32). A blastn analysis (https://blast.ncbi.nlm. nih.gov/Blast.cgi) comparing genomes of different SLEV strains, which we carried out for this review, indicates that the SLEV strains from Brazil are closely related to SLEV genotype V. This finding indicates that genotype V SLEV strains are also pathogenic for humans, as shown in previous studies in murine models (26). Because genotype $\mathrm{V}$ strains are widespread in South America, human SLEV cases could be misdiagnosed as dengue virus infection. A detailed analysis of South America human SLE cases is shown in Figure 1.

\section{Reemergence in Western United States}

SLEV was recognized in 1937 in California; cases were documented during 1940-1950 and in Kern County during a 1952 outbreak of Western equine encephalitis virus (33). SLEV caused periodic epidemics in humans and epizootics in equines during the 1950s1990s. However, expansive epidemics similar to those observed in Missouri and Texas were not detected in California, although concurrent activity in mosquitoes and seroconversions in birds were repeatedly documented in the San Joaquin Valley, Los Angeles Basin, and Imperial Valley (1,34-36). An outbreak resulting in 26 human cases occurred in 1984 in Los Angeles, (34), leading to avian studies (37), vector competence experiments (38), and vectoral capacity studies (39). A SLEV serosurvey following the Los Angeles outbreak found that $1.6 \%$ of 1,803 serum samples tested from residents of Los Angeles were seropositive. A subsequent outbreak of 26 confirmed SLEV cases was centered in Kern County but disappeared the following summer $(35,36)$. Envelope gene sequencing from mosquito isolates suggested that different SLEV strains were most likely responsible for these outbreaks (40). Sequencing of longitudinal SLEV isolates from California showed that isolates were genetically similar from 1952 through the 1970s, after which genetic changes were observed (41).

Even in the absence of epidemics since 1989, SLEV activity was documented continuously in California from the 1990s through 2003 by human cases, detections in mosquitoes, or seroconversions in sentinel chickens (42). In 2003, WNV activity was first detected in the state (17). During 2003-2015, no SLEV activity was detected in California, despite ongoing SLEV surveillance in Culex mosquitoes and sentinel chicken seroconversions (17). The absence of SLEV activity during that period was likely not the result of a lack of surveillance because the invasion of WNV led to a $\approx 6$-fold statewide increase in mosquito pool testing, from $\approx 5,000$ pools in 2003 to 30,000-35,000 pools annually since 2007 .
Instead, the absence of SLEV activity suggested extirpation from California.

Beginning in July 2015, SLEV activity was detected by the presence of viral RNA in mosquito pools and sentinel chicken seroconversions in the Coachella Valley of Riverside County, California (43). In 2016, SLEV spread northward to 6 additional counties in California (44); in 2017, a total of 15 California counties reported activity (45). A clinical study used unbiased clinical testing by metagenomic next-generation sequencing to diagnose a fatal case of meningoencephalitis from SLEV in a patient from Kern County, California, in September 2016 (46).

In Arizona, SLEV detection was historically less frequent than in California; low enzootic activity was reported most years during 1972-2006 and only a single human case during 2010-2014 (47). In Maricopa County (which includes Phoenix), a human SLEV outbreak during July-October 2015 resulted in 23 confirmed cases (48). Three patients in that outbreak were organ transplant recipients who experienced fever, rigors, diarrhea, headache, and confusion; all developed meningoencephalitis, and 1 patient died (48). SLEV infection in the 2 surviving transfusion recipients was confirmed by plaque reduction neutralization tests (48). Retrospective testing of archived mosquito pools from Phoenix collected in 2014 revealed a single SLEV isolate, indicating that SLEV was present in Arizona the summer before the 2015 outbreak (48).

To define the genetic relatedness of reemerging SLEV from Arizona and California to SLEV from elsewhere to infer a possible origin and pattern of spread, we performed phylogenetic analyses of genomes from mosquitoes in 2014-2016 and the fatal case in 2016 in Kern County. The 2014 and 2015 California and Arizona SLEV isolates share $>99 \%$ nucleotide identity with each other and also with their closest published relative isolated from $C x$. pipiens quinquefasciatus mosquitoes collected in the 2005 epidemic in Córdoba City (49). The SLEV genome sequence from the fatal case in Kern County from September 2016 was $>99 \%$ identical with 2014-2015 SLEV isolates from mosquitoes in California and Arizona, suggesting that the patient was infected by the reemergent genotype circulating in the southwestern United States (46). The 2014 and 2015 SLEV isolates are genetically distinct from the 2003 Imperial Valley, California, strain that was isolated before the 11-year absence of SLEV activity in the state (49). These results suggest there was likely a single introduction of SLEV into the United States from South America, and possibly Argentina, no later than November 2014, the earliest dated sample from which SLEV was isolated in Arizona and that the virus spread in the summer of 2015 from Arizona to California (49). 


\section{Conclusion and Future Perspectives}

Arthropodborne virus infections are emerging and reemerging infectious diseases worldwide. Mosquitoborne viruses, including dengue (emerged in 1990), WNV (emerged in 1999), chikungunya (2013), and Zika viruses (2015), have emerged as public health threats in the Western Hemisphere. SLEV is maintained in a bird-mosquito transmission network involving multiple species in a broad range of ecosystems encompassing a wide geographic distribution that ranges from southern Canada to southern Argentina. Biotic factors, including vector and host abundance and population age structure, as well as abiotic factors, including rainfall and drought dynamics and elevated summer temperatures, combine to produce conditions favorable for transmission of SLEV. A better understanding of how SLEV circulates between enzootic transmission cycles in nature and epidemic transmission in human populations is needed to more accurately predict where and when human SLEV epidemics will emerge.

The reemergence of SLEV in central Argentina is associated with several factors, including the recent introduction of a more virulent strain of SLEV into a naive bird community and increased populations of eared doves, a highly susceptible amplification species in Argentina. Argentina has experienced intense land use changes primarily because of the expansion of agricultural and urbanized habitats. More research is needed to define the effects of environmental change on avian reservoir and vector populations to clarify the dynamics of SLEV transmission, introduction, reintroduction, and reemergence in susceptible habitats throughout the Western Hemisphere.

The reemergence of SLEV in California and Arizona resulted from introduction of a South American strain of SLEV. The genetic diversity of SLEV in the Americas is spatially influenced, with wide genetic variation across the space, but some SLEV strains from North and South America show high genetic similarity, indicating long-range dispersal. Similar to WNV, long-range SLEV dispersal is likely mediated by migrating SLEV-infected birds. A better understanding of SLEV in wild birds and avian host migration patterns is necessary to predict the spread of SLEV.

\section{About the Author}

Dr. Diaz, a biologist, is an adjunct researcher at the National Council for Scientific and Technical Researches (CONICET) and associate professor at the Instituto de Virología Dr. J.M. Vanella, Universidad Nacional de Córdoba, Argentina, where he is in charge of the Arbovirus Laboratory. His research interests include ecology and phylogeography of viral zoonoses and biological interactions among vectors, hosts, and viruses.

\section{References}

1. Reisen WK. Epidemiology of St. Louis encephalitis virus. Adv Virus Res. 2003;61:139-83. http://dx.doi.org/10.1016/ S0065-3527(03)61004-3

2. Rodrigues SG, Nunes MR, Casseb SM, Prazeres AS, Rodrigues DS, Silva MO, et al. Molecular epidemiology of Saint Louis encephalitis virus in the Brazilian Amazon: genetic divergence and dispersal. J Gen Virol. 2010;91:2420-7. http://dx.doi.org/10.1099/vir.0.019117-0

3. Chamberlain RW. History of St. Louis encephalitis. In: Monath TP, editor. St. Louis encephalitis. Washington (DC): American Public Health Association;1980. p. 680.

4. Monath TP, Tsai TF. Flaviviruses. In: Richman DD, Whitley RJ, Hayden FG, editors. Clinical Virology. New York: ChurchillLivingstone;1997. p. 1133-86.

5. Day JF, Stark LM. Frequency of Saint Louis encephalitis virus in humans from Florida, USA: 1990-1999. J Med Entomol. 2000;37:626-33. http://dx.doi.org/10.1603/0022-2585-37.4.626

6. Day JF, Curtis GA. Blood feeding and oviposition by Culex nigripalpus (Diptera: Culicidae) blood feeing and oviposition before, during and after a widespread St. Louis encephalitis epidemic in Florida.J Med Entomol. 1999;36:176-81. http://dx.doi.org/10.1093/jmedent/36.2.176

7. Day JF. Predicting St. Louis encephalitis virus epidemics: lessons from recent, and not so recent, outbreaks. Annu Rev Entomol. 2001;46:111-38.http://dx.doi.org/10.1146/ annurev.ento.46.1.111

8. Shaman J, Day JF, Stieglitz M. Drought-induced amplification of Saint Louis encephalitis virus, Florida.Emerg Infect Dis. 2002;8:575-80. http://dx.doi.org/10.3201/eid0806.010417

9. Epstein PR, Defilippo C. West Nile virus and drought Glob Change Hum Health. 2001;2:105-7. http://dx.doi.org/ 10.1023/A:1015089901425

10. Curtis GA. Habitat selection strategies of mosquitoes Inhabiting citrus irrigation furrows.J Am Mosq Control Assoc. 1985;1:169-73.

11 Day JF, Curtis GA. When it rains, they soar-and that makes Culex nigripalpus a dangerous mosquito.Am Entomol. 1994;40:162-7. http://dx.doi.org/10.1093/ae/40.3.162

12. Fang Y, Reisen WK. Previous infection with West Nile or St. Louis encephalitis viruses provides cross protection during reinfection in house finches. Am J Trop Med Hyg. 2006;75:480-5. http://dx.doi.org/10.4269/ajtmh.2006.75.480

13. Ottendorfer CL. Impact of the West Nile virus on the natural history of St. Louis encephalitis virus in Florida [ddissertation]. Tampa (FL): University of South Florida; 2008 [cited 2018 Oct 2]. https://scholarcommons.usf.edu/cgi/viewcontent.cgi?referer= https://www.google.com/\&httpsredir $=1$ \&article=1437\&context=etd

14. Day JF, Stark LM. Avian serology in a St. Louis encephalitis epicenter before, during, and after a widespread epidemic in south Florida, USA.J Med Entomol. 1999;36:614-24. http://dx.doi.org/ 10.1093/jmedent/36.5.614

15. Komar N, Langevin S, Hinten S, Nemeth N, Edwards E, Hettler D, et al. Experimental infection of North American birds with the New York 1999 strain of West Nile virus.Emerg Infect Dis. 2003; 9:311-22.http://dx.doi.org/10.3201/eid0903.020628

16. Reisen WK, Chiles RE, Martinez VM, Fang Y, Green EN. Experimental infection of California birds with western equine encephalomyelitis and St. Louis encephalitis viruses. J Med Entomol. 2003;40:968-82.http://dx.doi.org/10.1603/ 0022-2585-40.6.968

17. Reisen WK, Lothrop HD, Wheeler SS, Kennsington M, Gutierrez A, Fang Y, et al. Persistent West Nile virus transmission and the apparent displacement St. Louis encephalitis virus in southeastern California, 2003-2006.J Med Entomol. 2008; 45:494-508. 
18. Centers for Disease Control and Prevention. Saint Louis encephalitis; 2015[cited 2018 Jul 27]. https://www.cdc.gov/sle/ technical/epi.html

19. Centers for Disease Control and Prevention. Saint Louis encephalitis virus: epidemiology and geographic distribution; 2017 [cited 2018 Jul 27]. https://www.cdc.gov/sle/technical/epi.html

20. Charosky L, Baldassari EL, KorenF, Mettler NE, Loizaga C. Síndrome encefálico a posible etiología por virus San Luis. Rev Asoc Med Argent. 1968;82:267-9.

21. Mettler NE, Casals J. Isolation of St. Louis encephalitis virus from man in Argentina.Acta Virol. 1971;15:148-54.

22. Spinsanti LI, Díaz LA, Glatstein N, Arselán S, Morales MA, Farías AA, et al.Human outbreak of St. Louis encephalitis detected in Argentina, 2005.J Clin Virol. 2008;42:27-33. http://dx.doi.org/10.1016/j.jcv.2007.11.022

23. DiazL A, Ré V, Almirón WR, Farías A, Vázquez A, Paz Sanchez-Seco M, et al.Genotype III Saint Louis encephalitis virus outbreak, Argentina, 2005.Emerg Infect Dis. 2006; 12:1752-4.http://dx.doi.org/10.3201/eid1211.060486

24. Díaz LA, Albrieu Llinás G, Vázquez A, Tenorio A, Contigiani MS. Silent circulation of St. Louis encephalitis virus prior to an encephalitis outbreak in Cordoba, Argentina (2005).PLoS Negl Trop Dis. 2012;6:e1489.http://dx.doi.org/10.1371/ journal.pntd.0001489

25. Diaz LA, Nemeth NM, Bowen RA, Almiron WR, Contigiani MS. Comparison of Argentinean Saint Louis encephalitis virus non-epidemic and epidemic strain infections in an avian model. PLoS Neg1 Trop Dis. 2011;5:e1177.http://dx.doi.org/10.1371/ journal.pntd.0001177

26. Rivarola ME, Tauro LB, Llinás GA, Contigiani MS. Virulence variation among epidemic and non-epidemic strains of Saint Louis encephalitis virus circulating in Argentina. Mem Inst Oswaldo Cruz. 2014;109:197-201.http://dx.doi.org/10.1590/ 0074-0276130475

27. Diaz LA, Quaglia AI, Konigheim BS, Boris AS, Aguilar JJ, Komar N, et al. Activity patterns of St. Louis encephalitis and West Nile viruses in free ranging birds during a human encephalitis outbreak in Argentina.PLoS One. 2016;11:e0161871. http://dx.doi.org/10.1371/journal.pone.0161871

28. Díaz A, Flores FS, Quaglia AI, Contigiani MS. Evaluation of Argentinean bird species as amplifying hosts for St. Louis encephalitis virus (Flavivirus, Flaviviridae). Am J Trop Med Hyg. 2018; 99:216-21.http://dx.doi.org/10.4269/ ajtmh.17-0856

29. Calamari NC, Dardanelli S, Canavelli SB. Variaciones en la abundancia poblacional de palomas medianas a lo largo del tiempo. INTA EEA Paraná.Serie Extensión. 2011;64:23-8.

30. Valinotto LE, Barrero PR, Viegas M, Alvarez López MC, Mistchenko AS. Molecular evidence of St. Louis encephalitis virus infection in patients in Buenos Aires, Argentina.J Clin Virol. 2012;54:349-51. http://dx.doi.org/10.1016/j.jcv.2012.04.005

31. Santos CL, Sallum MA, Franco HM, Oshiro FM, Rocco IM. Genetic characterization of St. Louis encephalitis virus isolated from human in São Paulo, Brazil.Mem Inst Oswaldo Cruz. 2006; 101:57-63. http://dx.doi.org/10.1590/S0074-02762006000100011

32. Mondini A, Cardeal IL, Lázaro E, Nunes SH, Moreira CC, Rahal P, et al.Saint Louis encephalitis virus, Brazil.Emerg Infect Dis. 2007;13:176-8.http://dx.doi.org/10.3201/eid1301.060905

33. ReevesWC, Hammon WM, Longshore WA Jr, McCLUREHE, Geib AF. Epidemiology of the arthropod-borne viral encephalitides in Kern County, California, 1943-1952. Publ Public Health Univ Calif. 1962;4:1-257.

34. Murray RA, Habel LA, Mackey KJ, Wallace HG, Peck BA, Mora SJ, et al. Epidemiological aspects of the 1984 St Louis encephalitis epidemic in southern California. Proc Calif Mosq Vector Control Assoc.1985;53:5-9.
35. Reisen WK, Meyer RP, Milby MM, Presser SB, Emmons RW, Hardy JL, et al. Ecological observations on the 1989 outbreak of St. Louis encephalitis virus in the southern San Joaquin Valley of California.J Med Entomol. 1992;29:472-82. http://dx.doi.org/10.1093/jmedent/29.3.472

36. Tueller JE. An outbreak of illness due to St. Louis encephalitis virus in the southern San Joaquin Valley, California, 1989.Proc Calif Mosq Vector Control Assoc.1990;58:12.

37. McLean RG, Webb JP, Campos EG, Gruwell J, Francy DB, Womeldorf D, et al. Antibody prevalence of St. Louis encephalitis virus in avian hosts in Los Angeles, California, 1986. J Am Mosq Control Assoc. 1988;4:524-8.

38. Hardy JL, Meyer RP, Reisen WK, Presser SB. A further evaluation of Culex mosquitoes in the greater Los Angeles area for their ability to vector St. Louis encephalitis virus. Proc Calif Mosq Vector Control Assoc.1986;54:9-10.

39. Reisen WK, Milby MM, Presser SB, Hardy JL. Ecology of mosquitoes and St. Louis encephalitis virus in the Los Angeles Basin of California, 1987-1990. J Med Entomol. 1992;29:582-98. http://dx.doi.org/10.1093/jmedent/29.4.582

40. Reisen WK, Lothrop HD, Chiles RE, Cusack R, Green EG, Fang Y, et al.Persistence and amplification of St. Louis encephalitis virus in the Coachella Valley of California, 2000-2001.J Med Entomol. 2002;39:793-805. http://dx.doi.org/ 10.1603/0022-2585-39.5.793

41. Kramer LD, Presser SB, Hardy JL, Jackson AO. Genotypic and phenotypic variation of selected Saint Louis encephalitis viral strains isolated in California. Am J Trop Med Hyg. 1997;57:222-9. http://dx.doi.org/10.4269/ajtmh.1997.57.222

42. Reisen W, Lothrop H, Chiles R, Madon M, Cossen C, Woods L, et al. West Nile virus in California.Emerg Infect Dis. 2004; 10:1369-78. http://dx.doi.org/10.3201/eid1008.040077

43. Feiszli T, Padgett K, Simpson J, Barker CM, Fang Y, Salas M, et al. Surveillance for mosquito-borne encephalitis virus activity in California, 2015.Proc Papers Mosq Vector Control Assoc Calif. 2016;84:124-9.

44. Feiszli T, Padgett K, Simpson J, Barker CM, Fang Y, Salas M, et al. Surveillance for mosquito-borne encephalitis virus activity in California, 2016. Proc Papers Mosq Vector Control Assoc Calif. 2017;85:9-14.

45. Saint Louis encephalitis virus (SLEV) in California counties 2017 YTD; updated Jan 2018[cited 2018 Jul 27]. http://westnile.ca.gov/ downloads.php?download_id $=3839 \&$ filename $=2017$ _slev county_map.pdf

46. Chiu CY, Coffey LL, Murkey J, Symmes K, Sample HA, Wilson MR, et al. Diagnosis of fatal human case of St. Louis encephalitis virus infection by metagenomic sequencing, California, 2016.Emerg Infect Dis. 2017;23:1964-8. http://dx.doi.org/10.3201/eid2310.161986

47. Arizona State Public Health Laboratory. Epidemiology and disease control.2014 [cited 2018 Jul 27]. https://www.azdhs.gov/preparedness/epidemiology-disease-control/index.php.

48. Venkat H, Krow-Lucal E, Hennessey M, Jones J, Adams L, Fischer M, et al. Concurrent outbreaks of St. Louis encephalitis virus and West Nile virus disease-Arizona, 2015. MMWR Morb Mortal Wkly Rep. 2015;64:1349-50.

49. White GS, Symmes K, Sun P, Fang Y, Garcia S, Steiner C, et al. Reemergence of St. Louis encephalitis virus, California, 2015.Emerg Infect Dis. 2016;22:2185-8.http://dx.doi.org/10.3201/ eid2212.160805

Address for correspondence: Adrián Diaz, National University of Córdoba-Arbovirus Laboratory, Institute of Virology Dr. J.M. Vanella, School of Medicine, Enfermera Gordillo Gómez s/n, CPX5016GCA, Córdoba, Argentina; email: adrian.diaz@conicet.gov.ar 


\title{
Autochthonous Human Case of Seoul Virus I nfection, the Netherlands
}

\author{
Caroline Swanink, Johan Reimerink, Jet Gisolf, Ankje de Vries, Mark Claassen, Liesbeth Martens, \\ Toos Waegemaekers, Harry Rozendaal, Stasja Valkenburgh, Tabitha Hoornweg, Miriam Maas
}

\begin{abstract}
Orthohantaviruses are a group of rodentborne viruses with a worldwide distribution. The orthohantavirus Seoul virus (SEOV) can cause hemorrhagic fever with renal syndrome in humans and is distributed worldwide, like its reservoir host, the rat. Cases of SEOV in wild and pet rats have been described in several countries, and human cases have been reported in the United Kingdom, France, Canada, and the United States. In the Netherlands, SEOV has previously been found in wild brown rats. We describe an autochthonous human case of SEOV infection in the Netherlands. This patient had nonspecific clinical symptoms of an orthohantavirus infection (gastrointestinal symptoms and distinct elevation of liver enzymes). Subsequent source investigation revealed 2 potential sources, the patient's feeder rats and a feeder rat farm. At both sources, a high prevalence of SEOV was found in the rats. The virus closely resembled the Cherwell and Turckheim SEOV strains that were previously found in Europe.
\end{abstract}

$\mathrm{O}$ rthohantaviruses are a family of rodentborne viruses with a worldwide distribution. Human infection can occur when virus-contaminated aerosols of rodent excreta are inhaled while entering or cleaning rodent-infested areas (1). Infection can also be transmitted by rodent bites or when orthohantavirus contaminated materials are directly introduced into broken skin or conjunctiva. The clinical syndromes that are associated with severe disease are hemorrhagic fever with renal syndrome (HFRS) and orthohantavirus cardiopulmonary syndrome (HCPS). HFRS cases are found in large parts of Europe and Asia, whereas HCPS cases are found in North America and South America (2). The orthohantavirus Seoul virus (SEOV) causes HFRS of medium severity and was originally found in Asia. In Europe,

Author affiliations: Rijnstate Hospital, Arnhem, the Netherlands (C. Swanink, J. Gisolf, M. Claassen); National Institute for Public Health and the Environment, Bilthoven, the Netherlands

(J. Reimerink, A. de Vries, T. Hoornweg, M. Maas); Municipal Health Service Gelderland-Midden, Arnhem (L. Martens,

T. Waegemaekers); Dutch Food and Consumer Products Safety Authority, Utrecht, the Netherlands ( $H$. Rozendaal, S. Valkenburgh)

DOI: https://doi.org/10.3201/eid2412.180229 cases of SEOV in wild and pet rats have been described in the United Kingdom, Belgium, Sweden, and France (3-6). Additional outbreaks have been reported in Canada and the United States (7). The first non-laboratory-related human infections with SEOV in Europe were reported in the United Kingdom and France in 2012, although retrospectively earlier cases might have occurred (8-10). From 2013 on, multiple additional human cases were reported in the United Kingdom and France $(3,11,12)$. In the United States, domestic cases of HFRS attributable to SEOV have been described since 1994 (13). In the Netherlands, SEOV has been reported in wild rats $(14,15)$. In this article, we describe an autochthonous human case of SEOV infection in the Netherlands and the subsequent source investigation.

\section{Case Description}

In September 2016, a 28-year-old man sought medical care at Rijnstate Hospital (Arnhem, the Netherlands); he reported having fever, vomiting, abdominal cramps, and diarrhea of 7 days' duration. He had a history of gamma-hydroxubutyrate (GHB) addiction and tobacco use (10 cigarettes/d). He denied drinking alcohol. He had no history of travel. The patient mentioned that he had been bitten regularly while handling live rats at his work at a rat breeding farm and by live rats that he kept for his reptiles at home. He also mentioned he had been swimming in the Rhine River, in which rats can be found. Physical examination revealed a sweating, obese, ill patient with normal blood pressure, tachycardia (113 beats/min), and a temperature of $38.6^{\circ} \mathrm{C}$. No abnormalities on auscultation of heart and lungs or lymphadenopathy were found. Abdominal examination revealed a painful enlarged liver. No abnormalities of the skin were recorded. We have summarized the patient's laboratory test results (Table 1). Ultrasound revealed normal aspect of liver and gall bladder and a slightly enlarged spleen $(17.2 \mathrm{~cm})$. The patient was hospitalized with an initial diagnosis of gastroenteritis, colitis, or leptospirosis. Antibiotic treatment with cefuroxime, metronidazole, and doxycycline was started. Blood cultures remained negative. A serum sample from the patient, taken at admission, tested 
Table 1. Laboratory test results for a patient diagnosed with Seoul virus infection, the Netherlands, September $2016^{*}$

\begin{tabular}{|c|c|c|c|}
\hline Laboratory test & Reference range & Day 1† & Day 3‡ \\
\hline C-reactive protein, $\mathrm{mg} / \mathrm{L}$ & $<10$ & 32 & 59 \\
\hline Leukocytes, $10^{9}$ cells/L & $4.0-11.0$ & 5.0 & 12.3 \\
\hline Lymphocytes, $10^{9}$ cells/L & $1.0-3.5$ & NT & 8.27 \\
\hline Atypical lymphocytes & - & NT & + \\
\hline Platelets, $10^{9}$ cells $/ \mathrm{L}$ & $150-400$ & 72 & 79 \\
\hline Creatinine, $\mu \mathrm{mol} / \mathrm{L}$ & $60-110$ & 78 & 72 \\
\hline Alanine aminotransferase, U/L & $<45$ & 114 & 211 \\
\hline Aspartate aminotransferase, U/L & $<35$ & 123 & 283 \\
\hline Lactate dehydrogenase, U/L & $<250$ & 753 & 1906 \\
\hline Bilirubin, $\mu \mathrm{mol} / \mathrm{L}$ & $<17$ & 12 & 10 \\
\hline Creatinine kinase, U/L & $<170$ & NT & 677 \\
\hline
\end{tabular}

*NT, not tested; -, negative; +, positive.

tDay 1 of hospitalization, 7 days after onset of symptoms.

‡Day 3 of hospitalization, 9 days after onset of symptoms.

negative for hepatitis A, B C, and E viruses; HIV; Treponema pallidum (syphilis); cytomegalovirus; Epstein-Barr virus; and Leptospira spp. The patient did not have signs of acute kidney injury and showed only a mild proteinuria of $0.25 \mathrm{~g} / \mathrm{L}$ in a single urine sample. He was tested for orthohantavirus infection because he mentioned that he was bitten by rats regularly. The father of the patient, who took care of the reptiles occasionally, and the patient's partner, who did not have any contact with the reptiles, were not feeling ill.

\section{Material and Methods}

\section{Human SEOV Diagnostics}

For detection of hantavirus IgG and IgM, we used an immunofluorescent assay (IFA) with mosaic slides containing SEOV and other orthohantaviruses Puumala virus, Sin Nombre virus, Hantaan virus, Dobrava-Belgrade virus, and Saaremaa virus (Euroimmun, Lübeck, Germany), according to the manufacturer's instructions (16). The titer was defined as the last sample dilution for which the fluorescence was identifiable, and a titer $>1: 32$ was considered positive. Serum samples of the patient and 2 close contacts were tested for hantavirus antibodies. Total nucleic acid was extracted from patient serum by using the MagNAPure 96 system (Roche, Basel, Switzerland) and tested for SEOV RNA by using a hantavirus genus-specific real-time reverse transcription PCR (rRT-PCR), as described by Kramski et al. (17).

\section{Investigation of Feeder Rats Owned by the Patient}

Because the patient kept feeder rats at home and these rats are a known source of SEOV infection, the rats were collected for source investigation. At the time of investigation, the patient had 5 live and 5 frozen feeder rats at home. The rats were housed in a domestic residence and were 7-13 months old. All likely originated from a feeder rat breeding farm, where the patient worked regularly as a volunteer, although the patient gave contradicting information about this. All available rats were tested for SEOV virus. The 5 live rats were euthanized, and serum and lung tissues were collected. For the frozen rats, serum was collected by vortexing and centrifugation of the rat hearts, as described previously (15). Antibodies in rat serum were detected by using a human SEOV ELISA (Hantavirus Dobrava/Hantaan IgG Elisa; Progen Biotechnik GmbH, Heidelberg, Germany), which was adapted to enable detection of IgG in rats. Rabbit- $\alpha$-rat horseradish peroxidase-labeled IgG (Sigma-Aldrich Chemie B.V., Zwijndrecht, the Netherlands) was used as conjugate at a 1:5,000 dilution. A cutoff value was based on the average OD of negative control rat serum $+3 \times$ SD (in this case, a value of $0.2-0.3$ ).

For euthanized and frozen rats, lung tissue was collected in RNAlater (Applied Biosystems, Foster City, CA, USA) and stored at $-80^{\circ} \mathrm{C}$. Lung tissue was disrupted in MagNA Pure 96 External Lysis Buffer (Roche) by using Lysis matrix D (MP Biomedicals, Santa Ana, CA, USA) and Fast Prep FP120 homogenizer (Thermo Savant, Carlsbad, CA, USA). Total nucleic acid was isolated by automated nucleic acid extraction by using the MagNA Pure 96 system (Roche). As a first screening, a hantavirus genus-specific rRT-PCR was performed on lung tissue, as described previously. Subsequently, a selection of the samples was confirmed with a nested rRT-PCR assay of the large (L) segment, as described by Klempa et al. (18). The resulting fragments were purified with ExoSAP-IT PCR clean-up (Isogen Life Science, Utrecht, the Netherlands) and sequenced by Baseclear (Leiden, the Netherlands). For clarity, details of the selection of rats and the subsequent experimental procedures are summarized in Figure 1.

\section{Investigation of Rats from the Feeder Rat Breeding Farm}

The feeder rats from the patient's home likely originated from the feeder rat breeding farm where the patient volunteered. At the time of the investigation, the feeder rat breeding farm housed 8,000-9,000 rats, 7 rabbits, and approximately 30 gerbils, 100 mice, and 60 snakes (constrictors). Adult rats at the farm were housed in 40 open boxes that measured $1 \mathrm{~m} \times 1 \mathrm{~m}$. About 30 rats were kept per box, of which $\approx 5-10$ were male and 20-25 were female. Smaller boxes were available for pregnant female rats, female rats with pups, and juvenile rats. 


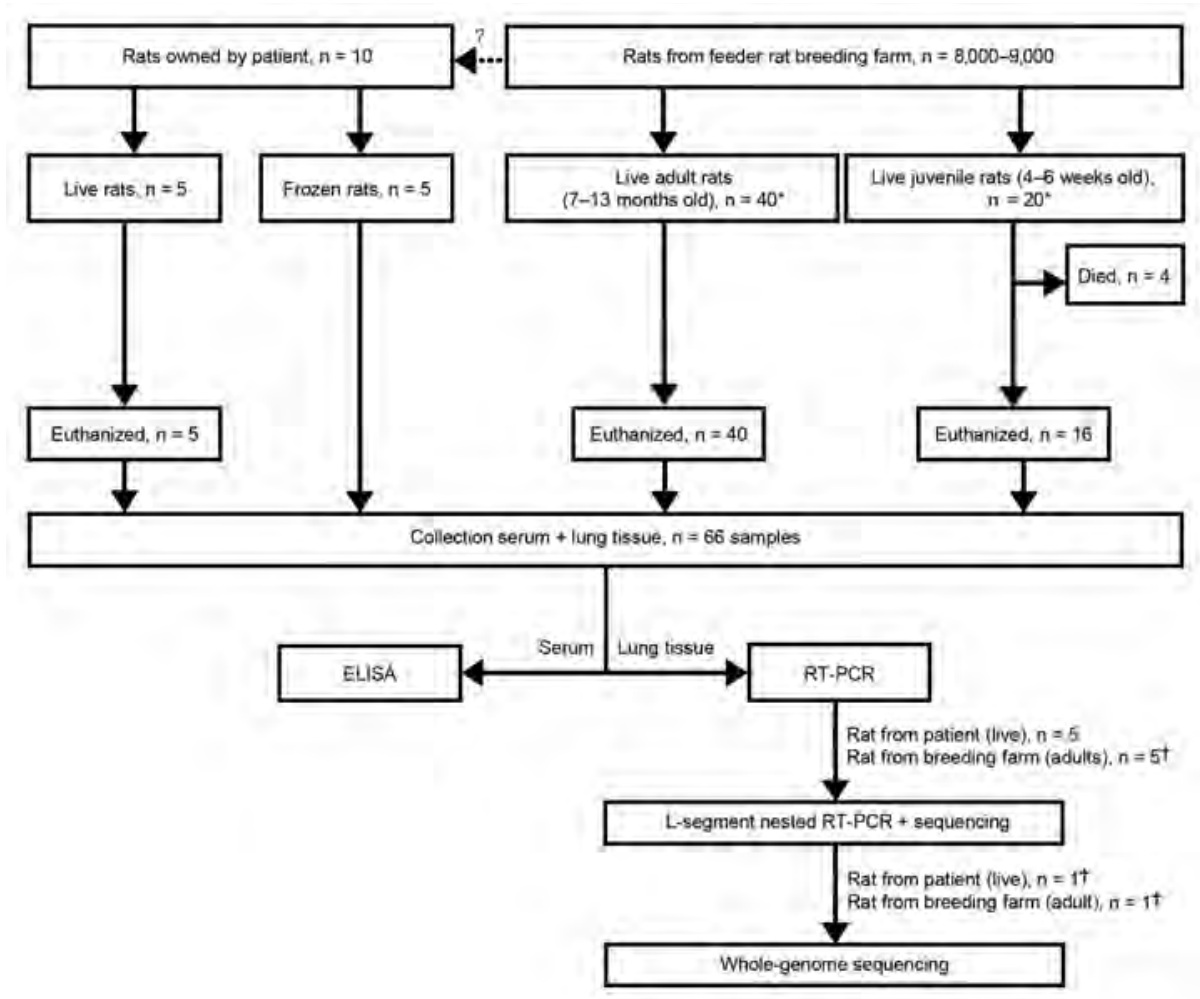

Figure 1. Flowchart depicting the selection and subsequent testing of feeder rats in a source investigation following detection of a human case of Seoul virus infection, the Netherlands, September, 2016. ${ }^{*}$ Rats were randomly picked; trats were randomly picked from among Seoul virus-positive animals. RTPCR, reverse transcription PCR.

Sixty rats, of which 40 were adults (age 7-13 months) and 20 were juveniles (age 4-6 weeks), were collected. One adult rat per box was picked randomly out of each box for this study. Five juvenile rats were collected randomly from 4 different boxes that contained the juvenile rats. When handling the rats, researchers used face masks (protective class FFP-2), disposable gloves, and coveralls. Serum and lung samples of the adults and juveniles were collected and analyzed as described previously.

The owner of the rat farm did not keep a record of where he bought and sold his rats. He reported that he regularly sold rats to several feeder rat breeding farms within the Netherlands. These farms were subsequently contacted, but their management would not cooperate with the investigation.

\section{Sequencing}

From 1 rat owned by the patient and 1 rat from the breeding farm, the complete SEOV genome (i.e., the small, medium $[\mathrm{M}]$, and L segments) was sequenced. Primers were developed based on published sequences and are available on request. All fragments were purified with ExoSAP-IT PCR clean-up (Isogen Life Science) and sequenced by Baseclear.

\section{Results}

\section{Patient and Close Contacts}

Testing found high antibody titers to the orthohantaviruses, especially SEOV, in the patient. The patient improved in 4 days, platelet count and liver enzyme test improved, and he was discharged. A second serum sample for detection of antibodies to orthohantaviruses was taken 3 weeks later when the patient had made a full recovery.

The patient was found to be positive in the orthohantavirus IFA with the highest IgG and IgM titers to SEOV (Table 2). Orthohantavirus RNA was not detected in the serum sample. Two close contacts of the patient tested negative in serologic testing for orthohantaviruses. The first close contact was the father of the index patient. While the index patient was hospitalized, his father had fed several rats to the reptiles. The second close contact was the cohabiting partner of the index patient and reported no contact with the rats.

\section{Feeder Rats from Patient}

Of the 10 rats collected from the patient's home, 6 (2/5 fresh and $4 / 5$ frozen) rats were found positive by serologic testing and rRT-PCR (Table 3). All 5 fresh rats were tested in the nested rRT-PCR assay of the L segment, and again, the same 2 rats were positive.

\section{Feeder Rats Breeding Farm}

Of 60 rats purchased from the rat breeding farm, 4 juveniles died from poor condition before they could be euthanized. The remaining 40 adults and 16 juveniles were tested using ELISA and rRT-PCR. All 40 adult rats were seropositive for orthohantaviruses. Lung tissues of all adult rats tested 
Table 2. Serologic orthohantavirus results for the patient with Seoul virus infection and 2 close contacts, the Netherlands, September $2016^{\star}$

\begin{tabular}{|c|c|c|c|c|c|c|c|c|c|c|c|c|c|}
\hline Sample & $\begin{array}{c}\text { Age, } \\
y\end{array}$ & $\begin{array}{l}\text { SEOV } \\
\text { IgG }\end{array}$ & $\begin{array}{l}\text { SEOV } \\
\text { IgM }\end{array}$ & $\begin{array}{l}\text { DOBV } \\
\operatorname{lgG}\end{array}$ & $\begin{array}{l}\text { DOBV } \\
\lg M\end{array}$ & $\begin{array}{l}\text { SAAV } \\
\lg G\end{array}$ & $\begin{array}{l}\text { SAAV } \\
\text { IgM }\end{array}$ & $\begin{array}{l}\text { HNTV } \\
\text { IgG }\end{array}$ & $\begin{array}{l}\text { HNTV } \\
\text { IgM }\end{array}$ & $\begin{array}{l}\text { PUUV } \\
\text { IgG }\end{array}$ & $\begin{array}{l}\text { PUUV } \\
\text { IgM }\end{array}$ & $\begin{array}{l}\text { SNV } \\
\text { IgG }\end{array}$ & $\begin{array}{l}\text { SNV } \\
\text { IgM }\end{array}$ \\
\hline $\begin{array}{l}\text { Patient } \\
\text { sample } 1 \dagger\end{array}$ & 28 & $1: 16,384$ & $1: 4,096$ & $1: 2,048$ & $1: 2,048$ & $1: 512$ & $1: 1,024$ & $1: 16,384$ & $1: 512$ & $1: 64$ & $1: 32$ & $1: 512$ & $1: 64$ \\
\hline $\begin{array}{l}\text { Patient } \\
\text { sample } 2 \ddagger\end{array}$ & & $1: 32,768$ & $1: 2,048$ & $1: 8,192$ & $1: 512$ & $1: 2,048$ & $1: 256$ & $1: 16,384$ & $1: 1,024$ & $1: 512$ & $1: 64$ & $1: 512$ & $1: 128$ \\
\hline $\begin{array}{l}\text { Close } \\
\text { contact } 1 \\
\text { (father) }\end{array}$ & 59 & - & - & - & - & - & - & - & - & - & - & - & - \\
\hline $\begin{array}{l}\text { Close } \\
\text { contact } 2 \\
\text { (partner) }\end{array}$ & 27 & - & - & - & - & - & - & - & - & - & - & - & - \\
\hline $\begin{array}{l}\text { *DOBV, Dob } \\
\text { Seoul orthoh } \\
\text { †Taken } 7 \mathrm{da} \\
\text { †Taken } 31 \mathrm{~d}\end{array}$ & $\begin{array}{l}\text {-Belgr } \\
\text { virus; } \\
\text { ter on } \\
\text { after o }\end{array}$ & $\begin{array}{l}\text { le orthohan } \\
\text { VV, Sin No } \\
\text { t of symptc } \\
\text { et of symp }\end{array}$ & $\begin{array}{l}\text { virus; HN } \\
\text { ore orthor } \\
\text { s. } \\
\text { ms (recon }\end{array}$ & $\begin{array}{l}\text { V, Hantaa } \\
\text { antavirus; } \\
\text { /alescent). }\end{array}$ & $\begin{array}{l}\text { orthoha } \\
\text { negativ }\end{array}$ & & & & & & & & SEOV, \\
\hline
\end{tabular}

positive for SEOV RNA by rRT-PCR. A selection of 5 adult rats was tested with the nested rRT-PCR assay of the $\mathrm{L}$ segment, and all 5 rats were positive.

Of the juveniles, 1 of the 16 was found to be seropositive. However, all 16 were orthohantavirus negative by rRT-PCR on lung tissues (Table 3).

\section{Sequencing}

The 7 SEOV-positive rats (2 from the patient and 5 from the rat breeding farm) tested by nested rRT-PCR showed identical sequences of the L segment. The complete SEOV genome was sequenced from 1 of the patient's rats and 1 breeding farm rat. Sequences were submitted to GenBank (accession nos. MG764078-83). The phylogenetic tree of the small segments (Figure 2) shows the strains are 100\% identical. Furthermore, these strains are $100 \%$ identical to the Turckheim strain isolated from pet rats in France (11) and $99.6 \%$ identical to the Cherwell strain (3) isolated from pet rats in the United Kingdom. Also, the M segment were identical to each other and $99.8 \%$ identical to the Cherwell strain. The L segment was $99.6 \%$ identical to the Cherwell strain. The sequence of the $\mathrm{M}$ and $\mathrm{L}$ segments of the Turckheim strain were not available for comparison.

\section{Discussion}

SEOV was detected in wild brown rats in the Netherlands in 2013, but no human cases had been reported. In this article, we report an autochthonous case of SEOV infection in the Netherlands. The case-patient had nonspecific clinical symptoms of an orthohantavirus infection, showing gastrointestinal symptoms and distinct elevation of liver enzymes. Although the patient did not develop acute kidney injury, we found mild proteinuria and thrombocytopenia, which might also be found in SEOV infection. Because SEOV infections are related to a mild form of HFRS, with low incidence of hemorrhagic manifestations and low mortality rates, previous cases might have been missed or misdiagnosed as viral infection or gastroenteritis. Elevation of liver enzymes has been reported to occur to a greater extent in SEOV infections but in this patient might have been attributable to use of medication (acetaminophen) or gamma-hydroxubutyrate. Diagnosis of HFRS attributable to SEOV infection is largely based on serologic testing.

The patient had high SEOV IgG and IgM titers (1:16,384 for IgG and 1:4,096 for IgM) by IFA. Cross-reactions exist within 2 defined serogroups of orthohantaviruses but are limited between the serogroups. Antibodies against Puumala virus and Sin Nombre virus cross-react, whereas SEOV as member of the other serogroup cross-reacts with Hantaan virus, Dobrava-Belgrade virus, and Saaremaa virus. This investigation found a clear link from the patient to the SEOV-infected rats at home and at the breeder farm. Combined with the high antibody titers against SEOV, we concluded that the patient's positive IFA result was due to a recent SEOV infection.

The patient's rats probably originated from the breeding farm where the patient worked as a volunteer, which is also suggested by the sequence results. Management at the farm facilitated spread of SEOV by frequently moving rats from one box to the other and randomly returning female rats to boxes after they had weaned their pups. No registration of these movements was recorded. The tested rats

\begin{tabular}{|c|c|c|c|}
\hline Source & Tested rats & No. (\%) seropositive rats & $\begin{array}{l}\text { No. (\%) rats found to be } \\
\text { positive by rRT-PCR }\end{array}$ \\
\hline Feeder rats of the patient, $n=10$ & $\begin{array}{l}5 \text { fresh adults } \\
5 \text { frozen adults }\end{array}$ & $\begin{array}{l}2(40) \\
4(80)\end{array}$ & $\begin{array}{l}2(40) \\
4(80)\end{array}$ \\
\hline Feeder rats from the farm, $n=8,000-9,000$ & $\begin{array}{c}40 \text { adults } \\
16 \text { juveniles }\end{array}$ & $\begin{array}{c}40(100) \\
1(6)\end{array}$ & $\begin{array}{c}40(100) \\
0(0)\end{array}$ \\
\hline
\end{tabular}




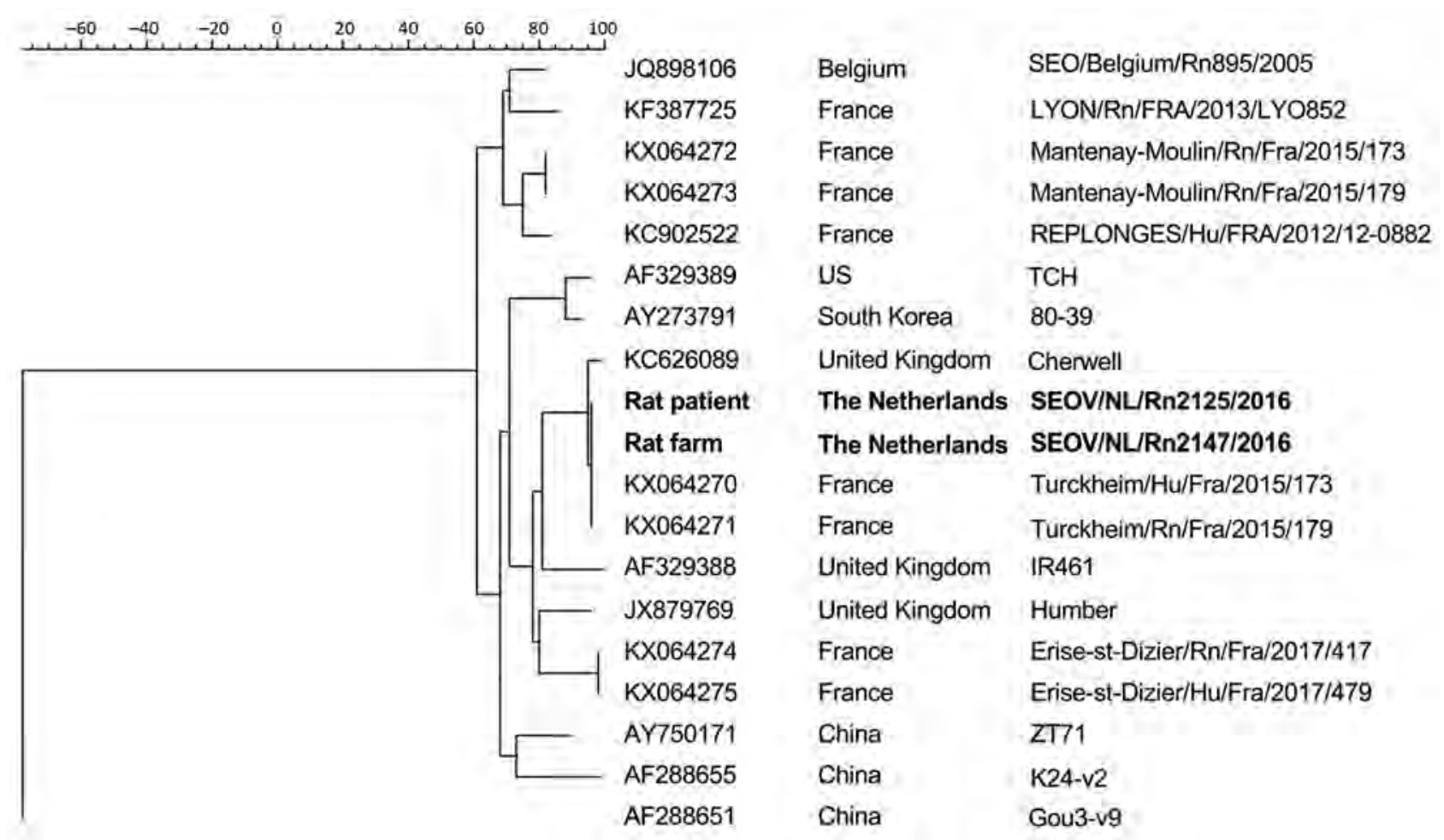

Figure 2. Phylogenetic tree calculated for the coding region (1,290 bp) of the small segment of the nucleocapsid protein in the Seoul virus strain implicated in a human case infection in the Netherlands, September 2016, compared with reference viruses. Boldface indicates isolates from this study; GenBank accession numbers are provided for reference viruses.

were in a poor body condition, and many had bite wounds on their ears, providing ideal transmission conditions for SEOV (19). Several health risks (including public health risks) were identified with regard to the SEOV-positive feeder rat breeding farm, including the possibility of infection of personnel and visitors to the farm and the potential spread of the pathogen by regular trade and exchange with other feeder rat breeding farms and occasionally with unknown parties, possibly including pet stores. Also, SEOV might spread from the feeder rats to wild rats living around the farm through direct contact or through contaminated materials (e.g., bedding material). Anyone entering the breeding farm was advised to wear disposable gloves, shoe sleeves, an apron, and a face mask (protective class FFP-2) and to wash their hands immediately after leaving the barn. They were also warned to avoid being bitten by the rats. All employees and volunteers were offered a blood test to see if they had been infected; no one accepted. Anyone buying or otherwise taking rats from the breeding farm was given a letter, signed by the Municipal Health Services, informing them of the possibility of the rats being infected. Pest control measures were set up, preventing contact of wild rats with feeder rats. The distribution of the used sawdust over nearby farm lands was discontinued. Used sawdust was thereafter brought to the municipal facility to be burnt in a waste disposal facility. By law in the Netherlands, notification of orthohantavirus infection in humans is mandatory but not in animals. Therefore, no legislation on control measures (e.g., enforced quarantine of infected animals, a ban on selling rats, or a forced closure of the breeding farm) was in effect. These limitations complicated source investigation because testing of the breeder rat farm was based on voluntary cooperation of the farm owner; they also severely complicated our efforts to contain this virus.

Duggan et al. showed that the seroprevalence of antibodies to SEOV in persons with a high contact rate with rats, such as rat owners, is $34 \%$, compared with $3 \%$ in controls with occupational exposure to pet fancy rats or wild rats (12). Naturally, this probability depends on the spread of SEOV in the domestic rat populations in the Netherlands. To what extent SEOV is present in rat populations in the Netherlands is unknown. Hantavirus-infected rodents do not show any overt symptoms and might spread orthohantavirus for a prolonged period, possibly lifelong (2023). However, the presence in feeder rats and anecdotal information about exchange between rat populations suggest that SEOV might be present in captive rat populations in the Netherlands. Also, exchange of pet and feeder rats between countries in Europe might be extensive, which is supported by the close resemblance of the SEOV strain in the Netherlands to the Cherwell strain in the United Kingdom and the Turckheim strain in France. In Europe, SEOV 
has been detected in pet rats in England and Wales (13), Sweden (5), and France (11).

This case illustrates the importance of clinical awareness for orthohantavirus infections after contact with rodents, including in patients with nonspecific symptoms, and the challenges that arise when source investigation and implementation of control measures are hampered by lack of legislation. The source investigation and implementation of control measures required multidisciplinary, constructive cooperation between research institutions and authorities. Future studies to assess the extent of SEOV infection in the domestic rat populations in the Netherlands are needed to inform the general public concerning the risk for contracting this virus by handling rats and the related health risks.

\section{About the Author}

Dr. Swanink is a clinical microbiologist at Rijnstate Hospital, Arnhem and Velp, the Netherlands. Her primary research interests are clinical virology, hepatitis, HIV, immunecompromised patients, and epidemiology.

\section{References}

1. de St Maurice A, Ervin E, Schumacher M, Yaglom H, VinHatton E, Melman S, et al. Exposure characteristics of hantavirus pulmonary syndrome patients, United States, 19932015. Emerg Infect Dis. 2017;23:733-9. http://dx.doi.org/10.3201/ eid2305.161770

2. Peters CJ, Simpson GL, Levy H. Spectrum of hantavirus infection: hemorrhagic fever with renal syndrome and hantavirus pulmonary syndrome. Annu Rev Med. 1999;50:531-45. http://dx.doi.org/10.1146/annurev.med.50.1.531

3. Jameson LJ, Taori SK, Atkinson B, Levick P, Featherstone CA, van der Burgt G, et al. Pet rats as a source of hantavirus in England and Wales, 2013. Euro Surveill. 2013;18:20415.

4. Plyusnina A, Heyman P, Baert K, Stuyck J, Cochez C, Plyusnin A. Genetic characterization of Seoul hantavirus originated from Norway rats (Rattus norvegicus) captured in Belgium. J Med Virol. 2012;84:1298-303. http://dx.doi.org/10.1002/ jmv.23321

5. Lundkvist A, Verner-Carlsson J, Plyusnina A, Forslund L, Feinstein R, Plyusnin A. Pet rat harbouring Seoul hantavirus in Sweden, June 2013. Euro Surveill. 2013;18:20521. http://dx.doi.org/ 10.2807/1560-7917.ES2013.18.7.20521

6. Dupinay T, Pounder KC, Ayral F, Laaberki MH, Marston DA, Lacôte S, et al. Detection and genetic characterization of Seoul virus from commensal brown rats in France. Virol J. 2014;11:32. http://dx.doi.org/10.1186/1743-422X-11-32

7. Kerins JL, Koske SE, Kazmierczak J, Austin C, Gowdy K, Dibernardo A, et al.; Seoul Virus Working Group; Canadian Seoul Virus Investigation Group (Federal); Canadian Seoul Virus Investigation Group (Provincial); Contributors. Outbreak of Seoul virus among rats and rat owners-United States and Canada, 2017. MMWR Morb Mortal Wkly Rep. 2018;67:131-4. http://dx.doi.org/ 10.15585/mmwr.mm6704a5

8. Jameson LJ, Logue CH, Atkinson B, Baker N, Galbraith SE, Carroll MW, et al. The continued emergence of hantaviruses: isolation of a Seoul virus implicated in human disease, United Kingdom, October 2012. Euro Surveill. 2013;18:4-7.
9. Macé G, Feyeux C, Mollard N, Chantegret C, Audia S, Rebibou JM, et al. Severe Seoul hantavirus infection in a pregnant woman, France, October 2012. Euro Surveill. 2013;18:20464.

10. McElhinney LM, Marston DA, Pounder KC, Goharriz H, Wise EL, Verner-Carlsson J, et al. High prevalence of Seoul hantavirus in a breeding colony of pet rats. Epidemiol Infect. 2017;145:3115-24. http://dx.doi.org/10.1017/S0950268817001819

11. Reynes JM, Carli D, Bour JB, Boudjeltia S, Dewilde A, Gerbier G, et al. Seoul virus infection in humans, France, 20142016. Emerg Infect Dis. 2017;23:973-7. http://dx.doi.org/10.3201/ eid2306.160927

12. Duggan JM, Close R, McCann L, Wright D, Keys M, McCarthy N, et al. A seroprevalence study to determine the frequency of hantavirus infection in people exposed to wild and pet fancy rats in England. Epidemiol Infect. 2017;145:2458-65. http://dx.doi.org/ 10.1017/S0950268817001480

13. Glass GE, Watson AJ, LeDuc JW, Childs JE. Domestic cases of hemorrhagic fever with renal syndrome in the United States. Nephron. 1994;68:48-51. http://dx.doi.org/10.1159/000188086

14. Goeijenbier M, Verner-Carlsson J, van Gorp EC, Rockx B, Koopmans MP, Lundkvist $\AA$, et al. Seoul hantavirus in brown rats in the Netherlands: implications for physicians - epidemiology, clinical aspects, treatment and diagnostics. Neth J Med. 2015; 73:155-60.

15. Verner-Carlsson J, Lõhmus M, Sundström K, Strand TM, Verkerk M, Reusken C, et al. First evidence of Seoul hantavirus in the wild rat population in the Netherlands. Infect Ecol Epidemiol. 2015;5:27215. http://dx.doi.org/10.3402/iee.v5.27215

16. Lederer S, Lattwein E, Hanke M, Sonnenberg K, Stoecker W, Lundkvist $\AA$, et al. Indirect immunofluorescence assay for the simultaneous detection of antibodies against clinically important old and new world hantaviruses. PLoS Negl Trop Dis. 2013;7:e2157. http://dx.doi.org/10.1371/journal.pntd.0002157

17. Kramski M, Meisel H, Klempa B, Krüger DH, Pauli G, Nitsche A. Detection and typing of human pathogenic hantaviruses by real-time reverse transcription-PCR and pyrosequencing. Clin Chem. 2007;53:1899-905. http://dx.doi.org/10.1373/clinchem. 2007.093245

18. Klempa B, Fichet-Calvet E, Lecompte E, Auste B, Aniskin V, Meisel H, et al. Hantavirus in African wood mouse, Guinea. Emerg Infect Dis. 2006;12:838-40. http://dx.doi.org/10.3201/eid1205.051487

19. Glass GE, Childs JE, Korch GW, LeDuc JW. Association of intraspecific wounding with hantaviral infection in wild rats (Rattus norvegicus). Epidemiol Infect. 1988;101:459-72. http://dx.doi.org/10.1017/S0950268800054418

20. Heyman P, Vaheri A, Lundkvist A, Avsic-Zupanc T. Hantavirus infections in Europe: from virus carriers to a major public-health problem. Expert Rev Anti Infect Ther. 2009;7:205-17. http://dx.doi.org/10.1586/14787210.7.2.205

21. Meyer BJ, Schmaljohn CS. Persistent hantavirus infections: characteristics and mechanisms. Trends Microbiol. 2000;8:61-7. http://dx.doi.org/10.1016/S0966-842X(99)01658-3

22. Easterbrook JD, Klein SL. Immunological mechanisms mediating hantavirus persistence in rodent reservoirs. PLoS Pathog. 2008;4:e1000172. http://dx.doi.org/10.1371/journal.ppat.1000172

23. Voutilainen L, Sironen T, Tonteri E, Bäck AT, Razzauti M, Karlsson M, et al. Life-long shedding of Puumala hantavirus in wild bank voles (Myodes glareolus). J Gen Virol. 2015;96:123847. http://dx.doi.org/10.1099/vir.0.000076

Address for correspondence: Caroline Swanink, Rijnstate Hospital, Department of Medical Microbiology and Immunology, President Kennedylaan 100, 6883 AZ, Velp, the Netherlands; email: cswanink@rijnstate.nl 


\title{
Restaurant I nspection Letter Grades and Salmonella I nfections, New York, New York, USA
}

\author{
Melanie J. Firestone, Craig W. Hedberg
}

Rates of Salmonella infection in the United States have not changed over the past 20 years. Restaurants are frequent settings for Salmonella outbreaks and sporadic infections. Few studies have examined the effect of posting letter grades for restaurant inspections on the incidence of foodborne illness. We compared Salmonella infection rates in New York, New York, USA (NYC), with those in the rest of New York state before and after implementation of a letter grade system for restaurant inspections in NYC. We calculated a segmented regression model for interrupted time series data. After implementation of letter grading, the rate of Salmonella infections decreased 5.3\% per year in NYC versus the rest of New York state during 2011-2015, compared with the period before implementation, 2006-2010. Posting restaurant inspection results as letter grades at the point of service was associated with a decline in Salmonella infections in NYC and warrants consideration for broader use.

E ach year, an estimated 48 million persons get sick, 128,000 are hospitalized, and 3,000 die from foodborne illness in the United States (1). Consumption of food prepared away from home, such as at restaurants and for takeout, has continuously increased, accounting for $50 \%$ of food expenditures in 2014, up from 33\% in 1970 (2). Restaurants are frequent settings for transmission of foodborne illness; in $2015,60 \%$ of foodborne illness outbreaks were associated with restaurants (3). There is also evidence that commercial food service establishments, such as restaurants, play a role in sporadic (nonoutbreak) cases of foodborne illness (4). Given our dependence on food prepared away from home, reducing the risk for foodborne illness from commercial food service establishments is of critical importance.

Food service establishments play a role in the epidemiology of Salmonella infections; Salmonella may contaminate a wide range of raw ingredients, infect food workers, survive on contaminated surfaces, and grow in improperly held food items. Nontyphoidal Salmonella infections are the second most common foodborne illness and the leading

Author affiliations: University of Minnesota School of Public

Health, Minneapolis, Minnesota, USA; Minnesota Integrated Food

Safety Center of Excellence, Minneapolis

DOI: https://doi.org/10.3201/eid2412.180544 cause of foodborne illness hospitalizations and death (1). Reducing Salmonella infections is a Healthy People 2020 objective (5), yet rates have not substantially changed over the last 20 years (6). Inspection of food service establishments to protect food safety is a core function of state and local health departments; the inspections help to identify risk factors for foodborne illness, such as those associated with Salmonella transmission, and to correct them, thus protecting consumers and industry.

New York does not have a statewide policy for reporting restaurant inspection results. In 2005, the Department of Health and Mental Hygiene (DOHMH) in New York, NY, USA (NYC), began using a point-scoring system for food service establishment inspections to weight violations to reflect risk factors for foodborne illness. In 2010, the NYC DOHMH implemented a letter grade program that converted these scores into categorical rankings of $\mathrm{A}, \mathrm{B}$, or $\mathrm{C}$, or grade pending, in an effort to improve restaurant food safety, increase transparency of inspection information, and reduce the risk for foodborne illness transmission in restaurants (7). The DOHMH required establishments to post a sign with the letter grade in its window so that consumers could see it before entering. Public disclosure programs like this one seek to provide information to consumers when and where they need it so they can make informed decisions about potential risks (8). These consumer decisions encourage restaurant operators to improve and maintain sanitary standards, thus improving sanitary conditions in restaurants. The letter grade program has already been shown to lead to improvements in sanitary conditions in NYC: $35 \%$ more restaurants earned $\mathrm{A}$ grades in the 3 years after grading, compared with the 3 years before (9).

The value of posting restaurant inspection ratings at the point of service has been the subject of considerable debate. Few studies have looked at the impact of posting policies on the incidence of foodborne illness. Two studies of a letter grade program in Los Angeles County, California, USA, showed a reduction in foodborne-illness hospitalizations $(10,11)$. In NYC, a preliminary analysis of letter grading at 18 months suggested a decline in Salmonella infections. The goal of our study was to compare the incidence of Salmonella infections in NYC with incidence in the rest 
of the state before and after the implementation of posting letter grade placards at the point of service.

\section{Methods}

\section{Data}

Salmonella infections are nationally notifiable (12). We obtained yearly laboratory-confirmed case counts from 1994-2015 from the NYC DOHMH and the New York State Department of Health. Cases are reported by year of diagnosis and county of residence.

\section{Statistical Analyses}

To account for population changes, we calculated annual rates using intercensal population estimates for 1994-2015 from the US Census Bureau. We calculated the percent change from year to year. We compared mean rates of Salmonella infection before and after implementation of a point scoring system in 2005 and after implementation of grade cards in 2010 using $t$-tests for NYC and the rest of the state (NYS). We considered the year of implementation to be a part of the before period in both analyses because the policies were not implemented on January 1 of each year.

We used segmented regression to determine the trend before implementing the policy in Salmonella infections, an immediate change at the time of policy implementation, and the long-term trend after policy implementation (13). We hypothesized that the long-term trend would decline after policy implementation. We expected a delayed effect because restaurants are not all on the same inspection cycle and because underlying improvements in sanitation driving the decline are not likely to be immediate. Because there were indications of overdispersion and heteroskedasticity, we used a negative binomial regression model with robust SEs to quantify the effect of letter grade placards on Salmonella infections. We used an offset term to account for population changes across the period. Examination of autocorrelation and partial autocorrelation functions confirmed that the outcome was not autocorrelated. We calculated incidence rate ratios (IRR) comparing Salmonella infections in NYC to NYS before and after the implementation of the point scoring system and posting of letter grade placards.

Key variables included year, coded as a continuous variable starting with $1994=1$; variables representing the 2 policy periods (prepolicy $=0$, postpolicy $=1$ ); 2 variables representing the trends after policy implementation, coded as 0 before the policy and a continuous numerical function after the policy was implemented; and a variable (region) representing NYC versus NYS. The model also included 5 interaction terms: region by year, to control for regional secular trends; region by policy for each policy (before and after letter grade was implemented), to account for the mean level change in NYC after the new policy took effect; and region by trend before and after policy implementation, to determine if the average Salmonella infections in NYC changed after policy implementation versus NYS (14). We also conducted a subanalysis using data from 2000-2015 to compare NYC to surrounding counties (NYC metropolitan area) and NYS. We analyzed data using Stata version 14.2 (StataCorp LLC, College Station, TX, USA) and determined statistical significance as $\mathrm{p}<0.05$.

\section{Results}

The annual rate of Salmonella infections decreased in both NYC and NYS during 1994-2015 (Table 1; Figure). The period-to-period percent change after letter grading implementation was a decline of $32.6 \%$ in NYC, compared with a decline of $14.1 \%$ in NYS (Table 1). Mean Salmonella infection rates in NYC between 1994 and 2010 were significantly higher $(\mathrm{p}<0.01)$ than in NYS (Table 2). In the period after letter grading was implemented (2011-2015), the mean rate of Salmonella infection was no longer significantly different $(\mathrm{p}=0.37)$ in NYC (mean 12.6 cases/100,000 persons; 95\% CI 10.9-14.4) compared with NYS (mean 12.0 cases/100,000 persons; 95\% CI 11.4-12.6).

In NYC and NYS, Salmonella infections were decreasing before either policy was implemented in NYC (IRR $0.95 ; 95 \%$ CI $0.94-0.96 ; \mathrm{p}<0.01$ ). The interaction term for trend by region after letter grade implementation was statistically significant $(\mathrm{p}<0.01)$, which indicates that Salmonella infection rates declined on average in NYC versus NYS in the years after letter grading was implemented, compared with the period after the point scoring system was implemented (Table 3). After letter grading was implemented, the rate of Salmonella infections decreased $5.3 \%$ per year in NYC versus NYS (IRR $0.95 ; 95 \%$ CI 0.92-0.98; $\mathrm{p}<0.01$ ).

In a subanalysis of Salmonella infections from 20002015 comparing number of infections in NYC with that of other counties in the NYC metropolitan area and NYS separately, Salmonella infections declined in both NYC and the NYC metropolitan area compared with those in NYS. In contrast to the findings in NYS, in the period after letter grading was implemented, NYC Salmonella infections declined $8.8 \%$ per year and Salmonella infections in the NYC metropolitan area declined $7.5 \%$ per year compared with the period between the implementation of a point scoring system and the letter grade program.

\section{Discussion}

Overall, Salmonella infections declined in NYC and NYS between 1994 and 2015. Although NYS had declines in Salmonella infection rates after 2010, NYC saw declines greater than those in NYS. Inspection processes were largely unchanged in NYC with the implementation of the point scoring system in 2005 (15). The letter grade 
Table 1. Confirmed Salmonella infection case counts, rates, and percent changes for New York, NY, USA, and the rest of the state, 1994-2015*

\begin{tabular}{|c|c|c|c|c|c|c|}
\hline \multirow[b]{2}{*}{ Year } & \multicolumn{3}{|c|}{ NYC } & \multicolumn{3}{|c|}{ NYS } \\
\hline & No. cases & $\begin{array}{l}\text { Rate per } 100,000 \\
\text { population }\end{array}$ & $\begin{array}{l}\text { Year-to-year } \\
\text { change, \% }\end{array}$ & No. cases & $\begin{array}{c}\text { Rate per } 100,000 \\
\text { population }\end{array}$ & $\begin{array}{c}\text { Year-to-year } \\
\text { change, \% }\end{array}$ \\
\hline 1994 & 1,890 & 25.0 & - & 1,977 & 18.2 & - \\
\hline 1995 & 2,166 & 28.4 & 13.7 & 1,912 & 17.6 & -3.3 \\
\hline 1996 & 1,927 & 25.0 & -11.8 & 1,940 & 17.8 & 1.5 \\
\hline 1997 & 1,772 & 22.8 & -8.9 & 1,649 & 15.2 & -14.9 \\
\hline 1998 & 1,751 & 22.3 & -2.3 & 1,680 & 15.4 & 1.7 \\
\hline 1999 & 1,508 & 19.0 & -14.8 & 1,516 & 13.9 & -10.1 \\
\hline 2000 & 1,215 & 15.2 & -20.1 & 1,293 & 11.8 & -15.1 \\
\hline 2001 & 1,386 & 17.2 & 13.5 & 1,397 & 12.7 & 7.7 \\
\hline 2002 & 1,458 & 18.1 & 5.0 & 1,613 & 14.6 & 15.0 \\
\hline 2003 & 1,307 & 16.2 & -10.3 & 1,282 & 11.5 & -20.8 \\
\hline 2004 & 1,273 & 15.8 & -2.3 & 1,291 & 11.6 & 0.5 \\
\hline $2005 \dagger$ & 1,203 & 15.0 & -5.1 & 1,427 & 12.8 & 10.6 \\
\hline 2006 & 1,272 & 15.9 & 6.0 & 1,423 & 12.8 & -0.2 \\
\hline 2007 & 1,304 & 16.3 & 2.3 & 1,476 & 13.3 & 3.7 \\
\hline 2008 & 1,268 & 15.7 & -3.4 & 1,491 & 13.4 & 0.8 \\
\hline 2009 & 1,236 & 15.2 & -3.3 & 1,370 & 12.3 & -8.4 \\
\hline 2010‡ & 1,304 & 15.9 & 4.7 & 1,448 & 12.9 & 5.4 \\
\hline 2011 & 1,125 & 13.6 & -14.7 & 1,423 & 12.7 & -1.9 \\
\hline 2012 & 1,171 & 14.0 & 3.1 & 1,395 & 12.4 & -2.0 \\
\hline 2013 & 1,124 & 13.3 & -4.8 & 1,300 & 11.6 & -6.9 \\
\hline 2014 & 987 & 11.6 & -12.8 & 1,320 & 11.7 & 1.6 \\
\hline 2015 & 918 & 10.7 & -7.8 & 1,314 & 11.7 & 0.0 \\
\hline Period-t & hange, 1994 & 05 vs. $2006-2010$ & -22.8 & & & -10.6 \\
\hline Period-t & hange, 2006 & 10 vs. $2011-2014$ & -20.0 & & & -7.1 \\
\hline Period-t & hange, 1994 & 10 vs. $2011-2015$ & -32.6 & 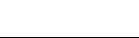 & - & -14.1 \\
\hline
\end{tabular}

placard program begun in 2010 did not change the point scoring system but rather used the points to create a readily comprehensible ranking system accessible at the point of service. Our analysis supports the hypothesis that having a point scoring system was not associated with declines in Salmonella infections but having a simple way to publicly disclose the results of the inspection was.

Although it appears that the implementation of a point scoring system in NYC was associated with a leveling off of declines in Salmonella infections, the system was

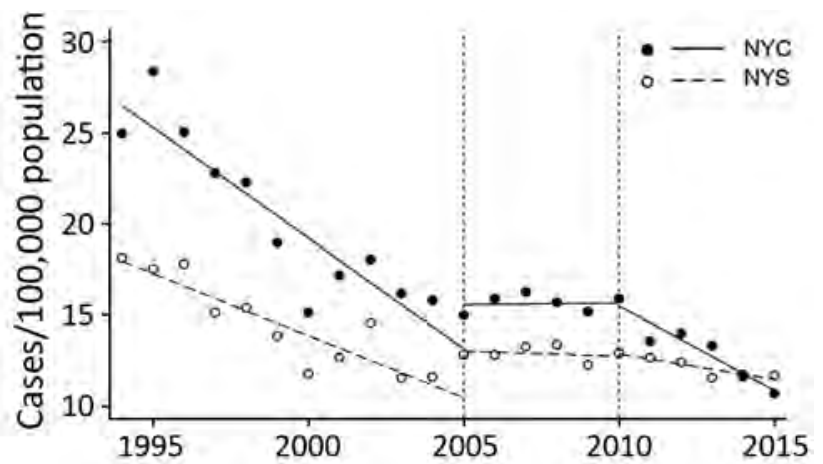

Figure. Confirmed Salmonella infection cases per 100,000 population in NYC and the rest of the state, 1994-2015. Dashed lines indicate implementation of point scores in 2005 and letter grades in 2010. NYC, New York City; NYS, rest of New York state. implemented at the end of a hyperendemic period of Salmonella enterica serotype Enteriditis infections. In the 1980s, high rates of Salmonella Enteriditis infections primarily associated with shell eggs were recognized, and a variety of prevention and control measures were put in place to combat these rising rates (16). The Northeast was particularly affected by this outbreak; New York state reported the highest number of outbreaks during 1985-1999 (16). Prevention measures appeared to have the greatest success in reducing rates in the Northeast compared with others (16). Furthermore, 2 notable changes in NYC during this time period led to improved surveillance, which typically results in better detection and reporting. In 2006, the NYC Board of Health mandated electronic laboratory reporting of notifiable diseases (17); in 2009, NYC became a Foodborne Diseases Centers for Outbreak Response Enhancement (FoodCORE) center with the goal of improving surveillance for Salmonella infections (18). To the extent that these efforts may have improved surveillance in NYC, they may also have been expected to increase case detection in NYC versus NYS.

The implementation of letter grading in 2010 marks the beginning of the current declining trend in Salmonella infections. By improving sanitation conditions in NYC food service establishments, the letter grade program can be expected to benefit other areas as well. In this study, 
Table 2. Mean Salmonella infection rates for New York, NY, USA, and the rest of the state, by policy implementation period, 1994-2015*

\begin{tabular}{|c|c|c|c|c|c|c|c|c|c|}
\hline \multirow[b]{2}{*}{ Location } & \multicolumn{3}{|c|}{ 1994-2005 } & \multicolumn{3}{|c|}{$2006-2010$} & \multicolumn{3}{|c|}{ 2011-2015 } \\
\hline & Mean (SE) & $95 \% \mathrm{Cl}$ & $p$ value & Mean (SE) & $95 \% \mathrm{Cl}$ & $p$ value & Mean (SE) & $95 \% \mathrm{Cl}$ & $p$ value \\
\hline NYC & $20.0(1.3)$ & $17.1-22.9$ & Ref & $15.8(0.2)$ & $15.3-16.3$ & $0.06 \ddagger$ & $12.6(0.6)$ & $10.9-14.4$ & $<0.01 \ddagger$ \\
\hline NYS & $14.4(0.7)$ & $12.9-16.0$ & Ref & $12.9(0.2)$ & $12.4-13.5$ & $0.21 \ddagger$ & $12.0(0.2)$ & $11.4-12.6$ & $0.01 \ddagger$ \\
\hline NYC vs. NYS & & & $<0.01$ & & & $<0.01$ & & & 0.37 \\
\hline
\end{tabular}

*NYC, New York City; Ref, referent; NYS, rest of New York state.

tComparison of mean rate between NYC and NYS within time period.

$\ddagger$ Comparison of mean rate within region to the preceding time period.

Salmonella infection were reported by county of residence, but NYC sees its population change daily due to commuting and tourism. Manhattan, one of the 5 NYC boroughs, sees its population nearly double during the workday. Of its commuters, $36 \%$ ( $\approx 550,000$ persons) travel from outside the other 4 boroughs (19). Additionally, NYC is a popular place for tourists; >60 million persons visited in 2016 (20). Our study showed that Salmonella infections in NYS declined after the letter grade program was implemented. This finding may be in part because improved sanitary conditions in NYC restaurants after the implementation of letter grades reduced risk for Salmonella exposure among NYS residents who commuted to or visited NYC.

This study had several limitations. First, this was a quasi-experimental, ecologic study that represents an association and not a causal relationship. Second, the NYC restaurant letter grade program involved multiple changes to sanitation enforcement in addition to letter grade posting; changes included inspection frequency, greater risk for fines, improvements to online resources, and additional training opportunities (21). As a result, we could not determine which factors contributed the most to the reduction in Salmonella infections. Furthermore, we were not able to assess whether Salmonella-infected persons had a known exposure to restaurants in the period before illness.

This study supports findings from an earlier NYC study (21) and previous studies of Los Angeles County that showed a decline in foodborne illness hospitalizations. Hospitalizations represent a subset of foodborne illnesses that may be caused by a variety of agents, such as Campylobacter, another leading cause of foodborne illness in the United States (1). However, Campylobacter rarely causes outbreaks in restaurant settings because its biology limits transmission to inadequate cooking of contaminated poultry or meats or cross-contamination from raw to ready-to-eat foods (22). As a result, improvements in restaurant sanitary conditions are unlikely to affect Campylobacter transmission in restaurants.

In contrast, the selection of Salmonella infections is a strength of this study because the biology of Salmonella makes it uniquely suited for transmission in food service establishments. In restaurants, Salmonella can cause illness from contaminated raw ingredients, through cross-contamination, or from infected food workers. Improper cooling of inadequately cooked foods, or failure to maintain cold or hot holding temperatures can amplify contamination. Thus,
Salmonella transmission serves as a good indicator of overall restaurant food safety practices.

Despite limitations, NYC's experience provides a useful case study of the beneficial effect of letter grading programs. Although the relationship between restaurant inspections and risk for foodborne illness is not well understood (23) and inspections represent a snapshot in time that may not represent the overall sanitary conditions in restaurants, factors related to food handling and preparation practices and food worker health and hygiene are frequent contributors to outbreaks in restaurants (24). These factors probably contribute to transmission of sporadic infections in restaurants, which are much more common; outbreak cases represent $<10 \%$ of all Salmonella infections $(4,6)$. The NYC restaurant letter grade program has been shown to be associated with sustained improvements in sanitary conditions in restaurants, including several factors associated with outbreaks (9). Furthermore, that study showed that after 18 months, $81 \%$ of adults in NYC had seen letter grade placards, and $88 \%$ of those persons considered the letter grades in their dining decisions (9). This finding suggests that consumer behavior helped support the program goal of driving improvements in sanitary conditions. Although future studies are needed to parse which restaurant inspection results may contribute most to declines in Salmonella infections, our findings support the hypothesis that the successful

\begin{tabular}{|c|c|c|c|c|}
\hline \multirow[b]{2}{*}{ Predictor variables } & \multicolumn{3}{|c|}{ Robust } & \multirow[b]{2}{*}{$p$ value } \\
\hline & IRR & SE & $95 \% \mathrm{Cl}$ & \\
\hline Year & 0.95 & 0.01 & $0.94-0.96$ & $<0.01$ \\
\hline Region & 1.50 & 0.08 & $1.35-1.68$ & $<0.01$ \\
\hline Region $\times$ year & 0.99 & 0.01 & $0.97-1.00$ & 0.16 \\
\hline $\begin{array}{l}\text { Point scoring policy } \\
\text { implementation }\end{array}$ & 1.20 & 0.06 & $1.08-1.32$ & $<0.01$ \\
\hline $\begin{array}{l}\text { Trend after point scoring } \\
\text { implementation }\end{array}$ & 1.04 & 0.01 & $1.02-1.07$ & $<0.01$ \\
\hline $\begin{array}{l}\text { Region } \times \text { point scoring } \\
\text { implementation }\end{array}$ & 0.92 & 0.07 & $0.80-1.07$ & 0.29 \\
\hline $\begin{array}{l}\text { Region } \times \text { trend after } \\
\text { points }\end{array}$ & 1.02 & 0.02 & 0.99-1.05 & 0.28 \\
\hline $\begin{array}{l}\text { Letter grade policy } \\
\text { implementation }\end{array}$ & 1.01 & 0.04 & $0.93-1.10$ & 0.81 \\
\hline Trend after grading & 0.98 & 0.01 & $0.96-1.00$ & 0.09 \\
\hline Region $\times$ grading & 0.99 & 0.06 & $0.88-1.11$ & 0.85 \\
\hline $\begin{array}{l}\text { Region } \times \text { trend after } \\
\text { grading }\end{array}$ & 0.95 & 0.02 & $0.92-0.98$ & $<0.01$ \\
\hline Intercept & 0.00 & 0.00 & $0.00-0.00$ & $<0.01$ \\
\hline
\end{tabular}

*IRR, incidence rate ratio. 
implementation of a letter grade program was associated with a reduction of Salmonella transmission in restaurants in NYC.

In conclusion, in the United States, considerable resources have been invested to prevent contamination of the food supply before the point of service. However, Salmonella infections remain unchanged at the national level. Previous studies have shown improvements in sanitary conditions after the implementation of the NYC restaurant letter grade program, and our study suggests a beneficial effect on the incidence of foodborne illnesses. Implementing a letter grade program is a feasible and relatively inexpensive tool to reduce Salmonella infections that warrants consideration for broader use. Other jurisdictions should consider adopting a letter grade program and decide on the form and location of the placard, frequency of inspections, and approaches to engage restaurant-industry and community support to ensure program success.

\section{Acknowledgments}

We thank Andrew Ryan and Joseph Servadio for analytic consultations.

\section{About the Author}

Ms. Firestone is a doctoral candidate at the University of Minnesota. Her primary research focuses on using surveillance data to inform policy targeted toward reducing foodborne illness.

Dr. Hedberg is a professor at the University of Minnesota. His primary research focuses on improving surveillance of foodborne illness and food safety hazards with the goal of reducing the burden of foodborne illness.

\section{References}

1. Scallan E, Hoekstra RM, Angulo FJ, Tauxe RV, Widdowson MA, Roy SL, et al. Foodborne illness acquired in the United Statesmajor pathogens. Emerg Infect Dis. 2011;17:7-15. http://dx.doi.org/10.3201/eid1701.P11101

2. US Department of Agriculture Economic Research Service. Food expenditures: table 10, food away from home as a share of food expenditures 2016 [cited 2018 Sep 17]. https://www.ers.usda.gov/ data-products/food-expenditures.aspx

3. Centers for Disease Control and Prevention. Surveillance for foodborne disease outbreaks, United States, 2014, Annual Report. Atlanta: US Department of Health and Human Services; 2016.

4. Jones TF, Angulo FJ. Eating in restaurants: a risk factor for foodborne disease? Clin Infect Dis. 2006;43:1324-8. http://dx.doi.org/10.1086/508540

5. Healthy People 2020. 2020 topics and objectives. 2018 Sep 21 [cited 2018 Sep 25]; https:/www.healthypeople.gov/2020/topics-objectives

6. Centers for Disease Control and Prevention. Foodborne Disease Active Surveillance Network (FoodNet): FoodNet 2015 surveillance report (final data). Atlanta: US Department of Health and Human Services; 2017.

7. New York City Department of Health and Mental Hygiene. Notice of adoption of amendments to Article 81 of the New York City Health Code. 2010 [cited 2018 Sep 25]. https://www1.nyc.gov/ assets/doh/downloads/pdf/notice/2010/Article-81.pdf
8. Fung A, Graham M, Weil D. Full disclosure: the perils and promise of transparency. Cambridge: Cambridge University Press; 2007.

9. Wong MR, McKelvey W, Ito K, Schiff C, Jacobson JB, Kass D. Impact of a letter-grade program on restaurant sanitary conditions and diner behavior in New York City. Am J Public Health. 2015;105:e81-7. http://dx.doi.org/10.2105/AJPH.2014.302404

10. Jin GZ, Leslie P. The effect of information on product quality: evidence from restaurant hygiene grade cards. Q J Econ. 2003;118:409-51. http://dx.doi.org/10.1162/003355303321675428

11. Simon PA, Leslie P, Run G, Jin GZ, Reporter R, Aguirre A, et al. Impact of restaurant hygiene grade cards on foodborne-disease hospitalizations in Los Angeles County. J Environ Health. 2005;67:32-6, 56, 59-60.

12. Centers for Disease Control and Prevention. 2018 National notifiable conditions. [cited 2018 July 14]. https://wwwn.cdc.gov/ nndss/conditions/notifiable/2018/

13. Wagner AK, Soumerai SB, Zhang F, Ross-Degnan D. Segmented regression analysis of interrupted time series studies in medication use research. J Clin Pharm Ther. 2002;27:299-309. http://dx.doi.org/ 10.1046/j.1365-2710.2002.00430.x

14. Johns M, Farley SM, Rajulu DT, Kansagra SM, Juster HR. Smoke-free parks and beaches: an interrupted time-series study of behavioural impact in New York City. Tob Control. 2015;24:497500. http://dx.doi.org/10.1136/tobaccocontrol-2013-051335

15. New York City Department of Health and Mental Hygiene. Notice of adoption of an amendment to Title 24 of the rules of the City of New York adding Chapter 23 ("Food Service Establishment Inspection Procedures"). New York: The Department; 2005.

16. Patrick ME, Adcock PM, Gomez TM, Altekruse SF, Holland BH, Tauxe RV, et al. Salmonella enteritidis infections, United States, 1985-1999. Emerg Infect Dis. 2004;10:1-7. http://dx.doi.org/ 10.3201/eid1001.020572

17. Nguyen TQ, Thorpe L, Makki HA, Mostashari F. Benefits and barriers to electronic laboratory results reporting for notifiable diseases: the New York City Department of Health and Mental Hygiene experience. Am J Public Health. 2007;97(Suppl 1): S142-5. http://dx.doi.org/10.2105/AJPH.2006.098996

18. Centers for Disease Control and Prevention. FoodCORE Center: New York City. 2016 [cited 2018 March 26]. https:/www.cdc.gov/ foodcore/centers/nyc.html

19. McKenzie B, Koerber W, Fields A, Benetsky M, Rapino M. Commuter-adjusted population estimates: ACS 2006-10. 2013 [cited 2018 Sep 17]. https://census.gov/library/working-papers/ 2013/acs/2013_McKenzie_02.html

20. NYC and Company. NYC travel and tourism visitation statistics [cited 2018 Feb 22]. http://www.nycandcompany.org/research/ nyc-statistics-page

21. New York City Department of Health and Mental Hygiene. Restaurant grading in New York City at 18 months. New York: The Department; 2012.

22. Silva J, Leite D, Fernandes M, Mena C, Gibbs PA, Teixeira P. Campylobacter spp. as a foodborne pathogen: a review. Front Microbiol. 2011;2:1-12. http://dx.doi.org/10.3389/fmicb.2011.00200

23. Lee P, Hedberg CW. Understanding the relationships between inspection results and risk of foodborne illness in restaurants. Foodborne Pathog Dis. 2016;13:582-6. http://dx.doi.org/10.1089/ fpd.2016.2137

24. Angelo KM, Nisler AL, Hall AJ, Brown LG, Gould LH. Epidemiology of restaurant-associated foodborne disease outbreaks, United States, 1998-2013. Epidemiol Infect. 2017;145:523-34. http://dx.doi.org/10.1017/S0950268816002314

Address for correspondence: Melanie J. Firestone, University of Minnesota School of Public Health, 420 Delaware St SE, Mayo Mail Code 807, Minneapolis, MN 55455, USA; email: fire0018@umn.edu 


\title{
Spatial Analysis of Wildlife Tuberculosis Based on a Serologic Survey Using Dried Blood Spots, Portugal
}

\author{
Nuno Santos, ${ }^{1}$ Telmo Nunes, Carlos Fonseca, Madalena Vieira-Pinto, \\ Virgílio Almeida, Christian Gortázar, Margarida Correia-Neves
}

We investigated the spatial epidemiology of bovine tuberculosis (TB) in wildlife in a multihost system. We surveyed bovine TB in Portugal by serologic analysis of elutes of dried blood spots obtained from hunted wild boar. We modeled spatial disease risk by using areal generalized linear mixed models with conditional autoregressive priors. Antibodies against Mycobaterium bovis were detected in 2.4\% $(95 \% \mathrm{Cl} 1.5 \%-3.8 \%)$ of 678 wild boar in 2 geographic clusters, and the predicted risk fits well with independent reports of $M$. bovis culture. Results show that elutes are an almost perfect substitute for serum (Cohen unweighted $\mathrm{k}$ $=0.818$ ), indicating that serologic tests coupled with dried blood spots are an effective strategy for large-scale bovine TB surveys, using wild boar as sentinel species. Results also show that bovine TB is an emerging wildlife disease and stress the need to prevent further geographic spread and prevalence increase.

$\mathrm{B}$ ovine tuberculosis (TB) is a zoonotic disease caused by Mycobacterium bovis and other members of the $M$. tuberculosis complex, whose natural hosts are wild and domestic mammals (1). Bovine TB is a disease of economic and public health relevance and is subjected to mandatory control programs in livestock in many countries. As a result of these programs, bovine TB has been eradicated in regions such as Australia and Scandinavia. However, in other regions, persistence of infection has been attributed to wildlife reservoirs, such as cervids in North America (2). In the Iberian Peninsula (Figure 1, panel A), bovine

Author affiliations: University of Minho School of Medicine (ICVS),

Braga, Portugal (N. Santos, M. Correia-Neves); ICVS/3B'S,

Portugal Government Associate Laboratory, Braga/Guimarães,

Portugal (N. Santos, M. Correia-Neves); University of Lisbon,

Lisbon, Portugal (T. Nunes, V. Almeida); University of Aveiro, Aveiro,

Portugal (C. Fonseca); University of Trás-os-Montes e Alto Douro,

Vila Real, Portugal (M. Vieira-Pinto); Instituto de Investigación en

Recursos Cinegéticos, Ciudad Real, Spain (C. Gortázar)

DOI: https://doi.org/10.3201/eid2412.171357
TB is maintained in a multihost pathogen system in which M. bovis and M. caprae circulate between sympatric wild ungulates (mainly wild boar [Sus scrofa] and red deer [Cervus elaphus]) and free-ranging domestic ungulates (1).

In Portugal, control of bovine TB has resulted in a low prevalence in livestock (4.5 cases/10,000 cattle and 2.9 cases/1,000 herds) in 2017 (3). Nevertheless, disease incidence has stabilized in recent years, and awareness of wildlife hosts has fueled the discussion over their role as reservoirs of bovine TB. In 2011, the Portuguese Animal Health Directorate (Lisbon, Portugal) established a surveillance area for bovine TB in large game species, encompassing regions where the disease was known to be present in wild ungulate populations (Figure 1, panel B).

Bovine TB in wildlife shows spatial structuring in the Iberian Peninsula. There is a core area in the central-southwestern region, in which the average prevalence of macroscopic lesions is $59 \%$ in wild boar (4). At the periphery of this core area, prevalence decreases, and becomes low to undetectable in eastern, northern, and western regions of the Iberian Peninsula (5-7). Nevertheless, spatial analyses of bovine TB on wildlife in the Iberian Peninsula, other than disease mapping, are notably lacking.

Large-scale disease surveys in wildlife require massscalable and inexpensive diagnostic tests; serologic methods are one of the most suitable techniques (8). An ELISA for detecting antibodies against the $M$. tuberculosis complex has been described and validated for use in wild boar $(9,10)$ and showed a moderately high estimated sensitivity of $79.2 \%$ and an excellent specificity of $100 \%$ (10). Another improvement for large-scale disease surveys in wildlife is a sampling protocol that might be used by nonspecialized personnel, such as hunters. One example is the dried blood spot technique, which was originally developed for human sampling but has been increasingly used for wildlife disease surveys (11).

${ }^{1}$ Current affiliation: Research Centre in Biodiversity and Genetic Resources, University of Porto,Vairão, Portugal. 


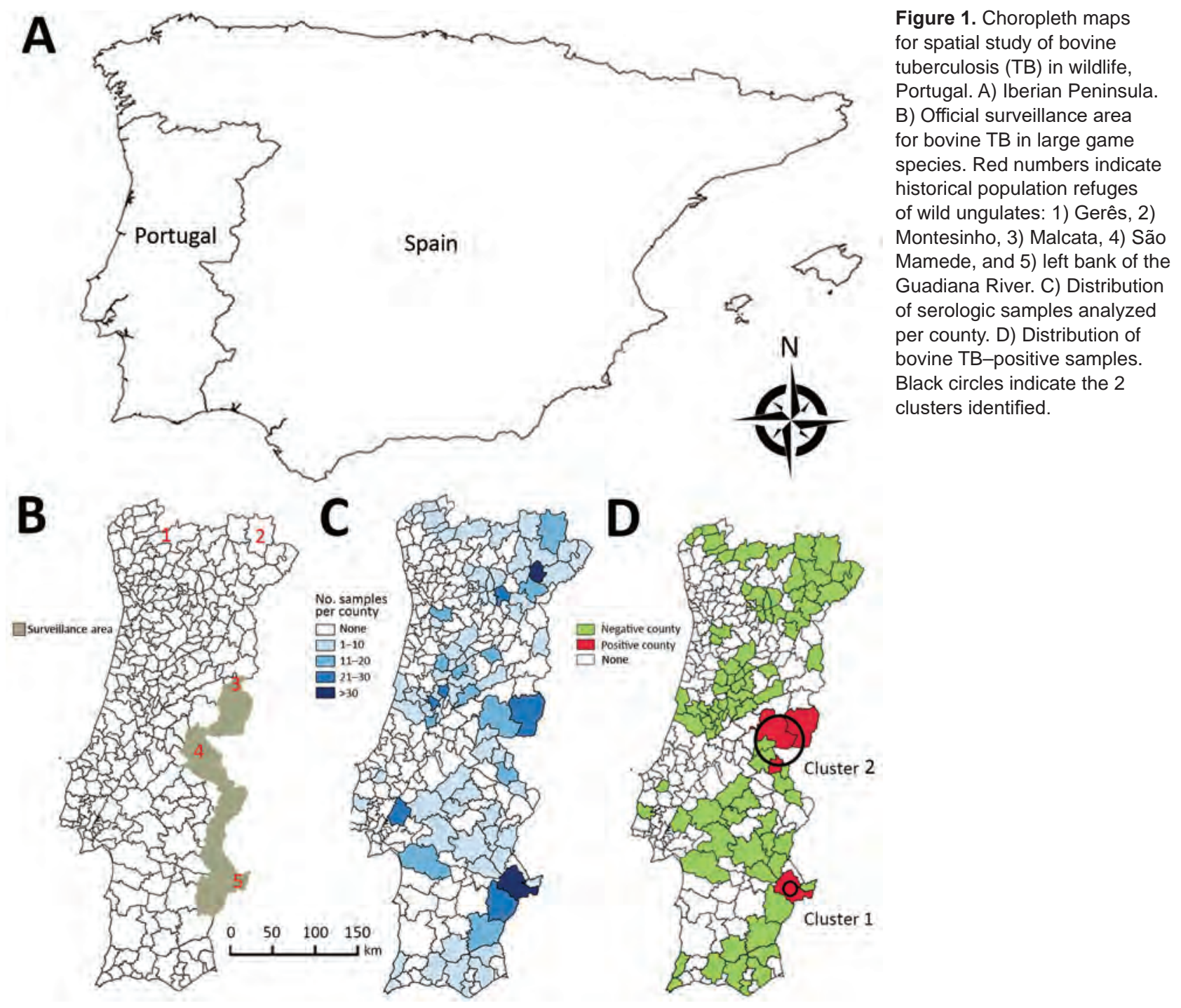

Wild boar have been shown to be a maintenance host for bovine TB in the Iberian Peninsula (12). Furthermore, wild boar have been used as a sentinel for bovine TB in wildlife because of their high susceptibility to infection with $M$. bovis and $M$. caprae and extensive exposure to these pathogens through direct contact, necrophagic habits, or fossorial habits (13-15). Wild boar populations in Portugal reached their nadir in the middle of the 20th century, when the species survived in only 5 populations or historical refuges $(16,17)$ (Figure 1, panel B). Since that time, wild boar populations have increased markedly and are currently found throughout Portugal $(16,17)$.

To assess the spatial epidemiology of bovine TB in wildlife, we analyzed the multihost pathogen system in the Iberian Peninsula by using the wild boar as a sentinel species. The main aims of this study were to assess the performance of dried blood spots as an alternative sample collection and storage technique for serologic surveys of bovine TB, map the distribution of wildlife bovine in Portugal on the basis of serologic methods, investigate spatial clustering of bovine TB in wildlife, and model the risk for bovine TB in Portugal.

\section{Methods}

\section{Collection of Samples}

Biological samples were obtained from 678 wild boar hunted during 2006-2013. Of these samples, 107 were serum and 571 were dried blood spots collected on Protein Saver (PS) 903 cards (Whatman, Maidstone, UK) $(\mathrm{n}=308)$ or Flinders Technology Associates (FTA) paper (Whatman) $(\mathrm{n}=263)$.

Serum samples were obtained from blood collected from the thoracic or abdominal cavities of hunted wild boar and stored at $-20^{\circ} \mathrm{C}$. Absorbent papers were distributed to hunters with instructions for the papers to be soaked in blood available from the thoracic cavity. Papers were dried in the shade and kept at room temperature in zipper-lock 
bags with data on the location of collection. Dried blood spots were recovered at the end of the hunting season and kept at $-20^{\circ} \mathrm{C}$ until processed. Elutes were obtained by cutting half a circle of PS card or a quarter of FTA cards. According to the manufacturer's instructions, a PS card absorbs $80 \mu \mathrm{L}$ of blood, and an FTA card absorbs $125 \mu \mathrm{L}$ of blood. These samples were further divided into 5 portions that were incubated overnight refrigerated in $200 \mu \mathrm{L}$ of phosphate-buffered saline (PBS), and the elute obtained was subjected to serologic analysis. Paired samples of serum and dried blood spots were collected from 22 wild boar that had macroscopic lesions compatible with bovine TB.

In 1 region of southeastern Portugal, tissue samples were collected from 340 hunted wild boar with either bovine TB-compatible lesions or pooled lymph nodes when lesions were absent. These samples were obtained during 2009-2014 and kept at $-20^{\circ} \mathrm{C}$ until bacteriological cultures were performed.

\section{Laboratory Analysis}

We tested serum samples by using an ELISA and bovine purified protein derivative (bovine PPD) as antigen and protein G-horseradish peroxidase as conjugate (10). In brief, we coated wells of ELISA plates with $100 \mu \mathrm{g}$ of bovine PPD for 18 hours at room temperature, washed the plates with PBS containing $0.05 \%$ Tween 20 (PBST), and incubated the plates for 1 hour at $37^{\circ} \mathrm{C}$ with $140 \mu \mathrm{L} /$ well of $5 \%$ skimmed milk in PBST to block potential free binding sites. We added serum or elute samples to plates $(10 \mu \mathrm{L} /$ well $)$ at dilutions of 1:200 in PBS for serum and 1:50 in PBS for elutes and incubated samples for 1 hour at $37^{\circ} \mathrm{C}$. Protein G-horseradish peroxidase conjugate was added $(100 \mu \mathrm{L} /$ well $)$ at a dilution of $2.5 \mu \mathrm{g} / \mathrm{mL}$ in PBST and incubated at $37^{\circ} \mathrm{C}$ for $80 \mathrm{~min}$. A total of $100 \mu \mathrm{L}$ of substrate (SigmaFast OPD; Sigma-Aldrich, St. Louis, MO, USA) was added to each well and incubated at room temperature in the dark. The reaction was stopped after 20 min by the addition of $50 \mu \mathrm{L} /$ well of $3 \mathrm{~N} \mathrm{H}_{2} \mathrm{SO}_{4}$.

We measured optical density (OD) by using a spectrophotometer at $450 \mathrm{~nm}$. Blanks and positive and negative controls were tested in duplicate in each plate, and samples were tested in triplicate. Results were calculated as mean sample OD divided by 2 times the mean negative control OD; the cutoff for positivity was 1 (10).

We prepared bacteriological cultures of tissue samples in a Biosafety Level 3 facility according to a described protocol (18). In brief, $3 \mathrm{~g}$ of tissue were homogenized and decontaminated for 2 hours with $0.75 \%$ hexa-decylpyridinium chloride, centrifuged at 2,566 $\times g$ for $30 \mathrm{~min}$, and the supernatant collected. We inoculated 2 tubes containing Coletsos medium (bioMerieux, Marcy l’Étoile, France) with $250 \mu \mathrm{L}$ of supernatant-sediment interface and incubated these tubes at $37^{\circ} \mathrm{C}$ for 15 wks. Isolates were identified by PCR for 16S rRNA, insertion sequence 1561, and
Rv1510 genes after DNA extraction by using the standard phenol-chloroform method after bead-beating with 100 $\mu \mathrm{L}$ of $0.1-\mathrm{mm}$ zirconia/silica beads (Biospec Products, Bartlesville, OK, USA) in a FastPrep 24 Homogenizer (MP Biomedicals, Santa Ana, CA, USA).

\section{Data Analysis}

To determine agreement between ELISA results (positive or negative) from paired serum and elute samples, we computed the Cohen unweighted $\kappa$ value (19) by using irr in $\mathrm{R}$ software (20) (R Development Core Team, Vienna, Austria). We obtained serologic data for 92 of 278 counties in Portugal (Figure 1, panel C). The area considered for each county excluded regions classified as urban or water bodies in the CORINE database (21). We created choropleth maps, which use differences in shading, coloring, or placing of symbols within predefined areas to indicate average values of a property or quantity in those areas, of regions with bovine TB and performed spatial interpolation by using QGIS version 2.6.1 Brighton software (https://www.qgis.org/en/site/getinvolved/governance/governance.html). To detect spatial aggregation of bovine TB, we performed cluster analysis on the basis of Kuldorff spatial scan statistics by using the Bernoulli distribution and setting the maximum cluster size at $50 \%$ with SatScan version 9.3.1 software (22).

We assessed the association between detection of bovine TB in wild boar in each county and independent variables (Table 1) by using spatial generalized linear mixed modeling of areal data with the localized conditional autoregressive priors proposed by Lee and Mitchel (23). We included the bioregions of the Iberian Peninsula (Atlantic/ Mediterranean) (24) as local conditional autoregressive priors because they have been shown to have distinct bovine TB epidemiologic scenarios (7). We implemented models by using the CARBayes version 5.0 package (25) in R software. We based inference on 20,000 Markov Chain Monte Carlo iterations (200,000 iterations with a thin factor of 10 to reduce autocorrelation) after an initial burn-in of 40,000 iterations. Taking into consideration the home ranges of wild boar (26), we decided to include counties $\leq 25 \mathrm{~km}$ apart in the neighborhood matrix of each other.

We estimated domestic host absolute densities on the basis of data from the Portuguese Livestock Movement Database (https://ifap.pt) with the following inclusion criteria: we considered only animals $>6$ months of age among freerange pigs, extensively reared sheep and goats, and meat production cattle. We excluded intensively reared animals to provide more realistic estimates of the livestock population at potential risk from contact with wildlife. Meatproduction cattle were selected as a proxy for extensive rearing because this is the predominant beef cattle production system in Portugal, and dairy cattle herds are almost exclusively housed indoors. 
Table 1. Independent variables included in initial models of bovine tuberculosis, Portugal*

\begin{tabular}{|c|c|c|}
\hline Variable & Specific variable & Unit \\
\hline \multirow[t]{3}{*}{ Wild host density } & Wild boar hunting bag & Wild boar hunted $/ \mathrm{km}^{2} \dagger$ \\
\hline & Red deer hunting bag & Red deer hunted $/ \mathrm{km}^{2} \dagger$ \\
\hline & Fallow deer hunting bag & Fallow deer hunted $/ \mathrm{km}^{2} \dagger$ \\
\hline Game management & $\begin{array}{l}\text { Intensity of management as proportion of area as tourist or } \\
\text { national hunting zones }\end{array}$ & Proportion \\
\hline \multirow[t]{4}{*}{ Domestic host density } & Cattle density $>6$ mo of age, meat & Cattle/km² \\
\hline & Sheep density $>6$ mo of age, extensive & Sheep $/ \mathrm{km}^{2}$ \\
\hline & Goat density $>6$-mo of age, extensive & Goats $/ \mathrm{km}^{2}$ \\
\hline & Pig density $>6$ mo of age, free range & $\mathrm{Pigs} / \mathrm{km}^{2}$ \\
\hline Bovine tuberculosis incidence in cattle & Bovine tuberculosis incidence in cattle & Proportion \\
\hline Historical population dynamics & Distance to the nearest historical refuge & $\mathrm{km}$ \\
\hline
\end{tabular}

We selected hunting bag (number of animals hunted per square kilometer in 1 year) as a proxy for wild ungulate density and calculated this density as the annual average of the number of hunted ungulates (wild boar, red deer, and fallow deer) from those hunting areas for which $\geq 2$ years of data were available for 2008-2012. Because hunting bag data were not available for $65 / 278$ counties, we performed an inverse distance weighted interpolation with power set at 3 to obtain estimates for the entire territory.

We selected the proportion of the area of each county dedicated to commercial hunting (tourism and national hunting areas usually intensively managed for maximizing profit) as a proxy for intensity of game management. Other types of hunting areas are dedicated to recreational hunting (i.e., usually no fencing, restocking, or large-scale artificial feeding of large game species). We included the historical presence of wild boar as the distance from the centroid of each county to the nearest historical refuge (Figure 1, panel B). We calculated the incidence of bovine TB in cattle as the average of the annual incidence rate for each county during 2008-2012 on the basis of data from the bovine TB eradication program.

We estimated a variance inflation factor to assess multicollinearity with a threshold of 5 . We used a nonspatial logistic regression with backward stepwise elimination and selected the final model on the basis of Akaike information criterion corrected for small samples and model weights (27). We calculated the McFadden pseudo- $\mathrm{R}^{2}$ value to measure the goodness of fit of each model. We used the posterior probabilities of the spatial model to predict the risk for bovine TB in wild boar populations in all counties in Portugal. We assessed model convergence on the basis of the Geweke statistic (28).

\section{Results}

We found that 11 paired serum samples and PS elutes were positive for antibodies against bovine PPD, 9 were negative for both, and 2 were positive in serum and negative in elutes. There was an almost perfect agreement between serologic results for both types of samples (mean \pm SD Cohen unweighted $\kappa$ value $=0.818 \pm 0.121)$.
We detected antibodies against bovine PPD in 16/678 wild boar $(2.4 \%, 95 \%$ CI 1.5\%-3.8\%). Antibody-positive wild boar originated from 4/92 counties tested (Figure 1, panel C). Kulldorff spatial scan statistics identified 2 clusters of bovine TB in wildlife. Cluster 1 was found in 1 county that had 8/36 positive wild boar (relative risk $=17.83, \mathrm{p}<0.001$ ), and cluster 2 was found in 5 counties (radius $=43 \mathrm{~km}$ ) that had $8 / 65$ positive wild boar (relative risk $=9.43, \mathrm{p}=0.011)$ (Figure 1, panel D).

The selected nonspatial logistic regression model with the presence of bovine TB as the dependent variable showed a McFadden pseudo- $\mathrm{R}^{2}$ value $=0.656$ (Table 2 ). The spatial generalized linear mixed model (deviance information criterion $=24.141$ ) that included the variables distance to historical refuge, bovine TB incidence in cattle, red deer hunting bag, intensity of management, and red deer hunting bag times intensity of management (Table 2) explained $45.5 \%$ of the deviance. The posterior probability of the presence of bovine TB in wildlife (Figure 2) was significantly higher for those counties with independent reports of isolation of $M$. bovis from free-ranging wildlife (Mann-Whitney-Wilcoxon $\mathrm{W}=320$; $\mathrm{p}<0.001)(26,29-32)$.

We further investigated bovine TB in southeastern Portugal. This investigation included cluster 1 , where $M$. bovis $(\mathrm{n}=51)$ and $M$. caprae $(\mathrm{n}=2)$ were isolated from $53 / 340$ wild boar in 6/17 hunting areas, which had an overall bacteriological culture prevalence of $15.6 \%$ (95\% CI $12.1 \%-$ $19.8 \%)$. In 1 nonfenced hunting area, culture prevalence of wild boar bovine TB increased significantly $(\mathrm{p}=0.049$ by Fisher exact test) from $46.2 \%$ (95\% CI $26.6 \%-66.6 \%)$ during 2005-2006 (6) to $67.7 \%$ (95\% CI 55.4\%-78.0\%) during 2009-2014 (Figure 3).

\section{Discussion}

We report a spatial analysis of bovine TB in wildlife in the multihost system of the Iberian Peninsula on the basis of serologic data for hunted wild boar. Serologic analysis was previously shown to have reasonably good sensitivity and excellent specificity for detecting bovine TB in wild boar (10), and this species is a suitable sentinel for bovine 
Table 2. Variables included in nonspatial binomial general linear and spatial generalized linear mixed models of bovine tuberculosis in wild boar, Portugal

\begin{tabular}{|c|c|c|c|c|}
\hline \multirow[b]{3}{*}{ Variable } & & & \multicolumn{2}{|c|}{ Spatial generalized linear mixed model } \\
\hline & \multicolumn{2}{|l|}{ Logistic regression model } & \multirow{2}{*}{$\begin{array}{c}\text { Median coefficient } \\
\text { (95\% credible interval) }\end{array}$} & \multirow{2}{*}{$\begin{array}{l}\text { Geweke } \\
\text { statistic }\end{array}$} \\
\hline & Coefficient $(95 \% \mathrm{Cl})$ & $\mathrm{p}$ value & & \\
\hline Intercept & $-8.561(-25.992$ to -2.644$)$ & 0.073 & $-12.886(-19.767$ to -7.985$)$ & 1.3 \\
\hline Wild boar historical refuge & $-0.114(-0.356$ to -0.020$)$ & 0.101 & $-0.088(-0.170$ to -0.022$)$ & 1.4 \\
\hline Bovine tuberculosis incidence in cattle & 73.719 (6.919 to 231.075$)$ & 0.126 & $35.797(-4.396$ to 82.742$)$ & -1.6 \\
\hline Management intensity & 29.296 (6.167 to 101.249$)$ & 0.126 & 25.009 (6.510 to 49.979$)$ & -1.3 \\
\hline Red deer hunting bag & 9.391 (1.884 to 34.953$)$ & 0.150 & $6.990(1.823$ to 11.814$)$ & -0.8 \\
\hline Red deer hunting bag $\times$ management intensity & $-30.922(-118.421$ to -7.887$)$ & 0.162 & $-23.221(-39.083$ to -8.343$)$ & 0.9 \\
\hline
\end{tabular}

TB (13-15). We provide evidence showing that dried blood spots collected on PS cards by nonspecialist personnel are appropriate for serologic surveys of bovine TB, as shown by the almost perfect agreement between serologic results for serum samples and elutes. These results were expected because dried blood spots have been extensively validated for many other human or animal host-pathogen systems and generally found to be suitable for serologic surveys, even when blood is collected in remote and demanding conditions $(11,33)$. This combination of dried blood spots and serologic analysis is a major advance for large-scale surveillance of bovine TB in wildlife anywhere wild boar are a suitable indicator species.

Our results support previous data suggesting a strong spatial structure of bovine TB in wildlife. Two clusters were identified in southern (cluster 1 ) and central-eastern (cluster 2) Portugal, which are located at the periphery of the high-prevalence core area in the central-southwestern region of the Iberian Peninsula $(4,5)$. A subsequent survey for bovine TB in cluster 1 relied on bacteriological culture of tissues collected from hunted wild boar, which is an established but more expensive and labor-intensive diagnostic technique than serologic analysis. This regional bacteriological survey confirmed bovine TB as an emerging disease in wildlife and documented a $46.2 \%$ increase in prevalence in less than a decade at 1 nonfenced hunting area, similar to other populations in the Iberian Peninsula (4).

The spatial risk model we reported identified some predicted high-risk counties not included in the surveillance area for bovine TB in large game species (Figure 1, panel B) and thus could be used for better allocation of resources for wildlife disease surveillance and public health protection. The spatial model of bovine TB risk in wildlife generally agrees with results of published independent surveys because most reported wildlife isolates of $M$. bovis and $M$. caprae overlap with predicted moderate-risk to high-risk areas. The single exception is $M$. bovis isolated from a wild boar in Coimbra in central-western Portugal (Figure 2), an area that has a low predicted risk for bovine TB in wildlife (30). Red deer were introduced into this region during 1995-1999 and some of the founder animals originated from bovine TB-infected areas included in cluster 1 , in which the same spoligotype was also found in wild boar and red deer $(17,30)$. No additional $M$. bovis isolates have been reported from this region, suggesting that after the initial introduction and spillover into local wild boar, the infection waned or persists at a low prevalence, in accordance with the predicted low risk for bovine TB in our model.

This observation suggests that relatively dense red deer populations are needed to maintain bovine TB at a high prevalence in wild ungulate populations. This finding also contrasts with the situation reported from south-central

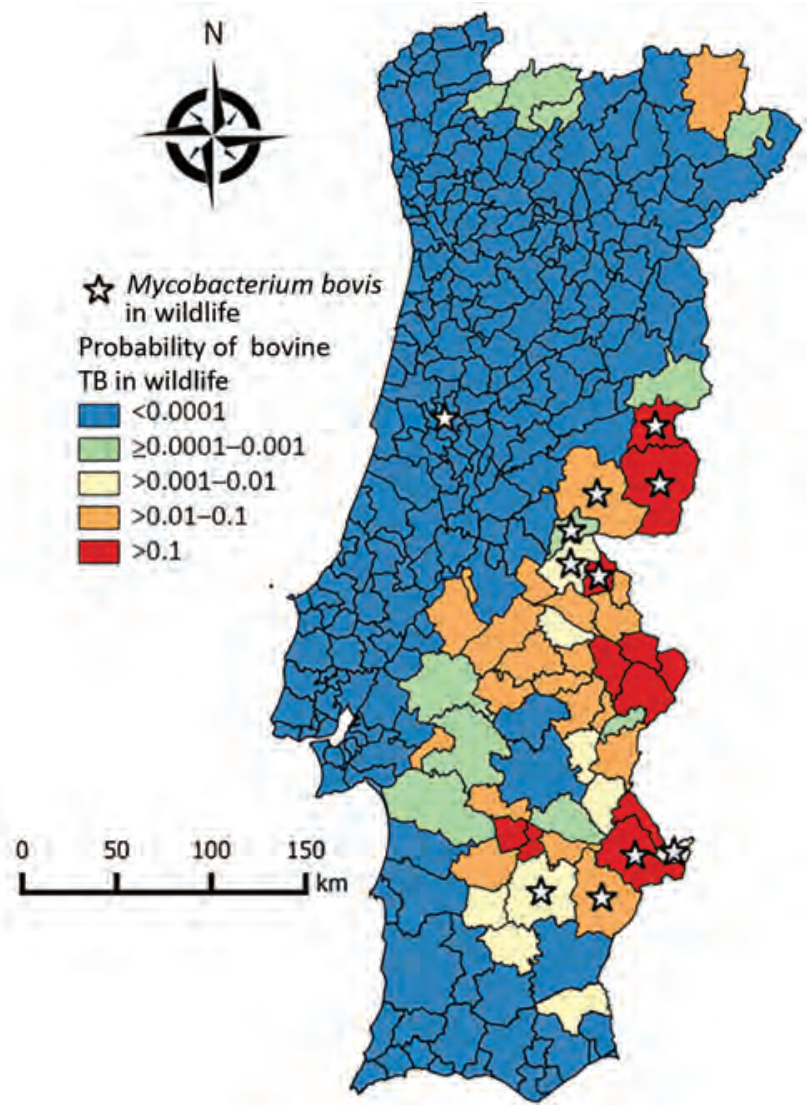

Figure 2. Choropleth map of risk for bovine TB in wildlife, Portugal, showing the probability of the presence of bovine TB in wildlife in counties based on the conditional autoregressive spatial generalized linear mixed model. Stars indicate counties in which Mycobacterium bovis was isolated from free-ranging wildlife, determined on the basis of independent published data (6,29-32). $\mathrm{TB}$, tuberculosis. 


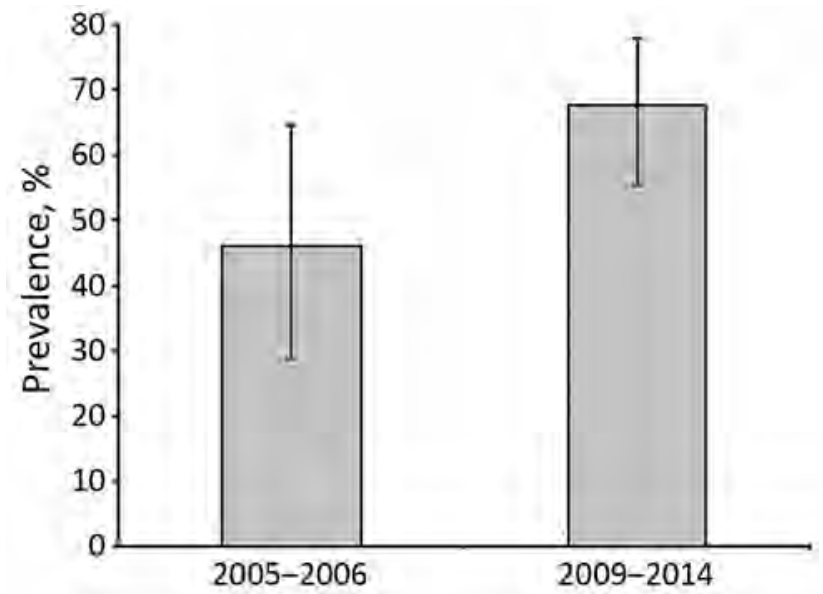

Figure 3. Temporal trend in prevalence of bovine tuberculosis in wild boar in 1 nonfenced hunting area, Portugal, by bacteriological culture during 2005-2006 (6) and 2009-2014 (this study). Error bars indicate $95 \% \mathrm{Cls}$.

Spain, where wild boar is considered the main maintenance host, probably because intensive game management is rare in Portugal, with similarities with what was described for the Atlantic bioregion of Spain $(14,17)$. Further studies involving spatially structured estimation of the abundance of wild ungulates and bovine TB prevalence along the edge of the infected area are warranted to identify the relative roles of wild boar and red deer densities in maintenance of bovine TB in this multihost system.

Red deer density and its interaction with the intensity of game management were associated with bovine TB in wild boar in the spatial risk model. Red deer density is a better predictor of bovine TB in wild boar populations than wild boar density, further strengthening the role of red deer as a key reservoir of bovine TB in wildlife in Portugal. The historical dynamics of wild boar populations are associated with contemporary distribution of bovine TB. Starting in the mid-20th century, wild boar populations expanded from historical refuges, and bovine TB in wildlife also seems to be increasing but at a much slower pace. One possible explanation for this pattern is that as wild boar populations spread, densities at the front of the expansion wave were low (34). Thus, bovine TB could not be maintained even with the addition of infected animals from infected historical refuges. As expansion continued and wild boar densities increased (34), the range of $M$. bovis seems to be slowly increasing.

The incidence of bovine TB in livestock was included in the selected model and highlighted the link between domestic and wild epidemiologic cycles in wildlife, which was strongly suspected on the basis of molecular epidemiology data (30). Although the directionality of such a link could not be inferred in our study, spillover from wildlife was shown to partially explain incidence of bovine TB in cattle in south-central Spain (35), suggesting that bovine
TB in livestock could be an indicator of local disease transmission in wildlife.

In conclusion, we report a spatial analysis of bovine TB in wildlife in Portugal that used wild boar as a sentinel species and assessed the relative performance of dried blood spots collected on PS cards as a new sampling tool for large-scale serologic surveys. We confirmed the strong spatial clustering of bovine TB in wildlife and identified risk factors related to red deer density, intensity of wild ungulate management, historical population dynamics of wild boar, and incidence of bovine TB in cattle. The risk maps developed provide new tools for the targeted control of bovine TB in wild ungulate populations and identify areas at high risk for spread of disease.

\section{Acknowledgments}

We thank the hunters for collecting blood samples from wild boar; Mariana Boadella, Paqui Talavera, and Natacha Melo for providing assistance with ELISAs; the Direcção Geral de Alimentação e Veterinária for providing data on populations of domestic hosts and bovine TB incidence in cattle; and the Instituto de Conservação da Natureza e Florestas for providing data on the number of hunted wild ungulates.

This study was supported by Plan Nacional (grant CGL201789866 from the Ministry of Economy and Competitiveness, Spain, and Fondo Europeo de Desarollo Regional) and Programa Operacional Regional do Norte (grant ON.2 O Novo Norte), Quadro de Referência Estratégico Nacional through the Fundo Europeu de Desenvolvimento Regional. N.S. was supported by $\mathrm{PhD}$ grant SFRH/BD/69390/2010 from Fundação para a Ciência e Tecnologia.

\section{About the Author}

Dr. Santos is a postdoctoral research scientist at the Research Centre in Biodiversity and Genetic Resources, Vairão, Portugal. His research interests are bovine tuberculosis epidemiology in wild ungulates, disease ecology at the wildlife-livestock interface, and conservation physiology using carnivores and ungulates as research models.

\section{References}

1. Gortázar C, Delahay RJ, Mcdonald RA, Boadella M, Wilson GJ, Gavier-Widen D, et al. The status of tuberculosis in European wild mammals. Mammal Review. 2012;42:193-206. http://dx.doi.org/10.1111/j.1365-2907.2011.00191.x

2. Palmer MV. Mycobacterium bovis: characteristics of wildlife reservoir hosts. Transbound Emerg Dis. 2013;60(Suppl 1):1-13. http://dx.doi.org/10.1111/tbed.12115

3. Directorate General for Food and Veterinary Affairs. Animal health technical report: bovine tuberculosis, 2017 [in Portuguese] [cited 2018 Jul 26]. http://www.dgv.min-agricultura.pt/portal/page/portal/ DGV.

4. Vicente J, Barasona JA, Acevedo P, Ruiz-Fons JF, Boadella M, Diez-Delgado I, et al. Temporal trend of tuberculosis in wild 
ungulates from Mediterranean Spain. Transbound Emerg Dis. 2013;60(Suppl 1):92-103. http://dx.doi.org/10.1111/tbed.12167

5. Boadella M, Acevedo P, Vicente J, Mentaberre G, Balseiro A, Arnal M, et al. Spatio-temporal trends of Iberian wild boar contact with Mycobacterium tuberculosis complex detected by ELISA. EcoHealth. 2011;8:478-84. http://dx.doi.org/10.1007/s10393-011-0713-y

6. Santos N, Correia-Neves M, Ghebremichael S, Källenius G, Svenson SB, Almeida V. Epidemiology of Mycobacterium bovis infection in wild boar (Sus scrofa) from Portugal. J Wildl Dis. 2009;45:1048-61. http://dx.doi.org/10.7589/0090-3558-45.4.1048

7. Muñoz-Mendoza M, Marreros N, Boadella M, Gortázar C, Menéndez S, de Juan L, et al. Wild boar tuberculosis in Iberian Atlantic Spain: a different picture from Mediterranean habitats. BMC Vet Res. 2013;9:176. http://dx.doi.org/10.1186/1746-6148-9-176

8. Gilbert AT, Fooks AR, Hayman DT, Horton DL, Müller T, Plowright R, et al. Deciphering serology to understand the ecology of infectious diseases in wildlife. EcoHealth. 2013;10:298-313. http://dx.doi.org/10.1007/s10393-013-0856-0

9. Aurtenetxe O, Barral M, Vicente J, de la Fuente J, Gortázar C, Juste RA. Development and validation of an enzyme-linked immunosorbent assay for antibodies against Mycobacterium bovis in European wild boar. BMC Vet Res. 2008;4:43. http://dx.doi.org/ 10.1186/1746-6148-4-43

10. Boadella M, Lyashchenko K, Greenwald R, Esfandiari J, Jaroso R, Carta T, et al. Serologic tests for detecting antibodies against Mycobacterium bovis and Mycobacterium avium subspecies paratuberculosis in Eurasian wild boar (Sus scrofa scrofa). J Vet Diagn Invest. 2011; 23:77-83. http://dx.doi.org/10.1177/104063871102300111

11. Curry PS, Ribble C, Sears WC, Hutchins W, Orsel K, Godson D, et al. Blood collected on filter paper for wildlife serology: detecting antibodies to Neospora caninum, West Nile virus, and five bovine viruses in reindeer. J Wildl Dis. 2014;50:297-307. http://dx.doi.org/ 10.7589/2012-02-047

12. Naranjo V, Gortázar C, Vicente J, de la Fuente J. Evidence of the role of European wild boar as a reservoir of Mycobacterium tuberculosis complex. Vet Microbiol. 2008;127:1-9. http://dx.doi.org/10.1016/j.vetmic.2007.10.002

13. Nugent G, Whitford J, Young N. Use of released pigs as sentinels for Mycobacterium bovis. J Wildl Dis. 2002;38:665-77. http://dx.doi.org/10.7589/0090-3558-38.4.665

14. Gortázar C, Vicente J, Boadella M, Ballesteros C, Galindo RC, Garrido J, et al. Progress in the control of bovine tuberculosis in Spanish wildlife. Vet Microbiol. 2011;151:170-8. http://dx.doi. org/10.1016/j.vetmic.2011.02.041

15. Yockney IJ, Nugent G, Latham MC, Perry M, Cross ML, Byrom AE. Comparison of ranging behaviour in a multi-species complex of free-ranging hosts of bovine tuberculosis in relation to their use as disease sentinels. Epidemiol Infect. 2013;141:1407-16. http://dx.doi.org/10.1017/S0950268813000289

16. Lopes F, Borges J. Wild boar in Portugal. Galemys. 2004;16:243-51.

17. Vingada J, Fonseca C, Cancela J, Ferreira J, Eira C. Ungulates and their management in Portugal. In: Appolonio M, Andersen R, Putman R, editors. European ungulates and their management in the 21st century. Cambridge (UK): Cambridge University Press; 2010. p. 392-418.

18. Santos N, Geraldes M, Afonso A, Almeida V, Correia-Neves M. Diagnosis of tuberculosis in the wild boar (Sus scrofa): a comparison of methods applicable to hunter-harvested animals. PLoS One. 2010;5:e12663. http://dx.doi.org/10.1371/journal.pone.0012663

19. Fleiss JL, Cohen J, Everitt BS. Large sample standard errors of kappa and weighted kappa. Psychological Bulletin. 1969;72:323-7. http://dx.doi.org/10.1037/h0028106

20. Gamer M, Lemon J, Fellows I, Singh P. Various coefficients of interrater reliability and agreement. $\mathrm{R}$ package version 0.84 ;
2012 [cited 2018 Sep 4]. https://cran.r-project.org/web/packages/irr/

21. European Environmental Agency. Luxembourg NATLAN. Nature/land cover information package; 2006 [cited 2016 Sep 11]. https://www.eea.europa.eu/publications/COR0-landcover

22. Kulldorff M. A spatial scan statistic. Communications Statistics Theory Methods. 1997;26:1481-96. http://dx.doi.org/10.1080/ 03610929708831995

23. Lee D, Mitchell R. Boundary detection in disease mapping studies. Biostatistics. 2012;13:415-26. http://dx.doi.org/10.1093/ biostatistics/kxr036

24. European Environmental Agency. Luxembourg. Biogeograhical regions; 2016 [cited 2016 Sep 11]. www.eea.europa.eu/data-andmaps/data/biogeographical-regions-europe-3\#tab-gis-data

25. Lee D. CARBayes: an R package for Bayesian spatial modeling with conditional autoregressive priors. Journal of Statistical Software. 2013;55:1-24. http://dx.doi.org/10.18637/jss.v055.i13

26. Bosch J, Peris S, Fonseca C, Martínez M, De La Torre A, Iglesias I. Distribution, abundance and density of the wild boar on the Iberian Peninsula, based on the CORINE program and hunting statistics. Folia Zoologica (Brno). 2012;61:138-51.

http://dx.doi.org/10.25225/fozo.v61.i2.a7.2012

27 Burnham KP, Anderson DR. Model selection and multimodel inference: a practical information-theoretical approach. 2nd ed. New York: Springer; 2002.

28. Geweke J. Evaluating the accuracy of sampling-based approaches to the calculation of posterior moments. In: Berger JO, Bernardo JM, Dawid AP, Smith AF, editors. Bayesian statistics. Oxford (UK): Oxford University Press; 2002. p. 169-193.

29. Vieira-Pinto M, Alberto J, Aranha J, Serejo J, Canto A, Cunha MV, et al. Combined evaluation of bovine tuberculosis in wild boar (Sus scrofa) and red deer (Cervus elaphus) from central-east Portugal. European Journal of Wildlife Research. 2011;57:1189-201. http://dx.doi.org/10.1007/s10344-011-0532-z

30. Cunha MV, Matos F, Canto A, Albuquerque T, Alberto JR, Aranha JM, et al. Implications and challenges of tuberculosis in wildlife ungulates in Portugal: a molecular epidemiology perspective. Res Vet Sci. 2012;92:225-35. http://dx.doi.org/10.1016/ j.rvsc.2011.03.009

31. Matos AC, Figueira L, Martins MH, Pinto ML, Matos M, Coelho AC. New insights into Mycobacterium bovis prevalence in wild mammals in Portugal. Transbound Emerg Dis. 2016;63:e313-22. http://dx.doi.org/10.1111/tbed.12306

32. Madeira S, Manteigas A, Ribeiro R, Otte J, Fonseca AP, Caetano P, et al. Factors that influence Mycobacterium bovis infection in red deer and wild boar in an epidemiological risk area for tuberculosis of game species in Portugal. Transbound Emerg Dis. 2017;64:793804. http://dx.doi.org/10.1111/tbed.12439

33. Parker SP, Cubitt WD. The use of the dried blood spot sample in epidemiological studies. J Clin Pathol. 1999;52:633-9. http://dx.doi.org/10.1136/jcp.52.9.633

34. Holland E, Aegerter JN, Smith GC. Spatial sensitivity of a generic population model, using wild boar (Sus scrofa) as a test case. Ecological Modelling. 2007;205:146-58. http://dx.doi.org/10.1016/ j.ecolmodel.2007.02.026

35. LaHue NP, Baños JV, Acevedo P, Gortázar C, Martínez-López B. Spatially explicit modeling of animal tuberculosis at the wildlifelivestock interface in Ciudad Real Province, Spain. Prev Vet Med. 2016;128:101-11. http://dx.doi.org/10.1016/j.prevetmed.2016.04.011

Address for correspondence: Nuno Santos, Research Centre in Biodiversity and Genetic Resources, University of Porto, Campus de Vairão, 4485-661 Vairão, Portugal; email: nuno.santos@cibio.up.pt 


\title{
Rat Lungworm I nfection in Rodents across Post-Katrina New Orleans, Louisiana, USA
}

\author{
Rosalyn C. Rael, Anna C. Peterson, Bruno Ghersi-Chavez, Claudia Riegel, Amy E. Lesen, Michael J. Blum
}

Rat lungworm (Angiostrongylus cantonensis), a parasitic nematode that can cause eosinophilic meningitis in humans, was first detected in New Orleans, Louisiana, USA, in the mid-1980s and now appears to be widespread in the southeastern United States. We assessed the distribution, prevalence, and intensity of $A$. cantonensis infection in New Orleans by examining lung biopsy samples of rodents trapped at 96 sites in 9 areas in Orleans Parish and 1 area in neighboring St. Bernard Parish during May 2015 through February 2017. These areas were selected to capture contrasting levels of income, flooding, and post-disaster landscape management after Hurricane Katrina in 2005. We detected $A$. cantonensis in all areas and in 3 of the 4 rat species trapped. Overall prevalence was $\approx 38 \%$ but varied by area, host species, and host species co-occurrence. Infection intensity also varied by host species. These findings suggest that socioecological analysis of heterogeneity in definitive and intermediate host infection could improve understanding of health risks across the city.

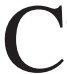
oncern is increasing about the spread of rat lungworm (Angiostrongylus cantonensis), especially in the southeastern United States (1-5). A parasitic nematode carried by intermediate mollusk hosts and definitive rat hosts $(6,7)$, rat lungworm can cause eosinophilic meningitis in humans who become infected by ingesting intermediate hosts or paratenic hosts, such as freshwater shrimp and frogs $(6,7)$. A. cantonensis was first reported in North America from Norway rats (Rattus norvegicus) trapped in New Orleans, Louisiana, USA, along the Mississippi River during April 1986 through February 1987 (8). Later surveys suggest the parasite has since become more widespread in Louisiana. Surveys of intermediate apple snail (Pomacea canaliculata) hosts, for example, detected the parasite in suburban areas of

Author affiliations: Tulane University, New Orleans, Louisiana, USA (R.C. Rael, A.C. Peterson, B. Ghersi-Chavez, A.E. Lesen, M.J. Blum); University of Tennessee, Knoxville, Tennessee, USA (A.C. Peterson, B. Ghersi-Chavez, M.J. Blum); The City of New Orleans Mosquito, Termite, and Rodent Control Board, New Orleans (C. Riegel)

DOI: https://doi.org/10.3201/eid2412.180056
New Orleans $(3,9)$. Infections have also since been reported in nonhuman incidental mammal hosts $(9,10)$, and 2 cases of human eosinophilic meningitis from rat lungworm infection were diagnosed in nearby areas of Louisiana $(11,12)$. Rat lungworm also appears to have become widespread across Florida (9) and has been recently detected in Oklahoma (11).

We trapped rodents across New Orleans to characterize the current distribution, prevalence, and intensity of $A$. cantonensis infection and to determine how these aspects vary according to organismal and ecological characteristics of definitive hosts, including species co-occurrence. This study enabled us to identify factors associated with definitive host infection, which might affect transmission risk across the city and offer further insight into the global progression, surveillance, and control of rodent-associated infectious disease.

\section{Methods}

\section{Study Animals}

We collected rats during May 2015 through February 2017 (following Tulane University [New Orleans, LA, USA] Institutional Animal Care and Use Committee [IACUC] protocol \#0451) during a quantitative population survey across 96 city blocks in 8 neighborhoods in New Orleans, a natural area in Orleans Parish, and an area in neighboring St. Bernard Parish. These areas were selected to capture contrasting levels of income, flooding, and postdisaster landscape management after Hurricane Katrina in 2005 (Figure 1; online Technical Appendix Table 1, https://wwwnc.cdc.gov/EID/ article/24/12/18-0056-Techapp1.pdf) $(13,14)$. We selected 8-10 sites in each study area by random stratification across a 1-km resolution grid spanning the city. Each site was visited 4 times during May 2015 through January 2017: once during summer and once during winter months each year, except for sites in St. Bernard Parish, which were visited only twice (summer 2016 and winter 2016-17). During each trapping period, 30 Tomahawk Live traps (Hazelhurst, WI, USA) were set in locations with potential or evident rodent activity for a minimum of 3 consecutive nights. Trapping efforts continued at each site until no additional rats were 
captured. Sherman traps (H.B. Sherman Traps, Inc., Tallahassee, FL, USA) were also placed at a subset of 48 sites to capture smaller rodents following the same approach (Tulane University IACUC protocol \#0460).

All rodents were necropsied after euthanasia following Tulane University IACUC protocols \#0451 and \#0460. We recorded standard weight and length measurements, as well as species identity; sex; sexual maturity; and, in females, parity. We categorized all Norway rats and roof rats ( $R$. rattus) into 3 age classes (juvenile, subadult, adult) according to body weight $(15,16)$. Urine, lung, liver, spleen, kidney, and tail tissues were sampled and archived in $-80^{\circ} \mathrm{C}$ freezers. We visually screened lung tissues for parasites, which were isolated, counted, and preserved in $95 \%$ ethanol. Representative lung parasites were identified through PCR (17) (online Technical Appendix; online Technical Appendix Table 2).

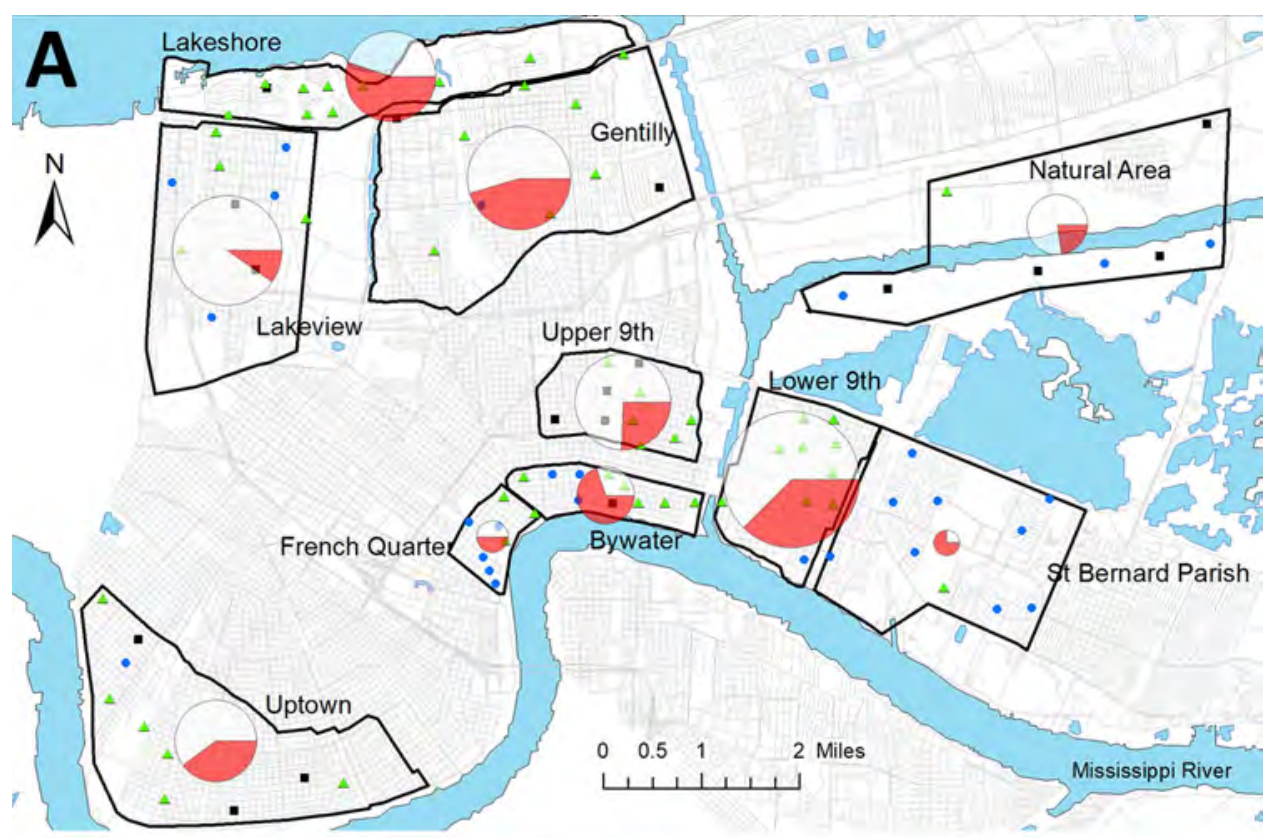

Figure 1. Prevalence of rat lungworm (Angiostrongylus cantonensis) in rodents, New Orleans, Louisiana, USA, May 2015-February 2017. A) Roof rats (Rattus rattus); B) Norway rats (R. norvegicus).

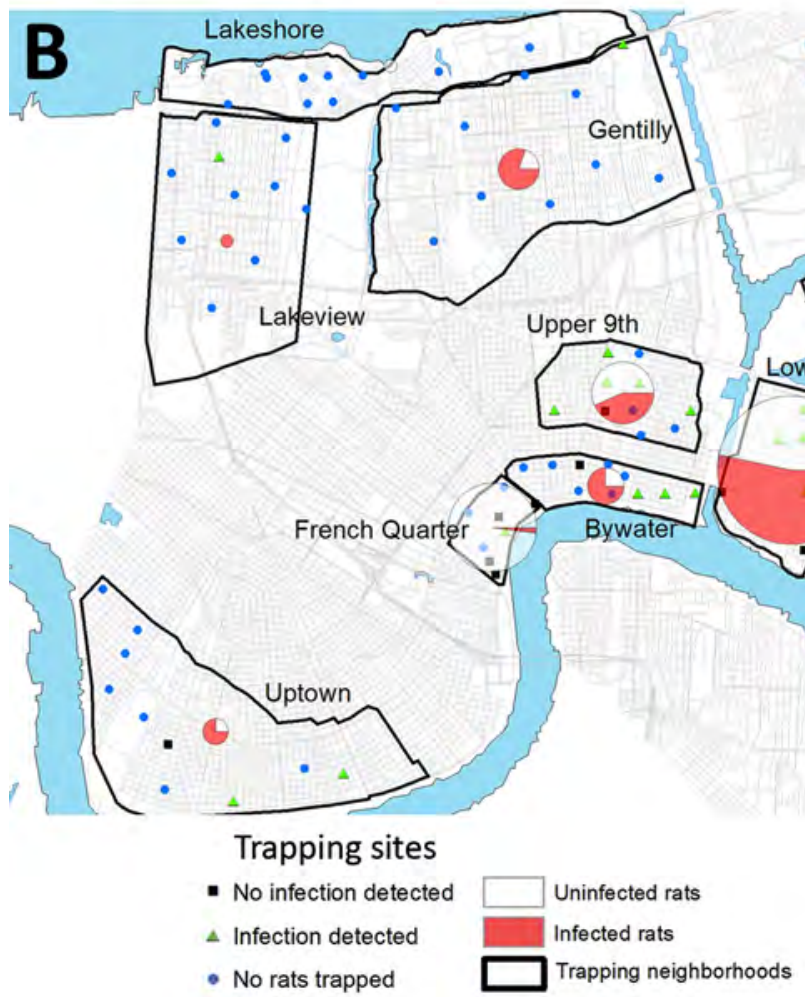




\section{Statistical Analyses}

We report on the distribution and prevalence of $A$. cantonensis according to all species trapped, but additional statistical analyses considered data only from Norway rats and roof rats because of small sample sizes or because the parasite was not detected in other species. Generalized linear models (GLMs) were constructed with a quasibinomial error distribution to determine whether sex or age class was a significant predictor of infection status (i.e., infected, not infected) in Norway rats and roof rats. We ran 3 GLMs: 1 with both Rattus species together and 1 for each species. The same predictors were used in 3 GLMs with a quasi-Poisson distribution to examine relationships with infection intensity (i.e., number of parasites per infected rodent) in Norway rats and roof rats together and separately by species.

We used $\chi^{2}$ tests to determine whether infection prevalence in Norway rats and roof rats differed among study areas, and among sites with 1 versus $>1$ species present. We ran a subset of pairwise tests to compare prevalence among study areas, correcting for multiple comparisons.

We used a Kruskal-Wallis test to compare infection intensity among areas, combining data from Norway rats and roof rats. We used Mann-Whitney U tests to compare infection intensity between Norway rats and roof rats and to compare intensity among sites with 1 or $>1$ species present. We conducted all statistical analyses using $\mathrm{R}$ version 3.4.2 (https://www.r-project.org/).

\section{Results}

\section{Rodent Trapping and Overall Prevalence of A. cantonensis}

A total of 696 rats were trapped at 78 of the 96 sampling sites. Both Norway rats and roof rats were found in all 10 areas, whereas hispid cotton rats (Sigmodon hispidus) were found in the natural area and the Lower 9th Ward, and rice rats (Oryzomys palustris) were found only in the natural area.

We detected $A$. cantonensis in all 10 areas (Figure 1) and in 3 of the 4 rat species sampled. Of the 444 roof rats necropsied, 160 (36.0\%) were positive for A. cantonensis, whereas 101 (44.1\%) of 229 Norway rats, 4 (21.1\%) of 19 S. hispidus, and 0 of $4 \mathrm{O}$. palustris were positive (Table 1). Prevalence differed between Norway rats and roof rats $\left(\chi^{2}=3.810, p=0.051\right)$. Median site-level prevalence estimates for roof rats $(33.3 \%)$ and Norway rats $(47.2 \%)$ (online Technical Appendix Figure) were similar to those estimated by pooling rats by species and area (Table 1).

A total of 488 house mice (Mus musculus) were collected at 48 locations with Sherman traps. Lungworms were not detected in any house mice, affirming that they do not serve as definitive hosts (18).

\section{Geographic Variation in Prevalence}

Excluding the St. Bernard Parish sites because of small sample sizes $(n=5)$, we found that overall prevalence in both Norway rats and roof rats differed among the sampled areas $\left(\chi^{2}=81.21, \mathrm{p}<0.001\right)$. Rats from the Bywater area exhibited the highest overall prevalence of $A$. cantonensis infection (71\%), whereas rats from the French Quarter exhibited the lowest (8\%) (Table 1; online Technical Appendix; online Technical Appendix Table 3). Most median site-level prevalence values (Figure 2, panel A) were similar to those estimated by pooling rats by species and area (Table 1 ).

Considering roof rats and Norway rats separately in areas with $\geq 10$ samples, we found that prevalence differed among areas for both species (roof rats, $\chi^{2}=46.755$, $\mathrm{p}<0.0001$; Norway rats, $\left.\chi^{2}=43.62, \mathrm{p}<0.0001\right)$. Prevalence in roof rats was lowest for rats trapped in the Lakeview area $(10 \%)$ and highest for those from the Bywater area (70\%), although pairwise comparisons showed that prevalence differed only among a subset of the study areas (online Technical Appendix; online Technical Appendix Table 4). Prevalence in Norway rats was lowest for rats trapped in the French Quarter (2\%) and highest among those from the Gentilly area (80\%). As with roof rats, prevalence in Norway rats differed only among a subset of the study areas (online Technical Appendix; online Technical Appendix Table 5).

\begin{tabular}{|c|c|c|c|c|c|}
\hline \multirow[b]{2}{*}{ Area } & \multicolumn{5}{|c|}{ No. positive/no. trapped (\%) } \\
\hline & Roof rats & Norway rats & Cotton rats & Rice rats & Total \\
\hline Uptown & $16 / 40(40)$ & $3 / 4(75)$ & - & - & $19 / 44(43)$ \\
\hline Lakeview & $7 / 72(10)$ & $1 / 1(100)$ & - & - & $8 / 73(11)$ \\
\hline Lakeshore & $27 / 49(55)$ & $0 / 1(0)$ & - & - & $27 / 50(54)$ \\
\hline Gentilly & $29 / 64(45)$ & $8 / 10(80)$ & - & - & $37 / 74(50)$ \\
\hline French Quarter & $3 / 6(50)$ & $1 / 47(2)$ & - & - & $4 / 53(8)$ \\
\hline Bywater & $14 / 20(70)$ & $6 / 8(75)$ & - & - & $20 / 28(71)$ \\
\hline Upper 9th & $14 / 55(25)$ & $10 / 23(43)$ & - & - & $24 / 78(31)$ \\
\hline Lower 9th & $42 / 112(38)$ & $71 / 133(53)$ & $0 / 2(0)$ & - & $113 / 247(46)$ \\
\hline Natural area & $5 / 22(23)$ & $0 / 1(0)$ & $4 / 17(24)$ & $0 / 4(0)$ & $9 / 44(20)$ \\
\hline St. Bernard Parish & $3 / 4(75)$ & $1 / 1(1)$ & - & - & $4 / 5(80)$ \\
\hline Total & $160 / 444(36)$ & $101 / 229(44)$ & $4 / 19(21)$ & $0 / 4(0)$ & $265 / 696(38)$ \\
\hline
\end{tabular}




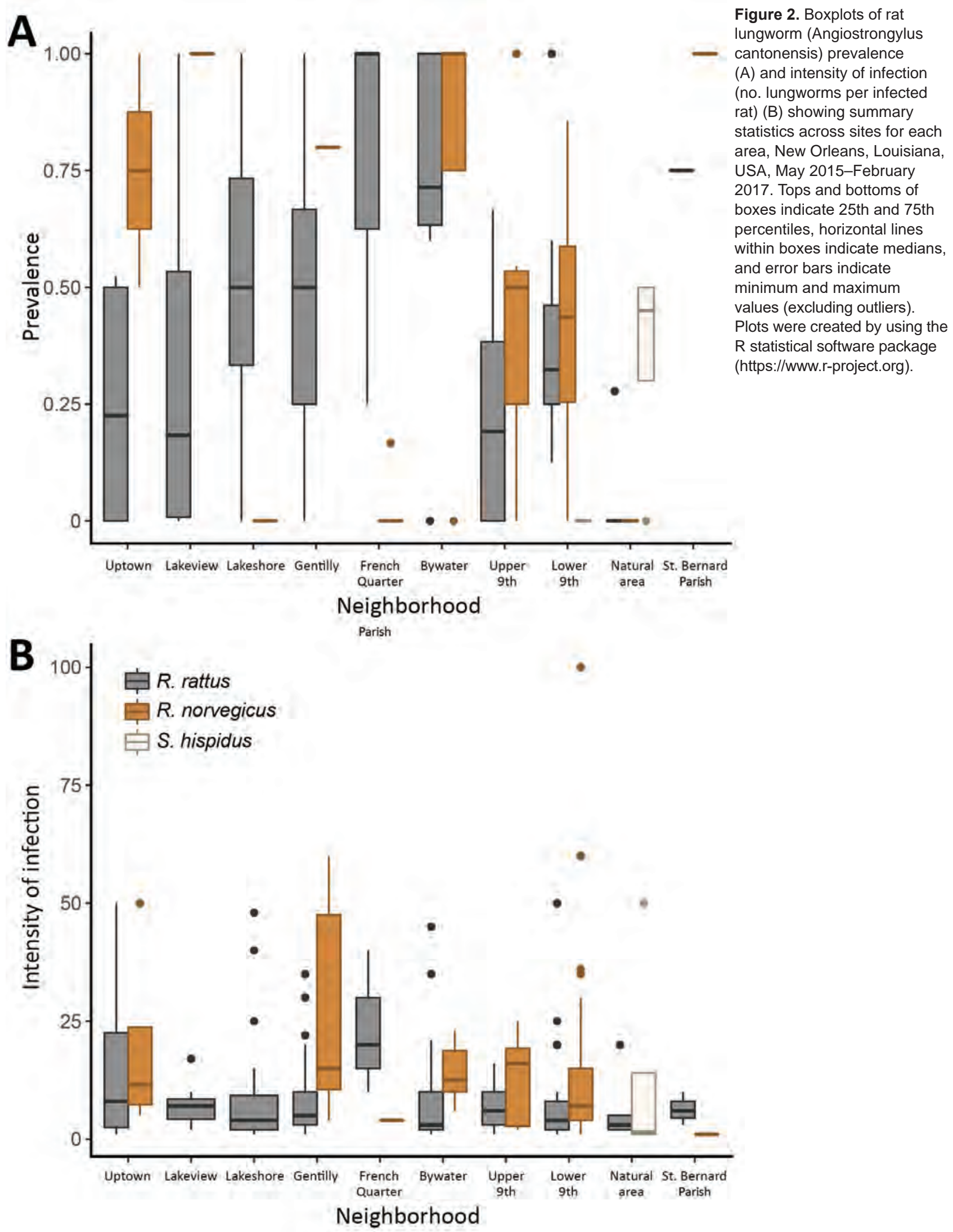


Infection prevalence was significantly lower in sites where only 1 rat species was trapped $(30 \%, \mathrm{n}=267,48$ sites) than in sites that harbored multiple rat species $(44 \%, \mathrm{n}$ $=429,30$ sites $)\left(\chi^{2}=11.654, p<0.001\right)$. However, sites with 1 species had significantly fewer rodents (mean 5.6) than sites with multiple species (mean 14.3) (Mann-Whitney U test, $\mathrm{p}<0.0001$ ).

\section{Likelihood of Infection}

Considering Norway rats and roof rats together, the likelihood of infection did not differ by sex ( $\mathrm{df}=656$, coefficient $=0.13133, \mathrm{p}=0.42$ ), but adults were significantly more likely to be infected than juveniles $(\mathrm{df}=656$, coefficient $=$ $-1.26201, \mathrm{p}<0.0001)$ and subadults $(\mathrm{df}=656$, coefficient $=$ -0.42601, $\mathrm{p}<0.05$ ) (online Technical Appendix Table 6).

When we considered species separately, we also detected differences in the likelihood of infection according to age class. The likelihood of infection in Norway rats did not differ by sex $(\mathrm{df}=224$, coefficient $=-0.04621$, $\mathrm{p}$ $=0.86$ ), but adults were more likely to be infected than either juveniles $(\mathrm{df}=224$, coefficient $=-1.88906, \mathrm{p}=$ $0.003)$ or subadults $(\mathrm{df}=224$, coefficient $=-0.82707, \mathrm{p}$ $=0.007$ ) (online Technical Appendix Table 6). Among roof rats, likelihood of infection did not differ by sex $(\mathrm{df}=$ 427 , coefficient $=0.2305, p=0.26$ ) or between adults and subadults $(\mathrm{df}=427$, coefficient $=-0.1541, \mathrm{p}=0.54)$, but adults were more likely than juveniles to be infected $(\mathrm{df}=$ 427 , coefficient $=-0.9550, p=0.004)$ (online Technical Appendix Table 6).

\section{Infection Intensity}

Infection intensity (Table 2) significantly differed between roof rats and Norway rats (Mann-Whitney U test, $\mathrm{p}<0.01$ ). Considering Norway rats and roof rats together across all areas, infection intensity did not differ by sex $(\mathrm{df}=240$, coefficient $=0.01331, \mathrm{p}=0.972)$. Subadults had lower infection intensity than adults ( $\mathrm{df}=240$, coefficient $=-0.44999, \mathrm{p}=0.0124)$, but juveniles did not $(\mathrm{df}=240$, coefficient $=0.0595, \mathrm{p}=0.82)$ (online Technical Appendix Table 7). Excluding the St. Bernard and French Quarter areas, each of which had only 4 infected rodents, we did not detect significant differences in infection intensity across the sampled areas (Kruskal-Wallis test, $\mathrm{p}=0.484)$. Also, we found no differences in infection intensity between sites with 1 species $(8.75, \mathrm{n}=79)$ versus multiple species present $(11.74, \mathrm{n}=186)$ (MannWhitney $U$ test, $p=0.072)$. Some median site-level intensities within areas (Figure 2, panel B) differed from intensities estimated by pooling all rats of each species within each area (Table 2). When we considered species separately, infection intensity did not differ according to sex or age class for Norway rats (all $\mathrm{p}>0.05$ ) or roof rats (all p>0.05) (online Technical Appendix Table 7).

\section{Discussion}

We assessed the current distribution and prevalence of $A$. cantonensis in definitive rat hosts across New Orleans, where the parasite has been present since at least 1986 (8). Our overall estimate of $38 \%$ infection prevalence in New Orleans is comparable to count-based estimates reported for other areas where $A$. cantonensis is considered endemic $(19,20$, but see 21$)$. We also found $A$. cantonensis in rats across New Orleans and in neighboring St. Bernard Parish, which contrasts with the patchy distributions exhibited by other rodent-associated pathogens in cities (22-25). Although rat lungworm is present across New Orleans, infection prevalence varied according to geography and rodent host species, suggesting the risk for transmission to humans might be mediated in part by geographically variable landscape features that affect commensal rats $(13,14)$. It is also likely, however, that the distributions of intermediate hosts and human population densities moderate transmission risk.

The first record of A. cantonensis in New Orleans reported lower prevalence in rat hosts than those observed in our study. An overall count-based prevalence of $18 \%$ was

\begin{tabular}{|c|c|c|c|c|c|}
\hline \multirow[b]{2}{*}{ Area } & \multicolumn{5}{|c|}{ Intensity (no. positive) } \\
\hline & Roof rats & Norway rats & Cotton rats & Rice rats & Total \\
\hline Uptown & $13.7(19)$ & $19.5(4)$ & - & - & $14.7(23)$ \\
\hline Lakeview & $8.0(7)$ & - & - & - & $8.0(7)$ \\
\hline Lakeshore & $8.7(23)$ & - & - & - & $8.7(23)$ \\
\hline Gentilly & $8.5(29)$ & $27.9(7)$ & - & - & $12.3(36)$ \\
\hline French Quarter & $23.3(3)$ & $4.0(1)$ & - & - & $18.5(4)$ \\
\hline Bywater & $11.1(12)$ & $12.2(5)$ & - & - & $11.4(17)$ \\
\hline Upper 9th & $7.1(15)$ & $12.3(7)$ & - & - & $8.7(22)$ \\
\hline Lower 9th & $7.7(42)$ & $13.3(65)$ & - & - & 11.1 (107) \\
\hline Natural area & $6.4(5)$ & - & $13.5(4)$ & - & $9.6(9)$ \\
\hline St. Bernard Parish & $6.3(3)$ & $1(1)$ & - & - & $5.0(4)$ \\
\hline Total & $9.2(158)$ & $14.3(90)$ & $13.5(4)$ & - & $11.1(252)$ \\
\hline
\end{tabular}


found for Norway rats and roof rats trapped in 1986-1987 (8). However, evidence of infection was found only in Norway rats; A. cantonensis was detected in $20(21 \%)$ of 94 trapped Norway rats and in 0 of 19 trapped roof rats (8). In comparison, we found overall prevalence $>18 \%$ in 8 of the 10 trapping areas in our study (Table 1), and we detected infected roof rats in all trapping areas. However, it is unclear whether the distribution of rodent host infections has changed, because the 1986-1987 surveys were limited to trapping on wharves along the Mississippi River (8). Thus, although we can affirm that infection prevalence varies by geography and definitive host (8), we cannot conclude that A. cantonensis became more broadly distributed across the city during the past 3 decades.

Evidence of greater prevalence and infection intensity of $A$. cantonensis in adult rats most likely reflects the increasing probability over time that an individual rat will consume an infected intermediate host and that infection becomes more evident in lung tissue. Waugh et al. (19) similarly reported differences in intensity and prevalence in rats from Jamaica according to size but not sex, although an earlier study found that female Norway rats were more likely to be infected (26). Evidence that $A$. cantonensis infection differs by host age contrasts with findings for other urban rodent-associated pathogens, including flea-vectored Bartonella bacteria (25). The finding of distinct Bartonella species in Norway rats and roof rats (25) suggests that co-occurrence does not facilitate pathogen transmission, whereas our results indicate otherwise. Contrasting patterns in the demography of definitive host infection may reflect pathogen-specific differences in transmission pathways. Further study is therefore warranted to determine the roles of definitive host abundance and diversity in pathogen transmission.

It has been more than a decade since the last diagnosed case of rat lungworm infection in the New Orleans area (12), which suggests that factors unrelated to rodent hosts mitigate the risk for transmission to humans. Although the most recent case in Louisiana resulted from consumption of a paratenic host (12), work elsewhere suggests that transmission to humans most likely occurs through accidental consumption of raw or undercooked infected snails on produce (7). Accordingly, the distribution and abundance of infected intermediate hosts are probably key factors affecting transmission risk, especially in cities such as New Orleans, where interest in urban agriculture is on the rise $(27,28)$. It is also possible that risk is influenced by climate-driven spread of invasive mollusks $(29,30)$, such as apple snails, that can serve as reservoirs $(1,3)$. Further study of infection prevalence in intermediate hosts would thus probably improve understanding of transmission risk across New Orleans and other cities that are vulnerable to climate change.
We intentionally diagnosed infection through a visual and count-based survey to draw comparisons to historical records, but implementing complementary approaches could have provided further understanding of $A$. cantonensis infection. As has been noted in prior studies $(19,20)$, count-based approaches probably yield conservative estimates relative to PCR-based approaches of infection. For example, in Hawaii, a count-based approach yielded a prevalence estimate of $54 \%$, whereas PCR yielded an estimate of $100 \%$ (20). More extensive use of PCR-based approaches in our study probably would have afforded additional perspective on parasitism and could also have excluded possible errors due to misidentification (31-33).

Our results indicate that cross-disciplinary analysis of A. cantonensis infection could shed further light on the risk for transmission to humans (34). As has been found with other zoonoses, human risk might correspond to socioecological disparities in habitat and resources favored by infected definitive and intermediate hosts $(22,23,35-38)$. For example, Rael et al. (13) detected a positive correlation between land abandonment and rat abundance in New Orleans across only low-income neighborhoods. The contrasting landscapes (14) and rodent assemblages (Table 1) found in the Lower 9th Ward in New Orleans and in adjacent St. Bernard Parish highlight the possibility that public health risks have been shaped by differences in postdisaster (i.e., Hurricane Katrina) land management policies (29). Although too few rats were trapped in St. Bernard Parish (n $=5$ ) to confidently estimate rat lungworm prevalence (Table 1), the observed differences in rat abundance suggest that transmission risk sharply differs between the Lower 9th Ward and St. Bernard Parish. Thus, further study of associations between definitive and intermediate host infection and socioecological factors probably would better define transmission risk across the city $(14,39)$.

Although rat lungworm is just 1 of many pathogens harbored by urban rats $(40,41)$, citywide estimates of host infection like those presented here can provide an epidemiologic baseline that can improve understanding of infectious disease dynamics in cities. Baselines are particularly informative for cities where zoonotic pathogens are likely to (re) emerge because of shifting climate conditions $(30,40,42)$ or extreme events, such as hurricanes, that can foster disease outbreaks (13). New Orleans unenviably straddles both sets of circumstances (43-45). Accordingly, further study of rat lungworm could help inform public health policies, surveillance programs, and intervention to safeguard the well-being of vulnerable communities in New Orleans and elsewhere.

\section{Acknowledgments}

We thank the investigators of the Coupled Natural and Human Systems-New Orleans project team for collaborations that supported this work. 
The Tulane-Xavier Center for Bioenvironmental Research, the Tulane ByWater Institute, the National Science Foundation (BCS-0948993, BCS-1313703), and the Louisiana Board of Regents provided funding for this work.

\section{About the Author}

Dr. Rael is a mathematical biologist involved in research on coupled human and natural systems at the ByWater Institute of Tulane University. Her research interests include modeling ecological interactions and infectious disease epidemiology.

\section{References}

1. Stockdale Walden HD, Slapcinsky JD, Roff S, Mendieta Calle J, Diaz Goodwin Z, Stern J, et al. Geographic distribution of Angiostrongylus cantonensis in wild rats (Rattus rattus) and terrestrial snails in Florida, USA. PLoS One. 2017;12:e177910. http://dx.doi.org/10.1371/journal.pone.0177910

2. Al Hammoud R, Nayes SL, Murphy JR, Heresi GP, Butler IJ, Pérez N. Angiostrongylus cantonensis meningitis and myelitis, Texas, USA. Emerg Infect Dis. 2017;23:1037-8. http://dx.doi.org/ 10.3201/eid2306.161683

3. Teem JL, Qvarnstrom Y, Bishop HS, da Silva AJ, Carter J, White-McLean J, et al. The occurrence of the rat lungworm, Angiostrongylus cantonensis, in nonindigenous snails in the Gulf of Mexico region of the United States. Hawaii J Med Public Health. 2013;72(Suppl 2):11-4.

4. York EM, Creecy JP, Lord WD, Caire W. Geographic range expansion for rat lungworm in North America. Emerg Infect Dis. 2015;21:1234-6. http://dx.doi.org/10.3201/eid2107.141980

5. Lv S, Zhang Y, Steinmann P, Yang GJ, Yang K, Zhou XN, et al. The emergence of angiostrongyliasis in the People's Republic of China: the interplay between invasive snails, climate change and transmission dynamics. Freshw Biol. 2011;56:717-34. http://dx.doi.org/10.1111/j.1365-2427.2011.02579.x

6. Alicata JE. Biology and distribution of the rat lungworm, Angiostrongylus cantonensis, and its relationship to eosinophilic meningoencephalitis and other neurological disorders of man and animals. Adv Parasitol. 1965;3:223-48. http://dx.doi.org/10.1016/ S0065-308X(08)60366-8

7. Cowie RH. Biology, systematics, life cycle, and distribution of Angiostrongylus cantonensis, the cause of rat lungworm disease. Hawaii J Med Public Health. 2013;72(Suppl 2):6-9.

8. Campbell BG, Little MD. The finding of Angiostrongylus cantonensis in rats in New Orleans. Am J Trop Med Hyg. 1988;38:568-73. http://dx.doi.org/10.4269/ajtmh.1988.38.568

9. Kim DY, Stewart TB, Bauer RW, Mitchell M. Parastrongylus (=Angiostrongylus) cantonensis now endemic in Louisiana wildlife. J Parasitol. 2002;88:1024-6. http://dx.doi.org/10.1645/ 0022-3395(2002)088[1024:PACNEI]2.0.CO;2

10. Gardiner CH, Wells S, Gutter AE, Fitzgerald L, Anderson DC, Harris RK, et al. Eosinophilic meningoencephalitis due to Angiostrongylus cantonensis as the cause of death in captive nonhuman primates. Am J Trop Med Hyg. 1990;42:70-4. http://dx.doi.org/10.4269/ajtmh.1990.42.70

11. New D, Little MD, Cross J. Angiostrongylus cantonensis infection from eating raw snails. N Engl J Med. 1995;332:1105-6. http://dx.doi.org/10.1056/NEJM199504203321619

12. Louisiana Office of Public Health. Angiostrongylus cantonensis annual report 2016 [cited 2017 Nov 30]. http://new.dhh.louisiana. gov/assets/oph/Center-PHCH/Center-CH/infectious-epi/ Annuals/Acantonensis_LaIDAnnual.pdf

13. Rael RC, Peterson AC, Ghersi BM, Childs J, Blum MJ. Disturbance, reassembly, and disease risk in socioecological systems.
EcoHealth. 2016;13:450-5. http://dx.doi.org/10.1007/ s10393-016-1157-1

14. Lewis JA, Zipperer WC, Ernstson H, Bernik B, Hazen R, Elmqvist T, et al. Socioecological disparities in New Orleans following Hurricane Katrina. Ecosphere. 2017;8:e01922. http://dx.doi.org/10.1002/ecs2.1922

15. McGuire B, Pizzuto T, Bemis WE, Getz LL. General ecology of a rural population of Norway rats (Rattus norvegicus) based on intensive live trapping. Am Midl Nat. 2006;155:221-36. http://dx.doi. org/ 10.1674/0003-0031(2006)155[0221:GEOARP]2.0.CO;2

16. King CM, Innes JG, Gleeson D, Fitzgerald N, Winstanley T, O’Brien B, et al. Reinvasion by ship rats (Rattus rattus) of forest fragments after eradication. Biol Invasions. 2011;13:2391-408. http://dx.doi.org/10.1007/s10530-011-0051-6

17. Eamsobhana P, Lim PE, Solano G, Zhang H, Gan X, Yong HS. Molecular differentiation of Angiostrongylus taxa (Nematoda: Angiostrongylidae) by cytochrome c oxidase subunit I (COI) gene sequences. Acta Trop. 2010;116:152-6. http://dx.doi.org/10.1016/ j.actatropica.2010.07.005

18. Yamashita T, Sato Y, Shiraki T, Otsuru M, Suzuki T. Development of Angiostrongylus cantonensis in rats, mice and guinea-pigs. Japanese J Parasitol. 1975;24:114-21.

19. Waugh CA, Lindo JF, Lorenzo-Morales J, Robinson RD. An epidemiological study of $A$. cantonensis in Jamaica subsequent to an outbreak of human cases of eosinophilic meningitis in 2000. Parasitology. 2016;143:1211-7. http://dx.doi.org/10.1017/ S0031182016000640

20. Qvarnstrom Y, Bishop HS, da Silva AJ. Detection of rat lungworm in intermediate, definitive, and paratenic hosts obtained from environmental sources. Hawaii J Med Public Health. 2013; 72(Suppl 2):63-9.

21. Zhang RL, Chen MX, Gao ST, Geng YJ, Huang DN, Liu JP, et al. Enzootic angiostrongyliasis in Shenzhen, China. Emerg Infect Dis. 2008;14:1955-6. http://dx.doi.org/10.3201/eid1412.080695

22. Costa F, Ribeiro GS, Felzemburgh RDM, Santos N, Reis RB, Santos AC, et al. Influence of household rat infestation on Leptospira transmission in the urban slum environment. PLoS Negl Trop Dis. 2014;8:e3338. http://dx.doi.org/10.1371/journal.pntd.0003338

23. Ko AI, Galvão Reis M, Ribeiro Dourado CM, Johnson WD Jr, Riley LW; Salvador Leptospirosis Study Group. Urban epidemic of severe leptospirosis in Brazil. Lancet. 1999;354:820-5. http://dx.doi.org/10.1016/S0140-6736(99)80012-9

24. Himsworth CG, Bai Y, Kosoy MY, Wood H, DiBernardo A, Lindsay R, et al. An investigation of Bartonella spp., Rickettsia typhi, and Seoul hantavirus in rats (Rattus spp.) from an inner-city neighborhood of Vancouver, Canada: is pathogen presence a reflection of global and local rat population structure? Vector Borne Zoonotic Dis. 2015;15:21-6. http://dx.doi.org/10.1089/ vbz.2014.1657

25. Peterson AC, Ghersi BM, Alda F, Firth C, Frye MJ, Bai Y, et al. Rodent-borne Bartonella infection varies according to host species within and among cities. EcoHealth. 2017;14:771-82. http://dx.doi.org/10.1007/s10393-017-1291-4

26. Waugh CA, Lindo JF, Foronda P, Ángeles-Santana M, Lorenzo-Morales J, Robinson RD. Population distribution and zoonotic potential of gastrointestinal helminths of wild rats Rattus rattus and $R$. norvegicus from Jamaica. J Parasitol. 2006;92:10148. http://dx.doi.org/10.1645/GE-795R1.1

27. McMillan T. 5 Urban farms reshaping the food world in New Orleans. The Atlantic. 2010 Nov 12 [cited 2018 Jan 1] https://www.theatlantic.com/health/archive/2010/11/5-urbanfarms-reshaping-the-food-world-in-new-orleans/66473/

28. Langenhennig S. Seeds and the city: urban lots are sprouting farms across New Orleans. NOLA.com. 2015 Jun 17 [cited 2018 Jan 01]. http://www.nola.com/homegarden/index.ssf/2015/06/seeds_and_ the_city_a_network_o.html 
29. Morand S, Bordes F, Chen HW, Claude J, Cosson JF, Galan M, et al. Global parasite and Rattus rodent invasions: The consequences for rodent-borne diseases. Integr Zool. 2015;10:409-23. http://dx.doi.org/10.1111/1749-4877.12143

30. Mazza G, Tricarico E, Genovesi P, Gherardi F. Biological invaders are threats to human health: An overview. Ethol Ecol Evol. 2014;26:112-29. http://dx.doi.org/10.1080/03949370.2013.863225

31. Kinsella JM. Studies on the life cycle and host specificity of Parastrongylus schmidti (Nematoda: Angiostrongylidae). Proc Helminthol Soc Wash. 1987;54:245-8.

32. Spratt DM. Species of Angiostrongylus (Nematoda: Metastrongyloidea) in wildlife: A review. Int J Parasitol Parasites Wildl. 2015;4:178-89. http://dx.doi.org/10.1016/j.ijppaw.2015.02.006

33. Miller CL, Kinsella JM, Garner MM, Evans S, Gullett PA, Schmidt RE. Endemic infections of Parastrongylus (=Angiostrongylus) costaricensis in two species of nonhuman primates, raccoons, and an opossum from Miami, Florida. J Parasitol. 2006;92:406-8. http://dx.doi.org/10.1645/GE-653R.1

34. Grisotti M, Avila-Pires FD. Worms, slugs and humans: the medical and popular construction of an emerging infectious disease. Hist Cienc Saude Manguinhos. 2011;18:877-91. http://dx.doi.org/10.1590/S0104-59702011000300016

35. Mills JN, Childs JE. Ecologic studies of rodent reservoirs: their relevance for human health. Emerg Infect Dis. 1998;4:529-37. http://dx.doi.org/10.3201/eid0404.980403

36. Macpherson CNL. Human behaviour and the epidemiology of parasitic zoonoses. Int J Parasitol. 2005;35:1319-31. http://dx.doi.org/10.1016/j.ijpara.2005.06.004

37. Neiderud C-J. How urbanization affects the epidemiology of emerging infectious diseases. Infect Ecol Epidemiol. 2015;5:27060. http://dx.doi.org/10.3402/iee.v5.27060

38. Bradley CA, Altizer S. Urbanization and the ecology of wildlife diseases. Trends Ecol Evol. 2007;22:95-102. http://dx.doi.org/ 10.1016/j.tree.2006.11.001

39. Gulachenski A, Ghersi BM, Lesen AE, Blum MJ. Abandonment, ecological assembly and public health risks in counter-urbanizing cities. Sustainability. 2016;8:491. http://dx.doi.org/10.3390/ su8050491

40. Jones KE, Patel NG, Levy MA, Storeygard A, Balk D, Gittleman JL, et al. Global trends in emerging infectious diseases. Nature. 2008;451:990-3. http://dx.doi.org/10.1038/nature06536

41. Lloyd-Smith JO, George D, Pepin KM, Pitzer VE, Pulliam JRC, Dobson AP, et al. Epidemic dynamics at the human-animal interface. Science. 2009;326:1362-7. http://dx.doi.org/10.1126/ science. 1177345

42. Gubler DJ, Reiter P, Ebi KL, Yap W, Nasci R, Patz JA. Climate variability and change in the United States: potential impacts on vector- and rodent-borne diseases. Environ Health Perspect. 2001;109(Suppl 2):223-33. http://dx.doi.org/10.2307/3435012

43. Dorn PL, Perniciaro L, Yabsley MJ, Roellig DM, Balsamo G, Diaz J, et al. Autochthonous transmission of Trypanosoma cruzi, Louisiana. Emerg Infect Dis. 2007;13:605-7. http://dx.doi.org/ 10.3201/eid1304.061002

44. Allen T, Murray KA, Zambrana-Torrelio C, Morse SS, Rondinini C, Di Marco M, et al. Global hotspots and correlates of emerging zoonotic diseases. Nat Commun. 2017;8:1124. http://dx.doi.org/10.1038/s41467-017-00923-8

45. Hotez PJ, Jackson Lee S. US Gulf Coast states: The rise of neglected tropical diseases in "flyover nation". PLoS Neg1 Trop Dis. 2017;11:e0005744. http://dx.doi.org/10.1371/ journal.pntd.0005744

Address for correspondence: Rosalyn C. Rael, The ByWater Institute, 627 Lindy Boggs Center, 6823 St. Charles Ave, Tulane University, New Orleans, LA 70118, USA; email: rosalyn.rael@gmail.com

\section{EID Podcast Rat Lungworm Expands into North America}

\section{The rat lungworm, Angostronglis (Paratrongylut cantonenis causes} eosinophilic meningitis in humans and various disease symptoms in atypical host species, including wildlife and captive animals.

Emily York, integrated pest management specialist at the Sam Noble Museum of Natural History, discusses the rat lungworm expansion in North America.

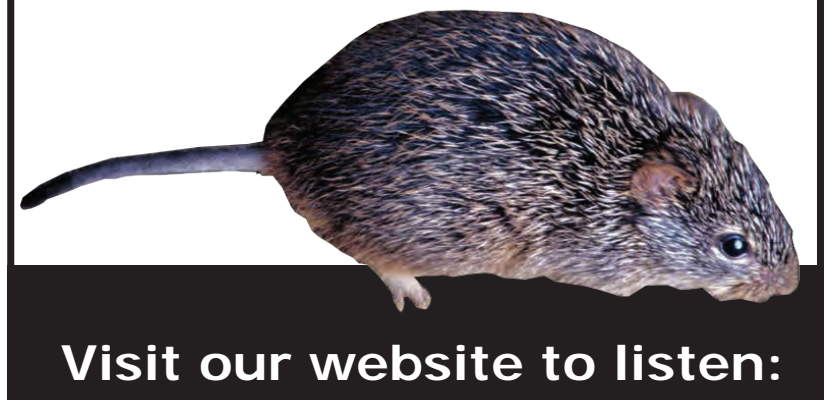

http:/ / www 2c.cdc.gov/ podcasts/ player.asp?f=8640172 EMERGING INFECTIOUS DISEASES 


\title{
Terrestrial Bird Migration and West Nile Virus Circulation, United States
}

\author{
Daniele Swetnam, Steven G. Widen, Thomas G. Wood, Martin Reyna, \\ Lauren Wilkerson, Mustapha Debboun, Dreda A. Symonds, Daniel G. Mead, \\ Barry J. Beaty, Hilda Guzman, Robert B. Tesh, Alan D.T. Barrett
}

Host migration and emerging pathogens are strongly associated, especially with regard to zoonotic diseases. West Nile virus (WNV), a mosquitoborne pathogen capable of causing severe, sometimes fatal, neuroinvasive disease in humans, is maintained in highly mobile avian hosts. Using phylogeographic approaches, we investigated the relationship between WNV circulation in the United States and the flight paths of terrestrial birds. We demonstrated southward migration of WNV in the eastern flyway and northward migration in the central flyway, which is consistent with the looped flight paths of many terrestrial birds. We also identified 3 optimal locations for targeted WNV surveillance campaigns in the United States-Illinois, New York, and Texas. These results illustrate the value of multidisciplinary approaches to surveillance of infectious diseases, especially zoonotic diseases.

$\mathrm{W}$ Test Nile virus (WNV) is a mosquitoborne virus that can cause severe and even fatal disease in humans. After WNV introduction into New York, NY, USA, its geographic range expanded quickly, reaching the West Coast in 2003. Previous studies have shown that the spread of WNV occurred faster than could be explained by contiguous diffusion (1-4) and demonstrated that its expansion occurred heterogeneously, consisting of contiguous diffusion and long distance translocations $(2,5)$. Since then, phylogeographic studies have reported frequent mixing of WNV strains from local and distant locations. The most notable exception is California, where several genetic studies have shown limited movement into and out of the state $(6,7)$.

Author affiliations: University of California at Davis, Davis, California, USA (D. Swetnam); University of Texas Medical Branch, Galveston, Texas, USA (D. Swetnam, S.G. Widen, T.G. Wood, H. Guzman, R.B. Tesh, A.D.T. Barrett); Harris County Public Health, Houston, Texas, USA (M. Reyna, L. Wilkerson, M. Debboun); Chesapeake Mosquito Control Commission, Chesapeake, Virginia, USA (D.A. Symonds); University of Georgia, Athens, Georgia, USA (D.G. Mead); Colorado State University, Fort Collins, Colorado, USA (B.J. Beaty)

DOI: https://doi.org/10.3201/eid2412.180382
The rapid expansion of WNV in the United States probably cannot be attributed to the movement of humans because humans are dead-end hosts. However, in nature, WNV is maintained in an enzootic transmission cycle involving mosquito vectors and highly mobile avian reservoirs. Hyalomma marginatum ticks have also been implicated in the transmission of WNV (8).

Although evidence of WNV infection has been identified in many species of birds, deaths and disease among birds vary greatly, ranging from asymptomatic to fatal infections; peak viremia potentially reaches $>10^{12} \mathrm{PFU} / \mathrm{mL}$ (9). WNV RNA has been detected in bird spleen and kidneys as long as 36 weeks after infection (10) and in brains of Nestor notabilis kea up to 72 months after infection (11).

Although phylogenetic evidence of geographic clustering by location is limited, a recent study reported that WNV isolates clustered according to avian flyway (12). Because birds are the primary reservoirs for $\mathrm{WNV}$, this finding was not surprising, but it is relevant because bird migration has also been implicated in the movement of influenza A virus (13), Borrelia burgdorferi (Lyme disease agent) (14), other pathogenic organisms (1), and even invasive invertebrate organisms (15). Several serologic studies (e.g., ELISA, plaque reduction neutralization test) have been used to determine the direction of WNV movement within the Atlantic, Mississippi, and Pacific flyways and demonstrated WNV in birds migrating southward, whereas evidence of the virus in birds during northward migration is limited $(16,17)$.

Studies of virus movement associated with avian hosts in the United States have concentrated on the migration of waterfowl and excluded terrestrial birds, largely because the migratory patterns of waterfowl have been thoroughly characterized by banding studies. However, passerine birds, the primary reservoir for WNV, are terrestrial birds, not waterfowl. Terrestrial birds and waterfowl fly along similar but distinct flyways. Although waterfowl follow regular paths bounded by mountains and rivers, terrestrial birds often follow looped routes that enable them to maximize tail winds, avoid head winds, and correlate with seasonal fluctuations in food availability $(18,19)$. Although looped migration paths have been described for several 
species of birds (Selasphorus rufus hummingbirds [20], Circus aeruginosus western marsh harriers [21], Falco eleonorae Eleonora's falcons [22], Cuculus canorus common cuckoos [23]), the general flyways of terrestrial birds have been inadequately studied. However, in 2014, La Sorte et al. provided a general description of terrestrial bird flyways in North America (18). They defined 3 flyways: the single distinct Western flyway and 2 overlapping flyways, the Central and Eastern flyways. A similar 3-flyways system (Pacific, Central, and Atlantic flyways) has been described for waterfowl (24); however, most studies have relied on the more common 4-flyways system (Pacific, Central, Mississippi, and Atlantic flyways). In this study, we used phylogeographic approaches to investigate the relationship between WNV circulation in the United States and the flight paths of terrestrial birds.

\section{Methods}

\section{Generation of Alignments}

We identified all unique sequences of natural and laboratory WNV strains by using the Virus Variation Resource (25). Virus sequences meeting the following criteria were included in this study: 1) the nucleotide sequence spanned the complete open reading frame, 2) the sequence was derived from natural isolates and not laboratory strains, 3) the sequence was unique (i.e., all sequences differed by $\geq 1 \mathrm{nt}$ ), and 4) the sequence contained no degenerate nucleotides. All sequences were manually aligned in BioEdit version 7.1.3 (http://www.mbio.ncsu.edu/BioEdit/bioedit.html) or MEGA7 (https://www.megasoftware.net/), and noncoding regions were removed when necessary (i.e., the open reading frame was used for analyses).

\section{Isolation of Viral RNA and Next-Generation Sequencing}

We obtained additional WNV isolates from the World Reference Center for Emerging Viruses and Arboviruses at the University of Texas Medical Branch at Galveston (Galveston, TX, USA) (26). Isolates were originally collected from Virginia, Georgia, Texas, and Colorado. We extracted viral RNA from the supernatant of infected Vero cells by using a QIAamp Viral RNA Mini Kit (QIAGEN, Germantown, MD, USA) according to the manufacturer's instructions.
We generated libraries with a TruSeq RNA version 2 kit (Illumina, San Diego, CA, USA) and samples sequenced by the University of Texas Medical Branch at Galveston Next Generation Sequencing Core on an Illumina $1500 \mathrm{Hi}$-Seq platform. Adaptor sequences and poor quality reads $(\mathrm{Q}$ score $<20)$ were removed with Trimmomatic (27). Reads were aligned with Bowtie2 (28) under the sensitive local parameter against the prototypical strain of WNV (NY99 flamingo 382-99, GenBank accession no. AF196835). Consensus sequences were generated by using SAMtools (29).

\section{Phylogeny}

To evaluate temporal structure, we generated a time-naive phylogeny (i.e., a maximum-likelihood phylogeny) to enable determination of the patristic distance between all isolates on the phylogeny. We generated maximum-likelihood trees with RAxML-HPC Black Box on Cyberinfrastructure for Phylogenetic Research version 3.3 (30) and determined automatic halting by bootstrapping. We determined the root-to-tip distance, which is a phylogenetic measure of genetic distance, for each isolate of the maximum-likelihood phylogenies by using TempEst (formerly Path-o-gen) (31). We evaluated the correlation (Pearson method) between root-to-tip distance and collection date in R (https://www.r-project.org/).

We used a Bayesian Markov chain Monte Carlo (MCMC) approach to infer phylogeographic relationships and selected the most appropriate phylogenetic model by using standard path sampling and stepping-stone approaches. XML files were generated in BEAUti version 1.8.3 or 1.8.4 and run with BEAST version 1.8.4 (32) on Cyberinfrastructure for Phylogenetic Research (30). We used the $\mathrm{GTR}+\Gamma+\mathrm{I}$ (general time reversible with gamma rate distribution and invariable sites) model to infer nucleotide substitution frequencies, an uncorrelated lognormal clock model to infer the mutation rate, and a Bayesian Skyline tree prior to model changes in population size. The evolution rate mean was restricted to $10^{-4}$ through $9 \times 10^{-4}$ substitutions/site/year, consistent with previously reported rates for WNV evolution $(7,33)$.

We ran trees with an MCMC length of 100 million and sampled every 5,000 steps. Log files were reviewed in Tracer (http://tree.bio.ed.ac.uk/software/tracer/) to determine burn-in, which ranged from $5 \%$ through $10 \%$. We ran

\begin{tabular}{|c|c|c|c|c|c|c|c|c|c|c|c|c|c|c|c|c|}
\hline Location & 1999 & 2000 & 2001 & 2002 & 2003 & 2004 & 2005 & 2006 & 2007 & 2008 & 2009 & 2010 & 2011 & 2012 & 2013 & 2014 \\
\hline NY & $x$ & & $x$ & $x$ & $x$ & $x$ & $x$ & & $x$ & $x$ & & & $x$ & & & \\
\hline VA & & $x$ & $x$ & $x$ & $x$ & $x$ & $x$ & $x$ & $x$ & $x$ & $x$ & $x$ & & & & \\
\hline GA & & & $x$ & $x$ & & $x$ & $x$ & & $x$ & $x$ & $x$ & & & & & \\
\hline IL & & & & $\mathrm{x}$ & $\mathrm{x}$ & $x$ & $x$ & $x$ & $x$ & & & & & & & \\
\hline TX & & & & $x$ & $x$ & & $x$ & $x$ & $x$ & & $x$ & $x$ & $x$ & $x$ & $x$ & $x$ \\
\hline $\mathrm{CO}$ & & & & $x$ & $\mathrm{x}$ & $x$ & & $\mathrm{x}$ & $x$ & $x$ & $\mathrm{x}$ & & & & & \\
\hline ND & & & & $x$ & $\mathrm{x}$ & $x$ & $x$ & $\mathrm{x}$ & & $x$ & $\mathrm{x}$ & & & & & \\
\hline SD & & & & & $x$ & $x$ & $x$ & $x$ & $\mathrm{x}$ & $x$ & $x$ & & & & & \\
\hline
\end{tabular}




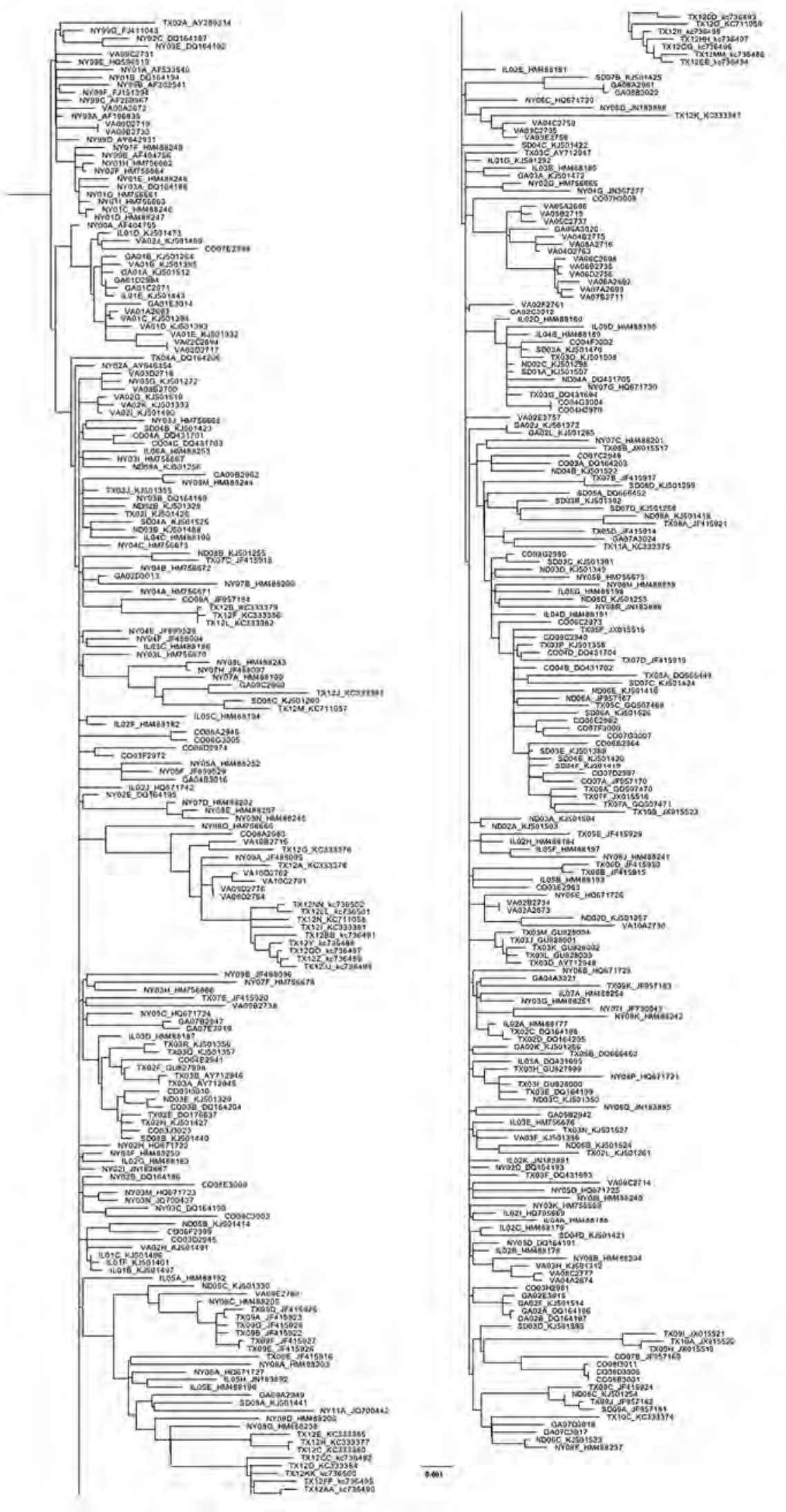

Figure 1. Maximum-likelihood phylogeny generated with all West Nile virus sequences from New York, Virginia, Georgia, Illinois, North Dakota, South Dakota, Texas, and Colorado $(n=379)$ in study of terrestrial bird migration and West Nile virus circulation, United States. Sequence names include the 2-letter state abbreviation to indicate the origin of isolation, followed by the year. Multiple isolates collected from the same state within the same year are differentiated by letter. GenBank accession numbers are provided for all taxa that were not sequenced in this study. Scale bar indicates nucleotide substitutions per site. 


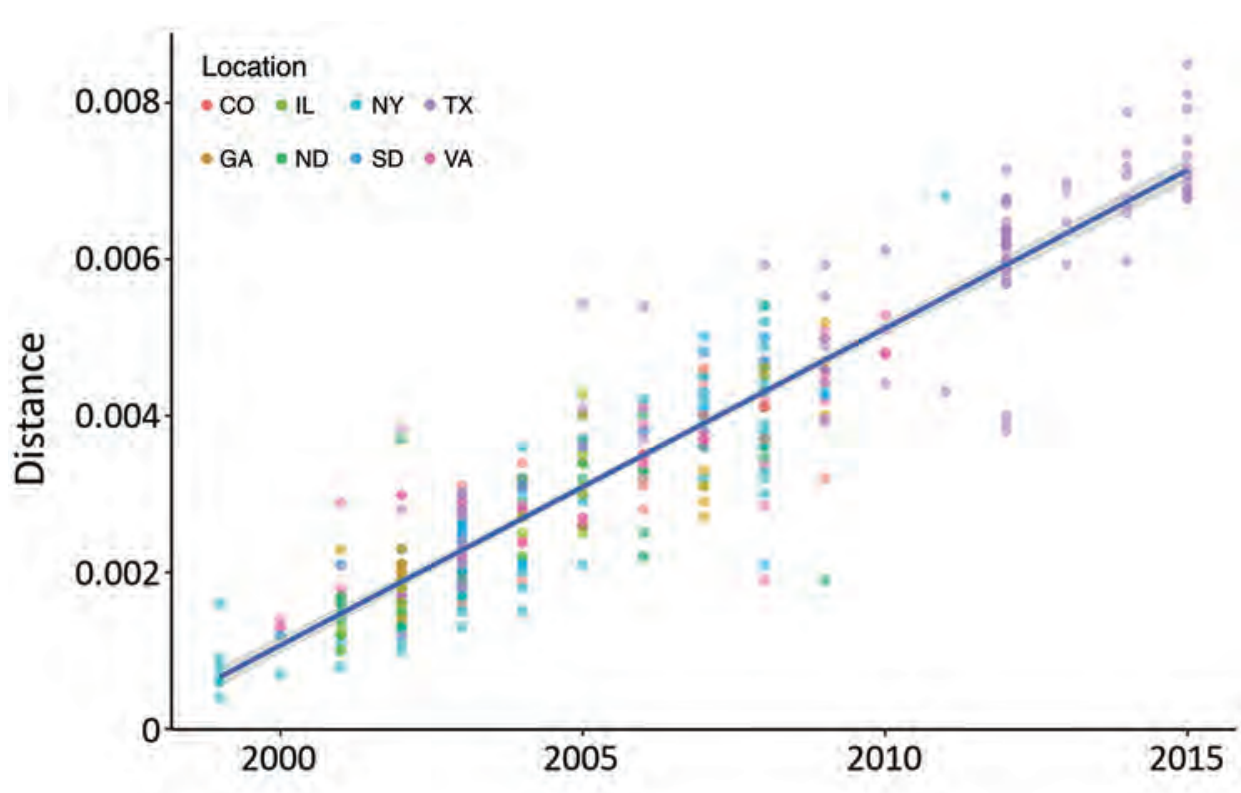

Figure 2. Analysis of correlation between virus isolation date and genetic diversity in study of terrestrial bird migration and West Nile virus circulation, United States. Root-to-tip distances of all sequences were determined for each isolate by using the maximumlikelihood tree shown in Figure 1 (https://wwwnc cdc.gov/EID/ article/24/12/18-0382-F1.htm) and plotted against the year. Dots are colored by location of isolation. The correlation between the root-to-tip distance and year of isolation was determined with linear regression shown in blue. $95 \% \mathrm{Cls}$ are shown in gray. The equation of the linear regression line was used to estimate the year of the most recent common ancestor (MRCA) and the mutation rate $(m): y=m x+M R C A$. multiple independent MCMC chains until effective sample size values exceeded 200. Log and tree files were combined in LogCombiner version 1.8, and a maximum clade credibility tree was generated in TreeAnnotator (32). Locations were inferred by using ancestral state reconstruction with an asymmetric discrete trait substitution model (34).

\section{Analysis of Migration}

After the XML files were generated in BEAUti, we manually edited them to enable counting of all Markov jumps (MJ) (which described the relative magnitude of migration out of source locations and into sink locations) for 2001 through 2009 (35). This method for evaluating migration, first described by Minin and Suchard (35), has been used to characterize migration of several major pathogens including rabies virus (36), dengue virus (37), HIV (38), influenza virus $(39,40)$, and Rift Valley fever virus $(41)$.

As expected for an emerging zoonotic disease, the annual West Nile neurologic disease (WNND) incidence and sample collection efforts varied dramatically among states over time, adding substantial complexity to the model. To mitigate the effects of inconsistent sampling and to confirm the observed results, we applied a stricter inclusion criterion to confirm the results obtained by using the full dataset. The sequences were randomly down-sampled such that the number of sequences used correlated ( $p<0.05$ by Pearson method) with the incidence of WNND reported to the Centers for Disease Control and Prevention (CDC) in a particular year (the most accurate record of relative WNV activity). Our analysis ensured that the dataset was representative of the WNV activity of each region in a particular year.

We calculated incidence by using the number of WNND cases reported to CDC from each state during each year and dividing that number by the estimated population of each state. The population estimates were obtained from the Time Series of Intercensal State Population Estimates available at the Population Division of the US Census Bureau (42). States with insufficient sequences to represent the WNND incidence were excluded. Down-sampling was undertaken in at least duplicate to ensure that reduction in sample size and diversity did not remove important relationships.

\section{Results}

\section{Sequence Collection}

All previously published sequences of natural WNV isolates collected in the United States were obtained from GenBank on January 1, 2016. The number of WNV sequences varied substantially over time and among locations, which

\begin{tabular}{lccc}
\hline \multicolumn{4}{l}{ Table 3. Statistical support for phylogeny in study of terrestrial bird } \\
\hline Variable & Mean & ESS & 95\% HPD interval \\
\hline Posterior & -49722.50 & 1370 & -49803.21 to -49640.55 \\
Prior & -3987.09 & 1110 & -4051.92 to -3916.56 \\
Likelihood & -45735.41 & 2179 & -45780.00 to -45691.43 \\
MRCA & 11.92 & 3119 & 10.82 to 13.08 \\
UCLD.mean & $3.92 \times 10^{-4}$ & 1604 & $3.55 \times 10^{-4}$ to $4.49 \times 10^{-4}$ \\
\hline
\end{tabular}

${ }^{*} \mathrm{ESS}$, effective sample size; HPD, highest posterior density; MRCA, most recent common ancestor (years before 2009); UCLD.mean, evolution rate inferred with an uncorrelated clock model with lognormal distribution. 
presented statistical challenges. In particular, although GenBank has $>900 \mathrm{WNV}$ open reading frames, most come from a few states where laboratories were actively involved in WNV surveillance and research: California, New York, and Texas. The ability to compare multiple isolates over

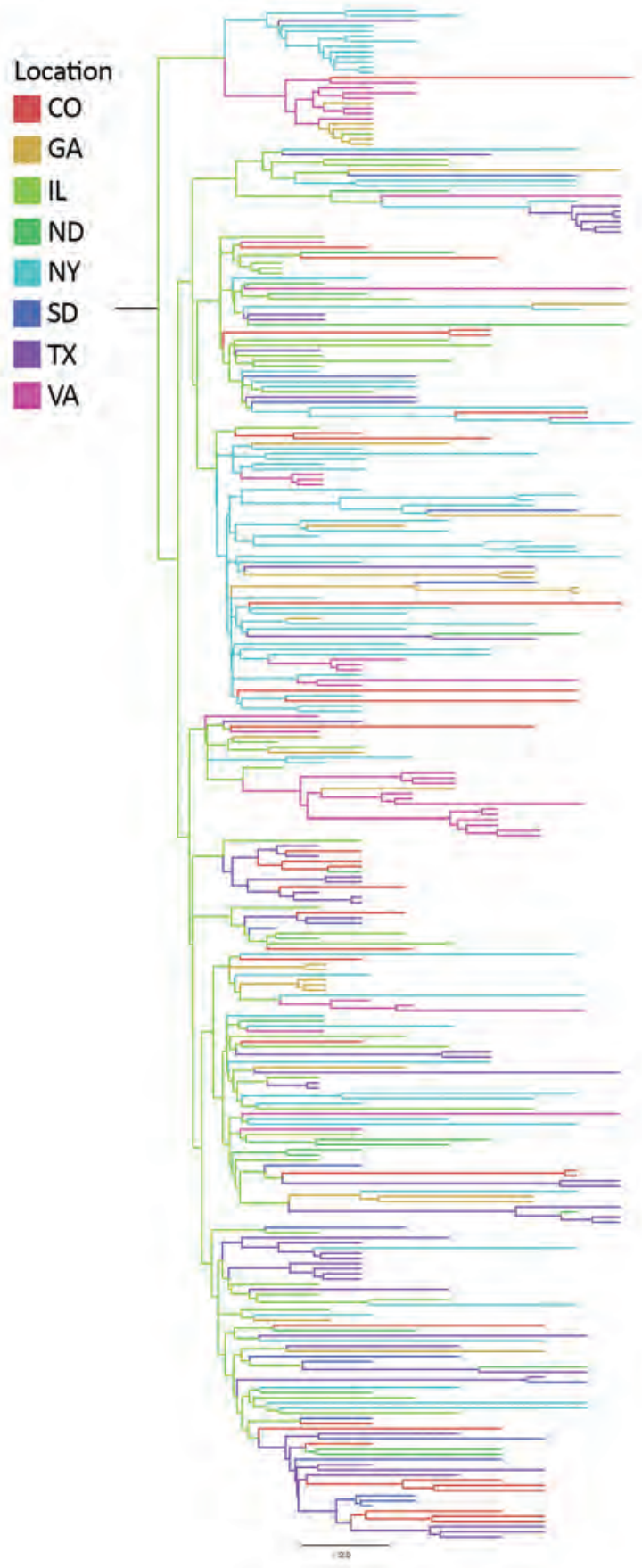

Figure 3. Bayesian phylogeny of West Nile virus isolates collected in representative regions along the Eastern and Central flyways between 2001 and 2009, United States. Maximum-clade credibility tree was obtained by using a Bayesian approach. The location of each isolate and the inferred location of each ancestor are depicted by color. multiple years was critical to the analysis. Only a few states had sufficient numbers of WNV sequences available in GenBank to enable analysis for multiple consecutive years: New York, Connecticut, Illinois, North Dakota, South Dakota, Texas, and California.

To mitigate the influence of sampling bias, we obtained additional WNV isolates from the World Reference Center for Emerging Viruses and Arboviruses for 3 states and sequences to support the analysis: Virginia (n =39), Georgia $(\mathrm{n}=20)$, and Colorado $(\mathrm{n}=31)$ (Table 1, https://wwwnc.cdc.gov/EID/article/24/12/18-0382-T1. $\mathrm{htm})$. Given that previous studies have demonstrated limited WNV movement into or out of California $(6,7)$, we did not include isolates from California in the analysis. Similarly, because of the proximity of New York and Connecticut, we chose New York to represent WNV in the Northeast because Connecticut is a small state. Last, to ensure that each location was represented across a similar time frame, we included only isolates collected during 2001-2009 in the Bayesian phylogeny and migration analysis. Table 2 shows the states and availability of yearly isolates.

\section{Model Selection}

We compared 203 nucleotide substitution models by using the Bayesian and Akaike Information Criteria in JModelTest2 (https://github/com/ddariba/jmodeltest2) and found the GTR $+\Gamma+\mathrm{I}$ model to be the most appropriate. For assessing temporal signature, we used a maximum-likelihood tree with sequences of WNV strains from New York, Virginia, Georgia, Illinois, North Dakota, South Dakota, Texas, and Colorado $(\mathrm{n}=379)$ (Figure 1, https://wwwnc.cdc.gov/EID/ article/24/12/18-0382-F1.htm). We identified a statistically significant positive correlation $(\mathrm{r}=0.93,95 \%$ highest posterior density $\left.[\mathrm{HPD}]=0.92-0.94 ; \mathrm{p}<2.2 \times 10^{-16}\right)$ between the root-to-tip distance and the date of isolation in Temp-Est (formerly known as Path-O-gen) (Figure 2). The mutation rate was estimated to be $4.05 \times 10^{-4}$ substitutions/site/year, and the most recent common ancestor (MRCA) was in 1997. Together these results indicated a strong temporal signal in the dataset. Finally, we evaluated Bayesian tree priors (skyride, skygrid, and skyline) and uncorrelated clock models (lognormal and exponential) by using path-sampling and steppingstone approaches. The uncorrelated lognormal clock model with the Bayesian skyline tree prior was the most appropriate.

\section{Phylogeographic Analysis for the United States}

Analysis of all WNV sequences collected from New York, Virginia, Georgia, Illinois, North Dakota, South Dakota, Texas, and Colorado during 2001-2009 provided estimates of the introduction date of the MRCA and mean evolution rate that were consistent with the estimates of the root-totip distance analysis (Table 3; Figure 3, https://wwwnc. cdc.gov/EID/article/24/12/18-0382-F3.htm). The date of 
Terrestrial Bird Migration and West Nile Virus

Table 4. Source and sink analysis in study of terrestrial bird migration and West Nile virus circulation, United States*

\begin{tabular}{|c|c|c|c|c|}
\hline Source & Sink & Markov jumps, mean & ESS & 95\% HPD interval \\
\hline $\mathrm{CO}$ & ND & 1.368 & 8603 & $0-3$ \\
\hline GA & IL & 1.31 & 31284 & $1-3$ \\
\hline $\mathrm{IL}$ & $\mathrm{CO}$ & 8.376 & 3311 & $1-14$ \\
\hline IL & $\mathrm{GA}$ & 8.226 & 3046 & $3-13$ \\
\hline IL & ND & 10.43 & 15423 & $6-14$ \\
\hline IL & NY & 29.965 & 1355 & $20-40$ \\
\hline IL & SD & 6.691 & 3635 & $1-11$ \\
\hline IL & $\mathrm{TX}$ & 22.872 & 4545 & $14-30$ \\
\hline IL & VA & 11.449 & 1631 & $6-16$ \\
\hline NY & $\mathrm{CO}$ & 4.362 & 2143 & $0-8$ \\
\hline NY & GA & 7.039 & 2170 & $2-11$ \\
\hline NY & $\mathrm{IL}$ & 1.012 & 3023 & $0-4$ \\
\hline NY & SD & 2.177 & 2740 & $0-5$ \\
\hline NY & $\mathrm{TX}$ & 4.564 & 2149 & $0-9$ \\
\hline NY & VA & 4.24 & 1171 & $0-8$ \\
\hline SD & $\mathrm{CO}$ & 1.328 & 3964 & $0-5$ \\
\hline TX & $\mathrm{CO}$ & 9.775 & 6039 & $3-16$ \\
\hline $\mathrm{TX}$ & ND & 5.177 & 7617 & $2-9$ \\
\hline TX & NY & 1.606 & 9410 & $0-4$ \\
\hline $\mathrm{TX}$ & SD & 7.557 & 5113 & $2-12$ \\
\hline VA & $\mathrm{CO}$ & 1.16 & 9807 & $0-3$ \\
\hline VA & GA & 3.616 & 14054 & $2-6$ \\
\hline
\end{tabular}

MRCA introduction was estimated as 1997, and the average evolution rate was $3.92 \times 10^{-4}$ substitutions/site/year.

We used MJ between reconstructed ancestral states to estimate the magnitude of relative migration out of, or into, each of the 8 regions (Table 4; Table 5, https:// wwwnc.cdc.gov/EID/article/24/12/18-0382-T5.htm). Frequent migration ( $>2 \mathrm{MJ}$ ) was detected from Illinois to Colorado (8.38 MJ), Illinois to Georgia (8.23 MJ), Illinois to North Dakota (10.43 MJ), Illinois to New York (29.97 MJ), Illinois to South Dakota (6.69 MJ), Illinois to Texas (22.87 MJ), Illinois to Virginia (11.45 MJ), New York to Colorado (4.36 MJ), New York to Georgia (7.04 MJ), New York to South Dakota (2.18 MJ), New York to Texas (4.56 MJ), New York to Virginia (4.24 MJ), Texas to Colorado (9.78 MJ), Texas to North Dakota (5.18 MJ), Texas to South Dakota (7.56 MJ), and Virginia to Georgia (3.62 MJ).

Overall, 3 major sources of WNV circulation (New York, Illinois, and Texas) seemed to be the origin of $88.5 \%$ of the total migration events observed (Table 4; Figure 4). Southward and westward movements were detected along the East Coast, but only northward movement was observed within the central United States. A notable exception was observed in Illinois, where evidence of WNV movement in all directions was demonstrated.

\section{Incidence-Controlled Phylogeny}

To mitigate the effects of inconsistent sampling, we applied a stricter inclusion criterion to ensure that the dataset was representative of WNV activity in each region in a particular year. In this approach, the sequences were randomly down-sampled by using the sample command in $\mathrm{R}$, such that the number of sequences was proportional to the incidence of WNND reported to CDC (Table 6). Illinois, North Dakota, and South Dakota were not included in the downsampled datasets because there were insufficient sequences to represent WNND incidence in these states. To ensure that reduction in sample size and diversity did not remove
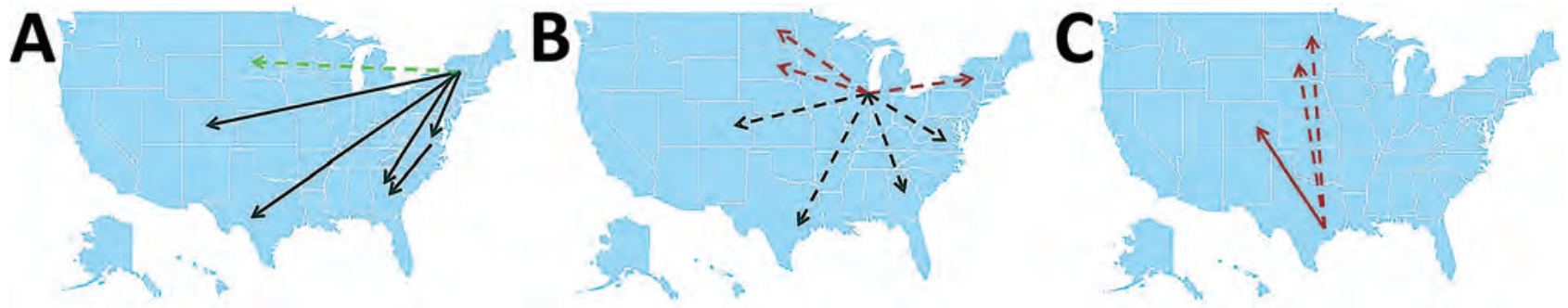

Figure 4. Summary of source/sink analysis in study of terrestrial bird migration and West Nile virus circulation, United States. Minimum number of migration events detected from A) the Eastern flyway, B) Illinois, and C) the Central flyway. Only events that occurred at least twice are depicted. Red arrows, northward migration; black arrows, southward migration; green arrow, lateral migration; dotted arrows, migration that could not be confirmed by incident-controlled down sampling because of an insufficient number of sequences. 
Table 6. Incidence-controlled down-sampling strategy used in study of of terrestrial bird migration and WNV circulation, United States*

\begin{tabular}{|c|c|c|c|}
\hline Location, year & WNV incidence & Sequences available & Sequences used \\
\hline \multicolumn{4}{|l|}{ GA } \\
\hline 2001 & $7.16 \times 10^{-7}$ & 5 & 3 \\
\hline 2002 & $3.29 \times 10^{-6}$ & 11 & 6 \\
\hline 2003 & $3.13 \times 10^{-6}$ & 1 & 1 \\
\hline 2004 & $1.60 \times 10^{-6}$ & 2 & 2 \\
\hline 2005 & $1.01 \times 10^{-6}$ & 1 & 1 \\
\hline 2006 & $2.18 \times 10^{-7}$ & 0 & 0 \\
\hline 2007 & $2.46 \times 10^{-6}$ & 5 & 5 \\
\hline 2008 & $4.21 \times 10^{-7}$ & 3 & 3 \\
\hline 2009 & $4.16 \times 10^{-7}$ & 3 & 3 \\
\hline \multicolumn{4}{|l|}{ NY } \\
\hline 2001 & $6.81 \times 10^{-7}$ & 10 & 3 \\
\hline 2002 & $3.55 \times 10^{-6}$ & 10 & 6 \\
\hline 2003 & $2.97 \times 10^{-6}$ & 15 & 5 \\
\hline 2004 & $3.65 \times 10^{-7}$ & 10 & 3 \\
\hline 2005 & $1.57 \times 10^{-6}$ & 7 & 4 \\
\hline 2006 & $8.37 \times 10^{-7}$ & 5 & 3 \\
\hline 2007 & $8.36 \times 10^{-7}$ & 9 & 3 \\
\hline 2008 & $1.67 \times 10^{-6}$ & 18 & 4 \\
\hline 2009 & $3.11 \times 10^{-7}$ & 2 & 2 \\
\hline \multicolumn{4}{|l|}{ VA } \\
\hline 2001 & 0 & 6 & 2 \\
\hline 2002 & $2.20 \times 10^{-6}$ & 10 & 5 \\
\hline 2003 & $2.58 \times 10^{-6}$ & 6 & 5 \\
\hline 2004 & $6.69 \times 10^{-7}$ & 4 & 3 \\
\hline 2005 & 0 & 3 & 2 \\
\hline 2006 & 0 & 4 & 2 \\
\hline 2007 & $3.87 \times 10^{-7}$ & 2 & 2 \\
\hline 2008 & 0 & 4 & 2 \\
\hline 2009 & $6.31 \times 10^{-7}$ & 5 & 3 \\
\hline
\end{tabular}

important relationships, the down-sampling was independently performed twice.

According to the 2 incidence-controlled datasets, the MRCA was $\approx 1997$ in both down-sampling exercises (95\% HPD 1996.001998 .52 and 95\% HPD 1995.7 1998.25), and the overall mutation rates were estimated to be $4.02 \times$ $10^{-4}$ and $3.83 \times 10^{-4}$ substitutions/site/year (Table 7; Figure 5, https://wwwnc.cdc.gov/EID/article/24/12/18-0382-F5. htm). As with the full dataset, the Markov analysis demonstrated that New York and Texas were strong sources of WNV circulation. Significant movement (mean $>2 \mathrm{MJ}$ ) was detected from Texas to Colorado (20.42 and 20.44 MJ); Texas to New York (12.36 and 11.77 MJ); Texas to Georgia (8.28 and 9.55 MJ); Texas to Virginia (7.14 and 7.732 MJ); New York to Georgia (6.1 and 5.38 MJ); New York to Virginia (4.95 and $3.65 \mathrm{MJ}$ ); New York to Colorado
(4.04 and 2.66 MJ); New York to Texas (2.66 and 2.73 $\mathrm{MJ}$ ); Virginia to Georgia (1.55 and 3.62 MJ); and, in dataset 2 only, Virginia to Colorado (1.31 MJ) (Figure 6).

Together, the MJ analyses of the incidence-controlled dataset and the full dataset illustrate a consistent pattern of WNV circulation. All southward movement originated in the eastern United States (New York and Virginia), and most of the northward movement originated in the central United States (Texas) (Figure 7).

\section{Discussion}

In recent years, emerging zoonotic diseases caused by Ebola, Zika, Nipah, Middle Eastern respiratory syndrome, and influenza A viruses have become major public health problems, devastating communities and costing millions for public health interventions. Decisive,

Table 7. Statistical support for the incidence-controlled phylogenies determined in study of terrestrial bird migration and West Nile virus circulation, United States*

\begin{tabular}{lcccccc}
\hline & \multicolumn{2}{c}{ Dataset 1 } & \multicolumn{2}{c}{ Dataset 2 } \\
\cline { 2 - 7 } Variable & Mean & ESS & 95\% HPD interval & Mean & ESS & 95\% HPD interval \\
\cline { 2 - 7 } Posterior & -36338.60 & 1385 & -36394.36 to -36280.37 & -36798.90 & 1803 & -36853.19 to -36740.97 \\
Prior & -3141.96 & 1187 & -3189.54 to -3091.38 & -3153.08 & 1387 & -3201.84 to -3104.50 \\
Likelihood & -33196.64 & 1367 & -33229.23 to -33165.79 & -33645.82 & 2115 & -33678.80 to -33614.39 \\
MRCA & 11.66 & 4720 & 10.47 to 12.10 & 11.97 & 6985 & 10.75 to 13.30 \\
UCLD.mean & $4.02 \times 10^{-4}$ & 2336 & $3.53 \times 10^{-4}$ to $4.55 \times 10^{-4}$ & $3.83 \times 10^{-4}$ & 1749 & $3.31 \times 10^{-4}$ to $4.39 \times 10^{-4}$ \\
\hline
\end{tabular}

*ESS, effective sample size; HPD, highest posterior density; MRCA, most recent common ancestor (years before 2009); UCLD.mean, evolution rate inferred with an uncorrelated clock model with lognormal distribution. 

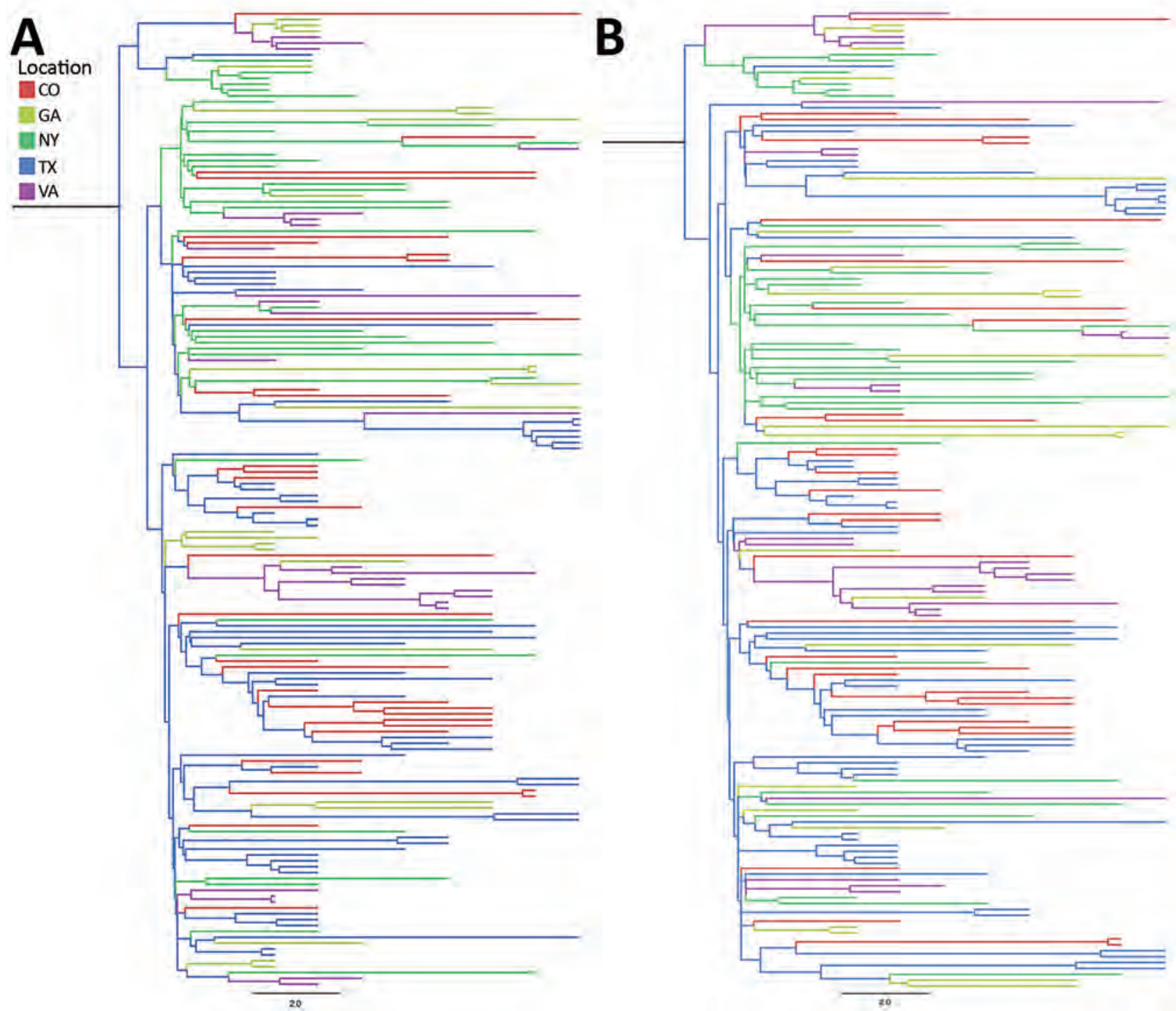

Figure 5. Incidence-controlled phylogeny of Eastern and Central flyways, United States. Sequences were down-sampled such that the number of sequences was proportional to the annual incidence of West Nile neurologic disease incidence for each location between 2001 and 2009. Down-sampling was undertaken twice (A and B) to ensure that the reduction in sequences did not result in a substantial loss of diversity. Illinois, North Dakota, and South Dakota were not included in the incidence-control analysis because too few sequences were available to support down-sampling. Bayesian approaches were used to generate maximum-clade credibility trees. Scale bars indicate nucleotide substitutions per site.

evidence-based approaches are critical for managing emerging infectious diseases, but effective and efficient countermeasures will be possible only after the relationships between pathogens and their hosts have been thoroughly characterized.

Bird migration has been implicated in the movement of a variety of pathogens (1). In particular, characterization of the relationship between avian influenza virus movement and waterfowl migration has supported surveillance and early warning programs $(1,43)$. However, studies of avian hosts in the Americas have mainly concentrated on the migration of waterfowl to the exclusion of terrestrial birds because waterfowl are easily tracked with banding; thus, their migration has been thoroughly characterized.

The introduction and subsequent spread of WNV into the Americas underscores the invasive potential of emerging pathogens in the New World, as has been recently exemplified by Zika virus, another mosquitoborne flavivirus. Dramatic variations in the location, timing, and intensity of WNV strain collection and sequencing has left the field with a limited understanding of virus circulation patterns and no reliable way of predicting the geographic spread of WNV outbreaks. We have addressed this knowledge gap 
A

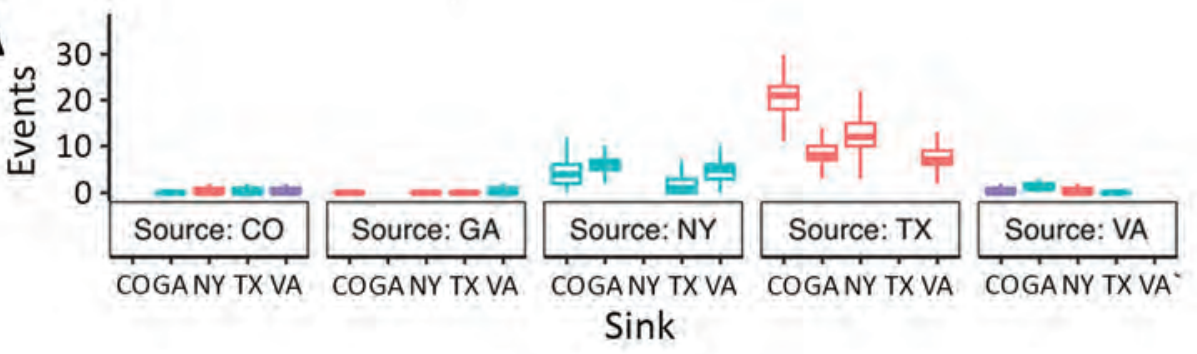

B
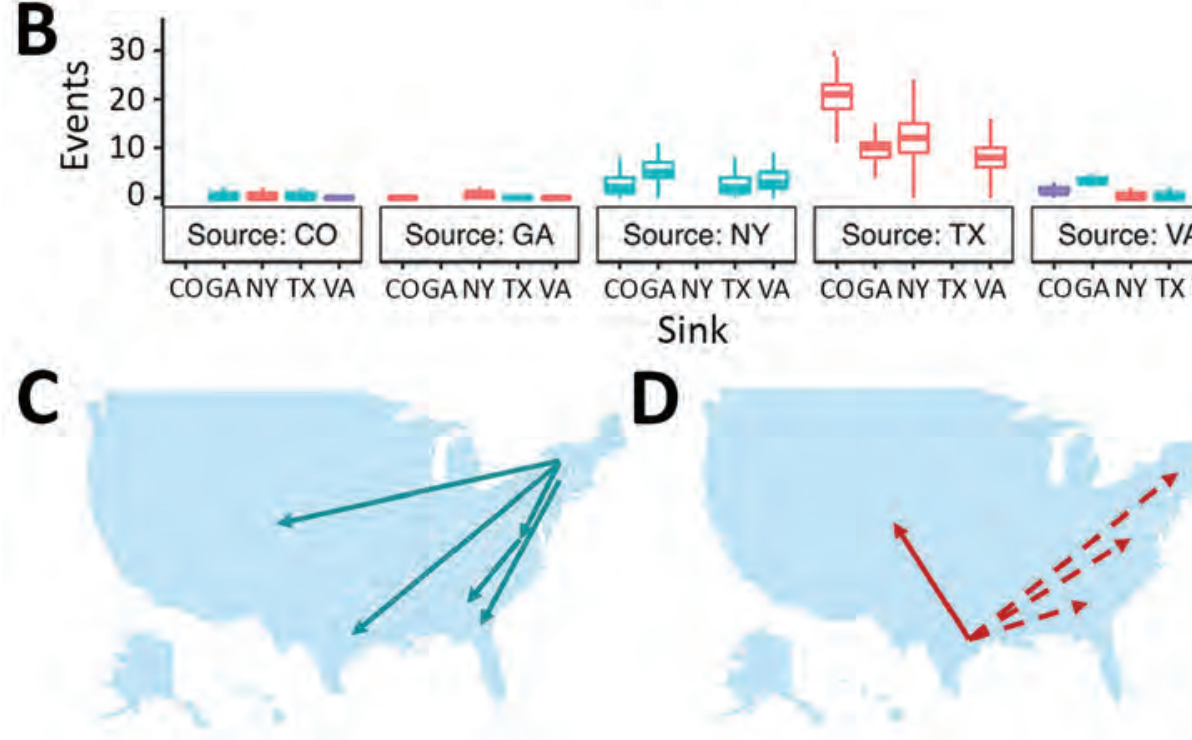

D

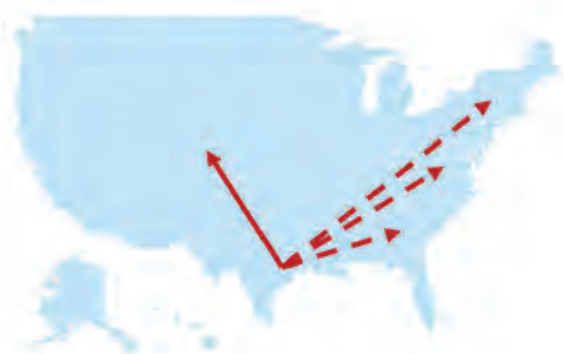

Figure 6. Summary of Markov jump analysis performed on the incidentcontrolled phylogeny. A, B) The results of the Markov jump analysis for each down-sampled dataset are summarized as box plots. Box tops indicate third quartiles, box bottoms indicate first quartiles; horizontal bars within boxes indicate medians; error bars indicate maximums and minimums. Red, northward movement; teal, southward movement; purple, movement that is neither north nor south; dotted arrows, movement that was not observed in the incident-controlled down-sampling because of an insufficient number of sequences. C, D) Movement originating in the eastern and central United States. Only Markov jumps that occurred $\geq 2$ times are depicted. by characterizing the movement of WNV with regard to the migratory patterns of its natural hosts, terrestrial birds. We compiled 379 virus sequences for analysis, including 289 previously reported sequences from New York, Virginia, Georgia, Illinois, North Dakota, South Dakota, Texas, and Colorado, plus 90 novel sequences from Virginia, Georgia, and Colorado.

Phylogeographic analysis revealed that 3 locationsNew York, Illinois, and Texas - accounted for $88.5 \%$ of the total WNV MJ inferred. Because New York is the presumed original introduction point for WNV into the United States, its role as a major source of WNV movement was expected. However, $74.2 \%$ of the observed MJ originated in Illinois and Texas only. Of note, North Dakota and South Dakota, which are 2 of the states with the highest annual WNND incidence, seem to be strong sinks for WNV moving out of Illinois and Texas.

The contributions of Illinois and Texas to WNV circulation are not surprising because both locations are situated at major convergence points between the Eastern and Central flyways. In the case of Texas, birds from both flyways may avoid long-distance flights across the Gulf of Mexico by traveling along the circa-Gulf route that follows the Gulf Coast through Texas into Mexico. In the case of Illinois, seasonal shifts in terrestrial bird migration routes ensure that Illinois supports birds from the Eastern and Central flyways during annual migrations.

Of note, although mosquito and WNV activity occurs earlier in the southern than in the northern United States, southward migration was detected along the East Coast during our sampling period, 2002-2009, indicating that the southeastern United States is probably a dead end for WNV circulation. Indeed, low-level transmission probably occurs during the winter in warmer locations such as Florida, Texas, and Louisiana. This possibility is supported by isolations of WNV from mosquitoes and birds during December and January in Harris County, Texas (44), and suggests that ecologic factors not related to mosquito abundance and WNV activity drive WNV movement along the East Coast. Instead, movement of WNV into the northeastern United States (New York) from Illinois and Texas was observed (in the incident-controlled analysis). These results suggest that introduction of WNV into the northeastern United States originated from the central United States.

Overall, we have defined the pattern of WNV circulation in the United States (Figure 7) and demonstrated looped virus movement patterns in the Eastern and Central flyways that are bridged by Illinois, a region shared between the 


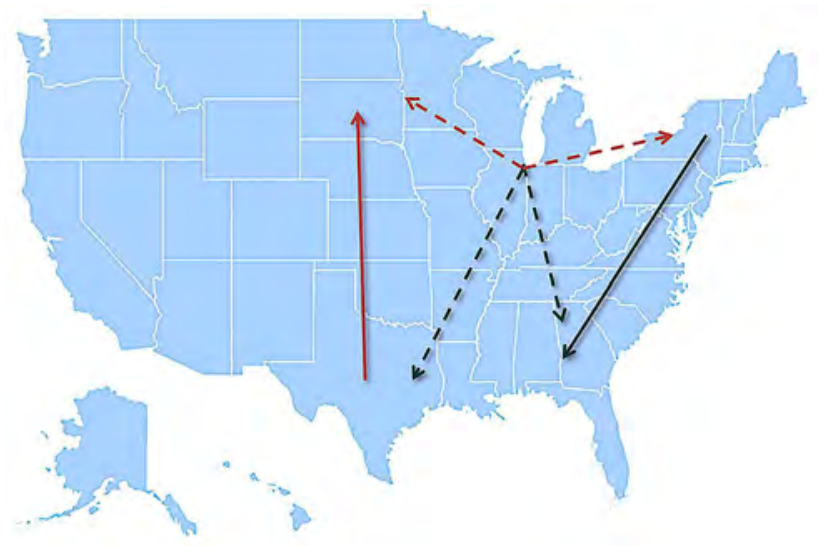

Figure 7. Model summarizing the general patterns of West Nile virus movement in the United States. Red, northward movement; teal, southward movement; dotted arrows, relationships that could not be confirmed in incident-controlled datasets because of an insufficient number of sequences.

2 flyways. This specific pattern correlates with the looped migration patterns of terrestrial birds. Although other geographic regions may contribute to virus movement, there were insufficient virus sequences available from other states to incorporate into this analysis. Thus, on the basis of available information, 3 of the 8 locations considered (New York, Illinois, and Texas) seem to be the preferred sites for efficiently monitoring ongoing WNV evolution.

As new WNV sequences become available, similar phylogeographic methods can be used to develop more detailed information about WNV circulation in the United States. For example, on the East Coast, WNV circulation occurs southward, so surveillance efforts in the Northeast are likely to be more informative than surveillance in the Southeast. Conversely, WNV in the central United States travels northward, so surveillance in the south-central United States is more likely than surveillance in the northcentral United States to be informative. Last, the region of overlap between the Eastern and Central flyways is the most likely location for deriving surveillance information because WNV in this area travels in multiple directions.

Collectively, the results of this study illustrate the value of using multidisciplinary approaches to surveillance of infectious diseases, especially zoonotic diseases. Animal migration is shaped by a delicate balance of ecologic factors and anthropomorphic barriers. Natural and manmade events (e.g., climate change, atmospheric fluctuations, habitat destruction) can drastically alter host behavior, which in turn affects the circulation patterns of infectious agents such as WNV. In this study, we defined the patterns of WNV circulation and key areas for surveillance and correlated them with the migratory patterns of their primary reservoir, terrestrial birds. Although this information does not enable prediction of the size of annual WNV outbreaks, these advancements support the construction of targeted surveillance and vector mitigation strategies to predict the annual flow of WNV strains and to enable public health officials to anticipate changes in WNV circulation resulting from altered bird migration.

This study was supported in part by National Institutes of Health contracts N01-AI 25489, N01-AI30027, and HHSN272201000040I/HHSN27200004/D04 to R.B.T. and grant AI 067847 to A.D.T.B.

\section{About the Author}

Dr. Swetnam is a postdoctoral fellow at the University of California at Davis. Her research interests include the evolution and molecular epidemiology of zoonotic and emerging diseases.

\section{References}

1. Reed KD, Meece JK, Henkel JS, Shukla SK. Birds, migration and emerging zoonoses: West Nile virus, lyme disease, influenza A and enteropathogens. Clin Med Res. 2003;1:5-12. http://dx.doi.org/10.3121/cmr.1.1.5

2. Pybus OG, Suchard MA, Lemey P, Bernardin FJ, Rambaut A, Crawford FW, et al. Unifying the spatial epidemiology and molecular evolution of emerging epidemics. Proc Natl Acad Sci U S A. 2012;109:15066-71. http://dx.doi.org/10.1073/ pnas.1206598109

3. Lewis M, Rencławowicz J, van den Driessche P. Traveling waves and spread rates for a West Nile virus model. Bull Math Biol. 2006;68:3-23. http://dx.doi.org/10.1007/s11538-005-9018-z

4. Maidana NA, Yang HM. Spatial spreading of West Nile Virus described by traveling waves. J Theor Biol. 2009;258:403-17. http://dx.doi.org/10.1016/j.jtbi.2008.12.032

5. Duggal NK, Reisen WK, Fang Y, Newman RM, Yang X, Ebel GD, et al. Genotype-specific variation in West Nile virus dispersal in California. Virology. 2015;485:79-85. http://dx.doi.org/10.1016/ j.virol.2015.07.004

6. Duggal NK, Bosco-Lauth A, Bowen RA, Wheeler SS, Reisen WK, Felix TA, et al. Evidence for co-evolution of West Nile Virus and house sparrows in North America. PLoS Negl Trop Dis. 2014;8:e3262. http://dx.doi.org/10.1371/journal.pntd.0003262

7. Añez G, Grinev A, Chancey C, Ball C, Akolkar N, Land KJ, et al. Evolutionary dynamics of West Nile virus in the United States, 1999-2011: phylogeny, selection pressure and evolutionary time-scale analysis [cited 2013 Dec 13]. http://dx.plos.org/10.1371/ journal.pntd.0002245

8. Formosinho P, Santos-Silva MM. Experimental infection of Hyalomma marginatum ticks with West Nile virus. Acta Virol. 2006; 50:175-80.

9. Komar N, Langevin S, Hinten S, Nemeth N, Edwards E, Hettler D, et al. Experimental infection of North American birds with the New York 1999 strain of West Nile virus. Emerg Infect Dis. 2003;9:311-22. http://dx.doi.org/10.3201/eid0903.020628

10. Wheeler SS, Langevin SA, Brault AC, Woods L, Carroll BD, Reisen WK. Detection of persistent West Nile virus RNA in experimentally and naturally infected avian hosts. Am J Trop Med Hyg. 2012;87:559-64. http://dx.doi.org/10.4269/ ajtmh.2012.11-0654

11. Bakonyi T, Gajdon GK, Schwing R, Vogl W, Häbich AC, Thaller D, et al. Chronic West Nile virus infection in kea (Nestor notabilis). Vet Microbiol. 2016;183:135-9. http://dx.doi.org/10.1016/j.vetmic.2015.12.012 
12. Di Giallonardo F, Geoghegan JL, Docherty DE, McLean RG, Zody MC, Qu J, et al. Fluid spatial dynamics of West Nile virus in the United States: rapid spread in a permissive host environment. J Virol. 2015;90:862-72. http://dx.doi.org/10.1128/JVI.02305-15

13. Bahl J, Krauss S, Kühnert D, Fourment M, Raven G, Pryor SP, et al. Influenza a virus migration and persistence in North American wild birds. PLoS Pathog. 2013;9:e1003570. http://dx.doi.org/10.1371/journal.ppat.1003570

14. Vollmer SA, Bormane A, Dinnis RE, Seelig F, Dobson ADM, Aanensen DM, et al. Host migration impacts on the phylogeography of Lyme borreliosis spirochaete species in Europe. Environ Microbiol. 2011;13:184-92. http://dx.doi.org/10.1111/ j.1462-2920.2010.02319.x

15. Muñoz J, Amat F, Green AJ, Figuerola J, Gómez A. Bird migratory flyways influence the phylogeography of the invasive brine shrimp Artemia franciscana in its native American range. PeerJ. 2013;1:e200. http://dx.doi.org/10.7717/peerj.200

16. Dusek RJ, McLean RG, Kramer LD, Ubico SR, Dupuis AP II, Ebel GD, et al. Prevalence of West Nile virus in migratory birds during spring and fall migration. Am J Trop Med Hyg. 2009;81:1151-8. http://dx.doi.org/10.4269/ajtmh.2009.09-0106

17. Reisen WK, Wheeler SS, Garcia S, Fang Y. Migratory birds and the dispersal of arboviruses in California. Am J Trop Med Hyg. 2010;83:808-15. http://dx.doi.org/10.4269/ajtmh.2010.10-0200

18. La Sorte FA, Fink D, Hochachka WM, Farnsworth A, Rodewald AD, Rosenberg KV, et al. The role of atmospheric conditions in the seasonal dynamics of North American migration flyways. J Biogeogr. 2014;41:1685-96. http://dx.doi.org/10.1111/jbi.12328

19. La Sorte FA, Fink D, Hochachka WM, DeLong JP, Kelling S. Spring phenology of ecological productivity contributes to the use of looped migration strategies by birds. Proc Biol Sci. 2014;281:20140984-20140984. http://dx.doi.org/10.1098/ rspb.2014.0984

20. Phillips AR. Migrations of Allen's and other hummingbirds. Condor. 1975;77:196-205. http://dx.doi.org/10.2307/1365790

21. Klaassen RHG, Strandberg R, Hake M, Olofsson P, Tøttrup AP, Alerstam T. Loop migration in adult marsh harriers Circus aeruginosus, as revealed by satellite telemetry. J Avian Biol. 2010; 41:200-7. http://dx.doi.org/10.1111/j.1600-048X.2010.05058.x

22. Mellone U, López-López P, Limiñana R, Piasevoli G, Urios V. The trans-equatorial loop migration system of Eleonora's falcon: differences in migration patterns between age classes, regions and seasons. J Avian Biol. 2013;44 [cited 2018 Oct 5]. http://doi.wiley.com/10.1111/j.1600-048X.2013.00139.x

23. Willemoes M, Strandberg R, Klaassen RHG, Tøttrup AP, Vardanis Y, Howey PW, et al. Narrow-front loop migration in a population of the common cuckoo Cuculus canorus, as revealed by satellite telemetry. PLoS One. 2014;9:e83515. http://dx.doi.org/ 10.1371/journal.pone.0083515

24. Boere GC, Stroud DA. The flyway concept: what it is and what it isn't. In: Boere GC, Galbraith C., Stroud DA, editors. Waterbirds around the World. Edinburgh: The Stationery Office; 2006. p. 40-4.

25. Hatcher EL, Zhdanov SA, Bao Y, Blinkova O, Nawrocki EP, Ostapchuck Y, et al. Virus Variation Resource-improved response to emergent viral outbreaks. Nucleic Acids Res. 2017;45:D482-90. http://dx.doi.org/10.1093/nar/gkw1065

26. Health UTMB. World Reference Center for Emerging Viruses and Arboviruses [cited 2018 Feb 20]. https://www.utmb.edu/wrceva/ home

27. Bolger AM, Lohse M, Usadel B. Trimmomatic: a flexible trimmer for Illumina sequence data. Bioinformatics. 2014;30:2114-20. http://dx.doi.org/10.1093/bioinformatics/btu170

28. Langmead B, Salzberg SL. Fast gapped-read alignment with Bowtie 2 [cited 2014 Mar 19]. http://www.central.nih.gov/ articlerender.fcgi? artid $=3322381 \&$ tool $=$ pmcentrez\&rendertype $=$ abstract
29. Li H, Handsaker B, Wysoker A, Fennell T, Ruan J, Homer N, et al. 1000 Genome Project Data Processing Subgroup. The Sequence Alignment/Map format and SAMtools. Bioinformatics. 2009;25:2078-9. http://dx.doi.org/10.1093/bioinformatics/btp352

30. Miller MA, Pfeiffer W, Schwartz T. Creating the CIPRES Science Gateway for inference of large phylogenetic trees [cited 2018 Oct 5]. http://ieeexplore.ieee.org/document/5676129/

31. Rambaut A, Lam TT, Max Carvalho L, Pybus OG. Exploring the temporal structure of heterochronous sequences using TempEst (formerly Path-O-Gen) [cited 2018 Oct 5]. http://ve.oxfordjournals.org/lookup/doi/10.1093/ve/vew007

32. Drummond AJ, Suchard MA, Xie D, Rambaut A. Bayesian phylogenetics with BEAUti and the BEAST 1.7. Mol Biol Evol. 2012;29:1969-73. http://dx.doi.org/10.1093/molbev/mss075

33. Gray RR, Veras NMC, Santos LA, Salemi M. Evolutionary characterization of the West Nile virus complete genome. Mol Phylogenet Evol. 2010;56:195-200. http://dx.doi.org/10.1016/ j.ympev.2010.01.019

34. Lemey P, Rambaut A, Drummond AJ, Suchard MA. Bayesian phylogeography finds its roots. PLOS Comput Biol. 2009;5:e1000520. http://dx.doi.org/10.1371/journal.pcbi.1000520

35. Minin VN, Suchard MA. Counting labeled transitions in continuous-time Markov models of evolution. J Math Biol. 2008;56:391-412. http://dx.doi.org/10.1007/s00285-007-0120-8

36. Tohma K, Saito M, Kamigaki T, Tuason LT, Demetria CS, Orbina JRC, et al. Phylogeographic analysis of rabies viruses in the Philippines. Infect Genet Evol. 2014;23:86-94. http://dx.doi.org/10.1016/j.meegid.2014.01.026

37. Raghwani J, Rambaut A, Holmes EC, Hang VT, Hien TT, Farrar J, et al. Endemic dengue associated with the co-circulation of multiple viral lineages and localized density-dependent transmission. PLoS Pathog. 2011;7:e1002064. http://dx.doi.org/ 10.1371/journal.ppat.1002064

38. Ward MJ, Lycett SJ, Kalish ML, Rambaut A, Leigh Brown AJ. Estimating the rate of intersubtype recombination in early HIV-1 group M strains. J Virol. 2013;87:1967-73. http://dx.doi.org/10.1128/JVI.02478-12

39. Lemey P, Rambaut A, Bedford T, Faria N, Bielejec F, Baele G, et al. Unifying viral genetics and human transportation data to predict the global transmission dynamics of human influenza H3N2. PLoS Pathog. 2014;10:e1003932. http://dx.doi.org/10.1371/ journal.ppat.1003932

40. Nelson MI, Viboud C, Vincent AL, Culhane MR, Detmer SE, Wentworth DE, et al. Global migration of influenza A viruses in swine. Nat Commun. 2015;6:6696. http://dx.doi.org/10.1038/ ncomms7696

41. Samy AM, Peterson AT, Hall M. Phylogeography of Rift Valley fever virus in Africa and the Arabian Peninsula. PLoS Negl Trop Dis. 2017;11:e0005226. http://dx.doi.org/10.1371/ journal.pntd.0005226

42. US Census Bureau. State intercensal tables: 2000-2010 [cited 2018 Oct 3]. https://www.census.gov/data/tables/time-series/demo/ popest/intercensal-2000-2010-state. html

43. Global Consortium for H5N8 and Related Influenza Viruses. Role for migratory wild birds in the global spread of avian influenza H5N8. Science. 2016;354:213-7. http://dx.doi.org/ 10.1126/science.aaf8852

44. Tesh RB, Parsons R, Siirin M, Randle Y, Sargent C, Guzman H, et al. Year-round West Nile virus activity, Gulf Coast region, Texas and Louisiana. Emerg Infect Dis. 2004;10:1649-52. http://dx.doi.org/10.3201/eid1009.040203

Address for correspondence: Alan D.T. Barrett, University of Texas Medical Branch, 301 University Blvd, Galveston, TX 77555-0436, USA; email: abarrett@utmb.edu 


\title{
Capnocytophaga canimorsus Capsular Serovar and Disease Severity, Helsinki Hospital District, Finland, 2000-2017
}

\author{
Estelle Hess, ${ }^{1}$ Francesco Renzi, ${ }^{1}$ Panu Karhunen, ${ }^{1}$ Mélanie Dol, Adrien Lefèvre, \\ Jenni Antikainen, Elodie Carlier, Johanna Hästbacka, ${ }^{2}$ and Guy R. Cornelis ${ }^{2}$
}

\begin{abstract}
We assembled a collection of 73 Capnocytophaga canimorsus isolates obtained from blood cultures taken from patients treated at Helsinki University Hospital (Helsinki, Finland) during 2000-2017. We serotyped these isolates by PCR and Western blot and attempted to correlate pathogen serovar with patient characteristics. Our analyses showed, in agreement with previous research, that $3 \mathrm{C}$. canimorsus serovars (A-C) caused most (91.8\%) human infections, despite constituting only $7.6 \%$ of isolates found in dogs. The 3 fatalities that occurred in our cohort were equally represented by these serovars. We found 2 untypeable isolates, which we designated serovars $\mathrm{J}$ and $\mathrm{K}$. We did not detect an association between serovar and disease severity, immune status, alcohol abuse, or smoking status, but dog bites occurred more frequently among patients infected with non-A-C serovars. Future research is needed to confirm serovar virulence and develop strategies to reduce risk for these infections in humans.
\end{abstract}

C apnocytophaga canimorsus is a gram-negative, rod-shaped, usually commensal bacteria of dog and cat oral flora that causes rare but potentially severe infections in humans $(1,2)$. Even with administration of adequate antimicrobial therapy, C. canimorsus-induced septicemia can progress to a debilitating disease or septic shock and can cause a mortality rate as high as $30 \%$. Annual incidence of $C$. canimorsus infections has been estimated at $0.5-0.67$ cases/ 1 million persons $(3,4)$, but in a retrospective study, a prevalence of 4.1 cases/ 1 million persons was estimated (5); this discrepancy probably resulted from the choice of diagnostics. The clinical manifestation of C. canimorsus infection might be mild,

Author affiliations: University of Namur, Namur, Belgium (E. Hess, F. Renzi, M. Dol, A. Lefèvre, E. Carlier, G.R. Cornelis); University of Eastern Finland, Kuopio, Finland (P. Karhunen); University of Helsinki and Helsinki University Hospital, Helsinki, Finland (J. Antikainen, J. Hästbacka)

DOI: https://doi.org/10.3201/eid2412.172060 with influenza-like symptoms and intestinal complaints (1), a disease severity not always reaching the threshold for a blood culture. Moreover, C. canimorsus is a fastidious and slow-growing organism, rendering its culture and isolation difficult (2).

Human exposure to a dog's oral flora can occur through a bite or scratch or even through just being in close proximity to the animal $(1,5)$. Although splenectomy, asplenia, alcohol abuse, smoking, and advanced age are often described as predisposing factors for severe illness caused by this bacterium, up to $40 \%$ of patients have no obvious risk factor (1); thus, C. canimorsus should not be considered exclusively an opportunistic pathogen.

C. canimorsus is enveloped by a lipooligosaccharide and a capsule consisting of units of the same $\mathrm{O}$ antigen but assembled by different polymerases (6). The capsule confers to $C$. canimorsus resistance to the bactericidal effects of human serum and phagocytosis by macrophages (6). One study showed that despite the seemingly vast repertoire of capsular serovars among $C$. canimorsus isolates from dog mouths, 3 serovars (A, B, and C) are associated with most human infections (7). However, this finding was from a study carried out with just 25 isolates from patients worldwide. To validate this finding, we evaluated the serovars present in a collection of 73 isolates from patients treated at Helsinki University Hospital (Helsinki, Finland) during 2000-2017.

\section{Materials and Methods}

\section{Study Setting}

HUSLAB (Helsinki) is a central laboratory that offers microbiological services to the whole Helsinki Hospital District, which encompasses the city of Helsinki and surrounding municipalities. The laboratory maintains a

${ }^{1}$ These first authors contributed equally to this article.

${ }^{2}$ These authors were co-principal investigators. 
frozen archive of bacterial isolates obtained from patient blood cultures. For the purposes of this study, we searched laboratory records for blood cultures positive for C. canimorsus during 2000-2017; a corresponding frozen bacteria isolate could be found for 78 patients. Of these frozen isolates, we could grow and analyze 73. To correlate analyses with clinical data, we searched patient journals, electronic patient records, and laboratory databases for patient characteristics, clinical information, and laboratory data. We recorded patient age, sex, concurrent medical conditions, medications administered, immune status, lifestyle factors, and type of contact with dogs (bitten, contact but not bitten, or not known), whenever the information was available. Of the clinical data, we recorded the level of care, length of stay in the hospital, complications, 30-day and 1-year mortality rates, and registered coagulation and fibrinolysis laboratory variables. We analyzed partial thromboplastin time according to the Owren method (8).

The Administrative Department of Helsinki Hospital District and Helsinki City College of Social and Health Care gave approval for obtaining this data from patient medical records. Because only data registers were used for acquiring data, obtaining informed consent from patients was waived.

\section{Bacterial Isolates and Growth Conditions}

We cultured C. canimorsus bacterial isolates (Table 1) obtained from HUSLAB, which were originally obtained from blood samples of patients in Finland, as described previously (9). In brief, we incubated aerobic and anaerobic blood culture bottles with BacT/ALERT 3D (bioMérieux, Marcy l'Etoile, France) for 6 days or until the cultures became positive. We used Gram staining and cultivated all positive samples on chocolate agar, fastidious anaerobe agar, or heart infusion agar plates. For serotyping, we grew bacteria on heart infusion agar plates (BD Difco, Franklin Lakes, NJ, USA) supplemented with $5 \%$ sheep blood (Oxoid, Basingstoke, UK) and $20 \mu \mathrm{g} / \mathrm{mL}$ gentamicin (Sigma-Aldrich, Darmstadt, Germany) for 48 $\mathrm{h}$ at $37^{\circ} \mathrm{C}$ with $5 \% \mathrm{CO}_{2}$.

\section{C. canimorsus Identification by $16 \mathrm{~S}$ rDNA Sequencing}

We extracted genomic DNA directly from blood culture bottles or by boiling of a single colony (online Technical Appendix Table 1, https://wwwnc.cdc.gov/EID/ article/24/12/17-2060-Techapp1.pdf). We used 4 different amplification methods involving 8 different primers to sequence 16S rDNA from bacterial isolates (online Technical Appendix Tables 2, 3). When $>1$ primer was used to sequence a PCR product, we obtained the consensus sequence using Bioedit (https://bioedit.software.informer. com), and we analyzed sequences using BLAST (https:// blast.ncbi.nlm.nih.gov/Blast.cgi).

\section{Antisera Production and Adsorption}

The production of antisera to serovars A-I has previously been described (7). We produced rabbit polyclonal anti-J (against isolate H12) and anti-K (against isolate H24) likewise (7). Immunizations were carried out at the Centre d'Economie Rurale (Aye, Belgium). The Centre d'Economie Rurale animal welfare committee approved our animal handling protocols and procedures. We adsorbed anti-J and anti-K sera with a mixture of 25 isolates from patients (Cc1-Cc25; online Technical Appendix Table 4) to obtain polyclonal antibodies specifically recognizing $\mathrm{J}$ or $\mathrm{K}$ capsular serovars. We performed adsorptions by incubating $250 \mu \mathrm{L}$ of antiserum with $6 \times$ $10^{9}$ paraformaldehyde-fixed bacteria on a rotating wheel at room temperature for $\geq 2$ hours. We removed bacteria by successive centrifugations. We repeated the incubations and centrifugations 4 times. We performed capsular

\begin{tabular}{|c|c|c|c|c|c|c|c|c|c|c|c|c|c|c|c|c|}
\hline \multirow[b]{2}{*}{ Isolates } & \multicolumn{6}{|c|}{ PCR typing $\dagger$} & \multicolumn{9}{|c|}{ Western blot typing $\neq$} & \multirow[b]{2}{*}{ Serovar } \\
\hline & $\mathrm{ABC}$ & $\mathrm{A}$ & $\mathrm{B}$ & $\mathrm{C}$ & $\mathrm{D}$ & $E$ & $\mathrm{~A}$ & $\mathrm{~B}$ & $\mathrm{C}$ & $\mathrm{D}$ & $E$ & $\mathrm{~F}$ & $\mathrm{G}$ & $\mathrm{H}$ & 1 & \\
\hline $\begin{array}{l}\text { H11, H16, H23, H37, H39, H42, H48, H52, H56, } \\
\mathrm{H} 60, \mathrm{H} 62, \mathrm{H} 70, \mathrm{H} 74, \mathrm{H} 75, \mathrm{H} 76, \mathrm{H} 78, \mathrm{H} 80\end{array}$ & + & + & + & - & - & - & + & - & - & ND & ND & ND & ND & ND & ND & $A$ \\
\hline $\begin{array}{l}\mathrm{H} 3, \mathrm{H} 4, \mathrm{H} 5, \mathrm{H} 6, \mathrm{H} 9, \mathrm{H} 14, \mathrm{H} 22, \mathrm{H} 25, \mathrm{H} 26, \mathrm{H} 30, \mathrm{H} 35 \text {, } \\
\mathrm{H} 38, \mathrm{H} 49, \mathrm{H} 50, \mathrm{H} 53, \mathrm{H} 55, \mathrm{H} 57, \mathrm{H} 58, \mathrm{H} 63, \mathrm{H} 65 \\
\mathrm{H} 67, \mathrm{H} 68, \mathrm{H} 69, \mathrm{H} 71, \mathrm{H} 72, \mathrm{H} 73, \mathrm{H} 79\end{array}$ & + & - & + & - & - & - & - & + & - & ND & ND & ND & ND & ND & ND & B \\
\hline $\mathrm{H} 27$ & + & - & + & - & - & - & + & + & - & ND & ND & ND & ND & ND & ND & B \\
\hline $\begin{array}{l}\mathrm{H} 1, \mathrm{H} 7, \mathrm{H} 8, \mathrm{H} 10, \mathrm{H} 13, \mathrm{H} 15, \mathrm{H} 17, \mathrm{H} 18, \mathrm{H} 19, \mathrm{H} 20 \text {, } \\
\mathrm{H} 28, \mathrm{H} 29, \mathrm{H} 33, \mathrm{H} 34, \mathrm{H} 36, \mathrm{H} 43, \mathrm{H} 44, \mathrm{H} 45, \mathrm{H} 46 \text {, } \\
\mathrm{H} 47, \mathrm{H} 51, \mathrm{H} 59\end{array}$ & + & - & - & + & - & - & - & - & + & ND & ND & ND & ND & ND & ND & $\mathrm{C}$ \\
\hline $\mathrm{H} 41, \mathrm{H} 64$ & - & - & - & - & + & - & - & - & - & + & ND & ND & ND & ND & ND & D \\
\hline H31 & - & - & - & - & - & + & - & - & - & ND & + & ND & ND & ND & ND & $E$ \\
\hline H21 & - & - & - & - & - & - & - & - & - & - & - & - & - & - & + & 1 \\
\hline $\mathrm{H} 12, \mathrm{H} 24$ & - & - & - & - & - & - & - & - & - & - & - & - & - & - & - & NT \\
\hline
\end{tabular}


typing of C. canimorsus by Western blot, ELISA, and PCR as previously described (7).

\section{Statistical Analysis}

We expressed categorical data as counts and percentages and continuous data as medians and interquartile ranges. We compared categorical data between groups by Fisher exact test. We assumed continuous data were nonnormally distributed and analyzed data using Mann-Whitney U-test for 2 groups and Kruskal-Wallis nonparametric test for $\geq 3$ groups. Because of the retrospective nature of the study, many data points were unavailable for many cases (data were more complete for severely ill patients and less complete for mildly ill patients), so we provided the number of patients included in each analysis. We considered $\mathrm{p}$ values $<0.05$ statistically significant and performed analyses using SPSS version 22 (https://www.ibm.com/analytics/ spss-statistics-software).

\section{Results}

\section{Capsular Typing Collection of $\mathbf{7 3}$ Isolates from Finland}

We identified the 73 isolates originating from Helsinki University Hospital (Table 1) as C. canimorsus through 16S rDNA sequencing (online Technical Appendix Tables 1, 3). We subjected isolates to a PCR designed to detect capsular serovars A, B, and C (7); 67 of 73 isolates were $\mathrm{ABC}$ positive (Table 1; online Technical Appendix Figure 1). We also typed these 67 strains using A-, B-, and C-specific PCR tests (7). To validate the PCR typing results, we performed Western blot analyses with polysaccharide samples of the 73 isolates using antiserum specifically recognizing $\mathrm{A}, \mathrm{B}$, or $\mathrm{C}$ capsular serovars (online Technical Appendix Figure 2). This analysis confirmed the PCR typing results and interpretation of all isolates tested, except H27. According to Western blot analyses, isolate H27 could be considered serovar A or B, but in agreement with the PCR results, we considered this isolate a B capsular serovar only. In short, $91.8 \%(67 / 73)$ of isolates tested were serovars A $(\mathrm{n}=17), \mathrm{B}(\mathrm{n}=28)$, or $\mathrm{C}(\mathrm{n}=22)$.

We then subjected isolates to PCR analyses for the detection of capsular types $\mathrm{D}$ and $\mathrm{E}$, which have previously been detected among $C$. canimorsus isolates from human infections (7). Two isolates were serovar D and 1 serovar E (online Technical Appendix Figure 1), findings that were confirmed by Western blot analyses (Table 1; online Technical Appendix Figure 2). We tested the 3 remaining nontypeable (non-A-E) isolates by Western blot for capsular types $\mathrm{F}-\mathrm{I}$, which have only been detected in isolates obtained from dogs (7). Isolate H21 was typed as serovar I, leaving only 2 strains (H12 and H24) not typed of the 73 tested.

We raised rabbit antisera against $\mathrm{H} 12$ and H24 bacteria and adsorbed antisera with related bacteria strains. The 2 new antisera recognized only the capsule of the isolate against which they were raised, indicating the 2 isolates belonged to 2 new serovars, which we named $\mathrm{J}$ and $\mathrm{K}$ (online Technical Appendix Figure 2). Thus, the 73 C. canimorsus isolates from the Helsinki University Hospital collection comprised 8 serovars (Figure 1, panel A); A, B, and C dominated $(91.8 \%)$, consistent with the findings of the previous study involving 25 worldwide isolates (Figure 1, panel B) (7). The distribution of serovars A $(p=0.071), B(p=0.47)$, $\mathrm{C}(\mathrm{p}=0.20), \mathrm{D}(\mathrm{p}=0.27), \mathrm{E}(\mathrm{p}=0.45)$, and $\mathrm{I}-\mathrm{K}(\mathrm{p}=1)$ was not significantly different between the 2 collections (all p values analyzed by Fisher exact test; Figure 1, panel C).

\section{Screening of Dog Isolates for Capsular Serovars $\mathrm{J}$ and $\mathrm{K}$}

We next tested for the prevalence of the $\mathrm{J}$ and $\mathrm{K}$ capsular serovars in a previously described collection of $C$. canimorsus isolates obtained from mouths of healthy dogs $(7,10)$. We screened these 52 dog isolates by ELISA using
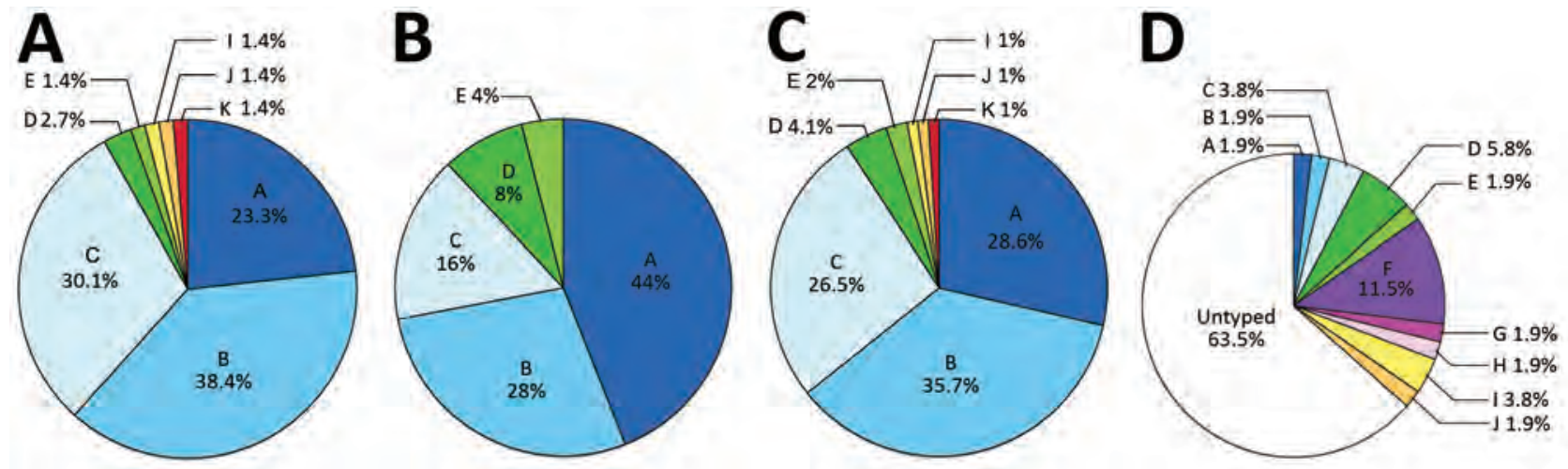

Figure 1. Prevalence of capsular serovars among Capnocytophaga canimorsus isolates from patients and dogs. A) Prevalence among 73 isolates from patients in Helsinki, Finland, 2000-2017. B) Prevalence among 25 isolates acquired from patients worldwide. C) Prevalence among pooled samples $(n=98)$. D) Prevalence among 52 isolates from dog mouths, Switzerland and Belgium. Percentages do not add up to $100 \%$ because of rounding. A portion of the data presented in panels B and D were previously published (7). 
the antisera we produced. Although no isolates reacted with the anti-K serum, isolate CcD35 from a dog in Switzerland reacted with the anti-J serum (Figure 1, panel D; online Technical Appendix Table 5). We confirmed this result by Western blot analysis of the polysaccharidic structures (online Technical Appendix Figure 2), which showed that capsular serovar $\mathrm{J}$ is thus not limited to Finland.

\section{Correlation between Disease Severity and Capsular Type}

We also tested the association between serovar and disease severity. For this investigation, the level of care was used as a surrogate; patients treated in a regular ward or who had only visited the emergency department were regarded as having a mild course of disease, and patients treated in a high-dependency or intensive care unit were regarded as severely ill. No statistically significant difference could be found in the proportions of any serovar between patients with mild and severe disease ( $p=0.76$; Figure 2, panel A). Among the 73 cases of $C$. canimorsus infection included in this study, 3 were fatal (Table 2). The isolates from these 3 patients were serovars A (H80), B (H26), and C (H28). Extensive amputations were reported in 6 cases, among which included the nonsurviving patient infected with the capsular B isolate H26. The 5 other capsular types associated with amputations were A ( $\mathrm{n}=2, \mathrm{H} 48$ and H56), B (n $=1, \mathrm{H} 79)$, and $\mathrm{C}(\mathrm{n}=2, \mathrm{H} 46$ and H59). Therefore, capsular serovars $\mathrm{A}, \mathrm{B}$, and $\mathrm{C}$ are all capable of causing severe disease in humans.

We looked for an association between capsular serovar and patient immune status or lifestyle factors but found no statistically significant link between serovar and immune compromised state $(\mathrm{p}=0.682)$, alcohol abuse $(\mathrm{p}=0.982)$, or smoking $(p=0.713)$ (Figure 2, panels $B-D)$. We defined patients as immune compromised if they had been on immune suppressive medication or had recently received chemotherapy, had a concurrent medical condition associated with impaired immunity or active cancer, or had undergone splenectomy. One of the 2 splenectomized patients had a severe course of disease, but both survived.

Severe C. canimorsus infections are often associated with purpura or petechiae, disseminated intravascular coagulation, and gangrene of extremities (1). In particular, coagulation disorders were found to be associated with $94 \%$ of patients having C. canimorsus-induced septic shock in a 10-year retrospective study in Helsinki (5). In our study, no statistically significant association could be found between coagulation and fibrinolysis laboratory variables (platelet count, partial thromboplastin time, fibrin D-dimers) and capsular serovars (Table 3). Given the low number of cases associated with some serovars, we could assess only the 3 dominant serovars (A, B, and C). We compared coagulation and fibrinolysis disorder markers between patients with mild and severe clinical course.
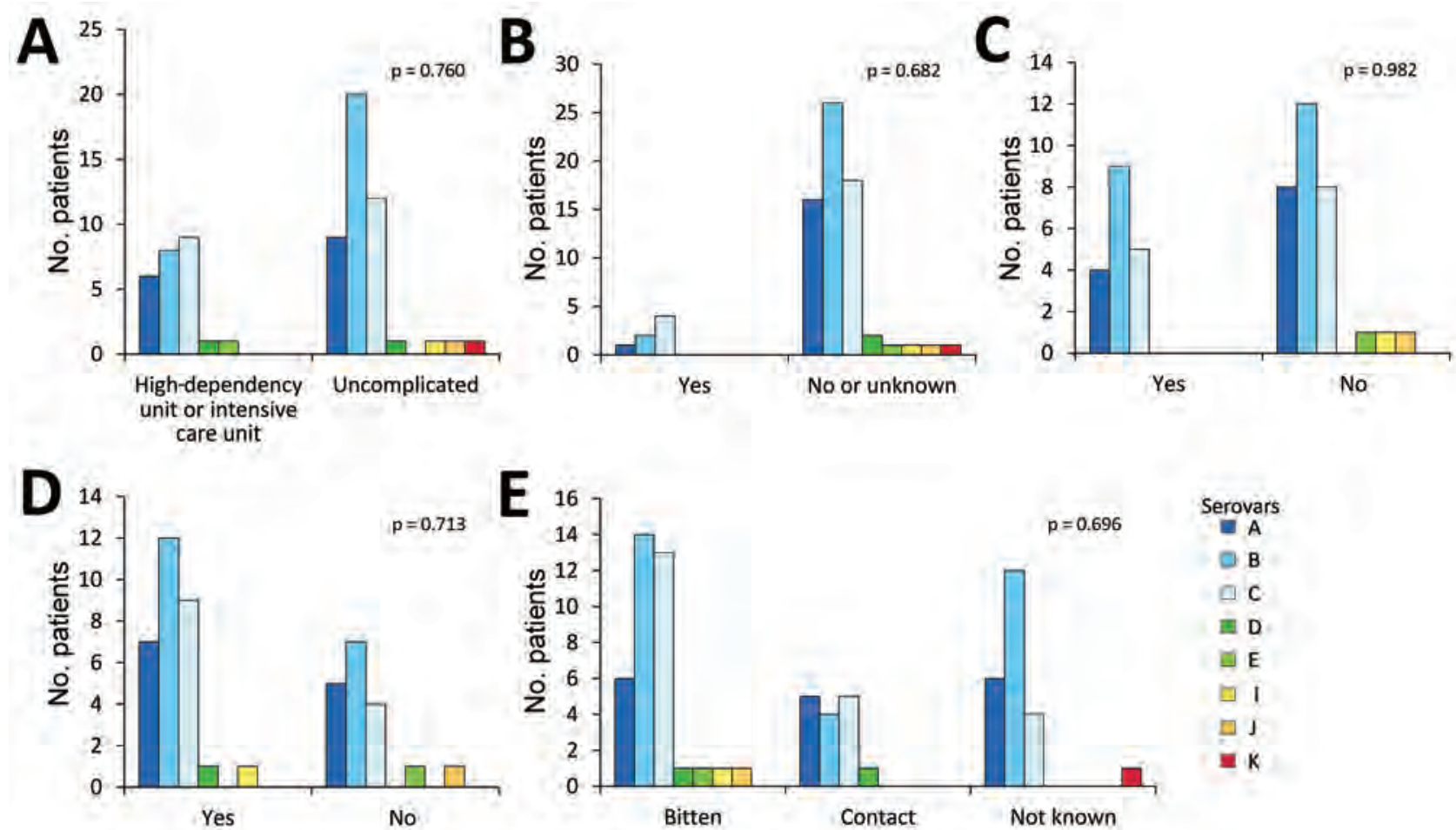

Figure 2. Association between Capnocytophaga canimorsus capsular serovar and various patient factors, Helsinki, Finland, $2000-2017$. A) Disease severity $(n=70)$; B) immune compromised $(n=73)$; C) alcohol abuse $(n=49)$; D) smoking status $(n=48)$; and E) contact with dogs $(n=73)$. Fisher exact test was used for statistical analysis. 
As expected, the analyzed variables were more affected in patients with a severe course of infection (Table 3). Of note, deviating coagulation and fibrinolysis variables were frequently present in patients with mild courses of disease, further strengthening the previously reported close association of $C$. canimorsus infection and coagulation disorders.

\section{Correlation between Type of Contact with Dog and Capsular Type}

The type of contact with dogs did not differ among infections with any of the dominant serovars, but 4 of the 5 patients infected with serovar D, E, I, or J had been bitten (Figure 2, panel E). The contact type was not known for the patient infected with the serovar $\mathrm{K}$ isolate, the fifth rare serotype.

\section{Discussion}

In this study, we analyzed 73 C. canimorsus isolates obtained from patients treated at Helsinki University Hospital. All isolates were serotyped and found to be endowed with a capsular polysaccharide (CPS), further confirming the commonality of the presence of a CPS in C. canimorsus isolates $(6,7)$. We confirmed the high prevalence of capsular serovars $\mathrm{A}, \mathrm{B}$, and $\mathrm{C}$ among isolates from human infections; $67(91.8 \%)$ of 73 isolates were typed as 1 of these 3 serovars. No significant difference was found in the prevalence of these serovars between this collection of 73 isolates from Finland and a previously studied collection of 25 isolates obtained from cases worldwide (7). Among the 98 C. canimorsus isolates from these 2 studies, 89 (90.8\%) were capsular types A, B, or C. Our data confirmed that serovars $\mathrm{A}, \mathrm{B}$, and $\mathrm{C}$ are significantly more common among clinical isolates than dog isolates $(4 / 52 ; 7.6 \%)$, suggesting these serovars are more virulent than the others. Our data also confirmed that serovars $\mathrm{A}, \mathrm{B}$, and $\mathrm{C}$ are present in different geographic areas.

Besides the A, B, and C serovars, the Helsinki collection contained 2 other serovars: 2 isolates of serovar $\mathrm{D}$ and 1 of serovar E. This observation is of high interest because
Table 2. Patient demographics, clinical characteristics, and contact with dogs, Helsinki Hospital District, Finland, 2000-2017*

\begin{tabular}{lcc}
\hline Characteristic & $\begin{array}{c}\text { No. } \\
\text { patients } \dagger\end{array}$ & Value \\
\hline Age, y, median (IQR) & 73 & $55(48.3-$ \\
& & $64.8)$ \\
\hline Sex & 73 & \\
M & & $38(52.1)$ \\
F & 73 & $75(47.9)$ \\
\hline Immune compromised & 48 & $30(62.5)$ \\
Smoking & 49 & $18(36.7)$ \\
Alcohol abuse & 73 & \\
Contact with dog & & $21(28.8)$ \\
$\quad$ Not known & & $15(20.5)$ \\
$\quad$ Contact but not bitten & 70 & $37(50.7)$ \\
$\quad$ Bitten & & $45(64.3)$ \\
Disease severity & & \\
$\quad$ Regular ward or emergency & & $11(15.7)$ \\
department & & $14(20.0)$ \\
$\quad$ High surveillance unit & & $6(3-13.3)$ \\
$\quad$ Intensive care unit & 62 & $3(4.1)$ \\
Length of hospital stay, d, median (IQR) & 73 & $4(6.6)$ \\
Deaths at day 30 & 61 & $6(8.2)$ \\
Deaths at 1 y & 73 & \\
Amputation &
\end{tabular}

*Values are no. (\%) patients except as indicated. IQR, interquartile range. †Because of missing data, number of patients in each category varied.

serovars $\mathrm{D}$ and $\mathrm{E}$ were previously isolated from patients in the United States $(n=1)$, Belgium $(n=1)$, and Switzerland $(n=1)(7)$. Thus, although serovars D and E represent only $4.1 \%$ and $2 \%$, respectively, of the total clinical isolates in this study, these serovars should be considered virulent and taken into account in prophylaxis.

One patient in our cohort was infected with a serovar I strain. This serovar had not been encountered before among humans but was found in dogs (1 in Belgium and 1 in Switzerland) (7). These findings suggest that not only serovars A-E but also rare serovars are widely distributed.

Last, we describe 2 new capsular serovars, $\mathrm{J}$ and $\mathrm{K}$, each with a limited (1\%) prevalence in human infections. We tested these 2 new antisera against our collection of isolates obtained from dogs in Switzerland and Belgium (10) and found 1 C. canimorsus isolate had a J-type CPS. Thus, using the 11 antisera we have that are specific to serovars

Table 3. Coagulation and fibrinolysis laboratory variables, by Capnocytophaga canimorsus serovar and disease severity, Helsinki, Finland, 2000-2017*

\begin{tabular}{|c|c|c|c|c|c|c|c|c|}
\hline \multirow[b]{2}{*}{ Variable } & \multirow{2}{*}{$\begin{array}{l}\text { Reference } \\
\text { range }\end{array}$} & \multicolumn{3}{|c|}{ Serovar } & \multirow[b]{2}{*}{$p$ value } & \multicolumn{2}{|c|}{ Severity of illness } & \multirow[b]{2}{*}{$p$ value } \\
\hline & & $A, n=17$ & $\mathrm{~B}, \mathrm{n}=28$ & $C, n=22$ & & Severe, $\mathrm{n}=25$ & Mild, $\mathrm{n}=45$ & \\
\hline Platelets, $10^{9} / \mathrm{L}$ & $150-360$ & 109 (29-137) [2] & $\begin{array}{c}109(28-140) \\
\text { [1] }\end{array}$ & $93(23-166)[1]$ & 0.98 & $23(9.5-89)[0]$ & $\begin{array}{c}117 \text { (95-154.3) } \\
{[4]}\end{array}$ & $<0.001$ \\
\hline PTT, \%† & $70-130$ & $56(24-71)[10]$ & $\begin{array}{c}78(56-86) \\
{[11]}\end{array}$ & $\begin{array}{c}58(44.5-75) \\
{[12]}\end{array}$ & 0.284 & $54(39-66)[2]$ & $87(70.5-109.5)$ & $<0.001$ \\
\hline FiDD, mg/L & $<0.5$ & $\begin{array}{c}47.9(5.05-83.8) \\
{[12]}\end{array}$ & $\begin{array}{c}9.1(3.4-85.7) \\
{[19]}\end{array}$ & $\begin{array}{c}14.5(4.1-80.7) \\
{[15]}\end{array}$ & 0.888 & $\begin{array}{c}32.9(5.6-81) \\
{[5]}\end{array}$ & $\begin{array}{c}1.6(0.6-74.7) \\
{[40]}\end{array}$ & 0.057 \\
\hline
\end{tabular}

*Values are given as median (interquartile range) [no. missing values] except as indicated. The comparison between patients with mild and severe courses of disease was defined by the level of care they needed. Patients with mild disease were those who were treated in a regular ward or the emergency department, and patients with severe disease were those treated in high surveillance or intensive care units. FiDD, fibrin D-dimers; PTT, partial thromboplastin time.

†PTT was analyzed according to the Owren method (8). PTT was calculated as the ratio of the result (in seconds) from normal plasma to the result (in seconds) from the patient sample $\times 100$. 
A-K, which identified 98 human clinical isolates, we can only type $36.5 \%(19 / 52)$ of our collection of dog isolates from Switzerland and Belgium. This finding, again, reinforces the hypothesis of the existence of a large repertoire of CPS serovars in C. canimorsus among dog isolates.

Because $C$. canimorsus extensively deglycosylates human N-linked glycoproteins from cell surfaces (11-13), a given blood group might be a predisposing factor for $C$. canimorsus infection, but further research is needed to investigate an association between blood type and serovar. Blood group information was available for 55 patients in our cohort, and we found no enrichment in any blood groups among patients infected with $C$. canimorsus compared with the blood group distribution of the population of (data not shown).

The availability of clinical records associated with the isolates typed in this study gave us the opportunity to investigate the link between capsular serovar and disease severity, patient immune status, lifestyle, or type of contact with dogs. When comparing the most prevalent capsular types (A, B, and C) found in these 73 clinical isolates, we found no significant correlation between disease severity and capsular type. In the previous C. canimorsus capsular typing study, the authors suggested that strains belonging to capsular types of lower prevalence, like D and E, might preferentially infect immunocompromised patients (7); we could not draw such a conclusion here. In addition, alcohol abuse or smoking status could not be linked to infection by a specific capsular type. Alcohol abuse, smoking status, and immune suppression all were not significantly associated with disease severity or the 30-day mortality rate (data not shown), although the relatively low sample size and missing data preclude us from drawing conclusions regarding this matter.

The capsular serovars less frequently isolated in human infections, such as E, I, and J, were mainly found in patients who had been bitten, which could suggest that these serovars are less virulent than serovars A-D, perhaps requiring a deeper inoculation to provoke an infection. Unfortunately, the information on dog exposure was missing for the patient infected with the serovar K strain.

Two patients included in this study were reportedly bitten on the same day by the same dog. The isolates from these 2 patients (H44 and H46) were both typed as capsular serovar $\mathrm{C}$, suggesting that the same strain infected both patients. The 2 patients had a severe form of the infection, requiring treatment in an intensive care unit. This observation of 2 patients being infected by the same dog has not been reported previously and gives an indication of the epidemiology of disease.

The observation of so few cases of C. canimorsus infection is indeed striking, considering that up to $74 \%$ of dogs carry C. canimorsus bacteria (14). We hypothesize that only a few C. canimorsus strains are virulent in humans, and few dogs carry these dangerous strains. Indeed, the 3 most prevalent serovars in human infection (A-C), represent only $7.6 \%$ of the C. canimorsus isolates from dogs (7), suggesting that a minority of dogs represent a risk for humans. This disease might be preventable in humans by identifying the dogs that carry these dangerous serotypes and specifically vaccinating them to eliminate the pathogen or drastically reduce pathogen shedding.

This work was financed by grant SOC 1510582 from the Belgian Walloon Region.

\section{About the Author}

Dr. Hess is a researcher at the Research Unit in the Biology of Microoganisms at the University of Namur in Belgium and an immunologist with a research interest in hostpathogen interactions.

\section{References}

1. Butler T. Capnocytophaga canimorsus: an emerging cause of sepsis, meningitis, and post-splenectomy infection after dog bites. Eur J Clin Microbiol Infect Dis. 2015;34:1271-80. http://dx.doi.org/ 10.1007/s10096-015-2360-7

2. Butler T, Weaver RE, Ramani TK, Uyeda CT, Bobo RA, Ryu JS, et al. Unidentified gram-negative rod infection. A new disease of man. Ann Intern Med. 1977;86:1-5. http:/dx.doi.org/10.7326/ 0003-4819-86-1-1

3. Pers C, Gahrn-Hansen B, Frederiksen W. Capnocytophaga canimorsus septicemia in Denmark, 1982-1995: review of 39 cases. Clin Infect Dis. 1996;23:71-5. http://dx.doi.org/10.1093/ clinids/23.1.71

4. van Dam AP, Jansz A. Capnocytophaga canimorsus infections in The Netherlands: a nationwide survey. Clin Microbiol Infect. 2011;17:312-5. http://dx.doi.org/10.1111/j.1469-0691. 2010.03195.x

5. Hästbacka J, Hynninen M, Kolho E. Capnocytophaga canimorsus bacteremia: clinical features and outcomes from a Helsinki ICU cohort. Acta Anaesthesiol Scand. 2016;60:1437-43. http://dx.doi.org/10.1111/aas.12752

6. Renzi F, Ittig SJ, Sadovskaya I, Hess E, Lauber F, Dol M, et al. Evidence for a LOS and a capsular polysaccharide in Capnocytophaga canimorsus. Sci Rep. 2016;6:38914. http://dx.doi.org/10.1038/srep38914

7. Hess E, Renzi F, Koudad D, Dol M, Cornelis GR. Identification of virulent Capnocytophaga canimorsus isolates by capsular typing. J Clin Microbiol. 2017;55:1902-14. http://dx.doi.org/10.1128/ JCM.00249-17

8. Owren PA. Thrombotest a new method for controlling anticoagulant therapy. Lancet. 1959;274:754-8. http://dx.doi.org/ 10.1016/S0140-6736(59)90857-8

9. Tissari P, Zumla A, Tarkka E, Mero S, Savolainen L, Vaara M, et al. Accurate and rapid identification of bacterial species from positive blood cultures with a DNA-based microarray platform: an observational study. Lancet. 2010;375:224-30. http://dx.doi.org/ 10.1016/S0140-6736(09)61569-5

10. Renzi F, Dol M, Raymackers A, Manfredi P, Cornelis GR. Only a subset of $C$. canimorsus strains is dangerous for humans. Emerg Microbes Infect. 2016;5:e29. http://dx.doi.org/10.1038/emi.2016.43 
11. Mally M, Shin H, Paroz C, Landmann R, Cornelis GR. Capnocytophaga canimorsus: a human pathogen feeding at the surface of epithelial cells and phagocytes. PLoS Pathog. 2008;4:e1000164. http://dx.doi.org/10.1371/journal.ppat.1000164

12. Manfredi P, Renzi F, Mally M, Sauteur L, Schmaler M, Moes $\mathrm{S}$, et al. The genome and surface proteome of Capnocytophaga canimorsus reveal a key role of glycan foraging systems in host glycoproteins deglycosylation. Mol Microbiol. 2011;81:1050-60. http://dx.doi.org/10.1111/j.1365-2958.2011.07750.x

13. Renzi F, Manfredi P, Mally M, Moes S, Jenö P, Cornelis GR. The N-glycan glycoprotein deglycosylation complex (Gpd) from Capnocytophaga canimorsus deglycosylates human IgG. PLoS
Pathog. 2011;7:e1002118. http://dx.doi.org/10.1371/journal. ppat.1002118

14. Suzuki M, Kimura M, Imaoka K, Yamada A. Prevalence of Capnocytophaga canimorsus and Capnocytophaga cynodegmi in dogs and cats determined by using a newly established speciesspecific PCR. Vet Microbiol. 2010;144:172-6. http://dx.doi.org/ 10.1016/j.vetmic.2010.01.001

Address for correspondence: Guy R. Cornelis, Unité de Recherche en Biologie des Microorganismes, Université de Namur, 61 rue de Bruxelles, 5000 Namur, Belgium; email: guy.cornelis@unamur.be

\section{etymologia}

\section{Capnocytophaga canimorsus [kap"no-si-tof'ə-gə kan"ə-mor'səs]}

\section{Ronnie Henry}

- rom the Greek kapnos ("smoke") for its dependence on carbon dioxide, which is a large I component of smoke, Capnocytophaga canimorsus (Latin canis, “dog,” and morsus, “bite”) are gram-negative, facultatively anaerobic, rod-shaped bacteria that are part of the normal oral microbiota of dogs and cats. The genus was proposed to distinguish these bacteria from Cytophaga spp. (Greek kytos, “cell,” and phagein, “eat”), which also exhibit gliding motility. C. canimorsus was previously known as CDC group DF-2 (dysgonic fermenter type 2) and was first isolated from a man who had experienced multiple dog bites and developed septicemia and meningitis. C. canimorsus remains a major cause of septicemia in persons, particularly those who are asplenic or immunocompromised, who are bitten by dogs or cats.

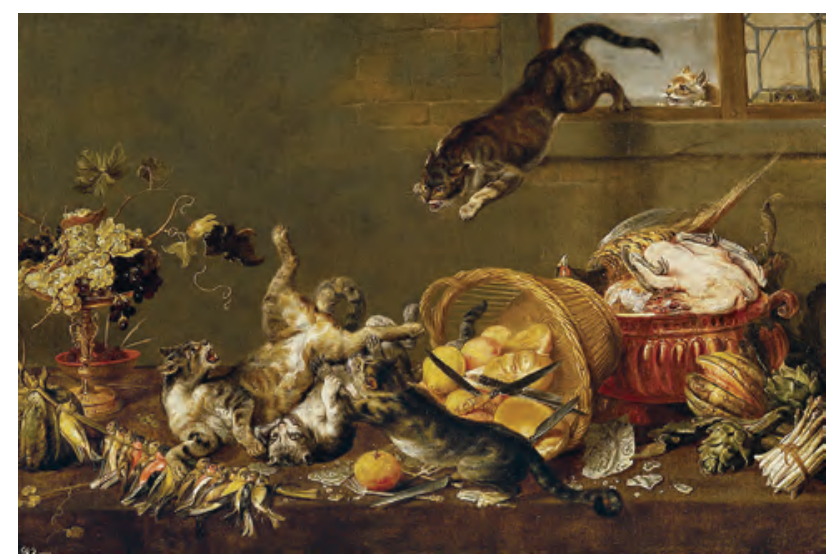

Paul de Vos, Cats Fighting in a Larder 1630-1640. Oil on canvas. Museo Nacional del Prado. https://www. museodelprado.es/ coleccion/galeria-online/galeria-on-line/ obra/pelea-de-gatosen-una-despensa/, Public Domain, https://commons. wikimedia.org/w/index. php? curid=39117357

\section{Sources}

1. Brenner DJ, Hollis DG, Fanning GR, Weaver RE. Capnocytophaga canimorsus sp. nov. (formerly CDC group DF-2), a cause of septicemia following dog bite, and C. cynodegmi sp. nov., a cause of localized wound infection following dog bite. J Clin Microbiol. 1989;27:231-5.

2. Leadbetter ER, Holt SC, Socransky SS. Capnocytophaga: new genus of gram-negative gliding bacteria. I. General characteristics, taxonomic considerations and significance. Arch Microbiol. 1979;122:9-16. http://dx.doi.org/10.1007/BF00408040

Address for correspondence: Ronnie Henry, Centers for Disease Control and Prevention, 1600 Clifton Rd NE, Mailstop E28, Atlanta, GA 30329-4027, USA; email: boq3@cdc.gov

DOI: https://doi.org/10.3201/eid2412.ET2412 


\title{
Crimean-Congo Hemorrhagic Fever Virus, Mongolia, 2013-2014
}

\author{
Matthew A. Voorhees, Susana L. Padilla, Dulamjav Jamsransuren, Jeffrey W. Koehler, \\ Korey L. Delp, Dolgorkhand Adiyadorj, Uyanga Baasandagwa, Battsetseg Jigjav, \\ Scott P. Olschner, Timothy D. Minogue, Randal J. Schoepp
}

During 2013-2014, we collected 1,926 serum samples from humans and 4,583 ticks (Hyalomma asiaticum or Dermacentor nuttalli) in select regions of Mongolia to determine the risk for Crimean-Congo hemorrhagic fever virus (CCHFV) infection among humans in this country. Testing of human serum samples by ELISA demonstrated an overall CCHFV antibody prevalence of 1.4\%; Bayankhongor Province had the highest prevalence, $2.63 \%$. We pooled and analyzed tick specimens by real-time reverse transcription PCR; 1 CCHFV-positive $H$. asiaticum tick pool from Ömnögovi was identified. In phylogenetic analyses, the virus's partial small segment clustered with CCHFV isolates from Central Asia, and the complete medium segment grouped with CCHFV isolates from Africa, Asia, and the Middle East. This study confirms CCHFV endemicity in Mongolia and provides information on risk for CCHFV infection. Further research is needed to better define the risk for CCHFV disease to improve risk mitigation, diagnostics, and surveillance.

Crimean-Congo hemorrhagic fever (CCHF) is a tick$U$ borne disease of humans that is prevalent over a wide geographic area, spanning from western China to southern Asia, from the Middle East to southeastern Europe, and over most of Africa (1-3). The causative agent is Crimean-Congo hemorrhagic fever virus (CCHFV), a member of the Bunyaviridae family and Orthonairovirus genus. The enveloped virus has a negative-sense RNA genome composed of 3 ambisense segments: small (S), medium (M), and large (L). The $\mathrm{S}$ segment encodes the nucleocapsid protein and the $\mathrm{L}$ segment the viral polymerase. The M segment encodes a glycoprotein precursor that is processed into 2 glycoproteins by the host cell. Genetic drift and segment reassortment of the CCHFV genome results in a high degree of phylogeographic diversity, with 5-7 designated genotypes $(2,4)$.

Author affiliations: United States Army Medical Research Institute of Infectious Diseases, Fort Detrick, Maryland, USA (M.A. Voorhees,

S.L. Padilla, J.W. Koehler, K.L. Delp, S.P. Olschner, T.D. Minogue, R.J. Schoepp); Ministry of Health National Center for Zoonotic Diseases, Ulaanbaatar, Mongolia (D. Jamsransuren, D. Adiyadorj, U. Baasandagwa, B. Jigjav)

DOI: https://doi.org/10.3201/eid2412.180175
CCHFV is maintained in the environment in a cycle between tick vectors, primarily Hyalomma ticks, and wild and domesticated animals. Birds generally appear to be refractory to CCHFV infection, although some avian species demonstrate infectivity (5). Widespread dispersion is most likely mediated by infected ticks carried by migrating birds or moving livestock $(1,6-8)$. Viremia in mammals is transient, but ticks remain infected throughout their lives (9). The human species is considered a dead-end host because virus transmission from infected humans to uninfected feeding ticks has not been reported. Humans can become infected when fed on by infected ticks, exposed to infectious bodily fluids during animal slaughter, or caring for infected patients $(1,10)$. Infection can result in an unapparent or mild disease of fever, chills, nausea, vomiting, headache, and body pains. In severe cases, hemorrhagic disease is characterized by petechial rashes of the skin, bleeding from mucosal membranes and muscles, organ failure, and cerebral hemorrhage. Mortality rates have been estimated to be $5 \%-30 \%$ but have reached as high as $80 \%$ in some outbreaks (10). One report describes CCHF survivors as having lasting effects, including prolonged vision and memory problems (11).

CCHFV prevalence in Mongolia is generally unknown. No published studies provide evidence of human infection or of virus circulating in tick vectors. A previous study in the Mongolia aimag (i.e., province) of Arkhangai demonstrated serologic evidence of virus infection in $1.8 \%$ of wildlife species tested (12), and a large study involving $>2,000$ samples collected from sheep demonstrated a CCHFV seroprevalence of $27.1 \%$ among sheep from multiple aimags across Mongolia (13). Furthermore, numerous CCHFV strains have been identified in Xinjiang Province, a region of China bordering Mongolia (14-16). Serologic evidence of CCHFV infections in wildlife and livestock suggests the virus circulates in Mongolia and that CCHFV infections are possible for persons exposed to biting ticks or infectious tissues or fluids. In this study, we sought to better characterize the risk for CCHFV infection among the population of Mongolia by determining antibody prevalence in humans associated with livestock 
and by surveying ticks collected from the environment and livestock for evidence of CCHFV infection.

\section{Materials and Methods}

\section{Human Samples}

During 2013-2014, we collected serum samples $(n=1,926)$ from persons in regions corresponding to the estimated geographic distribution of Hyalomma asiaticum ticks (17); we collected most samples in 2013. We collected samples from Dornod Aimag in eastern Mongolia; Dornogovi Aimag, Dundgovi Aimag, Ömnögovi Aimag, Bayankhongor Aimag, and Govi-Altai Aimag in southern Mongolia (the Gobi Desert landscapes); and Khovd Aimag in western Mongolia (Table 1; Figure 1). We collected blood from nomadic herders and local residents of both sexes from the selected regions by using standard blood sampling techniques. We centrifuged and stored serum samples at $-20^{\circ} \mathrm{C}$ until transporting them to the National Center for Zoonotic Diseases (Ulaanbaatar, Mongolia), where samples were stored at $-70^{\circ} \mathrm{C}$. We collected all human samples in accordance with Mongolia Ministry of Health-approved protocols and tested all human samples at the US Army Medical Research Institute of Infectious Diseases (USAMRIID) under approved human use protocol FY11-14.

\section{IgG ELISA}

We detected human CCHFV IgG by sandwich ELISA. We coated Immulon 2 HB plates (ThermoFisher Scientific, Waltham, MA, USA) with anti-CCHFV hyperimmune mouse ascitic fluid $(1: 1,000)$ in phosphate-buffered saline and $0.01 \%$ thimerosal. After incubating plates overnight at $4{ }^{\circ} \mathrm{C}$ and washing 3 times with wash buffer (phosphatebuffered saline with $0.1 \%$ tween 20 and $0.01 \%$ thimerosal), we added $100 \mu \mathrm{L}$ of inactivated CCHFV IbAr10200 supernatant (IgG capture antigen) or noninfected Vero E6 cell supernatant (negative control) diluted (1:20) in milk buffer (wash buffer plus 5\% skim milk) to each well. We incubated plates for $60 \mathrm{~min}$ at $37^{\circ} \mathrm{C}$, washed 3 times with wash buffer, and added serum samples diluted 1:100 in milk buffer to test wells and negative control wells. We incubated plates for $60 \mathrm{~min}$ at $37^{\circ} \mathrm{C}$, washed 3 times with wash buffer, and added $100 \mu \mathrm{L}$ of the detector antibody (horseradish peroxidase-labeled anti-human IgG $\mathrm{F}_{c}$; Accurate Chemical and Scientific, Westbury, NY, USA) diluted $1: 8,000$ in milk buffer to all wells. We incubated plates for $60 \mathrm{~min}$ at $37^{\circ} \mathrm{C}$, washed 3 times with wash buffer, and developed using $100 \mu \mathrm{L}$ of substrate 2, 2'-azino-di-(3-ethylbenzothiazoline-6-sulfonate) with a 30 -min incubation at $37^{\circ} \mathrm{C}$. We read plates using a TECAN Infinite M200 Pro (Tecan Group Ltd., Männedorf, Switzerland) plate reader at $405 \mathrm{~nm}$ and determined the optical density (OD) for each well. We calculated the adjusted OD for each sample by
Table 1. Prevalence of CCHFV IgG among healthy persons as determined by ELISA, by province and district, Mongolia, 2013-2014*

\begin{tabular}{|c|c|c|c|}
\hline \multirow[b]{2}{*}{ Province, district } & \multicolumn{3}{|c|}{ No. positive/tested (\%) } \\
\hline & 2013 & 2014 & Total \\
\hline Khovd & $0 / 229(0)$ & NA & $0 / 229(0)$ \\
\hline Üyench & $0 / 79(0)$ & & 0/79 (0) \\
\hline Bulgan & 0/72 (0) & & $0 / 72(0)$ \\
\hline Altai & $0 / 78(0)$ & & $0 / 78(0)$ \\
\hline Govi-Altai & $3 / 314(0.96)$ & $1 / 21(4.76)$ & 4/335 (1.19) \\
\hline Bugat & 2/84 (2.38) & & 2/84 (2.38) \\
\hline Altai & 1/84 (1.19) & & 1/84 (1.19) \\
\hline Tsogt & $0 / 74(0)$ & & $0 / 74(0)$ \\
\hline Erdene & $0 / 72(0)$ & & $0 / 72(0)$ \\
\hline Taishir & & 0/5 (0) & 0/5 (0) \\
\hline Yesönbulag & & $1 / 16(6.25)$ & 1/16 (6.25) \\
\hline Bayankhongor & 4/146 (2.74) & $1 / 44(2.27)$ & $5 / 190(2.63)$ \\
\hline Bayan-Öndör & 2/74 (2.70) & 0/1 (0) & 2/75 (2.67) \\
\hline Shinejinst & 2/72 (2.78) & & 2/72 (2.78) \\
\hline Jinst & & $1 / 43(2.33)$ & $1 / 43(2.33)$ \\
\hline Ömnögovi & $7 / 525$ (1.33) & $1 / 40(2.50)$ & $8 / 565$ (1.42) \\
\hline Gurvan-tes & $3 / 79$ (3.80) & 0/10 (0) & $3 / 89$ (3.37) \\
\hline Noyon & $1 / 71(1.41)$ & $1 / 10(10.00)$ & $2 / 81(2.47)$ \\
\hline Bayandalai & 0/74 (0) & 0/10 (0) & 0/84 (0) \\
\hline Khürmen & 1/75 (1.33) & $0 / 10(0)$ & 1/85 (1.18) \\
\hline Nomgon & $1 / 75(1.33)$ & & 1/75 (1.33) \\
\hline Bayan-Ovoo & $1 / 75(1.33)$ & & $1 / 75(1.33)$ \\
\hline Khanbogd & 0/76 (0) & & 0/76 (0) \\
\hline Dornogovi & $6 / 410(1.46)$ & $4 / 72(5.56)$ & 10/482 (2.07) \\
\hline Khatanbulag & 2/93 (2.15) & 2/17 (11.76) & $4 / 110(3.63)$ \\
\hline Khövsgöl & 3/91 (3.30) & $1 / 14(7.14)$ & 4/105 (3.81) \\
\hline Ulaanbadrakh & $0 / 60(0)$ & $1 / 16(6.25)$ & $1 / 76(1.31)$ \\
\hline Erdene & 0/80 (0) & $0 / 14(0)$ & $0 / 94(0)$ \\
\hline Zamyn-Üüd & $1 / 86(1.16)$ & $0 / 11(0)$ & 1/97 (1.03) \\
\hline Dundgovi & NA & $0 / 76(0)$ & $0 / 76(0)$ \\
\hline Khuld & & $0 / 33(0)$ & $0 / 33(0)$ \\
\hline Luus & & $0 / 39(0)$ & 0/39 (0) \\
\hline Saintsagaan & & $0 / 4(0)$ & $0 / 4(0)$ \\
\hline Dornod & NA & $0 / 49(0)$ & $0 / 49(0)$ \\
\hline Bayan-Uul & & $0 / 14(0)$ & $0 / 14(0)$ \\
\hline Bayandun & & $0 / 15(0)$ & $0 / 15(0)$ \\
\hline Dashbalbar & & $0 / 20(0)$ & $0 / 20(0)$ \\
\hline Total & 20/1,624 (1.23) & $7 / 302(2.32)$ & $27 / 1,926(1.40)$ \\
\hline
\end{tabular}

subtracting the average OD value of the Vero cell supernatant control wells from the average OD value of the IgG capture antigen wells. The positive-negative cutoff of each assay was the mean OD value plus 3 SDs of the 4 negative control samples (typically 0.2).

\section{Plaque Reduction Neutralization Test}

For the plaque reduction neutralization test (PRNT), we diluted all test samples 1:10 in minimum essential media (MEM) with 5\% heat-inactivated Hyclone Fetal Bovine Serum (FBS) (GE Healthcare Life Sciences, Pittsburgh, PA, USA) and then placed samples in a $56^{\circ} \mathrm{C}$ water bath for $30 \mathrm{~min}$. For test and positive control serum samples, we 2-fold serially diluted to 1:320 in MEM with 5\% FBS. For negative control serum samples, we 2-fold serially diluted to $1: 40$ in the same buffer. We diluted CCHFV IbAr10200 in MEM with 5\% FBS to a concentration of 1,000 plaqueforming units/mL. 


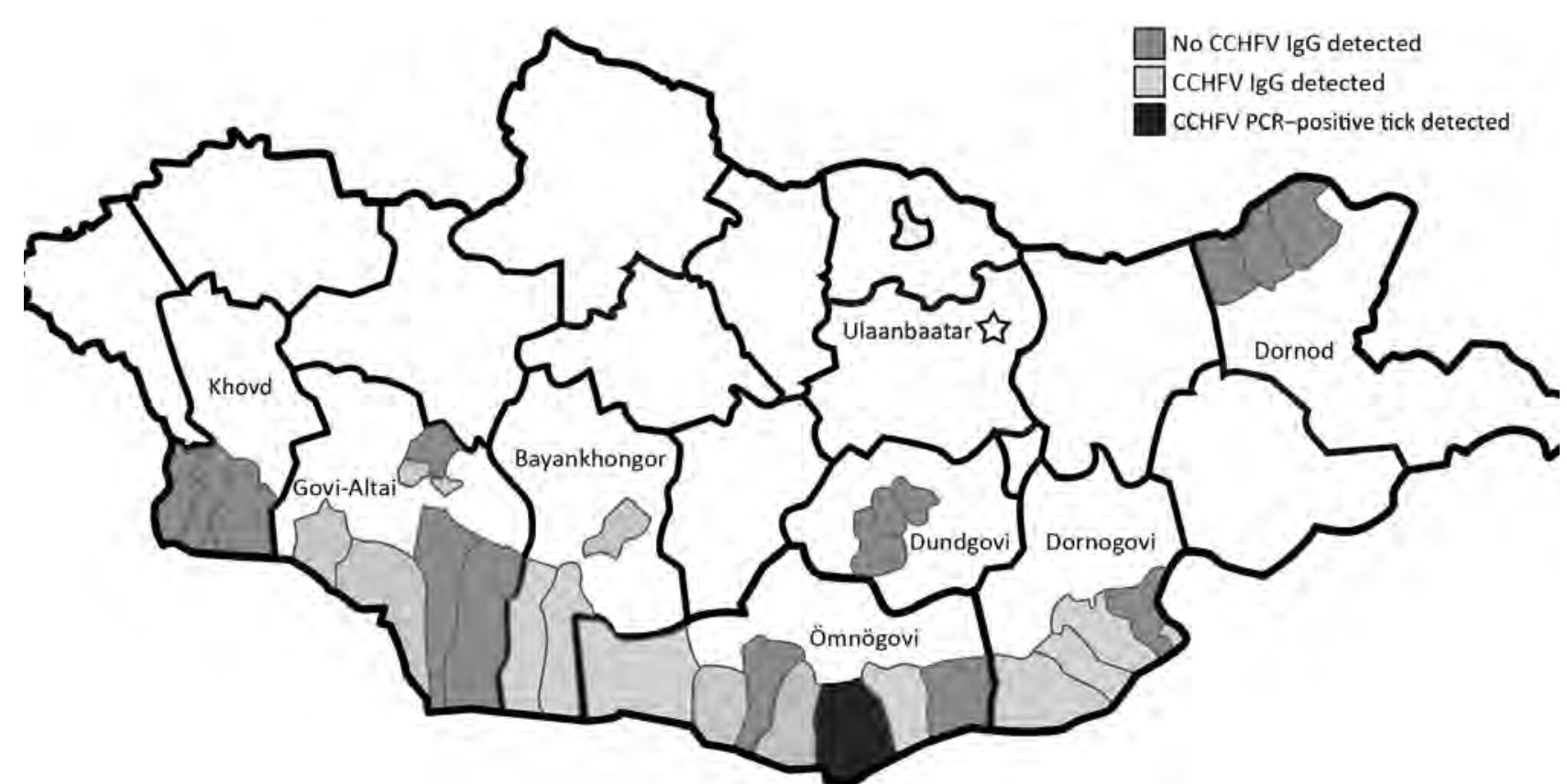

Figure 1. Geographic distribution of CCHFV-positive serum samples and tick and CCHFV-negative serum samples, Mongolia, 20132014. CCHFV, Crimean-Congo hemorrhagic fever virus.

We mixed positive control, negative control, and test serum samples with virus 1:1 in microtiter tubes, covered tubes with parafilm, and then placed them at $37^{\circ} \mathrm{C}$ for 1 h. We incubated $200 \mu \mathrm{L}$ of serum-virus mixture in duplicate wells of 6-well plates with SW-13 cells grown to a minimum of $85 \%$ confluence. We incubated plates at $37^{\circ} \mathrm{C}$ with $5 \% \mathrm{CO}_{2}$ for $1 \mathrm{~h}$ with gentle rocking every $15 \mathrm{~min}$. After incubation, we added $2 \mathrm{~mL}$ of $0.5 \%$ agar overlay $(2 \times$ basal medium Eagle with Earle salts with 10\% FBS, 1\% penicillin-streptomycin, $1 \%$ L-glutamine, and $1 \%$ nonessential amino acids) to each well and incubated at $37^{\circ} \mathrm{C}$ for $\approx 36 \mathrm{~h}$. Then, we added a second $0.5 \%$ agar overlay containing $5 \%$ neutral red to each well, incubated at $37^{\circ} \mathrm{C}$ for $4-5$ $\mathrm{h}$, and counted plaques. We reported the reciprocal of the highest serum dilutions reducing 50\% $\left(\mathrm{PRNT}_{50}\right)$ and $80 \%$ $\left(\mathrm{PRNT}_{80}\right)$ of the plaque assay dose as the titer and calculated the probit titer.

\section{Tick Collections and Processing}

We collected ticks from livestock and questing ticks from the environment in the same regions of Mongolia where we collected human serum samples. During the spring and early summer, we collected adult, unfed ticks by the drag cloth method (dragging a cotton cloth on a dowel) and by removing ticks from livestock with forceps. We placed each collection in a centrifuge tube with a moist cotton. In the field, we stored tick collections in portable thermal coolers at $4^{\circ} \mathrm{C}$. We sent ticks to the National Center for Zoonotic Diseases, where samples were stored at $-20^{\circ} \mathrm{C}$ until species identification was completed. Afterward, we transferred ticks to $-70^{\circ} \mathrm{C}$ storage until shipment to USAMRIID for processing. We collected 4,583 ticks for processing, of which 1,772 were $H$. asiaticum and 2,811 were Dermacentor nuttalli.

We sorted field-collected unfed specimens by sex and species and placed ticks in pairs into single polycarbonate vials containing prepared media and 3/8-inch steel balls. We homogenized ticks using the 1600 MiniG Automated Tissue Homogenizer and Cell Lyser (SPEX SamplePrep, Metuchen, NJ, USA), combined portions of homogenized subpools into larger pools (maximum 8 ticks/pool), and treated them with TRIzol LS (ThermoFisher Scientific) according to the manufacturer's instructions. We purified total nucleic acid using the KingFisher Flex Purification System (ThermoFisher Scientific) and the MagMax 96 for MicroArrays Total RNA Isolation Kit (ThermoFisher Scientific) according to the manufacturer's recommendations. We stored all tick pools and processed nucleic acids at $-70^{\circ} \mathrm{C}$ until needed for testing.

\section{Real-Time Reverse Transcription PCR}

We tested total nucleic acid extracted from tick pools for the CCHFV S segment using a real-time reverse transcription PCR (RT-PCR) assay optimized for CCHFV sequence diversity $(18,19)$. We performed this assay in triplicate in wells of 384-well plates using the SuperScript III One-Step RT-PCR Kit (Invitrogen, Carlsbad, CA, USA) with $2.5 \mu \mathrm{L}$ of sample in $10-\mu \mathrm{L}$ reactions run on the LightCycler 480 (Roche Applied Science, Penzberg, Germany). Cycling conditions were $50^{\circ} \mathrm{C}$ for $15 \mathrm{~min}$; $95^{\circ} \mathrm{C}$ for $5 \mathrm{~min}$; and 45 
cycles of $94^{\circ} \mathrm{C}$ for $1 \mathrm{~s}, 55^{\circ} \mathrm{C}$ for $20 \mathrm{~s}$, and $68^{\circ} \mathrm{C}$ for $5 \mathrm{~s}$. Fluorescence was measured after each extension step. We considered a sample negative if the quantification cycle was $>40$ cycles.

\section{Nested RT-PCR}

To confirm real-time RT-PCR results, we performed nested RT-PCR targeting the CCHFV S segment as previously described (20). In brief, we used $15 \mu \mathrm{L}$ of extracted tick homogenate and SuperScript III One-Step RT-PCR System with Platinum Taq High Fidelity DNA Polymerase (Invitrogen) in a $50-\mu \mathrm{L}$ reaction with outer primers CCHF-F2 (5'-TGGACACCTTCACAAACTC-3'), CCHF-F2C (5'-TGGATACTTTCACAAACTC-3'), and CCHF-R3 (5'-GACAAATTCCCTGCACCA-3'). Cycling conditions were $42^{\circ} \mathrm{C}$ for $30 \mathrm{~min} ; 94^{\circ} \mathrm{C}$ for $5 \mathrm{~min} ; 5$ cycles of $94^{\circ} \mathrm{C}$ for $30 \mathrm{~s}$, $37^{\circ} \mathrm{C}$ for $30 \mathrm{~s}$, and $72^{\circ} \mathrm{C}$ for $30 \mathrm{~s} ; 72^{\circ} \mathrm{C}$ for $2 \mathrm{~min} ; 30$ cycles of $94^{\circ} \mathrm{C}$ for $30 \mathrm{~s}, 52^{\circ} \mathrm{C}$ for $30 \mathrm{~s}, 72^{\circ} \mathrm{C}$ for $30 \mathrm{~s}$; and a final cycle of $72^{\circ} \mathrm{C}$ for $5 \mathrm{~min}$. We assessed for the presence of amplicons (536 bp) by performing electrophoresis on $2 \%$ agarose gels.

We performed nested PCR with $1 \mu \mathrm{L}$ of amplicon as template in a $50-\mu \mathrm{L}$ reaction with inner primers CCHFF3 (5'-GAATGTGCATGGGTTAGCTC-3'), CCHF-F3C (5'-GAGTGTGCCTGGGTTAGCTC-3'), CCHF-R2a (5'-GACATCACAATTTCACCAGG-3'), and CCHF-R2b (5'-GACATTACAATTTCGCCAGG-3'). Cycling conditions were $94^{\circ} \mathrm{C}$ for $5 \mathrm{~min} ; 30$ cycles of $94^{\circ} \mathrm{C}$ for $30 \mathrm{~s}, 52^{\circ} \mathrm{C}$ for $30 \mathrm{~s}, 72^{\circ} \mathrm{C}$ for $30 \mathrm{~s}$; and $72^{\circ} \mathrm{C}$ for $5 \mathrm{~min}$. We assessed for the presence of amplicons (260 bp) by performing electrophoresis on $2 \%$ agarose gels.

\section{CCHFV Sequencing and Analysis}

We performed a second RT-PCR with S segment outer primers with the only tick pool that was positive by realtime and nested RT-PCR (159A). We then used $5 \mu \mathrm{L}$ of the reaction for Sanger sequencing. We ran the amplicon (536 bp, near $5^{\prime}$ end of S segment) through a $2 \%$ agarose gel, cut the band out, and purified the amplicon using the QIAquick Gel Extraction Kit (QIAGEN, Desseldorf, Germany). We created sequencing libraries using the BigDye Terminator v3.1 Sequencing Kit (Applied Biosystems, Foster City, CA, USA) with $10 \mu \mathrm{L}$ of the purified amplicon in a $20-\mu \mathrm{L}$ reaction. Cycling conditions were $96^{\circ} \mathrm{C}$ for $1 \mathrm{~min}$ and 25 cycles of $96^{\circ} \mathrm{C}$ for $10 \mathrm{~s}, 50^{\circ} \mathrm{C}$ for $5 \mathrm{~s}$, and $60^{\circ} \mathrm{C}$ for $4 \mathrm{~min}$. We sequenced libraries using the 3500xL Genetic Analyzer (Applied Biosystems).

We attempted to amplify CCHFV segments L, M, and $\mathrm{S}$ from tick pool 159A using segment-specific primers modified for Nextera-based sequencing (Table 2) $(21,22)$; however, only the M segment amplified. In brief, we used $5 \mu \mathrm{L}$ of extracted nucleic acid from tick homogenate to reverse transcribe the genome and amplify the cDNA of the M segment using the SuperScript III One-Step RT-PCR System with Platinum Taq High Fidelity DNA Polymerase (Invitrogen). Cycling conditions were $52.5^{\circ} \mathrm{C}$ for $30 \mathrm{~min}$; $94^{\circ} \mathrm{C}$ for $2 \mathrm{~min} ; 40$ cycles of $94^{\circ} \mathrm{C}$ for $15 \mathrm{~s}, 50.5^{\circ} \mathrm{C}$ for $30 \mathrm{~s}$, and $68^{\circ} \mathrm{C}$ for $1 \mathrm{~min} / \mathrm{kb}$; and $68^{\circ} \mathrm{C}$ for $5 \mathrm{~min}$. We ran the amplified $\mathrm{M}$ segment through an agarose gel and purified the band using the QIAquick Gel Extraction Kit (QIAGEN).

We generated next-generation sequencing libraries using the Nextera XT DNA Library Preparation Kit (Illumina, San Diego, CA, USA) according to the manufacturer's instructions and sequenced using the 500-cycle MiSeq Reagent Kit v2 (Illumina). We analyzed sequencing reads with the CLC Genomics Workbench (QIAGEN), filtered and trimmed them for quality, and assembled the complete $\mathrm{M}$ segment de novo. We generated the final consensus sequence by remapping the trimmed reads to the de novo consensus sequence as previously described (22).

We identified the full-length $M$ segment sequence and partial S segment sequence from tick pool 159A by BLAST analysis (https://blast.ncbi.nlm.nih.gov/Blast.cgi). We aligned the segments from tick pool 159A with the available full-length $\mathrm{M}$ and near-full-length $\mathrm{S}$ segments in GenBank and generated a neighbor-joining phylogenetic tree (Jukes-Cantor model) with 1,000 bootstrap replicates.

\section{Virus Isolation}

To propagate virus in the RT-PCR-positive tick pool, we thawed $60 \mu \mathrm{L}$ of tick homogenates and mixed with $6 \mathrm{~mL}$ of freshly prepared MEM with 10\% FBS, 1\% nonessential amino acids, $1 \% \mathrm{~L}$-glutamine, $1 \%$ penicillin-streptomycin, and $0.4 \%$ fungizone. We inoculated confluent monolayers of SW-13 cells in 2 T75 flasks with $2.5 \mathrm{~mL}$ of the virus

\begin{tabular}{|c|c|}
\hline Primer name & Sequence, $5^{\prime} \rightarrow 3^{\prime} \dagger$ \\
\hline CCHFV-M F-next & TCGTCGGCAGCGTCAGATGTGTATAAGAGACAGtctcaaagaaatacttgc \\
\hline CCHFV-M R-next & GTCTCGTGGGCTCGGAGATGTGTATAAGAGACAGtctcaaagatatagtggc \\
\hline CCHFV-S F-next & TCGTCGGCAGCGTCAGATGTGTATAAGAGACAGtctcaagaaacacgtgccgc \\
\hline CCHFV-S R-next & GTCTCGTGGGCTCGGAGATGTGTATAAGAGACAGtctcaaagatatcgttgccgC \\
\hline CCHF-L 1F-next & TCGTCGGCAGCGTCAGATGTGTATAAGAGACAGtctcaaagatatcaatcccccC \\
\hline CCHF-L 1R-next & GTCTCGTGGGCTCGGAGATGTGTATAAGAGACAGttggcactatctttcatttga \\
\hline CCHF-L 2F-next & TCGTCGGCAGCGTCAGATGTGTATAAGAGACAGgaagagctatatgacataaggc \\
\hline CCHF-L 2R-next & GTCTCGTGGGCTCGGAGATGTGTATAAGAGACAGtctcaaagaaatcgttccccccac \\
\hline
\end{tabular}


mixture and incubated at $37^{\circ} \mathrm{C}$ for $1 \mathrm{~h}$; then, we added an additional $30 \mathrm{~mL}$ of MEM. We incubated flasks at $37^{\circ} \mathrm{C}$ with $5 \% \mathrm{CO}_{2}$ for 1 wk or until cytopathic effects (CPE) were evident. To test for unapparent infections (i.e., infections not resulting in $\mathrm{CPE}$ ), each inoculated culture was tested by real-time RT-PCR as previously described.

\section{Results}

\section{CCHFV Serologic Testing in Humans}

To assess the overall risk for CCHFV infection in Mongolia, we tested 1,926 serum samples collected from nomadic herders and local residents in select regions of the country by ELISA. In total, 27 samples tested positive for $\mathrm{CCH}$ FV IgG; the overall prevalence rate was $1.4 \%$ (Table 1 ). Bayankhongor Aimag had the highest prevalence (2.63\%); other aimags with CCHFV IgG-positive populations had prevalences of $2.07 \%$ (Dornogovi), 1.42\% (Ömnögovi), and $1.19 \%$ (Govi-Altai). The remaining 3 aimags tested (Khovd, Dundgovi, and Dornod) showed no evidence of CCHFV IgG.

We evaluated 12 positive and 7 randomly selected negative samples for their ability to neutralize CCHFV strain IbAr10200 (Table 3). In total, 8 (67\%) of 12 ELISA-positive samples and 2 (29\%) of 7 ELISA-negative samples neutralized CCHFV with PRNT $_{50}$ probit titers; 5 samples with PRNT ${ }_{50}$ probit titers (3 ELISA-positive and 2 ELISA-negative) had $\mathrm{PRNT}_{80}$ probit titers. Neutralization ability did not correlate with ELISA OD values.

\section{Molecular Testing for CCHFV in Ticks}

We collected questing ticks and ticks from livestock and identified and pooled them on the basis of tick species, sex, and geographic location; then, we screened tick pools by real-time RT-PCR using a broad CCHFV assay (19). Of the 893 tick pools tested, 1 pool (159A) tested positive by real-time RT-PCR and was confirmed CCHFV positive by nested RT-PCR; results for 6 other pools were indeterminate. Tick pool 159A, which contained $7 \mathrm{H}$. asiaticum ticks, was collected in the soum (i.e., district) Nomgon in Ömnögovi Aimag.

\section{Characterization of CCHFV-Positive Pool 159A}

To genetically characterize the CCHFV detected in tick pool 159A, we amplified and sequenced the full-length M segment and partial S segment. BLAST analysis of the nucleocapsid coding region located within the partial S segment identified K128_76 (GenBank no. KX013455.1) from Kazakhstan as the CCHFV isolate having the greatest percent identity at $98 \%$, and IbAr10200, the prototypical CCHFV isolate, had a percent identity of $87 \%$. BLAST analysis of the M segment identified SPU 97/85 (GenBank accession no. DQ211633.1) from South Africa as the
CCHFV isolate having the highest percent identity at 91\%, with IbAr10200 having a percent identity of $81 \%$. Phylogenetic analysis indicated that the partial S segment sequence clusters within the Asia 2 lineage (Figure 2), which contains virus strains from China and Russia (23). CCHFV M segments in general do not cluster well by geographic location on phylogenetic analyses; the $\mathrm{M}$ segment sequence tick 159A/Mongolia clustered with CCHFV strains from Asia, Africa, and the Middle East (Figure 3).

\section{Virus Isolation}

Attempts to isolate CCHFV from putative PCR-positive tick pools in SW-13 culture cells were made from multiple tick homogenate subpools $(\mathrm{N}=18)$, containing 2 ticks per subpool. None of the inoculated cultures showed evidence of virus growth by visible CPE or genomic signature by real-time RT-PCR in either the first or second passages.

\section{Discussion}

To better determine the risk for CCHFV infection among humans in Mongolia, we conducted an assessment that included a CCHFV IgG serosurvey and screening for CCHFV RNA in ticks collected in parallel with the serosurvey. Overall, this study provides evidence that CCHFV is circulating in $H$. asiaticum ticks and humans in Mongolia, provides sequence data on a virus circulating in the region, and extends the geographic distribution of CCHFV north to Mongolia.

Previous studies conducted in Mongolia revealed serologic evidence of CCHFV in wildlife species and livestock. Chumikhin et al. found serologic evidence for $\mathrm{CCH}-$ FV infection in small mammals, such as the Tolai hare, Mongolian pika, and long-tailed ground squirrel $(12,13)$. Livestock, such as cattle, sheep, goats, and camels, are amplifying hosts of the virus, and CCHFV infections in livestock correlate with the risk for infection of humans (24). Morikawa investigated CCHFV IgG seroprevalence in sheep in the southern aimags of Mongolia and found prevalences of 7\%-28\% (13). Evidence of CCHFV in wildlife and livestock in Mongolia suggests the possibility of virus circulation and human disease, but the actual occurrence was unclear.

We found an overall CCHFV IgG seroprevalence of $1.4 \%$ in Mongolia; Bayankhongor Aimag had the highest rate, $2.63 \%$. Each soum tested within Bayankhongor had IgG prevalences $>2 \%$, suggesting a persistent source of CCHFV infection is likely present in these soums. Bayankhongor is located in the southwestern part of the country and has an economy based on livestock, meat, and wool production. The greater interaction with animals among persons of this aimag could partially explain the higher antibody prevalence in the population. Dornogovi Aimag had the next highest antibody prevalence (2.07\%). Dornogovi 


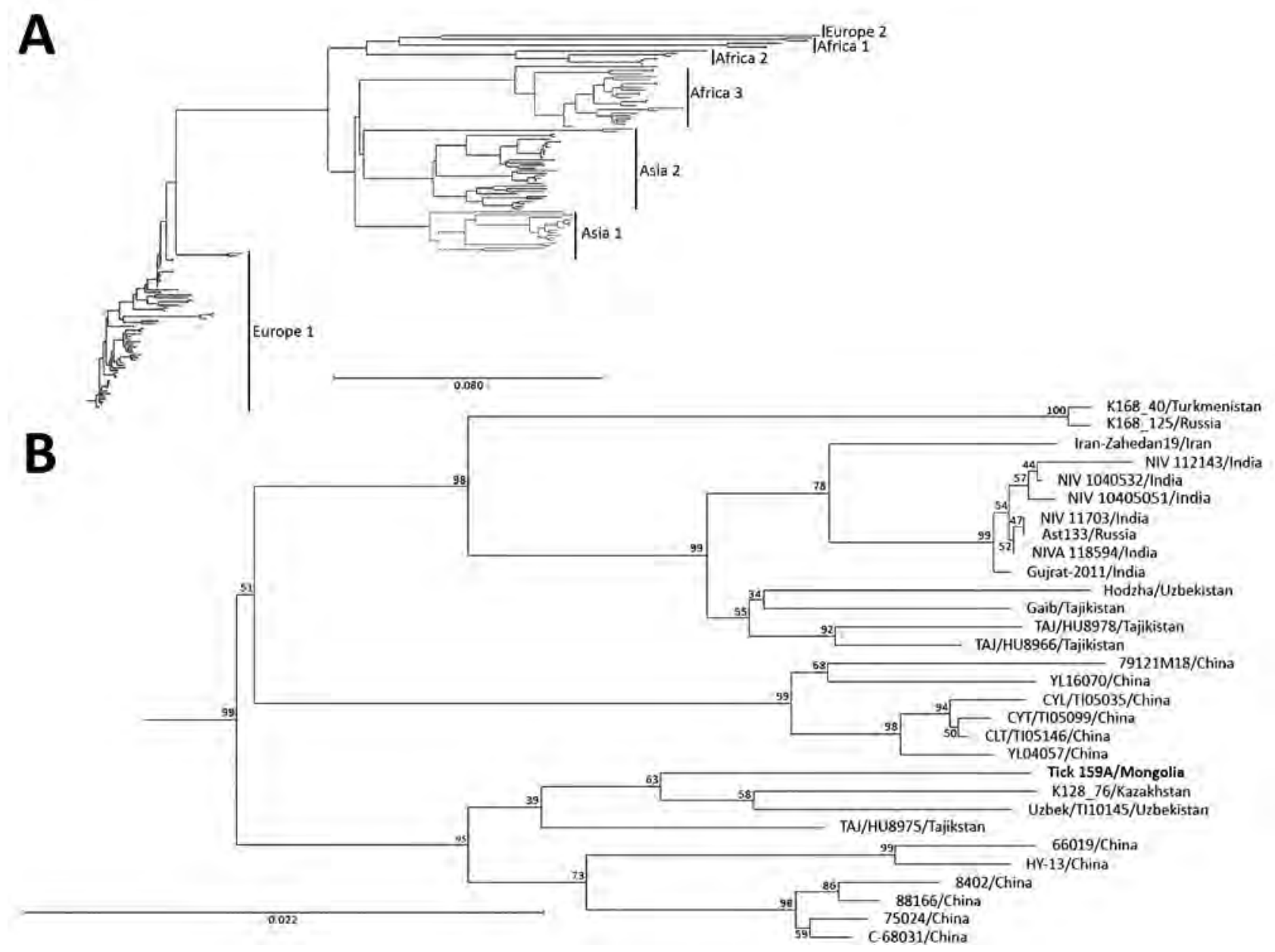

Figure 2. Phylogenetic characterization of partial small (S) segment sequence of Crimean-Congo hemorrhagic fever virus (CCHFV) isolate from tick pool 159A, Mongolia, 2013-2014. Near full-length CCHFV S segments from GenBank were aligned with the S segment sequence from tick pool 159A and a phylogenetic tree was generated. A) Genetic clusters are displayed as previously described (23). B) Detailed view of phylogenetic tree of Asia 2 lineage. S segment of the CCHFV isolate from this study (tick 159A/Mongolia; bold) clusters in the Asia 2 lineage. Scale bars indicate nucleotide substitutions per site.

is in the southeastern part of Mongolia and lies in the eastern part of the Gobi Desert. Raising livestock previously was the main economic driver of this aimag, but this activity has been replaced by mineral exploration. Soums Khatanbulag and Khövsgöl within Dornogovi had some of the highest CCHFV antibody prevalences of those sampled.

Ömnögovi Aimag had an antibody prevalence of $1.42 \%$, despite having the greatest number of samples tested and being the only aimag having a soum with a $\mathrm{CCH}-$ FV-positive tick pool sample. Ömnögovi, the largest aimag in Mongolia, is located in the southern part of the country and southern part of the Gobi Desert. Ömnögovi is rich in mineral deposits and depends heavily on mining, and agriculture is of minor importance. The lowest antibody prevalence, $1.19 \%$, occurred in the Govi-Altai Aimag, where the Gobi Desert, steppe grasslands, and mountains of western Mongolia converge. No CCHFV IgG was found in samples tested from the other 3 aimags, Khovd, Dundgovi, and Dornod. Dornod Aimag is outside the Hyalomma tick range, and thus, we did not expect to find evidence of $\mathrm{CCH}$ FV infection in this area.

Antibody neutralization tests were used to confirm the IgG ELISA data because antibody-mediated virus neutralization suggests specificity to the tested virus. We found that $67 \%$ of the ELISA-positive samples tested neutralized CCHFV IbAr10200. Because we used an isolate from West Africa in our PRNT, our results are probably an underestimate of the true percentage of samples specific for CCHFV. Nucleic acid differences could account for the reduced neutralization. On the other hand, ELISA-positive, PRNT-negative samples could indicate exposure to related viruses, such as Dugbe virus. Two of 7 randomly selected ELISA-negative samples neutralized CCHFV to some extent, which could have been the result 


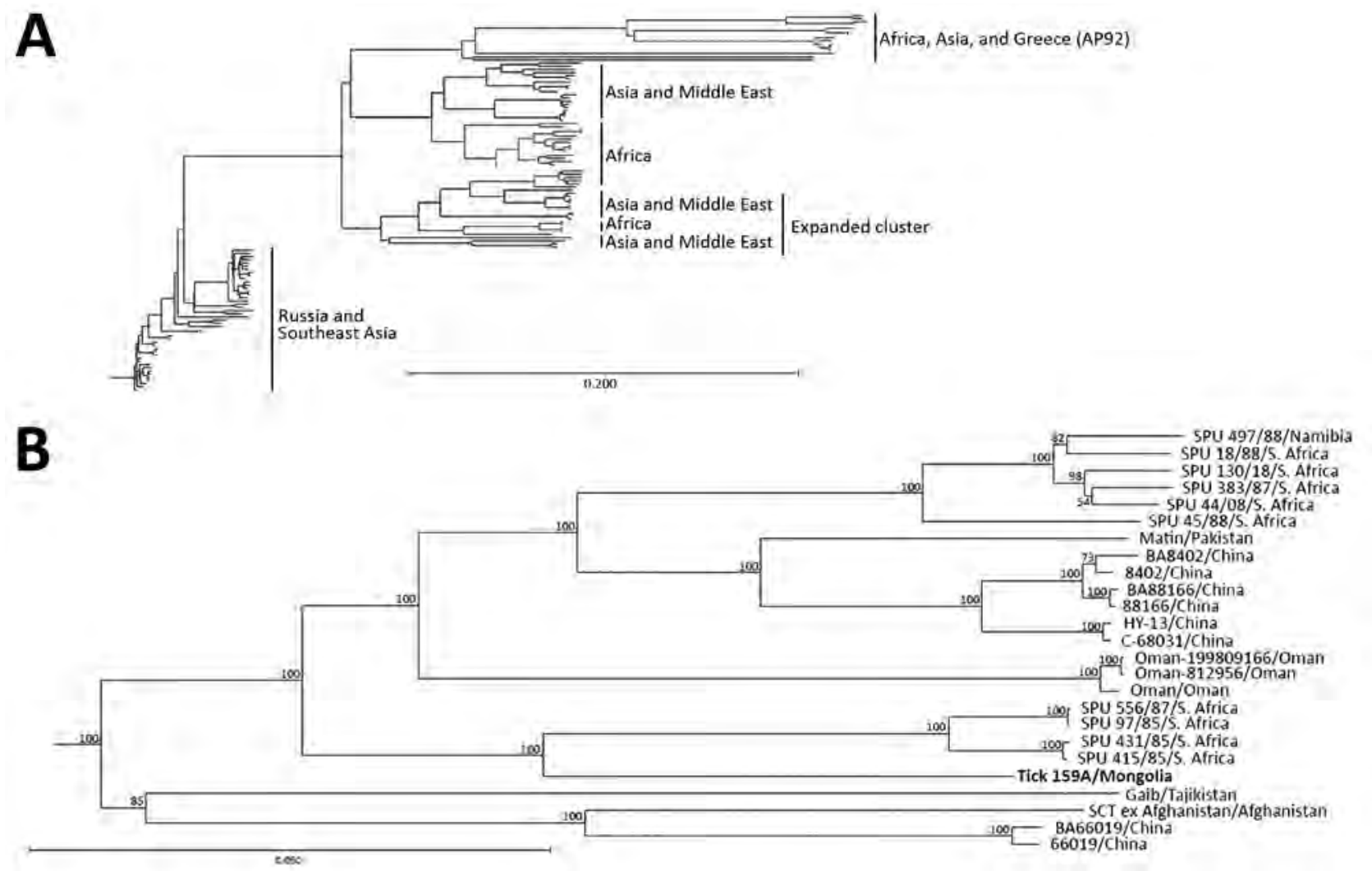

Figure 3. Phylogenetic characterization of full-length medium (M) segment sequence of Crimean-Congo hemorrhagic fever virus (CCHFV) isolate from tick pool 159A, Mongolia, 2013-2014. Full-length and near full-length CCHFV M segments from GenBank were aligned with the $\mathrm{M}$ segment sequence of tick pool 159A and a phylogenetic tree was generated. A) M segment lineages weakly cluster by geographic location. B) Detailed view of phylogenetic tree cluster including the isolate from this study (tick 159A/Mongolia; bold). Tick 159A/Mongolia groups with CCHFV isolates from Asia, the Middle East, and Africa. Scale bars indicate nucleotide substitutions per site.

of nonspecific binding or low IgG concentrations below the level of detection of our ELISA.

As further supporting evidence suggesting CCHFV circulation in Mongolia, we identified 1 tick pool positive for CCHFV genetic material, which enabled us to sequence the $\mathrm{S}$ and $\mathrm{M}$ segments of the isolate and perform phylogenetic analyses. The S segment, which generally clusters on the basis of geographic location in phylogenetic analyses, was similar to other sequenced CCHFV isolates found in countries of Central and East Asia, including Kazakhstan, Uzbekistan, Tajikistan, and China, corresponding to the Asia 2 CCHFV lineage or lineage IV $(21,23)$. The M segment, which has a higher mutation rate than the $\mathrm{S}$ segment, had the greatest homology to isolates from South Africa, the Middle East, and Asia, corresponding to the M segment lineage IV $(21,23)$.

Overall, our study demonstrated the presence of $\mathrm{CCH}$ FV in Mongolia, suggesting human infections are occurring in the area. Our findings are not surprising, considering that others have shown by serology and tick testing the presence of CCHFV in the region $(15,25)$. Because of Mongolia's size and diversity, additional studies are required to better characterize the virus and the disease severity it causes. Taken together, these data suggest CCHFV is likely endemic in Mongolia. Further investigations are needed to better define the risk for human infection and the genomic diversity of CCHFV in the region to improve risk mitigation, diagnostics, and surveillance.

\section{Acknowledgments}

The authors thank all the men and women of Mongolia whose contributions directly or indirectly made this work possible. We give a special acknowledgment to Shigeru Morikawa for his contributions to the CCHFV nested RT-PCR. Thanks to Cindy Rossi and Tamara Clements for their expert technical advice and ELISA assistance. Thanks to Lucas Bagley for assistance with sorting, identifying, and homogenizing ticks.

Laboratory work was funded in part by the Global Emerging Infections Surveillance Section of the Armed Forces Health Surveillance Branch research plans (ProMIS P00123-16-RD and P0017-17-RD) through USAMRIID and the World Bank project Capability Building for Emerging Infectious Disease Preparedness and Response, 2012-2013. 
Disclaimer: Opinions, interpretations, conclusions, and recommendations are those of the authors and are not necessarily endorsed by the US Army.

\section{About the Author}

Mr. Voorhees is a biological sciences laboratory technician working in the Diagnostic Systems Division at USAMRIID, Fort Detrick, Maryland. His research interests are in the development of immunologic assays and evaluation of platforms to detect and identify viruses requiring Biosafety Level 3 and 4 containment and their transition to austere settings for disease surveillance and diagnostics.

\section{References}

1. Bente DA, Forrester NL, Watts DM, McAuley AJ, Whitehouse CA, Bray M. Crimean-Congo hemorrhagic fever: history, epidemiology, pathogenesis, clinical syndrome and genetic diversity. Antiviral Res. 2013;100:159-89. http://dx.doi.org/10.1016/j.antiviral.2013. 07.006

2. Lukashev AN, Klimentov AS, Smirnova SE, Dzagurova TK, Drexler JF, Gmyl AP. Phylogeography of Crimean Congo hemorrhagic fever virus. PLoS One. 2016;11:e0166744. http://dx.doi.org/10.1371/journal.pone.0166744

3. Messina JP, Pigott DM, Golding N, Duda KA, Brownstein JS, Weiss DJ, et al. The global distribution of Crimean-Congo hemorrhagic fever. Trans R Soc Trop Med Hyg. 2015;109:503-13. http://dx.doi.org/10.1093/trstmh/trv050

4. Hewson R, Gmyl A, Gmyl L, Smirnova SE, Karganova G, Jamil B, et al. Evidence of segment reassortment in CrimeanCongo haemorrhagic fever virus. J Gen Virol. 2004;85:3059-70. http://dx.doi.org/10.1099/vir.0.80121-0

5. Mostafavi E, Chinikar S, Moradi M, Bayat N, Meshkat M, Fard MK, et al. A case report of Crimean Congo hemorrhagic fever in ostriches in Iran. Open Virol J. 2013;7:81-3. http://dx.doi.org/ 10.2174/1874357901307010081

6. Lindeborg M, Barboutis C, Ehrenborg C, Fransson T, Jaenson TG, Lindgren PE, et al. Migratory birds, ticks, and Crimean-Congo hemorrhagic fever virus. Emerg Infect Dis. 2012;18:2095-7. http://dx.doi.org/10.3201/eid1812.120718

7. Shepherd AJ, Swanepoel R, Leman PA, Shepherd SP. Field and laboratory investigation of Crimean-Congo haemorrhagic fever virus (Nairovirus, family Bunyaviridae) infection in birds. Trans R Soc Trop Med Hyg. 1987;81:1004-7. http://dx.doi.org/ 10.1016/0035-9203(87)90379-8

8. Mild M, Simon M, Albert J, Mirazimi A. Towards an understanding of the migration of Crimean-Congo hemorrhagic fever virus. J Gen Virol. 2010;91:199-207. http://dx.doi.org/10.1099/vir.0.014878-0

9. Papa A, Tsergouli K, Tsioka K, Mirazimi A. Crimean-Congo hemorrhagic fever: tick-host-virus interactions. Front Cell Infect Microbiol. 2017;7:213. http://dx.doi.org/10.3389/fcimb.2017.00213

10. Ergönül O. Crimean-Congo haemorrhagic fever. Lancet Infect Dis. 2006;6:203-14. http://dx.doi.org/10.1016/S1473-3099(06)70435-2

11. Swanepoel R, Gill DE, Shepherd AJ, Leman PA, Mynhardt JH, Harvey S. The clinical pathology of Crimean-Congo hemorrhagic fever. Rev Infect Dis. 1989;11(Suppl 4):S794-800. http://dx.doi.org/ 10.1093/clinids/11.Supplement_4.S794
12. Chumikhin S, Potshild E, Nyamadava P, Peshetnikov I, Tkachenko E, Dzagurova T. Serological and virological tests used for the detection of arboviruses in wild mammals and birds of Mongolia. Med Parazitol (Mosk). 1987:10-2.

13. Morikawa S. Serological surveillance of Crimean-Congo haemorrhagic fever infection among sheep in Mongolia. Tokyo: National Institute of Infectious Diseases; 2013.

14. Guo R, Shen S, Zhang Y, Shi J, Su Z, Liu D, et al. A new strain of Crimean-Congo hemorrhagic fever virus isolated from Xinjiang, China. Virol Sin. 2017;32:80-8. http://dx.doi.org/10.1007/ s12250-016-3936-9

15. Sun S, Dai X, Aishan M, Wang X, Meng W, Feng C, et al. Epidemiology and phylogenetic analysis of Crimean-Congo hemorrhagic fever viruses in Xinjiang, China. J Clin Microbiol. 2009;47:2536-43. http://dx.doi.org/10.1128/JCM.00265-09

16. Yu-Chen Y, Ling-Xiong K, Ling L, Yu-Qin Z, Feng L, Bao-Jian C, et al. Characteristics of Crimean-Congo hemorrhagic fever virus (Xinjiang strain) in China. Am J Trop Med Hyg. 1985;34:1179-82. http://dx.doi.org/10.4269/ajtmh.1985.34.1179

17. Papa A, Weber F, Hewson R, Weidmann M, Koksal I, Korukluoglu G, et al. Meeting report: First International Conference on Crimean-Congo Hemorrhagic Fever. Antiviral Res. 2015; 120:57-65. http://dx.doi.org/10.1016/j.antiviral.2015. 05.005

18. Garrison AR, Alakbarova S, Kulesh DA, Shezmukhamedova D, Khodjaev S, Endy TP, et al. Development of a TaqMan minor groove binding protein assay for the detection and quantification of Crimean-Congo hemorrhagic fever virus. Am J Trop Med Hyg. 2007;77:514-20.

19. Koehler JW, Delp KL, Hall AT, Olschner SP, Kearney BJ, Garrison AR, et al. Sequence optimized real-time reverse transcription polymerase chain reaction assay for detection of Crimean-Congo hemorrhagic fever virus. Am J Trop Med Hyg. 2018;98:211-5. http://dx.doi.org/10.4269/ajtmh.17-0165

20. Morikawa S. Laboratory diagnosis of Crimean-Congo hemorrhagic fever (CCHF). Tokyo: National Institute of Infectious Diseases; 2012. p. 1-15.

21. Deyde VM, Khristova ML, Rollin PE, Ksiazek TG, Nichol ST. Crimean-Congo hemorrhagic fever virus genomics and global diversity. J Virol. 2006;80:8834-42. http://dx.doi.org/10.1128/ JVI.00752-06

22. Koehler JW, Delp KL, Kearney BJ, Conrad TA, Schoepp RJ, Garrison AR, et al. Draft genome sequences of eight CrimeanCongo hemorrhagic fever virus strains. Genome Announc. 2017;5:e00240-17. http://dx.doi.org/10.1128/genomeA.00240-17

23. Anagnostou V, Papa A. Evolution of Crimean-Congo hemorrhagic fever virus. Infect Genet Evol. 2009;9:948-54. http://dx.doi.org/ 10.1016/j.meegid.2009.06.018

24. Spengler JR, Bergeron É, Rollin PE. Seroepidemiological studies of Crimean-Congo hemorrhagic fever virus in domestic and wild animals. PLoS Negl Trop Dis. 2016;10:e0004210. http://dx.doi.org/ 10.1371/journal.pntd.0004210

25. Xia H, Li P, Yang J, Pan L, Zhao J, Wang Z, et al. Epidemiological survey of Crimean-Congo hemorrhagic fever virus in Yunnan, China, 2008. Int J Infect Dis. 2011;15:e459-63. http://dx.doi.org/10.1016/j.ijid.2011.03.013

Address for correspondence: Randal J. Schoepp, USAMRIID, Diagnostic Systems Division, 1425 Porter St, Fort Detrick, MD 21702, USA; email: randal.j.schoepp.civ@mail.mil 


\title{
Novel Type of Chronic Wasting Disease Detected in Moose (Alces alces), Norway
}

\author{
Laura Pirisinu, Linh Tran, Barbara Chiappini, Ilaria Vanni, Michele A. Di Bari, \\ Gabriele Vaccari, Turid Vikøren, Knut Ivar Madslien, Jørn Våge, Terry Spraker, \\ Gordon Mitchell, Aru Balachandran, Thierry Baron, Cristina Casalone, \\ Christer M. Rolandsen, Knut H. Røed, Umberto Agrimi, Romolo Nonno, Sylvie L. Benestad
}

Chronic wasting disease (CWD) persists in cervid populations of North America and in 2016 was detected for the first time in Europe in a wild reindeer in Norway. We report the detection of CWD in 3 moose (Alces alces) in Norway, identified through a large-scale surveillance program. The cases occurred in 13-14-year-old female moose, and we detected an abnormal form of prion protein $\left(\mathrm{PrPs}^{\mathrm{Sc}}\right)$ in the brain but not in lymphoid tissues. Immunohistochemistry revealed that the moose shared the same neuropathologic phenotype, characterized by mostly intraneuronal deposition of PrPsc. This pattern differed from that observed in reindeer and has not been previously reported in CWD-infected cervids. Moreover, Western blot revealed a PrPsc type distinguishable from previous CWD cases and from known ruminant prion diseases in Europe, with the possible exception of sheep $\mathrm{CH} 1641$. These findings suggest that these cases in moose represent a novel type of CWD.

$\mathrm{T}$ ransmissible spongiform encephalopathies (TSEs), or prion diseases, are fatal and transmissible neurodegenerative diseases that include scrapie in sheep and goats, bovine spongiform encephalopathy (BSE) in cattle, chronic wasting disease (CWD) in cervids, and CreutzfeldtJakob disease in humans. TSEs are characterized by the

Author affiliations: Istituto Superiore di Sanità, Rome, Italy

(L. Pirisinu, B. Chiappini, I. Vanni, M.A. Di Bari, G. Vaccari, U. Agrimi, R. Nonno); Norwegian Veterinary Institute, Oslo, Norway (L. Tran, T. Vikøren, K.I. Madslien, J. Våge, S.L.

Benestad); Colorado State University, Fort Collins, Colorado, USA (T. Spraker); Canadian Food Inspection Agency, Ottawa, Ontario, Canada (G. Mitchell, A. Balachandran); Anses Lyon Unité "Maladies Neuro-Dégénératives," Lyon, France (T. Baron); Istituto Zooprofilattico Sperimentale del Piemonte Liguria e Valle d'Aosta, Torino, Italy (C. Casalone); Norwegian Institute for Nature Research, Trondheim, Norway (C.M. Rolandsen); Norwegian University of Life Sciences, Faculty of Veterinary Science, Oslo (K.H. Røed)

DOI: https://doi.org/10.3201/eid2412.180702 misfolding of the normal host-encoded cellular prion protein $\left(\mathrm{PrP}^{\mathrm{C}}\right)$ into an abnormal disease-associated isoform $\left(\mathrm{PrP}^{\mathrm{Sc}}\right)$. $\mathrm{PrP}^{\mathrm{Sc}}$ is considered to be the main or exclusive component of prions, the transmissible agents for TSEs (1). TSEs might have a genetic, infectious, or sporadic origin. Classical scrapie and CWD can be highly contagious, spreading directly among animals or through environmental contamination.

Since its first description in Colorado in 1967, CWD has been detected in new geographic areas and with increasing prevalence in captive and free-ranging cervids. Currently, the disease has been diagnosed in 25 states in the United States and in 2 Canada provinces (2,3), along with cases in South Korea associated with importation of infected cervids from Canada (4). High disease prevalence in some areas represents a challenge for preservation of wild cervids and mitigation of human exposure to CWD-related prions $\left(\mathrm{PrP}^{\mathrm{Sc}}\right)$. The high prevalence might be a plausible explanation for local deer population decline $(5,6)$.

Species naturally affected by CWD include white-tailed deer (Odocoileus virginianus), mule deer (O. hemionus), moose (Alces alces), elk or wapiti (Cervus canadensis), and red deer (C. elaphus). In 2016, CWD was reported for the first time in Europe in wild reindeer (Rangifer tarandus) (7), a species never previously found to be naturally infected. The biochemical analysis and immunohistochemical (IHC) distribution of $\mathrm{PrP}^{\mathrm{Sc}}$ from Norway reindeer revealed a pattern indistinguishable from North America isolates (7).

We report 3 cases of CWD detected in moose in Norway, characterized by biochemical and IHC features clearly different from CWD cases previously described in North America and Norway. Our findings suggest the involvement of a different type of CWD prion.

\section{Materials and Methods}

\section{Animals and Tissues}

The 3 moose were found in Trøndelag County in central Norway. The first case, moose no. 1 (ID P138), was 
emaciated and demonstrated abnormal behavior, showing reduced fear of humans. The second case, moose no. 2 (ID P153), was found dead in a river. Necropsy revealed normal body condition and pregnancy with twins; trauma was the cause of death. The third case, moose no. 3 (ID CD11399), was observed showing abnormal behaviors, including reduced fear of humans. Necropsy revealed a poor body condition and a severe dislocation of the left hip joint, which might have influenced the animal's behavior. All 3 were older female moose $(13,14$, and 13 years old, based on counts of cementum annuli in the root of the first incisor [8]).

Samples included in this study are described in the Table. We performed the primary diagnostic test (TeSeE ELISA; Bio-Rad Laboratories, Inc., Hercules, CA, USA) for detection of protease-resistant core of $\operatorname{PrP}^{\mathrm{Sc}}\left(\mathrm{PrP}^{\mathrm{res}}\right)$ on the 3 moose and 1 reindeer from Norway (7) in the medulla oblongata at the level of the obex. After the initial positive test results, the remaining brain tissues were divided; one half was fixed in $10 \%$ neutral buffered formalin, and the other half was frozen. In addition, lymph nodes (Ln) from moose no. 1 (retropharyngeal, submandibular, and jejunal Ln), moose no. 3 (retropharyngeal, parotid, prescapular, and submandibular Ln, and tonsils), and the reindeer (2 tracheobronchial Ln) were equally divided and formalin fixed or frozen.

\section{Genotyping of Moose PRNP}

DNA was extracted from $100 \mathrm{mg}$ of brain tissue by using a DNeasy Blood and Tissue Kit (QIAGEN, Hilden, Germany), according to the manufacturer's instructions. The PRNP coding sequence was amplified in a $50 \mu \mathrm{L}$ final volume by using $5 \mu \mathrm{L}$ of extracted DNA, eluate $1 \mathrm{X}$
AmpliTaq Gold 360 PCR Buffer (Life Technologies, Carlsbad, CA, USA), $2.5 \mathrm{mmol} / \mathrm{L} \mathrm{MgCl}_{2}, 1 \mathrm{X} 360 \mathrm{GC}$ Enhancer (Life Technologies), $200 \mu \mathrm{mol} / \mathrm{L}$ dNTPs, 0.25 $\mu \mathrm{mol} / \mathrm{L}$ of forward $\left(5^{\prime}\right.$-GCTGACACCCTCTTTATTTTGCAG- $\left.3^{\prime}\right)$ and reverse (5'-GATTAAGAAGATAATGAAAACAGGAAG -3') primers (11), and $0.5 \mu \mathrm{L}$ AmpliTaq Gold 360 (Life Technologies), according to the following amplification protocol: $5 \mathrm{~min}$ at $96^{\circ} \mathrm{C} ; 30 \mathrm{~s}$ at $96^{\circ} \mathrm{C} ; 15 \mathrm{~s}$ at $57^{\circ} \mathrm{C} ; 90 \mathrm{~s}$ at $72^{\circ} \mathrm{C}$ for 40 cycles; and $4 \mathrm{~min}$ at $72^{\circ} \mathrm{C}$. Amplicons were purified with the Illustra ExoProStar 1-Step clean-up kit (GE Healthcare Life Sciences, Little Chalfont, UK), sequenced using the Big Dye Terminator Cycle Sequencing Kit v1.1 (Life Technologies), and purified with the Big Dye XTerminator Purification Kit (Life Technologies), and detected by using an ABI PRISM 3130 apparatus (Life Technologies).

\section{Anti-Prion Protein Monoclonal Antibodies}

Several antibodies with different epitopes (sheep prion protein $[\mathrm{PrP}]$ numbering) were used for discriminatory Western blot (WB) and IHC. SAF84 (aa 167-173) was obtained from Bertin Pharma (Montigny-le-Bretonneux, France), L42 (aa 148-153) from R-Biopharm (Darmstadt, Germany), 9A2 (aa 102-104) and 12B2 (aa 93-97) from Wageningen Bioveterinary Research (Lelystad, Netherlands), and F99/97.6 (aa 220-225) from VMRD, Inc. (Pullman, WA, USA).

\section{Immunohistochemistry}

Brain, $\mathrm{Ln}$, and tonsil tissues were formalin fixed for $\geq 48 \mathrm{~h}$ and processed by standard histopathologic techniques. We used IHC to visualize the distribution of $\mathrm{PrP}^{\mathrm{Sc}}$ as previously described (7). We applied a commercially available kit

\begin{tabular}{|c|c|c|c|c|}
\hline Animal or species & Geographic origin & Age & Pathologic phenotype & PrP genotype $†$ \\
\hline Moose no. 1 & Norway & $13 \mathrm{y}$ & Atypical CWD & $\mathrm{KK}_{109} \mathrm{MM}_{209}$ \\
\hline Moose no. 2 & Norway & $14 \mathrm{y}$ & Atypical CWD & $\mathrm{KK}_{109} \mathrm{MM}_{209}$ \\
\hline Moose no. 3 & Norway & $13 y$ & Atypical CWD & $\mathrm{KK}_{109} \mathrm{MM}_{209}$ \\
\hline Reindeer & Norway & $3-4 y$ & CWD & $\mathrm{VV}_{2} \mathrm{GG}_{129} \mathrm{SS}_{138} \mathrm{VV}_{169}$ \\
\hline Elk or wapiti & Canada & $6 \mathrm{y}$ & CWD & $\mathrm{MM}_{132}$ \\
\hline Elk or wapiti & Canada & Adult & CWD & $\mathrm{MM}_{132}$ \\
\hline Elk or wapiti & Canada & $6 y$ & CWD & $\mathrm{MM}_{132}$ \\
\hline White-tailed deer & Canada & $4 y$ & CWD & $\mathrm{GG}_{96}$ \\
\hline Moose & Canada & Adult & CWD & $\mathrm{KK}_{109} \mathrm{II}_{209} \ddagger$ \\
\hline Sheep & Italy & $2.5 \mathrm{y}$ & Classical scrapie & $\mathrm{AA}_{136} \mathrm{RR}_{154} \mathrm{QQ}_{171}$ \\
\hline Sheep & Italy & Adult & Classical scrapie & $\mathrm{AA}_{136} \mathrm{RR}_{154} \mathrm{QQ}_{171}$ \\
\hline Sheep & Italy & $4.5 \mathrm{y}$ & Atypical/Nor98 scrapie & $\mathrm{AA}_{136} \mathrm{RH}_{154} \mathrm{QQ}_{171}$ \\
\hline Sheep & Experimental sample & NA & Scrapie CH1641§ & $\mathrm{AA}_{136} \mathrm{HH}_{154} \mathrm{QQ}_{171}$ \\
\hline Sheep & France & NA & Scrapie CH1641-likeף & $\mathrm{AA}_{136} \mathrm{RR}_{154} \mathrm{QQ}_{171}$ \\
\hline Cattle & Italy & $6 y$ & C-BSE & NA \\
\hline Cattle & Italy & $15 \mathrm{y}$ & L-BSE & NA \\
\hline Cattle & France & $11 \mathrm{y}$ & H-BSE & NA \\
\hline \multicolumn{5}{|c|}{ 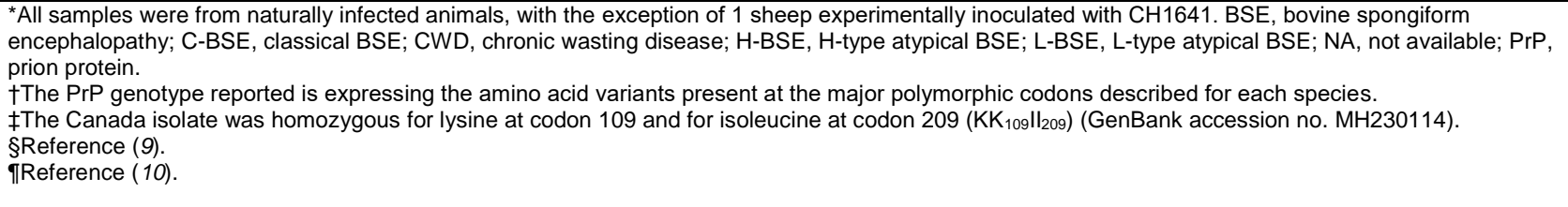 } \\
\hline
\end{tabular}


(EnVisionTM + System HRP [(AEC)] DAKO, Glostrup, Denmark) by using the monoclonal antibodies (mAbs) 12B2, 9A2, L42, SAF 84, or F99/97.6 for $30 \mathrm{~min}$ at $37^{\circ} \mathrm{C}$ and counterstained with hematoxylin. In each run, tissues from CWD-negative moose and reindeer were added as negative controls.

\section{PrPres Detection}

We initially tested the obex samples by using the TeSeE SAP ELISA and confirmed positive ELISA results by using the TeSeE WB. Both tests were performed as recommended by the manufacturer (Bio-Rad).

\section{Typing of PrPres}

We performed further characterization of PrPres by discriminatory immunoblotting, according to the ISS discriminatory WB method (12). Brain homogenates at 10\% (wt/vol) in $100 \mathrm{mmol} / \mathrm{L}$ Tris- $\mathrm{HCl}$ (pH 7.4) $2 \%$ sarkosyl were incubated for $1 \mathrm{~h}$ at $37^{\circ} \mathrm{C}$ with Proteinase K (Sigma-Aldrich, St. Louis, Missouri, USA) to a final concentration of 200 $\mu \mathrm{g} / \mathrm{mL}$. Protease treatment was stopped with $3 \mathrm{mmol} / \mathrm{L}$ PMSF (Sigma-Aldrich). Aliquots of samples were added with an equal volume of isopropanol/butanol (1:1 vol/vol) and centrifuged at $20,000 \times g$ for $10 \mathrm{~min}$. The pellets were resuspended in denaturing sample buffer (NuPAGE LDS Sample Buffer; Life Technologies) and heated for $10 \mathrm{~min}$ at $90^{\circ} \mathrm{C}$.

We loaded each sample onto $12 \%$ bis-Tris polyacrylamide gels (Invitrogen) for electrophoresis with subsequent WB on polyvinylidene fluoride membranes using the Trans-Blot Turbo Transfer System (Bio-Rad) according to the manufacturer's instructions. The blots were processed with anti-PrP mAbs by using the SNAP i.d. 2.0 system (Millipore, Burlington, MA, USA) according to the manufacturer's instructions. After incubation with horseradish peroxidase-conjugated anti-mouse immunoglobulin (Pierce Biotechnology, Waltham, MA, USA) at 1:20,000, the PrP bands were detected by using enhanced chemiluminescent substrate (SuperSignal Femto; Pierce Biotechnology) and ChemiDoc imaging system (Bio-Rad). The chemiluminescence signal was quantified by using Image Lab 5.2.1 (Bio-Rad).

We performed deglycosylation by adding $18 \mu \mathrm{L}$ of 0.2 $\mathrm{mmol} / \mathrm{L}$ sodium phosphate buffer ( $\mathrm{pH} 7.4$ ) containing $0.8 \%$ Nonidet P40 (Roche) and $2 \mu \mathrm{L}$ (80 U/ml) di N-Glycosidase F (Roche) to $5 \mu \mathrm{L}$ of proteinase $\mathrm{K}$-digested and denaturated samples. We then incubated the mixtures for $3 \mathrm{~h}$ at $37^{\circ} \mathrm{C}$ with gentle shaking.

\section{Results}

CWD was diagnosed in 2 moose in May 2016 in Norway's Selbu municipality and in 1 moose in October 2017 in Lierne municipality. Selbu and Lierne are respectively located $\approx 300$ and $\approx 450 \mathrm{~km}$ northeast of Nordfjella, where CWD in reindeer was detected in 2016. Norway is populated by several species of wild cervids with varying degrees of overlapping range. Seasonal migrations are common and distances might exceed $150 \mathrm{~km}$ (13-15). However, studies tracking global positioning satellitecollared moose have not documented regular seasonal migrations between Selbu and Lierne municipalities, suggesting that these can be considered different moose subpopulations.

We initially detected $\operatorname{PrP}^{\mathrm{sc}}$ in brain samples by using a rapid test and then confirmed by WB (data not shown) and IHC. Sequencing analysis of the entire PrP coding sequence revealed that the 3 moose had the wild type $\operatorname{PrP}$ genotype, homozygous for lysine at codon 109 and for methionine at codon $209\left(\mathrm{KK}_{109} \mathrm{MM}_{209}\right)(\mathrm{GenBank}$ accession no. MH230115).

\section{Discriminatory PrPs Immunohistochemistry Show Differences between Reindeer and Moose}

The distribution of $\mathrm{PrP}^{\mathrm{Sc}}$ staining was examined by IHC and compared in the tissues of the 3 moose and the reindeer by using 5 different antibodies (Figure 1). No staining was observed in CWD-negative reindeer and moose independently of the antibody used. The distribution of $\operatorname{PrP}^{\mathrm{Sc}}$ in the reindeer was identical for each of the 5 antibodies and did not differ from the description of $\mathrm{PrP}^{\mathrm{Sc}}$ distribution in North America cervids (16-18). The labeling was most consistent within the gray matter of the medulla oblongata, particularly in the dorsal motor of the vagus nerve (7). The thalamic and brain stem regions of the brain were most affected, with a minimal amount of $\operatorname{PrP}^{\mathrm{Sc}}$ identified dorsal to the corpus callosum.

$\mathrm{PrP}^{\mathrm{Sc}}$ labeling in the moose brains (Figure 1, panels F-J) was clearly different from that of the reindeer (Figure 1, panels A-E). In the moose, after staining with F99/97.6 and L42, PrP ${ }^{\mathrm{Sc}}$ was almost exclusively observed as intraneuronal aggregates, although intraastrocytic type (multiple small granules scattered in the cytoplasm of astrocyteresembling cells) and intramicroglial type (1 single or a few large granules in close proximity to microglia-like nuclei) were also observed in the cerebral cortices and olfactory bulb (online Technical Appendix Figure 1, panel A, https://wwwnc.cdc.gov/EID/article/24/12/18-0702Techapp1.pdf). The degree of $\operatorname{PrP}^{\mathrm{Sc}}$ staining was more intensive and appeared more widespread in the neuropil using SAF84.

At the level of the obex, we found stained neurons in all nuclei, whereas the dorsal motor of the vagus nerve was not remarkably stained, as observed in reindeer. The intensity of labeling varied among the 3 moose; no. 2 displayed sparse labeling, no. 3 widespread and abundant labeling, and no. 1 intermediate labeling intensity. We observed $\mathrm{PrP}^{\mathrm{Sc}}$ in all parts of the brain investigated except the 


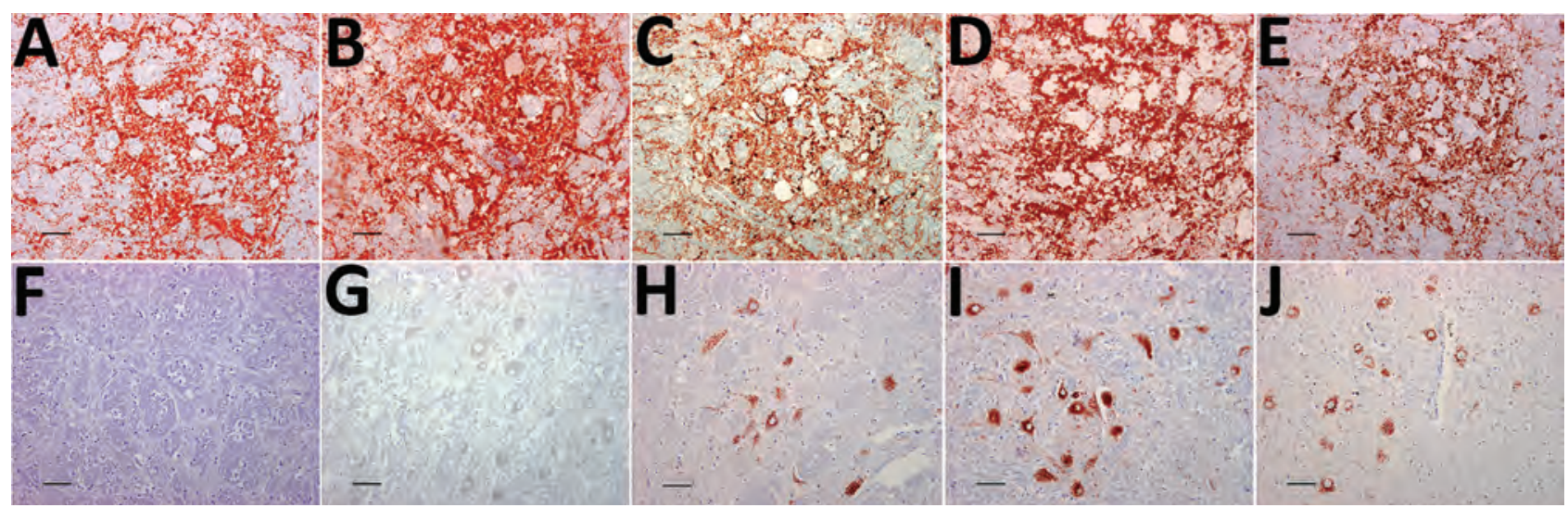

Figure 1. Immunohistochemical detection of disease-associated prion protein in brain sections at the level of the obex in cervids with chronic wasting disease, Norway. A-E) Reindeer; F-J) moose. mAbs used were 12B2 (A, F), $9 A 2$ (B, G), L42 (C, H), SAF 84 (D, I), and F99/97.6 (E, J). Staining obtained in the reindeer tissues is similar regardless of mAbs used (A-E). Conversely, for moose tissues, the staining was primarily observed intraneuronally with L42, SAF84, and F99/97.6 (H-J) but was not observed using the more $\mathrm{N}$-terminal mAbs 12B2 and 9A2 (F, G). Scale bars indicate $40 \mu \mathrm{m}$. mAbs, monoclonal antibodies.

cerebella of moose nos. 1 and 2. A diffuse or discrete punctate staining was observed in the granular layer of the cerebellum of moose no. 3, with stronger staining in some Golgi neurons (online Technical Appendix Figure 1 , panel B). In all 3 moose, the cortical regions showed laminar staining of neurons in all the cell layers, especially in fusiform-shaped neurons. The neurons of the olfactory tubercle from all 3 also stained strongly, and some gliaassociated staining could be observed.

In contrast to the reindeer, the downstream flexible tail mAbs 12B2 and 9A2 did not stain in the moose (Figure 1 , panels $F$ and $G$ ), suggesting that the moose $\operatorname{PrP}^{\mathrm{Sc}}$ was truncated by endogenous proteases further upstream in the $\mathrm{N}$ terminus than was reindeer $\operatorname{PrP}^{\mathrm{Pc}}$. Contrary to previous findings in reindeer, $\mathrm{PrP}^{\mathrm{Sc}}$ was not detected in the Ln from moose no. 1 or in the Ln and tonsils from moose no. 3 (lymphoid tissues were not available in moose no. 2) by either IHC or ELISA.

\section{PrPsc from Norway Moose Compared with Other CWD Isolates from Canada and Norway}

We compared the $\mathrm{PrP}^{\mathrm{Sc}}$ features in moose from Norway with those of other CWD isolates from Norway and Canada by discriminatory WB, which enabled comparison of $\mathrm{PrP}^{\mathrm{res}}$ by epitope mapping with different antibodies. Norway moose $\mathrm{PrP}^{\mathrm{res}}$ had a lower apparent molecular weight (MW) than $\mathrm{PrP}^{\mathrm{res}}$ from Norway reindeer (Figure 2, panel A) or from Canada isolates (Figure 2, panel B). This lower MW was explained by the occurrence of more C-terminal cleavage of $\mathrm{PrP}^{\mathrm{sc}}$ by protease $\mathrm{K}$, as confirmed by the partial loss of the 12B2 epitope (Figure 2, panel B).

Given the unusual pattern observed in moose isolates from Norway, we further investigated their biochemical characteristics with additional mAbs and by enzymatic deglycosylation (Figure 3). Moose samples showed a main C-terminal fragment of $\approx 17 \mathrm{kDa}$, detected with SAF84, L42, and 9A2, and an additional glycosylated C-terminal fragment of $\approx 13 \mathrm{kDa}$ (CTF13) detected only with SAF84. The $\mathrm{N}$ terminal 12B2 epitope was mainly lost, although a small amount of $\mathrm{PrP}^{\mathrm{res}}$ was still detectable in moose no. 1 (Figure 3) and no. 3 (online Technical Appendix Figure 2) with this antibody.
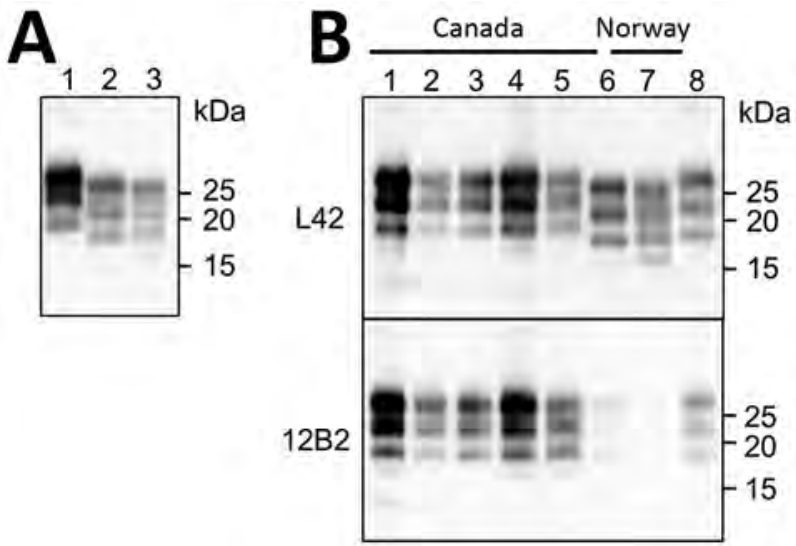

Figure 2. Western blot analysis of PrPres in brains of chronic wasting disease-affected cervids from Norway and Canada. A) Western blot analysis PrPres in brains of moose and reindeer from Norway. Membrane was probed with L42 monoclonal antibodies. Molecular weights $(\mathrm{kDa})$ are indicated on the right. Tissue equivalent loaded per lane was $1 \mathrm{mg}$. B) Western blot analysis of PrPres from moose isolates from Norway (lanes 6-7) compared with PrPres from chronic wasting disease-affected elk or wapiti (lanes 1-3), white-tailed deer (lane 4), and moose (lane 5) from Canada. Membranes were probed with L42 (upper) and 12B2 (lower) monoclonal antibodies. A scrapie sheep sample from Italy was added as control (lane 8). Molecular weights (kDa) are indicated on the right of each blot. Tissue equivalents loaded per lane were $1 \mathrm{mg}$ for Canadian isolates, $2 \mathrm{mg}$ for Norwegian isolates, and $0.15 \mathrm{mg}$ for scrapie sheep control. PrPres, proteaseresistant core of abnormal form of prion protein. 

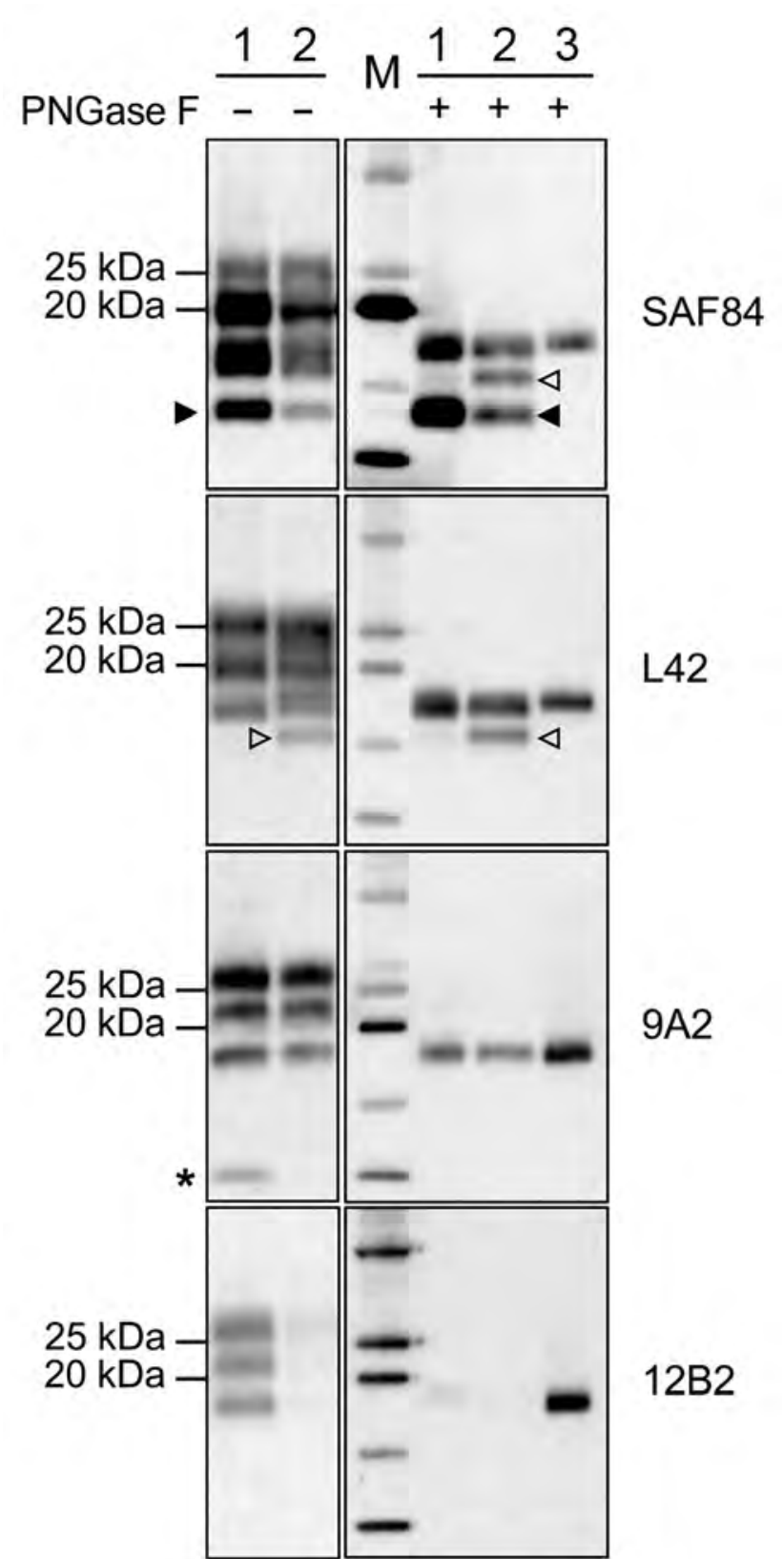

Figure 3. Characterization of PrPres fragments from moose (Alces alces) in Europe by epitope mapping. Mapping with mAbs spanning the whole prion protein enabled the analysis of PrPres in moose samples before (PNGase F-) and after (PNGase F+) deglycosylation, based on presence or absence of the epitopes and apparent molecular weight. Lanes 1, moose no. 1; lanes 2, moose no. 3; lane M, protein standards; lane 3, sheep scrapie sample. Solid arrowheads indicate C-terminal fragment of $\approx 13 \mathrm{kDa}$ fragment (present in both samples and detected with SAF84 mAbs). Open arrowheads indicate C-terminal fragment of $\approx 16 \mathrm{kDa}$ fragment in moose no. 2 with SAF84 and L42 mAbs. Asterisk indicates the internal fragment detected in moose no. 1 with 9A2 mAbs. Molecular weights are indicated on the left. In the blots on the right, protein standards are shown in lane $\mathrm{M}(10,15,20,25$, and $37 \mathrm{kDa})$. The mAbs used are indicated on the right. mAbs, monoclonal antibodies; PrPres, protease-resistant core of abnormal form of prion protein.
In moose nos. 2 and 3, an additional glycosylated C-terminal fragment of $\approx 16 \mathrm{kDa}$ (CTF16) was detected by SAF84 and L42 mAbs (Figure 3; online Technical Appendix Figure 2). We cannot exclude that a small amount of CTF16 was also present in moose no. 1, given that a weak PrPres fragment of $\approx 16 \mathrm{kDa}$ was detectable upon deglycosylation and long exposure of blots (Figure 3; online Technical Appendix Figure 3). Moose nos. 1 and 3 also had a nonglycosylated internal fragment of $\approx 10 \mathrm{kDa}$, cleaved at both $\mathrm{N}$ and $\mathrm{C}$ termini of $\mathrm{PrP}^{\mathrm{Sc}}$, which was recognized by using mAbs 9A2 (Figure 3). Moreover, the analysis of PrPres from different neuroanatomic regions showed that the slight differences observed among the 3 moose were not dependent on the area analyzed (online Technical Appendix Figure 2).

\section{Comparison of the PrPsc Features of the Norway Moose with Sheep and Cattle Prion Strains from Europe}

Comparison with ovine and bovine prions was performed to determine the $\mathrm{N}$ terminal cleavage of the main PrPres fragment by analyzing the different $\mathrm{PrP}^{\mathrm{res}}$ fragments in each sample, the MW of these fragments, and the L42/12B2 antibody ratio (Figure 4; online Technical Appendix Table). Among ovine prions, classical scrapie and atypical/Nor98 were easily discriminated from moose isolates (Figure 5). Classical scrapie $\mathrm{PrP}^{\mathrm{res}}$ had a higher MW than moose $\mathrm{PrP}^{\mathrm{res}}$,

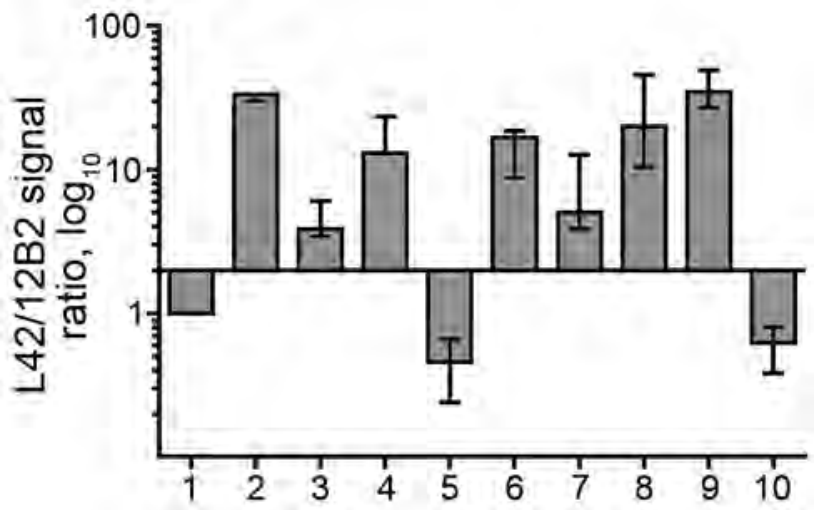

Figure 4. Bar graph of antibody-signal ratios (y-axis) showing discrimination of the ovine, bovine, moose, and reindeer samples (x-axis) analyzed in a study characterizing chronic wasting disease in moose (Alces alces), Norway. Numbers indicate sample type: 1 , scrapie; 2, CH1641; 3, CH1641-like; 4, classical bovine spongiform encephalopathy (BSE); 5, H-type atypical BSE; 6, L-type atypical BSE; 7, moose no. 1; 8, moose no. 2; 9, moose no. 3; 10, reindeer. The antibody ratio is the L42/12B2 ratio of the chemiluminescence signal relative to the $L 42 / 12 B 2$ ratio of the control scrapie loaded in each blot. Bars represent median values of $\geq 3$ independent determinations; error bars represent the range of observed values. Bars start at $y=2$, which is the cutoff value of the antibody ratio for the discrimination of low molecular weight samples (i.e., suspected bovine spongiform encephalopathy cases) from scrapie, according to discriminatory Western blot. Numbers indicate sample type: 1 , scrapie; 2, CH1641; 3, CH1641-like; 4, classical bovine spongiform encephalopathy (BSE); 5, H-type atypical BSE; 6, L-type atypical BSE; 7 , moose no. $1 ; 8$, moose no. 2; 9 , moose no. $3 ; 10$, reindeer. 


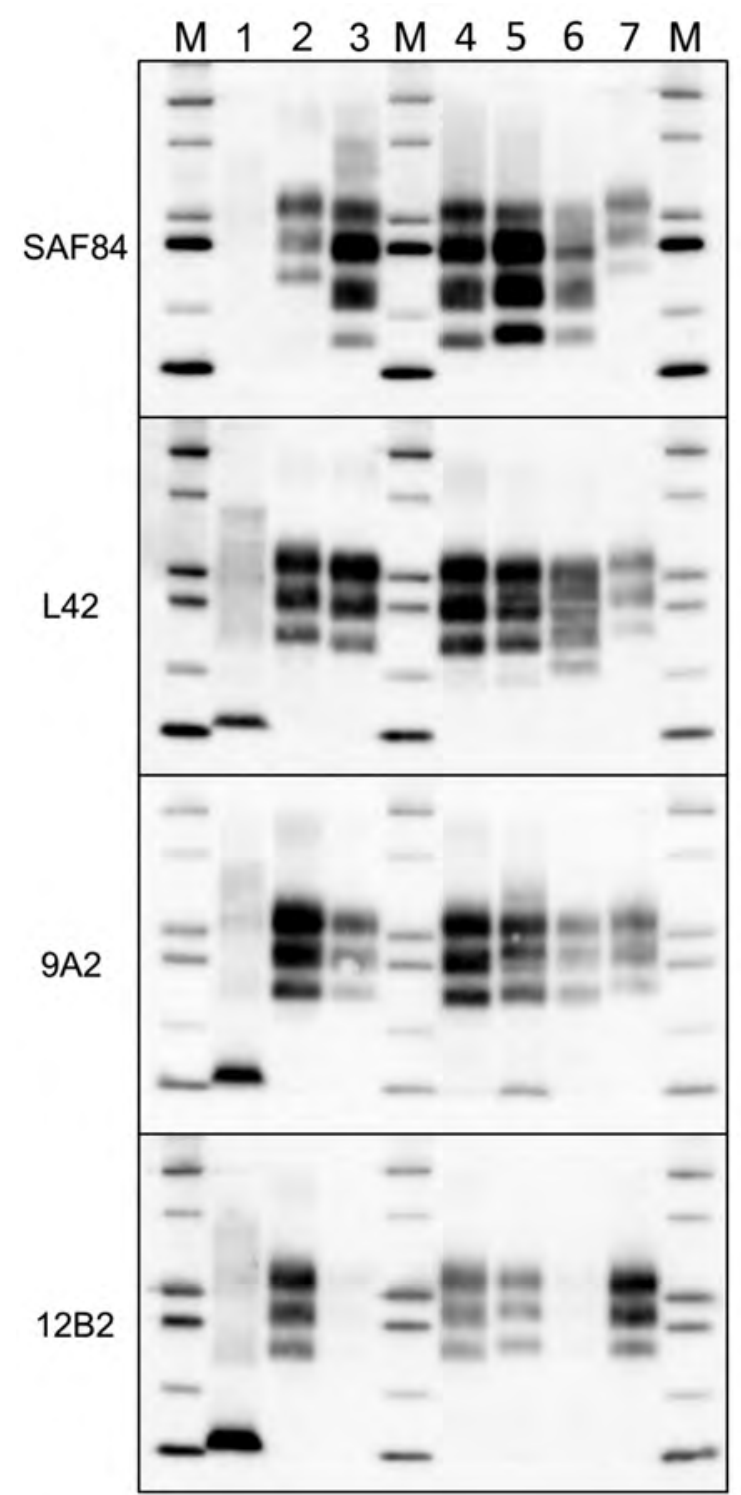

Figure 5. Comparison of protease-resistant PrPres from moose (Alces alces) with chronic wasting disease and from sheep with scrapie, Europe. Representative blots show epitope mapping analysis of PrPres (lane 4, CH1641; lane 5, moose no. 1; lane 6, moose no. 2) in comparison with different ovine transmissible spongiform encephalopathy isolates (lane 1, atypical/Nor98; lane 2, classical scrapie; and lane 3, CH1641). A chronic wasting disease isolate from Canada was loaded as control (lane 7). The antibodies used are indicated on the left. Protein standards are shown in lane $M(10,15,20,25,37$, and $50 \mathrm{kDa})$. The small amount of PrPres with intact 12B2 epitope in moose no.1 had a molecular weight higher than that observed with more $\mathrm{C}$-terminal monoclonal antibodies $(18.7 \pm 0.3 \mathrm{kDa}$ measured with $12 \mathrm{~B} 2 \mathrm{vs} .17 .2 \pm 0.1 \mathrm{kDa}$ measured with L42). Even if the increase of the apparent molecular weight might be a known behavior when proteinase $\mathrm{K}$ cleavage occurs near the epitope, we noted that, in the case of moose no. 1, the 12B2-positive PrPres had a molecular weight higher than scrapie (18.1 $\pm 0.1 \mathrm{kDa}$ measured with 12B2) and $\mathrm{CH} 1641$-like sample $(18.1 \pm 0.4 \mathrm{kDa}$ when detected with 12B2). PrPres, protease-resistant core of abnormal form of prion protein. as confirmed by the preservation of 12B2 epitope. As previously observed (19), Nor98 PrPres was cleaved at both the N and $\mathrm{C}$ termini, and the characteristic 11-12 $\mathrm{kDa}$ band was detected by L42, 9A2, and 12B2 mAbs (Figure 5). In contrast, CH1641 samples showed molecular features partially overlapping with the moose (Figure 5). CH1641 samples showed a $\mathrm{PrP}^{\mathrm{res}}$ of $\approx 17 \mathrm{kDa}$ and were accompanied by an additional C-terminal fragment of 13-14 kDa detected by using SAF84 mAbs (20). However, CTF16 and the internal PrP ${ }^{\text {res }}$ fragment of $10 \mathrm{kDa}$ could not be detected in $\mathrm{CH} 1641$ samples.

Moose $\mathrm{PrP}^{\mathrm{Sc}}$ did not overlap with any type of bovine $\mathrm{PrP}^{\mathrm{Sc}}$. The lack of the 12B2 epitope in moose $\mathrm{PrP}^{\mathrm{res}}$ was similar to C-type and atypical L-type BSE, but the 2 bovine prions had neither CTF13, CTF16, nor the internal fragment (Figure 6). H-type atypical BSE showed the CTF13 and the internal fragment similar to moose PrPres, but the main $\mathrm{PrP}^{\mathrm{res}}$ fragment showed a higher MW and preserved the 12B2 epitope (Figure 6).

The ratio of reactivity obtained with L42 and 12B2 antibodies reflected the $\mathrm{N}$ terminal cleavage of the main fragment of $\mathrm{PrP}^{\mathrm{Sc}}$, enabling confirmation that the differences observed in MW of PrPres actually depend on different $\mathrm{N}$ terminal proteinase $\mathrm{K}$ cleavages, irrespective of the host species (Figure 4). Values $>2$ are indicative of BSE-like cleavage, whereas values $<1$ indicate a better preservation of 12B2 epitope compared with scrapie. In this respect, the behavior of moose $\mathrm{PrP}^{\text {res }}$ was BSE-like (ratio $>2$ ). However, moose no. 1 had a ratio lower than moose nos. 2 and 3. The CH1641-like field sample was similar to moose no. 1 in this respect, whereas CH1641 was similar to moose nos. 2 and 3. Finally, the value $<1$ observed for $\mathrm{PrP}^{\text {res }}$ in H-type atypical BSE, CWD in reindeer, and CWD isolates from Canada reflected their higher MW compared with classical scrapie (online Technical Appendix Table).

\section{Discussion}

Although CWD has been detected in several captive and free-ranging cervid species from a large geographic area in North America, $<10$ cases in moose have been reported (21-23). We report 3 naturally occurring cases of prion disease in moose in Norway that showed molecular and IHC phenotypes differing from those previously described for classical CWD in North America, as well as in reindeer in Norway. The phenomenon of strain variation is well known in prion diseases and is often associated with phenotype variation in natural hosts, as observed in bovines with classical, H-type, or L-type BSE, and in sheep with classical or atypical/Nor98 scrapie. Identification of a new CWD phenotype in 3 moose in Norway can be suggestive of a new CWD strain. Although the existence of CWD strain variation in North America has been inferred from transmission studies (24-26), this phenomenon has not been directly associated with phenotypic variations in natural hosts so far. 
The phenotype variant found in moose from Norway could be hypothetically attributed to host species factors. To address this issue, we directly compared $\operatorname{PrP}^{\mathrm{sc}}$ characteristics in the Norway moose with those in a Canada moose with CWD. In agreement with the available evidence, we found that the Canada moose $\mathrm{PrP}^{\mathrm{Sc}}$ had

\section{$M \quad 1 \quad 2 \quad 3 \quad M \quad 4 \quad 5 \quad 6 \quad M$}

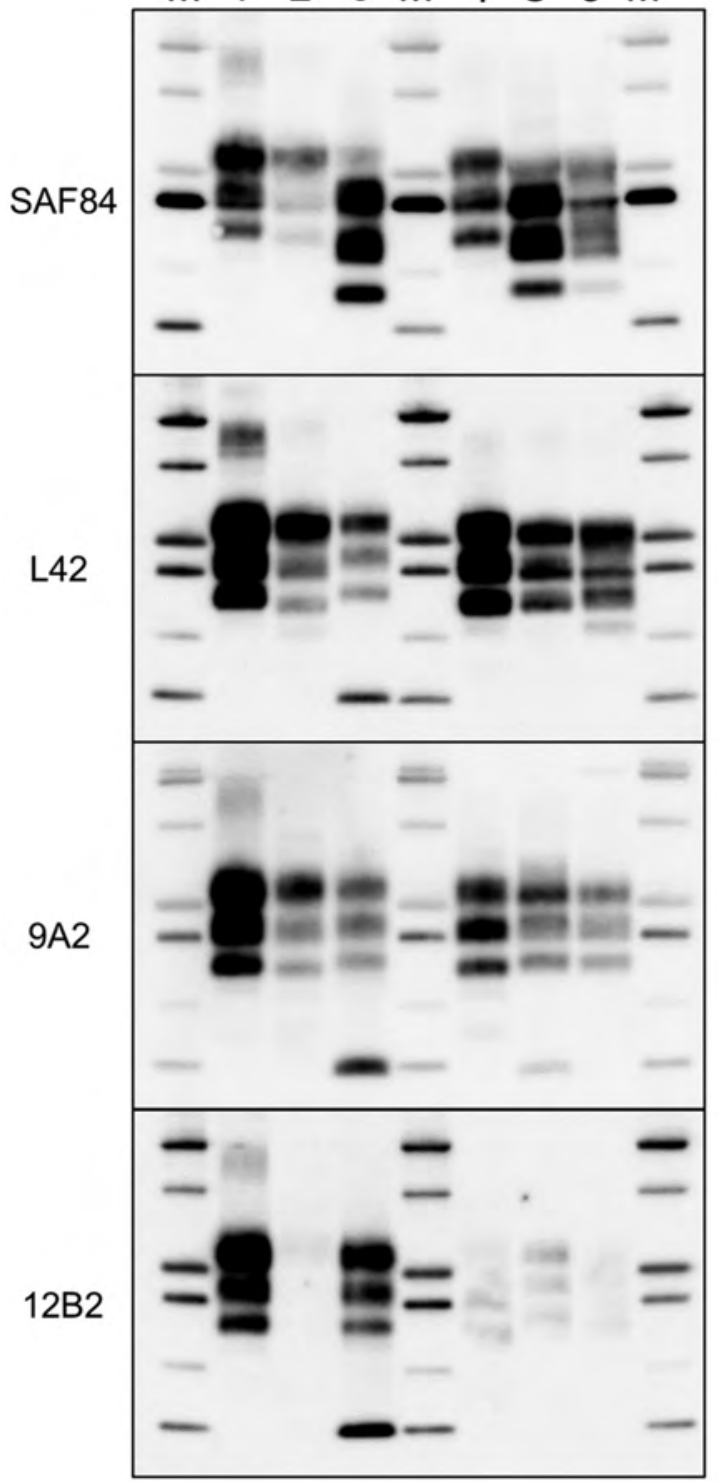

Figure 6. Comparison of protease-resistant core of abnormal form of prion protein from moose (Alces alces) in Europe with chronic wasting disease and from cattle with BSE. Representative blots show epitope mapping analysis of protease-resistant core of abnormal form of prion protein in moose (lane 5 , moose no. 1; lane 6, moose no. 2) in comparison with different BSE isolates (lane 2, classical BSE; lane 3, H-type BSE; and lane 4, L-type BSE). A sheep scrapie isolate was loaded as control (lane 1). The antibodies are indicated on the left. Protein standards are shown in lane $M(10,15,20,25,37$, and $50 \mathrm{kDa})$. BSE, bovine spongiform encephalopathy. features different from Norway moose $\mathrm{PrP}^{\mathrm{Sc}}$ and were indistinguishable from other cervids with classical CWD. This finding suggests that the variant $\mathrm{PrP}^{\mathrm{Sc}}$ type observed in Norway moose could not simply reflect a host species factor. Notably, in both natural and experimental conditions, CWD-affected moose in North America have been reported to display disease features indistinguishable from CWD in other cervids and had detectable $\mathrm{PrP}^{\mathrm{Sc}}$ in lymphoid tissues $(21,27)$.

Species-specific amino acid polymorphisms in the cervid PrP are associated with CWD susceptibility, incubation time, and pathology (28-30). In transmission experiments, atypical features were reported in elk or wapiti and mule deer with genotypes associated with a relative resistance to disease, extension of the incubation period, or both $(31,32)$. Moose $\operatorname{PrP}$ is polymorphic at codon 109 (K/Q) and 209 $(\mathrm{M} / \mathrm{I})$, combined in 3 alleles: $\mathrm{K}_{109} \mathrm{M}_{209}$ (observed in Europe and North America), $\mathrm{Q}_{109} \mathrm{M}_{209}$ (observed in Europe), and $\mathrm{K}_{109} \mathrm{I}_{209}$ (observed in North America) $(33,34)$. The 3 moose with CWD from Norway had the $\mathrm{KK}_{109} \mathrm{MM}_{209}$ genotype, whereas the moose case from Canada used for comparison had the $\mathrm{KK}_{109} \mathrm{II}_{209}$ genotype. Thus, we cannot exclude that the differences observed between Norway and Canada moose in our study are dependent on differences in PrP genotype. However, a classical CWD phenotype has been reported in naturally (21) and experimentally infected (27) moose with the $\mathrm{KK}_{100} \mathrm{MM}_{209}$ genotype, suggesting that a difference at PrP codon 209 is probably not the cause of the variant phenotype observed in moose in Norway. All of these findings suggest that neither the species nor the individual PrP genotypes are likely to have caused the variant phenotypes observed and imply that this variant phenotype could represent a novel CWD strain.

CWD is known to be a highly contagious disease in North America; however, data relating to the disease in moose are sparse and insufficient to understand the epidemiology and the implications of CWD in this species. The apparent low CWD prevalence reported for moose in North America compared with other cervid species might be attributable to the individual social behavior of moose and the minimal habitat overlap between moose and other cervids in areas with CWD. Additionally, surveillance program design, disease variability, and host genetics might influence the prevalence of the disease. Based on the epizootic dynamics in North America, CWD plausibly could have become established in reindeer in Norway more than a decade ago (35). In this scenario, the disease in moose could possibly be linked to the disease observed in reindeer, with strain mutation or phenotype shift putatively caused by interspecific transmission. However, a main cause of strain mutation after interspecies transmission (i.e., PrP amino acid differences between the donor and host species) is not relevant in this case because reindeer and moose 
share the same PrP primary sequence. An alternative hypothesis could be that moose have a prion disease that is independent of the reindeer epidemic, being either specific to the Norwegian moose or acquired by species other than the reindeer.

The 3 moose were 13, 14, and 13 years of age. Although moose can reach ages beyond 20 years, we consider these moose as old because female moose $>10-12$ years of age start to show signs of senescence and declining survival and reproduction rates $(36,37)$. The old age of the moose, the absence of lymphoid tissue involvement, and the low disease prevalence observed so far ( 3 of 10,531 moose tested) could suggest that CWD in moose is less contagious than classical CWD or could represent a spontaneous TSE. The finding that the affected moose were from the same geographic area does not seem to support a spontaneous origin of the disease; however, the actual evidence for geographic clustering could have been biased by oversampling in Trøndelag County, where the first positive moose was detected. Lack of detailed data on the ages of the moose tested so far in different geographic areas prevents any definitive conclusion. Still, the recent detection of a positive moose in Finland, several hundred kilometers from Trøndelag County, might indicate that the disease is not restricted to Norway (38). The ongoing intensive surveillance in Norway and several European Union countries with large moose populations will help to better clarify the actual geographic distribution and prevalence and will be critical for understanding the contagious or spontaneous nature of the disease.

The 3 moose analyzed shared a distinctive IHC pattern, mainly characterized by intraneuronal accumulation of $\mathrm{PrP}^{\mathrm{Sc}}$, and common $\mathrm{PrP}^{\mathrm{Sc}}$ features, such as the proteinase $\mathrm{K}$ N-terminal cleavage and the presence of an additional CTF13 fragment. However, we also observed unexpected differences among the 3 moose. By WB, the CTF16 fragment was observed in moose nos. 2 and 3 but not in moose no. 1, whereas the nonglycosylated internal fragment of $10 \mathrm{kDa}$ was evident in moose nos. 1 and 3 but could not be detected in moose no. 2. Furthermore, we also showed that these differences did not depend on the brain area investigated. We cannot rule out that these slight differences might depend on technical issues rather than represent actual $\mathrm{PrP}^{\mathrm{Sc}}$ variations. The outcome of the ongoing bioassay experiments will help to clarify the meaning of the observed variations.

By comparing the moose $\operatorname{PrP}^{\mathrm{Sc}}$ features with other animal TSEs circulating in Europe, we found no evidence of similarities with bovine and ovine prions. Minimal similarities were observed with CH1641 samples; however, CH1641 cases have not yet been detected in Norway. Bioassay in a large spectrum of rodent models will assist in determining whether these molecular similarities imply biologic association between the atypical CWD in moose and small ruminant CH1641. Transmission studies in several rodent models are under way and will help to clarify whether the different phenotype observed (designated Nor$16 \mathrm{CWD}$ ) could reflect the presence of a new cervid prion strain in moose from Norway.

\section{Acknowledgments}

The authors thank the Department of Pathology at the Norwegian Veterinary Institute for their excellent work with TSE testing and sample collection, Liv Midthjell for her skillful PRNP genotyping, Magne Haugum for the necropsy of 2 of the moose, and Jacques Grassi for kindly providing the Norwegian Veterinary Institute with SAF84 mAbs.

This work was supported in part by a grant from the Norwegian Ministry of Agriculture.

\section{About the Author}

Dr. Pirisinu is a researcher at the Istituto Superiore di Sanità in Rome, Italy. Her primary research interests include the prion strain characterization and zoonotic potential of animal prion diseases.

\section{References}

1. Prusiner SB. Molecular biology of prion diseases. Science. 1991;252:1515-22. http://dx.doi.org/10.1126/science.1675487

2. Benestad SL, Telling GC. Chronic wasting disease: an evolving prion disease of cervids. Handb Clin Neurol. 2018;153:135-51. http://dx.doi.org/10.1016/B978-0-444-63945-5.00008-8

3. EFSA Panel on Biological Hazards. Scientific opinion on chronic wasting disease (CWD) in cervids. EFSA J. 2017;15:4667.

4. Kim TY, Shon HJ, Joo YS, Mun UK, Kang KS, Lee YS. Additional cases of Chronic Wasting Disease in imported deer in Korea. J Vet Med Sci. 2005;67:753-9. http://dx.doi.org/10.1292/ jvms.67.753

5. Miller MW, Swanson HM, Wolfe LL, Quartarone FG, Huwer SL, Southwick CH, et al. Lions and prions and deer demise. PLoS One. 2008;3:e4019. http://dx.doi.org/10.1371/journal.pone.0004019

6. Edmunds DR, Kauffman MJ, Schumaker BA, Lindzey FG, Cook WE, Kreeger TJ, et al. Chronic wasting disease drives population decline of white-tailed deer. PLoS One. 2016; 11:e0161127. http://dx.doi.org/10.1371/journal.pone.0161127

7. Benestad SL, Mitchell G, Simmons M, Ytrehus B, Vikøren T. First case of chronic wasting disease in Europe in a Norwegian free-ranging reindeer. Vet Res (Faisalabad). 2016;47:88. http://dx.doi.org/10.1186/s13567-016-0375-4

8. Rolandsen CM, Solberg EJ, Heim M, Holmstrøm F, Solem MI, Sæther B-E. Accuracy and repeatability of moose (Alces alces) age as estimated from dental cement layers. Eur J Wildl Res. 2008;54:6-14. http://dx.doi.org/10.1007/s10344-007-0100-8

9. Pirisinu L, Migliore S, Di Bari MA, Esposito E, Baron T, D’Agostino C, et al. Molecular discrimination of sheep bovine spongiform encephalopathy from scrapie. Emerg Infect Dis. 2011;17:695-8. http://dx.doi.org/10.3201/eid1704.101215

10. Nicot S, Baron TG. Strain-specific proteolytic processing of the prion protein in prion diseases of ruminants transmitted in ovine transgenic mice. J Gen Virol. 2010;91:570-4. http://dx.doi.org/10.1099/vir.0.014464-0 
11. Kaluz S, Kaluzova M, Flint APF. Sequencing analysis of prion genes from red deer and camel. Gene. 1997;199:283-6. http://dx.doi.org/10.1016/S0378-1119(97)00382-X

12. Community Reference Laboratory of the European Union. TSE strain characterization in small ruminants-a technical handbook for national reference laboratories in the EU. Version 8. December 2016 [cited 2016 Dec 12]. https://science.vla.gov.uk/tse-lab-net/ documents/tse-oie-rl-handbook.pdf

13. Rolandsen CM, Solberg EJ, Saether B-E, Moorter BV, Herfindal I, Bjørneraas K. On fitness and partial migration in a large herbivore-migratory moose have higher reproductive performance than residents. Oikos. 2017;126:547-55. http://dx.doi.org/10.1111/ oik.02996

14. Bunnefeld N, Börger L, van Moorter B, Rolandsen CM, Dettki H, Solberg EJ, et al. A model-driven approach to quantify migration patterns: individual, regional and yearly differences. J Anim Ecol. 2011; 80:466-76. http://dx.doi.org/10.1111/j.1365-2656.2010. 01776.x

15. Meisingset EL, Loe LE, Brekkum Ø, Bischof R, Rivrud IM, Lande US, et al. Spatial mismatch between management units and movement ecology of a partially migratory ungulate. J Appl Ecol. 2018;55:745-53. http://dx.doi.org/10.1111/1365-2664.13003

16. Spraker TR, Miller MW, Williams ES, Getzy DM, Adrian WJ, Schoonveld GG, et al. Spongiform encephalopathy in free-ranging mule deer (Odocoileus hemionus), white-tailed deer (Odocoileus virginianus) and Rocky Mountain elk (Cervus elaphus nelsoni) in northcentral Colorado. J Wildl Dis. 1997;33:1-6. http://dx.doi.org/10.7589/0090-3558-33.1.1

17. Williams ES, Young S. Neuropathology of chronic wasting disease of mule deer (Odocoileus hemionus) and elk (Cervus elaphus nelsoni). Vet Pathol. 1993;30:36-45. http://dx.doi.org/10.1177/ 030098589303000105

18. Spraker TR, Zink RR, Cummings BA, Wild MA, Miller MW, O'Rourke KI. Comparison of histological lesions and immunohistochemical staining of proteinase-resistant prion protein in a naturally occurring spongiform encephalopathy of free-ranging mule deer (Odocoileus hemionus) with those of chronic wasting disease of captive mule deer. Vet Pathol. 2002;39:110-9. http://dx.doi.org/10.1354/vp.39-1-110

19. Pirisinu L, Nonno R, Esposito E, Benestad SL, Gambetti P, Agrimi U, et al. Small ruminant nor98 prions share biochemical features with human gerstmann-sträussler-scheinker disease and variably protease-sensitive prionopathy. PLoS One. 2013;8:e66405. http://dx.doi.org/10.1371/journal.pone.0066405

20. Baron T, Bencsik A, Vulin J, Biacabe AG, Morignat E, Verchere J, et al. A C-terminal protease-resistant prion fragment distinguishes ovine "CH1641-like" scrapie from bovine classical and L-Type BSE in ovine transgenic mice. PLoS Pathog. 2008;4:e1000137. http://dx.doi.org/10.1371/journal.ppat.1000137

21. Baeten LA, Powers BE, Jewell JE, Spraker TR, Miller MW. A natural case of chronic wasting disease in a free-ranging moose (Alces alces shirasi). J Wildl Dis. 2007;43:309-14. http://dx.doi.org/10.7589/0090-3558-43.2.309

22. Moreno JA, Telling GC. Molecular mechanisms of chronic wasting disease prion propagation. Cold Spring Harb Perspect Med. 2018;8:a024448. http://dx.doi.org/10.1101/cshperspect.a024448

23. Haley NJ, Hoover EA. Chronic wasting disease of cervids: current knowledge and future perspectives. Annu Rev Anim Biosci. 2015;3:305-25. http://dx.doi.org/10.1146/annurev-animal022114-111001

24. Raymond GJ, Raymond LD, Meade-White KD, Hughson AG, Favara C, Gardner D, et al. Transmission and adaptation of chronic wasting disease to hamsters and transgenic mice: evidence for strains. J Virol. 2007;81:4305-14. http://dx.doi.org/10.1128/ JVI.02474-06

25. Angers RC, Kang HE, Napier D, Browning S, Seward T, Mathiason C, et al. Prion strain mutation determined by prion protein conformational compatibility and primary structure. Science. 2010;328:1154-8. http://dx.doi.org/10.1126/ science. 1187107

26. Perrott MR, Sigurdson CJ, Mason GL, Hoover EA. Evidence for distinct chronic wasting disease (CWD) strains in experimental CWD in ferrets. J Gen Virol. 2012;93:212-21. http://dx.doi.org/ 10.1099/vir.0.035006-0

27. Kreeger TJ, Montgomery DL, Jewell JE, Schultz W, Williams ES. Oral transmission of chronic wasting disease in captive Shira's moose. J Wildl Dis. 2006;42:640-5. http://dx.doi.org/ 10.7589/0090-3558-42.3.640

28. Jewell JE, Conner MM, Wolfe LL, Miller MW, Williams ES. Low frequency of PrP genotype 225SF among free-ranging mule deer (Odocoileus hemionus) with chronic wasting disease. J Gen Virol. 2005;86:2127-34. http://dx.doi.org/10.1099/ vir.0.81077-0

29. Johnson CJ, Herbst A, Duque-Velasquez C, Vanderloo JP, Bochsler P, Chappell R, et al. Prion protein polymorphisms affect chronic wasting disease progression. PLoS One. 2011;6:e17450. http://dx.doi.org/10.1371/journal.pone.0017450

30. Robinson SJ, Samuel MD, O'Rourke KI, Johnson CJ. The role of genetics in chronic wasting disease of North American cervids. Prion. 2012;6:153-62. http://dx.doi.org/10.4161/pri.19640

31. O’Rourke KI, Spraker TR, Zhuang D, Greenlee JJ, Gidlewski TE, Hamir AN. Elk with a long incubation prion disease phenotype have a unique PrPd profile. Neuroreport. 2007;18:1935-8. http://dx.doi.org/10.1097/WNR.0b013e3282flca2f

32. Wolfe LL, Fox KA, Miller MW. "Atypical” chronic wasting disease in PRNP genotype 225FF mule deer. J Wildl Dis. 2014;50:660-5. http://dx.doi.org/10.7589/2013-10-274

33. Huson HJ, Happ GM. Polymorphisms of the prion protein gene (PRNP) in Alaskan moose (Alces alces gigas). Anim Genet. 2006; 37:425-6. http://dx.doi.org/10.1111/j.1365-2052.2006.01466.x

34. Wik L, Mikko S, Klingeborn M, Stéen M, Simonsson M, Linné T. Polymorphisms and variants in the prion protein sequence of European moose (Alces alces), reindeer (Rangifer tarandus), roe deer (Capreolus capreolus) and fallow deer (Dama dama) in Scandinavia. Prion. 2012;6:256-60. http://dx.doi.org/10.4161/ pri.19641

35. EFSA Panel on Biological Hazards. Scientific opinion on chronic wasting disease (II). EFSA J. 2018;16:5132.

36. Ericsson G, Wallin K. Age-specific moose (Alces alces) mortality in a predator-free environment: evidence for senescence in females. Ecoscience. 2001;8:157-63. http://dx.doi.org/10.1080/11956860.20 01.11682641

37. Ericsson G, Wallin K, Ball JP, Broberg M. Age-related reproductive effort and senescence in free-ranging moose, Alces alces. Ecology. 2001;82:1613. http://dx.doi.org/10.2307/2679804

38. Finnish Food Safety Authority (Evira). Moose found dead in forest with chronic wasting disease [cited 2018 Mar 8]. https://www.evira.fi/en/animals/current_issues/2018/moose-founddead-in-forest-with-chronic-wasting-disease

Address for correspondence: Sylvie L. Benestad, Norwegian Veterinary Institute, Pathology, PO Box 750 Sentrum, 0106 Oslo, Norway; email: sylvie.benestad@vetinst.no 


\title{
Genomic Characterization of B-Glucuronidase-Positive Escherichia coli 0157:H7 Producing Stx2a
}

\author{
Yoshitoshi Ogura, Kazuko Seto, Yo Morimoto, Keiji Nakamura, Mitsuhiko P. Sato, \\ Yasuhiro Gotoh, Takehiko Itoh, Atsushi Toyoda, Makoto Ohnishi, Tetsuya Hayashi
}

\begin{abstract}
Among Shiga toxin (Stx)-producing Escherichia coli (STEC) O157:H7 strains, those producing Stx2a cause more severe diseases. Atypical STEC O157:H7 strains showing a $\beta$-glucuronidase-positive phenotype (GP STEC O157:H7) have rarely been isolated from humans, mostly from persons with asymptomatic or mild infections; Stx2aproducing strains have not been reported. We isolated, from a patient with bloody diarrhea, a GP STEC 0157:H7 strain (PV15-279) that produces Stx2a in addition to Stx1a and Stx2c. Genomic comparison with other STEC 0157 strains revealed that PV15-279 recently emerged from the stx1a/ stx2c-positive GP STEC 0157:H7 clone circulating in Japan. Major virulence genes are shared between typical ( $\beta$-glucuronidase-negative) and GP STEC 0157:H7 strains, and the Stx2-producing ability of PV15-279 is comparable to that of typical STEC 0157:H7 strains; therefore, PV15279 presents a virulence potential similar to that of typical STEC 0157:H7. This study reveals the importance of GP O157:H7 as a source of highly pathogenic STEC clones.
\end{abstract}

Shiga toxin (Stx)-producing Escherichia coli (STEC)

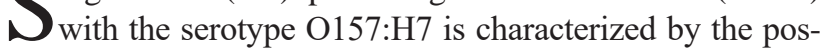
session of stx gene(s), the locus of enterocyte effacement (LEE) -encoded type 3 secretion system (T3SS), and a large virulence plasmid (pO157) that encodes enterohemolysin and other virulence factors, such as catalase-peroxidase KatP, a type II secretion system, and the protease EspP (1). Non-sorbitol-fermenting (NSF) and $\beta$-glucuronidasenegative (GN) STEC O157:H7 (hereafter referred to as typical STEC O157:H7), the major clone among currently

Author affiliations: Kyushu University, Fukuoka, Japan (Y. Ogura, K. Nakamura, M.P. Sato, Y. Gotoh, T. Hayashi); Osaka Institute of Public Health, Osaka, Japan (K. Seto); Hokkaido Institute of Public Health, Hokkaido, Japan (Y. Morimoto); Tokyo Institute of Technology, Tokyo, Japan (T. Itoh); National Institute of Genetics, Shizuoka, Japan (A. Toyoda); National Institute of Infectious Diseases, Tokyo (M. Ohnishi)

DOI: https://doi.org/10.3201/eid2412.180404 circulating STEC O157:H7 strains, frequently causes large outbreaks of severe enteric infections, including diarrhea, hemorrhagic colitis, and hemolytic uremic syndrome. Stxs are classified as Stx 1 or Stx2; Stx 1 currently includes 3 subtypes (stx1a, stx1c, and stx1d) and Stx2 includes 7 subtypes (stx2a to $s t x 2 g$ ) (2). The stx genes are encoded by lambdalike phages and have been acquired by STEC strains through phage transduction (3). Typical STEC O157:H7 produces Stx1a, Stx2a, and Stx2c, either alone or in combination. Stx2a-producing strains cause more severe infections than do Stx1a-producing strains (4). In addition, the levels of Stx 2 production among the STEC O157:H7 strains carrying stx2c, but not stx2a, are typically very low (5-7).

According to the stepwise evolution model $(8,9)$, STEC O157:H7 evolved from ancestral enteropathogenic E. coli (EPEC) O55:H7 (sorbitol-fermenting [SF] and $\beta$-glucuronidase-positive [GP]; clonal complex [CC] A1) through sequential acquisitions of virulence factors and phenotypic traits along with a serotype change (Figure 1). Two phenotypic variants of STEC O157 are known: SF/GP STEC O157:H- (nonmotile) (CC A4), known as the German clone (SF STEC O157:H-), and NSF/GP STEC O157:H7 (CC A5) (GP STEC O157:H7). Both variants are postulated to have emerged from a hypothetical intermediate, CC A3; typical STEC O157:H7 (CC A6) has further emerged from CC A5.

SF STEC O157:H- strains are usually stx2a positive (10); like typical STEC O157:H7, they have caused many outbreaks and sporadic cases of hemolytic uremic syndrome in Germany and other countries in Europe. Therefore, this clone is generally thought to be highly pathogenic (11-13). In contrast, although GP STEC O157:H7 strains have been reported to carry both stx 1 and stx 2 or only stx2 (14-16), strains producing the Stx2a subtype have not been described. Although GP STEC O157:H7 was first isolated from a patient with bloody diarrhea in 1994 (14), this variant has rarely been isolated from humans. Moreover, human isolates obtained to date have generally been isolated from patients with asymptomatic or mild infections (16). 


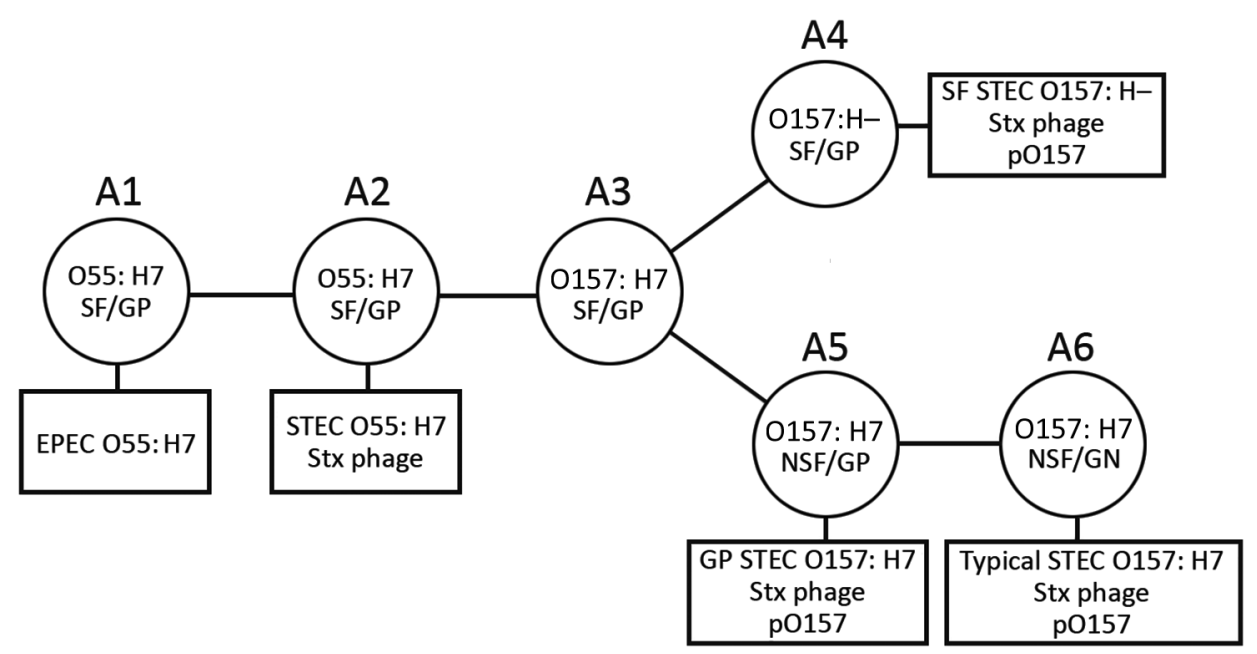

Figure 1. Schematic illustrating model of the stepwise evolution of STEC 0157. The proposed stepwise evolution model of STEC 0157 was schematically illustrated according to previous reports $(8,9)$. Clonal complexes (CCs) A1 to A6 are indicated, along with phenotypic changes, antigen shifts, and acquisitions of Stx phages and pO157. Squares indicate contemporary circulating STEC 0157 clones. EPEC, enteropathogenic E. coli; GP, $\beta$-glucuronidase-positive; NSF, non-sorbitol-fermenting; SF, sorbitol-fermenting; STEC, Shiga toxin-producing $E$. coli.
The genomic information available for GP STEC O157:H7 is also limited to strain G5101 $(9,17)$ and 4 strains in public databases. Thus, the virulence potential of GP STEC O157:H7 remains to be fully elucidated.

In this study, we isolated a GP STEC O157:H7 strain that produces Stx2a, in addition to Stx1a and Stx2c, from a patient with bloody diarrhea in Japan and determined its complete genome sequence. To reveal the genomic features of the Stx2a-producing strain, we determined the draft genome sequences of an additional 13 GP STEC O157:H7 isolates, which are all Stx2a negative but Stx1a/Stx2c (or Stx2c) positive, and performed fine phylogenetic and genomic comparisons of these GP STEC O157:H7 strains with typical STEC O157:H7 and SF STEC O157:Hstrains. We also analyzed the Stx production levels of GP STEC O157:H7 strains.

\section{Methods}

\section{Bacterial Strains}

The strains used in this study are listed in online Technical Appendix Table 1 (http://wwwnc.cdc.gov/EID/ article/24/12/18-0404-Techapp1.pdf). The GP STEC O157:H7 strain PV15-279 was isolated from an adult patient in Japan who was hospitalized in 2015 with severe symptoms, including bloody diarrhea. The other 13 GP STEC O157:H7 strains sequenced in this study were isolated in Japan during 1988-2013 from humans with or without symptoms. We obtained genome sequence information for 3 E. coli O55:H7 (18-20), 2 SF STEC O157:H-, 14 typical STEC O157:H7 (21-26), and 2 GP STEC O157:H7 strains (17) from public databases.

\section{Subtyping of stx Genes}

We performed in silico subtyping of $s t x 1$ and stx2 in all strains analyzed in this study. We used blastn
(https://blast.ncbi.nlm.nih.gov/Blast; $>99 \%$ identity and $>99 \%$ coverage) for comparisons with previously reported reference sequences (2).

\section{Genome Sequencing, Assembly, and Annotation}

We determined the complete genome sequence of PV15279 using PacBio reads obtained with an RS II system (PacBio, Menlo Park, CA, USA). We assembled the reads with Canu version 1.5 (27) and circularized them using Circlator (28). We obtained Illumina paired-end reads $(300 \mathrm{bp} \times 2)$ with a MiSeq sequencer (Illumina, San Diego, CA, USA) and mapped them to the assembly using the Burrows-Wheeler aligner (29) for sequence-error correction with Pilon (30). We completed further corrections to the sequences corresponding to Stx phage regions using MiSeq reads obtained by sequencing long-range PCR products covering each Stx phage. We performed annotations with the DDBJ Fast Annotation and Submission Tool (31), followed by manual curation using the IMC-GE software (In Silico Biology, Kanagawa, Japan).

We obtained the draft genome sequences of $13 \mathrm{GP}$ STEC O157:H7 strains by assembling Illumina paired-end reads using Platanus (32). The Illumina reads for strain LB473017, for which the only read data available were from public databases, were also assembled with Platanus.

The genome sequences of PV15-279 and the 13 GP STEC O157:H7 strains have been deposited in DDBJ/ EMBL/GenBank under the Bioproject accession numbers PRJDB6584 and PRJDB6498, respectively. The accession numbers of each sample, including the reference data, are listed in online Technical Appendix Table 1.

\section{Single-Nucleotide Polymorphism Detection and Phylogenetic Analysis}

We performed single-nucleotide polymorphism (SNP) detection and phylogenetic analyses as described previously 
(33). The genome sequences to be examined were aligned with the phage- and insertion sequence (IS)-masked chromosome sequence of the STEC O157:H7 strain Sakai (22) using MUMmer (34) to identify conserved regions (cutoff thresholds $>98 \%$ sequence identity and $>1,000$-bp alignment length) in each strain and the SNP sites located therein. We then determined the core genome sequence that was conserved in all strains examined. Only SNPs located in the core genome were subjected to further analysis. After reconstructing the genome sequences of each strain using the SNPs and removing recombinogenic SNP sites using Gubbins (35), we constructed a maximum-likelihood phylogenetic tree using RAxML (36) with the general timereversible plus gamma model of nucleotide substitution and 500 bootstrap replicates. The tree was displayed and annotated using iTOL (37).

\section{Repertoire Analysis of Genes Encoding T3SS Effectors and Plasmid-Encoded Virulence Factors}

We analyzed the conservation of genes encoding T3SS effectors and plasmid-encoded virulence factors with blastn ( $>90 \%$ identity and $>30 \%$ coverage). All intact effector genes and plasmid virulence genes identified in strains Sakai and PV15-279 were clustered using CD-HIT (38) at $\geq 90 \%$ identity and $\geq 30 \%$ alignment coverage of the longer sequences, and representative sequences of each cluster were used to create a database for blastn analysis.

\section{Stx Phage Sequencing}

We determined the complete sequences of the Stx1a and Stx2c phages from strain 980938 (from an asymptomatic carrier) and the Stx2c phage from strain 981447 (from a patient with bloody diarrhea) as described previously (39). We constructed fosmid libraries of the 2 strains using a CopyControl fosmid library production kit (Epicenter Biotechnologies, Madison, WI, USA). We screened the stx1or stx2-containing clones using PCR and sequenced them by the shotgun sequencing strategy using an ABI3730 sequencer (Applied Biosystems, Foster City, CA, USA).

\section{Stx Production Assay}

We inoculated bacterial cells into $40 \mathrm{~mL}$ of CAYE broth (Merck, Darmstadt, Germany) and grew them to mid-log phase at $37^{\circ} \mathrm{C}$ with shaking. We then added mitomycin $\mathrm{C}$ (MMC) to the culture at a final concentration of $500 \mathrm{ng} /$ $\mathrm{mL}$. After MMC addition, we collected $100 \mu \mathrm{L}$ of the culture every hour and immediately subjected it to sonication using a Bioruptor (Cosmo Bio, Tokyo, Japan). We obtained the soluble fractions of each cell lysate via centrifugation at $14,000 \times g$ for $10 \mathrm{~min}$ at $4^{\circ} \mathrm{C}$. We determined Stx1 and Stx2 concentrations in each cell lysate using a previously described sandwich ELISA (40). We captured Stx using RIDASCREEN Verotoxin microtiter plates (R-Biopharm,
Darmstadt, Germany) coated with capture antibodies that recognize both Stx1 and Stx2. We conjugated monoclonal antibodies against Stx1 and Stx2 (LSBio, Seattle, WA, USA) with horseradish peroxidase using the Peroxidase Labeling Kit- $\mathrm{NH}_{2}$ (Dojindo, Kumamoto, Japan) and employed them as detection antibodies. We used reagents supplied in the RIDASCREEN Verotoxin kit for detection. Finally, we measured the absorbance at $450 \mathrm{~nm}\left(\mathrm{~A}_{450}\right)$ using Tecan Infinite 200 PRO (Tecan, Männedorf, Switzerland).

\section{Results}

\section{Isolation and Genome Sequencing of GP 0157:H7 Strains}

In PV15-279, we detected stx2a in addition to stx1a and stx2c (online Technical Appendix Table 1). Determination of the complete genome sequence of PV15-279 revealed that the genome consisted of a 5,598,151-bp chromosome and a 94,391-bp plasmid. The draft genome sequences of 13 additional GP STEC O157:H7 strains were also determined for comparison (online Technical Appendix Table 1). These strains were all stx1a/stx2c-positive and stx2a-negative. The uidA gene, which encodes $\beta$-glucuronidase, was intact in all the GP STEC O157:H7 strains sequenced in this study, as well as in 2 GP STEC O157:H7 strains whose draft genome sequences were publicly available (online Technical Appendix Table 1). In typical STEC O157:H7, uidA has been inactivated by a frameshift mutation (41).

\section{Comparisons of General Genomic Features and Mobile Genetic Elements}

Although the chromosome of PV15-279 was $\approx 100 \mathrm{~kb}$ larger than that of the typical GP STEC O157:H7 strain Sakai (Table and online Technical Appendix Figure 1), the chromosome backbone was highly conserved; $97.2 \%$ of

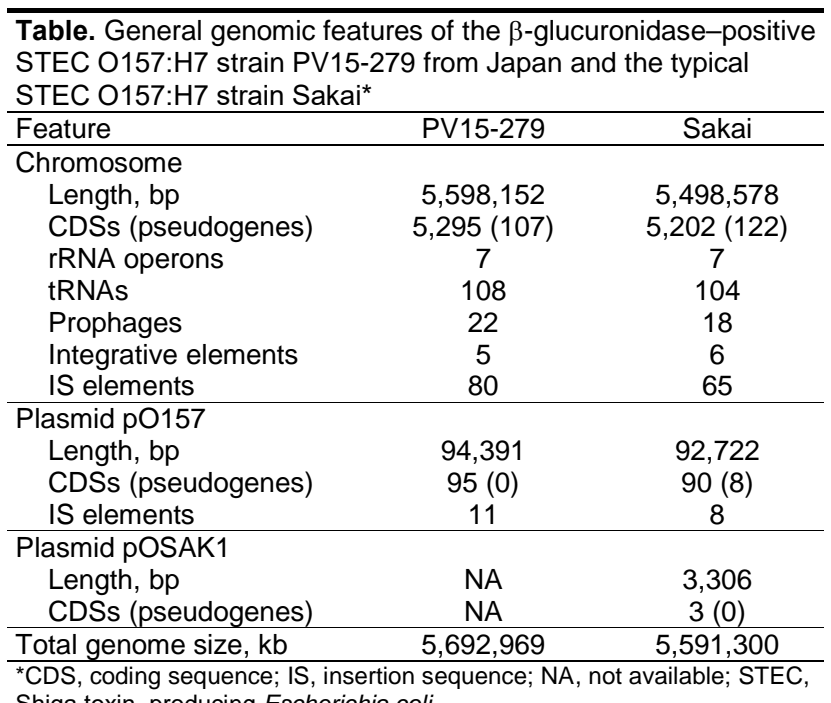


the coding sequences (CDSs) identified in Sakai were conserved in PV15-279. Among the various types of mobile genetic elements (MGEs), integrative elements (defined as elements that contain an integrase gene but no other MGErelated genes) were well conserved and showed only minor variations. Among the 5 integrative elements identified in Sakai, 1 small element (SpLE2) was missing in PV15-279, and multiple small structural variations were observed in 3 elements (online Technical Appendix Table 2, Figure 2, panel B). In contrast, the prophage contents exhibited notable differences. PV15-279 contained 22 prophages, whereas Sakai contained 18 prophages. This difference is attributable mainly to the difference in chromosome sizes between the 2 strains. Although most of the integration sites used by Sakai prophages (13/16) are also used by PV15-279 prophages (online Technical Appendix Table 2, Figure 2, panel A), in most instances prophages that had integrated into the same site showed notable variations between the 2 strains, suggesting the existence of different evolutionary histories. Furthermore, PV15-279 carried as many as 4 Stx phages: 1 Stx 1a phage, 2 Stx2a phages, and 1 Stx2c phage (online Technical Appendix Figure 1). In contrast, Sakai carries 1 Stx1a phage and 1 Stx2a phage (see subsequent sections for a comparison of these phages with Stx phages from various STEC O157:H7 strains). Surprisingly, 1 of the 2 Stx2a phages (PV15p10) was integrated together with 3 prophages at the $y d f J$ locus in PV15-279 in tandem (online Technical Appendix Table 2, Figure 1, Figure 2, panel A).

The repertoires and total copy numbers of IS elements also exhibited notable differences (online Technical Appendix Table 3). Although 24 types of IS elements were identified in Sakai and 18 types were identified in PV15279 , only 14 were shared by the 2 strains. A greater number of total copies of IS elements was detected in PV15-279 (91, compared with 73 in Sakai), primarily because of the proliferation of IS1203 (also annotated as IS629) in PV15279. The virulence plasmid pO157 also showed notable differences, as described in the next section.

\section{Phylogenetic Characterization of GP STEC 0157:H7 Strains}

To determine the precise phylogenetic position of PV15-279 in STEC O157, we performed high-resolution phylogenetic analysis using the genome sequences of various typical and atypical STEC O157 strains (Figure 2). The results clearly indicate that PV15-279 formed a distinct cluster with all other GP STEC O157:H7 strains, including 2 US isolates, and is a strain that recently emerged from the stx1a/stx2c-positive clone circulating in Japan. The phylogenetic relationship of this cluster with other STEC O157 lineages was concordant with the stepwise evolution model (Figure 1).

In our phylogenetic tree, the GP STEC O157:H7 strains were relatively clonal compared with the typical

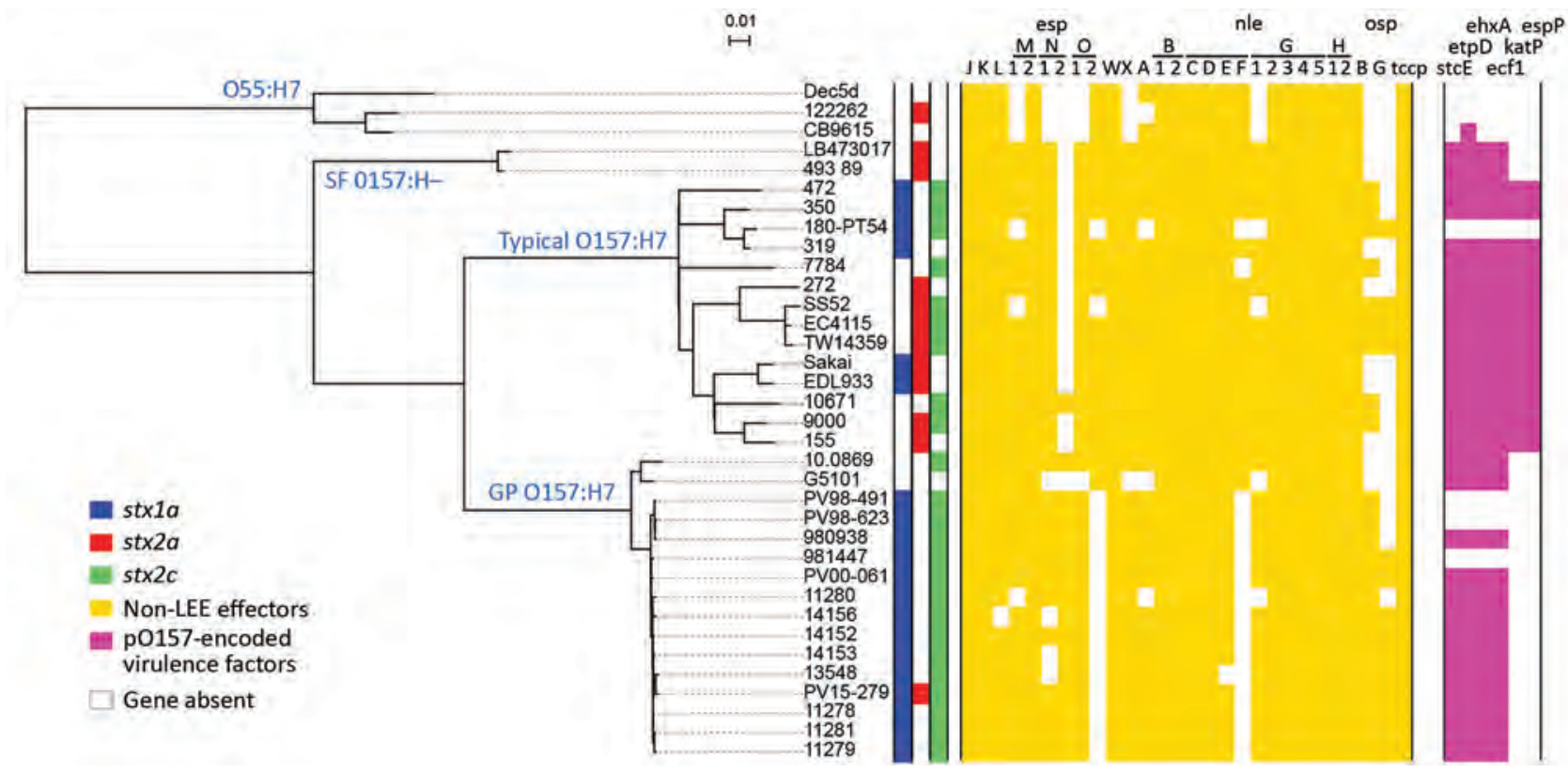

Figure 2. Whole-genome sequence-based phylogenetic analysis and repertoires of T3SS effectors and plasmid-encoding virulence factors from the study of Stx-producing E. coli O157:H7. The genome sequences of all the strains used in this study were aligned with the complete chromosome sequence of Sakai, and the single-nucleotide polymorphisms located in the 4,074,209-bp backbone sequence that were conserved in all the test strains were identified. After removing the recombinogenic single-nucleotide polymorphisms sites, we performed a concatenated alignment of 5,803 informative sites to generate the maximum-likelihood phylogeny. The conservation of T3SS effectors and plasmid-encoding virulence factors is shown in the tree. Colored boxes indicate the presence, and open boxes the absence, of each gene. GP, $\beta$-glucuronidase-positive; LEE, locus of enterocyte effacement; SF, sorbitol-fermenting. Scale bars indicate nucleotide substitutions per site. 
STEC O157:H7 strains, although these typical STEC O157:H7 strains were selected to represent their currently known phylogenetic diversity (26) (Figure 2). Despite the close phylogenetic relationship between the GP STEC O157:H7 strains, only PV15-279 was stx2a-positive, indicating that PV15-279 acquired stx2a very recently. No stx genes were detected in the publicly available sequence data for the GP STEC O157:H7 strain G5101, although this strain was previously reported to contain stx1 and stx2 (14).

\section{Distribution of T3SS Effectors in the GP 0157:H7 Lineage}

In addition to 5 LEE-encoded T3SS effectors, 18 families of effectors are encoded at non-LEE genomic loci (non-LEE effectors) in Sakai (42). In PV15-279, we identified most of the effector families found in Sakai (online Technical Appendix Table 4). The major differences were the absence of nleF in PV15-279 and the presence of $о s p B$ and $o s p G$ in PV15-279, both of which are absent in Sakai. After subgrouping the 4 effector families (espM, espN, espO, and nleG) into 2-5 subgroups based on their sequence diversity, we analyzed the effector repertoires of the STEC O157 isolates used in the phylogenetic analysis (Figure 2). This analysis revealed that the effectors identified in PV15-279 were mostly conserved in other GP STEC O157:H7 strains, although some variations were detected.

\section{Virulence Plasmid p0157 of GP 0157:H7 Lineage}

The pO157 plasmid of PV15-279 was nearly identical to the plasmid from the GP STEC O157:H7 strain G5101 (43), with several small variations that were apparently generated by mechanisms involving IS (online Technical Appendix Figure 3). The plasmid-encoded virulence genes identified in PV15-279 were well conserved in other GP STEC O157:H7 strains, except for 3 strains from which pO157 has apparently been deleted (Figure 2). It is unknown whether this deletion occurred before or after strain isolation.

As previously reported (43), the pO157 plasmids from GP STEC O157:H7 showed high similarity to those from typical STEC O157:H7 and SF STEC O157:H-. Thus, a pO157-like plasmid was likely acquired by the common ancestor of the 3 STEC 0157 lineages. A notable difference between the pO157 plasmids of the 3 STEC 0157 lineages was the distribution of katP and espP. These genes were detected only in typical STEC O157:H7 strains, suggesting that these genes may have been specifically acquired by the typical STEC O157:H7 lineage. Although the roles of katP and espP in STEC virulence in humans have not yet been elucidated, at least the SF STEC O157:H- strain causes severe infections even without these genes.

\section{Stx Phages of GP STEC 0157:H7}

As shown in Figure 3, we performed fine genomic comparisons of the Stx phages from PV15-279 with the Stx1a, Stx2a, and Stx2c phages from other STEC O157:H7 strains used in the phylogenetic analysis shown in Figure 2 (phages were included only when complete sequences were available). The Stx1a and Stx2c phages of the GP STEC O157:H7 strain 980938 and the Stx2c phage of the GP STEC O157:H7 strain 981447 were sequenced individually and included in the analysis.

Stx1a phages were integrated in $s b c B$ in the 2 GP STEC O157:H7 strains but were integrated in yehV or $\arg W$ in typical STEC O157:H7 strains. According to the dot plot analysis, the 2 Stxla phages from GP STEC O157:H7 were nearly identical (Figure 3). The phages from typical STEC 0157:H7 strains were classified into 3 groups based on sequence similarity. The Stx1a phages from GP STEC O157:H7 showed various levels of sequence similarity to each group. The highest similarity was observed for the 3 phages integrated in yeh $V$, but a clear difference was observed in the early region.

The 2 Stx2a phages from PV15-279 that integrated in yecE and $y d f J$ were nearly identical (only 2 SNPs), indicating that these phages were recently duplicated in this strain. With 1 exception (the phage from strain 155), the Stx2a phages from the typical STEC O157:H7 strains analyzed in this study were integrated in either wrbA or $\arg W$, and all shared a similar genomic structure, although considerable variations were observed in the early region, as reported previously (7). The Stx2a phages from PV15-279 exhibited a genetic structure similar to that of strain 155 , which was also integrated in yecE. However, those phages showed high sequence similarity only in limited regions.

The Stx2c phages from GP STEC O157:H7 strains (all integrated in yehV) were nearly identical, showing only minor variations, such as IS integration. The Stx2c phages from typical STEC O157:H7 strains, which were all integrated in $s b c B$, also exhibited very similar genomic structures and sequences. Although notable sequence similarity was observed between the Stx2c phages from GP STEC O157:H7 and typical STEC O157:H7, the early regions were very different.

\section{Stx Production by GP STEC 0157:H7 Strains}

Stx1 production is partially dependent on phage induction, whereas Stx2 production is strongly dependent on phage induction (44-46). We compared Stx production levels between GP STEC O157:H7 and typical STEC O157:H7 using MMC as a phage induction agent; we analyzed Stx1 and Stx2 production by representative strains of the 2 lineages (PV15-279, 981447, and 980938 for GP STEC O157:H7; Sakai and EDL933 for typical STEC O157:H7). MMC exhibited different levels of effectiveness in phage 

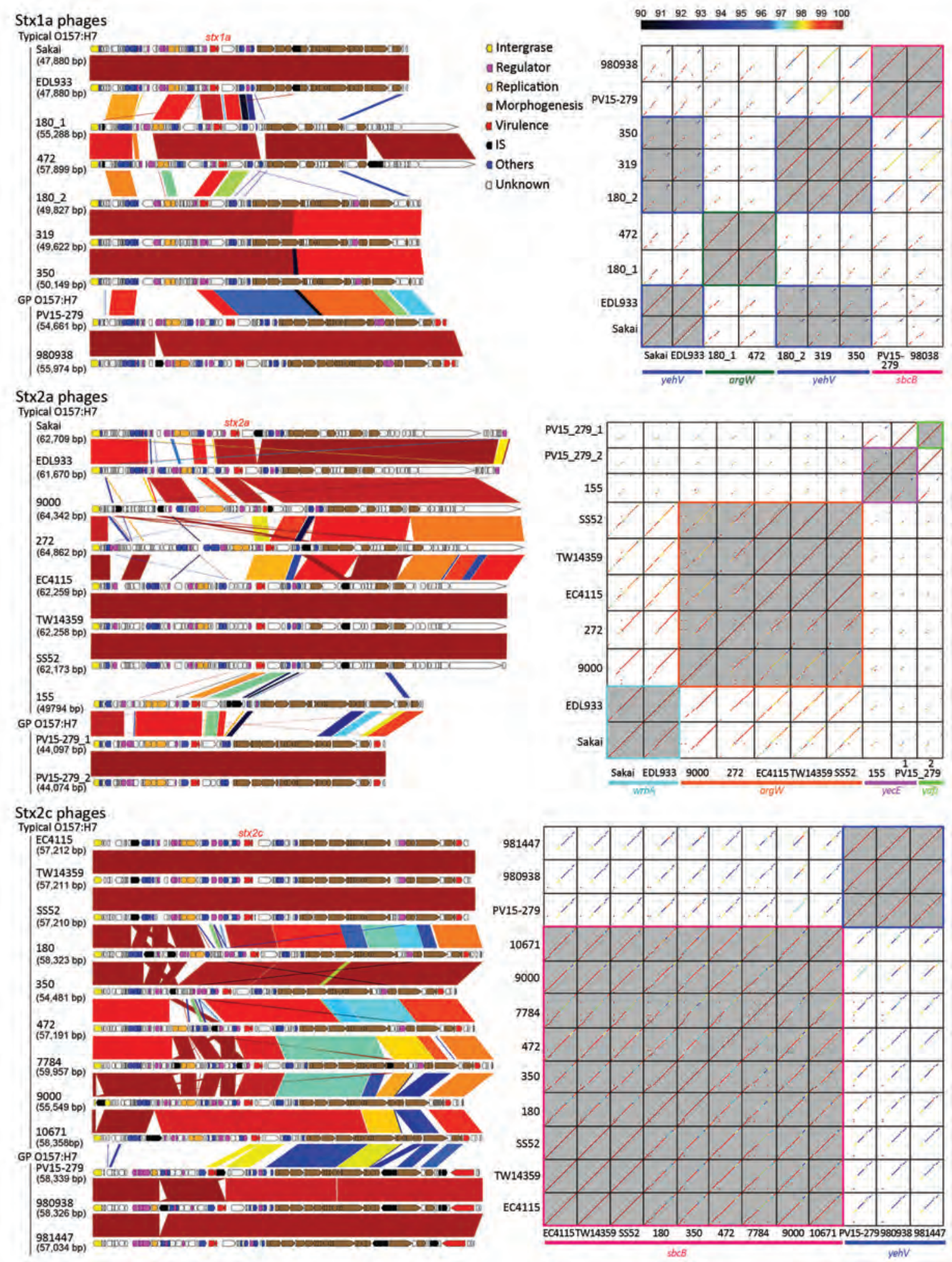

Figure 3. Genome comparisons of Stx phages from the study of Stx-producing E. coli O157:H7. The results of the comparison of the genome structure (left) and dot-plot sequence comparisons (right) of the Stx phages are shown. Sequence identities are indicated by different colors. In the dot-plot matrices, phages integrated in the same integration sites are highlighted by gray shading and colored frames. GP, $\beta$-glucuronidase-positive; IS, insertion sequence. 
induction in each strain, and clear cell lysis (a clear reduction in $\mathrm{OD}_{600}$ values) was observed only in EDL933 and PV15-279 (Figure 4, panel A).

Consistent with a previous report (47), the Stx1 concentration was not notably elevated by phage induction in any of the strains, and no clear difference was observed between the GP STEC O157:H7 and typical STEC O157:H7 strains (Figure 4, panel B). In contrast, levels of Stx2 production were highly variable, as previously shown for typical STEC O157:H7 strains (7). Although Stx2 production was poorly induced in 2 GP STEC O157:H7 strains carrying stx $2 c$ alone, it was strongly induced in the stx2a-positive PV15-279 and typical STEC O157:H7 strains (Figure 4, panel C). Therefore, stx2a was strongly induced in PV15279 , as in typical STEC O157:H7, but stx2c was poorly induced in PV15-279, as in the other GP STEC O157:H7 strains. The level of Stx2 production by PV15-279 was comparable to that of typical STEC 0157:H7 strains and similar to that of the Sakai strain.

\section{Discussion}

In this study, we isolated a GP STEC O157:H7 strain (PV15-279) that produces Stx2a in addition to Stx1a and Stx2c. The whole-genome sequence-based phylogenetic analysis, which included additional GP STEC O157:H7 strains and representative strains belonging to other $E$. coli O55/O157 lineages, revealed that PV15-279 recently emerged by the acquisition of Stx2a phage from the stx1a/ stx2c-positive GP STEC O157:H7 clone circulating in Japan (Figure 2). Most of the major virulence genes identified in typical STEC O157:H7, such as T3SS effector genes and plasmid-encoded virulence genes, were well conserved in
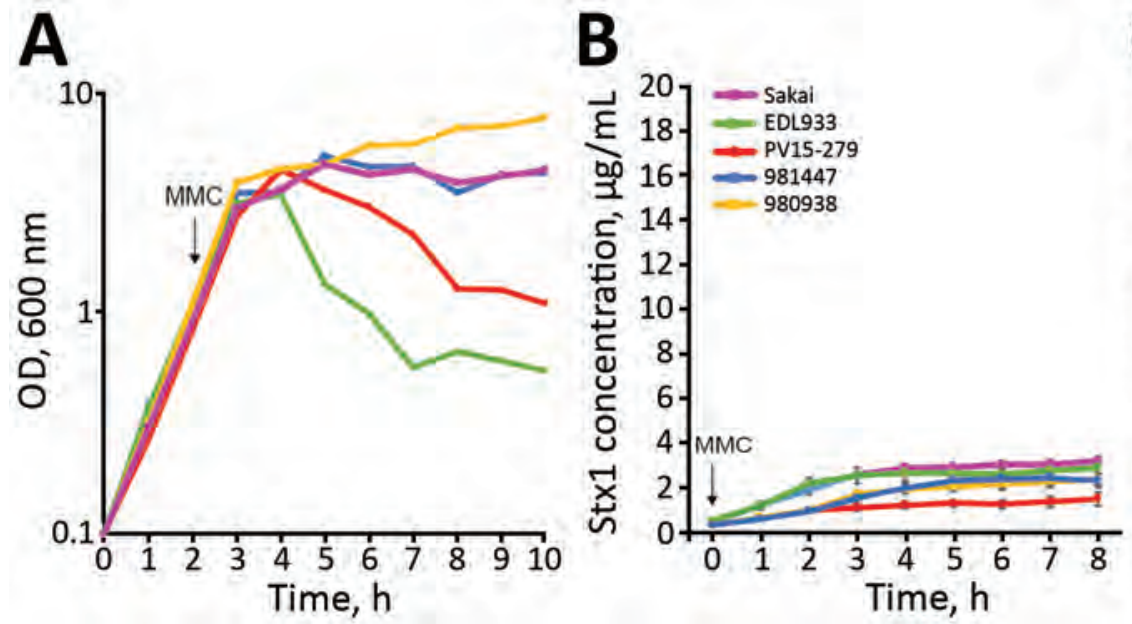

PV15-279 and other GP STEC O157:H7 strains, although some variations were detected (Figure 2). Moreover, the ability of PV15-279 to produce Stx2a was comparable to that of typical STEC O157:H7 (Figure 4). These findings suggest that PV15-279 presents a virulence potential similar to that of typical STEC O157:H7. In fact, PV15-279 was isolated from a patient who had severe enteric symptoms, including bloody diarrhea. However, further investigations are necessary to determine how the variations in the virulence gene repertoire detected in the comparison with other STEC O157 lineages affect the potential virulence of GP STEC O157:H7. The repertoires of the minor virulence genes of GP STEC O157:H7 must also be investigated.

We also obtained several noteworthy findings concerning the evolution of GP O157:H7 in this study. For example, fine genomic comparisons of Stx phages revealed that Stx phages differ notably between GP STEC O157:H7 and typical STEC O157:H7 (Figure 3). Although many of these variations may have been generated through extensive recombination with various resident or incoming phages (48), the acquisition of the Stx2a phage by the GP STEC 0157:H7 strain PV15-279 was apparently an independent genetic event. The clear differences in the genetic structure, sequence, and integration sites of Stx2c phages between GP and typical STEC O157:H7 may suggest that Stx2c phages were also acquired independently by the 2 lineages after they separated. In contrast, the similar genetic structures and sequences of the pO157 plasmids from GP and typical STEC O157:H7 and SF STEC O157:H- suggest that a pO157-like plasmid might have been acquired by their common ancestor. Although more extensive studies are needed to obtain a complete understanding of these

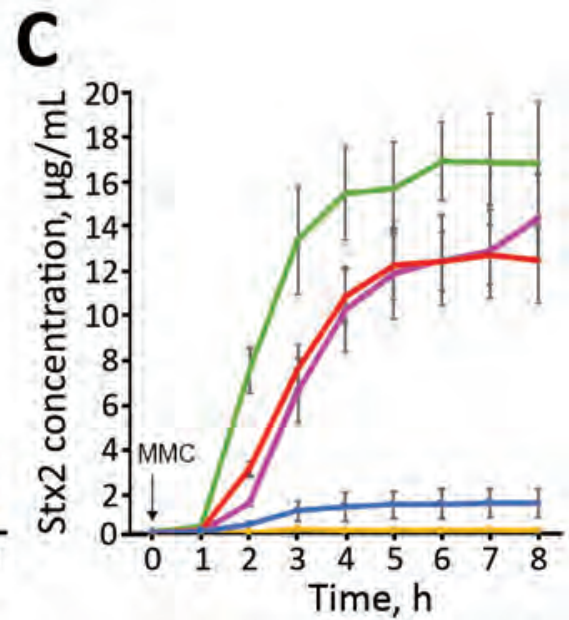

Figure 4. Lysis curves and levels of Stx produced by STEC 0157:H7 strains after MMC treatment in study of Stx-producing E. coli. The lysis curves (A) and levels of Stx1 (B) and Stx2 (C) production by 5 STEC 0157:H7 strains after the MMC treatment are shown. After the addition of $\mathrm{MMC}$, the $\mathrm{OD}_{600}$ of each strain was measured every hour for 8 hours, and $100 \mu \mathrm{L}$ of the culture was collected at each time point. Cell lysates were prepared, and the Stx 1 and Stx 2 concentrations in the soluble fractions were analyzed using a sandwich ELISA. All experiments were performed 3 times, and the averages and SEs of the Stx 1 and Stx2 concentrations in each sample are plotted. Strains Sakai and EDL933 belong to typical STEC 0157:H7, and the other 3 strains belong to GP STEC O157:H7. GP, $\beta$-glucuronidase-positive; MMC, mitomycin C; OD, optical density; STEC, Stx-producing E. coli. 
issues, only a limited number of SF STEC O157:H- genome sequences and no complete sequences of the Stx phages of this lineage are currently available. The available genome sequence information of GP STEC O157:H7 is also highly biased toward Japanese isolates.

In conclusion, we isolated a Stx2a-producing GP STEC 0157:H7 strain that emerged from the stx1a/stx2cpositive GP STEC O157:H7 clone circulating in Japan; the virulence potential of this isolate is similar to that of typical STEC O157:H7. Researchers should pay more attention to this less commonly reported STEC O157 lineage, particularly the spread of this Stx2a-producing GP STEC O157:H7 clone and the emergence of additional stx2apositive clones. Larger-scale genomic analyses including more GP STEC O157:H7 strains from various geographic regions and more SF STEC O157:H- strains are required to obtain a better understanding of the evolution and genomic diversity of GP O157:H7.

\section{Acknowledgments}

We thank A. Yoshida, Y. Inoue, M. Shimbara, N. Kanemaru, N. Kawano, N. Sakamoto, H. Iguchi, M. Horiguchi, M. Kumagai, and Y. Morita for providing technical assistance.

This work was funded by the Japan Society for the Promotion of Science (KAKENHI) under grant nos. 16K15278 and 17H04077 to Y.O. and 16H05190 to T.H. and by the Japan Agency for Medical Research and Development under grant nos. JP17efk0108127h0001 to Y.O. and JP17fk0108308j0003 to T.H.

\section{About the Author}

Dr. Ogura is an associate professor in the department of bacteriology at the Faculty of Medical Sciences, Kyushu University, Kyushu, Japan. His primary research interests include bacterial genomics and pathogenicity.

\section{References}

1. Pennington H. Escherichia coli O157. Lancet. 2010;376:1428-35. http://dx.doi.org/10.1016/S0140-6736(10)60963-4

2. Scheutz F, Teel LD, Beutin L, Piérard D, Buvens G, Karch H, et al. Multicenter evaluation of a sequence-based protocol for subtyping Shiga toxins and standardizing Stx nomenclature. J Clin Microbiol. 2012;50:2951-63. http://dx.doi.org/10.1128/JCM.00860-12

3. O'Brien AD, Newland JW, Miller SF, Holmes RK, Smith HW, Formal SB. Shiga-like toxin-converting phages from Escherichia coli strains that cause hemorrhagic colitis or infantile diarrhea. Science. 1984;226:694-6. http://dx.doi.org/10.1126/science.6387911

4. Boerlin P, McEwen SA, Boerlin-Petzold F, Wilson JB, Johnson RP, Gyles CL. Associations between virulence factors of Shiga toxinproducing Escherichia coli and disease in humans. J Clin Microbiol. 1999;37:497-503.

5. de Sablet T, Bertin Y, Vareille M, Girardeau JP, Garrivier A, Gobert AP, et al. Differential expression of stx2 variants in Shiga toxin-producing Escherichia coli belonging to seropathotypes A and C. Microbiology. 2008;154:176-86. http://dx.doi.org/10.1099/ mic.0.2007/009704-0
6. Kawano K, Okada M, Haga T, Maeda K, Goto Y. Relationship between pathogenicity for humans and stx genotype in Shiga toxin-producing Escherichia coli serotype O157. Eur J Clin Microbiol Infect Dis. 2008;27:227-32. http://dx.doi.org/10.1007/ s10096-007-0420-3

7. Ogura Y, Mondal SI, Islam MR, Mako T, Arisawa K, Katsura K, et al. The Shiga toxin 2 production level in enterohemorrhagic Escherichia coli O157:H7 is correlated with the subtypes of toxin-encoding phage. Sci Rep. 2015;5:16663. http://dx.doi.org/ 10.1038/srep16663

8. Feng PC, Monday SR, Lacher DW, Allison L, Siitonen A, Keys C, et al. Genetic diversity among clonal lineages within Escherichia coli O157:H7 stepwise evolutionary model. Emerg Infect Dis. 2007;13:1701-6. http://dx.doi.org/10.3201/eid1311.070381

9. Wick LM, Qi W, Lacher DW, Whittam TS. Evolution of genomic content in the stepwise emergence of Escherichia coli O157:H7. J Bacteriol. 2005;187:1783-91. http://dx.doi.org/10.1128/ JB.187.5.1783-1791.2005

10. Karch H, Bielaszewska M. Sorbitol-fermenting Shiga toxinproducing Escherichia coli O157:H(-) strains: epidemiology, phenotypic and molecular characteristics, and microbiological diagnosis. J Clin Microbiol. 2001;39:2043-9. http://dx.doi.org/ 10.1128/JCM.39.6.2043-2049.2001

11. Ammon A, Petersen LR, Karch H. A large outbreak of hemolytic uremic syndrome caused by an unusual sorbitol-fermenting strain of Escherichia coli O157:H-. J Infect Dis. 1999;179:1274-7. http://dx.doi.org/10.1086/314715

12. Karch H, Böhm H, Schmidt H, Gunzer F, Aleksic S, Heesemann J. Clonal structure and pathogenicity of Shiga-like toxin-producing, sorbitol-fermenting Escherichia coli O157:H-. J Clin Microbiol. 1993;31:1200-5.

13. Rosser T, Dransfield T, Allison L, Hanson M, Holden N, Evans J, et al. Pathogenic potential of emergent sorbitol-fermenting Escherichia coli O157:NM. Infect Immun. 2008;76:5598-607. http://dx.doi.org/10.1128/IAI.01180-08

14. Hayes PS, Blom K, Feng P, Lewis J, Strockbine NA, Swaminathan B. Isolation and characterization of a beta-Dglucuronidase-producing strain of Escherichia coli serotype O157:H7 in the United States. J Clin Microbiol. 1995;33:3347-8.

15. Nagano H, Hirochi T, Fujita K, Wakamori Y, Takeshi K, Yano S. Phenotypic and genotypic characterization of beta-D-glucuronidasepositive Shiga toxin-producing Escherichia coli O157:H7 isolates from deer. J Med Microbiol. 2004;53:1037-43. http://dx.doi.org/ 10.1099/jmm.0.05381-0

16. Nagano H, Okui T, Fujiwara O, Uchiyama Y, Tamate N, Kumada H, et al. Clonal structure of Shiga toxin (Stx)-producing and betaD-glucuronidase-positive Escherichia coli O157:H7 strains isolated from outbreaks and sporadic cases in Hokkaido, Japan. J Med Microbiol. 2002;51:405-16. http://dx.doi.org/10.1099/0022-131751-5-405

17. Rump LV, Strain EA, Cao G, Allard MW, Fischer M, Brown EW, et al. Draft genome sequences of six Escherichia coli isolates from the stepwise model of emergence of Escherichia coli O157:H7. J Bacteriol. 2011;193:2058-9. http://dx.doi.org/ 10.1128/JB.00118-11

18. Hazen TH, Sahl JW, Redman JC, Morris CR, Daugherty SC, Chibucos MC, et al. Draft genome sequences of the diarrheagenic Escherichia coli collection. J Bacteriol. 2012;194:3026-7. http://dx.doi.org/10.1128/JB.00426-12

19. Schutz K, Cowley LA, Shaaban S, Carroll A, McNamara E, Gally DL, et al. Evolutionary context of non-sorbitol-fermenting Shiga toxin-producing Escherichia coli O55:H7. Emerg Infect Dis. 2017;23:1966-73. http://dx.doi.org/10.3201/eid2312.170628

20. Zhou Z, Li X, Liu B, Beutin L, Xu J, Ren Y, et al. Derivation of Escherichia coli O157:H7 from its O55:H7 precursor. PLoS One. 2010;5:e8700. http://dx.doi.org/10.1371/journal.pone.0008700 
21. Eppinger M, Mammel MK, Leclerc JE, Ravel J, Cebula TA. Genomic anatomy of Escherichia coli O157:H7 outbreaks. Proc Natl Acad Sci U S A. 2011;108:20142-7. http://dx.doi.org/10.1073/ pnas. 1107176108

22. Hayashi T, Makino K, Ohnishi M, Kurokawa K, Ishii K, Yokoyama K, et al. Complete genome sequence of enterohemorrhagic Escherichia coli O157:H7 and genomic comparison with a laboratory strain K-12. DNA Res. 2001;8:11-22. http://dx.doi.org/10.1093/dnares/8.1.11

23. Katani R, Cote R, Raygoza Garay JA, Li L, Arthur TM, DebRoy C, et al. Complete genome sequence of SS52, a strain of Escherichia coli O157:H7 recovered from supershedder cattle. Genome Announc. 2015;3:e01569-14. http://dx.doi.org/10.1128/ genomeA.01569-14

24. Kulasekara BR, Jacobs M, Zhou Y, Wu Z, Sims E, Saenphimmachak C, et al. Analysis of the genome of the Escherichia coli O157:H7 2006 spinach-associated outbreak isolate indicates candidate genes that may enhance virulence. Infect Immun. 2009;77:3713-21. http://dx.doi.org/10.1128/IAI.00198-09

25. Latif H, Li HJ, Charusanti P, Palsson BO, Aziz RKA. A gapless, unambiguous genome squence of the enterohemorrhagic Escherichia coli O157:H7 strain EDL933. Genome Announc. 2014;2:e0821-14. http://dx.doi.org/10.1128/genomeA.00821-14

26. Shaaban S, Cowley LA, McAteer SP, Jenkins C, Dallman TJ, Bono JL, et al. Evolution of a zoonotic pathogen: investigating prophage diversity in enterohaemorrhagic Escherichia coli $\mathrm{O} 157$ by long-read sequencing. Microb Genom. 2016;2:e000096.

27. Koren S, Walenz BP, Berlin K, Miller JR, Bergman NH, Phillippy AM. Canu: scalable and accurate long-read assembly via adaptive $k$-mer weighting and repeat separation. Genome Res. 2017;27:722-36. http://dx.doi.org/10.1101/gr.215087.116

28. Hunt M, Silva ND, Otto TD, Parkhill J, Keane JA, Harris SR. Circlator: automated circularization of genome assemblies using long sequencing reads. Genome Biol. 2015;16:294. http://dx.doi.org/10.1186/s13059-015-0849-0

29. Li H, Durbin R. Fast and accurate short read alignment with Burrows-Wheeler transform. Bioinformatics. 2009;25:1754-60. http://dx.doi.org/10.1093/bioinformatics/btp324

30. Walker BJ, Abeel T, Shea T, Priest M, Abouelliel A, Sakthikumar S, et al. Pilon: an integrated tool for comprehensive microbial variant detection and genome assembly improvement. PLoS One. 2014;9:e112963. http://dx.doi.org/10.1371/journal.pone.0112963

31. Tanizawa Y, Fujisawa T, Kaminuma E, Nakamura Y, Arita M. DFAST and DAGA: web-based integrated genome annotation tools and resources. Biosci Microbiota Food Health. 2016;35:173-84. http://dx.doi.org/10.12938/bmfh.16-003

32. Kajitani R, Toshimoto K, Noguchi H, Toyoda A, Ogura Y, Okuno M, et al. Efficient de novo assembly of highly heterozygous genomes from whole-genome shotgun short reads. Genome Res. 2014;24:1384-95. http://dx.doi.org/10.1101/gr.170720.113

33. Ogura Y, Gotoh Y, Itoh T, Sato MP, Seto K, Yoshino S, et al. Population structure of Escherichia coli O26: H11 with recent and repeated stx2 acquisition in multiple lineages. Microb Genom. 2017;3:e00141. http://dx.doi.org/10.1099/mgen.0.000141

34. Kurtz S, Phillippy A, Delcher AL, Smoot M, Shumway M, Antonescu C, et al. Versatile and open software for comparing large genomes. Genome Biol. 2004;5:R12. http://dx.doi.org/10.1186/ gb-2004-5-2-r12

35. Croucher NJ, Page AJ, Connor TR, Delaney AJ, Keane JA, Bentley SD, et al. Rapid phylogenetic analysis of large samples of recombinant bacterial whole genome sequences using Gubbins. Nucleic Acids Res. 2015;43:e15. http://dx.doi.org/10.1093/nar/gku1196
36. Stamatakis A. RAxML-VI-HPC: maximum likelihood-based phylogenetic analyses with thousands of taxa and mixed models. Bioinformatics. 2006;22:2688-90. http://dx.doi.org/10.1093/ bioinformatics/btl446

37. Letunic I, Bork P. Interactive tree of life (iTOL) v3: an online tool for the display and annotation of phylogenetic and other trees. Nucleic Acids Res. 2016;44(W1):W242-5. http://dx.doi.org/ 10.1093/nar/gkw290

38. Li W, Godzik A. Cd-hit: a fast program for clustering and comparing large sets of protein or nucleotide sequences. Bioinformatics. 2006;22:1658-9. http://dx.doi.org/10.1093/ bioinformatics/btl158

39. Ogura Y, Abe H, Katsura K, Kurokawa K, Asadulghani M, Iguchi A, et al. Systematic identification and sequence analysis of the genomic islands of the enteropathogenic Escherichia coli strain B171-8 by the combined use of whole-genome PCR scanning and fosmid mapping. J Bacteriol. 2008;190:6948-60. http://dx.doi.org/ 10.1128/JB.00625-08

40. Ishijima N, Lee KI, Kuwahara T, Nakayama-Imaohji H, Yoneda S, Iguchi A, et al. Identification of a new virulent clade in enterohemorrhagic Escherichia coli O26:H11/H- sequence type 29. Sci Rep. 2017;7:43136. http://dx.doi.org/10.1038/srep43136

41. Monday SR, Whittam TS, Feng PC. Genetic and evolutionary analysis of mutations in the gusA gene that cause the absence of beta-glucuronidase activity in Escherichia coli O157:H7. J Infect Dis. 2001;184:918-21. http://dx.doi.org/10.1086/323154

42. Tobe T, Beatson SA, Taniguchi H, Abe H, Bailey CM, Fivian A, et al. An extensive repertoire of type III secretion effectors in Escherichia coli $\mathrm{O} 157$ and the role of lambdoid phages in their dissemination. Proc Natl Acad Sci U S A. 2006;103:14941-6. http://dx.doi.org/10.1073/pnas.0604891103

43. Rump LV, Meng J, Strain EA, Cao G, Allard MW, GonzalezEscalona N. Complete DNA sequence analysis of enterohemorrhagic Escherichia coli plasmid pO157_2 in $\beta$-glucuronidase-positive E. coli O157:H7 reveals a novel evolutionary path. J Bacteriol. 2012;194:3457-63. http://dx.doi.org/10.1128/JB.00197-12

44. Calderwood SB, Mekalanos JJ. Iron regulation of Shiga-like toxin expression in Escherichia coli is mediated by the fur locus. J Bacteriol. 1987;169:4759-64. http://dx.doi.org/10.1128/ jb.169.10.4759-4764.1987

45. Tyler JS, Mills MJ, Friedman DI. The operator and early promoter region of the Shiga toxin type 2-encoding bacteriophage 933W and control of toxin expression. J Bacteriol. 2004;186:7670-9. http://dx.doi.org/10.1128/JB.186.22.7670-7679.2004

46. Wagner PL, Livny J, Neely MN, Acheson DW, Friedman DI, Waldor MK. Bacteriophage control of Shiga toxin 1 production and release by Escherichia coli. Mol Microbiol. 2002;44:957-70. http://dx.doi.org/10.1046/j.1365-2958.2002.02950.x

47. Shimizu T, Ohta Y, Noda M. Shiga toxin 2 is specifically released from bacterial cells by two different mechanisms. Infect Immun. 2009;77:2813-23. http://dx.doi.org/10.1128/IAI.00060-09

48. Asadulghani M, Ogura Y, Ooka T, Itoh T, Sawaguchi A, Iguchi A, et al. The defective prophage pool of Escherichia coli O157: prophage-prophage interactions potentiate horizontal transfer of virulence determinants. PLoS Pathog. 2009;5:e1000408. http://dx.doi.org/10.1371/journal.ppat.1000408

Address for correspondence: Yoshitoshi Ogura, Kyushu University, Department of Bacteriology, Faculty of Medicine Sciences, 3-1-1

Maidashi Higashi-ku, Fukuoka 812-8582, Japan; email:

y-ogura@bact.med.kyushu-u.ac.jp 


\title{
Survey of Ebola Viruses in Frugivorous and Insectivorous Bats in Guinea, Cameroon, and the Democratic Republic of the Congo, 2015-2017
}

\author{
Helene M. De Nys, ${ }^{1}$ Placide Mbala Kingebeni, ${ }^{1}$ Alpha K. Keita, ${ }^{1}$ Christelle Butel, \\ Guillaume Thaurignac, Christian-Julian Villabona-Arenas, Thomas Lemarcis, Mare Geraerts, \\ Nicole Vidal, Amandine Esteban, Mathieu Bourgarel, François Roger, Fabian Leendertz, \\ Ramadan Diallo, Simon-Pierre Ndimbo-Kumugo, Justus Nsio-Mbeta, Nikki Tagg, \\ Lamine Koivogui, Abdoulaye Toure, Eric Delaporte, Steve Ahuka-Mundeke, \\ Jean-Jacques Muyembe Tamfum, Eitel Mpoudi-Ngole, Ahidjo Ayouba, ${ }^{2}$ Martine Peeters ${ }^{2}$
}

To clarify the role of bats in the ecology of Ebola viruses, we assessed the prevalence of Ebola virus antibodies in a large-scale sample of bats collected during 2015-2017 from countries in Africa that have had previous Ebola outbreaks (Guinea, the Democratic Republic of the Congo) or are at high risk for outbreaks (Cameroon). We analyzed 4,022 blood samples of bats from $\geq 12$ frugivorous and

Author affiliations: TransVIHMI of Institut de Recherche pour le Développement, Institut National de la Santé et de la Recherche Médicale and University of Montpellier, Montpellier, France (H.M. De Nys, P. Mbala Kingebeni, A.K. Keita, C. Butel, G. Thaurignac, C.-J. Villabona-Arenas, T. Lemarcis, M. Geraerts, N. Vidal, A. Esteban, A. Toure, E. Delaporte, A. Ayouba, M. Peeters); National Institute of Biomedical Research, Kinshasa, Democratic Republic of the Congo (P. Mbala Kingebeni, S.-P. Ndimbo-Kumugo, S. Ahuka-Mundeke, J.-J. Muyembe Tamfum); Cliniques Universitaires de Kinshasa, Kinshasa (P. Mbala Kingebeni, S. Ahuka-Mundeke, J.-J. Muyembe Tamfum); Centre de Recherche et de Formation en Infectiologie de Guinée, Conakry, Guinea (A.K. Keita, A. Toure); ASTRE of Centre de coopération internationale en recherche agronomique pour le développement, Institut national de la Recherche Agronomique and Univerisity of Montpellier, Montpellier (M. Bourgarel, F. Roger); Robert Koch-Institute, Berlin, Germany (F. Leendertz); Ministère de l'Elevage et des Productions Animales, Conakry (R. Diallo); Direction de Lutte contre la Maladie, Kinshasa (J. Nsio-Mbeta); Royal Zoological Society of Antwerp, Antwerp, Belgium (N. Tagg); Université de Conakry, Conakry (L. Koivogui); Institut National de Sante Publique, Conakry (A. Toure); Institut de Recherches Médicales et d'Études des Plantes Médicinales, Yaoundé, Cameroon (E. Mpoudi-Ngole); Cameroon Institut de Recherche pout le Développement, Yaoundé (E. Mpoudi-Ngole)

DOI: https://doi.org/10.3201/eid2412.180740
27 insectivorous species; $2-37(0.05 \%-0.92 \%)$ bats were seropositive for Zaire and $0-30(0 \%-0.75 \%)$ bats for Sudan Ebola viruses. We observed Ebola virus antibodies in 1 insectivorous bat genus and 6 frugivorous bat species. Certain bat species widespread across Africa had serologic evidence of Zaire and Sudan Ebola viruses. No viral RNA was detected in the subset of samples tested $(n=665)$. Ongoing surveillance of bats and other potential animal reservoirs are required to predict and prepare for future outbreaks.

ince the first outbreak of Ebola virus disease (EVD) in $\checkmark 1976$ in the northern part of the Democratic Republic of the Congo (DRC), 26 recognized outbreaks have occurred in humans across Africa; fatality rates of outbreaks have been 25\%-90\% (1-4). Each EVD outbreak most likely resulted from independent zoonotic events.

Bats are believed to play a role in the ecology of Ebola viruses as a reservoir species (5). Bats might infect humans directly or via intermediate amplifying hosts, like nonhuman primates or duikers $(6,7)$. Bats might serve as a source of infection in certain areas where bats are hunted and eaten as bushmeat, but infection could also occur after consumption of fruits contaminated with saliva, urine, or feces from Ebola virus-infected bats $(8,9)$. Ebola virus emergence through exposure to bats was suspected for at least 2 outbreaks: Luebo (the DRC) in 2007 and West Africa in $2013(10,11)$.

Relatively few data are available to support the role of bats in the ecology of Ebola viruses. During the EVD outbreaks of 2003 in Gabon and the Congo, Zaire Ebola

${ }^{1}$ These first authors contributed equally to this article.

${ }^{2}$ These senior authors contributed equally to this article. 
virus RNA and antibodies were detected in live-caught specimens from 3 fruit bat species (Epomops franqueti, Hypsignathus monstrosus, Myonycteris torquata); virus sequences were found in the livers or spleens of a few bats (6). In subsequent studies in Gabon, the Congo, Ghana, and Zambia, antibodies were detected in additional frugivorous bat species (Eidolon helvum, Epomophorus gambianus, Rousettus aegyptiacus, Micropteropus pusillus) and 1 insectivorous species (Mops condylurus) (12-16). The amplification and sequencing of viral RNA of other filoviruses in bats, such as Marburg virus in bats from Africa (17-20), Lloviu virus in bats from Europe (21), and new filoviruses in bats from China (22), has provided additional evidence for a possible role of bats in Ebola virus ecology.

In general, EVD outbreaks have been limited in terms of their geographic spread and chains of human-to-human transmission (1). However, during the 2013-2016 outbreak, virus spread to the urban areas of 3 countries, infecting $\approx 30,000$ persons in Guinea, Sierra Leone, and Liberia, and $\approx 11,000$ deaths were recorded (23). This outbreak illustrated the potential for epidemic spread from a single zoonotic transmission, with severe public health and socioeconomic impact (24). Additional studies are urgently needed to identify the animal reservoir, predict EVD outbreak risks, and improve our capacity to control epidemics.

In previous modeling studies, areas were defined as at risk for EVD outbreaks on the basis of data collected from a limited number of wildlife bat species from a few geographic regions $(5,25)$. Also, a wide variety of serologic assays and interpretation criteria have been used, making comparison of results challenging $(12-16,26,27)$. For this study, we performed a large serosurvey with a highly specific and sensitive high-throughput assay to assess Ebola virus prevalence in bats from Africa (28). We studied bats from Guinea and the DRC, countries with previous EVD outbreaks, and Cameroon, a country considered at high risk for future EVD outbreaks $(5,25)$.

\section{Materials and Methods}

\section{Study Sites and Sample Collection}

During November 2015-August 2017, we collected samples from free-ranging frugivorous and insectivorous bats in Guinea, Cameroon, and the DRC. We captured bats at night using ground mist nets or harp traps in roosting and foraging sites. We set up ground mist nets $(12 \times 3.2 \mathrm{~m})$ of 30-mm and 60-mm mesh sizes at different heights (1-7 m) to maximize capture of different species. We opened nets or harp traps just before sunset and checked for bats every 1-2 hours. Captured bats were released the same night immediately after sampling. Using bat whole blood taken by venipuncture of the propatagial or brachial vein, we dropped blood samples directly onto Whatman 903 filter paper (GE Healthcare, Feasterville-Trevose, PA, USA). We air-dried and preserved samples individually in plastic bags containing silica desiccant and stored them in hermetic boxes; $2-3$ weeks later, we transferred dried blood spots to $-20^{\circ} \mathrm{C}$ until needed for analysis. Data recorded in the field included information on capture site (global positioning system coordinates, ecologic environment), capture method, morphology (body measurements, weight, color), sex, age class (adult, juvenile), and species (identified visually). We collected negative control samples $(n=145)$ from a captive-born insectivorous bat species (103 Carollia perspicillata bats) hosted at the Parc Zoologique de Montpellier (Montpellier, France) and 2 frugivorous bat species (19 Pteropus giganteus bats, 23 R. aegyptiacus bats) hosted at Wilhelma Zoo and Botanical Garden (Stuttgart, Germany). We collected and preserved samples the same way we did for free-ranging bats.

\section{Screening for Ebola Virus Antibodies}

We tested dried blood spots with a Luminex-based serologic assay adapted for bats (28) (online Technical Appendix, https://wwwnc.cdc.gov/EID/article/24/12/18-0740Techapp1.pdf). The assay included recombinant Ebola virus proteins glycoprotein, nucleoprotein, or viral protein 40 for different lineages: Zaire, Sudan, Bundibugyo, and Reston. We reconstituted plasma from dried blood spots as previously described (28) and incubated $100 \mu \mathrm{L}$ of sample (final plasma dilution $1: 2,000$ ) with $50 \mu \mathrm{L}$ of recombinant protein-coated beads ( $2 \mu \mathrm{g}$ protein $/ 1.25 \times 10^{6}$ beads) in 96-well flat-bottom filter plates (Millipore, Tullagreen, Ireland) on a plate shaker at $300 \mathrm{rpm}$ for $16 \mathrm{~h}$ at $4^{\circ} \mathrm{C}$ in the dark. After washing, we added $0.1 \mu \mathrm{g} / \mathrm{mL}$ of goat antibat biotin-labeled IgG (Euromedex, Souffelweyersheim, France) per well and incubated for $30 \mathrm{~min}$ at $300 \mathrm{rpm}$. After another round of washing, we added $50 \mu \mathrm{L}$ of $4 \mu \mathrm{g} / \mathrm{mL}$ streptavidin-R-phycoerythrin (Fisher Scientific, Illkirch, France) per well and incubated for $10 \mathrm{~min}$ at $300 \mathrm{rpm}$. Reactions were read with BioPlex-200 (BioRad, Marnes-laCoquette, France). We expressed results as median fluorescence intensity (MFI) per 100 beads. We included 3 samples on every plate to validate interassay repeatability.

\section{Determination of Cutoffs}

In the absence of positive control samples, we used 4 different statistical methods to determine the MFI cutoff value for each antigen $(29,30)$ (online Technical Appendix Table 1). First, we used a general formula that involved the MFI of the 145 negative control samples, and we assigned the cutoff as mean plus 4 times the SD (mean $+4 \times \mathrm{SD}$ ). Second, we used a change point analysis (31) to identify the value at which statistical properties of the underlying probability distribution changed. This value was used to identify outliers and classify them as reactive. We used the R package 
Table 1. Bat samples collected for Ebola virus serology by study site, Guinea, Cameroon, and the Democratic Republic of the Congo, 2015-2017

\begin{tabular}{lc}
\hline Country, site & No. samples \\
\hline Democratic Republic of the Congo & \\
Boma & 156 \\
Kimpese & 202 \\
Zongo & 472 \\
Subtotal & 830 \\
\hline Cameroon & \\
Yaoundé & 126 \\
Libellengoi Sud & 44 \\
Mbalmayo & 48 \\
Bipindi & 479 \\
Campo M'an & 344 \\
North Dja & 295 \\
Ekom & 122 \\
Djoum & 56 \\
Mambele & 348 \\
Mbam Djerem & 156 \\
Subtotal & 2,018 \\
\hline Guinea & \\
Conakry & 107 \\
Kindia & 323 \\
Kankan & 378 \\
Koundara & 90 \\
Mamou & 147 \\
Gueckedou & 49 \\
Macenta & 9 \\
Nzerekore & 71 \\
Subtotal & 1,174 \\
\hline Total & 4,022 \\
\hline
\end{tabular}

changepoint (32) to calculate a single shift in the arithmetic mean with the at-most-1-change method (33). Third, we fitted univariate distributions to our data and defined the cutoff as a 0.001 risk for error, as was used in other virus serology studies $(13,34)$. We reduced the set of candidate distributions following a bootstrapped skewness-kurtosis analysis (35). We performed fitting by maximum-likelihood estimation and selected the best-fit distribution on the basis of the Akaike information criteria with the R library fitdistrplus (36). A negative binomial distribution best-fit the data; however, we also used the negative exponential distribution as in Pourrut et al. and Laing et al. $(13,34)$. For every antigen, we computed bootstrap values using 10,000 replicates and averaged. We performed analyses with $\mathrm{R}$ version 3.3.2 software (https://www.r-project.org/). We considered a blood sample reactive if the MFI of the reaction was above the cutoff. We defined Ebola virus antibody positivity as reactivity to glycoprotein and nucleoprotein of the same lineage, as was done in our previous study (28).

\section{Nucleic Acid Extraction and PCR Screening for Ebola Virus RNA}

We extracted total DNA and RNA from dried blood spots as previously described using Nuclisens (bioMerieux, Marcyl'Etoile, France) or m2000sp methods (Abbott Molecular Inc., Des Plaines, IL, USA), which are known for a high performance recovering nucleic acids from dried blood spots
$(37,38)$. For bat species from Cameroon and Guinea, we screened for Zaire Ebola virus RNA by seminested reverse transcription PCR (RT-PCR) targeting the nucleoprotein region of the virus genome. We amplified a 126-bp fragment of Zaire Ebola virus using primers NP1F1 (forward, 5'-CGGACACACAAAAAGAAWGAA-3') and NP1R-ZR (reverse, 5'-CTCTATCTTKGTGATRTGGCTCTGA-3') in the first round of PCR and NP1F2 (forward, 5'- TTGTGTGCGARTAACTAYGAGGAAG-3') plus NP1R-ZR in the second round. For species from the DRC, we performed seminested RT-PCR targeting the viral protein 35 region of the genome using the protocol of He et al. with modifications (41). In the first round, we amplified a 217bp fragment with primers VP35-F (5'-ATYATGTATGATCACYTVCCWGG-3') and VP35-R (52-AGCGRATGTGGATSACRGGT-32) and, in the second round, a 184-bp product with primers VP35-R and VP35-in-F (5'-GCTTTYCAYCAAYTAGTRCAAG-3').

\section{Molecular Confirmation of Bat Species}

We confirmed bat species identification recorded in the field on a subset of samples by using molecular tests. We amplified an $\approx 800$-bp fragment of mitochondrial cytochrome b using primers cytb-L14724 (forward) and cytb-H15506 (reverse) $(11,39,40)$. We substituted the cytb-L14724 primer with cytb-L140217 (5'-ATGACCAACATCCGAAAATCNCAC-3') to improve PCR performance for certain species. We purified PCR products through agarose gel (1\%) and directly sequenced on an ABI 3500 sequencer (Applied Biosystems, Courtaboeuf, France). We performed BLAST analyses (https://blast.ncbi.nlm.nih.gov/Blast.cgi) to identify the most similar bat species. For samples with no or low similarity $(<97 \%)$ hits with species in GenBank, we performed phylogenetic analyses with newly obtained sequences and reference sequences for different bat species using maximum-likelihood methods implemented with PhyML (http://www.atgc-montpellier.fr/phyml/) to determine genus.

\section{Results}

\section{Bat Species and Sampling}

We analyzed blood samples from 4,022 wild bats from 21 different regions in Cameroon $(\mathrm{n}=10)$, Guinea $(\mathrm{n}=8)$, and the DRC $(\mathrm{n}=3)$ (Figure 1; Table 1). To increase species diversity, we captured bats in multiple ecologic settings: forests $(49 \%)$, open fields $(10 \%)$, villages $(29 \%)$, plantations $(7 \%)$, and urban areas (5\%). For 1,470 (36.5\%) samples, species identification in the field was confirmed by sequence analysis. At each site, $\geq 1$ sample was confirmed per sampling date, capture method, and morphologic description. For the remaining samples, species identification was extrapolated by combining molecular and morphologic data, including photographs whenever available. For some insectivorous bat 


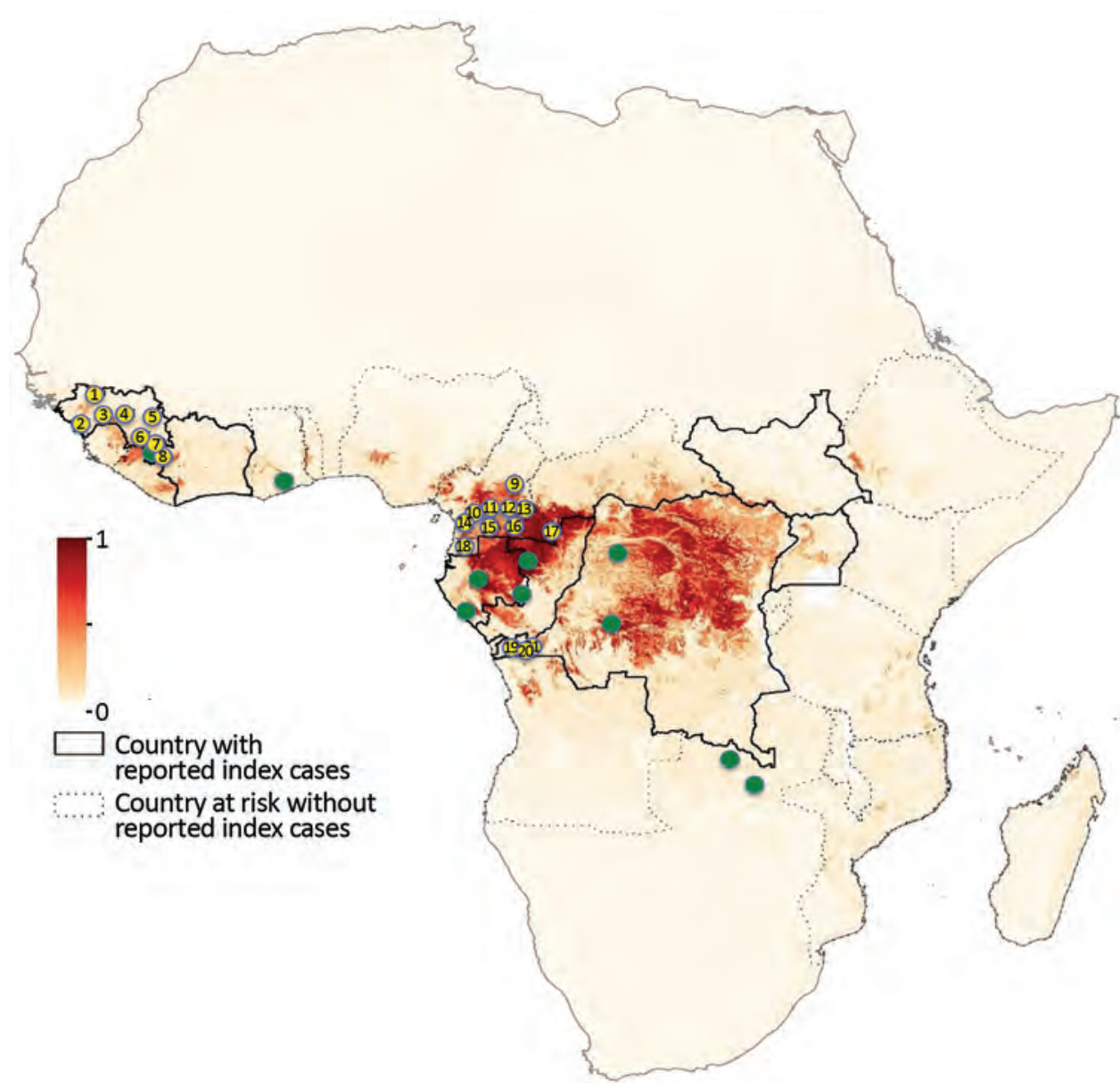

Figure 1. Study sites for bat blood sample collection for Ebola virus serology, Guinea, Cameroon, and the Democratic Republic of the Congo, 20152017. Yellow dots indicate sampling sites for bats in our study, and green dots indicate sampling sites in previously published studies. Dark red shading indicates highest and light yellow lowest risk for Ebola virus spillover events. Study sites are numbered: 1 , Koundara; 2 , Conakry; 3, Kindia; 4, Mamou; 5, Kankan; 6, Gueckedou; 7, Macenta; 8, Nzerekore; 9, Mbam Djerem; 10, Libellengoi Sud; 11, Yaoundé; 12, Ekom; 13, North Dja; 14, Bipindi; 15, Mbalmayo; 16, Djoum; 17, Mambele; 18, Campo M'an; 19, Boma; 20, Kimpese; 21, Zongo. Countries with reported index Ebola cases and countries without such cases but deemed at risk are indicated. Map of Africa adapted from Pigott et al. (5) (https://creativecommons.org/ licenses/by/4.0/) by adding locations of collection sites.

families (Miniopteridae, Molossidae, Nycteridae, Rhinolophidae), identification was possible only at the genus level; for some Molossidae bats, we could not distinguish between Mops and Chaerephon genera because of the lack of sequences in GenBank (Table 2). For 87 (2.16\%) samples, species identification was not possible because incomplete data were recorded in the field, and available biologic materials were insufficient for molecular confirmation. We collected samples from 1,736 (43.2\%) frugivorous bats (family Pteropodidae) of 12 species and 2,199 (54.7\%) insectivorous bats (7 families) of $\geq 27$ species. The insectivorous bat families sampled, in order of decreasing frequency, were Hipposideridae (31.9\%), Molossidae (13.4\%), Miniopteridae (5.8\%), Rhinolophidae (2.1\%), Vespertilionidae (0.8\%), Nycteridae (0.5\%), and Emballonuridae (0.12\%). Overall, $54.7 \%$ of bats were female and $43.8 \%$ were male; for $1.5 \%(n=60)$ of bats, sex was unknown. Most (77.9\%) bats were adults, and $9.6 \%$ were juveniles; for $12.5 \%(n=502)$ of bats, age could not be determined or was not recorded.

\section{Bats Antibodies against Different Ebola Virus Antigens}

We tested all samples for Ebola virus antibodies. The number of samples reacting with $\geq 1$ antigen was 734 (18.2\%) by the mean $+4 \times \mathrm{SD}$ method, $274(6.8 \%)$ for the changepoint method, 175 (4.4\%) for the binomial method, and 457 $(11.4 \%)$ for the exponential method. Blood samples frequently reacted with glycoprotein antigens; samples reacted most with Zaire and Sudan Ebola virus antigens and least with Reston (Table 3). Simultaneous reactivity to $>1$ antigen (i.e., glycoprotein, nucleoprotein, viral protein 40) from the same virus lineage was rare. Simultaneous reactivity to the same antigen from different virus lineages was frequent; $32.3 \%-76.7 \%$ of blood samples were reactive to glycoprotein from $\geq 2$ Ebola virus species, $18.4 \%-34.0 \%$ to viral protein 40 , and $1.5 \%-4.4 \%$ to nucleoprotein (online Technical Appendix Table 2). When using the criterion simultaneous presence of antibodies to nucleoprotein and glycoprotein, the antibody positivity for Zaire or Sudan Ebola virus antibodies was generally $<1 \%$ for all bats tested, regardless of cutoff method, and was lower among insectivorous than frugivorous bats: $0.05 \%-0.27 \%$ (insectivorous) and $0.06 \%-1.79 \%$ (frugivorous) for Zaire Ebola virus versus $0 \%-0.09 \%$ (insectivorous) and 0\%-1.61\% (frugivorous) for Sudan Ebola virus (Table 3; Figure 2). Three samples were positive for Zaire and Sudan Ebola viruses, but only by less stringent cutoff methods (i.e., mean $+4 \times \mathrm{SD}$ ). 
Table 2. Bat species sampled for Ebola virus serology, Guinea, Cameroon, and the DRC, 2015-2017*

\begin{tabular}{|c|c|c|c|c|c|}
\hline Family & Species & DRC, no. & Cameroon, no. & Guinea, no. & Total, no. \\
\hline Emballonuridae & Coleura afra & 0 & 5 & 0 & 5 \\
\hline \multirow[t]{8}{*}{ Hipposideridae } & Hipposideros abae & 0 & 0 & 37 & 37 \\
\hline & H. beatus & 0 & 4 & 0 & 4 \\
\hline & H. cyclops & 0 & 14 & 0 & 14 \\
\hline & H. fuliginosus & 0 & 2 & 0 & 2 \\
\hline & H. gigas & 2 & 9 & 2 & 13 \\
\hline & H. jonesi & 0 & 1 & 12 & 13 \\
\hline & H. ruber/caffer & 127 & 807 & 237 & 1,171 \\
\hline & Hipposideros sp. & 28 & 0 & 0 & 28 \\
\hline Subtotal & & 157 & 837 & 288 & 1,282 \\
\hline Miniopteridae & Miniopterus sp. & 205 & 0 & 27 & 232 \\
\hline \multirow[t]{4}{*}{ Molossidae } & Chaerephon sp. & 0 & 0 & 44 & 44 \\
\hline & Mops condylurus & 0 & 0 & 110 & 110 \\
\hline & Mops sp. & 0 & 256 & 0 & 256 \\
\hline & Mops/Chaerephon sp. & 0 & 8 & 120 & 128 \\
\hline Subtotal & & 0 & 264 & 274 & 538 \\
\hline Nycteridae & Nycteris sp. & 0 & 7 & 15 & 22 \\
\hline \multirow[t]{5}{*}{ Rhinolophidae } & Rhinolophus alcyone & 0 & 16 & 0 & 16 \\
\hline & R. darlingii & 3 & 0 & 0 & 3 \\
\hline & R. fumigatus & 0 & 0 & 19 & 19 \\
\hline & R. landeri & 0 & 0 & 6 & 6 \\
\hline & Rhinolophus sp. & 3 & 38 & 1 & 42 \\
\hline Subtotal & & 6 & 54 & 26 & 86 \\
\hline \multirow[t]{7}{*}{ Vespertilionidae } & Glauconycteris variegata & 0 & 3 & 0 & 3 \\
\hline & Kerivoula sp. & 0 & 1 & 0 & 1 \\
\hline & Myotis bocagii & 0 & 3 & 0 & 3 \\
\hline & Neoromicia sp. & 0 & 5 & 0 & 5 \\
\hline & Scotophilus leucogaster & 0 & 0 & 15 & 15 \\
\hline & S. nigrita & 0 & 0 & 1 & 1 \\
\hline & S. nux & 0 & 6 & 0 & 6 \\
\hline Subtotal & & 0 & 18 & 16 & 34 \\
\hline \multirow[t]{12}{*}{ Pteropodidae } & Eidolon helvum & 305 & 158 & 17 & 480 \\
\hline & Epomophorus gambianus & 0 & 0 & 191 & 191 \\
\hline & Epomophorus wahlbergi & 0 & 16 & 0 & 16 \\
\hline & Epomops buettikoferi & 0 & 0 & 4 & 4 \\
\hline & Epomops franqueti & 20 & 256 & 0 & 276 \\
\hline & Hypsignathus monstrosus & 1 & 176 & 8 & 185 \\
\hline & Lissonycteris angolensis & 22 & 30 & 32 & 84 \\
\hline & Megaloglossus woermanni & 1 & 19 & 0 & 20 \\
\hline & Micropteropus pusillus & 44 & 2 & 18 & 64 \\
\hline & Myonycteris torquata & 35 & 21 & 0 & 56 \\
\hline & Rousettus aegyptiacus & 0 & 131 & 228 & 359 \\
\hline & Scotonycteris zenkeri & 0 & 1 & 0 & 1 \\
\hline Subtotal & & 428 & 810 & 498 & 1,736 \\
\hline Inderminate species & & 34 & 23 & 30 & 87 \\
\hline Total & & 830 & 2,018 & 1,174 & 4,022 \\
\hline
\end{tabular}

\section{Zaire and Sudan Ebola Virus Reactivity of Different Bat Species}

We estimated specific reactivity to Zaire and Sudan Ebola viruses by bat species. We did not include Bundibugyo and Reston because recombinant nucleoproteins were not available. Among insectivorous bats, only blood samples from Mops sp. bats (1-6/494) were positive for Zaire or Sudan Ebola virus antibodies (Table 4). Among frugivorous bats, samples from E. helvum, H. monstrosus, and $R$. aegyptiacus bats had the highest reactivity. We observed Zaire and Sudan Ebola virus seropositivity in these 3 species with almost all cutoff methods: $0.2 \%$ $3.3 \%$ for Zaire Ebola virus and $1.0 \%-2.9 \%$ for Sudan Ebola virus in E. helvum bat samples, $0.5 \%-1.6 \%$ for
Zaire Ebola virus and 1.1\%-4.3\% for Sudan Ebola virus in $H$. monstrosus bat samples, and $0.6 \%-2.5 \%$ for Zaire Ebola virus and $0.8 \%-1.4 \%$ for Sudan Ebola virus in $R$. aegyptiacus bat samples. We observed 2.4\% Zaire Ebola virus-seropositive samples for Lissonycteris angolensis bats and $0.5 \%$ for Epomophorus sp. bats, but only by less stringent cutoff methods. One sample from M. pusillus bats was seropositive for Sudan Ebola virus. No samples from $E$. franqueti or $M$. torquata bats were reactive with any Ebola virus antigens. Samples from the 1 Scotonycteris zenkeri bat and 20 Megaloglossus woermanni bats were seronegative. Overall, Zaire or Sudan Ebola virus antibodies were observed in 7 (1 insectivorous and 6 frugivorous) bat species. 
Ebola Virus in Frugivorous and Insectivorous Bats

Table 3. Blood samples from bats reactive with Ebola virus antigens in Luminex assay, by antigen, bat type, and statistical method used to determine cutoff, Guinea, Cameroon, and the Democratic Republic of the Congo, 2015-2017*

\begin{tabular}{|c|c|c|c|c|c|c|}
\hline \multirow[b]{2}{*}{ Ebola virus species, antigen } & \multirow[b]{2}{*}{ Bat type } & \multicolumn{4}{|c|}{ Statistical method, no. (\%) } & \multirow{2}{*}{$\begin{array}{c}\text { Estimated } \\
\text { range, \% }\end{array}$} \\
\hline & & Mean $+4 \times \mathrm{SD}$ & Change point & Binomial & Exponential & \\
\hline \multicolumn{7}{|l|}{ Zaire } \\
\hline NP & Frugivorous & $57(3.28)$ & $8(0.46)$ & $24(1.38)$ & $51(2.94)$ & $0.46-3.28$ \\
\hline NP & Insectivorous & $15(0.68)$ & $1(0.05)$ & $6(0.27)$ & $15(0.68)$ & $0.05-0.68$ \\
\hline NP & Total & 72 (1.79) & $9(0.22)$ & $30(0.75)$ & $66(1.64)$ & $0.23-1.79$ \\
\hline GP-K & Frugivorous & 365 (21.03) & $141(8.12)$ & $20(1.15)$ & $113(6.51)$ & $1.15-21.03$ \\
\hline GP-K & Insectivorous & 73 (3.32) & $18(0.82)$ & $2(0.09)$ & $12(0.55)$ & $0.09-3.32$ \\
\hline GP-K & Total & $440(10.94)$ & $160(3.98)$ & $22(0.55)$ & $125(3.11)$ & $0.55-10.94$ \\
\hline GP-M & Frugivorous & $226(13.02)$ & $128(7.37)$ & $16(0.92)$ & $103(5.93)$ & $0.92-13.02$ \\
\hline GP-M & Insectivorous & $31(1.41)$ & $14(0.64)$ & $2(0.09)$ & $12(0.55)$ & $0.09-1.41$ \\
\hline GP-M & Total & $259(6.44)$ & $143(3.56)$ & $18(0.45)$ & $115(2.86)$ & $0.45-6.44$ \\
\hline VP & Frugivorous & $55(3.17)$ & $8(0.46)$ & 24 (1.38) & $44(2.53)$ & $0.46-3.17$ \\
\hline VP & Insectivorous & $19(0.86)$ & $5(0.23)$ & $6(0.27)$ & $14(0.64)$ & $0.23-0.86$ \\
\hline VP & Total & 75 (1.86) & $14(0.35)$ & $30(0.75)$ & 59 (1.47) & $0.35-1.86$ \\
\hline $\mathrm{NP}+\mathrm{GP}$ & Frugivorous & 31 (1.79) & 31 (1.79) & $1(0.06)$ & $7(0.40)$ & $0.06-1.79$ \\
\hline $\mathrm{NP}+\mathrm{GP}$ & Insectivorous & $6(0.27)$ & $6(0.27)$ & $1(0.05)$ & $1(0.05)$ & $0.05-0.27$ \\
\hline $\mathrm{NP}+\mathrm{GP}$ & Total & $37(0.92)$ & $37(0.92)$ & $2(0.05)$ & $8(0.20)$ & $0.05-0.92$ \\
\hline \multicolumn{7}{|l|}{ Sudan } \\
\hline $\mathrm{NP}$ & Frugivorous & $71(4.09)$ & $15(0.86)$ & $34(1.96)$ & 77 (4.44) & $0.86-4.44$ \\
\hline NP & Insectivorous & $12(0.55)$ & $1(0.05)$ & $5(0.23)$ & $18(0.82)$ & $0.05-0.82$ \\
\hline NP & Total & 84 (2.09) & $17(0.42)$ & 39 (0.97) & 96 (2.39) & $0.42-2.39$ \\
\hline GP & Frugivorous & 459 (26.44) & $147(8.47)$ & $17(0.98)$ & $121(6.97)$ & $0.98-26.44$ \\
\hline GP & Insectivorous & $49(2.23)$ & $6(0.27)$ & $1(0.05)$ & $1(0.05)$ & $0.05-2.23$ \\
\hline GP & Total & 509 (12.66) & $154(3.83)$ & $18(0.45)$ & $125(3.11)$ & $0.45-12.66$ \\
\hline VP & Frugivorous & $102(5.88)$ & $20(1.15)$ & 28 (1.61) & $61(3.51)$ & $1.15-5.88$ \\
\hline VP & Insectivorous & $19(0.86)$ & $4(0.18)^{\prime}$ & $6(0.27)^{\prime}$ & $18(0.82)$ & $0.18-0.86$ \\
\hline VP & Total & $121(3.01)$ & $24(0.60)$ & $34(0.85)$ & $80(1.99)$ & $0.60-3.01$ \\
\hline $\mathrm{NP}+\mathrm{GP}$ & Frugivorous & $28(1.61)$ & $28(1.61)$ & 0 & $10(0.58)$ & $0-1.61$ \\
\hline $\mathrm{NP}+\mathrm{GP}$ & Insectivorous & $2(0.09)$ & $2(0.09)$ & 0 & 0 & $0-0.09$ \\
\hline $\mathrm{NP}+\mathrm{GP}$ & Total & $30(0.75)$ & $30(0.75)$ & 0 & $10(0.25)$ & $0-0.75$ \\
\hline \multicolumn{7}{|l|}{ Bundibugyo } \\
\hline GP & Frugivorous & 301 (17.34) & $59(3.40)$ & 0 & $93(5.36)$ & 0-17.34 \\
\hline GP & Insectivorous & $58(2.64)$ & $8(0.36)$ & $5(0.23)$ & $13(0.59)$ & $0.23-2.64$ \\
\hline GP & Total & $361(8.98)$ & 68 (1.69) & $22(0.55)$ & $107(2.66)$ & $0.55-8.98$ \\
\hline VP & Frugivorous & $9(0.52)$ & $7(0.40)$ & $12(0.69)$ & $37(2.13)$ & $0.40-2.14$ \\
\hline VP & Insectivorous & 0 & $1(0.05)$ & $8(0.36)$ & $20(0.91)$ & $0-0.91$ \\
\hline VP & Total & $9(0.22)$ & $8(0.20)$ & $20(0.5)$ & $57(1.42)$ & $0.20-1.42$ \\
\hline \multicolumn{7}{|l|}{ Reston } \\
\hline GP & Frugivorous & $17(0.98)$ & 28 (1.61) & $26(1.50)$ & 61 (3.51) & $0.98-3.51$ \\
\hline GP & Insectivorous & $3(0.14)$ & $10(0.45)$ & $10(0.45)$ & $29(1.32)$ & $0.14-1.32$ \\
\hline GP & Total & $20(0.50)$ & $38(0.94)$ & $36(0.90)$ & $90(2.24)$ & $0.50-2.24$ \\
\hline
\end{tabular}

\section{Comparison of Zaire Ebola Virus Seroprevalence in Bats from Africa across Studies}

For comparison, we compiled data regarding Zaire Ebola virus serology in bats of known species from previous studies $(\mathrm{n}=4,493)$ and this study $(\mathrm{n}=3,935 ; 46.7 \%)$ (Tables 5 , 6). Data were available for 3,023 insectivorous bats of $\approx 30$ species from 7 different families; 2,199 (72.7\%) were from this study (Table 5). Insectivorous bat samples originated from Guinea, Cameroon, the DRC, and Gabon. Zaire Ebola virus reactivity has been observed only in $M$. condylurus bat samples from Gabon and Mops sp. bat samples from Cameroon. Data were available for 5,405 frugivorous bats of 17 species from 12 genera from West (Guinea, Ghana), West Central (Cameroon, Gabon, the Congo, the DRC), and East (Zambia) Africa (Table 6). No Zaire Ebola virus reactivity has been seen in blood samples from bat species
Casinycteris, Megaloglossus, Nanonycteris, and Scotonycteris, but only a limited number of samples $(\mathrm{n}=152)$ have been tested. Overall, blood samples from 8 frugivorous bat species have been found reactive with Zaire Ebola virus antigens. Blood samples from E. helvum, H. monstrosus, and $R$. aegyptiacus bats from several countries across Africa have been reported to be seropositive. Reactivity has been observed with samples from E. gambianus bats in Ghana $(10.8 \%)$ and Guinea. Reactivity was observed with large sample sets from $E$. franqueti bats derived from Gabon and the Congo and a small sample set from Ghana but not Guinea, Cameroon, or the DRC. M. pusillus and M. torquata bats tested positive for Zaire Ebola virus antibodies in studies in which large sample sets were collected. Among L. angolensis bat samples, only those from Cameroon have tested positive for antibodies. 


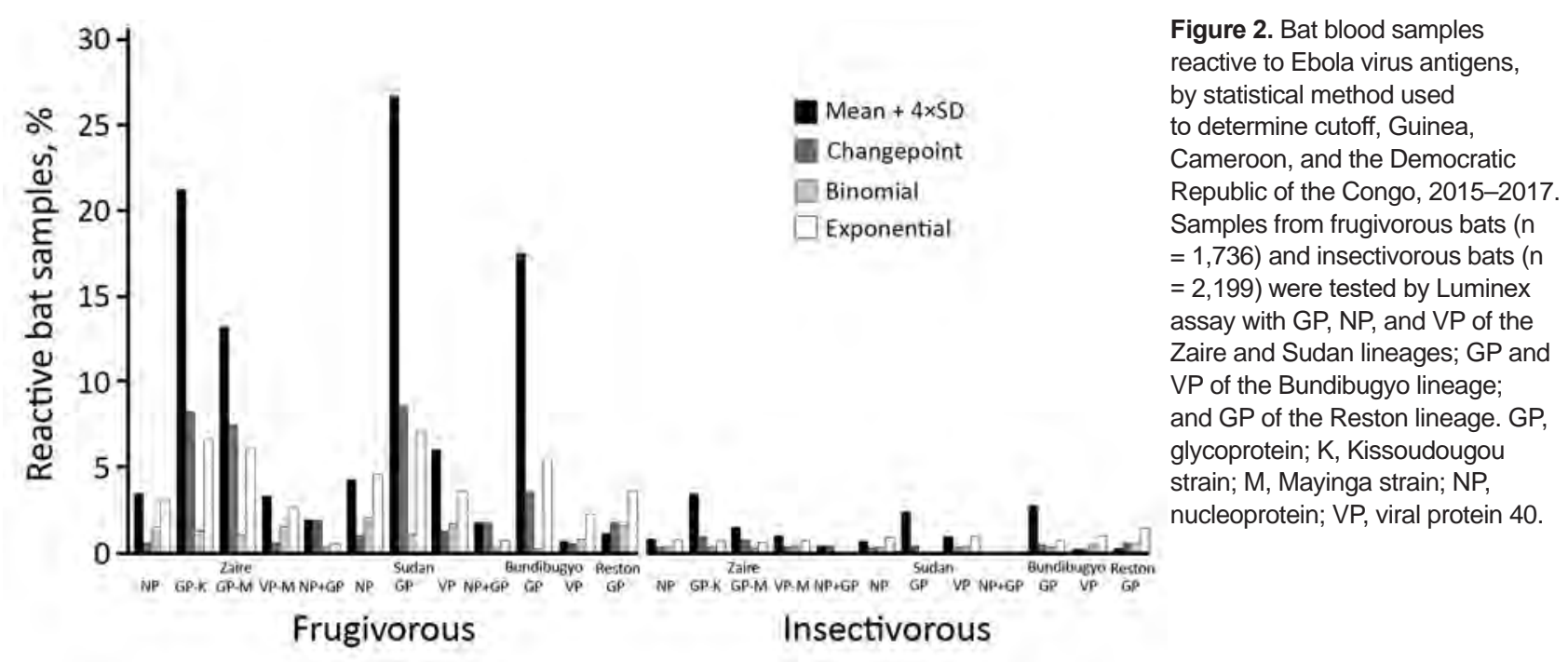

\section{RT-PCR Screening for Zaire Ebola Virus RNA}

We screened 665 samples from the DRC $(n=193)$, Cameroon $(\mathrm{n}=399)$, and Guinea $(\mathrm{n}=73)$ by RT-PCR for the presence of Zaire Ebola virus RNA. Of the 294 samples originating from bats previously documented to carry Zaire Ebola virus RNA (6) (i.e., H. monstrosus [132 from Cameroon, 1 from the DRC], M. torquata [20 from Cameroon, 25 from the DRC], and E. franqueti [116 from Cameroon]), all were negative for Zaire Ebola virus RNA. Of the 371 samples from bat species E. helvum (58 from Cameroon, 165 from the DRC, 3 from Guinea), $L$. angolensis (8 from Cameroon, 4 from Guinea), M. pusillus (2 from the DRC, 1 from Guinea), R. aegyptiacus (45 from Cameroon, 40 from Guinea), E. gambianus (25 from Guinea), and Mops sp. (20 from Cameroon), all were negative for Zaire Ebola virus RNA.

\section{Discussion}

To clarify the role of bats in Ebola virus ecology and identify where the virus circulates between outbreaks, we tested $>4,000$ bats, almost doubling the total number of samples tested in all previous studies in Africa $(5-7,42)$. We provided data on bats from Cameroon, added to the existing data on bats from Guinea and the DRC, and substantially increased the data available on insectivorous bats. We tested samples with the same assay, enabling comparison across species and countries. We used different statistical methods to determine positive sample numbers and expressed the proportion of reactive samples as a range on the basis of the different cutoff values proposed by those methods. As has been done in studies of human Zaire Ebola virus survivors $(28,43)$, we defined Zaire and Sudan Ebola virus positivity as the presence of antibodies to both nucleoprotein and glycoprotein. As such, we estimated that $2-37(0.05 \%-0.92 \%)$ bats were seropositive for Zaire Ebola virus and 0-30 (0\%-0.75\%) bats were seropositive for Sudan Ebola virus (Table 3). Among insectivorous bats, we observed Zaire and Sudan Ebola virus antibodies only in Mops sp. bats, an observation that has previously been observed (13). We provided information on insectivorous Miniopterus and Rhinolophus bats and extended knowledge on Mops and Hipposideros bats; all 1,200 Hipposideros samples were seronegative. We confirmed the presence of Zaire Ebola virus antibodies in only 1 of 3 frugivorous species in which Zaire Ebola virus RNA has been reported, that is, in $H$. monstrosus but not $E$. franqueti or $M$. torquata bats (6). However, this result might have been influenced by sample size, test used, and interpretation criteria. We confirmed antibodies in E. helvum bats and showed that Zaire Ebola virus antibodies are widespread among this species across Africa: Ghana and Zambia, and with our data, also Cameroon, Guinea, and the DRC $(13,14,16)$. We confirmed antibodies in $R$. aegyptiacus bats from Cameroon and Guinea, in agreement with previous findings in these bats from the Congo and Gabon (13). For E. gambianus bats from Ghana, we also observed Zaire Ebola virus reactivity of samples from this species in Guinea (15). In contrast with a previous study, we observed Sudan Ebola virus antibodies (not Zaire Ebola virus antibodies) in M. pusillus bats (13). We also identified Zaire Ebola virus antibodies in L. angolensis bats from Cameroon, although only when using less stringent cutoff calculations.

When combining data from previous Zaire Ebola virus seroprevalence studies in bats with data from our study, only 1 insectivorous bat species (Mops sp.) and 8 frugivorous bat species (E. helvum, E. gambianus, E. franqueti, $H$. monstrosus, L. angolensis, M. pusillus, M. torquata, R. aegyptiacus) exhibited Zaire Ebola virus antibodies (13-16). As seen in bat samples from Zambia, we observed in this study Sudan Ebola virus antibodies in E. helvum bats from Guinea, Cameroon, and the DRC, suggesting that Zaire and Sudan Ebola viruses co-circulate and could be widespread among this species. However, only 1 other study has tested 
Table 4. Blood samples from bats reactive with both nucleoprotein and glycoprotein of Zaire or Sudan Ebola virus, by statistical method used to determine cutoff, Guinea, Cameroon, and the Democratic Republic of the Congo, 2015-2017

\begin{tabular}{|c|c|c|c|c|c|c|c|c|c|c|}
\hline \multirow[b]{3}{*}{ Bat family, genus } & \multirow{3}{*}{$\begin{array}{c}\text { No. } \\
\text { tested }\end{array}$} & \multirow{3}{*}{$\begin{array}{c}\text { Ebola } \\
\text { virus } \\
\text { species }\end{array}$} & \multicolumn{8}{|c|}{ Statistical method } \\
\hline & & & \multicolumn{2}{|c|}{ Mean $+4 \times$ SD } & \multicolumn{2}{|c|}{ Change-point } & \multicolumn{2}{|c|}{ Binomial } & \multicolumn{2}{|r|}{ Exponential } \\
\hline & & & No. & $\%(95 \% \mathrm{Cl})$ & No. & $\%(95 \% \mathrm{Cl})$ & No. & $\%(95 \% \mathrm{Cl})$ & No. & $\%(95 \% \mathrm{Cl})$ \\
\hline \multicolumn{11}{|l|}{ Hipposideridae } \\
\hline Hipposideros sp. & $\begin{array}{l}1,282 \\
1,282\end{array}$ & $\begin{array}{l}\text { Zaire } \\
\text { Sudan }\end{array}$ & $\begin{array}{l}0 \\
0\end{array}$ & $\begin{array}{l}0(0-0.3) \\
0(0-0.3)\end{array}$ & $\begin{array}{l}0 \\
0\end{array}$ & $\begin{array}{l}0(0-0.3) \\
0(0-0.3)\end{array}$ & $\begin{array}{l}0 \\
0\end{array}$ & $\begin{array}{l}0(0-0.3) \\
0(0-0.3)\end{array}$ & $\begin{array}{l}0 \\
0\end{array}$ & $\begin{array}{l}0(0-0.3) \\
0(0-0.3)\end{array}$ \\
\hline \multicolumn{11}{|l|}{ Miniopteridae } \\
\hline Miniopterus sp. & $\begin{array}{l}232 \\
232 \\
\end{array}$ & $\begin{array}{l}\text { Zaire } \\
\text { Sudan }\end{array}$ & $\begin{array}{l}0 \\
0\end{array}$ & $\begin{array}{l}0(0-1.6) \\
0(0-1.6)\end{array}$ & $\begin{array}{l}0 \\
0\end{array}$ & $\begin{array}{l}0(0-1.6) \\
0(0-1.6)\end{array}$ & $\begin{array}{l}0 \\
0\end{array}$ & $\begin{array}{l}0(0-1.6) \\
0(0-1.6)\end{array}$ & $\begin{array}{l}0 \\
0\end{array}$ & $\begin{array}{l}0(0-1.6) \\
0(0-1.6)\end{array}$ \\
\hline \multicolumn{11}{|l|}{ Molossidae } \\
\hline Chaerephon sp. & $\begin{array}{c}44 \\
44 \\
494 \\
494 \\
\end{array}$ & $\begin{array}{l}\text { Zaire } \\
\text { Sudan } \\
\text { Zaire } \\
\text { Sudan }\end{array}$ & $\begin{array}{l}0 \\
0 \\
6 \\
2 \\
\end{array}$ & $\begin{array}{c}0(0-8.0) \\
0(0-8.0) \\
1.2(0.6-2.6) \\
0.4(0.1-1.5)\end{array}$ & $\begin{array}{l}0 \\
0 \\
6 \\
2 \\
\end{array}$ & $\begin{array}{c}0(0-8.0) \\
0(0-8.0) \\
1.2(0.6-2.6) \\
0.4(0.1-1.5)\end{array}$ & $\begin{array}{l}0 \\
0 \\
1 \\
0\end{array}$ & $\begin{array}{c}0(0-8.0) \\
0(0-8.0) \\
0.2(0.03-1.1) \\
0(0-0.8)\end{array}$ & $\begin{array}{l}0 \\
0 \\
1 \\
0\end{array}$ & $\begin{array}{c}0(0-8.0) \\
0(0-8.0) \\
0.2(0.03-1.1) \\
0(0-0.8)\end{array}$ \\
\hline \multicolumn{11}{|l|}{ Nycteridae } \\
\hline Nycteris sp & $\begin{array}{l}22 \\
22\end{array}$ & $\begin{array}{l}\text { Zaire } \\
\text { Sudan }\end{array}$ & $\begin{array}{l}0 \\
0\end{array}$ & $\begin{array}{l}0(0-14.9) \\
0(0-14.9)\end{array}$ & $\begin{array}{l}0 \\
0\end{array}$ & $\begin{array}{l}0(0-14.9) \\
0(0-14.9)\end{array}$ & $\begin{array}{l}0 \\
0\end{array}$ & $\begin{array}{l}0(0-14.9) \\
0(0-14.9)\end{array}$ & $\begin{array}{l}0 \\
0\end{array}$ & $\begin{array}{l}0(0-14.9) \\
0(0-14.9)\end{array}$ \\
\hline \multicolumn{11}{|l|}{ Rhinolophidae } \\
\hline Rhinolophus sp. & $\begin{array}{l}86 \\
86 \\
\end{array}$ & $\begin{array}{l}\text { Zaire } \\
\text { Sudan }\end{array}$ & $\begin{array}{l}0 \\
0\end{array}$ & $\begin{array}{l}0(0-4.3) \\
0(0-4.3)\end{array}$ & $\begin{array}{l}0 \\
0\end{array}$ & $\begin{array}{l}0(0-4.3) \\
0(0-4.3)\end{array}$ & $\begin{array}{l}0 \\
0\end{array}$ & $\begin{array}{l}0(0-4.3) \\
0(0-4.3)\end{array}$ & $\begin{array}{l}0 \\
0\end{array}$ & $\begin{array}{l}0(0-4.3) \\
0(0-4.3)\end{array}$ \\
\hline \multicolumn{11}{|l|}{ Vespertilionidae } \\
\hline Glauconycteris sp.* & $\begin{array}{l}3 \\
3\end{array}$ & $\begin{array}{l}\text { Zaire } \\
\text { Sudan }\end{array}$ & $\begin{array}{l}0 \\
0\end{array}$ & & $\begin{array}{l}0 \\
0\end{array}$ & & $\begin{array}{l}0 \\
0\end{array}$ & & $\begin{array}{l}0 \\
0\end{array}$ & \\
\hline Kerivoula sp.* & $\begin{array}{l}1 \\
1\end{array}$ & $\begin{array}{l}\text { Zaire } \\
\text { Sudan }\end{array}$ & $\begin{array}{l}0 \\
0\end{array}$ & & $\begin{array}{l}0 \\
0\end{array}$ & & $\begin{array}{l}0 \\
0\end{array}$ & & $\begin{array}{l}0 \\
0\end{array}$ & \\
\hline Myotis bocagii* & $\begin{array}{l}3 \\
3\end{array}$ & $\begin{array}{l}\text { Zaire } \\
\text { Sudan }\end{array}$ & $\begin{array}{l}0 \\
0\end{array}$ & & $\begin{array}{l}0 \\
0\end{array}$ & & $\begin{array}{l}0 \\
0\end{array}$ & & $\begin{array}{l}0 \\
0\end{array}$ & \\
\hline Neoromicia sp.* & $\begin{array}{l}5 \\
5\end{array}$ & $\begin{array}{l}\text { Zaire } \\
\text { Sudan }\end{array}$ & $\begin{array}{l}0 \\
0\end{array}$ & & $\begin{array}{l}0 \\
0\end{array}$ & & $\begin{array}{l}0 \\
0\end{array}$ & & $\begin{array}{l}0 \\
0\end{array}$ & \\
\hline Scotophilus sp. & $\begin{array}{l}22 \\
22\end{array}$ & $\begin{array}{l}\text { Zaire } \\
\text { Sudan }\end{array}$ & $\begin{array}{l}0 \\
0\end{array}$ & $\begin{array}{l}0(0-14.9) \\
0(0-14.9)\end{array}$ & $\begin{array}{l}0 \\
0\end{array}$ & $\begin{array}{l}0(0-14.9) \\
0(0-14.9)\end{array}$ & $\begin{array}{l}0 \\
0\end{array}$ & $\begin{array}{l}0(0-14.9) \\
0(0-14.9)\end{array}$ & $\begin{array}{l}0 \\
0\end{array}$ & $\begin{array}{l}0(0-14.9) \\
0(0-14.9)\end{array}$ \\
\hline \multicolumn{11}{|l|}{ Pteropodidae } \\
\hline \multirow[t]{2}{*}{ Eidolon helvum } & 480 & Zaire & 16 & $3.3(2.1-5.4)$ & 16 & $3.3(2.1-5.4)$ & 1 & $0.2(0-1.2)$ & 4 & $0.8(0.3-2.1)$ \\
\hline & 480 & Sudan & 14 & $2.9(1.7-4.8)$ & 14 & $2.9(1.7-4.8)$ & 0 & $0(0-0.8)$ & 5 & $1.0(0.4-2.4)$ \\
\hline \multirow[t]{2}{*}{ Epomophorus sp. } & 207 & Zaire & 1 & $0.5(0.08-2.7)$ & 1 & $0.5(0.08-2.7)$ & 0 & $0(0-1.4)$ & 0 & $0(0-1.8)$ \\
\hline & 207 & Sudan & 0 & $0(0-1.8)$ & 0 & $0(0-1.8)$ & 0 & $0(0-1.8)$ & 0 & $0(0-1.8)$ \\
\hline Epomops sp. & $\begin{array}{l}280 \\
280\end{array}$ & $\begin{array}{l}\text { Zaire } \\
\text { Sudan }\end{array}$ & $\begin{array}{l}0 \\
0\end{array}$ & $0(0-1.4)$ & $\begin{array}{l}0 \\
0\end{array}$ & $0(0-1.4)$ & 0 & $\begin{array}{l}0(0-1.4) \\
0(0-1.4)\end{array}$ & $\begin{array}{l}0 \\
0\end{array}$ & $0(0-1.4)$ \\
\hline Hypsignathus & 185 & Zaire & 3 & $1.6(0.6-4.7)$ & 3 & $1.6(0.6-4.7)$ & 0 & $0(0-2.0)$ & 1 & $0.5(0.05-3.0)$ \\
\hline monstrosus & 185 & Sudan & 8 & $4.3(2.2-8.3)$ & 8 & $4.3(2.2-8.3)$ & 3 & $1.6(0.6-4.7)$ & 2 & $1.1(0.3-3.9)$ \\
\hline Lissonycteris & 84 & Zaire & 2 & $2.4(0.7-8.3)$ & 2 & $2.4(0.7-8.3)$ & 0 & $0(0-4.4)$ & 0 & $0(0-4.4)$ \\
\hline angolensis & 84 & Sudan & 0 & $0(0-4.4)$ & 0 & $0(0-4.4)$ & 0 & $0(0-4.4)$ & 0 & $0(0-4.4)$ \\
\hline Megaloglossus & 20 & Zaire & 0 & $0(0-16.1)$ & 0 & $0(0-16.1)$ & 0 & $0(0-16.1)$ & 0 & $0(0-16.1)$ \\
\hline woermanni & 20 & Sudan & 0 & $0(0-16.1)$ & 0 & $0(0-16.1)$ & 0 & $0(0-16.1)$ & 0 & 0 (0-16.1) \\
\hline Micropteropus & 64 & Zaire & 0 & $0(0-5.7)$ & 0 & $0(0-5.7)$ & 0 & $0(0-5.7)$ & 0 & $0(0-5.7)$ \\
\hline pusillus & 64 & Sudan & 1 & $1.6(0.3-8.3)$ & 1 & $1.6(0.3-8.3)$ & 0 & $0(0-5.7)$ & 0 & $0(0-5.7)$ \\
\hline Myonycteris & 56 & Zaire & 0 & $0(0-6.4)$ & 0 & $0(0-6.4)$ & 0 & $0(0-6.4)$ & 0 & $0(0-6.4)$ \\
\hline torquata & 56 & Sudan & 0 & $0(0-6.4)$ & 0 & $0(0-6.4)$ & 0 & $0(0-6.4)$ & 0 & $0(0-6.4)$ \\
\hline Rousettus & 359 & Zaire & 9 & $2.5(1.3-4.7)$ & 9 & $2.5(1.3-4.7)$ & 0 & $0(0-1.1)$ & 2 & $0.6(0.2-2.0)$ \\
\hline aegyptiacus & 359 & Sudan & 5 & $1.4(0.6-3.2)$ & 5 & $1.4(0.6-3.2)$ & 0 & $0(0-1.1)$ & 3 & $0.8(0.3-2.4)$ \\
\hline Scotonycteris & 1 & Zaire & 0 & & 0 & & 0 & & 0 & \\
\hline zenkeri* & 1 & Sudan & 0 & & 0 & & 0 & & 0 & \\
\hline
\end{tabular}

for Ebola viruses other than Zaire Ebola virus in E. helvum bats (16). In our study, we also observed Sudan Ebola virus antibodies in Mops sp., H. monstrosus, and R. aegyptiacus bats in Cameroon. Almost all samples were positive for either Zaire or Sudan Ebola virus but not for both.

Despite the presence of Ebola virus antibodies, the role of bats as reservoir species remains unclear because viral RNA detection is rare. In only 1 study Zaire Ebola virus RNA was amplified in a few bats (6). Thus, antibodies might reflect previous acute infection with viral clearance. Unlike inoculations with Marburg virus (44-46), experimental inoculation of $R$. aegyptiacus bats with Zaire Ebola virus leads to antibody development but infrequent or absent detection of viral RNA or shedding $(44,47)$. $R$. aegyptiacus bats are therefore able to clear Zaire Ebola virus after a short infectious period without viral shedding and with little or no transmission. No antibodies or viral RNA were detected in noninoculated 
Table 5. Zaire Ebola virus antibodies in insectivorous bats from our research, Guinea, Cameroon, and the DRC, 2015-2017, and other published studies*

\begin{tabular}{|c|c|c|c|c|c|c|c|}
\hline Family & Species & Country & $\begin{array}{l}\text { Year of study } \\
\text { (reference) }\end{array}$ & Test & $\begin{array}{c}\text { No. } \\
\text { tested }\end{array}$ & $\begin{array}{l}\text { No. }(\%) \\
\text { positive† }\end{array}$ & $\begin{array}{c}\text { Total, no. } \\
\text { positive/tested (\%)† }\end{array}$ \\
\hline Emballonuridae & $\begin{array}{c}\text { Coleura afra } \\
\text { Saccolaimus peli }\end{array}$ & $\begin{array}{l}\text { Cameroon } \\
\text { DRC }\end{array}$ & $\begin{array}{c}2015-2017 \ddagger \\
1979-1980(26)\end{array}$ & $\begin{array}{l}\text { Luminex } \\
\text { IFA }\end{array}$ & $\begin{array}{l}5 \\
9 \\
\end{array}$ & $\begin{array}{c}0-0(0-0) \\
0(0)\end{array}$ & $0 / 14(0)$ \\
\hline Hipposideridae & $\begin{array}{l}\text { Hipposideros sp. } \\
\text { Hipposideros sp. } \\
\text { Hipposideros sp. } \\
\text { Hipposideros sp. } \\
\text { Hipposideros sp. }\end{array}$ & $\begin{array}{c}\text { DRC } \\
\text { Cameroon } \\
\text { DRC } \\
\text { Guinea } \\
\text { Guinea }\end{array}$ & $\begin{array}{c}2015-2017 \ddagger \\
2015-2017 \ddagger \\
1979-1980(26) \\
2015-2017 \ddagger \\
2014(11)\end{array}$ & $\begin{array}{l}\text { Luminex } \\
\text { Luminex } \\
\text { IFA } \\
\text { Luminex } \\
\text { ELISA }\end{array}$ & $\begin{array}{c}157 \\
837 \\
69 \\
288 \\
44\end{array}$ & $\begin{array}{l}0-0(0-0) \\
0-0(0-0) \\
0(0) \\
0-0(0-0) \\
0(0)\end{array}$ & $0 / 1,395(0)$ \\
\hline Miniopteridae & $\begin{array}{l}\text { Miniopterus sp. } \\
\text { Miniopterus sp. } \\
\text { M. minor }\end{array}$ & $\begin{array}{l}\text { Guinea } \\
\text { DRC } \\
\text { DRC }\end{array}$ & $\begin{array}{c}2015-2017 \ddagger \\
2015-2017 \ddagger \\
1995(27)\end{array}$ & $\begin{array}{c}\text { Luminex } \\
\text { Luminex } \\
\text { ELISA }\end{array}$ & $\begin{array}{c}27 \\
205 \\
2\end{array}$ & $\begin{array}{c}0-0(0-0) \\
0-0(0-0) \\
0(0)\end{array}$ & $0 / 234(0)$ \\
\hline Molossidae & $\begin{array}{l}\text { Chaerephon sp. } \\
\text { C. pumilus } \\
\text { C. ansorgei } \\
\text { C. major } \\
\text { C. pumilus }\end{array}$ & $\begin{array}{c}\text { Guinea } \\
\text { Guinea } \\
\text { DRC } \\
\text { DRC } \\
\text { DRC }\end{array}$ & $\begin{array}{c}2015-2017 \ddagger \\
2014(11) \\
1995(27) \\
1979-1980(26) \\
1995(27)\end{array}$ & $\begin{array}{c}\text { Luminex } \\
\text { ELISA } \\
\text { ELISA } \\
\text { IFA } \\
\text { Elisa }\end{array}$ & $\begin{array}{c}44 \\
1 \\
120 \\
26 \\
210\end{array}$ & $\begin{array}{c}0-0(0-0) \\
0(0) \\
0(0) \\
0(0) \\
0(0)\end{array}$ & 0/401 (0) \\
\hline & $\begin{array}{c}\text { Mops sp. } \\
\text { Mops sp. } \\
\text { Mops sp. } \\
\text { Mops sp. } \\
\text { Mops condylurus } \\
\text { M. condylurus } \\
\text { Myopterus whitleyi }\end{array}$ & $\begin{array}{c}\text { Guinea } \\
\text { Cameroon } \\
\text { DRC } \\
\text { DRC } \\
\text { Gabon } \\
\text { Guinea } \\
\text { DRC } \\
\end{array}$ & $\begin{array}{c}2015-2017 \ddagger \\
2015-2017 \ddagger \\
1979-1980(26) \\
1995(27) \\
2003-2008(13) \\
2014(11) \\
1995(27)\end{array}$ & $\begin{array}{l}\text { Luminex } \\
\text { Luminex } \\
\text { IFA } \\
\text { ELISA } \\
\text { ELISA } \\
\text { ELISA } \\
\text { ELISA } \\
\end{array}$ & $\begin{array}{c}230 \\
264 \\
158 \\
28 \\
24 \\
1 \\
2 \\
\end{array}$ & $\begin{array}{c}0-0(0-0) \\
1-6(0.4-2.3) \\
0(0) \\
0(0) \\
3(12.5) \\
0(0) \\
0(0)\end{array}$ & 4-9/705 (0.6-1.3) \\
\hline Nycteridae & $\begin{array}{l}\text { Nycteris sp. } \\
\text { Nycteris sp. } \\
\text { Nycteris sp. } \\
\text { Nycteris sp. } \\
\text { Nycteris hispida }\end{array}$ & $\begin{array}{c}\text { Guinea } \\
\text { Guinea } \\
\text { Cameroon } \\
\text { DRC } \\
\text { DRC } \\
\end{array}$ & $\begin{array}{c}2015-2017 \ddagger \\
2014(11) \\
2015-2017 \ddagger \\
1979-1980(26) \\
1995(27)\end{array}$ & $\begin{array}{l}\text { Luminex } \\
\text { ELISA } \\
\text { Luminex } \\
\text { IFA } \\
\text { ELISA }\end{array}$ & $\begin{array}{c}15 \\
6 \\
7 \\
14 \\
1 \\
\end{array}$ & $\begin{array}{c}0-0(0-0) \\
0(0) \\
0-0(0-0) \\
0(0) \\
0(0)\end{array}$ & $0 / 43(0)$ \\
\hline Rhinolophidae & $\begin{array}{l}\text { Rhinolophus sp. } \\
\text { Rhinolophus sp. } \\
\text { Rhinolophus sp. }\end{array}$ & $\begin{array}{c}\text { Guinea } \\
\text { DRC } \\
\text { Cameroon } \\
\end{array}$ & $\begin{array}{l}2015-2017 \ddagger \\
2015-2017 \ddagger \\
2015-2017 \ddagger \\
\end{array}$ & $\begin{array}{l}\text { Luminex } \\
\text { Luminex } \\
\text { Luminex }\end{array}$ & $\begin{array}{c}26 \\
6 \\
54 \\
\end{array}$ & $\begin{array}{l}0-0(0-0) \\
0-0(0-0) \\
0-0(0-0)\end{array}$ & $0 / 86(0)$ \\
\hline Vespertilionidae & $\begin{array}{c}\text { Glauconycteris variegata } \\
\text { Chalinolobus sp. } \\
\text { Eptesicus sp. } \\
\text { Eptesicus tenuipinnis } \\
\text { Kerivoula sp. } \\
\text { Kerivoula sp. } \\
\text { Myotis bocagii } \\
\text { M. bocagii } \\
\text { M. bocagii } \\
\text { Neoromicia sp. } \\
\text { Pipistrellus nanus } \\
\text { Scotophilus nux } \\
\text { Scotophilus leucogaster } \\
\text { Scotophilus nigrita } \\
\text { Scotophilus dinganii } \\
\text { Scotophilus sp. }\end{array}$ & $\begin{array}{l}\text { Cameroon } \\
\text { DRC } \\
\text { DRC } \\
\text { DRC } \\
\text { Guinea } \\
\text { Cameroon } \\
\text { Cameroon } \\
\text { DRC } \\
\text { DRC } \\
\text { Cameroon } \\
\text { DRC } \\
\text { Cameroon } \\
\text { Guinea } \\
\text { Guinea } \\
\text { DRC } \\
\text { DRC } \\
\end{array}$ & $\begin{array}{c}2015-2017 \ddagger \\
1979-1980(26) \\
1979-1980(26) \\
1995(27) \\
2014(11) \\
2015-2017 \ddagger \\
2015-2017 \ddagger \\
1995(27) \\
1979-1980(26) \\
2015-2017 \ddagger \\
1995(27) \\
2015-2017 \ddagger \\
2015-2017 \ddagger \\
2015-2017 \ddagger \\
1995(27) \\
1979-1980(26) \\
\end{array}$ & $\begin{array}{l}\text { Luminex } \\
\text { IFA } \\
\text { IFA } \\
\text { ELISA } \\
\text { ELISA } \\
\text { Luminex } \\
\text { Luminex } \\
\text { ELISA } \\
\text { IFA } \\
\text { Luminex } \\
\text { ELISA } \\
\text { Luminex } \\
\text { Luminex } \\
\text { Luminex } \\
\text { ELISA } \\
\text { IFA } \\
\end{array}$ & $\begin{array}{c}3 \\
15 \\
22 \\
1 \\
1 \\
1 \\
3 \\
22 \\
17 \\
5 \\
2 \\
6 \\
15 \\
1 \\
19 \\
10 \\
\end{array}$ & $\begin{array}{l}0-0(0-0) \\
0(0) \\
0(0) \\
0(0) \\
0(0) \\
0-0(0-0) \\
0-0(0-0) \\
0(0) \\
0(0) \\
0-0(0-0) \\
0(0) \\
0-0(0-0) \\
0-0(0-0) \\
0-0(0-0) \\
0(0) \\
0(0) \\
\end{array}$ & $0 / 143(0)$ \\
\hline Total & & & & & & & $4-9 / 3,023(0.13-0.30)$ \\
\hline \multicolumn{8}{|c|}{$\begin{array}{l}\text { *DRC, the Democratic Republic of the Congo; IFA, immunofluorescence assay. } \\
\text { †For data from cited studies, the number of positive samples reported in the original study is indicated. For our results, we show the range in the number } \\
\text { of samples simultaneously reactive with glycoprotein and nucleoprotein of Zaire Ebola virus on the basis of } 4 \text { different statistical methods used to } \\
\text { determine cutoff values. } \\
\text { łThis study. }\end{array}$} \\
\hline
\end{tabular}

bats housed with experimentally Zaire Ebola virusinfected bats (44). Whether this low level of infectiousness also occurs for other bat species that carry Ebola virus antibodies remains to be determined. Zaire Ebola virus was experimentally inoculated in other bat species (M. condylurus, Chaerephon pumilus, and Epomophorus wahlbergi) in only 1 study; virus replication was seen in all species, and fecal shedding was seen in E. wahlbergi bats (48). $R$. aegyptiacus bats experimentally infected with Marburg virus were shown to develop antibodies that protect against reinfection (49). Long-term survival with Zaire Ebola virus antibodies has been reported with E. helvum bats from Ghana but without information on protection (14). Among insectivorous bats, the presence of Ebola virus antibodies in only Mops sp. is striking, suggesting higher exposure or susceptibility compared with other insectivorous bats.

In conclusion, we demonstrated higher rates of Ebola virus antibodies in frugivorous than in insectivorous 
Table 6. Zaire Ebola virus antibodies in frugivorous (Pteropodidae family) bats from our research, Guinea, Cameroon, and the DRC, 2015-2017, and published studies*

\begin{tabular}{|c|c|c|c|c|c|c|}
\hline Species & Country & $\begin{array}{c}\text { Year of study } \\
\text { (reference) }\end{array}$ & Test & $\begin{array}{c}\text { No. } \\
\text { tested }\end{array}$ & $\begin{array}{l}\text { No. (\%) } \\
\text { positive }\end{array}$ & Total, no. positive/tested (\%) \\
\hline Casinycteris ophiodon & Guinea & $2014(11)$ & ELISA & 1 & 0 & $0 / 20$ \\
\hline Casinycteris argynnis & Gabon, Congo & $2003-2008(13)$ & ELISA & 18 & 0 & \\
\hline C. argynnis & DRC & $1995(27)$ & ELISA & 1 & 0 & \\
\hline \multirow[t]{8}{*}{ Eidolon helvum $†$} & Guinea & $2014(11)$ & ELISA & 6 & 0 & $21-36 / 1,551$ (1.4-2.3) \\
\hline & Guinea & 2015-2017‡ & Luminex & 17 & $0-3(0-17.6)$ & \\
\hline & Ghana & $2008(14)$ & IFA & 262 & $1(0.39)$ & \\
\hline & Cameroon & 2015-2017‡ & Luminex & 158 & $1-9(0.6-5.7)$ & \\
\hline & Gabon, Congo & 2003-2008 (13) & ELISA & 49 & 0 & \\
\hline & DRC & 1979-1980 (26) & IFA & 6 & 0 & \\
\hline & DRC & 2015-2017‡ & Luminex & 305 & $0-4(0-1.3)$ & \\
\hline & Zambia & $2006-2013(16)$ & ELISA & 748 & $19(2.55)$ & \\
\hline \multirow[t]{2}{*}{ Epomophorus gambianus } & Guinea & $2015-2017 \ddagger$ & Luminex & 191 & $0-1(0-0.5)$ & $4-5 / 244(1.6-2.0)$ \\
\hline & Ghana & $2007(15)$ & ELISA & 37 & $4(10.82)$ & \\
\hline Epomophorus wahlbergi & Cameroon & 2015-2017 & Luminex & 16 & $0-0(0-0)$ & \\
\hline \multirow{2}{*}{ Epomops buettikoferi } & Guinea & $2014(11)$ & ELISA & 17 & 0 & $47 / 1,269(3.7)$ \\
\hline & Guinea & $2015-2017 \ddagger$ & Luminex & 4 & $0-0(0-0)$ & \\
\hline \multirow[t]{7}{*}{ Epomops franqueti } & Ghana & 2007 (15) & ELISA & 27 & $3(11.2)$ & \\
\hline & Cameroon & $2015-2017 \ddagger$ & Luminex & 256 & $0-0(0-0)$ & \\
\hline & Gabon, Congo & $2001-2005(6)$ & ELISA & 117 & $8(6.8)$ & \\
\hline & Gabon, Congo & 2003-2008 (13) & ELISA & 805 & $36(4.5)$ & \\
\hline & DRC & 2015-2017‡ & Luminex & 20 & $0-0(0-0)$ & \\
\hline & DRC & $1979-1980(26)$ & IFA & 21 & 0 & \\
\hline & DRC & $1995(27)$ & ELISA & 2 & 0 & \\
\hline \multirow[t]{8}{*}{ Hypsygnathus monstrosus } & Guinea & 2015-2017‡ & Luminex & 8 & $0-0(0-0)$ & $15-18 / 347$ (4.3-5.2) \\
\hline & Guinea & 2014 (13) & ELISA & 1 & 0 & \\
\hline & Ghana & $2008(14)$ & IFA & 3 & 0 & \\
\hline & Ghana & 2007 (15) & ELISA & 16 & 2 (12.5) & \\
\hline & Cameroon & 2015-2017 & Luminex & 176 & $0-3(0-1.7)$ & \\
\hline & Gabon, Congo & $2001-2005(6)$ & ELISA & 17 & $4(23.5)$ & \\
\hline & Gabon, Congo & 2003-2008 (13) & ELISA & 125 & $9(7.2)$ & \\
\hline & DRC & 2015-2017‡ & Luminex & 1 & $0-0(0-0)$ & \\
\hline \multirow[t]{4}{*}{ Lissonycteris angolensis } & Guinea & $2014(11)$ & ELISA & 45 & 0 & $0-2 / 129(0-1.6)$ \\
\hline & Guinea & 2015-2017 & Luminex & 32 & $0-0(0-0)$ & \\
\hline & DRC & 2015-2017 & Luminex & 22 & $0-0(0-0)$ & \\
\hline & Cameroon & $2015-2017 \ddagger$ & Luminex & 30 & $0-2(0-6.7)$ & \\
\hline Megaloglossus azagnyi & Guinea & $2014(11)$ & ELISA & 3 & 0 & $0 / 110$ \\
\hline \multirow[t]{4}{*}{ Megaloglossus woermanni } & Cameroon & 2015-2017‡ & Luminex & 19 & $0-0(0-0)$ & \\
\hline & Gabon, Congo & 2003-2008 (13) & ELISA & 49 & 0 & \\
\hline & DRC & 2015-2017‡ & Luminex & 1 & $0-0(0-0)$ & \\
\hline & DRC & $1995(27)$ & ELISA & 38 & 0 & \\
\hline \multirow[t]{5}{*}{ Micropteropus pusillus } & Guinea & 2015-2017‡ & Luminex & 18 & $0-0(0-0)$ & $4 / 339$ (1.2) \\
\hline & Cameroon & 2015-2017‡ & Luminex & 2 & $0-0(0-0)$ & \\
\hline & Gabon, Congo & $2003-2008$ (13) & ELISA & 197 & $4(2.04)$ & \\
\hline & DRC & 2015-2017 & Luminex & 44 & $0-0(0-0)$ & \\
\hline & DRC & $1995(27)$ & ELISA & 78 & 0 & \\
\hline Myonycteris leptodon & Guinea & $2014(11)$ & ELISA & 21 & 0 & $23-27 / 708$ (3.2-3.8) \\
\hline \multirow{4}{*}{ Myonycteris torquata } & Cameroon & 2015-2017‡ & Luminex & 21 & $0-0(0-0)$ & \\
\hline & Gabon, Congo & $2001-2005(6)$ & ELISA & 58 & $4(6.9)$ & \\
\hline & Gabon, Congo & 2003-2008 (13) & ELISA & 573 & $19(3.32)$ & \\
\hline & DRC & 2015-2017‡ & Luminex & 35 & $0-0(0-0)$ & \\
\hline \multirow[t]{2}{*}{ Nanonycteris veldkampii } & Guinea & $2014(11)$ & ELISA & 17 & 0 & $0 / 21$ \\
\hline & Ghana & $2007(15)$ & ELISA & 4 & 0 & \\
\hline \multirow[t]{3}{*}{ Rousettus aegyptiacus } & Guinea & 2015-2017 & Luminex & 228 & $0-1(0-0.4)$ & $24-33 / 666$ (3.6-5.0) \\
\hline & Cameroon & 2015-2017 & Luminex & 131 & $0-8(0-6.1)$ & \\
\hline & Gabon, Congo & $2003-2008$ (13) & ELISA & 307 & $24(7.8)$ & \\
\hline Scotonycteris zenkeri & Cameroon & 2015-2017 & Luminex & 1 & $0-0(0-0)$ & $0-0 / 1(0-0)$ \\
\hline Total & & & & & & $138-172 / 5,405(2.55-3.18)$ \\
\hline
\end{tabular}


bats. The total number of frugivorous species shown to be Zaire Ebola virus seropositive has increased to 8, and 1 insectivorous bat species (Mops sp.) was confirmed to be seropositive. Zaire and Sudan Ebola viruses circulate in different species across Africa, with potential co-circulation of both viruses in some species. Although we have data on $>8,000$ bats from $\geq 40$ species, this sample size is small, given the high numbers of bats that constitute colonies. This study illustrates the complexity of tracking the animal reservoir of Ebola viruses, not only because sampling of wild bats without performing euthanasia is difficult and time-consuming but also because of the absence of a reference standard for serologic tests. To clarify the significance of Ebola virus antibodies, documenting the extent to which viral RNA and shedding can be detected in species with antibodies is crucial for predicting and controlling the risk for new outbreaks. Efforts must continue not only to sample bats but also other animals to elucidate where the virus circulates in wildlife.

\section{Acknowledgments}

We thank the staffs from the Ministry of Health and Ministry of Environment and the national ethics committees from the DRC, Cameroon, and Guinea for permission to perform this study. We thank all the field staff from the DRC (Guy Midingi and Servet Kimbonza); Guinea (Souana Goumou, Mamadou Kalif Diallo, Pépé Justin Beavogui, Philippe Kolié, Michel Guilavogui); and Cameroon (Innocent Ndong Bass, Aime Mebenga, Joseph Moudindo, Thomas Atemkem) for the collection of bat samples. We thank the staffs of the National Institute of Biomedical Research (Kinshasa, the DRC), the Kongo Central Provincial Government (Matadi, the DRC), and Projet PRESICA; Donald Mbohli from Project Grand Singes; and the staff of the Institut National de Santé Publique (Conakry, Guinea) for logistical support in the field. We thank Seny Mane for his involvement and support in the implementation of this project and Daouda Bangoura for his constant support for this project and the facilitation of field missions. We thank the veterinary staff from the Parc Zoologique de Montpellier and Wilhelma Zoo and Botanical Garden for providing control samples.

This work was supported in part by grants from Institut National de la Santé et de la Recherche Médicale, the Ebola Task Force, REACTing, EBO-SURSY project funded by the European Union, Institut de Recherche pour le Développement (IRD), and Christophe Mérieux Prize 2015 (to J-.J.M.T.). A.K.K. was supported by a fellowship from the IRD and the University of Montpellier (MUSE, ANR-16-IDEX-0006). C.-J.V.-A. was supported by a fellowship from IRD, Labex EpiGenMed via the National Research Agency, Programme for Future Investment (ANR-10-LABX-12-01), and the University of Montpellier.

\section{About the Author}

Drs. Mbala Kingebeni and Keita are researchers from ASTRE of Centre de coopération internationale en recherche agronomique pour le développement, Institut national de la recherche agronomique and University of Montpellier, Montpellier, France. Their research interests include characterization of hosts that harbor zoonotic pathogens.

\section{References}

1. Mylne A, Brady OJ, Huang Z, Pigott DM, Golding N, Kraemer MU, et al. A comprehensive database of the geographic spread of past human Ebola outbreaks. Sci Data. 2014;1:140042. http://dx.doi.org/10.1038/sdata.2014.42

2. Baize S, Pannetier D, Oestereich L, Rieger T, Koivogui L, Magassouba N, et al. Emergence of Zaire Ebola virus disease in Guinea. N Engl J Med. 2014;371:1418-25. http://dx.doi.org/ 10.1056/NEJMoa1404505

3. Maganga GD, Kapetshi J, Berthet N, Kebela Ilunga B, Kabange F, Mbala Kingebeni P, et al. Ebola virus disease in the Democratic Republic of Congo. N Engl J Med. 2014;371:2083-91. http://dx.doi.org/10.1056/NEJMoa1411099

4. World Health Organization. Ebola outbreak Democratic Republic of the Congo 2017. 2017 Jul 2 [cited 2018 May 3]. http://www.who.int/emergencies/ebola-DRC-2017/en/

5. Pigott DM, Millear AI, Earl L, Morozoff C, Han BA, Shearer FM, et al. Updates to the zoonotic niche map of Ebola virus disease in Africa. eLife. 2016;5:e16412. http://dx.doi.org/10.7554/eLife.16412

6. Leroy EM, Kumulungui B, Pourrut X, Rouquet P, Hassanin A, Yaba P, et al. Fruit bats as reservoirs of Ebola virus. Nature. 2005;438:575-6. http://dx.doi.org/10.1038/438575a

7. Leendertz SA, Gogarten JF, Düx A, Calvignac-Spencer S, Leendertz FH. Assessing the evidence supporting fruit bats as the primary reservoirs for Ebola viruses. EcoHealth. 2016;13:18-25. http://dx.doi.org/10.1007/s10393-015-1053-0

8. Kamins AO, Rowcliffe JM, Ntiamoa-Baidu Y, Cunningham AA, Wood JL, Restif O. Characteristics and risk perceptions of Ghanaians potentially exposed to bat-borne zoonoses through bushmeat. EcoHealth. 2015;12:104-20. http://dx.doi.org/10.1007/ s10393-014-0977-0

9. Leroy E, Gonzalez JP, Pourrut X. Ebolavirus and other filoviruses. Curr Top Microbiol Immunol. 2007;315:363-87. http://dx.doi.org/ 10.1007/978-3-540-70962-6_15

10. Leroy EM, Epelboin A, Mondonge V, Pourrut X, Gonzalez JP, Muyembe-Tamfum JJ, et al. Human Ebola outbreak resulting from direct exposure to fruit bats in Luebo, Democratic Republic of Congo, 2007. Vector Borne Zoonotic Dis. 2009;9:723-8. http://dx.doi.org/10.1089/vbz.2008.0167

11. Marí Saéz A, Weiss S, Nowak K, Lapeyre V, Zimmermann F, Düx A, et al. Investigating the zoonotic origin of the West African Ebola epidemic. EMBO Mol Med. 2015;7:17-23. http://dx.doi.org/ 10.15252/emmm.201404792

12. Pourrut X, Délicat A, Rollin PE, Ksiazek TG, Gonzalez JP, Leroy EM. Spatial and temporal patterns of Zaire ebolavirus antibody prevalence in the possible reservoir bat species. J Infect Dis. 2007;196(Suppl 2):S176-83. http://dx.doi.org/ $10.1086 / 520541$

13. Pourrut X, Souris M, Towner JS, Rollin PE, Nichol ST, Gonzalez JP, et al. Large serological survey showing cocirculation of Ebola and Marburg viruses in Gabonese bat populations, and a high seroprevalence of both viruses in Rousettus aegyptiacus. BMC Infect Dis. 2009;9:159. http://dx.doi.org/10.1186/1471-2334-9-159

14. Hayman DT, Emmerich P, Yu M, Wang LF, Suu-Ire R, Fooks AR, et al. Long-term survival of an urban fruit bat seropositive for 
Ebola and Lagos bat viruses. PLoS One. 2010;5:e11978. http://dx.doi.org/10.1371/journal.pone.0011978

15. Hayman DT, Yu M, Crameri G, Wang LF, Suu-Ire R, Wood JL, et al. Ebola virus antibodies in fruit bats, Ghana, West Africa. Emerg Infect Dis. 2012;18:1207-9. http://dx.doi.org/10.3201/ eid1807.111654

16. Ogawa H, Miyamoto H, Nakayama E, Yoshida R, Nakamura I, Sawa H, et al. Seroepidemiological prevalence of multiple species of filoviruses in fruit bats (Eidolon helvum) migrating in Africa. J Infect Dis. 2015;212(Suppl 2):S101-8. http://dx.doi.org/10.1093/ infdis/jiv063

17. Towner JS, Pourrut X, Albariño CG, Nkogue CN, Bird BH, Grard G, et al. Marburg virus infection detected in a common African bat. PLoS One. 2007;2:e764. http://dx.doi.org/10.1371/ journal.pone. 0000764

18. Towner JS, Amman BR, Sealy TK, Carroll SA, Comer JA, Kemp A, et al. Isolation of genetically diverse Marburg viruses from Egyptian fruit bats. PLoS Pathog. 2009;5:e1000536. http://dx.doi.org/10.1371/journal.ppat.1000536

19. Kuzmin IV, Niezgoda M, Franka R, Agwanda B, Markotter W, Breiman RF, et al. Marburg virus in fruit bat, Kenya. Emerg Infect Dis. 2010;16:352-4. http://dx.doi.org/10.3201/eid1602.091269

20. Swanepoel R, Smit SB, Rollin PE, Formenty P, Leman PA, Kemp A, et al.; International Scientific and Technical Committee for Marburg Hemorrhagic Fever Control in the Democratic Republic of the Congo. Studies of reservoir hosts for Marburg virus. Emerg Infect Dis. 2007;13:1847-51. http://dx.doi.org/ 10.3201/eid1312.071115

21. Negredo A, Palacios G, Vázquez-Morón S, González F, Dopazo H, Molero F, et al. Discovery of an ebolavirus-like filovirus in europe. PLoS Pathog. 2011;7:e1002304. http://dx.doi.org/10.1371/ journal.ppat.1002304

22. Yang XL, Zhang YZ, Jiang RD, Guo H, Zhang W, Li B, et al. Genetically diverse filoviruses in Rousettus and Eonycteris spp. bats, China, 2009 and 2015. Emerg Infect Dis. 2017;23:482-6. http://dx.doi.org/10.3201/eid2303.161119

23. World Health Organization. Ebola situation report - 30 March 2016. 2016 [cited 2018 May 3]. http://apps.who.int./ebola/ current-situation/ebola-situation-report-30-march-2016

24. Dudas G, Carvalho LM, Bedford T, Tatem AJ, Baele G, Faria NR, et al. Virus genomes reveal factors that spread and sustained the Ebola epidemic. Nature. 2017;544:309-15. http://dx.doi.org/ 10.1038/nature22040

25. Pigott DM, Deshpande A, Letourneau I, Morozoff C, Reiner RC Jr, Kraemer MUG, et al. Local, national, and regional viral haemorrhagic fever pandemic potential in Africa: a multistage analysis. Lancet. 2017;390:2662-72. http://dx.doi.org/10.1016/ S0140-6736(17)32092-5

26. Breman JG, Johnson KM, van der Groen G, Robbins CB, Szczeniowski MV, Ruti K, et al.; Ebola Virus Study Teams. A search for Ebola virus in animals in the Democratic Republic of the Congo and Cameroon: ecologic, virologic, and serologic surveys, 1979-1980. J Infect Dis. 1999;179(Suppl 1):S139-47. http://dx.doi.org/10.1086/514278

27. Leirs H, Mills JN, Krebs JW, Childs JE, Akaibe D, Woollen N, et al. Search for the Ebola virus reservoir in Kikwit, Democratic Republic of the Congo: reflections on a vertebrate collection. J Infect Dis. 1999;179(Suppl 1):S155-63. http://dx.doi.org/ 10.1086/514299

28. Ayouba A, Touré A, Butel C, Keita AK, Binetruy F, Sow MS, et al. Development of a sensitive and specific serological assay based on luminex technology for detection of antibodies to Zaire Ebola virus. J Clin Microbiol. 2017;55:165-76. http://dx.doi.org/ 10.1128/JCM.01979-16

29. Peel AJ, McKinley TJ, Baker KS, Barr JA, Crameri G, Hayman DT, et al. Use of cross-reactive serological assays for detecting novel pathogens in wildlife: assessing an appropriate cutoff for henipavirus assays in African bats. J Virol Methods. 2013;193:295303. http://dx.doi.org/10.1016/j.jviromet.2013.06.030

30. Gilbert AT, Fooks AR, Hayman DT, Horton DL, Müller T, Plowright R, et al. Deciphering serology to understand the ecology of infectious diseases in wildlife. EcoHealth. 2013;10:298-313. http://dx.doi.org/10.1007/s10393-013-0856-0

31. Lardeux F, Torrico G, Aliaga C. Calculation of the ELISA's cut-off based on the change-point analysis method for detection of Trypanosoma cruzi infection in Bolivian dogs in the absence of controls. Mem Inst Oswaldo Cruz. 2016;111:501-4. http://dx.doi.org/10.1590/0074-02760160119

32. Killick R, Eckley IA. changepoint: an R package for changepoint analysis. J Stat Softw. 2014;58:1-19. http://dx.doi.org/10.18637/ jss.v058.i03

33. Hinkley DV. Inference about the change-point in a sequence of random variables. Biometrika. 1970;57:1-17. http://dx.doi.org/ 10.1093/biomet/57.1.1

34. Laing ED, Mendenhall IH, Linster M, Low DHW, Chen Y, Yan L, et al. Serologic evidence of fruit bat exposure to filoviruses, Singapore, 2011-2016. Emerg Infect Dis. 2018;24:114-7. http://dx.doi.org/10.3201/eid2401.170401

35. Cullen AC, Frey HC. Probabilistic techniques in exposure assessment. New York: Plenum Press; 1999. p. 81-159.

36. Delignette-Muller ML, Dutang C. fitdistrplus: an R package for fitting distributions. J Stat Softw. 2015;64:1-34. http://dx.doi.org/ 10.18637/jss.v064.i04

37. Monleau M, Montavon C, Laurent C, Segondy M, Montes B, Delaporte E, et al. Evaluation of different RNA extraction methods and storage conditions of dried plasma or blood spots for human immunodeficiency virus type 1 RNA quantification and PCR amplification for drug resistance testing. J Clin Microbiol. 2009;47:1107-18. http://dx.doi.org/10.1128/ JCM.02255-08

38. Guichet E, Serrano L, Laurent C, Eymard-Duvernay S, Kuaban C, Vidal L, et al. Comparison of different nucleic acid preparation methods to improve specific HIV-1 RNA isolation for viral load testing on dried blood spots. J Virol Methods. 2018;251:75-9. http://dx.doi.org/10.1016/j.jviromet.2017.10.014

39. Irwin DM, Kocher TD, Wilson AC. Evolution of the cytochrome b gene of mammals. J Mol Evol. 1991;32:128-44. http://dx.doi.org/ 10.1007/BF02515385

40. Kocher TD, Thomas WK, Meyer A, Edwards SV, Pääbo S, Villablanca FX, et al. Dynamics of mitochondrial DNA evolution in animals: amplification and sequencing with conserved primers. Proc Natl Acad Sci U S A. 1989;86:6196-200. http://dx.doi.org/10.1073/pnas.86.16.6196

41. He B, Feng Y, Zhang H, Xu L, Yang W, Zhang Y, et al. Filovirus RNA in fruit bats, China. Emerg Infect Dis. 2015;21:1675-7. http://dx.doi.org/10.3201/eid2109.150260

42. Han BA, Schmidt JP, Alexander LW, Bowden SE, Hayman DT, Drake JM. Undiscovered bat hosts of filoviruses. PLoS Negl Trop Dis. 2016;10:e004815. http://dx.doi.org/10.1371/ journal.pntd.0004815

43. Rimoin AW, Lu K, Bramble MS, Steffen I, Doshi RH, Hoff NA, et al. Ebola virus neutralizing antibodies detectable in survivors of theYambuku, Zaire outbreak 40 years after infection. J Infect Dis. 2018;217:223-31. http://dx.doi.org/10.1093/infdis/jix584

44. Paweska JT, Storm N, Grobbelaar AA, Markotter W, Kemp A, Jansen van Vuren P. Experimental inoculation of Egyptian fruit bats (Rousettus aegyptiacus) with Ebola virus. Viruses. 2016;8:29. http://dx.doi.org/10.3390/v8020029

45. Amman BR, Jones ME, Sealy TK, Uebelhoer LS, Schuh AJ, Bird BH, et al. Oral shedding of Marburg virus in experimentally infected Egyptian fruit bats (Rousettus aegyptiacus). J Wildl Dis. 2015;51:113-24. http://dx.doi.org/10.7589/2014-08-198 
46. Schuh AJ, Amman BR, Jones ME, Sealy TK, Uebelhoer LS, Spengler JR, et al. Modelling filovirus maintenance in nature by experimental transmission of Marburg virus between Egyptian rousette bats. Nat Commun. 2017;8:14446. http://dx.doi.org/10.1038/ncomms 14446

47. Jones ME, Schuh AJ, Amman BR, Sealy TK, Zaki SR, Nichol ST, et al. Experimental inoculation of Egyptian rousette bats (Rousettus aegyptiacus) with viruses of the Ebolavirus and Marburgvirus genera. Viruses. 2015;7:3420-42. http://dx.doi.org/10.3390/v7072779

48. Swanepoel R, Leman PA, Burt FJ, Zachariades NA, Braack LE, Ksiazek TG, et al. Experimental inoculation of plants and animals with Ebola virus. Emerg Infect Dis. 1996;2:321-5. http://dx.doi.org/10.3201/eid0204.960407

49. Storm N, Jansen Van Vuren P, Markotter W, Paweska JT. Antibody responses to Marburg virus in Egyptian rousette bats and their role in protection against infection. Viruses. 2018;10:73. http://dx.doi.org/10.3390/v10020073

Adress for correspondence: Martine Peeters, TransVIHMI, Institut de Recherche pour le Développement, 911 Ave Agropolis, BP34394 Montpellier CEDEX 1, France; e-mail: martine.peeters@ird.fr

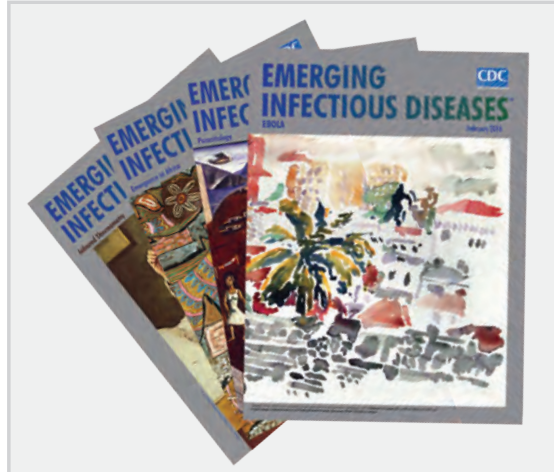

- Ebola and Its Control in Liberia, 2014-2015

- Epidemiology of Epidemic Ebola Virus Disease in Conakry and Surrounding Prefectures, Guinea, 2014-2015

- Hospital Preparations for Viral Hemorrhagic Fever Patients and Experience Gained from the Admission of an Ebola Patient

- Trematode Fluke Procerovum varium as Cause of Ocular Inflammation in Children, South India

- Association between Landscape Factors and Spatial Patterns of Plasmodium knowlesi Infections in Sabah, Malaysia

- Feasibility of Xpert Ebola Assay in Médecins Sans Frontières Ebola Program, Guinea

- Prognostic Indicators for Ebola Patient Survival

- Invasive Group A Streptococcus Infection among Children, Rural Kenya

- Randomized Controlled Trial of Hospital-Based Hygiene and Water Treatment Intervention ( $\mathrm{CHoBI}$ ) to Reduce Cholera

- Sustained Transmission of Pertussis in Vaccinated, 1-5-Year-Old Children in a Preschool, Florida, USA

- Molecular Characterization of Invasive Streptococcus dysgalactiae subsp. equisimilis, Japan

- Epidemiology of Serotype 1 Invasive Pneumococcal Disease, South Africa, 2003-2013

- Hemorrhagic Fever with Renal Syndrome, Zibo City, China, 2006-2014

\section{February 2016}

\section{Ebola}

- Dogs and Opossums Positive for Vaccinia Virus during Outbreak Affecting Cattle and Humans, São Paulo State, Brazil

- African Buffalo Movement and Zoonotic Disease Risk across Transfrontier Conservation Areas, Southern Africa

- Anaplasmataceae-Specific PCR for Diagnosis and Therapeutic Guidance for Symptomatic Neoehrlichiosis in Immunocompetent Host

- Candidatus Coxiella massiliensis Infection

- Ebola Virus Persistence in Semen Ex Vivo

- Ebola Virus RNA Stability in Human Blood and Urine in West Africa's Environmental Conditions

- Uveitis and Systemic Inflammatory Markers in Convalescent Phase of Ebola Virus Disease

- Louseborne Relapsing Fever among East African Refugees, Italy, 2015

- Mediterranean Fin Whales (Balaenoptera physalus) Threatened by Dolphin Morbillivirus

- Blastomyces gilchristii as Cause of Fatal Acute Respiratory Distress Syndrome

- Effectiveness of Meningococcal B Vaccine against Endemic Hypervirulent Neisseria meningitidis W Strain, England

- Frequency and Distribution of Rickettsiae, Borreliae, and Ehrlichiae Detected in Human-Parasitizing Ticks, Texas, USA

- High Prevalence of Borrelia miyamotoi among Adult Blacklegged Ticks from White-Tailed Deer 


\title{
Rat Hepatitis E Virus as Cause of Persistent Hepatitis after Liver Transplant
}

\author{
Siddharth Sridhar, Cyril C.Y. Yip, Shusheng Wu, Jianpiao Cai, Anna Jin-Xia Zhang, \\ Kit-Hang Leung, Tom W.H. Chung, Jasper F.W. Chan, Wan-Mui Chan, \\ Jade L.L. Teng, Rex K.H. Au-Yeung, Vincent C.C. Cheng, Honglin Chen, Susanna K.P. Lau, \\ Patrick C.Y. Woo, Ning-Shao Xia, Chung-Mau Lo, Kwok-Yung Yuen
}

\begin{abstract}
All hepatitis $E$ virus (HEV) variants reported to infect humans belong to the species Orthohepevirus $A$ (HEV-A). The zoonotic potential of the species Orthohepevirus $C$ (HEV-C), which circulates in rats and is highly divergent from HEV-A, is unknown. We report a liver transplant recipient with hepatitis caused by HEV-C infection. We detected HEV-C RNA in multiple clinical samples and HEV-C antigen in the liver. The complete genome of the HEV-C isolate had 93.7\% nt similarity to an HEV-C strain from Vietnam. The patient had preexisting HEV antibodies, which were not protective against HEV-C infection. Ribavirin was an effective treatment, resulting in resolution of hepatitis and clearance of HEV-C viremia. Testing for this zoonotic virus should be performed for immunocompromised and immunocompetent patients with unexplained hepatitis because routine hepatitis $\mathrm{E}$ diagnostic tests may miss HEV-C infection. HEV-C is also a potential threat to the blood product supply.
\end{abstract}

$\mathrm{H}$ epatitis E virus (HEV) infects 20 million humans worldwide annually (1). HEV-infected persons usually have self-limiting acute hepatitis. However, persistent hepatitis can occur in HEV-infected immunocompromised patients who acquire infection by eating undercooked pork, rabbit, deer, camel, or boar meat (2-6). HEV transmission through blood product transfusion also has been described (7).

The diverse Hepeviridae family, which incorporates all HEV variants, includes members whose primary host species are terrestrial mammals (genus Orthohepevirus) and fish (genus Piscihepevirus) (8). The Orthohepevirus genus is classified into 4 species; HEV variants that have

Author affiliations: The University of Hong Kong, Hong Kong,

China (S. Sridhar, C.C.Y. Yip, S. Wu, J. Cai, A.J.-X. Zhang,

K.-H. Leung, T.W.H. Chung, J.F.W. Chan, W.-M. Chan,

J.L.L. Teng, R.K.H. Au-Yeung, V.C.C. Cheng, H. Chen, S.K.P. Lau, P.C.Y. Woo, C.-M. Lo, K.-Y. Yuen); The University of Hong KongShenzhen Hospital, Shenzhen, China (J.F.W. Chan, C.-M. Lo, K.-Y. Yuen); Xiamen University, Xiamen, China (N.-S. Xia)

DOI: https://doi.org/10.3201/eid2412.180937 been reported to infect humans belong to Orthohepevirus A (HEV-A). Five genotypes within HEV-A (HEV-1-4 and -7) cause hepatitis in humans, and 3 genotypes (HEV-3, -4, and -7) can cause chronic hepatitis in immunocompromised patients after foodborne zoonotic transmission $(2,6,9,10)$.

In addition to HEV-A, the Orthohepevirus genus includes 3 other species: Orthohepevirus B circulates in chickens, Orthohepevirus C (HEV-C) in rats and ferrets, and Orthohepevirus $D$ in bats. HEV-C, also known as rat hepatitis E virus, shares only $50 \%-60 \%$ nt identity with HEV-A (8). The zoonotic potential of HEV-C is unknown; cases of clinical infection have not been reported. The substantial phylogenetic divergence between HEV-A and HEV-C, especially in critical receptor binding domains, forms a theoretical species barrier (11). Serologic and molecular tests for HEV are designed primarily to detect HEV-A, and they might miss HEV-C infections. Therefore, the threat to human health, including blood and organ supply safety, from HEV-C is unknown. We aimed to prove definitively that HEV-C can infect humans and describe the clinical, epidemiologic, genomic, and serologic features of this new zoonosis.

\section{Materials and Methods}

\section{Study Population}

We conducted this study in Queen Mary Hospital, a 1,700bed tertiary care hospital in Hong Kong. We assessed 518 solid-organ transplant recipients (kidney, liver, lung, and heart transplant) who were followed up in Queen Mary Hospital for persistent biochemical hepatitis from January 1, 2014, or date of transplant (whichever date was later) through December 31, 2017. We defined persistent hepatitis as elevation of alanine aminotransferase (ALT) $>1.5$ times the upper limit of the reference level for a continuous period of $\geq 6$ weeks. For patients whose ALT met this definition, we reviewed clinical records, ultrasonogram results, endoscopic retrograde cholangiopancreatography results, and laboratory results to identify the likely cause of hepatitis. We 
considered patients to have hepatitis B virus (HBV), hepatitis $\mathrm{C}$ virus (HCV), or cytomegalovirus (CMV) reactivation if any of these viruses were detected in blood during the hepatitis episode. In patients with no identifiable cause of hepatitis, HEV IgM ELISA screening was performed, in accordance with the usual practice in Queen Mary Hospital. HEV infection was diagnosed if the HEV IgM assay was positive, and persistent HEV infection was diagnosed if HEV viremia in patient plasma lasted for $\geq 3$ months. PCR sequencing was performed for speciation of HEV isolate. We obtained ethics approval from the Institutional Review Board of the University of Hong Kong/Hospital Authority West Cluster. We obtained written informed consent from all patients with persistent HEV infection.

\section{Nucleic Acid Detection for Hepatitis Viruses and HEV Complete Genome Sequencing}

We designed 3 in-house-developed reverse transcription PCRs (RT-PCRs) to detect HEV (online Technical Appendix Table 1, https://wwwnc.cdc.gov/EID/article/24/12/180937-Techapp1.pdf). Hepatitis A virus (HAV) RNA and CMV DNA detections were performed using in-house nucleic acid amplification tests. HBV and HCV viral loads were quantified using commercial kits (COBAS TaqMan, Roche, Basel, Switzerland; and RealTime HCV, Abbott, Chicago, IL, USA, respectively).

We sequenced the PCR product of the pan-Orthohepevirus RT-PCR using the RT-PCR primers. Because the RNA-dependent RNA polymerase sequences of patient HEV isolates clustered with rat HEV-C strains, primers for complete genome amplification were designed by multiple alignment of rat HEV-C genomes in GenBank (online Technical Appendix Table 2). We used these primers for complete genome sequencing of HEV in patient feces (strain LCK-3110). We constructed phylogenetic trees using MEGA6 with the general time reversible plus gamma model (12).

\section{Cloning and Purification of Recombinant HEV-A and HEV-C Open Reading Frame 2 Protein}

We used specific primers (online Technical Appendix) to amplify the genes encoding the 239 aa immunogenic recombinant peptides of HEV-A (genotype 4) and HEVC. Cloning the amplified genes into a bacterial expression vector, expression in Escherichia coli, and protein purification were performed as previously described $(13,14)$.

\section{Antibodies Against HEV-A and HEV-C}

Polyclonal antibodies against the HEV-C recombinant protein were raised in mice (online Technical Appendix). In addition, we used 2 murine monoclonal antibodies (mAbs) against open reading frame (ORF) 2 antigen of HEV-A in this study.

\section{Serologic Testing}

We conducted HEV antibody screening for patients with unexplained persistent hepatitis using HEV IgM and HEV IgG commercial ELISA kits (Wantai, Beijing, China) and detected hepatitis B surface antigen (HBsAg) using the ARCHITECT HBsAg chemiluminescent microparticle immunoassay (Abbott). HAV IgM and HCV antibodies were tested using VIDAS immunoassay kits (bioMérieux, Marcy-L’Étoile, France). For investigation of the HEV-C transmission event, we subjected patient and donor serum to HEV-A and HEV-C Western blots using polyclonal antiserum from mice inoculated with HEV-C protein and mAbs as controls. ELISAs using recombinant HEV-A and HEV-C proteincoated plates were designed based on the method described by Shimizu et al. with modifications (15). We set cutoffs and interpreted results to differentiate HEV$\mathrm{A}-$ and HEV-C-specific serologic responses (online Technical Appendix).

\section{Virus Culture}

We selected cell lines A549 (lung adenocarcinoma), Huh-7 (hepatocellular carcinoma), and Caco-2 (colorectal adenocarcinoma) to investigate whether human cell lines could support HEV-C growth. Cell lines were chosen if they supported growth of patient-derived HEV isolates or HEV infectious clones (16-18) (online Technical Appendix). We subjected supernatants and lysates to HEV-C quantitative RT-PCR (qRT-PCR) and immunostaining.

\section{Immunohistochemical and Immunofluorescence Staining} We conducted immunohistochemical staining of formalin-fixed paraffin-embedded liver tissue sections and infected A549 cell culture monolayers using HEV-C polyclonal serum antibodies and HEV-A mAbs. We performed immunofluorescence staining of permeabilized infected cells using HEV-C polyclonal antiserum (online Technical Appendix).

\section{Epidemiologic and Environmental Investigation}

We retrieved organ and blood donor serum for HEV ELISA, Western blot, and HEV-C qRT-PCR. To survey density of rat fecal contamination and collect environmental specimens for HEV-C qRT-PCR, we visited the patient's housing estate on November 22, 2017. Furthermore, from deep freezers we retrieved archived Rattus sp. liver, spleen, rectal swab, and kidney specimens collected during 2012-2017 within a 2.5-km radius around the patient's residence for preexisting pathogen surveillance programs and subjected them to HEV-C qRT-PCR. The HEV-C ORF2 fragment of qRT-PCR-positive specimens was sequenced using additional primers (online Technical Appendix Table 3). 


\section{Results}

\section{Hepatitis E Incidence in Transplant Recipient Cohort} Of 518 patients, $52(10.2 \%)$ had persistent hepatitis (Table $1)$. Five $(9.6 \%)$ patients with hepatitis tested positive for HEV IgM; 4 of these were kidney transplant recipients, and 1 was a liver transplant recipient. Together with reactivation of chronic HBV infection, HEV was the third most common cause of viral hepatitis in the local transplant population. Of the 5 patients, plasma HEV-A qRT-PCR of 3 renal transplant recipients was positive; another renal transplant recipient tested negative for HEV RNA. We have previously reported the clinical details of the $3 \mathrm{HEV}$ A-infected patients (9). Rat-derived HEV-C infection was diagnosed in the liver transplant recipient, which accounted for $1.9 \%(1 / 52)$ of persistent hepatitis in our cohort.

\section{Patient History}

A 56-year-old man underwent deceased-donor liver transplant on May 14, 2017, because of hepatocellular carcinoma complicating chronic HBV carriage. He received 1,000 mg hydrocortisone and $20 \mathrm{mg}$ basiliximab (anti-interleukin-2 receptor $\mathrm{mAb}$ ) as intraoperative antirejection prophylaxis and 4 units of platelets (derived from 4 separate blood donors) during the operation. His liver function tests

Table 1. Demographic and clinical characteristics of solid organ transplant recipients, Queen Mary Hospital, Hong Kong, January 1, 2014-December 31, 2017*

\begin{tabular}{lc}
\hline Characteristic & Result $\dagger$ \\
\hline No. transplant recipients & 518 \\
\hline Organ transplanted & \\
Kidney & $430(83.0)$ \\
Liver & $61(11.7)$ \\
Heart & $16(3.1)$ \\
Lung & $10(1.9)$ \\
Combined kidney and liver & $1(0.2)$ \\
\hline Median age, y & 56 \\
\hline Sex & $203(39.2)$ \\
F & $315(60.8)$ \\
M & $52(10.2)$ \\
hepatitis & \\
\hline Cause of biochemical hepatitis & \\
Viral hepatitis $\ddagger$ & \\
Reactivation of chronic HBV infection & $5(9.6)$ \\
Chronic HCV infection & $7(13.5)$ \\
Chronic HEV infection & $5(9.6)$ \\
CMV reactivation & $8(15.4)$ \\
Nonviral causes $\dagger$ & $7(13.5)$ \\
Drug toxicity & $3(5.8)$ \\
Nonalcoholic fatty liver disease & $7(13.5)$ \\
Liver graft rejection & $5(9.6)$ \\
Biliary anastomotic stricture & $2(3.8)$ \\
Liver malignancies & $2(3.8)$ \\
Septic cholestasis & $1(1.9)$ \\
Recurrent pyogenic cholangitis & \\
\hline
\end{tabular}

${ }^{*} \mathrm{CMV}$, cytomegalovirus; $\mathrm{HBV}$, hepatitis $\mathrm{B}$ virus; $\mathrm{HCV}$, hepatitis $\mathrm{C}$ virus; HEV, hepatitis E virus.

†All results are no. (\%) unless otherwise indicated.

‡All percentages based on no. patients with biochemical hepatitis.
(LFTs) reverted to normal, and he was discharged on posttransplant day 11 . He was taking mycophenolate mofetil (500 mg $2 \times / \mathrm{d}$ ), tacrolimus ( $1 \mathrm{mg} 2 \times / \mathrm{d}$ ), and prednisolone (5 mg $2 \times / \mathrm{d}$ ) as antirejection prophylaxis. He was also taking entecavir (0.5 mg $1 \times / \mathrm{d})$ for HBV suppression; serum HBsAg was negative 6 weeks after the transplant.

Routine phlebotomy on July 12 (day 59 posttransplant) revealed mild derangement of ALT to $74 \mathrm{U} / \mathrm{L}$ (reference 8-58 U/L). Other LFTs were normal. One week later, there was further derangement of parenchymal liver enzymes: ALT was $138 \mathrm{U} / \mathrm{L}$, aspartate aminotransferase was elevated to $65 \mathrm{U} / \mathrm{L}$ (reference $15-38 \mathrm{U} / \mathrm{L}$ ), $\gamma$-glutamyltransferase was $124 \mathrm{U} / \mathrm{L}$ (reference 11-62 U/L), and alkaline phosphatase was within reference limits at $70 \mathrm{U} / \mathrm{L}$ (reference 42-110 U/L). Complete blood count showed lymphopenia, at $0.88 \times 10^{9}$ cells/L, although total leukocyte count was within reference levels.

The patient was empirically managed for acute graft rejection with increased immunosuppression using a 3-day course of methylprednisolone. Valganciclovir was prescribed for low-level whole blood CMV viremia of 5.31 $\times 10^{2} \mathrm{IU} / \mathrm{mL}$. However, LFTs continued to deteriorate despite clearance of CMV viremia and increased immunosuppression. Liver biopsy showed nonspecific mild to moderate inflammatory infiltrate comprising small lymphocytes in the portal tracts. There were no viral inclusion bodies, and immunohistochemical staining for CMV and hepatitis $\mathrm{B}$ core antigens was negative. Results of testing for HBsAg in serum, HBV DNA in plasma, HCV antibody in serum, HAV IgM in serum, and HAV RNA in plasma and feces were all negative. HEV IgM was detected in serum collected on August 22 (day 100 posttransplant). Because of the serology result and ongoing LFT derangement, persistent HEV infection was suspected. A qRT-PCR targeting HEV-A was performed on patient fecal and plasma specimens; HEV-A RNA was not detected in either specimen. An RT-PCR capable of detecting all species within the Orthohepevirus genus detected amplicons (online Technical Appendix Figure 1) in plasma, feces, and liver tissue. Sequencing confirmed that the products clustered with rat HEV-C strains.

\section{Viral RNA Kinetics and Effect of Ribavirin Therapy}

The patient's archived serum, saliva, urine, feces, and nonfixed liver tissue samples were retrieved for HEVC RNA load testing using HEV-C qRT-PCR (Figure 1, panel A). Two pretransplant serum samples and 1 serum sample collected on day 17 after transplant did not contain HEV-C RNA. The first specimen with detectable HEV-C RNA was a serum sample collected 43 days after transplant, which contained an RNA load of $9.48 \times 10^{2}$ copies/mL; this result preceded onset of LFT derangement by 3 weeks. After heightened immunosuppression in July 

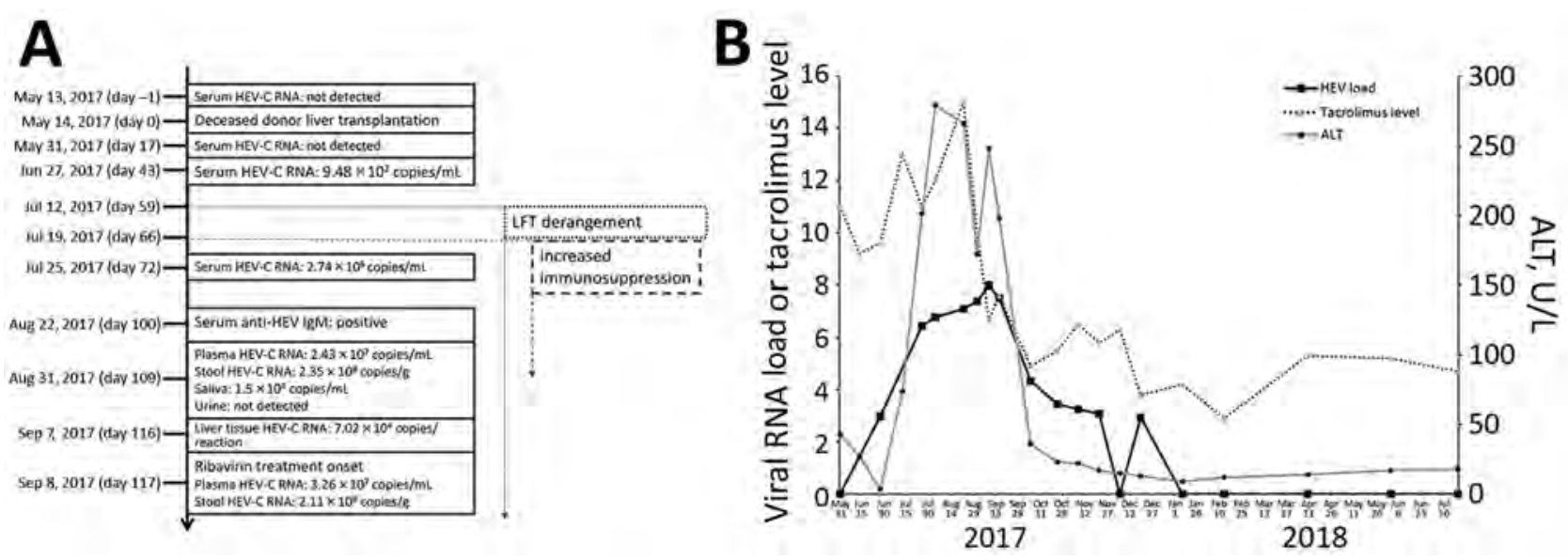

Figure 1. Natural course of HEV-C infection in a 56-year-old man at Queen Mary Hospital, Hong Kong. A) Timeline of major clinical events. All days are post transplant. B) Kinetics of liver function tests, tacrolimus levels ( $\mu \mathrm{g} / \mathrm{L})$, and plasma HEV-C RNA load $\left(\log _{10}\right.$ copies $\left./ \mathrm{mL}\right)$ with relation to ribavirin therapy. ALT, alanine aminotransferase; HEV-C, Orthohepevirus C; LFT, liver function test.

and August, the HEV-C RNA load in blood steadily rose along with ALT (Figure 1, panel B). Variation in ALT correlated with the HEV-C RNA viral load by linear regression $\left(\mathrm{R}^{2}=0.791\right)$. HEV-C RNA was also detected in feces, saliva, and liver tissue (Figure 1, panel A); feces contained the highest RNA load.

Immunosuppressant dosages were decreased after confirmation of HEV infection. However, ALT and HEV-C RNA loads continued to increase despite reduction of plasma tacrolimus levels by $55 \%$ and rebound of lymphocyte count to $2.27 \times 10^{9}$ cells/L. Therefore, oral ribavirin 400 mg twice daily was started on September 7. ALT decreased within the first week after start of therapy and normalized within 1 month after starting ribavirin (Figure 1, panel B). HEV-C RNA loads also decreased to undetectable levels in plasma obtained on February 13, 2018. Ribavirin was stopped in April 2018, and HEV-C RNA in serum remained undetectable as of August 21, 2018, confirming sustained virologic response.

\section{Serologic Analysis}

We retrospectively tested all available patient serum and plasma samples for HEV IgG and IgM ELISA using the Wantai ELISA kit. The patient's serum before transplant was HEV IgG positive and IgM negative. HEV IgG and IgM optical density rose sharply from June 27, when HEVC RNA was first detectable in blood, to July 25, when clinical hepatitis began (online Technical Appendix Figure 2). Despite high IgG levels, HEV-C RNA continued to rise until ribavirin was started.

To characterize the serologic response, Western blot using purified HEV-A and HEV-C recombinant proteins (Figure 2, panel A) was performed. Two mAbs raised against HEV-A were used: 1 produced a band in HEV-A
IgG blot but not in the HEV-C blot (lane 8; Figure 2, panels $\mathrm{B}, \mathrm{C})$ confirming specificity, and the other was crossreactive against HEV-A and HEV-C (lane 9; Figure 2, panels B, C). Polyclonal serum raised in mice inoculated with HEV-C protein reacted in both blots, showing that the serum was cross-reactive (lane 7). Patient serum collected on day 100 after transplant (lane 1) was tested against HEVA and HEV-C recombinant proteins. The serum specimen showed reactivity in both Western blots.

Two patient serum samples, 1 obtained 3 months before transplant and the other obtained on day 100 after transplant, were tested in IgG ELISAs using HEV-A and HEV-C protein-coated plates. The pretransplant serum (Figure 2, panel D) had cross-reactive antibodies against both HEV-A and HEV-C proteins $(<2$-fold difference in titer using OD cutoff of 0.3 ). However, the posttransplant serum (Figure 2, panel E) showed $>16$-fold rise in HEVA IgG titer and markedly higher reactivity against HEVA than against HEV-C ( $>4$-fold difference in titer using a cutoff OD of 0.3).

\section{Liver Histologic and Immunohistochemical Analyses}

Serial liver biopsies showed progressively worsening hepatocyte ballooning and degenerative changes (Figure 3, panels A, B). Apoptotic hepatocytes were identified in the biopsy obtained on day 98 posttransplant (Figure 3, panel B). Immunohistochemical staining with the cross-reactive $\mathrm{mAb}$ showed positive perinuclear cytoplasmic signals (Figure 3, panel C), and negative control with bovine serum albumin instead of mAb showed no signals (Figure 3, panel D).

\section{Genomic Description}

Complete genome sequencing of the patient's fecal HEV isolate (LCK-3110) showed that the genome was 6,942 bp 

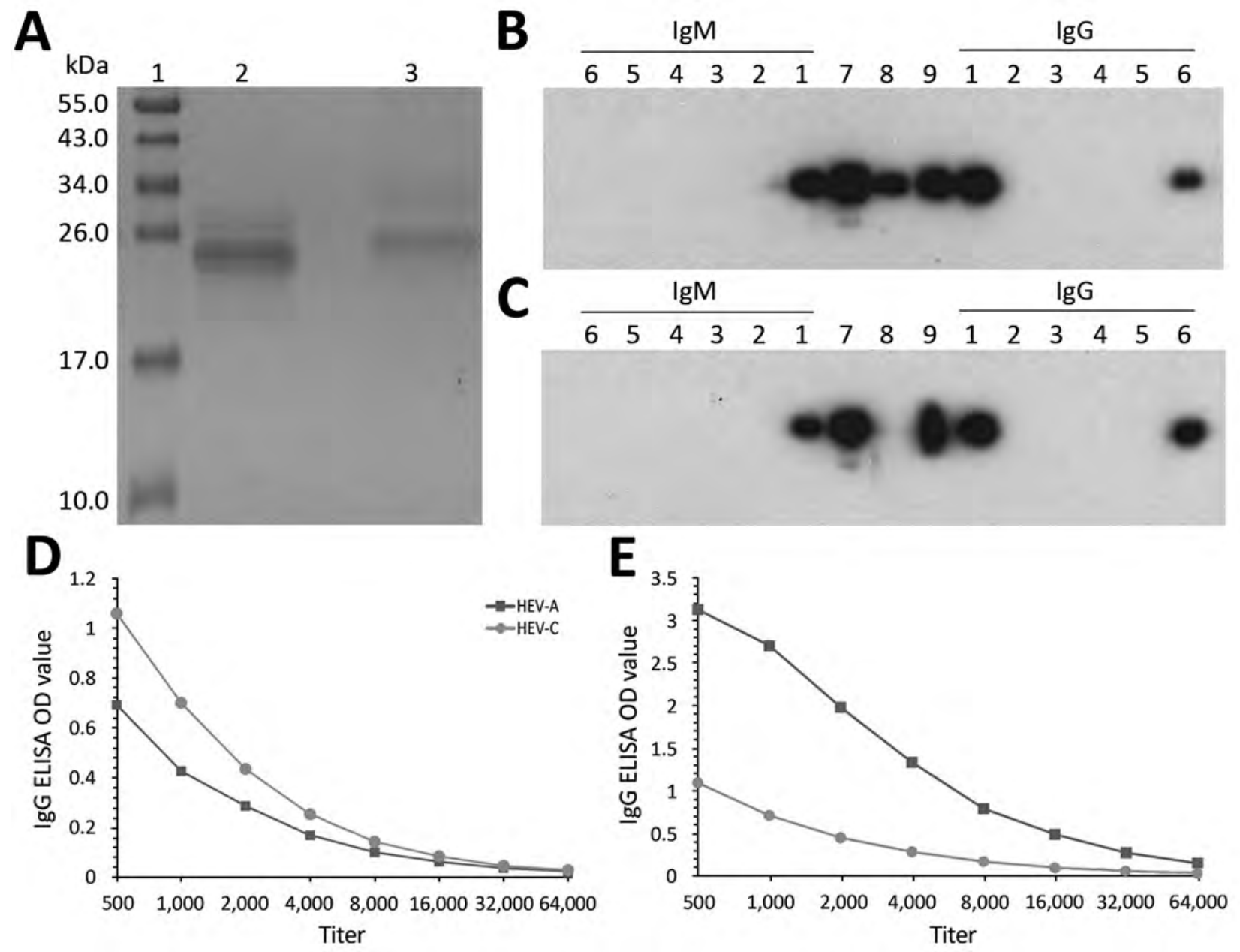

Figure 2. Serologic testing for HEV infection at Queen Mary Hospital, Hong Kong. A) Sodium dodecyl sulfate polyacrylamide gel electrophoresis gel showing purified HEV-A and HEV-C 239-aa recombinant proteins used in Western blot and ELISA. Lane 1, molecular weight marker; lane 2, HEV-A protein; lane 3, HEV-C protein. B-C) IgM and IgG Western blot using HEV-A protein (B) and HEV-C protein (C). Lane 1, patient serum (posttransplant day 100); lanes 2-5, individual platelet donor serum; lane 6, organ donor serum; lane 7, murine polyclonal serum against HEV-C; lane 8, specific monoclonal antibody against HEV-A; lane 9, cross-reactive monoclonal antibody against HEV-A and HEV-C. D, E) HEV-A and HEV-C ELISA IgG titers of patient pretransplant (D) and posttransplant serum (E) using an OD of 0.3 as assay cutoff as described in the online Technical Appendix (https://wwwnc.cdc.gov/EID/article/24/12/18-0937Techapp1.pdf). HEV, hepatitis E virus; HEV-A, Orthohepevirus A; HEV-C, Orthohepevirus C; OD, optical density.

long (GenBank accession no. MG813927). Phylogenetic trees of the nucleotide and amino acid sequences of ORF1, ORF2, and ORF3 of HEV strains showed that LCK-3110 is most closely related to the Vietnam-105 strain (Figure 4; online Technical Appendix Figure 3, panels A, B), sharing $93.7 \%$ nt identity. Because no phylogenetic incongruence was found on comparison of trees of the 3 genomic segments, recombination was unlikely (Table 2; online Technical Appendix). To determine whether commonly used RT-PCRs for HEV nucleic acid amplification could detect HEV-C, we aligned published primer/ probe sequences of HEV RT-PCRs (19-22) with complete genome sequences of HEV-A (genotype 1 reference strain) and HEV-C (strains LCK-3110, Vietnam-105, and
LA-B350) using ClustalX 2.0 (http://www.clustal.org/ clustal2/). Alignment revealed significant lack of homology with HEV-C at the $3^{\prime}$ end of either the forward or reverse primer for the assays described by Jothikumar et al. and Rolfe et al. (online Technical Appendix Figure 4, panels A, B) $(20,21)$. Our in-house HEV-A qRT-PCR is based on the primer/probe design of Jothikumar et al. and was unable to detect HEV-C in patient specimens (20). For the assays described by Mansuy et al. and Colson et al. $(19,22)$, there was significant lack of matching of probe sequence ( $40 \%-45 \%$ mismatch) to HEV-C genomes (online Technical Appendix Figure 4, panels C, D), which most likely would result in failure to detect any amplified nucleic acid. 


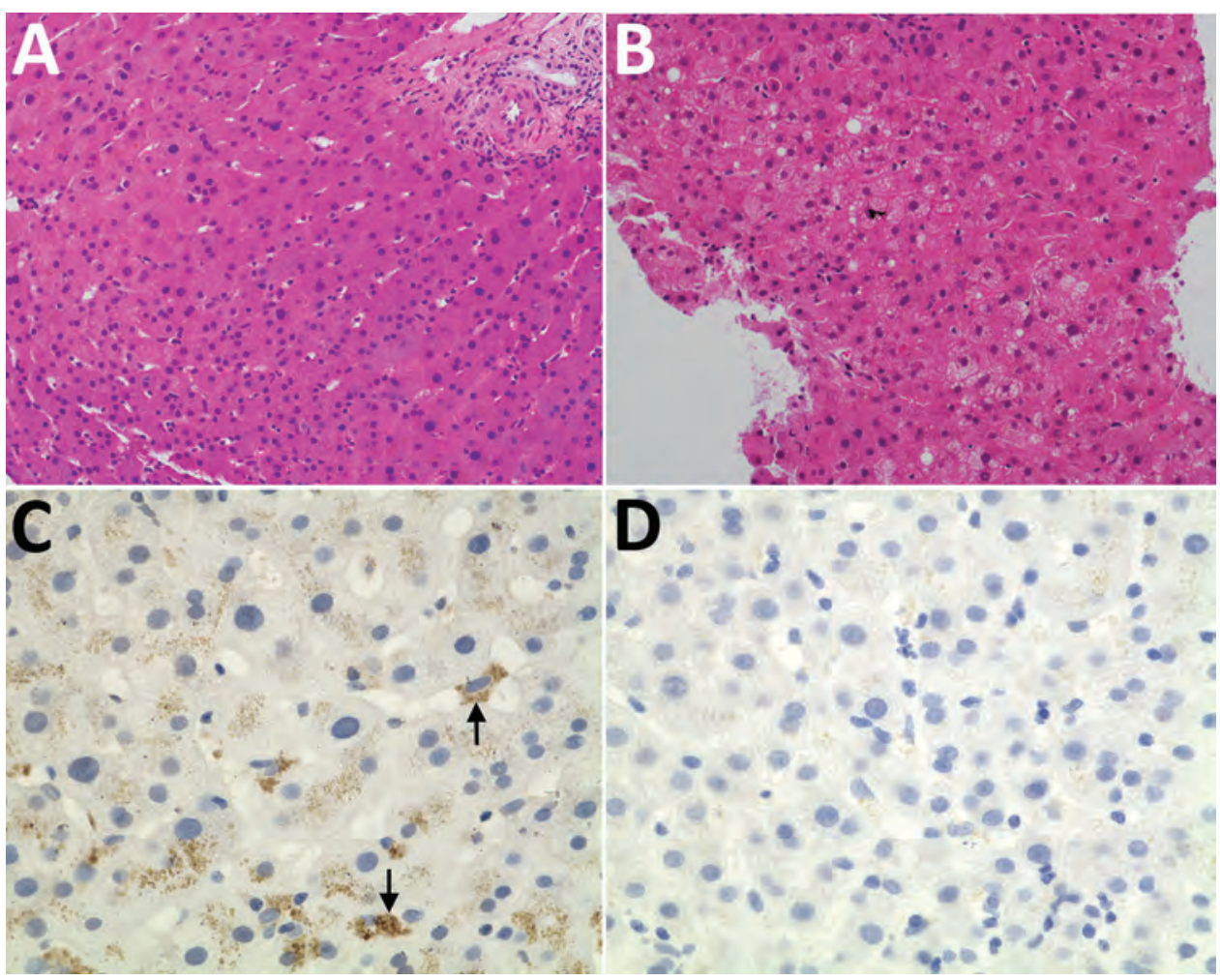

Figure 3. Histologic and immunohistochemical staining of liver tissue from a 56-yearold man at Queen Mary Hospital, Hong Kong. A, B) Liver tissue sections (original magnification $\times 200$ ) stained with hematoxylin and eosin obtained at day $0(A)$, showing normal hepatocyte architecture, and day 98 (B) after transplant showing progressive increase in hepatocyte ballooning and degenerative changes. C, D) Liver tissue section stained with cross-reactive monoclonal antibody (original magnification $\times 400$ ); arrows show perinuclear antigen staining $(\mathrm{C})$ and negative control with bovine serum albumin (D).

\section{Virus Culture}

We detected HEV-C RNA in supernatants from all 3 cell lines (Figure 5, panel A) inoculated with patient's feces at steady levels from day 3 to day 7 after inoculation. RNA loads in cell lysates were $\approx 1 \log$ higher than concomitantly harvested supernatants, suggesting successful viral cell entry. Immunohistochemical staining (Figure 5, panels B, C) of A549 cell monolayers and immunofluorescence staining of infected Huh-7 and Caco-2 cells (online Technical Appendix Figure 5) confirmed the presence of cytoplasmic HEV ORF2 antigen when stained with antiserum against $\mathrm{HEV}-\mathrm{C}$. These findings suggested abortive viral replication of HEV-C in human cell lines.

\section{Epidemiologic Investigation}

The first clinical sample with detectable HEV-C RNA was obtained 43 days after transplant. HEV-C was not detected in serum samples obtained before transplant. Serum samples from the organ donor and all 4 platelet donors tested negative by IgM Western blot against HEV-C recombinant protein (Figure 2, panel C, lanes 2-6) and HEV-C qRT-PCR.

The patient's house unit was located adjacent to a refuse chute. He had noticed rodent droppings but had never seen rats inside his home. A site visit to the housing estate was conducted on November 22, 2017. Rodent droppings were found around refuse collection bins on the ground floor and the floor where the patient lived. Twelve rodent fecal specimens, $2 \mathrm{swab}$ samples from the drain, and 2 swab samples from the refuse room floor tested negative for HEV-C RNA. To expand the investigation, we retrieved archived rodent samples collected from the area around the patient's housing estate $(\approx 2.5-\mathrm{km}$ radius) as part of preexisting pathogen surveillance programs. Spleen, kidney, liver, and rectal swab specimens from 27 rats were tested by qRT-PCR. The internal organs of 1 street rat (Rattus norvegicus) collected in 2012 tested positive for HEVC RNA (strain name SRN-02). The ORF2 aa sequence of this isolate had $90.9 \%$ identity to LCK-3110.

\section{Discussion}

Discovered in Germany in 2010, rat HEV variants have been detected in rodent samples in Asia, Europe, and North America (23-26). Because of high divergence from humanpathogenic HEV, rat HEV has been classified into a separate species, Orthohepevirus $C$, within the family Hepeviridae (27). The zoonotic potential of HEV-C is controversial. Virus-like protein ELISAs show possible subclinical infection among forestry workers in Germany and febrile inpatients in Vietnam, although interpretation of such studies is difficult because of serologic cross-reactivity between HEV-A and HEV-C $(15,28)$. Immunocompetent rhesus macaques do not appear to be susceptible to experimental infection with a North America HEV-C isolate (23).

In this study, we detected HEV-C RNA in multiple specimens from a transplant recipient. The HEV-C infection manifested as persistent hepatitis, as shown by temporal correlation between blood HEV-C RNA detection and 


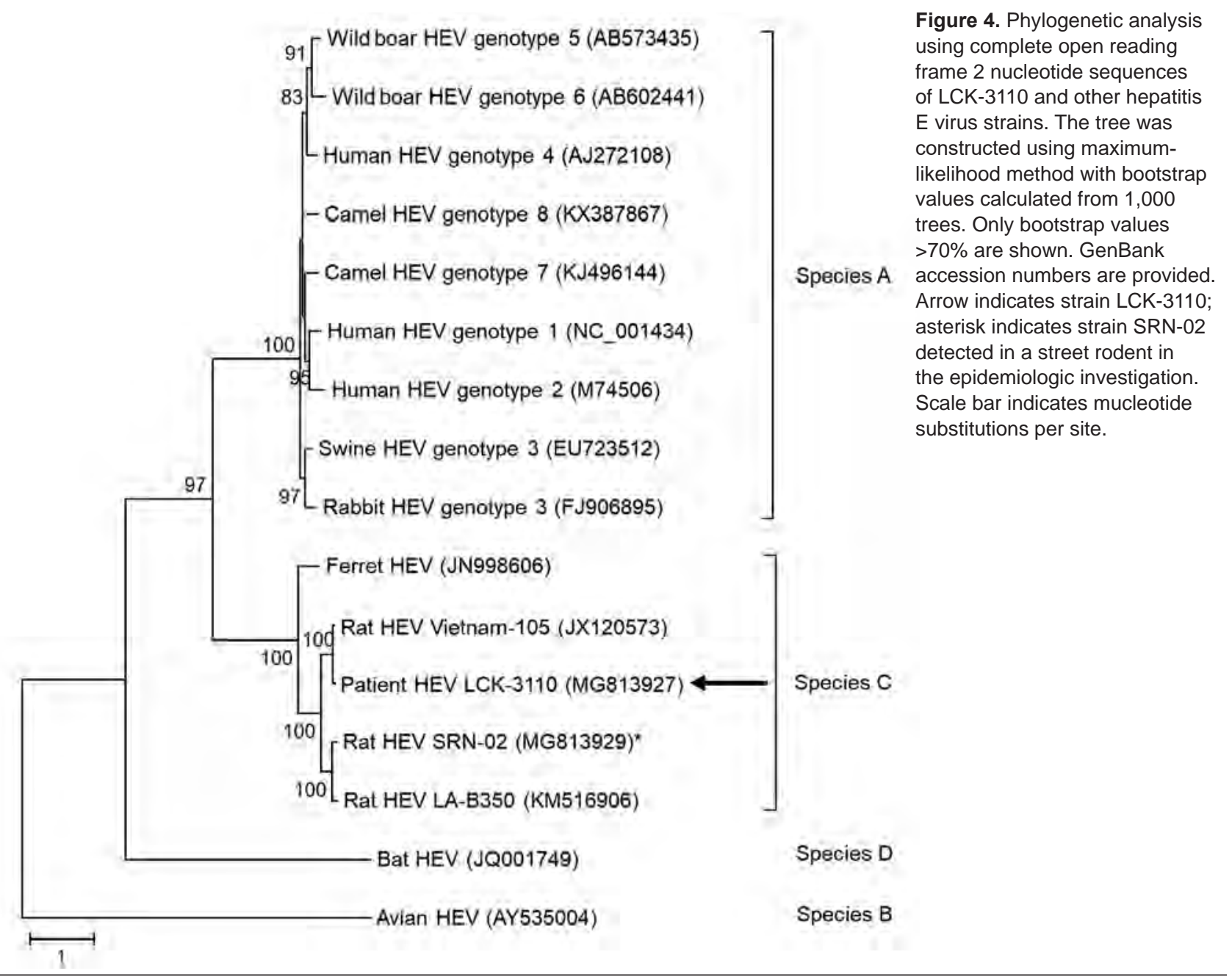

Figure 4. Phylogenetic analysis using complete open reading constructed using maximumlikelihood method with bootstrap from 1,000 trees. Only bootstrap values accession numbers are provided. Arrow indicates strain LCK-3110 the epidemiologic investigation. Scale bar indicates mucleotide substitutions per site.

hepatitis onset, presence of HEV-C RNA in liver tissue, and normalization of liver function tests with viral clearance. These findings prove that HEV-C can infect humans to cause clinically significant illness and signal a need to reevaluate the importance of $\mathrm{HEV}-\mathrm{C}$ as a human zoonosis among both immunocompromised and immunocompetent patients with hepatitis of unknown etiology.

The patient reported here acquired HEV-C infection despite having HEV IgG. Interpreted in parallel with the finding by Sanford et al. that inoculating pigs with HEV-C

Table 2. Comparison between nucleotide and deduced amino acid sequence identities of HEV strain LCK-3110 and other HEV strains

\begin{tabular}{|c|c|c|c|c|c|c|c|c|}
\hline \multirow[b]{3}{*}{ HEV strain (GenBank accession no.) } & \multirow[b]{3}{*}{ HEV species } & \multicolumn{7}{|c|}{ Rat HEV strain LCK-3110 } \\
\hline & & \multirow{2}{*}{$\begin{array}{c}\text { Entire } \\
\text { genome }\end{array}$} & \multicolumn{3}{|c|}{ Nucleotides, \% } & \multicolumn{3}{|c|}{ Amino acids, $\%$} \\
\hline & & & ORF1 & ORF2 & ORF3 & ORF1 & ORF2 & ORF3 \\
\hline Genotype 1 (NC_001434) & HEV-A & 57.6 & 56.4 & 60.9 & 55.0 & 50.0 & 56.3 & 31.0 \\
\hline Genotype 2 (M7 $\overline{4} 506)$ & HEV-A & 57.3 & 56.3 & 60.0 & 50.4 & 49.7 & 56.1 & 27.6 \\
\hline Genotype 3 (EU723512) & HEV-A & 56.6 & 55.4 & 60.7 & 51.9 & 50.3 & 56.5 & 30.7 \\
\hline Genotype 4 (AJ272108) & HEV-A & 56.5 & 55.4 & 59.8 & 55.4 & 49.7 & 56.4 & 31.0 \\
\hline Rabbit HEV (FJ906895) & HEV-A & 56.0 & 54.9 & 59.9 & 51.6 & 50.1 & 56.4 & 27.6 \\
\hline Wild boar HEV (AB573435) & HEV-A & 57.3 & 56.2 & 60.4 & 54.8 & 49.7 & 56.2 & 33.6 \\
\hline Wild boar HEV (AB602441) & HEV-A & 56.8 & 55.7 & 59.6 & 54.5 & 50.3 & 56.5 & 31.9 \\
\hline Camel HEV (KJ496144) & HEV-A & 55.9 & 54.9 & 59.4 & 53.5 & 50.5 & 56.2 & 32.2 \\
\hline Camel HEV (KX387867) & HEV-A & 55.6 & 54.3 & 59.7 & 53.7 & 50.1 & 55.8 & 29.6 \\
\hline Rat HEV Vietnam-105 (JX120573) & HEV-C & 93.7 & 93.3 & 95.2 & 96.4 & 98.2 & 98.0 & 95.1 \\
\hline Rat HEV LA-B350 (KM516906) & HEV-C & 77.3 & 76.3 & 79.7 & 79.3 & 88.0 & 92.1 & 64.7 \\
\hline Ferret HEV (JN998606) & HEV-C & 68.7 & 67.5 & 71.1 & 64.2 & 74.8 & 78.7 & 45.9 \\
\hline Bat HEV (JQ001749) & HEV-D & 53.8 & 53.8 & 54.3 & 44.7 & 45.7 & 47.9 & 18.1 \\
\hline Avian HEV (AY535004) & HEV-B & 53.5 & 54.0 & 53.0 & 46.4 & 45.6 & 43.5 & 24.8 \\
\hline
\end{tabular}



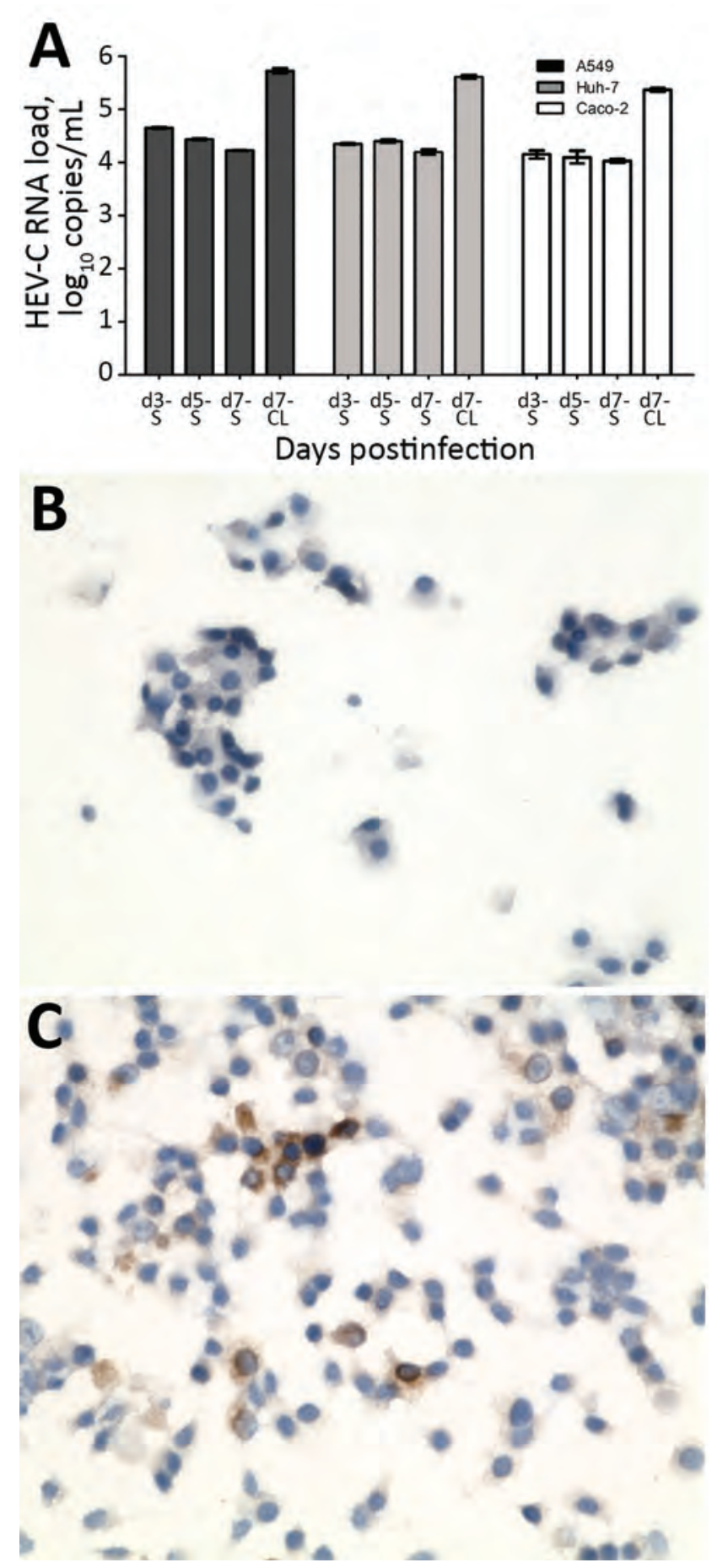

Figure 5. Isolation of HEV-C from 56-year-old male patient's feces in cell culture, Queen Mary Hospital, Hong Kong. A) HEV-C RNA loads in culture S and day-7 CL of A549, Huh-7, and Caco- 2 cell lines after inoculation by patient's filtered fecal suspension. Mean of 3 replicates; error bars indicate SEM. B) Uninfected A549 cell monolayer stained with anti-HEV-C polyclonal antiserum. C) Infected A549 cell monolayer stained with anti-HEV-C polyclonal antiserum. Original magnification $\times 400$. CL, cell lysate; HEV, hepatitis $E$ virus; $\mathrm{S}$, supernatant.
ORF2 protein did not protect them from HEV-A infection and low amino acid homology between HEV-A and HEV$\mathrm{C}$ in critical immunogenic domains (29), our data suggest that HEV-A antibodies do not protect against HEV-C infection. The patient's postinfection serum showed significantly higher reactivity in an HEV-A-specific ELISA than in an HEV-C ELISA; the humoral immune responses of persons with past HEV-A infection to de novo HEV-C infection are worthy of further study to identify whether anamnestic responses are mounted.

The patient's HEV isolate had high nucleotide similarity to the HEV-C Vietnam-105 strain. It shared less homology with the North America LA-B350 strain, especially in the ORF3 domain, which is important for viral egress (30). Interspecies transmission could not be attributed to specific viral mutations. Future studies will need to include differences in zoonotic potential between HEV-C strains from Asia and the Americas.

The patient's immunosuppression possibly enabled the virus to surmount the species barrier, as described previously for avian influenza $(31,32)$. HEV-C infections may go undiagnosed because of amplification failure in RT-PCRs, which are designed based on HEV-A sequences (online Technical Appendix Figure 3). The Wantai ELISA, based on HEV-A genotype 1, was able to detect IgM in this patient, but whether the assay is sensitive for $\mathrm{HEV}-\mathrm{C}$ infection or was detecting only HEV-A-specific antibodies is uncertain. Therefore, we believe that specific RT-PCR is the most reliable method to diagnose HEV-C infections.

Our findings are also relevant to blood and organ donation safety. Because of the inability of commonly used RTPCRs to detect HEV-C, transmission from asymptomatically infected immunocompetent donors may occur, even in countries that screen donated blood for HEV. Studies examining frequency of $\mathrm{HEV}-\mathrm{C}$ contamination in blood products are needed to quantify this threat.

The patient lived in a housing estate with evidence of rat infestation in the refuse bins outside his home. We identified HEV-C in street rodents from the area, but the isolate was not closely related to the patient's isolate. The route of transmission is unclear; we postulate that contamination of food by infected rat droppings in the food supply is possible. Other possibilities include reactivation of a subclinical infection in the patient posttransplant or a donor-derived infection from residual HEV-C in the transplanted organ. However, we found no serologic or virologic evidence of HEV-C infection in donor and recipient serum before transplant. An occult infection in the donated liver, which reactivated after transplant as described previously for HEV-A, cannot be completely excluded. Detailed studies are needed to ascertain the route of HEV-C infection in humans. 


\section{Acknowledgment}

We thank N.S. Xia for his kind gift of murine mAbs against HEV-A.

This study was supported by donations from Shaw Foundation and from Michael Seak-Kan Tong. The study was partially funded by the Consultancy Service for Enhancing Laboratory Surveillance of Emerging Infectious Disease for Department of Health of the Hong Kong Special Administrative Region of China, Seed Fund for Basic Research, and Enhanced New Staff Start-up Research Grant of the University of Hong Kong.

J.F.W.C. has received travel grants from Pfizer Corporation Hong Kong and Astellas Pharma Hong Kong Corporation Limited and was an invited speaker for Gilead Sciences Hong Kong Limited and Luminex Corporation.

\section{About the Author}

Dr. Sridhar is a clinical assistant professor at the Department of Microbiology, The University of Hong Kong. His major research interests include viral hepatitis and clinical virology.

\section{References}

1. Rein DB, Stevens GA, Theaker J, Wittenborn JS, Wiersma ST. The global burden of hepatitis E virus genotypes 1 and 2 in 2005. Hepatology. 2012;55:988-97. http://dx.doi.org/10.1002/hep.25505

2. Lee GH, Tan BH, Teo EC, Lim SG, Dan YY, Wee A, et al. Chronic infection with camelid hepatitis $\mathrm{E}$ virus in a liver transplant recipient who regularly consumes camel meat and milk. Gastroenterology. 2016;150:355-7.e3. http://dx.doi.org/10.1053/ j.gastro.2015.10.048

3. Abravanel F, Lhomme S, El Costa H, Schvartz B, Peron JM, Kamar N, et al. Rabbit hepatitis E virus infections in humans, France. Emerg Infect Dis. 2017;23:1191-3. http://dx.doi.org/ 10.3201/eid2307.170318

4. Li TC, Chijiwa K, Sera N, Ishibashi T, Etoh Y, Shinohara Y, et al. Hepatitis E virus transmission from wild boar meat. Emerg Infect Dis. 2005;11:1958-60. http://dx.doi.org/10.3201/eid1112.051041

5. Tei S, Kitajima N, Takahashi K, Mishiro S. Zoonotic transmission of hepatitis E virus from deer to human beings. Lancet. 2003; 362:371-3. http://dx.doi.org/10.1016/S0140-6736(03)14025-1

6. Kamar N, Selves J, Mansuy JM, Ouezzani L, Péron JM, Guitard J, et al. Hepatitis E virus and chronic hepatitis in organ-transplant recipients. N Engl J Med. 2008;358:811-7. http://dx.doi.org/ 10.1056/NEJMoa0706992

7. Hewitt PE, Ijaz S, Brailsford SR, Brett R, Dicks S, Haywood B, et al. Hepatitis E virus in blood components: a prevalence and transmission study in southeast England. Lancet. 2014;384: 1766-73. http://dx.doi.org/10.1016/S0140-6736(14)61034-5

8. Sridhar S, Teng JLL, Chiu TH, Lau SKP, Woo PCY. Hepatitis E virus genotypes and evolution: emergence of camel hepatitis $\mathrm{E}$ variants. Int J Mol Sci. 2017;18:E869. http://dx.doi.org/10.3390/ ijms18040869

9. Sridhar S, Chan JFW, Yap DYH, Teng JLL, Huang C, Yip CCY, et al. Genotype 4 hepatitis E virus is a cause of chronic hepatitis in renal transplant recipients in Hong Kong. J Viral Hepat. 2018;25:209-13. http://dx.doi.org/10.1111/jvh.12799

10. Sridhar S, Lo SK, Xing F, Yang J, Ye H, Chan JF, et al. Clinical characteristics and molecular epidemiology of hepatitis $\mathrm{E}$ in
Shenzhen, China: a shift toward foodborne transmission of hepatitis E virus infection. Emerg Microbes Infect. 2017;6:e115. http://dx.doi.org/10.1038/emi.2017.107

11. Zhang J, Li SW, Wu T, Zhao Q, Ng MH, Xia NS. Hepatitis E virus: neutralizing sites, diagnosis, and protective immunity. Rev Med Virol. 2012;22:339-49. http://dx.doi.org/10.1002/rmv.1719

12. Tamura K, Stecher G, Peterson D, Filipski A, Kumar S. MEGA6: Molecular Evolutionary Genetics Analysis version 6.0. Mol Biol Evol. 2013;30:2725-9. http://dx.doi.org/10.1093/molbev/mst197

13. Chan JF, Yip CC, Tsang JO, Tee KM, Cai JP, Chik KK, et al. Differential cell line susceptibility to the emerging Zika virus: implications for disease pathogenesis, non-vector-borne human transmission and animal reservoirs. Emerg Microbes Infect. 2016;5:e93. http://dx.doi.org/10.1038/emi.2016.99

14. Chan JF, Chan KH, Choi GK, To KK, Tse H, Cai JP, et al. Differential cell line susceptibility to the emerging novel human betacoronavirus 2c EMC/2012: implications for disease pathogenesis and clinical manifestation. J Infect Dis. 2013; 207:1743-52. http://dx.doi.org/10.1093/infdis/jit123

15. Shimizu K, Hamaguchi S, Ngo CC, Li TC, Ando S, Yoshimatsu K, et al. Serological evidence of infection with rodent-borne hepatitis E virus HEV-C1 or antigenically related virus in humans. J Vet Med Sci. 2016;78:1677-81. http://dx.doi.org/10.1292/ jvms.16-0200

16. Emerson SU, Nguyen HT, Torian U, Burke D, Engle R, Purcell RH. Release of genotype 1 hepatitis E virus from cultured hepatoma and polarized intestinal cells depends on open reading frame 3 protein and requires an intact PXXP motif. J Virol. 2010;84:9059-69. http://dx.doi.org/10.1128/JVI.00593-10

17. Emerson SU, Nguyen H, Graff J, Stephany DA, Brockington A, Purcell RH. In vitro replication of hepatitis E virus (HEV) genomes and of an HEV replicon expressing green fluorescent protein. J Virol. 2004;78:4838-46. http://dx.doi.org/10.1128/ JVI.78.9.4838-4846.2004

18. Tanaka T, Takahashi M, Kusano E, Okamoto H. Development and evaluation of an efficient cell-culture system for Hepatitis E virus. J Gen Virol. 2007;88:903-11. http://dx.doi.org/10.1099/vir.0.82535-0

19. Mansuy JM, Peron JM, Abravanel F, Poirson H, Dubois M, Miedouge M, et al. Hepatitis E in the south west of France in individuals who have never visited an endemic area. J Med Virol. 2004;74:419-24. http://dx.doi.org/10.1002/jmv.20206

20. Jothikumar N, Cromeans TL, Robertson BH, Meng XJ, Hill VR. A broadly reactive one-step real-time RT-PCR assay for rapid and sensitive detection of hepatitis E virus. J Virol Methods. 2006;131:65-71. http://dx.doi.org/10.1016/j.jviromet.2005.07.004

21. Rolfe KJ, Curran MD, Mangrolia N, Gelson W, Alexander GJ, L'estrange M, et al. First case of genotype 4 human hepatitis $\mathrm{E}$ virus infection acquired in India. J Clin Virol. 2010;48:58-61. http://dx.doi.org/10.1016/j.jcv.2010.02.004

22. Colson P, Coze C, Gallian P, Henry M, De Micco P, Tamalet C. Transfusion-associated hepatitis E, France. Emerg Infect Dis. 2007;13:648-9. http://dx.doi.org/10.3201/eid1304.061387

23. Purcell RH, Engle RE, Rood MP, Kabrane-Lazizi Y, Nguyen HT, Govindarajan S, et al. Hepatitis E virus in rats, Los Angeles, California, USA. Emerg Infect Dis. 2011;17:2216-22. http://dx.doi.org/10.3201/eid1712.110482

24. Wang B, Cai CL, Li B, Zhang W, Zhu Y, Chen WH, et al. Detection and characterization of three zoonotic viruses in wild rodents and shrews from Shenzhen city, China. Virol Sin. 2017;32:290-7. http://dx.doi.org/10.1007/s12250-017-3973-z

25. Mulyanto, Depamede SN, Sriasih M, Takahashi M, Nagashima S, Jirintai S, et al. Frequent detection and characterization of hepatitis E virus variants in wild rats (Rattus rattus) in Indonesia. Arch Virol. 2013;158:87-96.

26. Johne R, Plenge-Bönig A, Hess M, Ulrich RG, Reetz J, Schielke A. Detection of a novel hepatitis E-like virus in faeces of wild rats 
using a nested broad-spectrum RT-PCR. J Gen Virol. 2010;91:7508. http://dx.doi.org/10.1099/vir.0.016584-0

27. Smith DB, Simmonds P, Jameel S, Emerson SU, Harrison TJ, Meng XJ, et al.; International Committee on Taxonomy of Viruses Hepeviridae Study Group. Consensus proposals for classification of the family Hepeviridae. J Gen Virol. 2014;95:2223-32. http://dx.doi.org/10.1099/vir.0.068429-0

28. Dremsek P, Wenzel JJ, Johne R, Ziller M, Hofmann J, Groschup MH, et al. Seroprevalence study in forestry workers from eastern Germany using novel genotype 3- and rat hepatitis E virus-specific immunoglobulin G ELISAs. Med Microbiol Immunol (Berl). 2012;201:189-200. http://dx.doi.org/10.1007/s00430-011-0221-2

29. Sanford BJ, Opriessnig T, Kenney SP, Dryman BA, Córdoba L, Meng XJ. Assessment of the cross-protective capability of recombinant capsid proteins derived from pig, rat, and avian hepatitis E viruses (HEV) against challenge with a genotype $3 \mathrm{HEV}$ in pigs. Vaccine. 2012;30:6249-55.

30. Ding Q, Heller B, Capuccino JM, Song B, Nimgaonkar I, Hrebikova G, et al. Hepatitis E virus ORF3 is a functional ion channel required for release of infectious particles. Proc Natl Acad Sci U S A. 2017;114:1147-52. http://dx.doi.org/10.1073/ pnas.1614955114

31. Cheng VC, Chan JF, Wen X, Wu WL, Que TL, Chen H, et al. Infection of immunocompromised patients by avian H9N2 influenza A virus. [Erratum in: Proc Natl Acad Sci U S A. 2017]. J Infect. 2011;62:394-9. http://dx.doi.org/10.1016/j.jinf.2011.02.007

32. Chen Y, Liang W, Yang S, Wu N, Gao H, Sheng J, et al. Human infections with the emerging avian influenza A H7N9 virus from wet market poultry: clinical analysis and characterisation of viral genome. Lancet. 2013;381:1916-25. http://dx.doi.org/10.1016/ S0140-6736(13)60903-4

Address for correspondence: Kwok-Yung Yuen, The University of Hong Kong, Carol Yu Centre for Infection, State Key Laboratory of Emerging Infectious Diseases, Department of Microbiology, Li Ka Shing Faculty of Medicine, Queen Mary Hospital, 120 Pokfulam Rd, Pokfulam, Hong Kong HK1, China; email: kyyuen@hkucc.hku.hk

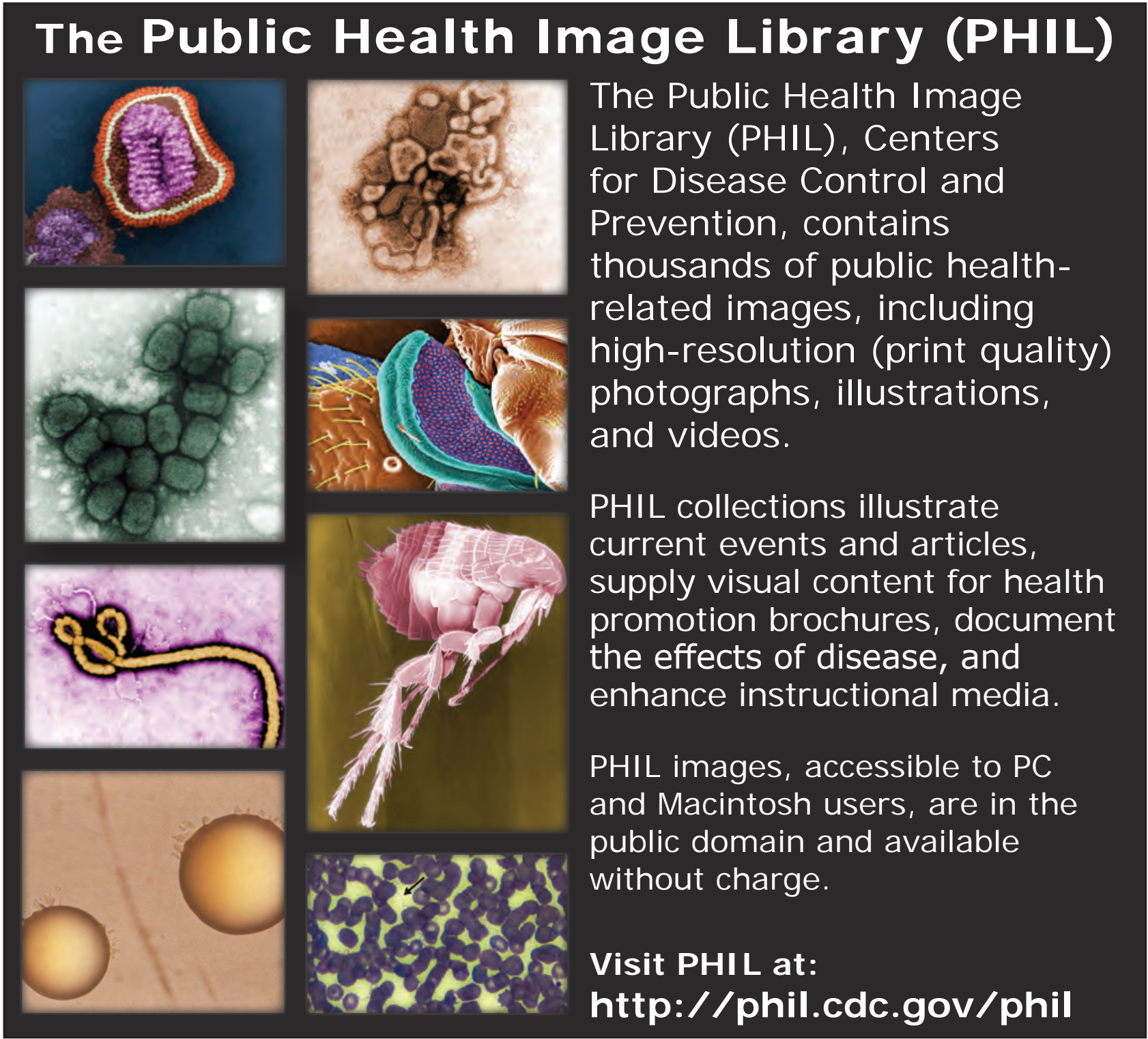




\title{
Influences of Community I nterventions on Zika Prevention Behaviors of Pregnant Women, Puerto Rico, J uly 2016-J une 2017
}

\author{
Giulia Earle-Richardson, Christine Prue, Khadija Turay, Dana Thomas
}

We assessed how community education efforts influenced pregnant women's Zika prevention behaviors during the 2016 Centers for Disease Control and Prevention-Puerto Rico Department of Health Zika virus response. Efforts included Zika virus training, distribution of Zika prevention kits, a mass media campaign, and free home mosquito spraying. We used telephone interview data from pregnant women participating in Puerto Rico's Women, Infants, and Children Program to test associations between program participation and Zika prevention behaviors. Behavior percentages ranged from $4 \%$ (wearing long-sleeved shirt) to $90 \%$ (removing standing water). Appropriate mosquito repellent use (28\%) and condom use (44\%) were common. Receiving a Zika prevention kit was significantly associated with larvicide application (odds ratio [OR] 8.0) and bed net use (OR 3.1), suggesting the kit's importance for lesser-known behaviors. Offer of free residential spraying was associated with spraying home for mosquitoes (OR 13.1), indicating that women supported home spraying when barriers were removed.

$I^{\prime}$ n early 2016, in response to the rising number of Zika virus infections in Puerto Rico and the devastating effects of Zika infection during pregnancy (1), the Puerto Rico Department of Health (PRDOH) activated its emergency operations center, with support from the US Centers for Disease Control and Prevention (CDC) (2). Because there is currently no Zika virus vaccine and no known measures can prevent prenatal mother-to-child transmission (3), personal protection measures and home vector control are the only feasible protections for most pregnant women. To maximize these self-protection behaviors, the response introduced 4 different community Zika prevention behavior promotion interventions. Health behavior interventions can change behavior by addressing behavioral barriers, by creating or enhancing incentives, and by increasing persons' capabilities and opportunities to perform the behavior (4).

Author affiliation: Centers for Disease Control and Prevention, Atlanta, Georgia, USA

DOI: https://doi.org/10.3201/eid2412.181056

\section{Interventions}

\section{PRDOH Women, Infants, and Children Program} Zika Orientation

During the tracking period, all newly enrolled pregnant women at 1 of the island's 92 Women, Infants, and Children (WIC) clinics were given a 20-30-minute presentation on Zika virus infection and prevention. Orientation (individually or in small groups) was provided by the nutrition educator or, during the peak of the epidemic, by a Zika educator provided by CDC. The primary advantages of this counseling approach are interpersonal communication (including answering questions) and how easily it can be integrated into existing trusted programs, such as WIC prenatal visits $(5,6)$.

\section{Zika Prevention Kit Distribution}

The Zika prevention kit (ZPK) was a tote bag containing insect repellent, condoms, a mosquito bed net, larvicide, and printed Zika education materials. Approximately 26,000 ZPKs were distributed in Puerto Rico (CDC-Puerto Rico Department of Health, unpub. data, April 26, 2017). Whenever possible, the ZPK was given to the pregnant woman at the same time as the WIC Zika orientation. Prevention kits enable healthy behavior by putting needed items in persons' hands but also by providing a visual reminder of the recommended behavior. Similar home infection prevention kits were used during the Zika response in the US Virgin Islands (7) and during the Ebola epidemic in West Africa (8-10) to provide home caregivers with tools to prevent virus transmission. Only HIV infection prevention kits have been evaluated to date; these preliminary evaluations indicate kit popularity and suggest supportive effects $(11,12)$.

\section{Detén el Zika Campaign}

The Detén el Zika (“This Is How We Stop Zika”) campaign disseminated strategically designed Zika prevention

${ }^{1}$ Preliminary results from this study were presented as a poster presentation at the International Conference on Emerging Infectious Diseases, August 29, 2018, Atlanta, Georgia, USA. 
Table 1. Demographic characteristics of all women giving birth in 2016 and interview participants, Puerto Rico, July 2016-June 2017*

\begin{tabular}{|c|c|c|c|}
\hline \multirow[b]{2}{*}{ Characteristic } & \multirow{2}{*}{$\begin{array}{c}\text { Sample size, } \\
\text { no. (\%) }\end{array}$} & \multicolumn{2}{|c|}{ Women who gave birth in 2016, no. (\%)† } \\
\hline & & $\geq 18$ y of age & All ages \\
\hline Total sample & $1,329(100)$ & $27,230(100)$ & $28,257(100)$ \\
\hline \multicolumn{4}{|l|}{ Age group, y } \\
\hline$<18 \ddagger$ & 0 & 0 & $1,027(4)$ \\
\hline $18-22$ & $353(27)$ & $7,963(29)$ & $7,963(28)$ \\
\hline $23-25$ & $324(24)$ & $5,436(20)$ & $5,436(19)$ \\
\hline $26-29$ & $319(24)$ & $5,884(22)$ & $5,884(21)$ \\
\hline$\geq 30$ & $333(25)$ & $7,947(29)$ & $7,947(28)$ \\
\hline Total sample & $1,329(100)$ & $27,230(100)$ & $28,257(100)$ \\
\hline \multicolumn{4}{|l|}{ Educational attainment } \\
\hline Some high school or less & $24(3)$ & $427(2)$ & $579(2)$ \\
\hline Attended or completed 12 th grade & $285(31)$ & $9,105(34) \S$ & $9,958 \S(35)$ \\
\hline Attended or completed university & $545(60)$ & $15,648(58)$ & $15,670(55)$ \\
\hline Attended or completed graduate program & $55(6)$ & $2031(8)$ & $2,031(7)$ \\
\hline Total sample & $909 \rrbracket(100)$ & $27,230(100)$ & $28,257(100)$ \\
\hline Participation in WIC program\# & $1,329(100)$ & $23,679(87)$ & $24,671(87)$ \\
\hline \multicolumn{4}{|l|}{ Geographic region of Puerto Rico } \\
\hline Metropolitan San Juan & $203(15)$ & $2,864(11)$ & 2,955 (10) \\
\hline Metropolitan Bayamon & $182(14)$ & $1,556(6)$ & $1,597(6)$ \\
\hline Nonmetropolitan regions & $941(71)$ & $22,810(83)$ & $23,705(84)$ \\
\hline Total sample & $1,327(100)$ & $27,230(100)$ & $28,257(100)$ \\
\hline \multicolumn{4}{|c|}{$\begin{array}{l}\text { *NCHS, National Center for Health Statistics (Centers for Disease Control and Prevention); WIC, Women, Infants, and Children Program (US Depa } \\
\text { of Agriculture Food and Nutrition Service). } \\
\text { tSource: NCHS's US Territories, } 2016 \text { natality public use file (https://www.cdc.gov/nchs/data_access/vitalstatsonline.htm). } \\
\text { fBecause women had to be } \geq 18 \text { years of age to participate, the }<18 \text { age category is empty for the WIC sample. } \\
\text { SIn the NCHS data, this group includes } 9 \text { th-12th grade, not just } 12 \text { th grade. } \\
\text { TThe educational attainment data in the WIC dataset }(n=909) \text { were incomplete. The data here represent } 68 \% \text { of the total sample of } 1,329 \text {. } \\
\text { \#Source: WIC (https://www.fns.usda.gov/wic/women-infants-and-children-wic). }\end{array}$} \\
\hline
\end{tabular}

messages through television, radio, print, and social media channels (13). The television advertisement included a montage showing couples or pregnant women and their families performing the following behaviors: using repellent, using condoms, using bed nets, removing standing water, and installing screens. Mass media campaigns have the advantage of reaching multiple audiences (including partners, families, and pregnant women not enrolled in WIC) with repeating messages that appeal cognitively and emotionally by showing relatable images of women taking preventive steps and by showing a healthy baby (14).

\section{Offer of Free Residential Mosquito Spraying Services}

When pregnant women attended their WIC appointments, they were also offered a free residential mosquito spraying service. Upon consent, WIC provided women's contact information to a contracted professional spraying service. Across the island, $\approx 3,400$ homes were sprayed through this program. For this analysis, this intervention is defined as the offer of free residential spraying services, meaning that women who report being offered the free service are classified as exposed to the intervention, regardless of whether they chose to use the service. In this way, we can determine whether having free residential spraying services available affected the overall frequency of spraying the home (or yard) for mosquitoes.

Although we might intuit that making residential spraying free would increase use, the literature contains inconsistent evidence about whether removing cost barriers increases vector control behavior (15-17). This offer of free residential mosquito spraying was discontinued in August 2016 after a CDC evaluation found that mosquito populations in and around sprayed homes had not changed, probably as a result of movement of mosquitoes from nearby homes (18).

\section{Intervention Implementation Monitoring}

As these interventions were being implemented, the response behavioral science team conducted monthly telephone interviews of a random sample of 300 pregnant women participating in WIC to provide feedback to the response leadership about intervention exposure and women's Zika prevention behavior. A subset of 150 respondents were asked about their performance of the following 10 CDC-recommended behaviors: using mosquito repellent, using condoms, abstaining from sex, wearing long-sleeved shirts, wearing long pants, sleeping under a bed net, removing or covering standing water, applying larvicide (in water that cannot be removed), putting screens on windows and doors, and spraying home and yard for mosquitoes. This assessment continued until June 2017, when PRDOH declared the Zika epidemic over (19). During 2016-2017, a total of 9 monthly (in 2017, bimonthly) interview rounds were conducted. Our analysis addresses the following: 1) the proportion of pregnant respondents reached by the 4 interventions and the factors associated with exposure; 2) the Zika prevention behaviors that were most widely practiced and that were most strongly associated with exposure to interventions; and 3) additional factors associated with 
Table 2. Respondents exposure to 4 Zika prevention interventions, by demographic characteristics and calendar month, Puerto Rico, July 2016-June $2017^{\star}$

\begin{tabular}{|c|c|c|c|c|c|c|c|c|c|}
\hline \multirow[b]{2}{*}{ Characteristic } & \multirow[b]{2}{*}{ Sample } & \multicolumn{2}{|c|}{$\begin{array}{l}\text { Received WIC } \\
\text { Zika orientation }\end{array}$} & \multicolumn{2}{|c|}{$\begin{array}{l}\text { Received } \\
\text { ZPK }\end{array}$} & \multicolumn{2}{|c|}{$\begin{array}{l}\text { Exposed to Detén } \\
\text { el Zika campaign }\end{array}$} & \multicolumn{2}{|c|}{$\begin{array}{c}\text { Offered free } \\
\text { home spraying }\end{array}$} \\
\hline & & No & Yes & No & Yes & No & Yes & No & Yes \\
\hline \multicolumn{10}{|l|}{ Pregnancy trimester at interview } \\
\hline 1st & 26.8 & 8.4 & 91.6 & 32.9 & 67.1 & 52.2 & 47.8 & 68.1 & 31.9 \\
\hline 2nd & 48.6 & 8.2 & 91.8 & 24.6 & 75.4 & 45.9 & 54.1 & 71.7 & 28.3 \\
\hline 3rd & 24.6 & 3.7 & 96.3 & 16.9 & 83.1 & 53.4 & 46.6 & 52.8 & 47.2 \\
\hline Total no. & 1,329 & 95 & 1,230 & 324 & 976 & 600 & 616 & 873 & 448 \\
\hline$p$ value & & \multicolumn{2}{|c|}{0.019} & \multicolumn{2}{|c|}{0.000} & \multicolumn{2}{|c|}{0.052} & \multicolumn{2}{|c|}{0.000} \\
\hline \multicolumn{10}{|l|}{ Calendar month of interview } \\
\hline Jul 2016 & 11.2 & 4.8 & 95.2 & 4.8 & 95.2 & 62.9 & 37.1 & 29.7 & 70.3 \\
\hline Aug 2016 & 11.1 & 8.2 & 91.8 & 23.8 & 76.2 & 59.2 & 40.8 & 29.3 & 70.7 \\
\hline Sep 2016 & 10.1 & 6.0 & 94.0 & 31.3 & 68.7 & 44.4 & 55.6 & 34.6 & 65.4 \\
\hline Oct 2016 & 11.3 & 10.7 & 89.3 & 41.3 & 58.7 & 31.6 & 68.4 & 65.8 & 34.2 \\
\hline Nov 2016 & 11.3 & 8.0 & 92.0 & 31.3 & 68.7 & 35.0 & 65.0 & 70.1 & 29.9 \\
\hline Dec 2016 & 11.3 & 4.0 & 96.0 & 30.0 & 70.0 & 36.1 & 63.9 & 72.7 & 27.3 \\
\hline Feb 2017 & 11.3 & 6.7 & 93.3 & 20.7 & 79.3 & 55.2 & 44.8 & 97.3 & 2.7 \\
\hline Apr 2017 & 11.3 & 5.3 & 94.7 & 16.0 & 84.0 & 68.1 & 31.9 & 94.7 & 5.3 \\
\hline Jun 2017 & 11.2 & 10.7 & 89.3 & 22.1 & 77.9 & 52.9 & 47.1 & 96.6 & 3.5 \\
\hline Total no. & 1,329 & 203 & 1,230 & 324 & 976 & 600 & 616 & 873 & 418 \\
\hline $\mathrm{p}$ value & & \multicolumn{2}{|c|}{0.225} & \multicolumn{2}{|c|}{0.000} & \multicolumn{2}{|c|}{0.000} & \multicolumn{2}{|c|}{0.000} \\
\hline \multicolumn{10}{|l|}{ Age group, y } \\
\hline $18-22$ & 26.6 & 6.8 & 93.2 & 22.3 & 77.7 & 51.0 & 49.0 & 66.2 & 33.8 \\
\hline $23-25$ & 24.4 & 7.4 & 92.6 & 23.5 & 76.5 & 52.1 & 47.9 & 68.5 & 68.5 \\
\hline $26-29$ & 24.0 & 6.9 & 93.1 & 27.0 & 73.0 & 44.1 & 55.9 & 64.9 & 35.1 \\
\hline$\geq 30$ & 25.1 & 7.5 & 92.5 & 27.2 & 72.8 & 49.8 & 50.2 & 64.8 & 35.2 \\
\hline Total no. & 1,329 & 95 & 1,230 & 324 & 976 & 600 & 616 & 873 & 448 \\
\hline $\mathrm{p}$ value & & \multicolumn{2}{|c|}{0.981} & \multicolumn{2}{|c|}{0.356} & \multicolumn{2}{|c|}{0.217} & & \\
\hline Educational attainment & & & & & & & & & \\
\hline Some high school or less & 2.6 & 0.0 & 100.0 & 21.7 & 78.3 & 54.5 & 45.5 & 66.7 & 33.3 \\
\hline Attended or completed 12 th grade & 31.4 & 7.4 & 92.6 & 22.3 & 77.7 & 49.4 & 50.6 & 66.1 & 33.9 \\
\hline Attended or completed university & 60.0 & 6.1 & 93.9 & 23.6 & 76.4 & 49.9 & 50.1 & 62.2 & 37.8 \\
\hline Attended or completed graduate program & 6.1 & 7.3 & 92.7 & 25.6 & 74.1 & 36.2 & 63.8 & 58.2 & 41.8 \\
\hline Total no. & 909 & 58 & 848 & 207 & 681 & 404 & 419 & 572 & 332 \\
\hline $\mathrm{p}$ value & & & & & & & & & \\
\hline Population in poverty in ZIP code, $\%$ quartiles & & & & & & & & & \\
\hline$>55$ below poverty & 25.0 & 5.1 & 94.9 & 22.8 & 77.2 & 49.5 & 50.5 & 65.2 & 34.8 \\
\hline$\overline{4} 9-54$ below poverty & 25.3 & 7.9 & 92.1 & 25.6 & 74.4 & 43.7 & 56.3 & 67.5 & 32.5 \\
\hline 43-48 below poverty & 25.1 & 6.7 & 93.3 & 23.1 & 76.9 & 51.6 & 48.4 & 64.2 & 35.8 \\
\hline$\leq 43$ below poverty & 24.5 & 8.8 & 91.2 & 29.2 & 70.8 & 53.1 & 46.9 & 68.1 & 31.9 \\
\hline Total no. & 1,255 & 89 & 1,163 & 309 & 918 & 566 & 579 & 826 & 421 \\
\hline$p$ value & & & & & & & & & \\
\hline Municipality population & & & & & & & & & \\
\hline$\geq 200,000$ & 63.5 & 6.1 & 93.9 & 23.1 & 76.9 & 48.6 & 51.4 & 66.0 & 34.0 \\
\hline$\geq 100,00-200,000$ & 9.9 & 10.6 & 89.4 & 31.3 & 68.7 & 50.4 & 49.6 & 61.8 & 38.2 \\
\hline$\geq 50,000-100,000$ & 12.6 & 7.7 & 92.3 & 27.9 & 72.1 & 46.8 & 53.2 & 63.3 & 36.7 \\
\hline$<50,000$ & 14.0 & 8.8 & 91.2 & 26.3 & 73.7 & 56.1 & 43.9 & 72.5 & 27.5 \\
\hline Total no. & 1,326 & 91 & 1,184 & 313 & 937 & 578 & 589 & 839 & 431 \\
\hline$p$ value & & & & 0. & & & & & \\
\hline
\end{tabular}

*All data are percentages unless otherwise indicated. Statistically significant differences $\left(p<0.05\right.$ by $\chi^{2}$ test) are shown in boldface. WIC, Women, Infants, and Children Program (US Department of Agriculture Food and Nutrition Service); ZPK, Zika prevention kit.

†Source: US Census Bureau American Community Survey, 2016. American Community Survey 5-year estimates, Table S1701 (generated by G.B.E. using American Fact Finder, 2018 Feb 24).

Zika prevention behavior that might provide insight into how the interventions influenced behavior.

\section{Methods}

\section{Interview Population and Sampling}

Each month during July-December 2016 and every 2 months during February-June 2017, a random sample of 950 pregnant women $\geq 18$ years of age (317 women per pregnancy trimester) was drawn from the WIC enrollment database of
10,000-12,000 women currently enrolled (and not previously contacted) for interviews. Vital statistics data indicate that $87 \%$ of women giving birth in Puerto Rico in 2016 were enrolled in WIC (Table 1). The calling list was divided among interviewers so that some began with first trimester women, some with second, and some with third. As part of the Zika response, these interviews were determined to be nonresearch public health practice and were approved by the US Office of Management and Budget (control no. 0920-1196). Before asking women for their verbal agreement to participate, 


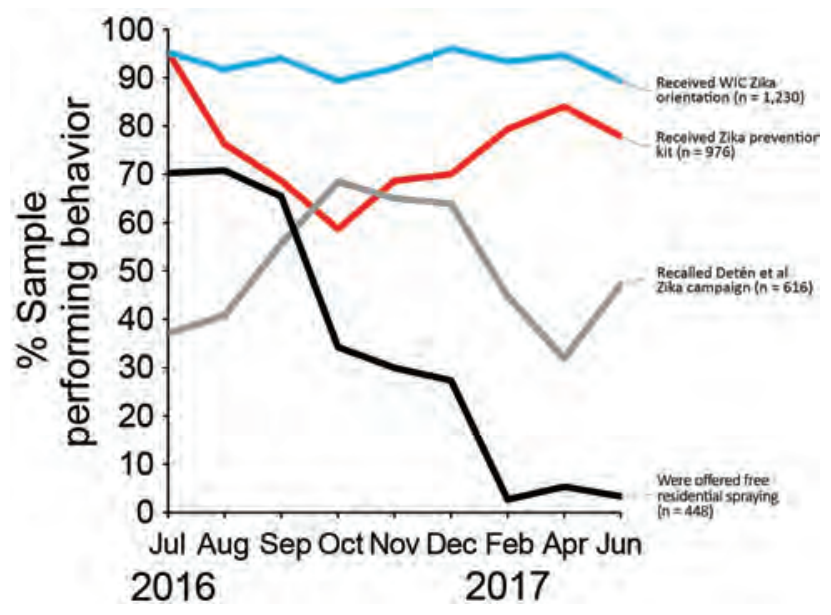

Figure 1. Percentage of pregnant women reporting exposure to 4 Zika prevention interventions, by interview month, Puerto Rico, 2016-2017. August 12, 2016: President declares Zika in Puerto Rico a "public health emergency" (https://www.reuters.com/article/ us-health-zika-usa/u-s-declares-a-zika-public-health-emergencyin-puerto-rico-idUSKCN10N2KA). September 30, 2016: Free residential spraying discontinued. Women who report the offer through December are referring to receiving the offer before September. October 28, 2016: First baby born with microcephaly in Puerto Rico (https://www.nytimes.com/2016/10/29/health/ zika-microcephaly-puerto-rico.html). June 5, 2017: Zika epidemic declared over by Puerto Rico Department of Health (https://www. businesswire.com/news/home/20170605006235/en/Puerto-RicoDepartment-Health-Declared-2016-Zika). WIC, Women, Infants, and Children Program (US Department of Agriculture Food and Nutrition Service).

interviewers explained the purpose of the data collection, the fact that their participation and all responses would be kept confidential, and that they could discontinue the interview any time without any penalty. The 3 groups of callers continued until 300 total interviews were completed. The interview had 2 parts, administered 2 weeks apart. Those women who consented to complete part 2 were called in the same order as for part 1 until 150 interviews were completed.

\section{Data Collection}

The interview consisted of questions about Zika knowledge, attitudes, sources of information, exposure to prevention interventions, and Zika prevention behaviors. Many of the questions involved binary (e.g., yes or no) or scaled (e.g., never, rarely, sometimes, frequently, or often) responses. Others were questions in which the interviewer did not provide response options to the participant but coded the response according to a checklist. Although Zika infection status was not an interview question, if a participant disclosed that she was Zika positive, the interview was excluded from the dataset. This exclusion was made because Zika virus infection confers immunity and therefore an already positive woman would have no reason to take prevention steps.

\section{Definition of Intervention Exposure}

Respondents were asked if they had received the WIC Zika orientation, the ZPK, or the offer of free home spraying. They were also asked if they had seen communications from the Detén el Zika campaign. Any woman answering affirmatively to any of these questions was defined as exposed to the corresponding intervention.

\section{Data Analysis}

\section{Calculation of Zika Prevention Behavior Variables}

Because the original interview instrument included multiple questions about each Zika prevention behavior without any clear formula for integrating question responses into a single variable (1 per behavior), analysts had to create such a formula. For example, some questions asked whether a woman performed the behavior any time during pregnancy (or during the previous day or week) (yes or no), whereas others used ordinal frequency scales (e.g., never, sometimes, or always). In addition, a Zika prevention behavior could be reported in response to the question, "What actions have you taken to protect yourself from being infected by the Zika virus?"

To describe women's Zika prevention behavior as completely as possible, analysts created behavior variables that incorporated 2, 3, or more questions. We prioritized timebound, behavior-specific questions, such as, "How often did you use mosquito repellent in the past week?" (never, sometimes, or always), over a more general question such as, "What actions have you taken to protect yourself from being infected with the Zika virus?" Among the behaviorspecific questions, those questions with multilevel response options were prioritized over yes or no or dichotomous response questions, given that the greater number of response options yielded more information. Zika prevention behavior variables were then created with ordinal scales, combining the most detailed behavior-specific question available for the behavior with other questions that might serve to increase the number of levels of Zika prevention behavior. Once preliminary scales were created, frequencies and plots were reviewed by behavioral scientists and epidemiologists involved with the Zika response to achieve a consensus on the final composition. We have compiled a list of all candidate questions and final variables (online Technical Appendix, https:// wwwnc.cdc.gov/EID/article/24/12/18-1056-Techapp1.pdf).

\section{Statistical Methods}

Analysts calculated frequencies of intervention exposure by interview month and demographic characteristics. In addition, because the interventions sought to increase Zika prevention behavior by increasing a woman's concern about Zika, her confidence in her ability to protect herself, and involvement of partners and families in Zika prevention, variables representing these constructs were tested for 
Table 3. Associations between Zika prevention intervention exposure and interpersonal communications about Zika and personal risk perceptions, Puerto Rico, July 2016-June 2017*

\begin{tabular}{|c|c|c|c|c|c|c|c|c|c|}
\hline \multirow[b]{2}{*}{ Variable } & \multirow[b]{2}{*}{ Sample } & \multicolumn{2}{|c|}{$\begin{array}{l}\text { Received WIC } \\
\text { Zika orientation }\end{array}$} & \multicolumn{2}{|c|}{ Received ZPK } & \multicolumn{2}{|c|}{$\begin{array}{l}\text { Exposed to Detén } \\
\text { el Zika campaign }\end{array}$} & \multicolumn{2}{|c|}{$\begin{array}{c}\text { Offered free } \\
\text { home spraying }\end{array}$} \\
\hline & & No & Yes & No & Yes & No & Yes & No & Yes \\
\hline \multicolumn{10}{|c|}{ Family and interpersonal communication } \\
\hline \multicolumn{10}{|c|}{ Frequency of talking to family and friends about Zika } \\
\hline Not at all & 10.7 & 8.4 & 10.8 & 9.9 & 11.1 & 14.5 & 7.3 & 12.7 & 6.7 \\
\hline Only once or twice & 16.2 & 21.1 & 15.9 & 16.7 & 16.1 & 17.7 & 15.3 & 17.4 & 13.6 \\
\hline Sometimes & 32.7 & 45.3 & 31.8 & 34.9 & 32.3 & 33.0 & 32.5 & 33.3 & 31.5 \\
\hline Often & 22.0 & 16.8 & 22.4 & 18.5 & 22.7 & 19.2 & 23.5 & 20.3 & 25.2 \\
\hline Every day & 18.4 & 8.4 & 19.2 & 20.1 & 17.8 & 15.7 & 21.4 & 16.3 & 23.0 \\
\hline Total no. & 1,329 & 79 & 1,230 & 600 & 616 & 324 & 976 & 873 & 448 \\
\hline $\mathrm{p}$ value & & \multicolumn{2}{|c|}{0.009} & \multicolumn{2}{|c|}{0.472} & \multicolumn{2}{|c|}{0.000} & \multicolumn{2}{|c|}{0.000} \\
\hline \multicolumn{10}{|c|}{ Aware of Zika prevention actions of family } \\
\hline No & 38.3 & 38.8 & 38.2 & 38.2 & 38.4 & 46.0 & 31.0 & 41.4 & 30.9 \\
\hline Yes & 61.7 & 61.2 & 61.8 & 61.8 & 61.6 & 54.0 & 69.0 & 58.6 & 69.1 \\
\hline Total no. & 1,168 & 85 & 1,081 & 511 & 561 & 314 & 850 & 818 & 343 \\
\hline $\mathrm{p}$ value & & \multicolumn{2}{|c|}{0.910} & \multicolumn{2}{|c|}{0.966} & \multicolumn{2}{|c|}{0.000} & \multicolumn{2}{|c|}{0.001} \\
\hline \multicolumn{10}{|c|}{ Individual risk perception } \\
\hline \multicolumn{10}{|c|}{ How concerned women feel about Zika } \\
\hline Not at all concerned & 8.2 & 7.4 & 8.3 & 5.6 & 9.0 & 8.8 & 7.8 & 8.9 & 6.7 \\
\hline Slightly concerned & 16.4 & 13.7 & 16.7 & 14.8 & 17.1 & 17.9 & 15.6 & 17.7 & 13.8 \\
\hline Somewhat concerned & 21.1 & 20.0 & 21.2 & 20.7 & 21.1 & 21.7 & 21.1 & 21.6 & 20.3 \\
\hline Moderately concerned & 27.3 & 33.7 & 26.6 & 30.2 & 26.4 & 27.4 & 26.8 & 27.5 & 26.8 \\
\hline Extremely concerned & 27.0 & 25.3 & 27.3 & 28.7 & 26.4 & 24.2 & 28.7 & 24.3 & 32.4 \\
\hline Total no. & 1,328 & 95 & 1,229 & 599 & 616 & 324 & 975 & 872 & 448 \\
\hline $\mathrm{p}$ value & & \multicolumn{2}{|c|}{0.665} & \multicolumn{2}{|c|}{0.182} & \multicolumn{2}{|c|}{0.435} & \multicolumn{2}{|c|}{0.019} \\
\hline \multicolumn{10}{|c|}{ How likely women feel they will become infected with Zika } \\
\hline Extremely unlikely & 10.0 & 9.7 & 10.0 & 8.1 & 10.8 & 10.2 & 9.9 & 12.0 & 5.9 \\
\hline Unlikely & 37.4 & 36.6 & 37.4 & 37.4 & 37.4 & 36.6 & 38.0 & 36.8 & 38.4 \\
\hline Neither likely nor unlikely & 30.6 & 32.3 & 30.5 & 31.5 & 30.2 & 30.3 & 31.4 & 31.0 & 30.2 \\
\hline Likely & 19.4 & 19.4 & 19.4 & 20.9 & 18.8 & 19.3 & 18.8 & 18.0 & 22.1 \\
\hline Extremely likely & 2.7 & 2.2 & 2.7 & 2.2 & 2.8 & 3.6 & 2.0 & 2.1 & 3.4 \\
\hline Total no. & 1,306 & 93 & 1,209 & 587 & 606 & 321 & 957 & 855 & 443 \\
\hline $\mathrm{p}$ value & & & & & & & & & \\
\hline Confidence in ability to protect $s$ & baby fron & Zika & & & & & & & \\
\hline Not confident at all & 1.1 & 2.1 & 1.1 & 1.9 & 0.9 & 1.2 & 1.3 & 1.3 & 0.9 \\
\hline Somewhat unconfident & 9.9 & 16.0 & 9.4 & 10.3 & 9.6 & 10.1 & 9.3 & 10.0 & 9.5 \\
\hline Not confident or unconfident & 22.3 & 27.7 & 21.8 & 20.6 & 22.3 & 24.7 & 20.2 & 21.1 & 24.8 \\
\hline Confident & 49.5 & 45.7 & 49.9 & 48.9 & 50.1 & 49.5 & 49.8 & 50.7 & 47.1 \\
\hline Very confident & 17.2 & 8.5 & 17.9 & 18.4 & 17.1 & 14.6 & 19.3 & 16.8 & 17.8 \\
\hline Total no. & 1,319 & 94 & 1,221 & 596 & 610 & 321 & 969 & 867 & 444 \\
\hline p value & & & & & & & & & \\
\hline
\end{tabular}

associations with intervention exposure and Zika prevention behaviors. All analyses were conducted with SPSS 21.0 (IBM Corp., Armonk, NY, USA).

Analysts used logistic regression modeling to estimate odds ratios (ORs) for the likelihood of performing recommended Zika prevention behaviors by exposure to 1 of the Zika prevention interventions while controlling for the effects of age, education, pregnancy trimester, poverty, calendar month of interview, and exposure to other interventions. For these models, Zika prevention behavior variable responses were collapsed into dichotomous (yes or no) variables, indicating whether a respondent had performed the ideal behavior (e.g., always uses a condom) or not. In the case of mosquito repellent use, the 2 top levels, which both include the response always, were combined to make the top level.
Because the WIC orientation reached nearly all respondents, the naturally occurring control group of unexposed women was very small, causing concerns about small cell size in models with many covariates (20). Conversely, a small exposure group was a concern with the offer of free residential mosquito spraying. Therefore, these 2 interventions were modeled separately from ZPK distribution and Detén el Zika, which were modeled together. In addition, sparsity concerns led us to consolidate the calendar month of interview variable into 1 representing 3-month intervals.

\section{Results}

\section{Participant Characteristics}

Our sample encompassed 1,329 pregnant WIC participants interviewed during July 2016-June 2017 (Table 1). 
Table 4. Zika personal protection behaviors among pregnant women, by exposure to 4 interventions, Puerto Rico, July 2016-June $2017^{*}$

\begin{tabular}{|c|c|c|c|c|c|c|c|c|c|}
\hline \multirow[b]{2}{*}{ Behavior } & \multirow{2}{*}{$\begin{array}{c}\text { Entire } \\
\text { sample }\end{array}$} & \multicolumn{2}{|c|}{$\begin{array}{l}\text { Received } \\
\text { WIC Zika } \\
\text { orientation }\end{array}$} & \multicolumn{2}{|c|}{$\begin{array}{c}\text { Received } \\
\text { ZPK }\end{array}$} & \multicolumn{2}{|c|}{$\begin{array}{l}\text { Exposed to } \\
\text { Detén el Zika } \\
\text { campaign }\end{array}$} & \multicolumn{2}{|c|}{$\begin{array}{c}\text { Offered free } \\
\text { home } \\
\text { spraying }\end{array}$} \\
\hline & & Yes & No & Yes & No & Yes & No & Yes & No \\
\hline \multicolumn{10}{|l|}{ Mosquito repellent use } \\
\hline Always, reported reapplying & 28.3 & 29.1 & 18.9 & 29.7 & 24.5 & 31.2 & 25 & 32.6 & 26.1 \\
\hline Always, did not report reapplying & 23.9 & 23.5 & 28.4 & 24.1 & 23.5 & 27.6 & 21.5 & 23.9 & 23.9 \\
\hline Usually or most of the time & 25.9 & 26.4 & 21.1 & 25.9 & 26 & 23.1 & 28.4 & 23.2 & 27.1 \\
\hline Sometimes & 13.0 & 12.8 & 14.7 & 12.2 & 15.2 & 11.7 & 14.5 & 13.2 & 13.1 \\
\hline Rarely or seldom & 4.6 & 4.4 & 7.4 & 4.7 & 4.0 & 3.9 & 4.7 & 4.0 & 4.9 \\
\hline Never & 4.2 & 3.8 & 9.5 & 3.4 & 6.8 & 2.6 & 5.8 & 3.1 & 4.8 \\
\hline Total no. & 1,328 & 1,229 & 95 & 976 & 323 & 614 & 597 & 448 & 873 \\
\hline $\mathrm{p}$ value & & \multicolumn{2}{|c|}{0.018} & \multicolumn{2}{|c|}{0.016} & \multicolumn{2}{|c|}{$<0.001$} & \multicolumn{2}{|c|}{0.012} \\
\hline \multicolumn{10}{|l|}{ Condom use $\dagger$} \\
\hline Always & 44.1 & 45.3 & 31.6 & 45.1 & 42.6 & 44.2 & 26.3 & 42.5 & 44.8 \\
\hline Sometimes & 29.3 & 29.5 & 24.1 & 30.6 & 25.8 & 28.7 & 26.3 & 28.3 & 29.9 \\
\hline Never & 26.6 & 25.2 & 44.3 & 24.3 & 31.6 & 27.2 & 47.4 & 29.2 & 25.3 \\
\hline Total no. & 1,047 & 964 & 79 & 768 & 256 & 491 & 464 & 353 & 689 \\
\hline $\mathrm{p}$ value & & \multicolumn{2}{|c|}{0.001} & \multicolumn{2}{|c|}{0.130} & \multicolumn{2}{|c|}{0.001} & \multicolumn{2}{|c|}{0.266} \\
\hline \multicolumn{10}{|l|}{ Bed net use } \\
\hline Slept under bed net yesterday & 14.8 & 15.4 & 7.4 & 17.7 & 6.8 & 16.1 & 13.8 & 13.8 & 15.3 \\
\hline Did not use yesterday, reports use generally & 4.9 & 5.2 & 1.1 & 5.7 & 2.5 & 4.2 & 4.7 & 3.1 & 5.8 \\
\hline Did not use yesterday, does not report use generally & 80.3 & 79.4 & 91.6 & 76.5 & 90.7 & 79.7 & 81.5 & 83 & 78.8 \\
\hline Total no. & 1,329 & \multirow{2}{*}{\multicolumn{2}{|c|}{$\begin{array}{l}1,230 \\
0.005\end{array}$}} & 976 & 324 & 616 & 600 & 448 & 873 \\
\hline$p$ value & & & & \multicolumn{2}{|c|}{$<0.001$} & \multicolumn{2}{|c|}{0.390} & \multicolumn{2}{|c|}{0.094} \\
\hline \multicolumn{10}{|l|}{ Wearing long pants } \\
\hline Wearing now, every day, all day & 21.3 & 21.4 & 21.1 & 20.6 & 23.5 & 21.2 & 20.8 & 20.6 & 21.5 \\
\hline Wearing now, every day, part of day & 19.2 & 19.5 & 15.8 & 18.7 & 21.0 & 20.4 & 18.3 & 19.7 & 19.0 \\
\hline Wearing now, does not wear every day & 20.0 & 20 & 21.1 & 20.0 & 19.4 & 20.5 & 20 & 17.7 & 21.3 \\
\hline Not wearing long pants now & 39.4 & 39.1 & 42.1 & 40.7 & 36.1 & 40.8 & 37.9 & 41.9 & 38.1 \\
\hline Total no. & 1,327 & 1,228 & 95 & 974 & 324 & 614 & 600 & 446 & 873 \\
\hline $\mathrm{p}$ value & & \multicolumn{2}{|c|}{0.549} & & & & & & \\
\hline Sexual abstinence & & & & & & & & & \\
\hline Had no sex during pregnancy & 20.2 & 20.7 & 15.8 & 20.3 & 19.9 & 31.2 & 25.0 & 20.6 & 19.9 \\
\hline Had sex during pregnancy & 79.8 & 79.3 & 84.2 & 79.7 & 80.1 & 80.6 & 78.2 & 79.4 & 80.1 \\
\hline Total no. & 1,324 & 1,225 & 95 & 973 & 322 & 614 & 597 & 447 & 869 \\
\hline $\mathrm{p}$ value & & 0.2 & & & & & & 0.7 & \\
\hline Wearing long-sleeved shirt & & & & & & & & & \\
\hline Wearing now, every day, all day & 3.9 & 3.8 & 5.3 & 3.7 & 4.7 & 77.7 & 79.3 & 4.0 & 3.8 \\
\hline Wearing now, every day, part of day & 6.7 & 6.7 & 7.4 & 7.2 & 5.6 & 6.4 & 7.2 & 6.9 & 6.7 \\
\hline Wearing now, does not wear every day & 10.6 & 10.8 & 7.4 & 9.9 & 13.7 & 11.1 & 10.4 & 8.9 & 11.5 \\
\hline Not wearing long sleeves now & 78.7 & 78.6 & 79.8 & 79.3 & 79.3 & 79.3 & 83.5 & 80.1 & 78 \\
\hline Total no. & 1,325 & 1,227 & 94 & 974 & 322 & 614 & 598 & 448 & 869 \\
\hline $\mathrm{p}$ value & & & & & & & & 0.4 & \\
\hline
\end{tabular}

${ }^{*}$ All data indicate percentages unless otherwise indicated. Statistically significant differences ( $p<0.05$ by Mann-Whitney $U$ nonparametric test) are shown in bold. WIC, Women, Infants, and Children Program (US Department of Agriculture Food and Nutrition Service); ZPK, Zika prevention kit.

†Among those reporting having had sex during pregnancy.

Among eligible women (i.e., $\geq 18$ years of age, pregnant, and not Zika positive), the response rate was $79 \%$. Age and educational attainment distributions of the sample were similar to the general population of women giving birth in Puerto Rico in 2016 (21), whereas urban residence is somewhat higher.

\section{Women's Exposure to 4 Zika Prevention Interventions}

Women reported exposure to the 4 interventions as follows: WIC Zika orientation (93\%), ZPK distribution (75\%), Detén el Zika campaign (51\%), and offer of free residential mosquito spraying $(68 \%$ for the months it was running and $34 \%$ over the entire period). Pregnancy trimester was statistically significant for association with exposure to all 4 interventions, whereas calendar month of interview was significantly associated with 3 interventions (Table 2). No significant associations were observed in terms of age, education, poverty, or rurality.

Graphed by calendar month of interview (Figure 1), exposure to the WIC Zika orientation remained consistently high (89\%-96\%). ZPK distribution began high (95\%), dropped in October, then rebounded. Detén el Zika campaign exposure began much lower (37\%), then steadily increased through October (68\%), dropped off, and rose again in 2017. Exposure to the offer of free residential mosquito spraying started at $70 \%$ in July 2016 , then dropped precipitously after September. 
Table 5. Zika home protection behaviors among pregnant women, by exposure to 4 interventions, Puerto Rico, July 2016-June 2017*

\begin{tabular}{|c|c|c|c|c|c|c|c|c|c|}
\hline \multirow[b]{2}{*}{ Behavior } & \multirow{2}{*}{$\begin{array}{l}\text { Samples, \% } \\
\text { (no.) }\end{array}$} & \multicolumn{2}{|c|}{$\begin{array}{l}\text { Received } \\
\text { WIC Zika } \\
\text { orientation }\end{array}$} & \multicolumn{2}{|c|}{$\begin{array}{l}\text { Received } \\
\text { ZPK }\end{array}$} & \multicolumn{2}{|c|}{$\begin{array}{l}\text { Exposed to } \\
\text { Detén el Zika } \\
\text { campaign }\end{array}$} & \multicolumn{2}{|c|}{$\begin{array}{c}\text { Offered free } \\
\text { home } \\
\text { spraying }\end{array}$} \\
\hline & & Yes & No & Yes & No & Yes & No & Yes & No \\
\hline \multicolumn{10}{|l|}{ Removing (or covering) standing water* } \\
\hline Removed standing water in past week & $90.3(531)$ & 90.5 & 87.2 & 91.8 & 85.5 & 93.9 & 85.6 & 91.3 & 90.2 \\
\hline Has not in past week; reports action generally & $1.2(7)$ & 1.1 & 2.6 & 0.9 & 2.2 & 1.3 & 1.2 & 1.9 & 0.8 \\
\hline Has not in past week; does report action generally & $8.5(50)$ & 8.4 & 10.3 & 7.3 & 12.3 & 4.7 & 13.2 & 6.8 & 9.0 \\
\hline Total no. & 588 & 546 & 39 & 438 & 138 & 297 & 243 & 377 & 206 \\
\hline $\mathrm{p}$ value & & \multicolumn{2}{|c|}{0.516} & \multicolumn{2}{|c|}{0.032} & \multicolumn{2}{|c|}{0.001} & \multicolumn{2}{|c|}{0.637} \\
\hline \multicolumn{10}{|l|}{ Spraying home (or yard) for mosquitoes } \\
\hline Sprayed for mosquitoes (self or service) & $43.1(569)$ & 43.7 & 33.7 & 44.4 & 37.7 & 42.6 & 43.2 & 82.3 & 22.9 \\
\hline No home spraying & $56.9(752)$ & 56.3 & 66.3 & 55.6 & 62.3 & 57.4 & 56.8 & 17.7 & 77.1 \\
\hline Total no. & 1,321 & 1,222 & 95 & 971 & 321 & 615 & 595 & 446 & 873 \\
\hline $\mathrm{p}$ value & & \multicolumn{2}{|c|}{0.058} & \multicolumn{2}{|c|}{0.036} & \multicolumn{2}{|c|}{0.835} & \multicolumn{2}{|c|}{$\begin{array}{l}440 \\
<0.001\end{array}$} \\
\hline \multicolumn{10}{|l|}{ Larvicide application $\dagger$} \\
\hline Has applied larvicide around home (self or family) & $31.3(308)$ & 24.2 & 10.8 & 40.5 & 7.9 & 30.0 & 32.9 & 20.1 & 37.3 \\
\hline Never applied larvicide around home (self & $68.7(675)$ & 75.8 & 89.2 & 59.5 & 92.1 & 70.0 & 67.1 & 79.9 & 62.7 \\
\hline Total no. & 983 & 1,229 & 93 & 708 & 253 & 476 & 423 & 334 & 641 \\
\hline p value & & \multicolumn{2}{|c|}{0.002} & \multicolumn{2}{|c|}{$<0.001$} & \multicolumn{2}{|c|}{0.364} & \multicolumn{2}{|c|}{$<0.001$} \\
\hline \multicolumn{10}{|l|}{ Installing window or door screens } \\
\hline Reports putting screens on windows, doors & $17.8(236)$ & 17.4 & 22.1 & 17.6 & 18.5 & 18.0 & 18.7 & 18.1 & 17.5 \\
\hline Does not report putting screens on windows, doors & $82.2(1,093)$ & 82.6 & 77.9 & 82.4 & 81.5 & 82.0 & 81.3 & 81.9 & 82.5 \\
\hline Total no. & 1,329 & 1,230 & 95 & 976 & 324 & 616 & 600 & 448 & 873 \\
\hline$p$ value & & \multicolumn{2}{|c|}{0.247} & \multicolumn{2}{|c|}{0.715} & \multicolumn{2}{|c|}{0.771} & \multicolumn{2}{|c|}{0.803} \\
\hline
\end{tabular}

*All data indicate percentages unless otherwise indicated. Statistically significant differences ( $<<0.05$ by Mann-Whitney U nonparametric test) are shown in boldface. WIC, Women, Infants, and Children Program (US Department of Agriculture Food and Nutrition Service); ZPK, Zika prevention kit.

†Among those having yards for which they are responsible, and where water was present.

Intervention exposure was more often significantly associated with family or interpersonal communication variables than with individual risk variables (Table 3 ). The same pattern was observed for associations with Zika prevention behaviors (data not shown): "frequency of talking to family and friends about Zika" was significantly associated with 10 behaviors and "aware of Zika prevention actions of family" with 5 behaviors, whereas all 3 individual risk perception-related variables were associated with $\leq 3$ behaviors.

\section{Pregnant Women's Zika Personal Protection Behaviors}

Frequencies of recommended personal protection behaviors (i.e., the top level on the ordinal scale) ranged from $4 \%$ (wearing long-sleeved shirt) to $44 \%$ (condom use) (Table 4). Although just over half of women reported using repellent always, fewer (28\%) reported the top category, "used always and reported reapplying it." Among the interventions, exposure to the WIC Zika orientation showed the greatest exposed versus not exposed frequency differences for the top behavior levels (Tables 4, 5).

Over the monthly interview cohorts, the top level of condom use rose steadily with a sustained peak at over $50 \%$, whereas mosquito repellent use rose to $42 \%$, declined, and peaked again in December (Figure 2). Wearing long pants had 2 peaks (in October and December) near 30\%, then a steep decline in 2017, whereas sexual abstinence stayed near 20\%. Bed net use peaked at 23\% in September, then fluctuated.

\section{Zika Home Protection Behaviors}

We ranked home protection behaviors from the most frequent (removing standing water [90\%]) to the least (installing window or door screens [18\%]) (Table 5). Over time, removing standing water declined slightly through September but then remained at $\geq 85 \%$, whereas spraying the home for mosquitoes had a steep decline during August-June 2017 (Figure 3). In contrast, larvicide application began low (13\%) and then increased through June 2017 (40\%).

\section{Independent Associations between Interventions and Zika Prevention Behaviors}

In multivariable logistic regression models, we observed a strong association between the offer of free residential mosquito spraying services and spraying the home for mosquitoes (Table 6). We also observed strong associations between ZPK receipt and larvicide application and between $\mathrm{ZPK}$ receipt and bed net use.

\section{Discussion}

For each intervention, exposure patterns corresponded with implementation history; WIC orientation exposure was consistently high, Detén el Zika campaign exposure grew over time, ZPK exposure faltered (because of logistical problems with kit distribution) and then recovered, and the free offer of home mosquito spraying was widely received during the offer period. These largely successful implementations illustrate the benefits of collaborating with a trusted local partner like WIC. WIC 


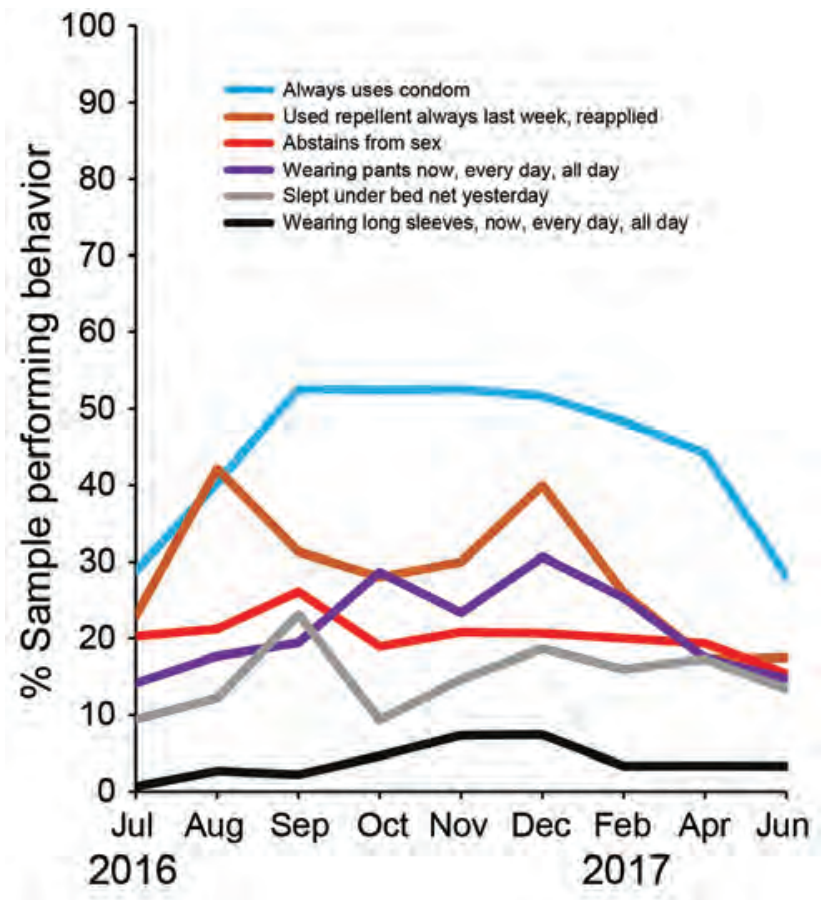

Figure 2. Percentage of women reporting highest levels of 6 Zika personal protection behaviors, by interview month, Puerto Rico, July 2016-June 2017. See Figure 1 legend for timeline of events.

was able to incorporate Zika orientations into its regular programming, distribute ZPKs effectively, and provide the free offer of home spraying during a WIC visit. WIC also played an important role in developing the Detén el Zika messaging.

Performance of Zika prevention behaviors varied widely. Nearly all women removed any standing water that they saw, and about three quarters usually or always used mosquito repellent, but very few wore long sleeves or put up screens. These findings are consistent with the Pregnancy Risk Assessment Monitoring System Zika Postpartum Emergency Response (PRAMS-ZPER) study of postpartum women in Puerto Rico (22). Despite important methodologic differences between PRAMSZPER and our analysis, reported frequencies were similar for mosquito repellent use, removing standing water, bed net use, and wearing long sleeves. Where frequencies diverged (condom use and spraying home for mosquitoes), WIC sample frequencies were more similar to PRAMS-ZPER when limited to women in their third trimester. In contrast, interview data from US Virgin Islands in late 2016 (7) showed lower frequencies of using repellent, using condoms, removing standing water, and spraying home for mosquitoes. Only data for bed net use were similar to the results of our analysis.

Overall, the ZPK distribution had the greatest number of independent positive associations with Zika prevention

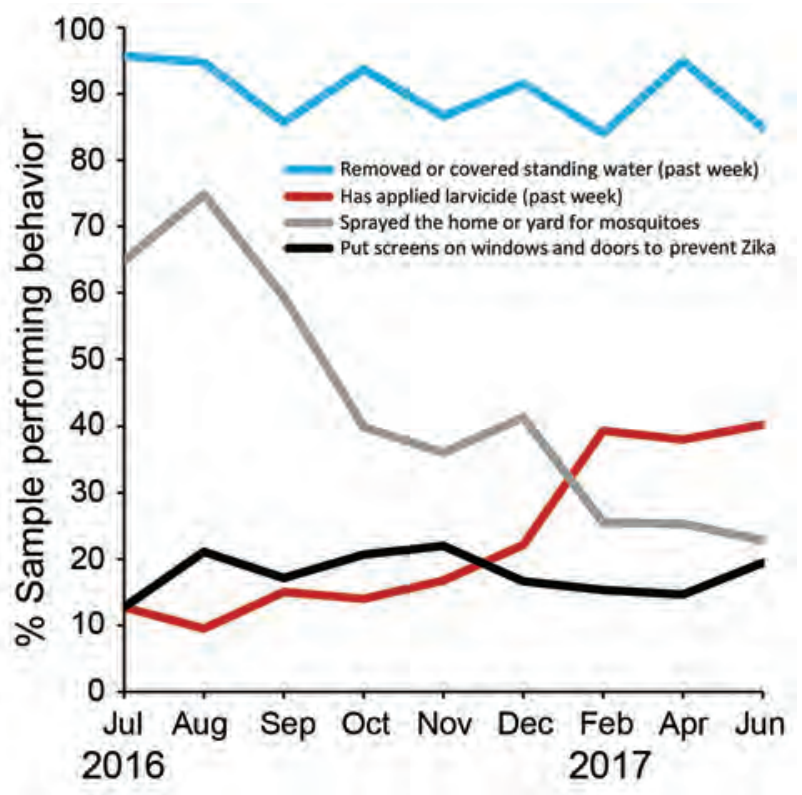

Figure 3. Percentage of women reporting highest levels of 4 Zika home protection behaviors, by interview month, Puerto Rico, July 2016-June 2017. See Figure 1 legend for timeline of events.

behavior and some of the strongest associations. This finding is consistent with a small but growing body of literature demonstrating the effectiveness of distributing items for encouraging prevention behavior $(11,23,24)$. Prevention kits containing prevention products for at-risk populations should be considered a best practice, particularly in lowresource settings.

Larvicide use and bed net use were independently associated with ZPK receipt, and distributing items associated with these 2 largely unfamiliar behaviors probably increased use because women were then able to try them. According to Rogers' diffusion of innovations theory (25), the ability to try a new behavior and observe the results enhances the likelihood of adoption. Larvicide application might have been further enhanced by what Rogers calls "relative advantage"; that is, the intervention might have been popular because it was easier to implement than the other 3 recommended home protection behaviors (removing standing water, installing screens, and spraying home for mosquitoes). Many of the ZPKs in the early months of tracking were missing larvicide tablets; thus, the dramatic increase in larvicide use over the period is not surprising. The finding also suggests that the actual association between ZPKs and larvicide use is stronger than what our results indicate, given that the incomplete kits might have diluted the observed association.

Offer of free residential mosquito spraying services was strongly associated with spraying the home 
Table 6. Logistic regression models for Zika prevention behaviors performed by pregnant women that were significantly associated with $\geq 1$ Zika prevention interventions, Puerto Rico, July 2016-June 2017*†

\begin{tabular}{|c|c|c|c|c|}
\hline \multirow[b]{2}{*}{ Behavior } & \multicolumn{4}{|c|}{ Odds ratio $(95 \% \mathrm{Cl})$} \\
\hline & Received ZPK & $\begin{array}{c}\text { Recalled Detén el } \\
\text { Zika campaign }\end{array}$ & $\begin{array}{c}\text { Received WIC } \\
\text { Zika orientation }\end{array}$ & $\begin{array}{c}\text { Offered free } \\
\text { residential spraying }\end{array}$ \\
\hline \multicolumn{5}{|l|}{ Personal protection behaviors } \\
\hline Bed net use & $3.1(1.9-5.1)$ & $1.2(0.8-1.7)$ & $2.2(1.0-4.8)$ & NA \\
\hline Condom use $\ddagger$ & $1.4(0.9-2.1)$ & $1.4(1.0-2.0)$ & $2.4(1.2-4.7)$ & NA \\
\hline Mosquito repellent use & $1.5(1.1-2.0)$ & $1.6(1.2-2.1)$ & $1.2(0.8-1.9)$ & NA \\
\hline Sexual abstinence & $0.9(0.6-1.4)$ & $0.9(0.6-1.3)$ & $1.2(0.5-2.5)$ & NA \\
\hline Wearing long sleeves & $1.9(0.6-6.2)$ & $2.9(0.9-8.8)$ & $1.9(0.2-14.9)$ & NA \\
\hline Wearing long pants & $1.1(0.7-1.7)$ & $1.0(0.7-1.5)$ & $1.4(0.6-$ & NA \\
\hline \multicolumn{5}{|l|}{ Home protection behaviors } \\
\hline Larvicide application & $8.0(4.8-13.3)$ & $0.8(0.6-1.1)$ & $2.7(1.4$ & $0.4(0.3-0.5)$ \\
\hline Spraying home or yard for mosquitoes & $1.5(1.1-2.3)$ & $1.0(0.7-1.4)$ & $1.6(0.9$ & $8.5-20.3)$ \\
\hline Removing or covering standing water & $2.2(0.8-5.7)$ & $2.7(1.1-6.5)$ & 0.5( & $1.1(0.4-2.9)$ \\
\hline Installing window or door screens & $0.8(0.6-1.2)$ & $0.8(0.6,1.2)$ & $0.7(0.4-1.5)$ & $0.9(0.6-1.5)$ \\
\hline \multicolumn{5}{|c|}{$\begin{array}{l}\text { *Bold indicates significant result. WIC, Women, Infants, and Children Program (US Department of Agriculture Food and Nutrition Service); ZPK, Zika } \\
\text { prevention kit. } \\
\text { †Models for WIC orientation and offer of free residential spraying were modeled separately, whereas ZPK distribution and Detén el Zika recall were } \\
\text { modeled together to measure independent effects. Thus, each Zika prevention behavior had } 3 \text { models. To reduce possible bias associated with sparse } \\
\text { data, calendar month of interview was consolidated into a 3-level, 3-month variable. All } 5 \text { demographic variables and consolidated calendar month of } \\
\text { interview were controlled for in each model, except for the following cases: 1) WIC orientation did not include any calendar month of interview variable; or } \\
\text { 2) very few respondents did not receive WIC orientation, thus the naturally occurring control group was very small. To not bias the models, no time of } \\
\text { interview variable was included in models of WIC orientation. Education was excluded from bed net, larvicide, and repellent use models. Because of the } \\
\text { substantial amount of missing data for education, additional testing was performed to determine whether women with missing education data performed } \\
\text { the } 10 \text { behaviors with significantly higher or lower frequency. Three behaviors (repellent, bed net, and larvicide use) were significantly associated with } \\
\text { whether education data were missing, so education was not included in these models. No calendar month or consolidated month variable was used for } \\
\text { any of the larvicide use models because of small cell sizes. } \\
\ddagger \text { Among those reporting having had sex during pregnancy. }\end{array}$} \\
\hline
\end{tabular}

for mosquitoes, enabling women to overcome both cost and logistical barriers. Although efficacy concerns led to discontinuation of the spraying program, the offer had a strong association with spraying behavior, a finding consistent high percentage $(81 \%)$ of respondents who rated the offer of insecticide spraying to pregnant women as very important.

The Detén el Zika campaign had the greatest independent effect on removing standing water, significant effects for repellent use, and modest (marginally significant) effects for condom use, whereas the WIC orientation appeared to have a slightly greater effect on condom use. Although WIC Zika orientation did not yield the same large number of positive associations in regression models as was observed in the bivariate analyses, its highly successful implementation left it with a very small natural control group, which might have limited the utility of modeling for this intervention.

As we consider the public health implications of these results, we should note that in the context of cross-sectional data with outcomes that are not rare, ORs do not equate to relative risk. Thus, we cannot say that women receiving the free offer of home mosquito spraying were 13 times more likely to spray their homes. Unfortunately, estimating relative risks from ORs is not straightforward. Simple conversion formulas (26) have been shown to be imprecise (27), but such conversions can provide at least a rough sense of the extent to which relative risk is more modest than odds with nonrare outcomes (28). For example, the ORs of 8.0 (ZPK exposure and larvicide application), 13.1 (offer of free residential spraying and spray- ing home for mosquitoes), and 3.1 (ZPK exposure and bed net use) roughly, convert to risk ratios of 5.2, 3.5, and 2.7, respectively, whereas the more modest ORs of 2.7 (Detén el Zika campaign exposure and removing standing water and WIC orientation and larvicide application) and 2.4 (WIC orientation and condom use) undergo a smaller adjustment (1.1, 2.2, and 1.7, respectively). Further research is needed to evaluate these associations more precisely.

In our exploration of intervention mechanisms, the 2 interpersonal communication variables showed stronger association with the interventions and to the Zika prevention behaviors than did the individual variables (Zika concern, perceived likelihood of infection, and self-confidence). This finding suggests that the interpersonal factors were more influential on behavior than individual risk perceptions. Interpersonal communication has long been recognized as an important mediator of the effects of educational campaigns on health-related behavior change (29-33), and our results confirm this assertion.

The main challenge of this analysis was that the data were collected during an emergency response for nonresearch purposes, meaning that much of the analysis design had to be created after the fact, particularly the creation of Zika behavior outcome variables. Further, this analysis did not use an optimal research design (i.e., there were no pre-post groups or predesignated control groups). The resulting imbalances in naturally occurring control groups prevented the use of a single logistic model for all 4 interventions. However, the use of random sampling from a frame representing $87 \%$ of the island's pregnant women 
and logistic regression modeling to control confounding by demographic factors provide a credible first look at possible effects of Zika prevention interventions during an epidemic response.

Among the 4 intervention strategies, ZPK distribution appears to have significant independent effects on the greatest number of Zika prevention behaviors. Consistent with the literature, this intervention should be considered a best practice for behavioral support in infectious disease outbreaks, particularly in low-resource settings. Social context factors appeared to be more influential in Zika prevention behavior than personal risk assessment and self-efficacy factors, whereas Zika prevention behaviors that enable women to try out lesserknown behaviors appeared to garner greater acceptance than other behaviors. Areas for future research include developing the evidence base for Zika prevention behavior effectiveness and more precise quantification of intervention mechanisms and effects.

\section{Acknowledgments}

We thank the pregnant women across Puerto Rico who gave their time and experience to participate in the interviews; the Puerto Rico Department of Health Women, Infants, and Children (WIC) Program leaders and staff; Brenda RiveraGarcia, Carla Espinet Crespo, Tyler Sharp, Steve Waterman, Katherine Lyon Daniel, Amy McMillen, John O’Connor, Carmen Perez, Nicki Pesik, Lyle Petersen, Lee Samuel, Eunice Soto, Laura Youngblood, Jeffrey Zirger, Mahmoud K. Aboukheir, Consuelo Abriles, Jorge Carlo, Pollyanna R. Chavez, Alexander Cruz-Benitez, Gabriela Escutia, Roberta Lugo, Gisela Medina, Brian D. Montalvo-Martínez, Carlos G. Grana-Morales, Rosalyn Plotzker, Clarissa Valdez, Santos Villarán, and Max Zarate-Bermudez.

\section{About the Author}

Dr. Earle-Richardson is an epidemiologist and behavioral scientist with CDC's National Center for Emerging and Zoonotic Infectious Diseases. Her research interests include the relationship between human behavior and health, the role of behavioral science in emergency response, and how culture affects health.

\section{References}

1. Rasmussen SA, Jamieson DJ, Honein MA, Petersen LR. Zika virus and birth defects - reviewing the evidence for causality. N Engl J Med. 2016;374:1981-7. http://dx.doi.org/10.1056/NEJMsr1604338

2. Thomas DL, Sharp TM, Torres J, Armstrong PA, Munoz-Jordan J, Ryff KR, et al. Local transmission of Zika virus-Puerto Rico, November 23, 2015-January 28, 2016. MMWR Morb Mortal Wkly Rep. 2016;65:154-8. http://dx.doi.org/10.15585/mmwr.mm6506e2

3. Petersen LR, Jamieson DJ, Powers AM, Honein MA. Zika Virus. N Engl J Med. 2016;374:1552-63. http://dx.doi.org/10.1056/ NEJMra1602113
4. Michie S, Atkins L, West R. The behaviour change wheel. A guide to designing interventions. London: Silverback Publishing; 2014.

5. Jones KE, Yan Y, Colditz GA, Herrick CJ. Prenatal counseling on type 2 diabetes risk, exercise, and nutrition affects the likelihood of postpartum diabetes screening after gestational diabetes. J Perinatol. 2018;38:315-23. http://dx.doi.org/10.1038/s41372-017-0035-1

6. Whitlock EP, Orleans CT, Pender N, Allan J. Evaluating primary care behavioral counseling interventions: an evidence-based approach. Am J Prev Med. 2002;22:267-84. http://dx.doi.org/ 10.1016/S0749-3797(02)00415-4

7. Prue CE, Roth JN Jr, Garcia-Williams A, Yoos A, Camperlengo L, DeWilde L, et al. Awareness, beliefs, and actions concerning Zika virus among pregnant women and community members-U.S. Virgin Islands, November-December 2016. MMWR Morb Mortal Wkly Rep. 2017;66:909-13. http://dx.doi.org/10.15585/ mmwr.mm6634a4

8. Samaritan's Purse. Samaritan's Purse launches bold new initiative to combat Ebola [cited 2018 Feb 28]. https://www.samaritanspurse. org/our-ministry/samaritans-purse-launches-bold-new-initiative-tocombat-ebola-10-07-14-press-release

9. Sifferlin A. This is how Ebola Patients are equipping their homes [cited $2018 \mathrm{Feb}$ 9]. http://time.com/3481394/equipping-homes-totreat-ebola-patients

10. Meltzer MI, Atkins CY, Santibanez S, Knust B, Petersen BW, Ervin ED, et al. Estimating the future number of cases in the Ebola epidemic-Liberia and Sierra Leone, 2014-2015. MMWR Suppl 2014;63:1-14.

11. Colindres P, Mermin J, Ezati E, Kambabazi S, Buyungo P, Sekabembe L, et al. Utilization of a basic care and prevention package by HIV-infected persons in Uganda. AIDS Care. 2008;20:139-45. http://dx.doi.org/10.1080/09540120701506804

12. Mabude ZA, Beksinska ME, Ramkissoon A, Wood S, Folsom M. A national survey of home-based care kits for palliative HIV/AIDS care in South Africa. AIDS Care. 2008;20:931-7. http://dx.doi.org/ 10.1080/09540120701768438

13. International RTI. Detén el Zika/Stop Zika Campaign, a comprehensive education campaign to fight the Zika epidemic in Puerto Rico [cited 2018 Feb 9]. https://www.rti.org/impact/ DeténDetén-el-zika-stop-zika-campaign

14. Wakefield MA, Loken B, Hornik RC. Use of mass media campaigns to change health behaviour. Lancet. 2010;376:1261-71. http://dx.doi.org/10.1016/S0140-6736(10)60809-4

15. Boene H, González R, Valá A, Rupérez M, Velasco C, Machevo S, et al. Perceptions of malaria in pregnancy and acceptability of preventive interventions among Mozambican pregnant women: implications for effectiveness of malaria control in pregnancy. PLoS One. 2014;9:e86038. http://dx.doi.org/10.1371/ journal.pone.0086038

16. Piltch-Loeb R, Abramson DM, Merdjanoff AA. Risk salience of a novel virus: US population risk perception, knowledge, and receptivity to public health interventions regarding the Zika virus prior to local transmission. PLoS One. 2017;12:e0188666. http://dx.doi.org/10.1371/journal.pone.0188666

17. Jambulingam P, Gunasekaran K, Sahu S, Vijayakumar T. Insecticide treated mosquito nets for malaria control in Indiaexperience from a tribal area on operational feasibility and uptake. Mem Inst Oswaldo Cruz. 2008;103:165-71. http://dx.doi.org/10.1590/S0074-02762008005000009

18. Adams L, Bello-Pagan M, Lozier M, Ryff KR, Espinet C, Torres J, et al. Update: ongoing Zika virus transmission-Puerto Rico, November 1, 2015-July 7, 2016. MMWR Morb Mortal Wkly Rep. 2016;65:774-9. http://dx.doi.org/10.15585/ mmwr.mm6530e1

19. BusinessWire. The Puerto Rico Department of health has declared that the 2016 Zika epidemic is over; Zika transmission has substantially decreased in Puerto Rico below epidemic levels [cited 2018 Feb 28]. 
https://www.businesswire.com/news/home/20170605006235/en/ Puerto-Rico-Department-Health-Declared-2016-Zika

20. Greenland S, Mansournia MA, Altman DG. Sparse data bias: a problem hiding in plain sight. BMJ. 2016;352:i1981. http://dx.doi.org/10.1136/bmj.i1981

21. Centers for Disease Control and Prevention National Center for Health Statistics. US Territories, 2016 natality public use file [cited $2018 \mathrm{Feb} 8$ ]. https://www.cdc.gov/nchs/data_access/ vitalstatsonline.htm

22. D'Angelo DV, Salvesen von Essen B, Lamias MJ, Shulman H, Hernandez-Virella WI, Taraporewalla AJ, et al. Measures taken to prevent Zika virus infection during pregnancy Puerto Rico, 2016. MMWR Morb Mortal Wkly Rep. 2017;66:5748. http://dx.doi.org/10.15585/mmwr.mm6622a2

23. Robinson MN, Tansil KA, Elder RW, Soler RE, Labre MP, Mercer SL, et al. Mass media health communication campaigns combined with health-related product distribution: a community guide systematic review. Am J Prev Med. 2014;47:360-71. http://dx.doi.org/10.1016/j.amepre.2014.05.034

24. Noar SM. A 10-year retrospective of research in health mass media campaigns: where do we go from here? J Health Commun. 2006;11:21-42. http://dx.doi.org/10.1080/10810730500461059

25. Rogers EM. Diffusion of innovations. Fifth edition. New York: Free Press; 2003.

26. Zhang J, Yu KF. What's the relative risk? A method of correcting the odds ratio in cohort studies of common outcomes. JAMA. 1998;280:1690-1. http://dx.doi.org/10.1001/ jama. 280.19.1690
27. McNutt LA, Hafner JP, Xue X. Correcting the odds ratio in cohort studies of common outcomes. JAMA. 1999;282:529. http://dx.doi.org/10.1001/jama.282.6.529

28. McNutt LA, Wu C, Xue X, Hafner JP. Estimating the relative risk in cohort studies and clinical trials of common outcomes. Am J Epidemiol. 2003;157:940-3. http://dx.doi.org/10.1093/aje/kwg074

29. Rogers EM. A prospective and retrospective look at the diffusion model. J Health Commun. 2004;9(Suppl 1):13-9. http://dx.doi.org/10.1080/10810730490271449

30. Bandura A. Health promotion by social cognitive means. Health Educ Behav. 2004;31:143-64. http://dx.doi.org/ 10.1177/ 1090198104263660

31. Jeong M, Bae RE. The effect of campaign-generated interpersonal communication on campaign-targeted health outcomes: a meta-analysis. Health Commun. 2018;33:988-1003. http://dx.doi.org/10.1080/10410236.2017.1331184

32. Gainforth HL, Latimer-Cheung AE, Athanasopoulos P, Moore S, Ginis KA. The role of interpersonal communication in the process of knowledge mobilization within a community-based organization: a network analysis. Implement Sci. 2014;9:59. http://dx.doi.org/10.1186/1748-5908-9-59

33. Katz E, Lazarsfeld P, Roper E. Personal influence. New York: Routledge; 2005.

Address for correspondence: Giulia Earle-Richardson, Centers for Disease Control and Prevention, 1600 Clifton Rd NE, Mailstop H16-5, Atlanta, GA 30329-4027, USA; email: evy8@cdc.gov
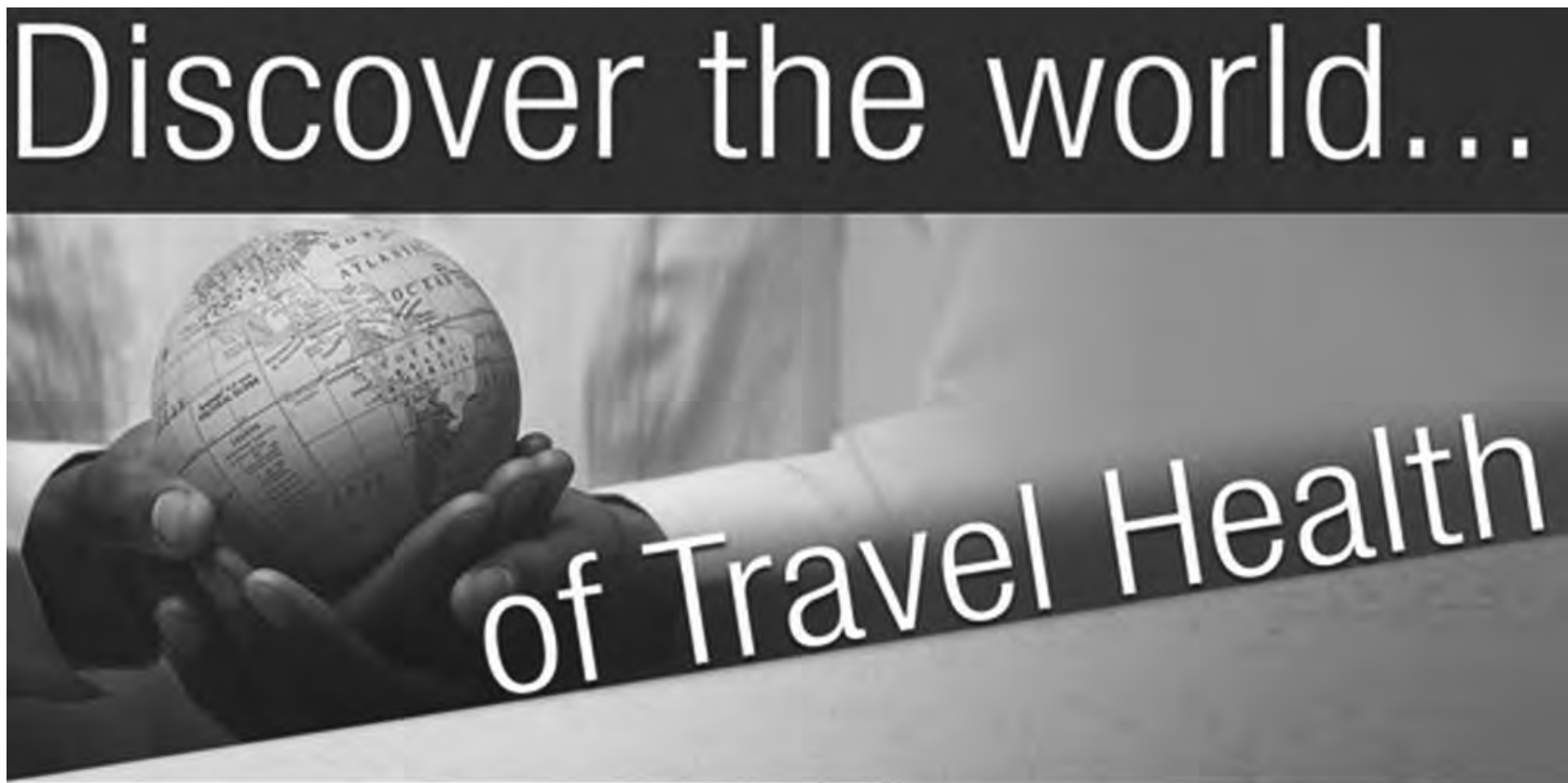

\section{www.cdc.gov/travel}

Visit the CDC Travelers' Health website for up-to-date information on global disease activity and international travel health recommendations.

\section{Deapartment of Health and Human Services Centers for Disease Control and Prevention}




\title{
Emerging Multidrug-Resistant Hybrid Pathotype Shiga Toxin-Producing Escherichia coli 080 and Related Strains of Clonal Complex 165, Europe
}

\author{
Aurélie Cointe, André Birgy, Patricia Mariani-Kurkdjian, Sandrine Liguori, Céline Courroux, \\ Jorge Blanco, Sabine Delannoy, Patrick Fach, Estelle Loukiadis, Philippe Bidet, Stéphane Bonacorsi
}

Enterohemorrhagic Escherichia coli serogroup O80, involved in hemolytic uremic syndrome associated with extraintestinal infections, has emerged in France. We obtained circularized sequences of the 080 strain RDEx444, responsible for hemolytic uremic syndrome with bacteremia, and noncircularized sequences of $35080 \mathrm{E}$. coli isolated from humans and animals in Europe with or without Shiga toxin genes. RDEx444 harbored a mosaic plasmid, pR444_A, combining extraintestinal virulence determinants and a multidrug resistanceencoding island. All strains belonged to clonal complex 165, which is distantly related to other major enterohemorrhagic $E$. coli lineages. All stx-positive strains contained eae- $\xi$, ehxA, and genes characteristic of pR444_A. Among stx-negative strains, 1 produced extended-spectrum $\beta$-lactamase, 1 harbored the colistin-resistance gene $m c r 1$, and 2 possessed genes characteristic of enteropathogenic and pyelonephritis E. coli. Because O80-clonal complex 165 strains can integrate intestinal and extraintestinal virulence factors in combination with diverse drug-resistance genes, they constitute dangerous and versatile multidrug-resistant pathogens.

— nterohemorrhagic Escherichia coli (EHEC), a subset of $\checkmark$ Shiga toxin-producing E. coli (STEC), are major foodborne pathogens responsible for outbreaks and sporadic cases of gastrointestinal diseases ranging from simple diarrhea to hemorrhagic colitis, characterized by bloody diarrhea. The

Author affiliations: Hôpital Robert-Debré (AP-HP), Paris, France (A. Cointe, A. Birgy, P. Mariani-Kurkdjian, S. Liguori, C. Courroux, P. Bidet, S. Bonacorsi); Université Paris Diderot, Sorbonne Paris Cité, Paris (A. Cointe, A. Birgy, P. Mariani-Kurkdjian, P. Bidet, S. Bonacorsi); Universidade de Santiago de Compostela, Lugo, Spain (J. Blanco); ANSES, Plateforme IdentyPath, Maisons-Alfort, France (S. Delannoy, P. Fach); Université de Lyon, CNRS, INRA, UCBL, VetAgro Sup, Laboratoire d'Écologie Microbienne, Villeurbanne, France (E. Loukiadis)

DOI: https://doi.org/10.3201/eid2412.180272 most serious complication, particularly in young children, is hemolytic uremic syndrome (HUS), defined by a combination of renal failure, thrombocytopenia, and hemolytic anemia (1). Post-STEC HUS is a major worldwide public health concern because it is the primary cause of acute renal failure in children (1). These clinical features result mainly from the action of the phage-encoded Shiga toxin (Stx), of which there are 2 types: Stx1, which has 3 subtypes, Stx1a, 1c, and 1d; and Stx2, which has 7 subtypes, Stx2a-g. In typical EHEC, adhesion to the intestinal epithelium is mediated by the locus of enterocyte effacement (LEE), a chromosomal pathogenicity island (PAI), shared with Enteropathogenic E. coli (EPEC) strains, which encodes a type III secretion system (T3SS), an adhesin called intimin, and its receptor Tir. Intimin, encoded by the eae gene, is a major virulence factor (VF) involved in the intimate attachment of typical EHEC to intestinal epithelium, causing characteristic attaching and effacing lesions. EHEC enterohemolysin $(e h x A)$ is a pore-forming cytolysin carried by a plasmid involved in EHEC virulence. This plasmid, initially described as part of the 0157 serogroup (pO157) (2), can carry 2 additional VFs, a catalase peroxidase, encoded by katP, and a serine protease, encoded by esp $P$, which can cleave human coagulation factor $\mathrm{V}$ and might be involved in the development of hemorrhagic colitis (3).

Serogroup O157 is the predominant STEC serogroup worldwide, but non-O157 serogroups are increasingly associated with post-STEC HUS, and the unusual serogroup O80 is emerging in France and Europe. In 2016, O80 represented the second most frequent serogroup isolated in France, after serogroup O26 (4). This phenomenon is no longer restricted to France; strains of serotype O80:H2, all belonging to sequence type (ST) 301 , have been identified in Spain (5), the Netherlands (6), and Switzerland (7).

This serogroup is unique for several reasons. First, it is always associated with multiple determinants of resistance 
(i.e., resistance to aminopenicillin, aminoglycoside, nalidixic acid, cotrimoxazole, tetracycline, or phenicols), whereas a resistance phenotype is uncommon among EHECs, which are generally fully susceptible to antibiotics, except for rare clones, such as the epidemic O104:H4 German clone carrying a bla $a_{\mathrm{CTX}-\mathrm{M}-15}$ gene (8). Furthermore, unusual extraintestinal infections have recently been described for this serogroup $(9,10)$, such as bacteremia, whereas EHEC is generally known to be a strictly intestinal pathogen. A recent case in the Netherlands illustrates the potential extreme pathogenicity of this serogroup; a 16-month-old boy died from multiorgan failure and extensive cerebral thrombotic microangiopathy attributable to an O80 Stx2d-producing E. coli strain (6). Finally, O80 EHEC appears to be a hybrid pathotype that combines intestinal VFs (Shiga toxin [stx], intimin [eae], enterohemolysin [ehxA]) and extraintestinal VFs (aerobactin [iucC]; salmochelin [iroN], an iron uptake protein encoded by sitABCD; serum resistance protein [issp]; a putative secretion system I [etsC]; omptin [ompTp]; hemolysin [hlyF], and 2 bacteriocins [cia and $c v a]$ ), suggesting the presence of a pS88-like plasmid (11). pS88 is a ColV plasmid, a key determinant of extraintestinal pathogenic E. coli virulence in poultry and humans. This plasmid is involved in neonatal meningitis (11) and could explain the occurrence of extraintestinal dissemination in these EHEC infections. The recent diffusion in Europe, high potential extraintestinal pathogenicity, and multidrug resistance (MDR) of this hybrid pathotype led us to further characterize these strains, which might represent a major public health concern.

\section{Methods}

We further characterized O80:H2 EHEC by fully sequencing a recent representative strain, called RDEx444. RDEx444 was responsible for a highly severe case of post-STEC HUS, complicated by bacteremia, in an 8-month-old infant in February 2016 in Bourg-en-Bresse, France. Initial symptoms were febrile diarrhea with signs of sepsis. Blood and stool cultures were positive for O80 EHEC, but urine cultures remained negative, suggesting gut translocation that led to bacteremia. The appearance of biologic signs of HUS with oliguria necessitated transfer to intensive care, several blood transfusions, and 5 days of peritoneal dialysis.

We performed sequencing of RDEx444 by using the PacBio single-molecule real-time method with RS II chemistry 2.4.0 (Pacific Biosciences, Menlo Park, CA, USA); we used 1 single-molecule real-time cell. De novo assembly was performed twice by using the HGAP pipeline (https://github.com/PacificBiosciences/BioinformaticsTraining/wiki/HGAP) and annotation by using the MAGE platform (http://www.genoscope.cns.fr/agc/microscope/ home/index.php) (Figure 1). For this strain, we performed plasmid typing by using databases (PlasmidFinder 1.3 and

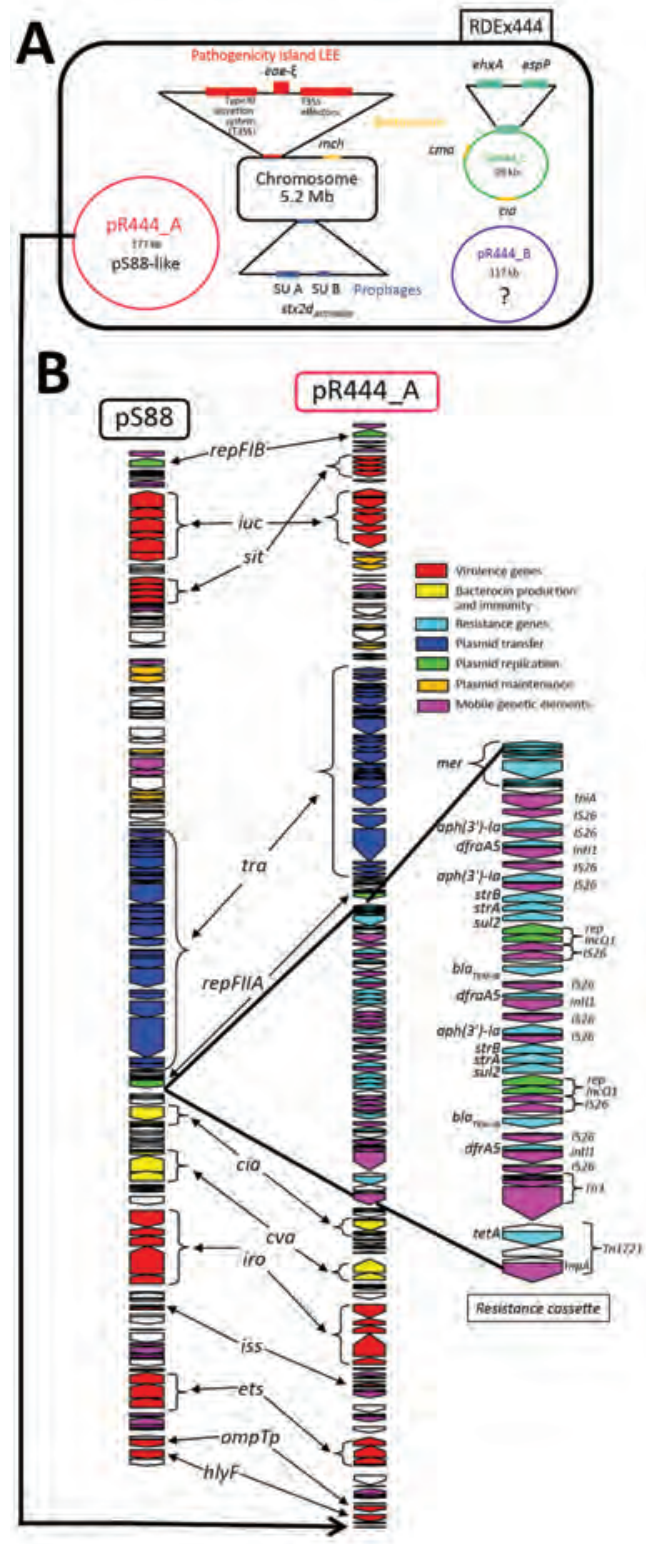

Figure 1. Genetic characterization of RDEx444, a strain of Escherichia coli serotype 080: $\mathrm{H} 2$ isolated in France in February 2016 and involved in hemolytic uremic syndrome with bacteremia, carrying both intestinal and extraintestinal virulence factors associated with (multidrug-resistance determinants (A) and genetic comparison between plasmid pS88 and mosaic plasmid pR444_A (B). A) Four circularized contigs (chromosome of 5,256,050 bp and the 3 plasmids pR444_A [176,500 bp], pR444_B [117,090 bp], and pR444_C [95,050 bp]) obtained by using PacBio (Pacific Biosciences, Meno Park, CA, USA) sequencing of RDEx444 are schematically represented. The main virulence factors are presented as colored rectangles. Intestinal virulence factors are indicated in red for the locus of enterocyte effacement genes, blue for prophage-encoded Shiga toxin genes, and green for VFs carried by pR444_C, a pO157-like plasmid. Bacteriocin genes ( $\mathrm{mch}$, cia, and $\mathrm{cma}$ ) are indicated in yellow. B) Comparison of the sequences of pR444_A and pS88, the plasmid of strain S88 involved in neonatal meningitis. LEE, locus of enterocyte effacement. 
pMLST 1.4 [12]) available on the Center for Genomic Epidemiology (CGE) website (http://www.genomicepidemiology.org). Plasmids were also characterized by S1 nuclease pulsed-field gel electrophoresis (PFGE) with Southern hybridization and conjugability of the largest confirmed by experiments using rifampin-resistant E. coli J53, as previously described (9).

We also sequenced a representative set of 35080 strains from various sources and countries using the Nextera kit (Illumina, San Diego, California, USA) to prepare the library. Sequencing was performed by using a MiSeq reagent kit V3 600 cycles (Illumina). This panel included human isolates from France $(\mathrm{n}=21)$, Spain $(\mathrm{n}=3)$, and Switzerland $(\mathrm{n}=2)$, as well as animal and environmental strains isolated in France $(n=3)$, Spain $(n=1)$, Slovakia ( $=1)$, and Germany $(n=4)$ (Figure 2). Some of the human strains from France and strains from Spain and Slovakia have been partially characterized previously (10). The strains from Spain and Slovakia were isolated during 1998-2012 and the strains from France during 2010-2016. The dates of isolation of the strains from Switzerland and Germany were not available. We performed assembly by using CLC Genomics Workbench software and SPAdes, also available on CGE website. The RAST server (http:// rast.nmpdr.org) was used for genome annotation and the PHASTER web server (http://phaster.ca) to identify and annotate prophage sequences within bacterial genomes and plasmids. We established phylogeny by single-nucleotide polymorphism alignments between the contigs generated by CLC Genomics of O80 strains and 9 reference EHEC strain sequences of major serotypes available in GenBank (O157:H7 EDL933, O26:H11 11368, O111:H- 11128,

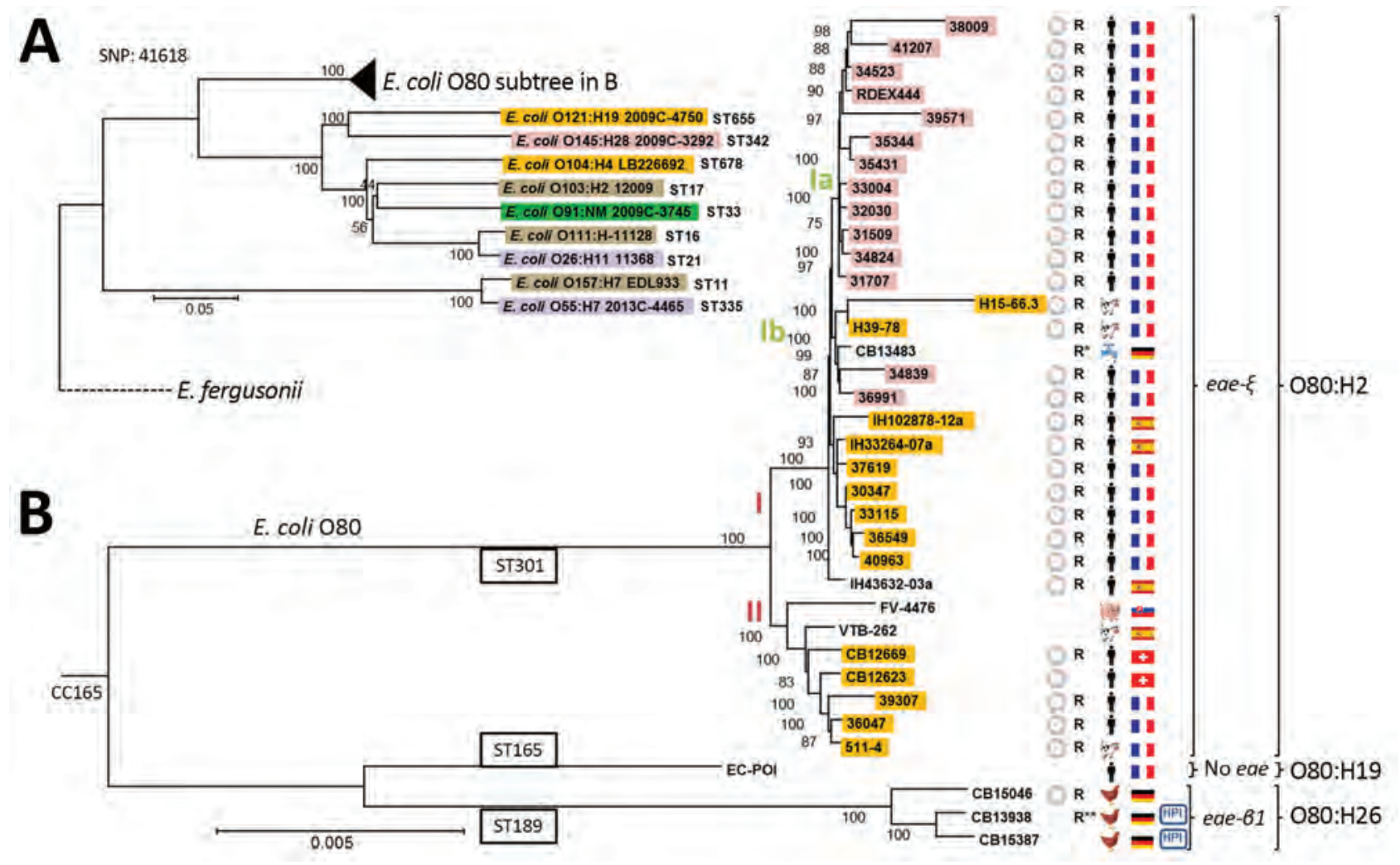

Figure 2. Phylogeny of 36 Escherichia coli serogroup 080 strains isolated from various sources and countries in Europe during 19982016 and their relationship to major enterohemorrhagic $E$. coli lineages. General phylogenic tree rooted on E. fergusonii (GenBank accession no. NC_011740), showing (A) the position of O80 strains among major enterohemorrhagic E. coli lineages (O157:H7 EDL933 NC_002655.2, O26:H11 11368 NC_013361.1, O111:H- 11128 NC_013364.1, O103:H2 12009 NC_013353.1, O55:H7 2013C-4465 CP015241, O91:NM 2009C-3745 JHGW00000000, O104:H4 LB226692 EO104H4LB.1, O145:H28 2009C-3292 JHHD00000000, and O121:H19 2009C-4750 JHGL00000000) and (B) a focused view of clonal complex 165, including O80 strains. The highlighted strains carry the Shiga toxin genes; the subtype of Stx is indicated by a color code as follows: purple, stx1a; yellow, stx2a; pink, stx2d $d_{\text {activable; }}$ brown, stx1a2a; dark green, stx1a2b. The presence of the pS88 like-plasmid is represented by using a plasmid scheme next to the strain number. $\mathrm{R}$ to the right of the plasmid indicates that the strain possesses $\geq 2$ resistance genes that confer resistance to $\beta$-lactams, kanamycin, or cotrimoxazole $\left(R^{*}\right.$ indicates presence of additional extended-spectrum $\beta$-lactamase gene; $R^{\star *}$ indicates presence of $m c r-1$ gene conferring additional resistance to colistin). The strain origin (country and source of isolation) is represented by flags and human, animal, or water symbols. CC, clonal complex; HPI, high-pathogenicity island (presence of chromosomal locus encoding the siderophore yersiniabactin); SNP, single-nucleotide polymorphism; ST, sequence type. Scale bar indicates nucleotide substitutions per site. 
O103:H2 12009, O55:H7 2013C-4465, O91:NM 2009C3745, O104:H4 LB226692, O145:H28 2009C-3292, and O121:H19 2009C-4750 [online Technical Appendix, https://wwwnc.cdc.gov/EID/article/24/12/18-0272Techapp1.pdf]) using CSIPhylogeny 1.4 (13). The neighbor-joining tree was built by using MEGA 3.1 (https:// www.megasoftware.net) using bootstraps calculated from 100 replicates. The maximum-likelihood method yielded the same results (online Technical Appendix Figure 1).

We performed multilocus sequence typing and identification of acquired antimicrobial resistance genes for all strains by using suitable databases available on the CGE website (SerotypeFinder 1.1 [14], MLST 1.8 [15], and ResFinder 3.0 [16]). Investigation of the resistome was completed by using the Resistance Gene Identifier at the Comprehensive Antibiotic Resistance Database website (https://card.mcmaster.ca/analyze/rgi). Moreover, 166 genes of intestinal and extraintestinal VFs (list available from authors) were assessed by local BLAST+ 2.2.31 analysis (https://blast.ncbi.nlm.nih.gov). Complete results are described in online Technical Appendix Figure 2. Nucleotide sequences of the 36 sequenced 080 strains (complete nucleotide sequence of RDEx444 and 35 draft nucleotide sequences) have been deposited in Genbank (project no. PRJNA449634); accession numbers are available in the online Technical Appendix.

\section{Results}

RDEx444 can be considered to be representative of hybrid pathotype strains because it was responsible for an extraintestinal infection and showed similar virulence and resistance profiles as other strains described elsewhere $(9,10)$. Complete sequencing yielded $2.3 \times 10^{9}$ bp with 165,041 reads and an N50 (the length of the smallest contig among the set of the largest contigs that together cover $>50 \%$ of the assembly) read length of 19,839 bp.

Four circularized contigs were obtained, including the chromosome of 5,256,050 bp, containing 5,146 coding sequences (CDSs), with an overall $\mathrm{G}+\mathrm{C}$ content of $50.7 \%$ and 3 plasmids of 176,500 (pR444_A), 117,090 (pR444_B), and 95,050 bp (pR444_C). The number and size of the RDEx444 plasmids were corroborated by S1 nuclease PFGE (data not shown). We compiled a schematic representation of the genetic content, including major VFs, of this strain (Figure 1, panel A).

As expected, RDEx444 belongs to serotype O80:H2 and ST301. Among the 12 prophage regions identified on its chromosome, this strain carries a complete Stx-converting bacteriophage of $43.9 \mathrm{~kb}$. RDEx444 carries the $s t \times 2 d_{a c-}$ tivable variant, which has been shown to be predictive of severe clinical outcomes and progression to HUS (17). The Stx prophage is integrated into the chromosomal yecE gene, a known integration site $(18,19)$, initially reported in a strain producing Stx2e, encoded by the phage $\varphi \mathrm{P} 27$ (20). The $\approx 15$ $\mathrm{kb}$ region between Stx subunit B and the phage integrase, comprising proteins for DNA replication and repression, shares strong homology with the phage $\varphi \mathrm{P} 27$. RDEx444 is otherwise a typical EHEC, given that it harbors a complete LEE and does not possess atypical adhesins, such as autoagglutinating adhesion (saa) or aggregative factors ( $a g g A / R)$. RDEx444 carries the rare variant of the intimin gene eae- $\xi$ (5), which has only very rarely been observed among other EHEC serotypes, but which is shared by all previously described O80 EHEC and EPEC isolates $(5-7,9,10)$. Two other chromosomal traits of RDEx444 might also confer a selective advantage: 1) a region encoding microcin $\mathrm{H} 47$ ( $m c h A, S, X, B, C, D, E$, and $F$ ) $\approx 35 \mathrm{~kb}$ downstream from the Stx prophage, and 2) a chromosomal mutation in DNA gyrase (gyrA S83L), which confers quinolone resistance.

Plasmid pR444_C is 95,050 bp long with a G+C content of $49.8 \%$ and was predicted to contain 114 CDSs. Plasmid pR444_C is comparable to pO157 because it contains enterohemolysin $(e h x A)$ and serine protease $(e s p P)$ but does not possess catalase peroxidase (katP). Moreover, 2

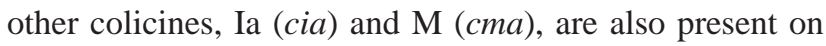
this plasmid.

Plasmid pR444_B is 117,090 bp long with a G+C content of $46.5 \%$ and contains $135 \mathrm{CDSs}$ and, unexpectedly, those for 3 tRNAs (threonine, asparagine, and tyrosine) redundant with those still present on the chromosome. This plasmid can be considered to be cryptic because it carries no drug resistance or virulence-associated genes, but several phage proteins are present. pR444_B shares high homology (99\% nucleotide identity and $89 \%$ coverage) with plasmid pECOH89 (21), encoding an extended-spectrum $\beta$-lactamase bla $a_{\text {Стх-м-15 }}$ identified in an E. coli strain and belonging to the family of phage-like plasmids. Members of this family are generally untypeable, nonconjugative, and cryptic plasmids, because no known virulence or resistance genes have been identified. Their function is unknown, but they all have strong homology to the Salmonella-specific bacteriophage SSU5 (21).

Plasmid pR444_A is 176,500 bp long, with a G+C content of 51.8\%, and carries 2 replicons: FII_A and FIB_1 (ST [F2:A-:B1]). We identified 202 CDSs. This plasmid carries virulence-associated genes characteristic of pS88 (described previously) and MDR genes, and thus can be considered to be a mosaic plasmid. We identified an MDRencoding region $(48,237 \mathrm{bp})$ in addition to the plasmid-related function (56,106 bp) and virulence-associated domain ( $72,157 \mathrm{bp)}$, closely related to pS88 (99\% nucleotide identity and $96 \%$ query coverage) (11). This MDR-encoding region contains genes encoding resistance tetracycline (tetA), trimethoprim ( $d f \mathrm{f} A 5$ ), sulfonamide (sul2), $\beta$-lactam (blaTEM-IB), kanamycin (aph[3']-Ia), and streptomycin (strA and $s t r B$ ) and resistance against heavy metals such as mercury 
(mer gene) (Figure 1, panel B). This resistance cassette has only been described for pO26-CRL ${ }_{125}$ (100\% nucleotide identity, 98\% coverage) from an O26 EHEC but without VFs (22). Co-localization of extraintestinal VFs and MDR genes on the same plasmid was confirmed by Southern hybridization (data not shown). Moreover, we confirmed that this plasmid is conjugative, suggested by the presence on the annotation of an almost complete F-like transfer region, by successfully conjugating it with rifampin-resistant $E$. coli J53 (data not shown). Thus, this plasmid can diffuse by horizontal transfer.

Accordingly, this mosaic plasmid shows high homology with plasmid pS88, responsible for extraintestinal virulence in neonatal meningitis strains within which a resistance cassette has been integrated (Figure 1, panel B). Furthermore, pR444_A also carries 2 bacteriocin genes (cia and $c v a$ ), such as pS88, which might confer a selective advantage by promoting intestinal establishment and colonization by killing other E. coli and freeing up their ecologic niches (Figure 1, panel B).

We sequenced a panel of strains consisting of representative O80 strains from France and all O80 strains from outside of France available at the beginning of the study to establish the genetic relationship between O80 strains isolated in several countries in Europe and analyze the diversity of their genetic content. We obtained an average of 233 contigs, with a mean depth of coverage of $58 \mathrm{X}$ and a mean N50 of 72,312. Statistics of each sequenced genome are summarized in online Technical Appendix Table 1. Singlenucleotide polymorphism analysis (41,618 sites total) between the 36 sequenced 080 strains and 9 EHEC strains of other major serotypes (O157:H7, O26:H11, O111:H-, O103:H2， O55:H7， O91:NM， O104:H4， O145:H28, O121:H19) enabled us to establish a phylogenic tree (Figure 2), which shows 2 main clusters. EHEC O157:H7 (EDL933) and O55:H7 (2013C-4465) group together, as expected by their common origin, demonstrated by Feng et al. (23), and are distantly related to the other major EHEC serotypes, including the O80 strains. However, O80 isolates clearly form a separate group, suggesting that O80 emerged independently from the other serogroups. All of the O80 strains belong to clonal complex 165 (CC165), containing ST301, ST165, and ST189 (Figure 2, panel B).

Almost all of the O80 strains (32/36), including RDEx444, belong to the ST301 clonal group of serotype O80:H2. All of these isolates have EHEC markers, including genes encoded by the LEE, containing the rare variant of intimin $e a e-\xi$, ehxA, and stx2a or stx2d genes, except for 4 strains missing the stx gene (CB13483, IH43632-03a, FV-4476, and VTB262) (online Technical Appendix Figure 2). However, the presence of the other EHEC markers in these 4 strains (eae, ehxA/espP, or katP) suggests that they were initially STEC and underwent subsequent elimination of the stx gene. This finding led us to search for a scar of the Stx-converting bacteriophage at the insertion site described in the RDEx444 strain (yecE). We first searched for the contig carrying the yecE gene. Then, we used the Phaster webserver system to detect phage regions, which were finally blasted against RDEx 444 . We found scars of $\approx 31 \mathrm{~kb}$ in the human strain from Spain (IH43632$03 \mathrm{a})$ and scars of $\approx 17 \mathrm{~kb}$ in the porcine strain from Slovakia (FV-4476), similar to the RDEx444 Stx-converting bacteriophage (92\% and 94\% nucleotide identity and $40 \%$ and $56 \%$ coverage, respectively) (data not shown). We found no scars for the other 2 strains, suggesting either complete prophage excision or insertion at another site.

All strains carrying the st $x$ gene (28/36) also possess VFs typical of pS88, and all but 1 (CB12623) also carry genes conferring resistance to $\geq 1$ of penicillin, tetracycline, kanamycin, or cotrimoxazole. The consistent association between extraintestinal VFs typical of pS88 and MDR genes, irrespective of the source or country of isolation, might suggest the presence of a mosaic plasmid, such as in the RDEx444 strain. Although it lacks pS88 markers, the water strain from Germany (CB13483) is nevertheless multidrug resistant and carries an extended spectrum $\beta$-lactamase gene $\left(b l a_{\text {Стх-м-1 }}\right)$, as well as the $m p h A$ gene, which confers resistance to azithromycin, the only antimicrobial drug that can be used for intestinal decontamination of EHEC (24; online Technical Appendix Figure 2).

Among our panel, 3 STEC strains from cattle in France clearly belong to this ST301 clonal group. Cattle might thus represent an animal reservoir for these hybrid pathotype strains, given that the isolates possess exactly the same VFs (eae, genes of T3SS, ehxA, stx, and determinants of the pS88-like plasmid) and resistance genes as human EHECs.

Two clusters (I and II) can be distinguished within the ST301 group we describe. The main difference between these 2 groups concerns the presence of the unknown cryptic plasmid (pR444_B) in RDEx444. All strains of cluster I $(n=25)$ possess $\geq 85 \%$ of the genetic determinants of this plasmid of unknown function. BLAST results are depicted for each strain in online Technical Appendix Table 2. Conversely, no strain of group II $(n=7)$, except 1 , has this plasmid. No strains of ST165 or ST189 carry it either.

Within cluster I, the pS88-like plasmid carries 2 distinct gene profiles, showing its plasticity. The $12 \mathrm{Stx} 2 \mathrm{~d}_{\text {acti- }}$ EHECs isolated from humans in France (designated as subcluster Ia in Figure 2) carry the most complete form of the plasmid, identical to pS88. The aerobactin iron-uptake system, encoded by iuc genes, and the type I secretion system, encoded by ets $C$, are absent from the pS88-like plasmid of the subcluster Ib strains.

The second clonal group, ST165, is formed by 1 strain (EC_POI) of serotype O80:H19, which is devoid of all VF and resistance genes. This isolate might reflect the ancestral 
origin from which serogroup O80 EHEC strains were derived after the acquisition of diverse VFs.

Finally, clonal group ST189 consists of the 3 avian strains of serotype O80:H26 from Germany. None has stx or ehxA genes, but all have a complete LEE with the variant $\beta 1$ of the intimin gene (eae). However, none of these strains carries the plasmid $b f p$ gene of typical EPEC (online Technical Appendix Figure 2). One strain (CB13938) has a region of $\approx 27 \mathrm{~kb}$ at the same insertion site (yecE gene) that shares homology with RDEx444 Stx-converting bacteriophage ( $88 \%$ nucleotide identity, $16 \%$ coverage), suggesting a potential scar of Stx-converting phage. Thus, this strain could have been originally an EHEC which secondarily lost its stx gene. No similar regions were found in the other 2 strains of this group. Otherwise, 1 strain (CB15046) has several VFs, confirming the presence of the pS88-like plasmid with MDR genes. The other 2 strains (CB15387 and CB13938) carry the locus encoding the siderophore yersiniabactin (fyuA), also called the high-pathogenicity island; these 2 strains also have pyelonephritis-associated pili with PapGII adhesin (online Technical Appendix Figure 2). These strains constitute another type of hybrid pathotype with intestinal and extraintestinal VFs. Although none of them has VFs typical of pS88 plasmids, CB13938 is multidrugresistant and carries the recently described $\mathrm{mcr}-1$ plasmid gene (25), conferring resistance to colistin. Blast analysis performed with the contig containing $\mathrm{mcr}-1$ (10.119 bp) shows strong homology (100\% coverage, $99 \%$ identity) with 2 chromosomal insertion sites previously described in strains Mbl323 and Mbl506 (26).

\section{Discussion}

We deciphered the molecular characteristics of O80:H2CC165 EHEC, an emerging hybrid pathotype diffusing throughout Europe. This pathotype is armed to spread by means of a conjugative plasmid combining extraintestinal virulence with resistance to nearly all major classes of antibiotics, improved by the presence of several plasmid and chromosomally encoded bacteriocins, such as colicines I, V, M, and H47. We used the same criteria of MDR as a recent study in England (27) (bla $a_{\mathrm{TEM}-1}$, strAstrB, sul1/sul2/dfrA, and tetA) and showed that $93 \%$ (26/28) of O80 STEC have this genotypic resistance profile, whereas only $5 \%$ of the strains identified within the O157 and O26 serogroups in the study in England had such a profile. MDR observed with this hybrid pathotype might complicate patient care, and the use of antimicrobial drugs during EHEC infections is still a subject of debate (28). However, the occurrence of invasive infections, such as bacteremia during EHEC infections, with this clone warrants antimicrobial treatment for such infections. In a previous study, the observed Stx rate was lower with a combination of azithromycin and ceftriaxone assays relative to basal secretion, and we proposed this association for the treatment of such infections (10).

Such a troubling plasmid has never been identified in human EHEC isolates. The only example of a similar mosaic plasmid was reported for S. enterica serovar Kentucky, in which an AmpC $\beta$-lactamase gene $\left(b l a_{\mathrm{CMY}-2}\right)$ was integrated into a pS88-like plasmid (29). The insertion of an MDR-encoding island in a pS88-like plasmid containing extraintestinal virulence genes is particularly worrisome. Massive and inappropriate use of veterinary antibiotics, such as tetracycline, in food-animal production promotes antimicrobial drug resistance among animals, known to be reservoirs for STEC. This practice can select and favor the spread of such MDR plasmids in human EHECs. Tetracycline still represented $36.5 \%$ of the tonnage of veterinary antibiotic use in 2015 in France (30). In our panel, all the sequenced O80 STEC strains carry the tetA gene, conferring resistance to tetracycline. Thus, large veterinary use of this drug might favor the selection of these hybrid strains and increase their diffusion.

We indicated a potential reservoir of these hybrid pathotype strains when we identified $3 \mathrm{O} 80: \mathrm{H} 2$ strains isolated from cattle that carry the same VFs and resistance genes as human strains. However, the presence of the CC165 strains in chickens suggest that this clonal complex is also adapted to poultry. An initial description of pS88-like plasmids in avian pathogenic $E$. coli strains reinforces this hypothesis (31). Moreover, the environmental survival of this clone in these potential reservoirs might be enhanced because of the resistance to mercury shared by all but 1 strain (36047), all carriers of the pS88-like plasmid, irrespective of their origin. Such resistance to heavy metals has been rarely described in EHEC strains (22).

We also detected an O80:H19-CC165 strain devoid of virulence genes, which might represent the ancestral precursor of CC165, and from which these hybrid pathotype strains might have been derived. This strain could be used for tracing the genetic history of this clone in future studies.

Our genetic description of the emerging hybrid pathotype E. coli $\mathrm{O} 80: \mathrm{H} 2$, associated with O80-related strains, reveals the outstanding capacity of O80-CC165 to acquire the combination of virulence genes involved in intestinal and extraintestinal pathogenicity and genes conferring broad antibiotic resistance, including extended-spectrum $\beta$-lactamase-encoding genes and those most recently identified, such as mcr-1. O80-CC165 strains, which are able to integrate multiple VFs with various consequences, MDR genes that encompass nearly all classes, and bacteriocins, represent a serious threat because of their exceptional versatility and should therefore be closely monitored in all countries in Europe. 


\section{Acknowledgments}

We thank Roger Stephan and Lothar Beutin for providing the strains from Switzerland and Germany, respectively.

This work was financed by Fonds d'Etudes et de Recherche du Corps Médical, AP-HP. Work in the Laboratorio de Referencia de Escherichia coli was financed by grant no. ED431C-2017-57 from Consellería de Cultura, Educación e Ordenación Universitaria (Xunta de Galicia) and the European Regional Development Fund. The funders had no role in study design, data collection and interpretation, or the decision to submit the work for publication.

\section{About the Author}

Dr. Cointe is a microbiologist working in the Parisian Public Hospital in France. Her research domain is pediatric infections, particularly the pathogenicity of intestinal Escherichia coli infections and the potential interplay between intraintestinal and extraintestinal E. coli.

\section{References}

1. Garg AX, Suri RS, Barrowman N, Rehman F, Matsell D, Rosas-Arellano MP, et al. Long-term renal prognosis of diarrheaassociated hemolytic uremic syndrome: a systematic review, meta-analysis, and meta-regression. JAMA. 2003;290:1360-70. http://dx.doi.org/10.1001/jama.290.10.1360

2. Schmidt H, Karch H, Beutin L. The large-sized plasmids of enterohemorrhagic Escherichia coli O157 strains encode hemolysins which are presumably members of the $E$. coli alpha-hemolysin family. FEMS Microbiol Lett. 1994;117:189-96.

3. Brunder W, Schmidt H, Karch H. EspP, a novel extracellular serine protease of enterohaemorrhagic Escherichia coli O157:H7 cleaves human coagulation factor V. Mol Microbiol. 1997;24:767-78. http://dx.doi.org/10.1046/j.1365-2958.1997.3871751.x

4. Bruyand M. Surveillance du syndrome hémolytique et urémique post-diarrhéique chez les enfants de moins de 15 ans en France en 2016 [cited $2016 \mathrm{Feb}$ 16]. http://invs.santepubliquefrance.fr/ content/download/138859/500759/version/1/file/Bilan SHU_2016.pdf

5. Blanco M, Blanco JE, Mora A, Dahbi G, Alonso MP, González EA, et al. Serotypes, virulence genes, and intimin types of Shiga toxin (verotoxin)-producing Escherichia coli isolates from cattle in Spain and identification of a new intimin variant gene $(e a e-\xi)$. J Clin Microbiol. 2004;42:645-51. http://dx.doi.org/10.1128/ JCM.42.2.645-651.2004

6. Wijnsma KL, Schijvens AM, Rossen JWA, Kooistra-Smid AMDM, Schreuder MF, van de Kar NCAJ. Unusual severe case of hemolytic uremic syndrome due to Shiga toxin 2d-producing E. coli O80:H2. Pediatr Nephrol. 2017;32:1263-8. http://dx.doi.org/ 10.1007/s00467-017-3642-3

7. Fierz L, Cernela N, Hauser E, Nüesch-Inderbinen M, Stephan R. Characteristics of Shigatoxin-producing Escherichia coli strains isolated during 2010-2014 from human infections in Switzerland. Front Microbiol. 2017;8:1471. http://dx.doi.org/10.3389/ fmicb.2017.01471

8. Bielaszewska M, Mellmann A, Zhang W, Köck R, Fruth A, Bauwens A, et al. Characterisation of the Escherichia coli strain associated with an outbreak of haemolytic uraemic syndrome in Germany, 2011: a microbiological study. Lancet Infect Dis. 2011;11:671-6. http://dx.doi.org/10.1016/S1473-3099(11)70165-7
9. Mariani-Kurkdjian P, Lemaître C, Bidet P, Perez D, Boggini L, Kwon T, et al. Haemolytic-uraemic syndrome with bacteraemia caused by a new hybrid Escherichia coli pathotype. New Microbes New Infect. 2014;2:127-31. http://dx.doi.org/10.1002/nmi2.49

10. Soysal N, Mariani-Kurkdjian P, Smail Y, Liguori S, Gouali M, Loukiadis E, et al. Enterohemorrhagic Escherichia coli hybrid pathotype O80:H2 as a new therapeutic challenge. Emerg Infect Dis. 2016;22:1604-12. http://dx.doi.org/10.3201/eid2209.160304

11. Peigne C, Bidet P, Mahjoub-Messai F, Plainvert C, Barbe V, Médigue C, et al. The plasmid of Escherichia coli strain S88 $(\mathrm{O} 45: \mathrm{K} 1: \mathrm{H} 7)$ that causes neonatal meningitis is closely related to avian pathogenic $E$. coli plasmids and is associated with high-level bacteremia in a neonatal rat meningitis model. Infect Immun. 2009;77:2272-84. http://dx.doi.org/10.1128/IAI.01333-08

12. Carattoli A, Zankari E, García-Fernández A, Voldby Larsen M, Lund O, Villa L, et al. In silico detection and typing of plasmids using PlasmidFinder and plasmid multilocus sequence typing. Antimicrob Agents Chemother. 2014;58:3895-903.

http://dx.doi.org/10.1128/AAC.02412-14

13. Kaas RS, Leekitcharoenphon P, Aarestrup FM, Lund O. Solving the problem of comparing whole bacterial genomes across different sequencing platforms. PLoS One. 2014;9:e104984. http://dx.doi.org/10.1371/journal.pone.0104984

14. Joensen KG, Tetzschner AMM, Iguchi A, Aarestrup FM, Scheutz F. Rapid and easy in silico serotyping of Escherichia coli isolates by use of whole-genome sequencing data. J Clin Microbiol. 2015;53:2410-26. http://dx.doi.org/10.1128/JCM.00008-15

15. Wirth T, Falush D, Lan R, Colles F, Mensa P, Wieler LH, et al. Sex and virulence in Escherichia coli: an evolutionary perspective. Mol Microbiol. 2006;60:1136-51. http://dx.doi.org/10.1111/ j.1365-2958.2006.05172.x

16. Zankari E, Hasman H, Cosentino S, Vestergaard M, Rasmussen S, Lund $\mathrm{O}$, et al. Identification of acquired antimicrobial resistance genes. J Antimicrob Chemother. 2012;67:2640-4. http://dx.doi.org/ 10.1093/jac/dks261

17. Bielaszewska M, Friedrich AW, Aldick T, Schürk-Bulgrin R, Karch H. Shiga toxin activatable by intestinal mucus in Escherichia coli isolated from humans: predictor for a severe clinical outcome. Clin Infect Dis. 2006;43:1160-7. http://dx.doi.org/10.1086/508195

18. Serra-Moreno R, Jofre J, Muniesa M. Insertion site occupancy by $s t x_{2}$ bacteriophages depends on the locus availability of the host strain chromosome. J Bacteriol. 2007;189:6645-54. http://dx.doi.org/10.1128/JB.00466-07

19. Bonanno L, Loukiadis E, Mariani-Kurkdjian P, Oswald E, Garnier L, Michel V, et al. Diversity of Shiga toxin-producing Escherichia coli (STEC) O26:H11 strains examined via stx subtypes and insertion sites of Stx and EspK bacteriophages. Appl Environ Microbiol. 2015;81:3712-21. http://dx.doi.org/10.1128/AEM.00077-15

20. Recktenwald J, Schmidt H. The nucleotide sequence of Shiga toxin (Stx) 2e-encoding phage phiP27 is not related to other Stx phage genomes, but the modular genetic structure is conserved. Infect Immun. 2002;70:1896-908. http://dx.doi.org/10.1128/ IAI.70.4.1896-1908.2002

21. Falgenhauer L, Yao Y, Fritzenwanker M, Schmiedel J, Imirzalioglu C, Chakraborty T. Complete genome sequence of phage-like plasmid pECOH89, encoding CTX-M-15. Genome Announc. 2014; 2:e00356-14. http://dx.doi.org/10.1128/genomeA.00356-14

22. Venturini C, Hassan KA, Roy Chowdhury P, Paulsen IT, Walker MJ, Djordjevic SP. Sequences of two related multiple antibiotic resistance virulence plasmids sharing a unique IS26-related molecular signature isolated from different Escherichia coli pathotypes from different hosts. PLoS One. 2013;8:e78862. http://dx.doi.org/10.1371/journal.pone.0078862

23. Feng P, Lampel KA, Karch H, Whittam TS. Genotypic and phenotypic changes in the emergence of Escherichia coli O157:H7. J Infect Dis. 1998;177:1750-3. http://dx.doi.org/10.1086/517438 
24. Jost C, Bidet P, Carrère T, Mariani-Kurkdjian P, Bonacorsi S. Susceptibility of enterohaemorrhagic Escherichia coli to azithromycin in France and analysis of resistance mechanisms. J Antimicrob Chemother. 2016;71:1183-7. http://dx.doi.org/ 10.1093/jac/dkv477

25. Liu Y-Y, Wang Y, Walsh TR, Yi L-X, Zhang R, Spencer J, et al. Emergence of plasmid-mediated colistin resistance mechanism MCR-1 in animals and human beings in China: a microbiological and molecular biological study. Lancet Infect Dis. 2016;16:161-8. http://dx.doi.org/10.1016/S1473-3099(15)00424-7

26. Donà V, Bernasconi OJ, Pires J, Collaud A, Overesch G, Ramette A, et al. Heterogeneous genetic location of $m c r-1$ in colistin-resistant Escherichia coli isolates from humans and retail chicken meat in Switzerland: emergence of mcr-1-carrying IncK2 plasmids. Antimicrob Agents Chemother. 2017;61:e01245-17. http://dx.doi.org/10.1128/AAC.01245-17

27. Day M, Doumith M, Jenkins C, Dallman TJ, Hopkins KL, Elson R, et al. Antimicrobial resistance in Shiga toxin-producing Escherichia coli serogroups O157 and O26 isolated from human cases of diarrhoeal disease in England, 2015. J Antimicrob Chemother. 2017;72:145-52. http://dx.doi.org/10.1093/jac/dkw371

28. Freedman SB, Xie J, Neufeld MS, Hamilton WL, Hartling L, Tarr PI; Alberta Provincial Pediatric Enteric Infection Team (APPETITE). Shiga toxin-producing Escherichia coli infection, antibiotics, and risk of developing hemolytic uremic syndrome: a meta-analysis. Clin Infect Dis. 2016;62:1251-8. http://dx.doi.org/10.1093/cid/ciw099

29. Fricke WF, McDermott PF, Mammel MK, Zhao S, Johnson TJ, Rasko DA, et al. Antimicrobial resistance-conferring plasmids with similarity to virulence plasmids from avian pathogenic Escherichia coli strains in Salmonella enterica serovar Kentucky isolates from poultry. Appl Environ Microbiol. 2009;75:5963-71. http://dx.doi.org/10.1128/AEM.00786-09

30. Agence Nationale de Sécurité Sanitaire de l'Alimentation, de l'Environnement et du Travail (ANSES). Suivi des ventes de médicaments vétérinaires contenant des antibiotiques en France en 2015 [cited 2016 Feb 16]. https://www.anses.fr/fr/system/files/ ANMV-Ra-Antibiotiques2015.pdf

31. Johnson TJ, Siek KE, Johnson SJ, Nolan LK. DNA sequence of a ColV plasmid and prevalence of selected plasmid-encoded virulence genes among avian Escherichia coli strains. J Bacteriol. 2006;188:745-58. http://dx.doi.org/10.1128/ JB.188.2.745-758.2006

Address for correspondence: Stéphane Bonacorsi, Service de Microbiologie, CHU Robert Debré, 48 boulevard Sérurier, 75019 Paris, France; email: stephane.bonacorsi@aphp.fr

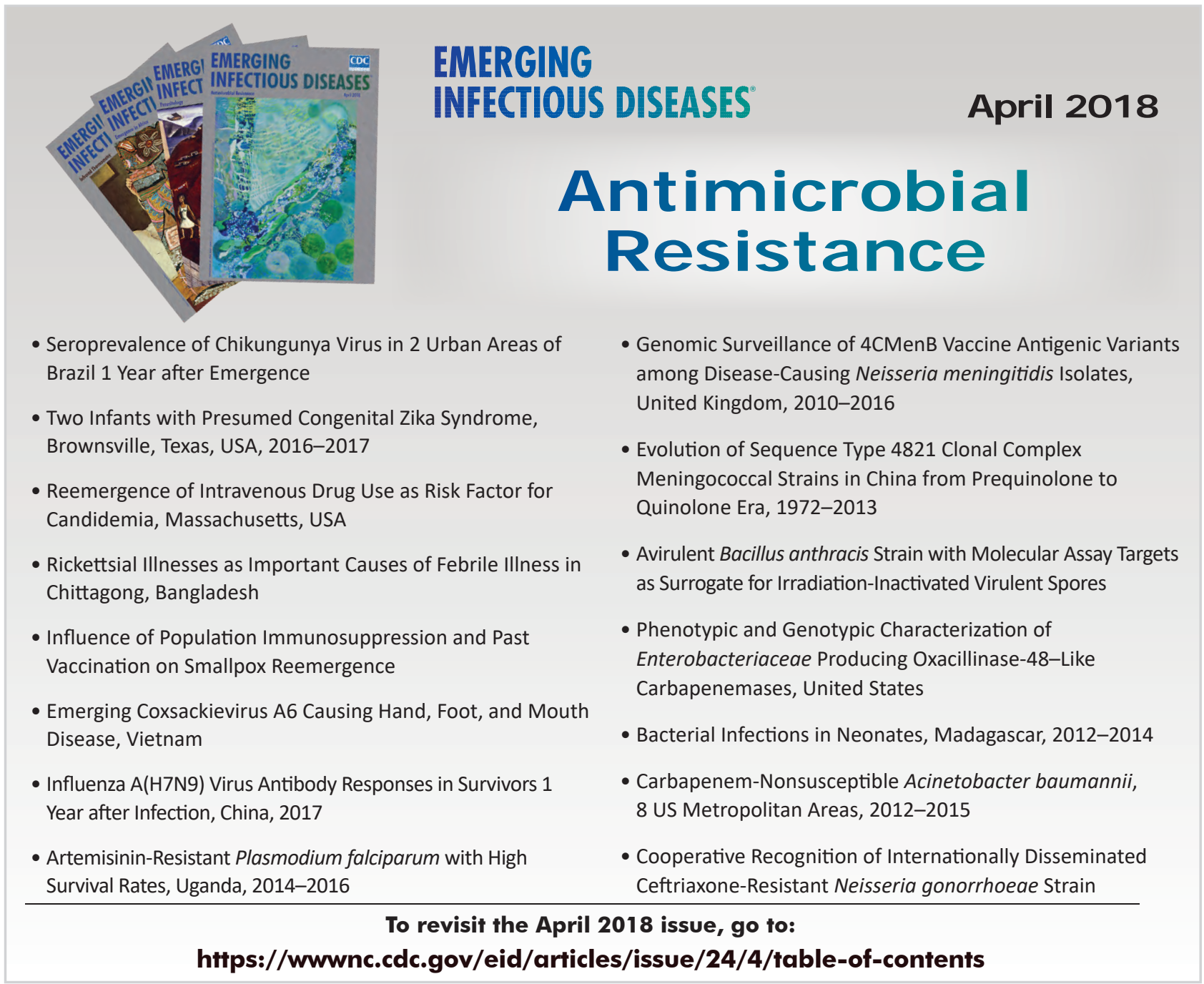




\title{
Comparison of 2016-17 and Previous Epizootics of Highly Pathogenic Avian Influenza H5 Guangdong Lineage in Europe
}

\author{
Pablo Alarcon, ${ }^{1}$ Adam Brouwer, ${ }^{1}$ Divya Venkatesh, Daisy Duncan, Chrysostomos I. Dovas, \\ George Georgiades, Isabella Monne, Alice Fusaro, Adam Dan, Krzysztof Śmietanka, \\ Vassilios Ragias, Andrew C. Breed, Taxiarchis Chassalevris, Gabriela Goujgoulova, \\ Charlotte Kristiane Hjulsager, Eoin Ryan, Azucena Sánchez, Eric Niqueux, Niina Tammiranta, \\ Siamak Zohari, David A. Stroud, Vladimir Savić, Nicola S. Lewis, lan H. Brown
}

We analyzed the highly pathogenic avian influenza (HPAI) H5 epizootic of 2016-17 in Europe by epidemiologic and genetic characteristics and compared it with 2 previous epizootics caused by the same $\mathrm{H} 5$ Guangdong lineage. The 2016-17 epizootic was the largest in Europe by number of countries and farms affected and greatest diversity of wild birds infected. We observed significant differences among the 3 epizootics regarding region affected, epidemic curve,

Author affiliations: Royal Veterinary College, London, UK (P. Alarcon); Animal and Plant Health Agency, Addlestone, UK (P. Alarcon, A. Brouwer, D. Duncan, A.C. Breed, N.S. Lewis, I.H. Brown); University of Cambridge, Cambridge, U K (D. Venkatesh); Aristotle University of Thessaloniki, Thessaloniki, Greece (C.I. Dovas, T. Chassalevris); Ministry of Rural Development and Food, Thessaloniki (G. Georgiades, V. Ragias); Istituto Zooprofilattico Sperimentale delle Venezie, Padova, Italy (I. Monne, A. Fusaro); Veterinary Diagnostic Institute, Budapest, Hungary (A. Dan); National Veterinary Research Institute, Pulawy, Poland (K. Śmietanka); Department of Agriculture and Water Resources, Canberra, Australian Capital Territory, Australia (A.C. Breed); University of Queensland, Brisbane, Queensland, Australia (A.C. Breed); NDRVMI, Sofia, Bulgaria (G. Goujgoulova); Technical University of Denmark, Lyngby, Denmark

(C.K. Hjulsager); Central Veterinary Research Laboratory, Celbridge, Ireland (E. Ryan); Central Veterinary Laboratory, Madrid, Spain (A. Sánchez); French Agency for Food, Environmental and Occupational Health \& Safety, Ploufragan, France (E. Niqueux); Finnish Food Safety Authority Evira, Helsinki, Finland (N. Tammiranta); National Veterinary Institute and World Organisation for Animal Health Collaborating Center, Uppsala, Sweden (S. Zohari); Joint Nature Conservation Committee, Peterborough, UK (D.A. Stroud); Croatian Veterinary Institute, Zagreb, Croatia (V. Savić)

DOI: https://doi.org/10.3201/eid2412.171860 seasonality, and outbreak duration, making it difficult to predict future HPAl epizootics. However, we know that in 2005-06 and 2016-17 the initial peak of wild bird detections preceded the peak of poultry outbreaks within Europe. Phylogenetic analysis of 2016-17 viruses indicates 2 main pathways into Europe. Our findings highlight the need for global surveillance of viral changes to inform disease preparedness, detection, and control.

Tighly pathogenic avian influenza (HPAI) is a zoonotic 1 notifiable disease that can cause high mortality rates in most domestic poultry and in some wild bird species. Since 2003, HPAI H5 viruses have been circulating in poultry in many countries (1). Periodically these poultry HPAI viruses have been reintroduced into the wild migratory bird population, representing a key risk pathway for its subsequent global spread (1-3). However, the effect of HPAI infection in both wild and domestic birds is variable and often strain-specific. Wild birds, particularly of the orders Anseriformes and Charadriiformes, are natural hosts of low pathogenicity avian influenza (4).

A passive surveillance system of testing wild birds found dead or sick for avian influenza has been in place in European Union (EU) member states since 2005 (Commission Decision 2005/94/EC, replaced with 2010/367/EU), with the objective of timely detection of HPAI subtype H5N1. Laboratory confirmation of HPAI infection following the development of clinical signs (passive surveillance) is the primary method of poultry surveillance in the EU member states, complemented by a serologic active surveillance program (5).

During epidemiologic year 2005-06 (epidemiologic years run from October to September of the next year),

${ }^{1}$ These authors are joint first authors. 
HPAI H5N1 clade 2.2 virus of the Guangdong H5 lineage spread to a number of countries in Europe, infecting poultry and wild bird populations (3). In 2014-15, another virus of the same lineage, HPAI H5N8 clade 2.3.4.4, was introduced into Europe and associated with variable disease severity, including subclinical infection in wild birds and domestic waterfowl (6). This H5N8 virus showed unprecedented intercontinental spread to the United States and Canada and was associated with both wild bird infection and, subsequent to local genetic reassortment, large HPAI H5N2 outbreaks in poultry (7).

In October 2016, a novel HPAI H5 clade 2.3.4.4 virus of the Guangdong lineage was detected in Hungary and was subsequently reported in other countries in Europe, infecting many poultry farms and causing both large-scale and sporadic deaths in wild bird populations. The hemagglutinin (HA) gene of this virus was considered phylogenetically distinct from the previous 2014 clade 2.3.4.4 viruses and was nominally suffixed by A (the 2016 clade) or B (the 2014 clade (8) but this subclade definition requires verification by the World Health Organization $\mathrm{H} 5$ nomenclature group. We describe the epidemiology and genetic characteristics of the 3 major wild-bird mediated epizootics in Europe associated with the Guangdong HPAI H5 lineage.

\section{Methods}

\section{Epidemiologic Data and Analyses}

We collected data from the 3 major HPAI H5 epizootics in Europe: HPAI H5N1 in epidemiologic year 2005-06 (2); HPAI H5N8 in 2014-15; and HPAI H5 in 2016-17. For 2016-17, we collected data through July 31, 2017. We obtained epidemiologic data from the Animal Disease Notification System and the Directorate-General for Health and Food Safety, managed by the European Commission, and from country notifications sent to the EU Reference Laboratory for avian influenza (Animal and Plant Health Agency, Weybridge, UK).

We conducted analyses to describe each epizootic, examined the geographic and temporal spread (epidemic curves), and assessed differences in clinical illness and death rates. For spatial analysis, we grouped countries into 4 regions (North, South-West, South-East, and Central Europe) on the basis of the broad migration patterns of the major migratory water bird species affected by HPAI (online Technical Appendix Figure 1, https://wwwnc.cdc.gov/ EID/article/24/12/17-1860-Techapp1.pdf) (9-14). A full description of the methods used is presented in the online Technical Appendix.

\section{Viruses' Sequence Data and Phylogenetic Analyses}

We obtained virus HA gene sequence data from countries' submissions to the EU Reference Laboratory and from GISAID (http://platform.gisaid.org.) We performed phylogenetic analyses on HA sequence data from each epizootic separately. We used IQ-TREE version 1.5.5 software (15) to infer maximum-likelihood trees with approximate likelihood ratio test (1,000 replicates) and bootstrap (100 replicates) support values for branches. We down-sampled each dataset using Cluster Database at High Identity with Tolerance to remove sequences with $>99.9 \%$ sequence identity (16). We performed root-totip regression analyses using Tempest version 1.5 on the downsampled datasets (17). Then, we inferred Bayesian phylogenetic trees from each downsampled dataset using BEAST version 1.8.4 to determine the mean substitution rate and TMRCA (time to most recent common ancestor) (18). We annotated the final trees using FigTree version 1.4 .3 (http://tree.bio.ed.ac.uk/software/figtree/). Details of criteria and priors used in the analyses are provided in the online Technical Appendix.

\section{Results}

\section{Epizootic Size}

In 2016-17, a total of 1,108 poultry outbreaks were reported in 21 countries in Europe. Extensive farm-to-farm spread, predominantly in ducks, seemed apparent in France, which had $>400$ farms affected, and Hungary, with $>200$ farms infected (19). Conversely, in 2005-06, a total of 230 poultry outbreaks occurred in 6 countries, mostly located in Romania $(86 \%)$ and Hungary (13\%). In 2014-15, only 13 poultry outbreaks were reported in 5 countries. The estimated number of poultry culled was 8 times higher in 2016-17 than in 2005-06 (Table 1).

The number of wild bird detections was substantially different between epizootics: 1,559 incidents in 27 countries in 2016-17, 487 in 18 countries in 2005-06, and only 5 in 3 countries in 2014-15. Almost half of the wild bird incidents reported in all 3 epizootics were in Germany.

\section{Wild Birds Species and Mass Mortality Events}

A total of 49 different wild bird species were reported infected with HPAI H5 virus of the Guangdong lineage in 2016-17, 28 in 2005-06, and 6 in 2014-15 (Table 2,3). Swans (Cygnus spp.), particularly mute swans (Cygnus olor), were the most frequent species infected in 200506 (41\% of all wild birds) and $2016-17$ (20\% of all wild birds). Ducks were the second most common type of wild birds infected. In 2005-06 and 2016-17, tufted duck (Aythya fuligula) was the most frequent duck species detected positive ( $5 \%$ of all wild birds). In 2005-06, a total of 28 (6\%) mass mortality events ( $>5$ birds dead in 1 location) were reported, whereas $112(7 \%)$ mass mortality events were reported in 2016-17; none were reported in 2014-15 (online Technical Appendix Figure 2). The number of wild 
birds found dead by incident was significantly different between epizootics ( $p<0.001$ by Mann-Whitney $U$ test).

\section{Type of Poultry Farm and Clinical Manifestations}

The types of poultry infected in each epizootic are shown in Table 4. In 2016-17, a large proportion of infected farms (40\%) kept ducks. In 2005-06, many affected backyard flocks in Romania $(176 / 230,77 \%)$ had $<100$ birds, whereas $70 \%(9 / 13)$ of poultry farms infected in 2014-15 had $>10,000$ birds and $>60 \%$ in 2016-17 had $>1,000$ birds (difference in flock size distribution, $\mathrm{p}<0.001$ by KruskalWallis test). When we excluded Romania from the comparison of flock size, there was no statistical difference in flock size between 2005-06 and 2016-17 (online Technical Appendix Figure 3).
Ducks, geese, turkeys, and broiler chickens on average had higher illness rates in 2005-06 than in the other epizootics (Figure 1). In 2016-17, average mortality rate was lowest in ducks $(7 \%)$ and turkeys $(6 \%)$; few farms $(<5 \%)$ reported a $>25 \%$ mortality rate. In contrast, $32 \%$ of affected broiler farms and $27 \%$ of affected layer farms reported mortality rates $>25 \%$. In 2005-06, more than half of broiler farms reported mortality rates $>25 \%$. When comparing overall estimates, we found the observed poultry illness and death rates to be substantially higher in 2005-06 than in 2016-17.

\section{Temporal Spread}

We determined the epidemiologic curves of the 3 epizootics (Figure 2, panels A-C). In 2016-17, H5 was first detected in Europe in a mute swan in Hungary; the first outbreak in

\begin{tabular}{|c|c|c|c|c|c|c|c|c|c|c|c|}
\hline \multirow[b]{2}{*}{ Country } & \multicolumn{3}{|c|}{ H5N1 2005-06 epizootic } & \multicolumn{4}{|c|}{ H5N8 2014-15 epizootic } & \multicolumn{4}{|c|}{ H5N8 2016-17 epizootic } \\
\hline & $\begin{array}{c}\text { No. } \\
\text { poultry } \\
\text { infected }\end{array}$ & $\begin{array}{c}\text { No. } \\
\text { wild } \\
\text { birds } \\
\text { infected }\end{array}$ & $\begin{array}{c}\text { No. } \\
\text { poultry } \\
\text { culled† }\end{array}$ & $\begin{array}{c}\text { No. } \\
\text { poultry } \\
\text { infected }\end{array}$ & $\begin{array}{c}\text { No. } \\
\text { wild } \\
\text { birds } \\
\text { infected }\end{array}$ & $\begin{array}{c}\text { No. } \\
\text { captive } \\
\text { birds } \\
\text { infected }\end{array}$ & $\begin{array}{c}\text { No. } \\
\text { poultry } \\
\text { culled† }\end{array}$ & $\begin{array}{c}\text { No. } \\
\text { poultry } \\
\text { infected }\end{array}$ & $\begin{array}{c}\text { No. } \\
\text { wild } \\
\text { birds } \\
\text { infected }\end{array}$ & $\begin{array}{c}\text { No. } \\
\text { captive } \\
\text { birds } \\
\text { infected }\end{array}$ & $\begin{array}{c}\text { No. } \\
\text { poultry } \\
\text { culled† }\end{array}$ \\
\hline France & 1 & 21 & 11,700 & - & - & - & - & 485 & 51 & 3 & $1,529,361$ \\
\hline Hungary & 29 & 12 & 251,948 & 1 & - & - & 22,000 & 238 & 86 & 5 & $2,678,191$ \\
\hline Germany & 1 & 220 & 14,300 & 5 & 2 & 1 & 58,964 & 89 & 738 & 15 & $1,150,631$ \\
\hline Bulgaria & - & 4 & - & - & - & - & - & 71 & 13 & 2 & 511,832 \\
\hline Poland & - & 29 & - & - & - & - & - & 65 & 66 & - & $1,167,282$ \\
\hline Romania & 197 & 17 & $755,372 \ddagger$ & - & - & - & - & 45 & 93 & 2 & 2,222 \\
\hline Czech & - & 14 & - & - & - & - & - & 38 & 39 & - & 79,308 \\
\hline \multicolumn{12}{|l|}{ Republic } \\
\hline Italy & - & 19 & - & 1 & - & - & 31,985 & 16 & 6 & - & 357,049 \\
\hline Spain & - & 1 & - & - & - & - & - & 10 & 2 & - & 28,330 \\
\hline Croatia & $\S$ & $\S$ & $\S$ & - & - & - & - & 9 & 12 & - & 1,546 \\
\hline United & - & 1 & - & 1 & - & - & 6,178 & 12 & 23 & - & 102,849 \\
\hline \multicolumn{12}{|l|}{ Kingdom } \\
\hline Netherlands & - & - & - & 5 & 1 & - & 245,600 & 8 & 48 & 10 & 202,004 \\
\hline Slovakia & - & 2 & - & - & - & - & - & 8 & 58 & 3 & 351 \\
\hline Greece & - & 25 & - & - & - & - & - & 5 & 8 & - & 28,275 \\
\hline Serbia & $\S$ & $\S$ & $\S$ & - & - & - & - & 4 & 20 & - & 289 \\
\hline Sweden & 1 & 13 & 692 & - & 2 & - & - & 4 & 30 & 2 & 203,053 \\
\hline Austria & - & 46 & & - & - & - & - & 2 & 55 & 1 & 1,258 \\
\hline Ukraine & $\S$ & $\S$ & $\S$ & - & - & - & - & 2 & 3 & 1 & 10,288 \\
\hline $\begin{array}{l}\text { Bosnia and } \\
\text { Herzegovina }\end{array}$ & $\S$ & $\S$ & $\S$ & - & - & - & - & 1 & 1 & 1 & 148 \\
\hline Denmark & 1 & 26 & 102 & - & - & - & - & 1 & 49 & 1 & 69 \\
\hline FYROM & $\S$ & $\S$ & $\S$ & - & - & - & - & 1 & 1 & - & 438 \\
\hline Belgium & - & - & - & - & - & - & - & 2 & 3 & 13 & 4,047 \\
\hline Finland & - & - & - & - & - & - & - & - & 15 & 2 & - \\
\hline Ireland & - & - & - & - & - & - & - & - & 10 & - & - \\
\hline Lithuania & - & - & - & - & - & - & - & - & 5 & - & - \\
\hline Portugal & - & - & - & - & - & - & - & - & 1 & - & - \\
\hline Slovenia & - & 28 & - & - & - & - & - & - & 41 & - & - \\
\hline Switzerland & - & 9 & - & - & - & - & - & - & 87 & - & - \\
\hline Luxembourg & - & - & - & - & - & - & - & - & - & 4 & - \\
\hline Totals & 230 & 487 & $1,034,114$ & 13 & 5 & 1 & 364,727 & 1,116 & 1,565 & 64 & $8,058,831$ \\
\hline Total infected & 73 & & & & 19 & & & & 2,745 & & \\
\hline \multicolumn{12}{|c|}{$\begin{array}{l}\text { *Table includes all reported HPAI H5N8 outbreaks through July } 31,2017 \text {. It excludes the new wave of secondary H5N8 outbreaks observed in Italy from } \\
\text { the beginning of July } 2017 \text { through September } 2017 \text {, which has different drivers and kinetics with maintenance in the poultry (primarily turkey) population } \\
\text { rather than through wild bird introduction. FYROM, the former Yugoslav Republic of Macedonia; HPAI, highly pathogenic avian influenza. } \\
\text { †lt is uncertain if for some outbreaks only the number of poultry in one farm building or if the poultry population in the area of the farm were reported. This } \\
\text { estimate should be used as an approximation and indicator of impact. } \\
\text { łOne observation contained } 600,000 \text { birds, representing the overall population of backyard flocks affected in Romania. This number is an approximation. } \\
\text { §These countries did not submit data to the Animal Disease Notification System in 2005-06; however, there is other evidence of H5N1 incursion in the } \\
\text { period. }\end{array}$} \\
\hline
\end{tabular}


poultry was detected 11 days later in a turkey farm, also in Hungary. We observed 3 major epidemic peaks on the incidence of poultry outbreaks (Figure 2, panel D): on day 54 (14.9 outbreaks/wk), following large farm-to-farm spread in Hungary; day 79 (12.1 outbreaks/wk) caused by farmto-farm transmission in France and Bulgaria; and on day 121 (16.9 outbreaks/wk), caused by the large farm-to-farm spread in France and Poland.

In 2005-06 and 2016-17, a peak in wild bird incidents preceded the peak in poultry outbreaks (Figure 2, panel A, C). Statistical analysis of the distribution of the epidemic curves indicates that the 2016-17 outbreak had significantly higher incidence values ( $\mathrm{p}<0.001$ by 2 -sample Kolmogorov-Smirnov test) than the other 2 epizootics;
2005-06 had significantly higher values $(p<0.001$ by 2-sample Kolmogorov-Smirnov test) than 2014-15. Temporal median of the poultry epizootic was substantially different between epizootics (mean/median distance for 2005-06, 189/223 days; for 2014-15, 33.5/26; for 201617, 92/90 days). Seasonal analysis of poultry outbreaks indicates significant differences $\left(\mathrm{p}<0.001\right.$ by Pearson $\chi^{2}$ test) between epizootics; $>50 \%$ of poultry outbreaks occurred in May in 2005-06, in November in 2014-15, and in December-February in 2016-17 (Figure 2, panel E).

\section{Spatial Spread}

We mapped a temporal-spatial analysis of the 3 epizootics (Figures 3-5). The data shown in Figure 5, panel B, suggest

Table 2. Wild bird species of the orders Podicipediformes, Anseriformes, and Charadriiformes, reported by event in 3 highly pathogenic avian influenza epizootics in Europe

\begin{tabular}{|c|c|c|c|c|}
\hline \multirow[b]{2}{*}{ Species group } & \multirow[b]{2}{*}{ Species } & \multicolumn{3}{|c|}{ No. (\%) events } \\
\hline & & $\begin{array}{l}\text { H5N1 2005-06 } \\
\text { epizootic }\end{array}$ & $\begin{array}{l}\text { H5N8 2014-15 } \\
\text { epizootic }\end{array}$ & $\begin{array}{c}\text { H5N8 2016-17 } \\
\text { epizootic }\end{array}$ \\
\hline \multirow[t]{5}{*}{ Rails } & Eurasian coot (Fulica atra) & $5(1)$ & & $8(0.5)$ \\
\hline & Crested coot (Fulica cristata) & & & $1(0.1)$ \\
\hline & Purple swamphen (Porphyrio porphyrio) & $4(1)$ & & \\
\hline & Common moorhen (Gallinula chloropus) & $1(0.2)$ & & $2(0.1)$ \\
\hline & Total & $10(2)$ & & $11(1)$ \\
\hline \multirow[t]{4}{*}{ Swans } & Unspecified. & $197(38)$ & $2(22)$ & $262(16)$ \\
\hline & Mute swan (Cygnus olor) & $92(18)$ & & $344(20)$ \\
\hline & Whooper swan (Cygnus cygnus) & $2(0.4)$ & & $80(5)$ \\
\hline & $\begin{array}{c}\text { Total } \\
\end{array}$ & $291(56)$ & $2(22)$ & $683(41)$ \\
\hline \multirow[t]{15}{*}{ Ducks } & Unspecified & $57(11)$ & & $143(9)$ \\
\hline & Northern pintail (Anas acuta) & $2(0.4)$ & & \\
\hline & Eurasian wigeon (Anas penelope) & & $1(11)$ & $21(1)$ \\
\hline & Mallard (Anas platyrhynchos) & $4(1)$ & $1(11)$ & $43(3)$ \\
\hline & Common pochard (Aythya farina) & $4(1)$ & & $8(0.5)$ \\
\hline & Red-crested pochard (Netta rufina) & & & $2(0.1)$ \\
\hline & Common goldeneye (Bucephala clangula) & & & $1(0.1)$ \\
\hline & Greater scaup (Aythya marila) & $2(0.4)$ & & \\
\hline & Common merganser (Mergus merganser) & $5(1)$ & & \\
\hline & Tufted duck (Aythya fuligula) & $18(3)$ & & $82(5)$ \\
\hline & Eurasian teal (Anas crecca) & & $1(11)$ & $3(0.2)$ \\
\hline & Smew (Mergus albellus) & $1(0.2)$ & & \\
\hline & Shelduck (Tadorna tadorna) & & & $2(0.1)$ \\
\hline & Common eider (Somateria mollissima) & & & $2(0.1)$ \\
\hline & Total & $93(18)$ & $3(33)$ & $307(18)$ \\
\hline \multirow[t]{10}{*}{ Geese } & Unspecified & $30(6)$ & & $94(6)$ \\
\hline & Canada goose (Branta canadensis) & & & $5(0.3$ \\
\hline & Barnacle goose (Branta leucopsis) & $1(0.2)$ & & \\
\hline & Greater white-fronted goose (Anser albifrons) & & & $9(1)$ \\
\hline & Lesser white-fronted goose (Anser erythropus) & $2(0.4)$ & & $4(0.2)$ \\
\hline & Greylag goose (Anser anser) & $1(0.2)$ & & $21(1)$ \\
\hline & Red-breasted goose (Branta ruficollis) & $1(0.2)$ & & \\
\hline & Bean goose (Anser fabalis) & & & $1(0.1)$ \\
\hline & Pink-footed goose (Anser brachyrhynchus) & & & $1(0.1)$ \\
\hline & Total & $35(7)$ & & $134(8)$ \\
\hline \multirow[t]{7}{*}{ Gulls } & Unspecified & $9(2)$ & & $89(5)$ \\
\hline & Great black-backed gull (Larus marinus) & & & $11(1)$ \\
\hline & Herring gull (Larus argentatus) & $1(0.2)$ & & $28(2)$ \\
\hline & Black-headed gull (Larus ridibundus) & $1(0.2)$ & $1(11)$ & $23(1)$ \\
\hline & Lesser black-backed gull (Larus fuscus) & & & $1(0.1)$ \\
\hline & Common gull (Larus canus) & & & $2(0.1)$ \\
\hline & Total & $11(2)$ & $1(11)$ & $154(9)$ \\
\hline \multirow[t]{3}{*}{ Waders } & Green sandpiper (Tringa ochropus) & & & $1(0.1)$ \\
\hline & Eurasian curlew (Numenius arquata) & & & $1(0.1)$ \\
\hline & Total & & & $2(0.1)$ \\
\hline
\end{tabular}


that, in the first 2 months of the 2016-17 epizootic, 2 different viral incursions may have occurred: one spreading through Hungary, Croatia, Switzerland, and southern Germany, and another spreading in northern Europe (Poland, Denmark, northern Germany, Sweden, and the Netherlands). The 2005-06 epizootic indicated a similar progression pattern, initiating in Romania and spreading up to northern Europe and down to southeastern Europe (Figure 3).

Comparison by region of Europe according to wild bird migratory patterns indicates poultry outbreaks were mostly observed in the South-East and South-West regions in 2005-06 and 2016-17 but in the North in 2014-15 (online Technical Appendix Figure 4). Most wild bird detections were reported in the North and Central regions.
Poultry detections by region were significantly different for the 3 epizootics ( $p<0.001$ by Pearson $\chi^{2}$ test), whereas wild bird detections by region were only significantly different ( $\mathrm{p}<0.001$ by Pearson $\chi^{2}$ test) between 2005-06 and 2016-17.

\section{Phylogenetic Analysis}

Genetic analysis of the HA gene for the 2014-15 and 201617 epizootics shows the involvement of $\mathrm{H} 5$ clade 2.3.4.4 in all cases where data were available (Figure 6). Patterns found in maximum-likelihood trees are largely in agreement with the Bayesian analysis; however, a greater proportion of the clades remain unresolved in the maximum-likelihood trees (Figure 6; online Technical Appendix Figure 8). The

\begin{tabular}{|c|c|c|c|c|}
\hline \multirow[b]{2}{*}{ Species group } & \multirow[b]{2}{*}{ Species } & \multicolumn{3}{|c|}{ No. (\%) events } \\
\hline & & $\begin{array}{l}\text { H5N1 2005-06 } \\
\text { epizootic }\end{array}$ & $\begin{array}{l}\text { H5N8 2014-15 } \\
\text { epizootic }\end{array}$ & $\begin{array}{c}\text { H5N8 2016-17 } \\
\text { epizootic }\end{array}$ \\
\hline \multirow[t]{16}{*}{ Birds of prey } & Unspecified & $30(6)$ & & \\
\hline & Buzzard & $1(0.2)$ & & $6(0.4)$ \\
\hline & Eagle & & & $1(0.1)$ \\
\hline & Falcon & $1(0.2)$ & & $3(0.2)$ \\
\hline & Hawk & $1(0.2)$ & & $3(0.2)$ \\
\hline & Owl. & $2(0.4)$ & & $4(0.2)$ \\
\hline & Barn owl (Tyto alba) & $1(0.2)$ & & \\
\hline & Peregrine falcon (Falco peregrinus) & $1(0.2)$ & & $8(0.5)$ \\
\hline & White-tailed eagle (Haliaeetus albicilla) & & & $24(1)$ \\
\hline & Common buzzard (Buteo buteo) & $7(1)$ & & $70(4)$ \\
\hline & Rough-legged buzzard (Buteo lagopus) & $1(0.2)$ & & \\
\hline & Eurasian eagle-owl (Bubo bubo) & $2(0.4)$ & & $1(0.1)$ \\
\hline & Eurasian sparrowhawk (Accipiter nisus) & & & $1(0.1)$ \\
\hline & Common kestrel (Falco tinnunculus) & & & $2(0.1)$ \\
\hline & Northern goshawk (Accipiter gentilis) & & & $1(0.1)$ \\
\hline & $\begin{array}{c}\text { Total } \\
\text { Totiter }\end{array}$ & $47(9)$ & & $124(7)$ \\
\hline \multirow[t]{7}{*}{ Crows } & Unspecified & $1(0.2)$ & & \\
\hline & Eurasian magpie (Pica pica) & $1(0.2)$ & & $4(0.3)$ \\
\hline & Hooded crow (Corvus cornix) & & & $3(0.2)$ \\
\hline & Rook (Corvus frugilegus) & & & $2(0.1)$ \\
\hline & Carrion crow (Corvus corone) & & & $1(0.1)$ \\
\hline & Common raven (Corvus corax) & & & $1(0.1)$ \\
\hline & Total & $2(0.4)$ & & $11(1)$ \\
\hline \multirow[t]{3}{*}{ Grebes } & Great crested grebe (Podiceps cristatus) & $7(1)$ & & $12(1)$ \\
\hline & Little grebe (Tachybaptus ruficollis) & $1(0.2)$ & & $4(0.2)$ \\
\hline & Total & $8(2)$ & & $16(1)$ \\
\hline \multirow[t]{3}{*}{ Thrushes } & Blackbird (Turdus merula) & & & $1(0.1$ \\
\hline & Song thrush (Turdus philomelos) & & & $2(0.1)$ \\
\hline & Total & & & $3(0.2$ \\
\hline \multirow[t]{4}{*}{ Pigeons, doves } & Wood pigeon (Columba palumbus) & & & $2(0.1)$ \\
\hline & Collared dove (Streptopelia decaocto) & $1(0.2)$ & & $1(0.1)$ \\
\hline & Rock dove (Coumbia livia) & & $1(11)$ & \\
\hline & Total & $1(0.2)$ & $1(11)$ & $3(0.2)$ \\
\hline \multirow[t]{3}{*}{ Herons } & Unspecified & $2(0.4)$ & & $16(1)$ \\
\hline & Grey heron (Ardea cinerea) & $4(1)$ & & $48(3)$ \\
\hline & Total & $6(1)$ & & $64(4)$ \\
\hline \multirow[t]{3}{*}{ Storks } & Unspecified & $2(0.4)$ & & \\
\hline & White stork (Ciconia ciconia) & & & $3(0.2)$ \\
\hline & Total & $2(0.4)$ & & $3(0.2)$ \\
\hline Pelicans & Unspecified. (Pelcanus spp.) & & & $2(0.1)$ \\
\hline Terns & Common tern (Sterna hirundo) & & & $2(0.1)$ \\
\hline Cormorants & Great cormorant (Phalacrocorax carbo) & $6(1)$ & & $17(1)$ \\
\hline Other & Unspecified & $9(2)$ & $2(22)$ & $140(8)$ \\
\hline
\end{tabular}


Table 4. Types of poultry on infected farms in 3 highly pathogenic avian influenza epizootics in Europe*

\begin{tabular}{|c|c|c|c|c|c|c|}
\hline \multirow[b]{3}{*}{ Type of poultry } & \multicolumn{2}{|c|}{ H5N1 2005-06 epizootic } & \multicolumn{2}{|c|}{ H5N8 2014-15 epizootic } & \multicolumn{2}{|c|}{ H5N8 2016-17 epizootic } \\
\hline & & No. with only & & No. with only & & No. with only \\
\hline & No. (\%) farms & 1 species & No. (\%) farms & 1 species & No. (\%) farms & 1 species \\
\hline Ducks & & & $3(23)$ & 0 & $495(44)$ & 433 \\
\hline Geese & & & & & $113(10)$ & 81 \\
\hline Ducks and geese & $29(13)$ & 0 & & & & \\
\hline Turkey & $5(2)$ & 1 & $3(23)$ & 0 & $91(8)$ & 82 \\
\hline Broilers & $23(10)$ & 17 & $4(31)$ & 0 & $93(8)$ & 48 \\
\hline Laying hens & & & & & $47(4)$ & 29 \\
\hline Pigeons & & & & & $9(1)$ & 1 \\
\hline Guinea fowl & & & & & $10(1)$ & 1 \\
\hline Peacocks & & & & & $2(0)$ & 0 \\
\hline Pheasants & & & & & $8(1)$ & 5 \\
\hline Quail & & & & & $2(0)$ & 1 \\
\hline Ostrich & & & & & $1(0)$ & 0 \\
\hline Backyard $†$ & $176(77)$ & NA & & & & \\
\hline Unknown & & & 2 & & $360(32)$ & NA \\
\hline Total infected farms & 230 & & 13 & & 1,116 & \\
\hline
\end{tabular}

2016-17 viruses form a distinct clade and can be clearly differentiated from the clade 2.3.4.4. viruses present in Europe in 2014-15. In agreement with the geospatial results, analysis of the HA gene of the viruses from the 2016-17 epizootic shows that most originate from a common progenitor (time to most recent common ancestor estimated May 2014-August 2015) (online Technical Appendix Figure 8). However, these viruses differ in their evolutionary pathway thereafter, evolving in 2 co-circulating subclades without clear geographic restriction (time to most recent common ancestor
March 2015-August 2016 [0.9 posterior probability] and November 2014-October 2015 [0.82 posterior probability]). This finding potentially indicates 2 major incursion pathways via wild birds.

We also found smaller clusters and singleton sequences including sequences from European viruses; viruses from 2014-15 form 1 subclade, estimated to have emerged in January-February 2014 (Figure 6; online Technical Appendix Figure 8). The 2005-06 data show viruses in several subclades, but the branching pattern in

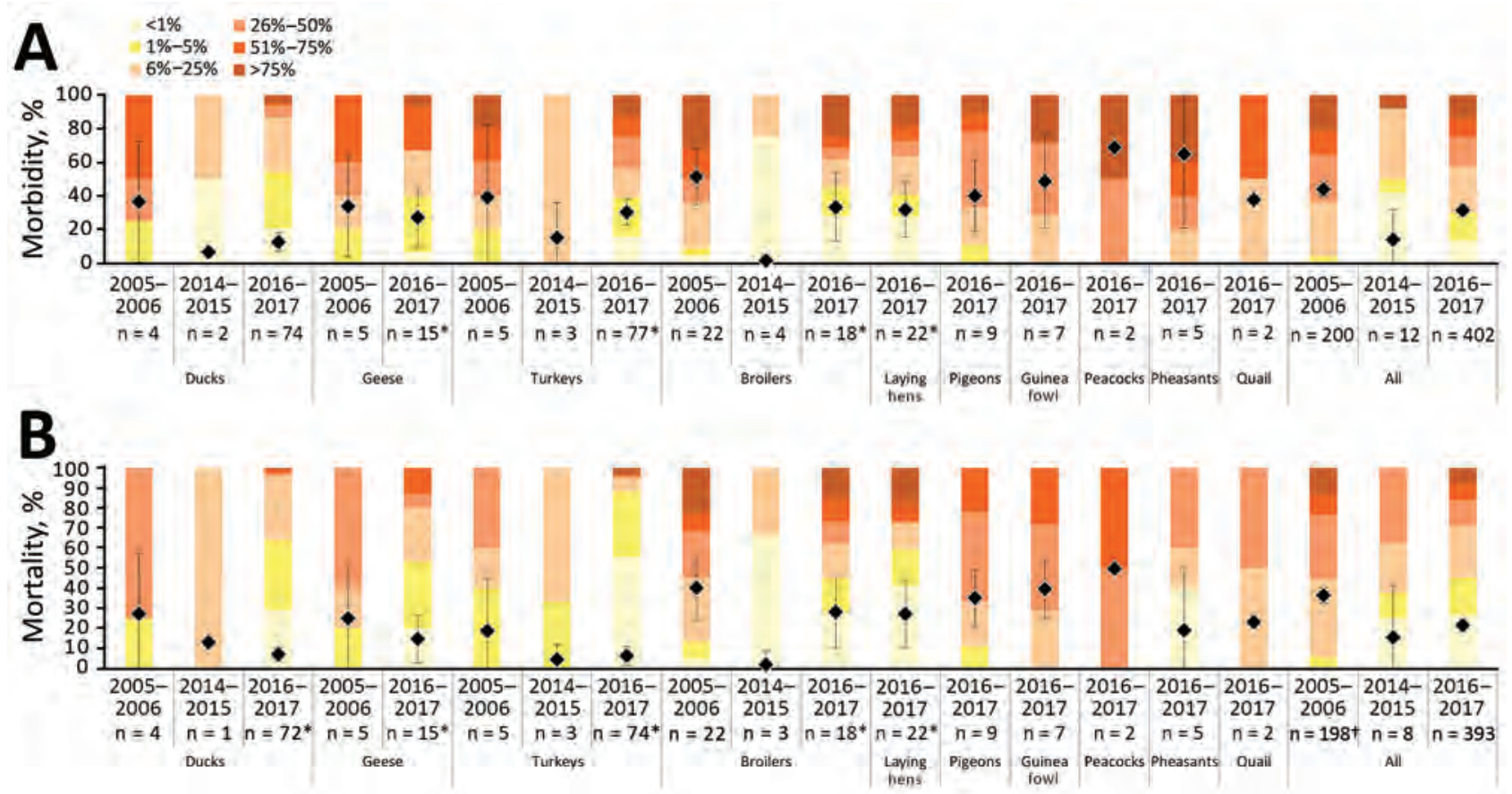

Figure 1. Morbidity (A) and mortality (B) rates as percentages of populations reported in infected poultry farms during 3 highly pathogenic avian influenza epizootics in Europe, 2005-06, 2014-15, and 2016-17. Years given are epidemiologic years (October through September of the next year). Diamonds with error bars indicate means and $95 \% \mathrm{Cls}$. Asterisks indicate farms with unique poultry species used for analysis; dagger indicates large majority of data from backyard farms reported in Romania. 


\section{A}

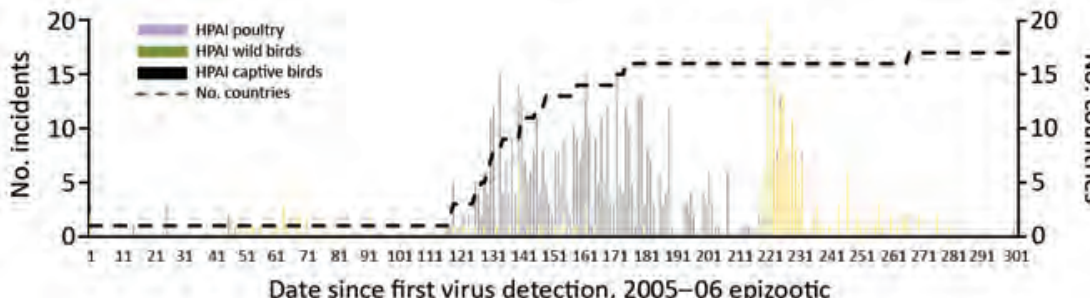

B

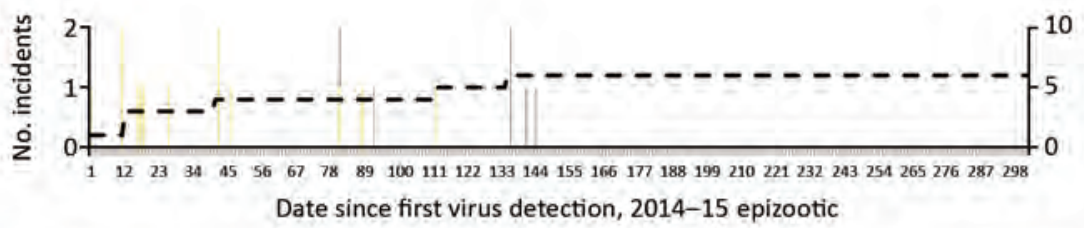

C

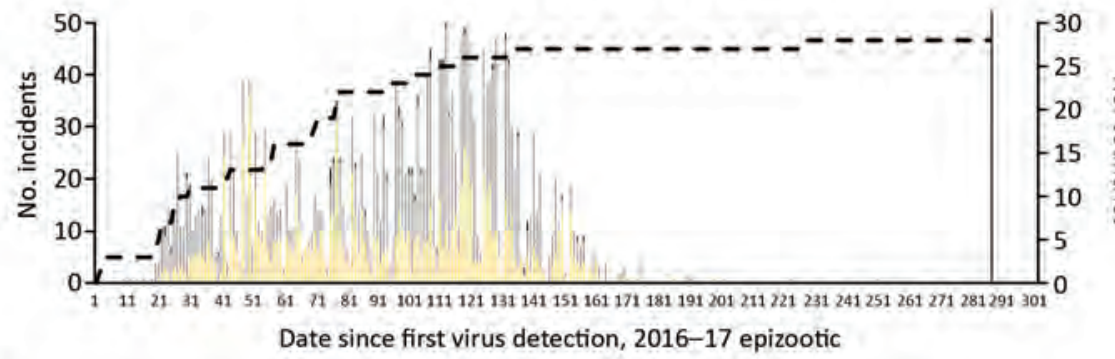

D

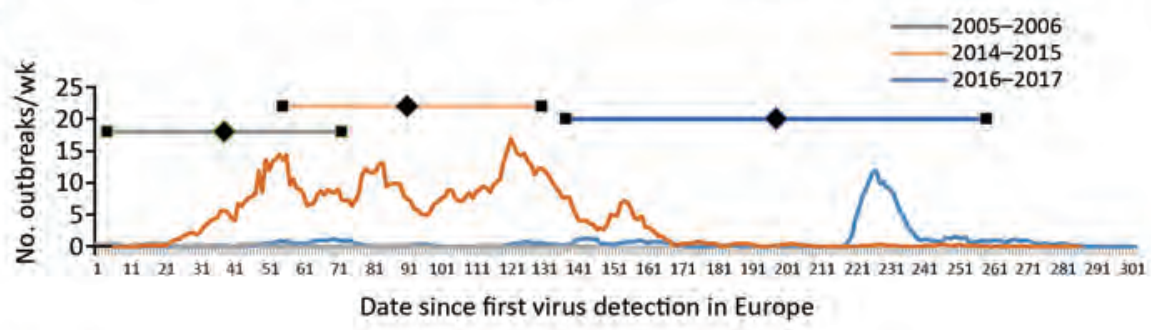

E

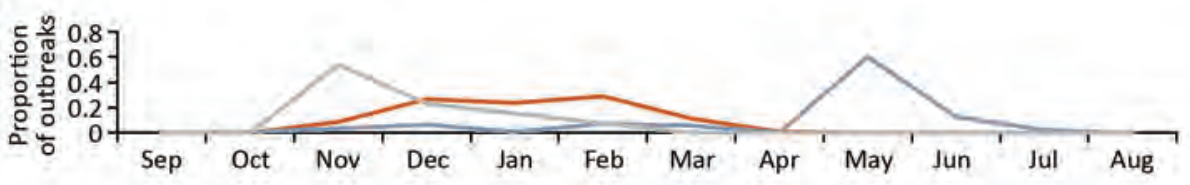

Figure 2. Epidemic curve of $3 \mathrm{HPAl}$ H5 virus epizootics in Europe: A) 2005-06 H5N1; B) 2014-15 H5N8; C) 2016-17 H5N8. Years given are epidemiologic years (October through September of the next year). Dashed lines indicate number of countries reporting an HPAl infection since the beginning of the epizootic; vertical line in panel $\mathrm{C}$ indicates data collected through July 31, 2017. D) Weekly average number of poultry outbreaks for each epizootic. Horizontal lines indicate the day at which half of the poultry outbreaks have occurred (diamonds); error bars indicate 1 SD. E) Number of poultry outbreaks for each month for the 3 epizootics. HPAI, highly pathogenic avian influenza. this dataset is generally less distinct and many sequences remain unresolved.

BEAST analyses (http://tree.bio.ed.ac.uk/software/ beast/) also revealed that the 2014-15 epizootic viruses show the highest mean substitution rate (measured per site per year), followed by 2016-17 and then by the 2005-06 epizootic, which is significantly lower (one-way analysis of variance $\mathrm{p}<0.001$ ) (online Technical Appendix Figure $6)$. These data are in agreement with the results of the rootto-tip regression analysis (online Technical Appendix Figure 7), which show a much steeper slope for the 2014-15 epizootic compared with the others. However, the spread of the data is high for the 2016-17 epizootic, where the SD of rates is an order of magnitude higher than that for the 2014-15 epizootic and 2 orders greater than for the
2005-06 outbreak. The nucleotide diversity for each epizootic (online Technical Appendix Figure 9) shows that persite diversity (average pairwise nucleotide differences in a population) is lowest in the 2005-06 epizootic (0.0038), consistent with the lower substitution rate inferred from BEAST. The 2014-15 epizootic has the highest diversity (0.0086); the rate for 2016-17, calculated from viruses collected through June 2017, is 0.0063 .

\section{Discussion}

The 2016-17 epizootic of HPAI H5 clade 2.3.4.4 viruses in Europe has 5 times more outbreaks in poultry than observed in the H5 clade 2.2 epizootic in 2005-06 and 80 times more than in the H5 clade 2.3.4.4 epizootic in 2014-15. This study highlights the unprecedented 


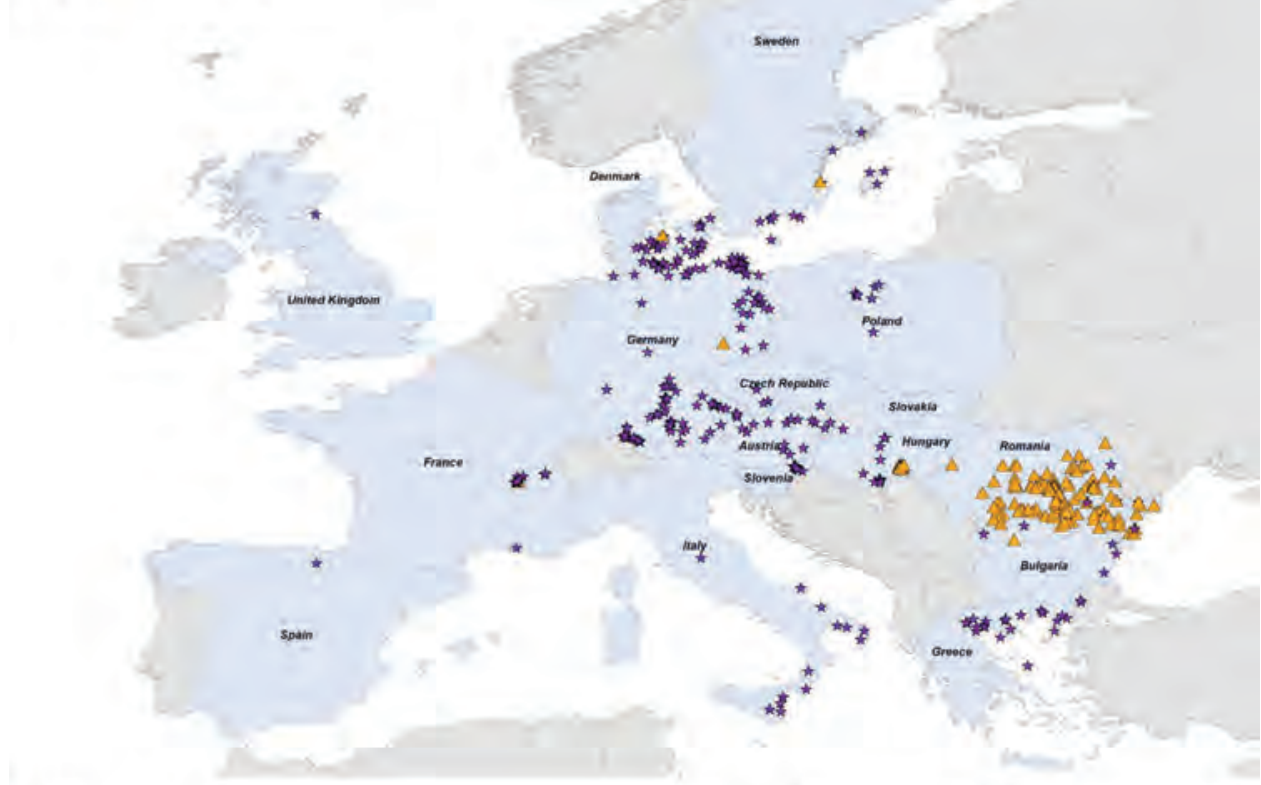

Figure 3. Geographic and temporal spread of the 2005-06 HPAI H5N1 epizootic. A) Location of each incident reported. Blue shading indicates countries where cases were reported. B) Month of first report of an HPAI H5N1 incident. Years given are epidemiologic years (October through September of the next year). HPAl, highly pathogenic avian influenza.

B

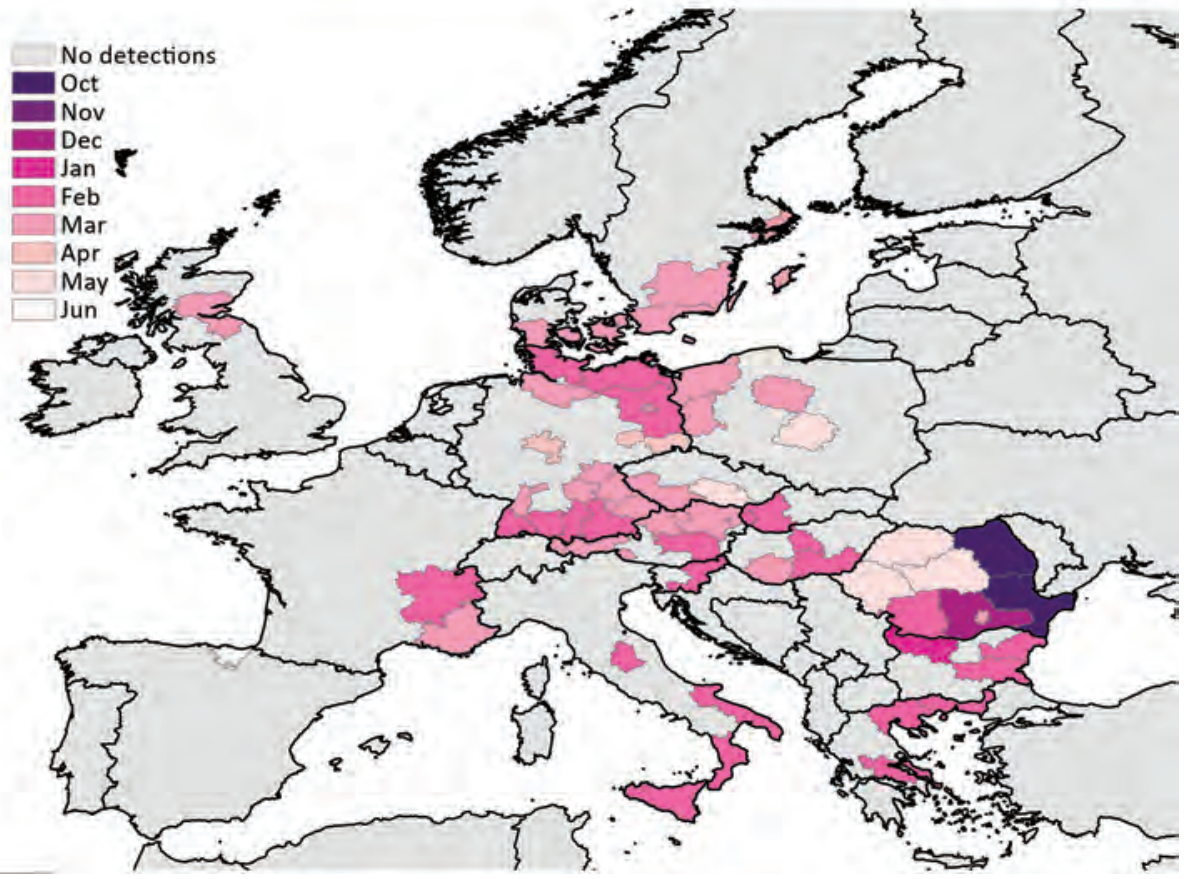

magnitude of the 2016-17 HPAI H5 epizootic in Europe, in terms of size (both number of poultry outbreaks and wild bird incidents), geographic spread, speed of incidents/outbreaks, and diversity of wild bird species reported infected. As a result, the economic impact is many times higher for 2016-17, which resulted in an $\geq 8$-fold increase in poultry that died or were culled.

A greater passive surveillance effort to detect influenza virus in wild birds was reported in the EU in 2006 than in
$2016(20,21)$. Despite reduced passive surveillance efforts in recent years, more virus detections were made in wild birds in calendar year 2016 compared with 2006, indicating a likely increase in viral burden within bird populations in Europe, leading to an increased risk for incursion into poultry. Although we found a lower rate of substitution and diversity in 2016-17 compared with 2014-15, the viruses in the 2016-17 epizootic might be more efficient in capacity to adapt and infect avian hosts. Different rates and diversity between 
A

$\triangle$ HPAI H5N8, poultry

HPAIH5N8, captive birds † HPAI H5N8, wild birds
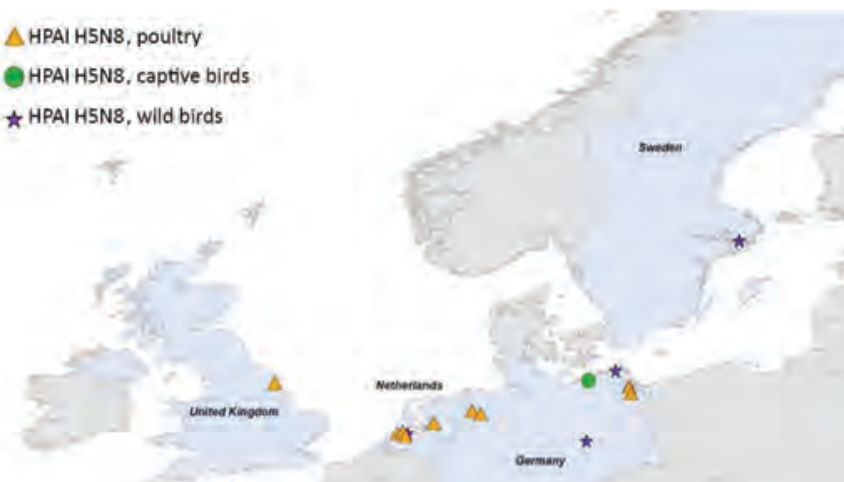

minger

B

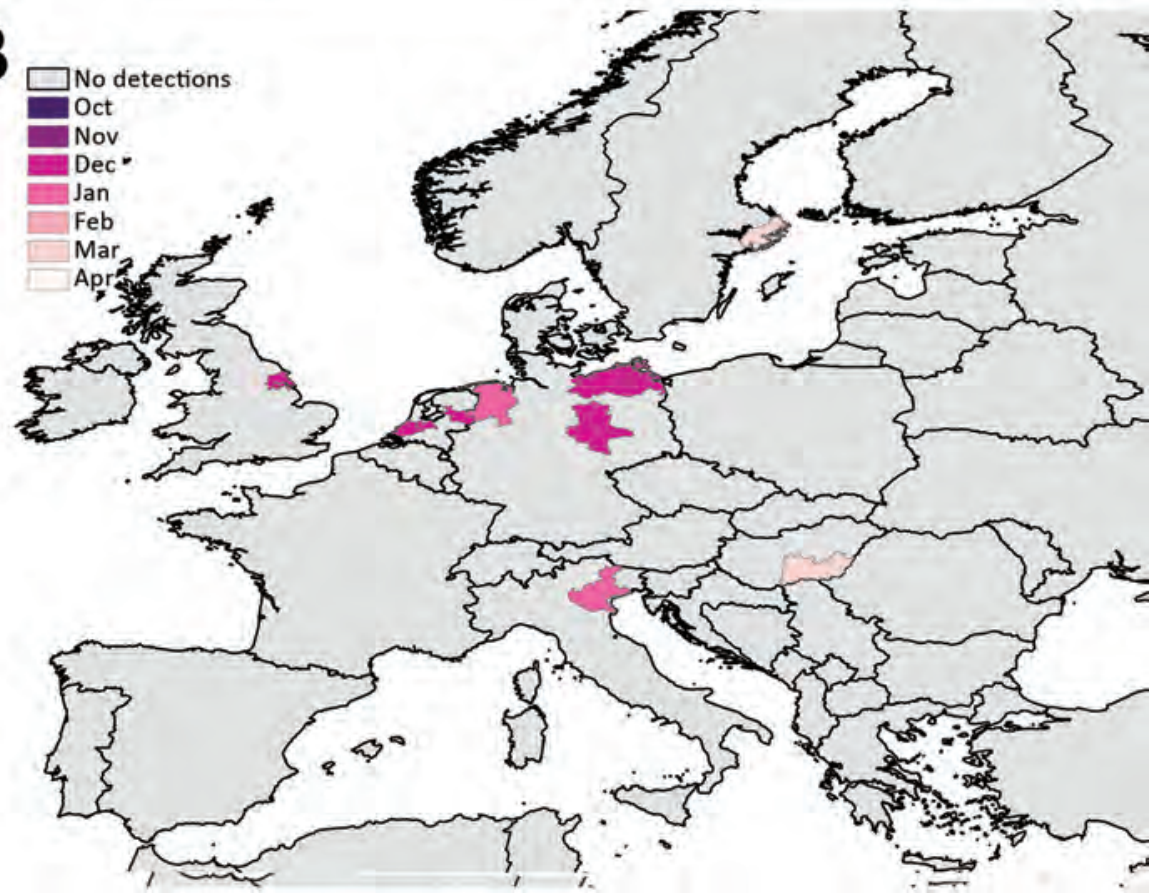

Figure 4. Geographic and temporal spread of the 2014-15 HPAI H5N8 epizootic. A) Location of each incident reported. Blue shading indicates countries where cases were reported. B) Month of first report of an HPAI H5N8 incident. Years given are epidemiologic years (October through September of the next year). HPAI, highly pathogenic avian influenza.
2005-06 and the 2 more recent epizootics may be caused by overall differences in the $\mathrm{H} 5$ lineages (clade 2.2 versus 2.3.4.4), which could influence viral spread. The greater genetic distances we observed in viruses detected in the 201415 epidemic could also be due to lower sensitivity of surveillance for this virus compared with the other 2 epidemics due to an apparently lower mortality rate in wild birds.

Extensive secondary spread is the most probable explanation for the large number of outbreaks reported in the farmed duck sector in 2016-17, possibly because of rapid attenuation of viral symptoms. Hence, on several farms with clinically healthy birds, we detected HPAI infections through active epidemiologic tracings and not on the basis of clinical signs, as reported in data from some member states. The results may also indicate that infection and transmission between domestic ducks is relatively easy for these viruses. The type of husbandry practices and frequent movement of birds, coupled with poor biosecurity and lack 


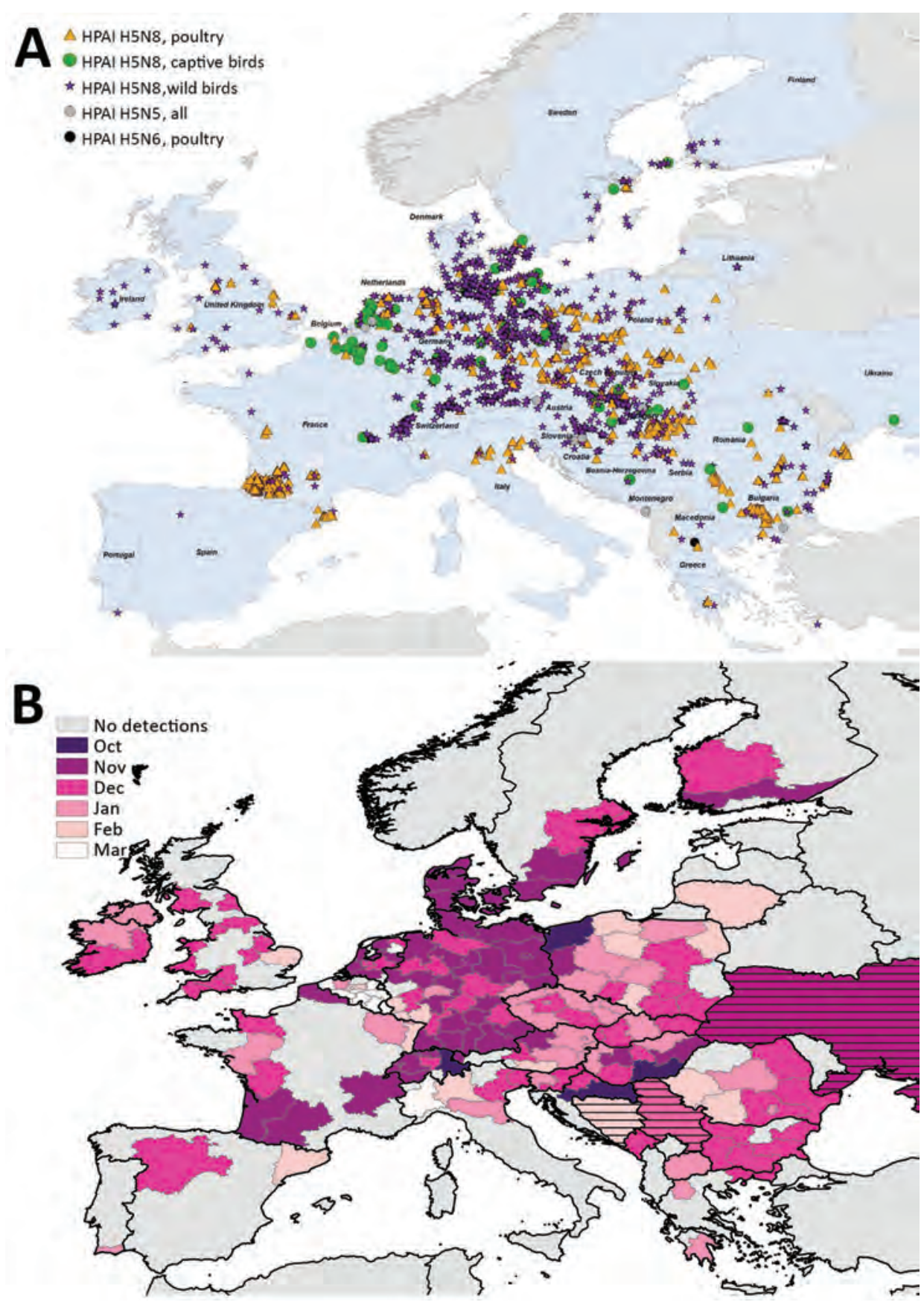

Figure 5. Geographic and temporal spread of the 2016-17 HPAI H5N8 epizootic. A) Location of each incident reported. Blue shading indicates countries where cases were reported. B) Month of first report of an HPAI H5N8 incident. Years given are epidemiologic years (October through September of the next year). HPAl, highly pathogenic avian influenza.

of robust hygiene practices, may also make the spread of the viruses between farms easier (22).

Swans and ducks were the predominant hosts infected in 2005-06 and 2016-17. Of interest, although mallards (Anas platyrhynchos) are the most frequently tested in EU passive surveillance (4), tufted ducks (Aythya fuligula) were the most commonly identified species of duck with HPAI in 2005-06 and 2016-17. In addition, the 2016-17 epizootic demonstrated a much expanded wild bird host range compared with previous outbreaks.
In light of these results, we recommend a review of the target species for avian influenza surveillance (5) to improve sensitivity of surveillance. Clarifying the precise origins of the current epizootic viruses from reported wild bird mortality data is problematic, because these data do not allow distinction between migratory carrier species and resident sentinel species. Many of the reported species are not migratory (e.g., mute swan or little grebes) and so might play a role as regional amplifiers of viruses but not in long-distance spread (23). 
Branch support (alrt)

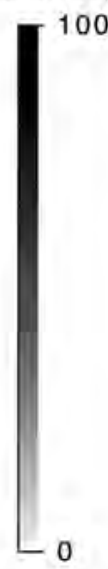

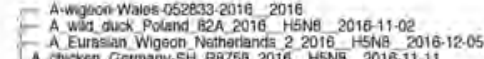

A choker Germany-SH RB756 2016 HSNB $2016-11-11$

- A chicken Germany.MV A8790 2016 H5NO 2016-11-11

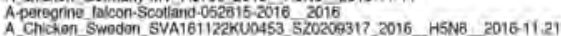

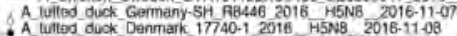

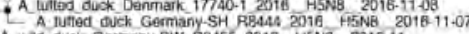

A wad duck Germany BW R9455 2016 195NG 2016.11

- A Common Goldeneye SWoder SVA161117KUOS22 H5NO_2016-11-14

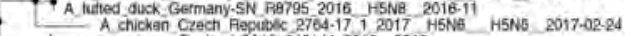
- A-eurasan wigeon-England-SA15 O19144 2016 - 2018

A Acockon Belgium 8072017 2017

A peracock Belgum 1017201720

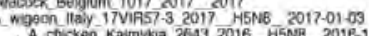

$\rightarrow$ A A creken Astraknan 3131 -2016 H5NB 2016-121

+ A turkey Aostow 112017 HENG 2017.01 .01 .2010

A goose krasnodar 3144.2017 HSNA 2017

1. A decoy duck Franos 1611046.2016 H5Ne $2016-11-20$

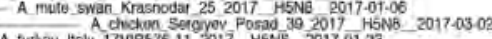

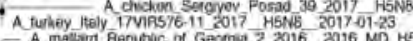

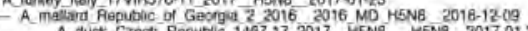

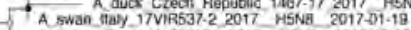

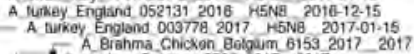

- A A Brahma Chicken Belgum 61592017

- - A A choken Luxambourg 171704472017 2017

A choken Exyland od $2299 \quad 2017 \quad 2017$

- A-ctucken-England-026832-2017 2017

A chichan ltaly 17VIF053-12 2017 H5NB 2017-01.2

- A-pheasant England 2441462016 . $2017 \mathrm{Na}$

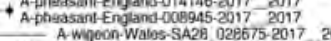

A-woon-lrotand PV16 04692320162016

A-chickan-England-0190241-2017-2017 201

A pochard duck England SA12 1578092016 H5NG 2016-12-19

A-chicken-England-038182-2017 2017

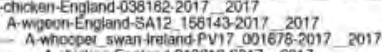

019219230172017

A domestic goose Poland 72 2016 MENG 2016-12-14

A chickon Crech Popublic 206-17222017. HSNA HENE 2017-01-06t

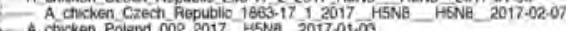

- A mule swar Czech Republic 581.17 2017 H5Na H5NO 2017.01.13

A wid duct Tatartant 30592016 H5Na 2016.10 .02

Cow

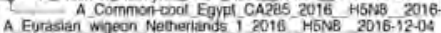

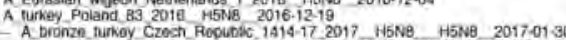

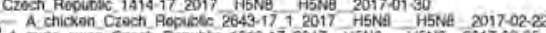

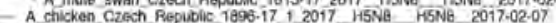

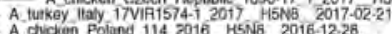

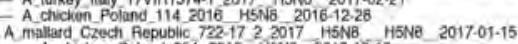
- A chickon Poland 85A, 2016 ASNB 2016-12

- A mallard Czech Repuolic 2706-17 2017 H5NB H5NE 2017-02-2

- A Indian Runner Dusk Czech Fepublic 749-17 2017 15NA HSNA 2017-01-16

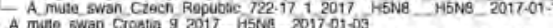

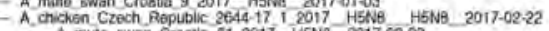

FA muse swan Croatia of 2017 HSNa $2017-02.02$

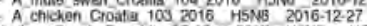

A chichor Czoch Rropubilic 55-17, 2017 H5NB H5NB 2017.01 .02

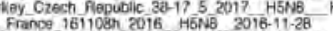

- A multe swan Croatia 302017 HiNe $2017-01-22$

- A chicker Croatia 70.2017 H5No $2017.02-09$

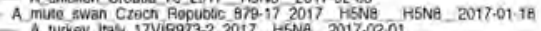

A brecder ouo Goatia 21 2017 HSNB 2017.01 .14

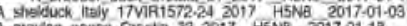

A greylag gouse Cloatla 332017 HIN 2017 . $2017.01-1$

A. mule swar Couetia 162017 HSNB 20170190

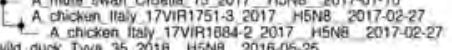

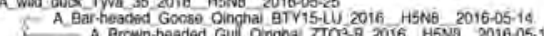

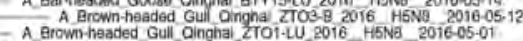

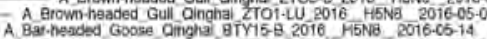

A Aar-headed Goose aimgna

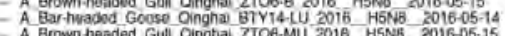

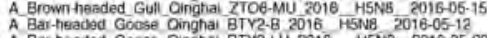

A Bar-headad Gocse Qinghal BTV9-LU 2016 H5NB 2016-05-10

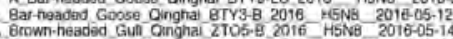

Brown-headed Guill ainghil ZTO5.K 2016 - HSNE-2016-05-14

headod Goose Oinghai BTY12.8 2010 H5NE 2016.05.13

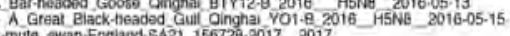

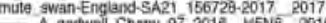

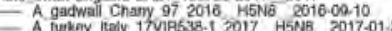

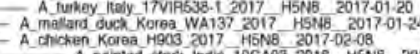

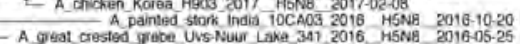

A grayiag goose Kazakhetan WR 2016 HSNA $2016-0815$

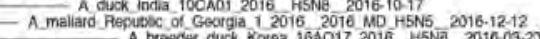

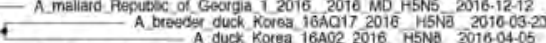

A chickon Taiwan $\times 37$. 2016 HIN Ho 2016.1221

0.0050

Figure 6. Maximum-likelihood tree from viral sequences of the 2016-17 highly pathogenic avian influenza H5 epizootic in Europe. Circles represent node support values, filled according to approximate likelihood ratio test values $0-100$. Light gray boxes indicate distinct clades with support $>50$ with isolates from Europe; dark gray boxes indicate clades with $<50$ or unresolved. Scale bar indicates nucleotide substitutions per site. An expanded figure showing trees for all 3 epizootic years is available online (https://wwwnc.cdc.gov/EID/article/24/12/17-1860-F6.htm). 
Epidemic curves for the 3 epizootics were significantly different. The incidence values in order of magnitude were 2016-17 > 2005-06 > 2014-15. In the period of the review, the mean temporal distances to the midpoint in the poultry epizootic were different; 2014-15 was relatively short, consistent with the incursion into the poultry sector and potentially lower virus infectivity present in the wild bird reservoir, whereas in 2005-06 and in 2016-17, epidemic curves show a clear peak of detection of wild bird incidence preceding the peak of poultry incidences, which demonstrates the importance of wild bird surveillance.

For the 2016-17 epizootic, the epidemic curve shows a long extended tail with small sporadic peaks relating to localized but limited detection and spread in both poultry and wild birds (Figure 2, panel C). These data might suggest greater infection pressure from migratory birds in 2016-17, leading to higher risks for incursion, greater environmental contamination, and exposure of local indigenous wild bird populations and poultry. The observed spatiotemporal relationships between poultry incursions and wild bird detections represent a complex dynamic. Exploration of the epidemic curves by country in 2016-17 shows important differences that relate to the type of poultry production infected (online Technical Appendix Figure 7). For example, we detected infections in Hungary relatively early in the epizootic; their rapid peak and decline may reflect extensive infection within the major duck-producing regions and less susceptible populations through infection and depopulation. In contrast, infection in Germany and Poland was more consistent and may reflect a more continuous exposure and incursion risk into a variety of poultry sectors.

The viruses showed close genetic similarity to viruses contemporaneously circulating in Central and Southeast Asia. The lower genetic diversity observed in 2016-17 was accompanied by reassortment of all gene segments, as shown in previous studies $(8,24,25)$. The high reassortment observed in the 2016-17 epizootic also resulted in novel NA reassortants such as the H5N6 and H5N5 viruses. The H5N6 viruses circulating in Europe were a reassortant of HPAI H5N8 and classical European LPAI present in wild birds (data not shown). We can clearly differentiate the genetic characteristics of this strain from viruses known to be circulating in poultry and wild birds in the Far East with occasional spillover to humans.

Epidemiologic results suggest 2 broad corridors of virus incursion in 2005-06 and 2016-17, through northern and central Europe with subsequent spread, later corroborated through phylogenetic analyses of the HA gene of the viruses from the 2016-17 epizootic. This dual incursion probably relates broadly to known postbreeding movements of northern duck species, which breed widely across northern Eurasia $(11,13,26)$. These movements occur on a broad front, but ringing recoveries and other analyses demonstrate movements from breeding areas from Siberia both southwest toward the Black and Aegean Seas and ultimately the coastal wetlands of the eastern Mediterranean, and further north and west through the Baltic Sea to coastal and other wetlands of the southern North Sea and northwestern countries (11-14). These represent migratory tendencies only; several studies have shown the high-level complexity of these movements and their variation due to both short-term weather patterns and longer-term climate change $(27,28)$. The fact that these corridors were apparent in 2 temporally distant epizootics suggests the need for further research to focus surveillance in these areas.

This study presents many limitations (online Technical Appendix). Differences in the implementation of passive wild bird surveillance between countries, which are implied in the EU avian influenza annual report for 2016 (20), suggest that sensitivity of wild bird surveillance varies across countries (29), which could affect the distribution of cases we observed. The true probability of detecting HPAI is dependent on many factors that may influence both the frequency of wild bird deaths and the likelihood of identification and sampling of wild bird carcasses in different regions and countries. Public awareness, the current avian influenza status of the country area, media coverage, prevailing climatic conditions, available food sources, and removal by predators may affect wild bird mortality, detection rates, or both (30). Furthermore, the efficacy of passive surveillance is difficult to measure because capturing the expended effort depends on observation and testing of deceased birds. On the other hand, surveillance has high sensitivity in farmed poultry, mainly because of higher virulence and much closer observation of these populations.

Despite apparent heavy infection pressure in wild birds in 2016-17, the virus was not detected early in the epizootic in areas in eastern Europe, such as the Danube Delta, with high density of early migratory waterfowl. There were significant incursions in poultry in northern Europe, particularly Germany and Poland, and these areas also reported the greatest number of infected wild birds. This finding may reflect the implementation of enhanced surveillance in wild bird populations rather than true increased risk. Southwestern Europe had relatively few wild bird detections compared to the number of poultry outbreaks, perhaps because of the establishment of the virus in the duck production sector in southwestern France, not as a result of increased introductions from wild birds (31).

The extent of the 2016-17 H5 epizootic indicates an urgent need to reappraise the effectiveness of surveillance strategies in both wild and domestic birds and to monitor key populations for emergence of viral variants. The differences we observed in the 3 epizootics illustrate the difficulty of predicting HPAI epizootics. However, the temporal peak of wild bird detections preceding the peak of 
poultry outbreaks at the EU level highlighted the utility of surveillance in wild birds, as observed in other studies (29). The spatial corridors of HPAI we identified may provide the basis for an increase in targeted surveillance to improve system sensitivity. Although the H5N8, H5N5, and H5N6 European-reassortant viruses have not been shown to infect humans and remain avian influenza-like strains with no evidence of key mammalian adaptation markers (27), their genetic volatility represents a potential threat that requires continuous monitoring and surveillance of virus incidence and genetics to continue to protect public safety.

\section{Acknowledgments}

The following laboratories supplied virus sequence data used in our analysis: National Food Chain Safety Office, Veterinary Diagnostic Directorate, Laboratory for Molecular Biology, Hungary; the Croatian Veterinary Institute, Croatia; the National Veterinary Research Institute, Poland; Wageningen UR, the Netherlands; the National Veterinary Institute, Denmark; the Friedrich-Loeffler Institute, Germany; National Veterinary Institute, Sweden; Wageningen University and Research Centre, the Netherlands.

The work at Animal and Plant Health Agency was jointly funded by the European Commission and the Department for Environment, Food and Rural Affairs, London, through the EU reference laboratory. This work was funded in part by National Institute of Allergy and Infectious Diseases (NIAID)-funded Centers of Excellence in Influenza Research and Surveillance (contract HHSN272201400008C) and a US Defense Threat Reduction Agency Broad Agency Announcement award (FRBAA09-6-2-0114).

\section{About the Author}

Dr. Alarcon is a lecturer in animal health economics at the Royal Veterinary College, London. During this study, he was a veterinary epidemiologist at the Animal and Plant Health Agency, United Kingdom, where his role and research focused on the analysis of avian influenza surveillance data in Europe.

\section{References}

1. Sims LD, Brown IH. Multi-continental panzootic of H5 highly pathogenic avian influenza (1996-2015). In: Swayne DE, editor. Animal influenza, 2nd ed. New York: Wiley \& Sons; 2016.

2. Global Consortium for H5N8 and Related Influenza Viruses. Role for migratory wild birds in the global spread of avian influenza H5N8. Science. 2016;354:213-7. http://dx.doi.org/10.1126/science. aaf8852

3. Hesterberg U, Harris K, Stroud D, Guberti V, Busani L, Pittman M, et al. Avian influenza surveillance in wild birds in the European Union in 2006. Influenza Other Respi Viruses. 2009;3:1-14. http://dx.doi.org/10.1111/j.1750-2659.2008.00058.x

4. European Union Reference Laboratory For Avian Influenza. Annual report on surveillance for avian influenza in poultry and in wild birds in member states of the European Union in 2015. 2016 [cited
2018 Sep 28]. https://ec.europa.eu/food/sites/food/files/ad control-measures_ai_surv-rslt_pltry-wld-brds_2015.pdf

5. European Union Occupational Health and Safety Information Service. Commission decision 2010/367/EU of 25 June 2010 on the implementation by Member States of surveillance programmes for avian influenza in poultry and wild birds. Official Journal of the European Union. 2010;166:22-32.

6. European Food Safety Authority. Highly pathogenic avian influenza A subtype H5N8. EFSA Journal. 2014;12:3941-32.

7. Lee, DH, Torchetti MK, Winker K, Ip HS, Song CS, Swayne DE, Intercontinental spread of Asian-origin H5N8 to North America through Beringia by migratory birds. J Virol. 2015;89:6521-4.

8. Lee DH, Sharshov K, Swayne DE, Kurskaya O, Sobolev I, Kabilov M, et al. Novel reassortant clade 2.3.4.4 avian influenza A(H5N8) virus in wild aquatic birds, Russia, 2016. Emerg Infect Dis. 2017;23:359-60. http://dx.doi.org/10.3201/eid2302.161252

9. Boere G, Galbraith C, Stroud D; Scottish Natural Heritage. The flyway concept: what it is and what it isn't. In: Waterbirds around the world: a global overview of the conservation, management, and research of the world's waterbird flyways, Edinburgh: The Stationery Office; 2006.

10. Delany S, Scott D, Dodman T, Stroud DA. An atlas of wader populations in Africa and western Eurasia. Wageningen (the Netherlands): Wetlands International; 2009.

11. Scott DA, Rose PM. Atlas of Anatidae populations in Africa and western Eurasia. Publication No. 41. Wageningen (the Netherlands): Wetlands International; 1996.

12. Wernham CV, Toms M, Marchant JH, Clark J, Siriwardena G, Baillie S, editors. The migration atlas: movements of the birds of Britain and Ireland. London: T. \& A.D. Poyser Ltd; 2002.

13. Viksne J, Švažas S, Czajkowski A, Janaus M, Mischenko A, Kozulin A, et al. Atlas of duck populations in eastern Europe. Vilnius (Lithuania): Oiseaux Migrateurs du Palearctique Occidental; 2010.

14. Veen J, Delany S. An atlas of movements of southwest Siberian waterbirds. Wageningen (the Netherlands): Wetlands International; 2005.

15. Nguyen LT, Schmidt HA, von Haeseler A, Minh BQ. IQ-TREE: a fast and effective stochastic algorithm for estimating maximumlikelihood phylogenies. Mol Biol Evol. 2015;32:268-74. http://dx.doi.org/10.1093/molbev/msu300

16. Fu L, Niu B, Zhu Z, Wu S, Li W. CD-HIT: accelerated for clustering the next-generation sequencing data. Bioinformatics. 2012;28:3150-2. http://dx.doi.org/10.1093/bioinformatics/bts565

17. Rambaut A, Carvalho LM. Exploring the temporal structure of heterochronous sequences using TempEst (formerly Path-O-Gen). Virus Evolution. 2016;2:vew007.

18. Drummond AJ, Suchard MA, Xie D, Rambaut A. Bayesian phylogenetics with BEAUti and the BEAST 1.7. Mol Biol Evol. 2012;29:1969-73. http://dx.doi.org/10.1093/molbev/mss075

19. Scoizec A, Niqueux E, Thomas R, Daniel P, Schmitz A, Le Bouquin S. Airborne detection of H5N8 highly pathogenic avian influenza virus genome in poultry farms, France. Front Vet Sci. 2018;5:15. http://dx.doi.org/10.3389/fvets.2018.00015

20. European Union Reference Laboratory For Avian Influenza. Annual report on surveillance for avian influenza in poultry and wild birds in member states of the European Union in 2016. 2017 [cited 2018 Apr 27]. https://ec.europa.eu/food/sites/food/files/animals/docs/ ad_control-measures_ai_surv-rslt_pltry-wld-brds_2016.pdf.

21. Hesterberg U, Harris K, Cook A, Brown I. Annual report of the EU avian influenza surveillance in wild birds 2006. Community Reference Laboratory for Avian Influenza and Newcastle Disease, European Commission 2007 [cited 2018 Apr 27]. https://ec.europa. $\mathrm{eu} /$ food/sites/food/files/animals/docs/ad_control-measures_ai surv-rslt_wld-brds_2006.pdf. 
22. Guinat C, Nicolas G, Vergne T, Bronner A, Durand B, Courcoul A, et al. Spatio-temporal patterns of highly pathogenic avian influenza virus subtype H5N8 spread, France, 2016 to 2017. Euro Surveill. 2018; 23. http://dx.doi.org/10.2807/1560-7917.ES.2018. 23.26.1700791

23. Hill NJ, Takekawa JY, Ackerman JT, Hobson KA, Herring G, Cardona CJ, et al. Migration strategy affects avian influenza dynamics in mallards (Anas platyrhynchos). Mol Ecol. 2012;21:598699. http://dx.doi.org/10.1111/j.1365-294X. 2012.05735.x

24. Pohlmann A, Starick E, Harder T, Grund C, Höper D, Globig A, et al. Outbreaks among wild birds and domestic poultry caused by reassorted influenza $\mathrm{A}(\mathrm{H} 5 \mathrm{~N} 8)$ clade 2.3.4.4 viruses, Germany, 2016. Emerg Infect Dis. 2017;23:633-6. http://dx.doi.org/10.3201/ eid2304.161949

25. Fusaro A, Monne I, Mulatti P, Zecchin B, Bonfanti L, Ormelli S, et al. Genetic diversity of highly pathogenic avian influenza A(H5N8/H5N5) viruses in Italy, 2016-17. Emerg Infect Dis. 2017;23:1543-7. http://dx.doi.org/10.3201/eid2309.170539

26. Atkinson PW, Robinson RA, Clark JA, Miyar T, Downie IS, du Feu CR, et al. Migratory movements of waterfowl: a web-based mapping tool. EURING report to the EU Commission. 2007. https://blx1.bto.org/ai-eu/main/data-home.jsp/

27. Lehikoinen A, Jaatinen K, Vähätalo AV, Clausen P, Crowe O, Deceuninck B, et al. Rapid climate-driven shifts in wintering distributions of three common waterbird species. Glob Change Biol. 2013;19:2071-81. http://dx.doi.org/10.1111/ gcb. 12200

28. Pavón-Jordán D, Fox AD, Clausen P, Dagys M, Deceuninck B, Devos K, et al. Climate-driven changes in winter abundance of a migratory waterbird in relation to EU protected areas. Divers Distrib. 2015;21:571-82. http://dx.doi.org/10.1111/ddi.12300

29. Breed AC, Harris K, Hesterberg U, Gould G, Londt BZ, Brown IH, et al. Surveillance for avian influenza in wild birds in the European Union in 2007. Avian Dis. 2010;54(Suppl):399-404. http://dx.doi.org/10.1637/8950-053109-Reg.1

30. Breed AC, Irvine RM, Duncan D, Rae D, Snow L, Cook AJ, et al. An evaluation of wild bird avian influenza surveillance in Great Britain. Avian Dis. 2012;56(Suppl):986-91. http://dx.doi.org/ 10.1637/10166-040912-Reg.1

31. Bahl J, Pham TT, Hill NJ, Hussein IT, Ma EJ, Easterday BC, et al. Ecosystem interactions underlie the spread of avian influenza A viruses with pandemic potential. PLoS Pathog. 2016;12:e1005620. http://dx.doi.org/10.1371/journal.ppat.1005620

Address for correspondence: Adam Brouwer, Animal and Plant Health Agency, Woodham Lane Addlestone, Surrey, KT15 3NB, UK; email: adam.brower@apha.gsi.gov.uk

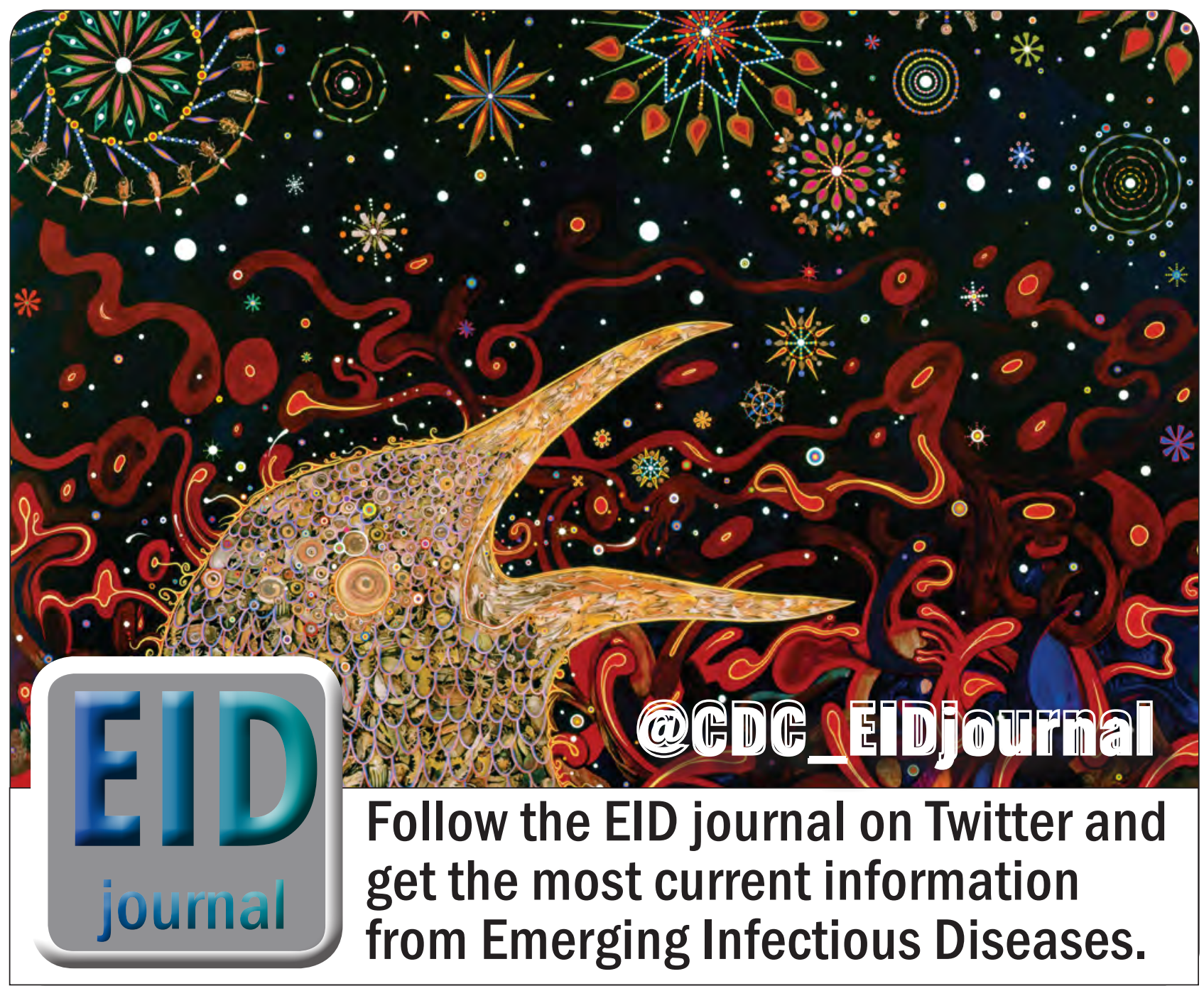




\title{
CTX-M-65 Extended-Spectrum $\beta$-Lactamase-Producing Salmonella enterica Serotype I nfantis, United States ${ }^{1}$
}

\author{
Allison C. Brown, Jessica C. Chen, Louise K. Francois Watkins, Davina Campbell, \\ Jason P. Folster, Heather Tate, Jamie Wasilenko, Christine Van Tubbergen, Cindy R. Friedman
}

Extended-spectrum $\beta$-lactamases (ESBLs) confer resistance to clinically important third-generation cephalosporins, which are often used to treat invasive salmonellosis. In the United States, ESBLs are rarely found in Salmone/la. However, in 2014, the US Food and Drug Administration found bla $a_{\text {CTX-M- } 65}$ ESBL-producing Salmonella enterica serotype Infantis in retail chicken meat. The isolate had a rare pulsed-field gel eletrophoresis pattern. To clarify the sources and potential effects on human health, we examined isolates with this pattern obtained from human surveillance and associated metadata. Using broth microdilution for antimicrobial susceptibility testing and whole-genome sequencing, we characterized the isolates. Of 34 isolates, 29 carried the bla $a_{\text {CTX-M-65 }}$ gene with $\leq 9$ additional resistance genes on 1 plasmid. Of 19 patients with travel information available, $12(63 \%)$ reported recent travel to South America. Genetically, isolates from travelers, nontravelers, and retail chicken meat were similar. Expanded surveillance is needed to determine domestic sources and potentially prevent spread of this ESBLcontaining plasmid.

A leading cause of bacterial foodborne disease in the United States is nontyphoidal Salmonella (1). Salmonella enterica serotype Infantis (hereafter called Salmonella Infantis) is one of the most common Salmonella serotypes in the United States and 1 of 3 serotypes for which incidence has substantially increased (by 60\%) in the past 10 years (2). Salmonella Infantis has been identified in a variety of foods, animals, and environmental settings.

Author affiliations: Centers for Disease Control and Prevention, Atlanta, Georgia, USA (A.C. Brown, J.C. Chen, L.K. Francois Watkins, D. Campbell, J.P. Folster, C. Van Tubbergen, C.R. Friedman); Food and Drug Administration, Laurel, Maryland, USA (H. Tate); US Department of Agriculture, Athens, Georgia, USA (J. Wasilenko)

DOI: https://doi.org/10.3201/eid2412.180500
Salmonellosis usually causes a self-limited gastroenteritis; however, current guidelines recommend that antimicrobial therapy be considered for groups of persons at increased risk for invasive infection. For those patients, treatment with ceftriaxone, ciprofloxacin, trimethoprim/ sulfamethoxazole, or amoxicillin is recommended (3). Extended-spectrum $\beta$-lactamases (ESBLs) confer resistance to most third-generation cephalosporins and penicillins, including ampicillin.

Some Enterobacteriaceae produce CTX-M ESBLs, which are encoded by bla $a_{\text {Стх-м }}$ genes that were discovered in 1989 (4). Since then, their prevalence has increased dramatically (5) and they have been isolated worldwide, primarily from Escherichia coli (6). Identification of ESBLs, including the CTX-M types, in Salmonella in the United States is relatively rare $(7,8)$.

In the United States, the National Antimicrobial Resistance Monitoring System (NARMS) is a surveillance system that tracks changes in the antimicrobial susceptibility of certain enteric bacteria isolated from ill persons, retail meats, and food animals. In July 2015, the US Food and Drug Administration (FDA) notified the Centers for Disease Control and Prevention (CDC) of a CTX-M-65producing Salmonella Infantis strain isolated from retail chicken meat in December 2014 (9). The isolate had a rare pulsed-field gel electrophoresis pattern, JFXX01.0787 (pattern 787). To clarify the sources and potential effects of this strain on human health, we analyzed data from several CDC surveillance systems to describe the prevalence, epidemiology, antimicrobial drug resistance, and molecular phylogenetics of Salmonella Infantis pattern 787 isolates from humans.

${ }^{1}$ Preliminary results from this analysis were presented at the International Salmonella and Salmonellosis Symposium, June 6-8, 2016, St. Malo, France, and at the International Association for Food Protection Annual Meeting, July 9-12, 2017, Tampa, Florida, USA. 


\section{Methods}

\section{Background Rates}

To determine the expected demographics, rates of hospitalization, and international travel among patients with Salmonella Infantis infections compared with patients with infections caused by other common nontyphoidal Salmonella serotypes, we analyzed data collected through the Foodborne Disease Active Surveillance Network (FoodNet; https://www.cdc.gov/foodnet/index.html) during 2012-2015. Begun in 1996, FoodNet has conducted active, population-based surveillance for culture-confirmed cases of infection caused by 9 pathogens transmitted commonly through food, including Salmonella. FoodNet is a collaboration of CDC, 10 state health departments, the US Department of Agriculture Food Safety and Inspection Service (USDA-FSIS), and the FDA. The FoodNet surveillance area includes $15 \%$ of the US population; these data are used to estimate the burden of US foodborne illnesses, hospitalizations, and deaths (1). We defined other common nontyphoidal Salmonella as the top 20 S. enterica serotypes (excluding Infantis) isolated from humans: Typhimurium, Enteritidis, Newport, Heidelberg, Javiana, Saintpaul, Montevideo, Agona, Oranienburg, Muenchen, Thompson, Hadar, Braenderup, Derby, I 4,[5],12:i:-, Paratyphi B var. $\mathrm{L}(+)$ tartrate+, Blockley, Anatum, Mississippi, and Panama. These 20 serotypes represented $69 \%$ of nontyphoidal Salmonella isolates reported to FoodNet in 2015.

\section{Case Finding}

We looked for pattern 787 isolates reported to the National Molecular Subtyping Network for Foodborne Disease Surveillance (PulseNet; https://www.cdc.gov/pulsenet/index. html) through 2017. The PulseNet database contains pulsedfield gel electrophoresis patterns from state and local public health laboratories and food regulatory agencies. Only the first isolate from each patient was included in case counts.

CDC also requested patient data and clinical isolates from state and local public health departments for any case with pattern 787 reported through October 2015. Patient data included age, sex, date of symptom onset, hospitalization, and recent international travel (defined as travel outside of the United States in the 7 days before symptom onset). Isolate data included specimen collection date(s) and source.

\section{Isolate Characterization}

We used the NARMS standard broth microdilution protocol (Sensititer; Thermo Fisher Scientific, Oakwood Village, OH, USA) to determine the MICs for 14 antimicrobial agents: gentamicin, streptomycin, ampicillin, amoxicillin/ clavulanic acid, ceftiofur, ceftriaxone, cefoxitin, azithromycin, sulfasoxazole, trimethoprim/sulfamethoxazole, chloramphenicol, ciprofloxacin, nalidixic acid, and tetracycline (10). These agents were categorized into 9 classes defined by the Clinical and Laboratory Standards Institute guidelines (https://clsi.org/); where available, CLSI interpretive criteria were used to define resistance. Transformation studies to confirm plasmid-associated genes were conducted by using plasmid purification and electroporation as previously described (11).

Whole-genome sequencing was performed according to the PulseNet protocol for the Illumina MiSeq (Illumina, La Jolla, CA, USA) (12). Closed PacBio genomes were generated as part of a previous study and used as references where indicated (8). Genome assemblies for short-read data were generated de novo and analyzed for acquired antimicrobial drug-resistance determinants and plasmid replicons by using ResFinder and PlasmidFinder $(13,14)$. To confirm the absence of certain genes, we performed read mapping in CLC Genomics Workbench version 8.5 (https://www.qiagenbioinformatics.com/products/clc-genomics-workbench/). To identify mutational resistance, we extracted the gyrA and parC genes from genome assemblies by using a perl script (https://github.com/lskatz/lskScripts/blob/master/blastAndExtract.pl). To identify mutations in the quinolone resistance-determining regions of these genes, we aligned gene sequences in CLC Workbench. To assess isolate relatedness, we generated high-quality single-nucleotide polymorphism (hqSNP) phylogenies. In brief, isolates were aligned to the closed chromosomal sequence of 2014AM-3028 (omitting the plasmid contig) by using Lyve-SET-v1.1.4f (https:// github.com/lskatz/lyve-SET/). Genome alignments were processed by using Gubbins (https://sanger-pathogens. github.io/gubbins/) to omit areas of recombination, uninformative sites were removed, and the resulting SNP alignment was used to calculate pairwise differences and generate hqSNP trees by using scripts bundled with Lyve-SET $(15,16)$. We performed a phylogeographic analysis (a type of molecular clock analysis) by using Bayesian Evolutionary Analysis Sampling Trees (BEAST; https://journals.plos.org/ ploscompbiol/article?id=10.1371/journal.pcbi.1003537).

To sample more diverse Salmonella Infantis isolates, we obtained sequence data on isolates from sources other than CDC NARMS assigned to the same National Center for Biotechnology Information (NCBI) SNP cluster PDS000003955.192 as our study isolates on the NCBI Pathogen Detection page (17). These additional genomes were from isolates recovered from hospitalized patients, meat, and environmental samples from Peru during 2010-2014 and collected by the Center for Food Safety and Applied Nutrition at FDA and the US Naval Medical Research Unit (18), and isolates from chicken samples obtained at slaughter by USDA-FSIS. These isolates were used to produce a time-measured maximum clade credibility tree to estimate the dates that study isolates in addition to all isolates (study 
isolates and isolates from Peru and cecal isolates from USDA-FSIS) shared most recent common ancestors (MRCAs), and the geographic location of these MRCAs (18). The date of the MRCA was estimated by reporting the height of the most ancestral node of the maximum clade credibility tree and the $95 \%$ highest posterior density (HPD) intervals for these estimates. More details on genetic analyses are available in the online Technical Appendix (https://wwwnc.cdc. gov/EID/article/24/12/18-0500-Techapp1.pdf).

\section{Statistical Analyses}

We used the $\chi^{2}$ test (or Fisher exact test when cell counts were $\leq 5$ ) for statistical comparisons. All denominators exclude persons for whom data were missing. We considered $\mathrm{p}<0.05$ to be significant. All $\mathrm{p}$ values were 2-tailed. We used SAS 9.3 or 9.4 (SAS Institute, Cary, NC, USA) to conduct our analyses.

\section{Results}

The first pattern 787 Salmonella Infantis isolate from a human in the United States was collected from a patient in June 2012. By the end of 2017, PulseNet contained 312 Salmonella Infantis pattern 787 isolates from persons living in 43 states; Washington, DC; and Puerto Rico. The number of cases detected each year increased from 5 in 2012 to 174 in 2017 and represented $8.4 \%$ of all Salmonella Infantis isolated that year (Figure 1).

State health departments submitted 34 pattern 787 isolates from humans to $\mathrm{CDC} ; 29$ (85\%) had resistance phenotypes consistent with ESBL-conferred resistance to ceftriaxone, ceftiofur, and ampicillin (Tables 1, 2). All 29 isolates with ESBL-resistant phenotypes had the bla $a_{\mathrm{CTX}}$ ${ }_{\mathrm{M}-65}$ gene. In addition to ESBL-conferred resistance, these isolates were also resistant to chloramphenicol, sulfisoxazole, tetracycline, nalidixic acid, and trimethoprim/sulfamethoxazole and intermediately susceptible to ciprofloxacin and gentamicin. Resistance to these drugs was plasmid mediated and transferable to E. coli, except for nalidixic acid and ciprofloxacin, for which resistance was caused by a chromosomal mutation in gyrA (D87Y). Transferable resistance resulted from a large IncFIB-like plasmid containing the resistance genes $a p h\left(3^{\prime}\right)-I c$, aph (4)-Ia, aadA1, aac (3)-IVa, bla ${ }_{\text {СтX-м-65 }}$, fosA, floR, sul1, tetA, and dfrA14. These observations are consistent with the organization of these genes on a single IncF1B-like plasmid closed by using long read sequencing, which was completed as part of a previous study by Tate et al., who recently published a further detailed description of this plasmid and a comparative genomics analysis of $b l a_{\text {СTX-M-65 }}$-positive IncFIB-like plasmids from Salmonella Infantis in the United States (9).

We focused our analysis on the 29 patients with Salmonella Infantis isolates containing the bla $a_{\text {Стх-м-65 }}$ gene (hereafter called CTX-M-65 Infantis). We compared patients

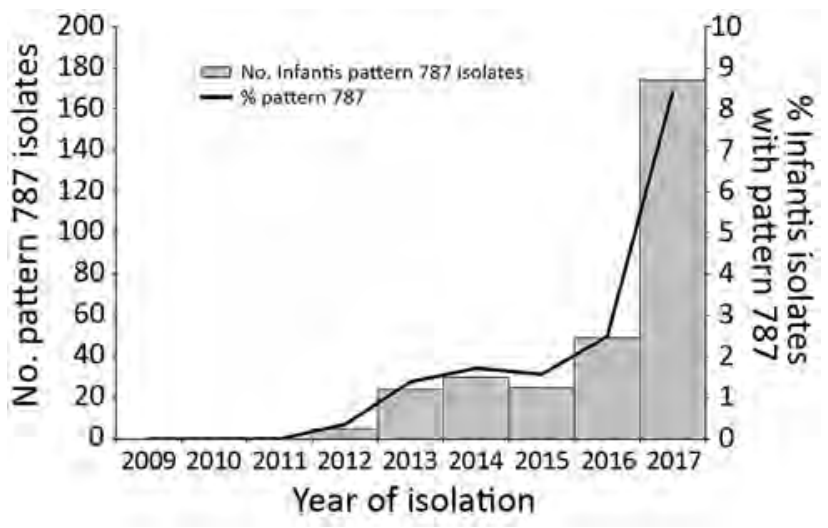

Figure 1. Total of 312 Salmonella enterica serotype Infantis isolates from humans with pattern JFXX01.0787 as a percentage of all Salmonella Infantis isolates by year, United States, 2012-2017. Source: PulseNet (https://www.cdc.gov/ pulsenet/index.html).

infected with CTX-M-65 Infantis with patients infected with all strains of Salmonella Infantis and with patients infected with common Salmonella serotypes other than Infantis. The median age of patients with CTX-M-65 Infantis was 25 years (interquartile range [IQR] $15-50$ years), and $69 \%$ were female (Table 3). The median age of patients with any strain of Salmonella Infantis was 37 years (IQR 12-58 years), and 56\% were female. The median age of patients infected with other common Salmonella serotypes was 29 years (IQR 6-54 years), and 52\% were female.

Of 18 patients infected with CTX-M-65 Infantis for whom outcome data were available, 8 (44\%) were hospitalized, compared with a hospitalization rate of $29 \%$ among patients in both comparison groups (those with Infantis and those with other Salmonella serotypes; Table 3). Patients with CTX-M-65 Infantis infection were more likely (17\%) to have the organism isolated from urine compared with all patients with Salmonella Infantis $(9 \%, \mathrm{p}=0.14)$ and patients with other common serotypes $(5 \%, \mathrm{p}<0.01)$.

For 20 CTX-M-65 Infantis-infected patients with information about symptom onset date and isolation date(s), Salmonella Infantis was recovered from 12 who provided fecal samples $>2$ weeks after their reported date of symptom onset. A total of 8 patients were still reporting symptoms and had positive cultures $>30$ days after symptom onset, including 3 patients who sought care and had multiple isolates recovered from 50 days to 8 months after symptom onset.

Twelve $(63 \%)$ patients with CTX-M-65 Infantis infections reported international travel in the 7 days before symptom onset. All reported travel to South America, 10 to Peru, and 2 to Ecuador (Table 3). Travel was less common among all Salmonella Infantis-infected patients (7\%, $\mathrm{p}<0.01)$ and those infected with other common serotypes $(8 \%, \mathrm{p}<0.01$; Table 3$)$. 
A maximum-likelihood hqSNP phylogeny of the 34 isolates from humans and that from retail chicken meat revealed that isolates from patients with travel-associated infections formed a well-supported clade (clade A) with isolates from patients with infections not associated with travel (Figure 2). Isolates in clade A differed by only 2-47 pairwise hqSNPs, suggesting that these isolates recently evolved from a common ancestor. Included in clade A was the isolate from retail chicken meat collected in 2014.

Of the 5 CTX-M-65-negative isolates, 2 grouped within clade $\mathrm{A}$ and contained a multidrug-resistance plasmid that was lacking the bla $a_{\text {СтХ-м-65 }}$ gene. An additional 3 CTXM-65-negative isolates lacked the IncFIB-like plasmid replicon, lacked resistance determinants, and differed by

\begin{tabular}{|c|c|c|c|c|c|c|c|c|c|}
\hline $\begin{array}{l}\text { Accession } \\
\text { no. } \dagger\end{array}$ & Year & $\begin{array}{l}\text { Specimen } \\
\text { source }\end{array}$ & Resistance profile & Plasmids & gyrA & parC & Hospitalized & $\begin{array}{l}\text { Recent } \\
\text { travel }\end{array}$ & $\begin{array}{l}\text { Prolonged } \\
\text { infection } \ddagger\end{array}$ \\
\hline SRR2485281 & 2012 & Urine & $\begin{array}{l}\text { ACSuTCxTioCip(I)NalCot } \\
\text { Gen(I) }\end{array}$ & IncFIB-like & D87Y & Wt & Yes & Peru & \\
\hline SRR2485278 & 2012 & Blood & $\begin{array}{l}\text { ACSuTCxTioFoxCip(I)NalCot } \\
\text { Gen(I) }\end{array}$ & IncFIB-like & D87Y & Wt & No data & Peru & Yes \\
\hline SRR4019593 & 2013 & Feces & ASuTCxTioCip(I)NalCotGen & IncFIB-like & D87Y & $\mathrm{Wt}$ & No data & No data & \\
\hline SRR4019592 & 2013 & Feces & ACSuTCxTioCip(I)NalCotGen & IncFIB-like & D87Y & Wt & No data & No data & \\
\hline SRR2485284 & 2013 & Feces & $\begin{array}{c}\text { ACSuTCxTioCip(I)NalCot } \\
\text { Gen(I) }\end{array}$ & IncFIB-like & D87Y & Wt & No data & No data & Yes \\
\hline SRR4025935 & 2013 & Feces & ACSuTCxTioCip(I)Nal & IncFIB-like & D87Y & Wt & No & Peru & \\
\hline SRR4025936 & 2013 & Feces & ACSuTCxTioCip(I)NalCot & IncFIB-like & D87Y & $\mathrm{Wt}$ & No data & Peru & Yes \\
\hline SRR3178069 & 2013 & Feces & ACSuTCxTioCip(I)NalCot & IncFIB-like & D87Y & $\mathrm{Wt}$ & No & Peru & Yes \\
\hline SRR4019589 & 2014 & Feces & $\begin{array}{l}\text { ACSSuTCxTioCip(I)NalCot } \\
\text { Gen }\end{array}$ & IncFIB-like & D87Y & Wt & No & Ecuador & \\
\hline SRR2407791 & 2014 & $\begin{array}{c}\text { Retail } \\
\text { chicken } \\
\text { meat }\end{array}$ & ACTCxTioCip(I)NalGen(I) & IncFIB-like & D87Y & Wt & NA & NA & NA \\
\hline SRR2353201 & 2014 & Feces & $\begin{array}{l}\text { ACSSuTCxTioCip(I)NalCot } \\
\text { Gen(I) }\end{array}$ & IncFIB-like & D87Y & Wt & No data & No data & \\
\hline SRR4025938 & 2014 & Feces & $\begin{array}{c}\text { ACSuTCxTioCip(I)NalCot } \\
\text { Gen(I) }\end{array}$ & IncFIB-like & D87Y & Wt & No data & No data & \\
\hline SRR2485287 & 2014 & Feces & $\begin{array}{l}\text { ACSSuTAug(I)CxTioFox } \\
\text { Cip(I)NalCotGen(I) }\end{array}$ & IncFIB-like & D87Y & Wt & No data & No data & Yes \\
\hline SRR4019601 & 2014 & Feces & ASSuTCxTioCip(I)NalCotGen & IncFIB-like & D87Y & Wt & No data & No data & Yes \\
\hline SRR4019595 & 2014 & Feces & ACSSuTCxTioCip(I)NalCot & IncFIB-like & D87Y & Wt & No & No travel & \\
\hline SRR2485288 & 2014 & Feces & $\begin{array}{l}\text { ACSSuTCxTioCip(I)NalCot } \\
\text { Gen(I) }\end{array}$ & IncFIB-like & D87Y & Wt & No data & No travel & \\
\hline SRR2485282 & 2014 & Feces & $\begin{array}{l}\text { ACSSuTCxTioCip(I)NalCot } \\
\text { Gen(I) }\end{array}$ & IncFIB-like & D87Y & Wt & No & Peru & Yes \\
\hline SRR2485286 & 2014 & Feces & ASSuTCxTioCip(I)NalCotGen & IncFIB-like & D87Y & $\mathrm{Wt}$ & Yes & Peru & \\
\hline SRR3178071 & 2014 & Feces & ACSSuTCxTioCip(I)NalCot & IncFIB-like & D87Y & Wt & Yes & Peru & \\
\hline SRR4019602 & 2015 & Feces & $\begin{array}{l}\text { ACSSuTCxTioFox(I)Cip(I)Nal } \\
\text { Cot }\end{array}$ & IncFIB-like & D87Y & Wt & No & Ecuador & \\
\hline SRR4019594 & 2015 & Feces & ACSSuTCxTioCip(I)NalCot & IncFIB-like & D87Y & Wt & No data & No data & \\
\hline SRR3185043 & 2015 & Urine & ACSSuTCxTioCip(I)NalCot & IncFIB-like & D87Y & $\mathrm{Wt}$ & No & No data & Yes \\
\hline SRR4019591 & 2015 & $\begin{array}{l}\text { Feces; } \\
\text { urine }\end{array}$ & ACSSuTCxTioCip(I)NalCot & IncFIB-like & D87Y & Wt & Yes & No data & Yes \\
\hline SRR4019588 & 2015 & Feces & $\begin{array}{l}\text { ACSuTCxTioCip(I)NalCot } \\
\text { Gen(I) }\end{array}$ & $\begin{array}{l}\text { IncFIB-like, } \\
\text { colE }\end{array}$ & D87Y & Wt & Yes & No travel & Yes \\
\hline SRR4019587 & 2015 & Feces & $\begin{array}{l}\text { ACSSuTCxTioCip(I)Nal } \\
\text { Gen(I) }\end{array}$ & IncFIB-like & D87Y & Wt & No & No travel & \\
\hline SRR3184311 & 2015 & Feces & $\begin{array}{l}\text { ACSSuTCXTioCip(I)NalCot } \\
\text { Gen(I) }\end{array}$ & IncFIB-like & D87Y & Wt & Yes & No travel & Yes \\
\hline SRR4019590 & 2015 & Feces & ACSuTCxTioCip(I)NalCot & $\begin{array}{l}\text { pXuzhou21, } \\
\text { IncFIB-like }\end{array}$ & D87Y & Wt & Yes & No travel & \\
\hline SRR4019603 & 2015 & Urine & ACSSuTCxTioCip(I)NalCot & IncFIB-like & D87Y & $\mathrm{Wt}$ & No & No travel & \\
\hline SRR3178070 & 2015 & Urine & ACSSuTCxTioCip(I)NalCot & IncFIB-like & D87Y & $\mathrm{Wt}$ & Yes & Peru & \\
\hline SRR4019600 & 2015 & Feces & ACSuTCxTioCip(I)NalCot & IncFIB-like & D87Y & $\mathrm{Wt}$ & No & Peru & Yes \\
\hline \multicolumn{10}{|c|}{$\begin{array}{l}\text { *A, ampicillin; Aug, amoxicillin-clavulanic acid; C, chloramphenicol; Cip, ciprofloxacin; Cot, trimethoprim/sulfamethoxazole; Cx, ceftriaxone; Cx, } \\
\text { ceftriaxone; Fox, cefoxitin; Gen, gentamicin; (I), intermediate susceptibility; NA, not applicable; Nal, nalidixic acid; S, streptomycin; Su, sulfisoxazole; T, } \\
\text { tetracycline; Tio, ceftiofur. } \\
\text { †Accession numbers are the sequence read archive run identification numbers from the National Center for Biotechnology Information. } \\
\text { fDocumented illness >2 wk after symptom onset. Blank cells indicate no evidence of prolonged infection (either no additional data were available or } \\
\text { additional data indicated no clear evidence of prolonged infection). }\end{array}$} \\
\hline
\end{tabular}


Table 2. Characteristics of patients and 5 Salmonella enterica serotype Infantis pattern JFXX01.0787 isolates without CTX-M-65, United States*

\begin{tabular}{|c|c|c|c|c|c|c|c|c|}
\hline Accession & & Specimel & & & & & & \\
\hline no.† & Year & source & Resistance profile & Plasmids & gyrA & parc & Hospitalized & Recent travel \\
\hline SRR2485280 & 2012 & Feces & No resistance detected & $\begin{array}{c}\text { No replicons } \\
\text { detected }\end{array}$ & None & $\mathrm{Wt}$ & No & No travel \\
\hline SRR4025941 & 2012 & Feces & CSuTCip(I)NalCotGen(I) & IncFIB-like & D87Y & Wt & No & $\begin{array}{c}\text { Peru, Bolivia, } \\
\text { Ecuador, } \\
\text { Chile }\end{array}$ \\
\hline SRR4019596 & 2014 & Urine & CSSuTFox(I)Cip(I)NalCot & IncFIB-like & D87Y & Wt & No & No data \\
\hline SRR3185042 & 2014 & Feces & No resistance detected & $\begin{array}{c}\text { No replicons } \\
\text { detected }\end{array}$ & None & Wt & Yes & No travel \\
\hline SRR2485289 & 2015 & Feces & No resistance detected & $\begin{array}{c}\text { No replicons } \\
\text { detected }\end{array}$ & None & Wt & No & No data \\
\hline
\end{tabular}

${ }^{\star}$ A, ampicillin; Aug, amoxicillin-clavulanic acid; C, chloramphenicol; Cip, ciprofloxacin; Cot, trimethoprim/sulfamethoxazole; Cx, ceftriaxone; Fox, cefoxitin; Gen, gentamicin; (I), intermediate susceptibility; Nal, nalidixic acid; S, streptomycin; Su, sulfisoxazole; T, tetracycline; Tio, ceftiofur.

$\dagger$ Accession numbers are the sequence read archive run identification numbers from the National Center for Biotechnology Information.

96-273 pairwise hqSNPs from isolates in clade A. Absence of the bla $a_{\text {СтХ-M- } 65}$ gene in these 5 isolates was confirmed by read-mapping procedures and supported by the observed phenotypic susceptibility to $\beta$-lactam antimicrobial drugs.

A phylogeographic analysis of the 32 isolates in the main clade and additional genomes from NCBI generated a time-measured phylogenetic tree of isolates from the United States and Peru (online Technical Appendix Figure 1). These isolates last shared a common ancestor around 2006 (95\% HPD interval 2003-2008). This analysis also suggests that the MRCA of these isolates existed in Peru with a probability of $98.7 \%$. The probability of the tree being rooted in the United States was $\approx 1.3 \%$. Clinical isolates from humans in the United States sequenced as part of our study last shared a common ancestor sometime around 2009 (95\% HPD interval 2008-2009), before the first isolation of pattern 787 in the United States in 2012.

\section{Discussion}

A new strain of Salmonella Infantis, which has pattern 787 and frequently carries a multidrug-resistant plasmid with a CTX-M-65 ESBL, has emerged in the United States. This strain possesses clinically important resistance associated with higher hospitalization rates. Using epidemiologic and phylogenetic methods, we demonstrated that the earliest cases of CTX-M-65 Infantis infection were among travelers returned from South America, whereas subsequent infections were acquired domestically.

The demographic and clinical characteristics among patients with CTX-M-65 Infantis infections differed from those of patients infected with all strains of Salmonella Infantis or other common Salmonella serotypes. CTX-M-65 Infantis-infected patients were younger and more frequently female, and rates of hospitalization were $50 \%$ higher for these patients than for those in the other 2 groups. Among patients with CTX-M-65 Infantis infections, 63\% reported recent travel to South America, predominantly to Peru, compared with $<10 \%$ in the comparison groups reporting travel. This finding is consistent with those of other studies that found foreign travel to be a risk factor for CTX-Mtype ESBLs (19-21). Studies have shown that CTX-M-65 has recently emerged in commensal E. coli in Bolivia (22) and in Salmonella Infantis in Ecuador (23), 2 countries to which patients in our study also traveled.

Our phylogeographic analysis, which included many isolates from Peru, suggested that isolates from patients in the United States last shared a common ancestor that existed in Peru in 2009. This finding makes sense, given the high proportion of patients in our study who reported travel to Peru and the known circulation of CTX-M-65-positive Enterobacteriaceae in South America. In our study, patients with CTX-M-65 Infantis infection and a history of travel

Table 3. Comparison of demographic and clinical characteristics of patients with CTX-M-65-positive isolates with patients infected with all Salmonella enterica serotype Infantis strains or other common Salmonella serotypes, FoodNet, United States, 2012-2015* CTX-M-65-positive Salmonella Infantis, pattern JFXX01.0787, $\mathrm{n}=29^{*}$

\begin{tabular}{|c|c|c|c|c|c|c|c|c|c|}
\hline \multirow[b]{2}{*}{ Characteristic } & \multicolumn{3}{|c|}{ 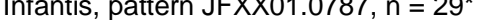 } & \multicolumn{3}{|c|}{ 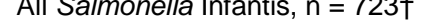 } & \multicolumn{3}{|c|}{ Unter sammenta, $n=\angle 1, \angle 85+$} \\
\hline & Proportion§̧ & $\%$ & $\mathrm{p}$ value & Proportion§ & $\%$ & $\mathrm{p}$ value & Proportion§ & $\%$ & $p$ value \\
\hline Female sex & $20 / 29$ & 69 & Referent & $403 / 722$ & 56 & 0.16 & $11,067 / 21,258$ & 52 & 0.07 \\
\hline Urine isolation & $5 / 29$ & 17 & Referent & $66 / 723$ & 9 & 0.14 & $1,076 / 21,214$ & 5 & $<0.01$ \\
\hline Hospitalization & $8 / 18$ & 44 & Referent & $199 / 695$ & 29 & 0.14 & $5,951 / 20,619$ & 29 & 0.14 \\
\hline International & $12 / 19$ & 63 & Referent & $51 / 611$ & 8 & $<0.01$ & $1,431 / 16,496$ & 9 & $<0.01$ \\
\hline
\end{tabular}

*Patient age range 0-82 y; median age (interquartile range) 25 (15-50) y.

†Patient age range 0-95 y; median age (interquartile range) 37 (12-58) y

łPatient age range 0-101 y; median age (interquartile range) 29 (6-54) y. Other Salmonella defined as the top 20 Salmonella enterica serotypes

(excluding Infantis): Typhimurium, Enteritidis, Newport, Heidelberg, Javiana, Saintpaul, Montevideo, Agona, Oranienburg, Muenchen, Thompson, Hadar,

Braenderup, Derby, I 4,[5],12:i:-, Paratyphi B var. L(+) tartrate+, Blockley, Anatum, Mississippi, Panama.

$\S$ Unknowns were excluded from the denominator. 
were reported every year from 2012 through 2015; patients with no history of travel were first reported in 2014.

One caveat is that genetic information for CTX-M Infantis from countries in South America other than Peru was not available; therefore, we could not distinguish the role that other countries in South America may have played in the spread of this Salmonella Infantis strain to the United States. We cannot definitively determine how patients with this strain of Salmonella Infantis who did not report travel to South America became infected; however, our analysis does show a close genetic relationship between clinical isolates from these patients and isolates collected by USDAFSIS from chickens in the United States.
Poultry consumption may be a source of CTX-M-65 Infantis infection in the United States and abroad. In our study, more than one third of patients with CTX-M-65 Infantis infections did not report recent international travel and thus were exposed via a domestic source. CTX-M genes have been linked to poultry, particularly broiler chickens (24-26). A study of 14 chicken farms in Henan Province, China, conducted during 2007-2008, was the first to describe detection of CTX-M-65-producing E. coli in chickens (27). Recently, CTX-M-65 Infantis was found to be transmitted from broiler chickens and chicken meat to humans in Italy (28).

The results of our initial hqSNP analysis demonstrated that the original isolate collected from retail chicken in

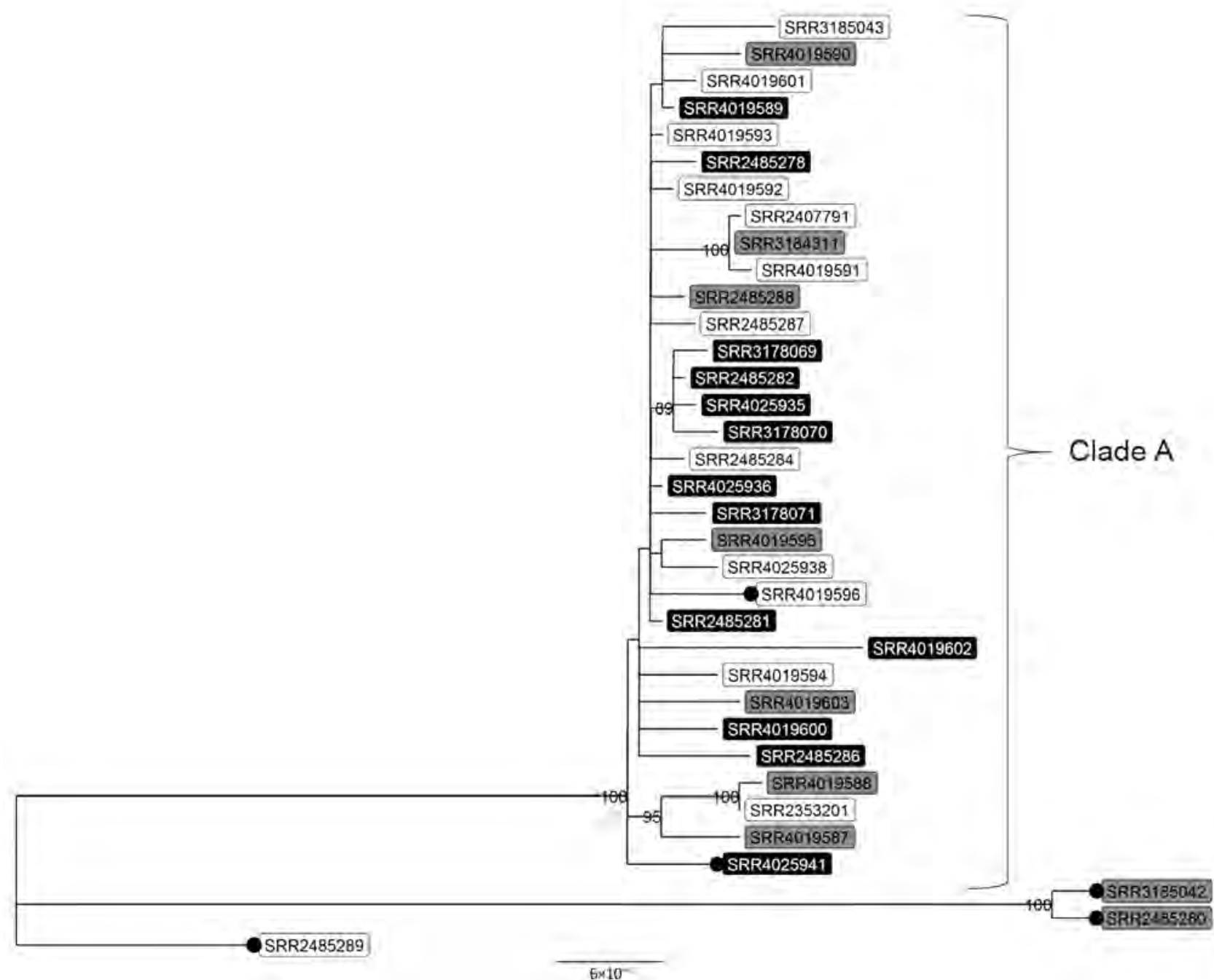

Figure 2. High-quality single-nucleotide polymorphism-based phylogenetic tree of clinical and retail meat isolates of Salmonella enterica serotype Infantis with pulsed-field gel electrophoresis pattern JFXX01.0787 collected in the United States and submitted to the National Antimicrobial Resistance Monitoring System for whole-genome sequencing. Tree tips are labeled with National Center for Biotechnology Information accession numbers (sequence read archive run identification numbers); shading indicates patients' international travel history for clinical isolates (black, recent international travel; gray, no recent international travel; white, travel data missing). Black circles indicate isolates that are missing the $b / a_{\text {СTX-M-65 }}$ gene. Isolates in top clade differed by $2-47$ high-quality single-nucleotide polymorphisms. Numbers displayed on nodes are bootstrap support values, an indication of the reliability of the tree. Only bootstrap values $>80$ are displayed. More information on patient and isolate characteristics are provided in Tables 1 and 2. Scale bar indicates nucleotide substitutions per site. 
2014 was genetically related to isolates from humans. In addition, our data show a clear partition between the dates of specimen collection from the first travel-associated infections detected in the United States (2012) and the dates of the first domestically acquired infections detected (2014) (online Technical Appendix Figure 2). Sampling in poultry production plants and sequence data from USDA-FSIS demonstrate that this strain was present in domestic food processing plants during the latter part of the study period (2014-2015) (29). We cannot, however, determine precisely when or how this strain was introduced into poultry stock in the United States because enhanced poultry plant sampling was not conducted before 2014. International distribution of infected breeder stocks, chicken feed, or feed additives contaminated with CTX-M-65 Infantis could help explain how these broiler-associated infections have spread globally during the same period.

CTX-M-65, in comparison with more well-characterized CTX-M enzymes, differs by only 2 substitutions (A77V, S272R) from CTX-M-14, one of the more commonly detected CTX-M variants worldwide $(30,31)$. The $b l a_{\text {CTX-M-65 }}$ gene was transmitted on a large IncFIB-like plasmid containing multiple resistance genes. The presence of multidrug resistance in CTX-M-65 Infantis isolates in our study suggests that a variety of antimicrobial drugs could provide positive selection pressure and thus promote persistence of this strain. These characteristics suggest that the potential for spread of the gene is high. Research conducted in Bolivia has shown that even in the absence of selective pressure from antimicrobial drug use, plasmid transfer of CTX-M-65 from E. coli to other pathogens was achieved at high frequency and shown to be stable (22).

Additional studies on antimicrobial drug use and management practices in food animals may help us understand which factors contribute most to the emergence, persistence, and spread of resistance genes such as $b l a_{\text {СTX-M-65 }}$. New efforts to perform whole-genome sequencing on all Salmonella isolates at public health laboratories nationwide will help determine whether plasmid-mediated $b l a_{\text {СТХ-м-65 }}$ has spread to other Salmonella serotypes. One limitation of our study was that we were unable to obtain epidemiologic data for all 312 cases. The data available from FoodNet enabled us to compare variables such as patient demographics, travel, and hospitalizations; however, we did not have a control population for evaluating other variables of interest to determine potential domestic sources of transmission.

The spread of CTX-M-65 is concerning because the presence of ESBLs eliminates 2 recommended treatment options, ceftriaxone and ampicillin, for the management of salmonellosis. Given the multidrug-resistant profile of CTX-M-65 Infantis, potential for plasmid-mediated transmission, increased hospitalization rate, and evidence of this strain in domestic poultry, action is needed to prevent widespread dissemination in the United States. Enhanced surveillance and additional studies in humans and food animals may help pinpoint the sources of infection for implementation of prevention and control measures. Meanwhile, travelers and healthcare providers should be aware of the risks and implications of infection with this strain, including the potential for antimicrobial treatment failure.

\section{Acknowledgments}

We thank Heather Carleton and Lee Katz for helpful discussion and guidance on some analyses, Beth Tolar for fulfilling repeated data requests, the USDA-FSIS Eastern Laboratory Microbiology Characterization Branch for their work on adding whole-genome sequencing data to NCBI, Lauren Ahart for manuscript preparation, and the FoodNet sites and public health departments in the following states for their collaboration on this investigation: Arizona, California, Colorado, Florida, Illinois, Kentucky, Louisiana, Massachusetts, Michigan, New York, Oregon, Pennsylvania, Texas, Utah, Virginia, and Wisconsin.

\section{About the Author}

Dr. Brown is team lead for AR Capacities and Special Studies in the Division of Healthcare Quality Promotion, National Center for Emerging and Zoonotic Infectious Diseases, CDC. Her research interests include emerging mechanisms of antimicrobial drug resistance in healthcare-associated infections.

\section{References}

1. Scallan E, Hoekstra RM, Angulo FJ, Tauxe RV, Widdowson MA, Roy SL, et al. Foodborne illness acquired in the United States - major pathogens. Emerg Infect Dis. 2011;17:7-15. http://dx.doi.org/10.3201/eid1701.P11101

2. Marder EP, Griffin PM, Cieslak PR, Dunn J, Hurd S, Jervis R, et al. Preliminary incidence and trends of infections with pathogens transmitted commonly through food-Foodborne Diseases Active Surveillance Network, 10 U.S. sites, 2006-2017. MMWR Morb Mortal Wkly Rep. 2018;66:397-403. http://dx.doi.org/10.15585/ mmwr.mm6615a1

3. Shane AL, Mody RK, Crump JA, Tarr PI, Steiner TS, Kotloff K, et al. 2017 Infectious Diseases Society of America clinical practice guidelines for the diagnosis and management of infectious diarrhea. Clin Infect Dis. 2017;65:1963-73. http://dx.doi.org/10.1093/cid/ cix959

4. Bauernfeind A, Casellas JM, Goldberg M, Holley M, Jungwirth R, Mangold P, et al. A new plasmidic cefotaximase from patients infected with Salmonella typhimurium. Infection. 1992;20:158-63. http://dx.doi.org/10.1007/BF01704610

5. Poirel L, Bonnin RA, Nordmann P. Genetic support and diversity of acquired extended-spectrum $\beta$-lactamases in Gram-negative rods. Infect Genet Evol. 2012;12:883-93. http://dx.doi.org/10.1016/ j.meegid.2012.02.008

6. Livermore DM, Canton R, Gniadkowski M, Nordmann P, Rossolini GM, Arlet G, et al. CTX-M: changing the face of ESBLs in Europe. J Antimicrob Chemother. 2007;59:165-74. http://dx.doi.org/10.1093/jac/dk1483

7. Sjölund-Karlsson M, Howie R, Krueger A, Rickert R, Pecic G, Lupoli K, et al. CTX-M-producing non-Typhi Salmonella spp. 
isolated from humans, United States. Emerg Infect Dis. 2011;17:97-9. http://dx.doi.org/10.3201/eid1701.100511

8. Sjölund-Karlsson M, Howie RL, Blickenstaff K, Boerlin P, Ball T, Chalmers $\mathrm{G}$, et al. Occurrence of $\beta$-lactamase genes among nonTyphi Salmonella enterica isolated from humans, food animals, and retail meats in the United States and Canada. Microb Drug Resist. 2013;19:191-7. http://dx.doi.org/10.1089/mdr.2012.0178

9. Tate H, Folster JP, Hsu CH, Chen J, Hoffmann M, Li C, et al. Comparative analysis of extended-spectrum-beta-lactamase CTX-M-65-producing Salmonella enterica serovar Infantis isolates from humans, food animals, and retail chickens in the United States. Antimicrob Agents Chemother. 2017;61:e0488-17. http://dx.doi.org/10.1128/AAC.00488-17

10. Centers for Disease Control and Prevention. National Antimicrobial Resistance Monitoring System for enteric bacteria (NARMS): human isolates surveillance report for 2015 (final report). Atlanta: The Centers; 2016. p. 22-5.

11. Folster JP, Pecic G, McCullough A, Rickert R, Whichard JM. Characterization of bla(CMY)-encoding plasmids among Salmonella isolated in the United States in 2007. Foodborne Pathog Dis. 2011;8:1289-94. http://dx.doi.org/10.1089/fpd.2011.0944

12. Centers for Disease Control and Prevention. Laboratory standard operating procedure for PulseNet Nextera XT Library Prep and Run Setup for the Illlumina MiSeq. Code PNL32. 2015 [cited 2016 Feb 1]. https://www.cdc.gov/pulsenet/pdf/pnl32-miseq-nextera-xt.pdf

13. Zankari E, Hasman H, Cosentino S, Vestergaard M, Rasmussen S, Lund $\mathrm{O}$, et al. Identification of acquired antimicrobial resistance genes. J Antimicrob Chemother. 2012;67:2640-4. http://dx.doi.org/ 10.1093/jac/dks261

14. Carattoli A, Zankari E, García-Fernández A, Voldby Larsen M, Lund $\mathrm{O}$, Villa L, et al. In silico detection and typing of plasmids using PlasmidFinder and plasmid multilocus sequence typing. Antimicrob Agents Chemother. 2014;58:3895-903. http://dx.doi.org/10.1128/AAC.02412-14

15. Katz LS, Petkau A, Beaulaurier J, Tyler S, Antonova ES, Turnsek MA, et al. Evolutionary dynamics of Vibrio cholerae O1 following a single-source introduction to Haiti. MBio. 2013; 4:e0398-13. http://dx.doi.org/10.1128/mBio.00398-13

16. Croucher NJ, Page AJ, Connor TR, Delaney AJ, Keane JA, Bentley SD, et al. Rapid phylogenetic analysis of large samples of recombinant bacterial whole genome sequences using Gubbins. Nucleic Acids Res. 2015;43:e15. http://dx.doi.org/10.1093/nar/gku1196

17. US National Library of Medicine. Pathogen detection [cited 2018 Jan 23]. https://www.ncbi.nlm.nih.gov/pathogens/

18. Gopinath G, Chase H, Gangiredla J, Patel I, Addy N, Beaubrun JJG, et al. Comparative genomics of blaCTX-M-65resistant clinical strains of Salmonella enterica serovar Infantis from Peru and resistant strains from chicken, cattle and humans. Poster presented at: International Association for Food Protection annual meeting; 2017 Jul 9-12; Tampa, FL, USA.

19. Tängdén T, Cars $O$, Melhus A, Löwdin E. Foreign travel is a major risk factor for colonization with Escherichia coli producing CTX-M-type extended-spectrum $\beta$-lactamases: a prospective study with Swedish volunteers. Antimicrob Agents Chemother. 2010;54:3564-8. http://dx.doi.org/10.1128/AAC.00220-10

20. Hopkins KL, Batchelor MJ, Liebana E, Deheer-Graham AP, Threlfall EJ. Characterisation of CTX-M and AmpC genes in human isolates of Escherichia coli identified between 1995 and 2003 in England and Wales. Int J Antimicrob Agents. 2006;28:180-92. http://dx.doi.org/10.1016/j.ijantimicag.2006.03.027
21. Arcilla MS, van Hattem JM, Haverkate MR, Bootsma MC, van Genderen PJ, Goorhuis A, et al. Import and spread of extended-spectrum $\beta$-lactamase-producing Enterobacteriaceae by international travellers (COMBAT study): a prospective, multicentre cohort study. Lancet Infect Dis. 2016.

22. Riccobono E, Di Pilato V, Di Maggio T, Revollo C, Bartoloni A, Pallecchi L, et al. Characterization of IncI1 sequence type 71 epidemic plasmid lineage responsible for the recent dissemination of CTX-M-65 extended-spectrum $\beta$-lactamase in the Bolivian Chaco region. Antimicrob Agents Chemother. 2015;59:5340-7. http://dx.doi.org/10.1128/AAC.00589-15

23. Cartelle Gestal M, Zurita J, Paz Y Mino A, Ortega-Paredes D, Alcocer I. Characterization of a small outbreak of Salmonella enterica serovar Infantis that harbour CTX-M-65 in Ecuador. Braz J Infect Dis. 2016;20:406-7. http://dx.doi.org/10.1016/ j.bjid.2016.03.007

24. Maciuca IE, Williams NJ, Tuchilus C, Dorneanu O, Guguianu E, Carp-Carare C, et al. High prevalence of Escherichia coliproducing CTX-M-15 extended-spectrum $\beta$-lactamases in poultry and human clinical isolates in Romania. Microb Drug Resist. 2015;21:651-62. http://dx.doi.org/10.1089/mdr.2014.0248

25. Silva KC, Fontes LC, Moreno AM, Astolfi-Ferreira CS, Ferreira AJ, Lincopan N. Emergence of extended-spectrum- $\beta$ lactamase CTX-M-2-producing Salmonella enterica serovars Schwarzengrund and Agona in poultry farms. Antimicrob Agents Chemother. 2013;57:3458-9. http://dx.doi.org/10.1128/ AAC.05992-11

26. Weill FX, Lailler R, Praud K, Kérouanton A, Fabre L, Brisabois A, et al. Emergence of extended-spectrum-beta-lactamase (CTX-M-9)-producing multiresistant strains of Salmonella enterica serotype Virchow in poultry and humans in France. J Clin Microbiol. 2004;42:5767-73. http://dx.doi.org/10.1128/ JCM.42.12.5767-5773.2004

27. Yuan L, Liu JH, Hu GZ, Pan YS, Liu ZM, Mo J, et al. Molecular characterization of extended-spectrum $\beta$-lactamase-producing Escherichia coli isolates from chickens in Henan Province, China. J Med Microbiol. 2009;58:1449-53. http://dx.doi.org/10.1099/ jmm.0.012229-0

28. Franco A, Leekitcharoenphon P, Feltrin F, Alba P, Cordaro G, Iurescia M, et al. Emergence of a clonal lineage of multidrugresistant ESBL-producing Salmonella Infantis transmitted from broilers and broiler meat to humans in Italy between 2011 and 2014. PLoS One. 2015;10:e0144802. http://dx.doi.org/10.1371/ journal.pone.0144802

29. Food and Drug Administration . NARMS Now [cited 2018 Jan 19]. https:/www.fda.gov/AnimalVeterinary/SafetyHealth/Antimicrobial Resistance/NationalAntimicrobialResistanceMonitoringSystem/ ucm416741.htm

30. Yin J, Cheng J, Sun Z, Ye Y, Gao YF, Li JB, et al. Characterization of two plasmid-encoded cefotaximases found in clinical Escherichia coli isolates: CTX-M-65 and a novel enzyme, CTX-M-87. J Med Microbiol. 2009;58:811-5. http://dx.doi.org/10.1099/jmm.0.006007-0

31. Bush K. Proliferation and significance of clinically relevant $\beta$-lactamases. Ann N Y Acad Sci. 2013;1277:84-90. http://dx.doi.org/10.1111/nyas.12023

Address for correspondence: Allison C. Brown, Centers for Disease Control and Prevention, 1600 Clifton Rd NE, Mailstop C16, Atlanta, GA 30329-4027, USA; email: acbrown1@cdc.gov 


\section{Substance Use and Adherence to HIV Preexposure Prophylaxis for Men Who Have Sex with Men ${ }^{1}$}

Martin Hoenigl, Sonia Jain, David Moore, Deborah Collins, Xiaoying Sun, Peter L. Anderson, Katya Corado, Jill S. Blumenthal, Eric S. Daar, Joel Milam, Michael P. Dubé, Sheldon Morris, for the California Collaborative Treatment Group 595 Team

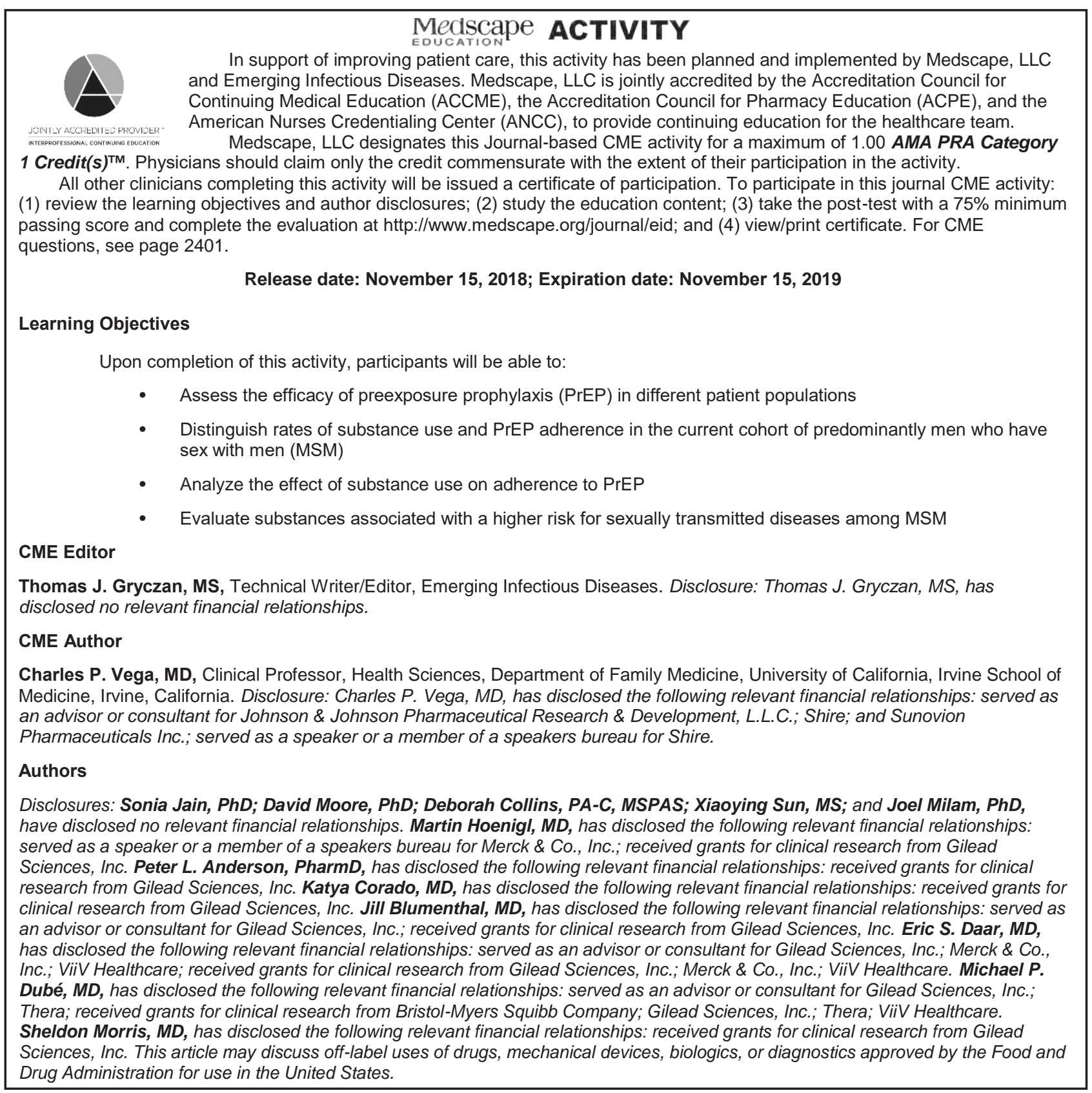

${ }^{1}$ Original data from this study were presented in part at the 2017 Conference on Retroviruses and Opportunistic Infections, February 13-16, 2017, Seattle, Washington, USA; and at the 12th International Conference on HIV Treatment and Prevention Adherence, June 4-6, 2017, Miami, Florida, USA. 
The effectiveness of oral HIV preexposure prophylaxis (PrEP) strongly depends on maintaining adherence. We investigated the association between substance use and PrEP adherence, as well as incident sexually transmitted infections (STIs) in a high-risk cohort of 394 participants (391 men who have sex with men and 3 transgender women) who were enrolled in a PrEP demonstration project. We assessed baseline and ongoing substance use over a 48-week period for stimulants and nonstimulant substances and for each substance separately. We measured PrEP adherence by using dried blood spots to obtain levels of tenofovir diphosphate. No differences in these levels were found between substance users and nonsubstance users. Baseline stimulant use was strongly associated (odds ratio $3.4 ; p<0.001$ ) with incident STIs during the study. Thus, PrEP adherence was not decreased by substance use. Because substance users had increased rates of STIs, indicating higher-risk behavior, they might be excellent candidates for PrEP.

$\mathrm{O}$ ver the past 2 decades, substance use, in particular use of stimulants, such as methamphetamine, remains prevalent among men who have sex with men (MSM) and transgender women in the United States (1). Alcohol, stimulant use, and injection drug use (IDU) are independently associated with increased risk behavior and HIV acquisition among MSM and transgender women (1-4). Thus, HIV-uninfected MSM and transgender women with substance use might represent ideal candidates for preexposure prophylaxis (PrEP).

The efficacy of tenofovir disoproxil fumarate (TDF) combined with emtricitibine (FTC) for HIV PrEP has been documented in several randomized and controlled trials (57). In the iPrEx study, TDF/FTC reduced the risk for HIV infection in MSM by $44 \%$ vs. placebo, and a $73 \%$ lower risk of HIV infection was reported for persons who had $\geq 90 \%$ self-reported adherence (5), and $>90 \%$ lower risk for persons who had adherence defined by tenofovir diphosphate (TFV-DP) drug levels commensurate with $\geq 4$ tablets per week (8).

The Bangkok TDF Study randomized 2,413 persons who injected drugs (PWIDs; $<5 \%$ were MSM) 1:1 to TDF or placebo, and results showed a $48 \%$ reduction in HIV

Author affiliations: Medical University of Graz, Graz, Austria (M. Hoenigl); University of California, San Diego, California, USA (M. Hoenigl, S. Jain, D. Moore, X. Sun, J.S. Blumenthal, S. Morris); Department of Health and Human Services, Long Beach, California, USA (D. Collins); University of Anschutz Medical Campus, Aurora, Colorado, USA (P.L. Anderson); Harbor UCLA Medical Center, Torrance, California, USA (K. Corado, E.S. Daar); University of Southern California Keck School of Medicine, Los Angeles, California, USA (J. Milam, M.P. Dubé)

DOI: https://doi.org/10.3201/eid2412.180400 seroconverison in the treatment arm $(9,10)$. However, in that study, study participants had daily observed dosing in conjunction with substance use disorder treatment. Therefore, adherence remains uncertain among substance users without observed therapy.

The effectiveness of TDF/FTC for HIV PrEP strongly depends on maintaining adherence $(11,12)$. Although studies have indicated that different strategies might be required for PrEP implementation for MSM who use stimulant substances and alcohol (13), comprehensive/demonstrative studies that evaluate adherence among MSM or transgender women using different classes of substances are lacking.

We hypothesized that, among MSM and transgender women enrolled in a randomized controlled PrEP demonstration trial, substance users would have lower levels of PrEP adherence. The objective of our study was to investigate the association between substance/alcohol use and adherence to PrEP, as well as sexually transmitted infections (STIs) and study completion, in a well-characterized high-risk cohort of MSM and transgender women who participated in the California Collaborative Treatment Group (CCTG) 595 Study.

\section{Materials and Methods}

CCTG 595 was a randomized controlled trial of individualized text messaging versus standard care for adherence to daily TDF/FTC PrEP (http://www.clinicaltrials.gov/ct2/ show/NCT01761643) (14). In CCTG 595, PrEP was given in combination with safety monitoring, HIV/STI testing, and risk reduction counseling. On a daily basis, participants in the intervention arm received a mixture of health promotion and factoid messages at a personally selected time consistent with when they planned to take PrEP. The study was conducted during February 2014-February 2016. Patients were enrolled at 4 medical centers in southern California (University of California San Diego, University of Southern California, Harbor-University of California Los Angeles, and Long Beach Health Department), and participants were provided with mobile phones in case they did not have a mobile phone (14).

Eligible participants for CCTG 595 were HIV-uninfected MSM and transgender women (age $>18$ years) confirmed by a negative result for an antigen/antibody assay or antibody assay plus HIV nucleic acid amplification test. Participants needed to have a persistent increased risk for HIV acquisition as determined by $\geq 1$ of the following criteria: 1) $\geq 1$ HIV-infected sexual partner for $\geq 4$ weeks; 2 ) condomless anal intercourse with $\geq 3$ male sex partners who were HIV positive or of unknown HIV status during the previous 3 months; or 3 ) condomless anal sex with $\geq 1$ male partner and an STI diagnosis during the previous 3 months. Participants were required to have acceptable laboratory test values during the previous 30 days; exclusion criteria 
included active hepatitis B. Study visits occurred at baseline and at weeks $4,12,24,36$, and 48 for the primary outcome. Study participants were allowed to continue receiving the study drug past week 48 until the last participant completed his or her week 48 visit.

At each visit, we collected data by using a confidential in-person interview and computer-assisted survey selfreport (e.g., Patient Health Questionnaire 9 [PHQ9]). We found no significant differences in the primary adherence outcome between the 2 study arms $(72.0 \%$ in text messaging arm vs. $69.2 \%$ in standard of care; $p=0.58)$, in adequate adherence at week $12(91.7 \%$ vs. $85.6 \% ; p=0.07)$ or week $48(83.4 \%$ vs. $81.6 \% ; p=0.77)$, or in baseline substance use $(\mathrm{p}=0.11)$ or Drug Abuse Screening Test (DAST10) result $(\mathrm{p}=0.30)(14)$.

For this analysis, we included randomized CCTG 595 participants who had completed the baseline substance use questionnaire $(\mathrm{n}=394)$ to examine associations with substance use over 48 weeks and used dried blood spot (DBS) intracellular TFV-DP levels as a biologic measure of PrEP adherence (15). We assessed substance use during the previous 3 months at baseline and week 4, 12, 24, 36, and 48 visits by using a Substance Use Screening Questionnaire (SCID). Each substance variable was categorized into no use, some use (1-4 times), and frequent use ( $\geq 5$ times) on the basis of the frequency of use during the previous 3 months. We also analyzed use of combined stimulant substances (i.e., poppers, methamphetamine, cocaine, ecstasy, amphetamine, and other stimulants); nonstimulant substances (i.e., heroin, other opioids [e.g., hydrocodone bitartrate/acetaminophen and oxycontin], sedatives, antianxiety drugs, hallucinogens, dissociative drugs, and inhalants); and any substances (i.e., stimulant and nonstimulant substances listed previously, not including alcohol and marijuana use).

We assessed problematic use at baseline by using the DAST10 and the Alcohol Use Disorders Identification Test (AUDIT). DAST10 score was grouped into 3 categories: no or low problems, DAST10 score $<3$; moderate problems, DAST10 score $\geq 3-<6$; and substantial or severe problems, DAST10 score $\geq 6$. AUDIT score was grouped into 3 categories: $<8, \geq 8-<16$, and $\geq 16$. Ongoing substance use was defined as $\geq 50 \%$ of completed study visits (study had 6 regular visits) with reported use in the SCID. Ongoing substance use was defined in a hierarchical way. We first defined frequent ongoing user as reporting frequent substance use on the SCID at $\geq 50 \%$ of visits; if this criterion was not reached, we looked further at whether the study participant reported any substance use (including some and frequent) at $\geq 50 \%$ of visits; if yes, we defined them as some ongoing user; otherwise, they were counted as not an ongoing user.

We determined adherence by measuring intracellular TFV-DP levels in DBS. A TFV-DP concentration $\geq 719$
fmoL/punch of a paper disk containing DBS (e.g., https:// www.analytical-sales.com/DBS.html) was defined as an average of $\geq 4$ tablets/week. This value is the unrounded level corresponding to $700 \mathrm{fmoL} /$ punch level used in the IPREX OLE study, which reported 0 of 28 seroconversions when the TFV-DP level was $\geq 700 \mathrm{fmoL} /$ punch (16). We determined intracellular TFV-DP concentrations at the week 12 visit and the last on-drug visit on or before the week 48 visit by using a validated method (15).

The primary DBS adherence outcome was a composite outcome for being adherent as defined by a DBS TFV-DP level $>719 \mathrm{fmoL} /$ punch (i.e., adequate adherence) at the week 12 visit and, if continued past week 12, the last study visit through week 48 (e.g., week 24, 36, or 48). Missing or not completing the visit at week 12 was considered nonadherence. If week 12 was the last study visit while receiving drug, then the adherence of the participant was based only on that 1 value. The secondary DBS near-perfect adherence composite outcome included the same composite outcome for DBS TFV-DP dose associated with taking 7 doses of TDF in the past week $(\geq 1,246 \mathrm{fmoL} /$ punch $)(15,16)$. In addition to the composite outcomes, we also performed crosssectional analyses at weeks 12 and 48 on the basis of available samples.

As a secondary objective, we assessed whether substance or alcohol use reported at baseline impacted study completion and incident STIs during the study (i.e., measure of sexual risk behavior). STI screening assessments at baseline and every 3 months over 12 months included syphilis (serum rapid plasma reagin, and if a positive result was obtained, a confirmatory treponemal test), nucleic acid amplification test of urine, and testing of pharyngeal and rectal swab specimens for Chlamydia spp. and gonorrhea (Aptima; Hologic, Marlborough, MA, USA). Information about newly diagnosed STIs was communicated to participants, and referrals were made to their providers or a local STI clinic for treatment. Incident STI was defined as having positive results for gonorrhea or infection with Chlamydia spp. at any site or a positive rapid plasma reagin result for syphilis during the study visits after baseline.

We compared baseline characteristics, DBS adherence composites, and incident STI during the study and at study completion regarding substance/alcohol use categories by using the Fisher exact test for categorical variables and analysis of variance test. We used the Wilcoxon rank-sum test to test continuous variables. To assess the association between substance/alcohol use and outcomes (i.e., adherence, study completion, and incident), we used separate logistic regression models adjusted for study arm and other baseline factors that were associated with outcome. We assessed model discrimination by using the goodness-of-fit Hosmer-Lemeshow statistic. In addition, we used Cox regression models to study the association between baseline 


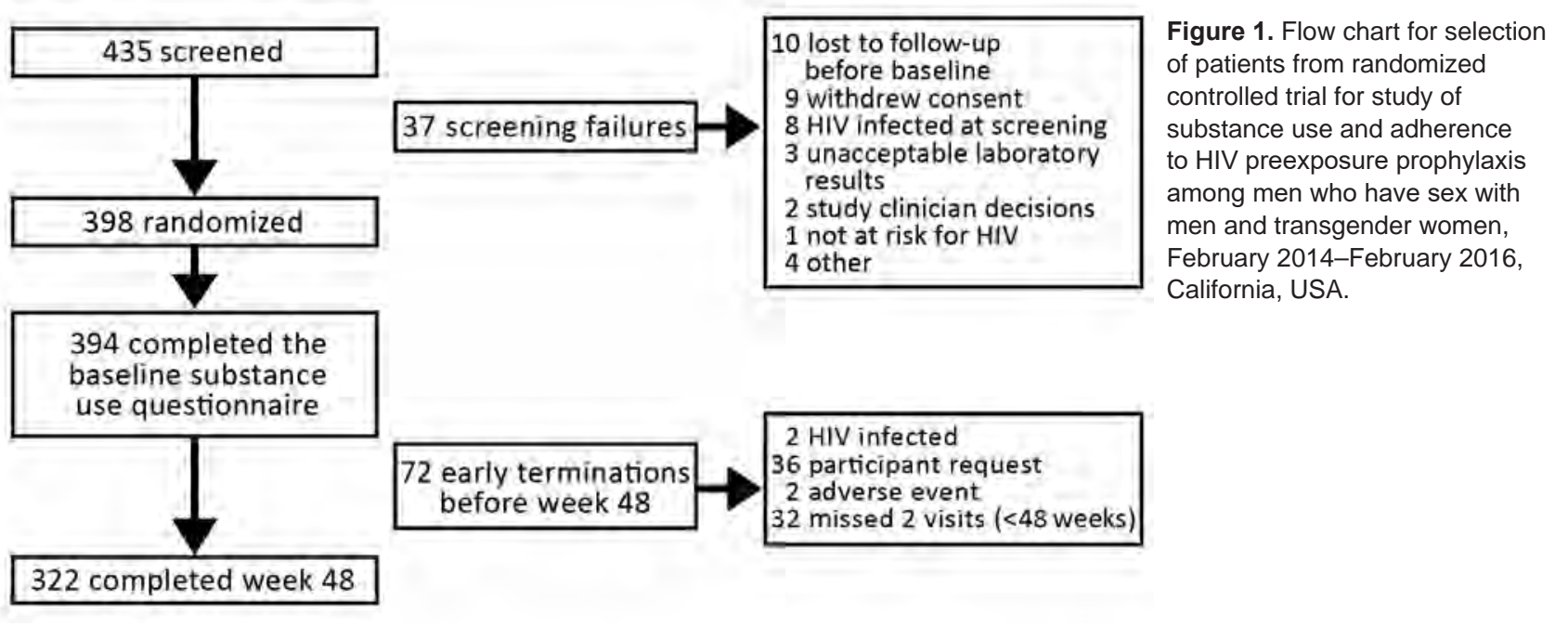

substance use and time to early study termination or time to first incident STI diagnosis and reported hazard ratios (HRs). We defined time to study termination as the last completed visit of a participant. Participants who did not leave the study early were censored at week 48 . Participants who did not reach the event were censored at their last visit before or at week 48 . A p value $<0.05$ was considered statistically significant. No adjustment was made for multiple comparisons. Statistical analyses were performed by using R software version 3.3.2 (http://cran.r-project.org).

\section{Results}

A total of 394 persons participated in the study and completed their baseline substance use questionnaire (Figure 1). Of these participants, any substance use was reported by 288 (73\%) and any alcohol use by 327 (83\%). Overall, substance use remained relatively stable over the course of the study (e.g., 39\% reported frequent substance use at baseline and $42 \%$ at week 48 ). Some ongoing substance use was reported by $37 \%$, and frequent ongoing substance was reported by $38 \%$. Participants with ongoing substance use had higher levels of depressive symptoms (PHQ9 scores) than those without ongoing substance use. We obtained demographic data and PHQ9 and DAST10 scores of subgroups with no, some, and frequent ongoing substance use (Table 1).

Overall, $89 \%$ of participants at week 12 and $83 \%$ of participants at week 48 had adequate DBS TFV-DP levels (i.e., $\geq 719 \mathrm{fmoL} /$ punch); $48 \%$ of participants at week 12 and $44 \%$ of participants at week 48 had estimated nearperfect DBS TFV-DP levels (i.e., $\geq 1,246$ fmoL/punch). A total of $279 / 394$ (71\%) study participants reached the primary DBS adherence composite (i.e., adequate adherence), 115/394 (29\%) reached the secondary DBS adherence composite (i.e., near-perfect adherence), and 322/394 (82\%) completed the study (i.e., the week 48 visit) (14).
Univariate analyses showed no significant difference in the primary or secondary DBS adherence outcomes between persons with or without ongoing substance/alcohol use (all $\mathrm{p}$ values $>0.2$; Table 2 ). There was also no significant association between baseline substance/alcohol use or between baseline DAST10 and AUDIT scores and adherence outcomes (all p values $>0.5$; Table 2 ). Similar results were confirmed in multivariable logistic regression models adjusted for study arm, race, and baseline PHQ9 scores (all $\mathrm{p}$ values $>0.1$ ).

We created boxplots of DBS TFV-DP levels at weeks 12 and 48 for those with no, some, and frequent ongoing substance use (alcohol and marijuana excluded), as well as boxplots of DBS TFV-DP levels at week 48 by AUDIT and DAST10 score categories (Figure 2). We also developed cross-sectional associations of previous 3 months substance use with adequate and perfect adherence at weeks 12 and 48 (online Technical Appendix Table 1, https://wwwnc.cdc.gov/EID/article/24/12/180400-Techapp1.pdf). Although at week 48 persons with the highest category of AUDIT scores were significantly less likely to have adequate adherence $(\mathrm{p}=0.03)$, persons who had substantial or severe substance use problems according to DAST10 were significantly more likely to reach near-perfect adherence $(\mathrm{p}=0.04)$. However, when we compared DBS TFV-DP as a continuous variable between the AUDIT and DAST10 groups, differences were not significant $(\mathrm{p}=0.847$ for AUDIT and $\mathrm{p}=0.099$ for DAST10; Figure 2).

Overall, 322/394 (82\%) participants completed the study (Table 3). In the Cox regression model adjusting for study arm (Table 4), we found that frequent baseline substance use was significantly associated with study completion (HR for early study termination 0.541; $\mathrm{p}=0.036$ ) compared with persons who had no substance 
use; $86 \%$ of persons who had frequent substance use completed the study compared with $81 \%$ who had some substance use and $76 \%$ who had no substance use). We also calculated the same model after replacing substance use with alcohol use and methamphetamine use. Although baseline alcohol use was not a strong predictor of study completion, we found that some methamphetamine use at baseline was associated with a significantly lower likelihood of study completion compared with no methamphetamine use, but frequent methamphetamine use at baseline was not associated with study completion (HR for early study termination for some methamphetamine use $1.885 ; \mathrm{p}=0.046 ; 70 \%$ study completion for some methamphetamine use vs. $83 \%$ study completion for no or frequent methamphetamine use). The logistic regression models yielded similar findings (online Technical Appendix Table 2).

In an explorative analysis, we focused on 39 (9.9\%) persons who left the study early (before the week 24 visit). We found that persons who had some baseline methamphetamine use had a greater tendency to leave the study early $(17.5 \%$ left the study early vs. $4.3 \%$ who had frequent methamphetamine use and $9.3 \%$ who had no methamphetamine use), but frequent baseline substance use tended to be associated with a lower tendency to leave the study early $(5.2 \%$; all $\mathrm{p}>0.05)$.

Overall, 152 (39\%) of 394 participants were given a diagnosis of an incident STI during the study. By using univariate analysis (Table 3), we found that incident STIs occurred more frequently in participants with some and frequent stimulant use at baseline (incident STIs occurred in $48 \%$ of both groups vs. $21 \%$ in persons with no stimulant use at baseline; $\mathrm{p}<0.001)$. This difference was driven by use of poppers ( $52 \%$ incident STIs in persons with frequent popper use and $50 \%$ in persons with some popper use vs. $26 \%$ in persons with no popper use; $p<0.001)$. We also found significantly higher rates of incident STIs in those with some methamphetamine use (58\% STI incidence vs. $36 \%$ in persons with no methamphetamine use and 39\% in persons with frequent methamphetamine use; $p=0.037$ ). No difference was found for alcohol use.

By using Cox regression models adjusting for study arm, age, and baseline STI status, we found that stimulant use was strongly associated with incident STI

\begin{tabular}{|c|c|c|c|c|c|}
\hline \multirow[b]{2}{*}{ Characteristic } & \multirow[b]{2}{*}{ Overall, $n=394$} & \multicolumn{3}{|c|}{ Substance use } & \multirow[b]{2}{*}{$\mathrm{p}$ value } \\
\hline & & None, $\mathrm{n}=102$ & Some, $n=144$ & Frequent, $\mathrm{n}=148$ & \\
\hline Sex & & & & & 0.191 \\
\hline M & 391 (99) & $100(98)$ & $143(99)$ & $148(100)$ & \\
\hline $\mathrm{F}$ & $3(1)$ & $2(2)$ & $1(1)$ & 0 & \\
\hline Median age, y (IQR) & $33(28-41)$ & $33(29-40)$ & $33(28-41)$ & $33.5(28-42)$ & 0.885 \\
\hline Race, $\mathrm{n}=386 \dagger$ & & & & & 0.238 \\
\hline Asian & $12(3)$ & $2(2)$ & $5(4)$ & $5(3)$ & \\
\hline Black & $51(13)$ & $20(20)$ & $19(13)$ & $12(8)$ & \\
\hline White & $292(76)$ & $72(72)$ & $104(73)$ & $116(81)$ & \\
\hline Multiple & $24(6)$ & $6(6)$ & $10(7)$ & $8(6)$ & \\
\hline Other & $7(2)$ & 0 & $4(3)$ & $3(2)$ & \\
\hline Hispanic ethnicity, $\mathrm{n}=391 \dagger$ & $119(30)$ & $30(29)$ & $54(38)$ & $35(24)$ & 0.048 \\
\hline English primary language & $3,786(95)$ & $98(96)$ & $133(92)$ & $145(98)$ & 0.066 \\
\hline Education & & & & & 0.296 \\
\hline High school or less & $35(9)$ & $12(12)$ & $15(10)$ & $8(5)$ & \\
\hline Some college & $146(37)$ & $36(35)$ & $58(40)$ & $52(35)$ & \\
\hline Bachelor's degree & $132(33)$ & $31(30)$ & $42(29)$ & $59(40)$ & \\
\hline Postgraduate or advanced degree & $81(21)$ & $23(23)$ & $29(20)$ & $29(20)$ & \\
\hline Household income/mo & & & & & 0.434 \\
\hline$<\$ 2,000$ & $84(21)$ & $27(26)$ & $31(22)$ & $26(18)$ & \\
\hline$\geq \$ 2,000$ & $248(63)$ & $63(62)$ & $89(62)$ & $96(65)$ & \\
\hline $\bar{N}$ ot known & $62(16)$ & $12(12)$ & $24(17)$ & $26(18)$ & \\
\hline Randomization arm & & & & & 0.019 \\
\hline Standard of care & $196(50)$ & $60(59)$ & $75(52)$ & $61(41)$ & \\
\hline Text messaging & $198(50)$ & $42(41)$ & $69(48)$ & $87(59)$ & \\
\hline Study site & & & & & 0.660 \\
\hline Harbor-UCLA & $48(12)$ & $11(11)$ & $15(10)$ & $22(15)$ & \\
\hline Long Beach & $46(12)$ & $15(15)$ & $17(12)$ & $14(9)$ & \\
\hline UCSD & $173(44)$ & $48(47)$ & $62(43)$ & $63(43)$ & \\
\hline USC & $127(32)$ & $28(27)$ & $50(35)$ & 49 (33) & \\
\hline Median PHQ9 for depression (IQR) & $3(1-7)$ & $2(0-5)$ & $3.5(1-7)$ & $5(2-8)$ & $<0.001$ \\
\hline Median baseline DAST10 (IQR) & $2(0-3)$ & $0(0-1)$ & $2(1-3)$ & $3(2-4)$ & $<0.001$ \\
\hline
\end{tabular}


during the study (HR 2.7 for some use, 2.6 for frequent use; both $\mathrm{p}<0.001)$ (Table 4). We also obtained significant results when stimulant use was replaced with popper use (HR 2.3 for some use, 2.5 for frequent use; both $\mathrm{p}<0.001$ ) or any substance use (HR 2.1 for some use, $\mathrm{p}=0.002$; HR 2.0 for frequent use, $\mathrm{p}=0.004)$. When we replaced stimulant use with methamphetamine use, some methamphetamine use was a significant predictor of incident STI (HR 1.9, p = 0.005), but frequent use was not a significant predictor. In contrast, alcohol use was not a strong predictor of incident STI. Logistic regression models yielded similar findings (online Technical Appendix Table 2).

\section{Discussion}

We investigated the association between substance/alcohol use and adherence to PrEP, as well as study completion and incident STIs, in a high-risk cohort of mostly MSM who participated in a randomized controlled PrEP adherence trial. Three main findings are evident. First, substance use was not associated with decreased adherence to PrEP, as measured by TFV-DP in DBS. Second, baseline frequent substance use was associated with higher likelihood of study completion. Third, baseline stimulant use was strongly associated with higher rates of incident STIs during the study, suggesting greater sexual risk behavior in users of stimulant substances. Taken together,

Table 2. Associations of DAST10 and AUDIT results at baseline and ongoing substance/alcohol use with primary and secondary DBS adherence endpoints for MSM and transgender women, California, USA*

\begin{tabular}{|c|c|c|c|c|c|c|}
\hline \multirow[b]{2}{*}{ Characteristic } & \multicolumn{2}{|c|}{ Primary endpoint } & \multirow[b]{2}{*}{$\mathrm{p}$ value } & \multicolumn{2}{|c|}{ Secondary endpoint } & \multirow[b]{2}{*}{$\mathrm{p}$ value } \\
\hline & No & Yes & & No & Yes & \\
\hline \multicolumn{7}{|l|}{ Substance use baseline, $n=394$} \\
\hline DAST10 problems & & & 0.80 & & & 0.55 \\
\hline No/low & $70(28)$ & $179(72)$ & & $173(69)$ & $76(31)$ & \\
\hline Moderate & 37 (31) & $83(69)$ & & $86(72)$ & $34(28)$ & \\
\hline Substantial/severe & $8(32)$ & 17 (68) & & $20(80)$ & $5(20)$ & \\
\hline AUDIT score & & & 0.09 & & & 0.84 \\
\hline$<8$ & 81 (29) & $201(71)$ & & $197(70)$ & $85(30)$ & \\
\hline $8-15$ & $25(26)$ & $70(74)$ & & $69(73)$ & $26(27)$ & \\
\hline$>15$ & $9(53)$ & $8(47)$ & & $13(76)$ & $4(24)$ & \\
\hline \multicolumn{7}{|l|}{ Ongoing substance use, $\mathrm{n}=394$} \\
\hline Methamphetamine & & & 0.82 & & & 0.32 \\
\hline No & 97 (29) & $238(71)$ & & $240(72)$ & $95(28)$ & \\
\hline Some & $10(28)$ & $26(72)$ & & $26(72)$ & $10(28)$ & \\
\hline Frequent & $8(35)$ & $15(65)$ & & $13(57)$ & $10(43)$ & \\
\hline Heroin & & & 0.79 & & & $>0.99$ \\
\hline No & $113(29)$ & $275(71)$ & & $274(71)$ & $114(29)$ & \\
\hline Some & $1(25)$ & $3(75)$ & & $3(75)$ & $1(25)$ & \\
\hline Frequent & $1(50)$ & $1(50)$ & & $2(100)$ & 0 & \\
\hline Poppers & & & 0.54 & & & 0.66 \\
\hline No & $62(31)$ & 139 (69) & & $146(73)$ & $55(27)$ & \\
\hline Some & $32(30)$ & $75(70)$ & & $75(70)$ & 32 (30) & \\
\hline Frequent & $21(24)$ & $65(76)$ & & $58(67)$ & 28 (33) & \\
\hline Cocaine & & & 0.48 & & & 0.33 \\
\hline No & 97 (29) & $241(71)$ & & $236(70)$ & $102(30)$ & \\
\hline Some & $13(29)$ & $32(71)$ & & $33(73)$ & $12(27)$ & \\
\hline Frequent & $5(45)$ & $6(55)$ & & $10(91)$ & $1(9)$ & \\
\hline Stimulant substances $\dagger$ & & & 0.37 & & & 0.75 \\
\hline No & $42(30)$ & $100(70)$ & & $98(69)$ & $44(31)$ & \\
\hline Some & $43(33)$ & $88(67)$ & & $96(73)$ & $35(27)$ & \\
\hline Frequent & $30(25)$ & $91(75)$ & & $85(71)$ & $36(29)$ & \\
\hline Nonstimulant substances $\ddagger$ & & & 0.96 & & & 0.32 \\
\hline No & $67(30)$ & $158(70)$ & & $166(74)$ & $59(26)$ & \\
\hline Some & $31(28)$ & $79(72)$ & & $74(67)$ & $36(33)$ & \\
\hline Frequent & 17 (29) & $42(71)$ & & 39 (66) & $20(34)$ & \\
\hline Any substance $\ddagger$ & & & 0.34 & & & $>0.99$ \\
\hline No & $31(30)$ & $71(70)$ & & $72(71)$ & $30(29)$ & \\
\hline Some & $47(33)$ & $97(67)$ & & $102(71)$ & $42(29)$ & \\
\hline Frequent & $37(25)$ & $111(75)$ & & $105(71)$ & $43(29)$ & \\
\hline Alcohol & & & 0.27 & & & 0.88 \\
\hline No & $23(38)$ & $38(62)$ & & 42 (69) & $19(31)$ & \\
\hline Some & 32 (29) & $79(71)$ & & $78(70)$ & $33(30)$ & \\
\hline Frequent & $60(27)$ & $162(73)$ & & $159(72)$ & $63(28)$ & \\
\hline
\end{tabular}

*Values are no. (\%). Primary endpoint value was TFV-DP $>719$ fmol/punch; secondary endpoint value was TFV-DP $>1,246$ fmoL/punch. For baseline use data for specific substances and substance classes not shown, all p values were $>0.2$. AUDIT, Alcohol Use Disorders Identification Test; DAST, Drug Abuse Screening Test; DBS, dried blood spot; MSM, men who have sex with men; TSF-DV, tenofovir diphosphate.

†Includes poppers, methamphetamine, cocaine, ecstasy, amphetamine, and other stimulants.

‡Marijuana and alcohol excluded. 


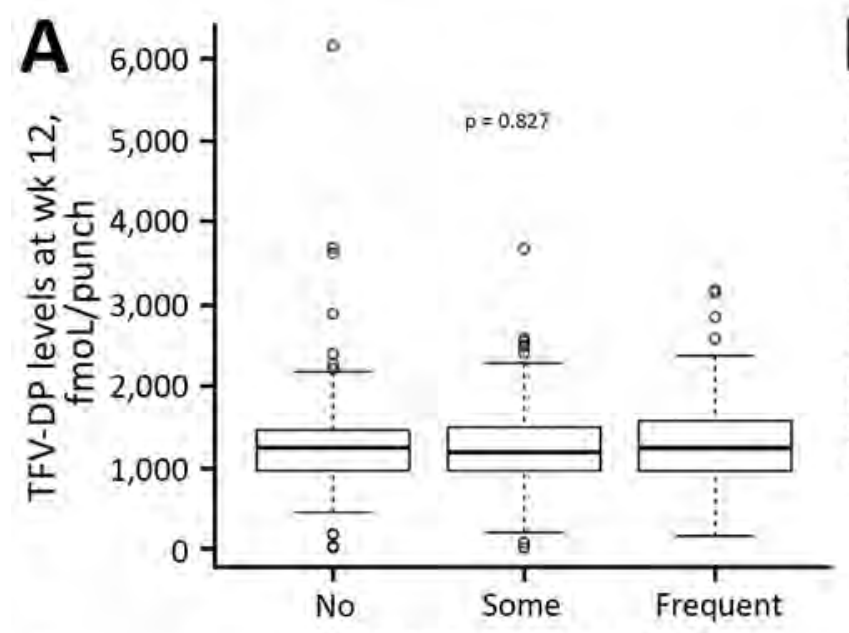

Substance use week 12

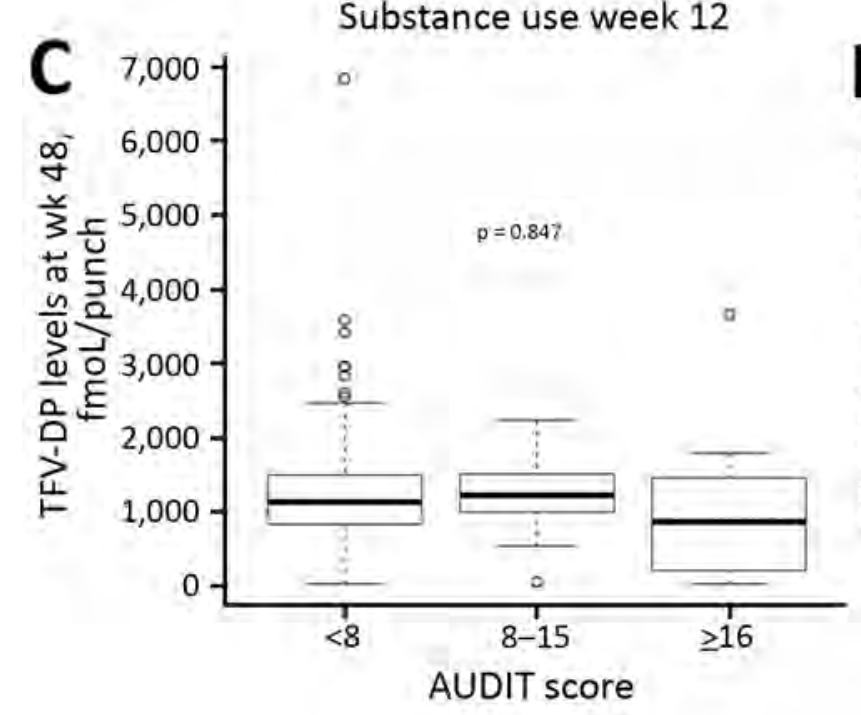

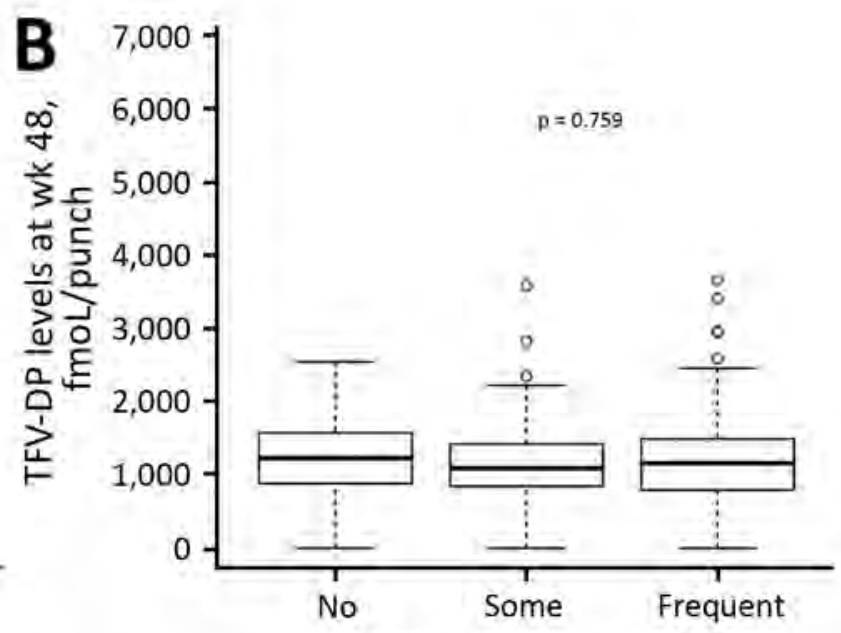

Substance use week 48

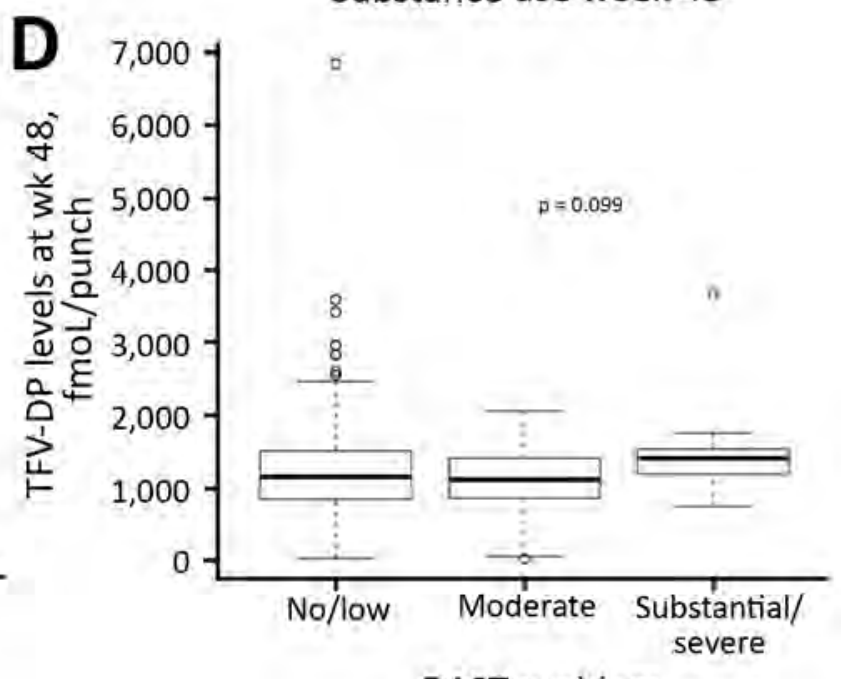

DAST problem

Figure 2. Substance use and adherence to HIV preexposure prophylaxis among transgender women and men who have sex with men, California, USA. A, B) Boxplots showing dried blood spot TFV-DP levels at weeks 12 (A) and 48 (B) for persons with no, some, and frequent ongoing substance use. C, D) Boxplots showing dried blood spot TFV-DP levels at week 48 in persons with and without alcohol (C) and substance use (D) problems, according to assessments with AUDIT (C) and DAST (D) (cross-sectional analysis). In each case, dried blood spot TFV-DP levels were compared among the 3 groups by using the analysis of variance test. Circles indicate outliers; horizontal lines within boxes indicate medians, box bottoms and tops indicate 25th and 75th quartiles; and error bars indicate levels within 1.5 times the interquartile range of the lower quartile and upper quartiles. AUDIT, Alcohol Use Disorders Identification Test; DAST, Drug Abuse Screening Test; TFV-DP, tenofovir diphosphate.

these findings indicate that substance use should not be used as a reason to withhold PrEP because of concerns about adherence.

We and others have shown that substance use in general, and methamphetamine and other stimulant use in particular, is a likely cause of increased sexual risk behavior among MSM and therefore a predictor for HIV acquisition (3,17-21). Thus, HIV-uninfected MSM who use substances should be considered a target population for PrEP. However, substance-using MSM often face major individual barriers (e.g., HIV-related stigma, substance use) and structural barriers (e.g., economic, healthcare) that might reduce linkage and adherence to PrEP (13,22-26).

Adherence is probably the major factor affecting PrEP effectiveness in those linked to PrEP, as outlined by a recently published mathematical model, which showed that increased adherence was the only factor resulting in reductions of the number needed to treat with PrEP to prevent 1 HIV infection (27). Our study indicates that substance use 
was not associated with decreased adherence to PrEP. Notably, in this study, persons who used methamphetamines did not have worse adherence than persons who did not use methamphetamines. A previous qualitative study indicated that barriers to PrEP uptake and adherence differ by type of substance used. In that study, stimulant drug users were more likely to be concerned that substance use would affect PrEP adherence, and were less concerned about HIV stigma as a barrier to PrEP uptake compared with alcohol users (13). However, in our study, we did not find an association between stimulant use and PrEP adherence.

Baseline frequent substance use was associated with higher likelihood of study completion, and no associations were found for alcohol use. Some methamphetamine use was associated with lower likelihood of study completion when compared with frequent or no methamphetamine use. Although some methamphetamine use might relate to MSM who use methamphetamines occasionally, in intermittent binges that are more likely to impart risk for loss to care, the reasons for the increased study dropout of these persons remains unknown.

Some or frequent stimulant use at baseline was strongly associated with contracting an incident STI during the study. This finding was driven mainly by popper use. We also found higher rates of incident STIs for persons with some methamphetamine use when compared with persons who had frequent or no methamphetamine use. One speculative explanation for this association is that many MSM take psychoactive drugs, in particular methamphetamine, and engage in sex at the same time. Also known as chemsex or party and play, this practice is associated with condomless anal sex, multiple sex partners, and the transmission of HIV and other STIs $(4,28,29)$. This intermittent methamphetamine use might not occur frequently (i.e., not fullfilling frequent methamphetamine use in an SCID questionnaire) but might be associated with high-risk sexual activities. Together with the finding that those with some methamphetamine use also have lower study completion rates, this finding might warrant further

\begin{tabular}{|c|c|c|c|c|c|c|}
\hline \multirow[b]{2}{*}{ Substance use baseline, $n=394$} & \multicolumn{2}{|c|}{ Study completion } & \multirow[b]{2}{*}{$\mathrm{p}$ value } & \multicolumn{2}{|c|}{ Incident STI } & \multirow[b]{2}{*}{$\mathrm{p}$ value } \\
\hline & No & Yes & & No & Yes & \\
\hline DAST10 problems & & & 0.59 & & & 0.043 \\
\hline No/low & $42(17)$ & 207 (83) & & $161(65)$ & $88(35)$ & \\
\hline Moderate & $25(21)$ & $95(79)$ & & $63(53)$ & 57 (48) & \\
\hline Substantial/severe & $5(20)$ & $20(80)$ & & $18(72)$ & $7(28)$ & \\
\hline Methamphetamine & & & 0.15 & & & 0.037 \\
\hline No & $56(17)$ & $275(83)$ & & $211(64)$ & $120(36)$ & \\
\hline Some & $12(30)$ & $28(70)$ & & $17(43)$ & $23(57)$ & \\
\hline Frequent & $4(17)^{\prime}$ & $19(83)$ & & $14(61)$ & $9(39)$ & \\
\hline Heroin & & & 0.24 & & & 0.80 \\
\hline No & $67(18)$ & $310(82)$ & & $230(61)$ & $115(39)$ & \\
\hline Some & $2(22)$ & $7(78)$ & & $6(67)$ & $1(33)$ & \\
\hline Frequent & $3(38)$ & $5(63)$ & & $6(75)$ & $2(25)$ & \\
\hline Poppers & & & 0.25 & & & $<0.001$ \\
\hline No & $41(21)$ & $150(79)$ & & $142(74)$ & $49(26)$ & \\
\hline Some & $19(17)$ & $94(83)$ & & $57(50)$ & $56(50)$ & \\
\hline Frequent & $12(13)$ & 78 (87) & & $43(48)$ & $47(52)$ & \\
\hline Cocaine & & & 0.31 & & & 0.18 \\
\hline No & $58(17)$ & $276(83)$ & & $211(63)$ & $123(37)$ & \\
\hline Some & $9(20)$ & $35(80)$ & & $24(55)$ & $20(45)$ & \\
\hline Frequent & $5(31)$ & $11(69)$ & & $7(44)$ & $9(56)$ & \\
\hline Stimulant substances $†$ & & & 0.40 & & & $<0.001$ \\
\hline No & $30(22)$ & $109(78)$ & & $110(79)$ & $29(21)$ & \\
\hline Some & $23(18)$ & $106(82)$ & & $67(52)$ & $62(48)$ & \\
\hline Frequent & $19(15)$ & $107(85)$ & & $65(52)$ & $61(48)$ & \\
\hline Nonstimulant substances $\ddagger$ & & & 0.95 & & & 0.33 \\
\hline No & $42(19)$ & $179(81)$ & & $140(63)$ & $81(37)$ & \\
\hline Some & $19(18)$ & $89(82)$ & & $60(56)$ & $48(44)$ & \\
\hline Frequent & $11(17)$ & $54(83)$ & & $42(65)$ & $23(35)$ & \\
\hline Any substance & & & 0.18 & & & $<0.001$ \\
\hline No & $25(24)$ & $81(76)$ & & $81(76)$ & $25(24)$ & \\
\hline Some & $25(19)$ & $110(81)$ & & $76(56)$ & $59(44)$ & \\
\hline Frequent & $22(14)$ & $131(86)$ & & $85(56)$ & $68(44)$ & \\
\hline Alcohol & & & 0.13 & & & 0.28 \\
\hline No & $16(24)$ & $51(76)$ & & $47(70)$ & $20(30)$ & \\
\hline Some & $21(22)$ & $74(78)$ & & $57(60)$ & $38(40)$ & \\
\hline Frequent & $35(15)$ & $197(85)$ & & $138(59)$ & $94(41)$ & \\
\hline
\end{tabular}


Table 4. Cox regression models used for assessing substance use and early study termination and incident STIs among MSM and transgender women, California, USA*

\begin{tabular}{|c|c|c|}
\hline Model & $\mathrm{HR}(95 \% \mathrm{Cl})$ & $\mathrm{p}$ value \\
\hline \multicolumn{3}{|l|}{ Model 1} \\
\hline Intervention arm (receiving individualized texting for adherence to daily TDF/FTC) & $1.377(0.862-2.200)$ & 0.180 \\
\hline Baseline some substance use (any) & $0.743(0.426-1.293)$ & 0.293 \\
\hline Baseline frequent substance use (any) & $0.541(0.304-0.961)$ & 0.036 \\
\hline \multicolumn{3}{|l|}{ Model 2} \\
\hline Intervention arm & $0.924(0.671-1.272)$ & 0.626 \\
\hline Age & $0.973(0.955-0.992)$ & 0.005 \\
\hline Baseline some stimulant use & $2.690(1.727-4.190)$ & $<0.001$ \\
\hline Baseline frequent stimulant use & $2.604(1.665-4.072)$ & $<0.001$ \\
\hline Positive STI test result at baseline & $1.450(1.031-2.039)$ & 0.033 \\
\hline
\end{tabular}

investigations into tailored HIV prevention counseling, as well as retention counseling, for this group of persons.

As a secondary finding, we found that substantial or severe problems with alcohol use, according to the AUDIT questionnaire at week 48, were associated with lower likelihood of adequate adherence in cross-sectional analysis, although we found no strong association when DBS TFV-DP levels were used as a continuous outcome. Also, we found no strong associations between baseline AUDIT scores and adherence composites.

Limitations of our study include that DBS TFV-DP levels were only measured at 2 time points, and that the composite adherence outcome logistic regression models did not account for missing follow-up data and time effects. In addition, we assessed frequency of substance use with validated SCID questionnaires that use categories (with the highest category being $\geq 5$ times) instead of assessing frequency as a continuous outcome. This limitation is applicable particularly to the assessment of alcohol use, in which the frequent use category (i.e., $\geq 5$ times within 3 months) might not seem appropriate. However, although our study did not look specifically into the effect of more frequent substance and alcohol use (e.g., $>10$ or $>20$ times in the previous 3 months), the study included AUDIT and DAST scores that have been accepted as measures of problematic alcohol and substance use.

Another limitation was that IDU was not assessed separately. However, when we analyzed heroin use as a proxy for IDU, we found no negative associations between heroin use and adherence, although these analyses were limited by small sample size. A recent dynamic compartmental cost model suggested oral PrEP for PWIDs as a potentially cost-effective strategy to control HIV in regions where IDU is a major driver of the substance use epidemic (30). Future studies are needed to evaluate PrEP adherence in PWIDs. Finally, there is a chance that drug interactions could increase TFV-DP concentrations in substance abusers, resulting in misclassification of substance users as adherent, but no evidence supports this hypothesis. In our study, drug interaction seems unlikely, given the consistency of findings across different drugs of abuse, including alcohol, that have different pharmacologic profiles.

In conclusion, for MSM who participated in a randomized controlled trial, we found that baseline substance users had increased STI rates, indicating higher risk behavior, but PrEP adherence was not decreased by substance use. Our findings suggest that substance-using persons are appropriately diligent with PrEP adherence and therefore are excellent candidates for PrEP.

\section{Acknowledgments}

We thank the volunteers for participating in this study; and study nurses Edward Seefried, Connie Funk, as well as other study staff, including Marvin Hanashiro, Kelly Walsh, Fang Wang, Daisy Villafuerte, Luis Mendez, Mario Guerrero, Romero Correa, Sadia Shaik, and Ruben Lopez, for their efforts during this study.

This study was supported by the California HIV Research Program (grants CHRP-MC08-SD-700 and EI-11-SD-005) and the National Institutes of Health (grants AI036214, MH113477, AI064086, MH081482, MH062512, DA026306, AI106039, and MH100974).

M.H. was principal investigator for this study, designed the analysis, assisted with analyzing data, and drafted the manuscript; D.M. and S.M. were the principal investigators of the parent randomized controlled trial and assisted with designing the analysis and drafting the manuscript; S.J. and X.S. analyzed data and assisted with designing the analysis; D.C., K.C., J.S.B., E.S.D., J.M., and M.P.D. were investigators at the participating centers of the randomized controlled trial, and helped with designing this analysis and drafting the manuscript; and P.L.A. analyzed dried blood spots and assisted in interpretation of data and drafting of the manuscript. All authors revised the manuscript critically for intellectual content, approved the final manuscript, and agreed to be accountable for all aspects of the study. 


\section{About the Author}

Dr. Hoenigl is a research professor at the University of California San Diego, San Diego, CA. His primary research interests are HIV prevention and primary HIV infection.

\section{References}

1. Freeman P, Walker BC, Harris DR, Garofalo R, Willard N, Ellen JM; Adolescent Trials Network for HIV/AIDS Interventions 016b Team. Methamphetamine use and risk for HIV among young men who have sex with men in 8 US cities. Arch Pediatr Adolesc Med. 2011;165:736-40. http://dx.doi.org/10.1001/archpediatrics.2011.118

2. Santos GM, Coffin PO, Das M, Matheson T, DeMicco E, Raiford JL, et al. Dose-response associations between number and frequency of substance use and high-risk sexual behaviors among HIV-negative substance-using men who have sex with men (SUMSM) in San Francisco. J Acquir Immune Defic Syndr. 2013;63:540-4. http://dx.doi.org/10.1097/ QAI.0b013e318293f10b

3. Hoenigl M, Chaillon A, Moore DJ, Morris SR, Smith DM, Little SJ. Clear links between starting methamphetamine and increasing sexual risk behavior: a cohort study among men who have sex with men. J Acquir Immune Defic Syndr. 2016;71:551-7. http://dx.doi.org/10.1097/QAI.0000000000000888

4. Hoenigl M, Chaillon A, Morris SR, Little SJ. HIV infection rates and risk behavior among young men undergoing community-based testing in San Diego. Sci Rep. 2016;6:25927. http://dx.doi.org/10.1038/srep25927

5. Grant RM, Lama JR, Anderson PL, McMahan V, Liu AY, Vargas L, et al.; iPrEx Study Team. Preexposure chemoprophylaxis for HIV prevention in men who have sex with men. N Engl J Med. 2010;363:2587-99. http://dx.doi.org/10.1056/NEJMoa1011205

6. Molina JM, Capitant C, Spire B, Pialoux G, Cotte L, Charreau I, et al.; ANRS IPERGAY Study Group. On-demand preexposure prophylaxis in men at high risk for HIV-1 infection. N Engl J Med. 2015;373:2237-46. http://dx.doi.org/10.1056/NEJMoa1506273

7. Baeten JM, Donnell D, Ndase P, Mugo NR, Campbell JD, Wangisi J, et al.; Partners PrEP Study Team. Antiretroviral prophylaxis for HIV prevention in heterosexual men and women. N Engl J Med. 2012;367:399-410. http://dx.doi.org/10.1056/NEJMoa1108524

8. Anderson PL, Glidden DV, Liu A, Buchbinder S, Lama JR, Guanira JV, et al.; iPrEx Study Team. Emtricitabine-tenofovir concentrations and pre-exposure prophylaxis efficacy in men who have sex with men. Sci Transl Med. 2012;4:151ra125. http://dx.doi.org/10.1126/scitranslmed.3004006

9. Choopanya K, Martin M, Suntharasamai P, Sangkum U, Mock PA, Leethochawalit M, et al.; Bangkok Tenofovir Study Group. Antiretroviral prophylaxis for HIV infection in injecting drug users in Bangkok, Thailand (the Bangkok Tenofovir Study): a randomised, double-blind, placebo-controlled phase 3 trial. Lancet. 2013;381:2083-90. http://dx.doi.org/10.1016/ S0140-6736(13)61127-7

10. Martin M, Vanichseni S, Suntharasamai P, Sangkum U, Mock PA, Chaipung B, et al. Factors associated with the uptake of and adherence to HIV pre-exposure prophylaxis in people who have injected drugs: an observational, open-label extension of the Bangkok Tenofovir Study. Lancet HIV. 2016.

11. Liu A, Glidden DV, Anderson PL, Amico KR, McMahan V, Mehrotra M, et al.; iPrEx Study Team. Patterns and correlates of PrEP drug detection among MSM and transgender women in the Global iPrEx Study. J Acquir Immune Defic Syndr. 2014;67:52837. http://dx.doi.org/10.1097/QAI.0000000000000351

12. Liu AY, Hessol NA, Vittinghoff E, Amico KR, Kroboth E, Fuchs J, et al. Medication adherence among men who have sex with men at risk for HIV infection in the United States: implications for pre-exposure prophylaxis implementation. AIDS Patient Care STDS. 2014;28:622-7. http://dx.doi.org/10.1089/apc.2014.0195

13. Oldenburg CE, Mitty JA, Biello KB, Closson EF, Safren SA, Mayer KH, et al. Differences in attitudes about HIV pre-exposure prophylaxis use among stimulant versus alcohol using men who have sex with men. AIDS Behav. 2016;20:1451-60. http://dx.doi.org/10.1007/s10461-015-1226-4

14. Moore DJ, Jain S, Dube MP, Daar E, Sun X, Yung J, et al. Randomized controlled trial of daily text messages to support adherence to PrEP in at-risk for HIV individuals: the TAPIR Study. Clin Infect Dis. 2018;66:1566-72. http://dx.doi.org/10.1093/cid/ cix1055

15. Castillo-Mancilla JR, Zheng JH, Rower JE, Meditz A, Gardner EM, Predhomme J, et al. Tenofovir, emtricitabine, and tenofovir diphosphate in dried blood spots for determining recent and cumulative drug exposure. AIDS Res Hum Retroviruses. 2013; 29:384-90.

16. Grant RM, Anderson PL, McMahan V, Liu A, Amico KR, Mehrotra M, et al.; iPrEx Study Team. Uptake of pre-exposure prophylaxis, sexual practices, and HIV incidence in men and transgender women who have sex with men: a cohort study. Lancet Infect Dis. 2014;14:820-9. http://dx.doi.org/10.1016/S1473-3099(14)70847-3

17. Buchbinder SP, Vittinghoff E, Heagerty PJ, Celum CL, Seage GR III, Judson FN, et al. Sexual risk, nitrite inhalant use, and lack of circumcision associated with HIV seroconversion in men who have sex with men in the United States. J Acquir Immune Defic Syndr. 2005;39:82-9. http://dx.doi.org/10.1097/ 01.qai.0000134740.41585.f4

18. Drumright LN, Little SJ, Strathdee SA, Slymen DJ, Araneta MR, Malcarne VL, et al. Unprotected anal intercourse and substance use among men who have sex with men with recent HIV infection. J Acquir Immune Defic Syndr. 2006;43:344-50. http://dx.doi.org/ 10.1097/01.qai.0000230530.02212.86

19. Koblin BA, Murrill C, Camacho M, Xu G, Liu KL, Raj-Singh S, et al. Amphetamine use and sexual risk among men who have sex with men: results from the National HIV Behavioral Surveillance study—New York City. Subst Use Misuse. 2007;42:1613-28. http://dx.doi.org/10.1080/10826080701212519

20. Pines HA, Gorbach PM, Weiss RE, Shoptaw S, Landovitz RJ, Javanbakht M, et al. Sexual risk trajectories among MSM in the United States: implications for pre-exposure prophylaxis delivery. J Acquir Immune Defic Syndr. 2014;65:579-86. http://dx.doi.org/ 10.1097/QAI.0000000000000101

21. Hoenigl M, Anderson CM, Green N, Mehta SR, Smith DM, Little SJ. Repeat HIV-testing is associated with an increase in behavioral risk among men who have sex with men: a cohort study. BMC Med. 2015;13:218. http://dx.doi.org/10.1186/ s12916-015-0458-5

22. Mimiaga MJ, Closson EF, Kothary V, Mitty JA. Sexual partnerships and considerations for HIV antiretroviral pre-exposure prophylaxis utilization among high-risk substance using men who have sex with men. Arch Sex Behav. 2014;43:99-106. http://dx.doi.org/10.1007/s10508-013-0208-8

23. Escudero DJ, Kerr T, Wood E, Nguyen P, Lurie MN, Sued O, et al. Acceptability of HIV pre-exposure prophylaxis (PREP) among people who inject drugs (PWID) in a Canadian setting. AIDS Behav. 2015;19:752-7. http://dx.doi.org/10.1007/s10461-014-0867-z

24. Guise A, Albers ER, Strathdee SA. 'PrEP is not ready for our community, and our community is not ready for PrEP': pre-exposure prophylaxis for HIV for people who inject drugs and limits to the HIV prevention response. Addiction. 2017;112:572-8. http://dx.doi.org/10.1111/add.13437

25. Edelman EJ, Moore BA, Calabrese SK, Berkenblit G, Cunningham C, Patel V, et al. Primary care physicians' willingness to prescribe HIV pre-exposure prophylaxis for people who inject drugs. AIDS Behav. 2017;21:1025-33. http://dx.doi.org/10.1007/ s10461-016-1612-6 
26. Marshall BD, Milloy MJ. Improving the effectiveness and delivery of pre-exposure prophylaxis (PrEP) to people who inject drugs. Addiction. 2016.

27. Jenness SM, Goodreau SM, Rosenberg E, Beylerian EN, Hoover KW, Smith DK, et al. Impact of the Centers for Disease Control's HIV preexposure prophylaxis guidelines for men who have sex with men in the United States. J Infect Dis. 2016;214:1800-7. http://dx.doi.org/10.1093/infdis/jiw223

28. Grov C, Rendina HJ, Breslow AS, Ventuneac A, Adelson S, Parsons JT. Characteristics of men who have sex with men (MSM) who attend sex parties: results from a national online sample in the USA. Sex Transm Infect. 2014;90:26-32. http://dx.doi.org/10.1136/ sextrans-2013-051094
29. Hegazi A, Lee MJ, Whittaker W, Green S, Simms R, Cutts R, et al. Chemsex and the city: sexualised substance use in gay bisexual and other men who have sex with men attending sexual health clinics. Int J STD AIDS. 2017;28:362-6. http://dx.doi.org/10.1177/0956462416651229

30. AIDSVu. Rollins School of Public Health, Emory University [cited 2017 Dec 2]. https://aidsvu.org

Address for correspondence: Martin Hoenigl, Antiviral Research Center, Division of Infectious Diseases, Department of Medicine, University of California, 200 W Arbor Dr, \#8208, San Diego, CA 92103, USA; email: mhoenigl@ucsd.edu

\section{EI D Podcast: Ending the HIV/ AI DS Pandemic}

\section{Dr. Anthony Fauci, Director of the National} Institute of Allergy and Infectious Diseases at the National Institutes of Health, discusses progress made toward ending HIV/AIDS and other pandemic infectious diseases.

\section{Visit our website to listen:} https:/ / tools.cdc.gov/ medialibrary/ index.aspx\#/ media/ id/ 380134 


\title{
Highly Pathogenic Clone of Shiga Toxin- Producing Escherichia coli 0157:H7, England and Wales
}

\author{
Lisa Byrne, ${ }^{1}$ Timothy J. Dallman, ${ }^{1}$ Natalie Adams, Amy F.W. Mikhail, Noel McCarthy, Claire Jenkins
}

We used whole-genome sequencing to investigate the evolutionary context of an emerging highly pathogenic strain of Shiga toxin-producing Escherichia coli (STEC) O157:H7 in England and Wales. A timed phylogeny of sublineage IIb revealed that the emerging clone evolved from a STEC 0157:H7 stx-negative ancestor $\approx 10$ years ago after acquisition of a bacteriophage encoding Shiga toxin (stx) 2a, which in turn had evolved from a stx $2 c$ progenitor $\approx 20$ years ago. Infection with the st $x 2 a$ clone was a significant risk factor for bloody diarrhea (OR 4.61, 95\% Cl 2.24-9.48; $p<0.001$ ), compared with infection with other strains within sublineage IIb. Clinical symptoms of cases infected with sublineage IIb stx2c and stx-negative clones were comparable, despite the loss of stx2c. Our analysis highlighted the highly dynamic nature of STEC 0157:H7 Stx-encoding bacteriophages and revealed the evolutionary history of a highly pathogenic clone emerging within sublineage IIb, a sublineage not previously associated with severe clinical symptoms.

Chiga toxin-producing Escherichia coli (STEC) 0157:H7 cause a wide range of gastrointestinal symptoms, including mild gastroenteritis, abdominal pain, vomiting, and bloody diarrhea (1). A subset of patients, most commonly the very old and the very young, go on to develop hemolytic uremic syndrome (HUS) (2). STEC O157:H7 are zoonotic, and transmission to humans is most commonly associated with ruminants, especially cattle and sheep. Transmission occurs by consumption of contaminated food or water or by direct contact with animals or their environment. The infectious dose is low (10-100 organisms), and person-to-person spread can occur in households, nurseries, and other institutional settings (1). The STEC pathotype is defined by the presence of the genes encoding Shiga toxin (Stx) type 1, type 2, or both, located on a bacteriophage incorporated into the bacterial genome (3). Stx1 and Stx2

Author affiliations: Public Health England, London, UK (L. Byrne, T.J. Dallman, N. Adams, A.F.W. Mikhail, C. Jenkins); National Institute for Health Research, London (T.J. Dallman, N. Adams, N. McCarthy); University of Warwick, Coventry, UK (N. McCarthy)

DOI: https://doi.org/10.3201/eid2412.180409 can be further divided into subtypes Stx1a-1d and Stx2a2g; Stx2a is strongly associated with causing severe disease $(4,5)$. The STEC O157:H7 population has previously been delineated into 3 main lineages (I, I/II, and II) (6), and 7 sublineages (Ia, Ib, Ic, IIa, IIb, IIc, and I/ll) (5).

In England, the most common STEC serotype is O157:H7, which causes an average of 800 cases/year (1). All STEC O157:H7 isolated at local hospital laboratories from fecal samples from hospitalized patients and all cases in the community are submitted to the Gastrointestinal Bacteria Reference Unit (GBRU) at Public Health England for confirmation of identification and typing. From 2000 through 2016, phage type (PT) 8 with the stx profile stx $1 a /$ stx $2 c$ and PT21/28 with the stx profile stx $2 a$ or stx2a/stx $2 c$ were detected most frequently in England, with PT21/28 the most frequently associated with severe disease $(2,7)$.

Since 2015, all isolates submitted to GBRU have been genome sequenced. Whole-genome sequencing (WGS) demonstrates unparalleled sensitivity and accuracy in identifying linked cases (8). Using WGS data during outbreak investigations has improved the robustness of case ascertainment and provided forensic evidence for linking human cases to the source of their infection $(9,10)$. Phylogenetic inference can also reveal how strains are related over time and space more accurately than other molecular typing methods and may provide insight into the evolutionary and epidemiologic context of emerging pathogenic clones $(8,10,11)$.

In 2015 , a total of 47 persons were affected by an outbreak in England of foodborne gastrointestinal illness caused by STEC 0157:H7 PT8 stx2a. The outbreak was associated with the consumption of contaminated prepacked salad leaves (11). The outbreak strain continued to cause sporadic infection and outbreaks of foodborne disease throughout 2016 and 2017 (11). The aim of our analysis was to investigate the evolutionary history of this newly emergent strain of STEC O157:H7 PT8 stx2a and assess the risk to public health.

\footnotetext{
${ }^{1}$ These first authors contributed equally to this article.
} 


\section{Materials and Methods}

\section{Bacterial Strains}

All isolates submitted to GBRU for confirmation and typing from local hospital laboratories in England and Wales during July 2015-December 2017 were sequenced for routine surveillance (National Center for Biotechnology Information Short Read Archive bioproject PRJNA248042). We included an additional 74 clinical isolates of STEC $\mathrm{O} 157: \mathrm{H} 7$ belonging to sublineage IIb, the lineage containing STEC O157:H7 PT8 stx2a, that were submitted to GBRU between January 2010-June 2015 from previous studies $(5,8)$ (online Technical Appendix Table, https:// wwwnc.cdc.gov/EID/article/24/12/18-0409-Techapp1. pdf). We selected these STEC O157:H7 isolates on the basis of stx subtype and phage type diversity to provide context as a sample of the background population. We defined STEC O157:H7 isolates from patients who were hospitalized as a result of their gastrointestinal symptoms or who reported bloody diarrhea as highly pathogenic or as having increased pathogenic potential compared with isolates from patients who were asymptomatic or reporting nonbloody diarrhea.

\section{Genome Sequence Analysis}

For WGS, we extracted DNA from cultures of STEC O157:H7 for sequencing on the HiSeq 2500 instrument (Illumina, San Diego, California, USA). We mapped quality trimmed Illumina reads (12) to the STEC O157:H7 reference genome Sakai (GenBank accession no. BA000007) using Burrows Wheeler Aligner-Maximum Exact Matching (BWA-MEM) (13). We identified single- nucleotide polymorphisms (SNPs) using Genome Analysis Toolkit version 2 (14) in unified genotyper mode and extracted core genome positions that had a high-quality SNP ( $>90 \%$ consensus, minimum depth $\times 10, \mathrm{GQ} \geq 30$ ) in $\geq 1$ isolate for further analysis. We performed hierarchical single linkage clustering on the pairwise SNP difference between all strains at various distance thresholds $(250,100,50,25,10,5,0)$. The result of the clustering is a SNP profile, or SNP address, that can be used to describe population structure based on clonal groups (15).

We performed recombination analysis using Gubbins (16) and reconstructed timed phylogenies using BEAST-MCMC version 2.4.7 (17). We computed alternative clock models and population priors and assessed their suitability on the basis of Bayes factor tests. The highest supported model was a relaxed log-normal clock rate with a Bayesian skyline population model. We ran all models with a chain length of 1 billion. We reconstructed a maximum clade credibility tree using TreeAnnotator version 1.75 (17).

We performed Stx subtyping as described by Ashton et al. (18). The integration of Stx-encoding prophage into the host genome has been characterized into 6 target genes: $w r b A$, encoding a NAD quinone oxidoreductase; yehV, a transcriptional regulator; $s b c B$, an exonuclease; yecE, a gene of unknown function; the tRNA gene $\operatorname{argW}$; and Z2577, which encodes an oxidoreductase (5). We mapped short

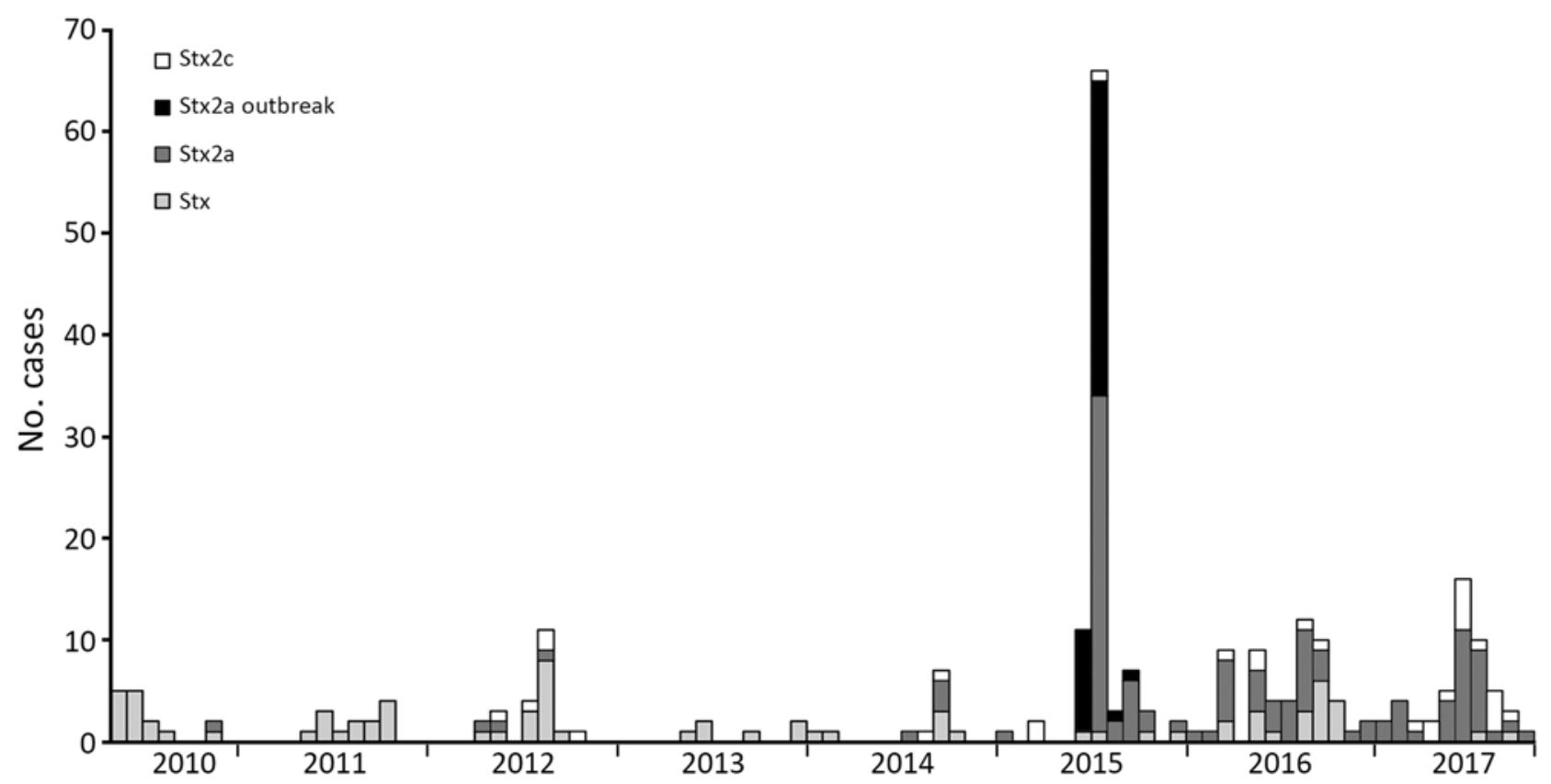

Figure 1. Cases of Shiga toxin-producing Escherichia coli $0157: \mathrm{H} 7$ belonging to sublineage Ilb, 250 single-nucleotide polymorphism single-linkage cluster $18 . \%$, by stx subtype profile, submitted to the Gastrointestinal Bacterial Reference Unit at Public Health England from England and Wales during June 1, 2010-December 31, 2017. 
reads from the STEC 0157:H7 genomes to intact reference sequences of these genes, and aligned them with BWA MEM (13). We defined occupied Stx bacteriophage insertion (SBI) sites as those strains that had disrupted alignments (5). We used Tablet to visualize read pileups (19).

\section{Data Analyses}

The National Enhanced Surveillance System for STEC (NESSS) in England was implemented on January 1, 2009, and has been described in detail elsewhere (1). For this study, we extracted data from NESSS for the cases identified as being infected with strains that had been sequenced and belonging to the sublineage IIb cluster of interest (containing the STEC 0157:H7 PT8 stx2a, outbreak strain). We excluded asymptomatic carriers detected through screening high-risk contacts of symptomatic patients as well as patients who did not return the enhanced surveillance questionnaire (ESQ) to NESSS. Data analyzed included age, gender, and whether the patient reported symptoms of nonbloody diarrhea, bloody diarrhea, and vomiting along with whether cases were hospitalized, developed typical HUS, or died. Cases were categorized into children ( $\leq 16$ years of age) or adults, based on a priori knowledge that children are most at risk for both STEC infection and progression to HUS (1). Where clinical symptoms were blank on the ESQ, we coded them as negative responses for these symptoms. We divided cases into 3 groups based on stx subtype: stx2a, stx2c, and stx-negative.

We first described patients' symptoms by stx subtype as well as by age group and sex and also examined the distribution of stx subtype by age and gender. We used Fisher exact tests to compare proportions among different groups. We assessed reporting of bloody diarrhea or hospitalization as a marker of disease severity by stx subtype. We used logistic regression to calculate odds ratios (ORs) to assess bloody diarrhea by stx subtype while adjusting for age (child/adult) and sex. We performed all analyses in Stata 13.0 (StataCorp LLC, College Station, TX, US).

\section{Results}

\section{Sublineage IIb}

The STEC O157:H7 stx2a clone analyzed in this study was located within sublineage IIb, and belonged to a 250 SNP single-linkage cluster, designated $18 \%$. This cluster comprised 251 clinical isolates: 138 of STEC O157:H7 stx2a, 77 of $s t x$-negative E. coli O157:H7, and 36 of STEC O157:H7 stx2c (Figure 1; online Technical Appendix Table). Since July 2015, when Public Health England implemented the use of WGS for STEC, the number of cases identified within sublineage IIb has remained stable $(\approx 60 / y)$. However, the number of cases of the stx-negative E. coli O157:H7 clone has declined, whereas the stx2a and stx2c clones are increasing (Figure 1).

\section{Evolutionary Timescale and Stx Prophage Insertion in STEC 0157:H7}

We reconstructed a timed phylogeny of sublineage IIb (Figure 2). We calculated the mutation rate of STEC O157:H7 within sublineage IIb to be $\approx 2$ mutations per genome per year $(95 \%$ highest posterior density [HPD] 1.7-2.4). This rate is less than the 2.6 mutations per genome per year previously calculated across the complete STEC 0157 population (5). Our analysis revealed that the emerging stx2a clone evolved from a st $x$-negative recent ancestor with the acquisition of $s t x 2 a \approx 10$ years ago $(95 \%$ HPD 9.0 years 12.7 years). Previously, this stx-negative clone had evolved from a st $x 2 c$ progenitor $\approx 20$ years ago (95\% HPD 17.6 years-24.6 years) after the loss of st $2 c$.

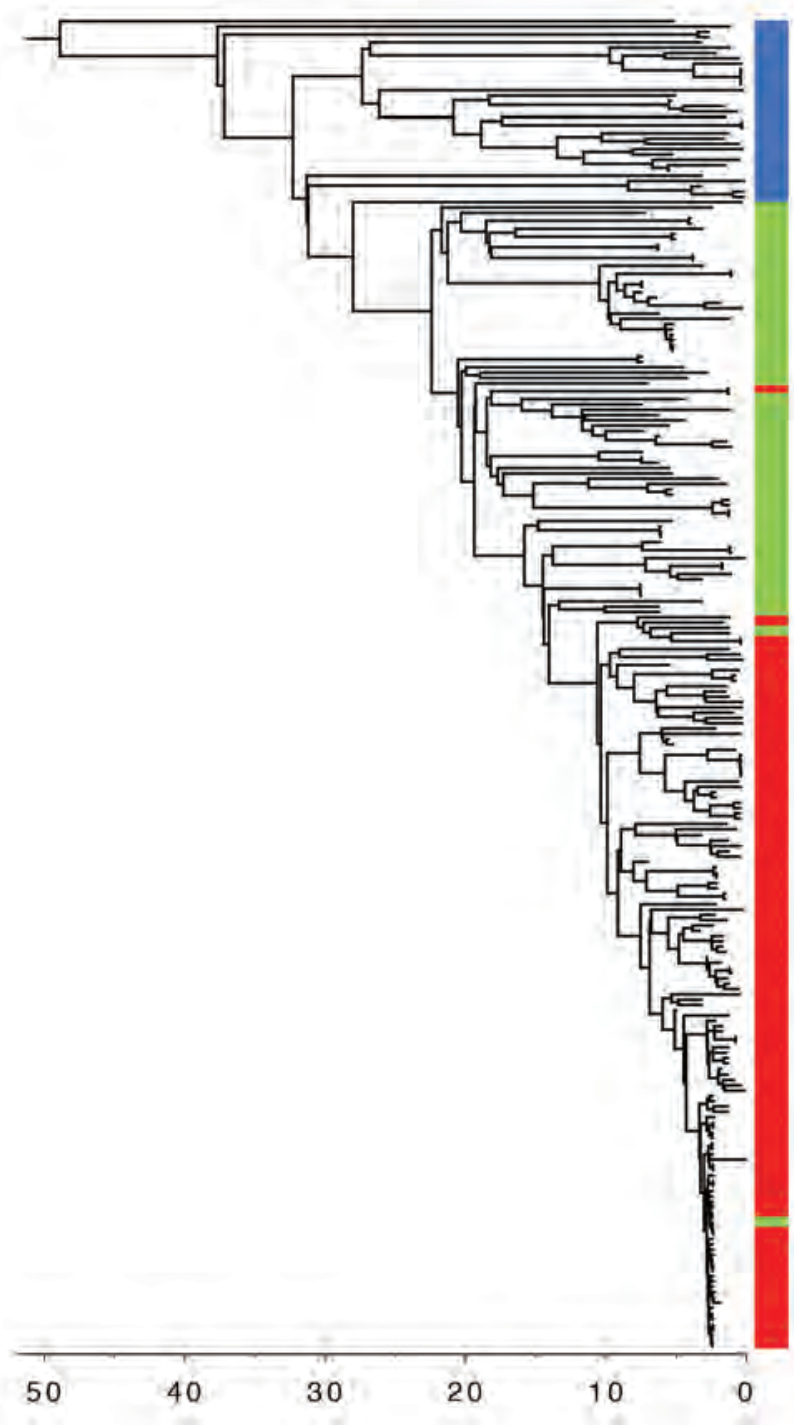

Figure 2. Timed phylogeny of Shiga toxin-producing Escherichia coli O157:H7 sublineage Ilb isolates illustrating the sequential loss of stx $2 c$ and subsequent gain of stx2a. Red indicates stx2a; green, stx negative; blue, stx2c. Scale bar indicates years in the past. 
Table 1. Clinical features of cases of Shiga toxin-producing Escherichia coli O157:H7 belonging to the IIb 250 SNP single-linkage cluster $18 . \%$ by stx subtype clone, England and Wales, July 2015-December $2017^{*}$

\begin{tabular}{|c|c|c|c|c|c|c|c|c|c|}
\hline \multirow[b]{2}{*}{ stx subtype } & \multirow[b]{2}{*}{$\begin{array}{c}\text { No. } \\
\text { patients }\end{array}$} & \multicolumn{2}{|c|}{ Diarrhea } & \multicolumn{2}{|c|}{ Bloody diarrhea } & \multicolumn{2}{|c|}{ Vomiting } & \multicolumn{2}{|c|}{ Hospitalization $\ddagger$} \\
\hline & & $\begin{array}{c}\text { No. } \\
\text { patients }\end{array}$ & $\begin{array}{c}\text { \% Patients } \\
(95 \% \mathrm{Cl})\end{array}$ & $\begin{array}{c}\text { No. } \\
\text { patients }\end{array}$ & $\begin{array}{c}\text { \% Patients } \\
(95 \% \mathrm{Cl})\end{array}$ & $\begin{array}{c}\text { No. } \\
\text { patients }\end{array}$ & $\begin{array}{c}\text { \% Patients } \\
(95 \% \mathrm{Cl})\end{array}$ & $\begin{array}{c}\text { No. } \\
\text { patients }\end{array}$ & $\begin{array}{c}\text { \% Patients } \\
(95 \% \mathrm{Cl})\end{array}$ \\
\hline$s t \times 2 c$ & 36 & 31 & $\begin{array}{c}86.1 \\
(74.2-98.0)\end{array}$ & 7 & $\begin{array}{c}22.6 \\
(5.9-33.0)\end{array}$ & 10 & $\begin{array}{c}32.3 \\
(12.4-43.1)\end{array}$ & 3 & $\begin{array}{c}8.3 \\
(-1.1 \text { to } 17.8)\end{array}$ \\
\hline stx-negative & 77 & 72 & $\begin{array}{c}93.5 \\
(87.8-99.1)\end{array}$ & 12 & $\begin{array}{c}16.7 \\
(7.3-23.9)\end{array}$ & 23 & $\begin{array}{c}31.9 \\
(19.4-40.3)\end{array}$ & 9 & $\begin{array}{c}11.7 \\
(4.3-19.0)\end{array}$ \\
\hline stx2a & 138 & 127 & $\begin{array}{c}92.0 \\
(87.4-96.6)\end{array}$ & 69 & $\begin{array}{c}54.3 \\
(41.5-58.4)\end{array}$ & 38 & $\begin{array}{c}29.9 \\
(20.0-35.1)\end{array}$ & 35 & $\begin{array}{c}25.4 \\
(18.0-32.7)\end{array}$ \\
\hline Total & 251 & 230 & $\begin{array}{c}91.6 \\
(88.1-95.1)\end{array}$ & 88 & $\begin{array}{c}38.3 \\
(29.1-41.0)\end{array}$ & 71 & $\begin{array}{c}30.9 \\
(22.7-33.9)\end{array}$ & 47 & $\begin{array}{c}18.7 \\
(13.9-23.5)\end{array}$ \\
\hline
\end{tabular}

*SNP, single-nucleotide polymorphism.

†Statistically significant difference in reporting by stx subtype using Fisher exact test $(p<0.001)$

¥Statistically significant difference in reporting by stx subtype using Fisher exact test $(p=0.011)$

Historically, the majority of strains in sublineage IIb harbored a Stx2c-encoding prophage at $s b c B$, with the yehV SBI site occupied by a truncated non-Stx-encoding prophage (5). Analysis of the short read data indicated that in the stx-negative sublineage IIb clone, yeh $V$ was disrupted but $s b c B$ was intact, indicating the loss of the Stx2c-encoding prophage from the SBI site. The more recently emerged sublineage IIb stx2a clone had disrupted SBI sites at $s b c B$ and yehV only, indicating that a Stx2a-encoding phage had been inserted into $s b c B$, the site left vacant in the $s t x$-negative clone after the loss of $s t \times 2 c$.

\section{Disease Severity of Clinical Cases within the Sublineage Ilb Cluster by stx Subtype}

Overall, 91.6\% patients (230/251, 95\% CI 88.1-95.1) had symptoms of diarrhea, and similar percentages were reported regardless of the stx subtype profile of the STEC O157:H7 causing the infection (Table 1). Rates of other symptoms varied; $28.3 \%$ of patients $(71 / 251,95 \%$ CI $22.7-$ $33.9)$ reported vomiting, $35.1 \%$ (88/251, $95 \%$ CI: $29.1-41.0)$ experienced bloody diarrhea, and $18.7 \%$ (47/251, 95\% CI: 13.9-23.5) were hospitalized. Hospitalization occurred more often for patients reporting bloody diarrhea $(35.2 \%$ [31/88, $95 \%$ CI $25.0-45.4]$ ) than those without bloody diarrhea
(9.8\% [16/163], 95\% CI 5.2-14.1; $<<0.001)$. Half $(50.0 \%)$ of patients infected with stx2a isolates reported bloody diarrhea (69/138, 95\% CI 41.5-58.4), compared with $15.6 \%$ of patients infected with $s t x$-negative isolates $(12 / 77,95 \%$ CI 7.3-23.9) and $19.4 \%$ of those infected with st $x 2 \mathrm{c}$ isolates $(7 / 36,95 \%$ CI 5.9-33.0; $<<0.001)$. No patients were known to experience HUS, and none died.

Among the 251 clinical cases, $141(56.2 \%, 95 \%$ CI $50.0 \%-62.3 \%)$ were adults and $136(54.2 \%, 95 \%$ CI $48.0 \%-60.4 \%)$ were female. Adult patients were infected with stx2a strains (61.0\% [86/141], 95\% CI 52.8\%-69.1\%) more often than children $(47.3 \%$ [52/110], 95\% CI 37.8\%$56.7 \% ; p=0.030)$. Conversely, children were more often infected with stx-negative strains than adults: $41.8 \%(46 / 110)$ of children ( $95 \%$ CI $32.4 \%-51.2 \%$ ) versus $22.0 \%(31 / 141)$ of adults (95\% CI 15.1\%-28.9\%; $\mathrm{p}=0.001)$. There was also variation in stx subtype by sex; proportionately more female patients were infected with stx2a strains $(61.0 \%$ [83/136], 95\% CI 52.7\%-69.3\%) than were male patients (47.8\% [55/115], 95\% CI 38.6\%-57.1\%; $\mathrm{p}=0.036)$. Adult patients reported bloody diarrhea $(46.8 \%$ [66/141], 95\% CI $38.5 \%-55.1 \%)$ more often than children (20.0\% [22/110], 95\% CI $12.2 \%-27.6 \% ; \mathrm{p}<0.001)$, as did female patients (40.4\% [55/136], 95\% CI 32.1\%-48.8\%) compared with

Table 2. Univariable and multivariable logistic regression analysis for reported bloody diarrhea in cases of Shiga toxin-producing Escherichia coli O157:H7 belonging to the Ilb, 250 SNP single-linkage cluster 18.\% by stx subtype clone, England and Wales, July 2015-December 2017*

\begin{tabular}{|c|c|c|c|c|c|c|}
\hline \multirow[b]{2}{*}{ Exposure } & \multirow{2}{*}{$\begin{array}{c}\text { No. (\%) } \\
\text { patients with } \\
\text { bloody diarrhea }\end{array}$} & \multirow{2}{*}{$\begin{array}{l}\text { No. }(\%) \\
\text { patients without } \\
\text { bloody diarrhea }\end{array}$} & \multicolumn{2}{|c|}{ Univariable analysis } & \multicolumn{2}{|c|}{ Multivariable analysis $\dagger$} \\
\hline & & & OR $(95 \% \mathrm{Cl})$ & $\mathrm{p}$ value & OR $(95 \% \mathrm{Cl})$ & $\mathrm{p}$ value \\
\hline \multicolumn{7}{|l|}{ stx subtype } \\
\hline stx negative & $12(16.7)$ & $65(83.3)$ & \multicolumn{2}{|c|}{ Reference } & \multicolumn{2}{|c|}{ Reference } \\
\hline$s t \times 2 c$ & $7(22.6)$ & $29(77.4)$ & $1.31(0.47-3.7)$ & 0.61 & $1.01(0.35-2.94)$ & 0.978 \\
\hline$s t \times 2 a$ & 69 (54.3) & $69(45.7)$ & $5.42(2.69-10.91)$ & $<0.001$ & $4.61(2.24-9.48)$ & $<0.001$ \\
\hline \multicolumn{7}{|l|}{ Age } \\
\hline Child, <16 y & $22(20.0)$ & $88(80.0)$ & $0.28(0.16-0.50)$ & $<0.001$ & $0.31(0.17-0.58)$ & $<0.001$ \\
\hline Adult, $>16$ y & $66(46.8)$ & $75(53.2)$ & \multicolumn{2}{|c|}{ Reference } & \multicolumn{2}{|c|}{ Reference } \\
\hline \multicolumn{7}{|l|}{ Sex } \\
\hline $\mathrm{F}$ & $55(40.4)$ & $81(59.6)$ & \multicolumn{2}{|c|}{ Reference } & \multicolumn{2}{|c|}{ Reference } \\
\hline$M$ & $33(28.7)$ & $82(71.3)$ & $0.59(0.35-1.01)$ & 0.053 & $0.81(0.45-1.47)$ & 0.494 \\
\hline
\end{tabular}

*OR, odds ratio; SNP, single-nucleotide polymorphism.

†Adjusted for each exposure variable (stx subtype, sex, age). 
male patients (28.7\% [33/115], 95\% CI 20.3\%-37.1\%), although the difference was not statistically significant $(\mathrm{p}=$ 0.05 ). The proportion of patients hospitalized did not differ significantly by sex or age group (data not shown).

After adjusting for age (adult or child) and sex, the odds ratio of experiencing bloody diarrhea was significantly higher in those infected with the stx2a clone compared with patients infected with the $s t x$-negative clone (Table 2). The odds of bloody diarrhea were no different for cases infected with the $s t x 2 c$ clone than for the stx-negative clone. Among the cases analyzed, being a child was protective for symptoms of bloody diarrhea.

\section{Discussion}

The data described here support previous studies that showed the acquisition and loss of the Stx-encoding phage is highly dynamic in STEC O157:H7 $(5,20)$. Most commonly described is the acquisition of stx $1 a$ or stx2a by a STEC 0157:H7 stx2c progenitor, followed by the subsequent loss of stx2c in strains that acquired stx2a. The involvement of a st $x$-negative intermediate in this process, as captured here, has not been previously described. The loss of the Stx2c-encoding phage appears to have facilitated the acquisition of the Stx2a-encoding phage because the latter was inserted into the same SBI site, $s b c B$, left vacant by the Stx2c-encoding phage.

Using phylogenetic analysis of variation at the wholegenome level, we reconstructed the recent evolutionary history of this emerging pathogenic clone within STEC O157:H7 sublineage IIb. We observed the loss of stx2c from the $s t x 2 c$ progenitor that caused a st $x$-negative clone $\approx 20$ years ago, followed by the acquisition of stx $2 a \approx 10$ years ago, and later expansion as shown in Figure 1. Previously, we showed that the historic acquisition of a Stx2aencoding bacteriophage by a population of STEC O157:H7 PT2 stx2c, belonging to lineage I/II indigenous in the UK cattle population, was associated with the first outbreaks of childhood HUS in England in the early 1980s (5,7). Subsequently, the increase in the incidence of STEC O157:H7 PT21/28 during the 1990s was linked to the acquisition of $s t \times 2 a$ by an indigenous population of STEC O157:H7 stx2c belonging to sublineage Ic, resulting in the highly pathogenic contemporary clone STEC PT21/28 stx2a/stx2c $(1,2,5,7)$. This clone has been associated with several outbreaks in the United Kingdom associated with a high incidence of HUS $(10,21-23)$. Here, we described an E.coli O157:H7 clone from yet another UK domestic lineage (sublineage IIb) that has recently acquired the Stx2a-encoding phage and is showing evidence of increasing pathogenic potential.

The analysis of disease severity of clinical cases by stx subtype of isolates of STEC O157:H7 within the same sublineage IIb cluster showed a significant association between the presence of $s t x 2 a$ and markers of disease severity; specifically, bloody diarrhea linked to higher rates of hospitalization. Previous studies have reported evidence of increased pathogenicity of STEC harboring stx2a $(4,5)$. However, these studies report on STEC from a wide range of different serotypes, exhibiting a wide variety of stx subtypes and are based on relatively small datasets. In this study, we present the analysis of a large dataset focusing on a specific clade within a single serotype characterized by a limited number of stx subtype combinations, specifically $s t x 2 c$, stx negative, and stx2a only. This analysis enabled us to make direct comparisons between specific stx profiles while limiting the influence of other factors in the genome.

Strains of Stx-negative E. coli O157:H7 are regarded as atypical enteropathogenic E. coli (EPEC), defined by the presence of the intimin gene (eae) and the absence of $s t x$ and the E. coli adherence factor (EAF) plasmid (24). EPEC are a common cause of infantile diarrhea and travelers' diarrhea and are known to cause mild diarrhea in adults (25). In this study, the fact that clinical cases infected with the E. coli O157:H7 stX-negative clone reported a similar frequency of symptoms, including bloody diarrhea and hospitalization, as those infected with STEC O157:H7 stx2c despite the loss of $s t x$ was an unexpected finding that requires further investigation.

A timed phylogenetic reconstruction of the evolutionary history of a cluster of sublineage IIb charted the recent emergence of a highly pathogenic clone of STEC O157:H7 stx2a. The symptom of bloody diarrhea, a marker of severity and predictor of HUS development (2), was strongly associated with cases infected with isolates of STEC O157:H7 harboring stx2a compared with those isolates without stx or those with stx2c. Our analysis also illustrated the highly dynamic nature of the Stx-encoding phages. In contrast to the observed excision events of stx2c-encoding phages in $\mathrm{O} 157: \mathrm{H} 7$, there is evidence to suggest that once a Stx2a-encoding phage is integrated into a population it tends to be maintained (5). As such, the emergence of yet another sublineage of STEC O157:H7 acquiring stx2a is of public health concern. Through this study, we demonstrate that STEC O157:H7 WGS surveillance data have a role in monitoring and anticipating emerging threats to public health and in contributing to our understanding of the underlying pathogenic mechanisms associated with severe gastrointestinal illness.

\section{Acknowledgments}

We thank Florence Aromona, Lukeki Kaindama, Nalini Purohit, and Mike Harte for their contributions to the National Enhanced Surveillance System for STEC in England, and all public health practitioners who report to the system. We also thank all the laboratory staff in the Gastrointestinal Bacteria Reference Unit, especially Michela Wright, Amy Gentle, Neil Perry, and Dawn Hedges. 
The research was funded by the National Institute for Health Research (NIHR) Health Protection Research Unit (in Gastrointestinal Infections at University of Liverpool in partnership with Public Health England, in collaboration with University of East Anglia, University of Oxford, and the Quadram Institute. The views expressed are those of the authors and not necessarily those of the National Health Service, the NIHR, the Department of Health, or Public Health England.

\section{About the Author}

Dr. Byrne is the lead epidemiologist for STEC at the National Infection Service, Public Health England, London, UK. Her interests include public health surveillance of STEC and hemolytic uremic syndrome and the investigation of outbreaks of foodborne gastrointestinal disease.

\section{References}

1. Byrne L, Jenkins C, Launders N, Elson R, Adak GK. The epidemiology, microbiology and clinical impact of Shiga toxinproducing Escherichia coli in England, 2009-2012. Epidemiol Infect. 2015;143:3475-87. http://dx.doi.org/10.1017/ S0950268815000746

2. Launders N, Byrne L, Jenkins C, Harker K, Charlett A, Adak GK. Disease severity of Shiga toxin-producing E. coli O157 and factors influencing the development of typical haemolytic uraemic syndrome: a retrospective cohort study, 2009-2012. BMJ Open. 2016;6:e009933. http://dx.doi.org/10.1136/bmjopen-2015-009933

3. Croxen MA, Law RJ, Scholz R, Keeney KM, Wlodarska M, Finlay BB. Recent advances in understanding enteric pathogenic Escherichia coli. Clin Microbiol Rev. 2013;26:822-80. http://dx.doi.org/10.1128/CMR.00022-13

4. Persson S, Olsen KE, Ethelberg S, Scheutz F. Subtyping method for Escherichia coli shiga toxin (verocytotoxin) 2 variants and correlations to clinical manifestations. J Clin Microbiol. 2007;45:2020-4. http://dx.doi.org/10.1128/JCM.02591-06

5. Dallman TJ, Ashton PM, Byrne L, Perry NT, Petrovska L, Ellis R, et al. Applying phylogenomics to understand the emergence of Shiga toxin-producing Escherichia coli O157:H7 strains causing severe human disease in the UK. Microb Genom. 2015;1:e000029. http://dx.doi.org/10.1099/mgen.0.000029

6. Zhang Y, Laing C, Steele M, Ziebell K, Johnson R, Benson AK, et al. Genome evolution in major Escherichia coli O157:H7 lineages. BMC Genomics. 2007;8:121. http://dx.doi.org/10.1186/1471-2164-8-121

7. Adams NL, Byrne L, Smith GA, Elson R, Harris JP, Salmon R, et al. Shiga toxin-producing Escherichia coli O157, England and Wales, 1983-2012. Emerg Infect Dis. 2016;22:590-7. http://dx.doi.org/10.3201/eid2204.151485

8. Dallman TJ, Byrne L, Ashton PM, Cowley LA, Perry NT, Adak G, et al. Whole-genome sequencing for national surveillance of Shiga toxin-producing Escherichia coli O157. Clin Infect Dis. 2015;61:305-12. http://dx.doi.org/10.1093/cid/civ318

9. Jenkins C, Dallman TJ, Launders N, Willis C, Byrne L, Jorgensen F, et al. Public health investigation of two outbreaks of Shiga toxin-producing Escherichia coli $\mathrm{O} 157$ associated with consumption of watercress. Appl Environ Microbiol. 2015;81:3946-52. http://dx.doi.org/10.1128/AEM.04188-14

10. Butcher H, Elson R, Chattaway MA, Featherstone CA, Willis $\mathrm{C}$, Jorgensen $\mathrm{F}$, et al. Whole genome sequencing improved case ascertainment in an outbreak of Shiga toxin-producing Escherichia coli $\mathrm{O} 157$ associated with raw drinking milk. Epidemiol Infect. 2016;144:2812-23. http://dx.doi.org/10.1017/S0950268816000509
11. Mikhail AFW, Jenkins C, Dallman TJ, Inns T, Martín AIC, Fox A, et al. An outbreak of Shiga toxin-producing Escherichia coli O157:H7 associated with contaminated salad leaves: epidemiological, genomic, and food trace back investigations. Epidemiol Infect. 2018;146:187-96.

12. Bolger AM, Lohse M, Usadel B. Trimmomatic: a flexible trimmer for Illumina sequence data. Bioinformatics. 2014;30:2114-20 https://doi.org/10.1093/bioinformatics/btu170. http://dx.doi.org/ 10.1093/bioinformatics/btu170

13. Li H, Durbin R. Fast and accurate long-read alignment with Burrows-Wheeler transform. Bioinformatics. 2010;26:589-95. http://dx.doi.org/10.1093/bioinformatics/btp698

14. McKenna A, Hanna M, Banks E, Sivachenko A, Cibulskis K, Kernytsky A, et al. The Genome Analysis Toolkit: a MapReduce framework for analyzing next-generation DNA sequencing data. Genome Res. 2010;20:1297-303. http://dx.doi.org/10.1101/ gr.107524.110

15. Dallman TJ, Ashton P, Schafer U, Jironkin A, Painset A, Shaaban S, et al. SnapperDB: A database solution for routine sequencing analysis of bacterial isolates. Bioinformatics. 2018;34:3028-29.

16. Croucher NJ, Page AJ, Connor TR, Delaney AJ, Keane JA, Bentley SD, et al. Rapid phylogenetic analysis of large samples of recombinant bacterial whole genome sequences using Gubbins. Nucleic Acids Res. 2015;43:e15. http://dx.doi.org/10.1093/nar/ gku1196

17. Drummond AJ, Suchard MA, Xie D, Rambaut A. Bayesian phylogenetics with BEAUti and the BEAST 1.7. Mol Biol Evol. 2012;29:1969-73. http://dx.doi.org/10.1093/molbev/mss075

18. Ashton PM, Perry N, Ellis R, Petrovska L, Wain J, Grant KA, et al. Insight into Shiga toxin genes encoded by Escherichia coli O157 from whole genome sequencing. PeerJ. 2015;3:e739. http://dx.doi.org/10.7717/peerj.739

19. Milne I, Bayer M, Stephen G, Cardle L, Marshall D. Tablet: visualizing next-generation sequence assemblies and mappings. Methods Mol Biol. 2016;1374:253-68. http://dx.doi.org/10.1007/ 978-1-4939-3167-5_14

20. Kyle JL, Cummings CA, Parker CT, Quiñones B, Vatta P, Newton E, et al. Escherichia coli serotype O55:H7 diversity supports parallel acquisition of bacteriophage at Shiga toxin phage insertion sites during evolution of the O157:H7 lineage. J Bacteriol. 2012;194:1885-96. http://dx.doi.org/10.1128/JB.00120-12

21. Ihekweazu C, Carroll K, Adak B, Smith G, Pritchard GC, Gillespie IA, et al. Large outbreak of verocytotoxin-producing Escherichia coli O157 infection in visitors to a petting farm in South East England, 2009. Epidemiol Infect. 2012;140:1400-13. http://dx.doi.org/10.1017/S0950268811002111

22. Rowell S, King C, Jenkins C, Dallman TJ, Decraene V, Lamden K, et al. An outbreak of Shiga toxin-producing Escherichia coli serogroup O157 linked to a lamb-feeding event. Epidemiol Infect. 2016;144:2494-500. http://dx.doi.org/10.1017/S0950268816001229

23. Wilson D, Dolan G, Aird H, Sorrell S, Dallman TJ, Jenkins C, et al. Farm-to-fork investigation of an outbreak of Shiga toxinproducing Escherichia coli O157. Microb Genom. 2018;4. http://dx.doi.org/10.1099/mgen.0.000160

24. Trabulsi LR, Keller R, Gomes TA. Typical and atypical enteropathogenic Escherichia coli. [Review]. Emerg Infect Dis. 2002;8:508-13. http://dx.doi.org/10.3201/eid0805.010385

25. Sakkejha H, Byrne L, Lawson AJ, Jenkins C. An update on the microbiology and epidemiology of enteropathogenic Escherichia coli in England 2010-2012. J Med Microbiol. 2013;62:1531-4. http://dx.doi.org/10.1099/jmm.0.062380-0

Address for correspondence: Claire Jenkins, Public Health England, Gastrointestinal Bacteria Reference Unit, 61 Colindale Ave, London, NW9 5HT, UK; email: claire.jenkins@phe.gov.uk 


\title{
Prevalence of Avian Influenza $A(H 5)$ and $A(H 9)$ Viruses in Live Bird Markets, Bangladesh
}

\author{
Younjung Kim,, ${ }^{1}$ Paritosh K. Biswas, ${ }^{1}$ Mohammad Giasuddin, ${ }^{1}$ Mahmudul Hasan, Rashed Mahmud, \\ Yu-Mei Chang, Steve Essen, Mohammed A. Samad, Nicola S. Lewis, lan H. Brown, \\ Natalie Moyen, Md. Ahasanul Hoque, Nitish C. Debnath, Dirk U. Pfeiffer, Guillaume Fournié
}

We conducted a cross-sectional study in live bird markets (LBMs) in Dhaka and Chittagong, Bangladesh, to estimate the prevalence of avian influenza $\mathrm{A}(\mathrm{H} 5)$ and $\mathrm{A}(\mathrm{H} 9)$ viruses in different types of poultry and environmental areas by using Bayesian hierarchical logistic regression models. We detected these viruses in nearly all LBMs. Prevalence of $A(H 5)$ virus was higher in waterfowl than in chickens, whereas prevalence of $A(H 9)$ virus was higher in chickens than in waterfowl and, among chicken types, in industrial broilers than in cross-breeds and indigenous breeds. LBMs with $\geq 1$ wholesaler were more frequently contaminated by $A(H 5)$ virus than retail-only LBMs. Prevalence of $A(H 9)$ virus in poultry and level of environmental contamination were also higher in LBMs with $\geq 1$ wholesaler. We found a high level of circulation of both avian influenza viruses in surveyed LBMs. Prevalence was influenced by type of poultry, environmental site, and trading patterns.

$\mathrm{L}$ ow pathogenicity avian influenza $\mathrm{A}(\mathrm{H} 9 \mathrm{~N} 2)$ virus and highly pathogenic avian influenza $\mathrm{A}(\mathrm{H} 5 \mathrm{~N} 1)$ virus are endemic in poultry populations in Bangladesh (1-4). In addition to their adverse effect on poultry production, these viruses have resulted in sporadic influenza cases in humans $(2,3)$. Because there is potential for generating novel reassortant variants between them or with other virus subtypes, their persistent circulation in poultry poses a serious threat to animal and human health globally (5-9).

Author affiliations: The Royal Veterinary College, Hatfield, UK (Y. Kim, Y.-M. Chang, N. Moyen, D.U. Pfeiffer, G. Fournié);

City University of Hong Kong, Hong Kong, China (Y. Kim,

D.U. Pfeiffer); Chittagong Veterinary and Animal Sciences University, Chittagong, Bangladesh (P.K. Biswas, R. Mahmud, M.A. Hoque); Bangladesh Livestock Research Institute, Dhaka, Bangladesh (M. Giasuddin, M. Hasan, M.A. Samad); Animal Health and Plant Agency, Weybridge, UK (S. Essen, N.S. Lewis, I.H. Brown); Food and Agriculture Organization of the United Nations, Dhaka (N.C. Debnath)

DOI: https://doi.org/10.3201/eid2412.180879
Live bird markets (LBMs) form the backbone of poultry trade in many countries in Asia. Birds of different types and from different geographic areas are introduced daily into LBMs and might be caged together, promoting local transmission of multiple virus subtypes and generating opportunities for reassortment (10-12). Surveys and routine surveillance have described the abundance and diversity of avian influenza A viruses (AIVs) in LBMs in AIV-endemic countries, including Bangladesh $(1,4,11,13-21)$. However, only the proportion of positive samples is usually reported, without accounting for the hierarchical data structure, especially the clustering of sampled poultry per LBM. Therefore, a robust assessment of AIV prevalence in LBMs is lacking, although this knowledge is essential to understand AIV epidemiology and optimize surveillance design.

Multiple poultry species and, for each poultry species, multiple breeds are offered for sale in LBMs in Bangladesh. Desi, Sonali, and broiler are the most commonly traded chicken types. Desi, which means "local" in Bengali, are indigenous chicken breeds raised in backyard farms. Sonali is a cross-breed of the Rhode Island Red cocks and Fayoumi hens. Broilers are industrial whitefeathered breeds. In addition to varying levels of susceptibility, different poultry types might be raised in different farming systems and traded through different value chains (i.e., the range of activities that businesses perform to deliver products to customers), therefore being exposed to different pathogens and pathogen loads $(22,23)$. However, the proportion of AIV-positive samples is generally reported as an overall estimate or stratified only by poultry species. Likewise, the relative position of individual LBMs in a regional or national live poultry trading network might also affect AIV prevalence; the sources from which poultry are supplied to traders and the time they spend in LBMs influence the likelihood of virus introduction and amplification in LBMs and vary depending on traders being wholesalers or retailers $(12,24)$.

${ }^{1}$ These authors contributed equally to this article. 
However, such information is generally poorly documented, or even ignored.

To address these issues, we conducted a cross-sectional survey in the 2 largest cities in Bangladesh, Dhaka and Chittagong, during February-March 2016. First, we estimated prevalence of influenza $\mathrm{A}(\mathrm{H} 5)$ and $\mathrm{A}(\mathrm{H} 9)$ viruses in marketed poultry and the LBM environment. We also accounted for the clustering effect at LBM level by using Bayesian hierarchical logistic regression models. Second, we assessed the effect of type of poultry and environmental site, and the position of LBMs in the poultry value chain on AIV prevalence.

\section{Materials and Methods}

\section{Sample Collection}

An LBM was defined as an open space in which $\geq 2$ poultry stalls sell live poultry at least once a week, and only those selling $>400$ poultry/day were considered eligible for this study. We aimed to sample $40 \mathrm{LBMs}$, and from each of these LBMs, 60 birds and 50 environmental sites (sample size calculations in online Technical Appendix 1, https:// wwwnc.cdc.gov/EID/article/24/12/18-0879-Techapp1. pdf). We used a stratified cluster sampling design. For poultry, LBMs, stalls within selected LBMs, and birds within selected stalls constituted the primary, secondary, and tertiary sampling units, respectively. For environmental sites, LBMs constituted primary sampling units and environmental sites within selected LBMs constituted secondary sampling units.

We stratified LBMs by city for Dhaka and Chittagong and, within each city, by poultry sales into large and small LBMs, hypothesizing that the risk for AIV infection varies between geographic locations and the number of poultry traded. Also, simple random sampling with too small a sample size of LBMs was not likely to capture diversity of LBM types because the distribution of LBMs as a function of their size tended to be right-skewed; the largest LBMs were often wholesale markets $(24,25)$. We hypothesized that samples of different origins have different AIV prevalences and thus stratified birds and environmental sites into 5 types of poultry and 10 types of environmental sites commonly found to be contaminated with AIV (26) (online Technical Appendix 2 Tables 1, 2, https://wwwnc.cdc.gov/ EID/article/24/12/18-0879-Techapp2.pdf).

At the first sampling stage, we sampled 40 LBMs. The number of LBMs selected in Dhaka $(\mathrm{n}=26)$ and Chittagong $(n=14)$ was proportional to the number of LBMs eligible in each city ( $\mathrm{n}=80$ for Dhaka and $\mathrm{n}=36$ for Chittagong). In each city, we further stratified LBMs by size: $50 \%$ of the selected LBMs were large, trading the highest number of poultry (13 largest LBMs in Dhaka and 7 largest LBMs in Chittagong); $50 \%$ were small, randomly selected from the bottom $50 \%$ of eligible LBMs in terms of number of poultry traded.

At the second sampling stage, we randomly selected stalls and environmental sites in each LBM independently for each type of poultry and environmental site. We created a list of stalls selling each poultry type for each LBM. We then selected stalls from these lists by using a random number generator. Likewise, for each type of environmental site, we selected sites from a list of sites identified in each LBM by using a random number generator. We collected 1 swab specimen from each environmental site. We pooled 5 swab specimens collected from the same LBM and site type.

At the third sampling stage, for each poultry type, we randomly selected 5 birds from each of the stalls selected for that type and collected cloacal and oropharyngeal swab specimens from each of the selected birds. We pooled 5 swab specimens collected from the same stall and poultry type separately for cloacal and oropharyngeal swab specimens. We transported samples collected in Chittagong on the day of sampling to the Chittagong Veterinary and Animal Sciences University (Chittagong) and samples collected in Dhaka on the day of sampling to the Bangladesh Livestock Research Institute (Dhaka). Samples were stored at $-80^{\circ} \mathrm{C}$ until diagnostic laboratory processing.

\section{Sample Screening}

We screened pools for AIVs by using a real-time reverse transcription PCR (RT-PCR) and specific primers and probes $(27,28)$. We extracted virus RNA by using the MagMAX RNA Isolation Kit (QIAGEN, Hilden, Germany) and reverse transcribed and amplified virus RNA by using the AgPath-ID One-Step RT-PCR (ThermoFisher Scientific, Waltham, MA, USA). We then screened a pool with a cycle threshold $\left(C_{t}\right)<40$ for the AIV matrix gene for the H5 and $\mathrm{H} 9$ genes. Results were considered positive for the $\mathrm{H} 5$ subtype if $\mathrm{C}_{\mathrm{t}}<38$ and positive for the $\mathrm{H} 9$ subtype if $\mathrm{C}_{\mathrm{t}}<40$ $(27,28)$. A pool was considered positive for AIV if its $C_{t}$ for the AIV matrix gene $<38$ or if it was positive for any of the $\mathrm{H} 5$ and $\mathrm{H} 9$ subtypes. A given group of 5 birds was considered positive if any of its cloacal and oropharyngeal pools showed a positive result.

\section{Bayesian Hierarchical Logistic Regression Models}

We developed 2-level Bayesian hierarchical logistic regression models to estimate LBM-level, bird-level, and environmental swab specimen-level prevalence from pooled swab samples, accounting for lower-level (swab specimens) and higher-level (LBMs) risk factors. We developed separate models for poultry and environmental samples to avoid parameters related to different sampling units interfering with each other (29). We used LBM type (retail or mixed), city (Chittagong or Dhaka), and size (small or large) as LBM- 
level risk factors. We defined an LBM with only retailers (i.e., trader selling poultry to end-users only) as retail and an LBM with $\geq 1$ wholesaler (i.e., trader selling poultry to other traders) as mixed. Each LBM-level risk factor (LBM type, city, and size) was assessed separately because we could not include them simultaneously in any given model due to the small number of sampled LBMs. In models for poultry samples, we differentiated birds into 1) chicken and waterfowl or 2) broiler, Desi, Sonali (i.e., chicken types), and waterfowl. Waterfowl were not differentiated further because of the small number of pools collected from ducks and geese. In models for environmental samples, we differentiated environmental sites into stall and slaughter areas or classified as environmental area without differentiation. We ran models (online Technical Appendix 1) by using a Markov Chain Monte Carlo simulation in JAGS (30) and R.3.4.2 (31).

\section{Results}

\section{Descriptive Results for Pooled Swab Samples}

We collected 477 pairs of cloacal and oropharyngeal pooled samples from 2,384 birds, and 400 environmental pooled samples from 2,000 environmental sites in 40 LBMs in Chittagong and Dhaka. Each pool was composed of $5 \mathrm{swab}$ specimens, except for 1 pair of cloacal and oropharyngeal pools made from 4 swab specimens collected from geese. We collected 12 pairs of cloacal and oropharyngeal pooled samples from all LBMs, except for 11 pairs from 3 LBMs. We sampled chickens in all LBMs (8-12 pairs/LBM), and waterfowl in 25 LBMs (0-4 pairs/LBM). Broilers accounted for most samples (32.1\%), followed by Desi $(26.6 \%)$ and Sonali $(25.6 \%)$. Ducks accounted for $76 \%$ of 75 pool pairs collected from waterfowl and geese accounted for $24 \%$. We collected 10 environmental pools in each LBM (stall areas: 4-8 pools, slaughter areas, 2-6 pools).

Of $47.4 \%$ (416/877) pools considered positive for AIV, $6.5 \%$ pools were negative for the AIV matrix gene but positive for any of the $\mathrm{H} 9$ and $\mathrm{H} 5$ subtypes. The H9 subtype $(63.2 \%$ positive pools) was detected more frequently than the H5 subtype (21.6\%), and $12.3 \%$ of pools were positive for both subtypes and $27.4 \%$ of pools were negative for both subtypes. Although $80.0 \%$ of the LBMs had $>1 \mathrm{~A}(\mathrm{H} 5)$ virus-positive poultry or environmental pool, $97.5 \%$ had $\geq 1 \mathrm{~A}(\mathrm{H} 9)$ virus-positive poultry or environmental pool. We determined the prevalence of pools that were positive for $\mathrm{A}(\mathrm{H} 5)$ and $\mathrm{A}(\mathrm{H} 9)$ viruses according to sample and LBM type (Table 1).

Approximately $33.3 \%$ of pools collected from waterfowl were positive for $\mathrm{A}(\mathrm{H} 5)$ virus, whereas only $5.5 \%$ of those collected from chickens were positive. In contrast, the prevalence of $\mathrm{A}(\mathrm{H} 9)$ virus-positive pools was higher in chickens $(36.3 \%)$ than in waterfowl $(18.7 \%)$. Among waterfowl, ducks (19.3\%) and geese (16.7\%) had a similar prevalence of $\mathrm{A}(\mathrm{H} 9)$ virus-positive pools, but the prevalence of $\mathrm{A}(\mathrm{H} 5)$ virus-positive pools was higher in ducks $(36.8 \%)$ than in geese $(22.2 \%)$. For both $\mathrm{H} 5$ and H9 subtypes, the prevalence of positive pools was higher for oropharyngeal samples $(8.6 \%$ for $\mathrm{H} 5$ and $31.9 \%$ for $\mathrm{H} 9)$ than for cloacal samples $(3.6 \%$ and $9.9 \%)$ in all surveyed poultry types (online Technical Appendix 2 Table 1).

Approximately $25 \%$ of environmental pools were positive for A(H9) virus, and the prevalence of positive pools was higher in slaughter areas $(31.5 \%)$, especially knives and boards used for slaughter and processing, than stall areas $(20.2 \%)$. The prevalence of $\mathrm{A}(\mathrm{H} 5)$ virus-positive environmental pools was lower $(10.8 \%)$ and did not vary between slaughter and stall areas (online Technical Appendix 2 Table 2).

\section{Bayesian Model Results}

Convergence was achieved for all models; the Gelman and Rubin statistic was $<1.001$ and the effective sample size was $>10,000$ for all parameters. For each AIV subtype, the best models reasonably predicted the number of positive pools (online Technical Appendix 2 Figure 1). In the best H5 models (i.e., lowest deviance information criterion), A(H5) virus prevalence differed according to poultry species (chicken, waterfowl), but not according to the type of environmental site. In contrast, in the best H9 models, A(H9) virus prevalence differed according to type of poultry (broiler, Desi, Sonali, waterfowl) and environmental site (slaughter and stall area). For both subtypes, LBM size and city did not improve model fit when compared with LBM type. For ease of comparison between the 2 AIV subtypes, we report LBM-level, bird-level, and environmental swab specimen-level prevalences of $\mathrm{A}(\mathrm{H} 5)$ and $\mathrm{A}(\mathrm{H} 9)$ viruses on the basis of the best H9 models with LBM type (Tables $1,2)$. This reporting did not affect interpretation of results, and we provide estimates obtained with more parsimonious models (online Technical Appendix 2 Tables 3-6).

LBM-level A(H5) virus prevalence was lower in retail LBMs than in mixed LBMs, and the posterior median estimate was $\approx 100 \%$ for mixed LBMs. However, among contaminated LBMs, levels of virus detection in birds and environmental areas did not vary between LBM types, but $\mathrm{A}(\mathrm{H} 5)$ virus prevalence in waterfowl was $\approx 6$ times higher than in chickens (Figure). The prevalence did not vary between chicken breeds or environmental areas.

In contrast to that for $\mathrm{A}(\mathrm{H} 5)$ virus, we found that the posterior median estimate of the LBM-level A(H9) virus prevalence was $\approx 100 \%$ for retail and mixed LBM groups, but the level of virus detection in birds and environmental areas was higher for mixed LBMs than for retail LBMs. A(H9) virus prevalence was highest in broilers and lowest in waterfowl. The prevalence in broilers was 3.8 times as 
Table 1. Prevalence of avian influenza $A(H 5)$ and $A(H 9)$ viruses in pooled poultry and environmental samples, Chittagong and Dhaka, Bangladesh*

\begin{tabular}{|c|c|c|c|c|c|}
\hline \multirow[b]{2}{*}{ Sample type } & \multirow[b]{2}{*}{ No. pools } & \multicolumn{2}{|c|}{$\mathrm{A}(\mathrm{H} 5)$ virus prevalence, $\%$} & \multicolumn{2}{|c|}{$\mathrm{A}(\mathrm{H} 9)$ virus prevalence, $\%$} \\
\hline & & $\begin{array}{c}\text { Observed pool } \\
\text { level }\end{array}$ & $\begin{array}{c}\text { Estimated bird level } \\
(95 \% \mathrm{HDI}) \dagger\end{array}$ & $\begin{array}{c}\text { Observed pool } \\
\text { level }\end{array}$ & $\begin{array}{c}\text { Estimated bird level } \\
(95 \% \mathrm{HDI}) \dagger\end{array}$ \\
\hline \multicolumn{6}{|l|}{ Poultry } \\
\hline \multicolumn{6}{|l|}{ Retail LBM } \\
\hline Broiler & 96 & 5.2 & $0.9(0-9.4)$ & 37.5 & $10.8(2.3-22.5)$ \\
\hline Sonali & 62 & 3.2 & $1.4(0-13.2)$ & 32.3 & $6.6(1.2-14.5)$ \\
\hline Desi & 61 & 3.4 & $1.3(0-12.2)$ & 29.5 & $6.8(1.3-14.9)$ \\
\hline Waterfowl & 20 & 50.0 & $8.1(0-46.8)$ & 25.0 & $2.8(0.3-7.0)$ \\
\hline \multicolumn{6}{|l|}{ Mixed LBM } \\
\hline Broiler & 57 & 1.8 & $0.9(0-4.0)$ & 47.4 & $13.1(1.2-30.1)$ \\
\hline Sonali & 60 & 10.0 & $1.4(0-5.7)$ & 31.7 & $8.0(0.4-19.8)$ \\
\hline Desi & 66 & 9.1 & $1.3(0-5.2)$ & 39.4 & $8.3(0.5-20.4)$ \\
\hline Waterfowl & 55 & 27.3 & $7.6(0-24.6)$ & 16.4 & $3.4(0.1-9.7)$ \\
\hline \multicolumn{6}{|l|}{ Environmental site } \\
\hline \multicolumn{6}{|l|}{ Retail LBM } \\
\hline Stall area & 101 & 5.9 & $1.5(0-10.4)$ & 16.8 & $3.2(0.1-9.1)$ \\
\hline Slaughter area & 99 & 7.1 & $1.4(0-10.1)$ & 25.3 & $6.2(0.2-16.6)$ \\
\hline \multicolumn{6}{|l|}{ Mixed LBM } \\
\hline Stall area & 102 & 15.7 & $3.1(0-11.3)$ & 23.5 & $5.2(0.1-14.1)$ \\
\hline Slaughter area & 98 & 14.3 & $3.0(0-11.0)$ & 37.8 & $9.9(0.4-25.0)$ \\
\hline
\end{tabular}

high as that in waterfowl and 1.6 times as high as that in Desi and Sonali (Figure). The environmental swab specimen-level prevalence was $\approx 2$ times as high for slaughter areas than for stall areas (Figure).

\section{Discussion}

We detected A(H5) and A(H9) viruses in marketed poultry and environmental sites in nearly all LBMs sampled in Chittagong and Dhaka. The prevalence of A(H5) virus was higher in waterfowl than in chickens, whereas the prevalence of $\mathrm{A}(\mathrm{H} 9)$ virus was higher in chickens than waterfowl and also varied among chicken types, being more prevalent in broilers than in Desi and Sonali breeds. Slaughter areas were more frequently contaminated by A(H9) virus than stall areas. Whereas mixed LBMs were more frequently contaminated by $\mathrm{A}(\mathrm{H} 5)$ virus than were retail LBMs, prevalence of $\mathrm{A}(\mathrm{H} 9)$ virus was higher in mixed LBMs than in retail LBMs for birds and environmental areas.

AIVs were ubiquitous in surveyed LBMs. The LBMlevel prevalence of $\mathrm{A}(\mathrm{H} 5)$ virus in Bangladesh was higher than in other AIV-endemic countries, including Egypt (32) and Vietnam (16). For both AIV subtypes, LBM-level prevalence was also higher than in another study conducted in Chittagong (21), which found that $17.5 \%$ of LBMs had $\geq 1$ environmental sample pool contaminated by A(H5) virus and $12.5 \%$ of LBMs had $\geq 1$ environmental sample pool contaminated by $\mathrm{A}(\mathrm{H} 9)$ virus. This difference might have been caused by different sampling schemes; in our study, we collected a larger number of pools per LBM.

Bird-level prevalence was also higher than that reported in other AIV-endemic countries, including Bangladesh $(1,4,16,19)$. However, care must be taken when comparing these results because studies used different study designs and sample screening protocols over different periods. Bird-level prevalence for contaminated LBMs was much higher than for virologic surveys conducted in backyard and commercial farms in Bangladesh $(1,4,33,34)$. This finding suggests that virus transmission was amplified along the value chain from farms to LBMs. Overcrowding and continuous supply of susceptible birds of different species and breeds might have created conditions promoting the silent transmission of AIVs within these markets (10).

Our results suggest that birds in LBMs with a mixture of wholesalers and retailers were at higher risk for infection than birds in LBMs with primarily retail poultry businesses. Poultry value chains supplying different business types might differ structurally, thereby affecting the risk for

\begin{tabular}{lccc}
\hline \multicolumn{4}{l}{ Table 2. Prevalence of avian influenza A(H5) and A(H9) viruses in LBMs, Chittagong and Dhaka, Bangladesh* } \\
\hline \multicolumn{5}{l}{ Sample type } & No. LBMs & H5 virus median prevalence, \% (95\% HDI) & H9 virus median prevalence, \% (95\% HDI) \\
\hline Poultry & & & \\
$\quad$ Retail LBM & 20 & $69.9(40.2-100.0)$ & $96.4(85.5-100.0)$ \\
$\quad$ Mixed LBM & 20 & $92.0(72.3-100.0)$ & $96.0(84.0-100.0)$ \\
Environmental sites & & & $94.9(80.5-100.0)$ \\
$\quad$ Retail LBM & 20 & $76.5(47.2-100.0)$ & $96.0(84.0-100.0)$ \\
$\quad$ Mixed LBM & 20 & $93.2(75.5-100.0)$ &
\end{tabular}

*Prevalence estimates were made by using the best models. HDI, high-density interval; LBM, live bird market. 


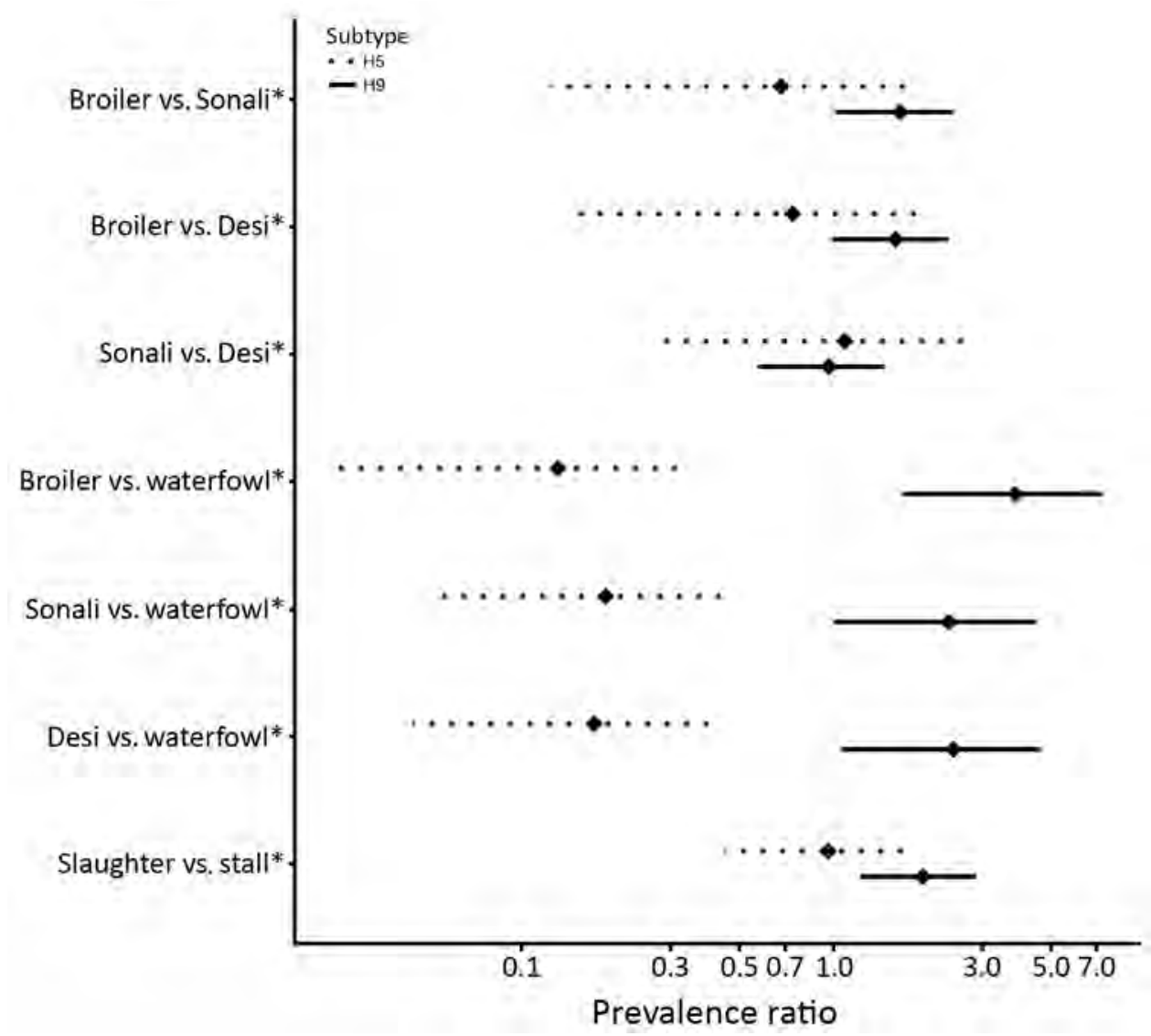

Figure. Bird and environmental swab specimen-level avian influenza $\mathrm{A}(\mathrm{H} 5)$ and $\mathrm{A}(\mathrm{H} 9)$ virus prevalence ratios, Bangladesh. Dotted lines indicate H5 subtypes, and solid lines indicate H9 subtypes. Diamonds indicate median values, and horizontal bars indicate $95 \%$ high-density interval of a given prevalence ratio. Asterisks (*) indicate reference groups for each comparison. Desi, which means "local" in Bengali, are indigenous chicken breeds raised in backyard farms. Sonali is a cross-breed of the Rhode Island Red cocks and Fayoumi hens.

introduced birds being already infected. Wholesalers generally trade a larger number of birds from more diverse geographic origins than do retailers (23) and therefore might have increased likelihood of virus introduction into mixed LBMs. Moreover, because wholesalers might sell birds to retailers in the same LBM (23), virus amplification might be increased through the presence of wholesalers.

The higher prevalence of $\mathrm{A}(\mathrm{H} 9)$ virus in broilers than in Sonali and Desi might result from differences in the structure of their respective value chains (23). Depending on the chicken type, different value chain actors might be involved and their trading practices might differ (23). The amount of time chickens spend with traders, the density at which chickens are kept in flocks of traders, and the frequency of contact with chickens from other flocks might vary with chicken type. The greater number of broilers marketed in surveyed LBMs might mean that broilers are more likely than Desi and Sonali to be sourced from large numbers of flocks, which are then mixed in densely populated trucks during transport to LBMs, promoting AIV transmission.
However, these prevalence patterns might also be caused by varying levels of genetic susceptibility to AIV infection $(35,36)$. Further investigations are needed to disentangle the possible influences of trade-related and genetic factors on AIV transmission in these chicken types. The higher level of contamination with A(H9) virus in slaughter areas than in stall areas suggests that, in the absence of appropriate biosecurity measures, slaughtering is likely to expose humans to AIVs by fomite transmission (37).

Co-circulation of $\mathrm{A}(\mathrm{H} 5)$ and $\mathrm{A}(\mathrm{H} 9)$ viruses arouses concerns over evolution of novel reassortant variants (58 ). Detection of both subtypes in some poultry pools suggests that these subtypes co-circulated near each other or in the same host during the study period. Although A(H5) viruses have considerable variability in their ability to infect, cause disease, and be transmitted among waterfowl (38), waterfowl are generally known to be less susceptible to highly pathogenic avian influenza $\mathrm{A}(\mathrm{H} 5 \mathrm{~N} 1)$ viruses (39). Therefore, waterfowl could harbor this virus but remain asymptomatic and serve as a potential host 
for genesis of novel AIVs in the presence of other virus subtypes. Also, the high level of A(H9) virus circulation among chickens could provide an ideal environment for virus diversification and selection in the LBM system. The different prevalence patterns in chickens and waterfowl observed suggest that these poultry species should be separated in LBMs and that active surveillance of novel reassortant variants should be implemented.

This study had some limitations. First, our models only accounted for clustering of sampled birds at the LBM level, but not at stall level. It is plausible that clustering of samples at stall level has less influence on AIV infection probability across the study population than clustering at the LBM level because stallholders in a given LBM in Bangladesh are likely to be supplied by the same traders and trade between each another (23). However, potential risk factors at stall level, such as ducks and hygiene level (21), might cause heterogeneous levels of AIV infection across stalls.

Second, our models did not account for the fact that sampling units in each stratum were selected with unequal probabilities. Although we selected different numbers of birds for each poultry type to account for variations in poultry populations, birds were still selected with different probabilities because their populations varied between clusters and strata. This selection might have resulted in larger SEs and thus less precise estimates compared with what could have been obtained with proportional sample sizes. Moreover, the overall prevalence might have been biased toward prevalence in samples selected with higher probabilities.

Third, our models assumed perfect sensitivity and specificity of real-time RT-PCR for pooled samples. The assays used in this study are considered highly sensitive and specific $(27,28)$, and previous studies did not report any differences in virus detection for pooled and individual samples (4042). Furthermore, virus detection in our study was based on parallel interpretation of cloacal or oropharyngeal sample test results (i.e., positive if $\geq 1$ was positive). However, pools that were negative for the AIV matrix gene but positive for any of the $\mathrm{H} 5$ and $\mathrm{H} 9$ subtypes indicate that accounting for actual test sensitivity and specificity would enable more robust prevalence estimation. Virus isolation might be attempted for RT-PCR-positive pools to assess the viability of virus material. However, this testing was not attempted in our study. Each pool consisted of swab specimens from different birds or environmental sites. Thus, multiple AIV subtypes and virus species, including Newcastle disease viruses, could be present in the same pool and interfere with growth of each virus in chicken eggs (43). Should such studies be replicated, the collection of individual swab specimens and their pooling at the laboratory is recommended to enable analysis of individual swab specimens that formed a virus-positive pool.
Fourth, we collected samples over a short period to reduce variability that could arise from seasonal variations in AIV prevalence. We focused on winter months, which are often reported to be periods of higher risk for AIV infection (44). Therefore, our estimates only represented AIV prevalence during that period and did not capture seasonal changes.

Contrary to previous cross-sectional studies, our approach enabled us to estimate AIV prevalence not only by poultry species but also by chicken type and account for the type of LBMs in which sampled poultry were marketed. Despite most AIV surveys and surveillance activities being based on multistage sampling, single-level analytic methods are generally used to analyze their results, while ignoring within-market correlation in poultry infection status. Accounting for this effect by incorporating LBM-specific random effects in a hierarchical model, and enabling mutual influence between bird-level, environmental swab specimen-level, and LBM-level parameters, improved the reliability of prevalence estimates (29). When applied to other settings, this approach needs to be adapted on the basis of an understanding of the variety of poultry value chains. Information about LBM locations and about trading practices and numbers and types of poultry sold within these LBMs is rarely readily available and would need to be collected to inform the study design.

In conclusion, LBMs surveyed in Bangladesh were highly contaminated by A(H5) and A(H9) viruses. The level of virus detection was associated with the type of poultry and environmental area and the presence of wholesalers in LBMs. These findings need to be included in the design of risk-based surveillance and control interventions aimed at reducing AIV prevalence, human exposure, and the risk for emergence of novel virus reassortant variants.

\section{Acknowledgments}

We thank Eric Brum for his support during study implementation and the participants involved in the study.

This study was supported by the BALZAC research program "Behavioural adaptations in live poultry trading and farming systems and zoonoses control in Bangladesh” (BB/L018993/1) and is 1 of 11 programs supported by the Zoonoses and Emerging Livestock Systems, a joint research initiative between the Biotechnology and Biological Sciences Research Council, the Defence Science and Technology Laboratory, the Department for International Development, the Economic and Social Sciences Research Council, the Medical Research Council, and the Natural Environment Research Council.

\section{About the Author \\ Mr. Kim is a doctoral student at the College of Veterinary Medicine and Life Sciences, City University of Hong Kong,}


Hong Kong, China. His primary research interests are the socioeconomic, cultural, and epidemiologic factors that shape zoonotic and animal infectious disease transmission, including avian influenza.

\section{References}

1. Negovetich NJ, Feeroz MM, Jones-Engel L, Walker D, Alam SM, Hasan K, et al. Live bird markets of Bangladesh: H9N2 viruses and the near absence of highly pathogenic H5N1 influenza. PLoS One. 2011;6:e19311. http://dx.doi.org/10.1371/journal.pone.0019311

2. Chakraborty A. Outbreak of mild respiratory disease caused by H5N1 and H9N2 infections among young children in Dhaka, Bangladesh, 2011. Health Science Bulletin. 2011;9:5-12.

3. World Health Organization. Cumulative number of confirmed human cases for avian influenza $\mathrm{A}(\mathrm{H} 5 \mathrm{~N} 1)$ reported to $\mathrm{WHO}$, 2003-2017; 2017 [cited 2017 Oct 9]. http://www.who.int/influenza/ human_animal_interface/H5N1_cumulative_table_archives/en/

4. Turner JC, Feeroz MM, Hasan MK, Akhtar S, Walker D, Seiler P, et al. Insight into live bird markets of Bangladesh: an overview of the dynamics of transmission of H5N1 and H9N2 avian influenza viruses. Emerg Microbes Infect. 2017;6:e12. http://dx.doi.org/10.1038/emi.2016.142

5. Lin YP, Shaw M, Gregory V, Cameron K, Lim W, Klimov A, et al. Avian-to-human transmission of H9N2 subtype influenza A viruses: relationship between H9N2 and H5N1 human isolates. Proc Natl Acad Sci U S A. 2000;97:9654-8. http://dx.doi.org/10.1073/ pnas.160270697

6. Monne I, Yamage M, Dauphin G, Claes F, Ahmed G, Giasuddin M, et al. Reassortant avian influenza $\mathrm{A}(\mathrm{H} 5 \mathrm{~N} 1)$ viruses with $\mathrm{H} 9 \mathrm{~N} 2-\mathrm{PB} 1$ gene in poultry, Bangladesh. Emerg Infect Dis. 2013;19:1630-4. http://dx.doi.org/10.3201/eid1910.130534

7. Gao R, Cao B, Hu Y, Feng Z, Wang D, Hu W, et al. Human infection with a novel avian-origin influenza A (H7N9) virus. N Engl J Med. 2013;368:1888-97. http://dx.doi.org/10.1056/ NEJMoa1304459

8. Chen H, Yuan H, Gao R, Zhang J, Wang D, Xiong Y, et al. Clinical and epidemiological characteristics of a fatal case of avian influenza A H10N8 virus infection: a descriptive study. Lancet. 2014;383:714-21. http://dx.doi.org/10.1016/S0140-6736 (14)60111-2

9. Lee DH, Bertran K, Kwon JH, Swayne DE. Evolution, global spread, and pathogenicity of highly pathogenic avian influenza H5Nx clade 2.3.4.4. J Vet Sci. 2017;18(S1):269-80. http://dx.doi.org/10.4142/jvs.2017.18.S1.269

10. Webster RG. Wet markets-a continuing source of severe acute respiratory syndrome and influenza? Lancet. 2004;363:234-6. http://dx.doi.org/10.1016/S0140-6736(03)15329-9

11. Nguyen DC, Uyeki TM, Jadhao S, Maines T, Shaw M, Matsuoka Y, et al. Isolation and characterization of avian influenza viruses, including highly pathogenic H5N1, from poultry in live bird markets in Hanoi, Vietnam, in 2001. J Virol. 2005;79:4201-12. http://dx.doi.org/10.1128/JVI.79.7.4201-4212.2005

12. Fournié G, Guitian FJ, Mangtani P, Ghani AC. Impact of the implementation of rest days in live bird markets on the dynamics of H5N1 highly pathogenic avian influenza. J R Soc Interface. 2011;8:1079-89. http://dx.doi.org/10.1098/rsif.2010.0510

13. Liu M, He S, Walker D, Zhou N, Perez DR, Mo B, et al. The influenza virus gene pool in a poultry market in South central china. Virology. 2003;305:267-75. http://dx.doi.org/10.1006/ viro.2002.1762

14. Okamatsu M, Nishi T, Nomura N, Yamamoto N, Sakoda Y, Sakurai K, et al. The genetic and antigenic diversity of avian influenza viruses isolated from domestic ducks, muscovy ducks, and chickens in northern and southern Vietnam, 2010-2012.
Virus Genes. 2013;47:317-29. http://dx.doi.org/10.1007/ s11262-013-0954-7

15. Phan MQ, Henry W, Bui CB, Do DH, Hoang NV, Thu NT, et al. Detection of HPAI H5N1 viruses in ducks sampled from live bird markets in Vietnam. Epidemiol Infect. 2013;141:601-11. http://dx.doi.org/10.1017/S0950268812001112

16. Nguyen DT, Bryant JE, Davis CT, Nguyen LV, Pham LT, Loth L, et al. Prevalence and distribution of avian influenza A(H5N1) virus clade variants in live bird markets of Vietnam, 2011-2013. Avian Dis. 2014;58:599-608. http://dx.doi.org/10.1637/ 10814-030814-Reg

17. Huang Y, Zhang H, Li X, Hu S, Cai L, Sun Q, et al. Detection and genetic characteristics of H9N2 avian influenza viruses from live poultry markets in Hunan Province, China. PLoS One. 2015;10:e0142584. http://dx.doi.org/10.1371/journal.pone.0142584

18. Chen LJ, Lin XD, Guo WP, Tian JH, Wang W, Ying XH, et al. Diversity and evolution of avian influenza viruses in live poultry markets, free-range poultry and wild wetland birds in China. J Gen Virol. 2016;97:844-54. http://dx.doi.org/10.1099/jgv.0.000399

19. Thuy DM, Peacock TP, Bich VTN, Fabrizio T, Hoang DN, Tho ND, et al. Prevalence and diversity of H9N2 avian influenza in chickens of Northern Vietnam, 2014. Infect Genet Evol. 2016;44:530-40. http://dx.doi.org/10.1016/j.meegid.2016.06.038

20. Wang X, Wang Q, Cheng W, Yu Z, Ling F, Mao H, et al. Risk factors for avian influenza virus contamination of live poultry markets in Zhejiang, China during the 2015-2016 human influenza season. Sci Rep. 2017;7:42722. http://dx.doi.org/10.1038/ srep42722

21. Sayeed MA, Smallwood C, Imam T, Mahmud R, Hasan RB, Hasan M, et al. Assessment of hygienic conditions of live bird markets on avian influenza in Chittagong metro, Bangladesh. Prev Vet Med. 2017;142:7-15. http://dx.doi.org/10.1016/ j.prevetmed.2017.04.009

22. Biswas PK, Biswas D, Ahmed S, Rahman A, Debnath NC. A longitudinal study of the incidence of major endemic and epidemic diseases affecting semi-scavenging chickens reared under the Participatory Livestock Development Project areas in Bangladesh. Avian Pathol. 2005;34:303-12. http://dx.doi.org/ 10.1080/03079450500178972

23. Moyen N, Ahmed G, Gupta S, Tenzin T, Khan R, Khan T, et al. A large-scale study of a poultry trading network in Bangladesh: implications for control and surveillance of avian influenza viruses. BMC Vet Res. 2018;14:12. http://dx.doi.org/10.1186/s12917-0181331-5

24. Fournié G, Tripodi A, Nguyen TT, Nguyen VT, Tran TT, Bisson A, et al. Investigating poultry trade patterns to guide avian influenza surveillance and control: a case study in Vietnam. Sci Rep. 2016;6:29463. http://dx.doi.org/10.1038/srep29463

25. Molia S, Boly IA, Duboz R, Coulibaly B, Guitian J, Grosbois V, et al. Live bird markets characterization and trading network analysis in Mali: implications for the surveillance and control of avian influenza and Newcastle disease. Acta Trop. 2016;155:77-88. http://dx.doi.org/10.1016/j.actatropica.2015.12.003

26. Indriani R, Samaan G, Gultom A, Loth L, Irianti S, Adjid R, et al. Environmental sampling for avian influenza virus A (H5N1) in live-bird markets, Indonesia. Emerg Infect Dis. 2010;16:1889-95. http://dx.doi.org/10.3201/eid1612.100402

27. Monne I, Ormelli S, Salviato A, De Battisti C, Bettini F, Salomoni A, et al. Development and validation of a one-step real-time PCR assay for simultaneous detection of subtype H5, $\mathrm{H} 7$, and $\mathrm{H} 9$ avian influenza viruses. J Clin Microbiol. 2008;46:1769-73. http://dx.doi.org/10.1128/JCM.02204-07

28. Heine HG, Foord AJ, Wang J, Valdeter S, Walker S, Morrissy C, et al. Detection of highly pathogenic zoonotic influenza virus H5N6 by reverse-transcriptase quantitative polymerase chain reaction. Virol J. 2015;12:18. http://dx.doi.org/10.1186/s12985-015-0250-3 
29. Kruschke JK. Doing Bayesian data analysis: a tutorial with R, JAGS, and Stan. 2nd ed. New York: Academic Press; 2014.

30. Plummer M. JAGS version 3.4.0 user manual, 2013 [cited 2018 Aug 23]. http://www.stats.ox.ac.uk/ nicholls/MScMCMC15/ jags_user_manual.pdf

31. R Core Team. R: a language and environment for statistical computing. Vienna: R Foundation for Statistical Computing, 2016 [cited 2018 Aug 23]. https://www.r-project.org/

32. Abdelwhab EM, Selim AA, Arafa A, Galal S, Kilany WH, Hassan MK, et al. Circulation of avian influenza H5N1 in live bird markets in Egypt. Avian Dis. 2010;54:911-4. http://dx.doi.org/ 10.1637/9099-100809-RESNOTE.1

33. Khatun A, Giasuddin M, Islam KM, Khanom S, Samad MA, Islam MR, et al. Surveillance of avian influenza virus type A in semi-scavenging ducks in Bangladesh. BMC Vet Res. 2013;9:196. http://dx.doi.org/10.1186/1746-6148-9-196

34. Sarkar S, Khan SU, Mikolon A, Rahman MZ, Abedin J, Zeidner N, et al. An epidemiological study of avian influenza A (H5) virus in nomadic ducks and their raising practices in northeastern Bangladesh, 2011-2012. Influenza Other Respi Viruses. 2017;11:275-82. http://dx.doi.org/10.1111/irv.12438

35. Blohm U, Weigend S, Preisinger R, Beer M, Hoffmann D. Immunological competence of different domestic chicken breeds against avian influenza infection. Avian Dis. 2016;60(Suppl): 262-8. http://dx.doi.org/10.1637/11159-051615-RegR

36. Ruiz-Hernandez R, Mwangi W, Peroval M, Sadeyen JR, Ascough $\mathrm{S}$, Balkissoon D, et al. Host genetics determine susceptibility to avian influenza infection and transmission dynamics. Sci Rep. 2016;6:26787. http://dx.doi.org/10.1038/srep26787

37. Fournié G, Høg E, Barnett T, Pfeiffer DU, Mangtani P. A systematic review and meta-analysis of practices exposing humans to avian influenza viruses, their prevalence, and rationale. Am J Trop Med Hyg. 2017;97:376-88. http://dx.doi.org/10.4269/ajtmh.17-0014

38. Hulse-Post DJ, Sturm-Ramirez KM, Humberd J, Seiler P, Govorkova EA, Krauss S, et al. Role of domestic ducks in the propagation and biological evolution of highly pathogenic H5N1 influenza viruses in Asia. Proc Natl Acad Sci U S A. 2005;102:10682-7. http://dx.doi.org/10.1073/pnas.0504662102

39. Kim JK, Negovetich NJ, Forrest HL, Webster RG. Ducks: the "Trojan horses" of H5N1 influenza. Influenza Other Respi Viruses. 2009;3:121-8. http://dx.doi.org/10.1111/j.1750-2659.2009.00084.x

40. Ladman BS, Spackman E, Gelb J Jr. Comparison of pooling 11 or 5 oropharyngeal swabbings for detecting avian influenza virus by real-time reverse transcription-PCR in broiler chickens. Avian Dis. 2012;56:227-9. http://dx.doi.org/10.1637/9839-062011-ResNote.1

41. Arnold ME, Slomka MJ, Coward VJ, Mahmood S, Raleigh PJ, Brown IH. Evaluation of the pooling of swabs for real-time PCR detection of low titre shedding of low pathogenicity avian influenza in turkeys. Epidemiol Infect. 2013;141:1286-97. http://dx.doi.org/ $10.1017 /$ S0950268812001811

42. Spackman E, Pedersen JC, McKinley ET, Gelb J Jr. Optimal specimen collection and transport methods for the detection of avian influenza virus and Newcastle disease virus. BMC Vet Res. 2013;9:35. http://dx.doi.org/10.1186/1746-6148-9-35

43. Ge S, Zheng D, Zhao Y, Liu H, Liu W, Sun Q, et al. Evaluating viral interference between Influenza virus and Newcastle disease virus using real-time reverse transcription-polymerase chain reaction in chicken eggs. Virol J. 2012;9:128. http://dx.doi.org/10.1186/1743-422X-9-128

44. Park AW, Glass K. Dynamic patterns of avian and human influenza in east and southeast Asia. Lancet Infect Dis. 2007;7:543-8. http://dx.doi.org/10.1016/S1473-3099(07)70186-X

Address for correspondence: Younjung Kim, City University of Hong Kong, Rm 1B-410, 4/F, Block 1, To Yuen Bldg, 31 To Yuen St, Kowloon, Hong Kong, China; email: younjung.kim@my.cityu.edu.hk

\section{EI D Podcast:} Emerging I nfectious Diseases Cover Art Byron Breedlove, managing editor of the journal, elaborates on aesthetic considerations and historical factors, as well as the complexities of obtaining artwork for Emerging Infectious Diseases.
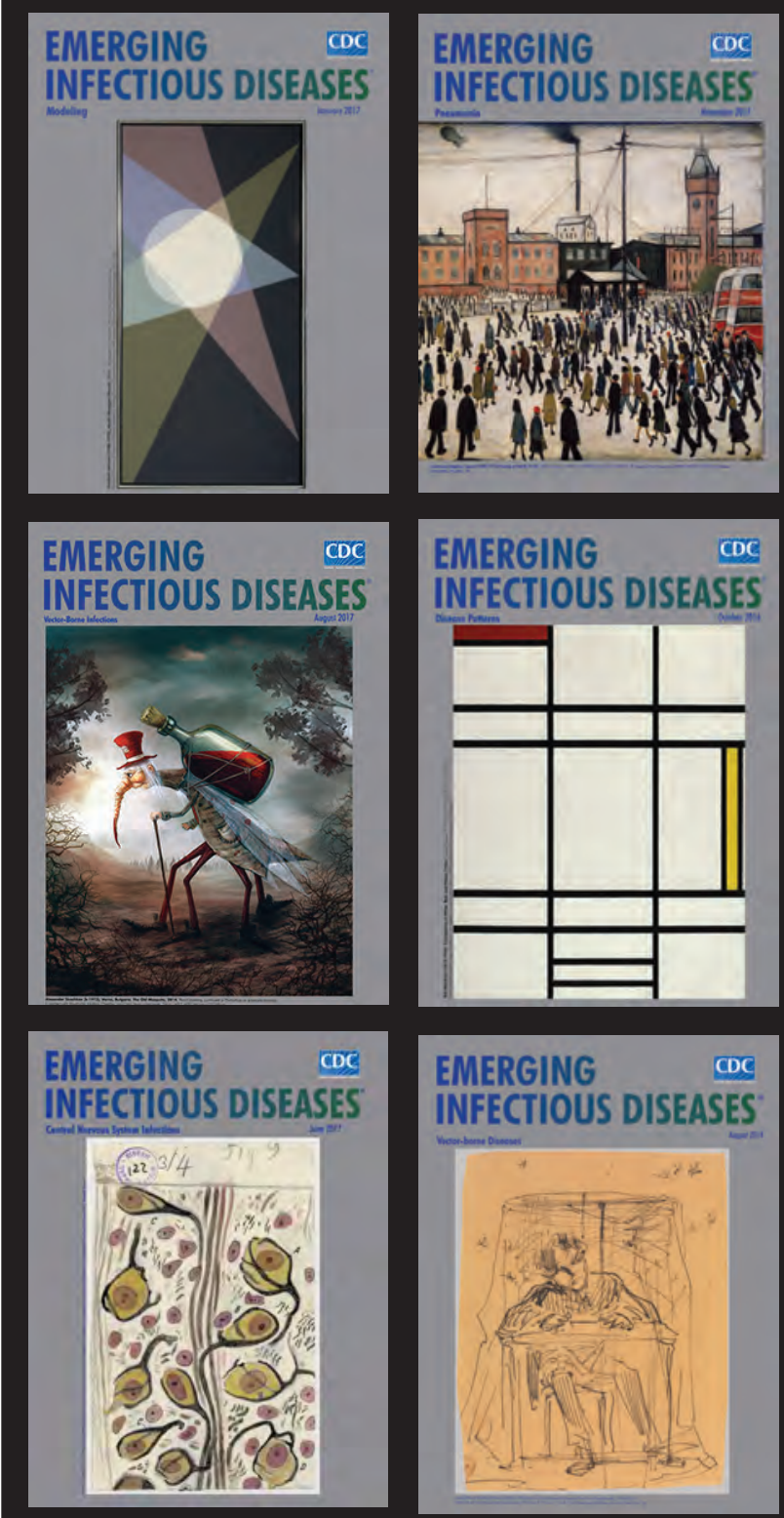

Visit our website to listen:

EMERCING INFECTIOUS DISEASES 


\title{
Human Exposure to Novel Bartonella Species from Contact with Fruit Bats
}

\author{
Ying Bai, Modupe O.V. Osinubi, Lynn Osikowicz, Clifton McKee, Neil M. Vora, Maria Rosales Rizzo, \\ Sergio Recuenco, Lora Davis, Mike Niezgoda, Ajoke M. Ehimiyein, Grace S.N. Kia, Akin Oyemakinde, \\ Olufunmilayo Sanni Adeniyi, Yemi H. Gbadegesin, Olugbon A. Saliman, Abiodun Ogunniyi, \\ Albert B. Ogunkoya, Michael Y. Kosoy; Idanre Bat Festival Investigation Team ${ }^{1}$
}

Twice a year in southwestern Nigeria, during a traditional bat festival, community participants enter designated caves to capture bats, which are then consumed for food or traded. We investigated the presence of Bartonella species in Egyptian fruit bats (Rousettus aegyptiacus) and bat flies (Eucampsipoda africana) from these caves and assessed whether Bartonella infections had occurred in persons from the surrounding communities. Our results indicate that these bats and flies harbor Bartonella strains, which multilocus sequence typing indicated probably represent a novel Bartonella species, proposed as Bartonella rousetti. In serum from 8 of 204 persons, we detected antibodies to $B$. rousetti without cross-reactivity to other Bartonella species. This work suggests that bat-associated Bartonella strains might be capable of infecting humans.

$\mathrm{B}$ ats are natural reservoirs for a variety of pathogens (1). However, despite the risk to human health, persons around the world still intentionally handle bats, often without taking appropriate precautions. This lack of precautions is particularly evident in the tropics, where bats are

Author affiliations: Centers for Disease Control and Prevention, Fort Collins, Colorado, USA (Y. Bai, L. Osikowicz, C. McKee, M.R. Rizzo, M.Y. Kosoy); Centers for Disease Control and Prevention, Atlanta, Georgia, USA (M.O.V. Osinubi, N.M. Vora, L. Davis, M. Niezgoda); Colorado State University, Fort Collins (C. McKee); Universidad Nacional Mayor de San Marcos, Lima, Peru (S. Recuenco); Ahmadu Bello University, Zaria, Nigeria (A.M. Ehimiyein, G.S.N. Kia); African Field Epidemiology Network, Abuja, Nigeria (A. Oyemakinde); Federal Ministry of Health, Abuja (O.S. Adeniyi); Federal Ministry of Science and Technology, Ibadan, Nigeria (Y.H. Gbadegesin); Ministry of Agriculture and Natural Resources, Ilorin, Nigeria (O.A. Saliman); Nigeria Center for Disease Control, Abuja (A. Ogunniyi); Centre for Control and Prevention of Zoonoses/Rabies in West Africa International, Ibadan (A.B. Ogunkoya)

DOI: https://doi.org/10.3201/eid2412.181204 abundant and frequently roost within or in close proximity to humans and domestic animals. In Asia and Africa, larger fruit bats (family Pteropodidae) are used as food, for either cultural reasons or subsistence (2). In some cultures, bat caves serve as spiritual sanctuaries (3).

One particular situation that has attracted the attention of scientists is a bat festival that takes place biannually in the Idanre Hills area of Nigeria. During the festival, which has occurred for many years, men enter designated caves, often without appropriate personal protective equipment, to capture bats. Local customs forbid persons from entering the caves outside of these festivities without permission from the community leadership. The captured bats are then eaten, used in cultural rituals, or sold as bushmeat (3). The predominant bat species within the caves is the Egyptian fruit bat (Rousettus aegyptiacus); colony sizes can reach $>1,000$ (4). Egyptian fruit bats are known reservoirs of zoonotic pathogens including Lagos bat virus, Marburg virus, and Yersinia pseudotuberculosis (5-8). Given the close human-to-bat contact that occurs during the festival, there is a risk for spillover of batborne pathogens to humans.

The genus Bartonella currently includes $>30$ species of bacteria (9), many of which have been described only recently. Various arthropod vectors seem to play an essential role in the maintenance and transmission of most known Bartonella species $(9,10)$. In recent years, recognition of multiple Bartonella species as human pathogens responsible for a wide range of clinical manifestations has grown. Numerous novel strains of Bartonella have been discovered in bats of various species around the globe, including the human pathogen Candidatus Bartonella mayotimonensis, which was originally detected in aortic valve tissue of a person with endocarditis (11-13). In addition, a novel Bartonella genotype found in bats from the country of Georgia clustered with genotypes found in human forest workers from Poland (14).

\footnotetext{
${ }^{1}$ Team members are listed at the end of this article.
} 
During 2010 and 2013, we researched the health risk to humans participating in the Idanre bat festival. We sampled bats and their ectoparasites from the caves and used them to identify a variety of zoonotic pathogens, including Bartonella. We recruited human participants from the surrounding community and surveyed them (through an orally administered questionnaire and serologic testing) to understand risk factors and the occurrence of pathogen spillover from bats to humans. We examined whether bats and ectoparasites of these bats within the caves used in the Idanre bat festival are infected with Bartonella, characterized any Bartonella species identified in bats or bat flies, and screened human serum samples for evidence of Bartonella infection.

\section{Materials and Methods}

Human subjects work was approved by the Centers for Disease Control and Prevention (CDC) Institutional Review Board, the Ahmadu Bello University Human Ethics Board, and the National Health Research Ethics Committee of Nigeria. All animal procedures were conducted in compliance with a field protocol approved by the CDC Animal Institutional Care and Use Committee.

\section{Field Sites, Bat Capture, and Sample Collection}

We captured bats by nets in 2 caves in Idanre Hills, Ondo State, southwestern Nigeria, in September $2010(\mathrm{n}=106)$ and February $2013(\mathrm{n}=71)$. We identified all bats by morphologic characteristics as Egyptian fruit bats $(R$. aegyptiacus). Captured bats were anesthetized by intramuscular injection of ketamine hydrochloride $(0.05-0.1 \mathrm{mg} / \mathrm{g}$ bat weight) and exsanguinated via cardiac puncture after surface sterilization with $75 \%$ alcohol. Serum and blood clots were separated by centrifugation. Clots were stored at $-80^{\circ} \mathrm{C}$ except while still in the field or being shipped, during which time they were stored on dry ice.

\section{Bat Blood Culture and Characterization of Bartonella Strain}

We plated bat blood clots on heart infusion agar containing $10 \%$ rabbit blood and incubated in an aerobic atmosphere with $5 \%$ carbon dioxide at $35^{\circ} \mathrm{C}$ for up to 4 weeks. Bacterial colonies morphologically identified as Bartonella were subcultured to obtain pure cultures.

We prepared crude genomic DNA by heating a heavy suspension of pure culture for 10 minutes at $95^{\circ} \mathrm{C}$, followed by centrifugation of the lysed cells for 1 minute at 3,000 rpm. The supernatant was then transferred to a clean centrifuge tube to be used as the template DNA. We first verified all isolates obtained from the blood clots as Bartonella spp. by PCR amplification targeting a fragment of the citrate synthase gene (gltA) (15). Positive (B. doshiae) and negative (deionized water) controls were included to ensure that the PCR worked properly.
We purified and sequenced all PCR products of gltA in both directions by using an ABI 3130 Genetic Analyzer (Applied Biosystems, Foster City, CA, USA). We used the Lasergene software package (DNASTAR, Madison, WI, USA) to compare the generated gltA sequences with all available Bartonella species/genotypes. Once the sequences were identified, we selected 1 representative strain (R191) for further characterization with multilocus sequence typing on the basis of sequence analysis of 8 molecular markers (ftsZ, gltA, nuoG, ribC, rpoB, ssrA, 16S rRNA, and internal transcribed spacers [ITS]) (16). For phylogenetic analyses, we used the neighbor-joining method by the Kimura 2-parameter distance method and bootstrap calculations with 1,000 replicates.

\section{Bat Ectoparasite Collection and Detection of Bartonella DNA}

We collected ectoparasites from the skin and pelage of bats and stored them in microcentrifuge tubes with $70 \%$ ethanol. Ectoparasite species were identified by using available morphologic keys (17), and identifications were later confirmed by sequencing of the mitochondrial $16 \mathrm{~S}$ rRNA and cytochrome oxidase I (COI) genes $(18,19)$.

Using a Bullet Blender Gold homogenizer (Next Advance, Averill Park, NY, USA), we homogenized whole ectoparasites in Navy Eppendorf bead tubes (Next Advance) containing $400 \mu \mathrm{L}$ brain-heart infusion broth (CDC, Atlanta, GA, USA). We extracted DNA from the homogenates by using the KingFisher Flex Purification System and the associated MagMAX Pathogen RNA/DNA Kit (both ThermoFisher, Waltham, MA, USA) according to the manufacturer's protocols. Detection of Bartonella DNA in ectoparasite samples was performed by nested PCR for gltA (20) because of low concentrations of DNA and by conventional PCR for ITS (21), followed by sequencing and sequence analysis of amplicons.

\section{Preparation of Antigen from the Bartonella Strain Obtained from Bats}

We produced a whole-cell antigen by co-cultivating Vero E6 cells with the pure culture $\left(\approx 10^{6}\right.$ agar-grown organisms) of the Bartonella strain (R-191) obtained from Egyptian fruit bats. Both were put into T-150 flasks that contained minimum essential medium supplemented with $10 \%$ fetal calf serum, $10 \mathrm{mmol}$ HEPES buffer solution, $10 \mathrm{mmol}$ nonessential amino acids, and $2 \mathrm{mmol} \mathrm{L-glutamine.} \mathrm{The} \mathrm{flasks} \mathrm{were}$ incubated at $35^{\circ} \mathrm{C}$ and harvested on postinoculation day 4 . At harvest, all but $2 \mathrm{~mL}$ of the medium was removed from the flask, sterile glass beads were added, and the flask was gently rocked to remove the Vero E6 cell monolayer. Drops $(\approx 15 \mu \mathrm{L})$ of the cell suspension were mounted on each well of 12-well glass slides, which were then air dried, fixed in acetone for 15 minutes, and stored at $-70^{\circ} \mathrm{C}$ until use. 


\section{Human Serum Collection and Testing for Antibodies}

Persons in communities surrounding the caves who gave consent were enrolled in the study 11-15 days after the first bat festival of 2013 (February 19, 2013); not all of these persons had participated in all bat festival activities. Participants were asked about their contact with bats and their role in the festival, and some provided a blood sample (considered an acute-phase specimen). About 69-78 days later, a follow-up survey was conducted and a second blood sample (considered a convalescent-phase specimen) was collected (the second bat festival of 2013 did not take place between collection of the acute- and convalescent-phase samples). Serum and blood clots were separated by centrifugation; serum was stored at $-80^{\circ} \mathrm{C}$ except while in the field or being shipped, during which time it was stored on dry ice.

To screen human serum, we used an indirect immunofluorescence assay at an initial dilution of 1:32 for IgG against the specific Bartonella antigen from the bat-associated isolate. Antigen-covered wells of the slide were overlaid with dilutions of human serum. Separate slides were included with positive and negative controls. The positive control against the Egyptian fruit bat-associated Bartonella species was produced in laboratory mice via mouse immunization with heat-inactivated bacterium (ProSci Incorporated, Poway, CA, USA). All slides were incubated at $35^{\circ} \mathrm{C}$ for 30 minutes and then washed in phosphate-buffered saline for 15 minutes. We used anti-human and antimouse conjugates (Kirkegaard \& Perry Laboratories Inc., Gaithersburg, MD, USA) for human and control serum samples, respectively. Each human serum sample reactive at the initial dilution was further titrated in 2-fold dilutions to endpoint; to check for cross-reactivity, we tested the final positive samples (defined as a titer $\geq 1: 64$ ) for 3 other Bartonella antigens (B. elizabethae, B. henselae, and B. quintana) previously reported in Africa (22-24).

\section{Results}

\section{Bartonella in Egyptian Fruit Bats}

We recovered Bartonella isolates from 22 of 177 Egyptian fruit bat blood clots, giving an overall prevalence of $12.4 \%$. The gltA sequences of all Bartonella strains obtained from Egyptian fruit bats were identical or similar ( $>97 \%$ identity) to each other and represented 4 unique variants (GenBank accession nos. HM363764, MH069693-MH069695). A variant is defined when it differs by $\geq 1 \mathrm{nt}$ from others. Together with Bartonella strains obtained from Egyptian fruit bats in Kenya (25), these variants constitute a monophyletic genogroup that is distant from all other genotypes previously found in other bat species and any other described Bartonella species.

Multilocus sequence typing of the type strain ( $\mathrm{glt} A$; GenBank accession no. HM363764) with 7 additional genetic loci (ftsZ, nuoG, ribC, rpoB, ssrA, 16S rRNA, and ITS) further confirmed the uniqueness of this strain. Sequencing information for each genetic marker demonstrated that the Bartonella strain from the Egyptian fruit bats was distant from all other known Bartonella species and genotypes, including those reported from other bats from Africa. Sequences of all genetic loci obtained during the analyses were deposited in GenBank (accession nos. HM363769, KM387321, HM363779, HM363774, KM382247, HM363784, and KM382255). We compared the fragment sequences of each target with those from other Bartonella species/genotypes. The Egyptian fruit bat-associated Bartonella formed a separate genetic group that was distant from all other Bartonella species with $\geq 20 \%$ genetic distance and probably represents a novel Bartonella species, according to the definition of La Scola et al. (26). We proposed that this bacterial species be named Bartonella rousetti, to reflect the Egyptian fruit bat (Rousettus aegyptiacus) as the natural host. A phylogenetic tree based on the ITS locus illustrates the relationship of this proposed novel species to other Bartonella species (Figure).

\section{Identification of Bat Flies and Detection of Bartonella DNA}

In 2013, we collected 51 ectoparasites from Egyptian fruit bats. With the exception of 1 unidentified mite, all arthropods were identified as the bat fly Eucampsipoda africana Theodor (Diptera: Nycteribiidae). The morphologic identification of every bat fly was confirmed by 1 or both mitochondrial markers (16S rRNA or COI). Representative 16S rRNA (accession nos. MH138030-MH138037) and COI (accession nos. MH151059-MH151066) sequences have been deposited in GenBank.

Of the 50 DNA extracts from bat flies, 21 (42\%) produced $\geq 1$ ITS or gltA sequence that was confirmed via BLAST (https://blast.ncbi.nlm.nih.gov/Blast.cgi) as Bartonella. Positive samples yielded 19 ITS sequences and 18 gltA sequences; 16 samples yielded sequences for both loci and 5 samples yielded only 1 sequence. All but 1 of the 19 ITS sequences matched closely to the proposed $B$. rousetti ( $>95 \%$ sequence identity) (Figure); the remaining sequence was identical to B. tamiae (DQ395180). Of the 18 (66.7\%) gltA sequences, 12 were close matches for $B$. rousetti ( $>98.3 \%$ sequence identity); all 12 of these samples also produced ITS sequences matching this strain.

The remaining 6 gltA sequences were identical to Bartonella sequences detected in a louse (Neohaematopinus sciuri) collected from a dead Eastern gray squirrel (Sciurus carolinensis) at a zoo in Greenville, SC, USA (GenBank accession no. EU368000) and an unidentified tick collected from a sheep in Peru (GenBank accession no. AF415209). These sequences were also closely ( $>99 \%$ sequence identity) related to other sequences from fleas (Ctenocephalides 


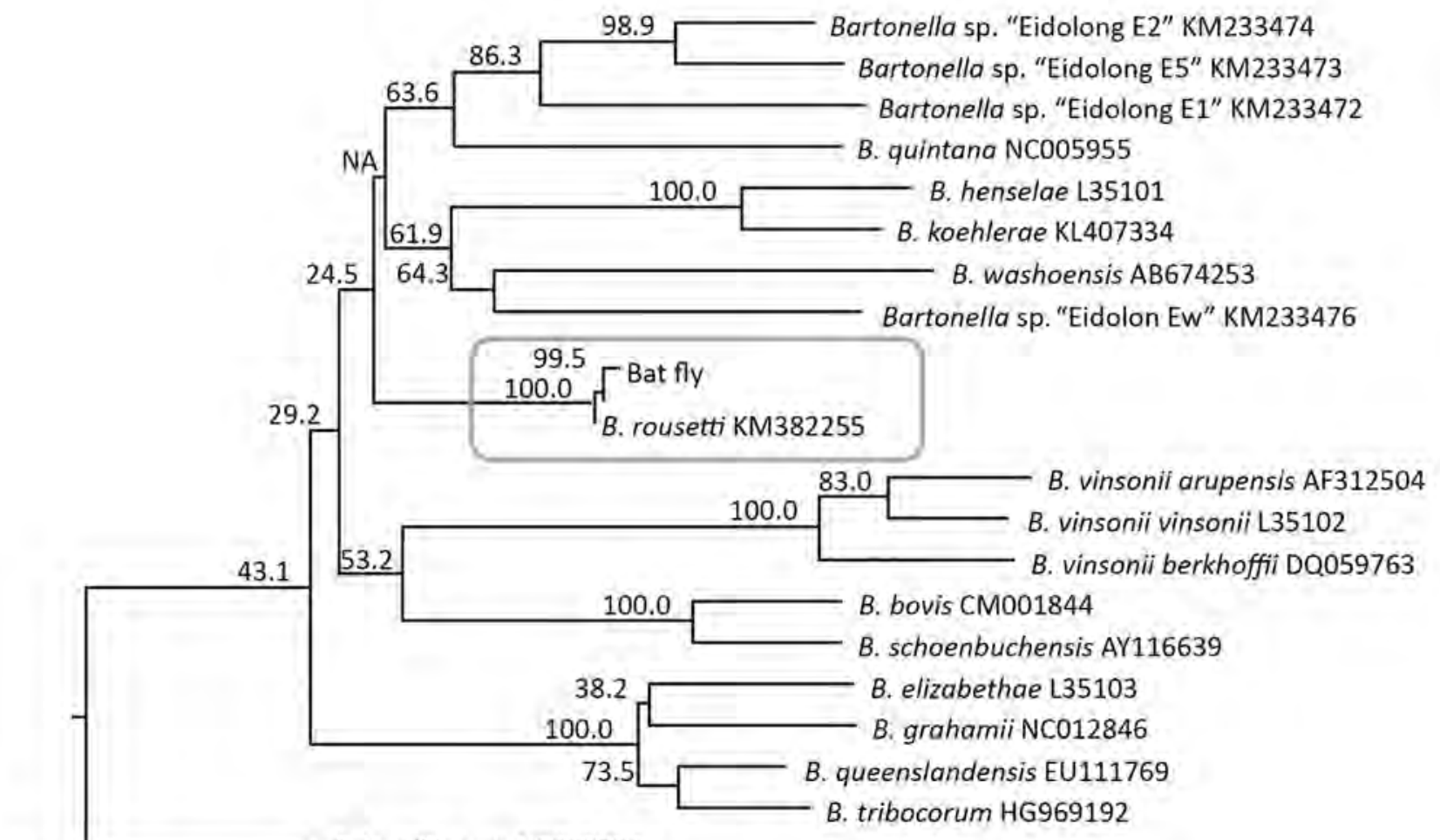

33.9

B. bacilliformis NC008783

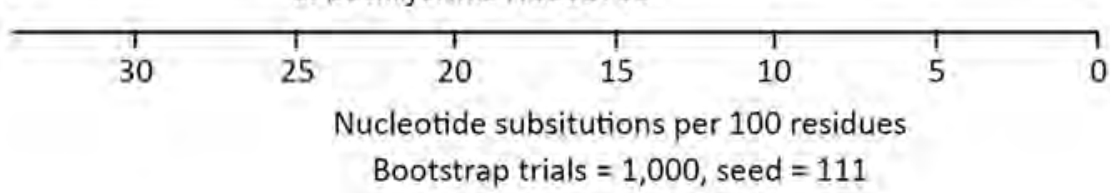

Figure. Phylogenetic relationships of Bartonella rousetti (proposed name) obtained from Egyptian fruit bats (Rousettus aegyptiacus) collected in Nigeria, 2010 and 2013, and other Bartonella species and bat-associated Bartonella based on internal transcribed spacer sequences. The neighbor-joining method by the Kimura 2-parameter distance method and bootstrap calculation was conducted with 1,000 replicates for phylogenetic analysis. The internal transcribed spacer sequence obtained from the bat flies was closely clustered with $B$. rousetti. GenBank accession numbers are provided for the $B$. rousetti sequence and the comparison sequences.

felis and C. canis) collected from dogs in Tunisia (GenBank accession nos. KP126468-74), a louse pool (Polyplax spp. and Hoplopleura spp.) collected from rodents in Thailand (GenBank accession no. KT324560), and an unidentified flea collected from a dog in Peru (GenBank accession no. GU583843). Of the specimens with this particular gltA sequence, 2 yielded no ITS sequence, 3 yielded ITS sequences matching $B$. rousetti, and 1 yielded the single $B$. tamiae sequence. Representative ITS (GenBank accession nos. MH14262-MH142639) and gltA (GenBank accession nos. MH151067-79) sequences for each novel sequence variant were submitted to GenBank.

\section{Human Exposure to B. rousetti}

A total of 305 serum samples from 204 participants were tested for IgG against B. rousetti; 12 samples from different persons showed reactivity at an initial dilution of 1:32. Further 2-fold titration confirmed that 8 were positive, with titers $\geq 1: 64$ (Table). The positive samples were retested for 3 other Bartonella species-B. henselae, B. quintana, and B. elizabethae, all of which have been reported in Africa (22-24); antibodies against these Bartonella species were not detected in any of the samples. Five seropositive participants reported having eaten bats and having either touched bats or been scratched or bitten by them, although not all reported having ever participated in the bat festival. Three seropositive participants reported never having eaten bats, touched bats, or been scratched or bitten by bats; in addition, these 3 participants claimed to have never participated in the bat festival. Of the 8 seropositive participants, only 1 reported having experienced a febrile illness since the bat festival that had taken place earlier in the year.

\section{Discussion}

We made several observations during this investigation. First, Egyptian fruit bats carry a unique Bartonella strain 
Human Exposure to Novel Bartonella Species

Table. Epidemiologic data for persons with antibodies to Bartonella rousetti detected in study of human exposure to a novel Bartonella species from contact with fruit bats, Nigeria, 2013*

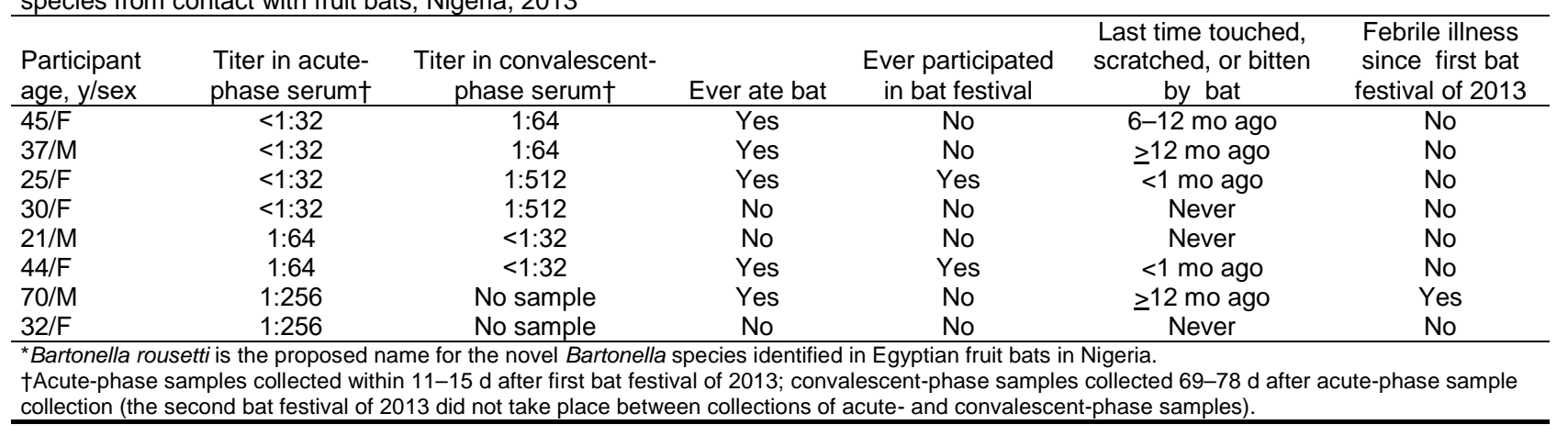

that probably represents a new species, for which we propose the name Bartonella rousetti. Second, bat flies, the common ectoparasites of bats, carry this same strain of Bartonella. Because this organism was detected by PCR only, the presence of the DNA does not necessarily indicate that the organism is viable. Last, persons from the communities surrounding the bat caves were exposed to this particular Bartonella strain, which might cause human infection.

Since 2010, several reports have described finding diverse Bartonella genotypes in bats of many species $(25,27-$ 30). The relationships between Bartonella genotypes and bat species that harbored these bacteria are not always simple. The same Bartonella species may circulate among different bat species, showing no specific relationship between the bats and the Bartonella species (27). Sometimes, multiple Bartonella species are associated with bats of only 1 species. For example, 6 Bartonella species have been identified in straw-colored fruit bats (Eidolon helvum) in Africa $(16,29)$. Our study indicates that Egyptian fruit bats carry a specific Bartonella strain that has not been identified in bats of other species.

Similarly, we found that the most prevalent Bartonella species found in bat flies parasitizing Egyptian fruit bats is $B$. rousetti. The ectoparasite bat flies E. africana are predominantly associated with Egyptian fruit bats $(17,31,32)$. Although sequences matching other Bartonella species were identified in the bat flies, these genogroups may be primarily associated with arthropods and not mammals. One sequence from a bat fly was identified as B. tamiae. The presence of $B$. tamiae in bat flies from Algeria has been recorded (33), and the bacterium reportedly has been identified in chigger mites collected from rodents in Thailand (34). It is possible that Bartonella species found only in arthropods and not their associated mammal hosts may represent facultative symbionts that are uniquely adapted to live in the arthropod gut or other body system $(35,36)$. The risks posed to humans by these primarily arthropod-associated Bartonella species are still unclear, although $B$. tamiae is a reported human pathogen that may cause febrile illness and other clinical signs and symptoms (37).
Detection of antibodies against $B$. rousetti in serum samples from several study participants indicates their exposure to the bacteria. However, with serologic results, cross-reactivity is a concern. For example, phylogenetically closely related $B$. henselae and B. quintana (the causative agents of cat-scratch disease and trench fever, respectively) exhibit a high level of serologic cross-reactivity $(26,38,39)$. We tested the positive human serum samples for 3 other Bartonella species (B. henselae, B. quintana, and B. elizabethae) that circulate in Africa, and we did not detect any positive results. Given that immunofluorescence assays have good discriminatory ability for a wide range of antigens (40-42), the results lead us to conclude that the antibodies in these participants were indeed reactive with B. rousetti but not the other Bartonella species tested, although cross-reactivity with other non-Bartonella agents cannot be ruled out.

Our study is not the first attempt to identify antibodies against bat-associated Bartonella in humans. Mannerings et al. (43) conducted a serologic survey of 335 volunteers from Ghana for antibodies against 6 species of Bartonella, including Bartonella strains isolated from straw-colored fruit bats. In that study, only 2 serum samples were positive for $B$. henselae antibodies at low titers, whereas none was positive against the bat strains.

All known species of Bartonella are transmitted between natural animal hosts by arthropods $(29,44)$. The presence of B. rousetti DNA in E. africana bat flies parasitizing Egyptian fruit bats suggests that these ectoparasites may act as vectors for the transmission of Bartonella infection among bats, but it is unclear how bat flies would play a role in transmitting the bacterium to humans because bat flies do not commonly bite humans (C. McKee, unpub. data). Instead, human exposure may potentially occur via other routes, such as 1 ) directly by bat bites or scratches, which is similar to how humans acquire infections with $B$. henselae through cat scratches $(10,45) ; 2)$ indirectly by contamination of open wounds with blood or other materials (e.g., saliva, urine, feces) of infected bats; or 3 ) indirectly by contamination of open wounds with bat fly 
excreta. Several studies have reported detecting Bartonella DNA in bat feces $(12,46,47)$, and Dietrich et al. (47) detected Bartonella DNA in bat saliva and urine, providing support for routes 1 and 2 above, although no attempts have been made to culture viable bacteria from these fluids. However, viable Bartonella bacteria have been cultured from experimentally infected ectoparasites, including fleas and bedbugs $(48,49)$, although such studies have yet to be performed for bat flies. Nevertheless, evidence is accumulating that Bartonella could spread from infected mammalian hosts through multiple routes. Therefore, it may not be necessary for humans to interact directly with live bats to be exposed to bat-associated Bartonella. Persons might be at risk when interacting with bat carcasses, guano, or other contaminated products. Of note, we do not provide definitive evidence of the route of exposure for any of the 8 seropositive participants. Indeed, 3 of these participants reported no interactions at all with bats.

Future studies should continue to evaluate the relative correlations of exposure routes, the pathobiology of batborne $B$. rousetti in humans, and vector competency of bat flies for transmitting Bartonella. Results should provide guidance to communities for mitigating the risks to humans interacting with animals and their arthropod vectors.

Idanre Bat Festival Investigation Team members: Ivan V. Kuzmin, Dianna Blau, James Ellison, Lauren Greenberg, Marissa Person, Ryan Wallace, Panayampalli S. Satheshkumar, Abimbola Aman-Oloniyo, Elizabeth B. Adedire, Mariat O. Soleye, Gloria C. Okara, Sebastian Yennan, Mohammed Abdurrahman, Munir A. Sani, Solomon. W Audu, Maruf Lawal, and Philip P. Mshelbwala.

\section{Acknowledgments}

We thank J.D. Kirby, Mary Reynolds, Todd Smith, the Vice Chancellor and Management of Ahmadu Bello University, the Federal Ministry of Health (Abuja, Nigeria), the Owa of Idanre Oba Fredrick Adegunle Aroloye IV, and the chiefs of the Idanre community, Ondo State, Nigeria, for their helpful comments and assistance with logistics.

This study was supported by the Biosecurity Engagement Program of the US Department of State, Bureau of International Security and Nonproliferation, and the Office of Cooperative Threat Reduction's Global Threat Reduction Programs; One Health funding; and the Global Disease Detection Program of the Center for Global Health at CDC.

\section{About the Author}

Dr. Bai is a microbiologist in the Division of Vector-Borne Infectious Diseases, National Center for Emerging and Zoonotic Infectious Diseases, CDC, Fort Collins. Her research interests include microbiology, epidemiology, and ecology of zoonotic infectious diseases.

\section{References}

1. Calisher CH, Childs JE, Field HE, Holmes KV, Schountz T. Bats: important reservoir hosts of emerging viruses. Clin Microbiol Rev. 2006;19:531-45. http://dx.doi.org/10.1128/CMR.00017-06

2. Mickleburgh S, Waylen K, Racey P. Bats as bushmeat: a global review. Oryx. 2009;43:217-34. http://dx.doi.org/10.1017/ S0030605308000938

3. Osinubi MO, Recuenco S, Kuzmin I, Haberling DL, Blau DM, Davis LB, et al. Knowledge, attitudes and practices among populations exposed to bats in southern Nigeria. Revista de Educação Continuada em Medicina Veterinária e Zootecnia do CRMV-SP. 2012;10, n. 2/3.

4. Kwiecinski GG, Griffiths TA. Rousettus egyptiacus. Mammalian Species. 1999;611:1-9. http://dx.doi.org/10.2307/3504411

5. Kuzmin IV, Niezgoda M, Franka R, Agwanda B, Markotter W, Beagley JC, et al. Lagos bat virus in Kenya. J Clin Microbiol. 2008;46:1451-61. http://dx.doi.org/10.1128/JCM.00016-08

6. Towner JS, Amman BR, Sealy TK, Carroll SA, Comer JA, Kemp A, et al. Isolation of genetically diverse Marburg viruses from Egyptian fruit bats. PLoS Pathog. 2009;5:e1000536. http://dx.doi.org/10.1371/journal.ppat.1000536

7. Nakamura S, Settai S, Hayashidani H, Urabe T, Namai S, Une Y. Outbreak of yersiniosis in Egyptian rousette bats (Rousettus aegyptiacus) caused by Yersinia pseudotuberculosis serotype 4b. J Comp Pathol. 2013;148:410-3. http://dx.doi.org/ 10.1016/j.jcpa.2012.07.007

8. Amman BR, Jones ME, Sealy TK, Uebelhoer LS, Schuh AJ, Bird BH, et al. Oral shedding of Marburg virus in experimentally infected Egyptian fruit bats (Rousettus aegyptiacus). J Wildl Dis. 2015;51:113-24. http://dx.doi.org/10.7589/2014-08-198

9. Chomel BB, Boulouis HJ, Breitschwerdt EB, Kasten RW, Vayssier-Taussat M, Birtles RJ, et al. Ecological fitness and strategies of adaptation of Bartonella species to their hosts and vectors. Vet Res. 2009;40:29. http://dx.doi.org/10.1051/ vetres/2009011

10. Chomel BB, Kasten RW, Floyd-Hawkins K, Chi B, Yamamoto K, Roberts-Wilson J, et al. Experimental transmission of Bartonella henselae by the cat flea. J Clin Microbiol. 1996;34:1952-6.

11. Lin EY, Tsigrelis C, Baddour LM, Lepidi H, Rolain JM, Patel R, et al. Candidatus Bartonella mayotimonensis and endocarditis. Emerg Infect Dis. 2010;16:500-3. http://dx.doi.org/10.3201/ eid1603.081673

12. Veikkolainen V, Vesterinen EJ, Lilley TM, Pulliainen AT. Bats as reservoir hosts of human bacterial pathogen, Bartonella mayotimonensis. Emerg Infect Dis. 2014;20:960-7. http://dx.doi.org/10.3201/eid2006.130956

13. Lilley TM, Wilson CA, Bernard RF, Willcox EV, Vesterinen EJ, Webber QM, et al. Molecular detection of Candidatus Bartonella mayotimonensis in North American bats. Vector Borne Zoonotic Dis. 2017;17:243-6. http://dx.doi.org/10.1089/vbz.2016.2080

14. Urushadze L, Bai Y, Osikowicz L, McKee C, Sidamonidze K, Putkaradze D, et al. Prevalence, diversity, and host associations of Bartonella strains in bats from Georgia (Caucasus). PLoS Negl Trop Dis. 2017;11:e0005428. http://dx.doi.org/10.1371/ journal.pntd.0005428

15. Norman AF, Regnery R, Jameson P, Greene C, Krause DC. Differentiation of Bartonella-like isolates at the species level by PCR-restriction fragment length polymorphism in the citrate synthase gene. J Clin Microbiol. 1995;33:1797-803.

16. Bai Y, Hayman DTS, McKee CD, Kosoy MY. Classification of Bartonella strains associated with straw-colored fruit bats (Eidolon helvum) across Africa using a multi-locus sequence typing platform. PLoS Negl Trop Dis. 2015;9:e0003478. http://dx.doi.org/ 10.1371/journal.pntd.0003478

17. Theodor O. The Nycteribiidae of the Ethiopian region and Madagascar. Parasitology. 1957;47:457-543. 
18. Quetglas J, Balvín O, Lučan RK, Benda P. First records of the bat bug Cacodmus vicinus (Heteroptera: Cimicidae) from Europe and further data on its distribution. Vespertilio. 2012;16:243-8.

19. Szalanski AL, Austin JW, Scheffrahn RH, Messenger MT. Molecular diagnostics of the Formosan subterranean termite (Isoptera: Rhinotermitidae). Fla Entomol. 2004;87:145-51. http://dx.doi.org/10.1653/0015-4040(2004)087[0145: MDOTFS]2.0.CO;2

20. Bai Y, Gilbert A, Fox K, Osikowicz L, Kosoy M. Bartonella rochalimae and $B$. vinsonii subsp. berkhoffii in wild carnivores from Colorado, USA. J Wildl Dis. 2016;52:844-9. http://dx.doi.org/ 10.7589/2016-01-015

21. Diniz PP, Maggi RG, Schwartz DS, Cadenas MB, Bradley JM, Hegarty B, et al. Canine bartonellosis: serological and molecular prevalence in Brazil and evidence of co-infection with Bartonella henselae and Bartonella vinsonii subsp. berkhoffii. Vet Res. 2007;38:697-710. http://dx.doi.org/10.1051/vetres:2007023

22. Kelly PJ, Rooney JJ, Marston EL, Jones DC, Regnery RL. Bartonella henselae isolated from cats in Zimbabwe. Lancet. 1998;351:1706. http://dx.doi.org/10.1016/S0140-6736(05)77744-8

23. Boutellis A, Veracx A, Angelakis E, Diatta G, Mediannikov O, Trape JF, et al. Bartonella quintana in head lice from Sénégal. Vector Borne Zoonotic Dis. 2012;12:564-7. http://dx.doi.org/ 10.1089/vbz.2011.0845

24. Kamani J, Morick D, Mumcuoglu KY, Harrus S. Prevalence and diversity of Bartonella species in commensal rodents and ectoparasites from Nigeria, West Africa. PLoS Negl Trop Dis. 2013;7:e2246. http://dx.doi.org/10.1371/journal.pntd.0002246

25. Kosoy M, Bai Y, Lynch T, Kuzmin IV, Niezgoda M, Franka R, et al. Bartonella spp. in bats, Kenya. Emerg Infect Dis. 2010;16:1875-81. http://dx.doi.org/10.3201/eid1612.100601

26. La Scola B, Raoult D. Serological cross-reactions between Bartonella quintana, Bartonella henselae, and Coxiella burnetii. J Clin Microbiol. 1996;34:2270-4.

27. Bai Y, Kosoy M, Recuenco S, Alvarez D, Moran D, Turmelle A, et al. Bartonella spp. in bats, Guatemala. Emerg Infect Dis. 2011;17:1269-72. http://dx.doi.org/10.3201/eid1707.101867

28. Bai Y, Recuenco S, Gilbert AT, Osikowicz LM, Gómez J, Rupprecht C, et al. Prevalence and diversity of Bartonella spp. in bats in Peru. Am J Trop Med Hyg. 2012;87:518-23. http://dx.doi.org/10.4269/ajtmh.2012.12-0097

29. Kamani J, Baneth G, Mitchell M, Mumcuoglu KY, Gutiérrez R, Harrus S. Bartonella species in bats (Chiroptera) and bat flies (Nycteribiidae) from Nigeria, West Africa. Vector Borne Zoonotic Dis. 2014;14:625-32. http://dx.doi.org/10.1089/vbz.2013.1541

30. Brook CE, Bai Y, Dobson AP, Osikowicz LM, Ranaivoson HC, Zhu Q, et al. Bartonella spp. in fruit bats and blood-feeding ectoparasites in Madagascar. PLoS Negl Trop Dis. 2015;9:e0003532. http://dx.doi.org/10.1371/journal.pntd.0003532

31. Nartey NAN. Common parasites of fruit-eating bats in southern Ghana. Accra (Ghana): University of Ghana; 2015. p. 1-144.

32. Charles AN. Haemoparasites and ectoparasites of fruit bat species in Amurum Forest Reserve and their effects on host physiologic and morphometric parameters. Jos (Nigeria): University of Jos; 2015. p. 1-54.

33. Leulmi H, Aouadi A, Bitam I, Bessas A, Benakhla A, Raoult D, et al. Detection of Bartonella tamiae, Coxiella burnetii and rickettsiae in arthropods and tissues from wild and domestic animals in northeastern Algeria. Parasit Vectors. 2016;9:27. http://dx.doi.org/10.1186/s13071-016-1316-9

34. Kabeya H, Colborn JM, Bai Y, Lerdthusnee K, Richardson JH, Maruyama S, et al. Detection of Bartonella tamiae DNA in ectoparasites from rodents in Thailand and their sequence similarity with bacterial cultures from Thai patients. Vector Borne Zoonotic Dis. 2010;10:429-34. http://dx.doi.org/10.1089/ vbz.2009.0124
35. Neuvonen MM, Tamarit D, Näslund K, Liebig J, Feldhaar H, Moran NA, et al. The genome of Rhizobiales bacteria in predatory ants reveals urease gene functions but no genes for nitrogen fixation. Sci Rep. 2016;6:39197. http://dx.doi.org/10.1038/ srep39197

36. Segers FH, Kešnerová L, Kosoy M, Engel P. Genomic changes associated with the evolutionary transition of an insect gut symbiont into a blood-borne pathogen. ISME J. 2017;11:1232-44. http://dx.doi.org/10.1038/ismej.2016.201

37. Kosoy M, Morway C, Sheff KW, Bai Y, Colborn J, Chalcraft L, et al. Bartonella tamiae sp. nov., a newly recognized pathogen isolated from three human patients from Thailand. J Clin Microbiol. 2008;46:772-5. http://dx.doi.org/10.1128/JCM.02120-07

38. Baneth G, Kordick DL, Hegarty BC, Breitschwerdt EB. Comparative seroreactivity to Bartonella henselae and Bartonella quintana among cats from Israel and North Carolina. Vet Microbiol. 1996;50:95-103. http://dx.doi.org/10.1016/0378-1135(96)00006-5

39. Vermeulen MJ, Verbakel H, Notermans DW, Reimerink JH, Peeters MF. Evaluation of sensitivity, specificity and crossreactivity in Bartonella henselae serology. J Med Microbiol. 2010;59:743-5. http://dx.doi.org/10.1099/jmm.0.015248-0

40. Iralu J, Bai Y, Crook L, Tempest B, Simpson G, Mckenzie T, et al. Rodent-associated Bartonella febrile illness, southwestern United States. Emerg Infect Dis. 2006;12:1081-6. http://dx.doi.org/ 10.3201/eid1207.040397

41. Laudisoit A, Iverson J, Neerinckx S, Shako JC, Nsabimana JM, Kersh G, et al. Human seroreactivity against Bartonella species in the Democratic Republic of Congo. Asian Pac J Trop Med. 2011;4:320-2. http://dx.doi.org/10.1016/S1995-7645(11)60094-1

42. Myint KS, Gibbons RV, Iverson J, Shrestha SK, Pavlin JA, Mongkolsirichaikul D, et al. Serological response to Bartonella species in febrile patients from Nepal. Trans R Soc Trop Med Hyg. 2011;105:740-2. http://dx.doi.org/10.1016/j.trstmh.2011.08.002

43. Mannerings AO, Osikowicz LM, Restif O, Nyarko E, Suu-Ire R, Cunningham AA, et al. Exposure to bat-associated Bartonella spp. among humans and other animals, Ghana. Emerg Infect Dis. 2016;22:922-4. http://dx.doi.org/10.3201/eid2205.151908

44. Billeter SA, Hayman DT, Peel AJ, Baker K, Wood JL, Cunningham A, et al. Bartonella species in bat flies (Diptera: Nycteribiidae) from western Africa. Parasitology. 2012;139:324-9. http://dx.doi.org/10.1017/S0031182011002113

45. Mosbacher M, Elliott SP, Shehab Z, Pinnas JL, Klotz JH, Klotz SA. Cat scratch disease and arthropod vectors: more to it than a scratch? J Am Board Fam Med. 2010;23:685-6. http://dx.doi.org/10.3122/ jabfm.2010.05.100025

46. Banskar S, Bhute SS, Suryavanshi MV, Punekar S, Shouche YS. Microbiome analysis reveals the abundance of bacterial pathogens in Rousettus leschenaultii guano. Sci Rep. 2016;6:36948. http://dx.doi.org/10.1038/srep36948

47. Dietrich M, Kearney T, Seamark ECJ, Markotter W. The excreted microbiota of bats: evidence of niche specialisation based on multiple body habitats. FEMS Microbiol Lett. 2017;364:fnw284.

48. Kernif T, Leulmi H, Socolovschi C, Berenger J-M, Lepidi H, Bitam I, et al. Acquisition and excretion of Bartonella quintana by the cat flea, Ctenocephalides felis felis. Mol Ecol. 2014;23: 1204-12. http://dx.doi.org/10.1111/mec.12663

49. Leulmi H, Bitam I, Berenger J-M, Lepidi H, Rolain J-M, Almeras L, et al. Competence of Cimex lectularius bed bugs for the transmission of Bartonella quintana, the agent of trench fever. PLoS Negl Trop Dis. 2015;9:e0003789. http://dx.doi.org/10.1371/ journal.pntd.0003789

Address for correspondence: Ying Bai, Centers for Disease Control and Prevention, 3150 Rampart Rd, Fort Collins, CO 80521, USA; email: ybai1@cdc.gov 


\title{
HISTORI CAL REVIEW
}

\section{Emergent Sand Fly- Borne Phleboviruses in the Balkan Region}

\author{
Nazli Ayhan, Remi N. Charrel
}

\begin{abstract}
Sand fly-borne phleboviruses are associated with febrile diseases and nervous system infections in the Mediterranean basin. Sandfly fever was first reported in the Balkan Peninsula at the end of the 19th century. Since then, accumulating data show that the Balkan Peninsula, as a transboundary region between Asia and Europe, plays a major role in the emergence of vectorborne diseases in Europe. To provide an inclusive approach, we collected published data on phleboviruses in the Balkan countries and used them to evaluate the impact of these pathogens from virologic, epidemiologic, and public health perspectives. Recent findings show a high diversity of phleboviruses belonging to 3 species or serocomplexes circulating heavily in the Balkans. Focusing on undisputable human pathogens, we found direct and indirect laboratory documentation for Toscana virus, Sandfly fever Sicilian virus, and Adria virus. These data demonstrate that the Balkans are a hotspot for phleboviruses transmitted by sand flies.
\end{abstract}

$\mathrm{P}$ hleboviruses (genus Phlebovirus, family Phenuiviridae, order Bunyavirales) are $80-120 \mathrm{~nm}$ in length and display helical symmetry. Their genome consists of 3 segmented negative-sense single-stranded RNA: the large segment encodes the viral RNA polymerase (RdRp), the medium segment encodes envelope glycoproteins (Gn and $\mathrm{Gc}$ ), and the small segment encodes nucleocapsid protein (N) and nonstructural protein (NS) $(1,2)$. The segmented nature of the genome allows recombination and reassortment to occur with the potential to generate new viruses with distinct ancestors $(3,4)$. Segment reassortment in $\mathrm{Bu}-$ nyavirales has been reported with increasing frequency, especially in the genus Orthobunyavirus (5). Specifically, reassortant viruses have been described in both Candiru and Rift Valley fever species $(5,6)$.

Two sand fly-borne phleboviruses in the Old World were historically associated with cases of sandfly fever: Sicilian virus and Naples virus (7). Later, Naples virus was renamed sandfly fever Naples virus (SFNV), which

Author affiliations: Unité des Virus Emergents, Marseille, France (N. Ayhan, R.N. Charrel); University of Florida, Gainesville,

Florida, USA (R.N. Charrel)

DOI: https://doi.org/10.3201/eid2412.171626 is included in the Sandfly fever Naples virus species. Likewise, Sicilian virus was renamed sandfly fever Sicilian virus (SFSV), which is still a tentative species. SFSV and SFNV are both responsible for sandfly fever, a self-limiting but incapacitating febrile illness. Toscana virus (TOSV), discovered in 1971, was incriminated as causing central and peripheral nervous system infections in $1983(8,9)$. TOSV can cause aseptic meningitis and meningoencephalitis (912), as well as a number of other manifestations affecting the central and peripheral nervous system. These viruses are transmitted via bites of Phlebotomus spp. sand flies.

Data concerning the geographic distribution of SFSV, SFNV, and TOSV have drastically increased during the past 2 decades, resulting in a more accurate cartography of their presence in the Mediterranean basin, the Middle East, and central Asia (12-16).

The Balkan Peninsula is a principal region for sandfly fever. It is located in southeastern Europe, and consists of Slovenia, Croatia, Bosnia-Herzegovina, the Republic of Macedonia, Albania, Bulgaria, Greece, Montenegro, Romania, Serbia, and Kosovo. The Balkan region is composed of 3 very different natural entities: the Adriatic littoral in the southwest, the Pannonian plain in the northeast, and a broad expanse of mountainous regions in between. The first record of sandfly fever originated in Bosnia-Herzegovina at the end of the 19th century (online Technical Appendix Table, http://wwwnc.cdc.gov/EID/article/24/12/171626-Techapp1.pdf). During World War I and World War II, sandfly fever affected great numbers of soldiers in the region $(17,18)$ (online Technical Appendix Table). In addition to historical data, recent reports show the activity of several novel viruses with severe human infections. We reviewed all the published data for sand fly-borne phleboviruses in the Balkan Peninsula to provide a comprehensive view of the current situation and of the public health effect on humans and vertebrate animals in the region.

\section{Methods}

We searched global web-based resources (PubMed [www. ncbi.nlm.nih.gov/pubmed], Google Scholar [https://scholar.google.com/], and Web of Science [https://isiknowledge.coml) to collect all the sand fly-borne phlebovirus data from the Balkan region. In addition, we investigated 
libraries and other national resources to identify books and conference reports that are not accessible on webbased resources. We used the keywords "sand fly," "Phlebovirus," "Bunyaviridae," "Phenuiviridae," "sand fly fever," "pappataci fever," "three-day fever," "sandfly fever," "Toscana virus," "Sicilian virus," "Naples virus," "SFSV," and "SFNV" matched with "Balkan," "Balkan Peninsula," "Yugoslavia," "Slovenia," "Croatia," "Bosnia-Herzegovina," "Macedonia," "Republic of Macedonia," "Former Yugoslav Republic of Macedonia," "FYROM,” "RoM,” “Albania,” "Bulgaria,” “Greece,” "Montenegro," "Romania," “Moldova," "Serbia,” and "Kosovo" for the research. After gathering all the data, we discarded the irrelevant publications. We put the collected data in order based on country, year, and the phlebovirus species complex.

We obtained all the accessible virus sequences from Balkan countries from GenBank. We aligned 589 nt partial nucleoprotein sequences and analyzed them with MEGA software version 6 (https://www.megasoftware.net/). We constructed a phylogenetic tree using the neighbor-joining method (Figure 1) and tested the robustness of each node by 1,000 bootstrap replicates.

\section{Results}

We collected 51 published articles: 2 articles from Albania, 7 from Bosnia-Herzegovina, 11 from Croatia, 17 from Greece, 5 from Kosovo, 1 from Republic of Macedonia, and 7 from Serbia (online Technical Appendix Table). One reference from Bulgaria was not available (online Technical Appendix Table). We found no published data from Montenegro and Romania. Most of the references included data concerning seroprevalence studies conducted in humans or animals (online Technical Appendix Table). Several articles reported results about either virus characterization or case reports/outbreak investigations (19-25) (online Technical Appendix Table).

\section{Historical Data on Phleboviruses in the Balkans}

Alois Pick made a clinical description of sandfly fever in Bosnia-Herzegovina military barracks from foreign soldiers at the end of the 19th century (Online Technical Appendix Table ). Pick, an Austro-Hungarian military doctor working in Trebinje (Herzegovina), characterized the syndrome observed in cases of sandfly fever (26). Sandfly fever was observed both in local populations and in visitors, specifically foreign soldiers. In 1904, Taussig noticed the presence of "pappataci" sand flies in army barracks in Herzegovina and conducted a large clinical and epidemiologic study in the region (27). The "endemic disease" emerged only in places where sand flies were present. Subsequently, the causative agent was discovered as a filterable agent (virus) that used pappataci sand flies as a vector (18).
During World War II, sandfly fever affected great numbers of foreign soldiers in all Mediterranean region and Balkan countries during the summer seasons, when sand fly activity peaks (17). The disease was called phlebotomus fever, pappataci fever, or three-day fever. In 1937, a massive outbreak occurred in Athens, Greece (online Technical Appendix Table). After World War II, sandfly fever epidemics were recorded in Belgrade, Serbia, affecting thousands of persons and then expanding into other regions of the Balkans (27) (online Technical Appendix Table). Although these articles could be the first record of sandfly fever based on clinical and epidemiologic grounds, there is no scientific evidence to demonstrate whether the disease described in the articles was sandfly fever caused by phlebovirus.

The seminal seroprevalence study using a neutralization assay, by Tesh et al. in 1976, showed that SFNV and SFSV had circulated and were likely to continue to infect human populations in the tested regions (online Technical Appendix Table). Also in 1976, Gligić et al. isolated a strain of SFNV (Yug Bogdanovac virus strain Yu 8/76) from P. perfiliewi sand flies in the Dobrič region of Serbia. Other strains of SFNV and SFSV were also isolated in Serbia from $P$. pappatasi sand flies, but no accessible sequence data are available (24). At the time of isolation, SFNV and SFSV identification was done using mouse hyperimmune ascitis fluid for neutralization assays and acetone sucrose antigens for complement fixation tests. SFNV strain YU-8-76 is available in the Yale University catalog, now stored at the University of Texas Medical Branch at Galveston. Partial sequence of this strain has been determined and confirmed the strain as belonging to the Sandfly fever Naples virus species $(28,29)$.

In 1985, Corfou virus, closely related to but distinct from SFSV, was isolated from $P$. neglectus sand flies collected in the island of Corfou, Greece (online Technical Appendix Table). Corfou and SFSV can be distinguished only by neutralization assays, unlike other serologic assays (ELISA, hemagglutination inhibition [HI], indirect immunofluorescence [IIF], complement fixation [CF]). Several studies have confirmed the presence of antibodies against both SFNV and SFSV in several areas of the Balkans (20) (online Technical Appendix Table).

\section{Toscana Virus in the Balkan Region}

In 1993, a German traveler was infected with TOSV after visiting Athens; diagnosis was established from immunofluorescence serology results, and it is therefore classified as a probable case rather than a laboratoryconfirmed case (online Technical Appendix Table). Recent serologic studies have provided evidence of TOSV presence in Bosnia-Herzegovina, Kosovo, Croatia, and 
Greece (online Technical Appendix Table). Several human cases documented serologically as TOSV infections have been reported in Greece (23) (online Technical Appendix Table).

In Croatia, TOSV RNA was detected in the cerebrospinal fluid of a patient infected with meningitis; sequence analysis showed that he was infected with a strain belonging to a genetic lineage that had not been previously recognized (subsequently named lineage $C$ ), which was clearly distinct from lineages A and B (on- line Technical Appendix Table). Subsequently, TOSV lineage $\mathrm{C}$ was detected from a patient in Greece (online Technical Appendix Table); unfortunately, the virus was not isolated in both cases and therefore only partial sequence data are available. Later, sequences obtained from $P$. neglectus sand flies confirmed the presence of lineage C TOSV but also showed that lineage B TOSV was present and that both genetic types were sympatric in Croatia (Figure 2; online Technical Appendix Table).

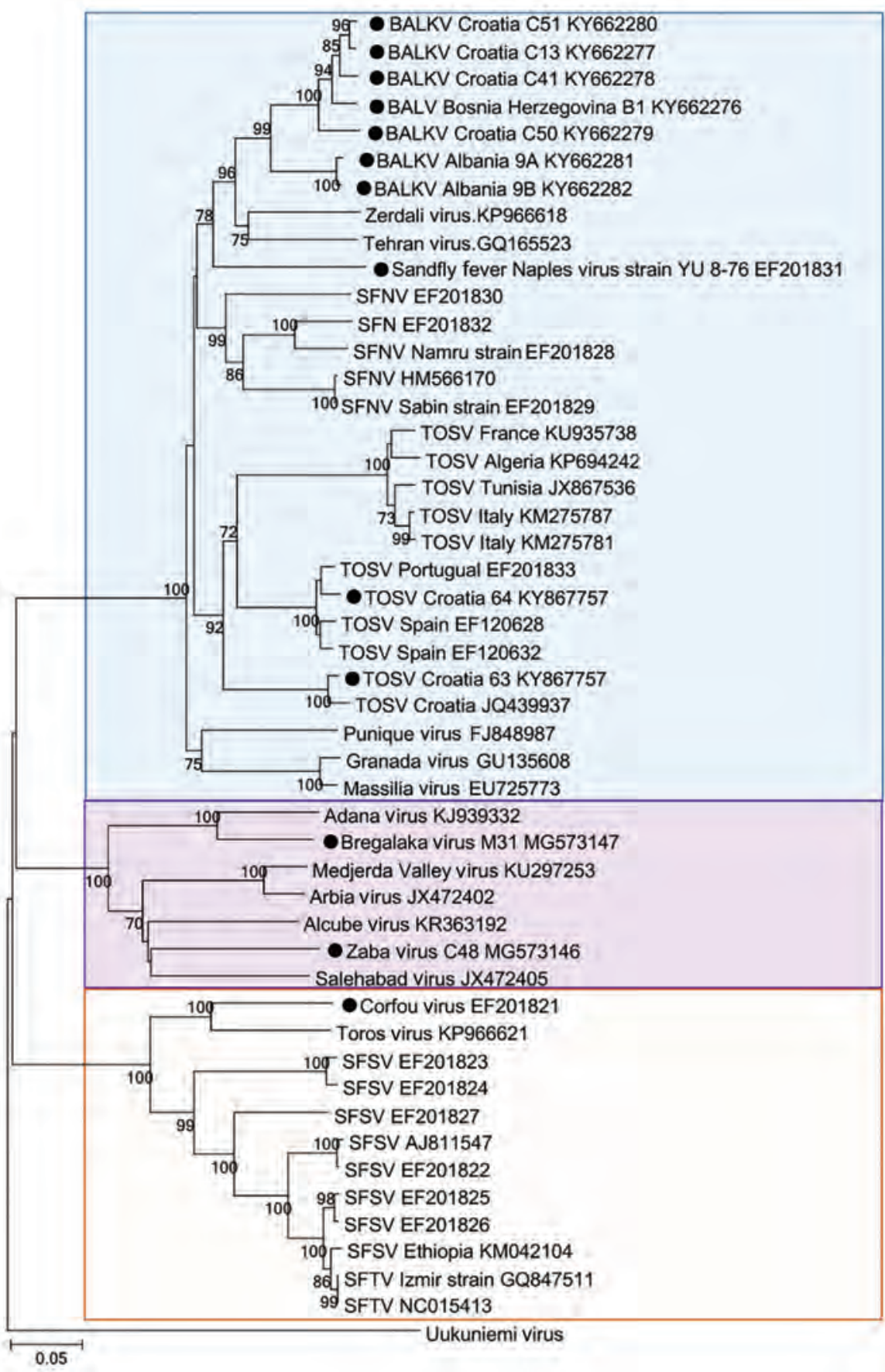

Figure 1. Phylogenetic relationships between sand fly-borne phleboviruses in the Old World based on $589 \mathrm{nt}$ partial nucleoprotein sequence. Phylogenetic tree was constructed using the neighbor-joining method with MEGA software version 6 (http://www.megasoftware.net). Black circles indicate viruses identified in the Balkan region. Boxes (top to bottom) include all viruses belonging to the Sandfly fever Naples virus species, viruses belonging to the Salehabad phlebovirus species, and viruses belonging to the Sandfly fever Sicilian and Corfou tentative virus species. The robustness of each node was tested by 1,000 bootstrap replicates. GenBank accession numbers are provided. Scale bar indicates nucleotide substitutions per site. 
Table. Characteristics of sand fly-borne phleboviruses in the Balkan region

\begin{tabular}{|c|c|c|c|c|c|c|}
\hline Virus & Taxonomy & Source & Virus isolation & Distribution & $\begin{array}{c}\text { Probable } \\
\text { vector species }\end{array}$ & $\begin{array}{c}\text { Human/animal } \\
\text { infections }\end{array}$ \\
\hline $\begin{array}{l}\text { Sand fly fever } \\
\text { Naples virus* }\end{array}$ & $\begin{array}{c}\text { Sandfly fever Naples } \\
\text { virus species }\end{array}$ & $\begin{array}{l}\text { Field-collected } \\
\text { sand flies }\end{array}$ & Yes, sand fly pools & Serbia & P. perfiliewi & Yes \\
\hline Corfou virus & $\begin{array}{c}\text { Sandfly fever Sicilian } \\
\text { virus species } \dagger\end{array}$ & $\begin{array}{l}\text { Field-collected } \\
\text { sand flies }\end{array}$ & Yes, sand fly pools & Greece & P. neglectus & Probable \\
\hline Adria virus & $\begin{array}{c}\text { Salahabad virus } \\
\text { species }\end{array}$ & $\begin{array}{l}\text { Field-collected } \\
\text { sand flies, } \\
\text { patient blood }\end{array}$ & $\begin{array}{c}\text { No, partial } \mathrm{L} \\
\text { segment sequence } \\
\text { available }\end{array}$ & Albania, Greece & $\begin{array}{l}\text { Phlebotomus } \\
\text { spp. }\end{array}$ & Yes \\
\hline Balkan virus & $\begin{array}{c}\text { Sandfly fever Naples } \\
\text { virus species }\end{array}$ & $\begin{array}{l}\text { Field-collected } \\
\text { sand flies }\end{array}$ & $\begin{array}{c}\text { No, partial } L \text { and } S \\
\text { segment sequences } \\
\text { available }\end{array}$ & $\begin{array}{l}\text { Albania, Bosnia- } \\
\text { Herzegovina, } \\
\text { Croatia }\end{array}$ & P. neglectus & Unknown \\
\hline Bregalaka virus & $\begin{array}{c}\text { Salahabad virus } \\
\text { species }\end{array}$ & $\begin{array}{l}\text { Field-collected } \\
\text { sand flies }\end{array}$ & Yes, sandfly pools & $\begin{array}{l}\text { Republic of } \\
\text { Macedonia }\end{array}$ & P. perfiliewi & Unknown \\
\hline Zaba virus & $\begin{array}{l}\text { Salahabad virus } \\
\text { species }\end{array}$ & $\begin{array}{l}\text { Field-collected } \\
\text { sand flies }\end{array}$ & Yes, sandfly pools & Croatia & P. neglectus & Unknown \\
\hline Toscana virus & $\begin{array}{c}\text { Sandfly fever Naples } \\
\text { virus species }\end{array}$ & $\begin{array}{l}\text { Field collected } \\
\text { sand-flies, CSF }\end{array}$ & $\begin{array}{c}\text { No, partial } L \text { and } S \\
\text { segment sequences } \\
\text { available }\end{array}$ & Croatia, Greece & P. neglectus & Yes \\
\hline
\end{tabular}

\section{New Phleboviruses Identified from Partial Genomic Sequences}

A novel phlebovirus, Adria virus, was detected in 2 pools of sand flies collected in Albania in 2005 (online Technical Appendix Table). Adria virus is most closely related to Arbia virus, which was isolated in Italy (8); both belong to the Salehabad phlebovirus species. Adria virus RNA was detected in the blood of a 2.5-year-old patient with febrile seizures in Greece (online Technical Appendix Table). This evidence showed that a virus within the Salehabad phlebovirus species could be associated with human disease (Figure 2).

Balkan virus (BALKV) was detected from 2 pools of $P$. neglectus sand flies in Albania in 2014, 1 pool from Bosnia-Herzegovina in 2014-15, and 4 pools from Croatia in 2015 (online Technical Appendix Table). Sequence data analysis showed that BALKV belongs to the Sandfly fever Naples virus species, where it clusters with subgroup I together with Tehran, Zerdali, Fermo, and SFNV YU 8-76 viruses respectively discovered in Iran, Turkey, Italy, and Serbia (30-32) (Figure 2; online Technical Appendix Table).

\section{New Phleboviruses Identified from Complete Genomic Sequences}

Bregalaka virus (BREV) was isolated in $P$. perfiliewi sand flies from the Republic of Macedonia in 2015. Sequence analysis demonstrated that BREV is most closely related to Adana virus, which was isolated in Turkey from field-collected sand flies in 2012. In Croatia, Zaba virus (ZABAV) was isolated from $P$. neglectus sand flies. ZABAV is most closely related to Adria virus and Salehabad virus. Both BREV and ZABAV belong to the Salehabad phlebovirus species (online Technical Appendix Table).

\section{Human and Animal Exposure to Phleboviruses}

SFSV and SFNV are both responsible for a febrile illness that is self-limited but incapacitating, with signs that are commonly observed in arboviral diseases, such as fever, headache, malaise, photophobia, myalgia, and retroorbital pain. From a clinical perspective, it is impossible to distinguish SFNV from SFSV infections, and also to discriminate between SFNV/SFSV and other arboviral infections. As mentioned previously, historic records were based on clinical and epidemiologic evidence, but virological documentation was lacking for studies before the 1950s (Figure 2; online Technical Appendix Table).

Although SFSV and SFNV infections are clinically indistinguishable from each other, they are caused by genetically and antigenically different viruses. Infection with SFNV does not induce cross-protection against SFSV and vice versa (33). As mentioned previously, neutralization test is the only technique that permits undisputable identification at the specific and intraspecific levels. Other techniques, such as ELISA, CF, HI, and IFA, which are prone to cross-reactions, cannot achieve unambiguous identification at the intraspecific or at the interspecific level.

Seroprevalence studies conducted in the Balkans from 1976 onward have described antibodies in human populations confirming exposure to several phleboviruses transmitted by sand flies. Complement-fixation tests showed antibodies against SFNV in Bosnia-Herzegovina (online Technical Appendix Table), and HI tests showed antibodies against SFNV and SFSV in the islands of Croatia (Table; online Technical Appendix Table). In Greece, neutralizing antibodies against SFNV and SFSV were described; $36 \%$ of persons $\geq 30$ of age showed positive results for SFSV, and 13\% showed positive results for SFNV. Persons $<30$ years of age had much lower rates, suggesting that the antimalarial campaign had drastically reduced 
the sand fly population and therefore the exposure to viruses transmitted by sand flies (online Technical Appendix Table). Presence of neutralizing antibodies against SFSV showed wide circulation (71.9\%) in mainland and island regions of Greece in dogs used as sentinel animals; in the same study, TOSV and Arbia virus neutralizing antibodies were also found at lower rates: $4.4 \%$ for TOSV and 2.6\% for Arbia virus (online Technical Appendix Table).
In Kosovo, 9.6\% of the 104 human serum samples tested were positive for neutralizing antibodies against SFSV and $27.9 \%$ of the serum samples were positive for neutralizing antibodies against SFNV (online Technical Appendix Table). With the same technique, $58.5 \%$ of cattle and $22.2 \%$ of sheep were positive (online Technical Appendix Table). CF antibodies were found for SFNV in $19.4 \%$ of human serum samples in Serbia (online Technical Appendix Table).
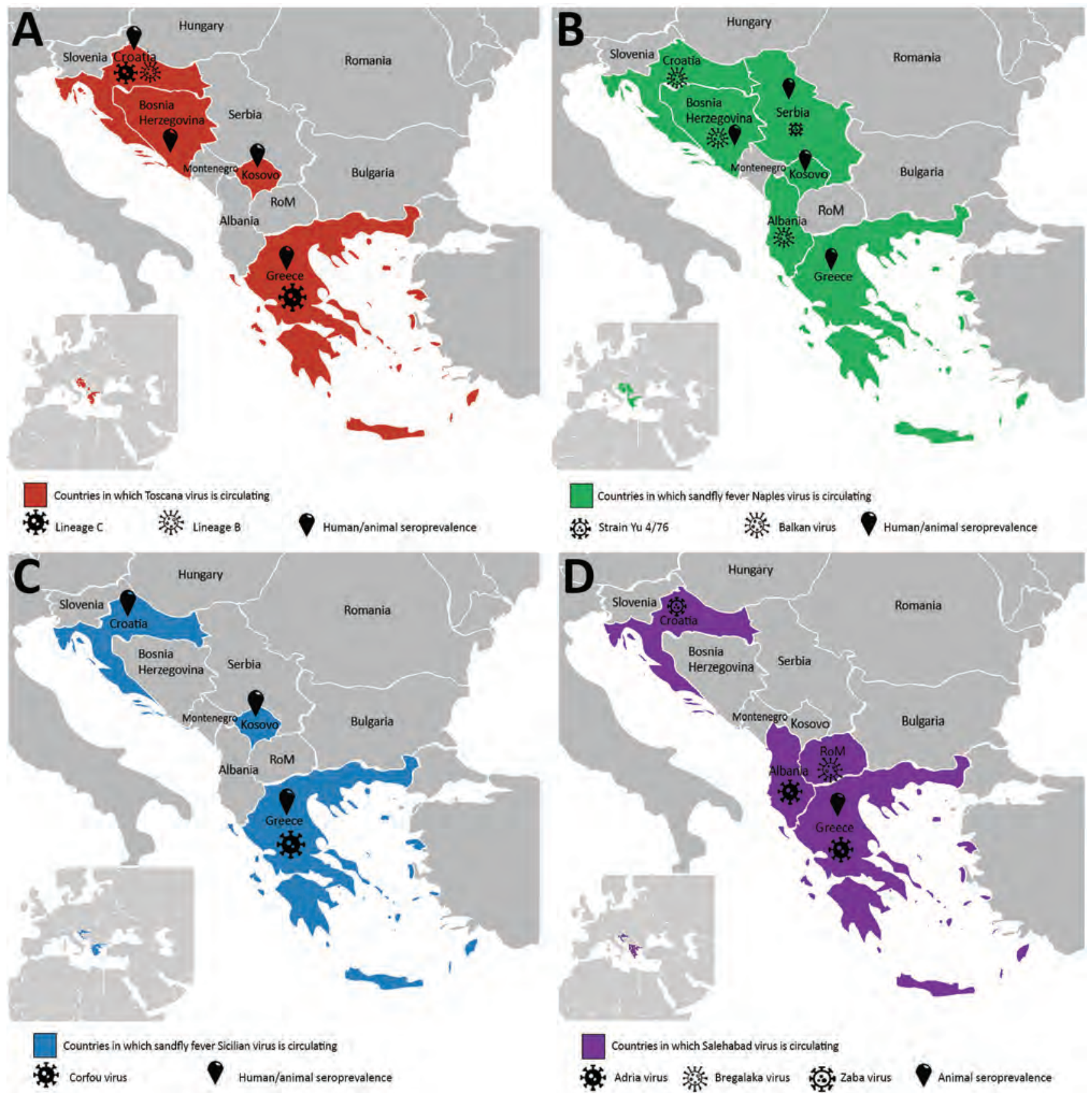

Figure 2. Current distribution of sand fly-borne phleboviruses in the Balkan region. A) Toscana virus, B) sandfly fever Naples virus, C) sandfly fever Sicilian virus, and D) Salehabad virus. Pictograms refer to virus isolation or sequence data demonstrating the presence of the virus in that area. Human/animal seroprevalence refers to studies reporting the presence of specific antibodies against the virus mentioned in the panel. 
TOSV was discovered in 1971 , but it was identified as a human pathogen 12 years later, which prevented early inclusion in the seroprevalence studies; thus, almost no data exist for TOSV before the 1990s.

Recent data have confirmed the circulation of TOSV and associated human cases in Kosovo, Greece, and Croatia. In Croatia, 2 risk factors were associated with TOSV positive serology: living on an island and age (online Technical Appendix Table). Possible presence of TOSV was assessed in Bosnia-Herzegovina through immune-line assays (online Technical Appendix Table). TOSV neutralizing antibodies were detected in cats and dogs in Greece and in cattle and sheep in Kosovo (Figure 2; online Technical Appendix Table).

\section{Discussion}

Sand fly-borne diseases are widespread in the Balkan region because of the favorable climate and socioeconomic conditions in that area. After the first record of sandfly fever in Bosnia-Herzegovina at the end of the 19th century (online Technical Appendix Table), several outbreaks occurred in the whole Balkan region. Epidemics of sandfly fever and leishmaniasis prompted faunistic and ecologic investigations of sand flies from 1947 through the 1970s (33). The number of studies on sand fly fauna has decreased since that time because of the decline in recorded sandfly fever cases. For some Balkan countries, almost nothing is known about sand fly distribution; when data are available, they are too old to reflect the current situation accurately. The collapse of the former Yugoslavia and subsequent armed conflicts have also contributed to the lack of sustained studies on sand fly-borne pathogens in this region. However, recent data show that the Balkan region is still a major hotspot for arboviral diseases.

Most virus studies are based on serosurveillance. The seminal neutralization-based seroprevalence study, performed by Tesh et al. in the 1970s, identified antibodies against SFNV and SFSV in human populations from Croatia, Greece, and Kosovo (online Technical Appendix Table). Successive studies confirmed the presence of antibodies against phleboviruses in most parts of the Balkans, and recent serologic studies show the circulation of TOSV in Bosnia-Herzegovina, Kosovo, Croatia, and Greece (online Technical Appendix Table).

SFNV Yu 8/76 was the first phlebovirus isolated in the Balkans (online Technical Appendix Table). It was isolated from $P$. perfiliewi sand flies; before this finding, other viruses had been isolated from $P$. papatasi sand flies, which were believed to be the unique vector competent for SFNV and SFSV (17). The recent discovery of BALKV should stimulate studies to address possible human pathogenicity (online Technical Appendix Table). Recent evidence for the presence of at least 2 different lineages of TOSV calls for studies to measure its involvement in summer meningitis and other neurologic infections, as were performed in
Italy after the discovery of TOSV neurotropism.

Corfou virus was isolated from $P$. major sand flies in the eponymous Greek island (online Technical Appendix Table). Although Corfou/SFSV circulation was assessed by seroprevalence studies, Corfou virus remains the only SFSV-like virus isolated in the Balkans.

Adria virus was the first member of the Salehabad virus complex to be associated with human disease. Because of this finding, and in light of the newly discovered viruses within this species (BREV and ZABAV), future actions should be directed at implementing direct and indirect diagnosis of Salehabad phlebovirus species in clinical microbiology laboratories to better understand their potential public health impact.

The fall of communism, the breakup of the former $\mathrm{Yu}$ goslavia, and the following civil war and other climatic and environmental changes resulted in an increase of zoonotic infections that emerged or reemerged in the Balkans (28). As previously suggested for North Africa and in Turkey, it is time to organize systematic testing of patients with CNS infections or unexplained febrile illness for such viruses in clinical microbiology laboratories in hospitals (35-37).

In summary, when historical and recent data are compiled, it appears that the Balkan region is a hotspot for viruses transmitted by sand flies, including those that cause diseases in humans. The variety of different viruses is higher than in other regions that were investigated, and certain areas display sympatric circulation of several viruses. Circulation of these viruses must be assessed by studies conducted in human populations and vertebrates, and diagnosis of human infections caused by sand fly-borne viruses must now be implemented using molecular and serologic tools in clinical microbiology laboratories.

This work was supported in part by the European Virus Archive Goes Global (EVAg) project, which has received funding from the European Union's Horizon 2020 research and innovation program under grant agreement no. 653316.

\section{About the Authors}

Dr. Ayhan is a postdoctoral fellow at the Institute of Research for Development in Marseille, France. Her primary research interest is the study of phleboviruses transmitted by sand flies in the Old World.

Dr. Charrel is a professor of virology at Aix Marseille University, Marseille, France. His primary research interest is in the field of arboviruses, with specific interest in viruses transmitted by sand flies, and, more generally, study of mechanisms of viral emergence.

\section{References}

1. Elliott RM. Molecular biology of the Bunyaviridae. J Gen Virol. 1990;71:501-22. http://dx.doi.org/10.1099/0022-1317-71-3-501 
2. Adams MJ, Lefkowitz EJ, King AMQ, Harrach B, Harrison RL, Knowles NJ, et al. Changes to taxonomy and the International Code of Virus Classification and Nomenclature ratified by the International Committee on Taxonomy of Viruses (2017). Arch Virol. 2017;162:2505-38. http://dx.doi.org/10.1007/s00705-017-3358-5

3. Steinhauer DA, Holland JJ. Rapid evolution of RNA viruses. Annu Rev Microbiol. 1987;41:409-31. http://dx.doi.org/10.1146/ annurev.mi.41.100187.002205

4. Briese T, Calisher $\mathrm{CH}$, Higgs S. Viruses of the family Bunyaviridae: are all available isolates reassortants? Virology. 2013;446:207-16. http://dx.doi.org/10.1016/j.virol.2013.07.030

5. Palacios G, Tesh R, Travassos da Rosa A, Savji N, Sze W, Jain K, et al. Characterization of the Candiru antigenic complex (Bunyaviridae: Phlebovirus), a highly diverse and reassorting group of viruses affecting humans in tropical America. J Virol. 2011;85:3811-20. http://dx.doi.org/10.1128/JVI.02275-10

6. Freire CC, Iamarino A, Soumaré POL, Faye O, Sall AA, Zanotto PM. Reassortment and distinct evolutionary dynamics of Rift Valley fever virus genomic segments. Sci Rep. 2015;5:11353. http://dx.doi.org/10.1038/srep11353

7. Sabin AB. Experimental studies on Phlebotomus (pappataci, sandfly) fever during World War II. Arch Gesamte Virusforsch. 1951;4:367-410. http://dx.doi.org/10.1007/BF01241161

8. Verani P, Ciufolini MG, Caciolli S, Renzi A, Nicoletti L, Sabatinelli G, et al. Ecology of viruses isolated from sand flies in Italy and characterized of a new Phlebovirus (Arbia virus). Am J Trop Med Hyg. 1988;38:433-9. http://dx.doi.org/10.4269/ ajtmh.1988.38.433

9. Ehrnst A, Peters CJ, Niklasson B, Svedmyr A, Holmgren B. Neurovirulent Toscana virus (a sandfly fever virus) in Swedish man after visit to Portugal. Lancet. 1985;325:1212-3. http://dx.doi.org/10.1016/S0140-6736(85)92886-7

10. Dionisio D, Esperti F, Vivarelli A, Valassina M. Epidemiological, clinical and laboratory aspects of sandfly fever. Curr Opin Infect Dis. 2003;16:383-8. http://dx.doi.org/10.1097/ 00001432-200310000-00003

11. Charrel RN, Gallian P, Navarro-Mari JM, Nicoletti L, Papa A, Sánchez-Seco MP, et al. Emergence of Toscana virus in Europe. Emerg Infect Dis. 2005;11:1657-63. http://dx.doi.org/10.3201/eid1111.050869

12. Depaquit J, Grandadam M, Fouque F, Andry PE, Peyrefitte C. Arthropod-borne viruses transmitted by Phlebotomine sandflies in Europe: a review. Euro Surveill. 2010;15:19507.

13. Gaidamovich SY, Khutoretskaya NV, Asyamov YV, et al. Sandfly fever in central Asia and Afghanistan. In: Calisher $\mathrm{CH}$, editor. Hemorrhagic fever with renal syndrome, tick- and mosquito-borne viruses. Basel (Switzerland): Springer; 1991. p. 287-93.

14. Al-Hazmi M, Ayoola EA, Abdurahman M, Banzal S, Ashraf J, El-Bushra A, et al. Epidemic Rift Valley fever in Saudi Arabia: a clinical study of severe illness in humans. Clin Infect Dis. 2003;36:245-52. http://dx.doi.org/10.1086/345671

15. Çarhan A, Uyar Y, Özkaya E, Ertek M, Dobler G, Dilcher M, et al. Characterization of a sandfly fever Sicilian virus isolated during a sandfly fever epidemic in Turkey. J Clin Virol. 2010;48:264-9. http://dx.doi.org/10.1016/j.jcv.2010.05.011

16. Ayhan N, Baklouti A, Prudhomme J, Walder G, Amaro F, Alten B, et al. Practical guidelines for studies on sandfly-bornephleboviruses: part I: important points to consider ante field work. Vector Borne Zoonotic Dis. 2017;17:73-80. http://dx.doi.org/10.1089/vbz.2016.1957

17. Alkan C, Bichaud L, de Lamballerie X, Alten B, Gould EA, Charrel RN. Sandfly-borne phleboviruses of Eurasia and Africa: epidemiology, genetic diversity, geographic range, control measures. Antiviral Res. 2013;100:54-74. http://dx.doi.org/ 10.1016/j.antiviral.2013.07.005

18. Doerr R, Franz K, Taussing S. Das Papatatsi Fieber. Leipzig-Wien: Franz Deuticke. 1909.
19. Gligić A, Mišcević Z, Tesh RB, et al. First isolations of Naples sandfly fever virus in Yugoslavia. Acta Biol Jug Mikrobiol. 1982;19:167-75.

20. Borcić B, Punda V. Sandfly fever epidemiology in Croatia. Acta Med Iugosl. 1987;41:89-97.

21. Hertig M, Sabin AB. Sandfly fever. In: Coates JB, editor. Preventive medicine in World War II, vol. VII. Communicable diseases. Washington: US Government Printing Office; 1964. p. 109-74.

22. Papadopoulos O. Arbovirus problems in Greece. In: Vesenjak-Hirjan J. editor Arboviruses in the Mediterranean countries. 6th FEMS symposium. Stuttgart: Gustav Fisher Verlag; 1980.

23. Papa A, Kontana A, Tsergouli K. Phlebovirus infections in Greece. J Med Virol. 2015;87:1072-6. http://dx.doi.org/10.1002/ jmv.24163

24. Guelmino DJ, Jevtic M. An epidemiological and hematological study of sandfly fever in Serbia. Acta Trop. 1955;12:179-82.

25. Drenski P, Drenski K. Contribution to the study of genus Phebotomus (Dipt.) and the three-day fever in Bulgaria. Proceed Bulg Entomol Soc. 1928;4:31-56.

26. Pick A. On the pathology and therapy of a peculiar endemic 1860 disease form [in German]. Wien Med Wochenschr. 1886;33:1141-5.

27. Taussig S. Die Hundskrankheit, endemischer Magenkatarrh in der Herzegowina. Wien Klin Wochenschr. 1905;50:164.

28. Hukić M, Numanović F, Siširak M, Moro A, Dervović E, Jakovec S, et al. Surveillance of wildlife zoonotic diseases in the Balkans region. Med Glas (Zenica). 2010;7:96-105.

29. Liu DY, Tesh RB, Travassos Da Rosa AP, Peters CJ, Yang Z, Guzman $\mathrm{H}$, et al. Phylogenetic relationships among members of the genus Phlebovirus (Bunyaviridae) based on partial M segment sequence analyses. J Gen Virol. 2003;84:465-73. http://dx.doi.org/10.1099/vir.0.18765-0

30. Karabatos N. International catalogue of arboviruses including certain other viruses of vertebrates, third ed. San Antonio (TX): American Society of Tropical Medicine and Hygiene; 1985.

31. Alkan C, Erisoz Kasap O, Alten B, de Lamballerie X, Charrel RN. Sandfly-borne phlebovirus isolations from Turkey: New insight into the sandfly fever Sicilian and sandfly fever Naples species. PLoS Negl Trop Dis. 2016;10:e0004519. http://dx.doi.org/10.1371/ journal.pntd.0004519

32. Remoli ME, Fortuna C, Marchi A, Bucci P, Argentini C, Bongiorno G, et al. Viral isolates of a novel putative phlebovirus in the Marche region of Italy. Am J Trop Med Hyg. 2014;90:760-3. http://dx.doi.org/10.4269/ajtmh.13-0457

33. Sabin AB. Recent advances in our knowledge of dengue and sandfly fever. Am J Trop Med Hyg. 1955;4:198-207. http://dx.doi.org/10.4269/ajtmh.1955.4.198

34. Simić Č, Živković V. Wildlife sandflies in Yugoslavia. I. The phlebotomids of Macedonia, southern Serbia and the Kosovo and Metohia region [in French]. Glas Srpske akademije nauka CXCIV, Odeljenje medicinskih nauka. 1949;1:151-81.

35. Alkan C, Allal-Ikhlef AB, Alwassouf S, Baklouti A, Piorkowski G, de Lamballerie X, et al. Virus isolation, genetic characterization and seroprevalence of Toscana virus in Algeria. Clin Microbiol Infect. 2015;21:1040.e1-9. http://dx.doi.org/10.1016/j.cmi. 2015.07.012

36. Charrel R. The tree that hides the forest: not only West Nile virus, but also Toscana virus and Usutu virus. Vet Ital. 2017; 53:367-8.

37. Ergunay K, Ayhan N, Charrel RN. Novel and emergent sandflyborne phleboviruses in Asia Minor: a systematic review. Rev Med Virol. 2017;27:e1898. http://dx.doi.org/10.1002/rmv.1898

Address for correspondence: Nazli Ayhan, Unite des Virus Emergents, School of Medicine, 27 blvd Jean Moulin, Marseille 13005, France; email: nazliayhann@gmail.com 


\section{I solation of Burkholderia pseudomallei from a Pet Green I guana, Belgium}

\author{
Tom Hellebuyck, Pierre Wattiau, Filip Boyen, \\ Ilse Moeremans, Nancy H. Roosens, \\ Kevin Vanneste, An Garmyn, Veronique Saey, \\ Frank Pasmans, Freddy Haesebrouck
}

We isolated Burkholderia pseudomallei, the causative agent of melioidosis, from liver granulomas of a pet green iguana (Iguana iguana) in Belgium. This case highlights a risk for imported green iguanas acting as a reservoir for introduction of this high-threat, zoonotic pathogen into nonendemic regions.

$\mathrm{T}$ he highly pathogenic, gram-negative bacterium Burkholderia pseudomallei is the causative agent of melioidosis, which is endemic to countries in Southeast Asia and in northern Australia and an emerging infectious disease in several tropical developing countries $(1,2)$. Human cases in Europe are limited to patients who traveled to disease-endemic regions. In Belgium, the last case was documented in 2013 in a 44-year-old man who had traveled to Madagascar (3). B. pseudomallei is classified as a tier 1 overlap select agent by the US Federal Select Agent Program (4). Postexposure prophylaxis and postexposure monitoring should be planned for persons who have had high-risk exposures, such as certain laboratory procedures with the organism that were not conducted under Biosafety Level 3 conditions (5).

Infected humans and importing of infected animals can introduce melioidosis into nonendemic areas $(2,6)$. However, importing of infected animals has not yet been associated with epizootic transmission. Infection commonly occurs through cutaneous inoculation, ingestion of contaminated soil or water, or inhalation of aerosolized bacteria $(7,8)$. Although the incubation period in humans is typically $1-21$ days, clinical disease might develop years after infection (9). The incubation period in naturally infected animals is not known (10).

Acute melioidosis results predominantly in pneumonia and septicemia. Chronic infection is associated with abscesses of the liver, lungs, spleen, and skin (9). Because

Author affiliations: Ghent University, Merelbeke, Belgium (T. Hellebuyck, F. Boyen, I. Moeremans, A. Garmyn, V. Saey, F. Pasmans, F. Haesebrouck); Veterinary and Agrochemical Research Centre, Brussels, Belgium (P. Wattiau); Sciensano, Ixelles, Belgium (N.H. Roosens, K. Vanneste)

DOI: https://doi.org/10.3201/eid2412.171661 of the intrinsic resistance of the bacterium to many antimicrobial drugs, combined with the inability to provide appropriate medical care in disease-endemic developing countries, overall case-fatality rates might exceed $70 \%$ $(1,4,9)$. B. pseudomallei is rarely reported in animals other than cattle, goats, and swine (10).

In reptiles, isolation of $B$. pseudomallei has been anecdotally documented in crocodiles (10), and clinical infections have been reported in 2 pet green iguanas in California, USA (11) and a pet green iguana in Prague (Czech Republic) (4). Dermal abscesses were observed in the iguana from the Czech Republic and in 1 of the iguanas from California, and hepatic masses were observed in the second iguana from California.

Because of the variable clinical manifestations of melioidosis and limited value of conventional bacterial methods for identification of B. pseudomallei, diagnosis of melioidosis can be challenging $(2,11)$. If one considers the highly pathogenic and zoonotic nature of B. pseudomallei, use of appropriate molecular detection methods is crucial to warrant correct identification and discrimination of $B$. pseudomallei from other Burkholderia species (11). Nextgeneration sequencing might be a valuable supplement to current identification and diagnostic methods.

\section{The Study}

A 5-year-old female green iguana (Iguana iguana) showed acute onset of lethargy, anorexia, and general weakness. The iguana had been purchased 4.5 years earlier by private owners from a pet shop in the Netherlands that imported the iguana from a captive breeding operation in Central America. Serum biochemical and hematologic tests showed hyperuricemia, hyperphosphatemia, an increased level of aspartate aminotransferase, hyperglobulinemia, nonregenerative anemia, and severe leukocytosis in comparison with physiologic reference ranges for these conditions (12). Ultrasonography showed hepatomegaly, multiple hyperechoic hepatic masses, and severely enlarged, hyperechoic kidneys. A presumptive diagnosis of hepatitis, kidney failure, and septicemia was made. Because the iguana did not respond to supportive treatment and its general condition continued to deteriorate, the owners agreed to euthanize the iguana on the fifth day after signs began.

During necropsy, pronounced renomegaly and hepatomegaly, as well as granulomatous hepatitis was observed. Histologic evaluation of hematoxylin and eosin-stained 
sections of liver showed coalescent granulomas throughout the liver parenchyma, with central necrosis surrounded by activated macrophages and giant cells (Figure). Gram, periodic acid-Schiff, and acid-fast staining did not show intralesional gram-positive or acid-fast bacteria or fungi. Microbiological examination of liver tissue yielded a pure and abundant culture of small colonies of gram-negative rods that could not be identified by using standard biochemical identification tests.

Sequencing of the 16S rRNA gene of the isolate showed $100 \%$ identity with B. pseudomallei (1,382 bp). Sequence data were deposited in GenBank under accession no. MF523223. After culturing of the isolate under Biosafety Level 3 conditions, we performed multilocus sequence typing-derived PCR and real-time PCR as described by Wattiau et al. (13), which confirmed identification of the isolate as $B$. pseudomallei.

We used next-generation sequencing to obtain the genome sequence of the isolate, which was deposited in the Short Read Archive under accession no. SRR6056996. The genome sequence was used to infer its multilocus sequence type (ST) as ST518. Isolate information was deposited accordingly in the B. pseudomallei database (https:// pubMLST.org) under ID 5121 (14). Of 5,600 deposited isolates that we accessed on June 18, 2018, ST518 matched only with $B$. pseudomallei isolates (ID 3330) obtained from a human who was infected in Costa Rica in 2009 (ID 1928) (14) and with 2 isolates obtained from abscesses in pet iguanas in 2007 (ID 5008) and 2012 (ID 3330) (11). We found that ID 3330 differed from the isolates from Central America by only 3 single-nucleotide polymorphisms, which strongly suggested that the iguanas were infected in Central America before transport abroad (15). However, whether captive iguanas might acquire infections through contact

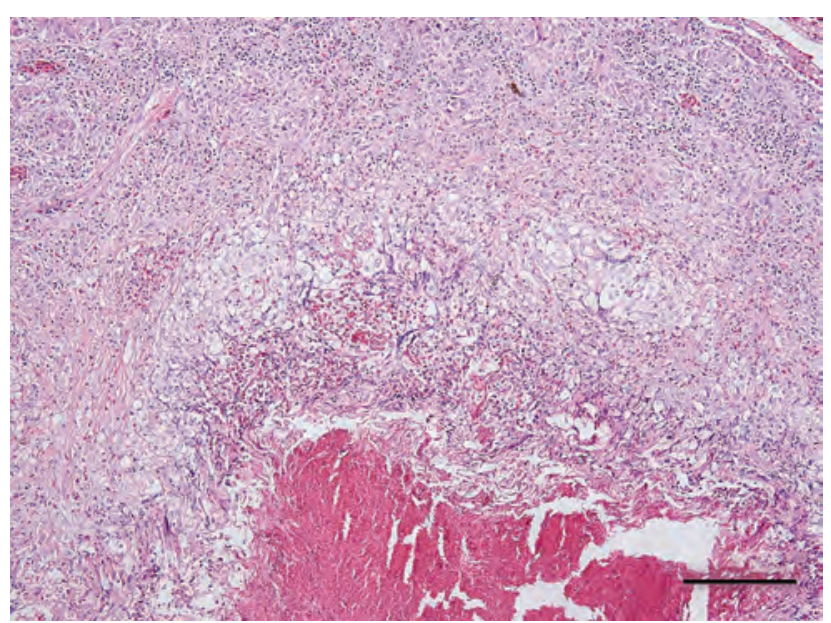

Figure. Coalescent granuloma in liver parenchyma of a pet green iguana (Iguana iguana) infected with Burkholderia pseudomallei, Belgium. Hematoxylin and eosin stain shows central necrosis surrounded by activated macrophages and giant cells. Scale bar indicates $200 \mu \mathrm{m}$. with other imported animals (e.g., in pet shops or in captive collections in nonendemic countries) cannot be excluded.

After we identified the isolate as B. pseudomallei, we collected $100 \mathrm{~mL}$ of water from a plastic water bath and a swab specimen from the emptied water reservoir from the housing of the iguana and tested these samples by using real-time PCR. All samples showed negative results.

The zoonotic potential of the bacterium was discussed with the owners and personnel who came into contact with the iguana or samples that were collected from the lizard. Although the owners did not have a clinical history that could indicate $B$. pseudomallei infection, they were advised to consult with their physicians about potential exposure. Although the owners refused postexposure prophylaxis and postexposure monitoring, postexposure prophylaxis was given to a selected number of staff members as recommended by Lipsitz et al. (5) because of potential exposure to the pathogen.

\section{Conclusions}

Previously reported B. pseudomallei-infected iguanas $(4,11)$ and the 1 reported in this study were presumably imported from disease-endemic regions, highlighting the potential role of this species as a reservoir of B. pseudomallei. On the basis of the relatedness of ST518 with an isolate obtained from a human in Costa Rica (14) and with 2 isolates from iguanas that presumably became infected in Central America (11), whose genomes were later confirmed to be closely related (15), our findings support the hypothesis that the iguana we report became infected in the captive breeding facility in Central America before importation to Europe.

If one considers the long incubation period observed for green iguanas, these reptiles might shed the bacterium unnoticed for years before nonspecific chronic disease develops. Transmission from infected captive iguanas to humans might occur through contact with stool, infected tissue, or biting and scratching lesions. Indirect infection might originate from wound infection through contaminated soil or water or inhalation of aerosolized bacteria.

In conclusion, traders, (para)veterinarians, and laboratory staff members who handle green iguanas or samples obtained from this species are susceptible to infection. In addition, physicians who are consulted by pet green iguana owners should be aware that these animals and their environment could potentially harbor B. pseudomallei.

\footnotetext{
About the Author

Dr. Hellebuyck is head of the clinic at the Division of Poultry, Exotic Animals, Wildlife, and Experimental Animals, Department of Pathology, Bacteriology and Avian Diseases, Faculty of Veterinary Medicine, Ghent University, Merelbeke, Belgium. His research interests are infectious and noninfectious diseases in reptiles, amphibians, and birds.
} 


\section{References}

1. Aldhous P. Tropical medicine: melioidosis? Never heard of it.... Nature. 2005;434:692-3. http://dx.doi.org/10.1038/434692a

2. Limmathurotsakul D, Golding N, Dance DA, Messina JP, Pigott DM, Moyes CL, et al. Predicted global distribution of Burkholderia pseudomallei and burden of melioidosis. Nat Microbiol. 2016; 1:15008. http://dx.doi.org/10.1038/ nmicrobiol.2015.8

3. ProMED-mail. Melioidosis-Belgium ex Madagascar. 2013 [cited 2018 Aug 1]. http://www.promedmail.org, archive no. 20130503.1687746

4. Elschner MC, Hnizdo J, Stamm I, El-Adawy H, Mertens K, Melzer F. Isolation of the highly pathogenic and zoonotic agent Burkholderia pseudomallei from a pet green iguana in Prague, Czech Republic. BMC Vet Res. 2014;10:283-7. http://dx.doi.org/ 10.1186/s12917-014-0283-7

5. Lipsitz R, Garges S, Aurigemma R, Baccam P, Blaney DD, Cheng AC, et al. Workshop on treatment of and postexposure prophylaxis for Burkholderia pseudomallei and B. mallei infection, 2010. Emerg Infect Dis. 2012;18:e2. http://dx.doi.org/10.3201/ eid1812.120638

6. Galimand M, Dodin A. Pseudomonas pseudomallei distribution in France and worldwide melioidosis [in French]. Bulletin de la Societé Vétérinaire Pratique de France. 1982;66:651-7.

7. Cheng AC, Jacups SP, Gal D, Mayo M, Currie BJ. Extreme weather events and environmental contamination are associated with case-clusters of melioidosis in the Northern Territory of Australia. Int J Epidemiol. 2006;35:323-9. http://dx.doi.org/10.1093/ije/ dyi271

8. Limmathurotsakul D, Kanoksil M, Wuthiekanun V, Kitphati R, deStavola B, Day NP, et al. Activities of daily living associated with acquisition of melioidosis in northeast Thailand: a matched case-control study. PLoS Negl Trop Dis. 2013;7:e2072. http://dx.doi.org/10.1371/journal.pntd.0002072

9. Limmathurotsakul D, Peacock SJ. Melioidosis: a clinical overview. Br Med Bull. 2011;99:125-39. http://dx.doi.org/10.1093/ bmb/ldr007

10. Sprague LD, Neubauer H. Melioidosis in animals: a review on epizootiology, diagnosis and clinical presentation. J Vet Med B Infect Dis Vet Public Health. 2004;51:305-20. http://dx.doi.org/ 10.1111/j.1439-0450.2004.00797.x

11. Zehnder AM, Hawkins MG, Koski MA, Lifland B, Byrne BA, Swanson AA, et al. Burkholderia pseudomallei isolates in 2 pet iguanas, California, USA. Emerg Infect Dis. 2014;20:304-6. http://dx.doi.org/10.3201/eid2002.131314

12. Divers SJ, Redmayne G, Aves EK. Haematological and biochemical values of 10 green iguanas (Iguana iguana). Vet Rec. 1996;138:203-5. http://dx.doi.org/10.1136/vr.138.9.203

13. Wattiau P, Van Hessche M, Neubauer H, Zachariah R, Wernery U, Imberechts H. Identification of Burkholderia pseudomallei and related bacteria by multiple-locus sequence typing-derived PCR and real-time PCR. J Clin Microbiol. 2007;45:1045-8. http://dx.doi.org/10.1128/JCM.02350-06

14. Aanensen DM, Spratt BG. The multilocus sequence typing network: mlst.net. Nucleic Acids Res. 2005;33:W728-33. http://dx.doi.org/10.1093/nar/gki415

15. Gee JE, Gulvik CA, Elrod MG, Batra D, Rowe LA, Sheth M, et al. Phylogeography of Burkholderia pseudomallei isolates, Western Hemisphere. Emerg Infect Dis. 2017;23:1133-8. http://dx.doi.org/10.3201/eid2307.161978

Address for correspondence: Tom Hellebuyck, Department of Pathology, Bacteriology and Avian Diseases, Faculty of Veterinary Medicine, Ghent University, Salisburylaan 133, B-9820 Merelbeke, Belgium; email: tom.hellebuyck@ugent.be

\section{EI D Podcast:}

Visions of Matchstick Men and I cons of Industrialization

Byron Breedlove, managing editor of the journal, discusses and reads his

November 2017 cover art essay.

This cover (Going to Work, 1943) is

by English artist Laurence Stephen

Lowry (1887-1976) who died of pneumonia in 1976.

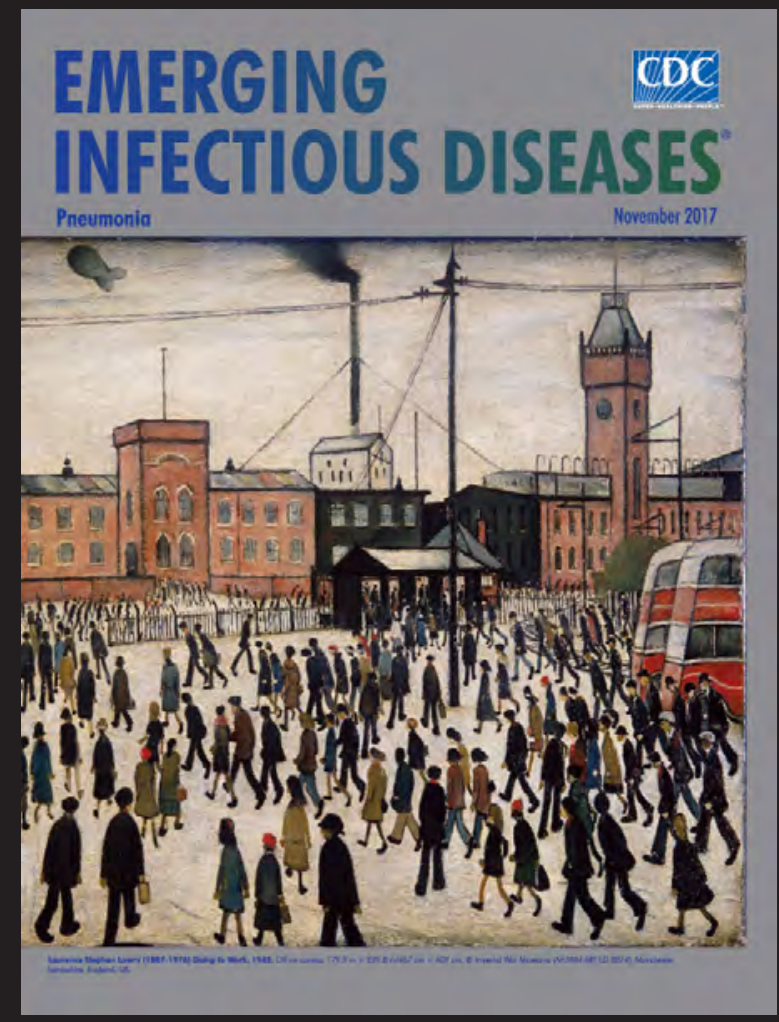

Visit our website to listen: https:/ / www2c.cdc.gov/ podcasts/ player.asp?f $=\mathbf{8 6 4 7 1 7 3}$

\section{EMERCING INFECTIOUS DISEASES}




\section{Neglected Hosts of Small Ruminant Morbillivirus}

\section{Claudia Schulz, ${ }^{1}$ Christine Fast, Kore Schlottau, Bernd Hoffmann, Martin Beer}

Eradication of small ruminant morbillivirus (PPRV) is targeted for 2030. PPRV lineage IV is found in much of Asia and Africa. We used PPRV lineage IV strain Kurdistan/2011 in transmission trials to investigate the role of pigs, wild boar, and small ruminants as PPRV reservoirs. Suids were a possible source of infection.

$\mathrm{P}$ este des petits ruminants is one of the most serious (economically and clinically) transboundary animal diseases (1-3). Of 4 lineages, small ruminant morbillivirus (previously called peste des petits ruminants virus; PPRV) lineage IV (PPRV-LIV) has spread the most widely in the past decade, particularly in Asia, and increasingly dominates the PPRV lineages in Africa $(2,4)$. Morbidity and mortality rates for goats are high, up to $100 \%$; however, sheep can be subclinically infected and play a major role in the silent spread of PPRV over large distances and across borders (3). The role of other wild and domestic Artiodactyls in the epidemiology of PPRV is unknown or insufficiently understood (3). Pigs are considered dead-end hosts for PPRV (5). In an experimental infection study, pigs infected with PPRV lineage II (LII) strains did not transmit PPRV to goats, but they can transmit the closely related Rinderpest morbillivirus to cattle (6). To determine the pathogenesis of PPRV-LIV infection in pigs and wild boar and the capability of these suids to transmit PPRV in comparison with that of goats and sheep, we conducted 4 independent transmission trials during 2015-2016. The experimental protocol was reviewed by a state ethics commission and approved by the State Office for Agriculture, Food Safety and Fisheries of Mecklenburg-Vorpommern, Rostock, Germany (LALLF M-V/TSD/7221.3-1-018/14).

\section{The Study}

In 4 trials (trials 1-4; Table), we intranasally inoculated suids with a recent PPRV-LIV strain (Kurdistan/2011 $[7,8])$. Contact control animals were added 2 days later. We recorded clinical signs and temperature regularly and collected samples to evaluate the suitability of different virologic, serologic, and pathological methods for detecting PPRV infection. We conducted statistical analyses to

Author affiliation: Friedrich-Loeffler-Institut, Greifswald-Insel

Riems, Germany

DOI: https://doi.org/10.3201/eid2412.180507 calculate whether PPRV RNA loads in secretions and excretions (oral, nasal, and fecal swab samples) collected over time from pigs, wild boar, goats, and sheep differed significantly and to determine correlations between the results of virus isolation and PCR assays by using swab samples and purified leukocytes as sample materials (online Technical Appendix, https://wwwnc.cdc.gov/EID/ article/24/12/18-0507- Techapp1.pdf).

As expected, goats showed the typical moderate to severe clinical signs (trials 1 and 3) reported previously (7-9). Clinical signs in PPRV-infected sheep (trial 4) were generally mild to moderate, as previously described $(3,8)$. Contact controls showed similar clinical signs. One PPRVinfected sheep showed severe clinical signs similar to those of the goats. Surprisingly, all PPRV infected pigs and wild boar (trials 1-3) showed various mild to moderate clinical signs, including fever and reduced general condition (all suids), diarrhea (pig 1, boar 1-4), and ocular (pigs 1-3) and nasal (boar 4) discharge typical for PPRV infection (Figure 1; Figure 2, panels A-C; online Technical Appendix). PPRV-induced immunosuppression may predispose affected animals to secondary infections $(3,9)$ as reflected by distinct severe leukocytopenia in pigs and goats a few days after inoculation. Different expressions of clinical signs after PPRV infection might have been caused by concurrent infections with other pathogens or differences in individual resistance to PPRV infection (9). In the 4 wild boar, for example, Balantidium coli, detected by histopathologic examination (data not shown), might have been an additional factor causing the diarrhea (10). Nevertheless, similar to the lack of clinical signs reported for pigs infected with a PPRV-LII strain (6), the 2 pigs in trial 3 showed only mild clinical signs.

Contact transmission of PPRV from intranasally infected pigs to 1 contact goat and 1 pig was noted (trial 1). This pig was refractory to intranasal infection but was infected by contact at a similar time as one of the goats. Furthermore, PPRV was transmitted from intranasally infected goats to contact pigs (trial 3) (Table). Hence, in contrast to the findings of Nawathe and Taylor (6), who reported contact transmission of a PPRV-LII strain from experimentally infected goats to contact pigs but not vice versa, our transmission trials demonstrated that a complete interspecies transmission cycle of a PPRV-LIV strain between goats and pigs, and possibly also intraspecies transmission between pigs, can be maintained. The virulence of ${ }^{1}$ Current affiliation: University of Veterinary Medicine Hannover, Hannover, Germany. 
Table. Design and outcomes of PPRV transmission trials, Germany*

\begin{tabular}{|c|c|c|c|c|c|c|c|}
\hline \multirow[b]{2}{*}{$\begin{array}{l}\text { Trial } \\
\text { no.† }\end{array}$} & \multirow[b]{2}{*}{ Trial } & \multirow[b]{2}{*}{$\begin{array}{l}\text { No. } \\
\text { inoculated } \\
\text { animals }\end{array}$} & \multirow[b]{2}{*}{$\begin{array}{l}\text { No. contact } \\
\text { controls }\end{array}$} & \multicolumn{4}{|c|}{ Outcomes } \\
\hline & & & & $\begin{array}{l}\text { Seroconversion, } \\
\text { total no. by } \\
\text { species }\end{array}$ & $\begin{array}{c}\text { Excretion of } \\
\text { PPRV RNA, total } \\
\text { no. by species }\end{array}$ & $\begin{array}{c}\text { Excretion of } \\
\text { infectious PPRV, } \\
\text { total no. by species }\end{array}$ & $\begin{array}{l}\text { Contact tran } \\
\text { contact-infe }\end{array}$ \\
\hline 1 & P-GP & $3 P \ddagger$ & 2G, 1P‡ & $3 P, \ddagger 2 \mathrm{G}$ & $3 P, \ddagger 2 G$ & $1 \mathrm{P}, 2 \mathrm{G}$ & Yes (1/2 \\
\hline 2 & & & & & $4 W$ & & $/ 2 P)$ \\
\hline & & & & $2 \mathrm{P}$ & $2 \mathrm{G}, 2 \mathrm{P}$ & $2 G$ & \\
\hline $4 \rrbracket$ & S-S & $5 S$ & & & $5 S$ & $5 S$ & No $(0 / 5 S)$ \\
\hline \multicolumn{8}{|c|}{$\begin{array}{l}\text { *P, pig; PPRV, small ruminant morbillivirus (formerly called peste des petits ruminants virus); W, wild boar; G, goat; GP, goats and pigs; S, sheep. } \\
\text { tFor trials } 1-3 \text {, animals were experimentally infected by intranasal inoculation with PPRV strain Kurdistan/2011 for independent transmission trials } \\
\text { conducted in the containment facility of the Friedrich-Loeffler-Institut, Isle of Riems, Germany. Contact control animals were added } 2 \mathrm{~d} \text { after experimental } \\
\text { infection. In } 2 \text { of the trials, PPRV transmission was documented from pigs to } 1 \text { goat and } 1 \text { pig (trial } 1 \text { ) and from goats to } 2 \text { pigs (trial } 3 \text { ). Infectious PPRV } \\
\text { excretion was detected in } \geq 1 \text { animal of each species, and PPRV RNA and seroconversion were detected in all experimentally infected or contact-infected } \\
\text { animals (further details in online Technical Appendix Figure } 1 \text {, https://wwwnc.cdc.gov/EID/article/24/12/18-0507-Techapp1.pdf). For trial } 4 \text {, a } 1 \text {-to-1 } \\
\text { (pairwise) study design was chosen to estimate the reproductive ratio. The results of the sheep trial are presented in this study to enable comprehensive } \\
\text { comparison with the PPRV pathogenesis in suids. } \\
\text { fOne of } 3 \text { pigs was probably not infected by experimental intranasal PPRV inoculation but by contact infection. } \\
\text { \$One contact goat was infected by pigs; however the source of infection (goat or pig) cannot be determined for the second contact goat. } \\
\text { ๆIn each of } 5 \text { stables, } 2 \text { sheep were kept together: } 1 \text { experimentally infected sheep and } 1 \text { contact control sheep. }\end{array}$} \\
\hline
\end{tabular}

the PPRV lineage or strain is possibly a factor influencing the susceptibility to PPRV infection and the probability of PPRV transmission $(9,11)$.

From 2 of 4 wild boar (trial 2), PPRV was isolated from a few fecal swab samples but was not transmitted to the contact goats or pigs. Unexpectedly, none of the intranasally infected sheep transmitted PPRV to any of the contact sheep. The considerable differences in transmission efficiency between goats and the other Artiodactyls can be explained by higher PPRV loads excreted by goats
(Figure 1). Statistically significantly higher PPRV RNA loads over time were found in PPRV-infected goats than in suids and sheep. Peak viral loads in goat samples were up to 1 log step (PCR) and $2.5 \log$ steps (virus isolation) higher $\left(9.3 \times 10^{7}\right.$ copies $/ \mathrm{mL}$; $10^{6.0}$ TCID $_{50}[50 \%$ tissue culture infective dose $/ \mathrm{mL}$ ) than in pig and wild boar samples $\left(1.5 \times 10^{7}\right.$ copies $\left./ \mathrm{mL} ; 10^{3.5} \mathrm{TCID}_{50} / \mathrm{mL}\right)$. Of note, peak viral loads in sheep $\left(10^{4} \mathrm{TCID}_{50} / \mathrm{mL}\right)$ were only slightly higher than those in pigs and wild boar, which may explain why none of the sheep transmitted PPRV to the contact
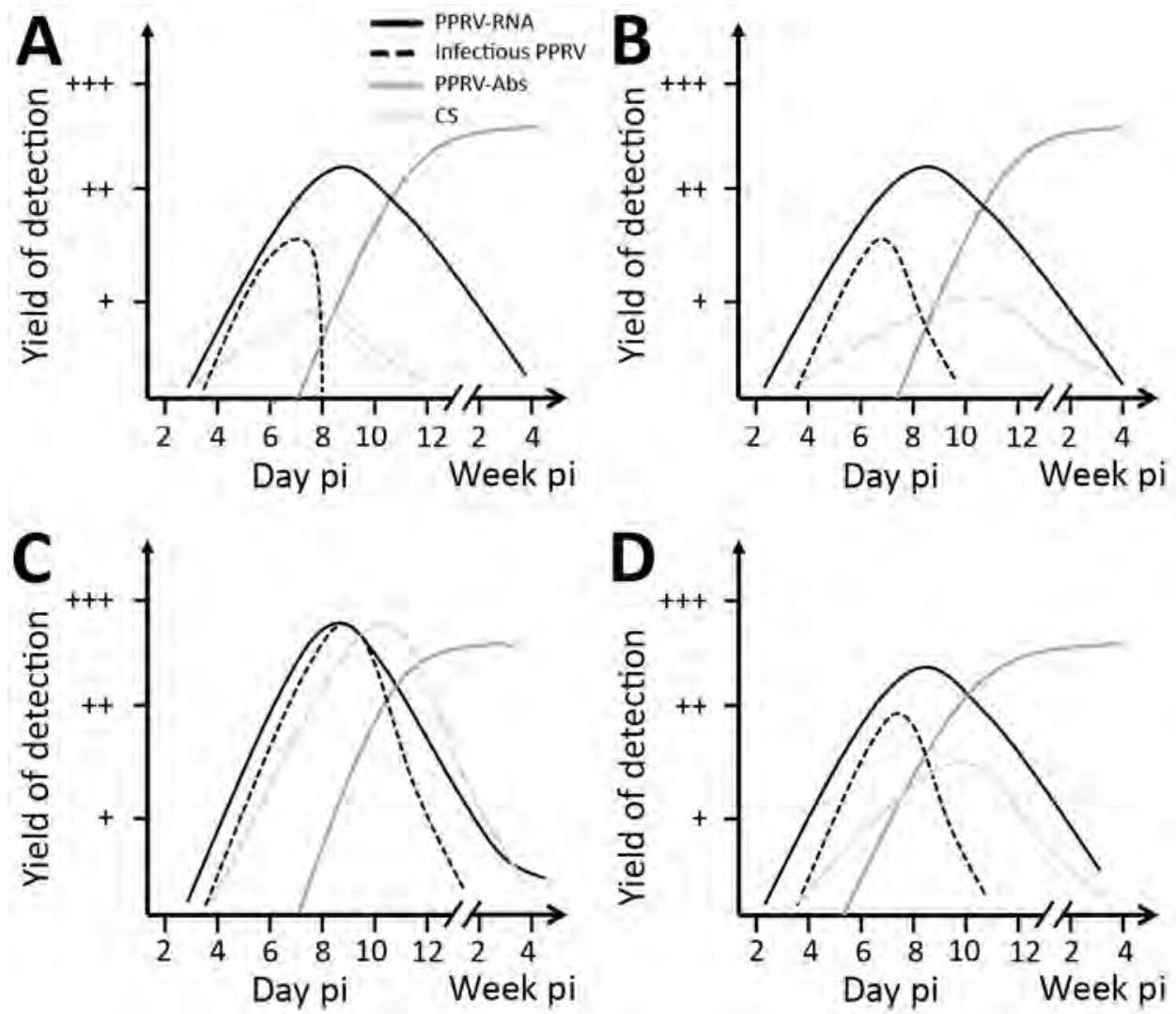

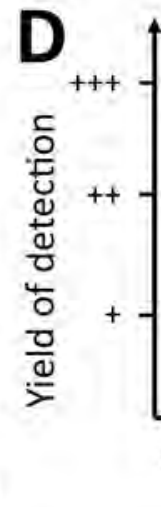

Figure 1. Progression of virologic, serologic, and clinical parameters analyzed in pigs (A), wild boar (B), goats (C), and sheep (D) in Germany after experimental infection with PPRV lineage IV strain Kurdistan/2011. Results are shown for reverse transcription quantitative PCR (solid black lines), endpoint dilution assay (dashed black lines), competitive ELISA (dark gray lines), and clinical score sheets (light gray lines). A detailed description of the infection experiment is provided in the online Technical Appendix (https://wwwnc.cdc.gov/EID/ article/24/12/18-0507-Techapp1. pdf). Abs, antibodies; CS, clinical signs; pi, postinfection; PPRV, small ruminant morbillivirus (formerly called peste des petits ruminants virus). 
Figure 2. Clinical signs observed in wild boar and pigs and small ruminant morbillivirus (formerly called peste des petits ruminants virus; PPRV) antigen detection in a pig tonsil in experimental study of PPRV transmission, Germany.

A) Purulent nasal discharge in wild boar 4 at 8 days after infection; B) diarrhea in wild boar 4 at 7 days after infection; C) swollen eyelids in pig 3 at 10 days after infection; D) PPRV antigen (red) in the tonsil of pig 1 at 30 days after infection $(\approx 22$ days after contact infection of pig 1), by immunohistochemical staining with monocloncal mouse anti-PPRV; scale bar indicates $50 \mu \mathrm{m}$. Clinical signs in the 3 pigs in trial 1 included a transient rise in body temperature, ruffling bristles, diarrhea, reduced activity and food intake/ slight emaciation, swelling of the eyelids, mild to severe conjunctivitis, and mucous to purulent ocular discharge in the
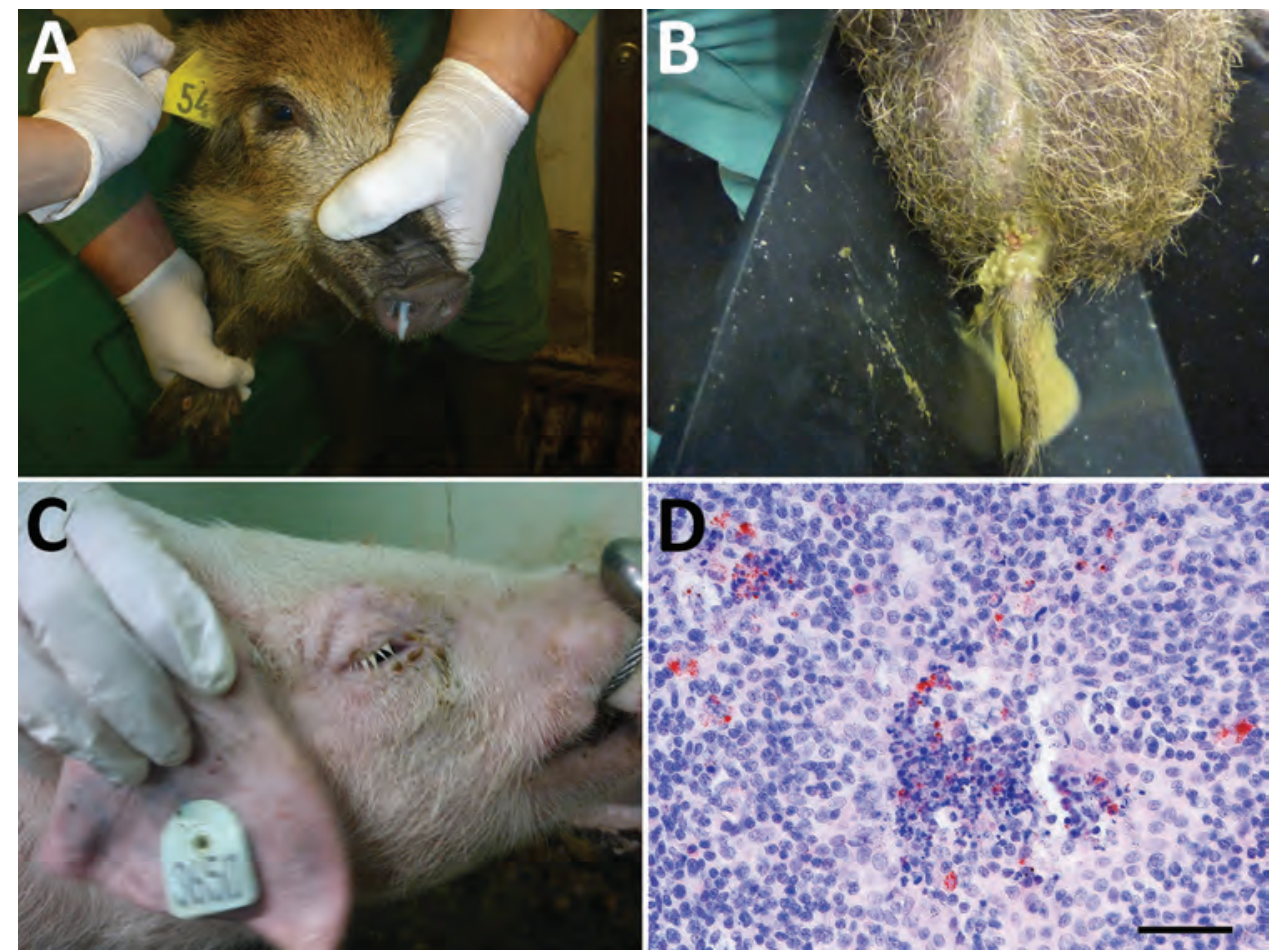

first days after infection. In the 4 wild boar in trial 2, clinical signs included transiently increased body temperature, diarrhea (including fresh blood), reduced general condition, inappetence, and mucopurulent nasal discharge. A detailed description of the infection experiments is provided in the online Technical Appendix (https://wwwnc.cdc.gov/EID/article/24/12/18-0507-Techapp1.pdf).

control sheep. The higher viral loads in goats could also explain the $\approx 4$ days earlier contact infection of the contact pigs in trial 3 than the contact goat and pig in trial 1 . Besides a higher innate susceptibility to PPRV infection observed for goats compared with sheep and suids, the infective dose may play a role in the efficiency of transmission and infection dynamics of PPRV in suids as previously reported for goats (9) and camelids (12).

We detected seroconversion in all PPRV-infected animals by using competitive ELISA and neutralization tests. Neutralizing antibody titers were moderate to high in suids and goats (2.16-2.96 $\log _{10} \mathrm{ND}_{50}$ [virus neutralization in 50\% of replicates]) and slightly lower in sheep (1.76-2.56 $\log _{10} \mathrm{ND}_{50}$ ). After seroconversion, no PPRV could be isolated from swab and purified leukocyte samples, but PPRV RNA was detected in swab samples for at least 3-4 weeks after infection in all species, with individual differences (Figure 1; online Technical Appendix). Correlation analyses revealed a poor to excellent correlation of PCR and virus isolation results before seroconversion, depending on the animal species. Possible reasons for (transient) PPRV RNA persistence are infection of neurons followed by transsynaptic spread (13). Indeed, PPRV RNA was detected in single or multiple brain samples of 2 sheep, 4 goats, and in the choroid plexus of 1 pig, 1 wild boar, and 3 goats. PPRV RNA in the choroid plexus might have been associated with PPRV-infected immune cells, as has been reported for ferrets infected with closely related canine morbillivirus (14). Immunohistochemistry demonstrated that PPRV antigens in other tissues were often associated with immune cells. For PPRV diagnosis in the examined species, tissue of the lymphoreticular system, in particular tonsils (Figure 2, panel D), head and lung-associated lymph nodes, mesenteric lymph nodes, and small intestinal Peyer's patches, were found most suitable for postmortem diagnosis with PCR and immunohistochemistry. PCR was the most sensitive virologic method independent from the sample material, and competitive ELISA proved reliable for serologic PPRV diagnosis (online Technical Appendix).

\section{Conclusions}

Transmission trials with a virulent PPRV-LIV strain revealed that suids are an unexpected possible source for PPRV infection. Therefore, domestic pigs and wild boar should be considered as possible PPRV reservoir hosts. This finding is especially relevant to stringent control programs. The epidemiologic role of suids in the spread of PPRV, as maintenance or spillover hosts (15), should be further investigated in field and experimental studies using different PPRV lineages and strains at different environmental and experimental conditions. 


\section{Acknowledgments}

We thank Holger Freese, Christian Korthase, Isabell Metz, Karin Pinger, Anja Schulz, and Susanne Zahnow for their excellent technical assistance; the staff of the experimental animal facilities for great assistance and dedicated animal care; Sandra Blome, Bernd Haas, and Jana Pietschmann for scientific and organizational advice; Reiner Ulrich and Jan Schinköthe for excellent support with gross pathology; and Jörn Gethmann, Mario Ziller, Carola Sauter-Louis, and Hans-Hermann Thulke for biostatistical advice.

We also thank the European Commission Animal Health, the Welfare European Research Area Network Fund, and the German Federal Office for Agriculture and Food (grant no. 2813ERA074) for providing support for epidemiologic research under the Improved Understanding of the Epidemiology of Peste-des-Petits Ruminants project.

\section{About the Author}

Dr. Schulz is a postdoctoral researcher at the University of Veterinary Medicine Hannover, Germany. Her primary research interests are the pathogenesis and epidemiology of emerging and vectorborne diseases in wild and domestic animals.

\section{References}

1. Banyard AC, Parida S, Batten C, Oura C, Kwiatek O, Libeau G. Global distribution of peste des petits ruminants virus and prospects for improved diagnosis and control. J Gen Virol. 2010;91:2885-97. http://dx.doi.org/10.1099/vir.0.025841-0

2. Munir M. Role of wild small ruminants in the epidemiology of peste des petits ruminants. Transbound Emerg Dis. 2014;61:411-24.

3. European Food Safety Authority. Scientific opinion on peste des petits ruminants: EFSA Panel on Animal Health and Welfare (AHAW). EFSA J. 2015;13:3985.

4. Baron MD, Parida S, Oura CA. Peste des petits ruminants: a suitable candidate for eradication? Vet Rec. 2011;169:16-21. http://dx.doi.org/10.1136/vr.d3947

5. World Organisation for Animal Health. Peste des petits ruminants [cited 2018 Oct 5]. http://www.oie.int/fileadmin/Home/eng/
Animal_Health_in_the_World/docs/pdf/Disease_cards/PESTE_ DES_PETITS_RUMINANTS.pdf

6. Nawathe DR, Taylor WP. Experimental infection of domestic pigs with the virus of peste des petits ruminants. Trop Anim Health Prod. 1979;11:120-2. http://dx.doi.org/10.1007/BF02237785

7. Hoffmann B, Wiesner H, Maltzan J, Mustefa R, Eschbaumer M, Arif FA, et al. Fatalities in wild goats in Kurdistan associated with peste des petits ruminants virus. Transbound Emerg Dis. 2012; 59:173-6. http://dx.doi.org/10.1111/j.1865-1682.2011.01270.x

8. Wernike K, Eschbaumer M, Breithaupt A, Maltzan J, Wiesner H, Beer M, et al. Experimental infection of sheep and goats with a recent isolate of peste des petits ruminants virus from Kurdistan. Vet Microbiol. 2014;172:140-5. http://dx.doi.org/10.1016/ j.vetmic.2014.05.010

9. Pope RA, Parida S, Bailey D, Brownlie J, Barrett T, Banyard AC. Early events following experimental infection with peste-des-petits ruminants virus suggest immune cell targeting. PLoS One. 2013;8:e55830. http://dx.doi.org/10.1371/ journal.pone.0055830

10. Deplazes P, Eckert J, Zahner H. Lehrbuch der Parasitologie für die Tiermedizin. 3rd ed. Stuttgart (Germany): EnkeVerlag; 2012.

11. Couacy-Hymann E, Bodjo C, Danho T, Libeau G, Diallo A. Evaluation of the virulence of some strains of peste-des-petitsruminants virus (PPRV) in experimentally infected West African dwarf goats. Vet J. 2007;173:178-83. http://dx.doi.org/10.1016/ j.tvjl.2005.08.020

12. Wernery U. Peste des petits ruminants (PPR) in camelids with own investigations. J Camel Pract Res. 2011;18:219-23.

13. Lin WH, Kouyos RD, Adams RJ, Grenfell BT, Griffin DE. Prolonged persistence of measles virus RNA is characteristic of primary infection dynamics. Proc Natl Acad Sci U S A. 2012;109: 14989-94. http://dx.doi.org/10.1073/pnas.1211138109

14. Ludlow M, Rennick LJ, Nambulli S, de Swart RL, Duprex WP. Using the ferret model to study morbillivirus entry, spread, transmission and cross-species infection. Curr Opin Virol. 2014;4:15-23. http://dx.doi.org/10.1016/j.coviro.2013.11.001

15. Palmer MV, Thacker TC, Waters WR, Gortazar C, Corner LA. Mycobacterium bovis: a model pathogen at the interface of livestock, wildlife, and humans. Vet Med Int. 2012;2012. http://dx.doi.org/10.1155/2012/236205

Address for correspondence: Claudia Schulz, University of Veterinary Medicine Hannover, Bünteweg 17, 30539 Hannover, Germany; email: claudia.schulz@tiho-hannover.de 


\section{Vaccinia Virus among Domestic Dogs and Wild Coatis, Brazil, 2013-2015}

\section{Galileu Barbosa Costa, Lara Ribeiro de Almeida, Aline Gabriele Ribeiro Cerqueira, Wander Ulisses Mesquita, Jaqueline Silva de Oliveira, Júlia Bahia Miranda, Ana Teresa Saraiva-Silva, Jônatas Santos Abrahão, Betânia Paiva Drumond, Erna Geessien Kroon, Pedro Lúcio Lithg Pereira, Danielle Ferreira de Magalhães Soares, Giliane de Souza Trindade}

To determine their potential role as a source of human infection, we tested domestic dogs (urban) and wild coatis (wild) in Brazil for vaccinia virus. Our findings of positive neutralizing antibodies and quantitative PCR results for $35 / 184$ dogs and $13 / 90$ coatis highlight a potential public health risk.

$\mathrm{S}$ ince smallpox was declared eradicated in 1980, after a massive effort led by the World Health Organization, other orthopoxviruses have gained notoriety as zoonotic agents worldwide (1). Over the past 17 years in Brazil, many zoonotic outbreaks of vaccinia virus (VACV) infection have been recorded throughout the country, becoming a burden for the dairy industry and public health (2). The most affected hosts during outbreaks are dairy cattle and humans (2). Recent studies assessing the role of wildlife in the maintenance cycle of VACV in nature have corroborated previous findings that rodents and marsupials serve as links between natural and anthropic environments (2-4).

Indeed, the increased frequency of reported VACV detection in several species of mammals points toward new insights into the circulation and maintenance of VACV in wild (forest) and rural (farm) environments (2,5-8). Studies conducted in Latin America suggest that wildlife, especially small and medium-sized mammals, plays a role in virus transmission and maintenance of orthopoxviruses in nature (9). Furthermore, some studies have shown the presence of VACV in urban environments, emphasizing the risks for humans (especially those not vaccinated against smallpox) $(10,11)$.

Author affiliations: Universidade Federal de Minas Gerais, Belo Horizonte, Brazil (G.B. Costa, L.R. de Almeida, A.G.R. Cerqueira, J.S. de Oliveira, J.B. Miranda, A.T. Saraiva-Silva, J.S. Abrahão, B.P. Drumond, E.G. Kroon, P.L.L. Pereira, D.F. de Magalhães Soares, G. de Souza Trindade); Universidade Federal de Ouro Preto, Ouro Preto, Brazil (W.U. Mesquita)

DOI: https://doi.org/10.3201/eid2412.171584
To determine the potential role of domestic and wild animals as a source of VACV infection for humans, we investigated VACV circulation among domestic dogs and wild coatis, animals that live at the intersection of urban and wild environments in Brazil. The capture of wild animals was authorized by the Brazilian Institute of Environment and Renewable Natural Resources, and the study was approved by the Ethics Committee in Animal Experimentation of Universidade Federal de Minas Gerais.

\section{The Study}

We analyzed serum and anal swab samples collected during 2013-2015 from 184 domestic dogs and 90 wild coatis in the city of Belo Horizonte $\left(19^{\circ} 55^{\prime} 15^{\prime \prime} \mathrm{S}, 43^{\circ} 56^{\prime} 16^{\prime \prime} \mathrm{W}\right)$ in the state of Minas Gerais, Brazil (Figure 1). Swab samples of lesions, if present, were also collected. To determine the presence of neutralizing antibodies in serum, we used an orthopoxvirus plaque reduction neutralization test as previously described (12). Serum titers were defined as the highest dilutions that inhibited $\geq 70 \%$ of virus plaques compared with negative controls.

To detect VACV DNA from serum and anal swab samples, we performed real-time PCR targeting the C11R or A56R gene (12). We directly sequenced A56R fragments in both orientations and in triplicate by using the ABI3130 platform (Applied Biosystems, Waltham, MA, USA). Sequences were aligned with other reference sequences from GenBank by using MEGA 7.0 (http://www.megasoftware. net). Statistical analyses were conducted by using Epi Info software version 7.2.1.0 (https://www.cdc.gov/epiinfo); $\chi^{2}$ and Fisher exact tests were applied with significance set at $5 \%$. We also calculated relative odds ratios (ORs) and $95 \%$ CIs.

We detected orthopoxvirus neutralizing antibodies in 35 dogs (prevalence rate 19.0\%, 95\% CI 14.0\%-25.5\%; titers 100-400 neutralizing units/mL) and in 13 coatis (prevalence rate $14.4 \%$, 95\% CI $8.5 \%-23.3 \%$; titers $100-800$ neutralizing units $/ \mathrm{mL}$ ) (Table 1). Univariate analyses indicated significant associations between presence of neutralizing antibodies and the following: male dogs (OR 2.6; p $=0.02)$, dogs $6-10$ years of age (OR 5.2; $\mathrm{p}=0.04)$, coatis captured in 2013 (OR 11.2; $\mathrm{p}=0.002)$, juvenile coatis $(<1$ $\mathrm{y}$ of age) (OR 35; $\mathrm{p}=0.001)$, and adult coatis ( $>2 \mathrm{y}$ of age) (OR 5.1; $\mathrm{p}=0.04)$.

Samples from all seropositive animals were submitted for quantitative PCR (qPCR) to detect VACV DNA (Table 


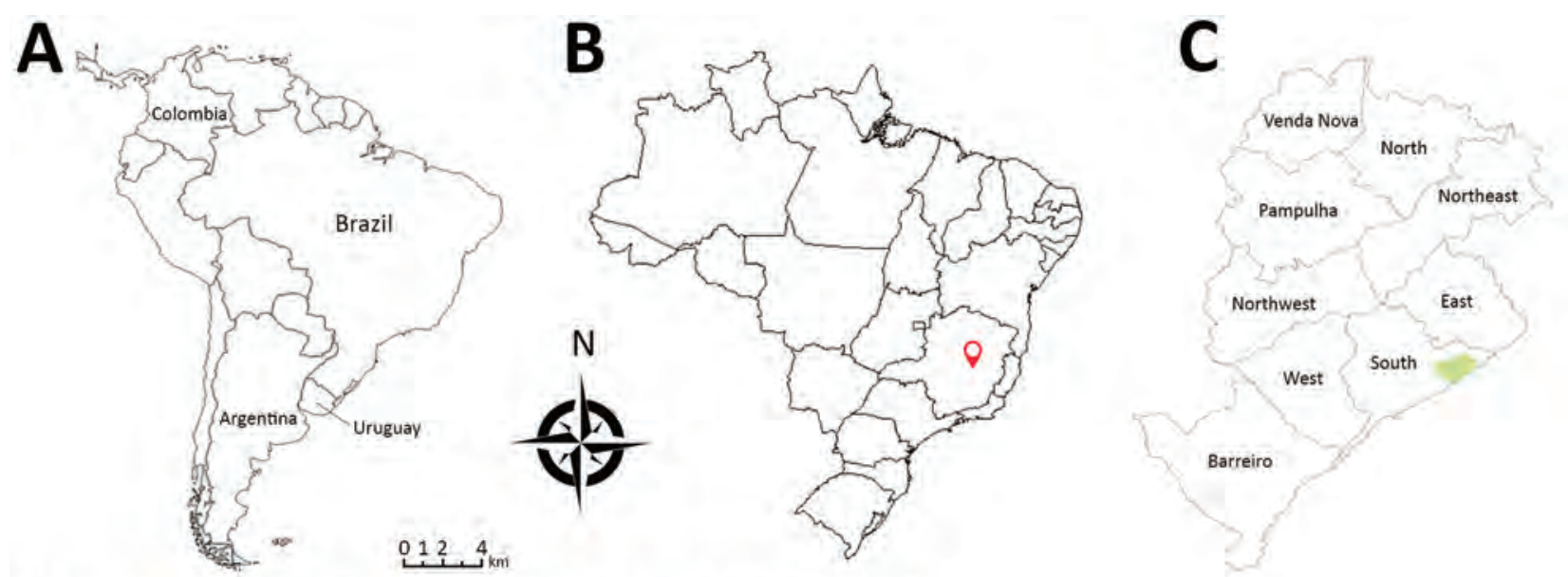

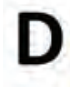

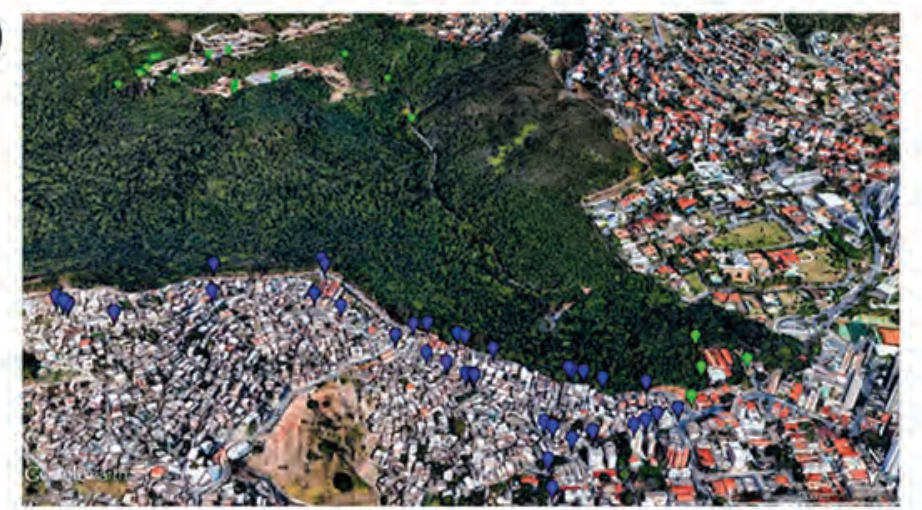

Figure 1. Area of study of vaccinia virus among domestic dogs and wild coatis, Brazil, 2013-2015. A) Countries in South America where vaccinia virus has been detected in recent years. B) Belo Horizonte (red locator), located in Minas Gerais state, Brazil. C) Regions of Belo Horizonte; green indicates area in wild environment where coatis were captured. D) Google Earth map from 2017 of studied area, showing details of the wild and urban environments. Green dots indicate where coatis were captured; blue dots indicate where dogs were sampled. Source: https://www.google.com/earth.
2). Overall, serum samples from 7 dogs and 6 coatis were positive for the C11R gene; of these, anal swab samples were positive for this gene for 3 dogs and 4 coatis. Samples from the C11R-positive animals were submitted for another qPCR targeting the A56R gene. Serum samples were positive for the A56R gene for 5 dogs and 4 coatis; of these, anal swab samples were positive for A56R for 1 dog and 3 coatis. No lesion swab samples were positive by qPCR for both C11R and A56R genes.

Alignment of the amplified A56R fragments showed high similarity to the homologous gene of VACV isolates from Brazil (online Technical Appendix Figure, https:// wwwnc.cdc.gov/EID/article/24/12/17-1584-Techapp1. pdf). Furthermore, 5 sequenced samples (from 4 dogs and 1 coatis) showed an 18-nt signature deletion, which is present in sequences of mouse nonvirulent VACV strains from Brazil (group $1 \mathrm{VACV}$ ). This deletion was not present in samples from 4 animals (1 dog and 3 coatis), grouping with mouse virulent VACV strains from Brazil (group 2 VACV).

\section{Conclusions}

We assessed VACV exposure of 2 interacting species of animal: domestic dogs from an urban area and coatis from a bordering wild area. In contrast to VACV infections, human cowpox virus infections have mostly occurred in urban areas of Europe. Cowpox virus is transmitted to humans mainly by domestic cats that are in contact with rodents, the natural cowpox virus reservoirs (13). However, some authors have hypothesized that domestic dogs could be implicated in the transmission cycle of VACV, acting as a link between the natural reservoirs and humans in urban environments $(14,15)$. Indeed, our molecular findings support exposure and possible VACV infection of these animals, thereby indicating that they are a potential source of VACV exposure for humans in urban areas.

The seroprevalence of orthopoxvirus neutralizing antibodies in dogs in Brazil has been described. Peres et al. found that, along with other farm animals, $22.8 \%$ of 114 dogs tested were seropositive for orthopoxviruses (7); this seroprevalence differs from that observed in our study, which was 3.8\% lower. In addition, most animals tested by Peres et al. were from rural areas where no bovine vaccinia outbreaks had been officially reported, and $96 \%$ of farmers declared that their domestic animals have contact with wild animals (9). These findings indicate that dogs could be exposed to VACV through contact with wild animals, corroborating our hypothesis.

We also detected orthopoxvirus neutralizing antibodies in wild coatis, which is consistent with results of a 
Table 1. Associations between neutralizing antibodies against Orthopoxvirus and demographic characteristics of domestic dogs and wild coatis, Belo Horizonte, Brazil, 2013-2015*

\begin{tabular}{|c|c|c|c|c|c|}
\hline Variable & No. (\%)† & No. (\%) positive $\neq$ & No. (\%) negative $\neq$ & $p$ value & Odds ratio $(95 \% \mathrm{Cl})$ \\
\hline \multicolumn{6}{|l|}{ Domestic dogs } \\
\hline \multicolumn{6}{|l|}{ Year of sampling } \\
\hline 2014 & $123(66.8)$ & $24(19.5)$ & 99 (80.5) & & \\
\hline 2015 & $61(33.2)$ & $11(18.0)$ & $50(82.0)$ & 1.00 & \\
\hline \multicolumn{6}{|l|}{ Sex } \\
\hline $\mathrm{F}$ & $85(46.4)$ & $23(27.1)$ & $62(72.9)$ & Reference & \\
\hline M & $96(52.5)$ & $12(12.5)$ & $84(87.5)$ & 0.02 & $2.6(1.2-5.6)$ \\
\hline \multicolumn{6}{|l|}{ Age, y } \\
\hline$\leq 1$ & $24(13.1)$ & $7(29.2)$ & $17(70.8)$ & Reference & \\
\hline$\overline{2}-5$ & $82(44.8)$ & $16(19.5)$ & $66(80.5)$ & 0.4 & \\
\hline $6-10$ & $41(22.4)$ & $3(7.3)$ & 38 (92.7) & 0.04 & $5.2(1.2-22.6)$ \\
\hline$>10$ & $18(9.8)$ & $4(22.2)$ & $14(87.8)$ & 0.9 & \\
\hline \multicolumn{6}{|l|}{ Size } \\
\hline Small & $75(41.0)$ & $13(18.8)$ & $56(81.2)$ & Reference & \\
\hline Medium & 69 (37.7) & $11(20.0)$ & $44(80.0)$ & 1.00 & \\
\hline Large & $30(16.4)$ & $4(14.3)$ & $24(85.7)$ & 0.8 & \\
\hline \multicolumn{6}{|l|}{ Confinement status } \\
\hline Always inside home & $41(22.4)$ & $8(19.5)$ & $33(80.5)$ & Reference & \\
\hline Always in backyard & $115(62.8)$ & $18(15.6)$ & 97 (84.3) & 0.7 & \\
\hline Home and backyard & $25(13.7)$ & $9(36.0)$ & $16(64.0)$ & 0.2 & \\
\hline \multicolumn{6}{|l|}{ Outdoors access $†$} \\
\hline Yes & $83(45.3)$ & $19(22.9)$ & $64(77.1)$ & 0.3 & \\
\hline No & $98(53.6)$ & $16(16.3)$ & $82(83.7)$ & Reference & \\
\hline \multicolumn{6}{|l|}{ Access to MMP } \\
\hline Yes & $18(9.8)$ & $6(33.3)$ & $12(66.7)$ & 0.2 & \\
\hline No & $101(55.2)$ & $18(17.8)$ & 83 (82.2) & Reference & \\
\hline \multicolumn{6}{|l|}{ Wild coatis } \\
\hline \multicolumn{6}{|l|}{ Year of capture } \\
\hline 2013 & $57(52.8)$ & $12(21.0)$ & $34(59.6)$ & 0.002 & $14.8(1.8-119.8)$ \\
\hline 2014 & $51(47.2)$ & $1(1.9)$ & $42(82.3)$ & Reference & \\
\hline \multicolumn{6}{|l|}{ Sex } \\
\hline $\mathrm{F}$ & $64(59.3)$ & $10(15.6)$ & $44(68.7)$ & Reference & \\
\hline M & $44(40.7)$ & $3(6.8)$ & 32 (72.7) & 0.3 & \\
\hline \multicolumn{6}{|l|}{ Age group } \\
\hline Juvenile, $\leq 1 \mathrm{y}$ & $44(40.7)$ & $1(2.3)$ & $35(79.5)$ & Reference & \\
\hline Subadult, $1-2$ y & $18(16.7)$ & $5(27.8)$ & $10(55.6)$ & 0.01 & $0.05(0.006-0.5)$ \\
\hline Adult, $>2$ y & $46(42.6)$ & $7(15.2)$ & $31(67.4)$ & 0.04 & $5.1(1.2-22.6)$ \\
\hline $\begin{array}{l}\text { *Totals may not add up to } 100 \\
\text { Mangabeiras Municipal Park. } \\
\text { †Includes access beyond bacl } \\
\text { †By plaque reduction neutraliz }\end{array}$ & sing informa & 3oldface indicates sig & icance; odds ratios a & nown only for & gnificant results. MMP, \\
\hline
\end{tabular}

previous study that described the seroprevalence of orthopoxviruses in procyonids from Mexico (9). Our detection of VACV DNA in anal swab samples from coatis indicate that these animals could act as a source of virus for domestic dogs and humans and serve as a link between wild and urban environments. However, future studies to determine if viable virus is shed are needed to confirm this possibility.

Table 2. Diagnostic results for 7 domestic dogs and 6 wild coatis with neutralizing antibodies for vaccinia virus, Belo Horizonte, Brazil, 2031-2015*

\begin{tabular}{|c|c|c|c|c|c|c|}
\hline \multirow[b]{2}{*}{ Animal } & \multirow[b]{2}{*}{$\mathrm{PRNT}_{70}$ titer $(\mathrm{NU} / \mathrm{mL})$} & \multicolumn{2}{|c|}{ qPCR C11R } & \multicolumn{2}{|c|}{ qPCR A56R } & \multirow[b]{2}{*}{ Strain } \\
\hline & & Serum sample & Anal swab sample & Serum sample & Anal swab sample & \\
\hline $\operatorname{Dog} 2$ & $1: 40(100)$ & + & - & + & - & Group 1 \\
\hline Dog 58 & $1: 80(200)$ & + & + & + & - & Group 1 \\
\hline Dog 41 & $1: 40(100)$ & + & - & - & - & \\
\hline Dog 77 & $1: 80(200)$ & + & + & + & - & Group 1 \\
\hline Dog 86 & $1: 40(100)$ & + & - & - & - & \\
\hline Dog 121 & $1: 160(400)$ & + & - & + & - & Group 1 \\
\hline Dog 128 & $1: 160(400)$ & + & + & + & + & Group 2 \\
\hline Coati 5 & $1: 40(100)$ & + & + & + & + & Group 2 \\
\hline Coatis 17 & $1: 40(100)$ & + & - & - & - & \\
\hline Coatis 27 & $1: 80$ (200) & + & - & - & - & \\
\hline Coatis 39 & $1: 160(400)$ & + & + & + & + & Group 2 \\
\hline Coatis 48 & $1: 40(100)$ & + & + & + & - & Group 1 \\
\hline Coatis 50 & 1:80 (200) & + & + & + & + & Group 2 \\
\hline
\end{tabular}

${ }^{*} \mathrm{NU}$, neutralizing units; $\mathrm{PRNT}_{70}, 70 \%$ plaque reduction neutralization test; $\mathrm{PPCR}$, quantitative PCR. 


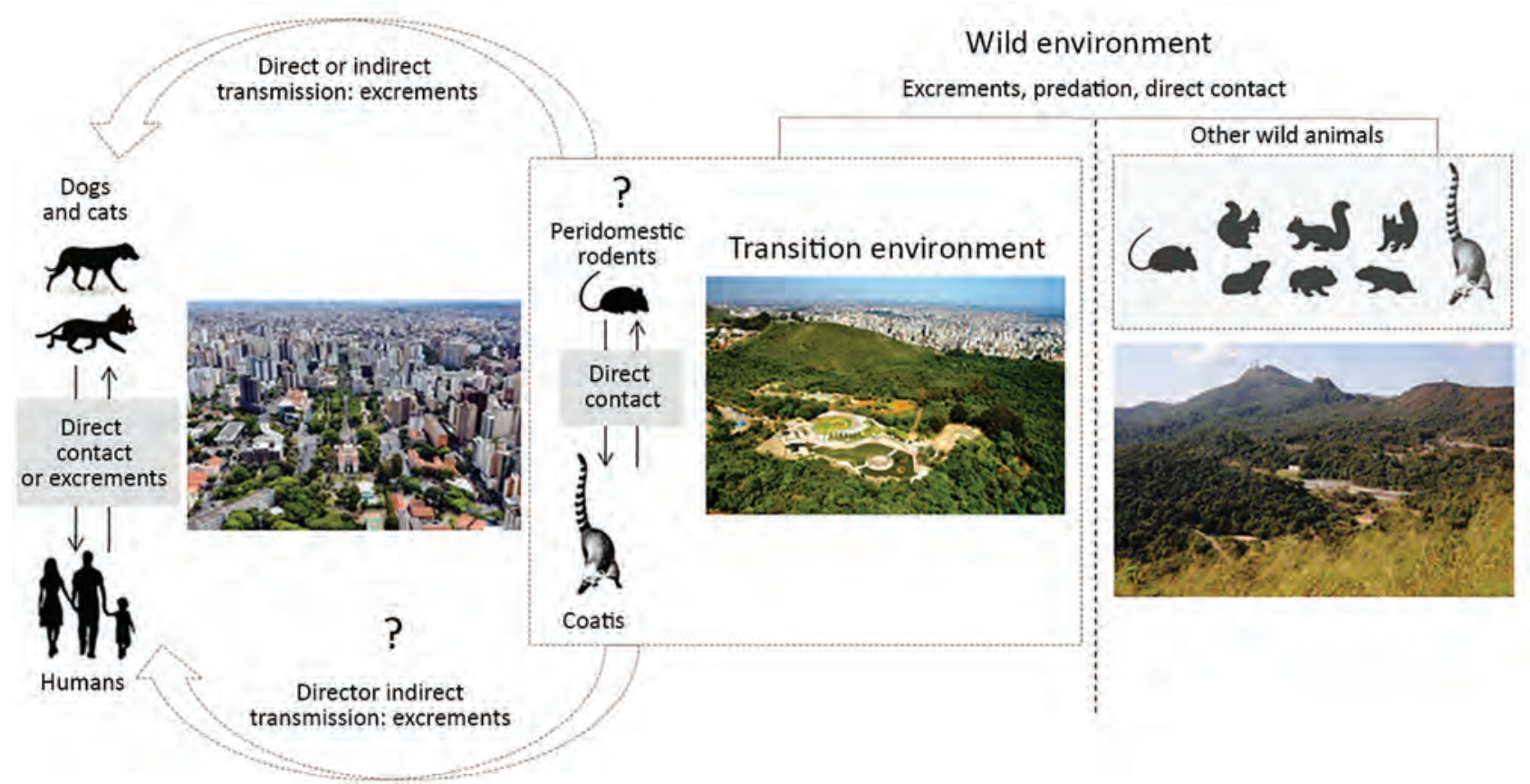

Figure 2. A hypothetical model developed to visualize the role of domestic animals and wildife in the natural cycle of vaccinia virus (VACV). The model illustrates the dynamics of VACV circulation in urban and wild areas of Brazil. In urban areas, wild coatis could promote the transmission of VACV between domestic animals or humans because they are in direct contact with domestic dogs and circulate among urban residences. Domestic dogs could also promote the transmission of VACV to humans because of direct contact or possibly indirect contact thought contaminated feces. In the wild environment, coatis can interact with other mammals such as wild rodents, which are believed to be VACV reservoirs, and acquire the infection (this potential interaction is still under investigation).

To impart information about the role of domestic animals and wildlife in the natural cycle of VACV, we developed a hypothetical model based on previous studies $(2,3,5)$, which could illustrate the dynamics of VACV circulation in urban areas (Figure 2). Because coatis can circulate in wild environments and surrounding urban areas, they could act as a bridge promoting the transmission of VACV between wild animals (mainly rodents) and dogs or humans. Domestic dogs could transmit VACV directly to humans through close contact or indirectly thought contaminated feces (Figure 2). These data raise questions about VACV circulation in Brazil and open discussions about the role of dogs and coatis in the VACV epidemiologic cycle.

\section{Acknowledgments}

We thank the Grupo de Epidemiologia e Conservação de Animais Silvestres and Residência Multiprofissional em Saúde, from Escola de Veterinária, Universidade Federal de Minas Gerais. We are thankful to the Gerência de Controle de Zoonoses team and Regional Centro Sul da Prefeitura Municipal de Belo Horizonte, members from Projeto Quatis, do Parque das Mangabeiras, and all the staff and partners for their support during sample collection. We also thank colleagues from the Laboratório de Vírus for their excellent technical support.
Financial support was provided by the Conselho Nacional de Desenvolvimento Científico e Tecnológico, Coordenação de Aperfeiçoamento de Pessoal de Nível Superior, Fundação de Amparo à Pesquisa do Estado de Minas Gerais, and Pró-Reitoria de Pesquisa/ Universidade Federal de Minas Gerais. J.S.A., E.G.K., and G.S.T. are researchers from Conselho Nacional de Desenvolvimento Científico e Tecnológico.

\section{About the Author}

Mr. Costa is a PhD candidate in microbiology at the Laboratório de Vírus, Microbiology Department, Instituto de Ciências Biológicas, Universidade Federal de Minas Gerais. His research is focused on the diagnosis, epidemiology, control, and prevention of infection with orthopoxviruses and other emerging viruses.

\section{References}

1. Shchelkunov SN. An increasing danger of zoonotic orthopoxvirus infections. PLoS Pathog. 2013;9:e1003756. http://dx.doi.org/10.1371/journal.ppat.1003756

2. Oliveira JS, Figueiredo PO, Costa GB, Assis FL, Drumond BP, da Fonseca FG, et al. Vaccinia virus natural infections in Brazil: the good, the bad, and the ugly. Viruses. 2017;9:E340. http://dx.doi.org/10.3390/v9110340 
3. Miranda JB, Borges IA, Campos SPS, Vieira FN, de Ázara TMF, Marques FA, et al. Serologic and molecular evidence of vaccinia virus circulation among small mammals from different biomes, Brazil. Emerg Infect Dis. 2017;23:931-8. http://dx.doi.org/10.3201/ eid2306.161643

4. Peres MG, Bacchiega TS, Appolinário CM, Vicente AF, Mioni MSR, Ribeiro BLD, et al. Vaccinia virus in feces and urine of wild rodents from São Paulo state, Brazil. Viruses. 2018;10:E51. http://dx.doi.org/10.3390/v10020051

5. Abrahão JS, Guedes MIM, Trindade GS, Fonseca FG, Campos RK, Mota BF, et al. One more piece in the VACV ecological puzzle: could peridomestic rodents be the link between wildlife and bovine vaccinia outbreaks in Brazil? PLoS One. 2009;4:e7428. http://dx.doi.org/10.1371/journal.pone.0007428

6. Abrahão JS, Silva-Fernandes AT, Lima LS, Campos RK, Guedes MI, Cota MM, et al. Vaccinia virus infection in monkeys, Brazilian Amazon. Emerg Infect Dis. 2010;16:976-9. http://dx.doi.org/10.3201/eid1606.091187

7. Peres MG, Bacchiega TS, Appolinário CM, Vicente AF, Allendorf SD, Antunes JM, et al. Serological study of vaccinia virus reservoirs in areas with and without official reports of outbreaks in cattle and humans in São Paulo, Brazil. Arch Virol. 2013;158:2433-41. http://dx.doi.org/10.1007/ s00705-013-1740-5

8. Peres MG, Barros CB, Appolinário CM, Antunes JM, Mioni MS, Bacchiega TS, et al. Dogs and opossums positive for vaccinia virus during outbreak affecting cattle and humans, São Paulo state, Brazil. Emerg Infect Dis. 2016;22:271-3. http://dx.doi.org/ 10.3201/eid2202.140747

9. Gallardo-Romero NF, Aréchiga-Ceballos N, Emerson GL, Martínez-Martínez FO, Doty JB, Nakazawa YJ, et al. Endemic orthopoxvirus circulating in procyonids in Mexico. J Wildl Dis. 2016;52:609-15. http://dx.doi.org/10.7589/ 2015-10-291

10. Dutra LA, de Freitas Almeida GM, Oliveira GP, Abrahão JS, Kroon EG, Trindade GS. Molecular evidence of orthopoxvirus DNA in capybara (Hydrochoerus hydrochaeris) stool samples. Arch Virol. 2017;162:439-48. http://dx.doi.org/10.1007/ s00705-016-3121-3

11. Costa GB, Miranda JB, Almeida GG, Silva de Oliveira J, Pinheiro MS, Gonçalves SA, et al. Detection of vaccinia virus in urban domestic cats, Brazil. Emerg Infect Dis. 2017;23:360-2. http://dx.doi.org/10.3201/eid2302.161341

12. Geessien Kroon E, Santos Abrahão J, de Souza Trindade GS, Pereira Oliveira G, Moreira Franco-Luiz AP, Barbosa Costa G, et al. Natural vaccinia virus infection: diagnosis, isolation, and characterization. Curr Protoc Microbiol. 2016;42:14A.5. 1-14A.5.43. http://dx.doi.org/10.1002/cpmc.13

13. Essbauer S, Pfeffer M, Meyer H. Zoonotic poxviruses. Vet Microbiol. 2010;140:229-36. http://dx.doi.org/10.1016/ j.vetmic.2009.08.026

14. Smith KC, Bennett M, Garrett DC. Skin lesions caused by orthopoxvirus infection in a dog. J Small Anim Pract. 1999; 40:495-7. http://dx.doi.org/10.1111/j.1748-5827.1999. tb03003.x

15. von Bomhard W, Mauldin EA, Breuer W, Pfleghaar S, Nitsche A. Localized cowpox infection in a 5-month-old Rottweiler. Vet Dermatol. 2011;22:111-4. http://dx.doi.org/ 10.1111/j.1365-3164.2010.00923.x

Address for correspondence: Galileu Barbosa Costa or Giliane de Souza Trinidade, Universidade Federal de Minas Gerais, Microbiologia, Avenida Presidente Antônio Carlos 6627, Campus Pampulha, Belo Horizonte, Minas Gerais, Brazil 31270-90; email: galileuk1@gmail.com or giliane@icb.ufmg.br

\section{Spotlight: HIV-AI DS}

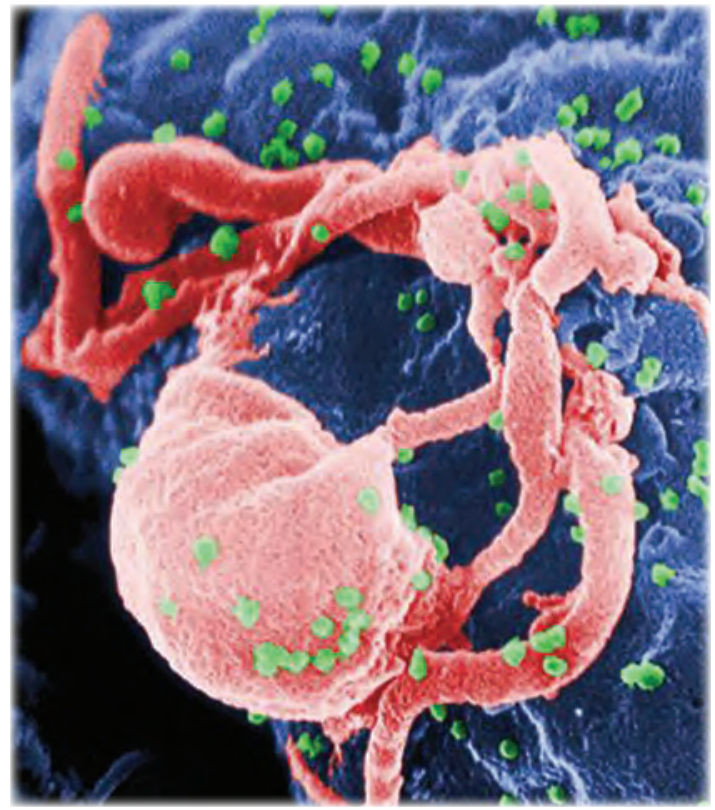

World AIDS Day is held on December 1 of each year and is an opportunity for people worldwide to unite in the fight against HIV, show their support for people living with HIV, and commemorate people who have died. World AIDS Day was the first ever global health day, held for the first time in 1988.

https://wwwnc.cdc.gov/eid/spotlight/hiv EMERGING INFECTIOUS DISEASES 


\section{Highly Pathogenic Avian Influenza A(H5N6) in Domestic Cats, South Korea}

\author{
KyungHyun Lee, ${ }^{1}$ Eun-Kyoung Lee, ${ }^{1}$ \\ HyunKyoung Lee, Gyeong-Beom Heo, Yu-Na Lee, \\ Ji-Youl Jung, You-chan Bae, ByungJae So, \\ Youn-Jeong Lee, Eun-Jin Choi
}

In December 2016, highly pathogenic avian influenza (HPAl) infection with systemic pathologic lesions was found in cats in South Korea. Genetic analyses indicated that the feline isolates were similar to HPAI H5N6 viruses isolated in chicken farms nearby. This finding highlights the need for monitoring of domestic mammals during HPAl outbreaks.

$\mathrm{H}$ ighly pathogenic avian influenza (HPAI) H5N6 has spread across Asia, Europe, and Africa. Since a novel influenza A(H5N6) virus emerged in China in late 2013 (1), H5N6 viruses have been subsequently reported in Southeast Asia. In China, HPAI A(H5N6) virus caused the earliest reported human infection in 2014 and became one of the dominant subtypes in poultry farms and live poultry markets (2). These viruses caused a potential threat to other mammals, including pigs and cats $(3,4)$. We report H5N6 virus infection in cats during 2016-17 HPAI outbreaks in domestic poultry in South Korea (5).

\section{The Study}

The 2016-17 winter season saw epidemics of HPAI A(H5N6) in domestic poultry and wild birds in South Korea (5). At the end of December 2016, three carcasses of cats were submitted from areas near H5N6 virus-infected chicken farms in Pocheon. The cats had shown sudden clinical signs of salivation, lethargy, convulsion, and bloody discharge around the mouth and jaws and died within 4 days after illness onset despite antimicrobial drug treatment. After necropsy, we processed representative tissues for histopathology and immunohistochemistry (online Technical Appendix, https://wwwnc.cdc.gov/ EID/article/24/12/18-0290-Techapp1.pdf). The necropsy findings included bloody nasal discharge (Figure 1, panel A), severe pulmonary congestion and edema, and whitecolored foci in the liver (Figure 1, panel B). The lungs were red and yellow in color and incompletely collapsed and had accumulated a small amount of frothy fluid. The spleen was

Author affiliation: Animal and Plant Quarantine Agency, Gimcheon, South Korea

DOI: https://doi.org/10.3201/eid2412.180290 enlarged 2-fold. The pancreas showed spotty hemorrhage and white pinpoint foci.

Histopathologic examination revealed severe lesions in brain, lungs, and liver in the examined cats. We observed necrosis and loss of neurons, lymphocytic perivascular cuffing, and gliosis (Figure 1, panel C) in the cerebellum and cerebrum, and especially severe necrosis in the hippocampus. The lungs showed marked congestion, edema, hemorrhage, and severe interstitial pneumonia (Figure 1, panel D), and thrombus in the alveolar capillaries. The liver showed severe necrotic foci and hepatitis. We observed influenza viral antigen in neurons (Figure 1, panel E), glial cells, and alveolar macrophages (Figure 1, panel F). Table 1 describes histopathologic lesions and Table 2 immunohistochemical reactivity.

We recognized H5N6 virus infection in a domestic male cat (cat 1) and juvenile outdoor cats (cats 2 and 3). We observed necrotic lesions and influenza viral antigens in multiple visceral organs, suggesting that the virus caused systemic infection. It seems likely that the neurotropism of H5N6 virus was a key factor contributing to the sudden death of these cats. The results of this study are consistent with those of other studies of HPAI pathogenicity in experimentally infected dogs $(6,7)$.

The histopathologic findings and the localization of H5N6 virus antigen to the lungs and liver, but not to the brain, in cats have been reported (8). In this case, we observed meningoencephalitis. Moreover, the 3 cats showed neurologic symptoms such as salivation and convulsion, which may be related to necrosis and loss of neurons. The severity of the lesions was consistent with the number of cells that reacted with influenza viral antigen. A few studies reported that $\mathrm{H} 9$ and $\mathrm{H} 10$ influenza viruses were nephrotropic in chickens with low pathogenicity $(9,10)$ and that HPAI H5 virus causes acute renal lesions in mammals and primates, including humans (11). The results of our study suggest that the HPAI H5N6 virus affects cats differently than do other HPAI viruses; therefore, further studies are needed to experimentally infect cats with other HPAI H5 subtypes, including the isolate from this study, for complete clarification.

Previous studies have shown that avian viruses preferentially recognize $\alpha-2,3$ linkage (SA $\alpha 2,3 \mathrm{Gal}$ ) and bind to type II alveolar cells, which are abundant in the lower

${ }^{1}$ These authors contributed equally to this article. 


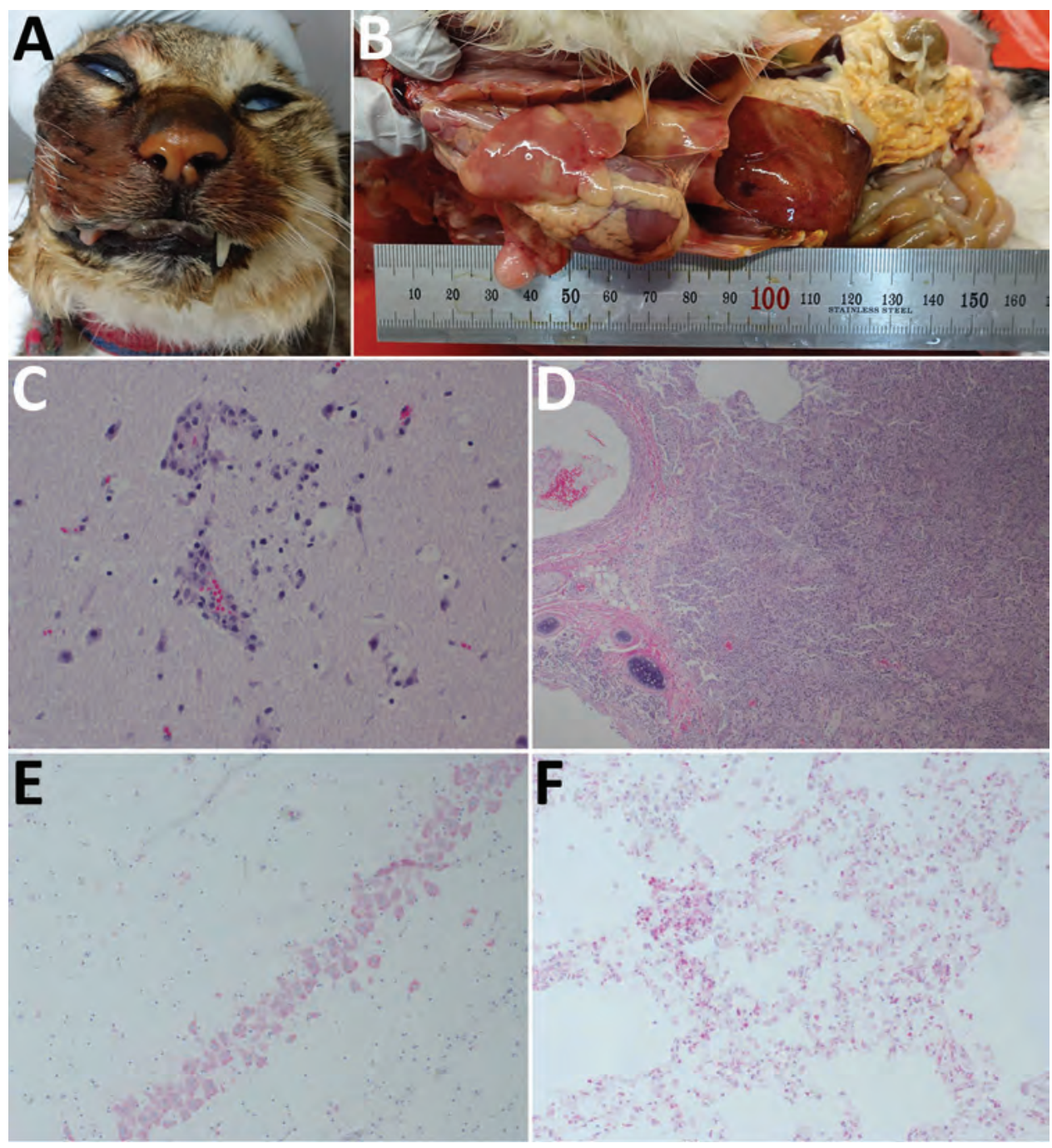

Figure 1. Gross, microscopic, and immunohistochemical (IHC) findings in 3 cats with highly pathogenic avian influenza $A(H 5 N 6)$ virus infection, South Korea. A) Bloody nasal discharge. B) Severe congestion and edema in the lungs and white-colored foci in the liver. C) Gliosis in the brain.

Hematoxylin and eosin stain; original magnification $\times 100$. D) Interstitial pneumonia with degenerated pneumocytes. Hematoxylin and eosin; original magnification $\times 40$. E) Influenza viral antigens in neurons. IHC testing; original magnification $\times 100$. F) Influenza viral antigens in alveolar macrophages. IHC testing; original magnification $\times 100$.

respiratory tract of mammals $(12,13)$. These findings are consistent with our observations of severe pneumonia with lung edema in the infected cats.
RNA samples extracted from organs of the cats were positive for influenza $\mathrm{H} 5$ and $\mathrm{N} 6$ subtypes by reverse transcription PCR. We selected 2 HPAI H5N6 viruses:

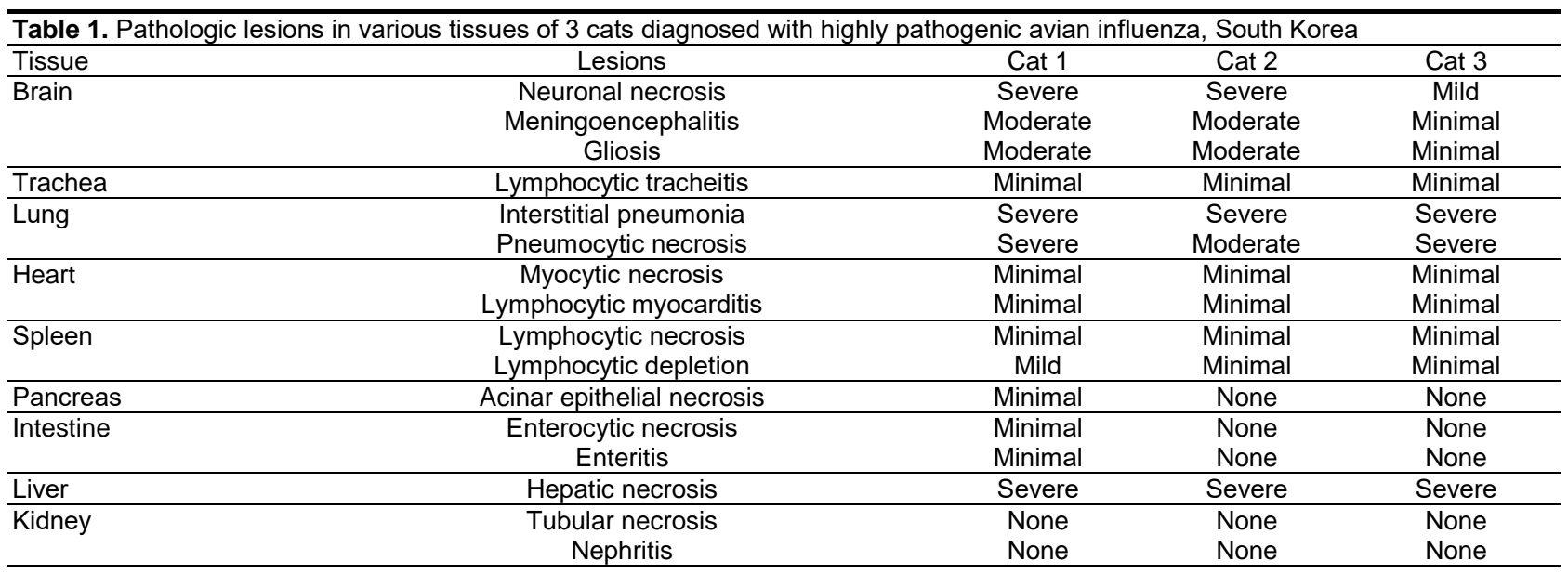


Table 2. Reactivity to influenza viral nucleoprotein in various tissues of 3 cats with highly pathogenic avian influenza, South Korea

\begin{tabular}{|c|c|c|c|c|}
\hline \multirow[b]{2}{*}{ Tissue } & \multirow[b]{2}{*}{ Cells } & \multicolumn{3}{|c|}{ Reactivity } \\
\hline & & Cat 1 & Cat 2 & Cat 3 \\
\hline \multirow[t]{3}{*}{ Brain } & Neurons & Numerous & Numerous & Moderate \\
\hline & Glial cells & Moderate & Numerous & Minimal \\
\hline & Ependymal cells & Numerous & Numerous & Minimal \\
\hline Trachea & Epithelial cells & Minimal & Minimal & Minimal \\
\hline \multirow[t]{2}{*}{ Lung } & Macrophages & Numerous & Numerous & Numerous \\
\hline & Vascular endothelial cells & Numerous & Numerous & Numerous \\
\hline \multirow[t]{2}{*}{ Heart } & Myocytes & Minimal & Minimal & Minimal \\
\hline & Epicardial cells & Minimal & Minimal & Minimal \\
\hline \multirow[t]{2}{*}{ Spleen } & Ellipsoid capillary endothelium & Minimal & Minimal & Minimal \\
\hline & Macrophages and necrotic debris & Moderate & Minimal & Minimal \\
\hline Pancreas & Acinar epithelium & Minimal & None & None \\
\hline \multirow[t]{2}{*}{ Intestine } & Crypt epithelium & Minimal & None & None \\
\hline & Mesenteric ganglial neurons & Minimal & None & None \\
\hline Liver & Kupffer cells and necrotic debris & Numerous & Numerous & Numerous \\
\hline \multirow[t]{2}{*}{ Kidney } & Tubule epithelium & Minimal & Minimal & Unknown \\
\hline & Glomeruli & Minimal & None & None \\
\hline
\end{tabular}
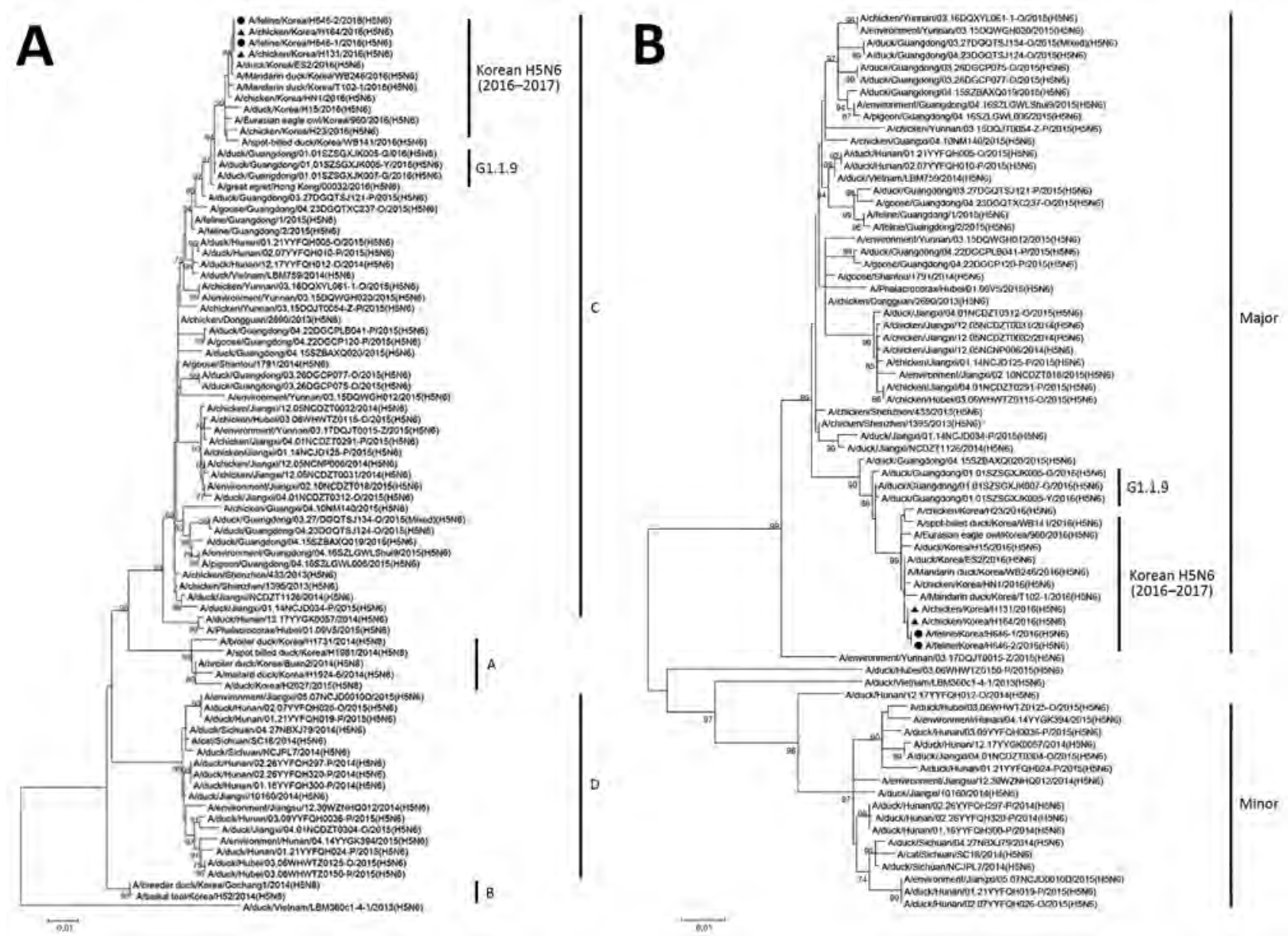

Figure 2. Maximum-likelihood phylogenetic tree of the hemagglutinin (A) and neuraminidase (B) gene segments for highly pathogenic avian influenza $A(\mathrm{H} 5 \mathrm{~N} 6)$ viruses from cats, South Korea, and comparison viruses. Black circles indicate isolates from cats and triangles indicate chicken isolates from this study. Virus sequences from the GISAID EpiFlu database (http://platform.gisaid.org) and GenBank were used for each phylogenetic comparison. The genetic subclades are annotated to the right of the tree. The genetic clusters major, minor, and G1.1.9, were designated according to the criteria of Bi et al. (2). The number at each branch indicates a bootstrap value. Scale bars indicate nucleotide substitutions per site. 
A/feline/Korea/H646-1/2016(H5N6) from the domestic male cat and A/feline/Korea/H646-2/2016(H5N6) from 1 juvenile outdoor cat. We performed virus isolation, sequencing, and phylogenetic analysis as described (online Technical Appendix). Phylogenetic analyses showed that the $\mathrm{H} 5$ genes of the cat isolates belonged to clade 2.3.4.4.C and were very closely related to $\mathrm{H} 5 \mathrm{~N} 6$ viruses detected in poultry in areas within a radius of $1 \mathrm{~km}$ of the cats' location (Figure 2, panel A). In a previous study, clade 2.3.4.4.C H5N6 viruses from the 2016-17 epidemic in South Korea were divided into 5 distinct genogroups (C-1 to C-5) (5). The feline isolates showed high similarity with H5N6 viruses in genogroup C-4, which was detected in domestic poultry nearby during 2016-17 HPAI A(H5N6) outbreaks (Figure 2; online Technical Appendix Figure).

Epidemiologic studies show that the cats might be infected by feeding on or by contact with infected wild birds, although the virus was not isolated from wild birds around this area. The affected domestic cat lived in a house near a small stream where migratory birds were observed and a wide main road. Across the main road, H5N6 virus-affected chicken farms were located within $1 \mathrm{~km}$. In previous reports, cats and tigers were naturally infected by feeding on infected bird carcasses $(8,14)$. In China, H5N6 virus infection in cats has been reported in regions such as Suchuan and Guangdong Provinces (3).

We compared each gene of the feline and chicken H5N6 isolates (online Technical Appendix Table). The hemagglutinin (HA) genes of the viruses contained multiple basic amino acid residues at the HA cleavage site (PLRERRRKR). The amino acid residues on the receptor binding sites of the HA gene of H5N6 viruses were Q226 and G228 (H3 numbering), indicating an avianlike $(\alpha 2,3-\mathrm{SA})$ receptor-binding preference. T160A mutation in the HA gene suggested a possible increased viral affinity for human-like ( $\alpha 2,6-\mathrm{SA})$ receptor binding, shown in feline isolates. The neuraminidase genes of feline isolates also had 11 aa deletions at positions 59-69, which were often observed in avian influenza virus lineages adapted to poultry and may increase the virulence to mammals (2). We did not observe amino acid substitution at position E627K of the polybasic 2 gene in the feline isolates.

\section{Conclusions}

Our results demonstrate that cats can be directly infected by HPAI H5N6 virus. Cats are companion animals and may act as a vector for influenza transmission to humans. Despite the low probability of H5N6 virus infection in companion animals, avian influenza surveillance will be needed for mammals, including cats, during H5N6 outbreaks.

\section{Acknowledgments}

We thank the Animal and Plant Quarantine Agency (APQA);

Ministry of Agriculture, Food and Rural Affairs (MAFRA); and regional office for animal disease control for their efforts to control HPAI.

This study was supported by a grant from APQA, MAFRA, Republic of Korea (project code nos. N-1543069-2015-99-01 and B-1543418-2018-19-01).

\section{About the Author}

Dr. KyungHyun Lee is a veterinary researcher at the Animal and Plant Quarantine Agency, South Korea. Her research interests include pathology and emerging infectious diseases in animals. Dr. Eun-Kyoung Lee is a veterinary researcher at the Animal and Plant Quarantine Agency. Her research interests include the molecular epidemiology and surveillance of avian influenza.

\section{References}

1. Qi X, Cui L, Yu H, Ge Y, Tang F. Whole-genome sequence of a reassortant $\mathrm{H} 5 \mathrm{~N} 6$ avian influenza virus isolated from a live poultry market in China, 2013. Genome Announc. 2014;2:e00706-14. http://dx.doi.org/10.1128/genomeA.00706-14

2. Bi Y, Chen Q, Wang Q, Chen J, Jin T, Wong G, et al. Genesis, evolution and prevalence of H5N6 avian influenza viruses in China. Cell Host Microbe. 2016;20:810-21. 10.1016/j.chom.2016.10.022 http://dx.doi.org/10.1016/j.chom.2016.10.022

3. Yu Z, Gao X, Wang T, Li Y, Li Y, Xu Y, et al. Fatal H5N6 avian influenza virus infection in a domestic cat and wild birds in China. Sci Rep. 2015;5:10704. http://dx.doi.org/10.1038/srep10704

4. Li X, Fu Y, Yang J, Guo J, He J, Guo J, et al. Genetic and biological characterization of two novel reassortant H5N6 swine influenza viruses in mice and chickens. Infect Genet Evol. 2015;36:462-6. http://dx.doi.org/10.1016/j.meegid.2015.08.017

5. Lee EK, Song BM, Lee YN, Heo GB, Bae YC, Joh SJ, et al. Multiple novel H5N6 highly pathogenic avian influenza viruses, South Korea, 2016. Infect Genet Evol. 2017;51:21-3. http://dx.doi.org/10.1016/j.meegid.2017.03.005

6. Son K, Kim YK, Oem JK, Jheong WH, Sleeman JM, Jeong J. Experimental infection of highly pathogenic avian influenza viruses, clade 2.3.4.4 H5N6 and H5N8, in mandarin ducks from South Korea. Transbound Emerg Dis. 2017;65:899-903.

7. Lyoo KS, Na W, Phan LV, Yoon SW, Yeom M, Song D, et al. Experimental infection of clade 1.1.2 (H5N1), clade 2.3.2.1c (H5N1) and clade 2.3.4.4 (H5N6) highly pathogenic avian influenza viruses in dogs. Transbound Emerg Dis. 2017;64:166975. http://dx.doi.org/10.1111/tbed.12731

8. Songserm T, Amonsin A, Jam-on R, Sae-Heng N, Meemak N, Pariyothorn N, et al. Avian influenza H5N1 in naturally infected domestic cat. Emerg Infect Dis. 2006;12:681-3. http://dx.doi.org/ 10.3201/eid1204.051396

9. Swayne DE, Alexander DJ. Confirmation of nephrotropism and nephropathogenicity of three low-pathogenic chicken-origin influenza viruses for chickens. Avian Pathol. 1994;23:345-52. http://dx.doi.org/10.1080/03079459408419002

10. Bonfante F, Fusaro A, Zanardello C, Patrono LV, De Nardi R, Maniero S, et al. Lethal nephrotropism of an H10N1 avian influenza virus stands out as an atypical pathotype. Vet Microbiol. 2014;173:189-200. http://dx.doi.org/10.1016/j.vetmic.2014.07.023

11. Cardona CJ, Xing Z, Sandrock CE, Davis CE. Avian influenza in birds and mammals. Comp Immunol Microbiol 
Infect Dis. 2009;32:255-73. http://dx.doi.org/10.1016/ j.cimid.2008.01.001

12. van Riel D, Munster VJ, de Wit E, Rimmelzwaan GF, Fouchier RA, Osterhaus AD, et al. H5N1 virus attachment to lower respiratory tract. Science. 2006;312:399. http://dx.doi.org/10.1126/ science. 1125548

13. Shinya K, Ebina M, Yamada S, Ono M, Kasai N, Kawaoka Y. Influenza virus receptors in the human airway. Nature. 2006;440:435-6. http://dx.doi.org/10.1038/440435a
14. Keawcharoen J, Oraveerakul K, Kuiken T, Fouchier RA, Amonsin A, Payungporn S, et al. Avian influenza H5N1 in tigers and leopards. Emerg Infect Dis. 2004;10:2189-91. http://dx.doi.org/10.3201/eid1012.040759

Address for correspondence: Youn-Jeong Lee and Eun-Jin Choi, Animal and Plant Quarantine Agency, hyuksin8ro 177 Gimcheon, Gimcheon 39660, South Korea; email: leeyj700@korea.kr; choiej@korea.kr

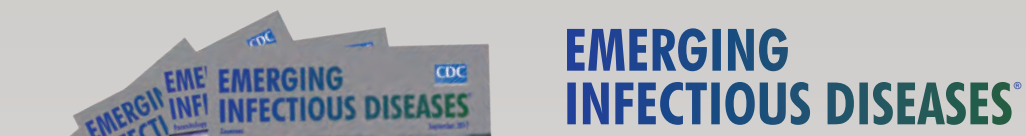
INFECTIOUS DISEASES
- Bioinformatic Analyses of Whole-Genome Sequence Data in a Public Health Laboratory

- Convergence of Humans, Bats, Trees, and Culture in Nipah Virus Transmission, Bangladesh

- Processes Underlying Rabies Virus Incursions across US-Canada Border as Revealed by Whole-Genome Phylogeography

- Real-Time Whole-Genome Sequencing for Surveillance of Listeria monocytogenes, France

- Role of Food Insecurity in Outbreak of Anthrax Infections among Humans and Hippopotamuses Living in a Game Reserve Area, Rural Zambia

- Molecular Antimicrobial Resistance Surveillance for Neisseria gonorrhoeae, Northern Territory, Australia

- Estimated Annual Numbers of Foodborne PathogenAssociated IIInesses, Hospitalizations, and Deaths, France, 2008-2013

- Epidemiology of Salmonella enterica Serotype Dublin Infections among Humans, United States,1968-2013

- Prevalence of Yersinia enterocolitica Bioserotype 3/0:3 among Children with Diarrhea, China, 2010-2015

- Risk for Low Pathogenicity Avian Influenza Virus on Poultry Farms, the Netherlands, 2007-2013

- Patterns of Human Plague in Uganda, 2008-2016

- Serologic Evidence for Influenza C and D Virus among Ruminants and Camelids, Africa, 1991-2015

- Norovirus in Bottled Water Associated with Gastroenteritis Outbreak, Spain, 2016

\section{Zoonoses}

\section{September 2017}

- Group A Rotavirus Associated with Encephalitis in Red Fox

- Protective Effect of $\mathrm{Val}_{129}$-PrP against Bovine Spongiform Encephalopathy but not Variant Creutzfeldt-Jakob Disease

- Imported Infections with Mansonella perstans Nematodes, Italy

- Genetic Diversity of Highly Pathogenic Avian Influenza $A(H 5 N 8 / H 5 N 5)$ Viruses in Italy, 2016-17

- Microcephaly Caused by Lymphocytic Choriomeningitis Virus

- Influenza A(H3N2) Virus in Swine at Agricultural Fairs and Transmission to Humans, Michigan and Ohio, USA, 2016

- Use of Blood Donor Screening to Monitor Prevalence of HIV and Hepatitis B and C Viruses, South Africa

- Emergence of Plasmid-Mediated Fosfomycin-Resistance Genes among Escherichia coli Isolates, France

- Determination of the Ferret Enteric Coronavirus Genome in Laboratory Ferrets

- Myocarditis Caused by Human Parechovirus in Adult

- Cost of Nosocomial Outbreak Caused by NDM-1-Containing Klebsiella pneumoniae in the Netherlands, 2015-2016

- Evaluation of 5 Commercially Available Zika Virus Immunoassays

- Epidemiology of Neisseria gonorrhoeae Gyrase A Genotype, Los Angeles, California, USA

- Conveyance Contact Investigation for Imported Middle East Respiratory Syndrome Cases, United States, May 2014

- Possible Role of Fish as Transport Hosts for Dracunculus spp. Larvae 


\section{Candidatus Cryptoplasma Associated with Green Lizards and I xodes ricinus Ticks, Slovakia, 2004-2011}

\author{
Božena Kočíková, Igor Majláth, \\ Bronislava Víchová, Lenka Maliničová, \\ Peter Pristaš, Vincent A. Connors, \\ Viktória Majláthová
}

During 2004-2011, we collected green lizards and Ixodes ricinus ticks in Slovak Karst National Park in Slovakia; $90 \%(36 / 40)$ of lizards and $37 \%$ of ticks removed from lizards were infected with family Anaplasmataceae bacteria. Only Candidatus Cryptoplasma sp. REP (reptile) was identified in these samples. Green lizards transmit this bacterium.

$\mathrm{T}$ he family Anaplasmataceae (Rickettsiales; Alphaproteobacteria) comprises bacteria that are able to invade and infect their vertebrate host's blood cells, bone marrow-derived phagocytic cells, and endothelial cells; these bacteria can also infect cells of insects, helminths, and arthropod reproductive tissues (1-3). Tickborne family members include bacteria of Anaplasma, Ehrlichia, Candidatus Neoehrlichia sp., and Candidatus Cryptoplasma californiense (4).

Although reptiles play a role as hosts for ixodid and argasid ticks, their role in maintaining tickborne Anaplasmataceae bacteria in the environment has not been described. Nieto et al. (5) suggested that lizards and snakes in the far western part of the United States could become exposed to Anaplasma phagocytophilum when fed on by infected ticks. Moreover, Rejmanek et al. detected 2 highly dissimilar strains of $A$. phagocytophilum in the same lizard species (6). In Europe, an undescribed Anaplasma sp. was detected in Ixodes ricinus ticks feeding on sand lizards and sand lizard blood samples $(7,8)$. In our study, we sought to confirm these previous findings by determining whether family Anaplasmataceae bacteria were present in lizards and their feeding ticks in Slovakia.

Author affiliations: Slovak Academy of Sciences, Košice, Slovakia (B. Kočíková, B. Víchová, P. Pristaš, V. Majláthová); Pavol Jozef Šafárik University in Košice, Košice (I. Majláth, L. Maliničová, P. Pristaš, V. Majláthová); University of South Carolina Upstate, Spartanburg, South Carolina, USA (V.A. Connors)

DOI: https://doi.org/10.3201/eid2412.161958

\section{The Study}

We conducted this study in the Slovak Karst National Park in Slovakia $\left(48^{\circ} 36^{\prime} \mathrm{N}, 20^{\circ} 52^{\prime} \mathrm{E}\right)$ during 2004-2011. We carried out lizard capture and sample collections with official permits (6103/2007-2.1 and 5498/2011-2.2) issued by the Ministry of Environment of the Slovak Republic. We captured 103 green lizards (Lacerta viridis) and collected blood from 40 (30 males and 10 females). We collected 235 I. ricinus ticks (118 larvae and 117 nymphs) from 63 green lizards and 271 questing I. ricinus ticks (132 nymphs, 76 males, and 63 females) from the same area and immediately stored them in $70 \%$ ethanol.

We isolated DNA from lizard blood using a DNeasy Blood \& Tissue Kit (QIAGEN, Hilden Germany) and isolated DNA from ticks by alkaline hydrolysis. We performed PCR amplification in $25-\mu \mathrm{L}$ (total) reaction mixtures using the MasterTaq DNA Polymerase Kit (Eppendorf AG, Hamburg, Germany). We amplified sequences using the primer combinations EHR747 plus EHR521 or fD1 plus rP2 (9), which spanned almost the entire 16S rRNA sequence (Table 1 ). We examined the $\approx 250$-bp gene fragment of 16S rRNA by single-strand conformation polymorphism (SSCP) analysis to determine Anaplasmataceae species type (10). We performed SSCP analysis following the protocol of Derdakova et al. (11). We ran positive control samples A. phagocytophilum, A. ovis, Wolbachia sp., and Candidatus $\mathrm{N}$. mikurensis with each reaction. We purified the PCR products obtained using the GenElute PCR Clean-Up Kit (Sigma-Aldrich, Buchs, Switzerland) and sequenced both strands. We edited variants obtained in this study (1,410 bp) using MEGA 4.0.2 (https://megasoftware.net/) and checked by eye. We made comparisons to sequences in GenBank with BLASTn 2.2.26 (https://pods.iplantcollaborative.org/wiki/display/DEapps/Blastn-2.2.26). For phylogenetic analysis of our variant (GenBank accession no. MG924904), we aligned 17 related sequences obtained from the GenBank database and constructed a phylogenetic tree using the Bayesian inference method (12).

We examined all blood and ticks collected for the presence of family Anaplasmataceae bacteria. Anaplasmataceae family members were present in 36 lizards (28 males and 8 females). Of the ticks removed from lizards, 87 (37\%) were infected, and of questing ticks, 18 (6.6\%) were infected (Table 2). 
Table 1. Primers used to amplify $16 \mathrm{~S}$ rRNA gene of Candidatus Cryptoplasma sp. found in green lizards and Ixodes ricinus ticks, Slovakia, 2004-2011

\begin{tabular}{lcccc}
\hline \multirow{2}{*}{ Organism } & $\begin{array}{c}\text { Primer } \\
\text { name }\end{array}$ & Sequences, 5' $\rightarrow 3^{\prime}$ & $\begin{array}{c}\text { Length of amplified } \\
\text { fragment, bp }\end{array}$ & Reference \\
\hline Family Anaplasmataceae & EHR747 & GCACTCATCGTTTACAGCGTG & 247 \\
& EHR521 & TGTAGGCGGTTCGGTAAGTAAAG & $(10)$ \\
\hline Most eubacteria & fD1 & CCGAATCGTCGACAACAGAGTTTGATCCTGGCTCAG & 1,500 \\
\hline
\end{tabular}

Denatured and electrophoresed PCR products from samples demonstrated several SSCP profiles, of which 1 was clearly distinguishable from the profiles of the Anaplasmataceae species used as controls (Figure 1). We detected this unique profile in all lizard blood samples, all ticks feeding on lizards, and some questing ticks. We sequenced representatives of this unidentified SSCP profile $(\approx 247 \mathrm{bp}$; GenBank accession nos. KY031322-3) and compared them with DNA fragments in the GenBank database. The closest related (99\% identity) 16S rRNA sequences were from uncultured Anaplasma sp. isolates from questing I. ricinus ticks from Morocco (GenBank accession no. AY672415), Tunisia (GenBank accession no. AY672420), and France (GenBank accession no. GU734325). Sequencing of a longer (1,410-bp) fragment of the 16S rRNA gene revealed 99.1\% similarity with the Candidatus C. californiense isolate from I. pacificus ticks in California (Figure 2). The 16S rRNA sequence obtained in this study was found to share a maximum of $94 \%$ identity with $A$. phagocytophilum Norway variant 2 (GenBank accession no. CP015376). The phylogenetic tree we constructed using 16S rRNA gene sequences showed that the reptile-associated Candidatus Cryptoplasma sp. REP (reptile) clustered in a separate branch with Candidatus C. californiense, indicating the isolate represents a lineage distinct from other known Anaplasmataceae species (e.g., A. phagocytophilum, A. marginale, A. platys, Ehrlichia muris, E. chaffeensis, and E. ewingii).

\section{Conclusions}

The role of ectotherm animals, especially lizards, in the maintenance of vectorborne pathogens is not clear. The interaction between reptiles and Anaplasmataceae family members has only been investigated in a few studies. Our findings expand knowledge on this research topic. Only limited information about the reptile-Anaplasma relationship exists. Ekner et al. suggested that sand lizards could potentially serve as a reservoir host for species of the Anaplasmataceae family when she discovered that ticks collected from these lizards in Poland were infected with Anaplasma-like pathogens (8). Although A. phagocytophilum might be transmitted by reptiles to a limited extent (5), the Anaplasma-like species detected in reptiles could also be a novel species, as suggested by Rejmanek et al. (6).

Despite the fact that lizards are exposed to a number of family Anaplasmataceae bacteria through infected ticks, our findings suggest that, except for Canditatus Cryptoplasma sp. REP, green lizards do not acquire infections with these species. In short, we detected Canditatus Cryptoplasma sp. REP in $90 \%$ of examined lizards, $37 \%$ of ticks feeding on lizards, and $6.6 \%$ of questing ticks in localities with lizards.

On the basis of our results, we cautiously speculate that Canditatus Cryptoplasma sp. REP is selected for and other genospecies selected against in ticks feeding on lizards. The Canditatus Cryptoplasma sp. REP variant had a high homology (100\%) with a sequence obtained from an Apodemus agrarius mouse from Slovakia (13), which indicates that rodents or other mammals might also become infected with this bacterium and contribute (to a lesser extent) to the circulation of these bacteria in nature.

In conclusion, we found a yet to be named species of Canditatus Cryptoplasma sp. (Canditatus Cryptoplasma sp.

Table 2. Prevalence of family Anaplasmataceae bacteria in Ixodes ricinus ticks collected from green lizards and surrounding vegetation, Slovakia, 2004-2011

\begin{tabular}{|c|c|c|c|c|c|c|}
\hline \multirow[b]{2}{*}{ Tick source, type } & \multirow[b]{2}{*}{$\begin{array}{l}\text { No. ticks } \\
\text { examined }\end{array}$} & \multirow[b]{2}{*}{$\begin{array}{l}\text { No. (\%) positive } \\
\text { ticks }\end{array}$} & \multicolumn{4}{|c|}{ No. (\%) positive ticks } \\
\hline & & & $\begin{array}{c}\text { Candidatus } \\
\text { Cryptoplasma }\end{array}$ & $\begin{array}{c}\text { Anaplasma } \\
\text { phagocytophilum }\end{array}$ & $\begin{array}{l}\text { Wolbachia } \\
\text { pipientis }\end{array}$ & $\begin{array}{c}\text { Candidatus } \\
\text { Neoehrlichia mikurensis }\end{array}$ \\
\hline \multicolumn{7}{|l|}{ Lizards } \\
\hline Larvae & 118 & $43(36.4)$ & $43(100)$ & - & - & - \\
\hline Nymphs & 117 & $44(37.6)$ & $44(100)$ & - & - & - \\
\hline Total & 235 & $87(37)$ & $87(100)$ & - & - & - \\
\hline \multicolumn{7}{|l|}{ Vegetation } \\
\hline Nymphs & 132 & $8(6.1)$ & 2 & 3 & 3 & 0 \\
\hline Males & 76 & $4(5.3)$ & 1 & 2 & 0 & 1 \\
\hline Females & 63 & $6(9.5)$ & 3 & 2 & 1 & 0 \\
\hline Adults & 139 & $10(7.2)$ & 4 & 4 & 1 & 1 \\
\hline Total & 271 & $18(6.6)$ & 6 & 7 & 4 & 1 \\
\hline
\end{tabular}




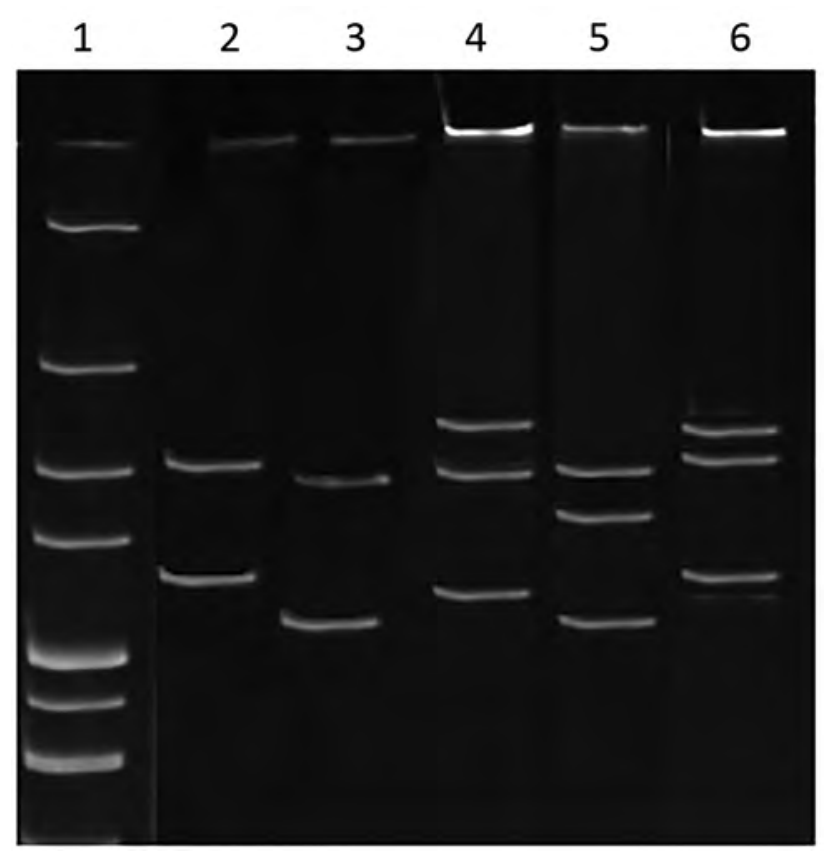

Figure 1. Single-strand conformation polymorphism profile of Anaplasmataceae isolate from reptiles, Slovakia, 20042011. The 247-bp 16S rRNA PCR fragments from the isolate from reptiles and known Anaplasmataceae species were denatured and electrophoresed. Lane 1, 100-bp ladder marker; lane 2, Candidatus Neoehrlichia mikurensis; lane 3, Anaplasma phagocytophilum; lane 4, isolate Candidatus Cryptoplasma sp. REP (reptile) obtained in this study; lane 5, A. ovis; and lane 6, Wolbachia.
REP) in questing $I$. ricinus ticks, $I$. ricinus ticks collected from and feeding on green lizards, and the blood of green lizards in Slovakia. These results indicate that green lizards serve as an intermediate host for this bacterium and that lizards can influence the enzootic maintenance and circulation of bacteria in the environment. However, other hosts besides reptiles could be involved in the Canditatus Cryptoplasma sp. REP lifecycle as well, though probably to a lesser extent.

This study was conducted within the framework of the project Environmental Protection against Parasitozoonoses under the Influence of Global Climate and Social Changes (project no. 26220220116), which is supported by the Research and Development Operating Program funded by the European Fund for Regional Development (0.4). Additional support was provided by the Scientific Grant Agency of the Ministry of Education of the Slovak Republic, Slovak Academy of Sciences VEGA 2/0113/18 (to I.M.), and EurNegVec COST Action TD1303. We thank the Fulbright Foundation (USA, to V.A.C.) for support.

\section{About the Author}

Dr. Kočíková is a postdoctoral researcher at the Department of Vector-Borne Diseases, Institute of Parasitology, Slovak Academy of Sciences, Košice, Slovakia. Her research interests include ecology and epidemiology of ticks and tickborne pathogens, the role of reptiles in transmission of vectorborne microorganisms, and molecular diversity of emerging parasitic and infectious disease agents in light of global changes.

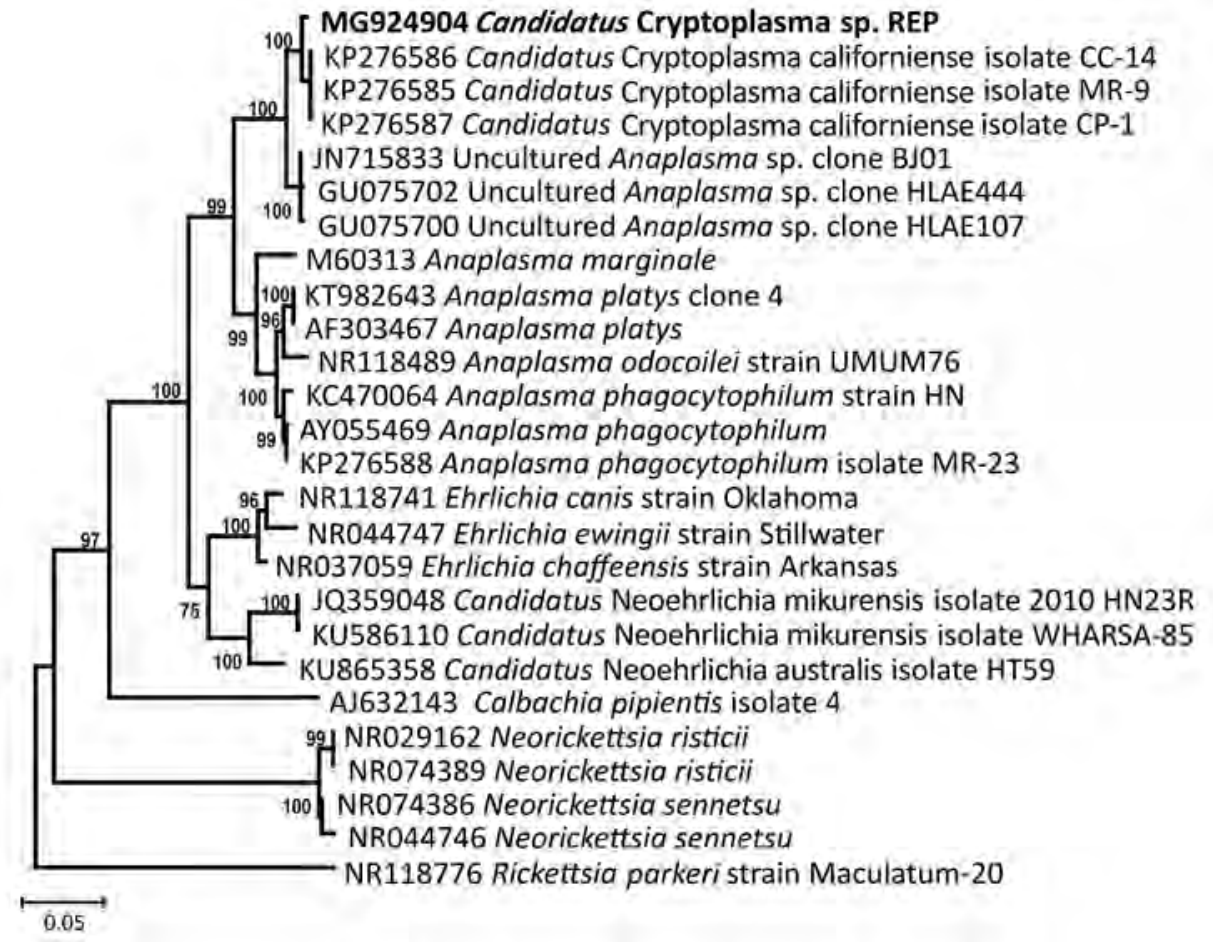

Figure 2. Phylogenetic relatedness of Candidatus Cryptoplasma sp. REP (reptile; bold), Slovakia, 2004-2011, to other Anaplasmataceae sp. family members. We constructed the tree using $16 S$ rRNA sequences and the Bayesian inference method. The Rickettsia parkeri sequence was used as an outgroup. Scale bar indicates nucleotide substitutions per site. 


\section{References}

1. Dumler JS. Anaplasma and Ehrlichia infection. Ann N Y Acad Sci. 2005;1063:361-73. http://dx.doi.org/10.1196/ annals.1355.069

2. Rikihisa Y. Mechanisms to create a safe haven by members of the family Anaplasmataceae. Ann N Y Acad Sci. 2003;990:548-55. http://dx.doi.org/10.1111/j.1749-6632.2003. tb07425.x

3. Rar V, Golovljova I. Anaplasma, Ehrlichia, and "Candidatus Neoehrlichia” bacteria: pathogenicity, biodiversity, and molecular genetic characteristics, a review. Infect Genet Evol. 2011;11:1842-61. http://dx.doi.org/10.1016/ j.meegid.2011.09.019

4. Eshoo MW, Carolan HE, Massire C, Chou DM, Crowder CD, Rounds MA, et al. Survey of Ixodes pacificus ticks in California reveals a diversity of microorganisms and a novel and widespread Anaplasmataceae species. PLoS One. 2015;10:e0135828. http://dx.doi.org/10.1371/ journal.pone. 0135828

5. Nieto NC, Foley JE, Bettaso J, Lane RS. Reptile infection with Anaplasma phagocytophilum, the causative agent of granulocytic anaplasmosis. J Parasitol. 2009;95:1165-70. http://dx.doi.org/10.1645/GE-1983.1

6. Rejmanek D, Bradburd G, Foley J. Molecular characterization reveals distinct genospecies of Anaplasma phagocytophilum from diverse North American hosts. J Med Microbiol. 2012;61:204-12. http://dx.doi.org/10.1099/jmm.0.034702-0

7. Tijsse-Klasen E, Fonville M, Reimerink JHJ, Spitzen-van der Sluijs A, Sprong H. Role of sand lizards in the ecology of Lyme and other tick-borne diseases in the Netherlands. Parasit Vectors. 2010;3:42. http://dx.doi.org/10.1186/ 1756-3305-3-42

8. Ekner A, Dudek K, Sajkowska Z, Majláthová V, Majláth I, Tryjanowski P. Anaplasmataceae and Borrelia burgdorferi sensu lato in the sand lizard Lacerta agilis and co-infection of these bacteria in hosted Ixodes ricinus ticks. Parasit Vectors. 2011;4:182. http://dx.doi.org/10.1186/1756-3305-4-182

9. Weisburg WG, Barns SM, Pelletier DA, Lane DJ. 16S ribosomal DNA amplification for phylogenetic study. J Bacteriol. 1991;173:697-703. http://dx.doi.org/10.1128/ jb.173.2.697-703.1991

10. Pancholi P, Kolbert CP, Mitchell PD, Reed KD Jr, Dumler JS, Bakken JS, et al. Ixodes dammini as a potential vector of human granulocytic ehrlichiosis. J Infect Dis. 1995;172:1007-12. http://dx.doi.org/10.1093/infdis/172.4.1007

11. Derdáková M, Beati L, Pet’ko B, Stanko M, Fish D. Genetic variability within Borrelia burgdorferi sensu lato genospecies established by PCR-single-strand conformation polymorphism analysis of the rrfA-rrlB intergenic spacer in Ixodes ricinus ticks from the Czech Republic. Appl Environ Microbiol. 2003;69:509-16. http://dx.doi.org/10.1128/AEM.69.1.509516.2003

12. Tamura K, Stecher G, Peterson D, Filipski A, Kumar S. MEGA6: Molecular Evolutionary Genetics Analysis version 6.0. Mol Biol Evol. 2013;30:2725-9. http://dx.doi.org/10.1093/ molbev/mst197

13. Štefančíková $A$, Derdáková $M$, Lenčáková $D$, Ivanová $R$, Stanko M, Čisláková L, et al. Serological and molecular detection of Borrelia burgdorferi sensu lato and Anaplasmataceae in rodents. Folia Microbiol (Praha). 2008;53:493-9. http://dx.doi.org/10.1007/s12223-008-0077-z

Address for correspondence: Viktória Majláthová, Institute of Parasitology, Slovak Academy of Sciences, Hlinkova 3, 04001 Košice, Slovakia; email: majlat@saske.sk

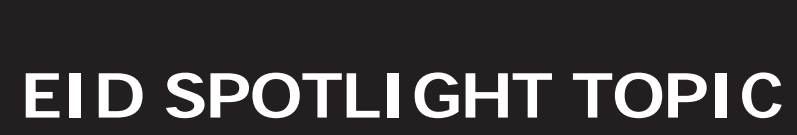 TICKS}

Ticks transmit a variety of different pathogens, including bacteria, protozoa, and viruses, which can produce serious and even fatal disease in humans and animals. Tens of thousands of cases of tickborne disease are reported each year, including Lyme disease.

Lyme disease is the most well-known tickborne disease. However, other tickborne illnesses such as Rocky Mountain spotted fever, tularemia, babesiosis, and ehrlichiosis also contribute to severe morbidity and more mortality each year.

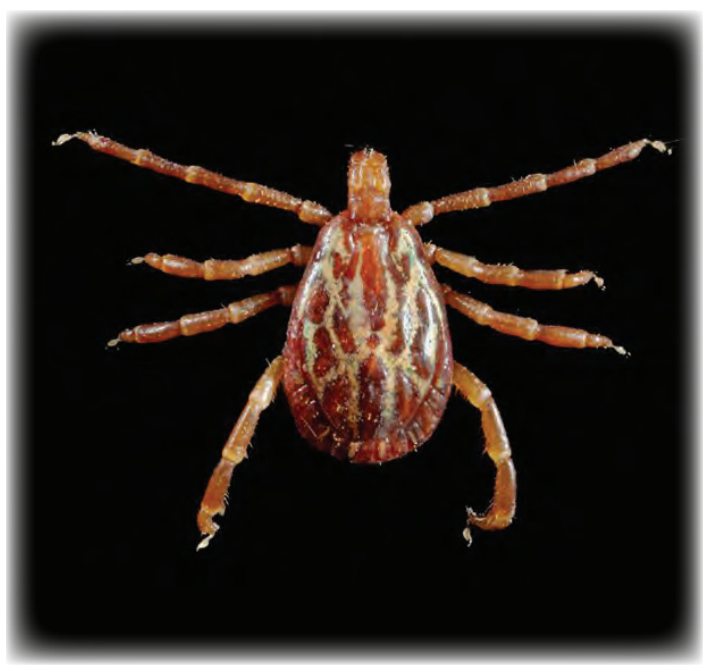

https://wwwnc.cdc.gov/eid/ page/tick-spotlight

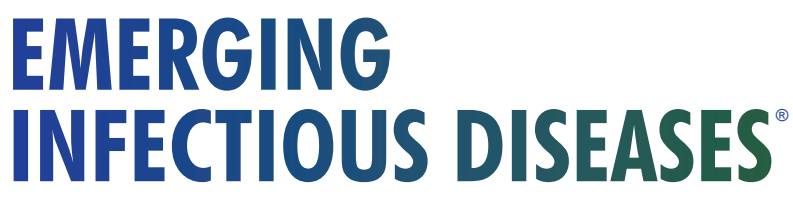




\section{Excess Mortality and Causes Associated with Chikungunya, Puerto Rico, 2014- 2015}

\section{André Ricardo Ribas Freitas, Maria Rita Donalisio, Pedro María Alarcón-Elbal}

During 2014-2015, a total of 31 deaths were associated with the first chikungunya epidemic in Puerto Rico. We analyzed excess mortality from various causes for the same months during the previous 4 years and detected 1,310 deaths possibly attributable to chikungunya. Our findings raise important questions about increased mortality rates associated with chikungunya.

$\mathrm{I}^{\mathrm{n}}$ December 2013, the first locally acquired chikungunya virus infections in the Americas were reported in Saint Martin. Since that report, the virus has spread to 45 countries and territories in North, Central, and South America, causing $\approx 2.4$ million suspected and confirmed cases and 440 deaths through December 2016 (1).

Chikungunya has emerged worldwide since 2004. Several gaps in knowledge exist about the disease and its consequences. Until recently chikungunya was considered a nonlethal disease, but severe forms and deaths have been described since an epidemic on Réunion Island during 2005-2006 (2).

In Puerto Rico, the chikungunya epidemic began in May 2014 as the first occurrence of the virus in the country (3). Official surveillance reported 28,327 suspected chikungunya cases, of which 4,339 were laboratoryconfirmed; 31 persons died (0.9 deaths/100,000 population). The chikungunya mortality rate was significantly lower than that observed in epidemics on other islands, such as Réunion Island (25.9 deaths/100,000 population in 2006), Martinique (20.5/100,000 population in 2014), and Guadaloupe $(14.4 / 100,000$ population in 2014) $(1,4)$. These differences could be a consequence of the difficulty of recognizing the etiology of severe clinical forms and deaths.

Author affiliations: Secretaria de Saúde de Campinas, Campinas, Brazil (A.R.R. Freitas); Faculdade de Medicina São Leopoldo Mandic, Instituto de Pesquisas São Leopoldo Mandic, Campinas (A.R.R. Freitas); Universidade Estadual de Campinas, Campinas (M.R. Donalisio); Universidad Iberoamericana, Instituto de Medicina Tropical \& Salud Global, Santo Domingo, Dominican Republic (P.M. Alarcón-Elbal)

DOI: https://doi.org/10.3201/eid2412.170639

\section{The Study}

We studied excess mortality associated with chikungunya in Puerto Rico by comparing monthly expected deaths and actual deaths during the epidemic (5-7). We calculated expected deaths by the average age-adjusted mortality rate for each month for 2010 to 2013 and projected them to the estimated population for 2014 and 2015 (6). We considered the difference between observed and expected deaths for the months in which observed deaths exceeded the upper limit of the $99 \% \mathrm{CI}(6)$ as excess deaths associated with the chikungunya epidemic (Figure 1).

We estimated the mortality rate expected for the second half of 2014, the worst period of the epidemic, and compared it with the observed mortality rate. This second group of calculations was based on the all-cause age-specific mortality rate for the 10 leading causes of death in Puerto Rico and main causes of deaths associated with severe chikungunya in Réunion Island (2).

We obtained data on estimated population from the US Census Bureau (8) and on deaths from the Centers for Disease Control and Prevention's National Center for Health Statistics (9). In mid-September 2014, Puerto Rico issued an official administrative order stating that only persons hospitalized with suspected chikungunya should be reported to health authorities in Puerto Rico (3), Thus, we monitored the occurrence of chikungunya, dengue, and other viral diseases in Puerto Rico using secondary data from published studies evaluating the etiology of acute febrile illness of any patient, such as sentinel surveillance at St. Luke's Episcopal Hospital in the cities of Ponce and Guayama $(10,11)$.

We determined an excess of 1,310 deaths concurrent with the peak of the chikungunya epidemic of 2014 in Puerto Rico. We found no substantial occurrence of dengue, influenza, or leptospirosis during the chikungunya epidemic, according to data from St. Luke's Episcopal Hospital (Figure 1) and official Puerto Rico surveillance $(10,12)$, reinforcing the possibility that chikungunya might be etiologically associated with the deaths. Although the most affected age group was persons $>75$ years of age, a statistically significant proportion of deaths occurred in persons 24-55 years of age, suggesting chikungunya-associated deaths are not exclusive to elderly persons (Figure 2).

The estimated mortality rate was 30.1 deaths/100,000 population, compatible with the excess deaths observed in 


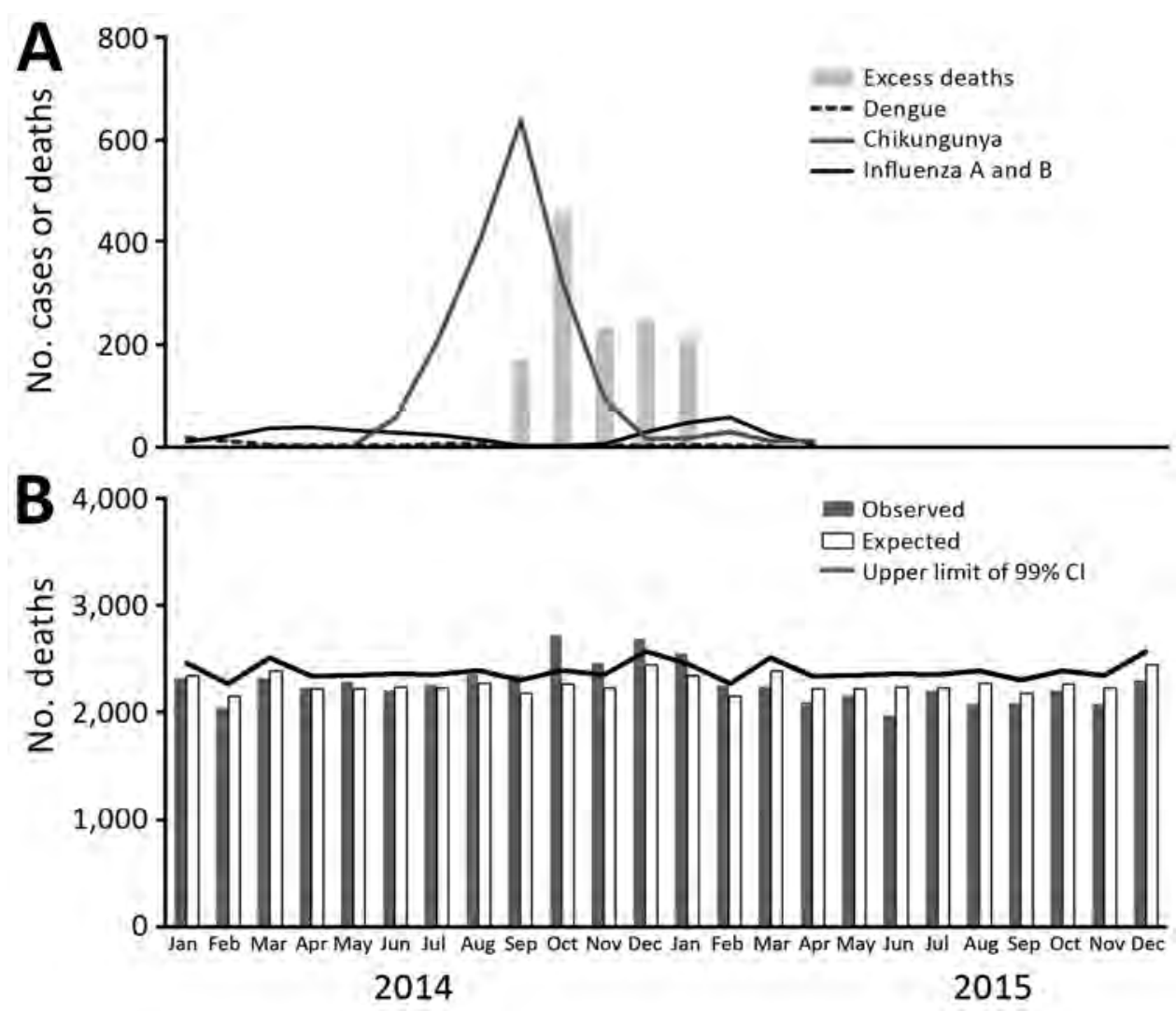

Figure 1. Expected and observed monthly deaths in Puerto Rico, 2014-2015. A) Excess number of monthly deaths and number of chikungunya, dengue, and influenza A and B cases diagnosed among patients with acute febrile illness in St. Luke's Episcopal Hospital, Puerto Rico, January 2014-December 2015. Data for acute febrile illness were discontinued in April 2015. B) Number of monthly deaths, observed deaths, and expected deaths and upper limit of $99 \%$ Cl, Puerto Rico, January 2014December 2015.

Réunion Island in 2006 (33.8/100,000 population) using similar methods (5) and slightly below the estimated rate of 47.9 deaths/100,000 population in Pernambuco, the most affected state of Brazil, during the chikungunya epidemic of 2016 (7). We estimated excess deaths as 42 times greater than the 31 deaths identified by the official surveillance developed during the chikungunya epidemic in Puerto Rico (3). In a similar study conducted in northeastern Brazil, we found 7,231 excess deaths in the region during the chikungunya epidemic of 2016, when the official surveillance system confirmed only 120 deaths, 60 times lower (7).

We detected a 1-month lag between the peak of chikungunya identified by St. Luke's Episcopal Hospital and the peak of excess deaths. Other lags also were observed in similar studies in Ahmedabad, India; Mauritius; and recently in Brazil $(6,7)$; these lags can be explained by prolonged hospitalization before death (2).

Deaths classified as "all other forms of chronic ischemic heart disease" (International Classification of Diseases, 10th Revision, codes I20, I25.1-I25.9) and diabetes mellitus (E10-E14) increased significantly, but deaths associated with other important causes of death, such as acute myocardial infarction (I21, I22) and neoplasms (C00-D49), did not (Table 1). These findings suggest that chikungunya contributes substantially to severity through its own pathophysiologic mechanisms and that some preexisting conditions participate only as additional risk factors for death. The most frequent clinically worsening conditions described in laboratory-confirmed chikungunya in other settings were heart failure, multiple organ failure, acute hepatitis, encephalitis, epidermolysis bullosa, myocarditis, respiratory failure, and renal failure (2). Many of these causes of death on Réunion Island (3) also increased over previous years in Puerto Rico during the epidemic (Table 2). In this study, volume depletion, emphysema, arrhythmias, asthma, diabetes mellitus and chronic ischemic heart disease led to a higher mortality rate during the epidemic than during previous years; all of these

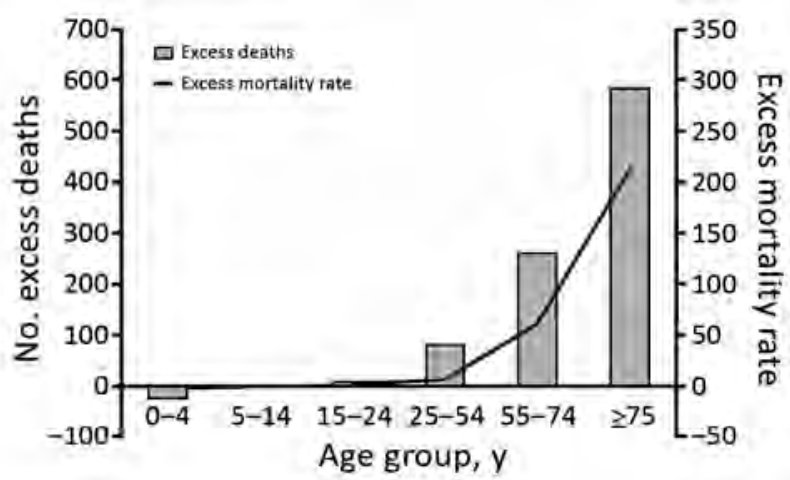

Figure 2. Excess deaths and difference between observed and expected deaths during chikungunya epidemic, Puerto Rico, JulyDecember 2014 . Mortality rate is deaths per 100,000 population. 
Table 1. Expected and observed deaths during a chikungunya epidemic, Puerto Rico, July-December 2014

\begin{tabular}{|c|c|c|c|c|c|c|c|}
\hline Cause of death (code) $)^{*}$ & $\begin{array}{l}\text { Observed } \\
\text { deaths }\end{array}$ & $\begin{array}{l}\text { Expected } \\
\text { deaths }\end{array}$ & Difference $†$ & $\begin{array}{c}\text { Relative } \\
\text { risk }\end{array}$ & $95 \% \mathrm{Cl}$ & $99 \% \mathrm{Cl}$ & $\mathrm{p}$ value \\
\hline Acute myocardial infarction (I21-I22) & 735 & 783 & -48 & 0.938 & $0.866-1.017$ & $0.844-1.043$ & 0.06 \\
\hline $\begin{array}{l}\text { All other forms of chronic ischemic heart } \\
\text { disease }(120,125.1-125.9)\end{array}$ & 963 & 787 & 176 & 1.224 & $1.139-1.315$ & $1.114-1.346$ & $<0.01$ \\
\hline Alzheimer's disease (G30) & 1,050 & 1,020 & 30 & 1.030 & $0.962-1.102$ & $0.942-1.126$ & 0.20 \\
\hline Diabetes mellitus (E10-E14) & 1,761 & 1,623 & 138 & 1.085 & $1.030-1.144$ & $1.012-1.163$ & $<0.01$ \\
\hline Hypertensive heart disease (I11) & 366 & 376 & -10 & 0.974 & 0.869-1.092 & $0.838-1.132$ & 0.33 \\
\hline $\begin{array}{l}\text { Other chronic obstructive pulmonary } \\
\text { disease (J44) }\end{array}$ & 382 & 410 & -28 & 0.932 & $0.833-1.041$ & $0.805-1.078$ & 0.11 \\
\hline $\begin{array}{l}\text { Pneumonia due to other or unspecified } \\
\text { organisms }(\mathrm{J} 16, \mathrm{~J} 18)\end{array}$ & 392 & 396 & -4 & 0.990 & $0.886-1.105$ & $0.856-1.145$ & 0.43 \\
\hline Renal failure (N17-N19) & 500 & 479 & 21 & 1.045 & $0.947-1.153$ & $0.918-1.189$ & 0.19 \\
\hline Septicemia (A40-A41) & 456 & 418 & 38 & 1.091 & $0.984-1.210$ & $0.952-1.250$ & 0.05 \\
\hline Neoplasms (C00-D49) & 2,849 & 2,849 & 0 & 1.000 & 0.960-1.042 & $0.947-1.055$ & 0.50 \\
\hline
\end{tabular}

diseases were found as cause of death or preexisting condition in patients with severe chikungunya $(2,13)$.

\section{Conclusions}

We found substantial excess mortality in Puerto Rico during the 2014 chikungunya epidemic, which should no longer be treated as a nonlethal disease. In addition to elderly persons, excess deaths occurred in other age groups. The main causes of death in patients with laboratory-confirmed chikungunya in hospital-based studies were similar to those in our study $(2,13)$.

Our study reinforces the hypothesis of the association of chikungunya with severe manifestations and deaths. Chikungunya-related death is critical to defining public health priorities, such as investment in research, vaccine development, and vector control. The evaluation of excess deaths is a tool that should be included in the assessment of chikungunya epidemics.

The results of our study are subject to several limitations. We conducted an ecologic analysis, which does not enable establishment of causality, and the investigation was based on secondary data, which may result in some inaccuracies. Therefore, some of the excess deaths we calculated might have resulted from other diseases, particularly vectorborne diseases, which have seasonal patterns of occurrence similar to those of chikungunya. However, we tried to reduce these limitations using several sources of information and accounting for other diseases that could interfere in the deaths in the region, including other viral diseases. The method used in this study is already well known and widely used to estimate deaths and hospitalizations associated with respiratory viruses and extreme weather phenomena $(14,15)$.

Table 2. Number of deaths from specific causes observed during chikungunya epidemic period, Puerto Rico, July-December 20102015, selected according to clinical presentation of severe chikungunya deaths in Réunion Island, 2005-2006*

\begin{tabular}{|c|c|c|c|c|c|c|c|c|}
\hline \multirow[b]{3}{*}{ Cause of death (code) $\dagger$} & \multirow{2}{*}{\multicolumn{6}{|c|}{ Year }} & \multirow{3}{*}{$\begin{array}{c}\text { Difference between } \\
2014 \text { and mean of } \\
2010-2013\end{array}$} & \multirow{3}{*}{$\begin{array}{c}\text { Change, } \\
\%\end{array}$} \\
\hline & & & & & & & & \\
\hline & 2010 & 2011 & 2012 & 2013 & 2014 & 2015 & & \\
\hline $\begin{array}{l}\text { Volume depletion, disorders of fluid, electrolyte and } \\
\text { acid-base balance (E86-E87) }\end{array}$ & 117 & 117 & 134 & 122 & 170 & 93 & 48 & 39 \\
\hline Emphysema (J43) & 82 & 66 & 76 & 51 & 104 & 70 & 35 & 51 \\
\hline $\begin{array}{l}\text { Conduction disorders and cardiac dysrhythmias (144- } \\
\text { 149) }\end{array}$ & 155 & 146 & 140 & 154 & 182 & 142 & 33 & 22 \\
\hline Asthma $(\mathrm{J} 45-\mathrm{J} 46)$ & 33 & 45 & 13 & 24 & 53 & 37 & 24 & 84 \\
\hline Pneumonia (J12-J18) & 419 & 401 & 339 & 364 & 402 & 283 & 21 & 6 \\
\hline $\begin{array}{l}\text { Other and unspecified diseases of skin and sub- } \\
\text { cutaneous tissue (L10-L98) }\end{array}$ & 85 & 98 & 81 & 95 & 106 & 90 & 16 & 18 \\
\hline Other chronic liver disease and cirrhosis (K73-K74) & 61 & 73 & 62 & 58 & 77 & 77 & 14 & 21 \\
\hline Alcoholic liver disease (K70) & 45 & 37 & 49 & 50 & 58 & 51 & 13 & 28 \\
\hline Other and unspecified heart failure (I50.1-I50.9) & 63 & 60 & 57 & 55 & 71 & 56 & 12 & 21 \\
\hline $\begin{array}{l}\text { Diseases of pericardium and acute myocarditis (I30- } \\
131,140)\end{array}$ & 3 & 2 & 2 & 4 & 6 & 3 & 3 & 118 \\
\hline $\begin{array}{l}\text { Meningitis and encephalitis (A83-A84, A85.2, G00, } \\
\text { G03) }\end{array}$ & 5 & 3 & 1 & 5 & 5 & 4 & 2 & 43 \\
\hline Congestive heart failure $(150.0)$ & 229 & 199 & 207 & 222 & 204 & 162 & -10 & -5 \\
\hline
\end{tabular}

*See reference (2).

†Except for pneumonia, the causes of death were grouped according to the List of 358 Selected Causes of Death of the National Center for Health Statistics (https://www.cdc.gov/nchs/data/dvs/Multiple_Cause_Record_Layout_2015.pdf). Codes are from the International Classification of Diseases, 10th Revision. 
Although limited, our results raise important questions about the occurrence of severe disease and increased mortality rates associated with chikungunya. Therefore, fundamental research is needed about chikungunya pathophysiologic mechanisms involving severe forms, exacerbation of preexisting conditions, and deaths. In addition to clinical studies, systematic diagnostic research of recent infection, including chikungunya, in all severe hospitalized patients during outbreaks could answer some important questions.

\section{Acknowledgments}

We thank Luana Hughes Freitas, Nicole Montenegro de Medeiros, Bruno Coelho, and Marcela Montenegro for their support in revising the manuscript.

\section{About the Author}

Dr. Freitas is a medical epidemiologist who works in the epidemiologic surveillance of Campinas, Campinas, São Paulo, Brazil, and is a professor of epidemiology, biostatistics, and scientific methodology at Faculdade de Medicina São Leopoldo Mandic de Campinas. His primary research interests include studies of mortality and communicable diseases, with emphasis on arboviruses and respiratory viruses.

\section{References}

1. Pan American Health Organization, World Health Organization. Chikungunya: communicable diseases and health analysis. Data, maps and statistics [cited 2016 Dec 13]. http://www.paho.org/ hq/index.php?option=com_topics\&view=readall\&cid=5927\&Itemi $\mathrm{d}=40931$ \&lang $=$ en

2. Economopoulou A, Dominguez M, Helynck B, Sissoko D, Wichmann O, Quenel P, et al. Atypical chikungunya virus infections: clinical manifestations, mortality and risk factors for severe disease during the 2005-2006 outbreak on Réunion. Epidemiol Infect. 2009;137:534-41. http://dx.doi.org/10.1017/ S0950268808001167

3. Sharp TM, Ryff KR, Alvarado L, Shieh W-J, Zaki SR, Margolis HS, et al. Surveillance for chikungunya and dengue during the first year of chikungunya virus circulation in Puerto Rico. J Infect Dis. 2016;214(suppl 5):S475-81. http://dx.doi.org/ 10.1093/infdis/jiw245

4. Renault P, Solet JL, Sissoko D, Balleydier E, Larrieu S, Filleul L, et al. A major epidemic of chikungunya virus infection on Reunion
Island, France, 2005-2006. Am J Trop Med Hyg. 2007;77:727-31. http://dx.doi.org/10.4269/ajtmh.2007.77.727

5. Josseran L, Paquet C, Zehgnoun A, Caillere N, Le Tertre A, Solet J-L, et al. Chikungunya disease outbreak, Reunion Island. Emerg Infect Dis. 2006;12:1994-5. http://dx.doi.org/10.3201/ eid1212.060710

6. Mavalankar D, Shastri P, Bandyopadhyay T, Parmar J, Ramani KV. Increased mortality rate associated with chikungunya epidemic, Ahmedabad, India. Emerg Infect Dis. 2008;14:412-5. http://dx.doi.org/10.3201/eid1403.070720

7. Freitas ARR, Cavalcanti L, Von Zuben AP, Donalisio MR. Excess mortality related to chikungunya epidemics in the context of co-circulation of other arboviruses in Brazil. PLoS Curr. 2017;9:pii: ecurrents.outbreaks.14608e586cd321d8d5088652d7a0d884.

8. US Census Bureau. Puerto Rico Commonwealth population totals tables: 2010-2016 [cited 2017 Apr 8]. https://www.census.gov/ data/tables/2016/demo/popest/total-puerto-rico.html

9. Centers for Disease Control and Prevention, National Center for Health Statistics. Vital statistics online. Mortality multiple cause files [cited 2017 Apr 8]. https://www.cdc.gov/nchs/data_access/ vitalstatsonline.htm\#Mortality_Multiple

10. Tomashek KM, Lorenzi OD, Andújar-Pérez DA, Torres-Velásquez BC, Hunsperger EA, Munoz-Jordan JL, et al. Clinical and epidemiologic characteristics of dengue and other etiologic agents among patients with acute febrile illness, Puerto Rico, 2012-2015. PLoS Negl Trop Dis. 2017;11:e0005859. http://dx.doi.org/10.1371/journal.pntd.0005859

11. Rohatgi A. WebPlotDigitizer [cited 2017 Apr 8]. https://automeris.io/WebPlotDigitizer

12. Departamento de Salud de Puerto Rico. Informe semanal de bigilancia del dengue—-semana 52/2014 [cited 2017 Apr 8]. http://www.salud.gov.pr/Estadisticas-Registros-y-Publicaciones/ Pages/Dengue.aspx

13. Crosby L, Perreau C, Madeux B, Cossic J, Armand C, Herrmann-Storke C, et al. Severe manifestations of chikungunya virus in critically ill patients during the 2013-2014 Caribbean outbreak. Int J Infect Dis. 2016;48:78-80. http://dx.doi.org/ 10.1016/j.ijid.2016.05.010

14. Freitas ARR, Donalisio MR. Excess of mortality in adults and elderly and circulation of subtypes of influenza virus in southern Brazil. Front Immunol. 2018;8:1903. http://dx.doi.org/10.3389/ fimmu.2017.01903

15. Hoshiko S, English P, Smith D, Trent R. A simple method for estimating excess mortality due to heat waves, as applied to the 2006 California heat wave. Int J Public Health. 2010;55:133-7. http://dx.doi.org/10.1007/s00038-009-0060-8

Address for correspondence: André Ricardo Ribas Freitas, Faculdade São Leopoldo Mandic, Instituto de Pesquisas São Leopoldo Mandic, área de Epidemiologia, R. Dr. José Rocha Junqueira, 13, Swift, Campinas, SP São Paulo 13045-755, Brazil; email: andre.freitas@slmandic.edu.br 


\section{Borrelia miyamotoi Infections in Small Mammals, California, USA}

\author{
Daniel J. Salkeld, ${ }^{1}$ Nathan C. Nieto, ${ }^{1}$ \\ Denise L. Bonilla, ${ }^{2}$ Melissa H. Yoshimizu, \\ Kerry A. Padgett
}

Surveillance to investigate the wildlife-vector transmission cycle of the human pathogen Borrelia miyamotoi in California, USA, revealed infections in dusky-footed woodrats, brush mice, and California mice. Phylogenetic analyses suggest a single, well-supported clade of $B$. miyamotoi is circulating in California.

$B$ orrelia miyamotoi is a spirochete that causes a relapsing febrile illness and is transmitted by hard Ixodes species ticks $(1,2)$. B. miyamotoi is prevalent in western black-legged tick (I. pacificus) populations in California, USA $(3,4)$; in some locations, B. miyamotoi prevalence in ticks is comparable with or higher than the prevalence of the Lyme disease agent, B. burgdorferi sensu stricto (3-5). There is mounting evidence that human infections occur in northern California (6).

Surveillance of B. miyamotoi in California has focused on ticks, and little is known about infection in wildlife hosts. B. miyamotoi has been identified from spleen samples of birds and rodents in Europe (7), from blood and bladder samples of rodents in Japan (8), and from white-footed mice (Peromyscus leucopus) in the eastern United States (9). In California, surveillance in Alameda County (east of the San Francisco Bay) observed B. miyamotoi in tick populations but failed to detect the spirochete in mammals (10). We investigated B. miyamotoi infection status in small mammals at 3 California sites where the bacterium is present in tick populations $(3,5)$.

\section{The Study}

We captured animals on 2 consecutive nights at each of 3 sites in the San Francisco Bay area of California during June 2014: Windy Hill $\left(37.37315^{\circ},-122.22466^{\circ}\right)$ and Thornewood $\left(37.39086^{\circ},-122.25066^{\circ}\right)$ Open Space Preserves (OSP) in San Mateo County, and Foothills Park (37.36243, $-122.17362^{\circ}$ ) in Santa Clara County. We chose these sites on

Author affiliations: Colorado State University, Fort Collins,

Colorado, USA (D.J. Salkeld); Northern Arizona University,

Flagstaff, Arizona, USA (N.C. Nieto); California Department of

Public Health, Richmond, California, USA (D.L. Bonilla,

M.H. Yoshimizu, K.A. Padgett)

DOI: https://doi.org/10.3201/eid2412.171632 the basis of local B. miyamotoi prevalence of $3.6 \%-10.7 \%$ in I. pacificus nymphs (5). Trapping occurred in June to coincide with the peak abundance of nymphal black-legged ticks and so perhaps increase the chance that $B$. miyamotoi would be circulating in animal populations (11). Animals were captured using Sherman live traps baited with peanut butter and oats.

We anesthetized captured animals with isoflurane, identified them by morphology using taxonomic guides, and examined them for ticks. We obtained blood and eartissue biopsy samples from each individual and tested both sample types for Borrelia spp. because different Borrelia species may vary in tissue tropism (9).

We extracted DNA from all samples (i.e., whole blood, ear punch biopsies, and ticks) using DNeasy Blood and Tissue Kits (QIAGEN, Valencia, CA, USA) and assayed for the presence of Borrelia using quantitative PCR (qPCR) (9), which is able to detect as few as 10 spirochetes. We sequenced all qPCR-positive samples using a primer set targeting the intergenic spacer rrs-rrlA locus, which allowed for differentiation of Borrelia genospecies (12). Alignments were made in ClustalX (http://www.clustal.org). We compared our sequences from I. pacificus ticks and wild-caught rodents (GenBank accession nos. MH342008-31) to representative GenBank sequences from isolates found in other sites in California (accession nos. KT343321, KT343334, KT343337, KU184505, KF957668), elsewhere in the United States (accession nos. HQ658901, HQ658902, AY374140, AY37139, AY374138, AY363706, GU993308, KY293400, KY293399, KY293398, KY293397, KY293396, GQ856588, GU993309, GQ856589), Japan (accession nos. AY363703, AY363704), and Sweden (accession no. AY363705). We conducted phylogenetic reconstruction using MrBayes (http://mrbayes.csit.fsu.edu/) and visualizations using FigTree (http:// tree.bio.ed.ac.uk/software/figtree/).

We captured a total of 117 small mammals from 5 species (Table 1). Our surveillance efforts demonstrate that $B$. miyamotoi infects woodrats (Neotoma fuscipes), brush mice (Peromyscus boylii), and California mice (P. californicus) (Table 2). At sites where B. miyamotoi was present in small mammals, B. miyamotoi prevalence was $6 \%-33 \%$ in different host species (Table 2). These data reflect B. miyamotoi prevalence in small-mammal hosts in other geographic

\footnotetext{
${ }^{1}$ These authors contributed equally to this article.

${ }^{2}$ Current affiliation: United States Department of Agriculture, Fort Collins, Colorado, USA.
} 
Table 1. Numbers of mammals captured and tested for Borrelia spp., by species and location, California, USA*

\begin{tabular}{|c|c|c|c|c|c|}
\hline \multirow[b]{2}{*}{ Species } & \multirow[b]{2}{*}{ Foothills Park } & \multicolumn{2}{|c|}{ Thornewood OSP } & \multirow[b]{2}{*}{ Windy Hill OSP } & \multirow[b]{2}{*}{ Total } \\
\hline & & $\begin{array}{c}\text { Redwood } \\
\text { habitat }\end{array}$ & $\begin{array}{l}\text { Oak-madrone } \\
\text { woodland }\end{array}$ & & \\
\hline Dusky-footed woodrat (Neotoma fuscipes) & 4 & 0 & 1 & 1 & 6 \\
\hline Brush mouse (Peromyscus boylii) & 27 & 9 & 17 & 18 & 71 \\
\hline California mouse (Peromyscus californicus) & 6 & 3 & 9 & 6 & 24 \\
\hline Deer mouse (Peromyscus maniculatus) & 6 & 4 & 2 & 1 & 13 \\
\hline Pinyon mouse (Peromyscus truei) & 3 & 0 & 0 & 0 & 3 \\
\hline Total & 46 & 16 & 29 & 26 & 117 \\
\hline
\end{tabular}

regions: $10.7 \%$ of voles and mice $(n=65)$ in the Netherlands, where nymphal infection prevalence (NIP) of B. miyamotoi in I. ricinus ticks is $2.5 \%$ (84/3360) (7), and $6.5 \%$ of whitefooted mice ( $P$. leucopus) in the northeastern United States, where NIP in I. scapularis ticks is 5.5\% (38/689) (9). We did not observe $B$. miyamotoi in pinyon mice or deer mice, either because of small sample sizes or because these species are not involved in B. miyamotoi transmission. In nearby Alameda County, B. miyamotoi was not observed in small mammals (10); possible reasons are that the spirochete is rarer in this locality (NIP $=0.4 \%$ in Alameda study sites), that mammal capture periods were dispersed across multiple years and not as coincident with nymphal tick activity, or that brush mice and California mice were not captured at that location.

Other identified Borrelia species included B. bissettiae in 1 pinyon mouse (P. truei) and 1 brush mouse, which mirrors earlier studies of $B$. bissettiae from farther north in California $(13,14)$. Woodrats, California voles (Microtus californicus), deer mice (P. maniculatus), and black rats (Rattus rattus) have also been observed infected with $B$. bissettiae $(10,13)$. During previous tick sampling efforts at our study sites, we did not detect $B$. bissettiae in questing western black-legged ticks $(3,5)$.

We did not find B. burgdorferi sensu stricto, although we observed uncharacterized $B$. burgdorferi sensu lato infection in 1 California mouse. None of the animals captured in the redwood habitat (Thornewood OSP) were infected with Borrelia spp., although the sample size was small at this location (Table 1). We found a co-infection of B. burgdorferi sl and B. miyamotoi in a brush mouse.
Co-infections of B. burgdorferi and B. miyamotoi have previously been reported from mice and ticks in the northeastern United States (9) and from ticks in Marin County, California (4).

Five mammals were infested with $I$. angustus ticks, of which we observed all 3 life stages. In redwood habitat at Thornewood OSP, 1 California mouse hosted 2 adult females and a nymph, 1 brush mouse was infested with 2 adult females and 2 nymphs, and 1 brush mouse carried 1 female and 1 nymphal tick. Of 2 brush mice captured at Windy Hill, 1 harbored 3 larvae and the other 5 larvae. None of the 17 I. angustus ticks tested positive for Borrelia spp., nor did the host animals from which the ticks were removed. We found no I. pacificus ticks infesting the small mammals.

Phylogenetic analyses suggest that $B$. miyamotoi in California is a single strain, separate from B. miyamotoi in the eastern United States and from strains circulating in Asia and Europe (Figure), corroborating an earlier study (15). Sequences from I. pacificus ticks previously collected in the San Francisco Bay area were identical to the sequences obtained from rodent infections.

\section{Conclusions}

The identification of B. miyamotoi in small mammals in California mirrors research from other locations that have documented the spirochete in small rodents. It is premature to claim these infected species as B. miyamotoi reservoirs (i.e., responsible for maintenance of the pathogen and acting as a source for of zoonotic transmission), in part because

\begin{tabular}{|c|c|c|c|c|c|}
\hline Borrelia species & Mammal species & Site & $\begin{array}{l}\text { Sample } \\
\text { source }\end{array}$ & $\begin{array}{l}\text { Prevalence at site, no. } \\
\text { tested/no. positive (\%) }\end{array}$ & $\begin{array}{c}\text { Prevalence across sites, } \\
\text { no. tested/no. positive (\%) }\end{array}$ \\
\hline \multirow[t]{5}{*}{ B. miyamotoi } & Dusky-footed woodrat & Foothills & Blood & $1 / 4(25)$ & $1 / 6(17)$ \\
\hline & Brush mouse & $\begin{array}{l}\text { Thornewood } \\
\text { woodland }\end{array}$ & Ear & $1 / 17(6)$ & $2 / 71(3)$ \\
\hline & Brush mouse* & Windy Hill & Ear & $1 / 18(6)$ & \\
\hline & California mouse & Foothills & Ear & $1 / 6(17)$ & $4 / 24(17)$ \\
\hline & California mouse & $\begin{array}{l}\text { Thornewood } \\
\text { woodland }\end{array}$ & 2 ear, 1 blood & $3 / 9(33)$ & \\
\hline \multirow[t]{2}{*}{ B. bissettiae } & Brush mouse & $\begin{array}{c}\text { Thornewood } \\
\text { woodland }\end{array}$ & Ear & $1 / 17(6)$ & $1 / 71(1)$ \\
\hline & Pinyon mouse & Foothills & Blood & $1 / 3(33)$ & 1/3 (33) \\
\hline B. burgdorferi sensu lato & California mouse & Windy Hill & Blood & $1 / 6(17)$ & $1 / 24(4)$ \\
\hline $\begin{array}{l}\text { Borrelia spp., not } \\
\text { sequenced }\end{array}$ & Brush mouse & $\begin{array}{l}\text { Thornewood } \\
\text { woodland }\end{array}$ & $\begin{array}{l}2 \text { blood, } 1 \\
\text { blood and ear }\end{array}$ & $3 / 17(18)$ & $3 / 71(4)$ \\
\hline
\end{tabular}




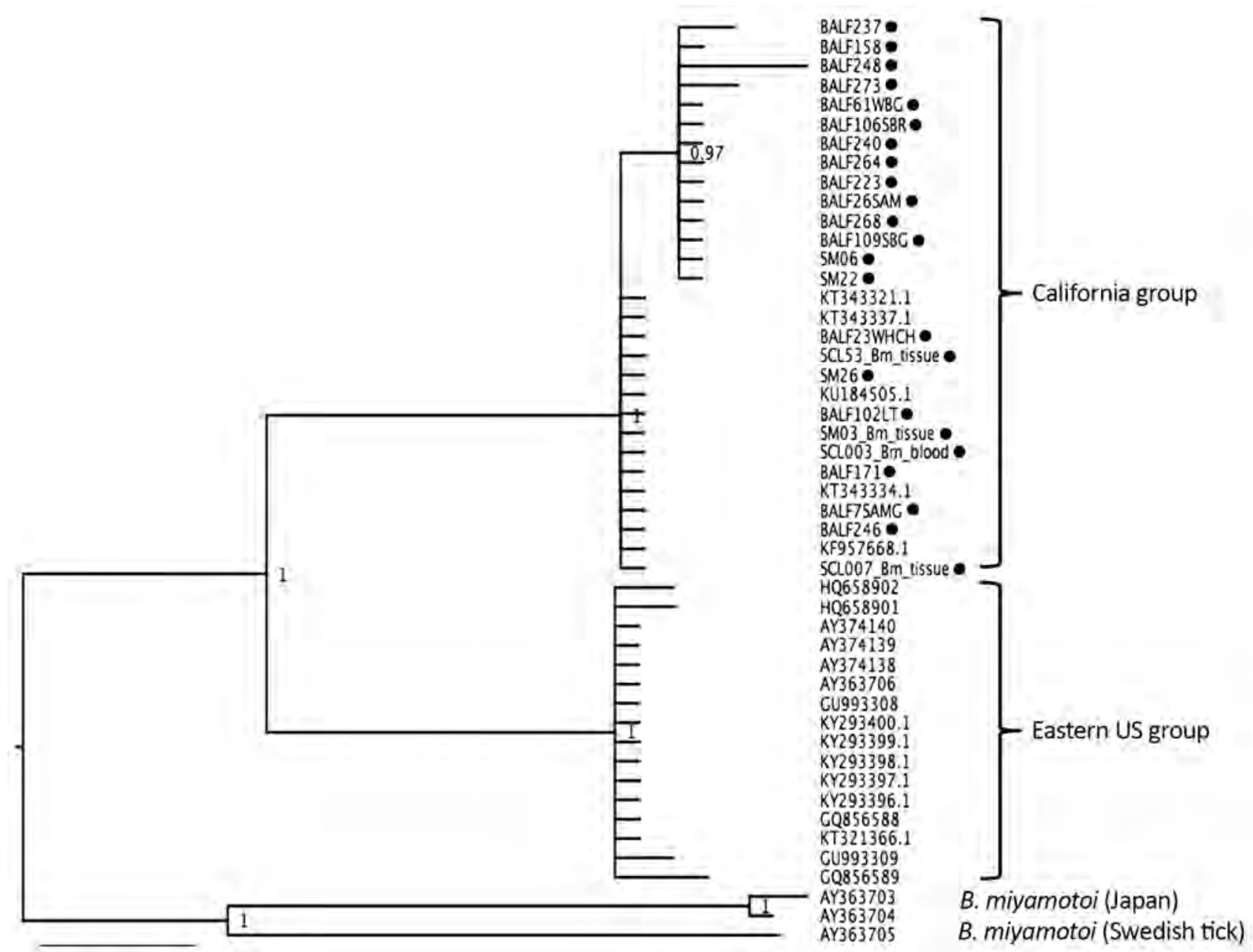

0.0050

Figure. Phylogenetic tree of Borrelia miyamotoi intergenic spacer ( $r$ rs-rrlA) sequences isolated from wild-caught rodents and ticks (black dots) from California, USA, in study of Borrelia spp. in small mammal species in the San Francisco Bay area, compared with reference samples from California, the eastern United States, Japan, and Sweden. Isolates are identified by isolate identification number or GenBank accession number. Scale bar indicates nucleotide substitutions per site.

B. miyamotoi can also be transmitted transovarially in the tick population; local maintenance of the spirochete may not require a reservoir host. Nonetheless, in California, where $B$. miyamotoi clusters into a single, well-supported phylogenetic clade, the spirochete appears to be circulating among rodent species. Xenodiagnostic investigation of these putative reservoir species, as well as more comprehensive investigations of the reservoir potential of other local fauna, including larger mammals (e.g., squirrels and deer) and birds, will further elucidate the ecology of B. miyamotoi in California.

\section{Acknowledgments}

We thank Foothills Park and the Midpeninsula Regional Open Space District for allowing us to conduct field research; Greg Hacker for help in the field; and W. Tanner Porter, Julie Wachara, and Thomas Lowrey for laboratory work.
This work was made possible by the generosity of the Bay Area Lyme Foundation.

\section{About the Author}

Dr. Salkeld at Colorado State University and Dr. Nieto at Northern Arizona University are interested in the ecology of tickborne diseases and the implications for human exposure and epidemiological risk.

\section{References}

1. Platonov AE, Karan LS, Kolyasnikova NM, Makhneva NA, Toporkova MG, Maleev VV, et al. Humans infected with relapsing fever spirochete Borrelia miyamotoi, Russia. Emerg Infect Dis. 2011;17:1816-23. http://dx.doi.org/10.3201/eid1710.101474

2. Krause PJ, Narasimhan S, Wormser GP, Rollend L, Fikrig E, Lepore T, et al. Human Borrelia miyamotoi infection in the United States. N Engl J Med. 2013;368:291-3. http://dx.doi.org/10.1056/ NEJMc1215469 
3. Salkeld DJ, Cinkovich S, Nieto NC. Tick-borne pathogens in northwestern California, USA. Emerg Infect Dis. 2014;20:493-4. http://dx.doi.org/10.3201/eid2003.130668

4. Padgett K, Bonilla D, Kjemtrup A, Vilcins I-M, Yoshimizu MH, Hui L, et al. Large scale spatial risk and comparative prevalence of Borrelia miyamotoi and Borrelia burgdorferi sensu lato in Ixodes pacificus. PLoS One. 2014;9:e110853. http://dx.doi.org/10.1371/ journal.pone.0110853

5. Salkeld DJ, Nieto NC, Carbajales-Dale P, Carbajales-Dale M, Cinkovich SS, Lambin EF. Disease risk and landscape attributes of tick-borne Borrelia pathogens in the San Francisco Bay area, California. PLoS One. 2015;10:e0134812. http://dx.doi.org/ 10.1371/journal.pone. 0134812

6. Krause PJ, Carroll M, Fedorova N, Brancato J, Dumouchel C, Akosa F, et al. Human Borrelia miyamotoi infection in California: serodiagnosis is complicated by multiple endemic Borrelia species. PLoS One. 2018;13:e0191725. http://dx.doi.org/10.1371/ journal.pone.0191725

7. Wagemakers A, Jahfari S, de Wever B, Spanjaard L, Starink MV, de Vries HJC, et al. Borrelia miyamotoi in vectors and hosts in the Netherlands. Ticks Tick Borne Dis. 2017;8:370-4. http://dx.doi.org/ 10.1016/j.ttbdis.2016.12.012

8. Taylor KR, Takano A, Konnai S, Shimozuru M, Kawabata H, Tsubota T. Borrelia miyamotoi infections among wild rodents show age and month independence and correlation with Ixodes persulcatus larval attachment in Hokkaido, Japan. Vector Borne Zoonotic Dis. 2013;13:92-7. http://dx.doi.org/10.1089/ vbz.2012.1027

9. Barbour AG, Bunikis J, Travinsky B, Hoen AG, Diuk-Wasser MA, Fish D, et al. Niche partitioning of Borrelia burgdorferi and Borrelia miyamotoi in the same tick vector and mammalian reservoir species. Am J Trop Med Hyg. 2009;81:1120-31. http://dx.doi.org/10.4269/ajtmh.2009.09-0208

10. Fedorova N, Kleinjan JE, James D, Hui LT, Peeters H, Lane RS. Remarkable diversity of tick or mammalian-associated Borreliae in the metropolitan San Francisco Bay area, California. Ticks Tick Borne Dis. 2014;5:951-61. http://dx.doi.org/10.1016/ j.ttbdis.2014.07.015

11. Salkeld DJ, Castro MB, Bonilla D, Kjemtrup A, Kramer VL, Lane RS, et al. Seasonal activity patterns of the western blacklegged tick, Ixodes pacificus, in relation to onset of human Lyme disease in northwestern California. Ticks Tick Borne Dis. 2014;5:790-6. http://dx.doi.org/10.1016/j.ttbdis.2014.05.002

12. Bunikis J, Garpmo U, Tsao J, Berglund J, Fish D, Barbour AG Sequence typing reveals extensive strain diversity of the Lyme borreliosis agents Borrelia burgdorferi in North America and Borrelia afzelii in Europe. Microbiology. 2004;150:1741-55. http://dx.doi.org/10.1099/mic.0.26944-0

13. Brown RN, Peot MA, Lane RS. Sylvatic maintenance of Borrelia burgdorferi (Spirochaetales) in northern California: untangling the web of transmission. J Med Entomol. 2006;43:743-51. http://dx.doi.org/10.1093/jmedent/43.4.743

14. Eisen L, Eisen RJ, Mun J, Salkeld DJ, Lane RS. Transmission cycles of Borrelia burgdorferi and B. bissettii in relation to habitat type in northwestern California. J Vector Ecol. 2009;34:81-91. http://dx.doi.org/10.1111/j.1948-7134. 2009.00010.x

15. Cook VJ, Fedorova N, Macdonald WP, Lane RS, Barbour AG. Unique strain of Borrelia miyamotoi in Ixodes pacificus ticks, California, USA. Emerg Infect Dis. 2016;22:2205-7. http://dx.doi.org/10.3201/eid2212.152046

Address for correspondence: Daniel J. Salkeld, Colorado State University, Department of Biology, 1878 Campus Delivery, Fort Collins, CO 80523, USA; email: dan.salkeld@colostate.edu

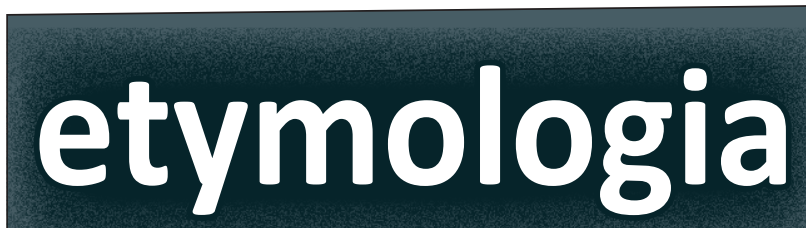

Etymology is

concerned with the

origin of words, how

they've evolved over time, and changed in form and meaning as they

were translated from one language to another.

Every month, EID publishes a feature highlighting the etymology of a word from medicine or public health.

\section{featured monthly in} EMERGING INFECTIOUS DISEASES

\section{http://wwwnc.edc.gov/eid/ articles/etymologia}




\section{I n-Host Adaptation of Salmonella enterica Serotype Dublin during Prosthetic Hip J oint I nfection}

\author{
Faten El Sayed, Guillaume Sapriel, Nizar Fawal, \\ Aurelia Gruber, Thomas Bauer, Beate Heym, \\ Caroline Dupont, Henri-Jean Garchon, \\ Jean-Louis Gaillard, Martin Rottman, \\ Simon Le Hello
}

Genome degradation has been central to the adaptation of Salmonella enterica serotypes to their hosts throughout evolution. We witnessed the patho-adaptation of a strain of Salmonella Dublin (a cattle-adapted serotype) to a human host during the course of a recurrent prosthetic hip joint infection evolving over several years.

Salmonella enterica serotype Dublin is a host-adapted Sbacterium with cattle as a predominant reservoir and is responsible for invasive, potentially life-threatening infections in humans $(1,2)$. In France, the epidemiology of Salmonella Dublin infections among humans corroborates surveillance data from the United States (1). Salmonella Dublin causes substantially more bloodstream infections and hospitalizations than other Salmonella serotypes. It is also much more likely to be isolated in metastatic foci of infection secondary to bacteremia. The prevalence of prosthetic joints in Salmonella Dublin patients is significantly greater than for other Salmonella cases (Table).

Host adaptation is central to pathogen evolution and is associated with gene acquisition, genome degradation (gene inactivation or deletion), or both. Genome degradation has played a major role in the adaptation of $S$. enterica serotypes Typhi and Paratyphi A to the human host throughout evolution (3). Degradation also has been recently reported in a severely immunocompromised patient in whom recurrent systemic Salmonella Enteritidis infections developed over 15 years (4). We report the phenotypic and genomic changes

Author affiliations: University of Versailles Saint-Quentin, Montigny-le-Bretonneux, France (F. El Sayed, G. Sapriel, B. Heym, H.-J. Garchon, J.-L. Gaillard); Hôpital Ambroise Paré, Boulogne-Billancourt, France (F. El Sayed, A. Gruber, T. Bauer,

B. Heym, C. Dupont, J.-L. Gaillard, M. Rottman); Atelier de Bioinformatique, Paris, France (G. Sapriel); Institut Pasteur, Paris (N. Fawal, S. Le Hello); Hôpital Raymond Poincaré,

Garches, France (M. Rottman)

DOI: https://doi.org/10.3201/eid2412.180214 undergone by Salmonella Dublin throughout a recurrent prosthetic hip infection in an immunocompetent patient.

\section{The Study}

In May 2011, a 74-year-old woman with bilateral hip prostheses (implanted in 1998 [right] and 2001 [left]) was admitted to intensive care for sepsis and left hip pain (online Technical Appendix Figure, https://wwwnc.cdc.gov/EID/ article/24/12/18-0214-Techapp1.pdf). Blood cultures and a joint aspiration yielded pure cultures of Salmonella spp. She underwent debridement and implant retention surgery, followed by a 6-week intravenous course of cefotaxime and ciprofloxacin. Three years later, she sought care at the emergency department with acute-onset fever and prosthesis joint infection of the right hip and underwent right hip debridement and implant retention surgery. Blood cultures, joint aspirates, and all interoperative periprosthetic tissue samples yielded Salmonella spp. The patient received 2 weeks of intravenous amoxicillin and oral ofloxacin, was discharged, and received oral antimicrobial drugs for 10 more weeks. Six weeks after surgery, the surgical wound was healed, and the patient walked normally. One year later (2015), her primary care physician referred her to the hospital because of night fevers without local signs or implant dysfunction. Radioleucoscintigraphy showed right hip inflammation. Bilateral hip biopsies were performed, and the right hip biopsy sample tested positive for Salmonella spp. A right hip 1-stage exchange procedure was performed. All intraoperative periprosthetic tissue samples yielded Salmonella spp. A 6-week course of intravenous therapy with ceftriaxone and ciprofloxacin was administered. One year later (2016), the patient appeared to be free from infection and walked normally.

We characterized isolates by matrix-assisted laser desorption/ionization time-of-flight mass spectrometry (Bruker Daltonik GmbH, Bremen, Germany) and serotyping (French National Reference Center for Salmonella). We assessed carbohydrate metabolic activity using the API50CH system (bioMérieux, Marcy-l'Etoile, France) and biofilm formation using crystal violet (5). Antimicrobial susceptibility testing was performed using 2015 EUCAST (European Committee on Antimicrobial Susceptibility Testing) guidelines (http:// www.eucast.org/clinical_breakpoints/). We conducted high-throughput whole-genome sequencing using the Illumina NextSeq 500 system (Illumina, San Diego, CA, USA). 
Table. Demographic characteristics of patients and sources for Salmonella enterica serotype Dublin and other Salmonella isolates, France, 2010-2016

\begin{tabular}{|c|c|c|c|}
\hline Patient characteristic & Salmonella Dublin, no (\%) & Other Salmonella, no. (\%) & $p$ value ${ }^{*}$ \\
\hline All cases, $N=63,264$ & 642 & 62,622 & \\
\hline \multicolumn{4}{|l|}{ Sex† } \\
\hline $\mathrm{F}$ & 266 & 30,762 & $<0.01$ \\
\hline M & 363 & 30,401 & \\
\hline \multicolumn{4}{|l|}{ Age group, y } \\
\hline$<1$ & $4(0.6)$ & $3,767(6.0)$ & $<0.01$ \\
\hline $1-5$ & $33(5.1)$ & $15,302(24.4)$ & $<0.01$ \\
\hline $6-14$ & $19(3.0)$ & $8,636(13.8)^{\prime}$ & $<0.01$ \\
\hline $15-64$ & $199(31.0)$ & $23,653(37.8)$ & NS \\
\hline$\geq 65$ & $375(58.4)$ & $9,801(15.7)$ & $<0.01$ \\
\hline Ūnknown & $12(1.9)$ & $1463(2.3)$ & NS \\
\hline \multicolumn{4}{|l|}{ Type of human sample§ा } \\
\hline Feces & $218(34.0)$ & $55,824(89.1)$ & $<0.01$ \\
\hline Blood & $264(41.1)$ & $3,268(5.2)$ & $<0.01$ \\
\hline Urine & $72(11.2)$ & $2,257(3.6)$ & $<0.01$ \\
\hline Articular & $20(3.1)$ & $30(0.05)$ & $<0.01$ \\
\hline Pus & $11(1.7)$ & $65(0.1)$ & $<0.01$ \\
\hline Bile & $1(0.2)$ & $32(<0.1)$ & NS \\
\hline Cerebrospinal fluid & 0 & $23(<0.1)$ & NS \\
\hline Other & $56(8.7)$ & $1123(1.8)$ & $<0.01$ \\
\hline Hospitalization & $368(57.3)$ & $20,556(32.8)$ & $<0.01$ \\
\hline \multicolumn{4}{|c|}{ 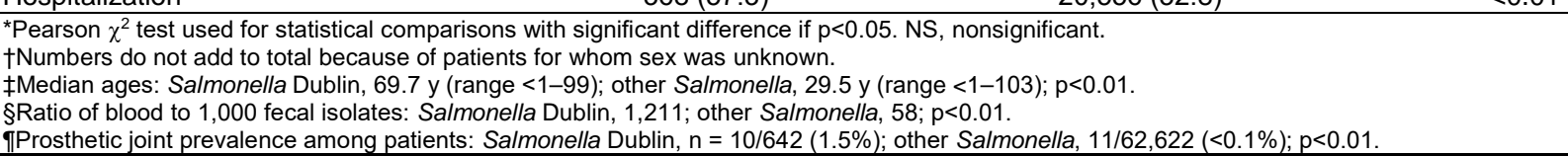 } \\
\hline
\end{tabular}

For each isolate, the paired-end reads were aligned against 2 Salmonella Dublin CT_020221853 and 3246 reference genomes to increase the single-nucleotide polymorphism (SNP) detection (GenBank accession nos. CP001144.1 and CM001151.1, respectively) (6) (online Technical Appendix). We annotated putative coding sequences using GeneMark (7) and performed ortholog identification, based on the prototypal human reference genome CT_020221853, using BLAST+ $(8)$ with a $1 \times 10^{-10}$ E-value threshold and confirmed by a synteny conservation check. We first identified candidate pseudogenized sequences using a proteome/ proteome BLASTp approach (https://blast.ncbi.nlm.nih.gov/ Blast.cgi): protein sequences of strains Str.2011, Str2014, and Str2015 with Query Coverage $<80 \%$ relative to the reference strain orthologs were selected as potentially pseudogenized sequences. We then analyzed nucleic acid level sequences corresponding to the previously selected candidates to confirm the change in coding sequences, and to precisely determine the genetic event kind of nucleic acid change involved (frame-shift mutation or indel).

We studied 3 patient strains: Str.2011 (May 2011, left hip intraoperative periprosthetic tissue [HIPT]), Str.2014 (April 2014, right HIPT), and Str.2015 (June 2015, right HIPT). Unlike Str.2011, Str.2014 and Str.2015 were nonmotile with no detectable $\mathrm{H}$ antigen and had lost the ability to use 9 of the 18 carbohydrates used by Str.2011, with slight profile variations (Figure). All strains could form biofilms and had identical wild-type antimicrobial drug susceptibility profiles and unchanged MICs for $\beta$-lactams and quinolones, without mutations in $\operatorname{gyr} A, \operatorname{gyr} B$, parC, and parE genes. All 3 strains belonged to the sequence type 10 Salmonella Dublin population (http://mlst.warwick.ac.uk/mlst/dbs/Senterica). The mapping of Str.2011, Str.2014, and Str.2015 genomes against the Salmonella Dublin CT_020221853 and str.3246 reference genomes detected 451 and 268 SNPs, respectively, whereas the 3 patient strains differed by only 6 SNPs, strongly suggesting they derived from a single infecting strain. Four SNPs were nonsynonymous in coding genes; 2 SNPs were in an intergenic region (Figure).

Comparative proteome/proteome analysis detected putative pseudogenized sequences that were due to indels within 3 coding sequences in Str.2014 and Str.2015. These pseudogenes were involved in carbohydrate transport (ptsA) (14-bp deletion), lipopolysaccharide (LPS) biosynthesis (waaY) (16-bp insertion), and protein synthesis (tufB) (790-bp deletion, total gene deletion); we identified no pseudogene among the genes of the flagellar regulon. The pseudogene involved in carbohydrate transport, ptsA, controls the import of carbohydrates, such as mannose, fructose, and N-acetyl-glucosamine (9), 3 carbohydrates used by Str.2011 but not Str.2014 or Str.2015. Mutation of the LPS biosynthesis gene waaY in Str.2014 and Str.2015 had apparently no effect on the expression of the Salmonella Dublin O antigen but might have affected flagellar assembly and function, as described for Salmonella Typhimurium and Escherichia coli $(10,11)$. The mutation of tufB in Str.2014 and Str.2015 leads to a one third decrease in the production of the translation elongation factor EF-Tu (the other two thirds is synthetized from tufA) (12). 
The patient in this study had no detectable gallbladder or intestinal carriage, which has been associated with recurrent Salmonella infections (13). She had no apparent immunodeficiency, a well-documented risk factor for invasive Salmonella Dublin infection. Salmonella Dublin most likely persisted as a biofilm at the surface of the implants, leading to recurrence of chronic disease despite prolonged antimicrobial therapy until the ablation of the infected material (14). The genomic changes we observed involved carbohydrate metabolism and LPS biosynthesis,

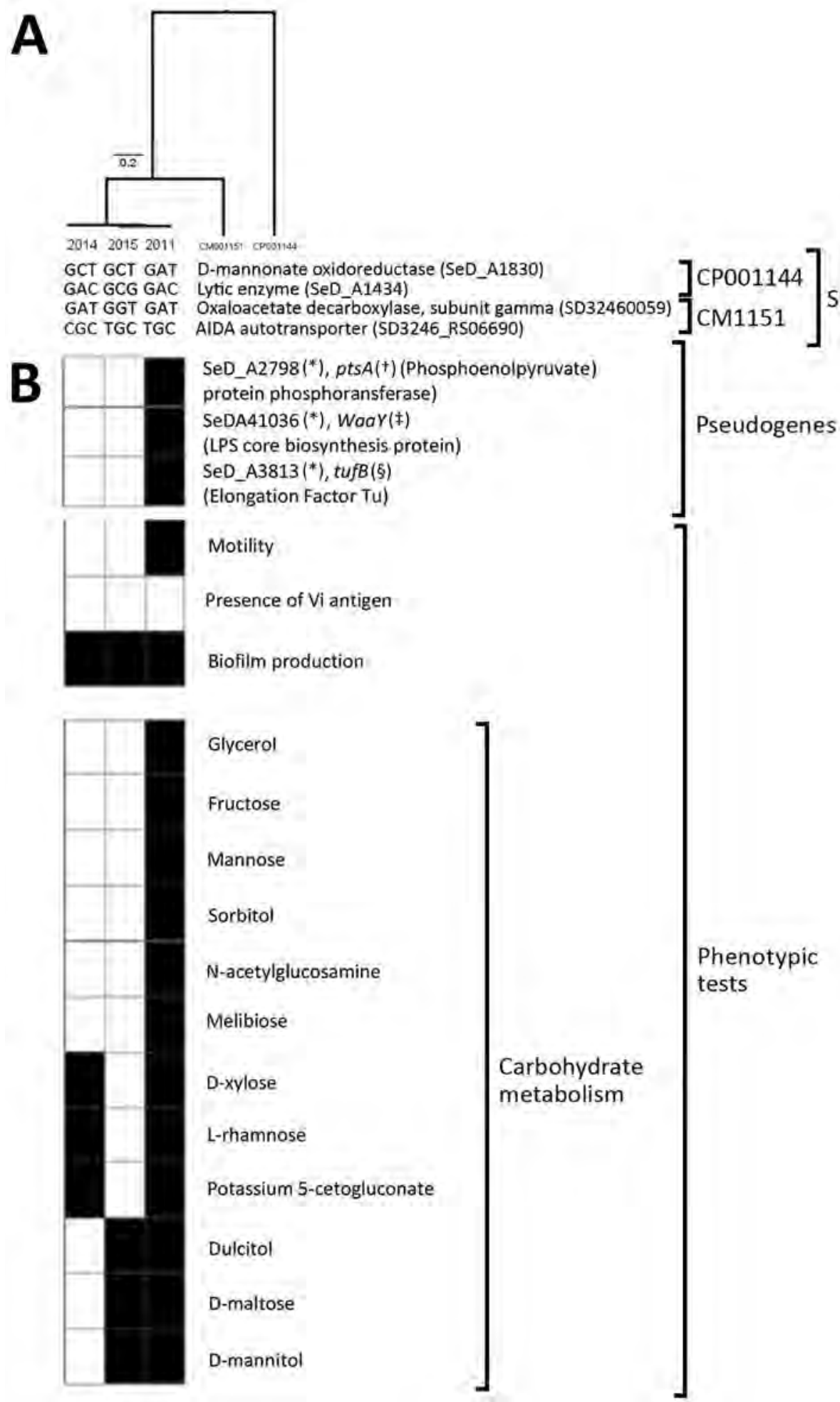

Figure. Genomic and phenotypic characteristics of Salmonella enterica serotype Dublin isolates Str.2011, Str.2014, and Str.2015 from a 74-year-old woman who had recurrent infections involving hip joint prostheses, France. A) Tree constructed after mapping the sequences of the isolates to reference genomes of Salmonella Dublin strains CT_020221853 and SNP 3246 (GenBank accession nos. CP001144.1 and CM001151.1, respectively). The 4 nonsynonymous SNPs and the corresponding coding genes are shown. Scale bar indicates genetic distance. B) Genes in Str.2011 and altered in Str.2014 and Str.2015 (reference strain Salmonella Dublin CT_02021853) are indicated by black and white boxes, respectively. Positive phenotypic tests are indicated as black boxes. For carbohydrate metabolism, only carbohydrates used by Str.2011 and not used by Str.2014 and/or Str.2015 are shown. *Gene ID annotation based on reference strain Salmonella Dublin str. CT 02021853 (GenBank accession no. CP001144.1). Genetic events found: †14-bp deletion; ‡16-bp insertion; §790-bp deletion (total gene deletion). SNP, singlenucleotide polymorphism.

Phenotypic tests 
as similarly reported for Salmonella Enteritidis isolates recovered from the bloodstream of a severely immunocompromised patient, years after the initial infection (4). Changes in carbon source availability are known to affect virulence gene regulators. In the presence/absence of a specific carbon source, specific virulence genes can be turned on or switched off, enabling pathogens to adapt to their new niche (9). Because LPS and flagellin are potent triggers of the inflammatory response, LPS alterations and the loss of flagellin expression (15) might have limited the host's innate immune response to Salmonella Dublin and facilitated its persistence at the implant interface.

\section{Conclusions}

This clinical case and other recent reports of patients with chronic infections highlight the remarkable adaptability of pathogens to a new niche. More specifically, these results show that the process of patho-adaptation of Salmonella serotypes may be extremely rapid and relies on mechanisms of genomic reshaping reminiscent of those found during the evolution of this pathogen in contact with humans. Enhanced awareness is warranted for Salmonella Dublin, especially in the elderly bearing prostheses.

\section{Acknowledgments}

We thank Vincent Enouf and Laetitia Fabre for processing the whole-genome sequencing and microbiological information, respectively. We are grateful to all the microbiological laboratories that participated in the human Salmonella network for processing the isolates.

This work was supported by the French National Reference Center for Escherichia coli, Shigella, and Salmonella, co-funded by Santé Publique France and belonging to the Integrative Biology of Emerging Infectious Diseases Laboratory of Excellence funded by the French Government Investissement d'Avenir program (grant no. ANR-10-LABX-62-IBEID).

Genomics sequence assemblies used in this project are available online on the National Center for Biotechnology Information network under accession no. PRJNA433355 (available at http://www.ncbi.nlm.nih.gov/bioproject/433355).

\section{About the Author}

Ms. El Sayed is a postgraduate fellow in clinical bacteriology of Ambroise Paré Teaching Hospital in Paris. Her research interests include bacteria adaptation and epidemiology in bone and joint infections context.

\section{References}

1. Harvey RR, Friedman CR, Crim SM, Judd M, Barrett KA, Tolar B, et al. Epidemiology of Salmonella enterica serotype Dublin infections among humans, United States, 1968-2013. Emerg Infect Dis. 2017;23:1493-501. http://dx.doi.org/10.3201/eid2309.170136
2. Crump JA, Sjölund-Karlsson M, Gordon MA, Parry CM. Epidemiology, clinical presentation, laboratory diagnosis, antimicrobial resistance, and antimicrobial management of invasive Salmonella infections. Clin Microbiol Rev. 2015;28:90137. http://dx.doi.org/10.1128/CMR.00002-15

3. McClelland M, Sanderson KE, Clifton SW, Latreille P, Porwollik S, Sabo A, et al. Comparison of genome degradation in Paratyphi A and Typhi, human-restricted serovars of Salmonella enterica that cause typhoid. Nat Genet. 2004;36:1268-74. http://dx.doi.org/10.1038/ng1470

4. Klemm EJ, Gkrania-Klotsas E, Hadfield J, Forbester JL, Harris SR, Hale C, et al. Emergence of host-adapted Salmonella Enteritidis through rapid evolution in an immunocompromised host. Nat Microbiol. 2016;1:1-6. http://dx.doi.org/10.1038/nmicrobiol.2015.23

5. Stepanović S, Cirković I, Ranin L, Svabić-Vlahović M. Biofilm formation by Salmonella spp. and Listeria monocytogenes on plastic surface. Lett Appl Microbiol. 2004;38:428-32. http://dx.doi. org/10.1111/j.1472-765X.2004.01513.x

6. Kuijpers LMF, Le Hello S, Fawal N, Fabre L, Tourdjman M, Dufour M, et al. Genomic analysis of Salmonella enterica serotype Paratyphi A during an outbreak in Cambodia, 2013-2015. Microb Genom. 2016;2:e000092. http://dx.doi.org/10.1099/mgen.0.000092

7. Besemer J, Lomsadze A, Borodovsky M. GeneMarkS: a selftraining method for prediction of gene starts in microbial genomes. Implications for finding sequence motifs in regulatory regions. Nucleic Acids Res. 2001;29:2607-18. http://dx.doi.org/10.1093/ nar/29.12.2607

8. Camacho C, Coulouris G, Avagyan V, Ma N, Papadopoulos J, Bealer K, et al. BLAST+: architecture and applications. BMC Bioinformatics. 2009;10:421. http://dx.doi.org/10.1186/1471-2105-10-421

9. Mitchell WJ. The phosphoenolpyruvate-dependent phosphotransferase system: a central feature of carbohydrate accumulation by enteric bacteria. Microbiol Sci. 1985;2:330-4, 339.

10. Deditius JA, Felgner S, Spöring I, Kühne C, Frahm M, Rohde M, et al. Characterization of novel factors involved in swimming and swarming motility in Salmonella enterica serovar Typhimurium. PLoS One. 2015;10:e0135351. http://dx.doi.org/10.1371/ journal.pone.0135351

11. Raetz CRH, Whitfield C. Lipopolysaccharide endotoxins. Annu Rev Biochem. 2002;71:635-700. http://dx.doi.org/10.1146/ annurev.biochem.71.110601.135414

12. Hughes D. Both genes for EF-Tu in Salmonella Typhimurium are individually dispensable for growth. J Mol Biol. 1990;215:41-51. http://dx.doi.org/10.1016/S0022-2836(05)80093-2

13. Crawford RW, Rosales-Reyes R, Ramírez-Aguilar ML, Chapa-Azuela O, Alpuche-Aranda C, Gunn JS. Gallstones play a significant role in Salmonella spp. gallbladder colonization and carriage. Proc Natl Acad Sci U S A. 2010;107:4353-8. http://dx.doi.org/10.1073/pnas.1000862107

14. McConoughey SJ, Howlin R, Granger JF, Manring MM, Calhoun JH, Shirtliff M, et al. Biofilms in periprosthetic orthopedic infections. Future Microbiol. 2014;9:987-1007. http://dx.doi.org/10.2217/fmb.14.64

15. Yim L, Sasías S, Martínez A, Betancor L, Estevez V, Scavone P, et al. Repression of flagella is a common trait in field isolates of Salmonella enterica serovar Dublin and is associated with invasive human infections. Infect Immun. 2014;82:1465-76. http://dx.doi.org/10.1128/IAI.01336-13

Address for correspondence: Simon Le Hello, Institut Pasteur, Unité de Recherche et d’Expertise des Bactéries Pathogènes Entériques, 28, rue du Docteur Roux, 75724 Paris CEDEX 15, France; email: simon. le-hello@pasteur.fr; Faten El Sayed, UFR des Sciences de la Santé Simone Veil, UVSQ, 2, avenue Source de la Bièvre, 78180 Montigny-leBretonneux, France; email: faten.elsayed@aphp.fr 


\section{Risk Factors for Acquiring Scrub Typhus among Children in Deoria and Gorakhpur Districts, Uttar Pradesh, I ndia, 2017}

\author{
Jeromie Wesley Vivian Thangaraj, \\ Ravi Vasanthapuram, Leonard Machado, \\ Govindakarnavar Arunkumar, Samir V. Sodha, \\ Kamran Zaman, Tarun Bhatnagar, \\ Shafeeq K. Shahul Hameed, Arun Kumar, \\ Jazeel Abdulmajeed, Anoop Velayudhan, \\ Avinash Deoshatwar, Anita S. Desai, \\ K. Hemanth Kumar, Nivedita Gupta, \\ Kayla Laserson, Manoj Murhekar; \\ Scrub Typhus Risk Factor Study Group ${ }^{1}$
}

Scrub typhus is associated with outbreaks of acute encephalitis syndrome in Uttar Pradesh, India. A case-control study indicated that children residing, playing, or visiting fields; living with firewood stored indoors; handling cattle fodder; and practicing open defecation were at increased risk for scrub typhus. Communication messages should focus on changing these behaviors.

$\mathrm{O}$ utbreaks of acute encephalitis syndrome (AES) with high case-fatality rates have been reported from Gorakhpur district, Uttar Pradesh, India, for $>2$ decades. These outbreaks occur during monsoon and postmonsoon seasons and predominantly affect children. Scrub typhus (ST) accounted for about two thirds of AES cases (1-4) and is also an important etiology of acute febrile illness (AFI) in the region (5). Untreated cases of ST-attributable AFI can progress to AES.

Author affiliations: Indian Council of Medical Research National Institute of Epidemiology, Chennai, India (J.W.V. Thangaraj, T. Bhatnagar, M. Murhekar); National Institute of Mental Health and Neurosciences, Bengaluru, India (R. Vasanthapuram, S.K.S. Hameed, A.S. Desai); World Health Organization, New Delhi, India (L. Machado, A. Kumar); Manipal Centre for Virus Research, Manipal Academy of Higher Education (Deemed to be University), Manipal, India (G. Arunkumar, J. Abdulmajeed, K.H. Kumar); US Centers for Disease Control and Prevention, New Delhi (S.V. Sodha, A. Velayudhan, K. Laserson); Indian Council of Medical Research National Institute of Virology, Gorakhpur, India (K. Zaman); Indian Council of Medical Research National Institute of Virology, Pune, India (A. Deoshatwar); Indian Council of Medical Research, New Delhi (N. Gupta)
ST, caused by the bacterium Orientia tsutsugamushi, is transmitted by the bite of trombiculid mites, which live in moist soil covered with vegetation (6). Several risk factors, including certain household characteristics, work-related practices, and behaviors, have been identified among adult ST patients (7-11). Household characteristics include location of the house near a grassland, vegetable field, or ditch; presence of mud floors; piled weeds inside the house; and scrub vegetation in the vicinity (7). Work-related practices include working in vegetable fields or hilly areas and working in short sleeves or with bare hands (8). Certain behaviors such as lying on grass and squatting to defecate or urinate also are associated with ST $(8,10,11)$. Because these risk factors are region specific, we conducted an exploratory case-control study among children in Gorakhpur and Deoria districts of Uttar Pradesh to identify factors associated with ST infection.

\section{The Study}

We conducted AFI surveillance in public health facilities in Deoria $(\mathrm{n}=5)$ and Gorakhpur $(\mathrm{n}=3)$ districts during October 3-November 11, 2017, a period coinciding with AES outbreaks in Gorakhpur district. We enrolled children 2-15 years of age with a $\geq 3$-day history of fever, from whom we collected $2 \mathrm{~mL}$ of blood after obtaining written informed consent from parents and assent from children 7-15 years of age. We screened serum samples for IgM and IgG against $O$. tsutugamushi by using ELISA kits (Scrub Typhus Detect; InBios International Inc., Seattle, WA, USA). For our study, an optical density value $\geq 0.5$ indicated IgM positivity. This cutoff has $93 \%$ sensitivity and $91 \%$ specificity for ST diagnosis (12). An optical density value $<0.5$ indicated IgM and IgG negativity.

Febrile children who were positive for O. tsutugamushi IgM were considered case-patients, whereas patients who were seronegative for IgM and IgG were considered controls. Case-patients and controls and their parents or guardians were interviewed in their houses by using a pretested structured questionnaire to collect information on sociodemographics, household characteristics, behaviors, and environmental exposures during the 2 weeks before fever onset. Interviewers were blinded to the case-patient or control status of children except during the first week of study. 
We calculated crude odds ratios (ORs) and 95\% CIs associated with different exposures. We included variables with $p$ value $\leq 0.2$ in univariate analysis in a stepwise backward elimination model to identify variables for inclusion in the final unconditional multiple logistic regression model by using Stata 13 (StataCorp LLC, College Station, TX, USA).

We recruited 819 AFI patients, of whom 155 (18.9\%) had O. tsutugamushi IgM (case-patients) and 409 (49.9\%) were seronegative (controls) (online Technical Appendix Table 1, https://wwwnc.cdc.gov/EID/article/24/12/18-0695Techapp1.pdf). We excluded 255 (31.1\%) patients who had only O. tsutugamushi IgG. We interviewed all case-patients and 406 controls. All case-patients and controls were from rural areas. The mean age of case-patients was higher than controls ( 7.0 vs. 6.3 years; $p=0.018$ ); $39 \%$ of case-patients and $51 \%$ controls were $\leq 5$ years of age. A higher proportion of case-patients than controls were from households that owned agricultural land (83\% vs. 73\%; $\mathrm{p}=0.017)($ Table 1$)$.

Aside from fever, clinical signs and symptoms among patients varied (online Technical Appendix Table 2). Five ST patients, who did not receive doxycycline/azithromycin, had onset of neurologic manifestations and required hospitalization; they recovered after administration of azithromycin.

Univariate analysis showed that ST patients were more likely than controls to live in houses located within or adjoining fields, houses with unpaved surroundings with mud or unkempt grass, and mud-floored houses (Table 2). In addition, a higher proportion of case-patients lived in households that used wood or cow dung for cooking and stored this fuel indoors. Compared with controls, children with ST were more likely to have played in agricultural fields, defecated in agricultural fields, bathed in a river or stream, carried grass bundles on their heads, handled fodder for cattle, or visited or accompanied parents to an agricultural field during the 2 weeks before fever onset (Table 2).
Multivariable analysis showed that children residing in houses within or adjoining fields (adjusted OR [aOR] $1.56,95 \%$ CI $1.02-2.38$ ) and that stored firewood indoors (aOR 1.61, 95\% CI 1.06-2.45) had higher odds of acquiring ST. Open defecation (aOR 2.0, 95\% CI 1.18-3.39), playing in (aOR 5.2, 95\% CI 1.92-14.2) or visiting (aOR $1.65,95 \%$ CI $1.07-2.52$ ) agricultural fields, and handling cattle fodder (aOR 2.05, 95\% CI 1.13-3.70) also were associated with ST (Table 2).

\section{Conclusions}

In Gorakhpur and Deoria districts, recent exposure to the outdoor environment, either while defecating or playing in agricultural fields, as well as visiting agricultural fields, storing firewood indoors, and handling fodder for cattle were associated with higher risk for acquiring ST among children. Of these risk factors, defecation in the agricultural field was the most common exposure at the population level. The observed association of ST and defecation in fields is consistent with the findings from an ST outbreak in Manipur, India, where persons who defecated or urinated in the jungle or bushy areas were found to be at higher risk for ST (11). In Gorakhpur and Deoria districts, about one third of the study population had toilets, a finding consistent with the 2015 national level survey, which indicated that $29.5 \%$ of the households in rural Uttar Pradesh had sanitary toilets and 27.3\% of households that had access to toilets were using them (http:// www.mdws.gov.in/sites/default/files/Swachh\%20Survekshan\%20Report\%20Eng.PDF). Efforts to prevent ST in the region therefore also need to focus on constructing household and community toilets, as well as behavior change communication about avoiding open defecation in the fields. In addition, storing firewood and fodder indoors attracts rodents that can harbor mites. Mites present on the firewood and fodder collected from fields might expose children to mite bites during storage or handling of the cattle fodder.

\begin{tabular}{|c|c|c|c|}
\hline Variables & $\%$ Case-patients, $n=155$ & $\%$ Controls, $n=406$ & $p$ value \\
\hline \multicolumn{4}{|l|}{ Age group, y } \\
\hline $2-5$ & 39 & 51 & \\
\hline $6-10$ & 46 & 35 & \\
\hline $11-15$ & 14 & 15 & \\
\hline Mean age, y (SD) & $7.0(3.2)$ & $6.3(3.5)$ & 0.0176 \\
\hline \multicolumn{4}{|l|}{ Sex } \\
\hline M & 61 & 61 & \\
\hline $\mathrm{F}$ & 39 & 39 & 0.882 \\
\hline \multicolumn{4}{|l|}{ District } \\
\hline Deoria & 56 & 55 & \\
\hline Gorakhpur & 44 & 45 & \\
\hline \multicolumn{4}{|l|}{ Religion } \\
\hline Hindu & 92 & 89 & \\
\hline Other & 8 & 11 & 0.348 \\
\hline Median duration of fever, $\mathrm{d}$ (IQR) & $7(4.5-10.0)$ & $5(4-8)$ & \\
\hline \multicolumn{4}{|l|}{ Household owns agricultural land } \\
\hline No & 17 & 27 & \\
\hline Yes & 83 & 73 & 0.017 \\
\hline
\end{tabular}

*Values are percentages unless otherwise indicated. IQR, interquartile range. 
Table 2. Risk factors associated with scrub typhus, Gorakhpur and Deoria districts, Uttar Pradesh, $2017^{\star}$

\begin{tabular}{|c|c|c|c|c|c|}
\hline Risk factor & $\begin{array}{c}\% \text { Cases, } \\
\mathrm{n}=155\end{array}$ & $\begin{array}{c}\% \text { Controls, } \\
n=406\end{array}$ & OR $(95 \% \mathrm{Cl})$ & $\begin{array}{c}\mathrm{p} \\
\text { value }\end{array}$ & $\operatorname{aOR}(95 \% \mathrm{Cl})$ \\
\hline Location of house within or adjoining field & 67 & 52 & $1.86(1.26-2.74)$ & 0.002 & $1.56(1.02-2.38)$ \\
\hline Mud or grassy approach road to house & 41 & 36 & $1.21(0.82-1.76)$ & 0.335 & \\
\hline Mud or grassy pavement in front of house & 81 & 71 & $1.73(1.09-2.74)$ & 0.018 & \\
\hline Mud house floor & 72 & 59 & $1.88(1.25-2.82)$ & 0.002 & \\
\hline Presence of waterbody within $100 \mathrm{~m}$ of house & 61 & 51 & $1.52(1.04-2.23)$ & 0.029 & \\
\hline \multicolumn{6}{|l|}{ Location of toilet } \\
\hline No toilet & 76 & 57 & $2.76(1.59-4.78)$ & 0.001 & \\
\hline Within house & 12 & 24 & 1 & & \\
\hline Outside house & 12 & 18 & $1.36(0.66-2.80)$ & & \\
\hline Wood or dung fuel & 55 & 34 & $2.32(1.59-3.39)$ & $<0.01$ & \\
\hline Storage of fuel (wood or dung) inside house or veranda & 53 & 34 & $2.16(1.48-3.14)$ & $<0.01$ & $1.61(1.06-2.45)$ \\
\hline Food waste given to cattle or disposed in common place & 30 & 25 & $1.27(0.84-1.92)$ & 0.248 & \\
\hline Spotted rats inside house, daily & 80 & 79 & $1.09(0.68-1.73)$ & 0.731 & \\
\hline Spotted rats outside house, daily & 43 & 48 & $0.81(0.56-1.17)$ & 0.263 & \\
\hline Presence of scrub vegetation around house & 79 & 73 & $1.38(0.88-2.17)$ & 0.154 & \\
\hline Storage of wet food produce inside house or veranda & 37 & 25 & $1.78(1.20-2.65)$ & 0.004 & \\
\hline Storage of dried food produce inside house or veranda & 83 & 72 & $1.87(1.17-2.99)$ & 0.009 & \\
\hline Livestock kept inside house or veranda & 32 & 22 & $1.65(1.09-2.48)$ & 0.017 & \\
\hline Fodder for livestock stored inside house or veranda & 32 & 24 & $1.49(0.99-2.24)$ & 0.055 & \\
\hline Place of drying clothes; bushes, ground, fence, or roof & 30 & 25 & $1.30(0.86-1.95)$ & 0.213 & \\
\hline Usual clothing at home for lower body; fully covered & 24 & 21 & $1.18(0.76-1.83)$ & 0.451 & \\
\hline Usual clothing at home for upper body; fully covered & 8 & 11 & $0.71(0.36-1.38)$ & 0.308 & \\
\hline Usual clothing for school for lower body; fully covered & 48 & 42 & $1.14(0.74-1.76)$ & 0.543 & \\
\hline Usual clothing for school for upper body; fully covered & 51 & 43 & $1.27(0.82-1.97)$ & 0.283 & \\
\hline Usual clothing during playing for lower body; fully covered & 21 & 17 & $1.26(0.79-1.99)$ & 0.334 & \\
\hline Usual clothing during playing for upper body; fully covered & 8 & 8 & $1.0(0.51-1.97)$ & 0.980 & \\
\hline Using clothing while sleeping for lower body; fully covered & 19 & 17 & $1.1(0.68-1.78)$ & 0.692 & \\
\hline Using clothing while sleeping for upper body; fully covered & 7 & 7 & $0.89(0.42-1.88)$ & 0.763 & \\
\hline Change of clothes before sleep; never or sometimes & 86 & 84 & $1.24(0.73-2.11)$ & 0.423 & \\
\hline Do not wear footwear during playing & 73 & 65 & $1.25(0.82-1.89)$ & 0.298 & \\
\hline Do not wear footwear while going to school & 6 & 4 & $1.37(0.59-3.19)$ & 0.465 & \\
\hline \multicolumn{6}{|l|}{ Place of play in 2 wks before illness } \\
\hline Indoor & 5 & 17 & 1 & 0.001 & \\
\hline Around house & 77 & 76 & $3.77(1.68-8.45)$ & & $2.68(1.15-6.27)$ \\
\hline Agricultural fields & 18 & 7 & $9.38(3.68-23.90)$ & & $5.20(1.92-14.15)$ \\
\hline \multicolumn{6}{|l|}{ Practice of washing after playing } \\
\hline Usually takes bath & 13 & 14 & 1 & & \\
\hline Usually washes hands or feet & 59 & 60 & $1.05(0.60-1.84)$ & 0.769 & \\
\hline None & 28 & 25 & $1.21(0.65-2.25)$ & 0.225 & \\
\hline Floor as usual place of sleeping & 14 & 11 & $1.41(0.81-2.44)$ & & \\
\hline \multicolumn{6}{|l|}{ Place of defecation in 2 wks before illness } \\
\hline Around house & 7 & 14 & $1.12(0.52-2.42)$ & 0.001 & $0.93(0.41-2.12)$ \\
\hline Field & 76 & 48 & $3.62(2.25-5.82)$ & & $2.00(1.18-3.39)$ \\
\hline Toilet & 17 & 38 & 1 & & 1 \\
\hline Early morning defecation & 30 & 25 & $1.28(0.85-1.93)$ & 0.237 & \\
\hline Swam or bathed in river or stream in 2 wks before illness & 11 & 5 & $2.37(1.21-4.67)$ & 0.012 & $1.74(0.80-3.77)$ \\
\hline Visited field in 2 wks before illness & 61 & 39 & $2.49(1.70-3.63)$ & $<0.01$ & $1.65(1.07-2.52)$ \\
\hline Carried bundle of grass over head in 2 wks before illness & 14 & 6 & $2.29(1.25-4.21)$ & $<0.01$ & $0.75(0.35-1.61)$ \\
\hline Handled cattle fodder in 2 wks before illness & 25 & 9 & $3.46(2.1-5.69)$ & $<0.01$ & $2.05(1.13-3.70)$ \\
\hline
\end{tabular}

Our study had limitations. First, although OT IgM is detectable as early as 4 days after fever onset (13), some ST patients in our study might have been negative for IgM and hence misclassified as controls. However, such nondifferential misclassification is likely to underestimate the actual association. Further, the median duration of fever was 7 days (interquartile range 4.5-10.0 days) among casepatients and 5 days (interquartile range 4-8 days) among controls (Table 1), indicating minimal possibility of such misclassification. Some controls might have been misclassified as case-patients because of persistence of IgM from previous infection (14). However, this possibility is less likely considering that IgM peaks by 4 weeks of infection and declines rapidly thereafter (15). Second, our controlpatients were AFI patients from healthcare facilities and not healthy children from the general population. Nevertheless, the behaviors of children visiting healthcare facilities before their febrile illness and children from the general population are less likely to be different.

For prevention of ST among children in Gorakhpur and Deoria districts, communication messages should focus on changing behaviors such as defecating or playing 
in agricultural fields and unnecessary visits to agricultural fields. Onset of central nervous system manifestations among untreated ST patients underscores the importance of early administration of doxycycline/azithromycin to ST patients in Gorakhpur and Deoria districts to prevent progression to AES.

Members of the Scrub Typhus Risk Factor Study Group: Vishal Shete, M. Prakash, Surya Prakash (Chennai, India); Reeta Mani, R. Vijayalakshmi, Narendra Kumar, Premanjali (Bangaluru, India); Ujjawal Sinha, Gagan Sharma, Hemant Kharnare, Abhishek Jain, Rachana Kathuria, A.K. Puttaraju, Manish K. Gawande, Ashish Tigga, Vishesh Kumar, Rajesh Badgal, Hamid Sayeed, Madhup Bajpai (New Delhi, India); Prasanth Pareet, Deepchand Agre, Nishikant Kumar (Manipal, India); Mohan Papanna, Ekta Saroha, Rajesh Yadav, Aslesh Prabhakaran (New Delhi, India); and Hirawati Deval and Gajanan Patil (Gorakhpur, India).

This study was funded by the Indian Council of Medical Research and a US Centers for Disease Control and Prevention (CDC) Cooperative Agreement for Global Health Security to the National Institute of Mental Health and Neurosciences.

The Institutional Ethics Committee of the Indian Council of Medical Research National Institute of Epidemiology approved the study protocol. The CDC Center for Global Health Associate Director for Science reviewed the protocol and determined that CDC was not engaged.

\section{About the Author}

Dr. Thangaraj currently works as a scientist with the Indian Council of Medical Research-National Institute of Epidemiology in Chennai, India. His primary research interests include the epidemiology of acute encephalitis syndrome.

\section{References}

1. Murhekar MV, Mittal M, Prakash JAJ, Pillai VM, Mittal M, Girish Kumar CP, et al. Acute encephalitis syndrome in Gorakhpur, Uttar Pradesh, India—role of scrub typhus. J Infect. 2016;73:6236. http://dx.doi.org/10.1016/j.jinf.2016.08.014

2. Mittal M, Thangaraj JWV, Rose W, Verghese VP, Kumar CPG, Mittal M, et al. Scrub typhus as a cause of acute encephalitis syndrome, Gorakhpur, Uttar Pradesh, India. Emerg Infect Dis. 2017;23:1414-6. http://dx.doi.org/10.3201/eid2308.170025

3. Pulla P. Disease sleuths unmask deadly encephalitis culprit. Science. 2017;357:344. http://dx.doi.org/10.1126/science.357. 6349.344
4. Mittal M, Bondre V, Murhekar M, Deval H, Rose W, Verghese VP, et al. Acute encephalitis syndrome in Gorakhpur, Uttar Pradesh, 2016: clinical and laboratory findings. Pediatr Infect Dis J. 2018;37:1101-6.

5. Vivian Thangaraj JW, Mittal M, Verghese VP, Kumar CPG, Rose W, Sabarinathan R, et al. Scrub typhus as an etiology of acute febrile illness in Gorakhpur, Uttar Pradesh, India, 2016. Am J Trop Med Hyg. 2017;97:1313-5. http://dx.doi.org/10.4269/ ajtmh.17-0135

6. Rahi M, Gupte MD, Bhargava A, Varghese GM, Arora R. DHR-ICMR Guidelines for diagnosis \& management of Rickettsial diseases in India. Indian J Med Res. 2015;141:417-22. http://dx.doi.org/10.4103/0971-5916.159279

7. Lyu Y, Tian L, Zhang L, Dou X, Wang X, Li W, et al. A casecontrol study of risk factors associated with scrub typhus infection in Beijing, China. PLoS One. 2013;8:e63668-63668. http://dx.doi.org/10.1371/journal.pone.0063668

8. Kweon S-S, Choi J-S, Lim H-S, Kim J-R, Kim K-Y, Ryu S-Y, et al. A community-based case-control study of behavioral factors associated with scrub typhus during the autumn epidemic season in South Korea. Am J Trop Med Hyg. 2009;80:442-6. http://dx.doi.org/10.4269/ajtmh.2009.80.442

9. Trowbridge P, P D, Premkumar PS, Varghese GM. Prevalence and risk factors for scrub typhus in South India. Trop Med Int Health. 2017;22:576-82. http://dx.doi.org/10.1111/tmi.12853

10. Varghese GM, Raj D, Francis MR, Sarkar R, Trowbridge P, Muliyil J. Epidemiology \& risk factors of scrub typhus in south India. Indian J Med Res. 2016;144:76-81. http://dx.doi.org/ 10.4103/0971-5916.193292

11. Singh SI, Devi KP, Tilotama R, Ningombam S, Gopalkrishna Y, Singh TB, et al. An outbreak of scrub typhus in Bishnupur district of Manipur, India, 2007. Trop Doct. 2010;40:169-70. http://dx.doi.org/10.1258/td.2010.090468

12. Blacksell SD, Tanganuchitcharnchai A, Nawtaisong P, Kantipong P, Laongnualpanich A, Day NPJ, et al. Diagnostic accuracy of the InBios Scrub Typhus Detect enzyme-linked immunoassay for the detection of IgM antibodies in Northern Thailand. Clin Vaccine Immunol. 2015;23:148-54. http://dx.doi.org/10.1128/CVI.00553-15

13. Ching WM, Wang H, Eamsila C, Kelly DJ, Dasch GA. Expression and refolding of truncated recombinant major outer membrane protein antigen (r56) of Orientia tsutsugamushi and its use in enzyme-linked immunosorbent assays. Clin Diagn Lab Immunol. 1998;5:519-26.

14. Varghese GM, Rajagopal VM, Trowbridge P, Purushothaman D, Martin SJ. Kinetics of IgM and IgG antibodies after scrub typhus infection and the clinical implications. Int J Infect Dis. 2018;71:535. http://dx.doi.org/10.1016/j.ijid.2018.03.018

15. Kim DM, Lee Y-M, Back J-H, Yang TY, Lee JH, Song H-J, et al. A serosurvey of Orientia tsutsugamushi from patients with scrub typhus. Clin Microbiol Infect. 2010;16:447-51. http://dx.doi.org/10.1111/j.1469-0691.2009.02865.x

Address for correspondence: Kayla Laserson, US Centers for Disease Control and Prevention, American Embassy, Shantipath, Chanakyapuri, New Delhi 110021, India; email: klaserson@cdc.gov 


\section{Using PCR-Based Sequencing to Diagnose Haycocknema perplexum Infection in Human Myositis Case, Australia}

\section{Anson V. Koehler, Peter Leung, Belinda McEwan, Robin B. Gasser}

We report a case of myositis in a male patient in Australia who had progressive weakness and wasting in his left lower limb. Although clinical, pathological, and laboratory assessments were inconclusive, a new, nested PCR-coupled sequencing method enabled the unequivocal diagnosis of myositis caused by the enigmatic nematode Haycocknema perplexum.

$I^{2}$ n 2017, a 37-year-old male resident of the eastern coast of Tasmania, Australia, arrived at Royal Hobart Hospital reporting a 2-year history of progressive muscle weakness and wasting in his left lower limb. He had also developed weakness in his left upper limb 6 months before clinical examination. Initially, he underwent a neurologic assessment for motor neurone disease before being referred to the hospital. The patient is a recreational hunter and consumes bushmeat; his only travel history was to Melbourne, Victoria, Australia.

\section{The Study}

Clinical examination revealed that this patient walked with a mild limp and a high stepping gait on the left. The Romberg test result was unremarkable, and tandem gait and coordination were normal. There was marked wasting of the left vastus lateralis and the gastrocnemius muscles and mild wasting of the left biceps and triceps muscles. There were no associated fasciculations. Muscle power was reduced on the left side, with weakness of hip flexion $(3+/ 5)$, knee extension $(4+/ 5)$, and flexion $(3+/ 5)$. The patient was unable to squat fully. Reflexes were reduced on the left compared with the right side, and plantar reflexes were recorded as normal bilaterally. No objective sensory deficit was detected. Cranial nerve function, specifically extraocular muscles, speech, and tongue power and movement, appeared normal. Axial muscles appeared to function normally.

Author affiliations: The University of Melbourne, Parkville, Victoria, Australia (A.V. Koehler, R.B. Gasser); Royal Hobart Hospital, Hobart, Tasmania, Australia (P. Leung, B. McEwan)

DOI: https://doi.org/10.3201/eid2412.181240
Complementary serologic tests revealed no evidence of antibodies for Toxocara, Toxoplasma, Trichinella, or Strongyloides. Test results for serum calcium, magnesium, and phosphate were within reference ranges, but serum creatinine kinase was elevated at 3,636 IU/L (reference range 22-198). Electrophoretic analysis for multiple myeloma revealed a diffuse increase in gamma globulin but no paraprotein. Liver function tests revealed that levels of alanine aminotransferase (139 IU/L) and aspartate aminotransferase (94 IU/L) were elevated, whereas urea electrolytes and creatinine were within reference ranges. Full blood examination revealed a transient mild eosinophilia $\left(0.54 \times 10^{9}\right.$ cells $\left./ \mathrm{L}\right)$.

Magnetic resonance imaging did not detect abnormalities in the brain or spinal cord, although a posterior disk protrusion relating to a slight central canal stenosis was observed. Nerve conduction results were within reference range, but electromyographic evaluations revealed active and chronic denervation changes confined to the left upper and lower limbs, consistent with a myopathic process. Histopathologic examination of a biopsy specimen $\left(20 \times 20 \times 5 \mathrm{~mm}^{2}\right)$ taken from the left vastus lateralis muscle revealed a chronic, destructive myopathy with inflammation, including eosinophils. This examination detected variation in muscle fiber size and patchy foci of inflammatory cell infiltrates, predominantly lymphocytic, but also with scattered eosinophils and some active myocyte destruction. There was an increase in interstitial fibrosis; many of the fibers appeared degenerative, some showed extensive vacuolation, and others were in the process of regenerating. Fibers appeared split, and some of them had internal nuclei. Occasional fibers had inclusions within the sarcoplasm, which appeared rounded, with central irregular eosinophilic and brown areas. No glycogen or lipid was found within vacuoles, and there was evidence of some cytochrome oxidase depletion. The muscle appeared to be type I dominant with atrophic type 1 and 2 fibers.

On the basis of these findings, we made a diagnosis of chronic destructive myopathy with inflammation (including eosinophils), with differential diagnoses of inclusion body myositis and parasitic myositis. We conducted examinations for autoimmune disorders, but results for dsDNA, 
antinuclear antibody, and extractable nuclear antigen tests were within reference ranges.

When the patient's condition showed no clinical improvement, we administered therapeutic doses of albendazole (400 mg $2 \times /$ d) for 3 months. The patient's clinical status improved, and creatinine kinase levels in serum decreased to $387 \mathrm{IU} / \mathrm{L}$. He remained on surveillance as an outpatient for 7 months. On the basis of this clinical evidence and the patient's history, we suspected an infection with $H$. perplexum nematodes.

From a 2- $\mathrm{mm}^{3}$ portion of the same muscle biopsy sample used for the previous histopathologic examination and frozen, we extracted genomic DNA using the DNeasy PowerSoil Kit (QIAGEN, Venlo, the Netherlands) and then subjected 2- $\mu \mathrm{L}$ aliquots of this DNA sample to 2 new nested PCR assays adapted from an established method (1-3) that had not amplified products from the same sample. The 2 optimized nested PCRs (Table 1 ) successfully amplified products of the expected sizes ( $\approx 400$ bp for cox- 1 and $\approx 830$ bp for $S S U$ ) from this sample, and these amplicons were directly sequenced (3).

We assessed the sequences obtained (GenBank accession nos. MH667568 [cox-1] and MH667568 [SSU]) for quality, compared them (using blastn, https://www.ncbi. nlm.nih.gov/BLAST) with publicly available sequences in the GenBank database to confirm the diagnosis of H. perplexum nematode infection, and then aligned them with respective reference sequences from this database, as described previously (3), to assess genetic variability. From the resultant cox-1 amplicon, we obtained 385 bp of clean sequence that varied by $1.3 \%-5.7 \%$ from previously determined cox-1 sequences from Haycocknema nematodes (GenBank accession nos. KU531719 and KU531720, representing Tasmania and Queensland, respectively [3]). As expected, the $c o x-1$ sequences from samples from Tasmania were more closely related to one another than to 1 from Queensland. A comparison of all three cox-1 sequences of $H$. perplexum nematodes (>348 nt) revealed 1 nonsynonymous and 24 synonymous mutations. From the resultant
SSU amplicon, we obtained 831 bp of clean sequence, which varied by $0.5 \%-0.6 \%$ from $S S U$ sequences reported previously for Haycocknema nematodes (GenBank accession nos. KU531721 and KU531722 representing Tasmania and Queensland, respectively [3]), whereas the sequences KU531721 and KU531722 differed by only $0.1 \%$ (1 nt).

\section{Conclusions}

This case was consistent clinically with some previous cases (3-9) associated with H. perplexum infection in Tasmania and Queensland (Table 2). PCR-coupled sequencing enabled an etiologic diagnosis, although this diagnosis was not possible based on clinical, clinicopathologic, and histopathologic findings. The analytical sensitivity of the nested PCRs allowed for the ready and specific detection of $H$. perplexum DNA within the muscle DNA sample. The genetic distinctiveness recorded here was also seen previously between samples from cases from Tasmania and Queensland. Although it is speculative, this information lends additional support for some population genetic substructuring within Haycocknema (because of geographic or reproductive isolation). It is possible that the 3 distinct genotypes recorded may differ in their biology, host affiliation, transmission pattern(s), or a combination of these factors if they turn out to be transmitted from animals to humans.

There has been considerable controversy surrounding the biology and transmission of $H$. perplexum nematodes $(3,5)$. It appears that only 4 of 9 cases of human infection recorded to date (the case we report and 3 previous cases $[7,8,10])$ had histories of the patients consuming bushmeat, which suggests the possibility of other routes of transmission. Other than locations in Tasmania and northern Queensland, the common thread among most clinical cases is an association with an exposure to native wildlife (e.g., through hunting, bush walking, caring for wildlife, keeping wildlife as pets, doing botanical fieldwork, or consuming native bushmeat $[3,4,6-9])$. However, Koehler et al. (3) suggested that $H$. perplexum nematodes might use an

\begin{tabular}{|c|c|c|c|c|c|}
\hline Designation & Primer pair & Oligonucleotide sequence, $5^{\prime} \rightarrow 3^{\prime}$ & $\begin{array}{c}\text { Annealing } \\
\text { temperature, }{ }^{\circ} \mathrm{C}\end{array}$ & $\begin{array}{l}\text { Expected } \\
\text { size, bp }\end{array}$ & Reference \\
\hline \multicolumn{6}{|l|}{$\operatorname{cox}-1$} \\
\hline \multirow{2}{*}{$1^{\circ} \mathrm{PCR}$} & RhigoCoxF & TTTTTTGGACATCCTGAGGTGTAT & & & (3) \\
\hline & RhigoCoxR & CAGACTCAACACATAATGAAAATG & 47 & 450 & (3) \\
\hline \multirow[t]{2}{*}{$2^{\circ} \mathrm{PCR}$} & HPC01F & GGACATCCTGAGGTGTATAT & & & This study \\
\hline & HPCO1R & AATGAAAATGTCCTACCACA & 50 & 400 & This study \\
\hline \multicolumn{6}{|c|}{ (2) } \\
\hline \multirow[t]{2}{*}{$1^{\circ} \mathrm{PCR}$} & Nem18sF & CGCGAATRGCTCATTACAACAGC & & & (1) \\
\hline & 1912R & TTTACGGTCAGAACTAGGG & 54 & 900 & (2) \\
\hline \multirow[t]{2}{*}{$2^{\circ} \mathrm{PCR}$} & $1096 \mathrm{~F}$ & GGTAATTCTGGAGCTAATAC & & & (2) \\
\hline & Nem18sR & GGGCGGTATCTGATCGCC & 54 & 830 & (1) \\
\hline
\end{tabular}

*All PCR used 35 cycles with initial extension of 5 min. All cycles were $30 \mathrm{~s}$ except that SSU had a 1 min extension for PCRs. cox-1, cytochrome $c$ oxidase 1; SSU, small subunit of nuclear ribosomal RNA. 
Table 2. Recorded human cases of Haycocknema perplexum infection in Australia $(8,9)^{\star}$

\begin{tabular}{|c|c|c|c|c|c|c|c|c|}
\hline $\begin{array}{l}\text { Patient } \\
\text { no., age, } \\
\text { y/sex }\end{array}$ & $\begin{array}{c}\text { Year of } \\
\text { diagnosis }\end{array}$ & State $\dagger$ & Travel & Animal contact & $\begin{array}{l}\text { Symptom } \\
\text { duration, y }\end{array}$ & $\begin{array}{l}\text { Maximum } \\
\text { CK, U/L } \ddagger\end{array}$ & $\begin{array}{l}\text { Eosinophils, } \\
\times 10^{9} \text { cells } / \mathrm{L}\end{array}$ & Reference \\
\hline $1,33 / \mathrm{F}$ & 1994 & TAS & Global, NT & $\begin{array}{l}\text { Botanist, fieldwork, } \\
\text { vegetarian, some } \\
\text { bushmeat consumption }\end{array}$ & 5 & 3,294 & 0.8 & $(4,6,10)$ \\
\hline $2,48 / \mathrm{M}$ & 1996 & TAS & $\begin{array}{c}\text { Far north QLD, } \\
\text { NT }\end{array}$ & NA & 1.5 & 1,586 & 2.0 & $(4-6)$ \\
\hline $3,61 / \mathrm{M}$ & 2004 & QLD & $\begin{array}{c}\text { None in } 20 \\
\text { years, raised in } \\
\text { TAS }\end{array}$ & NA & 3 & 1,263 & High & $(6,7)$ \\
\hline $4,23 / F$ & 2005 & QLD & WA, NSW, VIC & NA & 2 & 1,370 & 1.1 & (7) \\
\hline $5,61 / \mathrm{M}$ & 2006 & QLD & Regional & $\begin{array}{l}\text { Never consumed } \\
\text { bushmeat }\end{array}$ & 2 & 1,230 & 1.36 & $(7)$ \\
\hline $6,50 / \mathrm{M}$ & 2011 & TAS & $\begin{array}{l}\text { Born Tanzania; } \\
\text { Ireland, NSW }\end{array}$ & $\begin{array}{l}\text { Extensive contact with } \\
\text { native animals }\end{array}$ & 2 & 5,700 & Normal & (8) \\
\hline $7,72 / \mathrm{M}$ & 2015 & TAS & NA & Recreational hunter & $>2$ & 2,082 & 2.44 & (3) \\
\hline $8,30 / \mathrm{M}$ & 2015 & QLD & Regional & $\begin{array}{l}\text { Never consumed } \\
\text { bushmeat }\end{array}$ & 2 & 3,400 & 1.24 & (3) \\
\hline $9,80 / F$ & 2012 & QLD & Extensive TAS & Native animal carer & 1.5 & 270 & 0.7 & (9) \\
\hline $10,37 / \mathrm{M}$ & 2016 & TAS & VIC & Recreational hunter & 2 & 3,636 & 0.54 & This study \\
\hline $\begin{array}{l}{ }^{*} \mathrm{CK}, \text { creatir } \\
\text { Western Au } \\
\text { †All patient } \\
\text { †Reference } \\
\text { \&Reference }\end{array}$ & $\begin{array}{l}\text { inase; NA } \\
\text { a. } \\
\text { QLD wer } \\
\text { e }<170 \text {. } \\
\text { e }<0.4 \text {. }\end{array}$ & & $\begin{array}{l}\text { NSW, New South } \\
\text { n far north QLD. }\end{array}$ & & & & & \\
\hline
\end{tabular}

arthropod for transmission to humans. Clearly, the mysteries surrounding $H$. perplexum nematodes, including taxonomic classification, biology, reservoir host animals, and transmission to humans to cause disease, provide exciting paths for future research. The nested PCR-based sequencing approach we used will aid in such research.

\section{Acknowledgments}

We thank Dale Sebire for support and Catriona McLean for providing histopathologic service.

This work was supported by grants from the National Health and Medical Research Council (NHMRC) and Australian Research Council (ARC), Melbourne Water Corporation, and Yourgene Biosciences.

\section{About the Author}

Dr. Koehler is a molecular parasitologist in the Gasser Lab in the Department of Veterinary Biosciences at The University of Melbourne, Australia. His research interests include the phylogenetics of parasites.

\section{References}

1 Floyd RM, Rogers AD, Lambshead PJD, Smith CR. Nematode-specific PCR primers for the $18 \mathrm{~S}$ small subunit rRNA gene. Mol Ecol Notes. 2005;5:611-2. http://dx.doi.org/10.1111/ j.1471-8286.2005.01009.x

2. Holterman M, van der Wurff A, van den Elsen S, van Megen H, Bongers T, Holovachov O, et al. Phylum-wide analysis of SSU rDNA reveals deep phylogenetic relationships among nematodes and accelerated evolution toward crown clades.
Mol Biol Evol. 2006;23:1792-800. http://dx.doi.org/10.1093/ molbev/msl044

3. Koehler AV, Spratt DM, Norton R, Warren S, McEwan B, Urkude R, et al. More parasitic myositis cases in humans in Australia, and the definition of genetic markers for the causative agents as a basis for molecular diagnosis. Infect Genet Evol. 2016;44:69-75. http://dx.doi.org/10.1016/j.meegid.2016.06.026

4. Dennett X, Siejka SJ, Andrews JR, Beveridge I, Spratt DM. Polymyositis caused by a new genus of nematode. Med J Aust. 1998;168:226-7.

5. Spratt DM. Australian ecosystems, capricious food chains and parasitic consequences for people. Int J Parasitol. 2005;35:717-24. http://dx.doi.org/10.1016/j.ijpara.2005.01.014

6. Spratt DM, Beveridge I, Andrews JR, Dennett X. Haycocknema perplexum n. g., n. sp. (Nematoda: Robertdollfusidae): an intramyofibre parasite in man. Syst Parasitol. 1999;43:123-31. http://dx.doi.org/10.1023/A:1006158218854

7. Basuroy R, Pennisi R, Robertson T, Norton R, Stokes J, Reimers J, et al. Parasitic myositis in tropical Australia. Med J Aust. 2008;188:254-6.

8. McKelvie P, Reardon K, Bond K, Spratt DM, Gangell A, Zochling $\mathrm{J}$, et al. A further patient with parasitic myositis due to Haycocknema perplexum, a rare entity. J Clin Neurosci. 2013;20:1019-22. http://dx.doi.org/10.1016/j.jocn.2012.08.009

9. Vos LJ, Robertson T, Binotto E. Haycocknema perplexum: an emerging cause of parasitic myositis in Australia. Commun Dis Intell Q Rep. 2016;40:E496-9.

10. Andrews JR, Ainsworth R, Abernethy D. Trichinella pseudospiralis in humans: description of a case and its treatment. Trans R Soc Trop Med Hyg. 1994;88:200-3. http://dx.doi.org/10.1016/ 0035-9203(94)90295-X

Address for correspondence: Robin B. Gasser, The University of Melbourne, Department of Veterinary Biosciences, Melbourne Veterinary School, Faculty of Veterinary and Agricultural Sciences, Corner of Park Drive and Flemington Road, Parkville, Victoria 3010, Australia; email: robinbg@unimelb.edu.au 


\section{Chuzan Virus in Yaks, Qinghai-Tibetan Plateau, China}

\author{
Meng Wang, Yun Wang, Abdul Rasheed Baloch, \\ Yangyang Pan, Lili Tian, Fang $\mathrm{Xu}$, \\ Shaobo Chen, Qiaoying Zeng
}

Author affiliations: Gansu Agricultural University, Lanzhou, China (M. Wang, Y. Pan, F. Xu, Q. Zeng); Anning Branch Lanzhou General Hospital, Lanzhou (Y. Wang); University of South Bohemia in České Budějovice, Zátiší Vodňany, Czech Republic (A.R. Baloch); China Animal Health and Epidemiology Center, Qingdao, China (L. Tian); Veterinary Department of Gansu Province, Lanzhou (S. Chen)

DOI: https://doi.org/10.3201/eid2412.171414

We detected Chuzan virus (CHUV) in domestic yaks from the Qinghai-Tibetan Plateau, western China, indicating CHUV probably has been transmitted to yaks in recent years. Awareness for CHUV surveillance and transmission and livestock health management in these special regions should be raised to avoid outbreaks and animal loss.

C huzan virus (CHUV) belongs to the Palyam serogroup of genus Orbivirus, family Reoviridae. A CHUV outbreak, first reported in Japan in 1985, was the causative agent of disease that resulted in many reproductive disorders in cattle, including abortion, stillbirth, and congenital malformation (1) and in considerable economic loss in the cattle industry.

Like other orbiviruses, CHUV consists of 10 doublestranded RNA segments (Seg-1 to Seg-10), which encode 7 structural viral proteins (VP1-VP7) and 4 nonstructural proteins (NS1-NS4) (2-4). Seg-2 (VP2) and Seg-6 (VP5) of the Palyam serogroup show the highest levels of variation in genome sequence, which correlates with virus serotype specificity (2). These viruses usually are transmitted by arthropod vectors $(5,6)$. Therefore, CHUV has been widespread in many countries of Asia, such as South Korea (7) and mainland China $(8,9)$, which have reported CHUV infection in cattle. However, no information was available about CHUV in yaks (Bos grunniens) on China's QinghaiTibetan Plateau.

Yaks are an important livestock in the Qinghai-Tibetan Plateau. They have been farmed with other livestock, such as Tibetan sheep and Tibetan pigs. The high prevalence of bluetongue virus (BTV) infection, also belonging to genus Orbivirus, has been reported in yaks and Tibetan sheep (10). A study in 2016 found an abortion rate in yaks of $21.39 \%$ in part of Qinghai Province, presumably because of the high prevalence of BTV and other related pathogens (10). All these data and CHUV infection in cattle in China motivated us to study whether CHUV infects yaks.

During August 2016-April 2017, we randomly collected 208 blood samples from apparently healthy domestic yaks, 71 yaks from Gansu Province $(46<1$ year of age), 64 yaks from Qinghai Province $(23<1$ year of age), and 73 yaks from Sichuan Province $(29<1$ year of age). Soon after sampling, total RNAs were extracted and used as templates to amplify full-length cDNA by reverse transcription PCR (RT-PCR; SuperScript III Synthesis Kit, Invitrogen, Carlsbad, CA, USA). One pair of specific primers was designed based on VP2 genome sequence of CHUV (online Technical Appendix Table 1, https://wwwnc.cdc. gov/EID/article/24/12/17-1414-Techapp1.pdf) and used to detect CHUV in yaks. We also performed serologic assay by using the CHUV 2nd detection kit (iNtRON, IPC11028, Gyeonggi-do, South Korea), and the results of the assay were then authenticated by RT-PCR.

For phylogenetic and identity analysis of genome sequence of 10 segments from CHUV, we designed 10 pairs of primer based on known sequences deposited in GenBank (online Technical Appendix Table 1) to obtain the open reading frame genome of these proteins. Phylogenetic and identity analyses were performed based on these genome sequences and the corresponding sequences available in GenBank.

Five $(7 \%)$ of the 71 samples were positive for CHUV in Gansu Province, 4 of which were $<1$ year of age (Figure; online Technical Appendix Table 2). CHUV infection in yaks was not found in Qinghai and Sichuan provinces. Relatively low prevalence of CHUV infection in yaks is consistent with the report of CHUV infection in cattle in South Korea (7), which was also significantly lower than BTV infection in yaks (17.34\%) (10). We also obtained similar results in serologic investigation. We observed neither illness nor disease in these yaks; our results are consistent with CHUV infection in sentinel cattle (8) and thus suggest that subclinical infection of CHUV occurs in cattle. We used heparinized blood samples to inoculate baby hamster kidney 21 cells for 5 blind passages, as described previously $(6,8,9)$. Infected cells that exhibited a wrinkled morphology and were detached from the bottom of culture flasks within 5 days (online Technical Appendix Figure 1), and 19 segments of CHUV could also be detected by RT-PCR.

Sequence analysis revealed $100 \%$ identity of genomes for Seg-1 to Seg-10 of 3 CHUV sequences in yaks (CHN-GS-70). Identity analysis showed that genome sequences for Seg-1 to Seg-10 of CHUV shared $>98.38 \%$ nt identities and $>98.09 \%$ aa identities with CHUV strain KT887181/GX871/China in previous studies $(8,9)$ (online Technical Appendix Table 3). We constructed 2 phylogenetic trees based on VP2 and VP5 genome sequences 


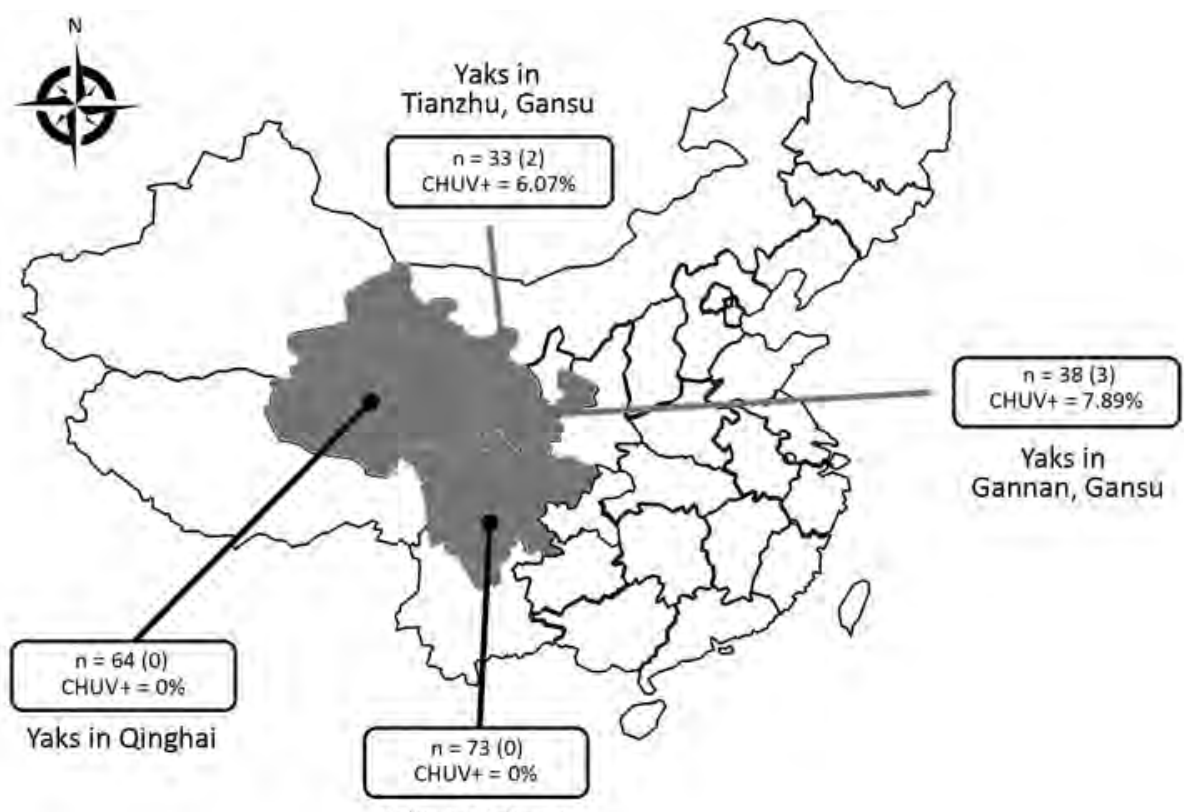

Figure. Number and species of yaks from provinces around the Qinghai Tibetan Plateau, China, 2016-2017. The 3 provinces where sampling was performed, yak species, and occurrence of CHUV are indicated. $n$ values indicate the total number of samples in each province; numbers in parentheses indicate the numbers of positive samples in each province; $\mathrm{CHUV}+$ percentages indicate the CHUV prevalence rate. CHUV, Chuzan virus.

Yaks in Sichuan

of CHUV and other members in genus Orbivirus (online Technical Appendix Figures 2, 3). All strains from our study were grouped in a new separate cluster and shared an ancestor with the strains KT002589/SZ187/China and KT887181/GX871/China. Furthermore, CHN-GS-26 and CHN-GS-70 were located in the same separate sub-cluster (online Technical Appendix Figures 2, 3), which demonstrated a complicated and transregional transmission cycle for CHUV in China.

The yaks that were positive for CHUV were located in 2 cities of Gansu Province $\approx 600 \mathrm{~km}$ apart, which indicates that transmission of CHUV has spread rapidly around the Qinghai-Tibetan Plateau. Further studies are needed to determine the epidemiology and evolution of CHUV in livestock with concomitant virus isolation and phylogenetic analysis. The awareness of livestock health management in these special regions should also be raised.

\section{Acknowledgment}

We thank the Veterinary Department of Gansu Province for its valuable assistance in sample collection.

This work was supported by a grant from the National Natural Science Foundation of China no. 31260616; the Fuxi Foundation of Exceptional Talent at Gansu Agricultural University and the grant from Gansu Agricultural University, College of Veterinary Medicine; the Scientific Research Foundation for New Scholars, Gansu Agricultural University (no. GSAURCZX201702); and the Innovation Foundation of the College of Veterinary Medicine, Gansu Agriculture University (no. JYCX-KX017).

\section{About the Author}

Dr. Wang is a veterinary pharmacologist and epidemiologist at the College of Veterinary Medicine from Gansu Agricultural University. Her primary research interests are veterinary pharmacology, animal disease impact in the Qinghai-Tibetan Plateau of China, and the role of livestock in infectious disease epidemiology.

\section{References}

1. Miura Y, Goto Y, Kubo M, Kono Y. Isolation of Chuzan virus, a new member of the Palyam subgroup of the genus Orbivirus, from cattle and Culicoides oxystoma in Japan. Am J Vet Res. 1988;49:2022-5.

2. Ohashi S, Matsumori Y, Yanase T, Yamakawa M, Kato T, Tsuda T. Evidence of an antigenic shift among Palyam serogroup orbiviruses. J Clin Microbiol. 2004;42:4610-4. http://dx.doi.org/ 10.1128/JCM.42.10.4610-4614.2004

3. Belhouchet M, Mohd Jaafar F, Firth AE, Grimes JM, Mertens PP, Attoui H. Detection of a fourth orbivirus non-structural protein. PLoS One. 2011;6:e25697. http://dx.doi.org/10.1371/ journal.pone. 0025697

4. Ratinier M, Caporale M, Golder M, Franzoni G, Allan K, Nunes SF, et al. Identification and characterization of a novel non-structural protein of bluetongue virus. PLoS Pathog. 2011;7:e1002477. http://dx.doi.org/10.1371/journal.ppat.1002477

5. Rao PP, Hegde NR, Reddy YN, Krishnajyothi Y, Reddy YV, Susmitha B, et al. Epidemiology of bluetongue in India. Transbound Emerg Dis. 2016;63:e151-64. http://dx.doi. org/10.1111/tbed.12258

6. Wang J, Li H, He Y, Zhou Y, Xin A, Liao D, et al. Isolation of Tibet orbivirus from Culicoides and associated infections in livestock in Yunnan, China. Virol J. 2017;14:105. http://dx.doi.org/10.1186/ s12985-017-0774-9

7. Lim SI, Kweon CH, Tark DS, Kim SH, Yang DK. Sero-survey on Aino, Akabane, Chuzan, bovine ephemeral fever and Japanese encephalitis virus of cattle and swine in Korea. J Vet Sci. 2007; 8:45-9. http://dx.doi.org/10.4142/jvs.2007.8.1.45 
8. Wang F, Lin J, Chang J, Cao Y, Qin S, Wu J, et al. Isolation, complete genome sequencing, and phylogenetic analysis of the first Chuzan virus in China. Virus Genes. 2016;52:138-41. http://dx.doi.org/10.1007/s11262-015-1282-x

9. Yang H, Xiao L, Meng J, Xiong H, Gao L, Liao D, et al. Complete genome sequence of a Chuzan virus strain isolated for the first time in mainland China. Arch Virol. 2016;161:1073-7. http://dx.doi.org/10.1007/s00705-015-2734-2

10. Ma J-G, Zhang X-X, Zheng W-B, Xu Y-T, Zhu X-Q, Hu G-X, et al. Seroprevalence and risk factors of bluetongue virus infection in Tibetan sheep and yaks in Tibetan Plateau, China. Biomed Res Int. 2017;2017:5139703.

Address for correspondence: Qiaoying Zeng, Gansu Agricultural University, The College of Veterinary Medicine, Lanzhou, China; email: zengqy@gsau.edu.cn

\section{Conservation of White Rhinoceroses Threatened by Bovine Tuberculosis, South Africa, 2016-2017}

\section{Michele A. Miller, ${ }^{1}$ Peter Buss, ${ }^{1}$ \\ Sven D.C. Parsons, Eduard Roos, Josephine Chileshe, Wynand J. Goosen, Louis van Schalkwyk, Lin-Mari de Klerk-Lorist, Markus Hofmeyr, ${ }^{2}$ Guy Hausler, Leana Rossouw, Tebogo Manamela, Emily P. Mitchell, ${ }^{3}$ Rob Warren, Paul van Helden}

\begin{abstract}
Author affiliations: Stellenbosch University, Department of Science and Technology-National Research Foundation Centre of Excellence for Biomedical Tuberculosis Research, South African Medical Research Council Centre for Tuberculosis Research, Cape Town, South Africa (M.A. Miller, S.D.C. Parsons, E. Roos, J. Chileshe, W.J. Goosen, G. Hausler, R. Warren, P. van Helden); South African National Parks, Skukuza, South Africa (P. Buss, M. Hofmeyr, L. Rossouw, T. Manamela); Office of the State Veterinarian, Skukuza (L. van Schalkwyk, L.-M. de Klerk-Lorist); National Zoological Gardens of South Africa, Pretoria, South Africa (E.P. Mitchell)
\end{abstract}

DOI: https://doi.org/10.3201/eid2412.180293

\footnotetext{
${ }^{1}$ These first authors contributed equally to this article.

${ }^{2}$ Current affiliation: Great Plains Conservation, Maun, Botswana.

${ }^{3}$ Current affiliation: University of Pretoria, Pretoria, South Africa.
}

During 2016-2017, when Kruger National Park, South Africa, was under quarantine to limit bovine tuberculosis spread, we examined 35 white and 5 black rhinoceroses for infection. We found 6 infected white rhinoceroses during times of nutritional stress. Further research on Mycobacterium bovis pathogenesis in white rhinoceroses is needed.

$\mathrm{T}$ uberculosis (TB) caused by Mycobacterium tuberculosis or M. bovis has been reported in captive rhinoceroses since the early 1800 s (1-3). Bovine TB is endemic in many wildlife populations worldwide, including among those in Kruger National Park (KNP), South Africa (4). KNP contains the largest free-living population of white rhinoceroses in the world (estimated at 6,6497,830). However, prolonged drought in South Africa (2015-2017) raised concerns that starvation and disease could increase the mortality rate and affect conservation efforts for this species (5).

In June 2016, a black rhinoceros (Diceros bicornis minor) with an $M$. bovis infection was discovered (6). Thereafter, a surveillance program was initiated to screen rhinoceros carcasses in KNP, leading to 35 white and 5 black rhinoceros carcasses being examined during June 2016-October 2017. To determine which animals were infected, we conducted macroscopic examinations and collected samples for histopathologic studies and mycobacterial culture, as previously described (7). Research protocols were approved by the South African National Park Animal Use and Care Committee and ethics committee of Stellenbosch University.

No additional cases of $M$. bovis infection were found in black rhinoceroses. However, we confirmed M. bovis infection in 6 white rhinoceroses (Table). Grossly visible lesions, mostly found in the retropharyngeal or tracheobronchial lymph nodes or lung, were typically small and localized and could easily be missed or mistaken for granulomas caused by other pathogens if careful dissections of tissues were not performed (online Technical Appendix, https:// wwwnc.cdc.gov/EID/article/24/12/18-0293-Techapp1. pdf). On histologic examination, we found granulomatous inflammation in lung or lymph node sections and rare acidfast organisms in some granulomas (Table). We typed these $M$. bovis isolates as strain SB0121, the most common strain found in KNP (8).

Four of the infected animals were found during September-November 2016, near the end of the drought, and the remaining 2 animals were found in September and October 2017, at the end of the next winter. The timing of infections suggests that animals under nutritional stress might be more susceptible to infection, similar to observations in other species (9). The low number of positive cases and localized paucibacillary lesions support the hypothesis that white rhinoceroses, although susceptible to infection, 
Table. Findings from 6 Mycobacterium bovis-infected white rhinoceroses, Kruger National Park, South Africa, 2016-2017*

\begin{tabular}{|c|c|c|c|c|c|c|c|c|c|}
\hline \multirow[b]{2}{*}{$\begin{array}{l}\text { Case } \\
\text { no. }\end{array}$} & \multirow[b]{2}{*}{$\begin{array}{c}\text { Age } \\
\text { category }\end{array}$} & \multirow[b]{2}{*}{ Sex } & \multirow[b]{2}{*}{ Date } & \multirow[b]{2}{*}{$\begin{array}{l}\text { Body } \\
\text { condition }\end{array}$} & \multicolumn{3}{|c|}{ Features of lesions consistent with bovine TB $\dagger$} & \multirow{2}{*}{$\begin{array}{l}\text { M. bovis culture- } \\
\text { positive tissue } \\
\text { pools } \dagger\end{array}$} & \multirow{2}{*}{$\begin{array}{l}\text { AFB on } \\
\text { cytology of } \\
\text { lesions } \dagger\end{array}$} \\
\hline & & & & & $\begin{array}{l}\text { Macroscopic, multifocal } \\
\text { mineralized granulomas }\end{array}$ & $\begin{array}{c}\text { Multifocal granulomas } \\
\text { on histology }\end{array}$ & $\begin{array}{l}\text { AFB on } \\
\text { histology }\end{array}$ & & \\
\hline 1 & Subadult & M & $\begin{array}{l}\text { Sep } \\
2016\end{array}$ & Thin & $\begin{array}{c}\text { + Lung and } \\
\text { retropharyngeal LN }\end{array}$ & $\begin{array}{c}\text { + Lung and } \\
\text { retropharyngeal LN }\end{array}$ & - & $\begin{array}{l}\text { Head, thoracic, } \\
\text { and peripheral } \\
\text { LNs and lung }\end{array}$ & $+\mathrm{LNs}$ \\
\hline 2 & Adult & $\mathrm{F}$ & $\begin{array}{c}\text { Sep } \\
2016\end{array}$ & Thin & - Lung and all LNs & ND & ND & $\begin{array}{l}\text { Thoracic } L N \text { and } \\
\text { lung }\end{array}$ & ND \\
\hline 3 & Adult & M & $\begin{array}{c}\text { Oct } \\
2016\end{array}$ & Thin & + Lung; - all LNs & + Lung; - LNs & - & $\begin{array}{l}\text { Thoracic and } \\
\text { abdominal LNs } \\
\text { and lung }\end{array}$ & + Lung \\
\hline 4 & Subadult & $\mathrm{F}$ & $\begin{array}{l}\text { Nov } \\
2016\end{array}$ & Thin & $\begin{array}{l}\text { - Lung; + } \\
\text { submandibular, } \\
\text { retropharyngeal, } \\
\text { tracheobronchial, and } \\
\text { mesenteric LNs }\end{array}$ & $\begin{array}{l}\text { - Lung; + } \\
\text { submandibular, } \\
\text { retropharyngeal, } \\
\text { tracheobronchial, and } \\
\text { mesenteric LNs }\end{array}$ & Rare & $\begin{array}{l}\text { Head and thoracic } \\
\text { LNs }\end{array}$ & + LNs \\
\hline 5 & Subadult & $\mathrm{F}$ & $\begin{array}{c}\text { Sep } \\
2017\end{array}$ & Normal & $\begin{array}{c}\text { + Lung and prescapular } \\
\text { and axillary LNs }\end{array}$ & ND & ND & Thoracic LN & + Lung \\
\hline 6 & Adult & M & $\begin{array}{c}\text { Oct } \\
2017\end{array}$ & Thin & $\begin{array}{l}\text { + Lung and } \\
\text { prescapular, } \\
\text { retropharyngeal, and } \\
\text { tracheobronchial LNs }\end{array}$ & ND & ND & $\begin{array}{l}\text { Tracheobronchial, } \\
\text { prescapular, and } \\
\text { retropharyngeal } \\
\text { LNs and lung }\end{array}$ & $\begin{array}{l}\text { + Lung } \\
\text { and LNs }\end{array}$ \\
\hline
\end{tabular}

*AFB, acid-fast bacilli; LN, lymph node; ND, not done; TB, tuberculosis; + feature present; - feature absent.

tLNs were pooled into 4 sets: head (retropharyngeal, submandibular, and cervical); thoracic (tracheobronchial and mediastinal); abdominal (mesenteric and hepatic); and peripheral (axillary, prescapular, and inguinal) LNs.

$\ddagger$ No clinically significant lesions other than inflammation (poaching case).

are able to limit disease progression (10). However, whether infected animals would develop disease if compromised is unknown. Location of lesions yielding positive cultures suggests an aerosol route of exposure, although $M$. bovis was also isolated from mesenteric and peripheral lymph nodes (Table). Although no data were available to evaluate transmission, a previous study has shown white rhinoceroses with localized $M$. bovis infection did not regularly shed bacilli (10). Further research is required to understand the pathogenesis and epidemiology of $M$. bovis infection in these animals.

Fresh samples from animals that die naturally are difficult to locate in a large ecosystem, especially before predators arrive at the carcass or decomposition occurs due to elevated temperatures. In our study, collection of samples with minimal degradation was facilitated by our examining only rhinoceroses dead for $<12$ hours and animals euthanized because of their severe state of debilitation, most often from poaching wounds. Bovine TB was not considered the cause of the poor condition or death in any of these animals. We found small, nonspecific culture-positive lesions histologically similar to those caused by helminths, foreign material, and fungi, and the paucibacillary nature of the infection could result in false-negative histopathologic results. Therefore, we needed to confirm infection by mycobacterial culture and species determination with every tissue set collected. However, low numbers of viable bacteria, sample handling, and the likelihood of overgrowth by contaminants could also lead to false-negative culture results. Positive culture results from $>1$ tissue sample in the same rhinoceros suggests infection rather than contamination. However, no cases of disseminated bovine TB have been observed in this species, supporting the authors' hypothesis that the disease in white rhinoceroses is self-limiting. Factors such as drought might play a role in altering susceptibility to infection, considering no positive culture results were obtained in $>20$ rhinoceros carcasses examined before June 2016.

Although disease and death associated with bovine TB have not been observed in white rhinoceroses, $M$. bovis infection nonetheless presents a threat to conservation of this species. Genetic management and translocation of rhinoceroses are essential components of in situ conservation; animals need to be moved from high-risk poaching areas to more secure locations (5). In addition, calves orphaned by poaching require intensive specialized care, which is only available outside KNP (5). Because M. bo$v i s$ is a controlled disease, premises with infected populations are placed under quarantine to prevent translocation of potentially infected animals. With a paucity of data to assess risks, movement restrictions are a substantial impediment to conservation and can threaten the survival of this population. Therefore, research into antemortem detection, pathogenesis, and epidemiology of $M$. bovis infection is essential for programs to conserve rhinoceroses of Africa.

\section{Acknowledgments}

We thank the staffs of the South African National Parks, State Veterinary Services of KNP, National Zoological Gardens of South Africa, and Faculty of Veterinary Science, University of Pretoria, for providing assistance with these cases. 
This study was supported by South African National Parks, National Research Foundation South African Research Chair Initiative in Animal Tuberculosis (grant no. 86949), the Department of Science and Technology-National Research Foundation Centre of Excellence for Biomedical Tuberculosis Research, and the South African Medical Research Council.

\section{About the Author}

Prof. Miller is the South African Research Chair in Animal TB at the Department of Science and Technology-National Research Foundation Centre of Excellence for Biomedical TB Research, and a member of the South African Medical Research Council, Centre for Tuberculosis Research, at Stellenbosch University, Cape Town, South Africa. Her research interests include infectious diseases that threaten animal conservation.

\section{References}

1. Miller M, Michel A, van Helden P, Buss P. Tuberculosis in rhinoceros: an underrecognized threat? Transbound Emerg Dis. 2017;64:1071-8. http://dx.doi.org/10.1111/tbed.12489

2. Stetter MD, Mikota SK, Gutter AF, Monterroso ER, Dalovisio JR, Degraw C, et al. Epizootic of Mycobacterium bovis in a zoologic park. J Am Vet Med Assoc. 1995;207:1618-21.

3. Espie IW, Hlokwe TM, Gey van Pittius NC, Lane E, Tordiffe ASW, Michel AL, et al. Pulmonary infection due to Mycobacterium bovis in a black rhinoceros (Diceros bicornis minor) in South Africa. J Wildl Dis. 2009;45:1187-93. http://dx.doi.org/10.7589/ 0090-3558-45.4.1187

4. Hlokwe TM, van Helden P, Michel AL. Evidence of increasing intra and inter-species transmission of Mycobacterium bovis in South Africa: are we losing the battle? Prev Vet Med. 2014;115:107. http://dx.doi.org/10.1016/j.prevetmed.2014.03.011

5. Department of Environmental Affairs, Republic of South Africa. Minister Molewa highlights process on integrated strategic management of rhinoceros. 2017 Feb 27 [cited 2018 Aug 14]. https://www.environment.gov.za/mediarelease/molewa_ progressonintegrated_strategicmanagement_ofrhinoceros

6. Miller M, Buss P, van Helden P, Parsons S. Mycobacterium bovis in a free-ranging black rhinoceros, Kruger National Park, South Africa, 2016. Emerg Infect Dis. 2017;23:557-8. http://dx.doi.org/ 10.3201/eid2303.161622

7. Goosen WJ, Miller MA, Chegou NN, Cooper D, Warren RM, van Helden PD, et al. Agreement between assays of cell-mediated immunity utilizing Mycobacterium bovis-specific antigens for the diagnosis of tuberculosis in African buffaloes (Syncerus caffer). Vet Immunol Immunopathol. 2014;160:133-8. http://dx.doi.org/10.1016/j.vetimm.2014.03.015

8. Dippenaar A, Parsons SDC, Miller MA, Hlokwe T, Gey van Pittius NC, Adroub SA, et al. Progenitor strain introduction of Mycobacterium bovis at the wildlife-livestock interface can lead to clonal expansion of the disease in a single ecosystem. Infect Genet Evol. 2017;51:235-8. http://dx.doi.org/ 10.1016/j.meegid.2017.04.012

9. Cegielski JP, McMurray DN. The relationship between malnutrition and tuberculosis: evidence from studies in humans and experimental animals. Int J Tuberc Lung Dis. 2004;8:286-98.

10. Michel AL, Lane EP, de Klerk-Lorist L-M, Hofmeyr M, van der Heijden EMDL, Botha L, et al. Experimental Mycobacterium bovis infection in three white rhinoceroses (Ceratotherium simum): susceptibility, clinical and anatomical pathology. PLoS One. 2017;12:e0179943. http://dx.doi.org/10.1371/journal.pone.0179943
Address for correspondence: Michele A. Miller, DST-NRF Centre of Excellence for Biomedical TB Research, MRC Centre for Tuberculosis Research, Division of Molecular Biology and Human Genetics, Faculty of Medicine and Health Sciences, Stellenbosch University, PO Box 241, Cape Town 8000, South Africa; email: miller@sun.ac.za

\section{Lung I nvolvement in Chronic Schistosomiasis with Bladder Squamous Cell Carcinoma}

\author{
Anastasia Saade, Edith Carton, \\ Audrey Mansuet-Lupo, Romain Jouffroy, \\ Diane Damotte, Hélène Yera, Marie-Pierre Revel, \\ François Goldwasser
}

Author affiliations: Hopital Cochin, Paris, France

(A. Saade, E. Carton, A. Mansuet-Lupo, D. Damotte,

H. Yera, M.-P. Revel, F. Goldwasser); Universite Paris Descartes,

Paris (A. Saade, R. Jouffroy, M.-P. Revel, F. Goldwasser)

DOI: https://doi.org/10.3201/eid2412.180355

We report a case of chronic Schistosoma haematobium infection with pseudometastatic pulmonary nodules and high-grade squamous cell carcinoma in a 30-year-old man in Mali. Lung biopsies revealed chronic pulmonary involvement of S. haematobium and ruled out lung metastases.

A 30-year-old man from Mali, who had immigrated to France a year before, was hospitalized for acute urinary retention. The patient reported isolated hematuria over the preceding month with recent dysuria. He was afebrile and had normal vital signs. Physical examination revealed pelvis tenderness and guarding. The only biologic abnormality was a hypereosinophilia $(1,640$ cells/ $\mathrm{mm}^{3}$ ). Unenhanced computed tomography (CT) revealed linear calcifications on the bladder wall, with a large intraluminal mass infiltrating the left ureter (Figure, panel A). Cystoscopy was typical of schistosomiasis. Anatomopathology revealed urinary schistosomiasis complicated by a high-grade, well-differentiated, keratinized squamous cell carcinoma (SCC) (Figure, panel B). Within the wall, ovoid structures, sometimes calcified, 
Figure. Schistosomiasis-induced squamous cell carcinoma of the bladder with pseudometastatic pulmonary nodules in a 30-year-old man from Mali. A) An unenhanced computed tomography axial image shows diffuse calcification of the bladder wall (arrow). A softtissue mass arises from the left posterolateral wall, breaching the calcifications and reaching the perivesical fat (asterisk). B) Anatomopathology slide stained with hematoxylin and eosin (original magnification $\times 10$ ) of the bladder wall showing massive infiltration by a carcinomatous proliferation of the epidermoid type arranged in islands, or in broad cords in depth (arrows). The cells are large and polygonal, with eosinophilic cytoplasm. The papillomatous malpighian epithelium infiltrates the entire thickness of the chorion and the muscularis into the perivesical fat with venous vascular emboli (asterisk). Within the wall are observed bilharzia eggs, sometimes calcified (small arrow). The chorion is, on the other hand, the seat of a moderate mononuclear inflammatory infiltrate. C) Eggs of Schistosoma haematobium in a wet mount of urine concentrate, showing their characteristic terminal spine (original magnification $\times 400$ ).
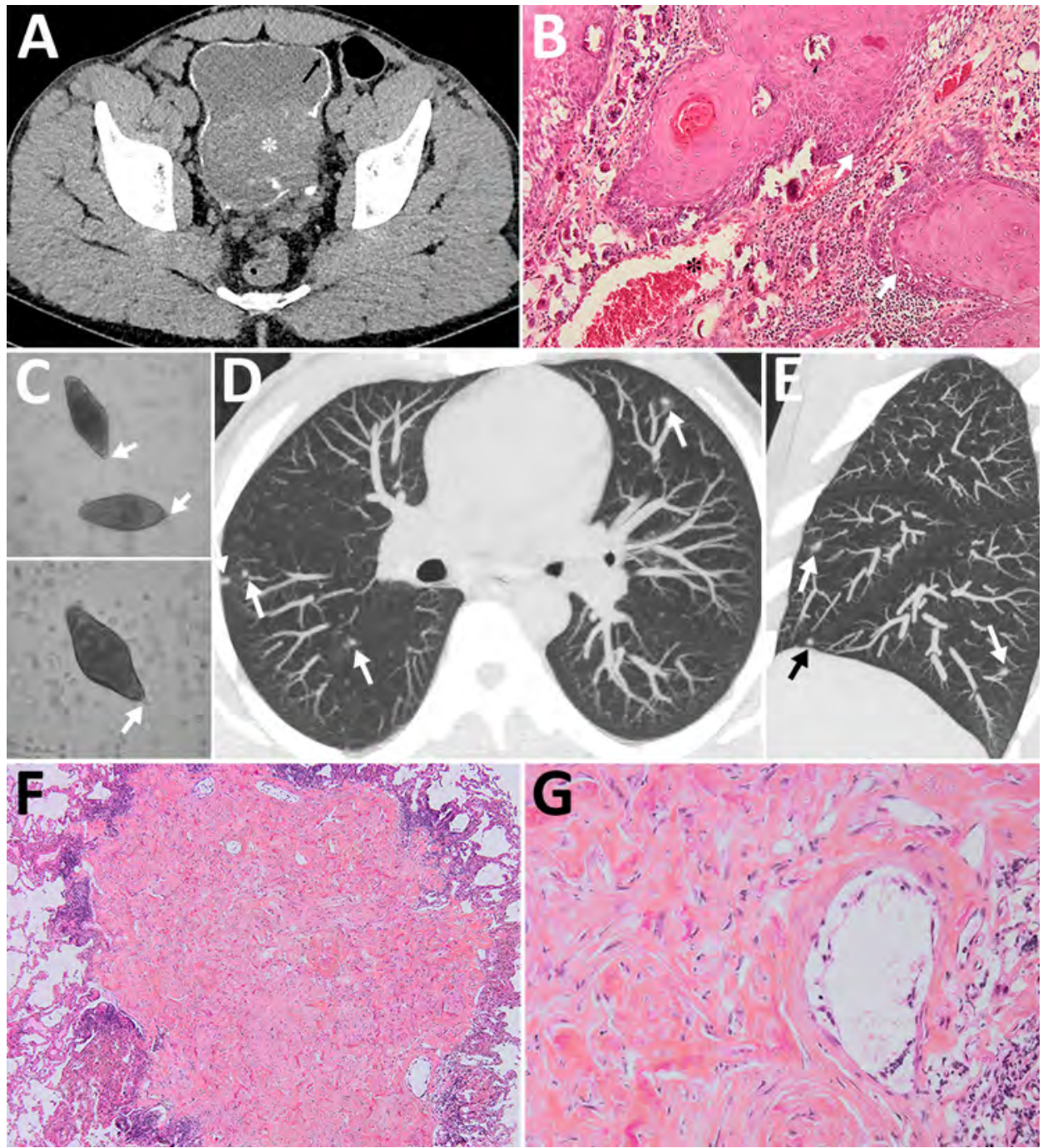

$D, E)$ Axial (D) and sagittal (E)

reconstruction chest computed tomography of the lungs showing blurred ground glass nodules scattered over both lungs. F) A lung subpleural nodule of $0.2 \mathrm{~cm}$ greater axis shows at low magnification (original magnification $\times 4$ ) a fibrous nodule surrounded by lymphoid follicles. G) Hematoxylin and eosin staining of the lung nodule (original magnification $\times 20$ ) shows inflammatory population, mainly represented by eosinophils and a structure corresponding to a bilharzia egg. No territory suspected of malignancy was visualized.

corresponded to bilharzia eggs. We found calcified eggs with a terminal spine resembling Schistosoma haematobium in the urine (Figure, panel C). Stool examinations were negative. Schistosomiasis serologic tests were positive in hemagglutination and ELISA and were confirmed by immunoblotting. Chest CT revealed multiple, bilateral, diffuse, infracentimetric, pulmonary, ill-defined nodules (Figure, panels D, E), suggesting metastases. Because secondary lesions contraindicate cystectomy and require aggressive chemotherapy, we performed further investigations.

The patient was HIV-negative. We excluded tuberculosis on the basis of direct examination and culture of sputum and bronchoalveolar lavage fluid. The latter showed
160,000 cells/mL with hypereosinophilia (11\%) and alveolar hemorrhage. Ziehl and Grocott stainings were negative. Bacteriologic tests were negative. We detected no tumor cell. We resected a nodule during video-assisted thoracoscopy. Anatomopathology revealed Bilharzia eggs with no carcinomatous lesions (Figure, panels F, G). Another nodule showed similar lesions. The eggshells appeared to be Ziehl-Neelson-negative, typical of S. haematobium. Echocardiography ruled out pulmonary hypertension.

We confirmed S. haematobium by using molecular analysis of mitochondrial cytochrome oxidase subunit I gene. We excluded S. haematobium-bovis hybrid species, frequent in Mali, by using internal transcribed spacer region 1 and 2 sequences (1). 
The patient received 2 courses of praziquantel $(40 \mathrm{mg} /$ $\mathrm{kg}$ ) at 8-week intervals. The tumor was considered free of node involvement and metastases. The patient underwent radical cystectomy with an orthotopic neobladder. Anatomopathology confirmed complete resection of the tumor reaching the muscle layer and the perivesical fat. At 3 months, blood eosinophils were normalized, and chest CT showed complete resolution of all pulmonary nodules. At 6-month follow-up, the patient was still in complete remission.

Schistosomiasis, the second most prevalent endemic parasitic disease, affects $\approx 230$ million persons (2). This helminthic infection is caused by a trematode and is also known as snail fever or Bilharzia. The larvae burrow into human skin after contact with contaminated water and migrate within the vascular system to the lung. Through the portal system, mature forms reach venous plexuses surrounding the bladder, and released eggs enter perivesical venules. Adult lifespan averages 3-5 years and can persist up to 40 years.

Acute schistosomiasis occurs 3-6 weeks after infection. Eosinophilia serves as a diagnostic clue. Acute lung lesions are common (3). S. haematobium, found in Africa and the Middle East, induces urinary schistosomiasis with frequent hematuria. The infection causes fibrosis of the bladder, wall calcification, and hydronephrosis. A major complication is the development of SCC. Schistosomiasisinduced SCC is overrepresented in endemic regions, accounting for only $2.5 \%$ of bladder cancer in Western countries $(4,5)$; it appears as a unique and voluminous mass with low incidence of lymph node involvement and distant metastases at $8 \%-10 \%(4,5)$. However, the prognosis remains poor, with $90 \%$ locoregional recurrence within 3 years. The effective treatment requires radical cystectomy.

Manifestations of chronic disease also involve the lung, and schistosomiasis is the leading cause of pulmonary hypertension, which occurs in $8 \%-25 \%$ of cases $(6,7)$. A less reported aspect of chronic lung involvement is nodular lesions, which are described in 5\% of schistosomiasis cases and are often asymptomatic when isolated (8). Belleli was the first to describe $S$. haematobium eggs in the lungs in 1885 , stipulating that eggs laying into perivesical plexuses migrate from portal and caval veins to the lungs. Embolized eggs can produce a granulomatous reaction leading to pulmonary fibrosis and obliterative arteriolitis, causing pulmonary hypertension. However, the exact pathophysiology remains unclear and involves different mechanisms. Pulmonary symptoms are more often described with S. mansoni and S. japonicum than S. haematobium, which preferentially transits through the genitourinary rather than the hepatic portal system (9). Radiographic features resemble coins or cavitations, whereas CT shows a ground glass-opacity halo or reticulonodular pattern (8). Nodules, considered to be ectopic sites, remain unspecific and indistinguishable from secondary lesions (6). The diagnosis of chronic lung involvement is oriented by symptoms of associated end-organ. Treatment with praziquantel is efficient on ectopic lesions.

This case is interesting because of the double presentation of schistosomiasis-induced SCC and chronic lung involvement. Although metastases are less frequent than chronic pulmonary nodules, lung metastases were highly expected in the context of invasive SCC. Each diagnosis involves a different therapeutic approach, and delayed diagnosis can affect the patient's prognosis. Lung biopsies might be necessary when severe disorders are suspected and avoided when suspicion for other causes, such as tuberculosis and malignancy, is low (8). In the latter scenario, PCR on bronchoalveolar lavage fluid might be an alternative in the diagnosis of chronic pulmonary schistosomiasis (10).

\section{Acknowledgments}

The authors thank Etienne Canoui and Alviset Sophie for their expertise on the topic, Yohann Rouscoff and Elie Saad for the surgical management of the patient, Emelyne Hamelin-Canny and Elena Garelli for performing lung biopsies, and Marie-Françoise Triller for her anatomopathology skills.

The patient approved the use of his medical record for this work and provided written informed consent.

\section{About the Author}

Dr. Saade started her work in medicine after she obtained her $\mathrm{PhD}$ in molecular biology and is currently a resident in infectious disease and hematology. Her primary research interests include the study of infections in immunosuppressed patients.

\section{References}

1. Soentjens P, Cnops L, Huyse T, Yansouni C, De Vos D, Bottieau E, et al. Diagnosis and clinical management of Schistosoma haematobium-Schistosoma bovis hybrid infection in a cluster of travelers returning from Mali. Clin Infect Dis. 2016;63:1626-9. http://dx.doi.org/10.1093/cid/ciw493

2. Colley DG, Bustinduy AL, Secor WE, King CH. Human schistosomiasis. Lancet. 2014;383:2253-64. http://dx.doi.org/ 10.1016/S0140-6736(13)61949-2

3. Ross AG, Vickers D, Olds GR, Shah SM, McManus DP. Katayama syndrome. Lancet Infect Dis. 2007; 7:218-24. http://dx.doi.org/10.1016/S1473-3099(07)70053-1

4. Shokeir AA. Squamous cell carcinoma of the bladder: pathology, diagnosis and treatment. BJU Int. 2004;93:216-20. http://dx.doi.org/10.1111/j.1464-410X.2004.04588.x

5. Martin JW, Carballido EM, Ahmed A, Farhan B, Dutta R, Smith C, et al. Squamous cell carcinoma of the urinary bladder: systematic review of clinical characteristics and therapeutic approaches. Arab J Urol. 2016;14:183-91. http://dx.doi.org/10.1016/ j.aju.2016.07.001

6. Niemann T, Marti HP, Duhnsen SH, Bongartz G. Pulmonary schistosomiasis_imaging features. J Radiol Case Rep. 2010;4:37-43. 
7. Papamatheakis DG, Mocumbi AOH, Kim NH, Mandel J. Schistosomiasis-associated pulmonary hypertension. Pulm Circ. 2014;4:596-611. http://dx.doi.org/10.1086/678507

8. Gobbi F, Buonfrate D, Angheben A, Beltrame A, Bassetti M, Bertolaccini L, et al. Pulmonary nodules in African migrants caused by chronic schistosomiasis. Lancet Infect Dis. 2017;17:e159-65. http://dx.doi.org/10.1016/S1473-3099(16)30530-8

9. Bethlem EP, Schettino GP, Carvalho CR. Pulmonary schistosomiasis. Curr Opin Pulm Med. 1997;3:361-5. http://dx.doi.org/10.1097/ 00063198-199709000-00008

10. Gobbi F, Formenti F, Perandin F, Buonfrate D, Angheben A, Paiano $\mathrm{S}$, et al. Real-time polymerase chain reaction assay on bronchoalveolar lavage: an alternative method for diagnosing chronic pulmonary schistosomiasis? Am J Trop Med Hyg. 2017;97:1808-9. http://dx.doi.org/10.4269/ajtmh.17-0499

Address for correspondence: Anastasia Saade, Hopital Cochin, 27 Rue du Faubourg Saint-Jacques, 75014 Paris, Île-de-France, France; email: anastasia.saade@gmail.com

\section{Strongyloidiasis and Culture-Negative Suppurative Meningitis, Japan, 1993- 2015}

\author{
Mitsuru Mukaigawara, Izumi Nakayama, \\ Koichiro Gibo
}

Author affiliation: Okinawa Chubu Hospital, Okinawa, Japan

DOI: https://doi.org/10.3201/eid2412.180375

Community-acquired Enterobacteriaceae infection and culture-negative meningitis are rare and atypical subtypes of meningitis in adults. Of 37 patients who had atypical suppurative meningitis during 1993-2015 in Okinawa, Japan, $54.5 \%$ had strongyloidiasis, of which $9.1 \%$ cases were hyperinfections and $3.0 \%$ dissemination. Strongyloidiasis should be considered an underlying cause of atypical suppurative meningitis.

A mong adults, suppurative meningitis caused by enteric organisms and suppurative meningitis that is culture negative are uncommon $(1,2)$. These types of meningitis with atypical features (hereafter atypical suppurative meningitis) remain a clinical challenge. The mortality rate among patients with community-acquired suppurative meningitis caused by gram-negative organisms is $52.5 \%$ (1). Treatment of culture-negative suppurative meningitis requires broad-spectrum antimicrobial drugs; however, the absence of detected pathogens increases the risk for development of antimicrobial drug resistance.

Strongyloidiasis, a nematode infection that occurs in the subtropics and tropics, is associated with Enterobacteriaceae meningitis (3). Previous reports of strongyloidiasis-associated meningitis also suggested potential links between strongyloidiasis and atypical suppurative meningitis on the basis of 9 cases of Enterobacteriaceae meningitis (not consecutive) (4) and 17 cases of culture-negative suppurative meningitis (5). Our aim was to investigate the association between strongyloidiasis and atypical suppurative meningitis.

We conducted a retrospective chart review of patients who consecutively received a diagnosis of atypical suppurative meningitis during January 1993-December 2015 at Okinawa Chubu Hospital, Okinawa, Japan. This hospital is one of the largest tertiary medical centers in Okinawa. Strongyloidiasis is endemic in Okinawa; reported prevalence is $5.2 \%(6)$.

We defined atypical suppurative meningitis as suppurative meningitis with positive cerebrospinal fluid (CSF) culture results for enteric organisms or with CSF leukocytosis $\geq 500$ cells $/ \mathrm{mm}^{3}$ and negative CSF culture results. We included in the study patients $\geq 18$ years of age with CSF that was culture positive for enteric organisms or negative with leukocytosis of $\geq 500 \mathrm{cells} / \mathrm{mm}^{3}$. Enteric organisms included in this study were Bacteroides spp., Enterococcus spp., Escherichia coli, Enterobacter spp., Klebsiella spp., Bifidobacterium bifidum, Clostridium perfringens, Proteus mirabilis, Streptococcus gallolyticus (bovis), and Campylobacter spp. (7). CSF of patients with bacterial meningitis typically shows leukocytosis of $\geq 1,000$ cells/ $\mathrm{mm}^{3}$; CSF of those with nonbacterial meningitis typically shows $<250$ cells $/ \mathrm{mm}^{3}$ (8). Considering the early phase of bacterial meningitis (9), the cutoff value (500 cells/ $\mathrm{mm}^{3}$ ) was defined to include suppurative meningitis and exclude most cases of nonbacterial meningitis. We excluded patients with nosocomial meningitis, prior use of antimicrobial drugs (within 7 days of lumbar puncture), negative CSF culture, and positive blood culture for nonenteric organisms.

We collected information about patient demographic and clinical characteristics, immunocompromised status, type of strongyloidiasis infection, outcomes, CSF analysis results, and culture results. Strongyloidiasis was classified into 3 categories: nonsystemic strongyloidiasis, hyperinfection, and dissemination. We defined these categories according to where larvae were detected: nonsystemic strongyloidiasis in fecal samples only, hyperinfection in sputum, and dissemination in samples other than feces or sputum (4). Samples were collected with regard to patients' clinical category. Identifying information was removed before 
analysis. This study was approved by the Okinawa Chubu Hospital Institutional Review Board (H29-76).

We identified 37 patients; CSF culture results were positive for Enterobacteriaceae for 14 and negative for 23. Strongyloidiasis was diagnosed by parasitologic examinations (Table; online Technical Appendix, https://wwwnc. cdc.gov/EID/article/24/12/18-0375-Techapp1.pdf).

Patients were 19-91 years of age (median 60 years of age); $59.5 \%$ (22/37) were male. Common chief complaints included headache (64.9\% [24/37]) and fever (59.5\% [22/37]). Human T-cell lymphotropic virus type 1 serologic test results were available for 31 patients, of which $22(71.0 \%)$ were positive. Of the 37 total patients, $3(8.1 \%)$ patients had diabetes mellitus, $3(8.1 \%)$ had cirrhosis, and $4(10.8 \%)$ used steroids on a regular basis. Parasitologic examinations were performed for 33 patients, and strongyloides were found in $18(54.5 \%): 14(42.4 \%)$ nonsystemic strongyloidiasis, 3 (9.1\%) hyperinfection, and 1 (3.0\%) dissemination.

Among 14 culture-positive patients, 5 (35.7\%) had nonsystemic strongyloidiasis and $3(21.4 \%)$ had hyperinfection. Among 19 culture-negative patients, 9 (47.4\%) had nonsystemic strongyloidiasis and 1 (5.3\%) had dissemination. When culture-positive patients with strongyloidiasis were compared with culture-negative patients with strongyloidiasis, the odds ratio was 1.71 (95\% CI $0.37-8.22)$. Of note, all strongyloidiasis-positive patients were born before 1960, suggesting changes in lifestyle and the environment since then (e.g., reduced exposure to contaminated soil during farming by not walking barefoot and improved farming environments).

\begin{tabular}{|c|c|c|c|}
\hline Characteristic & $\begin{array}{c}\text { All cases, no. }(\%) \\
n=37\end{array}$ & $\begin{array}{l}\text { Culture-positive, no. (\%), } \\
n=14\end{array}$ & $\begin{array}{l}\text { Culture-negative, no. (\%), } \\
n n=23\end{array}$ \\
\hline \multicolumn{4}{|l|}{ Demographic and clinical } \\
\hline \multicolumn{4}{|l|}{ Sex } \\
\hline $\mathrm{M}$ & $22(59.5)$ & $9(64.2)$ & $13(56.5)$ \\
\hline $\mathrm{F}$ & $15(40.5)$ & $5(35.8)$ & $10(43.5)$ \\
\hline \multicolumn{4}{|l|}{ Chief complaints } \\
\hline Headache & $24(64.9)$ & $6(42.9)$ & $18(78.3)$ \\
\hline Fever & $22(59.5)$ & $9(64.2)$ & $13(56.5)$ \\
\hline Altered mental status & $8(21.6)$ & $5(35.7)$ & $3(13.0)$ \\
\hline Nausea/vomiting & $8(21.6)$ & $1(7.1)$ & $7(30.4)$ \\
\hline \multicolumn{4}{|l|}{ Immunocompromised status } \\
\hline HTLV-1 infection $\dagger$ & $22(71.0)$ & $8(61.5)$ & $14(77.8)$ \\
\hline Diabetes mellitus & $3(8.1)$ & $2(14.3)$ & $1(4.3)$ \\
\hline Cirrhosis & $3(8.1)$ & $3(21.4)$ & 0 \\
\hline Steroid use & $4(10.8)$ & $1(7.1)$ & $3(13.0)$ \\
\hline \multicolumn{4}{|l|}{ Strongyloidiasis infection type } \\
\hline Nonsystemic strongyloidiasis & $14(42.4)$ & $5(35.7)$ & $9(47.4)$ \\
\hline Hyperinfection & $3(9.1)$ & $3(21.4)$ & 0 \\
\hline Dissemination & $1(3.0)$ & 0 & $1(5.3)$ \\
\hline Not analyzed & 4 & 0 & 4 \\
\hline Died & $5(13.5)$ & $3(21.4)$ & $2(8.7)$ \\
\hline \multicolumn{4}{|l|}{ CSF } \\
\hline \multicolumn{4}{|l|}{ Neutrophils $/ \mathrm{mm}^{3}$} \\
\hline $500-2,999$ & $24(64.9)$ & $6(42.9)$ & $18(78.3)$ \\
\hline $3,000-5,999$ & $4(10.8)$ & $1(7.1)$ & $3(13.0)$ \\
\hline $6,000-9,999$ & $2(5.4)$ & $1(7.1)$ & $1(4.3)$ \\
\hline$>10,000$ & $3(8.1)$ & $2(14.3)$ & $1(4.3)$ \\
\hline Glucose $<40 \mathrm{mg} / \mathrm{dL}$ & $14(37.8)$ & $7(50.0)$ & $7(30.4)$ \\
\hline \multicolumn{4}{|l|}{ Bacteriologic } \\
\hline \multicolumn{4}{|l|}{ Blood culture } \\
\hline Klebsiella pneumoniae & $7(18.9)$ & $5(35.7)$ & $2(8.7)$ \\
\hline Streptococcus gallolyticus & $4(10.8)$ & $4(28.6)$ & 0 \\
\hline Escherichia coli & $3(8.1)$ & $2(14.3)$ & $1(4.3)$ \\
\hline Streptococcus infantarius & $1(2.7)$ & $1(7.1)$ & 0 \\
\hline Campylobacter fetus & $1(2.7)$ & $1(7.1)$ & 0 \\
\hline Negative & $21(56.8)$ & $1(7.1)$ & $20(87.0)$ \\
\hline \multicolumn{4}{|l|}{ CSF culture } \\
\hline K. pneumoniae & $5(35.7)$ & $5(35.7)$ & 0 \\
\hline S. gallolyticus & $1(7.1)$ & $1(7.1)$ & 0 \\
\hline E. coli & $1(7.1)$ & $1(7.1)$ & 0 \\
\hline S. infantarius & $1(7.1)$ & $1(7.1)$ & 0 \\
\hline C. fetus & $1(7.1)$ & $1(7.1)$ & 0 \\
\hline Bacteroides fragilis & $1(7.1)$ & 1 (7.1) & 0 \\
\hline
\end{tabular}

${ }^{*}$ Patient median age (range): all, 60 y (19-91 y); culture-positive, 57 y (27-91 y); culture-negative, 58 y (19-90 y). CSF, cerebrospinal fluid; HTLV-1, human T-cell lymphotropic virus type 1 .

†HTLV-1 serology results were available for $93 \%$ (13/14) of culture-positive patients and $78 \%(18 / 23)$ of culture-negative patients. 
In patients with culture-positive meningitis, blood and CSF culture results were positive for Klebsiella pneumoniae, S. gallolyticus, E. coli, Streptococcus infantarius, and Campylobacter fetus. Among patients with culture-negative meningitis, blood culture results were positive for $K$. pneumoniae and E. coli.

Our investigation has several limitations because of the single-center, retrospective nature of this study. Also, the sensitivity of parasitologic examination is low (10). We potentially underestimated the prevalence of strongyloidiasis.

On the basis of previous reports of associations between strongyloidiasis and Enterobacteriaceae meningitis $(3,5)$, our analysis proposes an association between strongyloidiasis and culture-negative suppurative meningitis in Okinawa. Our findings suggest that atypical suppurative meningitis can occur as occult dissemination $(3,4)$, that anthelminthic treatment may be indicated, and that steroids should be administered with caution (5). The presence of atypical suppurative meningitis in adults should prompt consideration of occult disseminated strongyloidiasis; the index of suspicion for patients with atypical suppurative meningitis is high.

\section{About the Author \\ Dr. Mukaigawara is a chief medical resident at Okinawa Chubu Hospital. His primary research interests include the roles of international organizations in reducing global health inequities and clinical epidemiology of diseases in the Pacific region, including strongyloidiasis.}

\section{References}

1. Pomar V, Benito N, López-Contreras J, Coll P, Gurguí M, Domingo P. Spontaneous gram-negative bacillary meningitis in adult patients: characteristics and outcome. BMC Infect Dis. 2013;13:451. http://dx.doi.org/10.1186/1471-2334-13-451

2. Sigurdardóttir B, Björnsson OM, Jónsdóttir KE, Erlendsdóttir H, Gudmundsson S. Acute bacterial meningitis in adults. A 20-year overview. Arch Intern Med. 1997;157:425-30. http://dx.doi.org/ 10.1001/archinte.1997.00440250077009

3. Kishaba T, Uchihara T, Ueno K, Goeku C, Shimabukuro Y. Gram-negative rod meningitis probably caused by "occult" disseminated strongyloidiasis [in Japanese]. Okinawa Medical Journal. 1985;22:539-41.

4. Kishaba T, Suginohara K, Tamaki K, Miyara Y, Endo K, Taira Y, et al. Culture negative suppurative meningitis probably caused by occult disseminated strongyloidiasis [in Japanese]. Okinawa Medical Journal. 1989;26:219-21.

5. Sasaki Y, Taniguchi T, Kinjo M, McGill RL, McGill AT, Tsuha S, et al. Meningitis associated with strongyloidiasis in an area endemic for strongyloidiasis and human T-lymphotropic virus-1: a singlecenter experience in Japan between 1990 and 2010. Infection. 2013;41:1189-93. http://dx.doi.org/10.1007/s15010-013-0483-2

6. Tanaka T, Hirata T, Parrott G, Higashiarakawa M, Kinjo T, Kinjo T, et al. Relationship among Strongyloides stercoralis infection, human T-cell lymphotropic virus type 1 infection, and cancer: a 24-year cohort inpatient study in Okinawa, Japan. Am J Trop Med Hyg. 2016;94:365-70. http://dx.doi.org/10.4269/ ajtmh.15-0556
7. Cresci GA, Bawden E. Gut microbiome: what we do and don't know. Nutr Clin Pract. 2015;30:734-46. http://dx.doi.org/ $10.1177 / 0884533615609899$

8. Spanos A, Harrell FE Jr, Durack DT. Differential diagnosis of acute meningitis. An analysis of the predictive value of initial observations. JAMA. 1989;262:2700-7. http://dx.doi.org/10.1001/ jama.1989.03430190084036

9. Durand ML, Calderwood SB, Weber DJ, Miller SI, Southwick FS, Caviness VS Jr, et al. Acute bacterial meningitis in adults. A review of 493 episodes. N Engl J Med. 1993;328:21-8. http://dx.doi.org/ 10.1056/NEJM199301073280104

10. Siddiqui AA, Berk SL, Siddiqui A, Berk S. Diagnosis of Strongyloides stercoralis infection. Clin Infect Dis. 2001;33:1040 7. http://dx.doi.org/10.1086/322707

Address for correspondence: Mitsuru Mukaigawara, Department of Medicine, Okinawa Chubu Hospital, 281 Miyazato, Uruma, 9042293, Okinawa, Japan; email: mmukaigawara@gmail.com

\section{Use of Next-Generation Sequencing for Diagnosis of West Nile Virus I nfection in Patient Returning to Belgium from Hungary}

\author{
Elke Wollants, ${ }^{1}$ David Smolders, ${ }^{1}$ \\ Reinout Naesens, Peggy Bruynseels, \\ Katrien Lagrou, Jelle Matthijnssens, \\ Marc Van Ranst
}

Author affiliations: : KU Leuven Rega Institute, Leuven, Belgium

(E. Wollants, J. Matthijnssens, M. Van Ranst); ZNA Hospital

Middelheim, Antwerp, Belgium (D. Smolders, R. Naesens,

P. Bruynseels); University Hospital Leuven, Belgium (K. Lagrou,

M. Van Ranst)

DOI: https://doi.org/10.3201/eid2412.180494

An elderly patient in Belgium who became critically ill after returning from Hungary was tested for pathogens using routine diagnostic tests. All results were negative. However, using next-generation sequencing on a cultured respiratory sample, laboratorians detected a complete West Nile virus genome, similar to strains isolated in southeastern Europe.

\footnotetext{
${ }^{1}$ These authors contributed equally to this article.
} 
$\mathrm{W}$ est Nile virus (WNV) is a widespread reemerging global pathogen. Mosquito bites are the source for nearly all human infections. These infections usually remain asymptomatic; only $20 \%-40 \%$ of infected persons experience a mild influenza-like illness called West Nile fever. West Nile neuroinvasive disease, which can manifest as meningitis, encephalitis, or acute flaccid paralysis, develops in $1 \%$ of infected persons; immunosuppressed and elderly patients are at higher risk (1). WNV can be divided into 5 phylogenetic lineages, of which lineages 1 and 2 have been associated with many outbreaks in humans. Since 2010, occasional localized epidemics have been ongoing in European and Mediterranean countries (2). To date, no autochthonous WNV infections have been reported in Belgium, and imported cases are uncommon $(3,4)$.

An 83-year-old man residing in Antwerp, Belgium, who had a medical history of moderate chronic kidney disease traveled to Hungary from January through August 2017. One day after returning from Hungary, he went to the hospital emergency department. He had fever and sought treatment for pain in his mouth and throat, which had begun the day before. He reported having felt unusually fatigued over several days. Clinical examination showed the patient to be lethargic but having no abnormal neurologic signs.

Laboratory testing at admission revealed a normal leukocyte count of $6.8 \times 10^{9}$ cells $/ \mathrm{L}$ and a moderately elevated C-reactive protein level of $63.2 \mathrm{mg} / \mathrm{L}$. A urine sediment test, a chest radiograph, and an abdominal ultrasound revealed no signs of disease. Treatment with amoxicillin/clavulanate was started, and blood and urine samples for cultures were collected. After 5 days, the patient was transferred to the intensive care unit because of increasing lethargy, followed by loss of consciousness, and clinical and biochemical signs of multiple organ failure. He was intubated and his treatment changed to piperacillin/tazobactam and clarithromycin; new cultures were also performed. Fever persisted, and the patient developed pancytopenia (leukocytes 1.68 $\times 10^{9}$ cells $/ \mathrm{L}$; hemoglobin $8.6 \mathrm{~g} / \mathrm{dL}$; platelets $61 \times 10^{9} / \mathrm{L}$ ). Because kidney function deteriorated further, continuous venovenous ultrafiltration hemodialysis was begun. An electroencephalogram exam showed delayed response without signs of epilepsy. A cranial computed tomography scan showed no signs of acute disease, and autoimmune screening did not reveal any abnormalities.

The results from a multiplex PCR (RespiFinder22; PathoFinder, Maastricht, the Netherlands), performed on a broncheoalveolar lavage (BAL) sample, were negative for 22 different respiratory pathogens. The result from a singleplex PCR for Mycobacterium tuberculosis was negative. Test results from immunofluorescence and PCR for Pneumocystis jirovecii were also negative. Cerebrospinal fluid testing revealed an elevated $\mathrm{pH}$ of 7.95 , elevated total protein of $79 \mathrm{mg} / \mathrm{dL}$, normal glucose of $65 \mathrm{mg} / \mathrm{dL}$, slightly increased leukocytosis of $7 / \mathrm{mm}^{3}$, and elevated erythrocytes of $370 / \mathrm{mm}^{3}$. Results were negative for molecular diagnostics of cerebrospinal fluid for 13 pathogens

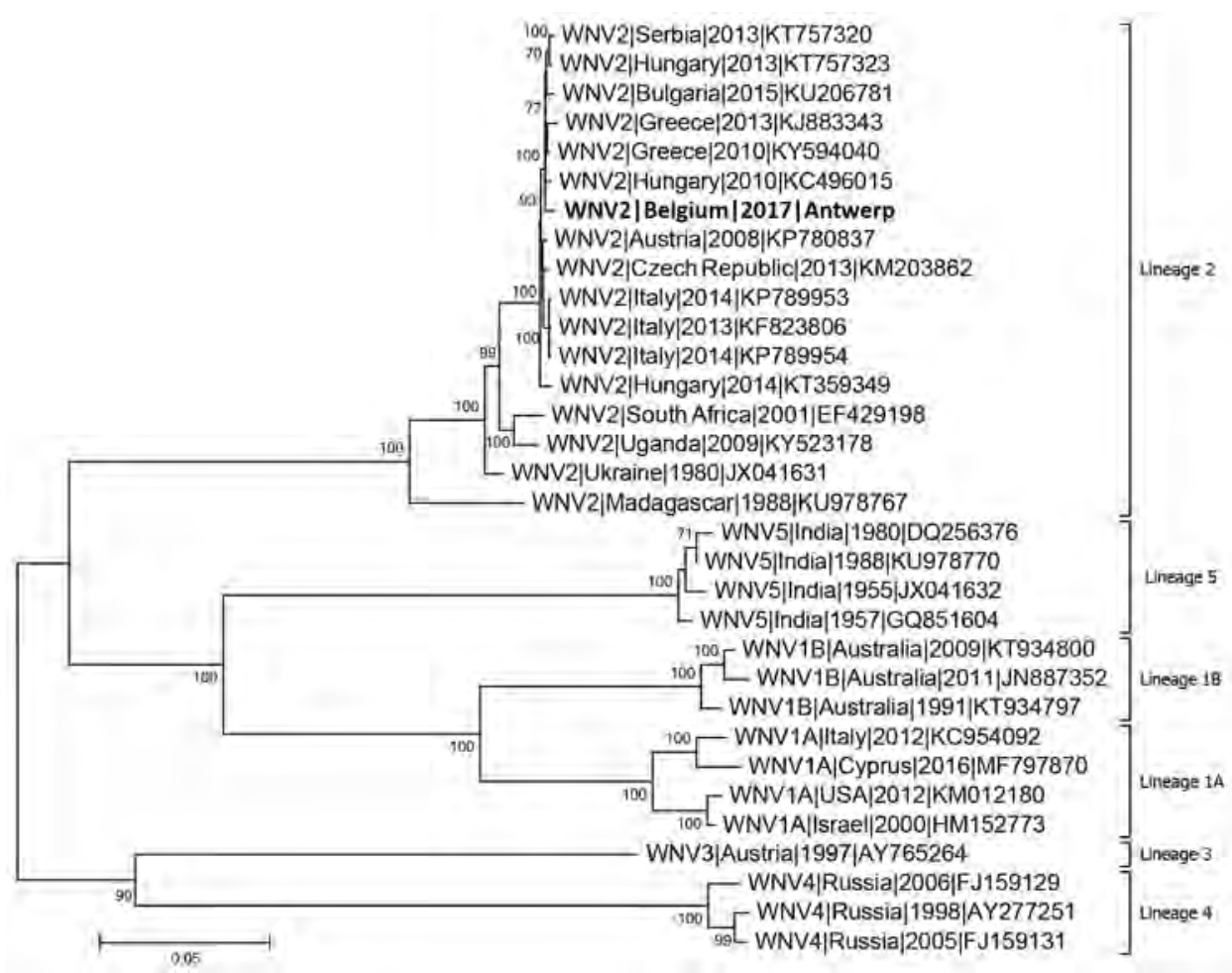

Figure. Alignments of complete WNV genome identified from patient in Belgium compared with 32 known reference strains from the 5 established WNV lineages. A phylogenetic tree was constructed using the maximum-likelihood method based on the Tamura-Nei model. Evolutionary analyses were conducted in MEGA7 (http://www.megasoftware. net). Bootstrapping was conducted with 1,000 bootstrap replicates; only bootstrap values over $70 \%$ are shown. The phylogenetic tree clusters the strain from this patient together with other WNV strains from southeastern Europe in lineage II. The patient isolate, named WNV-2|Belgium|2017|Antwerp (GenBank accession no. MH021189), is most similar to a strain from Hungary. GenBank accession numbers are provided for reference isolates. Scale bar represents genetic distance. 
and serology testing for 12 pathogens. Results from viral cultures on a BAL sample, taken 8 days after hospital admission, showed a cytopathogenic effect on 3 available cell lines (Vero, HEp-2, and MRC5) 7 days after incubation. Results from a PCR, administered in an effort to identify the positive viral culture, for herpes simplex virus and enterovirus were both negative.

Because the general condition of the patient had improved and stabilized, he was weaned from intubation 14 days after admission to the intensive care unit. He was discharged to the geriatric department and slowly recovered consciousness; his neurologic status also normalized. Three months after onset of the infection, the patient described having some memory loss and no memory at all of the days when the infection first started.

Because routine testing did not lead to a diagnosis, a BAL sample and a viral culture were sent to the University Hospital Leuven (Leuven, Belgium). The cell culture was subjected to next-generation sequencing (NGS) with the Novel enrichment technique of VIRomes protocol (5). This protocol includes purification of viral particles, nucleic acid extraction, and random PCR amplification, followed by library preparation and sequencing using NextSeq 500 system NGS technology (Illumina, San Diego, CA, USA) (6). The sequence reads obtained were filtered and trimmed, de novo assembled, and taxonomically classified $(7,8)$. Analysis of the NGS results identified a complete West Nile virus genome (11,060 nt). To confirm this result, a novel RNA extraction and a reverse transcription PCR (QIAGEN, Venlo, the Netherlands) with newly developed primers were performed on the BAL sample and cell culture, followed by Sanger sequencing (9). The sequence obtained from the positive sample was identical to the sequence from the complete genome obtained by NGS.

The WNV lineage 2 strain in this study, WNV2|Belgium|2017|Antwerp (GenBank accession number: MH021189), is closely related $(99.4 \%-99.6 \%$ nucleotide similarity) to a strain from Hungary and other Balkan countries identified between 2010 and 2015 (Figure), reinforcing the conclusion that this WNV infection was acquired in Hungary. This case illustrates that the combined use of cell cultures and NGS can be a powerful tool for identifying unknown pathogens in clinical specimens when results from routine tests are negative and the patient's condition is undiagnosed.

\footnotetext{
About the Author

Elke Wollants currently works as laboratory manager in the Clinical Virology Lab at the Rega Institute, KU Leuven. Her research interests are identifying pathogens not detected in routine diagnostic laboratory tests and nextgeneration sequencing.
}

\section{References}

1. Rizzoli A, Jimenez-Clavero MA, Barzon L, Cordioli P, Figuerola J, Koraka P, et al. The challenge of West Nile virus in Europe: knowledge gaps and research priorities. Euro Surv. 2015;20(20).

2. Petersen LR, Brault AC, Nasci RS. West Nile virus: review of the literature. JAMA. 2013;310:308-15. http://dx.doi.org/10.1001/ jama.2013.8042

3. Cnops L, Papa A, Lagra F, Weyers P, Meersman K, Patsouros N, et al. West Nile virus infection in Belgian traveler returning from Greece. Emerg Infect Dis. 2013;19:684-5. http://dx.doi.org/ 10.3201/eid1904.121594

4. Van Den Bossche D, Cnops L, Meersman K, Domingo C, Van Gompel A, Van Esbroeck M. Chikungunya virus and West Nile virus infections imported into Belgium, 2007-2012. Epidemiol Infect. 2015;143:2227-36. http://dx.doi.org/10.1017/ S0950268814000685

5. Conceição-Neto N, Zeller M, Lefrère H, De Bruyn P, Beller L, Deboutte W, et al. Modular approach to customise sample preparation procedures for viral metagenomics: a reproducible protocol for virome analysis. Sci Rep. 2015;5:16532. http://dx.doi.org/10.1038/srep16532

6. Conceição-Neto N, Zeller M, Lefrère H, De Bruyn P, Beller L, Deboutte W, et al. NetoVIR: a reproducible protocol for virome analysis. London: Nature Publishing Group; 2016.

7. Bankevich A, Nurk S, Antipov D, Gurevich AA, Dvorkin M, Kulikov AS, et al. SPAdes: a new genome assembly algorithm and its applications to single-cell sequencing. J Comput Biol. 2012;19:455-77.

8. Buchfink B, Xie C, Huson DH. Fast and sensitive protein alignment using DIAMOND. Nat Methods. 2015;12:59-60. http://dx.doi.org/10.1038/nmeth.3176

9. Wollants E, De Coster S, Van Ranst M, Maes P. A decade of norovirus genetic diversity in Belgium. Infect Genet Evol. 2015;30:37-44.

Address for correspondence: Elke Wollants, Rega Institute, KU Leuven, Laboratory of Clinical Virology, Herestraat 49 box 1040 Leuven 3000, Belgium; email: elke.wollants@kuleuven.be

\section{Disseminated Spiroplasma apis I nfection in Patient with Agammaglobulinemia, France}

\author{
Nicolas Etienne, Laurent Bret, \\ Cécile Le Brun, Hervé Lecuyer, Josquin Moraly, \\ Fanny Lanternier, Olivier Hermine, \\ Agnès Ferroni, Marc Lecuit, Sabine Pereyre, ${ }^{1}$ \\ Laure Beven, ${ }^{1}$ Olivier Lortholary ${ }^{1}$
}

Author affiliations: Paris Descartes University Hospital, Paris, France (N. Etienne, H. Lecuyer, F. Lanternier, O. Hermine,

A. Ferroni, M. Lecuit, O. Lortholary); Centre Hospitalier Régional, 
Orléans, France (L. Bret); Centre Hospitalier Régional Universitaire, Tours, France (C. Le Brun); Pasteur Institute, Paris (M. Lecuit); University of Bordeaux, Bordeaux, France (S. Pereyre); University of Bordeaux, Villenave d'Ornon, France (L. Beven)

DOI: https://doi.org/10.3201/eid2412.180567

We report a disseminated infection caused by Spiroplasma apis, a honeybee pathogen, in a patient in France who had $X$-linked agammaglobulinemia. Identification was challenging because initial bacterial cultures and direct examination by Gram staining were negative. Unexplained sepsis in patients with agammaglobulinemia warrants specific investigation to identify fastidious bacteria such as Spiroplasma spp.

I n February 2017, a 40-year-old man in France who was under immunoglobulin replacement therapy for X-linked agammaglobulinemia experienced a migrating nonitchy papular eruption. Skin biopsy revealed nonspecific lymphocytic dermatitis. One month later, he experienced distal interphalangeal arthritis. Histology of a synovial biopsy sample found a nonspecific lymphocytic infiltrate. Bacterial cultures were negative. In April 2017, febrile arthritis of the left ankle and right knee appeared, along with bilateral wrist and finger tenosynovitis (online Technical Appendix, https://wwwnc.cdc. gov/EID/article/24/12/18-0567-Techapp1.pdf). We initiated ceftriaxone $(1 \mathrm{~g} / \mathrm{d})$ and performed a left ankle joint aspiration 24 hours later. The patient remained febrile with a pulmonary valve murmur of tricuspid regurgitation and purpuric lesions, tenosynovitis in the left ankle and both wrists, and right knee and interphalangeal polyarthritis. Serum C-reactive protein was $136 \mathrm{mg} / \mathrm{dL}$ and IgG serum level $9.4 \mathrm{~g} / \mathrm{L}$. Transesophageal echography showed slight tricuspid regurgitation. Arthritis worsened, and we performed a right knee joint aspiration. Four of 6 blood cultures were positive after $55 \mathrm{~h}$ of incubation at $37^{\circ} \mathrm{C}$ (Bactec FX; Becton Dickinson, Franklin Lakes, NJ, USA). Results of direct examination by Gram staining of positive blood cultures and real-time multiplex PCR for blood culture identification (FilmArray BCID Panel; bioMérieux, Marcy l'Etoile, France) were negative. Small $\alpha$-hemolytic areas appeared on subcultures in horse blood agar after 5 days of aerobic culture; results of mass spectrometry performed on these areas were negative. Blood culture-specific PCR targeting Tropheryma whipplei, Bartonella spp., Brucella spp., Coxiella burnetii, and Rickettsia spp. showed negative results.

At this stage, we modified antimicrobial therapy to be optimal for Mycoplasma, Chlamydia, deficient streptococci, Campylobacter, and Helicobacter regarding the context of agammaglobulinemia. We introduced amoxicillin/clavulanic acid (12 g/1,200 mg/d) associated with levofloxacin (500 mg $2 \times / d$ ), leading to apyrexia and negative blood culture after 1 day. Results of testing for Chlamydia trachomatis (by PCR

${ }^{1}$ These authors contributed equally to this article. and culture) and Ureaplasma spp. and Mycoplasma hominis (by culture) in urine were negative. We seeded synovial fluid on blood culture, and small colonies developed on horse blood agar. We extracted DNA from the zone of hemolysis using EZ1 DNA tissue kit (QIAGEN, Valencia, CA, USA). We amplified and sequenced the 16S rRNA gene with the primer set $27 \mathrm{~F} / 16 \mathrm{~S} 1 \mathrm{RRB}$ as previously described (1) and performed bacterial identification using phylogenic analysis on the Bioinformatics Bacterial Identification tool (2). The 491-bp amplicon sequence was $100 \%$ identical to that of Spiroplasma apis strain ATCC 33834 (GenBank accession no. GU993267). We confirmed identification of $S$. apis by specific S. apis PCR (3) and culture (on modified SP4 agar) in synovial fluid from left ankle and right knee and in blood culture. Direct examination of synovial fluid and blood culture with dark field microscopy allowed identification of bacteria with a helical morphology and motility evocative of spiroplasmas (Figure). We obtained highly enriched Spiroplasma cultures starting from fluid and blood cultures. We retrospectively found specific PCR for $S$. apis positive in synovial biopsy from the distal interphalangeal sample. Antimicrobial drug susceptibility testing showed susceptibility to macrolides, tetracyclines, and fluoroquinolones and resistance to rifampin, cotrimoxazole, and penicillin G. Tetracycline and fluoroquinolone MIC values were similar to those reported for Ureaplasma spp.

Patient's condition gradually improved with 6-week administration of levofloxacin and iterative joint aspirations of the right knee. Because of severe tendinopathy, levofloxacin was stopped after 6 weeks and replaced by azithromycin $(500 \mathrm{mg} / \mathrm{d})$ and doxycycline $(200 \mathrm{mg} 2 \times / \mathrm{d})$ for 6 weeks.

Patients with agammaglobulinemia are susceptible to infections, including mycoplasma infections (4). Invasive infections such as endocarditis and septic arthritis have been reported with $M$. hominis and Ureaplasma urealyticum in hypogammaglobulinemic patients. Of note, our patient contracted mycoplasma infection despite an appropriate gammaglobulin serum level (IgG 9.4 g/L).

Spiroplasmas, arthropod-infecting bacteria of the class Mollicutes, are similar to mycoplasmas. Pathogenic species include S. apis, detected in hives in southwestern France (5). S. apis is associated with the lethal May disease of the European honeybee Apis mellifera. Its prevalence in bee colonies in France was not reported but was recently shown to reach high percentages in other countries (6). Only 3 cases of human infection due to Spiroplasma spp. have been reported: hepatitis in a lung transplant recipient (7), uveitis in a premature neonate (8), and bacteremia in a patient under certolizumab treatment (9). None of these infections correlated with any noticeable insect bite history. In contrast, the patient we report had multiple stings by a flying insect resembling a hornet in August 2016, in the Loiret department of France, leading to cutaneous blebs and transitory rash. This finding raises the possibility that $S$. apis might infect insects other 

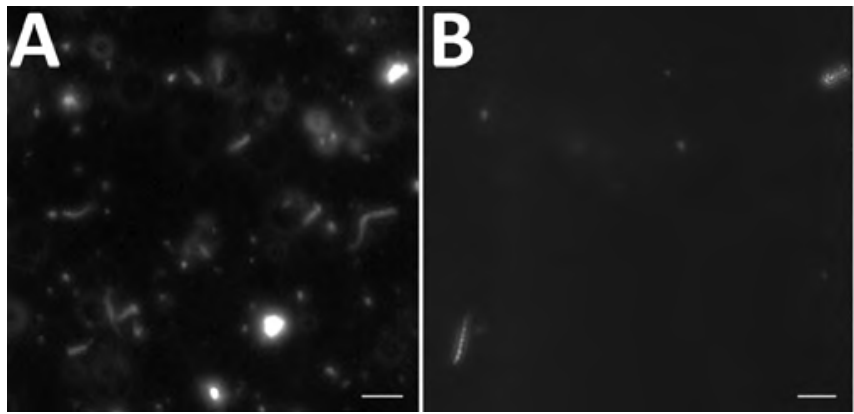

than honeybees. The insect stings in this patient are a likely gateway of the reported infection.

In summary, clinicians and microbiologists should be aware of fastidious organisms in atypical infections in immunocompromised patients. Our findings indicate a need for prolonged culture on specific agar on all joint fluids in patients with agammaglobulinemia and targeted molecular methods to identify S. apis organisms.

\section{Acknowledgments}

We thank Anne Laurence Thomi Georgelin, who referred the patient to the Necker-Pasteur Center for Infectious Diseases and Tropical Medicine; Valérie Zeller for the management of septic polyarthritis; and Philippe Lanotte and Marie-Pierre Dubrana, who participated in molecular analysis.

\section{About the Author}

Dr. Etienne is an infectious disease clinician and a clinical immunologist in Necker-Pasteur Center for Infectious Diseases, Paris. His research interest focuses on infections in immunocompromised patients.

\section{References}

1. Schabereiter-Gurtner C, Nehr M, Apfalter P, Makristathis A, Rotter ML, Hirschl AM. Evaluation of a protocol for molecular broad-range diagnosis of culture-negative bacterial infections in clinical routine diagnosis. J Appl Microbiol. 2008;104:1228-37. http://dx.doi.org/10.1111/j.1365-2672.2007.03648.x

2. Devulder G, Perrière G, Baty F, Flandrois JP. BIBI, a bioinformatics bacterial identification tool. J Clin Microbiol. 2003; 41:1785-7. http://dx.doi.org/10.1128/JCM.41.4.1785-1787.2003

3. Meeus I, Vercruysse V, Smagghe G. Molecular detection of Spiroplasma apis and Spiroplasma melliferum in bees. J Invertebr Pathol. 2012;109:172-4. http://dx.doi.org/10.1016/j.jip.2011.11.006

4. Blanchard A, Bébéar CM. Mycoplasmas of humans. In: Razin S, Herrmann R, editors. Molecular biology and pathogenicity of mycoplasmas. New York: Kluwer Academic/Plenum Publishers; 2002. p. $45-71 .</$ edb $>$

5. Mouches C, Bové JM, Tully JG, Rose DL, McCoy RE, Carle-Junca P, et al. Spiroplasma apis, a new species from the honey-bee Apis mellifera. Ann Microbiol (Paris). 1983;134A:383-97.

6. Schwarz RS, Teixeira ÉW, Tauber JP, Birke JM, Martins MF, Fonseca I, et al. Honey bee colonies act as reservoirs for two Spiroplasma facultative symbionts and incur complex, multiyear infection dynamics. MicrobiologyOpen. 2014;3:341-55. http://dx.doi.org/10.1002/mbo3.172

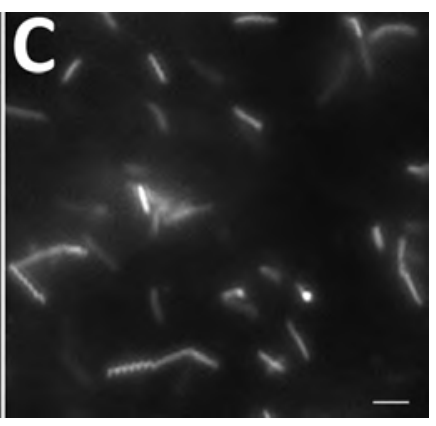

Figure. Direct examination with dark-field microscopy of specimens from a patient with agammaglobulinemia who had Spiroplasma apis infection, France. A) Helical and motile bacteria in blood culture. B) Elongated and coccoid bacteria in joint fluid. C) Helical and motile bacteria in culture from joint fluid in modified SP4 broth medium. Scale bar indicates $10 \mu \mathrm{m}$.
7. Mueller NJ, Tini GM, Weber A, Gaspert A, Husmann L, Bloemberg G, et al. Hepatitis from Spiroplasma sp. in an immunocompromised patient. Am J Transplant. 2015;15:2511-6. http://dx.doi.org/10.1111/ajt.13254

8. Lorenz B, Schroeder J, Reischl U. First evidence of an endogenous Spiroplasma sp. infection in humans manifesting as unilateral cataract associated with anterior uveitis in a premature baby. Graefes Arch Clin Exp Ophthalmol. 2002;240:348-53. http://dx.doi.org/10.1007/s00417-002-0453-3

9. Aquilino A, Masiá M, López P, Galiana AJ, Tovar J, Andrés M, et al. First human systemic infection caused by Spiroplasma. J Clin Microbiol. 2015;53:719-21. http://dx.doi.org/10.1128/JCM.02841-14

Address for correspondence: Olivier Lortholary, Necker-Enfants Malades University Hospital, 149 rue de Sevre 75743, Paris CEDEX, France; email: olivier.lortholary@aphp.fr

\section{Mycoplasma ovipneumoniae in Wildlife Species beyond Subfamily Caprinae}

\author{
Margaret A. Highland, David R. Herndon, Scott \\ C. Bender, Lisa Hansen, Robert F. Gerlach, \\ Kimberlee B. Beckmen
}

Author affiliations: Washington Animal Disease Diagnostic Laboratory, Pullman, Washington, USA (M.A. Highland); US Department of Agriculture, Pullman (M.A. Highland, D.R. Herndon); Navajo Technical University, Crownpoint, New Mexico, USA (S.C. Bender); Barron Veterinary Clinic, Barron, Wisconsin, USA (L. Hansen); Alaska Department of Environmental Conservation, Anchorage, Alaska, USA (R.F. Gerlach); Alaska Department of Fish and Game, Fairbanks, Alaska (K.B. Beckmen) DOI: https://doi.org/10.3201/eid2412.180632 
Elucidating the emergence of Mycoplasma ovipneumoniae-associated respiratory disease in ruminants requires identification of the pathogen host range. This bacterium was thought to be host restricted to subfamily Caprinae, but we describe its identification in healthy moose, caribou, and mule deer and diseased mule and white-tailed deer, all species in subfamily Capreolinae.

$M$ ycoplasma ovipneumoniae was identified in Queensland, Australia, in 1972 as an infectious agent associated with pneumonia in domestic sheep (Ovis aries) (1). Since then, it has most frequently been identified in healthy and diseased domestic sheep, domestic goats (Capra aegagrus hircus), and bighorn sheep (Ovis canadensis). Although M. ovipneumoniae was identified in respiratory disease outbreaks in bighorn sheep as early as 1980 (2), the past decade has brought it under scrutiny because of evidence supporting its association with bighorn sheep pneumonia in western North America (3). Because most reports have described this bacterium in sheep and goats, and fewer in muskoxen (Ovibos moschatus) (4), some have concluded that $M$. ovipneumoniae is specific to the subfamily Caprinae (5) or has a host range limited to Caprinae (6), despite publications describing M. ovipneumoniae in non-Caprinae species, including Beira antelope (Dorcatragus megalotis) with respiratory disease in Qatar (7) and in 9 cattle (Bos taurus) in Colorado, USA (8). Unfortunately, description of the method(s) used to identify M. ovipneumoniae in those reports was limited to stating the use of PCR with no supporting sequence data.

In general, definitive claims of host range restrictions are absent from mycoplasma literature, because "assumptions about restricted host range of mycoplasmas, based on the host from which they were first or frequently isolated, are usually made in the context of nearly complete absence of representative sampling of the vast majority of potential vertebrate hosts" (9). In addition to insufficient sampling of potential hosts, the fastidious and variably culturable nature of $M$. ovipneumoniae often requires molecular techniques for identification. We used molecular techniques to analyze multiple species from the subfamily Capreolinae for the presence of M. ovipneumoniae.

During July 2017-January 2018, the US Department of Agriculture Agricultural Research Service in Pullman, WA, USA, received nasal swab samples from 230 moose (Alces alces) and 243 caribou (Rangifer tarandus) from Alaska and 5 mule deer (Odocoileus hemionus) from Arizona (online Technical Appendix, https://wwwnc.cdc.gov/ EID/article/24/12/18-0632-Techapp1.pdf). Also received in February 2018 was an isolate of $M$. ovipneumoniae that had been cultured by Newport Laboratories (Worthington, MN, USA) from lung tissue from a white-tailed deer (Odocoileus virginianus) that died during a pneumonia outbreak at a captive facility in the upper Midwest region of the United States in 2016. We extracted DNA from swab samples and from the white-tailed deer isolate, performed PCR using a modified published PCR method (10) to amplify part of the 16S rRNA gene, and sequenced amplicons of the correct size (online Technical Appendix). Forward and reverse sequences were merged, manually inspected for errors, and trimmed to $290 \mathrm{bp}$ using Sequencher 5.2.2 (Gene Codes, Ann Arbor, MI, USA) corresponding to a 103-392-bp region of the 16S rRNA gene of type strain Y98 obtained from American Type Culture Collection (ATCC; Manassas, VA, USA). Sequences were blastn queried (https://www. ncbi.nlm.nih.gov/BLAST/) and identified as M. ovipneumoniae at $100 \%$ coverage and $\geq 97 \%$ identity to Y98 (GenBank accession no. NR_025989.1). The analyzed region represents the most divergent region of the 16S rRNA gene among strains of M. ovipneumoniae and between Mycoplasma spp. of the highest percentage identity to $M$. ovipneumoniae (online Technical Appendix).

We detected $M$. ovipneumoniae in 6 moose $(2.6 \%)$, including 3 from 2 captive facilities and 3 free-ranging; 5 free-ranging caribou (2.1\%); and 2 of 5 mule deer, 1 of which was coughing and had nasal discharge at the time of sample collection. The identity of the lung isolate, cultured from the white-tailed deer that had died from pneumonia, was confirmed as M. ovipneumoniae. For sequence comparison, we generated a percent identity matrix with the $M$. ovipneumoniae sequences from the Capreolinae species, nasal swabs collected from 2 healthy M. ovipneumoniaepositive wild sheep (online Technical Appendix), Y98, and bacteria of the closest identity to Y 98 and sequences generated in this study based on blastn queries (M. dispar, $M$. hyopneumoniae, and M. flocculare) (online Technical Appendix). The percent identity matrix revealed 2 groupings of $M$. ovipneumoniae and illustrates the divergence from the other Mycoplasma spp. of closest identity to $M$. ovipneumoniae. Sample sequences have been submitted to GenBank (online Technical Appendix).

This report describes $M$. ovipneumoniae carriage in multiple members of the subfamily Capreolinae (moose, caribou, and mule deer), and emergence of M. ovipneumoniae-associated respiratory disease in deer. These findings are of importance to epidemiologic investigations because current dogma regarding host specificity may dissuade laboratories from pursuing identification of this fastidious bacterium in hosts beyond the subfamily Caprinae. Improved diagnostic methods to increase detection sensitivity are warranted based on information provided in this report (online Technical Appendix). Full-length genome sequencing and phylogenetic analysis of $M$. ovipneumoni$a e$ isolates are necessary next steps in inferring evolutionary relationships and origin of this bacterium in identified host species. 


\section{Acknowledgments}

We thank Nicholas P. Durfee and Paige Grossman for technical support, Eric Roalson for assistance with sequence analysis, and Donald P. Knowles for final critical manuscript review. We acknowledge the following individuals and agencies for providing wildlife samples: Sara Longson, Anne Crane, John Crouse, Dominic Demma, Torsten Bentzen, Tony Hollis, Lincoln Parrett, Jason Caikoski, Warren Hansen, and the wildlife biologists and technicians at the Alaska Department of Fish and Game; Navajo Nation Zoological and Botanical Park-Navajo Nation Department of Fish and Wildlife; Ed Klein and Dan Love, Colorado Department of Agriculture; and Anthony Madrid and Lindsey Hansen, US Department of Agriculture Forest Service, San Juan National Forest.

Financial support for this project was provided by the US Department of Agriculture Agricultural Research Service, CRIS Project funds 2090-32000-036-00D.

\begin{abstract}
About the Author
Dr. Highland is a veterinary medical officer at the US Department of Agriculture Agricultural Research Service Animal Disease Research Unit in Pullman, WA, USA, and is an adjunct faculty member in the department of veterinary microbiology and pathology and the School for Global Animal Health at Washington State University. Her research interests include infectious diseases of small ruminants, with special focus on respiratory disease agents of domestic and wildlife species.
\end{abstract}

\section{References}

1. Carmichael LE, St George TD, Sullivan ND, Horsfall N. Isolation, propagation, and characterization studies of an ovine Mycoplasma responsible for proliferative interstitial pneumonia. Cornell Vet. 1972;62:654-79.

2. Bunch TD. Chronic sinusitis in desert bighorn sheep in northwestern Arizona. In: Douglas CL, Krausman PR, Leslie DM, editors. Desert Bighorn Council meeting 1985. Las Vegas (NV, USA): the Council; 1985. p. 1-3.

3. Besser TE, Highland MA, Baker K, Cassirer EF, Anderson NJ, Ramsey JM, et al. Causes of pneumonia epizootics among bighorn sheep, Western United States, 2008-2010. Emerg Infect Dis. 2012;18:406-14. http://dx.doi.org/10.3201/eid1803.111554

4. Handeland K, Tengs T, Kokotovic B, Vikøren T, Ayling RD, Bergsjø B, et al. Mycoplasma ovipneumoniae - a primary cause of severe pneumonia epizootics in the Norwegian muskox (Ovibos moschatus) population. PLoS One. 2014;9:e106116. http://dx.doi.org/10.1371/journal.pone.0106116

5. Cassirer EF, Manlove KR, Plowright RK, Besser TE. Evidence for strain-specific immunity to pneumonia in bighorn sheep. Journal Wildl Manag. 2017;81:133-43. http://dx.doi.org/10.1002/ jwmg. 21172

6. Besser TE, Cassirer EF, Potter KA, Foreyt WJ. Exposure of bighorn sheep to domestic goats colonized with Mycoplasma ovipneumoniae induces sub-lethal pneumonia. PLoS One. 2017;12:e178707. http://dx.doi.org/10.1371/journal.pone.0178707

7. Gull JM, Hebel C, Deb A, Arif A, Clauss M, Hatt JM, et al. Blood values of captive beira antelope (Dorcatragus megalotis) prior to and during an outbreak of fibrinous pleuropneumonia syndrome (FPPS). J Zoo Wildl Med. 2014;45:735-43. http://dx.doi.org/10.1638/2013-0073.1

8. Wolfe LL, Diamond B, Spraker TR, Sirochman MA, Walsh DP, Machin CM, et al. A bighorn sheep die-off in southern Colorado involving a Pasteurellaceae strain that may have originated from syntopic cattle. J Wildl Dis. 2010;46:1262-8. http://dx.doi.org/10.7589/0090-3558-46.4.1262

9. Brown DR, Zacher LA, Wendland LD, Brown MB. Emerging mycoplasmoses in wildlife. In: Blanchard A, Browning GF, editors. Mycoplasmas: molecular biology pathogenicity and strategies for control. Norfolk (UK): Horizon Bioscience; 2005. p. 382-414.

10. McAuliffe L, Hatchell FM, Ayling RD, King AI, Nicholas RA. Detection of Mycoplasma ovipneumoniae in Pasteurellavaccinated sheep flocks with respiratory disease in England. Vet Rec. 2003;153:687-8. http://dx.doi.org/10.1136/vr.153.22.687

Address for correspondence: Margaret A. Highland, US Department of Agriculture, Animal Disease Research Unit, Agricultural Research Service, ADBF 3003, Washington State University, Pullman, WA 99164 , USA; email: maggie.highland@ars.usda.gov

\section{Locally Acquired Leptospirosis in Expedition Racer, Manitoba, Canada}

\author{
Sameer S. Kassim, Antonia Dibernardo, \\ L. Robbin Lindsay, Terence C. Wuerz
}

Author affiliations: University of Manitoba, Winnipeg, Manitoba, Canada (S.S. Kassim, T.C. Wuerz); Public Health Agency of Canada, Winnipeg (A. Dibernardo, L.R. Lindsay); St. Boniface General Hospital, Winnipeg (T.C. Wuerz)

DOI: https://doi.org/10.3201/eid2412.181015

Leptospirosis is found worldwide, except in northern regions. We report a case associated with a backcountry adventure race in Manitoba, Canada. Initially, nonspecific symptomatology and diagnostic pitfalls contributed to a delay in identification. Careful attention needs to be paid to exposure to and risk for leptospirosis in northern and temperate climates.

T eptospirosis is a zoonotic disease caused by Leptospitransmission occurs by direct contact with contaminated urine or animals (1). The organism has a worldwide distribution outside of polar regions and is common during the rainy season in tropical and temperate climates (2). 
Occupational exposure is a reported risk factor in disease-endemic areas (3). Other risk factors are recreational exposure to water in temperate and tropical settings, walking barefoot through surface water, contact with wild rodents, and accidental laboratory exposure (3). Cases of leptospirosis have been identified at adventure races $(4,5)$.

Leptospirosis is not reported nationally in Canada or the United States. In the United States, 150-200 hospital admissions for leptospirosis were reported annually during 1998-2000 (6). In Canada, only 25 cases with supportive diagnostic results and 36 confirmed cases overall have been observed by the National Microbiology Laboratory (Winnipeg, Manitoba, Canada), which performs testing nationally (Table). In most instances, it is unclear whether these cases were acquired locally or abroad. We report a case of leptospirosis associated with a backcountry adventure race in Manitoba.

In October 2016, a 20-year-old man came to an emergency department in Winnipeg with a 7-day history of fever, dyspnea, cough, pleuritic chest pain, myalgias, sweats, nausea, vomiting, and diarrhea. These signs and symptoms had improved 1-2 days before admission. Rapid deterioration of the condition of the patient and progressive dyspnea and hypoxia were the reasons for his coming to the emergency department.

A complete blood count showed a leukocyte count of $12.1 \times 10^{9} \mathrm{cells} / \mathrm{L}$ (reference range $4.5 \times 10^{9}-11 \times 10^{9}$ cells $/ \mathrm{L}$ ), $84 \%$ neutrophils, and a platelet count of $34 \times 10^{9} / \mathrm{L}$ (reference range $140 \times 10^{9}-440 \times 10^{9} / \mathrm{L}$ ). Hemoglobin level was within the reference range. Aspartate aminotransferase level was $70 \mathrm{U} / \mathrm{L}$ (reference range $10 \mathrm{U} / \mathrm{L}-32 \mathrm{U} / \mathrm{L}$ ), alanine aminotransferase level $80 \mathrm{U} / \mathrm{L}$ (reference value $<30$ $\mathrm{U} / \mathrm{L}$ ), alkaline phosphatase level $144 \mathrm{U} / \mathrm{L}$ (reference range $30 \mathrm{U} / \mathrm{L}-120 \mathrm{U} / \mathrm{L}$ ), total bilirubin level $23 \mu \mathrm{mol} / \mathrm{L}$ (reference range $5 \mu \mathrm{mol} / \mathrm{L}-21 \mu \mathrm{mol} / \mathrm{L}$ ), and direct bilirubin level $13 \mu \mathrm{mol} / \mathrm{L}$ (reference value $<7.0 \mu \mathrm{mol} / \mathrm{L}$ ).
Blood smear and culture results were negative for bacteria and fungal elements. The patient had a urea level of $13.6 \mathrm{mmol} / \mathrm{L}$ (reference range $2.5 \mathrm{mmol} / \mathrm{L}-7.1 \mathrm{mmol} / \mathrm{L}$ ) and a creatinine level of $118 \mu \mathrm{mol} / \mathrm{L}$ (reference range 44 $\mu \mathrm{mol} / \mathrm{L}-106 \mu \mathrm{mol} / \mathrm{L})$. Results of urinalysis were within reference limits. Computed tomography of the abdomen and chest showed hepatosplenomegaly, nephromegaly, multifocal and subpleural nodular airspace opacities bilaterally with early cavitation, and a small left pleural effusion. There was no evidence of alveolar hemorrhage by imaging.

Within 12 hours, progressive hypoxemia developed; the patient was intubated and mechanically ventilated. He was given 2 doses of ceftriaxone initially, which was changed to meropenem. The patient also received vancomycin, ciprofloxacin, and liposomal amphotericin B for treatment of possible infection with Blastomyces gilchristii. A chest drain was inserted, and $1.5 \mathrm{~L}$ of serosanguinous exudative fluid was drained. Culture of pleural and bronchoalveolar fluid did not show pathogenic bacteria, mycobacteria, or fungi; PCR results for common respiratory viruses were negative.

Over the next 10 days, the patient's condition improved and he was extubated. Antimicrobial drugs were discontinued, amphotericin was replaced by oral itraconazole, and he was discharged. An IgM ELISA for leptospirosis performed 5 days after admission showed a positive result. Microscopic agglutination test (MAT) antibody titers for L. interrogans were 1:400 for serovar Australis and 1:100 for serovar Canicola. No appropriate clinical samples were available for retrospective confirmatory PCR testing. Itraconazole was discontinued at follow-up, 20 days after discharge.

Ten weeks after admission, the patient remained clinically asymptomatic, and results of a chest radiograph were uneventful. Serologic analysis showed persistently positive Leptospira IgM titers but negative MAT titers for all serovars. Repeat ultrasound of the abdomen showed near resolution of the organomegaly.

\begin{tabular}{|c|c|c|c|}
\hline Region and province & No. supportive cases $\dagger$ & No. confirmed cases $\ddagger$ & No. cases/region \\
\hline \multicolumn{4}{|l|}{ Western, including the Prairies } \\
\hline British Columbia & 5 & 6 & 20 \\
\hline Alberta & 2 & 4 & \\
\hline Saskatchewan & 0 & 0 & \\
\hline Manitoba & 3 & 0 & \\
\hline \multicolumn{4}{|l|}{ Central } \\
\hline Ontario & 9 & 9 & 37 \\
\hline Quebec & 6 & 13 & \\
\hline \multicolumn{4}{|l|}{ Atlantic } \\
\hline New Brunswick & 0 & 3 & 4 \\
\hline Nova Scotia & 0 & 1 & \\
\hline Prince Edward Island & 0 & 0 & \\
\hline Newfoundland and Labrador & 0 & 0 & \\
\hline Overall & 25 & 36 & 61 \\
\hline
\end{tabular}


We retrospectively identified a possible exposure 10 days before admission: the patient had participated in a 9-hour adventure race in the Whiteshell area of southeastern Manitoba. The patient trekked, biked, and paddled through swamps, bogs, and rivers and reported ingesting murky groundwater at various points during the race.

The Whiteshell area is predominantly a nature conservancy and home of many mammals. Most veterinary studies have focused on prevalence of leptospirosis in wildlife species in specific and limited geographic areas. Leptospirosis has been detected in beavers, coyotes, deer, foxes, opossums, and otters in Canada and is especially prevalent in raccoons and skunks in urban and rural environments $(8,9)$. There are limited published data on endemic leptospirosis in Canada, but sylvatic exposures have been reported among hunters, trappers, and indigenous peoples; the most northern exposure was identified in the Nunavik region of Quebec (10).

We report a case of endemically acquired leptospirosis, detected by using supportive laboratory evidence, from an adventure racer in western Canada. As the popularity of these events increases, clinicians should be vigilant for this life-threatening infection in regions not normally considered endemic for this disease. Early diagnosis of leptospirosis based on clinical features and exposure history in temperate and tropical climates should be considered. For suspected cases, an acute-phase serum sample should be collected for serologic testing by IgM ELISA and MAT. Urine or blood should be collected before treatment for PCR testing, followed by convalescent-phase serologic analysis where applicable to assist diagnosis. In addition, appropriate therapy with intravenous penicillin or ceftriaxone should be initiated empirically for severe disease.

\section{Acknowledgment}

We thank Katarina Bernat for performing diagnostic testing.

\footnotetext{
About the Author

Dr. Kassim is a resident medical microbiologist and clinical investigator trainee in the Department of Medical Microbiology and Infectious Disease, University of Manitoba, Winnipeg,
}

Manitoba, Canada. His research interests include infection prevention and control, application of molecular methods in infectious diseases, tropical medicine, and antimicrobial stewardship.

\section{References}

1. Adler B, de la Peña Moctezuma A. Leptospira and leptospirosis. Vet Microbiol. 2010;140:287-96. http://dx.doi.org/10.1016/ j.vetmic.2009.03.012

2. Haake DA, Levett PN. Leptospira species (leptospirosis). In: Bennett JE, Dolin R, Blaser MJ, eds. Mandell, Douglas, and Bennett's principles and practice of infectious diseases, 8th ed. Philadelphia: Elsevier Saunders; 2015. p. 2714-20.

3. Gundacker ND, Rolfe RJ, Rodriguez JM. Infections associated with adventure travel: A systematic review. Travel Med Infect Dis. 2017;16:3-10. http://dx.doi.org/10.1016/j.tmaid.2017.03.010

4. Teichmann D, Göbels K, Simon J, Grobusch MP, Suttorp N. A severe case of leptospirosis acquired during an iron man contest. Eur J Clin Microbiol Infect Dis. 2001;20:137-8. http://dx.doi.org/ $10.1007 / \mathrm{s} 100960000433$

5. Centers for Disease Control and Prevention. Update: leptospirosis and unexplained acute febrile illness among athletes participating in triathlons-Illinois and Wisconsin, 1998. MMWR Morb Mortal Wkly Rep. 1998;47:673-6.

6. Traxler RM, Callinan LS, Holman RC, Steiner C, Guerra MA. Leptospirosis-associated hospitalizations, United States, 1998-2009. Emerg Infect Dis. 2014;20:1273-9. http://dx.doi.org/ 10.3201/eid2008.130450

7. Centers for Disease Control and Prevention. Leptospirosis (Leptospira interrogans): case definition, 2013 [cited 2018 Jun 10]. https://www.cdc.gov/leptospirosis/index.html

8. Shearer KE, Harte MJ, Ojkic D, Delay J, Campbell D. Detection of Leptospira spp. in wildlife reservoir hosts in Ontario through comparison of immunohistochemical and polymerase chain reaction genotyping methods. Can Vet J. 2014;55:240-8.

9. Jardine C, Lindsay LR, Nicholson VM, Ojkic D, Prescott JF. Longitudinal study on the seroprevalence of avian influenza, leptospirosis, and tularemia in an urban population of raccoons (Procyon lotor) in Ontario, Canada. Vector Borne Zoonotic Dis. 2011;11:37-42. http://dx.doi.org/10.1089/vbz.2009.0209

10. Messier V, Lévesque B, Proulx JF, Rochette L, Serhir B, Couillard $\mathrm{M}$, et al. Seroprevalence of seven zoonotic infections in Nunavik, Quebec (Canada). Zoonoses Public Health. 2012;59:107-17. http://dx.doi.org/10.1111/j.1863-2378.2011.01424.x

Address for correspondence: Sameer S. Kassim, Department of Medical Microbiology and Infectious Diseases, University of Manitoba, Rm 540, Basic Medical Sciences Bldg, 745 Bannatyne Ave, Winnipeg, Manitoba R3E 0J9, Canada: email: kassims@umanitoba.ca 


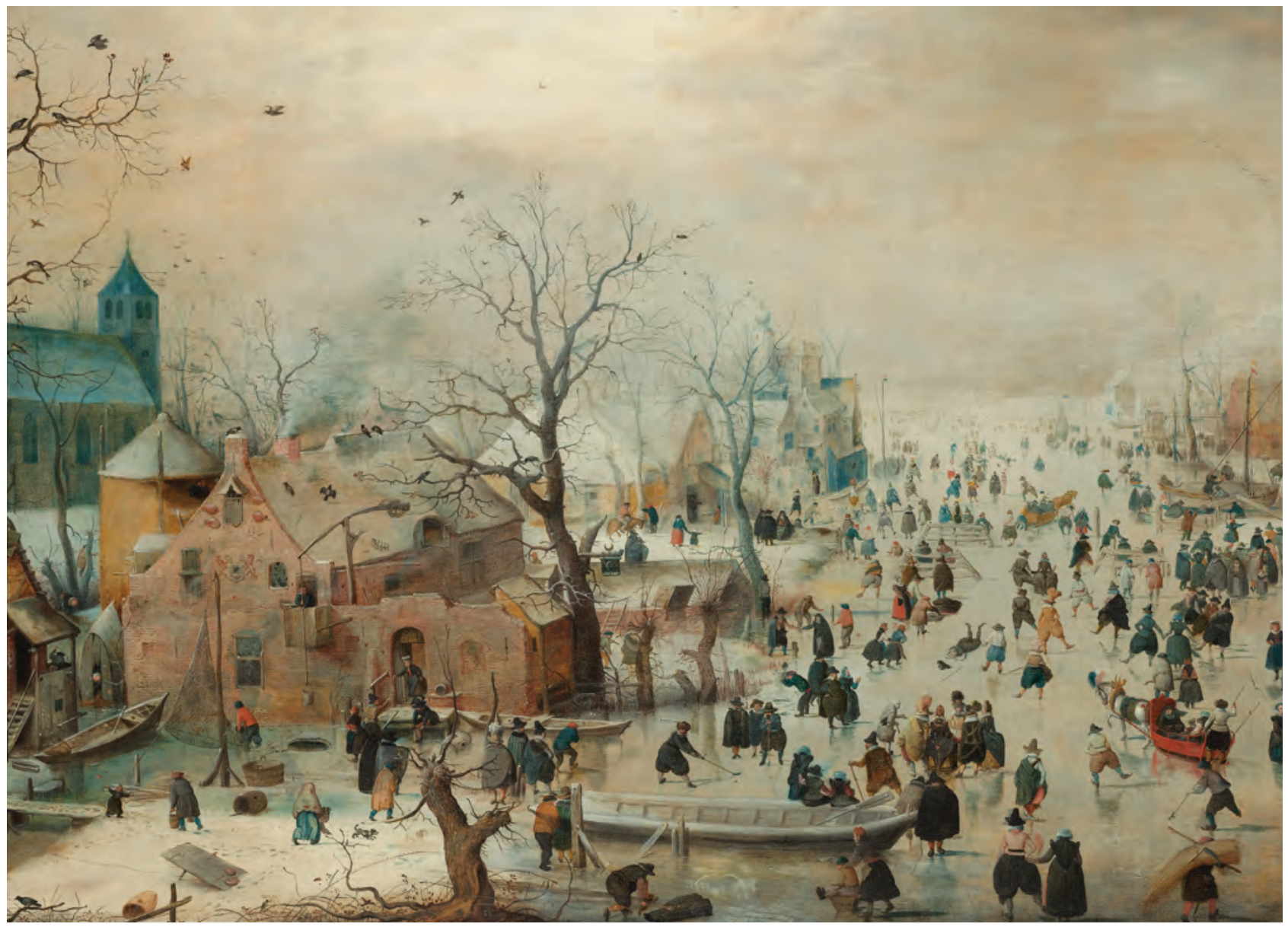

Hendrick Avercamp (1585-1634), Winter Landscape with Ice Skaters (detail) (c. 1608). Oil on panel, 30.4 in x 52 in/77.3 cm x 131.9 $\mathrm{cm}$. Digital image courtesy of Rijksmuseum, Amsterdam.

\section{An I cy Vista from a Golden Age}

\section{Byron Breedlove}

66 To other nation in the world looks forward to an 1 icy season the way the Dutch do," wrote Wim Pijbes and Earl A. Powell III in the preface to Hendrick Avercamp: Master of the Ice Scene. Wintery weather predominated across most of Europe during the Little Ice Age, which started around 1300 and lasted for about 350 years. The atypically cold, harsh weather affected agriculture, health, commerce, emigration, and social stability, precipitating unrest, revolt, warfare, and migration. Throughout

Author affiliation: Centers for Disease Control and Prevention, Atlanta, Georgia, USA

DOI: https://doi.org/10.3201/eid2412.AC2412 this time, epidemics of plague flared across Europe, and malaria persisted despite frigid conditions.

The coldest period of the Little Ice Age roughly overlapped the 90 years of the Dutch Golden Age. Unlike most of Europe, the Dutch Republic, which preceded the Netherlands of today, thrived during the icy 17 th century. Despite its small size and limited natural resources, the country achieved stability and prosperity, chiefly because of its unmatched commercial fishing and trading fleets and its agricultural practices. That confluence of "political, economic, religious, and social circumstances created a unique and fruitful climate for the arts," according to the National Gallery of Art, which also notes, "A remarkable number of 
pictures of extraordinary quality were produced during the Dutch Golden Age."

Perhaps less known than some of his contemporaries, Hendrick Avercamp is nonetheless considered to be among the great masters of that time. Avercamp painted vibrant, detailed winter landscapes during the first third of the 17th century. The United Sates National Gallery of Art states that he "did not invent the winter landscape, but he was the first artist to specialize in this quintessential Dutch subject."

Details of Avercamp's life are sparse. He was born in Amsterdam in 1585 , though the next year his family relocated to the smaller city of Kampen. His father, Barent, served as the town apothecary and later as its doctor until he died of the plague in 1602. His first art teacher and one of his brothers also died of the plague during that frigid 17 th century.

Apparently, Avercamp could not speak and was known as "the Mute of Kampen." He came from a learned family: his father and brothers were physicians and apothecaries, and his grandfather and uncle were headmasters of a prestigious Latin school. Avercamp probably lived in the house that his mother Beatrix owned. Art historian Arthur $\mathrm{K}$. Wheelock Jr. observed that "There, in relative isolation from the mainstreams of Dutch art, he devoted himself almost entirely to the painting of winter scenes and specifically to depictions of crowds of people engaging in a wide range of activities on frozen rivers." In total, Avercamp produced about a hundred paintings of winter scenes.

Avercamp completed Winter Landscape with Ice Skaters, this month's cover art, following the exceptionally frigid winter of 1607-1608. The painting, which is his largest known work, reveals an icy fog cloaking the winter sun and the edges of the village. A stark filigree of bare tree limbs claws toward the winter sky. The artist achieved an impression of depth by fading colors and blurring shapes in the distance. His placement of buildings and trees that flank the frozen canal also guides viewers toward the misty horizon. Pieter Roelofs, a curator at Rijksmuseum, Amsterdam, observes that Avercamp's use of vantage points and perspective served to "create a more natural space so that the viewer is involved more directly in the events on and around the ice."

The Rijksmuseum offers additional details: "The high vantage point of this painting turns it into a sampler of human-and animal-activity during a harsh winter. Hundreds of people are out on the ice, most of them for pleasure, others working out of dire necessity." A crowd of busy figures is scattered across the canvas. Among the working class, a beggar seeks bread, an angler balances a long trident and grips the day's catch, and a brewery worker fetches water from a hole cut into the ice. Knots of people conversing, couples skating, and sportsmen playing kolf on the ice (a hybrid golf and hockey) reveal that for many others this is a day for recreation and socializing.
Animals scattered throughout the painting include several horses pulling sledges; birds hovering in the silvery air or clinging to bare branches or sloping rooftops; domesticated fowl pecking for food; and a dog scampering over the ice. Roelofs notes, "Avercamp did not concentrate solely on winter fun and games and the sunny side of the cold season." For example, he placed crows and a dog feasting on a horse carcass in the foreground of this painting.

Environmental history professor Dagomar Degroot comments on the Little Ice Age: "Changing weather patterns altered the range of insects that carried pathogens, bringing new and deadly ailments to the previously unexposed. Because malnourished bodies have weak immune systems, farmers and their livestock soon fell sick. Refugees from the famine-stricken countryside spread disease to cities, where epidemic outbreaks often inflicted a fearsome toll." Winter Landscape with Ice Skaters provides a glimpse of village life during Europe's Little Ice Age and may stimulate viewers to consider the interrelationship of climate and human, animal, and environmental health.

\section{Bibliography}

1. Alfani G. Plague in seventeenth-century Europe and the decline of Italy: an epidemiological hypothesis. Eur Rev Econ Hist. 2013;17:408-30. http://dx.doi.org/10.1093/ereh/het013

2. Degroot D. Some places flourished in the Little Ice Age. There are lessons for us now [cited 2018 Sep 20]. https://www.washingtonpost.com/national/health-science/ some-places-flourished-in-the-little-ice-age-there-are-lessons-forus-now/2018/02/16/455fb2d8-0c25-11e. 8-8b0d-891602206fb7 story.html?utm term $=.026 \mathrm{a} 9 \mathrm{fdb} 48 \mathrm{e} 6$

3. de Kraker AM. The Little Ice Age. In: Roelofs P, editor. Hendrick Avercamp: master of the ice scene. Amsterdam: Rijksmuseum; 2010. p. 23-9.

4. National Gallery of Art. Hendrick Avercamp: The Little Ice Age [cited 2018 Sep 21]. https://www.nga.gov/content/dam/ngaweb/ exhibitions/pdfs/avercamp-brochure.pdf

5. Parker G. Lessons from the Little Ice Age [cited 2018 Sept 21] https://www.nytimes.com/2014/03/23/opinion/sunday/lessonsfrom-the-little-ice-age.html

6. Pijbes W, Powell EA. Foreword. In: Roelofs P, editor. Hendrick Avercamp: master of the ice scene. Amsterdam: Rijksmuseum; 2010. p. 7-8.

7. Reiter P. From Shakespeare to Defoe: malaria in England in the Little Ice Age. Emerg Infect Dis. 2000;6:1. http://dx.doi. org/10.3201/eid0601.000101

8. Rijksmuseum. Winter landscape with ice skaters, Hendrick Avercamp, c. 1608 [cited 2018 Sep 17]. https://www.rijksmuseum.nl/ en/collection/SK-A-1718

9. Roelofs P. The paintings. In: Roelofs P, editor. Hendrick Avercamp: master of the ice scene. Amsterdam: Rijksmuseum; 2010. p. 31-83.

10. Roosen J, Curtis DR. Dangers of noncritical use of historical plague data. Emerg Infect Dis. 2018;24:103-10. http://dx.doi.org/ 10.3201/eid2401.170477

11. Wheelock AK. National Gallery of Art. Hendrick Avercamp [cited 2018 Sep 17]. https://www.nga.gov/collection/artist-info.129.html

Address for correspondence: Byron Breedlove, EID Journal, Centers for Disease Control and Prevention, 1600 Clifton Rd NE, Mailstop H16-2, Atlanta, GA 30329-4027, USA; email: wbb1@cdc.gov 
John Aaskov

Preben Aavitsland

Raquel Abad

Winston Abara

Kaja Abbas

El-Sayed Mohammed

Abdel-Whab

Philip Abraham

Jay Achar

Joel Ackelsberg

Anna Acosta

Laura Adams

Alys Adamski

Tolulope Adebanjo

Toidi Adekambi

Bishwa Adhikari

Jennifer Adjemian

Cornelia Adlhoch

Zeeshan Afzal

Rakesh Aggarwal

Mahdis Aghazadeh

Patricia Aguilar

Michael Aidoo

Adam Akullian

Charbel Al Bayssari

Abraham Alabi

Munirul Alam

Belkisyolé Alarcón de Noya

Cesar Albariño

Kathleen Alexander

Margarete Alfonso

Afsar Ali

Ibne Ali

Jon-Patrick Allem

David Allen

Lesley Allison

Harvey Alter

Benjamin Althouse

Gerardo Álvarez-García

Brian Amman

Lucie Amoureux

Cheryl Andam

Danielle Anderson

Evan Anderson
Kathryn Anderson

Theodore Andreadis

Soa Fy Andriamandimby

Emmanouil Angelakis

Fred Angulo

Augustina Annan

Gloria Anyalechi

Charles Apperson

Amy Appril

Bob Arbeit

Maris Arcilla

Carmen Ardanuy

Paul Arguin

Cesar Arias

Kevin Ariën

Blas Armien

John Aronis

Nichole Arrigo

Bailey Arruda

Ray Arthur

Lawrence Ash

Edwin Asturias

Ricardo Ataíde

Katie Atkins

Barry Atkinson

Shiu Lun Ryan Au Yeung

Maite Aubry

Heidi Auerswald

John Austin

Paul Auwaerter

Tatjana Avsic

Abdu Azad

Taj Azarian

Laura Bachmann

P. Backenson

John Baddley

Edgar Badell

Sékéné Badiaga

Justin Bahl

Waheed Bajwa

Tamás Bakonyi

Andrew Ball

Bettina Bankamp

Krisztian Banyai
Lindley Barbee

Alan Barbour

Bridget Barker

Alex-Mikael Barkoff

Celine Barnadas

John Barnes

John Barnwell

Ian Barr

Joel Barratt

Anne-Sophie Barret

Alan Barrett

Drue Barrett

Lyric Bartholomay

Jason Bartz

Luisa Barzon

Sridhar Basavaraju

David Baud

Daniel Bausch

Frederique

Jacquerioz Bausch

Lauren Bayliss

Mariana Baz

Bernard Beall

David Beasley

Maria Serena Beato

Abdoul Beavogui

Paul Becher

Stephan Becker

J. David Beckham

Karlyn Beer

Martin Beer

Nancy Beerens

Meruyert Beknazarova.

Tom Belhorn

Jessica Belser

Daniel Beltran-Alcrudo

Jorge Benach

Jeff Bender

Kaitlin Benedict

Sylvie Benestad

John Bennett

Isaac Benowitz

Kimberley Benschop

Dennis Bente
Béatrice Berçot

John Berezowski

Nicholas Bergren

Sven Bergström

Vincent Beringue

James Berkley

Elizabeth Berkow

Robert Bernstein

Mabel Berois

Antoine Berry

Eric Bertherat

Khalid Beshir

Marianne Besnard

John Besser

Sonja Best

Julie Bettinger

Amrita Bharat

Anunay Bhattacharya

Dominique Bicout

Julia Bielicki

Dorothee Bienzle

Brad Biggerstaff

Matthew Biggerstaff

Holly Biggs

Bridgette Jeanne Billioux

Brian Bird

Zeno Bisoffi

Paritosh Biswas

Idir Bitam

Carolyn Black

Carina Blackmore

Stuart Blacksell

Carol Blair

David Blair

Stephane Blanche

Denis Blanchet

David Blaney

Lucas Blanton

Martina Bleyer

Alan Bloch

Evan Bloch

Debra Blog

Sandra Blome

Joseph Blondeau 


\begin{tabular}{|c|c|c|c|}
\hline Johannes Blum & Scott Bucher & Christopher Chase & Samuel Crowe \\
\hline Lucille Blumberg & Philippe Buchy & Man Ling Chau & Christiane Cuny \\
\hline Naomi Bock & Brian Buck & Luis Chaves & Aaron Curns \\
\hline Marleen Boelaert & Elizabeth Buckles & Nora Chea & Bart Currie \\
\hline Pierre-Yves Boelle & John Bullock & Judy Chen & Daniel Curtis \\
\hline Andrea Boggild & Marc Bulterys & Liang Chen & Sally Cutler \\
\hline John Bohnsack & Rebecca Bunnell & Mark Chen & Cristina Da Silva Carias \\
\hline Guy Boivin & Canio Buonavoglia & Sharon Chen & Juliana Da Silva \\
\hline Sameh Boktor & Dora Buonfrate & Kartik Cherabuddi & Charles Daley \\
\hline Manuel Borca & Anne Burdick & Harrell Chesson & Tim Dallman \\
\hline Ray Borrow & Andreas Burkovski & Michelle Chevalier & Clarissa Damaso \\
\hline Icaro Boszczowski & Joseph Burzynski & Charles Chiu & David Dance \\
\hline Graham Bothamley & Carlos Buscaglia & Eun Hwa Choi & Alexandra Dangel \\
\hline Teun Bouseman & Michael Busch & Young-Ki Choi & M. Carolina \\
\hline Donald Bouyer & Thomas Butler & B.B. Chomel & Danovaro-Holliday \\
\hline Anna Bowen & Carol Byerly & Terence Chorba & Masoud Dara \\
\hline Richard Bowen & Nate Byers & Gerardo Chowell & Jonathan Darbro \\
\hline Andrew Bowman & Diego Caceres & Henrik Christensen & Federica Dassoni \\
\hline Catherine Bozio & Lorenzo Cáceres & Rebecca Christofferson & Claudia Daubenberger \\
\hline Phillip Brachman, Jr. & Philippe Calain & Victoria Chu & Naval Daver \\
\hline Richard Bradbury & Charles Calisher & Kaw Bing Chua & Meghan Davis \\
\hline Oliver Brady & Mattia Calzolari & Hee Kyoung Chun & William Davis \\
\hline Marieta Braks & Jordi Camara & Holly Ciesielczuk & Fatimah Dawood \\
\hline Paulo Brandão & Floriana Campanile & Alexander Ciota & Xavier de Lamballerie \\
\hline Mary Brandt & Stefanie Campbell & Cliff Clark & Rob de Man \\
\hline Aaron Brault & Rafael Cantón & Oliver Clay & Daniela de Souza Rajao \\
\hline Mike Bray & Van-Mai Cao-Lormeau & Bill Coggin & Annabelle de St Maurice \\
\hline Byron Breedlove & Michael Cappello & Nicole Cohen & Henriette de Valk \\
\hline Edward B. Breitschwerdt & Helene Carabin & Samuel Cohn & Henry de Vries \\
\hline Joel Breman & Jonathan Carapetis & Charsey Cole Porse & Emilio DeBess \\
\hline Ben Brennan & Tamar Carter & Michelle Cole & Brooke Decker \\
\hline Carolyn Bridges & Pamela Cassiday & Samuel Collins & Oscar Del Brutto \\
\hline William Britt & Lucio Castellano & Simon Collin & Kevin Delaney \\
\hline Constança Britto & Jose Castillo-Mancilla & Philippe Colson & Mark Delorey \\
\hline Christopher Broder & Kenneth Castro & Iñaki Comas & Eric Delwart \\
\hline John Brooks & Marcio Castro & Andrew Comrie & Walter Demczuk \\
\hline Tal Brosh-Nissimov & Byron Caughey & Neeta Connally & Xiangyu Deng \\
\hline Florence Brossier & Joseph Cavanaugh & Andrea Conroy & David Denning \\
\hline Philippe Brouqui & Lindsay Caverly & Marta Contigiani & Dan Denoon \\
\hline Charles Brower & Peter Cegielski & Paul Cook & Christina Deschermeier \\
\hline Barbara Brown-Elliott & Maria Carolina Ceriani & Susan Cookson & Ulrich Desselberger \\
\hline Caroline Brown & Chrispin Chaguza & Victor Corman & Amélie Desvars-Larrive \\
\hline Catherine Brown & Jong-Yil Chai & Caitlin Cossaboom & Gregor Devine \\
\hline Corrie Brown & Victoria Chalker & Susan Cotmore & Han Di \\
\hline Daniel Brown & Thomas Chambers & Chris Coulter & Sabine Diedrich \\
\hline Kevin Brown & Chung-Hong Chan & David Couvin & Daniel Diekema \\
\hline Meredith Brown & Aileen Chang & Lauren Cowan & Linda Dixon \\
\hline Gary Brunette & Michelle Chang & Ben Cowling & Gerhard Dobler \\
\hline Fabrizio Bruschi & Caroline Charre & Matthew Crist & Ulrich Dobrindt \\
\hline Juliet Bryant & Rémi Charrel & Julio Croda & Pete Dodd \\
\hline
\end{tabular}


Roger Dodd

Sarah Doernberg

Yohei Doi

Sheila Dollard

Dragoslav Domanovic

Cristina Domingo

Libo Dong

Laurent Dortet

Ellen Dotson

Scott Dowell

Christine Doyle

Chris Drakeley

Angela Dramowski

Michel Drancourt

Michael Drebot

Jan Drexler

Robert Drillien

Christian Drosten

Mignon du Plessis

Grégory Dubourg

Mariette Ducatez

Elizabeth Dufort

John Stephen Dumler

William Dundon

Nicolas Dupin

Claire Duployez

Sophie Duraffour

Edison Durigon

Philippe Dussart

Linda Duval

Gregory Ebel

Hideki Ebihara

Juan Echevarria

Rose Eckhardt

John-Sebastian Eden

Sophie Edouard

Srilatha Edupuganti

Celeste Edwards

Paul Effler

Androulla Efstratiou

Daniel Eibach

Anna Maria Eis-Hübinger

Rebecca Eisen

Tobias Eisenberg

Elamin Elbasha

Armin Elbers

Randy Elder

Natalia Elguezabal

Robert Ellis

Richard Elson
Andrea Endimiani

Jeffrey Engel

J.A. Englund

Jonathan Epstein

Guliz Erdem

Dean Erdman

Ananias Escalante

Luis Escobar

Maria Esteve-Gassent

Steen Ethelberg

Shamsudeen Fagbo

Anna-Bella Failloux

Ann Falsey

Dennis Falzon

Hongxin Fan

Shufang Fan

Chi-Tai Fang

Seamus Fanning

Nuno Faria

Fernando Fariña

Heinz Feldmann

Leora Feldstein

Mariet Feltkamp

Peter Feng

Florence Fenollar

Kevin A Fenton

Ezio Ferroglio

Peter Figueroa

Maria Filippitzi

Paul Fine

Marc Fischer

Brian Fisher

Dale Fisher

Kiva Fisher

Matthew Fisher

Robert Fitzhenry

Katherine Fleming-Dutra

Anthony Flores

Colin Flynn

William Foege

Onikepe Folarin

Emanuela Foni

Arnaud Fontanet

Anthony Fooks

Pierre Formenty

Naomi Forrester

Monique Foster

Pierre-Edouard Fournier

Brian Foy

Rafael Franca
Carlos Franco-Paredes

Klaas Frankena

Eelco Franz

David Freedman

Celio Geraldo

Freire-de-Lima

Caroline Frey

Rachel Friedman

Matthew Frieman

Yongfeng Fu

Tsuguto Fujimoto

Isaac Chun-Hai Fung

Jennifer Furin

Luis Furuya-Kanamori

Alice Fusaro

Janet Fyfe

Simona Gabrielli

James Gaensbauer

Kirian Gajurel

Ohad Gal-Mor

John Galgiani

Jason Gallagher

Renee Galloway

Manoj Gambhir

Manoj Gambhir

Sumanth Gandra

Adolfo García-Sastre

Aysen Gargili

Mutien-Marie Garigliany

Rebecca Garten

Joy Gary

Joel Gaydos

Jay Gee

Thomas Geisbert

Rick Gelting

Kathleen Gensheimer

Emma Geoman

Patrick Gérardin

Sue Gerber

Dale Gerding

Nancy Gerloff

Peter Gerner-Smidt

Antoine Gessain

Katherine Gibney

Joseph Gillespie

Peter Gilligan

Andreas Gilsdorf

Christian Giske

John Glasser

Philippe Glaziou
Dieter Glebe

Richard Goering

Jerome Goddard

Wil Goessens

Tony Goldberg

Yann Gomard

Beatriz Gómez

Camila Gonzales

E. Gormley

Christian Gortazar

Rachel Gorwitz

Michael Gosciminski

Neela Goswami

Michihiko Goto

Ernest Gould

L. Hannah Gould

Joelle Gouy de Bellocq

Nelesh Govender

Katarzyna Goździk

Carlos Graeff-Teixeira

Marc Grandadam

Gavin Grant

Delphine Grau

Stephen Graves

Gregory Gray

Matthew Gray

Carolyn Greene

Justin Greenlee

Alexander Greninger

Carlos Grijalva

Ardath Grills

Mirjam Grobbel

Mirjam Groger

Allen Grolla

Jacques Grosset

Paolo Grossi

Nathan Grubaugh

Roland Grunow

Zemao Gu

Jeannette Guarner

Larisa Gubareva

Jonathan Gubbay

Duane Gubler

Humberto Guerra

Claire Guinat

Jesús Guinea

Nicole Guiso

Emily Gurley

Julie Gutman

Marjo Haanpera 
Michelle Haas

Andrew Haddow

Christos Hadjichristodoulou

Stephen Hadler

C.A. Hage

Ferry Hagen

Stéphane Haik

Aron Hall

Patricia Hall

Robert Hall

Robyn Hall

Eric Halsey

Scott Halstead

Davidson Hamer

Margaret Hammerschlag

Andreas Handel

Jennifer Harcourt

Timm Harder

Felicia Hardnett

Susan Hariri

Reid Harris

Shimon Harrus

Heli Harvala

Rodrigo Hasbun

Masahiro Hashizume

Ben Hause

Manon Haverkate

Harry Haverkos

Fiona Havers

Hal Hawkins

William Hawley

Tetsuya Hayashi

Amber Haynes

Craig Hedberg

Katrina Hedberg

Ian Hennessee

German Henostroza

Ronnie Henry

Christiane Herden

Juan Hernandez Garcia

Dionisio Herrera

Roger Hewson

David Heymann

Graham Hickling

Lauri Hicks

Steve Higgs

Susan Hills

Yuji Hirai

Yukihiro Hiramatsu

Christine Ho
Nicole Hoff

Alex Hoffmaster

Jörg Hofmann

Boris Hogema

Mike Holbrook

David Holland

F. Hollinger

Edward Holmes

Lori Holtz

Deborah Holtzman

Margaret Honein

Edward Hook

Donald Hopkins

C. Robert Horsburgh

Paul Horwood

Paul Hoskisson

M. Hossain

Joppe Hovius

Andrew Huang

Li-Ting Huang

Yhu-Chering Huang

Taoqi Huangfu

Holly Hughes

James Hughes

David S.C. Hui

Ralph Huits

Frederick Hull

John Humphrey

Yuen Wai Hung

Elizabeth Hunsperger

Yvan Hutin

Lars Hviid

Joseph Icenogle

Valerio Iebba

Allison Imrie

Tim Inglis

Jonathan Iredell

Badrul Islam

Shamim Islam

Michael Ison

Megumi Itoh

Danielle Iuliano

Jacques Izopet

Ashley Jackson

Brendan Jackson

Bart Jacobs

Anita Jacobson

Tomasz Jagielski

Denise Jamieson

J. Michael Janda
Yunho Jang

Julie Jarand

Claire Jenkins

Emily Jenkins

Helen Jenkins

Peter Jenkins

Christopher Jennelle

Jørgen Skov Jensen

Wei Jia

Baoming Jiang

Hai Jiang

Qi Jin

Michael Johansson

Nur Alia Johari

Reimar Johne

Nick Johnson

Michael Jones

Lisa Jones-Engel

Terry Jones

Jaume Jorba

Ferran Jori

Manish Joshi

Robin Jump

Sandra Junglen

Emily Kahn

Laura Kahn

Marion Kainer

Adriana Kajon

Pradeep Kakkattil

Naresh Kakker

Kazunari Kamachi

Nassim Kamar

Mary Kamb

Mini Kamboj

Saleem Kamili

Kenya Kamimura

Linda M. Kampschreur

Joseph Kanabrocki

Abraham Kandathil

Anu Kantele

Bryan Kapella

Ed Kaplan

Sheldon Kaplan

Amit Kapoor

Erik Karlsson

Sophia Kathariou

Midori Kato-Maeda

Alan Katz

Louis Katz

Daniel R. Kaul
Ghazi Kayali

Kevin Keel

Volkhard Kempf

Russell Kempker

Dervla Kenna

Joan Kenney

Janna Kerins

Arifa Khan

Ameneh Khatami

Mariana Kikuti

James Kile

Peter Kilmarx

Dong-Min Kim

Peter Kim

Sunkyung Kim

Kristine Kines

David Kingsley

Robert Kirkcaldy

Laura Kirkman

Boris Klempa

Kelly Kline

Albert Ko

Miwako Kobayashi

Tetsuro Kobayashi

Gary Kobinger

Guus Koch

Richard Kock

Aaron Kofman

Thomas Kohl

Anke Kohlenberg

Nicholas Komar

Marion Koopmans

Anita Koshy

Jasna Kovac

Colleen Kraft

Laura Kramer

Sarah Kramer

Florian Krammer

Gerard Krause

Peter Krause

Kristen Kreisel

Katharina Kreppel

S. Krishna

Pavla Krizova

Adam Kucharski

Roman Kuchta

Amy Kuenzi

Kiersten Kugeler

Sharon Kuhlmann-Berenzon Jens Kuhn 


\begin{tabular}{|c|c|c|c|}
\hline Edward Kuijper & Deborah Levine & Lawrence Madoff & Brendan McMullan \\
\hline Thijs Kuiken & Joseph Lewnard & Piet Maes & Jennifer McQuiston \\
\hline Sanjai Kumar & Feng Li & Younes Maged & John McQuiston \\
\hline Andreas Kurth & Hai Liang & Ricardo Maggi & Paul Mead \\
\hline Preeta Kutty & Geneviève Libeau & Alexandra Mailles & Oleg Mediannikov \\
\hline Ivan Kuzmin & Jean Lim & Richard Malik & Jennifer Meece \\
\hline Kyung Kwon-Chung & Chao-Nan Lin & Shannon Manning & Francis Mégraud \\
\hline Bernard La Scola & Jessica Lin & Janet Mans & Sanjay Mehta \\
\hline Marcelo Labruna & Michael Lin & Anna Mandra & Adam Meijer \\
\hline Tamar Lachish & Sally Lin & Lisa Manhart & Susan Meiring \\
\hline Shamez Ladhani & Robbin Lindsay & Oriol Manuel & Jacques Meis \\
\hline Jean-Christophe Lagier & Nicole Lindsey & Jean-Claude Manuguerra & Rojelio Mejia \\
\hline Kevin Lahmers & Rebecca Lindsey & Ben Marais & E. Mellado \\
\hline Elina Lahti & W. Lipkin & Jutta Marfurt & Eyal Meltzer \\
\hline Alexander Lai & John LiPuma & Wanda Markotter & Martin Meltzer \\
\hline Tsan Yuk Lam & Hannah Lishman & Michael Marks & Jingbo Meng \\
\hline Theresa Lamagni & Anastasia Litvintseva & Adriana Marques & Laina Mercer \\
\hline Amy Lambert & Qingyun Liu & Christina Marra & Matthias Merker \\
\hline Ben Lambert & Alejandro Llanos-Cuentas & Theodore Marras & Toby Merlin \\
\hline Laura Lamberti & Spencer Lloyd & Tom Marrie & Jonathan Mermin \\
\hline Ruiting Lan & Y.L. Lo & Nicola Marsden-Haug & Becky Merrill \\
\hline Christina Lancioni & Shawn Lockhart & Douglas Marthaler & Kevin Messacar \\
\hline Gabrielle Landolt & Catherine Logue & Joaquina Martin-Sanchez & Hermann Meyer \\
\hline Marie Landry & Igor Loncaric & Emily Martin & Daniella Michlmayr \\
\hline Christoph Lange & Maureen Long & Irene Martin & Claire Midgley \\
\hline Tatiana Lanzieri & Job Lopez & Stacey Martin & Susan Mikota \\
\hline Alfred Lardizabal & L. López-Cerero & Luis Martinez-Sobrido & M. Millar \\
\hline Don Latner & Anna Lena Lopez & Martin Martinot & Elizabeth Miller \\
\hline Eric Lau & Benjamin Lopman & Brian Maskery & Jackie Miller \\
\hline Max Siu Yin Lau & Alessio Lorusso & Alessandro Massolo & William Miller \\
\hline Susanna KP Lau & Miranda Loutet & Henry Masur & Mathieu Million \\
\hline Ana Lauer & David Lowe & Almea Matanock & James Mills \\
\hline Antje Lauer & Rachel Lowe & Amy Mathers & Paolo Miotto \\
\hline Tara Lavelle & Stephen Luby & Yasufumi Matsumura & Ali Mirazimi \\
\hline Kirsty Le Doare & Naomi Lucchi & Alberto Matteelli & Santiago Mirazo \\
\hline Tan Le Van & Jay Lucidarme & Sebastian Maurer-Stroh & Chad Mire \\
\hline Tuan Le Van & Martin Ludlow & Max Maurin & Maria Mistieri \\
\hline Karin Leder & Alexander Lukashev & Astrid Mayr & Toshihiro Mita \\
\hline James LeDuc & Tao Luo & Jerry Mazurek & Patrick Mitchell \\
\hline Dong-Hun Lee & Yaniv Lustig & Sandro Mazzariol & Tonya Mixson-Hayden \\
\hline Ellen Lee & Ulzii-Orshikh Luvsansharav & Placide Mbala-Kingebeni & Kenji Mizumoto \\
\hline Fan Lee & Samantha Lycett & Brian McCloskey & John Modlin \\
\hline Kyungwon Lee & Ruth Lynfield & Andrea McCollum & Christopher Mody \\
\hline Rogan Lee & Nkuchia M'ikanatha & Orion McCotter & Hamish Mohammed \\
\hline Fabian Leendertz & Miriam Maas & Tara McDaneld & Igor Mokrousov \\
\hline Florence & Kimberly Mace & Erin McDonald & Phillip Molloy \\
\hline Legrand-Abravanel & Alexandre Macedo & Peter McIntyre & Isabella Monne \\
\hline Fernanda Lessa & de Oliveira & James McKenna & Susan Montgomery \\
\hline Jessica Leung & C. Raina MacIntyre & Debbie McKenzie & Patrick Moonan \\
\hline Michael Levin & Susan Madison-Antenucci & Rita McManamon & Catherine Moore \\
\hline
\end{tabular}




\begin{tabular}{|c|c|c|c|}
\hline Cynthia Moore & Christopher Netherton & Donald Olson & Mary-Louise Penrith \\
\hline Stefano Morabito & Gabriele Neumann & Victoria Olson & Michael Pentella \\
\hline Danesh Moradigaravand & Paul Newton & Eng Eong Ooi & Pasi Penttinen \\
\hline Jacob Moran-Gilad & Lisa Ng & Walter Orenstein & Andrew Peregrine \\
\hline Gary Moran & Sophia Ng & Anthony Orvedahl & Lenore Pereira \\
\hline Gonzalo Moratorio & Terry Fei Fan Ng & Hitoshi Oshitani & RAPM Perera \\
\hline David Morens & Minh Ly Nguyen & Muhammad Osman & Sabine Pereyre \\
\hline Maria Moretti & Mya Ngwe-Tun & Albert Osterhaus & José-Antonio Pérez-Molina \\
\hline Oliver Morgan & Karamoko Niaré & Domenico Otranto & Andres Perez \\
\hline Kouichi Morita & Ainsley Nicholson & Stephen Owens & Daniel Perez \\
\hline Diane Morof & William Nicholson & Yusuf Ozbel & Alex Perkins \\
\hline Lillian Morris-Manahan & Matthias Niedrig & Aysegul Taylan Ozkan & Stanley Perlman \\
\hline J.Glenn Morris & Eva Møller Nielsen & Christopher Paddock & Patricia Pesavento \\
\hline Tem Morrison & Nathan Nieto & Kerry Padgett & C.J. Peters \\
\hline Irmgard Moser & Andreas Nitsche & Maria Clara Padoveze & Philip Peters \\
\hline Eric Mossel & Allan Nix & Stephen Page & Andreas Petersen \\
\hline Varvara Mouchtouri & Chimeremma Nnadi & Wendy Page & Emily Petersen \\
\hline Alexandra Moura & Rachel Noble & Amy Pakyz & Jeannine Petersen \\
\hline Scott Moye-Rowley & Maurício Nogueira & Gustavo Palacios & A. Townsend Peterson \\
\hline Yi Mu & Susan Noh & Peter Palese & Olivia Peuchant \\
\hline Atis Muehlenbachs & Melissa Nolan & Romain Palich & Michael Pfaller \\
\hline Lapo Mughini-Gras & Dawn Nolt & Mark Pallansch & Anastasia Pharris \\
\hline Elke Muhlberger & Johan Nordgren & Mitchell Palmer & Martine Piarroux \\
\hline Kamalich & Patrice Nordmann & Qiuwei Pan & Renata Picão \\
\hline Muniz-Rodriguez & Torbjörn Norén & Xiaoli Pang & David Pigott \\
\hline Carmen Muñoz-Almagro & Philip Norris & Alberto Paniz Mondolfi & Tamara Pilishvili \\
\hline César Muñoz-Fontela & Robert Norton & Marcus Panning & Segaran Pillai \\
\hline Vincent Munster & Robert Novak & Mary Pantin-Jackwood & Allan Pillay \\
\hline Pablo Murcia & Norbert Nowotny & Igor Paploski & Nils Pilotte \\
\hline Edward L. Murphy & Corrie Ntiforo & John Papp & Hernando Pinzon \\
\hline Kristy Murray & Max O’Donnell & Peter Pappas & Johann Pitout \\
\hline Darwin Murrell & Miguel O’Ryan & Manish Pareek & Mateusz Plucinski \\
\hline Michelle Murti & Ikwo Oboho & Philippe Parola & Alexander Plyusnin \\
\hline Didier Musso & Maria T. Ochoa & Gabriel Parra & Maurizio Pocchiari \\
\hline Jukka Mustonen & Sophie Octavia & Colin Parrish & Laura Podewils \\
\hline Jean-Paul Mutebi & Agricola Odoi & Michele Parsons & Laurent Poirel \\
\hline Nilson N. Mendes Neto & Titilope Oduyebo & Claudia Parvanta & Philippe Poirier \\
\hline Pierre Nabeth & John Oeltmann & Erica Pasini & Marjorie Pollack \\
\hline Samia Naccache & Lisa Oestereich & Jotam Pasipanyoda & Simon Pollett \\
\hline Steven Nadler & Sylvia Ofori & Daniel Pastula & Leo Poon \\
\hline Rajeshwari Nair & Kristen Ogden & Anita Patel & Yong Poovorawan \\
\hline Allyn Nakashima & Nick Ogden & Pragna Patel & Harry Porterfield \\
\hline Yoshinori Nakazawa & Chikara Ogimi & Richard Paul & Jamie Posey \\
\hline Srinivas Nanduri & Davor Ojkic & Thierry Paux & Sam Posner \\
\hline Roger Nasci & Nobuhiko Okabe & Nicole Pavio & Tonia Poteat \\
\hline Ruvandhi Nathavitharana & Liset Olarte & Lucia Pawloski & Rebecca Poulson \\
\hline Yuki Nawa & Kevin Olival & Daniel Payne & Ann Powers \\
\hline Maria Negron & Sara Oliver & Gabriela Paz-Bailey & Edoardo Pozio \\
\hline Noele Nelson & John Elmerdal Olsen & Michele Pearson & Bruno Pradines \\
\hline Nicole Nemeth & Sonja Olsen & Malik Peiris & Ranjan Premaratna \\
\hline
\end{tabular}


Rebecca Prevots

Erin Price

Lance Price

Marzia Puccioni-Sohler

Michael Purdy

Yvonne Qvarnstrom

Ingrid Rabe

Alan Radford

K.M. Rahman

Gowrisankar Rajam

M. Ramirez

Stéphane Ranque

Didier Raoult

Brian Raphael

Adrienne Rashford

Lasse Rasmussen

Sonja Rasmussen

Mario Raviglione

Adam Readhead

Paul Ready

Gil Redelman-Sidi

Jason Reed

Joanna Regan

Isabelle Régner

William Reisen

Paul Reiter

Adam Retchless

Chantal Reusken

Kelly Reveles

K. Reyes

Mary Reynolds

Allen Richards

Joachim Richter

Hans Rieder

Claudia Riegel

Kristian Riesbeck

Lee Riley

Pascal Ringwald

Suelee Robbe-Austerman

Florence Robert-Gangneux

Jacques Robert

Tamalee Roberts

Ignasi Roca

Barry Rockx

Guenael Rodier

Jesús Rodríguez-Baño

Eric Rogier

Amelie Rolle

Pierre Rollin

David Rollinson
Anne-Marie Roque-Afonso

Dale Rose

Benjamin Rosenthal

Shannan Rossi

Alan Rothman

Janell Routh

Sharon Roy

Steven Ruben

Erin Runcan

Charles Rupprecht

Colin Russell

Curtis Russell

Thomas Russo

Daniel Ruzek

Eoin Ryan

Raquel Sá-Leão

Nuria Sabe

Claude Sabeta

Claude Saegerman

Fabian Saenz

David Safronetz

Siddhartha Saha

Tais Saito

Takehiko Saito

Akihiko Saitoh

Yoshihiro Sakoda

Alex Salam

Vittorio Sambri

Orjan Samuelsen

Susan Sanchez

Vassilios Sandalakis

Dieudonne Sankara

Gilberto Santiago

Scott Santibanez

Sarah Sapp

Yuka Sasaki

R. Tedjo Sasmono

Ashley Satterfield-Nash

Paul Saunderson

Giovanni Savini

Maria Scaturro

William Schaffner

Frieder Schaumburg

Elizabeth Schiffman

Susanne Schjørring

Robert Schlaberg

William Schluter

Jonas Schmidt-Chanasit

Eileen Schneider

Paul Schnitzler
Monica Schoch-Spana

Randal Schoepp

Tomas Scholz

Angelica Schreiber

Peter Schreiber

Martin Schriefer

Amy Schuh

Tom Schwan

Ilan Schwartz

Martin Schwemmle

Jessica Schwind

Isaac See

Maikell Segovia

Mauricio Seguel

Karen Seiter

James Sejvar

Dejana Selenic

Rangaraj Selvarangan

Piseth Seng

Sang Heui Seo

Patricia Sequeira

Varadan Sevilimedu

Stephen Seligman

Daniel Sexton

Jessica Sexton

Andi Shane

Anupama Shankar

E.M. Shankar

Karen Shapiro

Aditya Sharma

Tyler Sharp

Beth Shaz

Heather Sheeley

Jianzhong Shen

Colin Shepard

John Shepard

Mika Shigematsu

Tom Shimabukuro

Nahoko Shindo

Kayoko Shioda

David Shlim

Carol Sibley

Kassim Sidibe

Eva Sierra

Roxane Silver

Peter Simmonds

Patricia Simner

Gustave Simo

Paul Simon

R.J. Simonds
Les Sims

Raj Singh

Ria Sinha

Theresa Sipe

Daouda Sissoko

Robert Skov

Owen Slater

Shirley Smart

Donald Smith

Duncan Smith

Gavin Smith

Grace Smith

Tara Smith

Eveline Snelders

Jesus Soares

Olusegun Soge

Anthony Solomon

Rami Sommerstein

Klara Sondén

Chang-Seon Song

Agnes Sonnevend

Uffe B. Skov Sørensen

Frank Sorvillo

Thiago Souza

John Spencer

Peter Spencer

Gianfranco Spiteri

Philip Spradling

Anon Srikiatkhachorn

Shiranee Sriskandan

Kirby Stafford

Karl Ståhl

David Stallknecht

J. Staples

Jeffrey Starke

Anneke Steens

Katarina Stefanidesova

Amir Steinman

Christen Stensvold

Werner Stenzel

Roger Stephan

O. Stine

Gregory Storch

Marc Strassburg

Daniel Streblow

Adrian Streit

Franc Strle

Jonathon Strysko

Jason Stull

Giorgia Sulis 


\begin{tabular}{|c|c|c|c|}
\hline Kelly Sullivan & Paul Trowbridge & David Vu & James Willig \\
\hline Xiang-Jie Sun & Jean Tsao & Duc Vugia & William Wilson \\
\hline Rebecca Sunenshine & Sharon Tsay & Dominique Vuitton & Jonas Winchell \\
\hline Mehul Suthar & Sarah Tschudin-Sutter & Supaporn Wacharapluesadee & Y.K. Wing \\
\hline Gerd Sutter & Mike Turell & Jun-ichi Wachino & Carla Winston \\
\hline Troy Sutton & Jane Turton & Jesse Waggoner & Amy Winter \\
\hline Patrick Suykerbuyk & Kenneth Tyler & Alpana Waghmare & Katja Wolthers \\
\hline Erik Svensson & Gregory Tyrrell & Niteen Wairagkar & Frank Wong \\
\hline David Swayne & Venkatachalam & David Walker & $\begin{array}{l}\text { Frank wong } \\
\text { Vanessa Wono }\end{array}$ \\
\hline William Switzer & Udhayakumar & Timothy Walker & Vanessa Wong \\
\hline Fabrizio Tagliavini & Anne-Catrin Uhlemann & Ryan Wallace & Gary Wormser \\
\hline Clarence Tam & David Ulaeto & Andrew Waller & Jonathan Wortham \\
\hline Kathrine Tan & Antti Vaheri & Xiu-Feng Wan & Henry Wu \\
\hline Shixing Tang & Ronald Valdiserri & Chengbin Wang & Linwan $\mathrm{Wu}$ \\
\hline Dennis Tappe & Marta Valenciano & David Wang & Qingping Wu \\
\hline Stefan Taube & Snigdha Vallabhaneni & Dayan Wang & Rosemary Wyber \\
\hline Maria Teixeira & Marie Francoise & Jianwei Wang & Guang Xu \\
\hline Sam Telford & Van Bressem & Lin-Fa Wang & Zhiwei Xu \\
\hline Michael Teng & Mark van der Linden & Tina Wang & Dafna Yahav \\
\hline Jeanette Teo & Marieke J. van der Werf & Yi-Chen Wang & Tatsuo Yamamoto \\
\hline Sanne Terryn & H. Rogier van Doorn & Kinley Wangdi & Richard Yanagihara \\
\hline Eyasu Teshale & Russell Van Gelder & Nasstasja Wassilew & Stephanie Yanow \\
\hline Saravanan Thangamani & Ted van Hinsbergh & Douglas Watts & Hui-Ling Yen \\
\hline Elitza Theel & Jakko van Ingen & Joel Watts & Jingjing Yin \\
\hline William Thompson & Kristien Van Reeth & Scott Weaver & Kevin Yin \\
\hline Sharmi Thor & Annelies van Rie & Richard Webby & Benediktus Yohan \\
\hline Julie Thwing & Alain Vanderplasschen & J. Todd Weber & \\
\hline Tejpratap Tiwari & Thomas Vanwolleghem & Scott Weese & Eun-Jeong Yoon \\
\hline Eugenia Tognotti & Olli Vapalahti & Manfred Weidmann & InKyu Yoon \\
\hline Evelyn Toh & Meagan Vaughn & Michael Weigand & Ian York \\
\hline Kentaro Tohma & Arun Venkatesan & Daniel Weinberger & Hongjie Yu \\
\hline Rafael Toledo & Marietjie Venter & Don Weiss & Xuejie Yu \\
\hline Mark Toleman & Rob M. Verdijk & Louis Weiss & Thomas Yuill \\
\hline Carlo Tomasini & Timothée Vergne & Renata Welc-Falêciak & Mark Zabel \\
\hline Oyewale Tomori & Marijke Verhegghe & Leisa Weld & Ruth Zadoks \\
\hline Stephen Tompkins & Andrew Vernon & Will Weldon & Gina Zanella \\
\hline Eric Toner & Guilherme Verocai & Eoin West & Aleksandra Zasada \\
\hline Mia Torchetti & Pablo Vial & Emily Whiston & Bianca Zecchin \\
\hline Paul Torgerson & Mariana Viegas & A. White & Hervé Zeller \\
\hline Alessandra Torina & Dhanasekaran Vijaykrishna & Jennifer White & Jing Zeng \\
\hline Alfredo Torres & Joseph Vinetz & Andrew Whitelaw & Dominik Zenner \\
\hline Fernando Torres & Jan Vinjé & Richard Whitley & $\begin{array}{l}\text { Dominik Zenner } \\
\text { Xinyan Zhang }\end{array}$ \\
\hline Elizabeth Torrone & Abinash Virk & Ryan Wiegand & \\
\hline Rita Traxler & Victor Virlogeux & Jeffrey Wiener & Hua Zhu \\
\hline Ana Cecilia Morice Trejos & Anastasia Vlasova & Joost Wiersinga & Rosalie Zimmerman \\
\hline David Tribble & Robert Vogel & Saman Wijesooriya & Dezemon Zingue \\
\hline William Trick & Michael von Fricken & Melisa Willby & Marko Zivcec \\
\hline Giliane Trindade & C. Fordham von Reyn & John Williams & Bruno Zorn \\
\hline
\end{tabular}




\section{EMERGING INFECTIOUS DISEASES Upcoming Issue}

- Nonsimplicity of Epidemiologic Metric Basic Reproduction Number $\left(R_{0}\right)$

- Clinical and Radiologic Characteristics of Human Metapneumovirus Infections in Adults

- Enterovirus A71 Infection and Neurologic Disease, Madrid, Spain, 2016

- Association of Increased Receptor-Binding Avidity of Influenza A(H9N2) Viruses with Escape from Antibody-Based Immunity and Enhanced Zoonotic Potential

- Epidemiology of Imported Infectious Diseases, China, 2005-2016

- Multiple Introductions of Feline Leukemia Virus in Endangered Florida Panthers

- Burdens of Invasive Methicillin-Susceptible and Methicillin-Resistant Staphylococcus aureus Disease, Minnesota, USA

- Dengue Virus IgM Serotyping by ELISA with Recombinant Mutant Envelope Proteins

- Lonepinella Identification in Koala Bite Wound Infections

- Isolation and Full-Genome Characterization of Nipah Viruses from Bats, Bangladesh

- Influenza Virus A(H3N2) with Antigenic Variation Prevalent in Dogs, China, 2016-2017

- Autochthonous Transmission of Coccidioides in Animals, Washington, USA

- Influenza H5/H7 Virus Vaccination in Poultry and Reduction of Zoonotic Infections, Guangdong Province, China, 2017-18

- Meat and Fish as Sources of Extended-Spectrum $\beta$-lactamaseProducing Escherichia coli, Cambodia

- Severe Disseminated Infection Caused by an Emerging Lineage of Methicillin-sensitive Staphylococcus aureus Clonal Complex 398 Lacking Classical Virulence Factors

- Severe Disease with Community-Associated MRSA Strain Chinese ST398, Australia, 2017

- Using the Health Belief Model to Analyze \#Zika on Instagram for Public Health Communications

- Case of Zoonotic Endocarditis, the Netherlands

Complete list of articles in the January issue at http://www.cdc.gov/eid/upcoming.htm
Upcoming Infectious

Disease Activities

March 4-7, 2019

Conference on Retroviruses and

Opportunistic Infections

Seattle, WA, USA

http://www.croiconference.org/

April 13-16, 2019

European Congress of Clinical

Microbiology and Infectious Diseases

29th Annual Congress

Amsterdam, the Netherlands

http://www.eccmid.org/

April 16-18, 2019

International Conference on

One Health Antimicrobial Resistance

Amsterdam, the Netherlands

https://www.escmid.org/

ICOHAR2019/

May 5-9, 2019

ASM Clinical Virology Symposium

Savannah, GA, USA

https://10times.com/clinical-virologysymposium

June 20-24, 2019

ASM Microbe 2019

San Francisco, CA, USA

https://www.asm.org/index.php/asm-

microbe-2018

\section{Announcements}

Email announcements to

EIDEditor (eideditor@cdc.gov).

Include the event's date, location,

sponsoring organization, and a

website. Some events may appear

only on EID's website, depending on

their dates. 


\section{Earning CME Credit}

To obtain credit, you should first read the journal article. After reading the article, you should be able to answer the following, related, multiple-choice questions. To complete the questions (with a minimum $75 \%$ passing score) and earn continuing medical education (CME) credit, please go to http://www.medscape.org/journal/eid. Credit cannot be obtained for tests completed on paper, although you may use the worksheet below to keep a record of your answers.

You must be a registered user on http://www.medscape.org. If you are not registered on http://www.medscape.org, please click on the "Register" link on the right hand side of the website.

Only one answer is correct for each question. Once you successfully answer all post-test questions, you will be able to view and/or print your certificate. For questions regarding this activity, contact the accredited provider, CME@medscape. net. For technical assistance, contact CME@medscape.net. American Medical Association's Physician's Recognition Award (AMA PRA) credits are accepted in the US as evidence of participation in CME activities. For further information on this award, please go to https://www.ama-assn.org. The AMA has determined that physicians not licensed in the US who participate in this CME activity are eligible for AMA PRA Category 1 Credits $^{\mathrm{TM}}$. Through agreements that the AMA has made with agencies in some countries, AMA PRA credit may be acceptable as evidence of participation in CME activities. If you are not licensed in the US, please complete the questions online, print the AMA PRA CME credit certificate, and present it to your national medical association for review.

\section{Article Title CME Questions}

1. You are advising a public health department in California regarding potential needs for St. Louis encephalitis virus (SLEV) detection and management. On the basis of the epidemiological review by Diaz and colleagues, which one of the following statements about the history of SLEV epidemiology in the United States is correct?

A. SLEV was first identified as the causative agent of a mosquito-borne viral epidemic in St. Louis, Missouri, during the summer of 1953

B. SLEV activity increased considerably in the United States after West Nile virus introduction in 1999

C. SLEV genotypes IV and V are most prevalent in the United States

D. SLEV is transmitted by numerous Culex mosquito species, and is amplified by passerine and columbiform avian species

2. According to the epidemiological review by Diaz and colleagues, which one of the following statements about the emergence and reemergence of SLEV in South America is correct?

A. Introduction of a more pathogenic strain and increasing populations of the eared dove as amplifying host led to the emergence of SLEV in Argentina
B. Human SLE epidemics have occurred every 2 to 3 years in Latin America since the 1980s

C. The 2005 outbreak of SLE in Córdoba City had worse outcomes in younger patients

D. SLEV strains isolated during the 2005 outbreak were closely related to a genotype $\mathrm{V}$ SLEV strain

3. On the basis of the epidemiological review by Diaz and colleagues, which one of the following statements about reemergence of SLEV in the western United States is correct?

A. In 2014 to 2015, SLEV caused a human outbreak in New Mexico

B. Emerging SLEV in the western United States is related to the strains isolated during sporadic transmission in Florida in 1993 and 1997

C. Reemerging SLEV in the western United States may have been mediated via migrating infected birds from southern South America, where it reemerged most recently in 2002

D. Longitudinal SLEV isolates from California were genetically similar from 1952 through 2015 


\section{Earning CME Credit}

To obtain credit, you should first read the journal article. After reading the article, you should be able to answer the following, related, multiple-choice questions. To complete the questions (with a minimum $75 \%$ passing score) and earn continuing medical education (CME) credit, please go to http://www.medscape.org/journal/eid. Credit cannot be obtained for tests completed on paper, although you may use the worksheet below to keep a record of your answers.

You must be a registered user on http://www.medscape.org. If you are not registered on http://www.medscape.org, please click on the "Register" link on the right hand side of the website.

Only one answer is correct for each question. Once you successfully answer all post-test questions, you will be able to view and/or print your certificate. For questions regarding this activity, contact the accredited provider, CME@medscape. net. For technical assistance, contact CME@medscape.net. American Medical Association's Physician's Recognition Award (AMA PRA) credits are accepted in the US as evidence of participation in CME activities. For further information on this award, please go to https://www.ama-assn.org. The AMA has determined that physicians not licensed in the US who participate in this CME activity are eligible for AMA PRA Category 1 Credits $^{\mathrm{TM}}$. Through agreements that the AMA has made with agencies in some countries, AMA PRA credit may be acceptable as evidence of participation in CME activities. If you are not licensed in the US, please complete the questions online, print the AMA PRA CME credit certificate, and present it to your national medical association for review.

\section{Article Title CME Questions}

1. You are seeing a 30-year-old man who has sex with men (MSM) who reports having sex with several partners per month with unknown HIV status. You note that he has had 2 episodes of sexually transmitted infections over the past year, and you ask him if he would consider preexposure prophylaxis (PrEP). What can you explain regarding research into the efficacy of PrEP?
A. Clinical studies have failed as yet to demonstrate a reduction in HIV transmission associated with PrEP among MSM
B. Adherence to PrEP does not appear to affect the risk for HIV acquisition
C. PrEP can be over $90 \%$ effective among MSM with adequate drug levels
D. No study has confirmed that PrEP can effective among adults who use intravenous drugs

2. The patient is hesitant to start PrEP because he is currently using multiple substances and is worried about treatment adherence. What can you tell him regarding the baseline characteristics of the current study cohort of MSM and transgender women?
A. Nearly $40 \%$ of participants reported any substance use
B. Rates of substance use declined by $50 \%$ during the study period

C. More than one third of participants reported frequent ongoing substance use

D. Adherence to PrEP declined by more than $40 \%$ between study weeks 12 and 48

3. What did the current study demonstrate regarding substance use and PrEP adherence?

A. There was no significant association between substance use and PrEP adherence

B. Only amphetamine use was significantly associated with worse PrEP adherence

C. Only opioid use was significantly associated with worse PrEP adherence

D. Only problematic substance use was associated with worse PrEP adherence

4. You take more history on this patient's substance use. Which of the following substances was most associated with a higher risk for STDs in the current study?
A. Opioids
B. Alcohol
C. Amphetamine
D. Prescription antianxiety drugs 


\section{DAVID J. SENCER
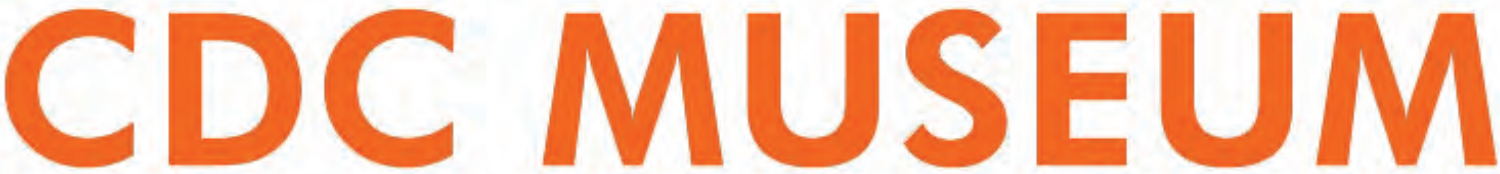 \\ History • Legacy • Innovation}

The David J. Sencer CDC Museum, a Smithsonian Affiliate, uses awardwinning exhibits and innovative programing to educate visitors about the value of public health, and presents the rich heritage and vast accomplishments of CDC.

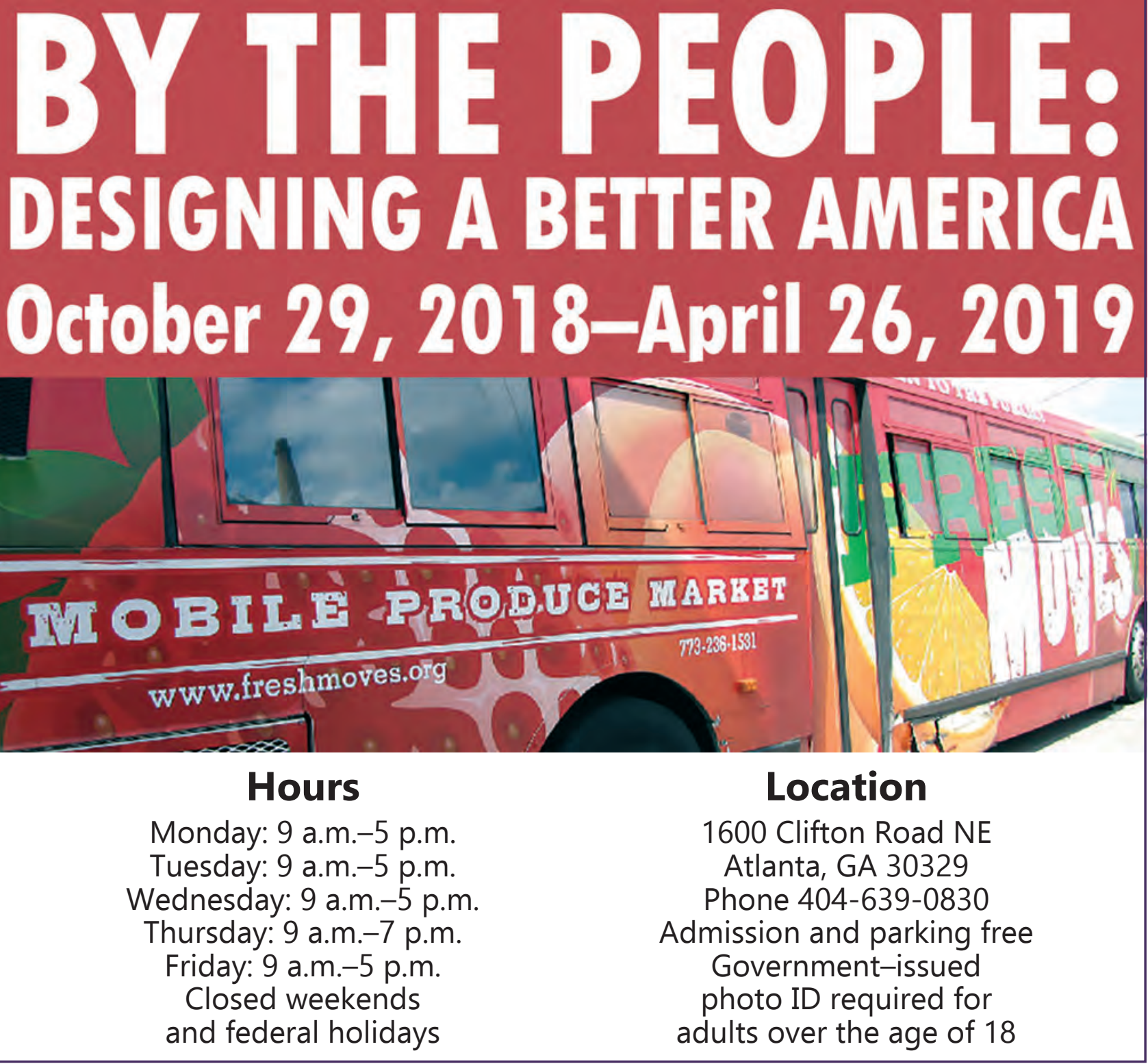




\section{CDC}

\section{YE}

HEALTH INFORMATION FOR INTERNATIONAL TRAVEL

\section{Available Now - New for 2018}

The fully revised and updated CDC Yellow Book 2018: Health Information for International Travel codifies the U.S. government's most current health guidelines and information for clinicians advising international travelers, including pretravel vaccine recommendations, destination-specific health advice, and easy-to-reference maps, tables, and charts.

ISBN: 9780190628611 | \$49.95 I May 2017 | Paperback | 704 pages

The 2018 Yellow Book includes important travel medicine updates:

- The latest information about emerging infectious disease threats such as Zika, Ebola, and sarcocystosis

- New cholera vaccine recommendations

- Updated guidance on the use of antibiotics in the treatment of travelers' diarrhea

- Special considerations for unique types of travel such as wilderness expeditions, work-related travel, and study abroad

IDSA members: log in via www.idsociety.org before purchasing this title to receive your $\mathbf{2 0} \%$ discount

\section{Order your copy at:}

WwW.oup.com/academic 


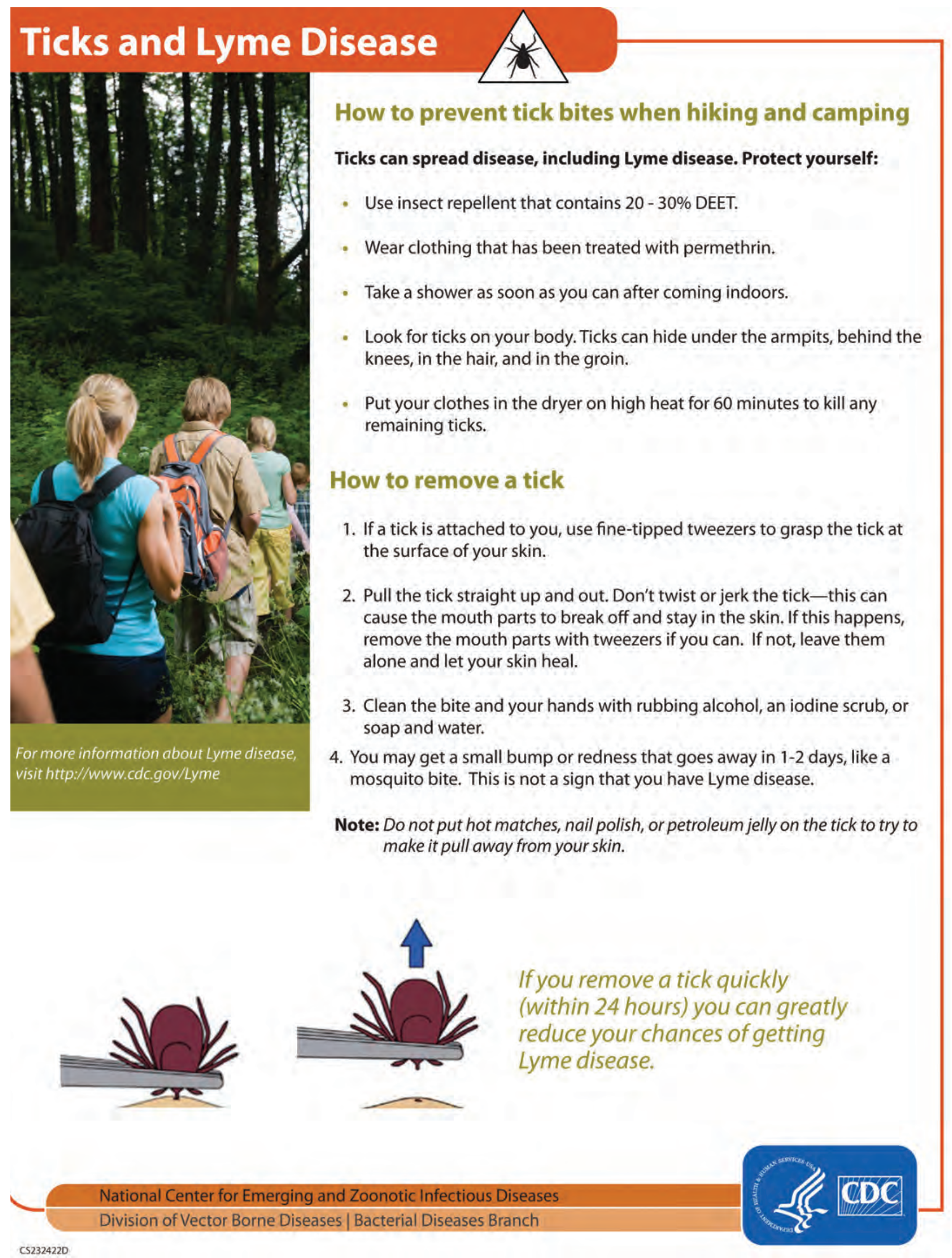


Emerging Infectious Diseases is a peer-reviewed journal established expressly to promote the recognition of new and reemerging infectious diseases around the world and improve the understanding of factors involved in disease emergence, prevention, and elimination.

The journal is intended for professionals in infectious diseases and related sciences. We welcome contributions from infectious disease specialists in academia, industry, clinical practice, and public health, as well as from specialists in economics, social sciences, and other disciplines. Manuscripts in all categories should explain the contents in public health terms. For information on manuscript categories and suitability of proposed articles, see below and visit http://wwwnc.cdc.gov/eid/pages/author-resource-center.htm.

\section{Summary of Authors' Instructions}

Authors' Instructions. For a complete list of EID's manuscript guidelines, see the author resource page: http://wwwnc.cdc.gov/eid/page/author-resource-center.

Manuscript Submission. To submit a manuscript, access Manuscript Central from the Emerging Infectious Diseases web page (www.cdc.gov/eid). Include a cover letter indicating the proposed category of the article (e.g., Research, Dispatch), verifying the word and reference counts, and confirming that the final manuscript has been seen and approved by all authors. Complete provided Authors Checklist.

Manuscript Preparation. For word processing, use MS Word. Set the document to show continuous line numbers. List the following information in this order: title page, article summary line, keywords, abstract, text, acknowledgments, biographical sketch, references, tables, and figure legends. Appendix materials and figures should be in separate files.

Title Page. Give complete information about each author (i.e., full name, graduate degree(s), affiliation, and the name of the institution in which the work was done). Clearly identify the corresponding author and provide that author's mailing address (include phone number, fax number, and email address). Include separate word counts for abstract and text.

Keywords. Use terms as listed in the National Library of Medicine Medical Subject Headings index (www.ncbi.nlm.nih.gov/mesh).

Text. Double-space everything, including the title page, abstract, references, tables, and figure legends. Indent paragraphs; leave no extra space between paragraphs. After a period, leave only one space before beginning the next sentence. Use 12-point Times New Roman font and format with ragged right margins (left align). Italicize (rather than underline) scientific names when needed.

Biographical Sketch. Include a short biographical sketch of the first author-both authors if only two. Include affiliations and the author's primary research interests.

References. Follow Uniform Requirements (www.icmje.org/index.html). Do not use endnotes for references. Place reference numbers in parentheses, not superscripts. Number citations in order of appearance (including in text, figures, and tables). Cite personal communications, unpublished data, and manuscripts in preparation or submitted for publication in parentheses in text. Consult List of Journals Indexed in Index Medicus for accepted journal abbreviations; if a journal is not listed, spell out the journal title. List the first six authors followed by "et al." Do not cite references in the abstract.

Tables. Provide tables within the manuscript file, not as separate files. Use the MS Word table tool, no columns, tabs, spaces, or other programs. Footnote any use of boldface. Tables should be no wider than $17 \mathrm{~cm}$. Condense or divide larger tables. Extensive tables may be made available online only.

Figures. Submit editable figures as separate files (e.g., Microsoft Excel, PowerPoint) Photographs should be submitted as high-resolution (600 dpi) .tif or .jpg files. Do not embed figures in the manuscript file. Use Arial $10 \mathrm{pt}$. or $12 \mathrm{pt}$. font for lettering so that figures, symbols, lettering, and numbering can remain legible when reduced to print size. Place figure keys within the figure. Figure legends should be placed at the end of the manuscript file.

Videos. Submit as AVI, MOV, MPG, MPEG, or WMV. Videos should not exceed 5 minutes and should include an audio description and complete captioning. If audio is not available, provide a description of the action in the video as a separate Word file. Published or copyrighted material (e.g., music) is discouraged and must be accompanied by written release. If video is part of a manuscript, files must be uploaded with manuscript submission. When uploading, choose "Video" file. Include a brief video legend in the manuscript file.

\section{Types of Articles}

Perspectives. Articles should not exceed 3,500 words and 50 references. Use of subheadings in the main body of the text is recommended. Photographs and illustrations are encouraged. Provide a short abstract (150 words), 1-sentence summary, and biographical sketch. Articles should provide insightful analysis and commentary about new and reemerging infectious diseases and related issues. Perspectives may address factors known to influence the emergence of diseases, including microbial adaptation and change, human demographics and behavior, technology and industry, economic development and land use, international travel and commerce, and the breakdown of public health measures.

Synopses. Articles should not exceed 3,500 words in the main body of the text or include more than 50 references. Use of subheadings in the main body of the text is recommended. Photographs and illustrations are encouraged. Provide a short abstract (not to exceed 150 words), a 1-line summary of the conclusions, and a brief biographical sketch of first author or of both authors if only 2 authors. This section comprises case series papers and concise reviews of infectious diseases or closely related topics. Preference is given to reviews of new and emerging diseases; however, timely updates of other diseases or topics are also welcome. If detailed methods are included, a separate section on experimental procedures should immediately follow the body of the text.

Research. Articles should not exceed 3,500 words and 50 references. Use of subheadings in the main body of the text is recommended. Photographs and illustrations are encouraged. Provide a short abstract (150 words), 1-sentence summary, and biographical sketch. Report laboratory and epidemiologic results within a public health perspective. Explain the value of the research in public health terms and place the findings in a larger perspective (i.e., "Here is what we found, and here is what the findings mean").

Policy and Historical Reviews. Articles should not exceed 3,500 words and 50 references. Use of subheadings in the main body of the text is recommended. Photographs and illustrations are encouraged. Provide a short abstract (150 words), 1-sentence summary, and biographical sketch. Articles in this section include public health policy or historical reports that are based on research and analysis of emerging disease issues.

Dispatches. Articles should be no more than 1,200 words and need not be divided into sections. If subheadings are used, they should be general, e.g., "The Study" and "Conclusions." Provide a brief abstract (50 words); references (not to exceed 15); figures or illustrations (not to exceed 2); tables (not to exceed 2); and biographical sketch. Dispatches are updates on infectious disease trends and research that include descriptions of new methods for detecting, characterizing, or subtyping new or reemerging pathogens. Developments in antimicrobial drugs, vaccines, or infectious disease prevention or elimination programs are appropriate. Case reports are also welcome.

Research Letters Reporting Cases, Outbreaks, or Original Research. EID publishes letters that report cases, outbreaks, or original research as Research Letters. Authors should provide a short abstract (50-word maximum), references (not to exceed 10 ), and a short biographical sketch. These letters should not exceed 800 words in the main body of the text and may include either 1 figure or 1 table. Do not divide Research Letters into sections.

Letters Commenting on Articles. Letters commenting on articles should contain a maximum of 300 words and 5 references; they are more likely to be published if submitted within 4 weeks of the original article's publication.

Commentaries. Thoughtful discussions (500-1,000 words) of current topics. Commentaries may contain references (not to exceed 15) but no abstract, figures, or tables. Include biographical sketch.

Another Dimension. Thoughtful essays, short stories, or poems on philosophical issues related to science, medical practice, and human health. Topics may include science and the human condition, the unanticipated side of epidemic investigations, or how people perceive and cope with infection and illness. This section is intended to evoke compassion for human suffering and to expand the science reader's literary scope. Manuscripts are selected for publication as much for their content (the experiences they describe) as for their literary merit. Include biographical sketch.

Books, Other Media. Reviews (250-500 words) of new books or other media on emerging disease issues are welcome. Title, author(s), publisher, number of pages, and other pertinent details should be included.

Conference Summaries. Summaries of emerging infectious disease conference activities (500-1,000 words) are published online only. They should be submitted no later than 6 months after the conference and focus on content rather than process. Provide illustrations, references, and links to full reports of conference activities.

Online Reports. Reports on consensus group meetings, workshops, and other activities in which suggestions for diagnostic, treatment, or reporting methods related to infectious disease topics are formulated may be published online only. These should not exceed 3,500 words and should be authored by the group. We do not publish official guidelines or policy recommendations.

Photo Quiz. The photo quiz (1,200 words) highlights a person who made notable contributions to public health and medicine. Provide a photo of the subject, a brief clue to the person's identity, and five possible answers, followed by an essay describing the person's life and his or her significance to public health, science, and infectious disease.

Etymologia. Etymologia (100 words, 5 references). We welcome thoroughly researched derivations of emerging disease terms. Historical and other context could be included.

Announcements. We welcome brief announcements of timely events of interest to our readers. Announcements may be posted online only, depending on the event date. Email to eideditor@cdc.gov. 


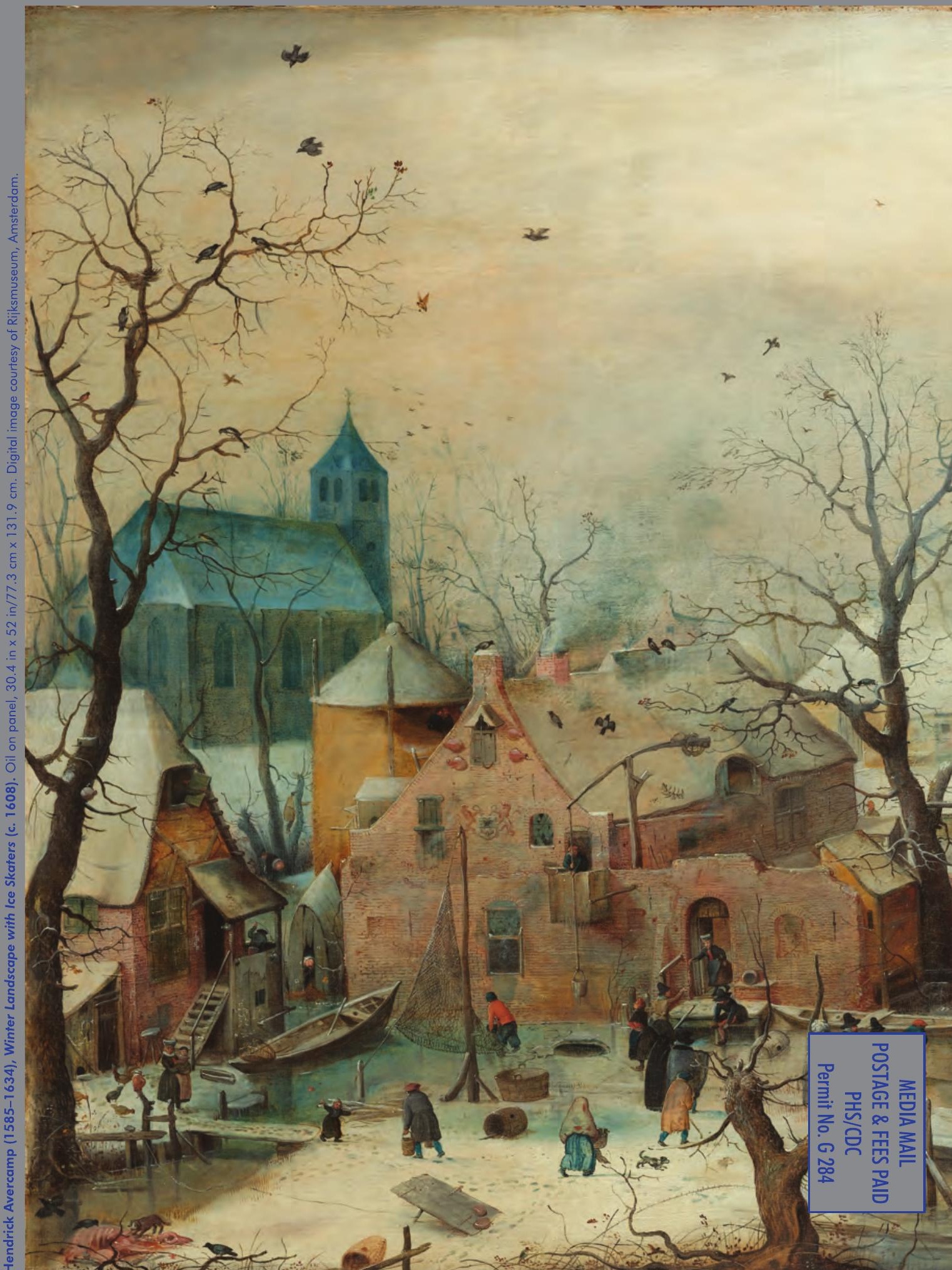

